DOE/OR/01-1273/V2\&D1

Y/ER-182/V2\%DI

Remedial Investigation Report on Bear Creek Valley Operable Unit 2

(Rust Spoil Area, Spoil Area 1, and SY-200 Yard) at the Oak Ridge Y-12 Plant,

Oak Ridge, Tennessee

Volume 2. Appendixes

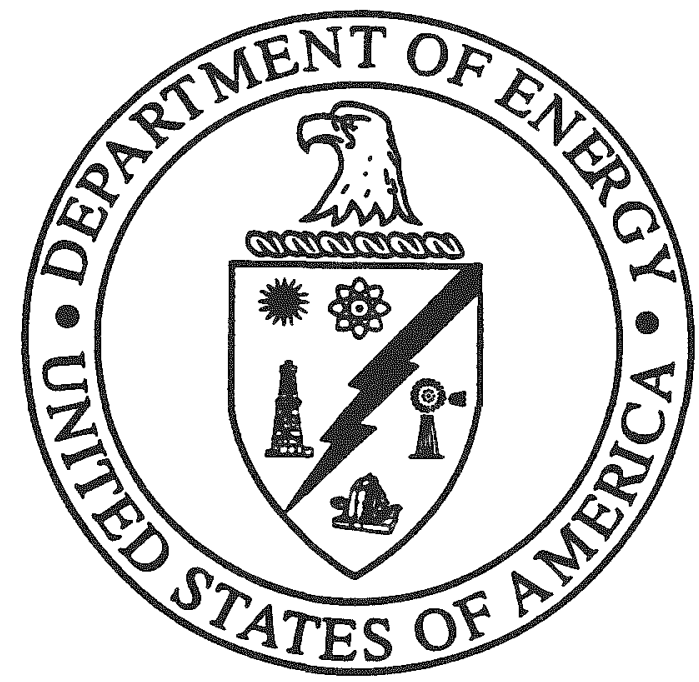




\section{Science Applications International Corporation}

contributed to the preparation of this document and should not be considered an eligible contractor for its review.

This report has been reproduced directly from the best available copy.

Available to DOE and DOE contractors from the Office of Scientific and Technical Information, P.O. Box 62, Oak Ridge. TN 37831; prices available from 615-576-8401, FTS 626-8401.

Available to the public from the National Technical information Service, U.S. Department of Commerce, 5285 Port Royal Rd., Springfield, VA 22161. 
DOE/OR/01-1273/V2\&D1

Y/ER-182/V2\&D1

Energy Systems Environmental Restoration Program

Y-12 Environmental Restoration Program

Remedial Investigation Report

on Bear Creek Valley Operable Unit 2

(Rust Spoil Area, Spoil Area 1, and SY-200 Yard)

at the Oak Ridge Y-12 Plant

Oak Ridge, Tennessee

Volume 2 Appendixes

Date Issued-August 1994

Appendixes A, B, D, and E prepared by

Science Applications International Corporation

Oak Ridge, Tennessee

under subcontract 43B-99069C, Y-05

Prepared for

U.S. Department of Energy

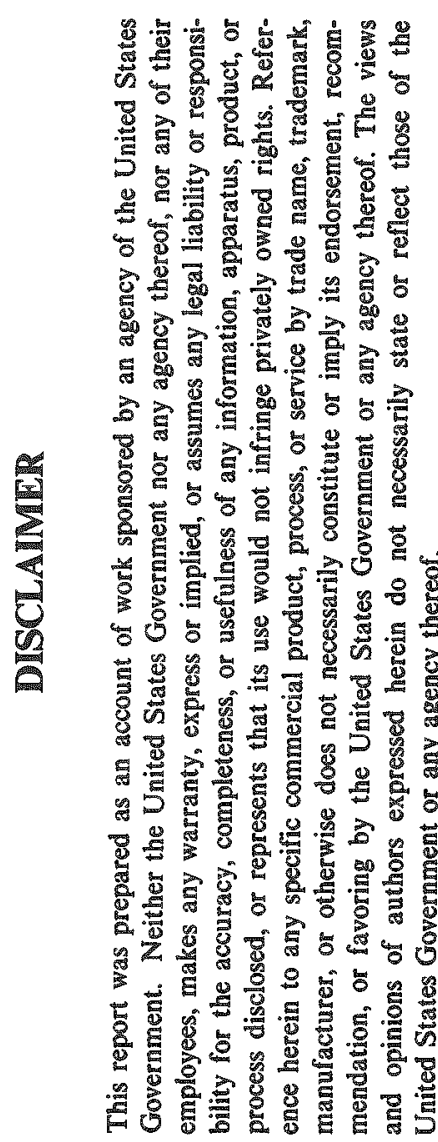

Office of Environmental Restoration and Waste Management under budget and reporting code EW 20

Environmental Restoration and Waste Management Programs

OAK RIDGE Y-12 PLANT

Oak Ridge, Tennessee 37831-8169

managed by

MARTIN MARIETTA ENERGY SYSTEMS, INC.

for the

U.S. DEPARTMENT OF ENERGY

under contract DE-AC05-84OR21400

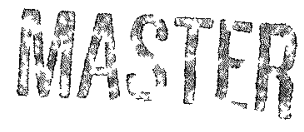





\section{DISCLAIMER}

Portions of this document may be illegible in electronic image products. Images are produced from the best available original document. 


\section{CONTENTS}

APPENDIX A: CURRENT AND HISTORICAL SOIL BORING AND GROUNDWATER MONITORING WELL INFORMATION, WELL CONSTRUCTION LOGS, AND FIELD CHANGE ORDERS ............. A-1

APPENDIX B: ANALYTICAL DATA .................. B-1

APPENDIX C: HUMAN HEALTH RISK ASSESSMENT DATA $\ldots \ldots \ldots$ C-1

APPENDIX D: ECOLOGICAL RISK ASSESSMENT DATA ......... D-1

APPENDIX E: DATA QUALITY ...................... E-1 



\section{APPENDIX A}

Current and Historical Soil Boring and Groundwater Monitoring Well Information, Well Construction Logs, and Field Change Orders 

Current Soil Boring Information 

BCV OU2 RI Soil Boring Log Key Sheet

\section{SYMBOLS}

\begin{tabular}{|c|c|}
\hline $\begin{array}{l}\mathbb{a} \\
\mathbb{a} \\
\mathbb{a} \\
\stackrel{b}{b}\end{array}$ & DESCRIPTION \\
\hline $\begin{aligned} E= & = \\
= & = \\
= & =\end{aligned}$ & CLAY \\
\hline $\begin{array}{l}= \\
=0 \\
0=0 \\
0=0\end{array}$ & CLAY WITH ROCK FRAGMENTS \\
\hline $\begin{aligned}= & =9 \\
E= & =3 \\
E= & =1\end{aligned}$ & CLAY WITH PLANT DETRITUS \\
\hline $\begin{aligned}= & = \\
= & =3 \\
= & =3\end{aligned}$ & SANDY CLAY \\
\hline $\begin{aligned} E= & =3 \\
E= & =3 \\
= & =3\end{aligned}$ & SILTY CLAY \\
\hline 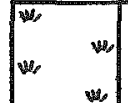 & SAND WITH PLANT DETRITUS \\
\hline $\begin{array}{ccc} & 0 & 9 \\
0 & 0 \\
0 & 0 \\
\end{array}$ & GRAVEL \\
\hline$=0$ & CLAYEY GRAVEL \\
\hline TI & LIMESTONE \\
\hline & SHALE \\
\hline n & CONSTRUCTION DEBRIS \\
\hline 11 & NO RECOVERY \\
\hline
\end{tabular}

\section{ABBREVIATIONS}

\begin{tabular}{|l|}
\hline LITHOLOGY DESCRIPTION \\
\hline \hline LMST - LIMESTONE \\
FRAGISI- FRAMENTISI \\
RAD - RADIOLOGICAL LEVEL \\
\hline SAMPLE TYPE \\
\hline $\begin{array}{c}\text { SPLT SPN - SPLIT SPOON } \\
\text { SHELBY - SHELBY TUBE } \\
\text { AUGER - HAND AUGER } \\
\text { CUTTINGS - HOLLOW STEM } \\
\text { AUGER CUTTINGS }\end{array}$ \\
\hline
\end{tabular}

Soil color based on Munsel Soil Color Chart 


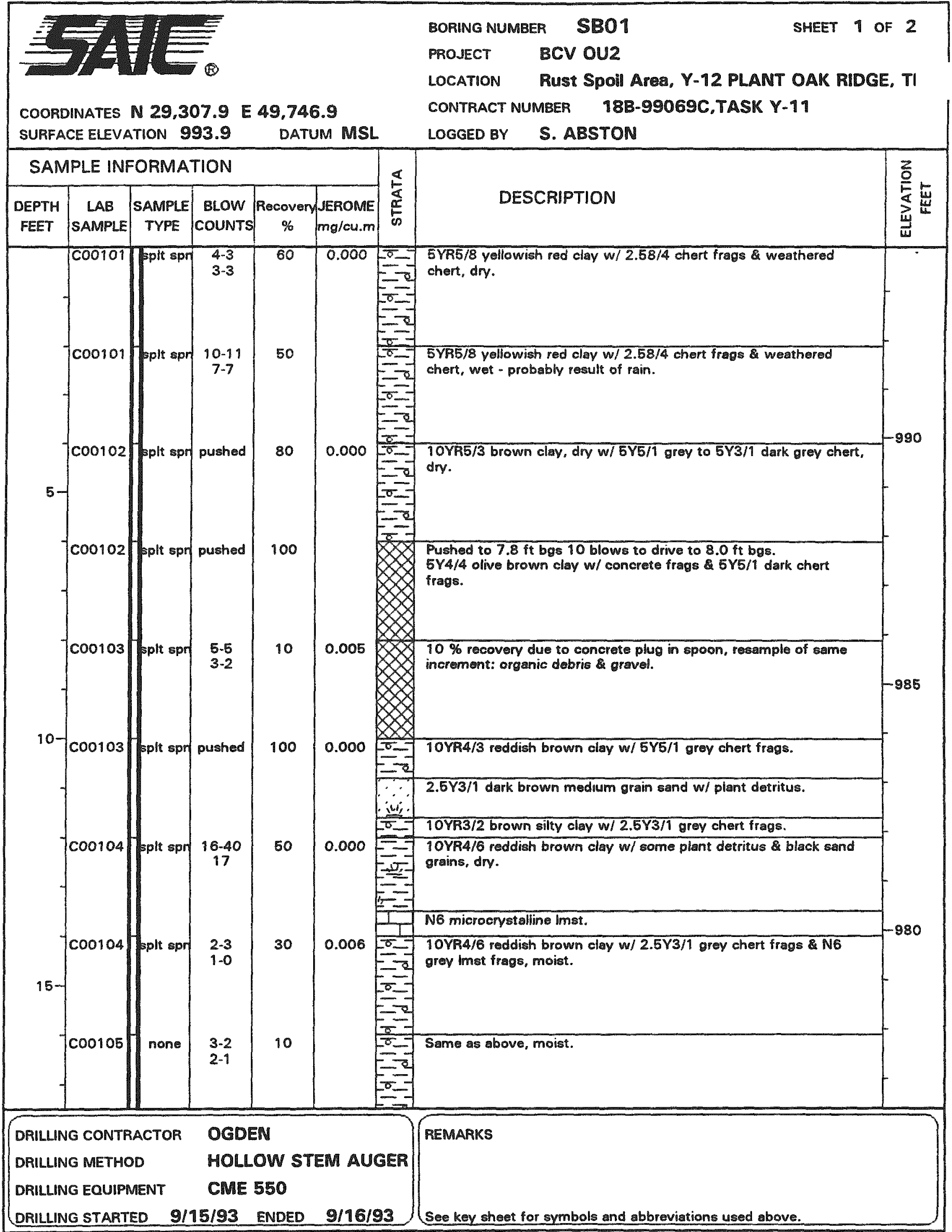


A-7

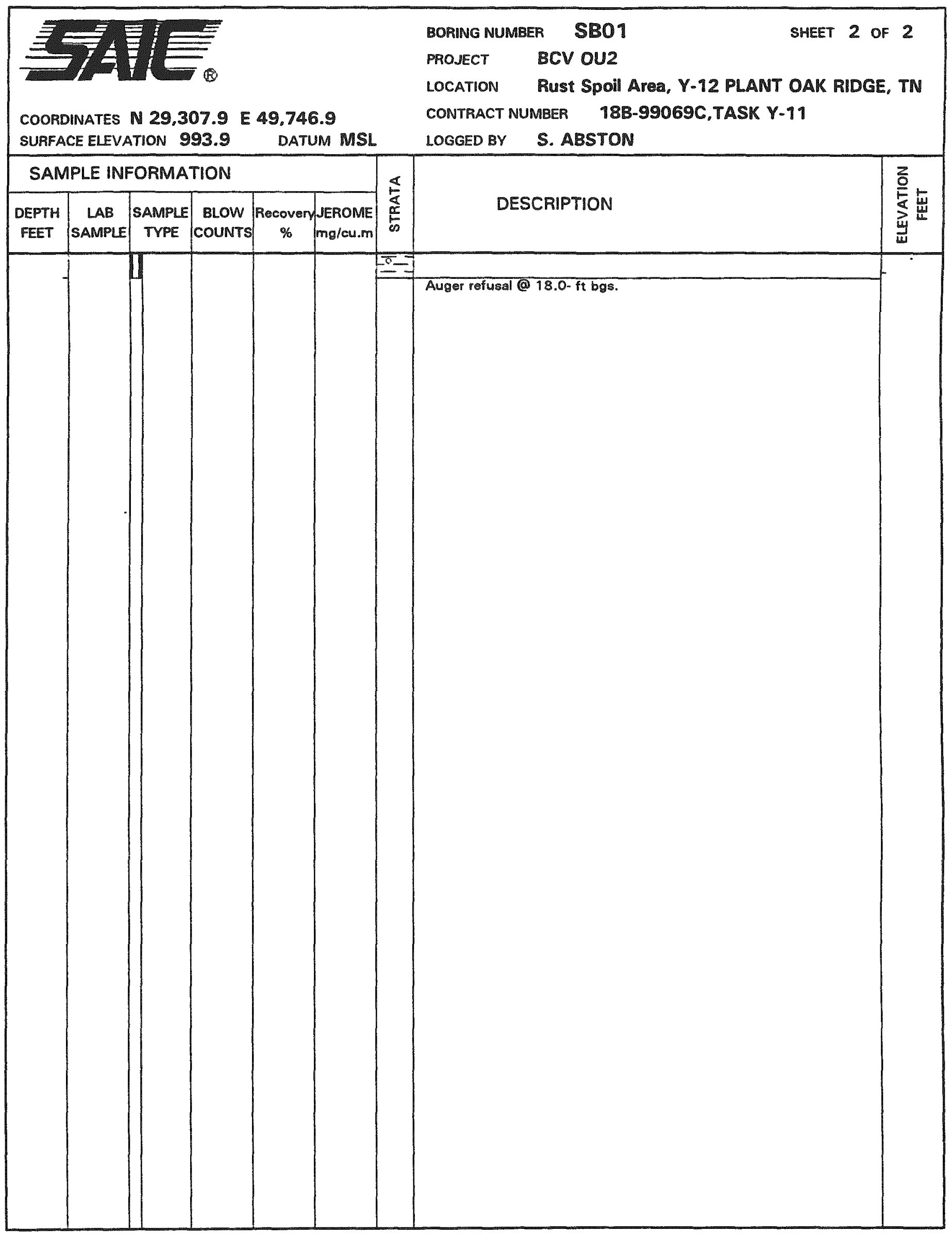




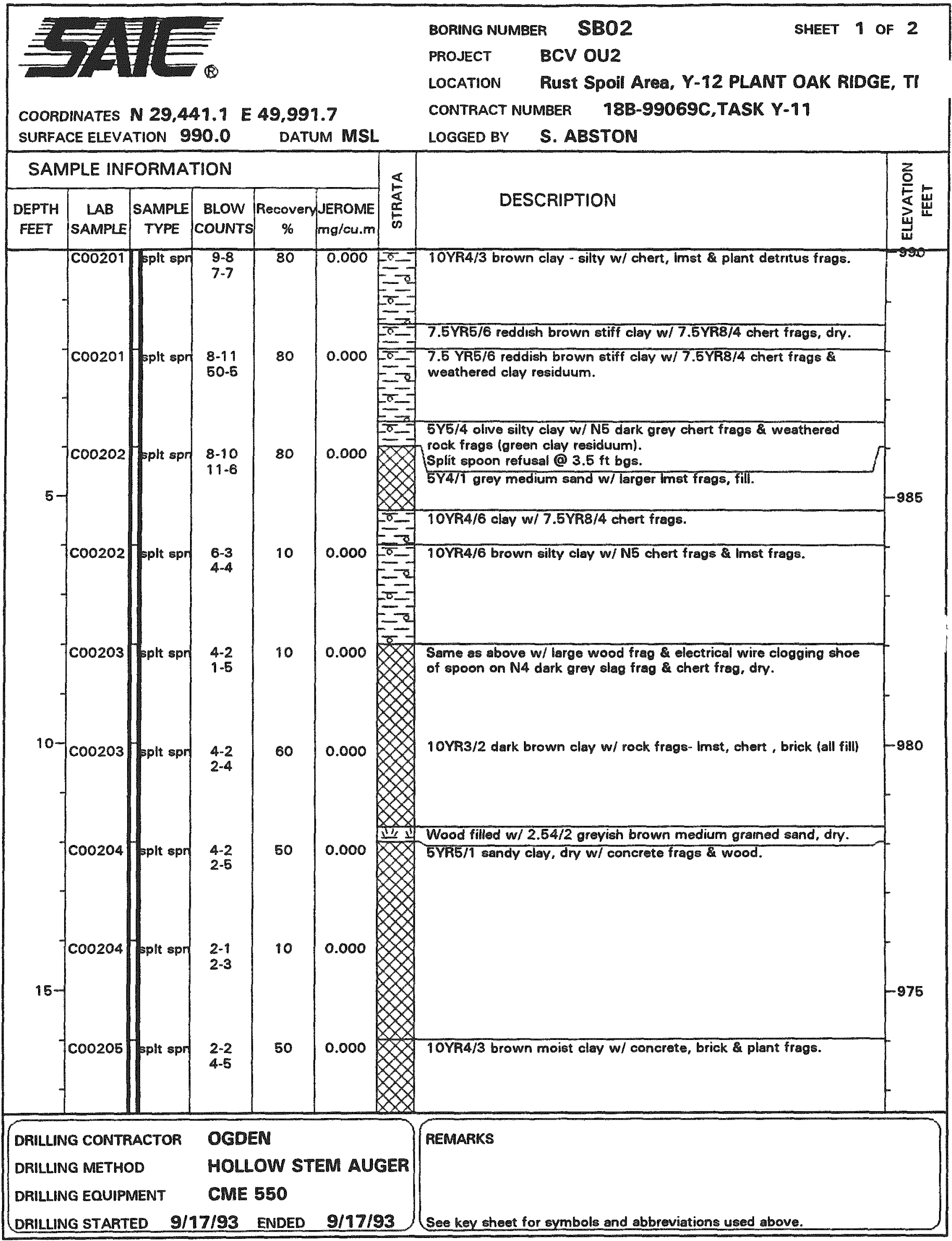


A-9

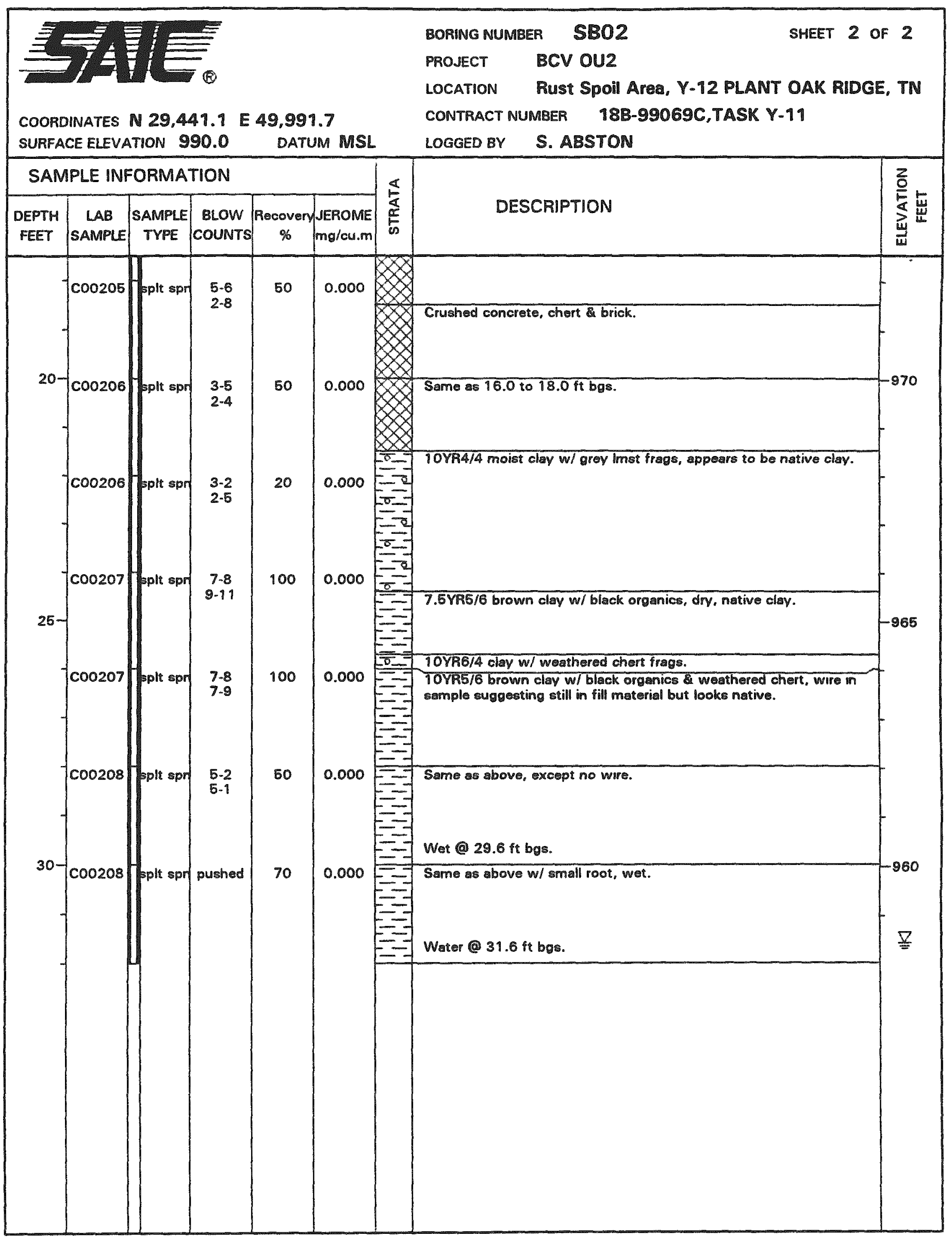




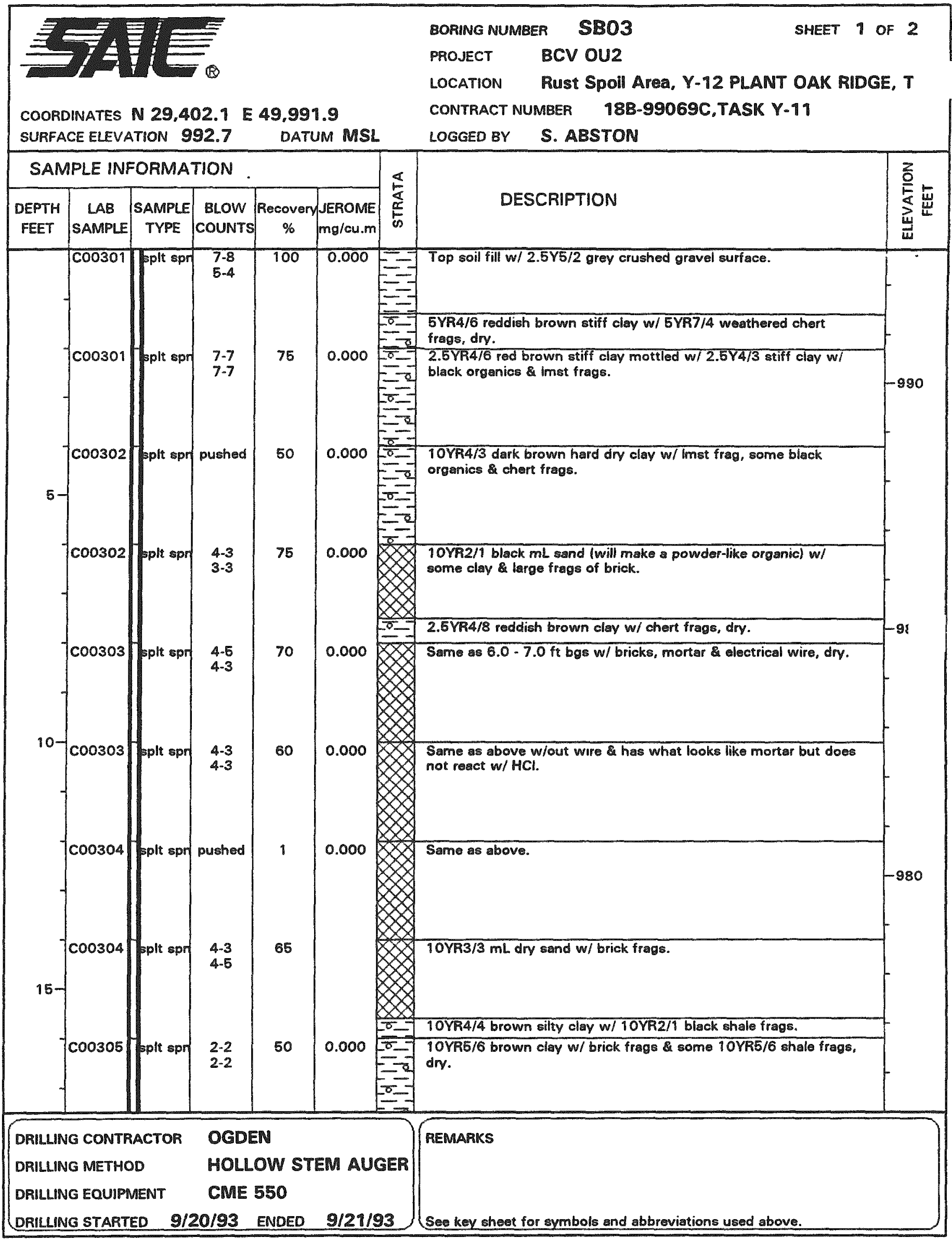


A-11

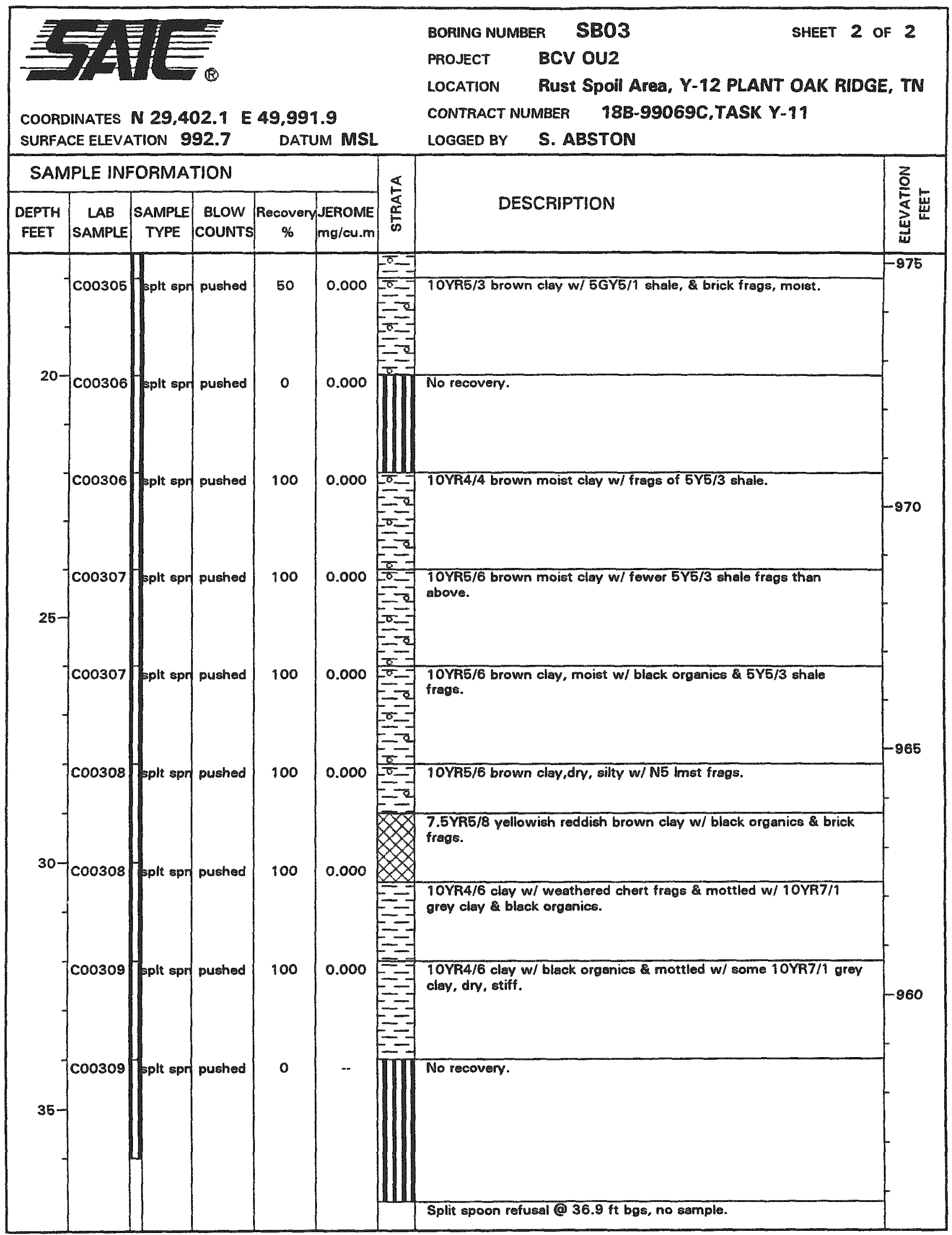




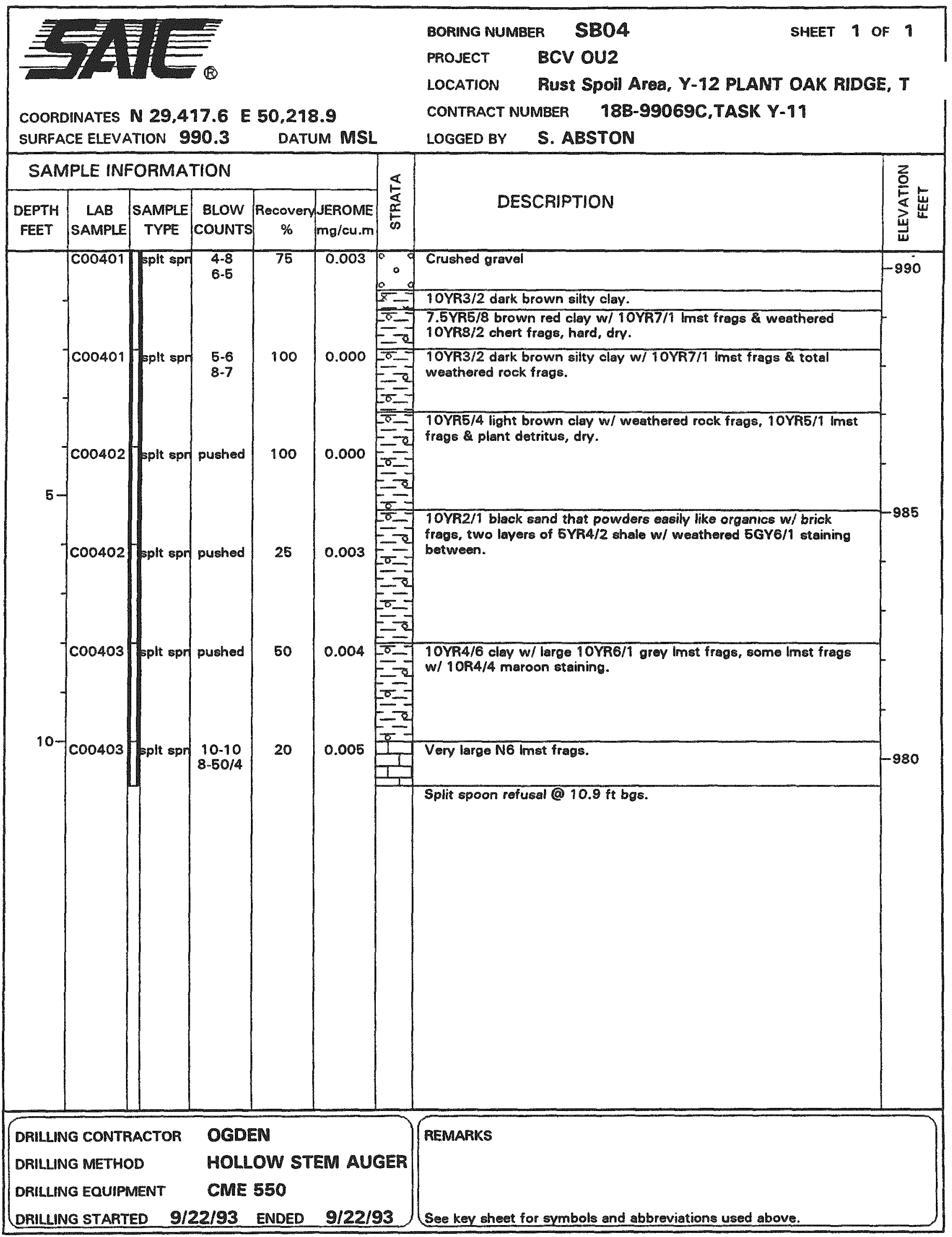


A-13

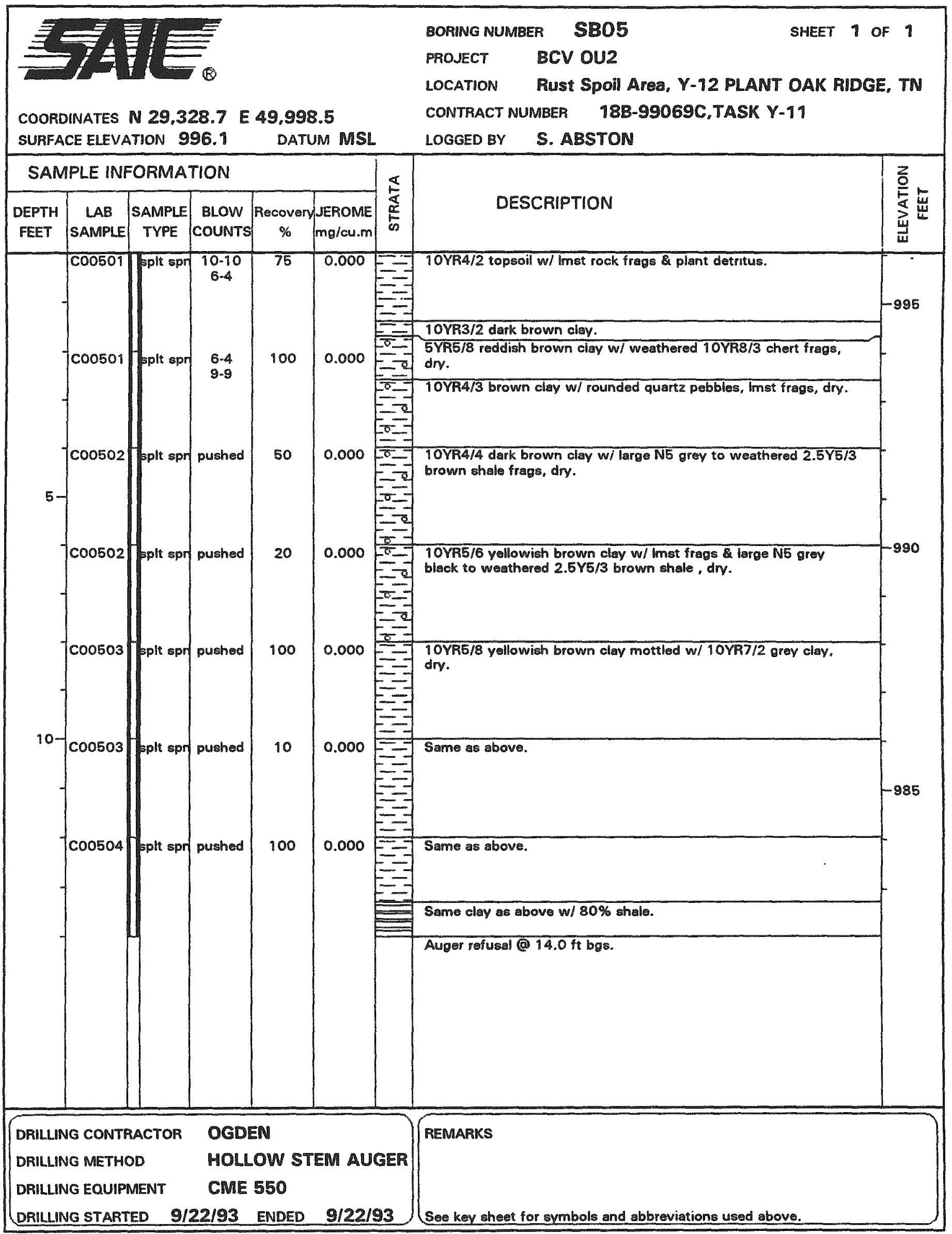


A-14

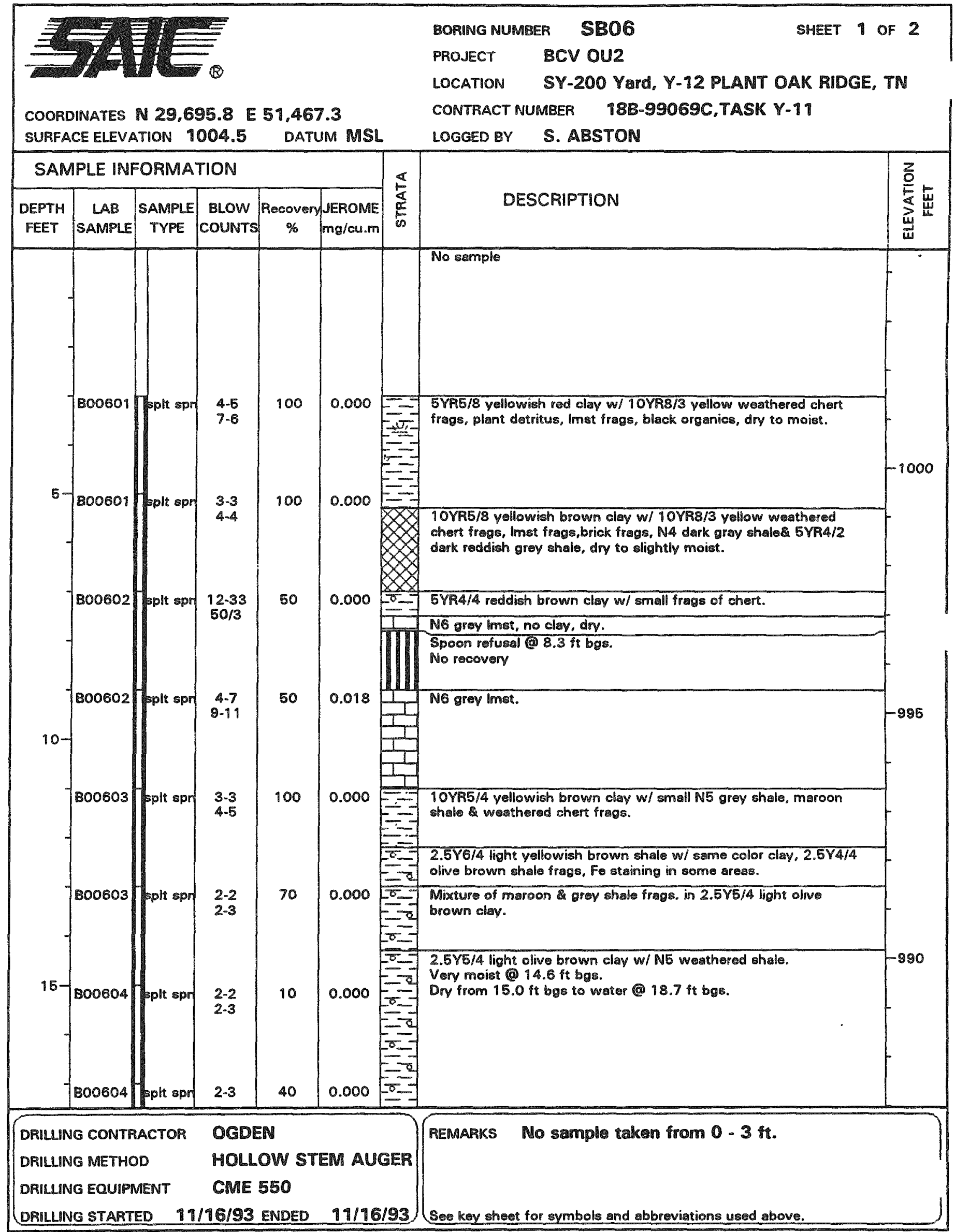


A-15

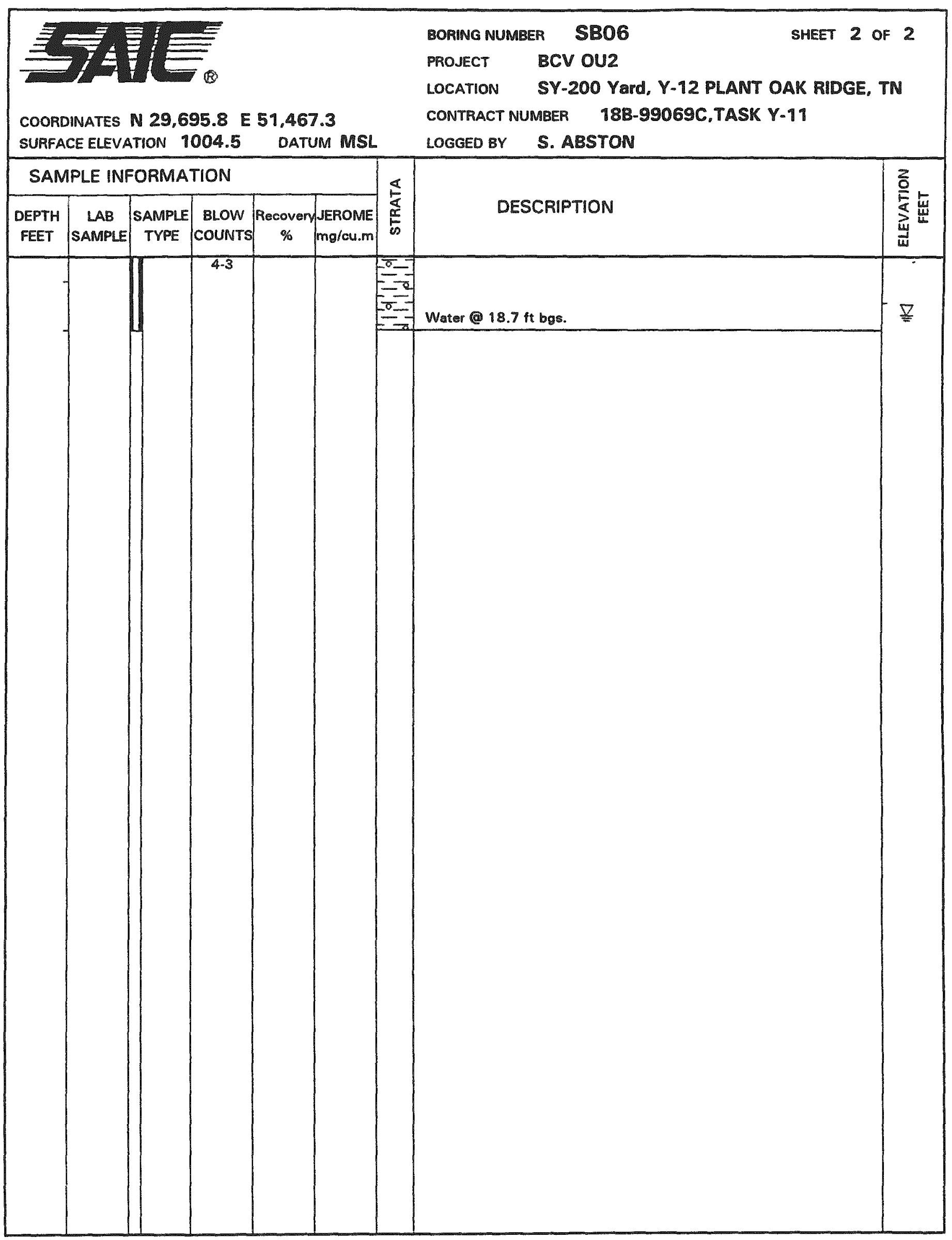




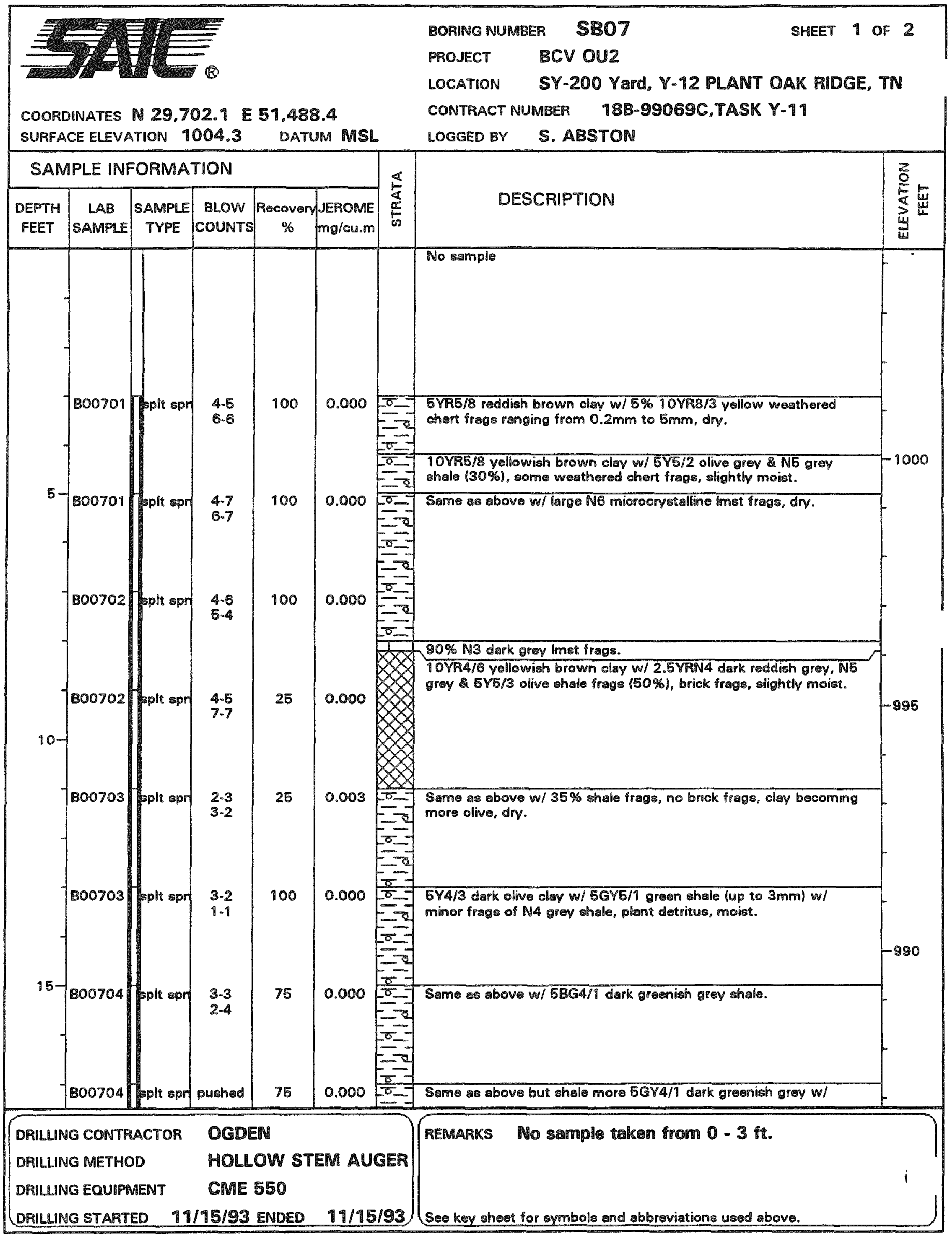


A-17

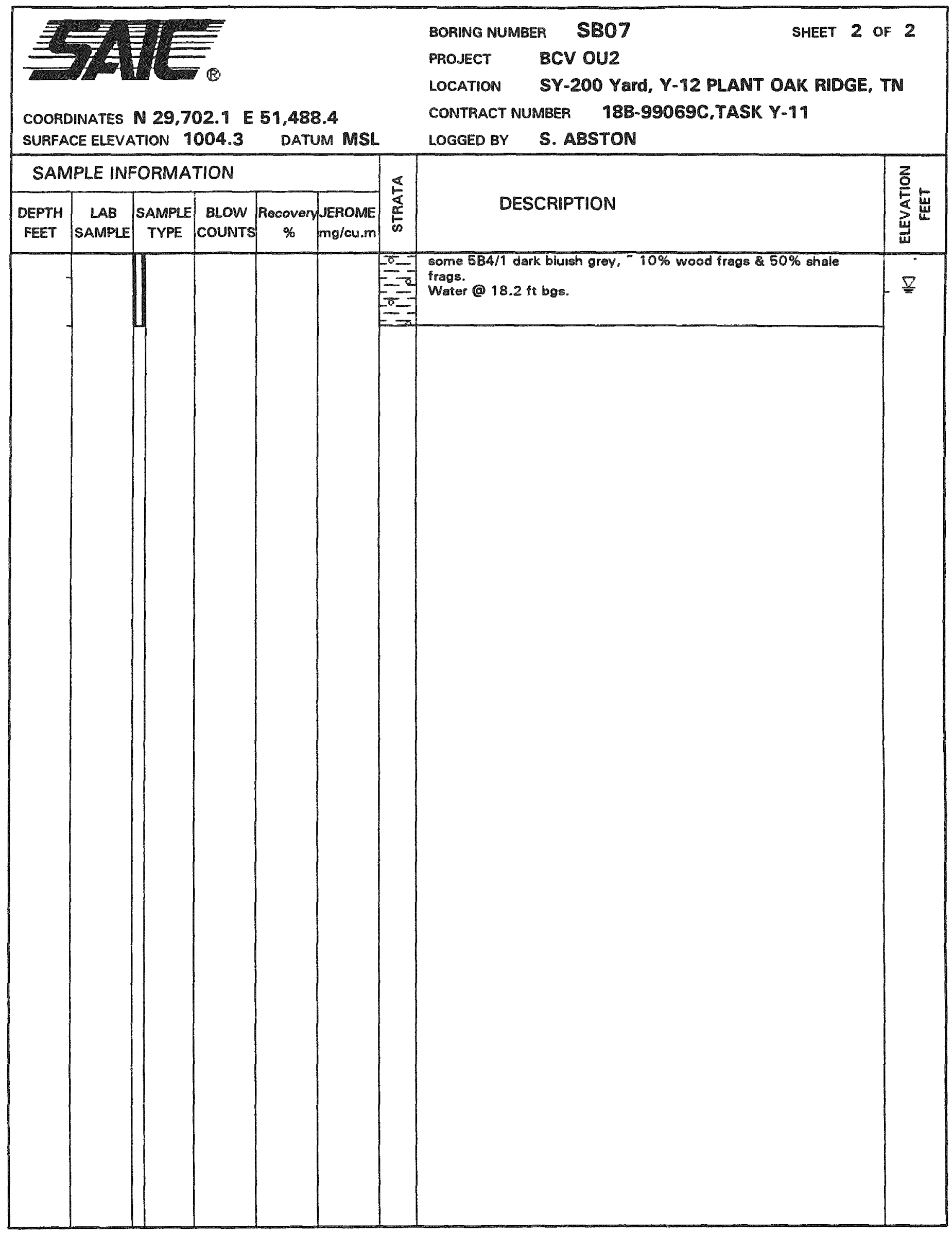


A-18

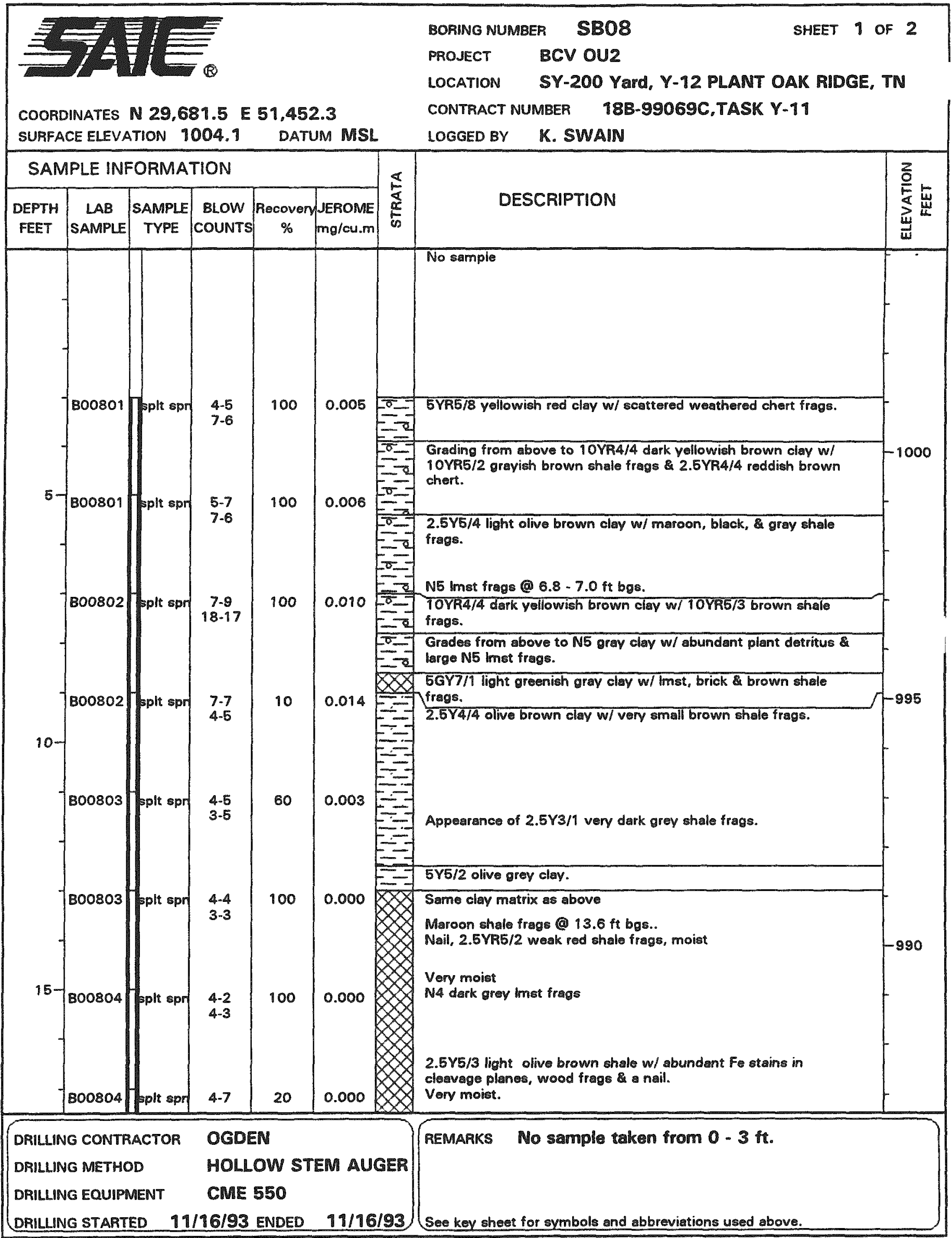


A-19

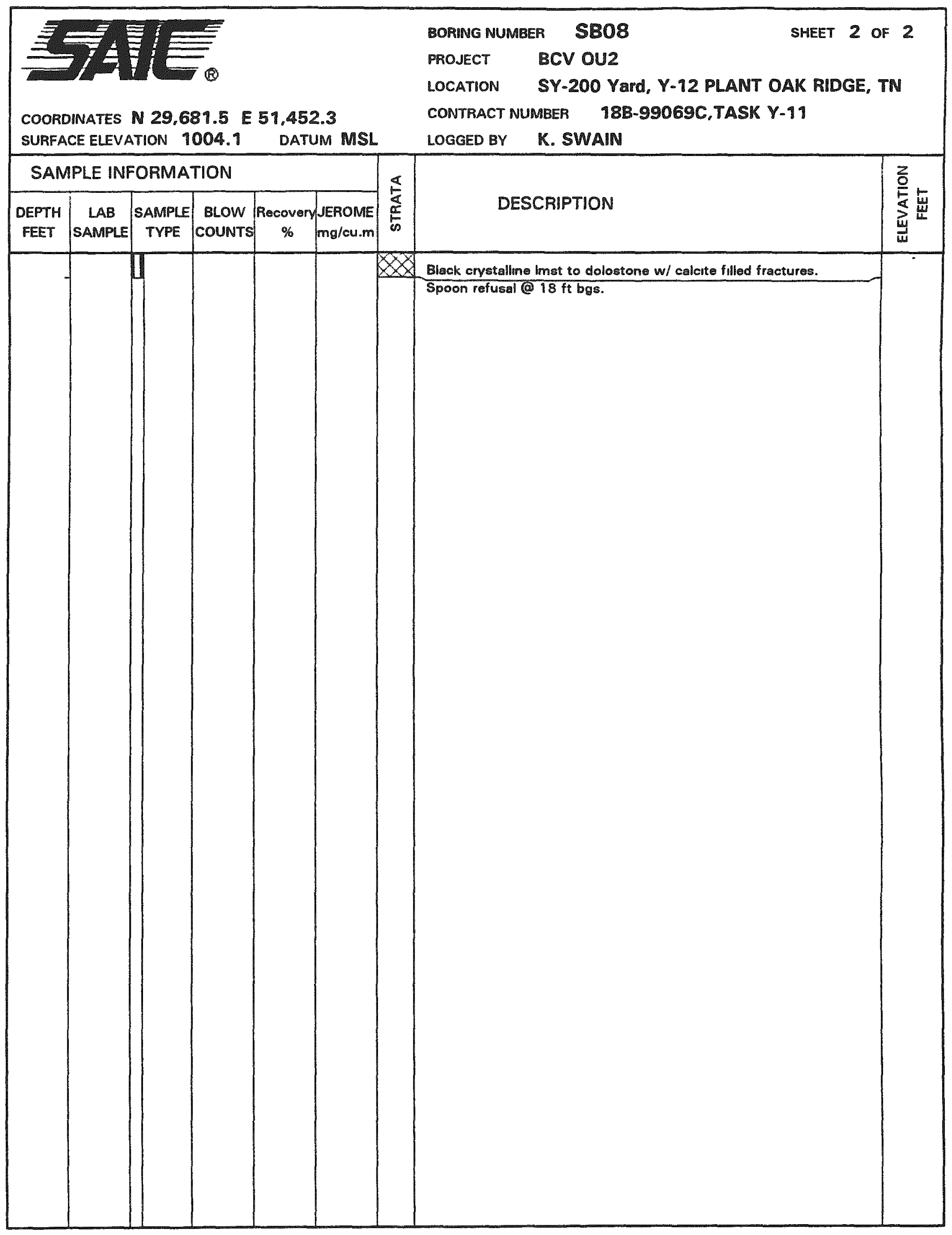




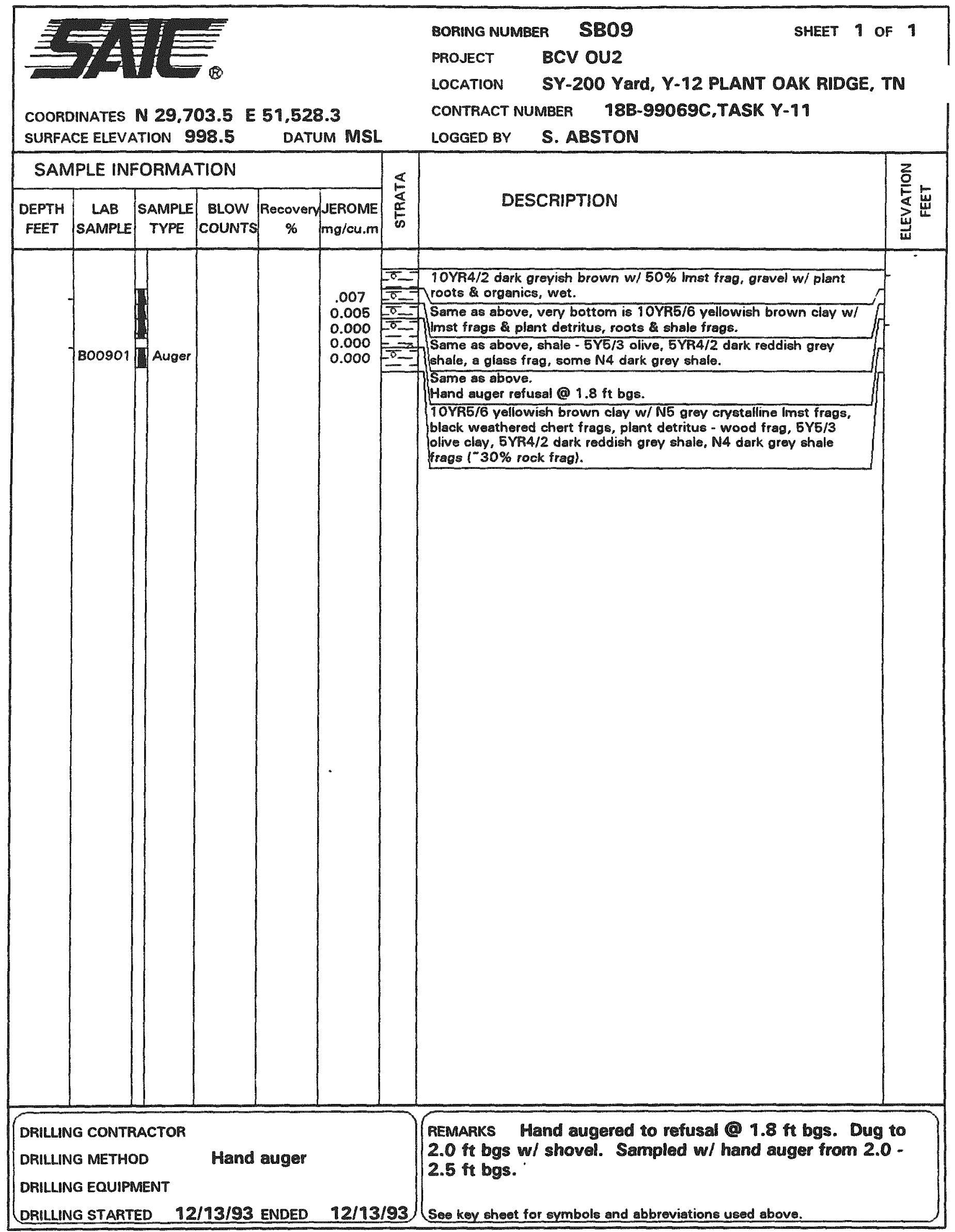




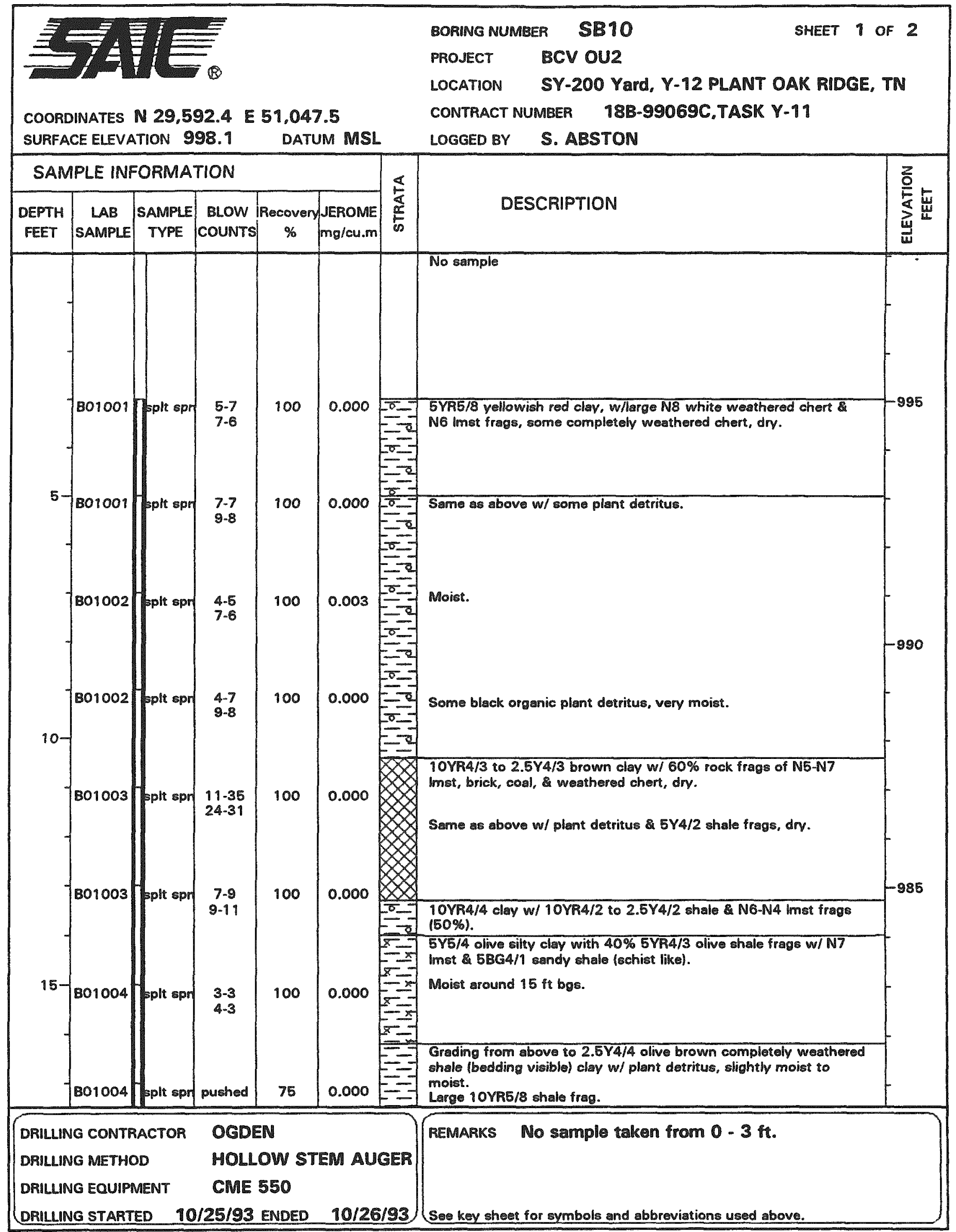


A-22

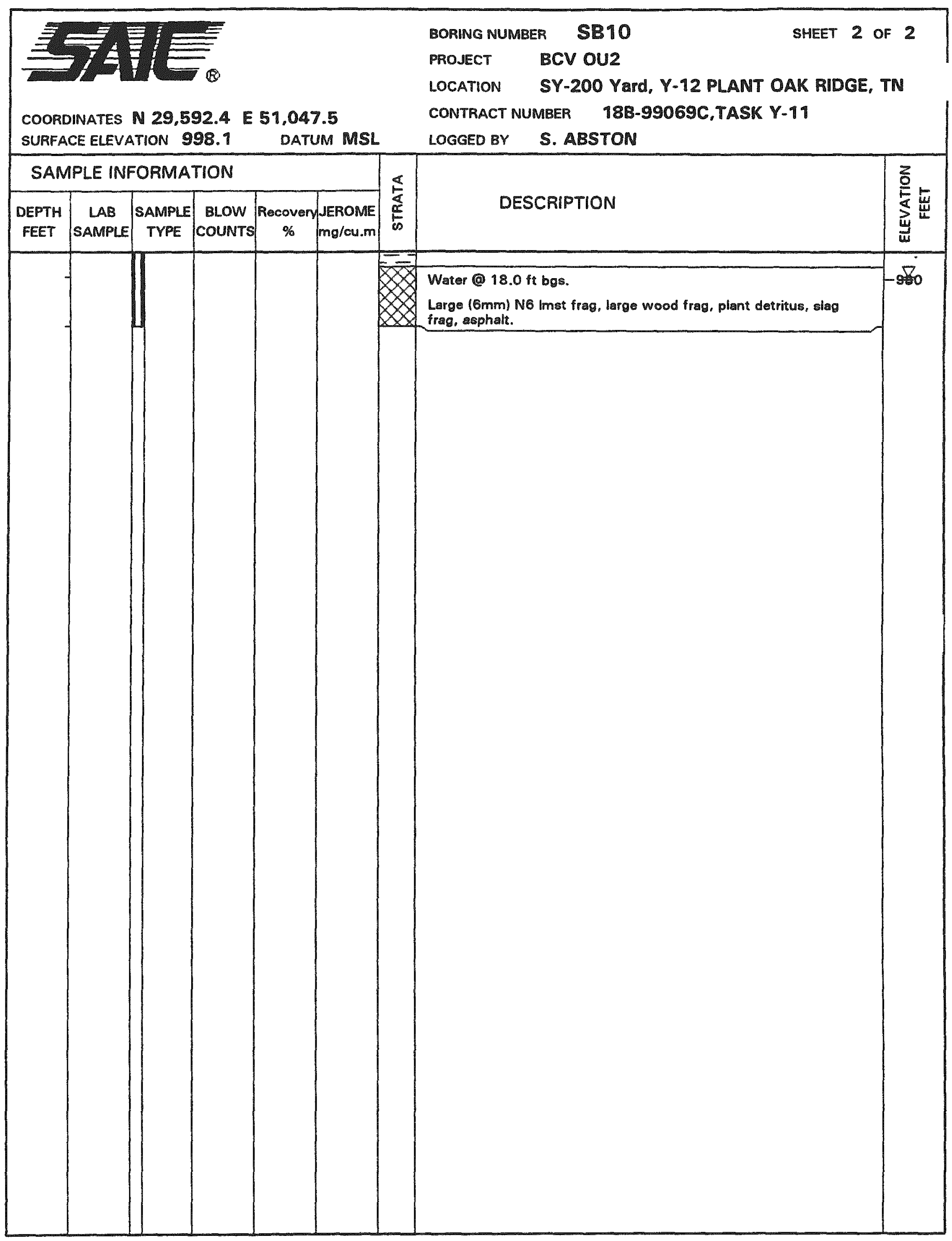




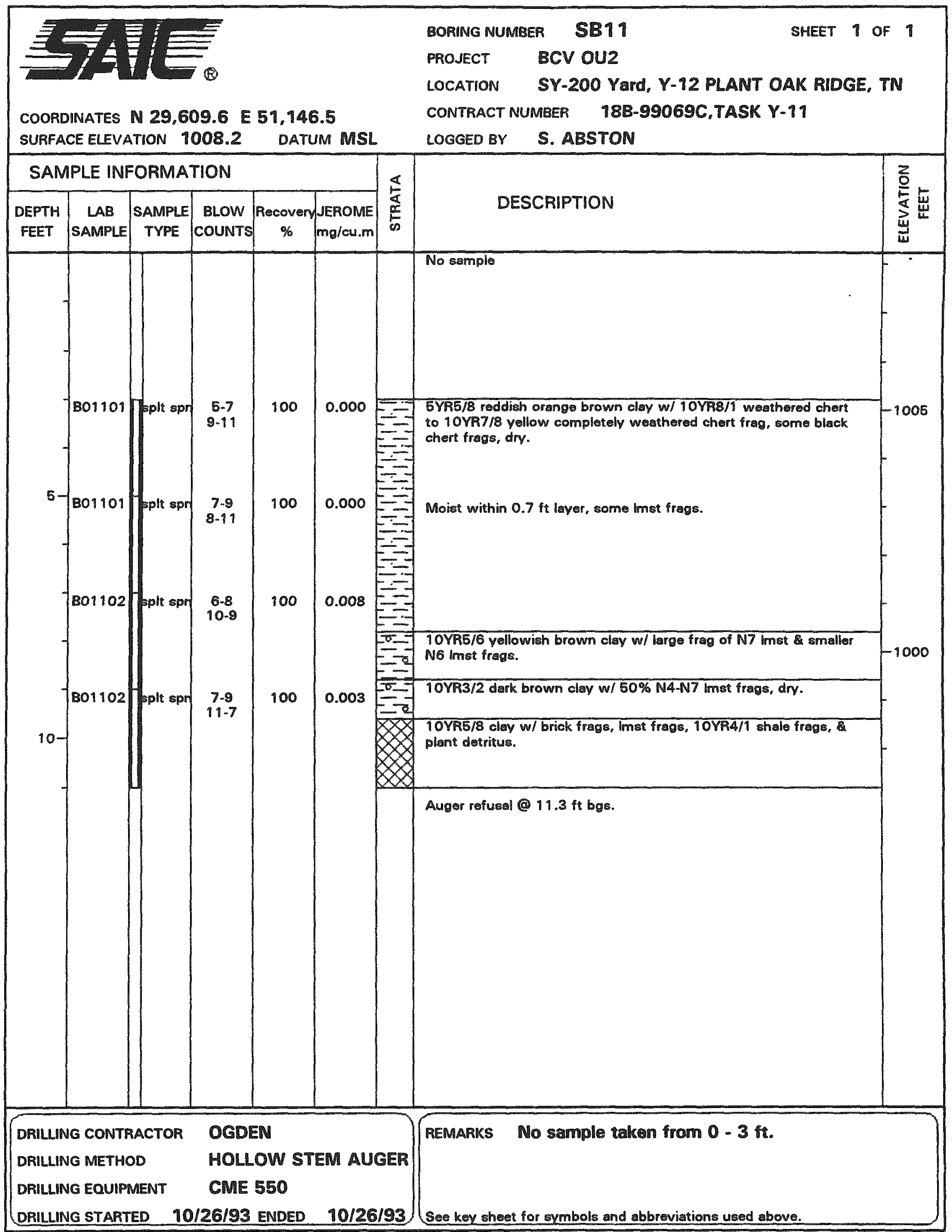




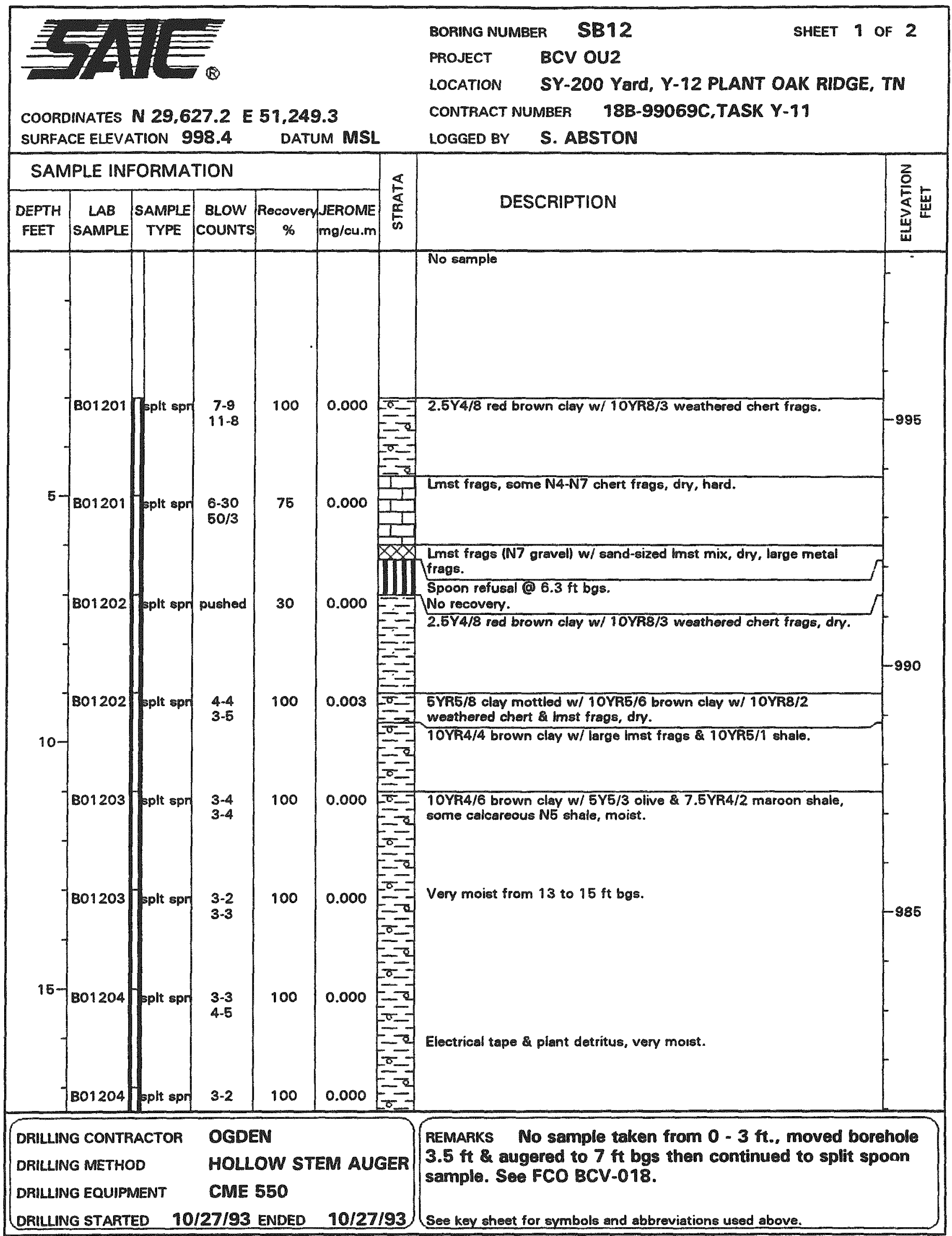


A-25

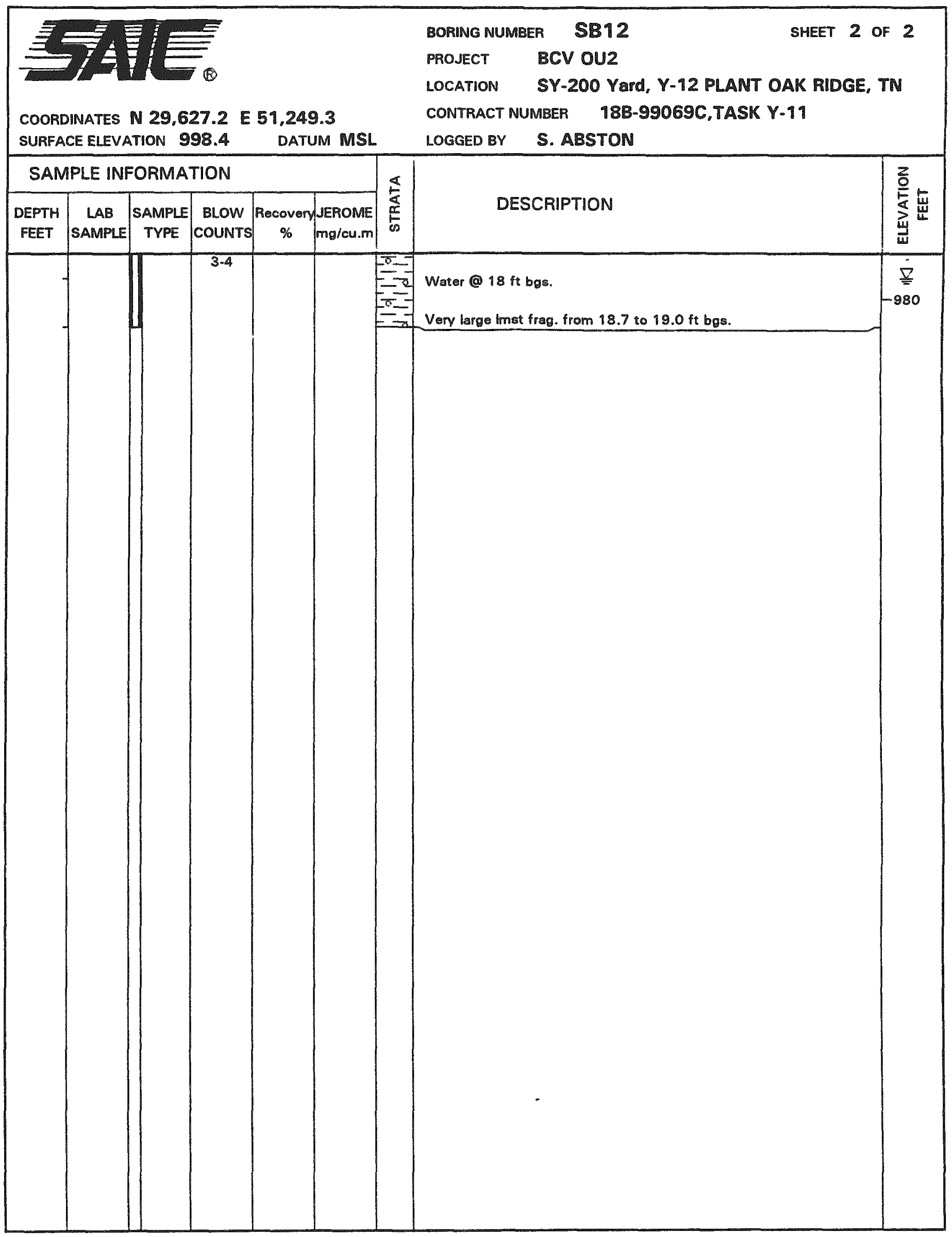




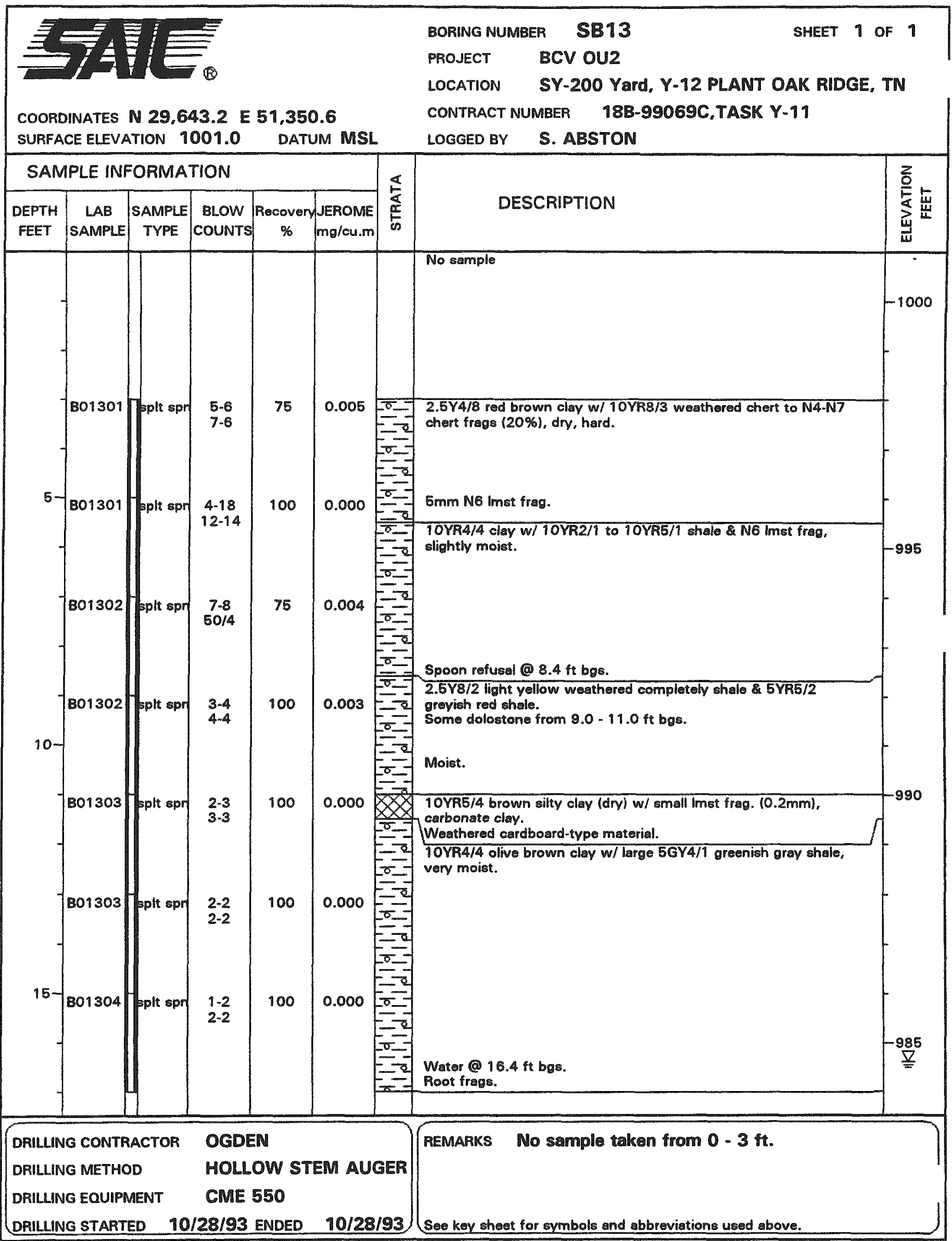




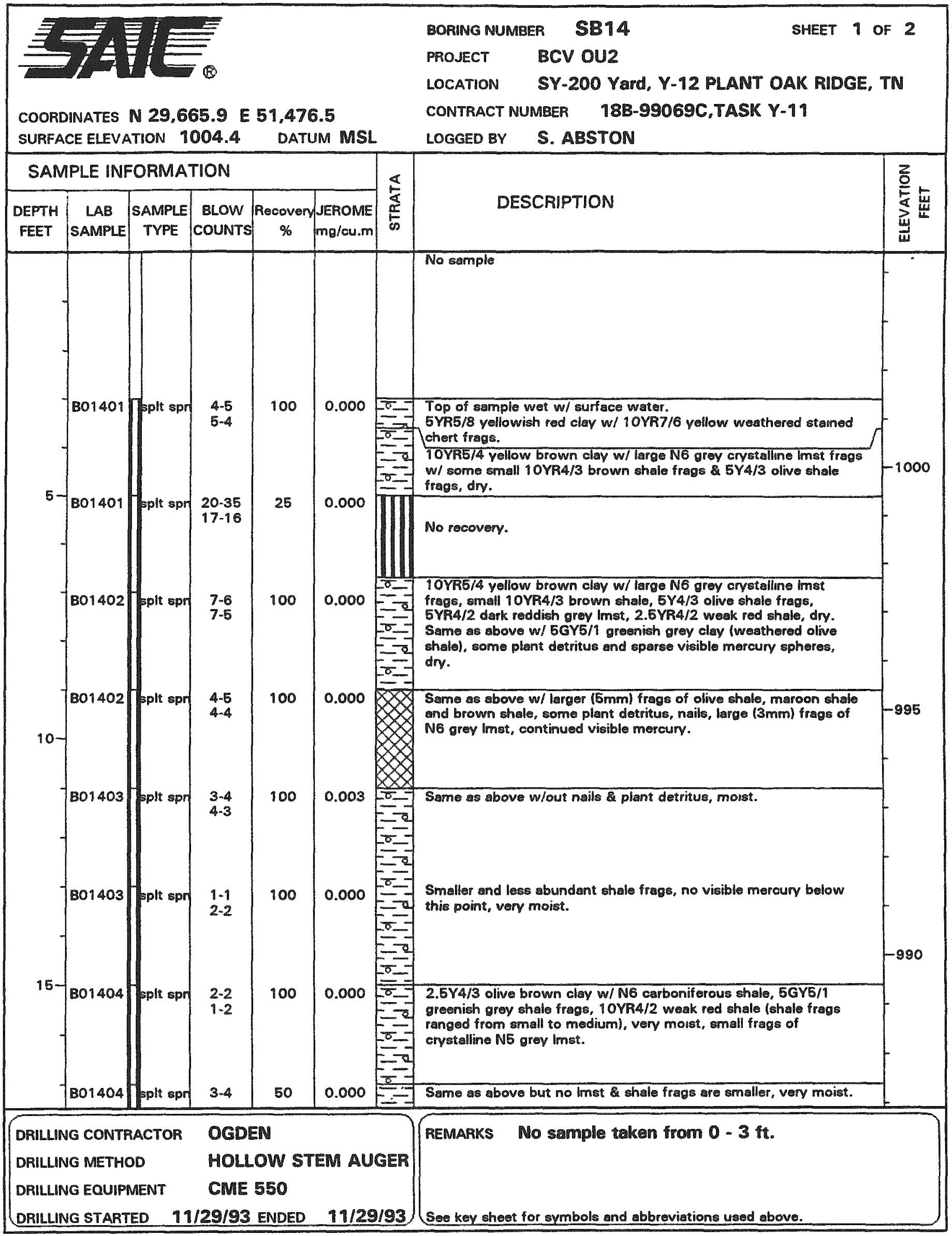


A-28

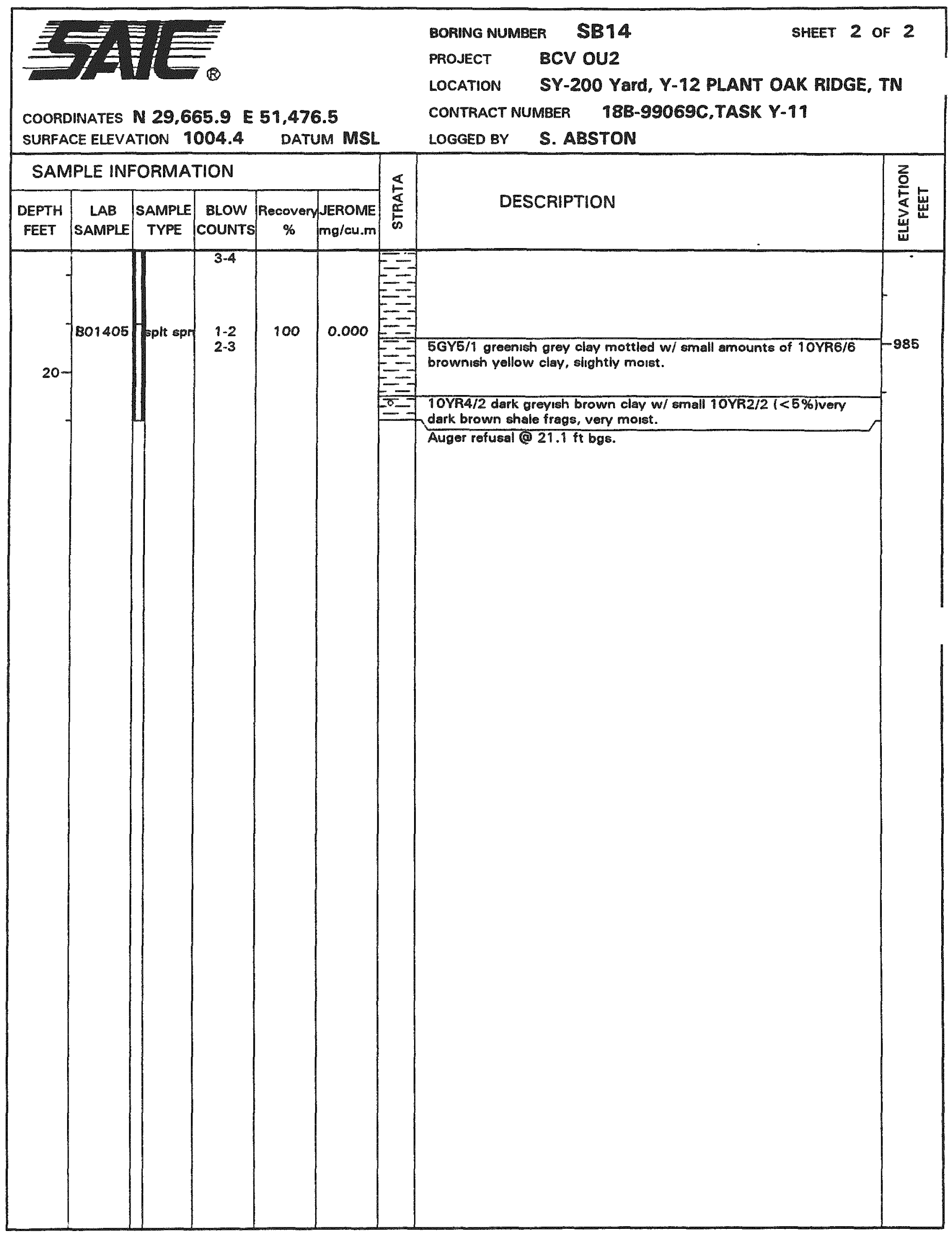




\begin{tabular}{|c|c|c|c|c|c|c|c|c|}
\hline \multicolumn{7}{|c|}{ 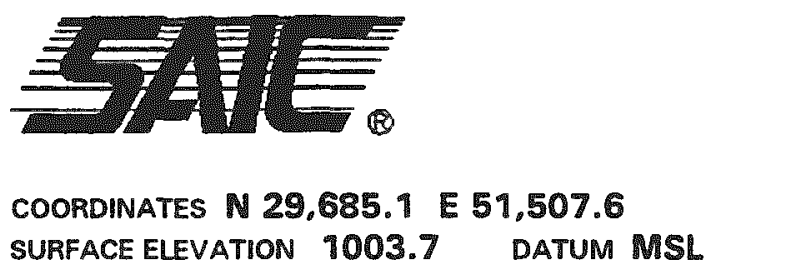 } & $\begin{array}{l}\text { BORING NUMBER SB15 } \\
\text { PROJECT } \quad \text { BCV OU2 } \\
\text { LOCATION } \quad \text { SY-200 YaId, Y-12 PLANT OAK RIDGE, } \\
\text { CONTRACT NUMBER 18B-99069C.TASK Y-11 } \\
\text { LOGGED BY } \quad \text { S.ABSTON }\end{array}$ & OF 1 \\
\hline $\begin{array}{l}\text { SAN } \\
\text { DEPTH } \\
\text { FEET }\end{array}$ & $\begin{array}{c}\text { LAB } \\
\text { SAMPLE }\end{array}$ & $\begin{array}{l}\text { ORMA } \\
\text { SAMPLE } \\
\text { TYPE }\end{array}$ & TION & $\begin{array}{c}\text { Recovery } \\
\%\end{array}$ & JEROME & $\mid \begin{array}{l}\frac{a}{6} \\
\frac{E}{\alpha} \\
\frac{a}{6}\end{array}$ & DESCRIPTION & $\sum_{\frac{\mathbb{w}}{4}}^{\frac{Z}{w}}$ \\
\hline $10-$ & Bo1502 & spit spr & $\begin{array}{l}5-7 \\
6-7\end{array}$ & 100 & 0.000 & 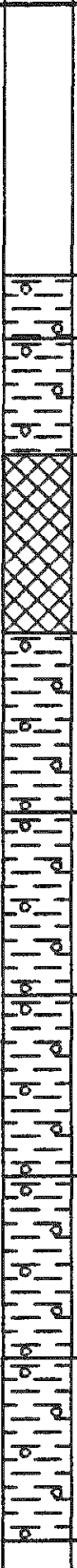 & 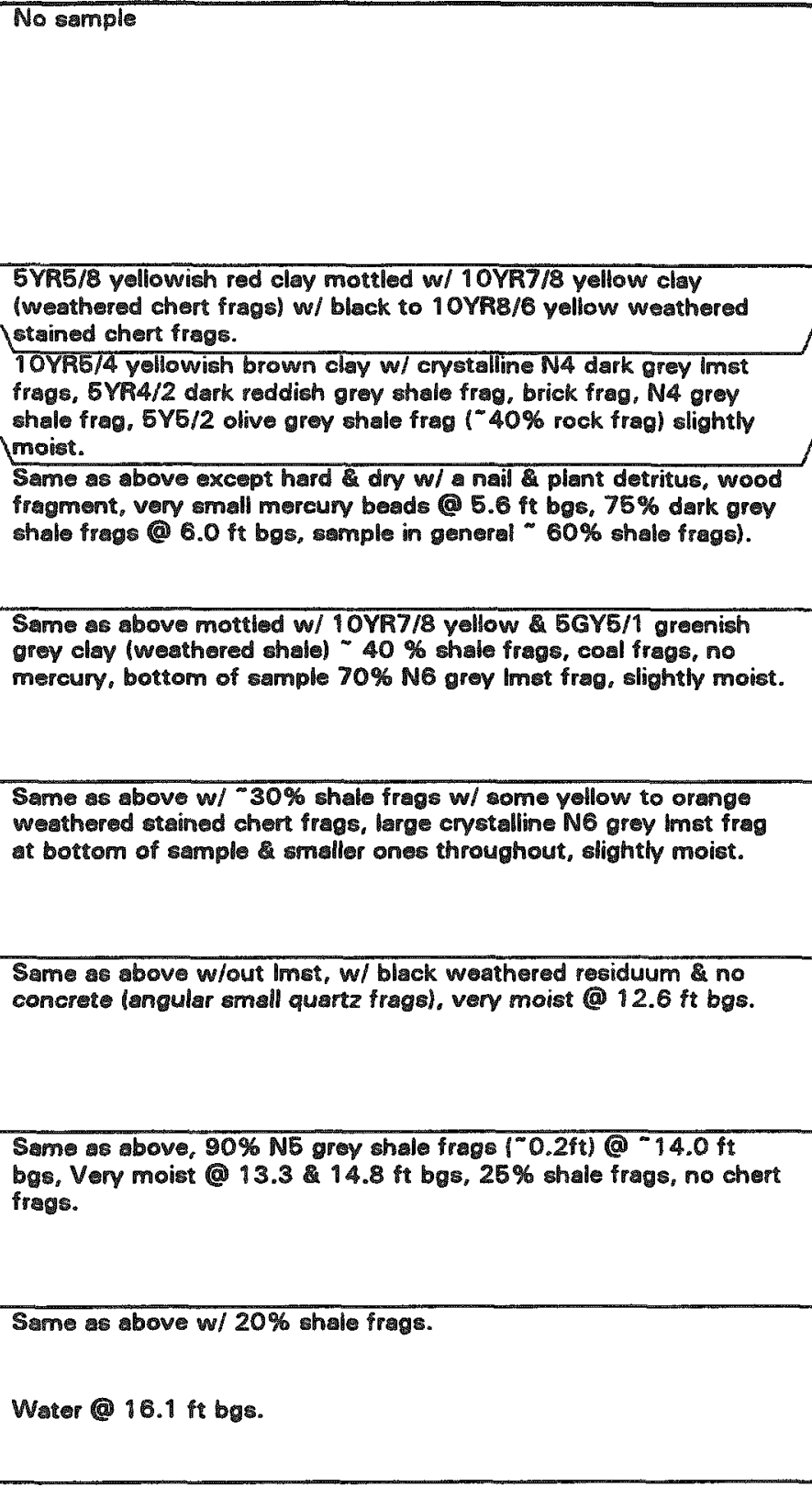 & \multirow[t]{2}{*}{-1000} \\
\hline \multicolumn{7}{|c|}{$\begin{array}{lll}\text { ORILLNG CONTRACTOR } & \text { OGDEN } \\
\text { DRILLING METHOD } & \text { HOLLOW STEM AUGER } \\
\text { DRILLING EQUIPMENT } & \text { CME } 550 \\
\text { DRILLING STARTED } & 12 / 17 / 93 \text { ENDED } & 12 / 17 / 93\end{array}$} & $\begin{array}{l}\text { REMARKS Hand augered to refrusal @ } 2.1 \mathrm{ft} \text { bgs. Moved } \\
\text { borthole to location accessible by drill rig. }\end{array}$ & \\
\hline
\end{tabular}


A-30

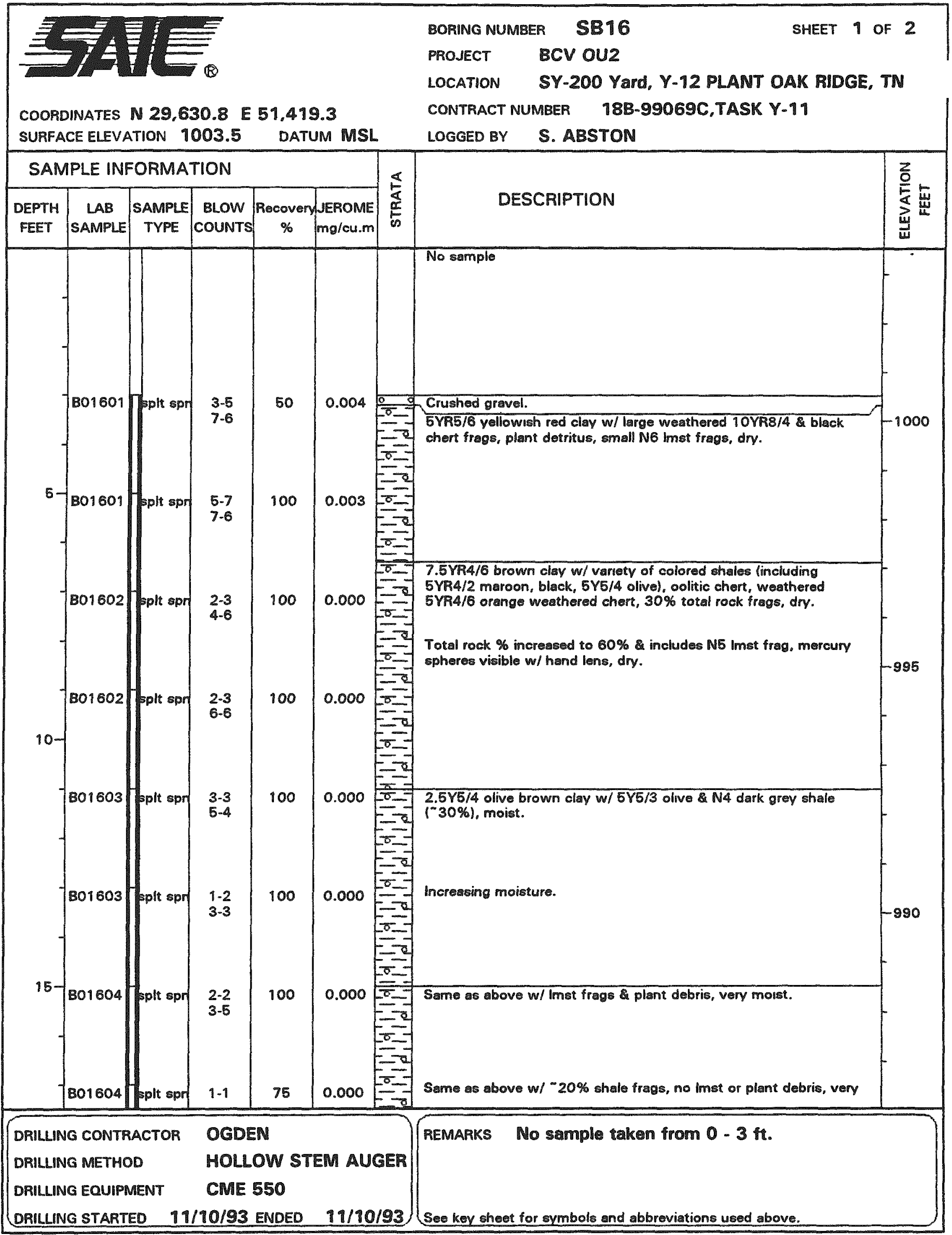


A-31

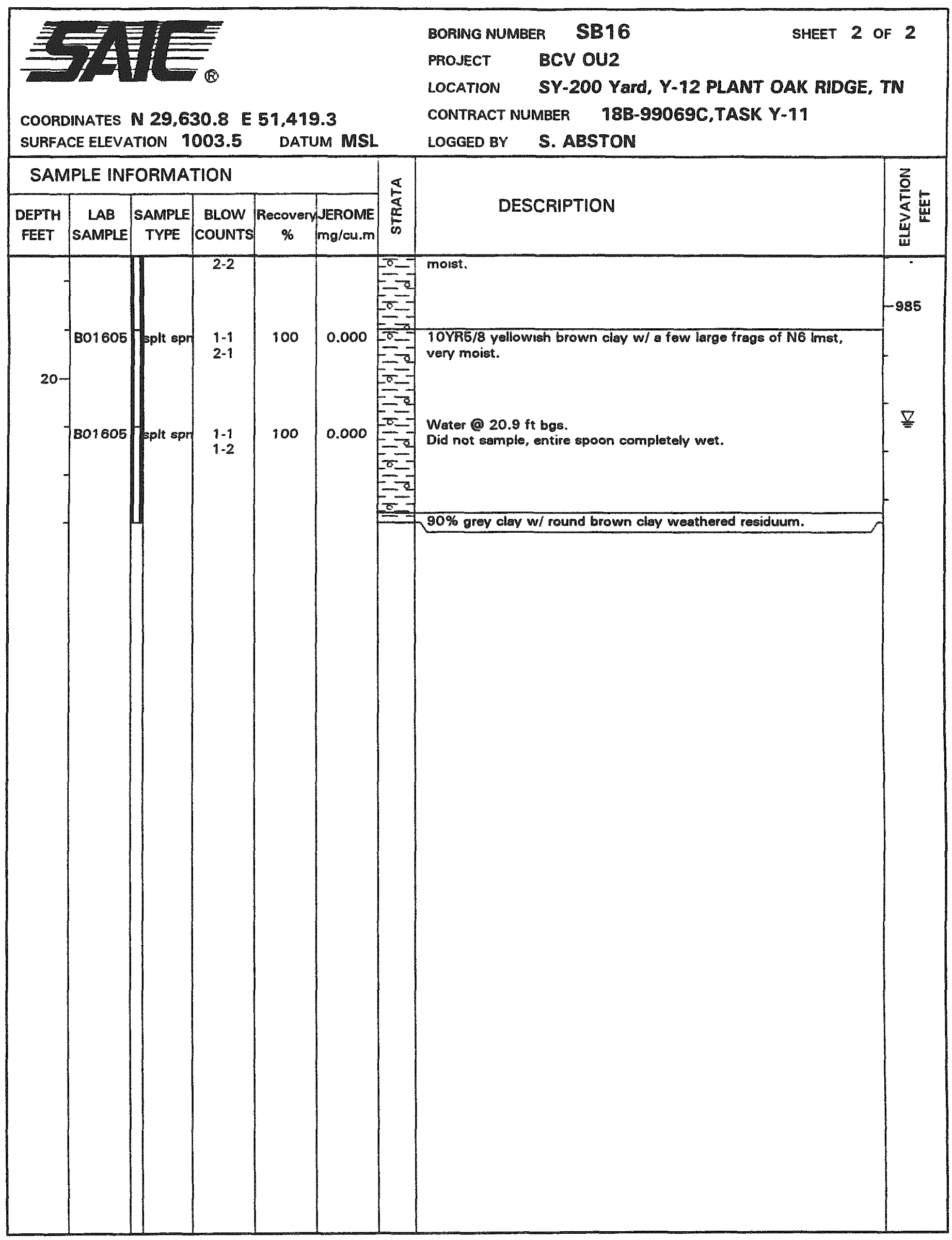




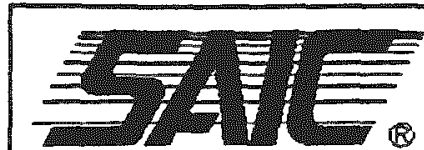

COORDINATES N 29,636.7 E $51,442.6$ SURFACE ELEVATION 1003.6
DATUM MSL

\section{SAMPLE INFORMATION}

\begin{tabular}{|c|c|c|c|c|c|}
\hline $\begin{array}{c}\text { DEPTH } \\
\text { FEET }\end{array}$ & $\begin{array}{c}\text { LAB } \\
\text { SAMPLE }\end{array}$ & $\begin{array}{c}\text { SAMPLE } \\
\text { TYPE }\end{array}$ & $\begin{array}{c}\text { BLOW } \\
\text { COUNTS }\end{array}$ & $\begin{array}{c}\text { Recoven } \\
\%\end{array}$ & $\begin{array}{l}\text { JEROME } \\
\text { mg/cu.m. }\end{array}$ \\
\hline
\end{tabular}

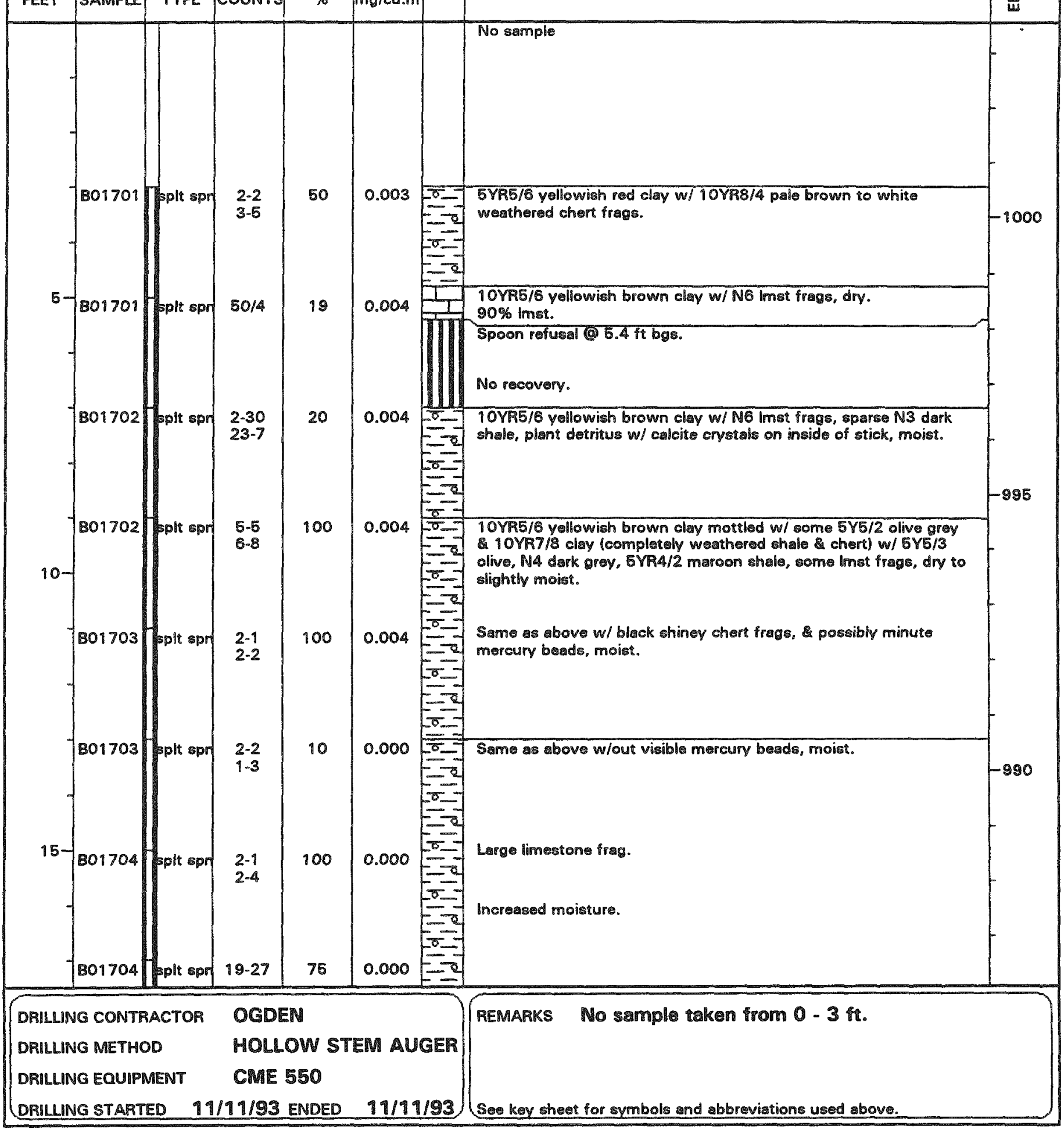

\section{$\stackrel{8}{8}$ DESCRIPTION}

SHEET 1 OF 2

PROJECT BCV OU2

LOCATION SY-200 Yard, Y-12 PLANT OAK RIDGE, TN CONTRACT NUMBER 18B-99069C.TASK Y-11 


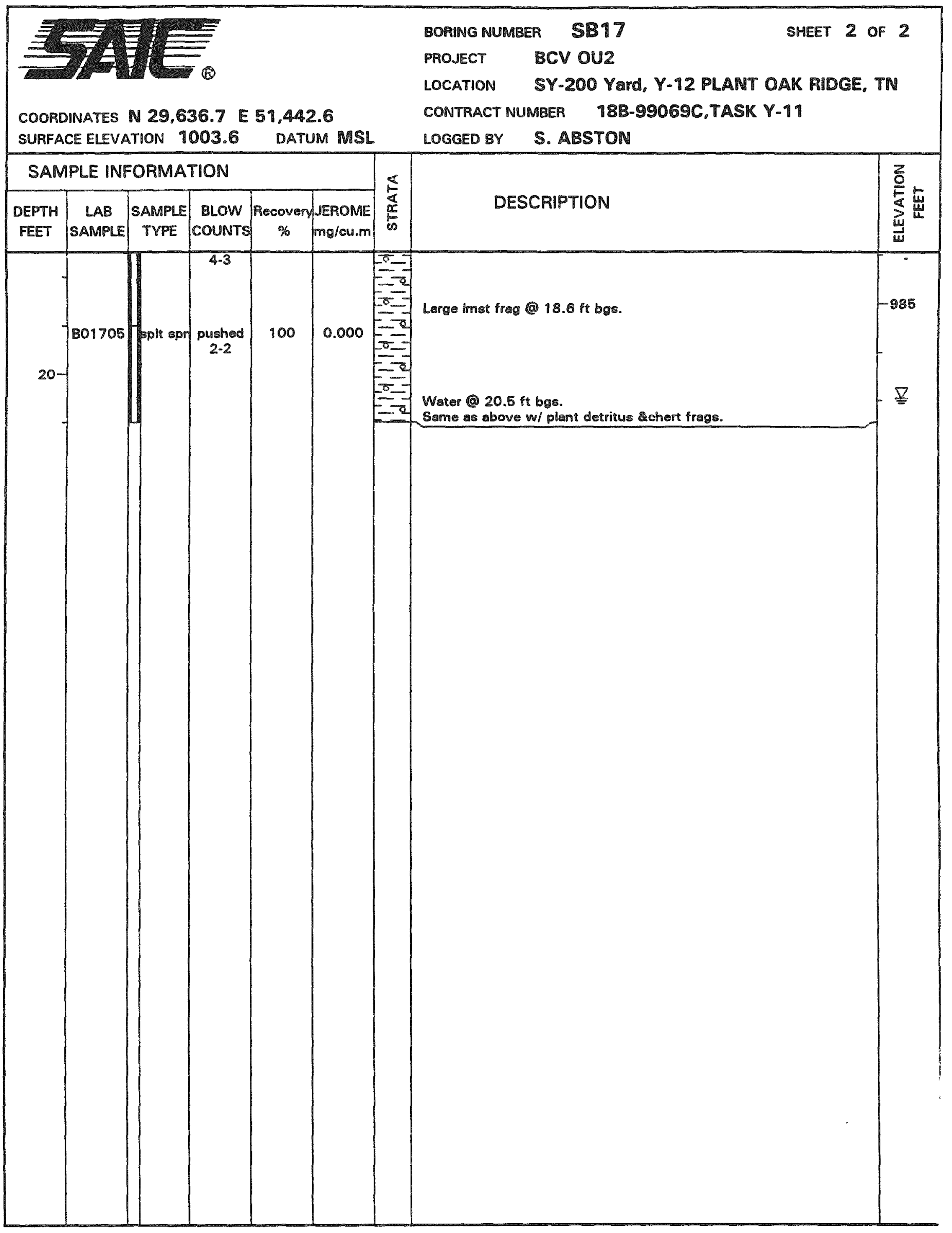




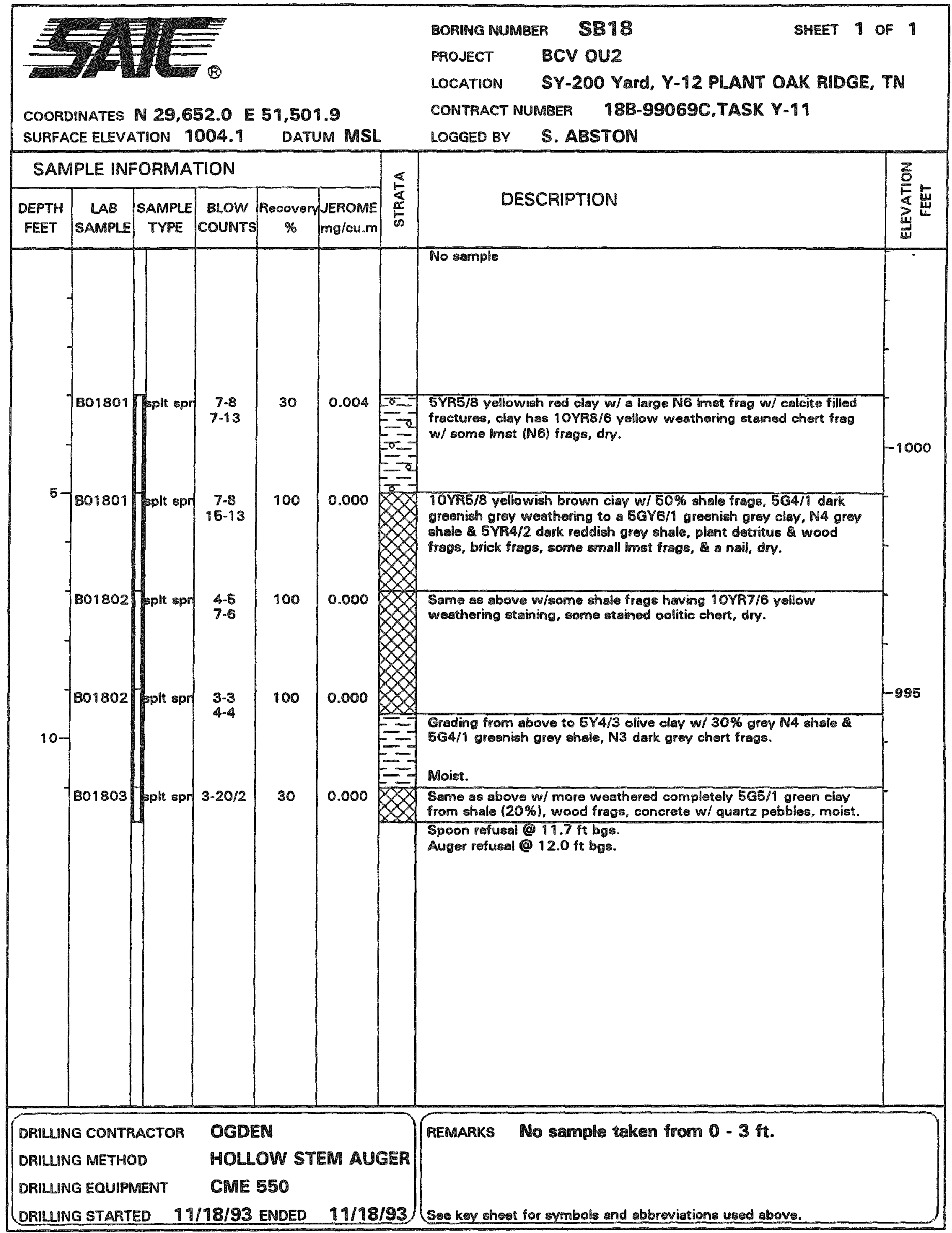




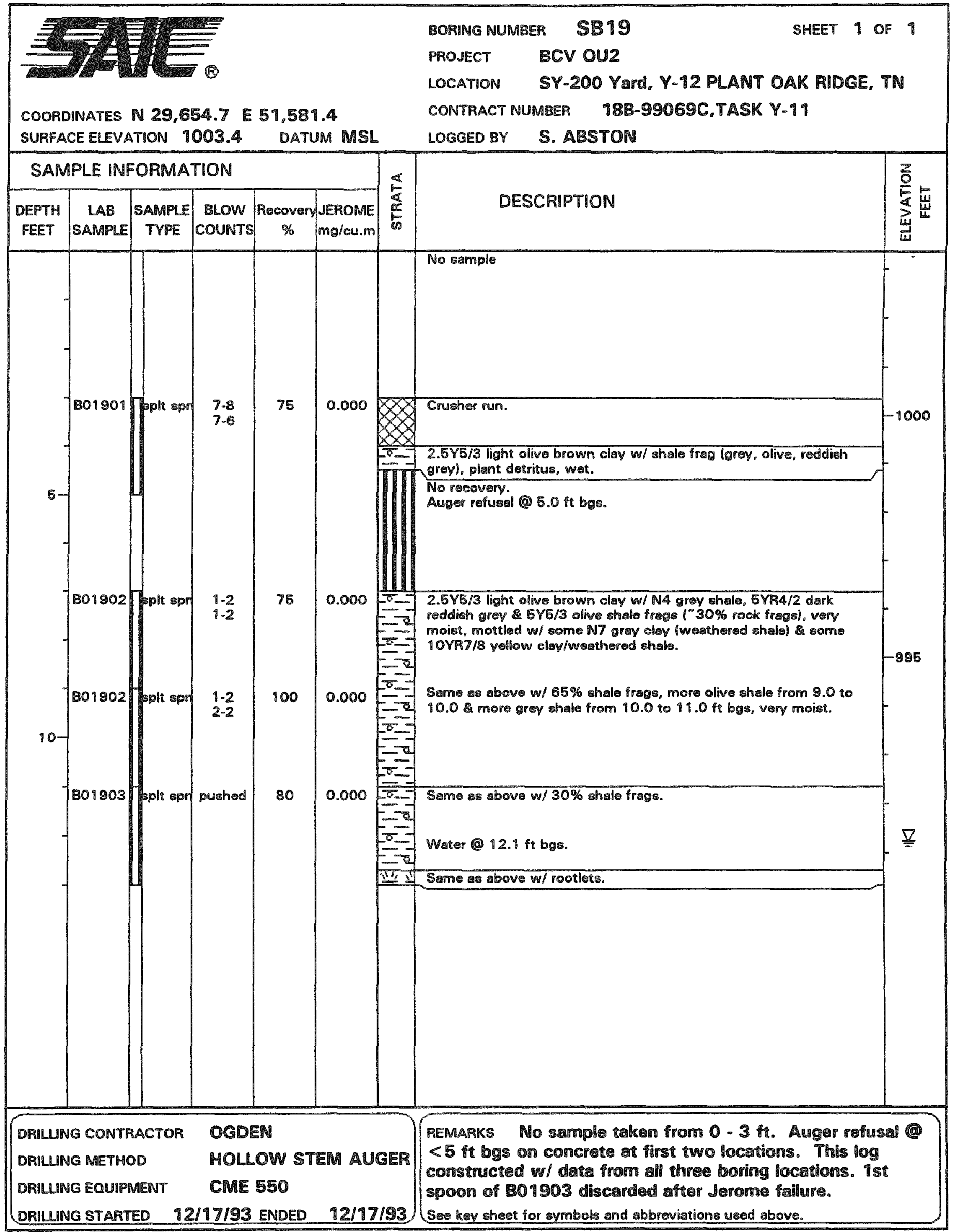




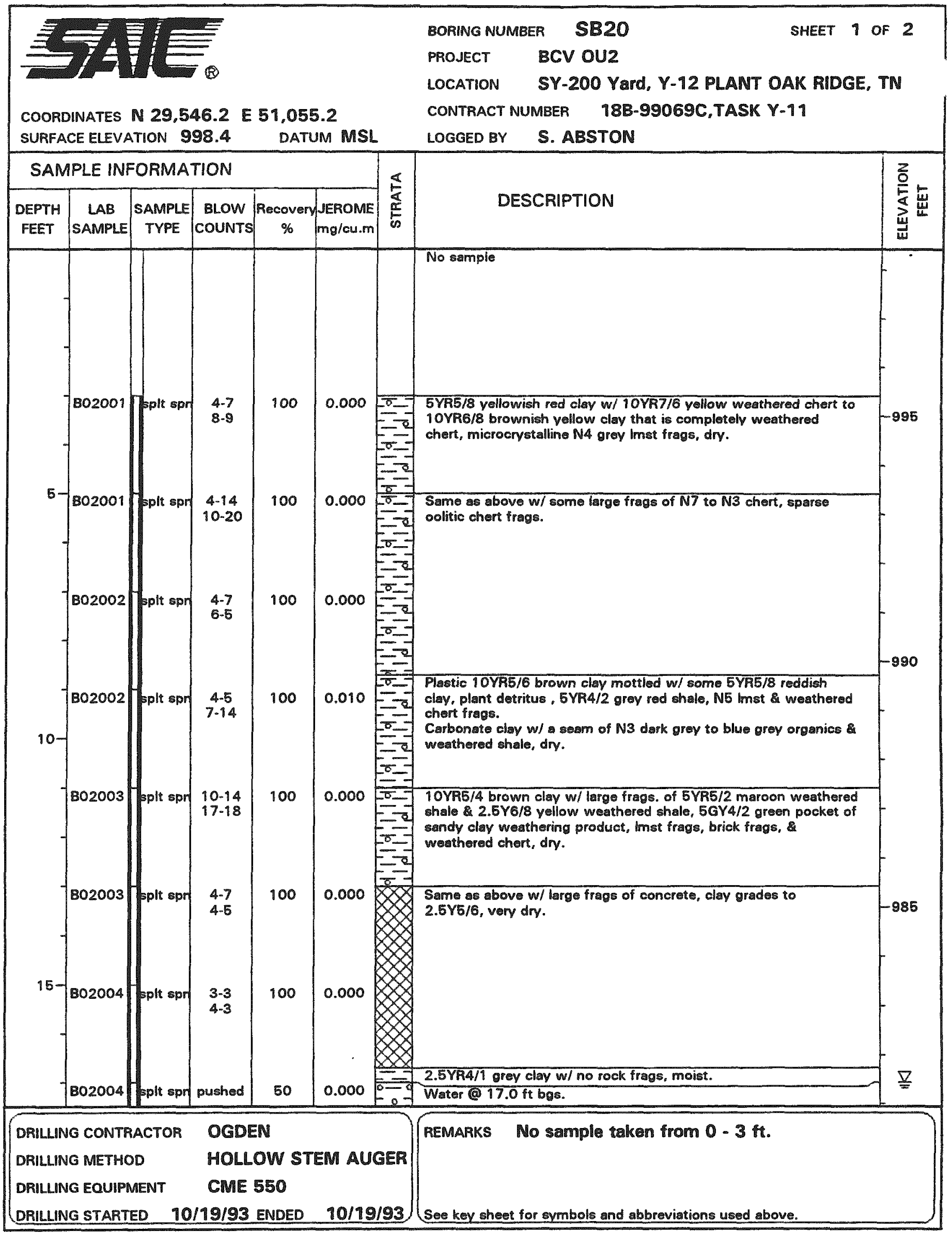


A-37

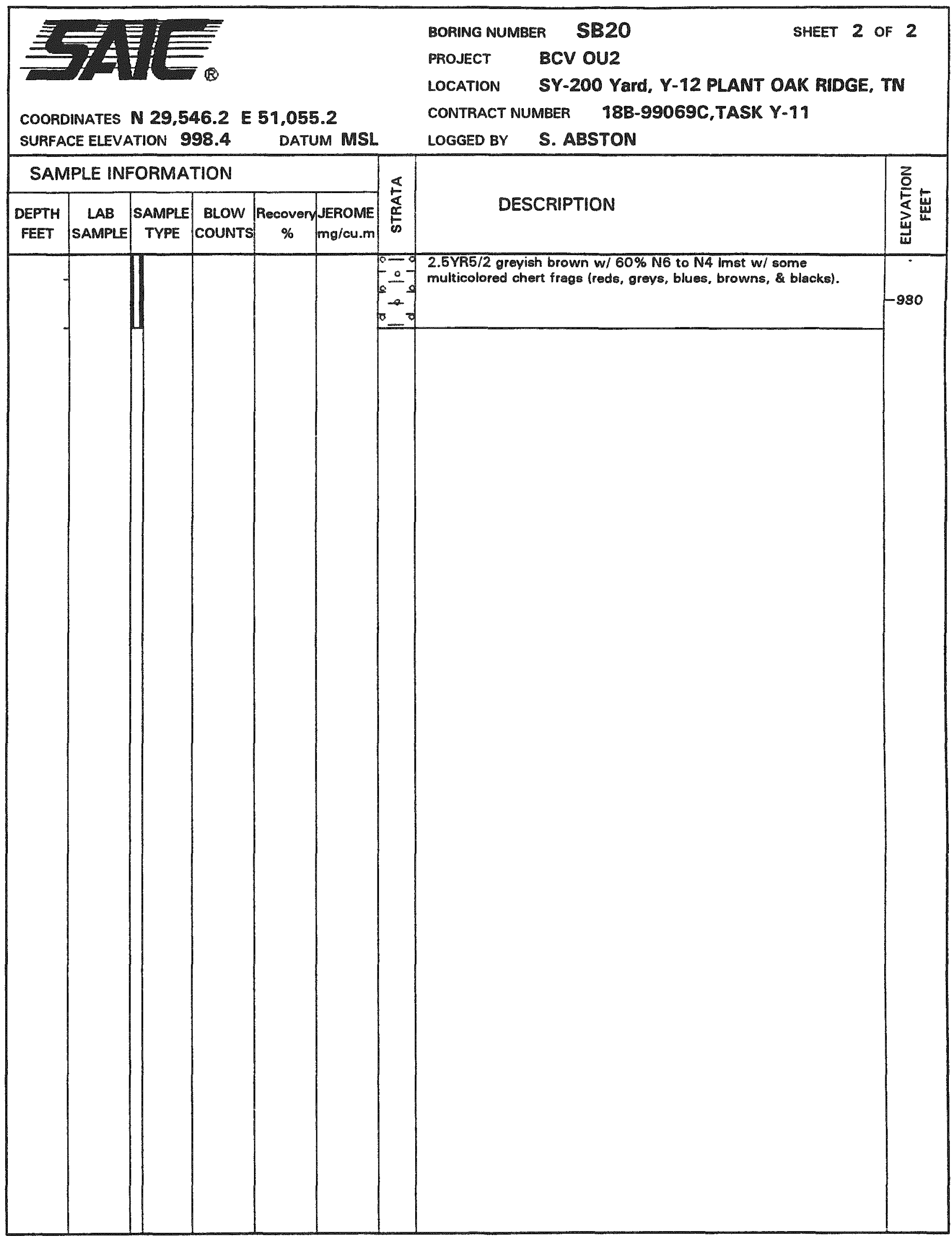




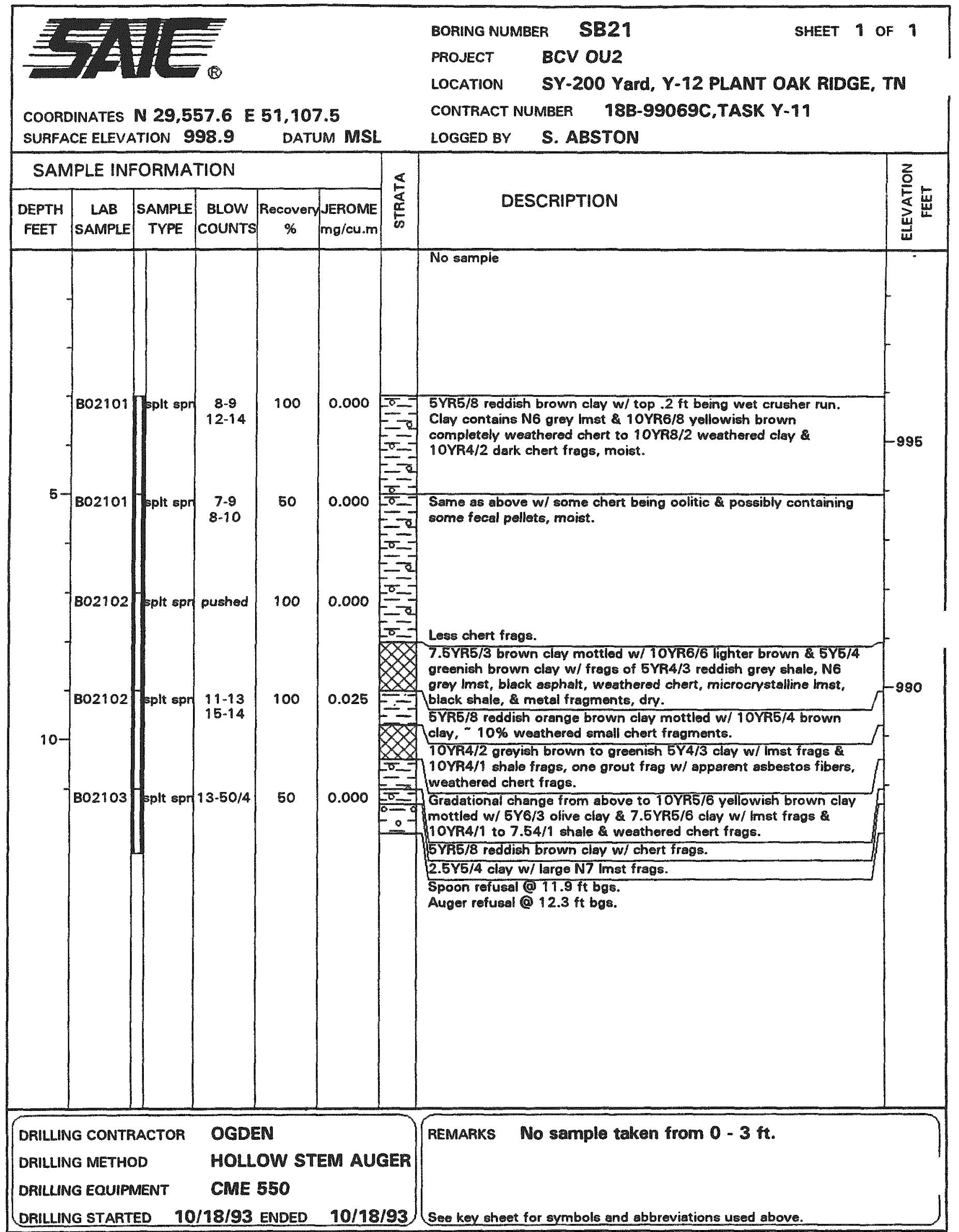




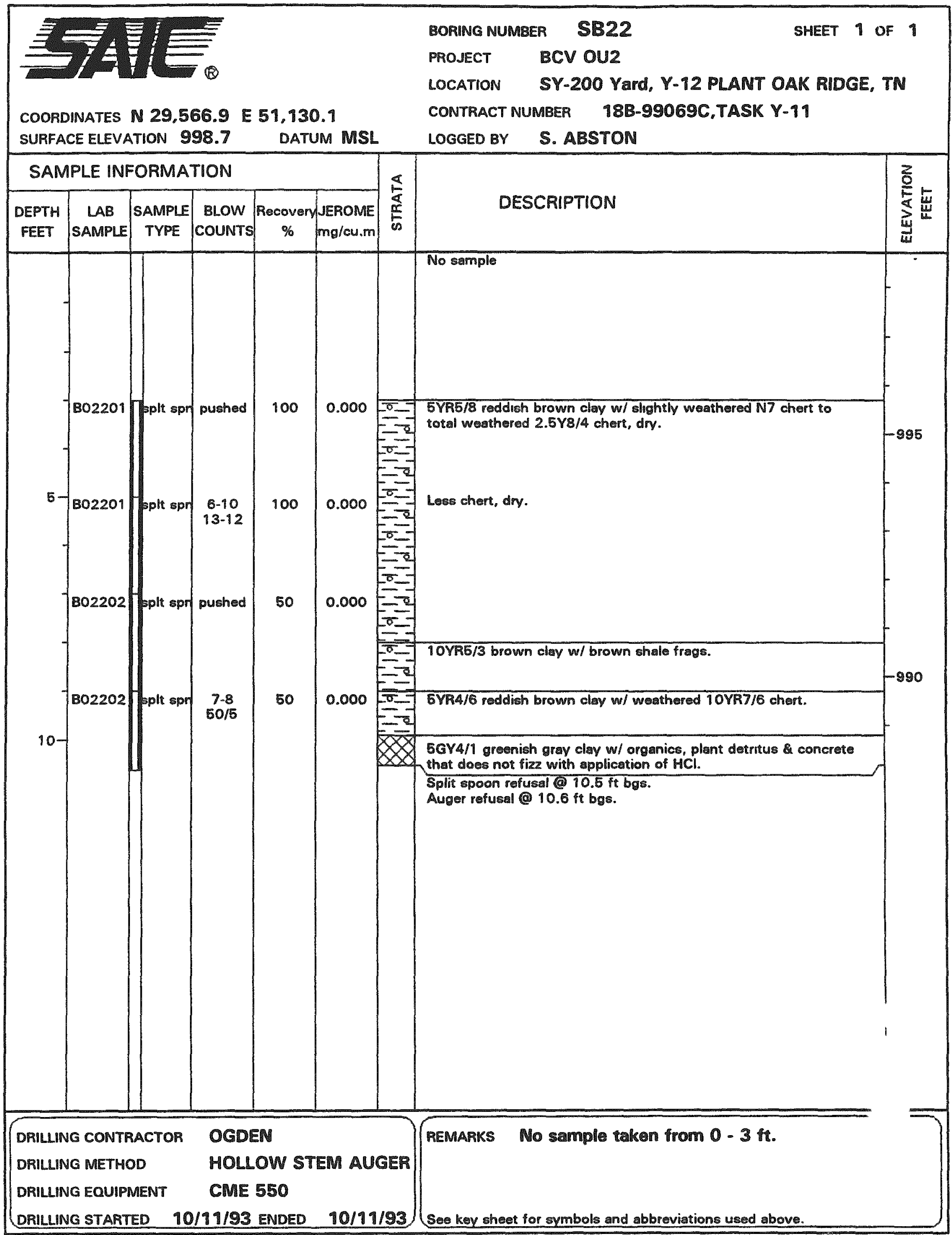




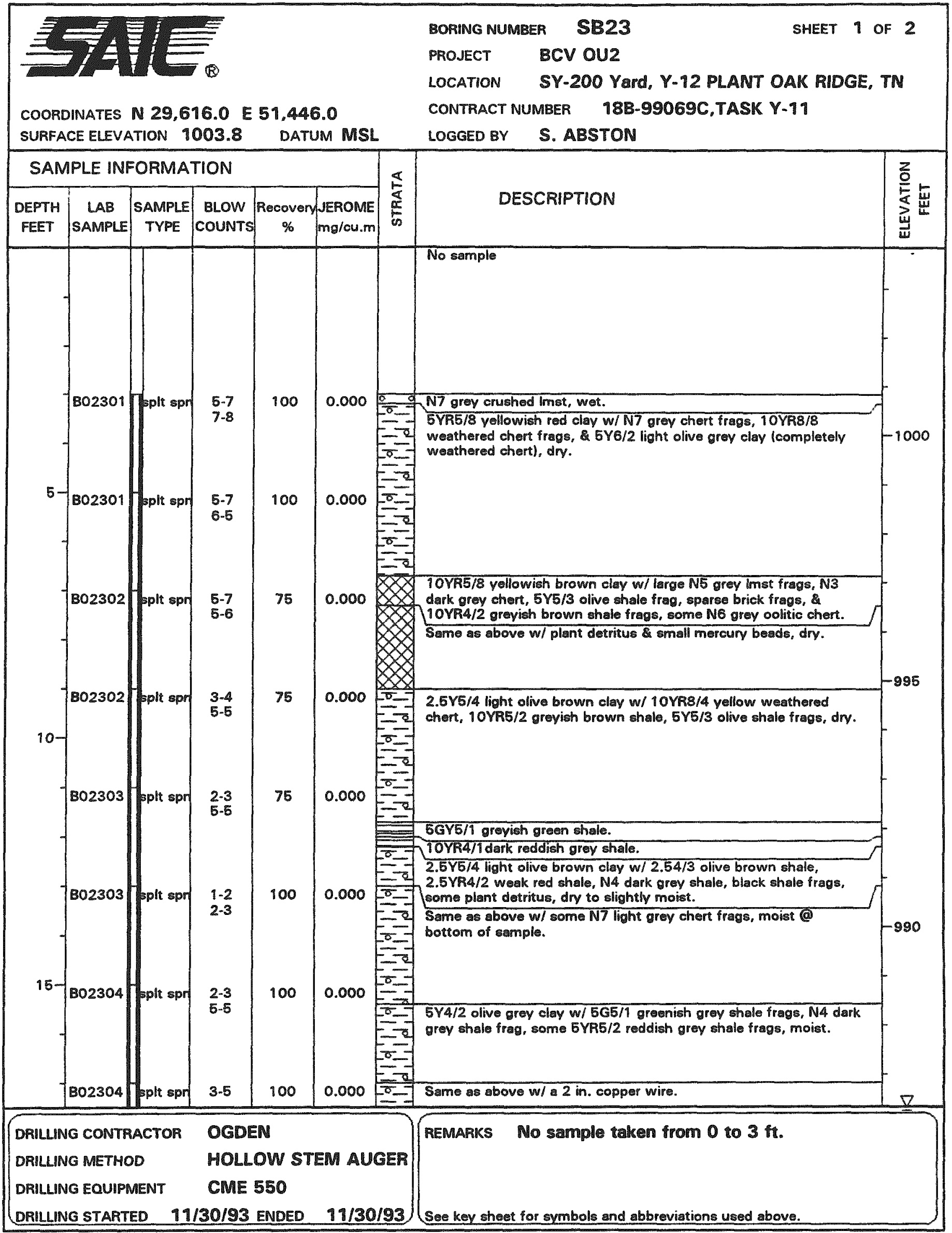


A-41

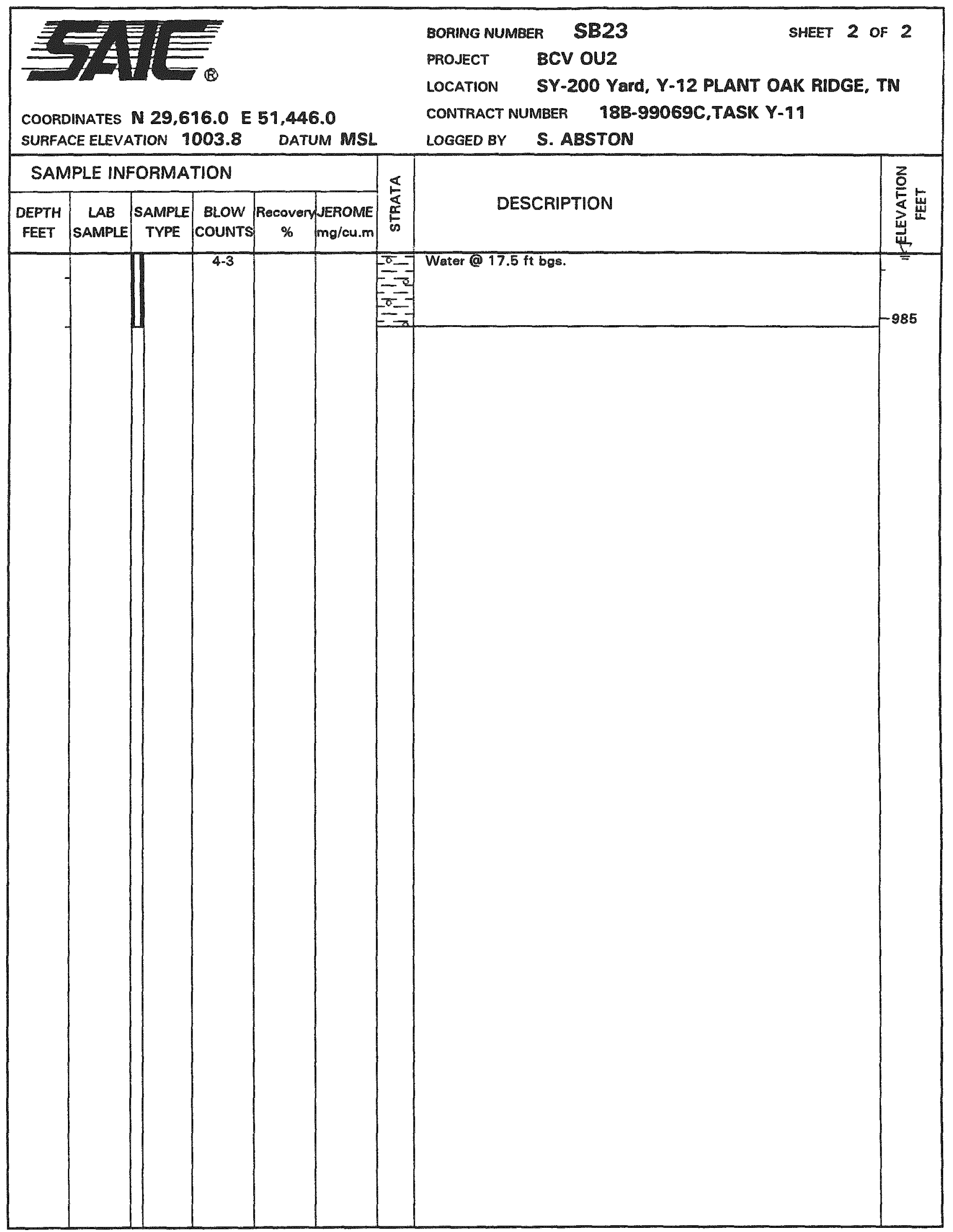




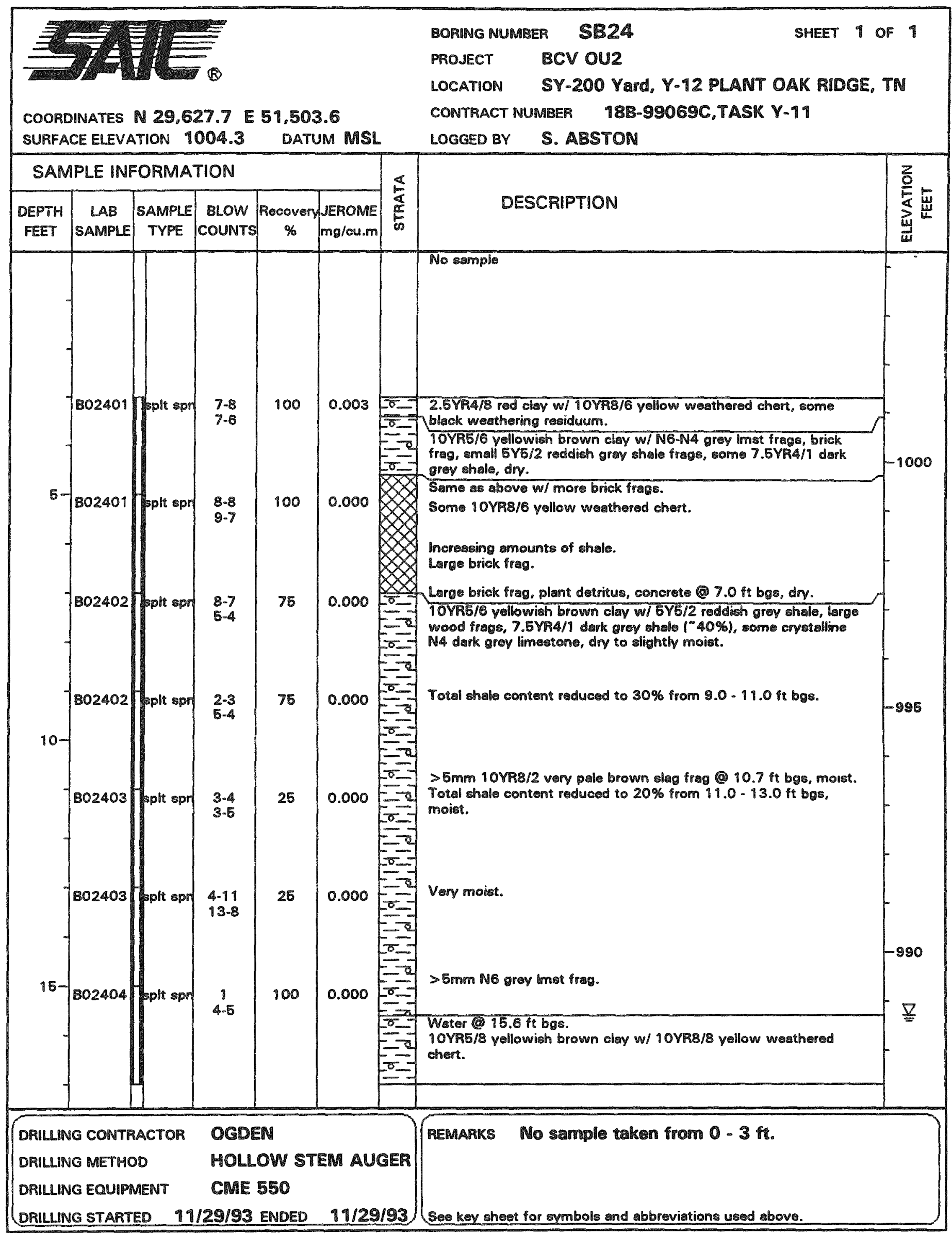




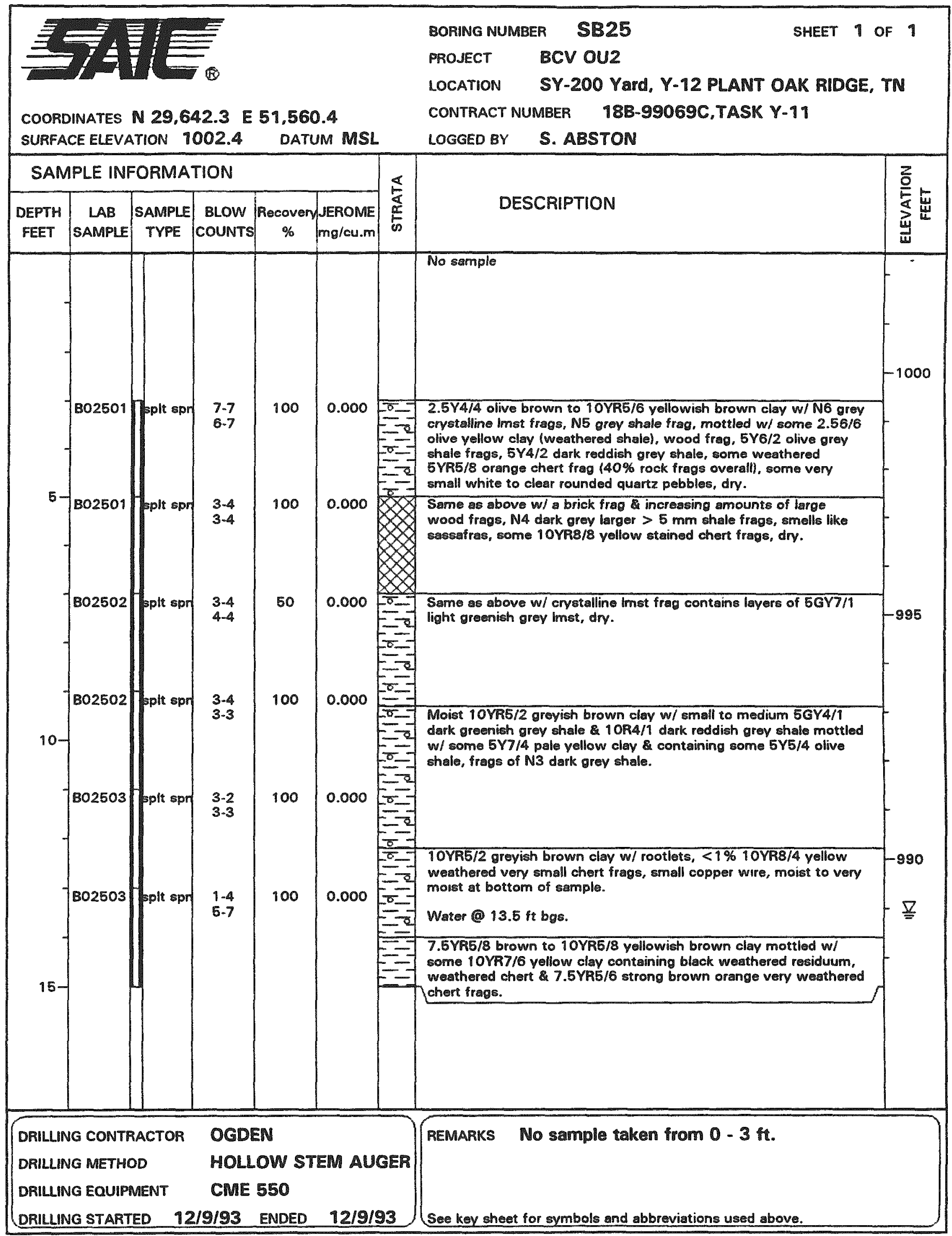




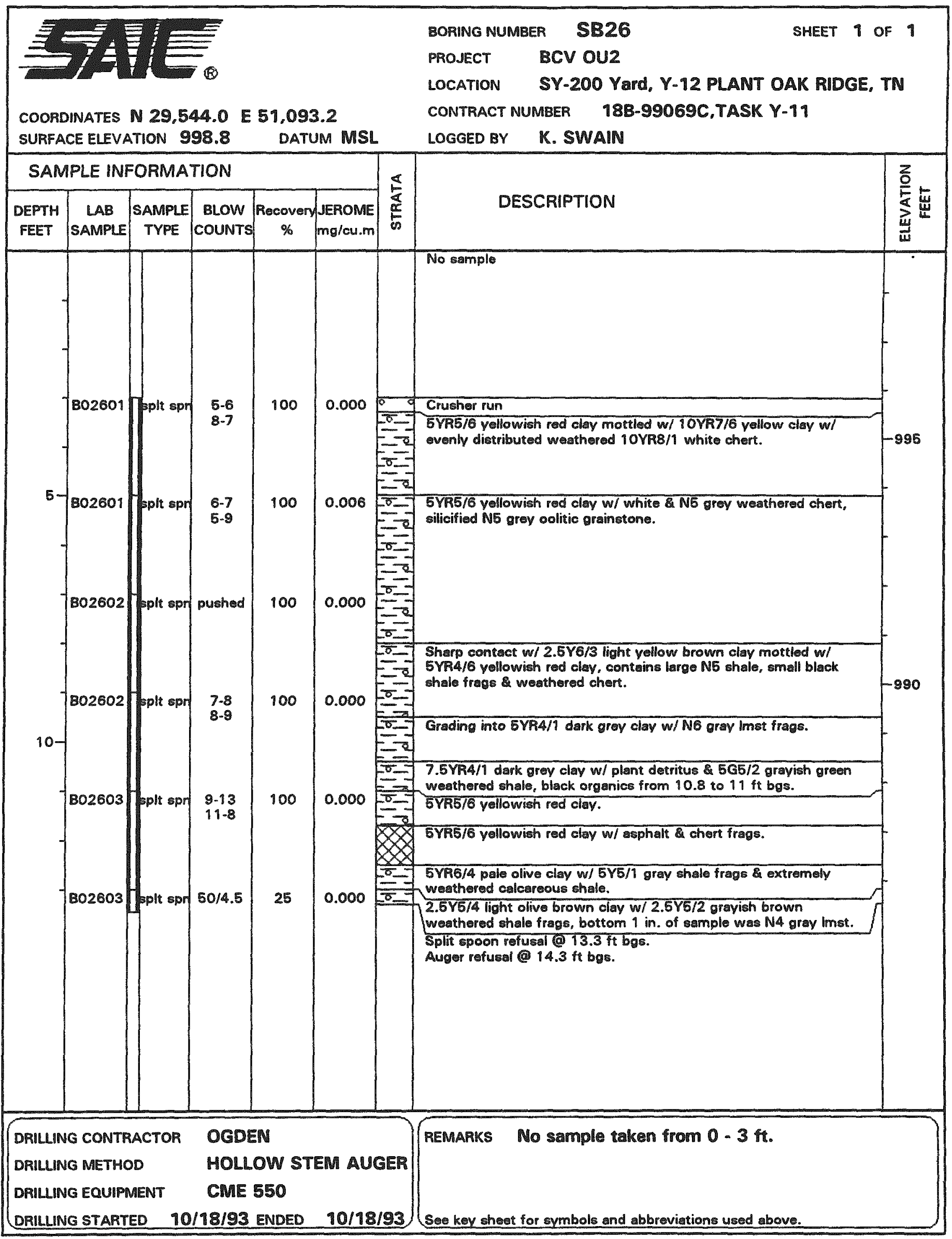




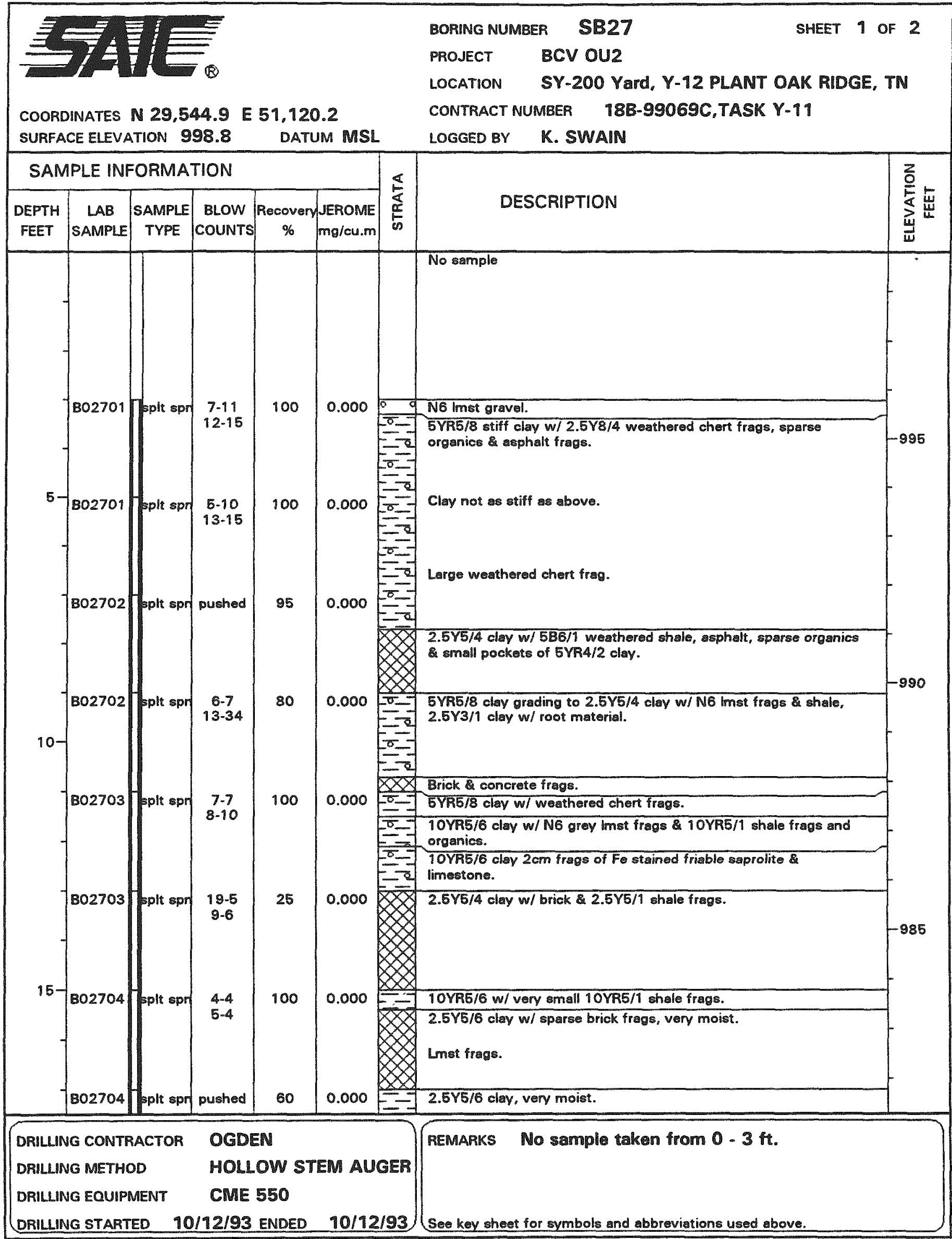




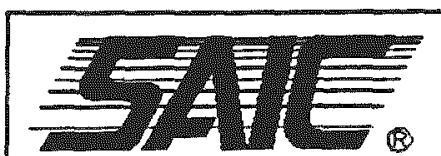

COORDINATES N 29,544.9 E $51,120.2$ SURFACE ELEVATION 998.8
BORING NUMBER

SB27

PROJECT

BCV OU2

LOCATION SY-200 Yard, Y-12 PLANT OAK RIDGE, TN CONTRACT NUMBER 18B-99069C,TASK Y-11
LOGGED BY
K. SWAIN

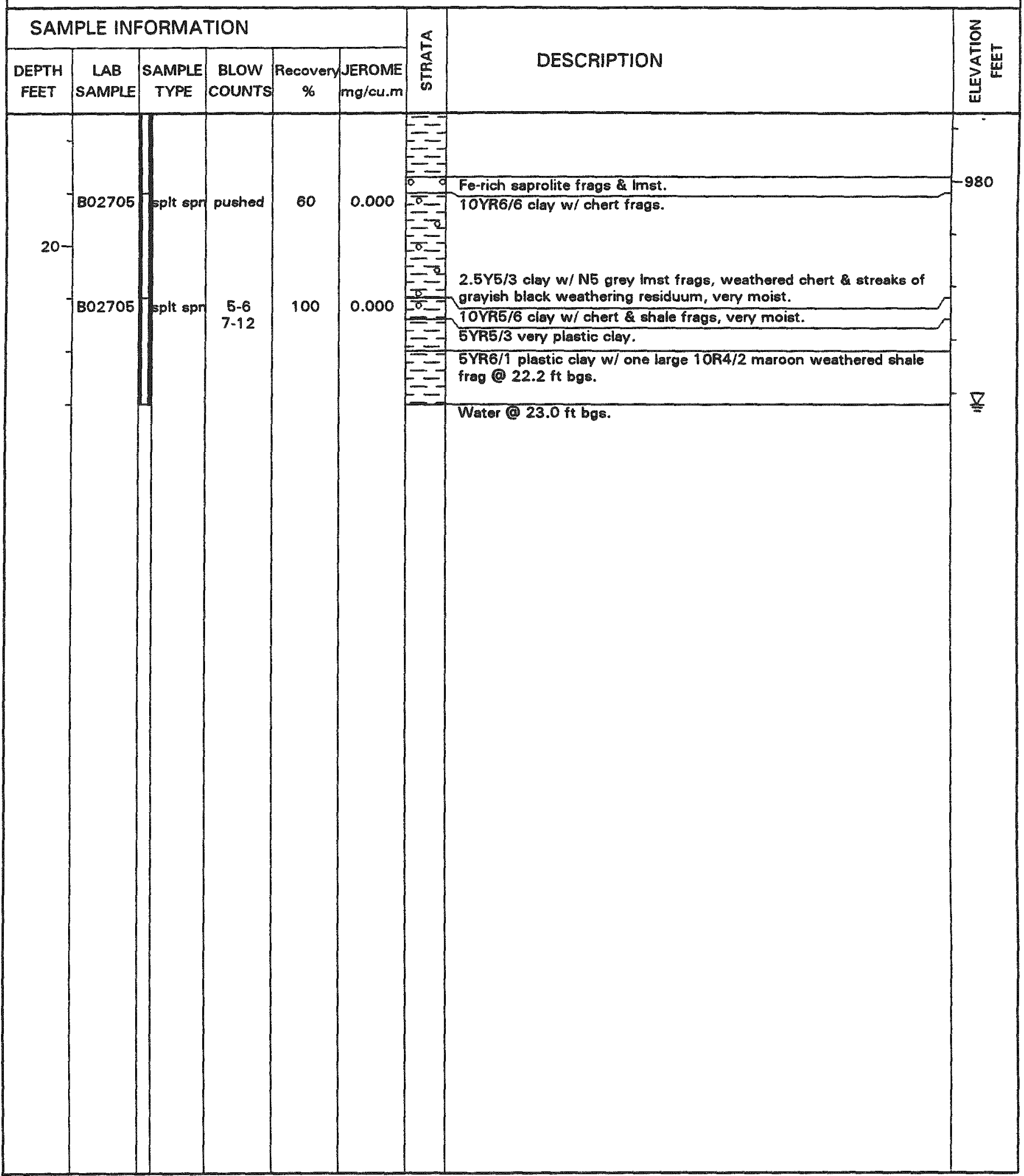




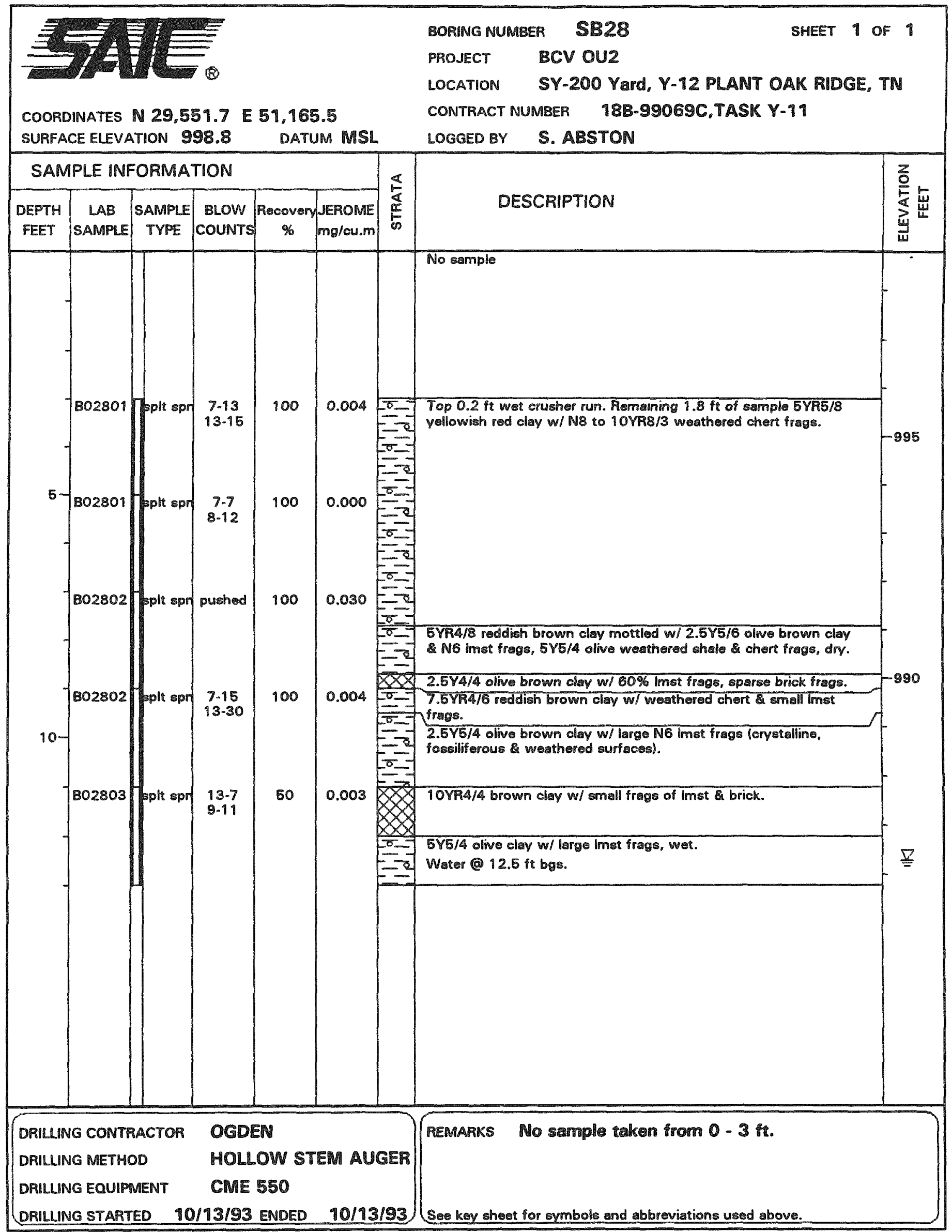




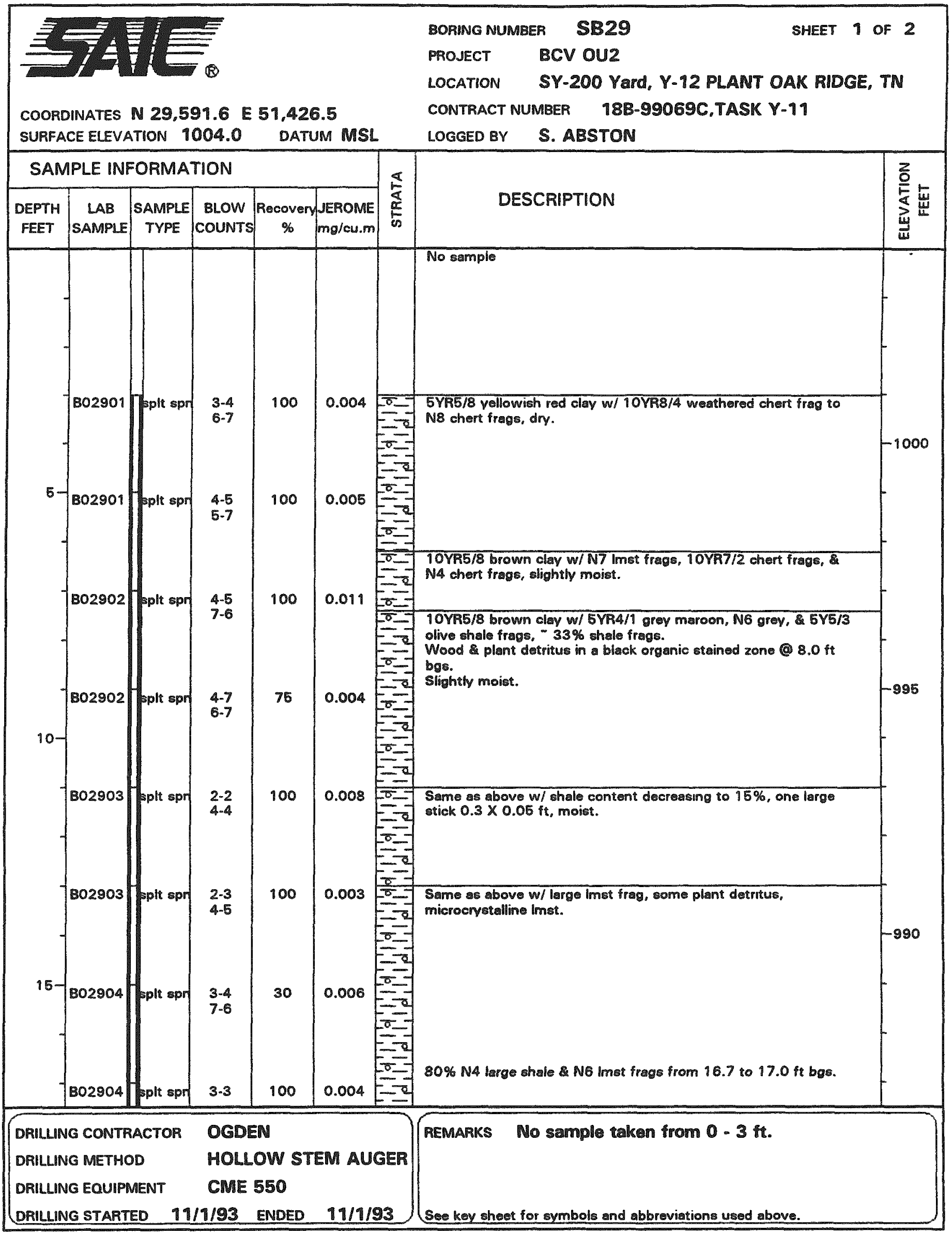




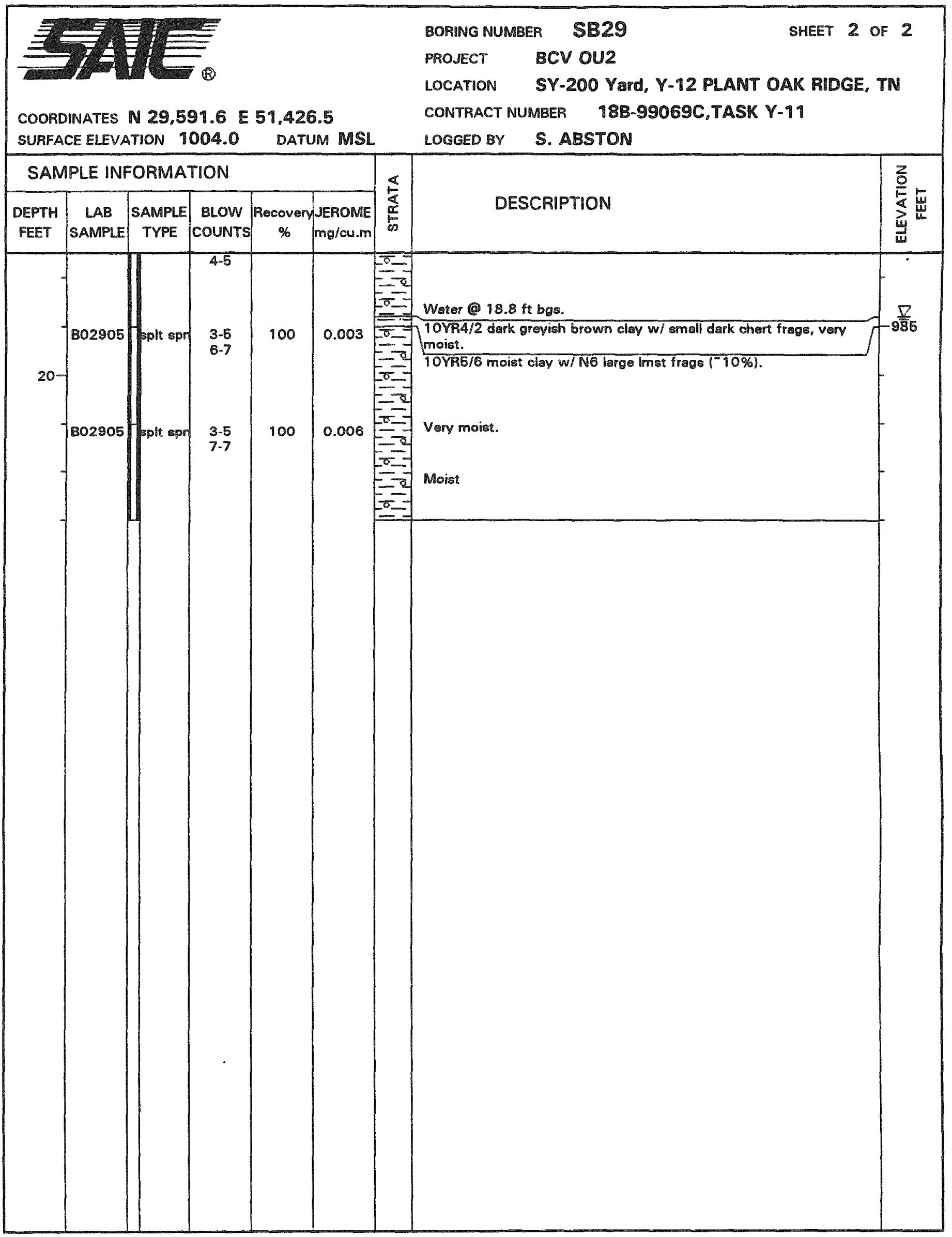




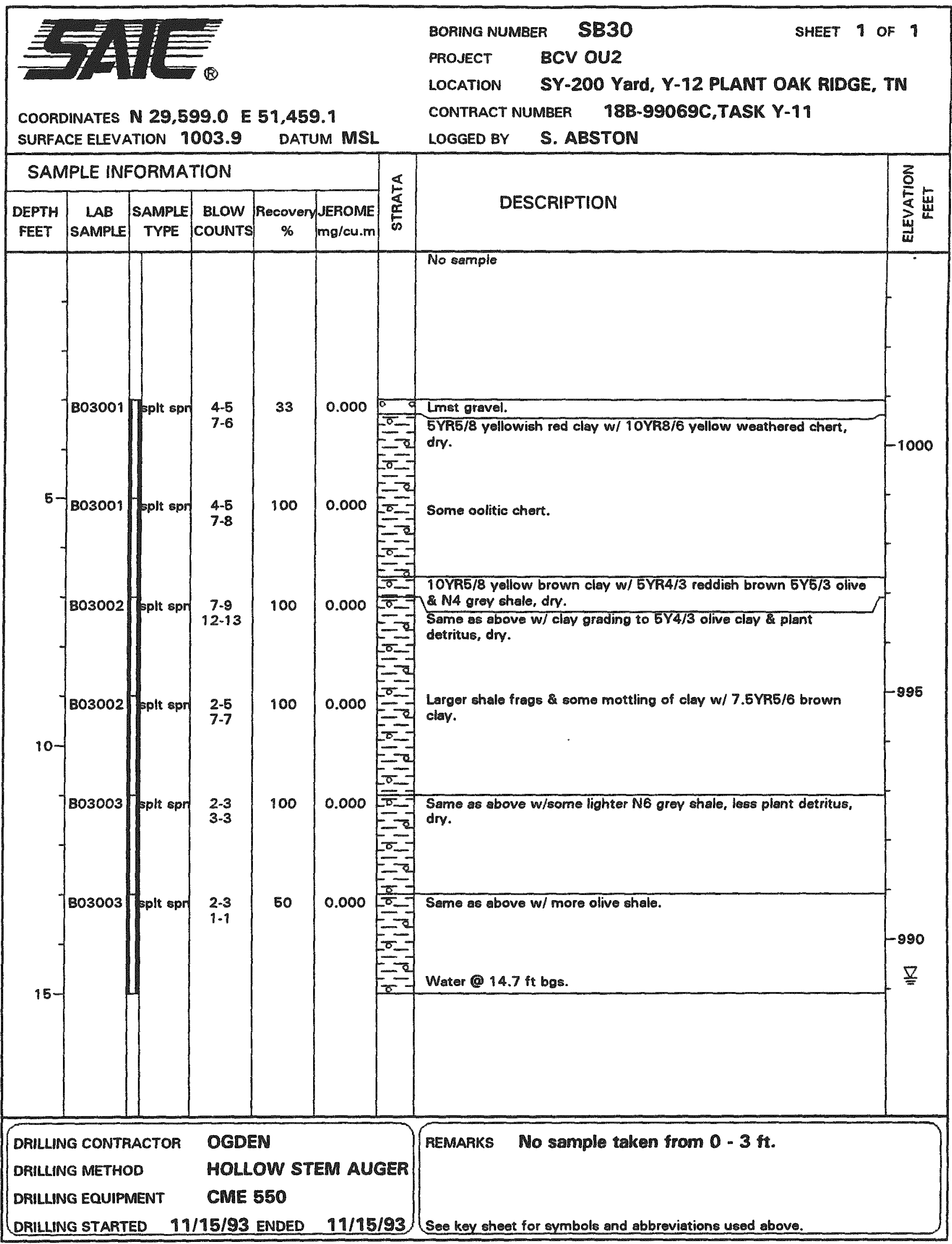




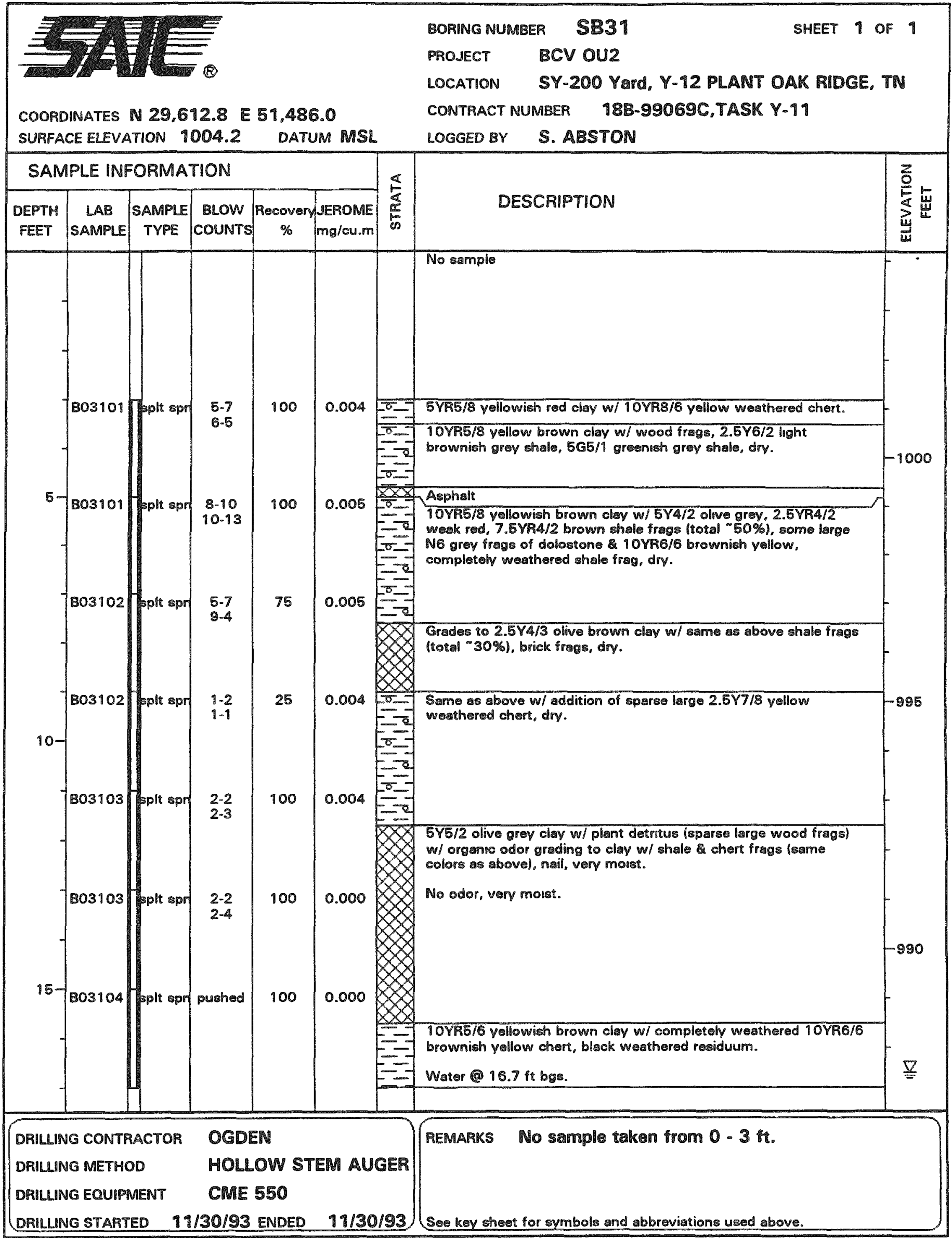




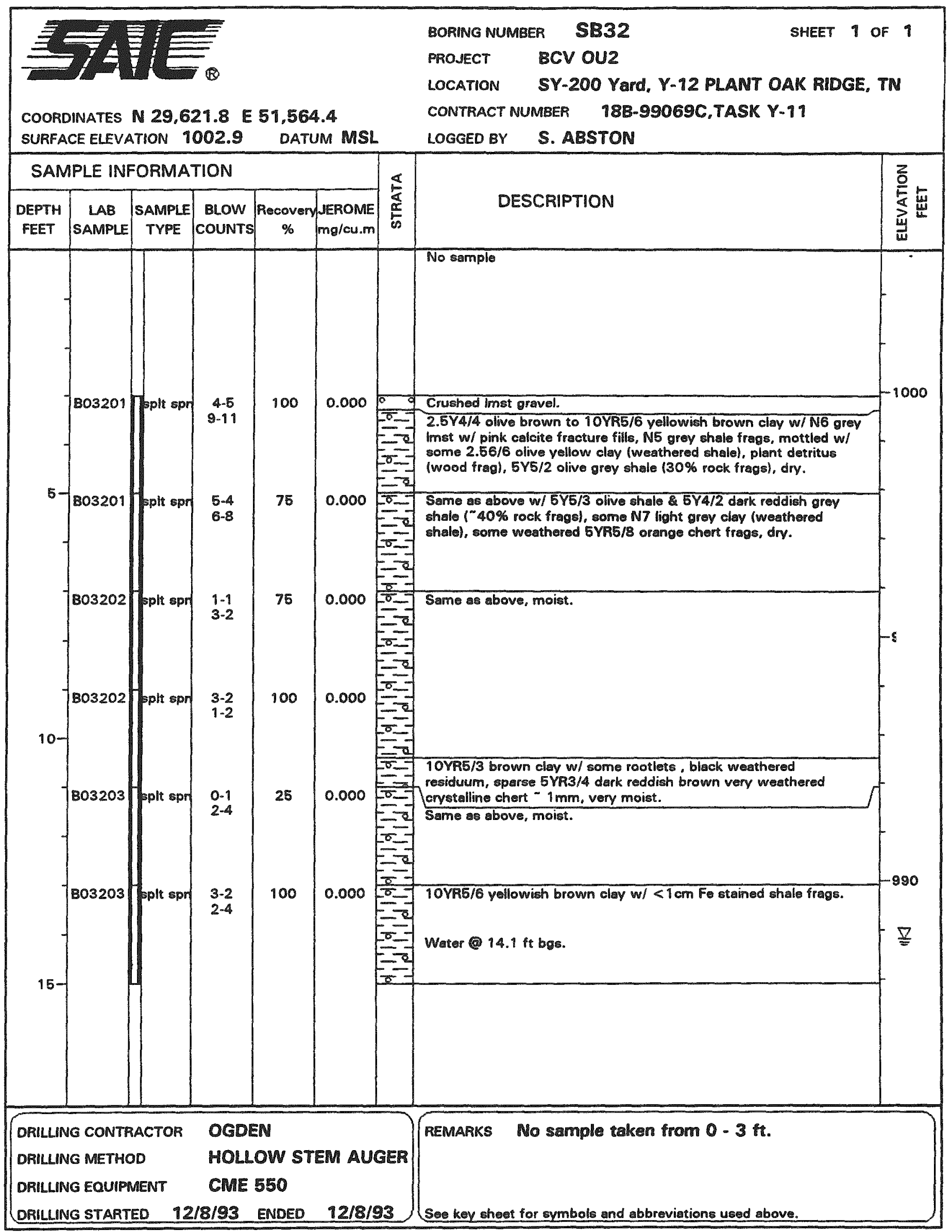




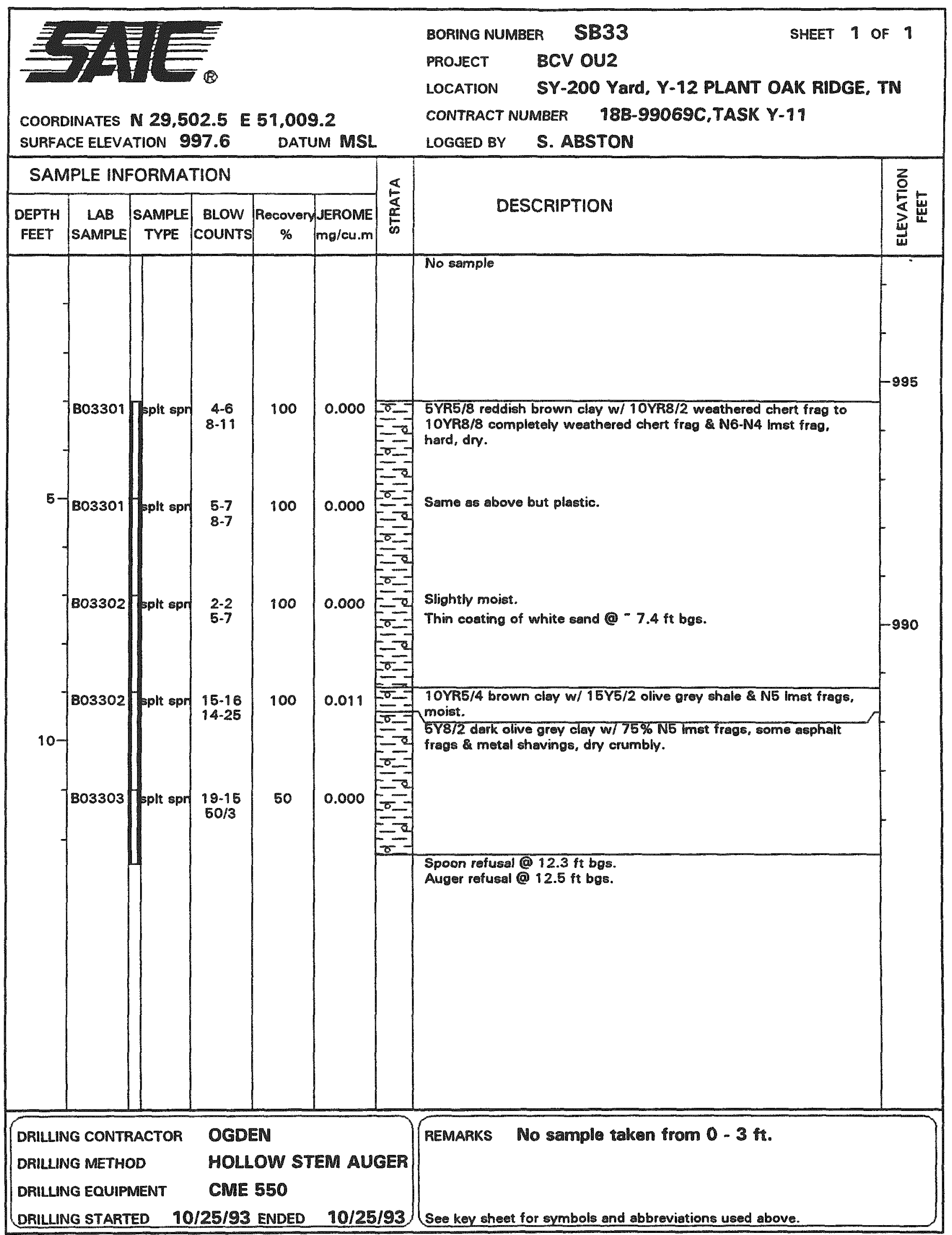




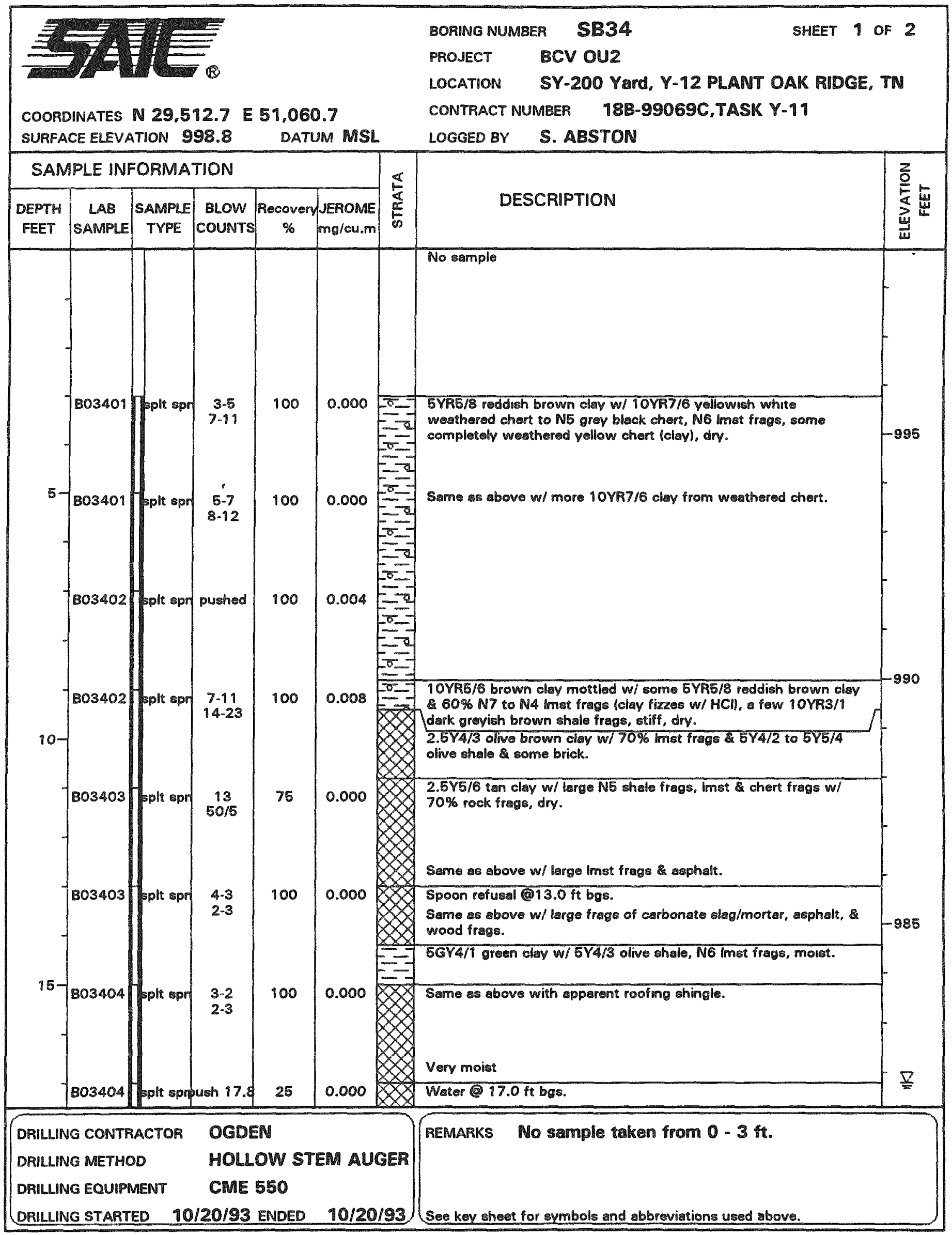




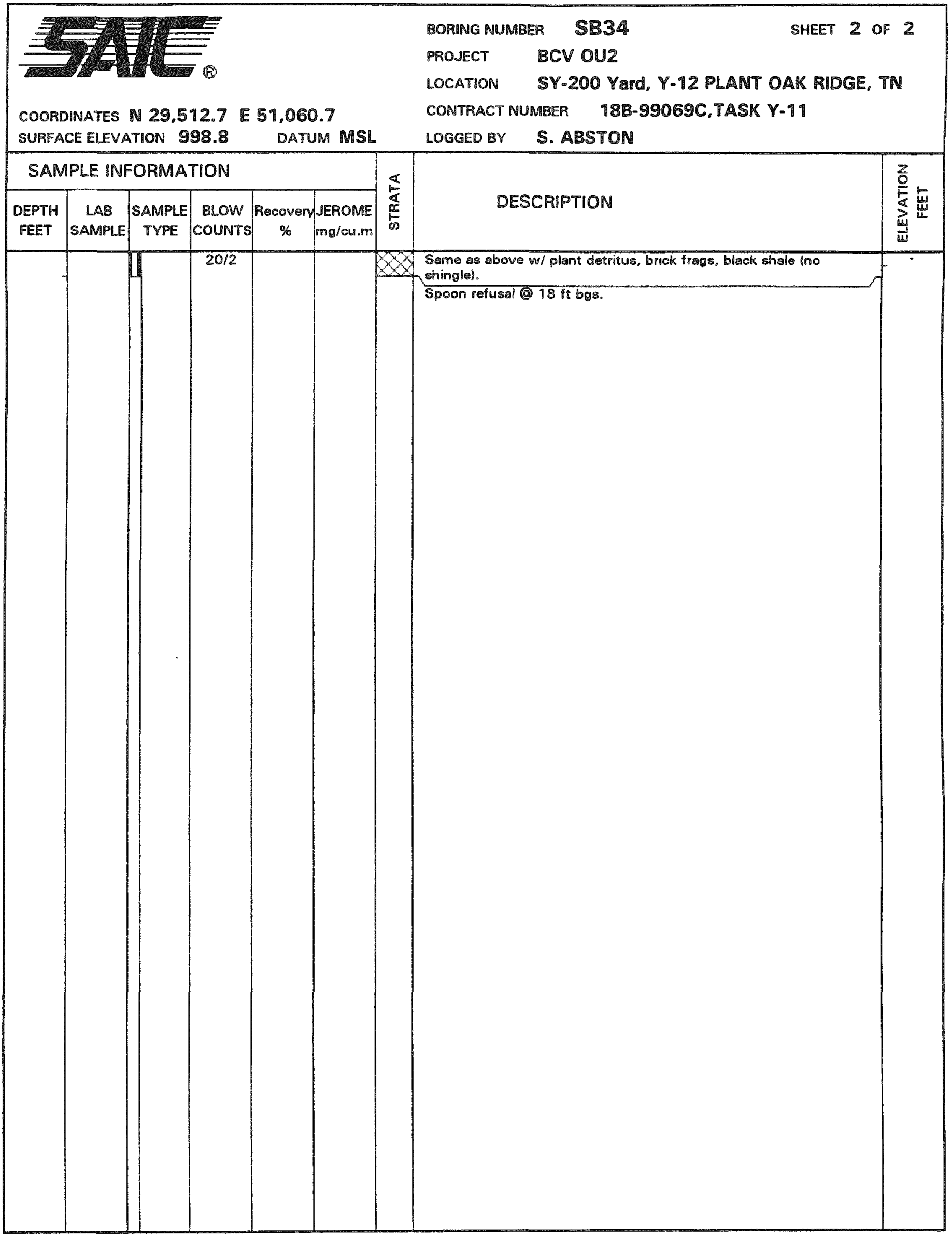




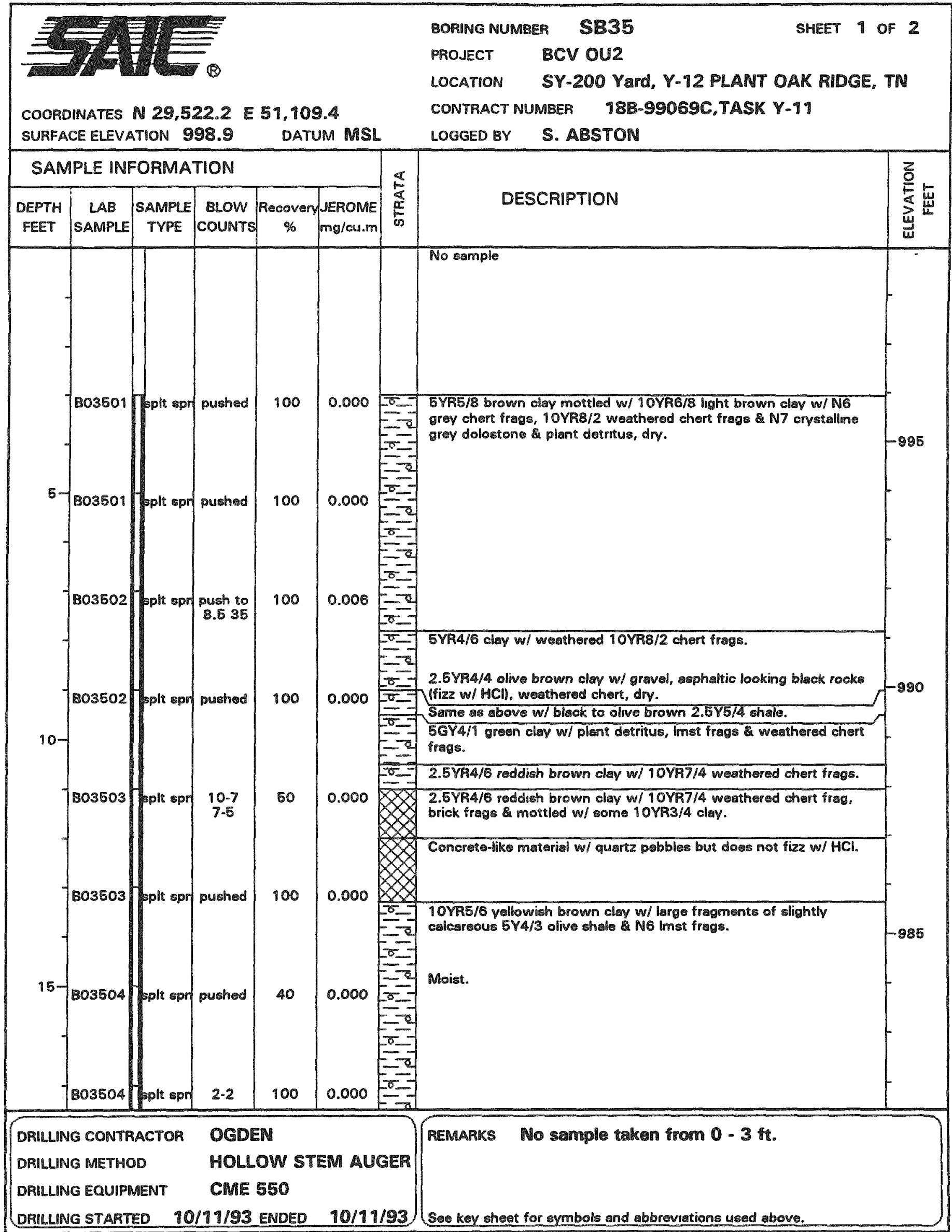




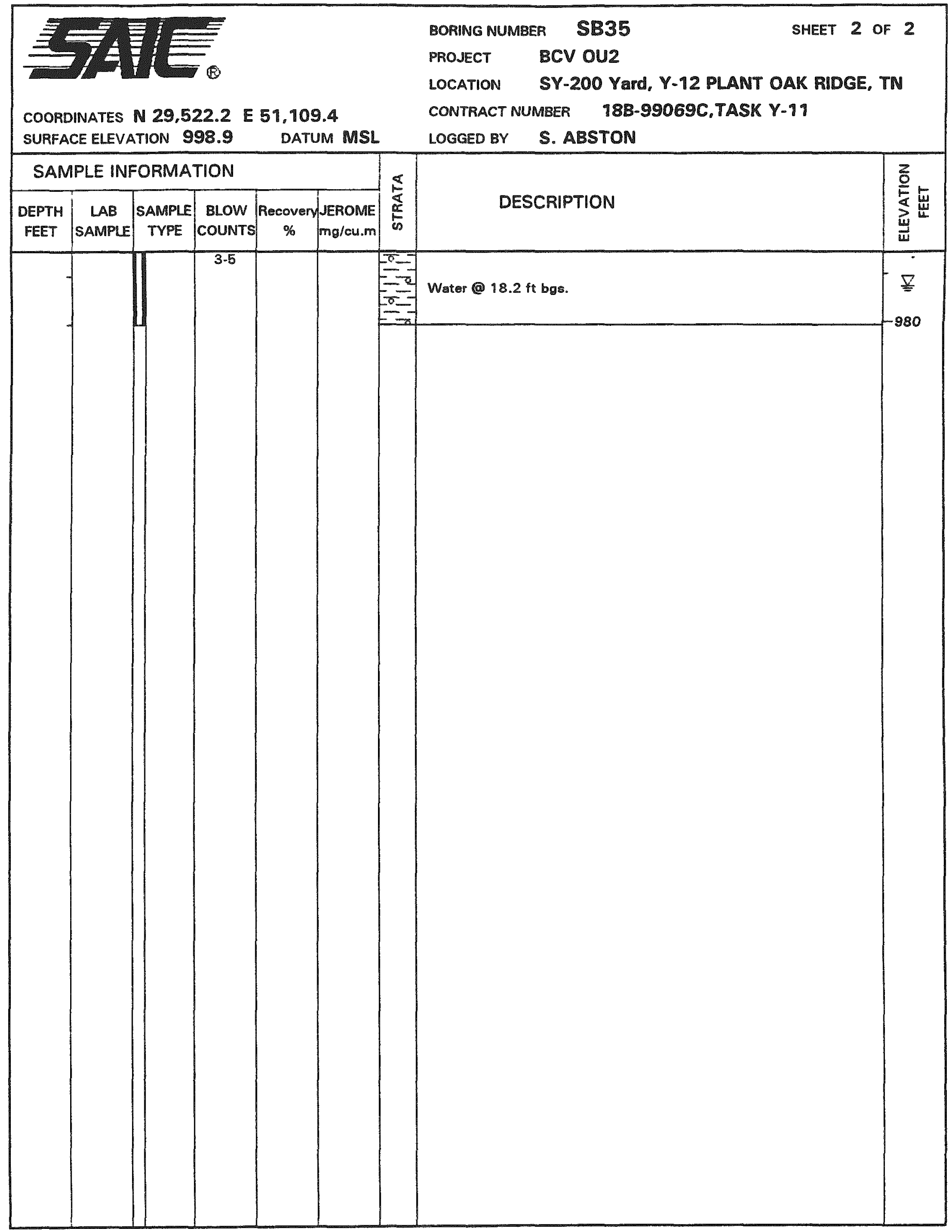




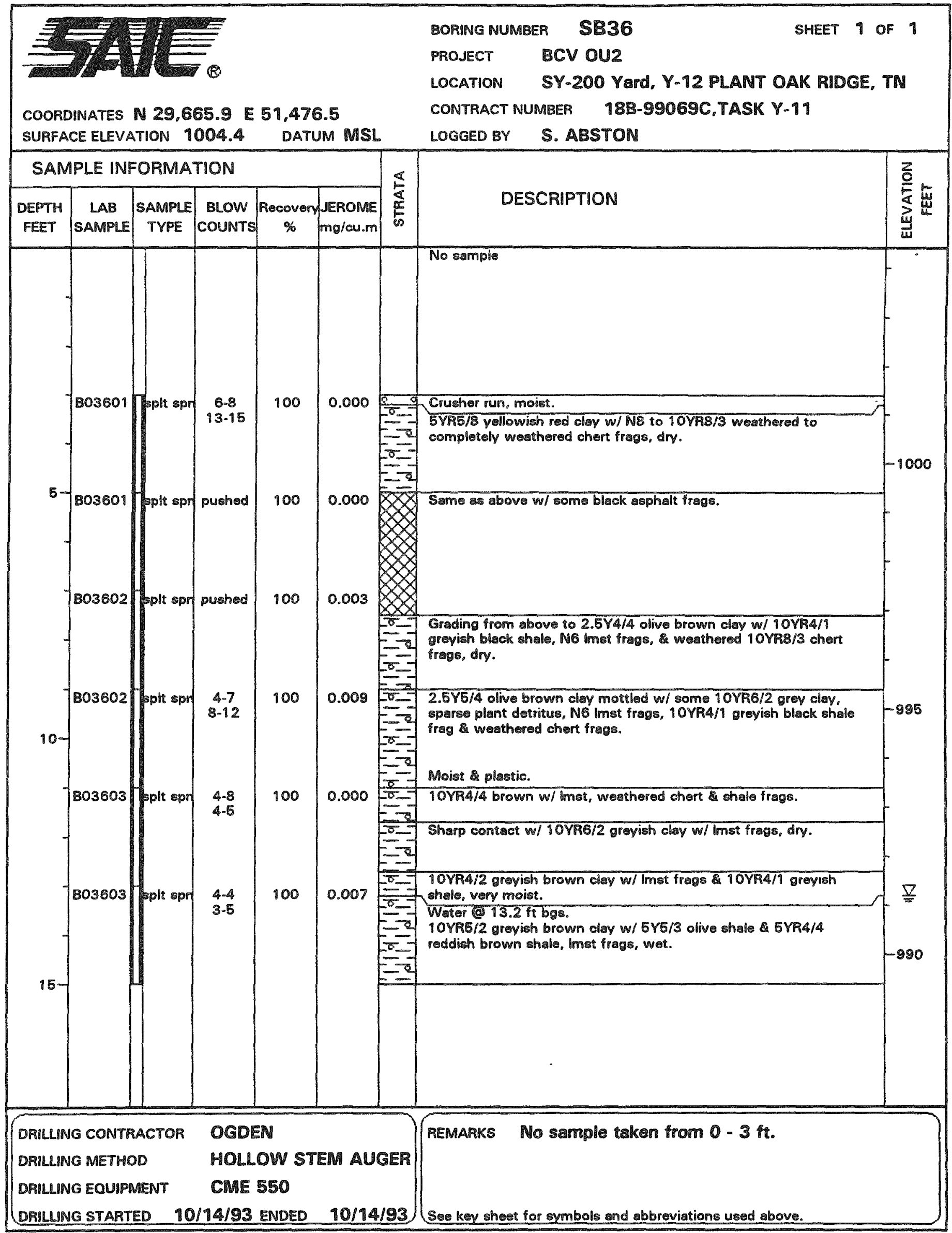




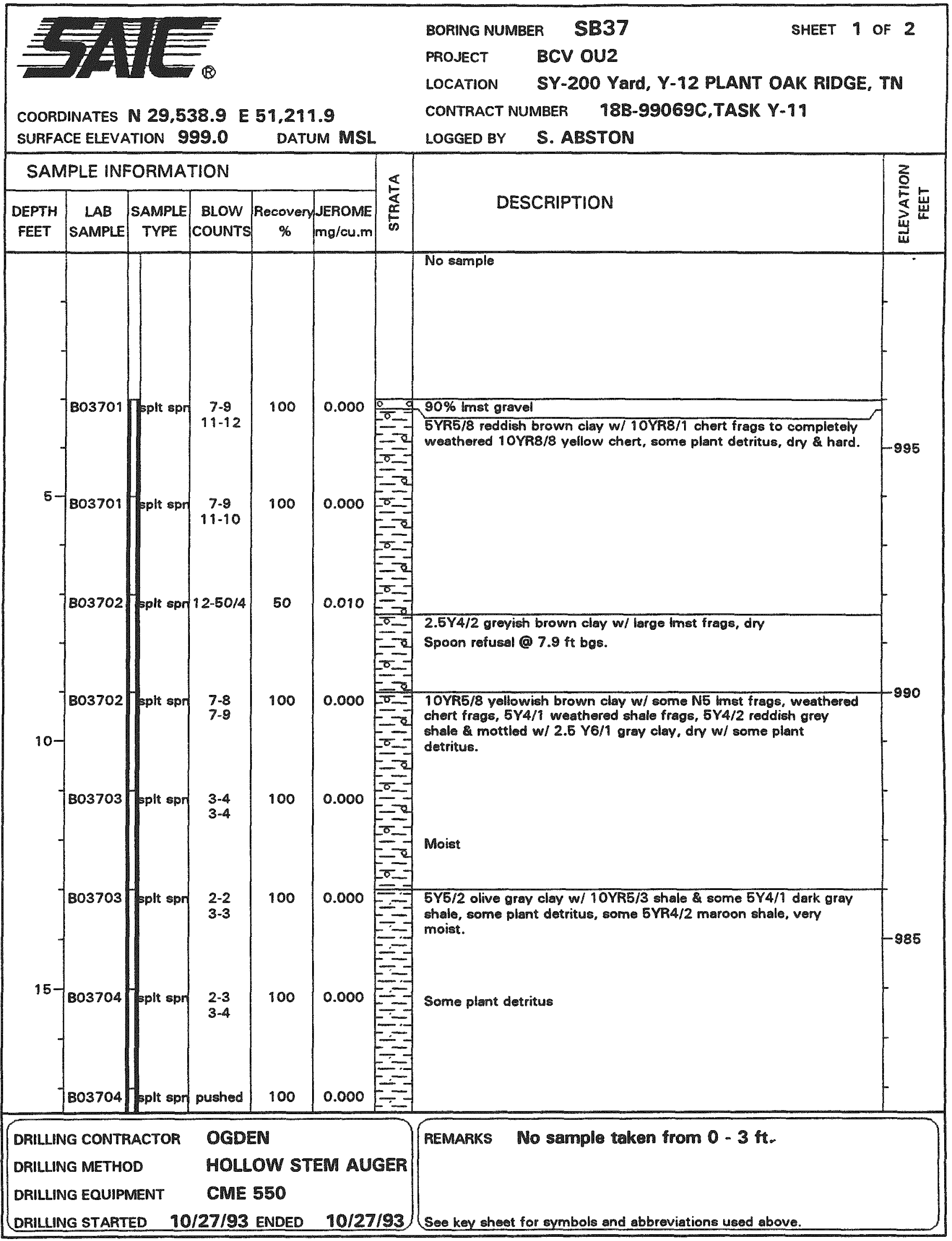




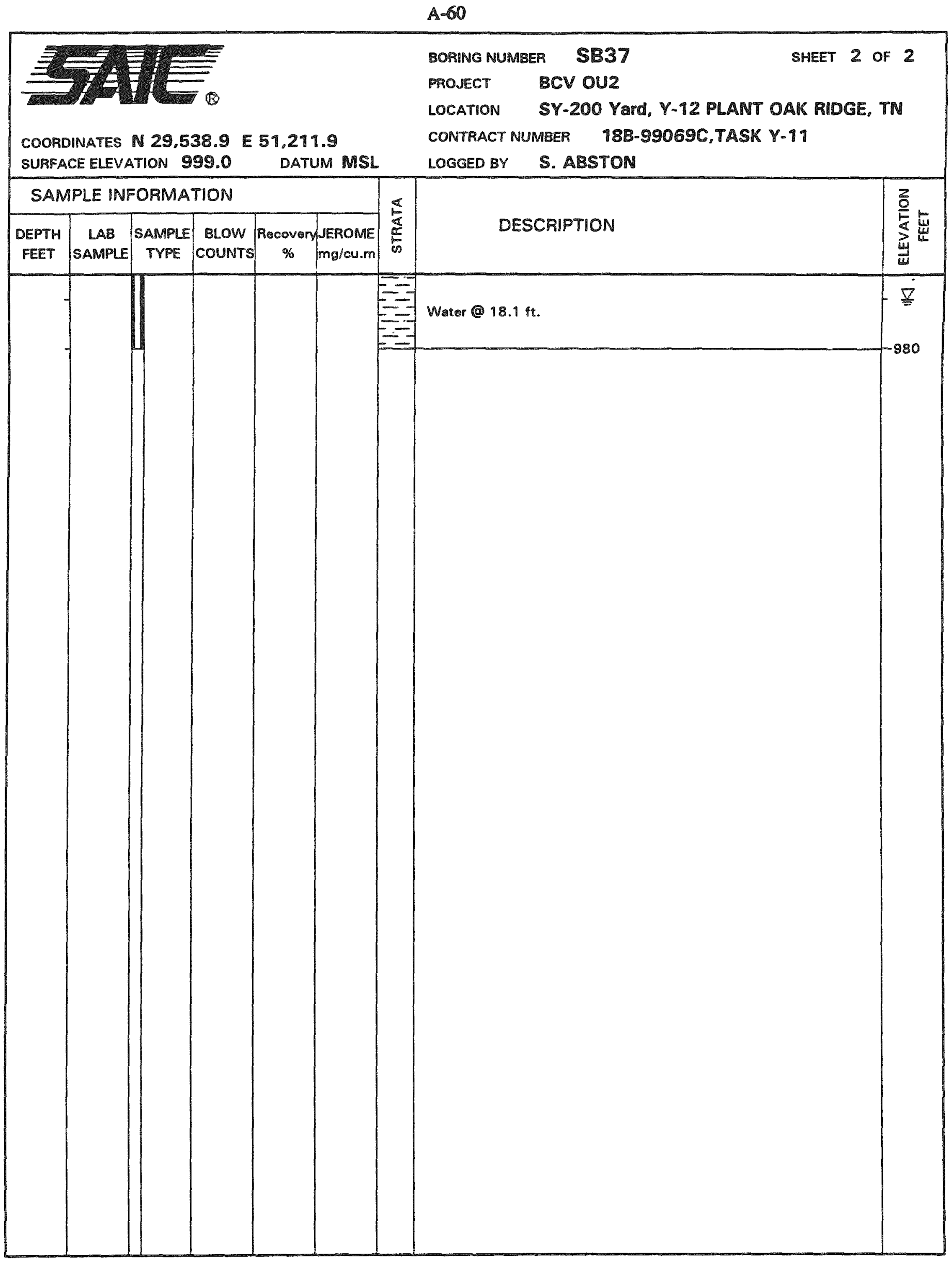




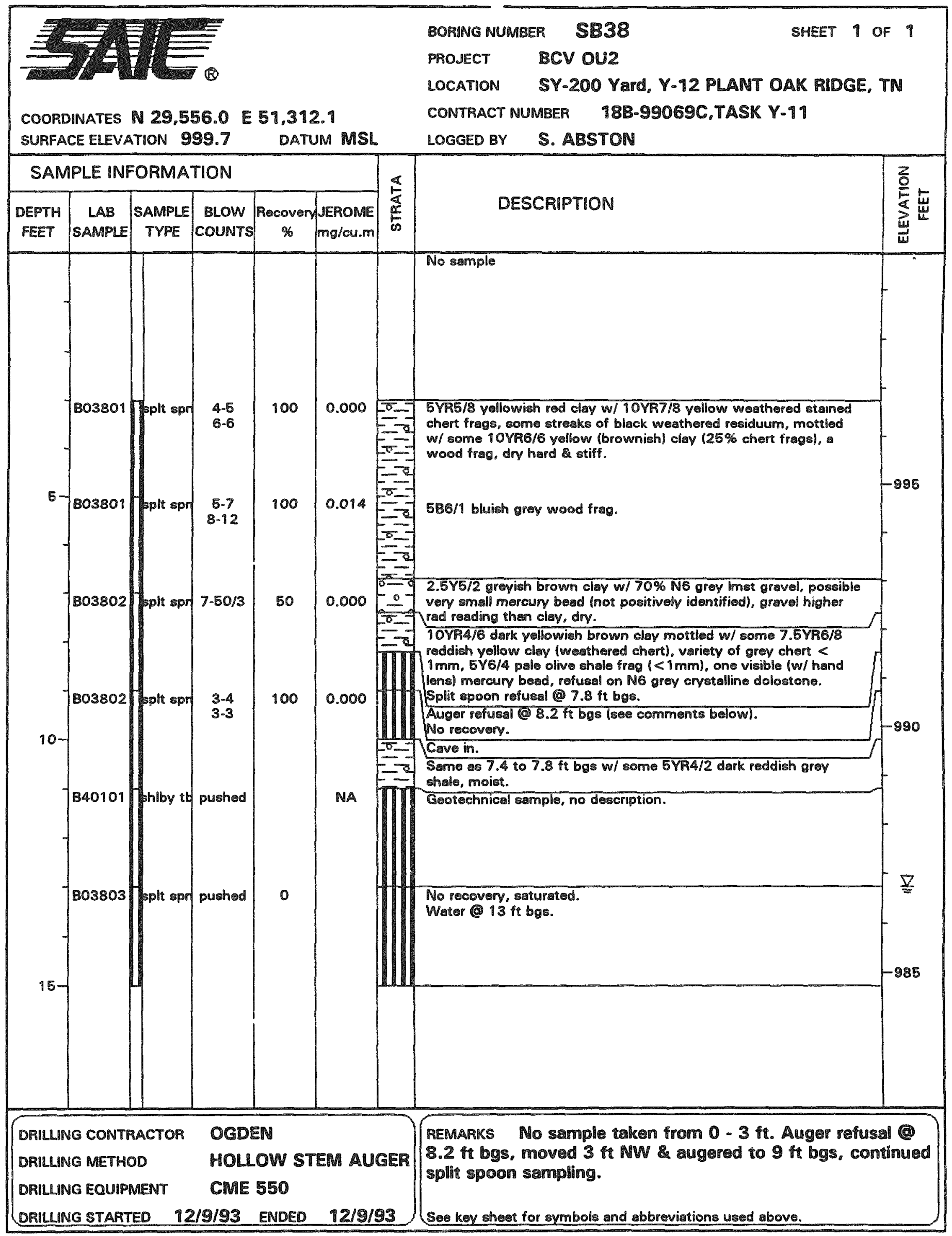




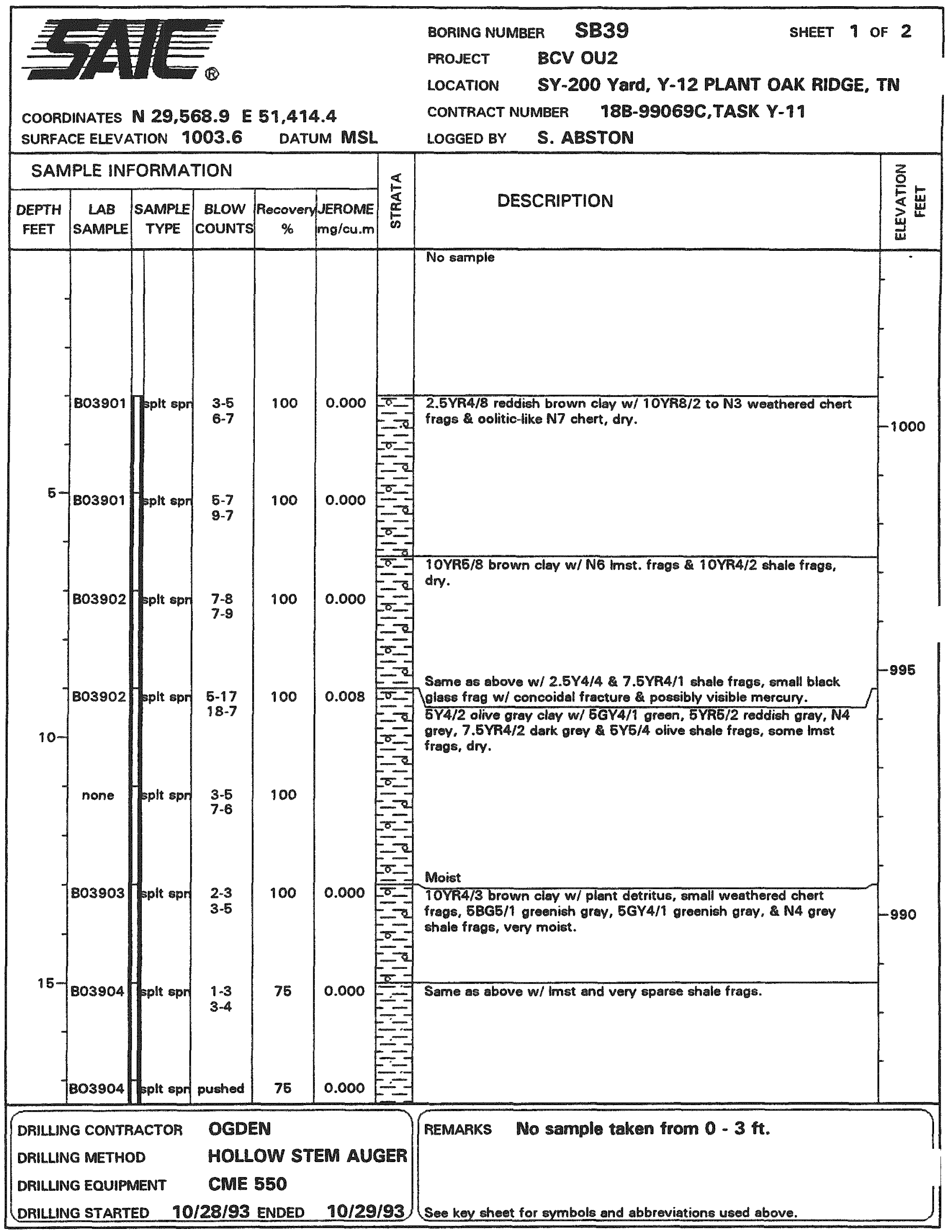




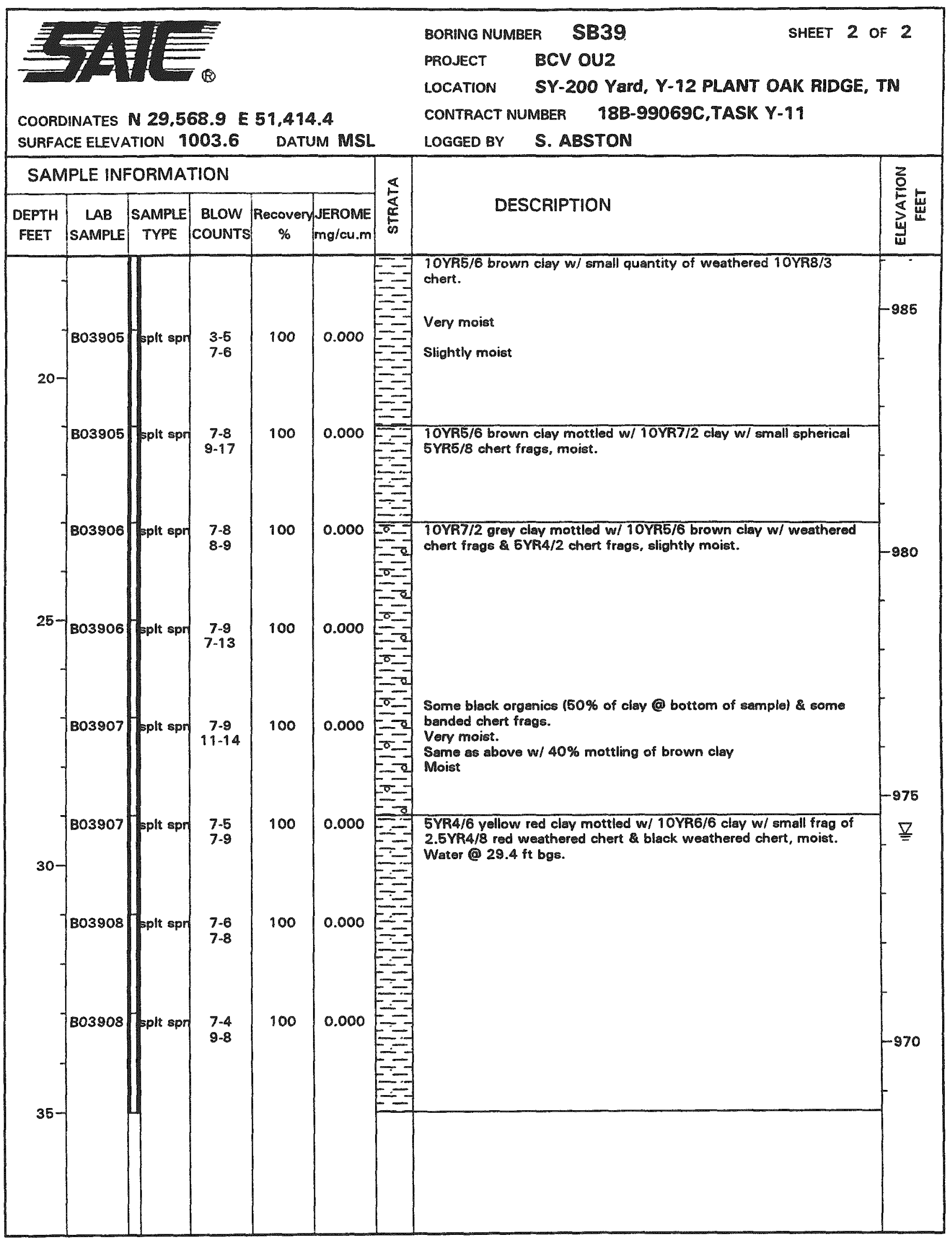




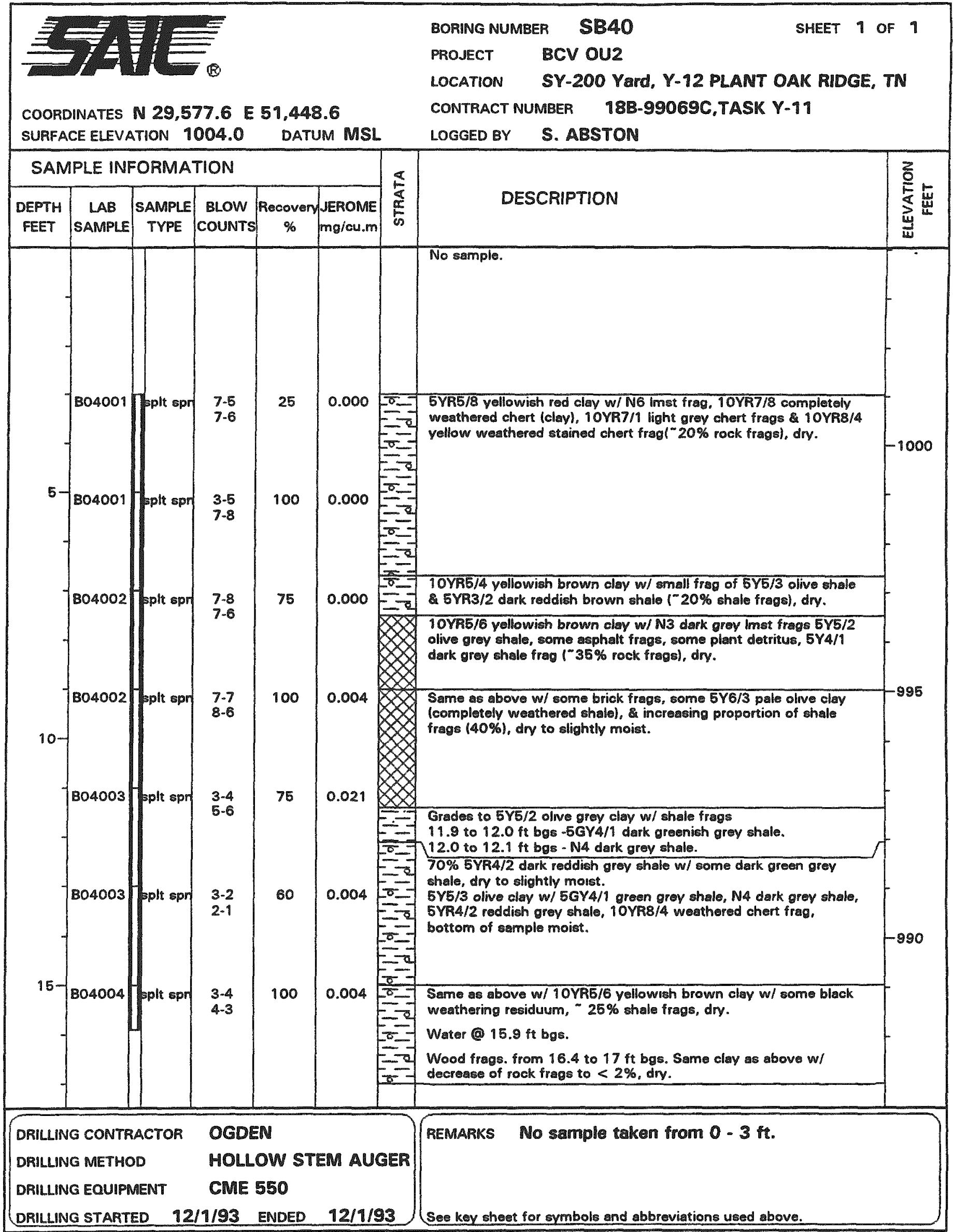




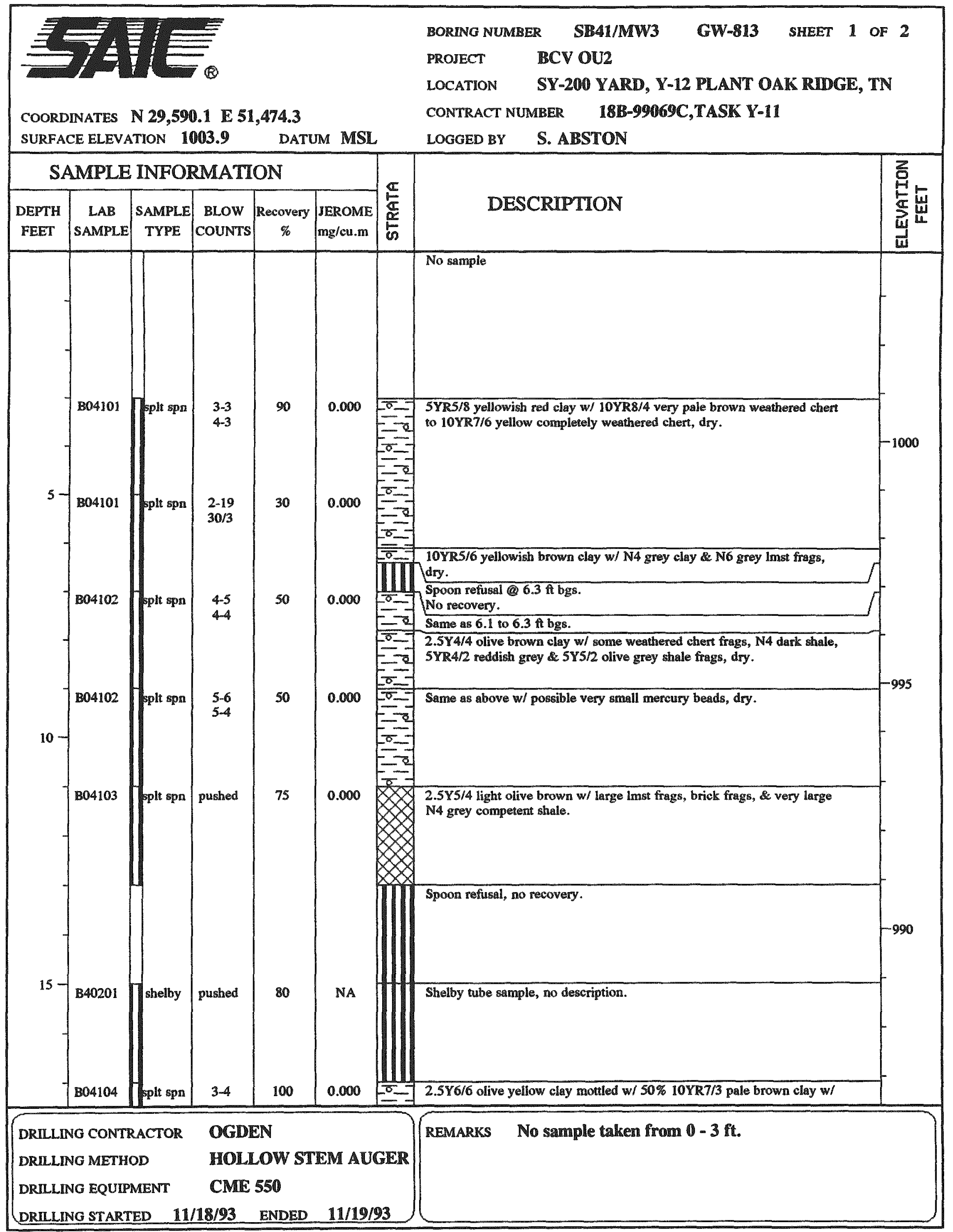




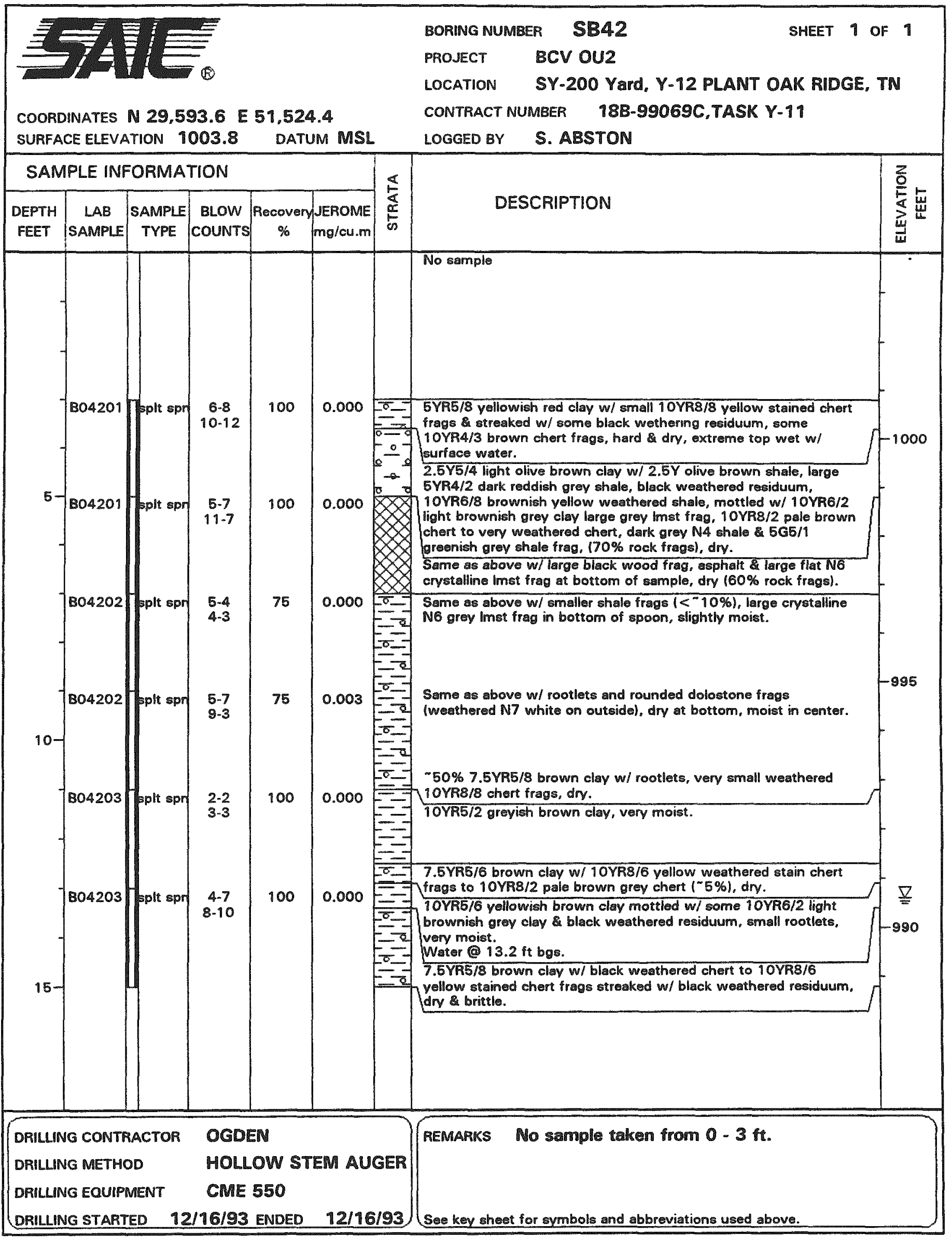




\begin{tabular}{|c|c|c|c|c|c|c|c|c|}
\hline \multicolumn{7}{|c|}{ 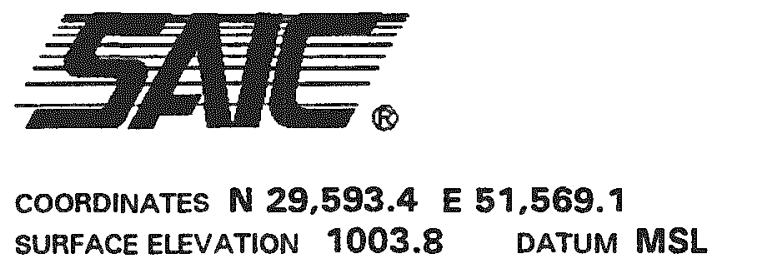 } & $\begin{array}{l}\text { BORING NUMBER } \quad \text { SB43 } \\
\text { PROJECT } \quad \text { BCV OU2 } \\
\text { LOCATION } \quad \text { SY-200 YaTd, Y-12 PLANT OAK RIDGE, } \\
\text { CONTRACT NUMBER 18B-99069C.TASK Y-11 } \\
\text { LOGGED BY } \quad \text { S. ABSTON }\end{array}$ & $\begin{array}{l}\text { OF } 2 \\
\text { TN }\end{array}$ \\
\hline $\begin{array}{c}\text { SAM } \\
\text { DEPTH } \\
\text { FEET }\end{array}$ & $\begin{array}{c}\text { LAB } \\
\text { SAMPLE }\end{array}$ & $\begin{array}{l}\text { OORMAT } \\
\begin{array}{c}\text { SAMPLE } \\
\text { TYPE }\end{array}\end{array}$ & $\left|\begin{array}{c}\text { BLOW } \\
\text { COUNTS }\end{array}\right|$ & $\mid \begin{array}{c}\text { Recovery } \\
\%\end{array}$ & $\begin{array}{l}\text { JEROME } \\
\text { mg/cu.m. }\end{array}$ & $\frac{5}{5}$ & DESCRIPTION & $\frac{Z}{O}$ \\
\hline $10-$ & B04304 & $H_{\text {pplt spr }}$ & $\begin{array}{l}5-7 \\
5.10\end{array}$ & 100 & $\begin{array}{l}0.000 \\
0.000\end{array}$ & 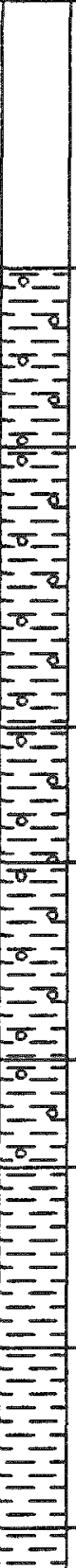 & 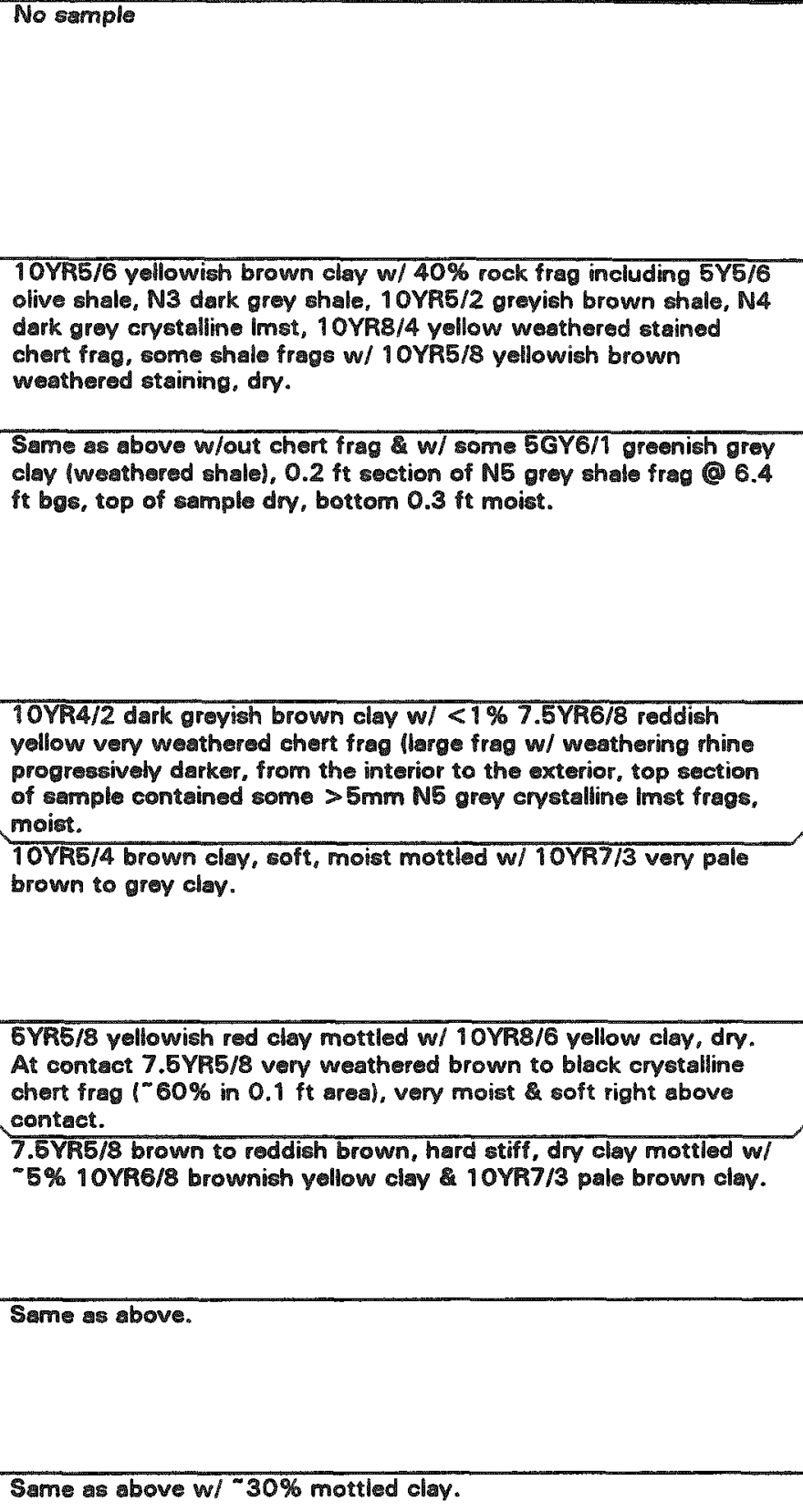 & -1000 \\
\hline $\begin{array}{l}\text { DRILLIN } \\
\text { DRILLIN } \\
\text { DRILLN } \\
\text { DRILLIN }\end{array}$ & $\begin{array}{l}\text { IG CONTR } \\
\text { IG METHO } \\
\text { IG EOUIPN } \\
\text { IG STARTE }\end{array}$ & $\begin{array}{l}\text { ACTOR } \\
\text { DD } \\
\text { MENT } \\
\text { ED } 121\end{array}$ & $\begin{array}{l}\text { OGDE } \\
\text { HOLL } \\
\text { CME } \\
\text { /8/93 }\end{array}$ & $\begin{array}{l}\text { ON } \\
\text { OW STI } \\
550 \\
\text { ENOED }\end{array}$ & И AUC & & See key sheet for symbois and abbreviations used above. & \\
\hline
\end{tabular}


A-69

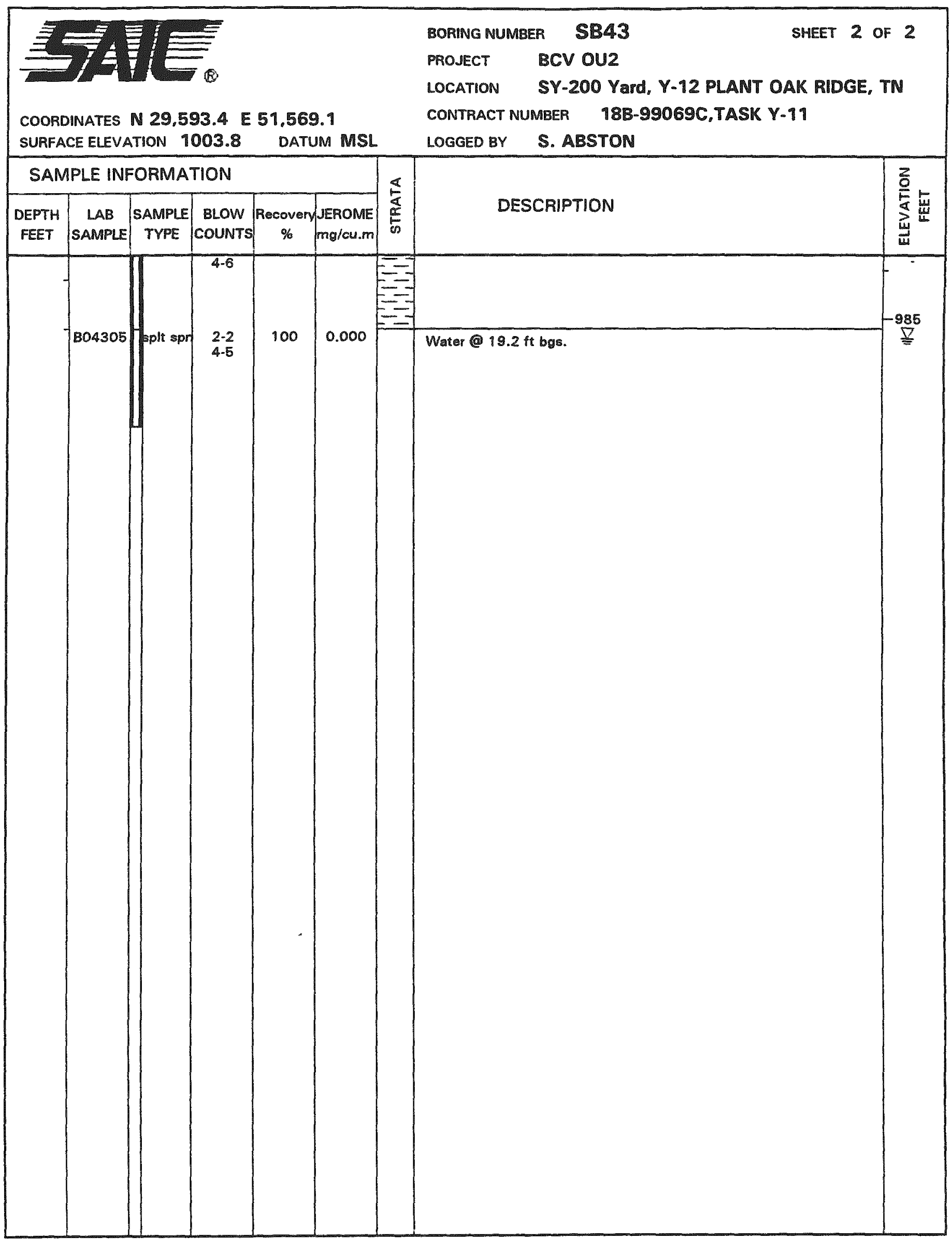




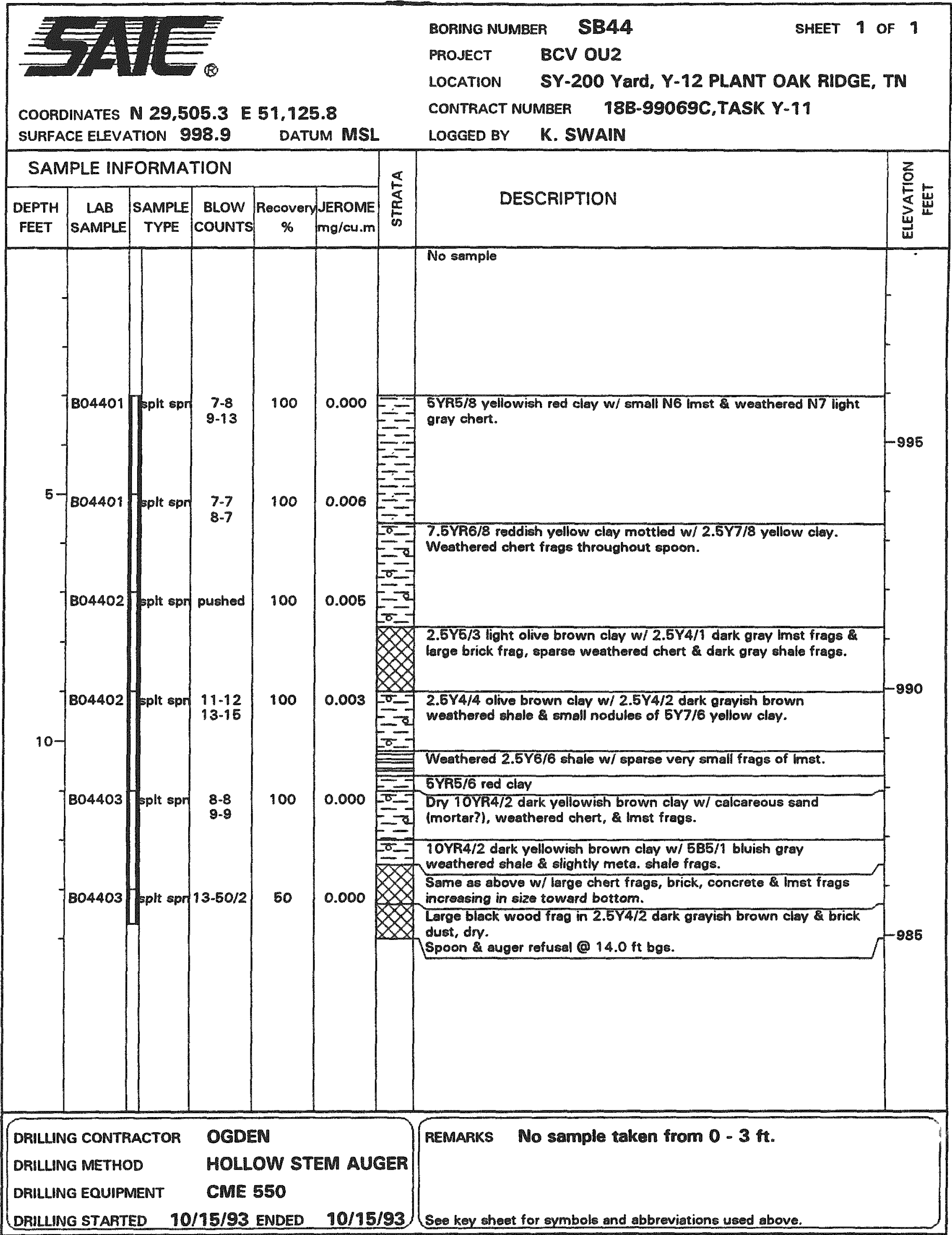




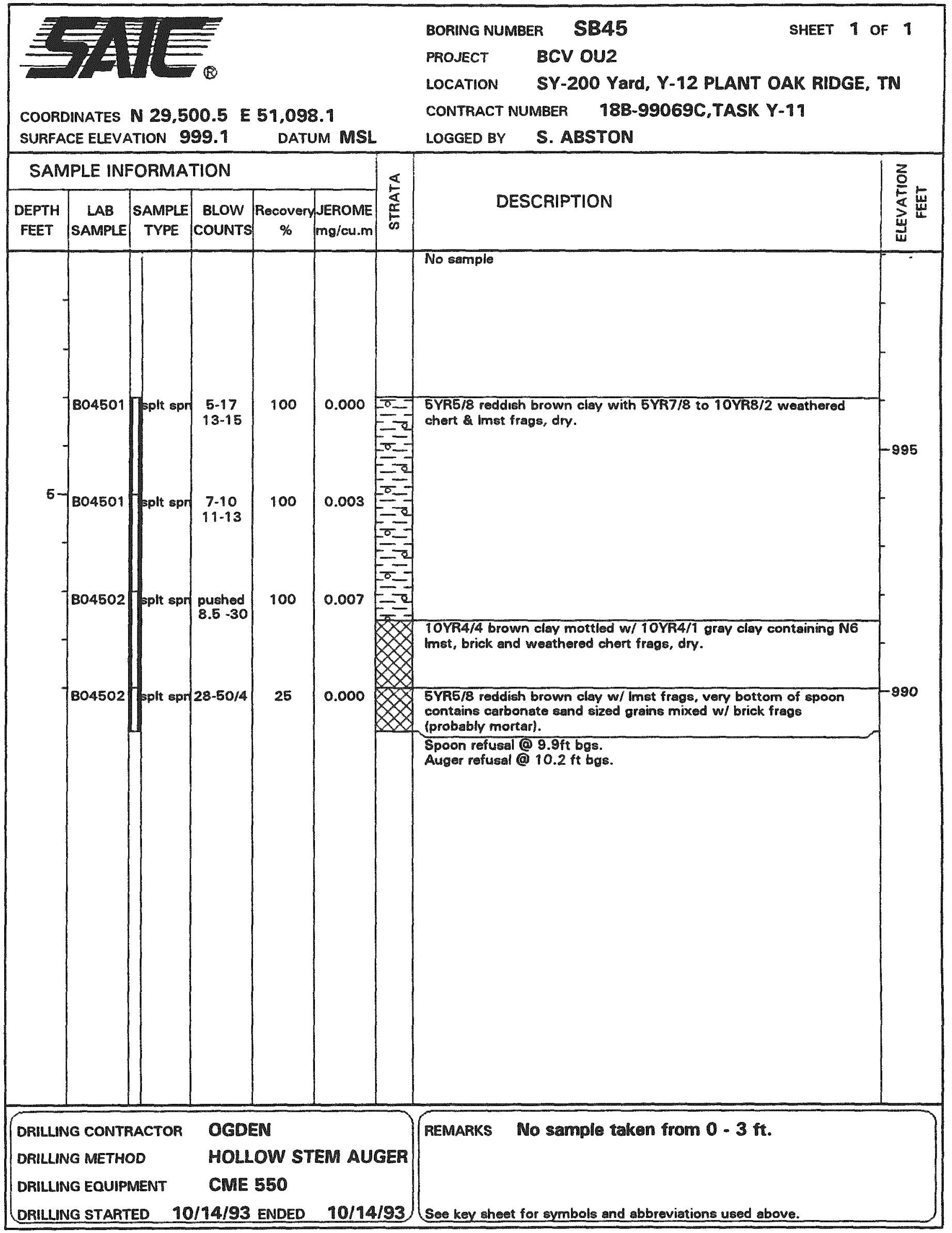


A-72

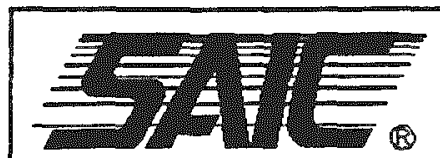

COORDINATES N 29.541.7 E $51,476.5$ SURFACE ELEVATION 1004.5 DATUM MSL
BORING NUMBER SB46

PROJECT BCV OU2

LOCATION SY-200 Yard, Y-12 PLANT OAK RIDGE, TN CONTRACT NUMBER 18B-99069C.TASK Y-11
S. ABSTON

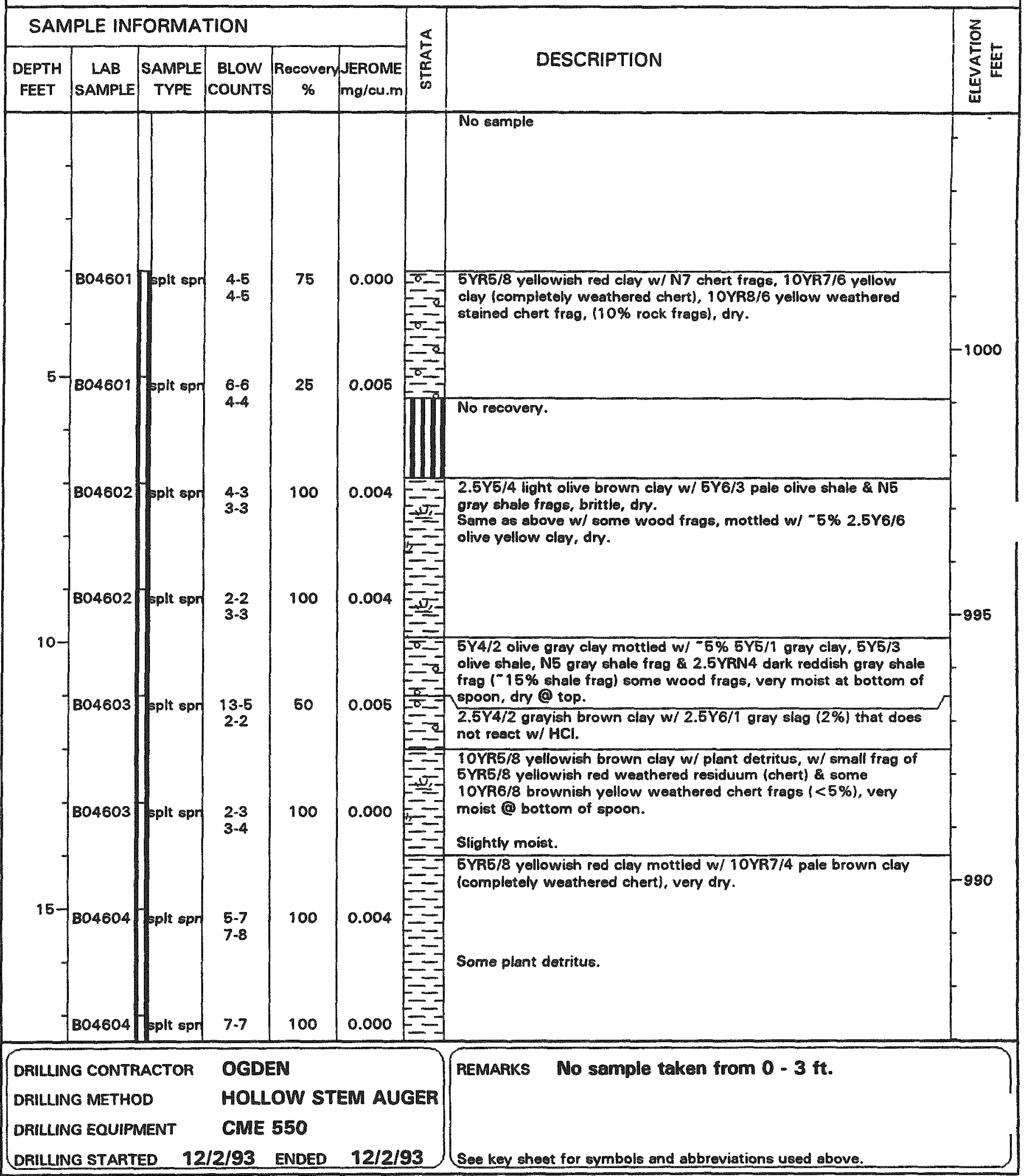


A-73

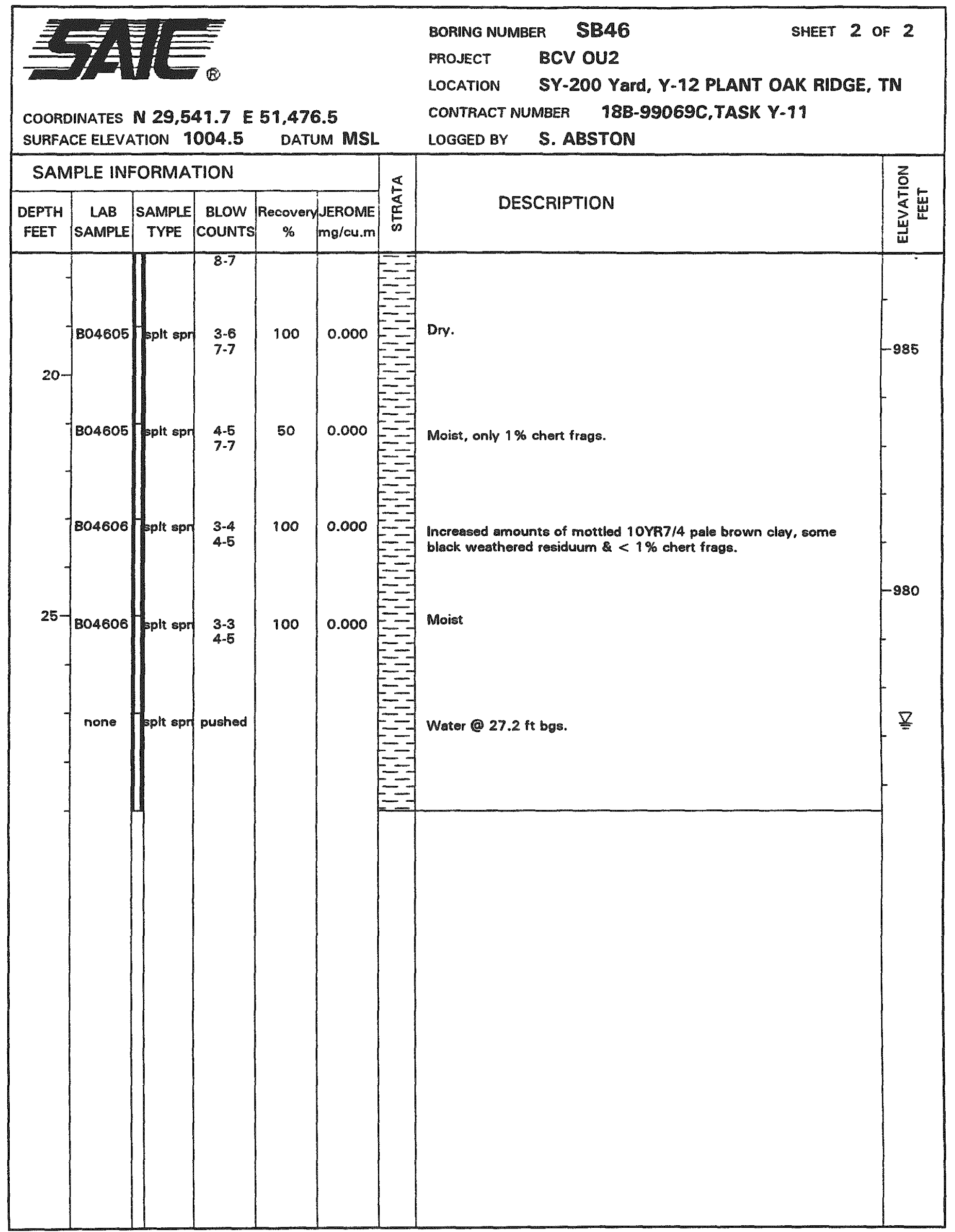




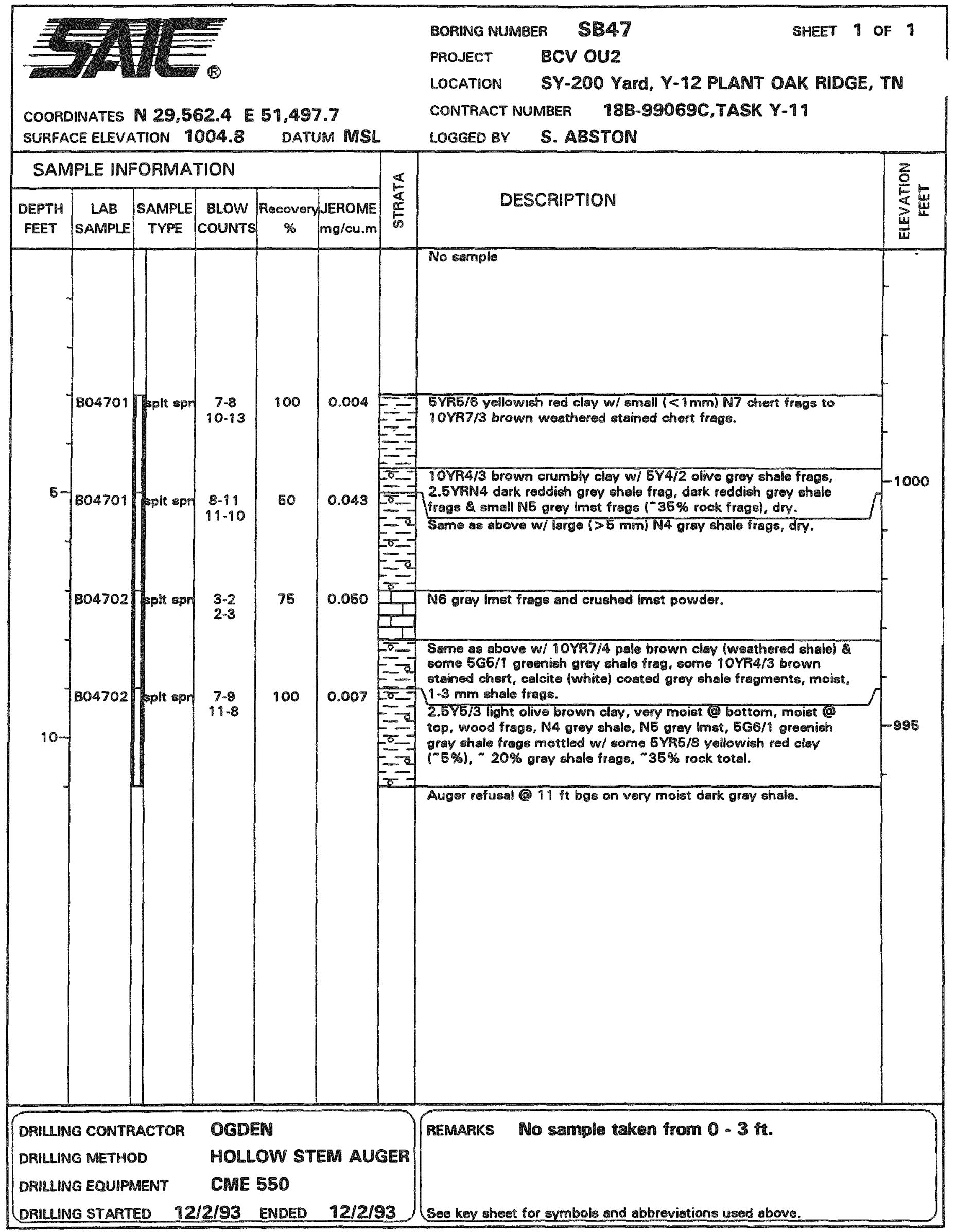




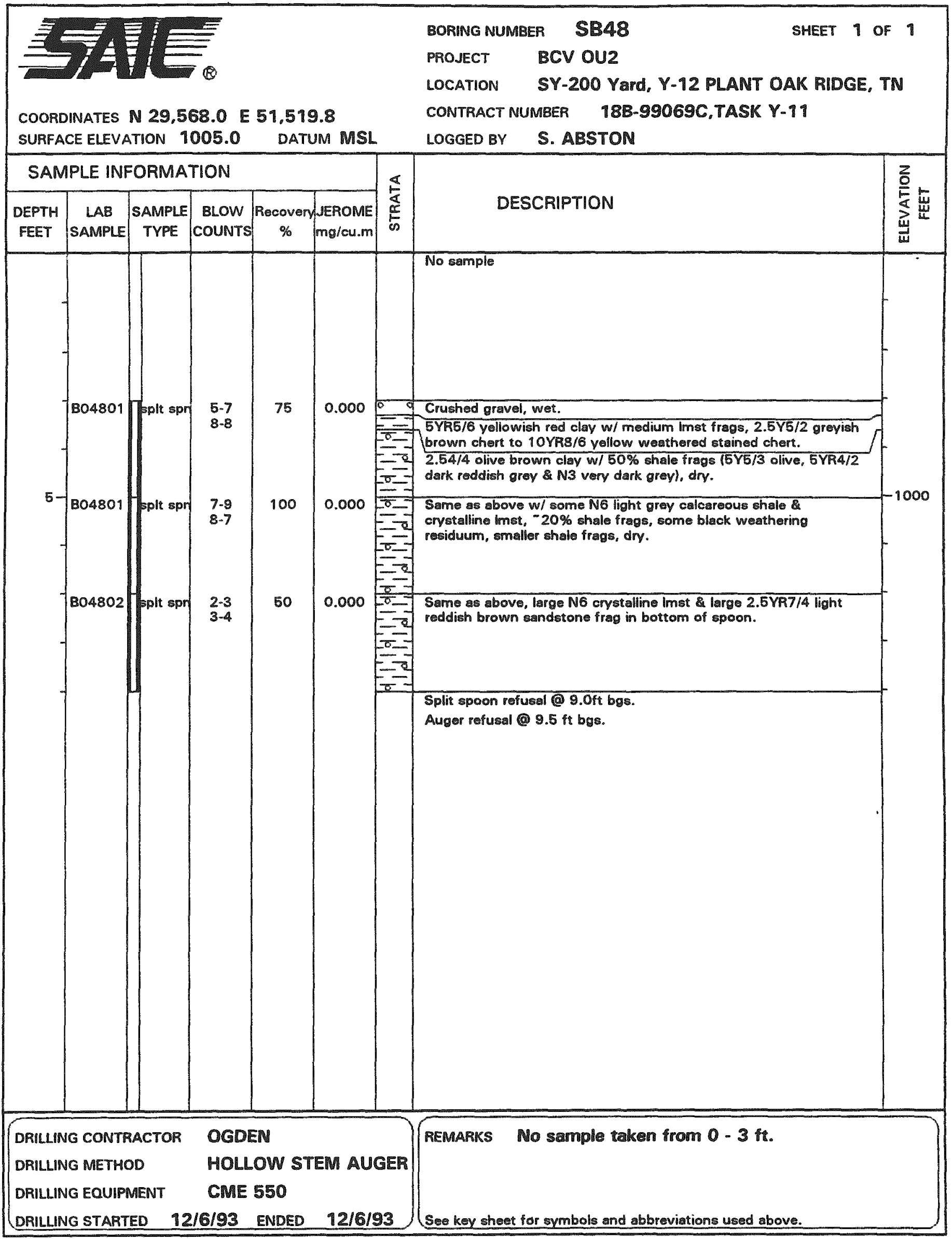




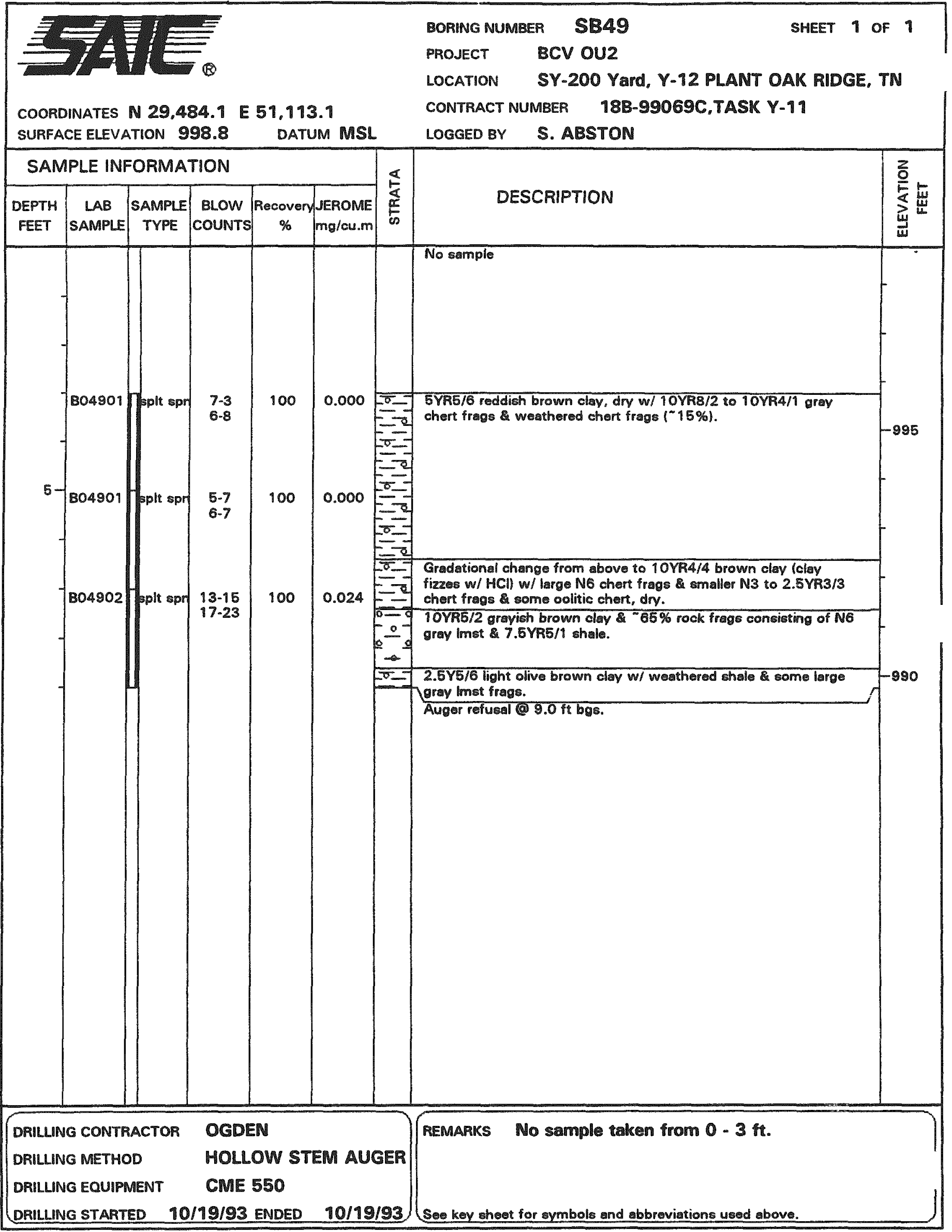




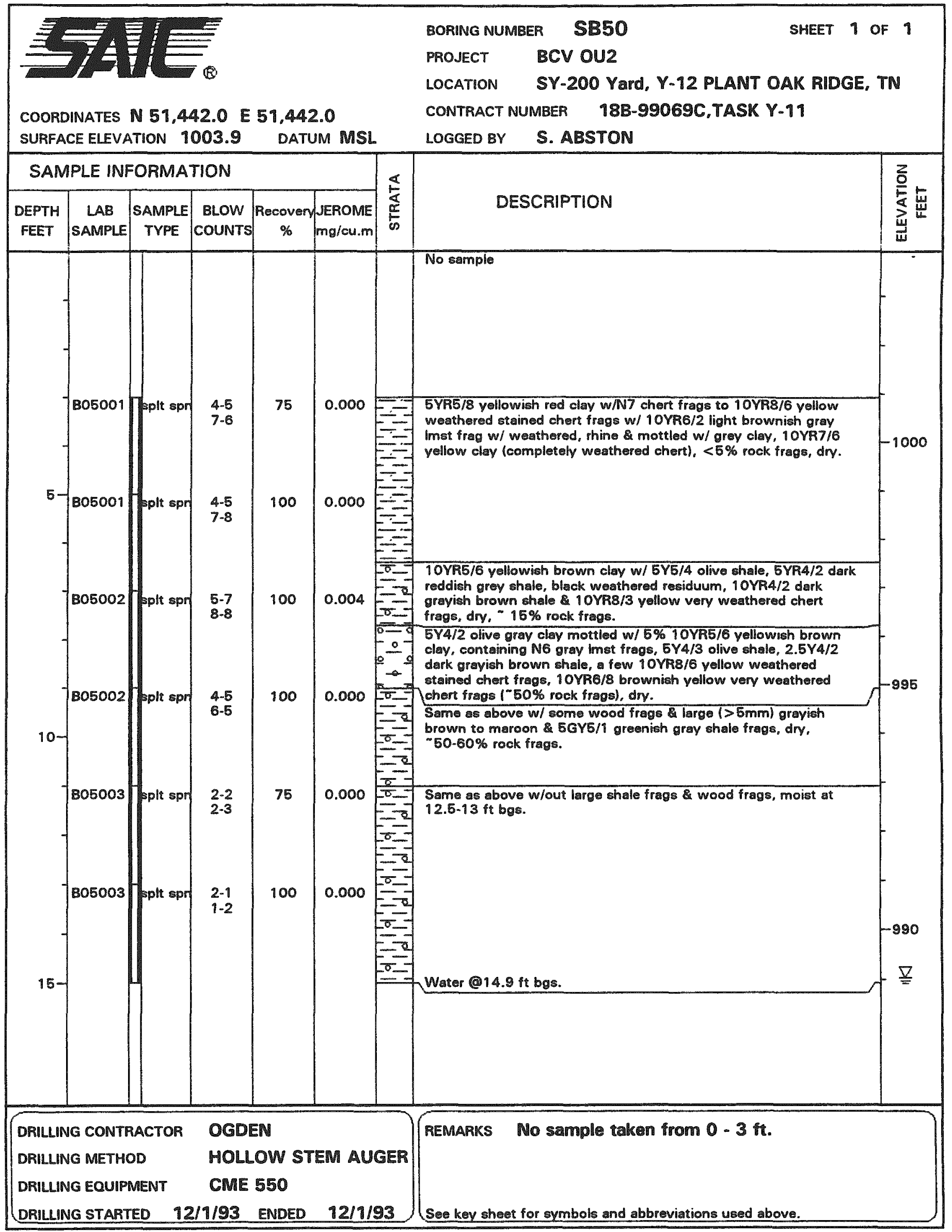




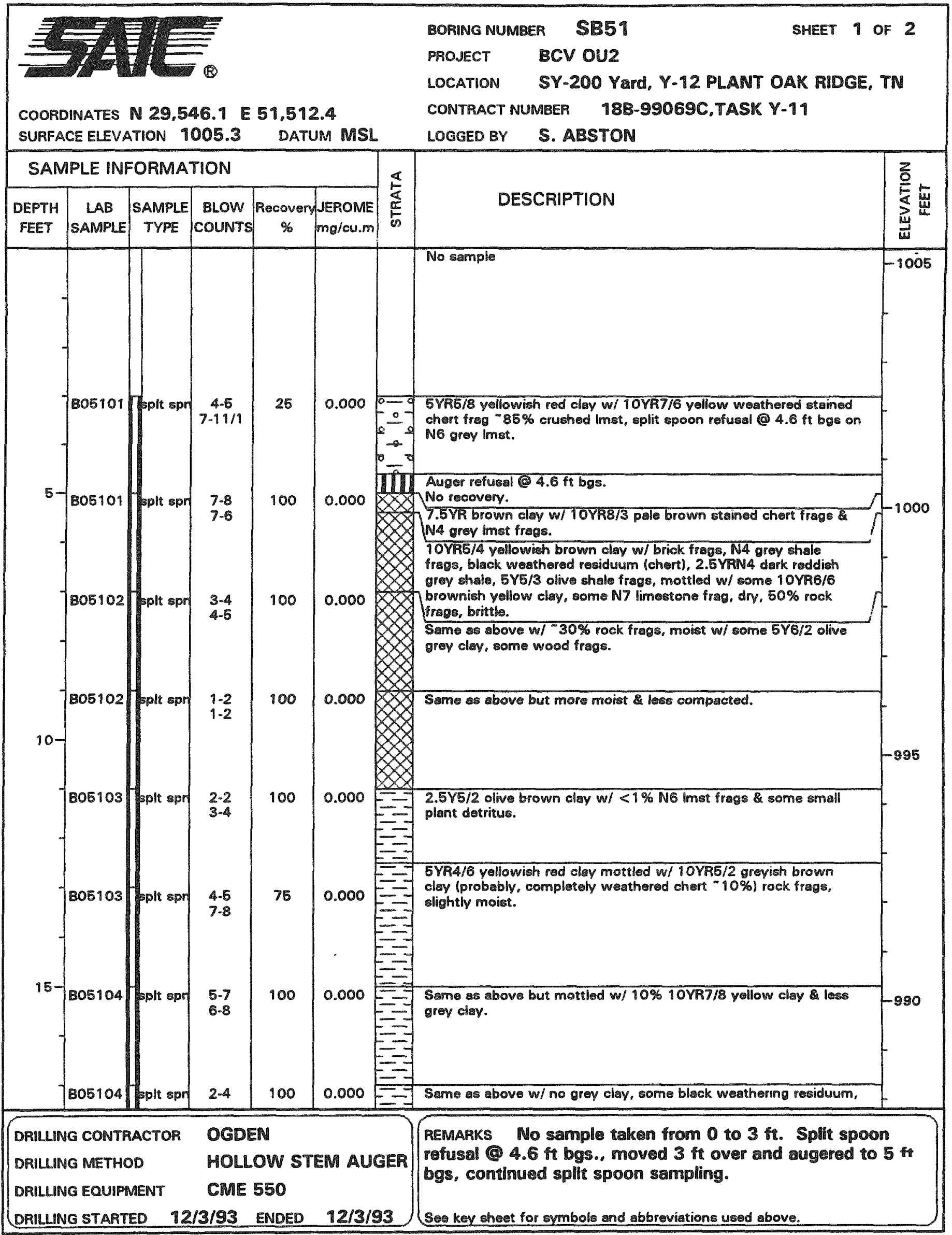




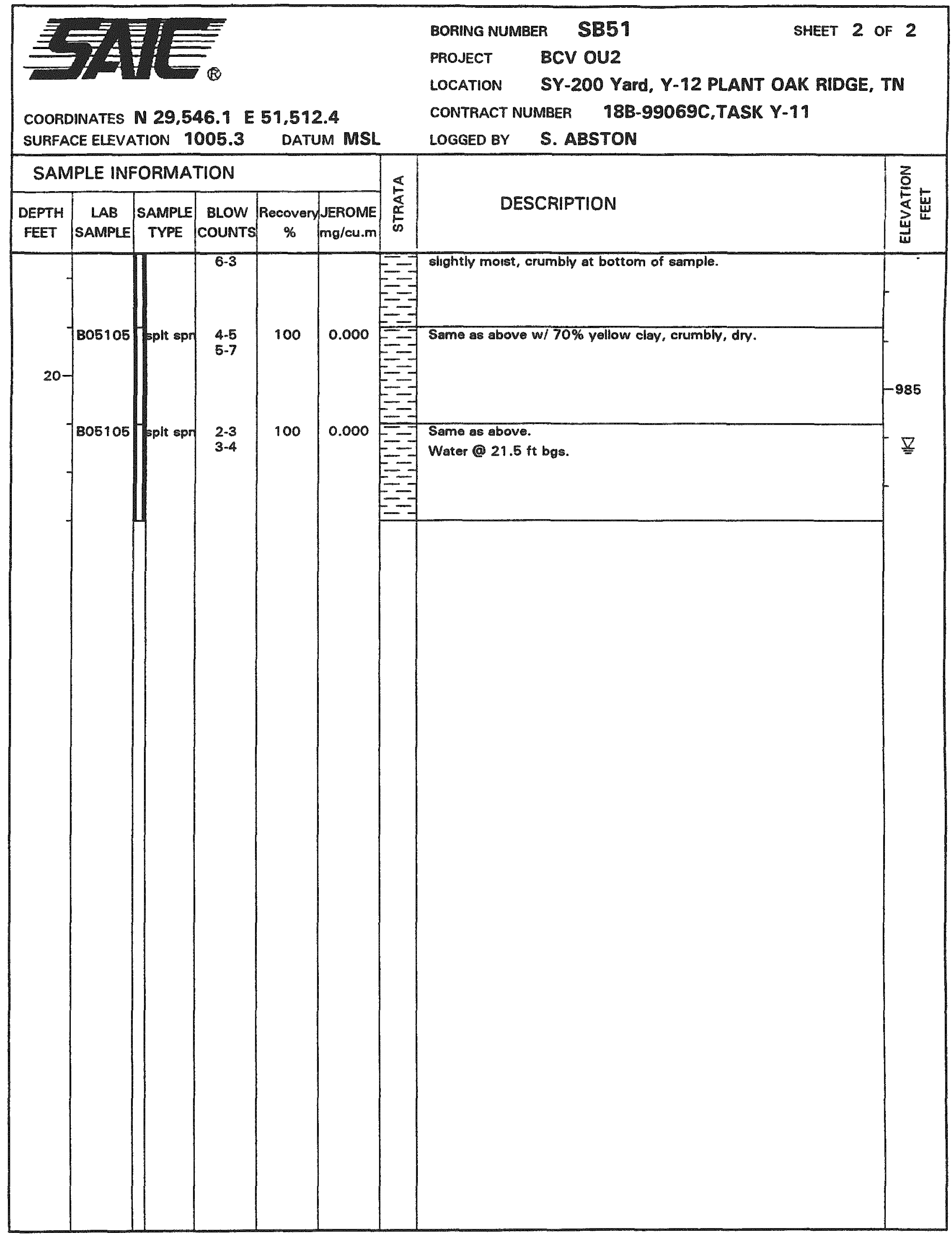




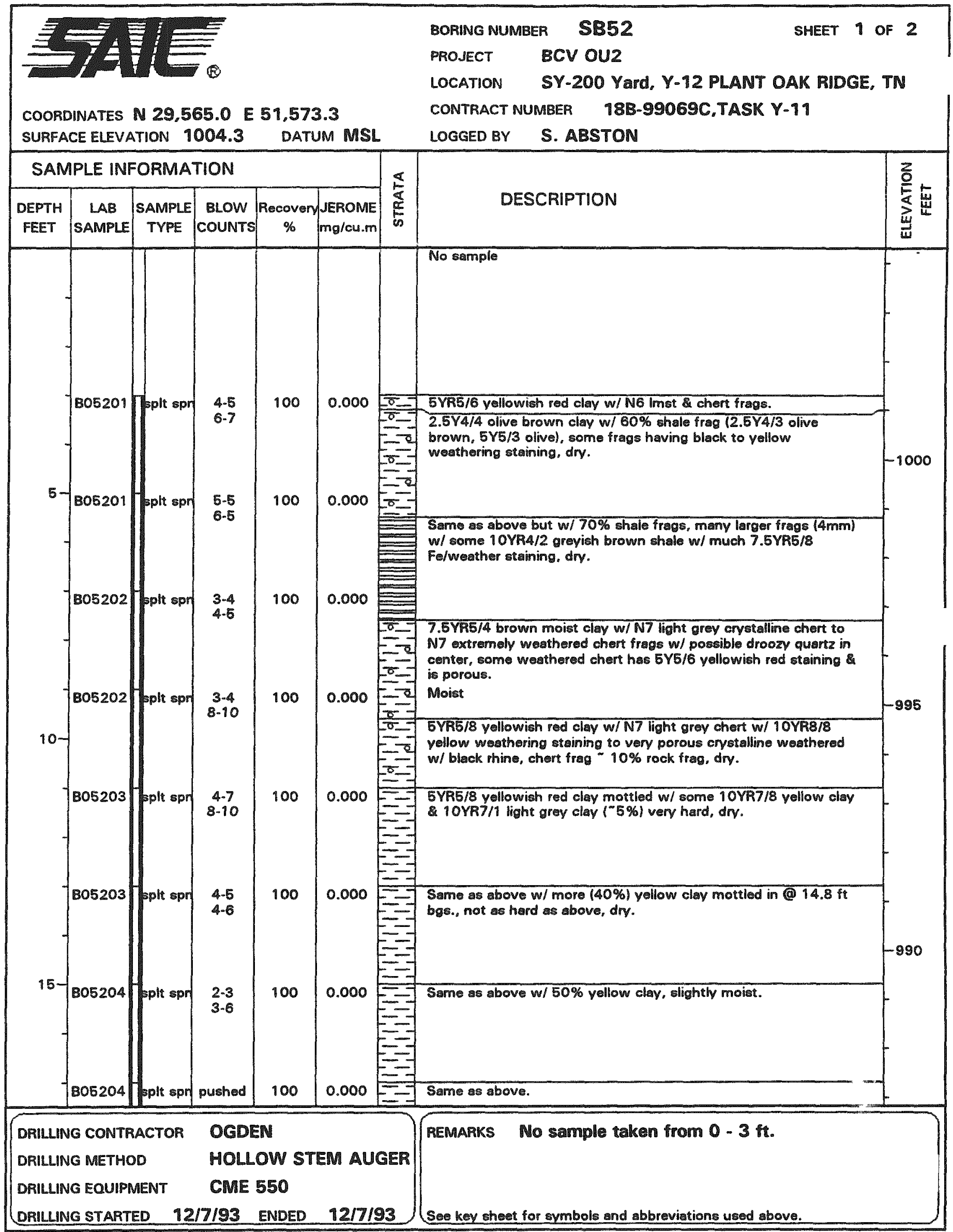




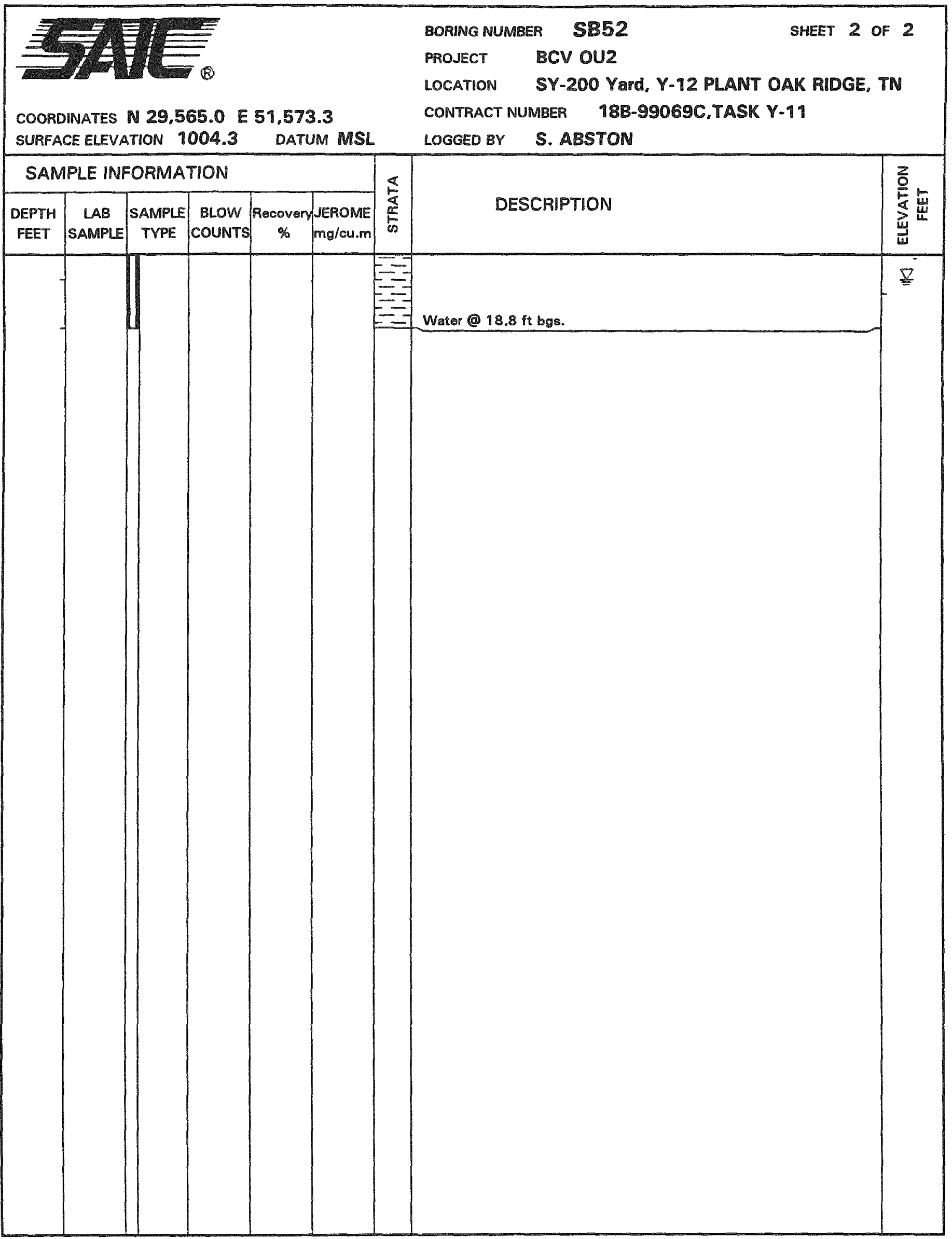




\begin{tabular}{|c|c|c|c|c|c|c|c|c|}
\hline \multicolumn{6}{|c|}{ 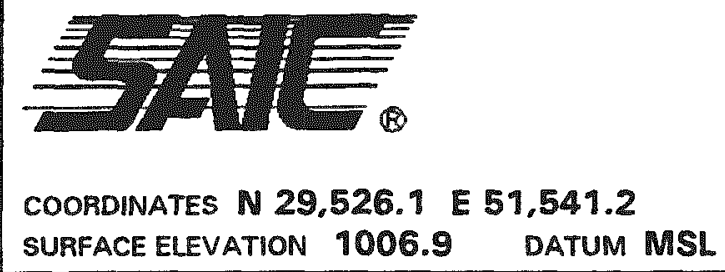 } & & $\begin{array}{ll}\text { BORING NUMBER } & \text { SB53 } \\
\text { PROJECT } & \text { BCV OU2 } \\
\text { LOCATION } & \text { SY-200 YaId, Y-12 PLANT OAK RIDGE. }\end{array}$ & OF 2 \\
\hline $10-$ & BO5302 & splt spn & $\begin{array}{l}4-5 \\
7-8\end{array}$ & 100 & 0.000 & 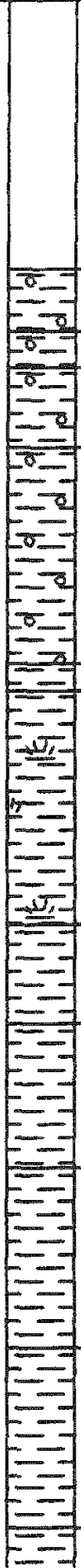 & 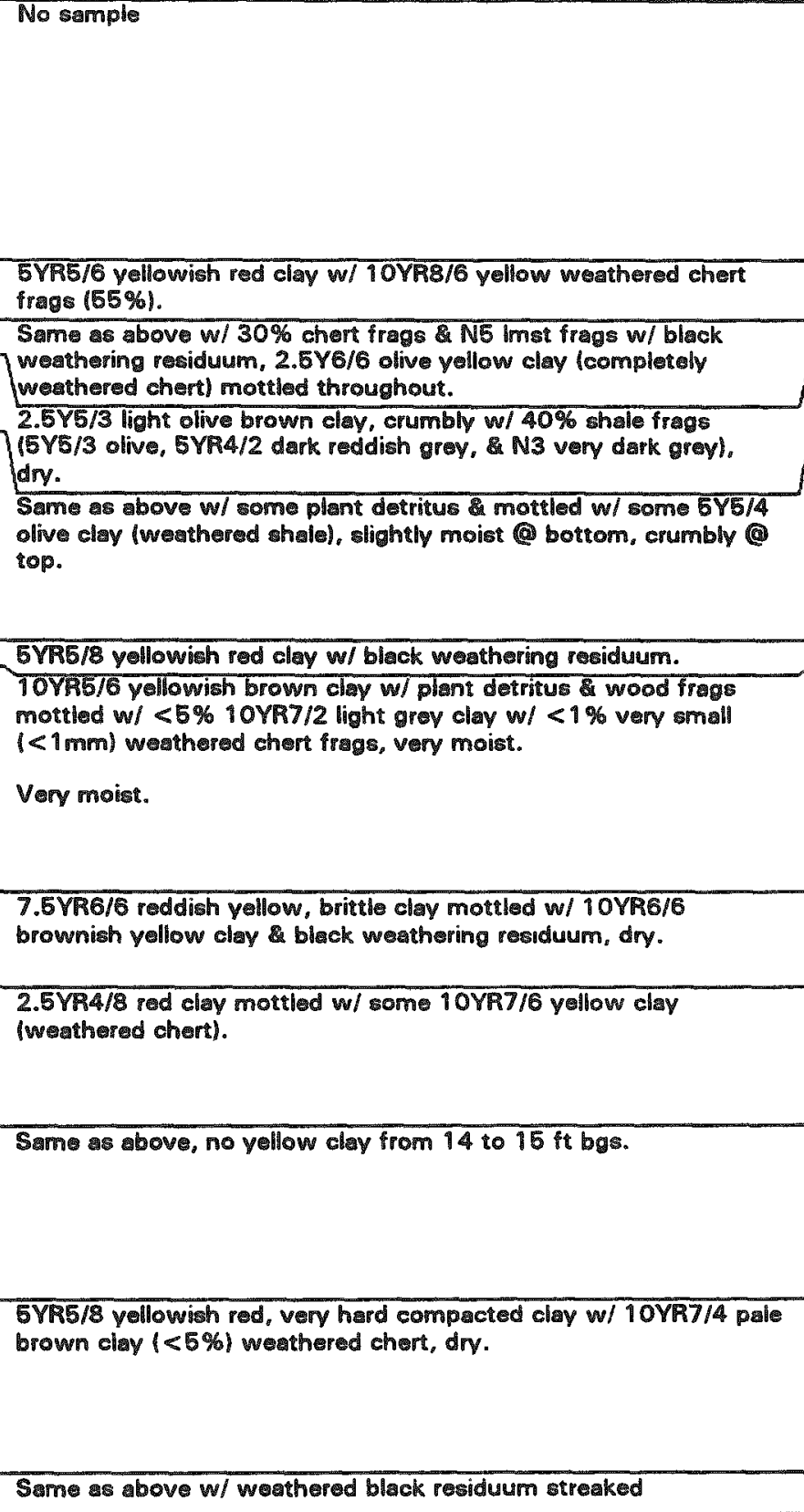 & -1005 \\
\hline \multicolumn{3}{|c|}{$\begin{array}{l}\text { DRILLING CONTRAC } \\
\text { DRILLING METHOD } \\
\text { DRILLING EQUIPMEN } \\
\text { DRILLING STARTED }\end{array}$} & $\begin{array}{l}\text { OGDE } \\
\text { HOLL } \\
\text { CME } \\
16 / 93 \\
\end{array}$ & $\begin{array}{l}\text { EN } \\
\text { OW ST } \\
550 \\
\text { ENDED }\end{array}$ & EM AU & GER & REMARKS No sample taken from 0.3 ft. & \\
\hline
\end{tabular}




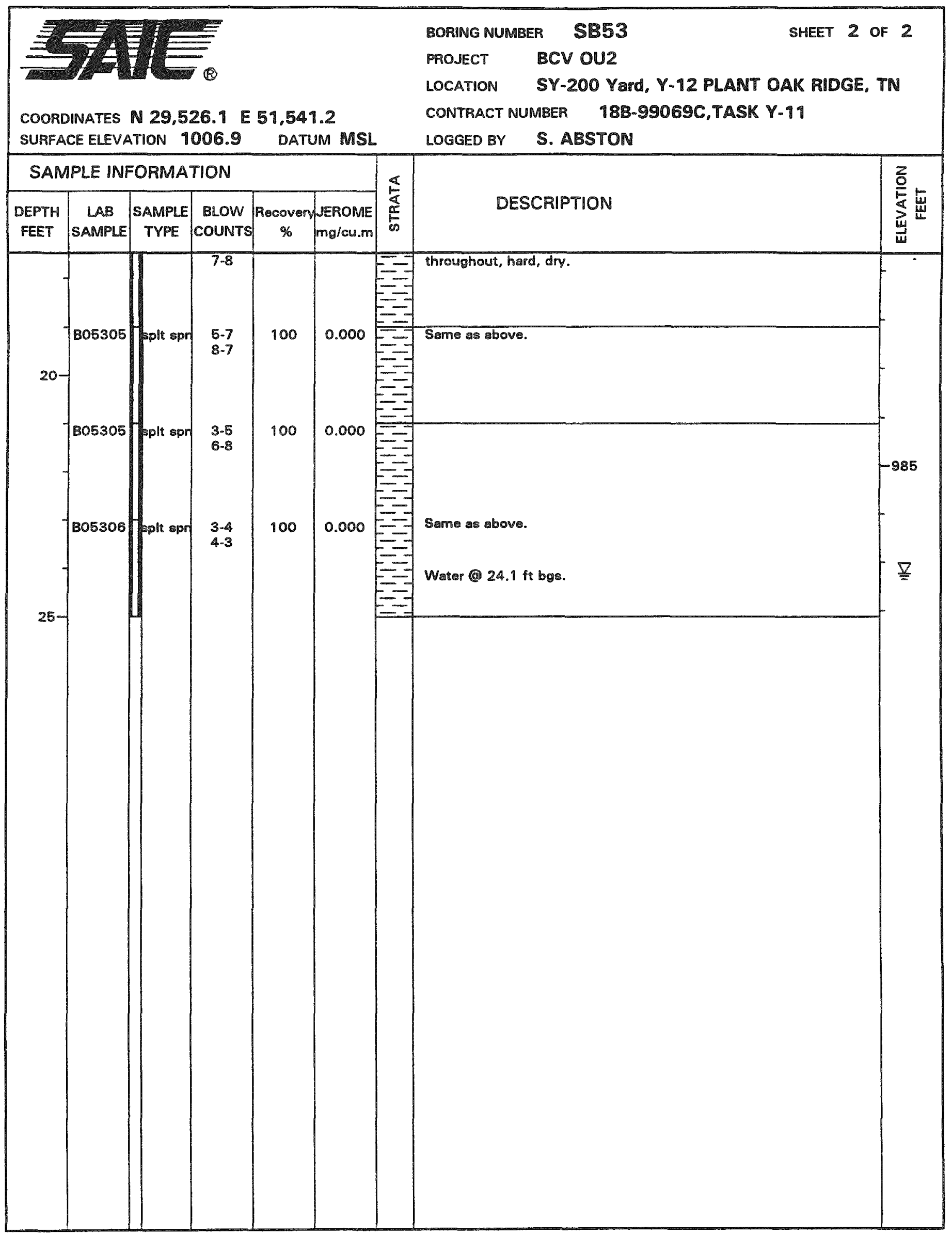




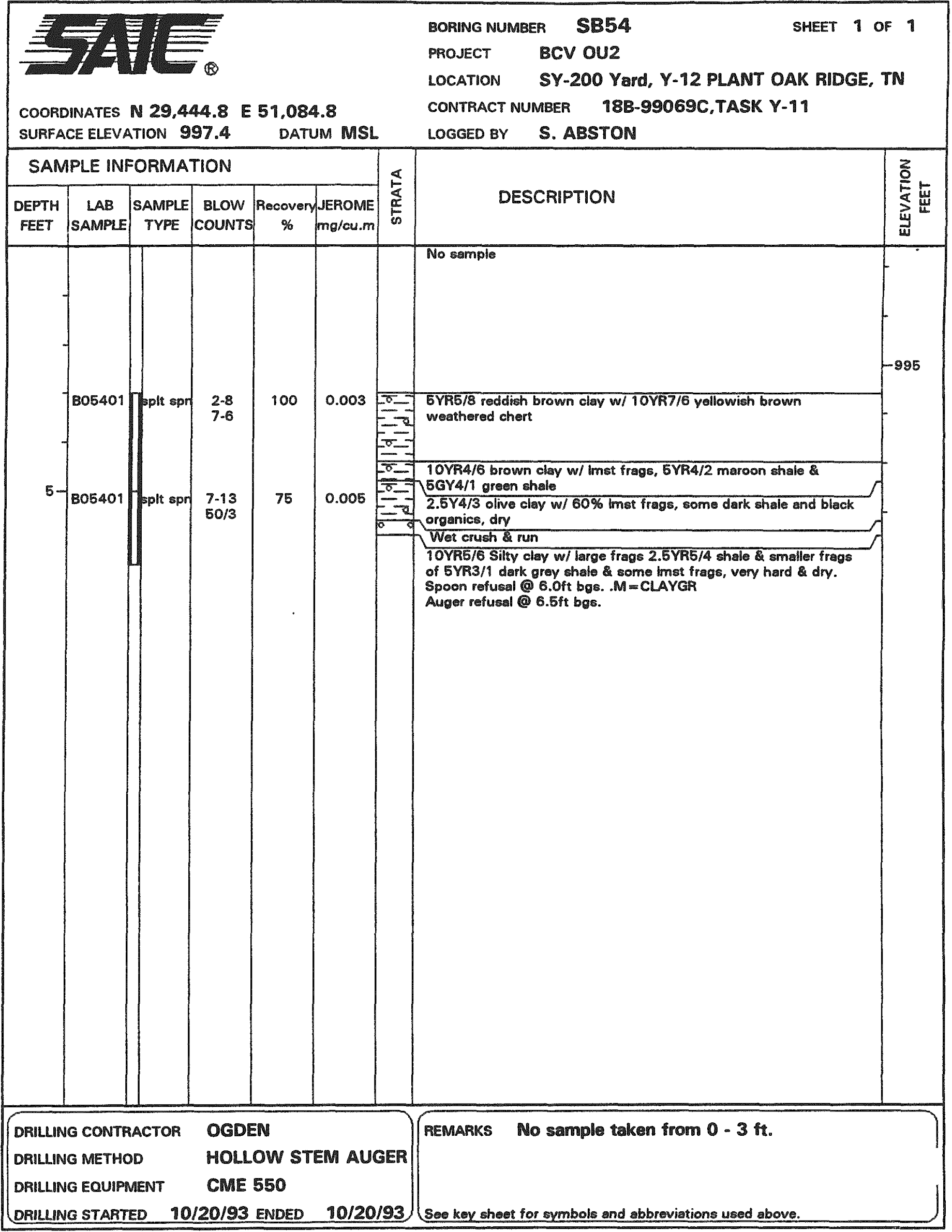




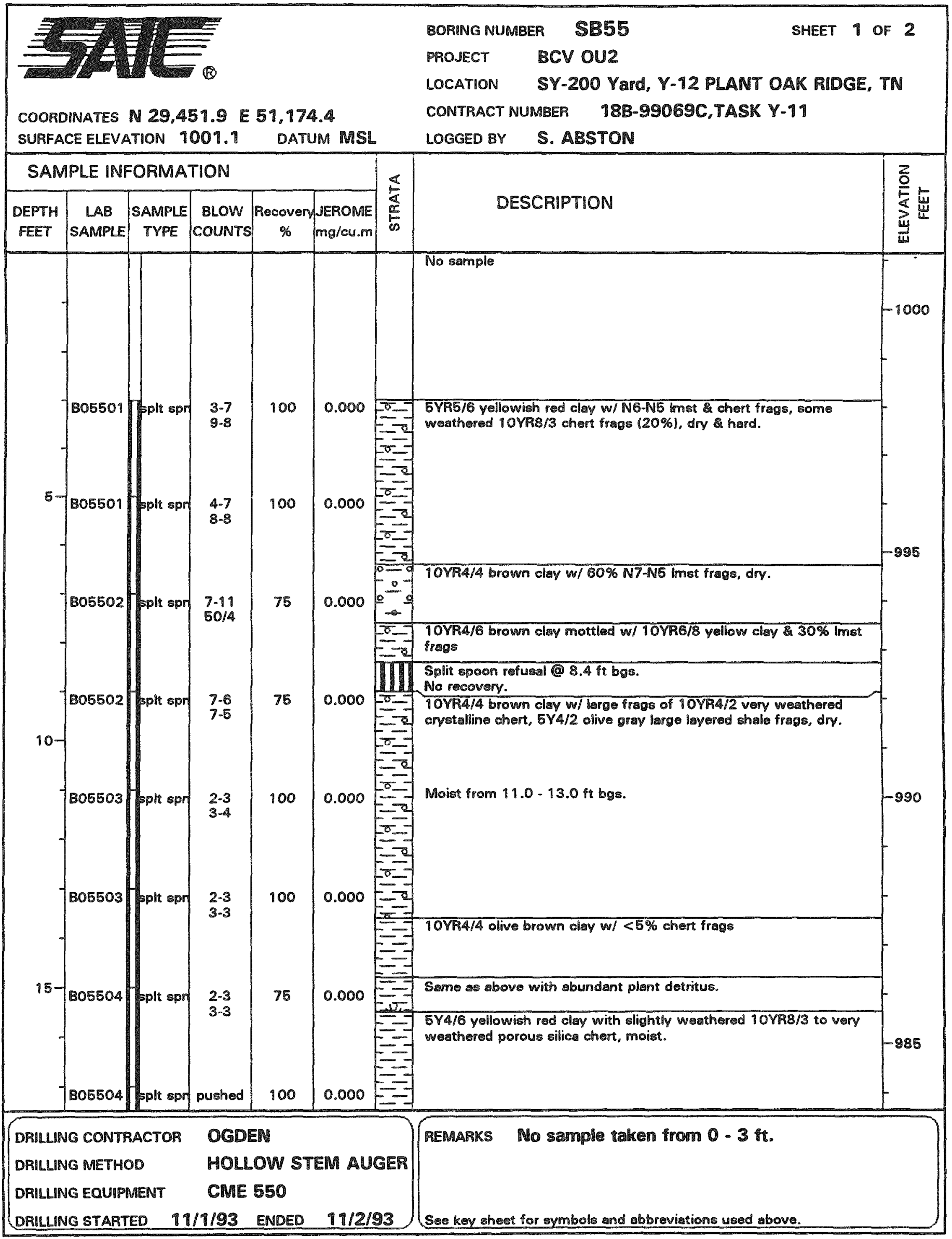


$0^{\infty 0}$

A-86

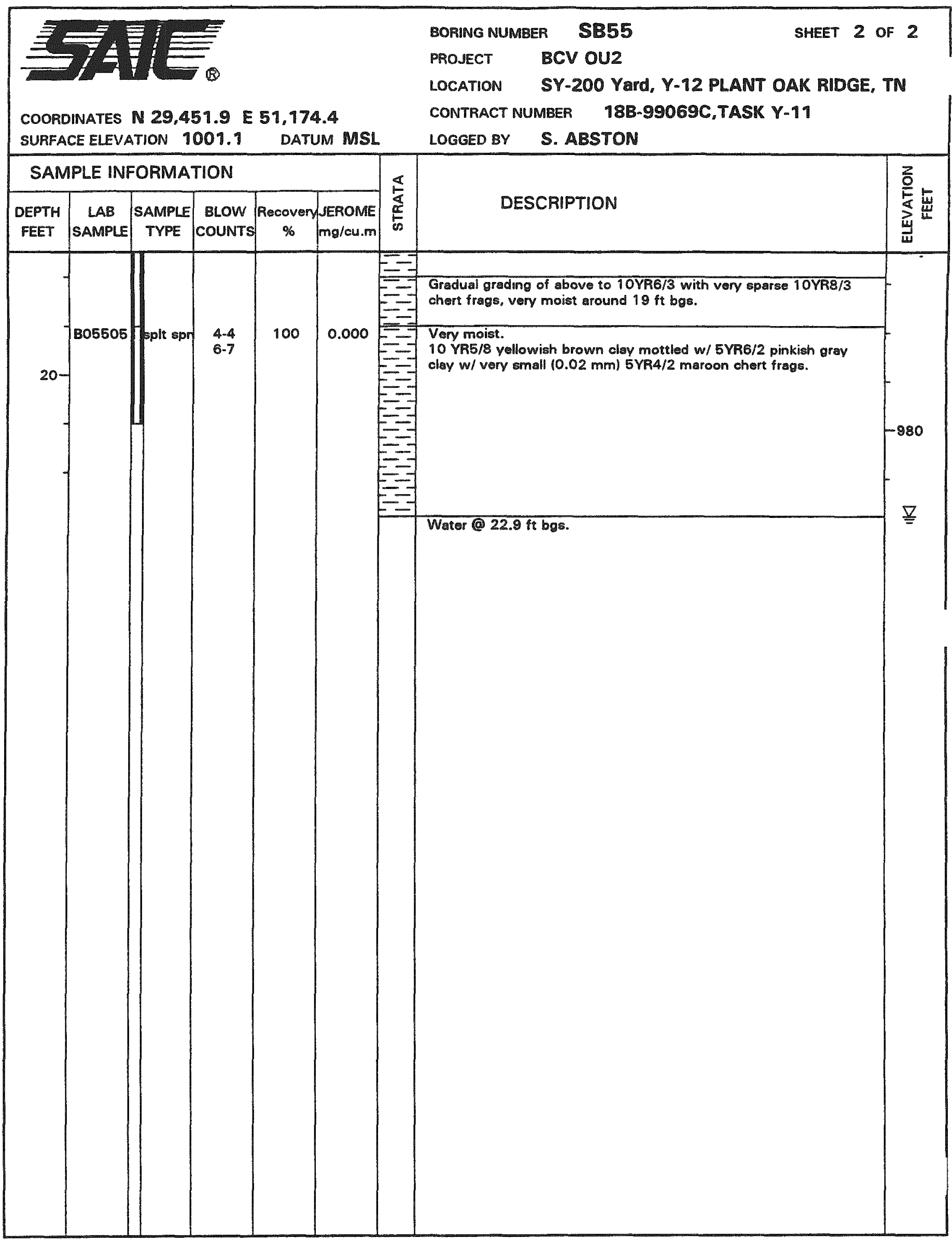




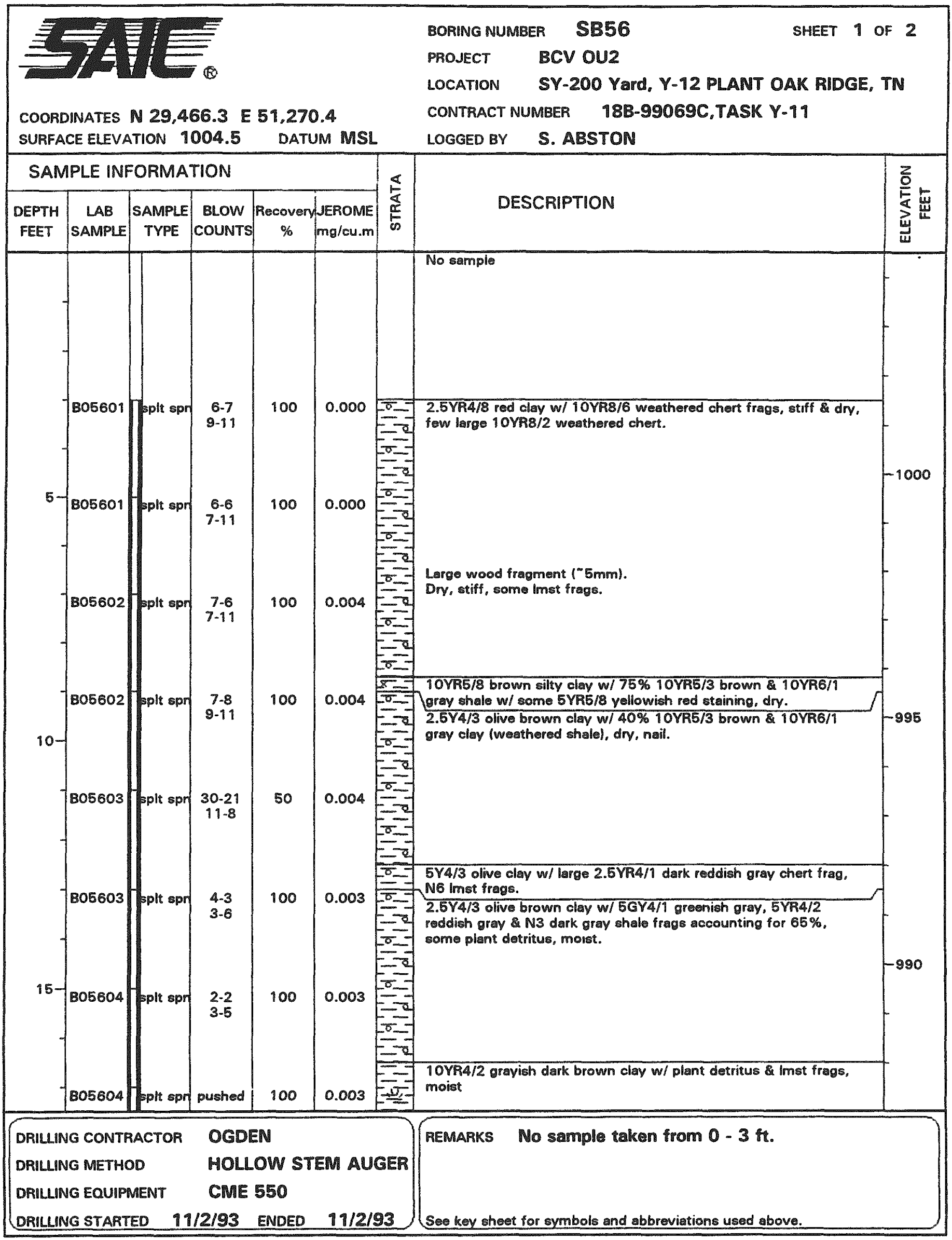




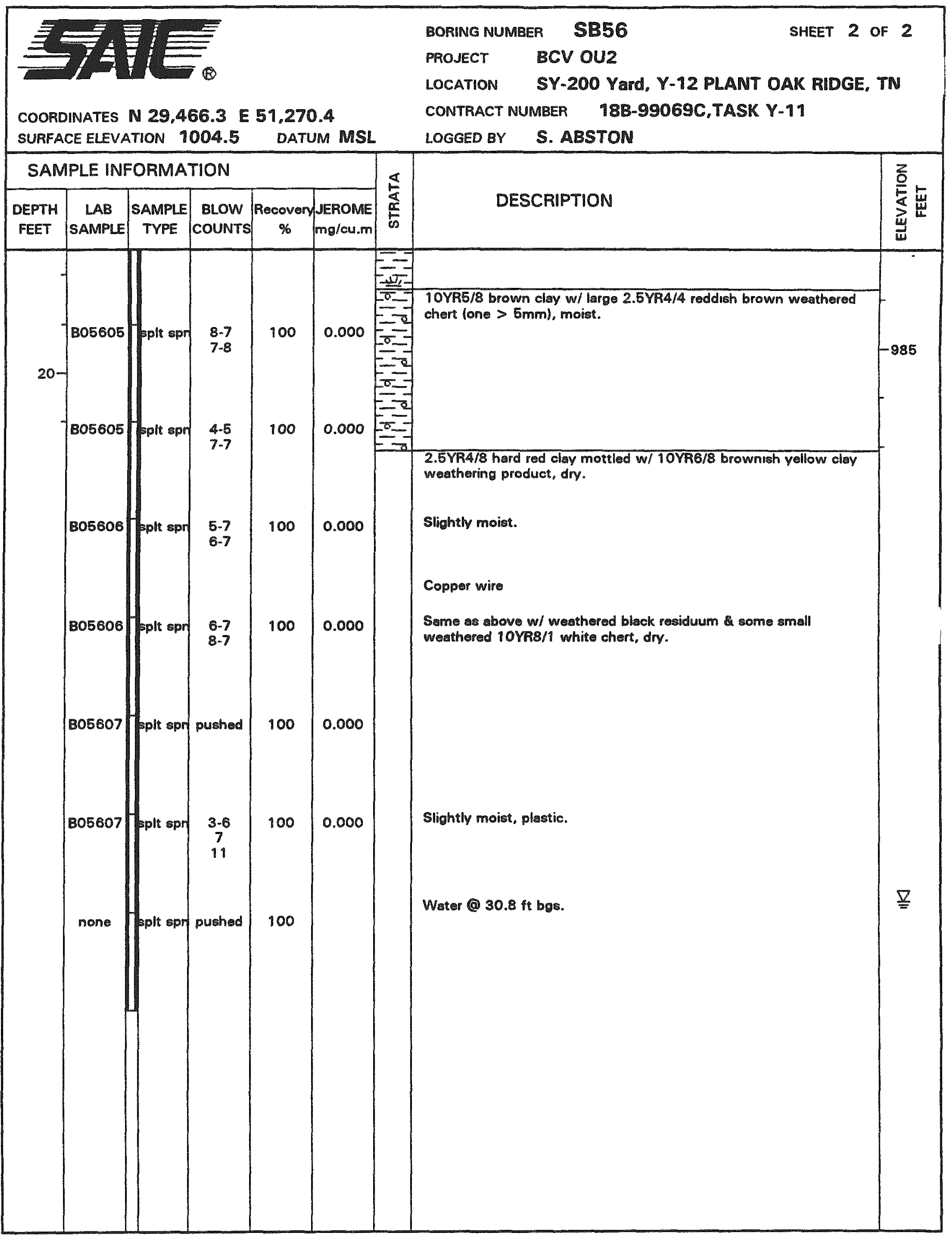




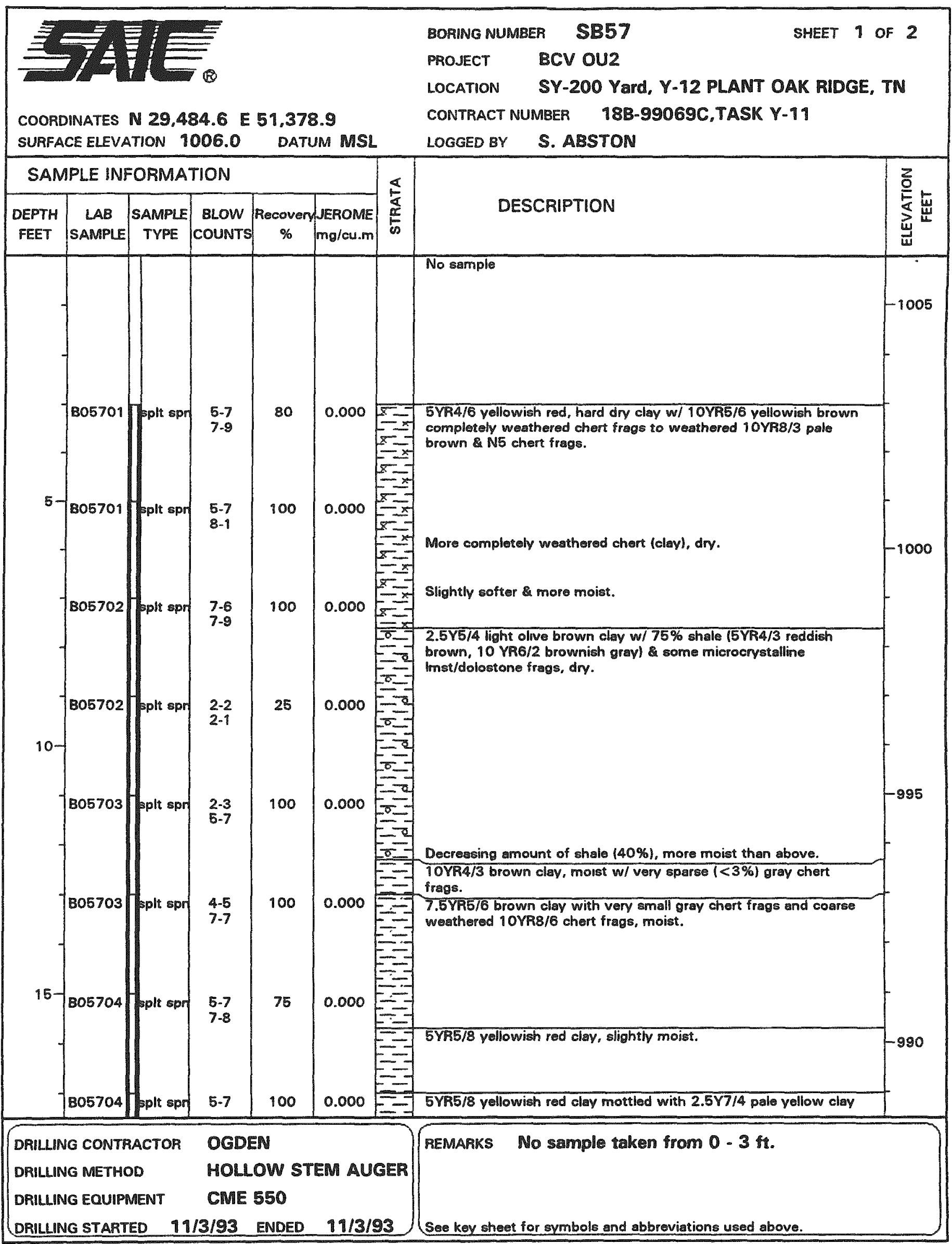




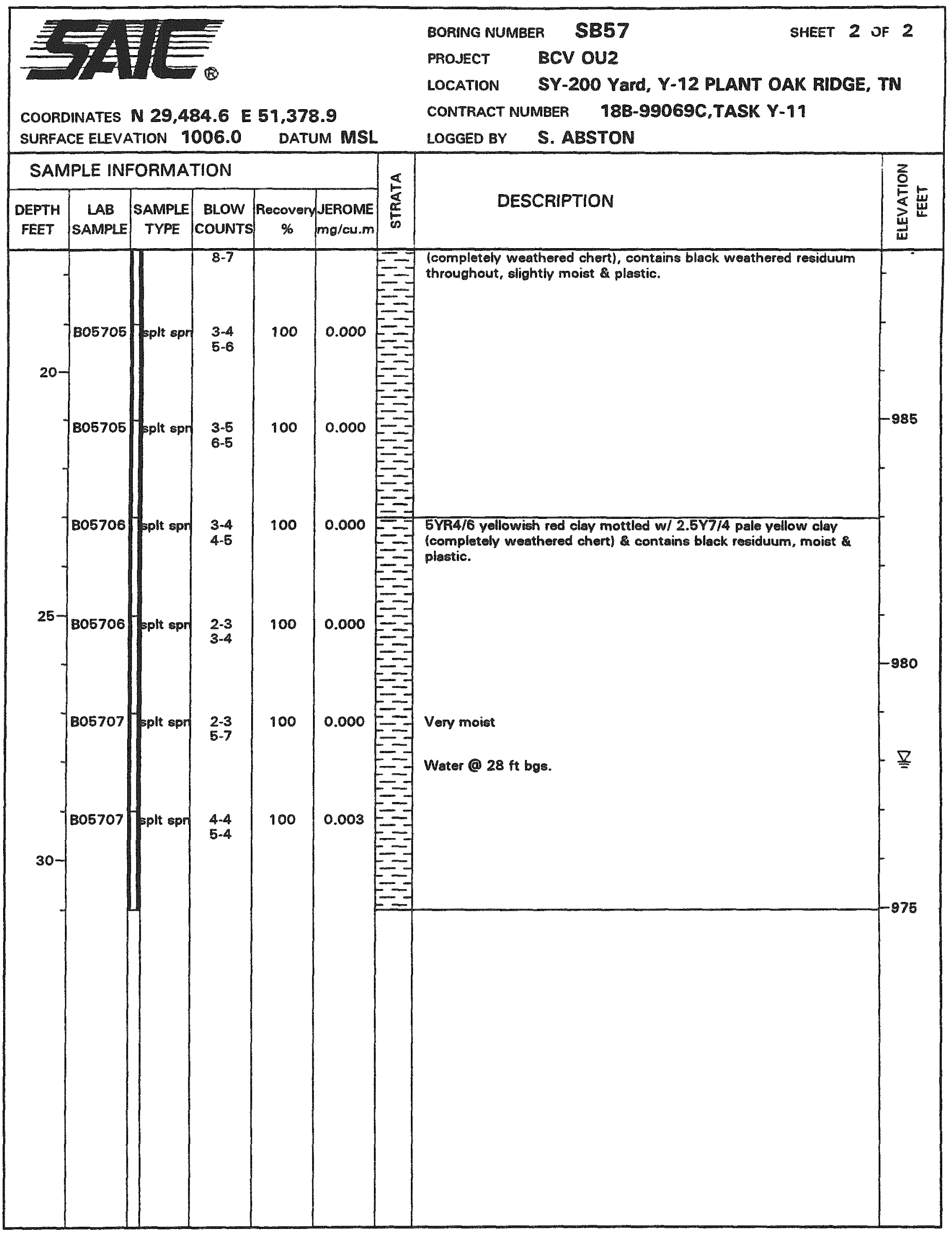




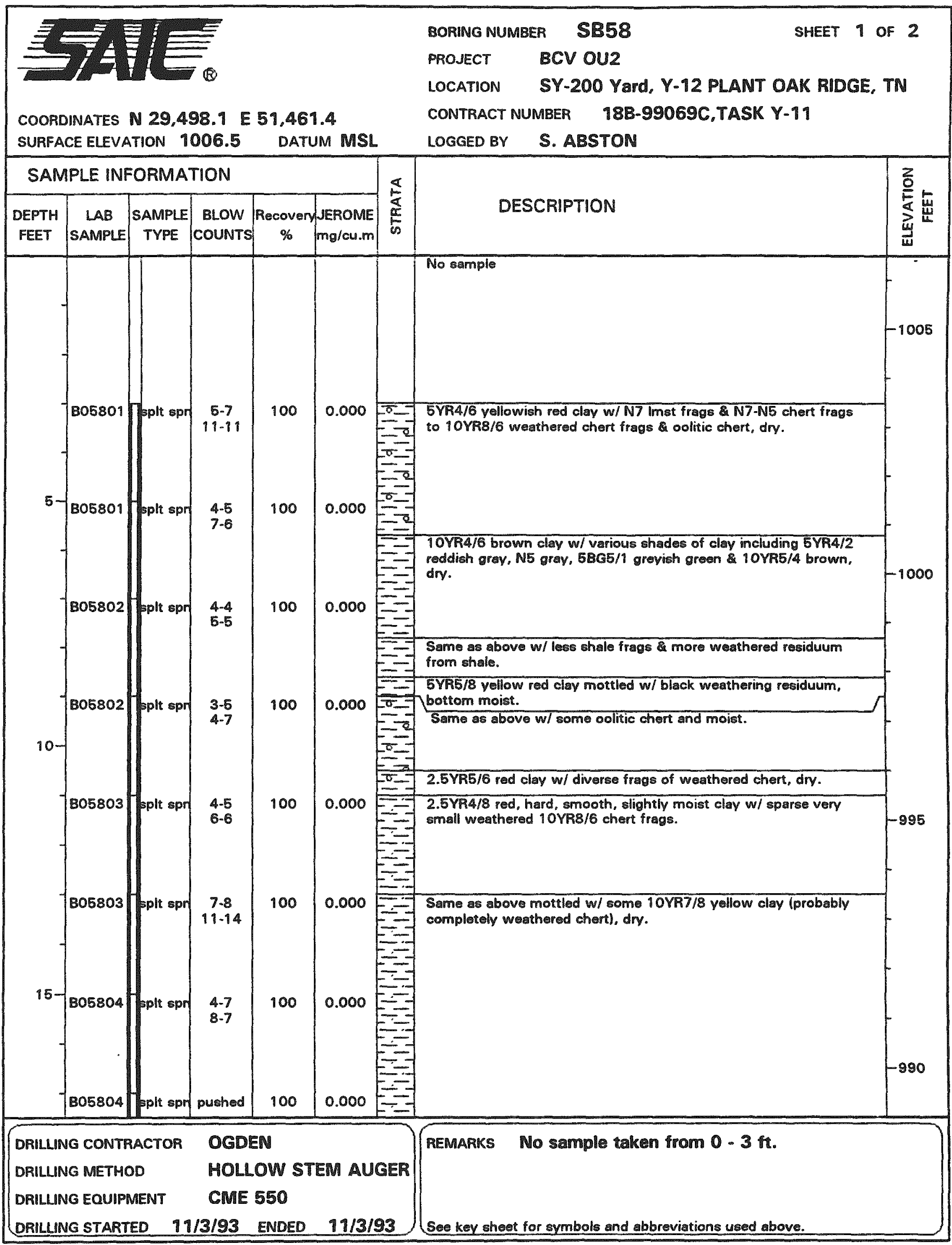




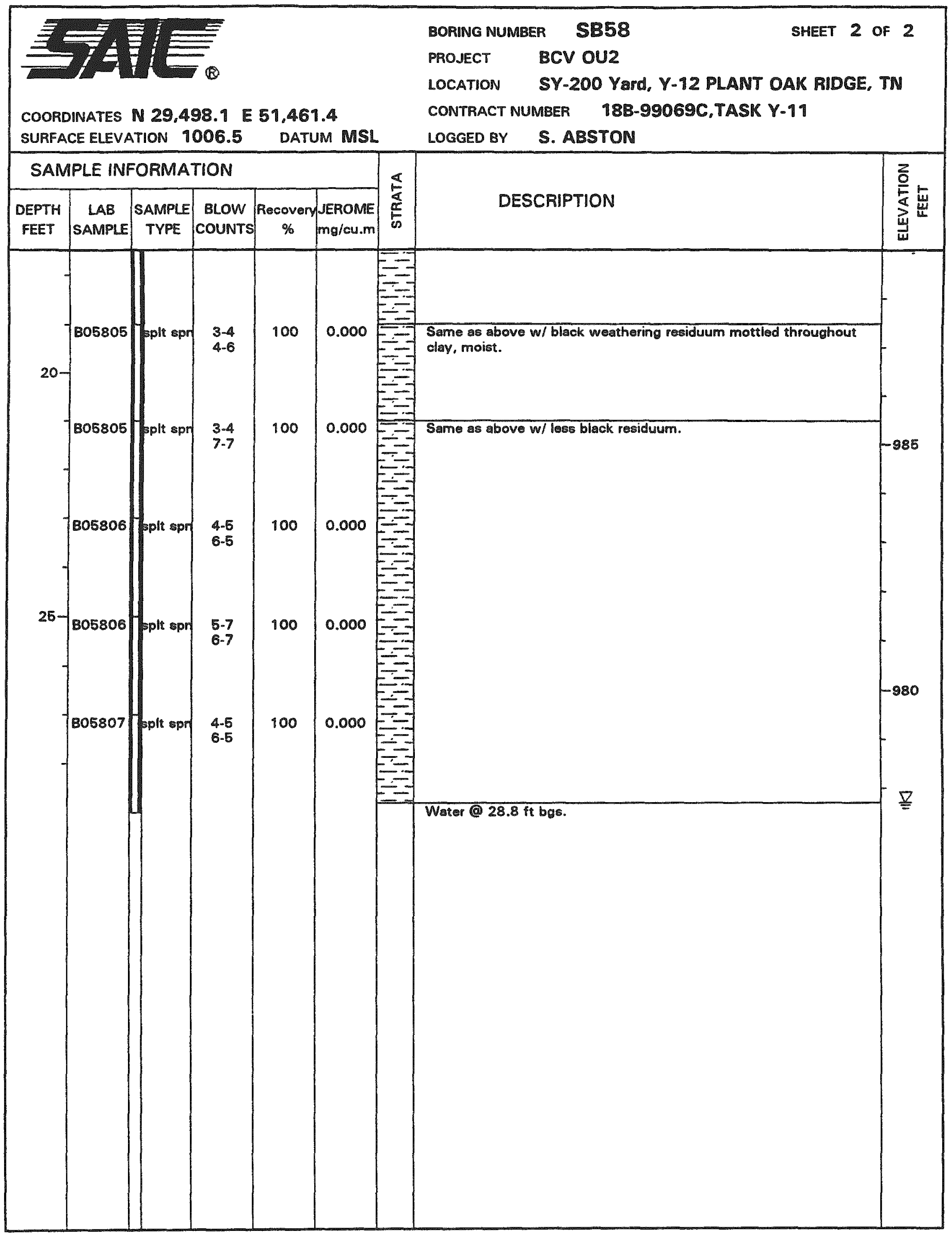




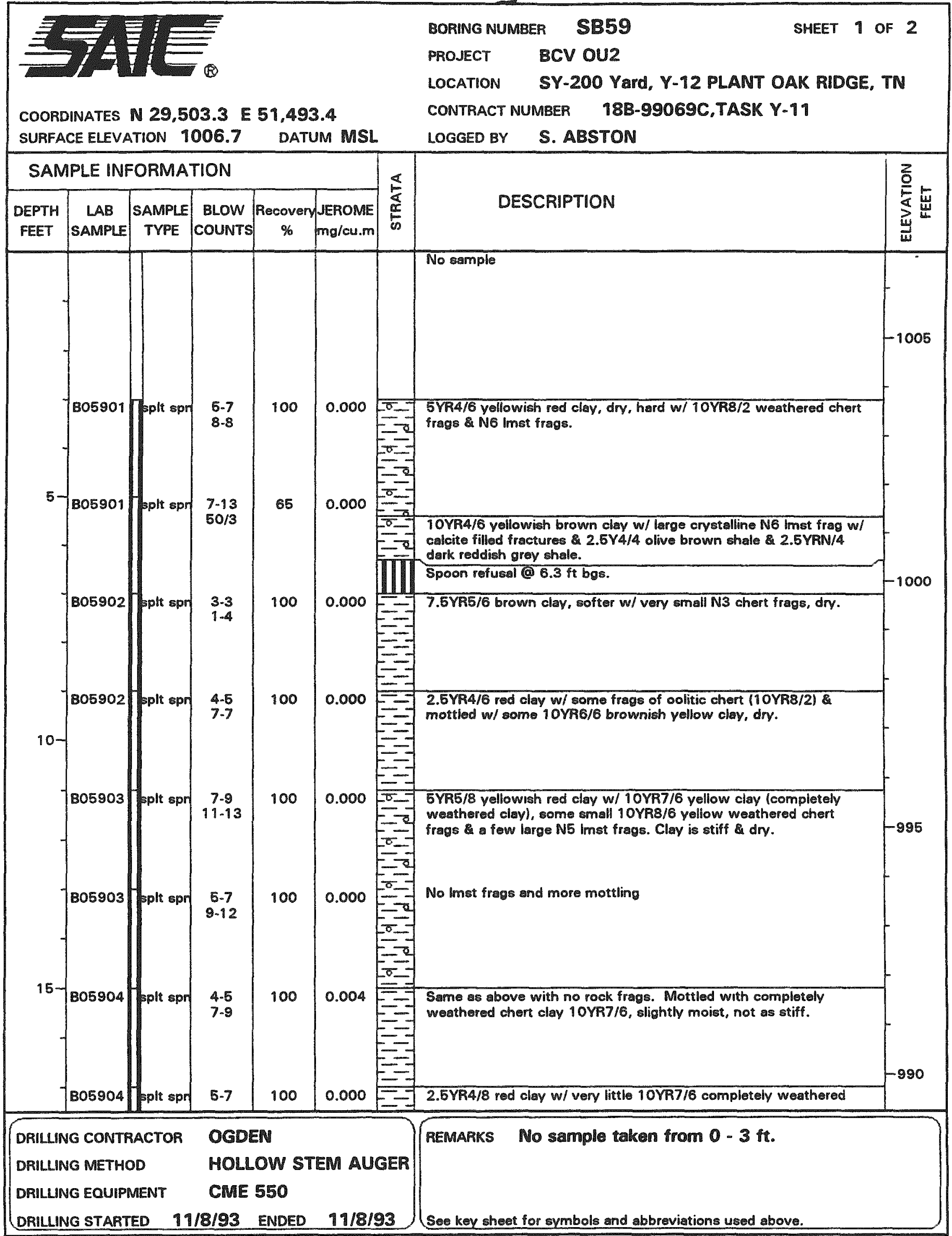




\section{A-94}

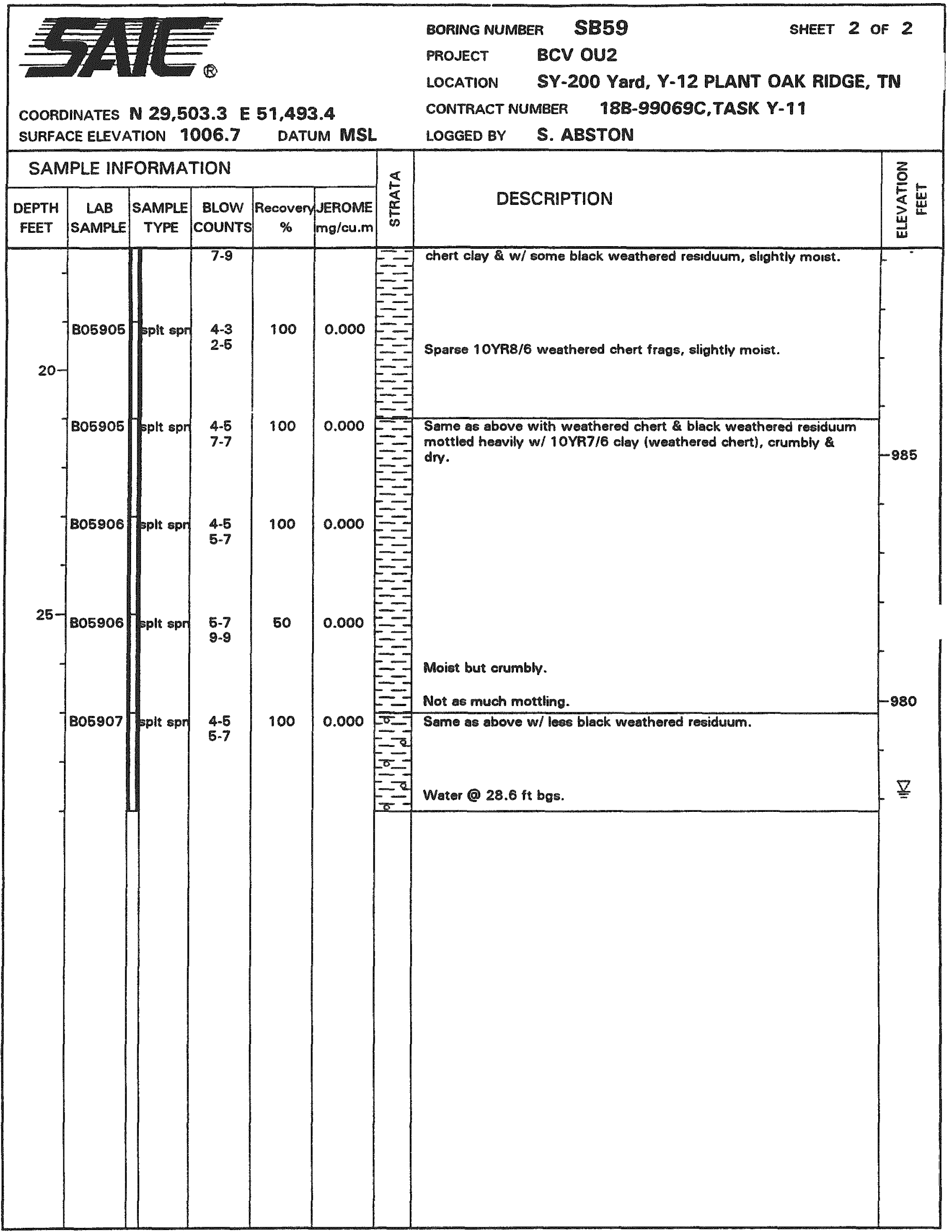




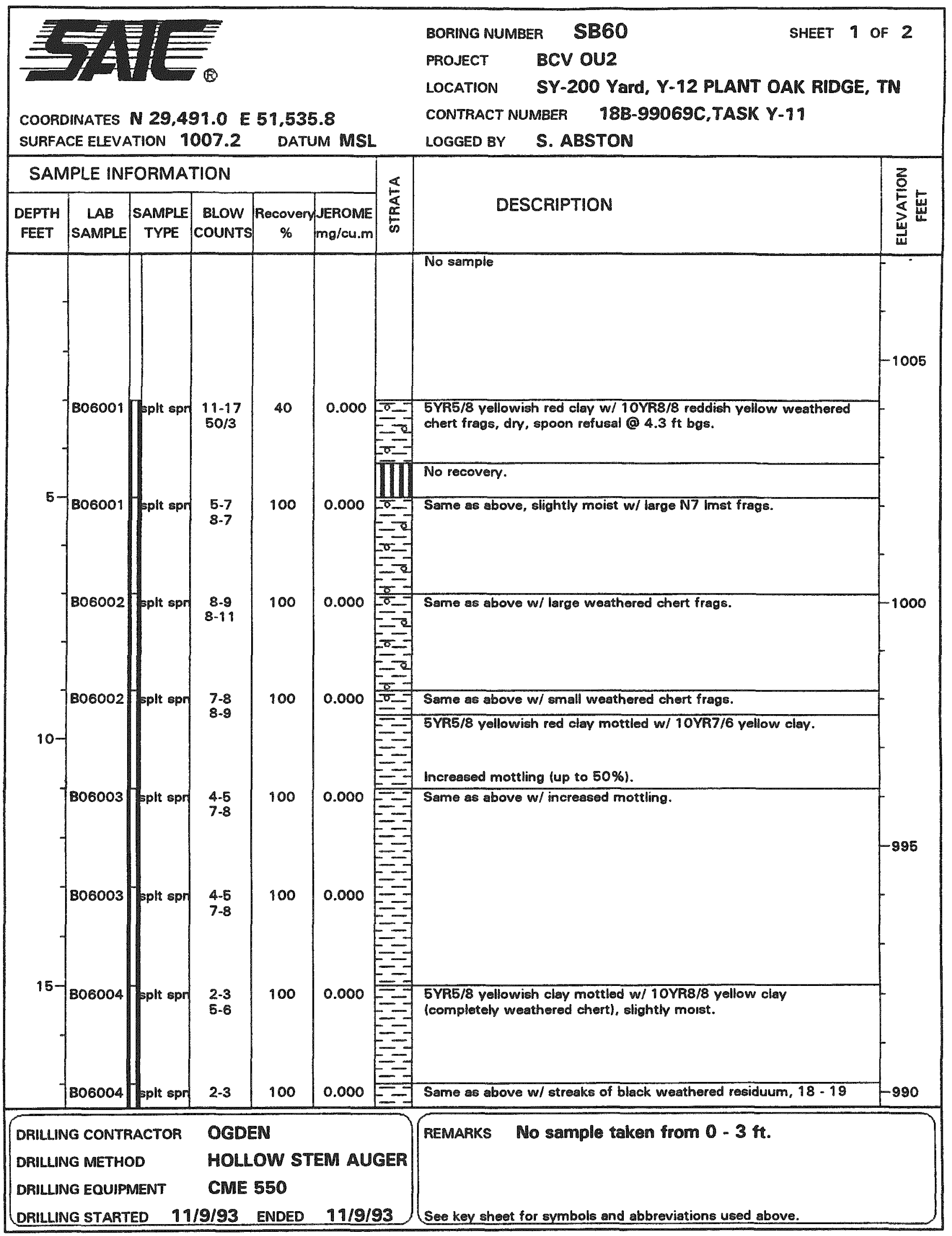


A-96

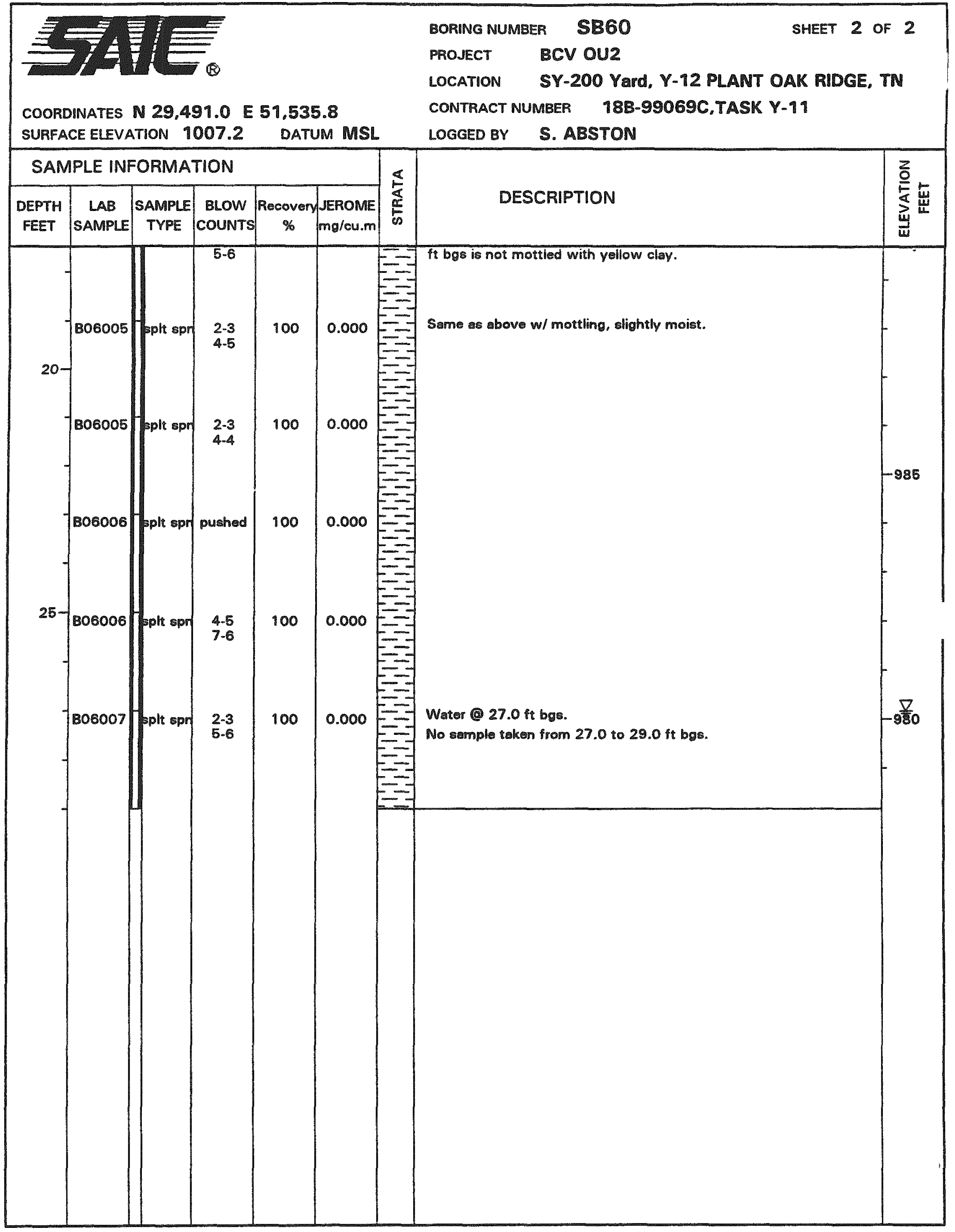




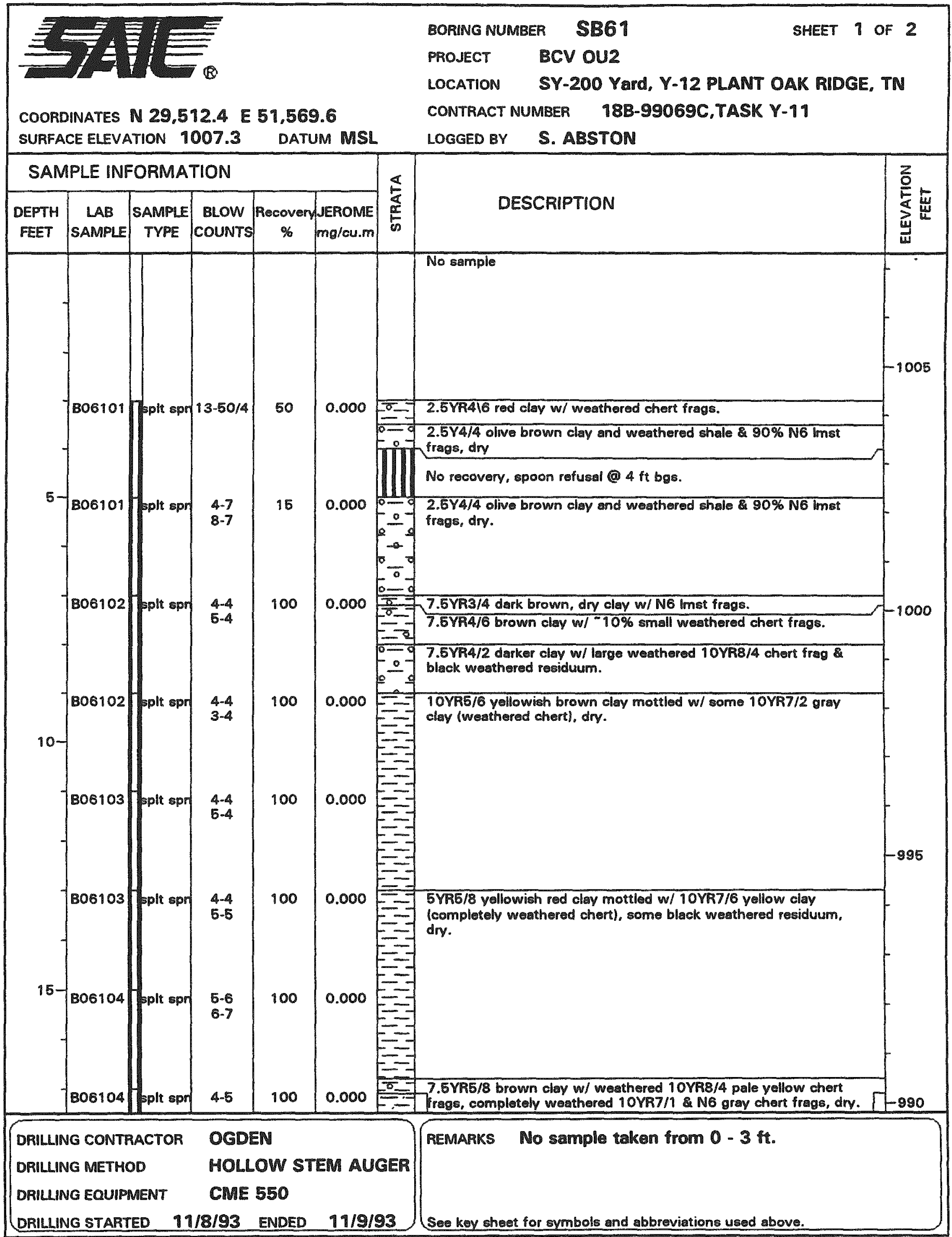




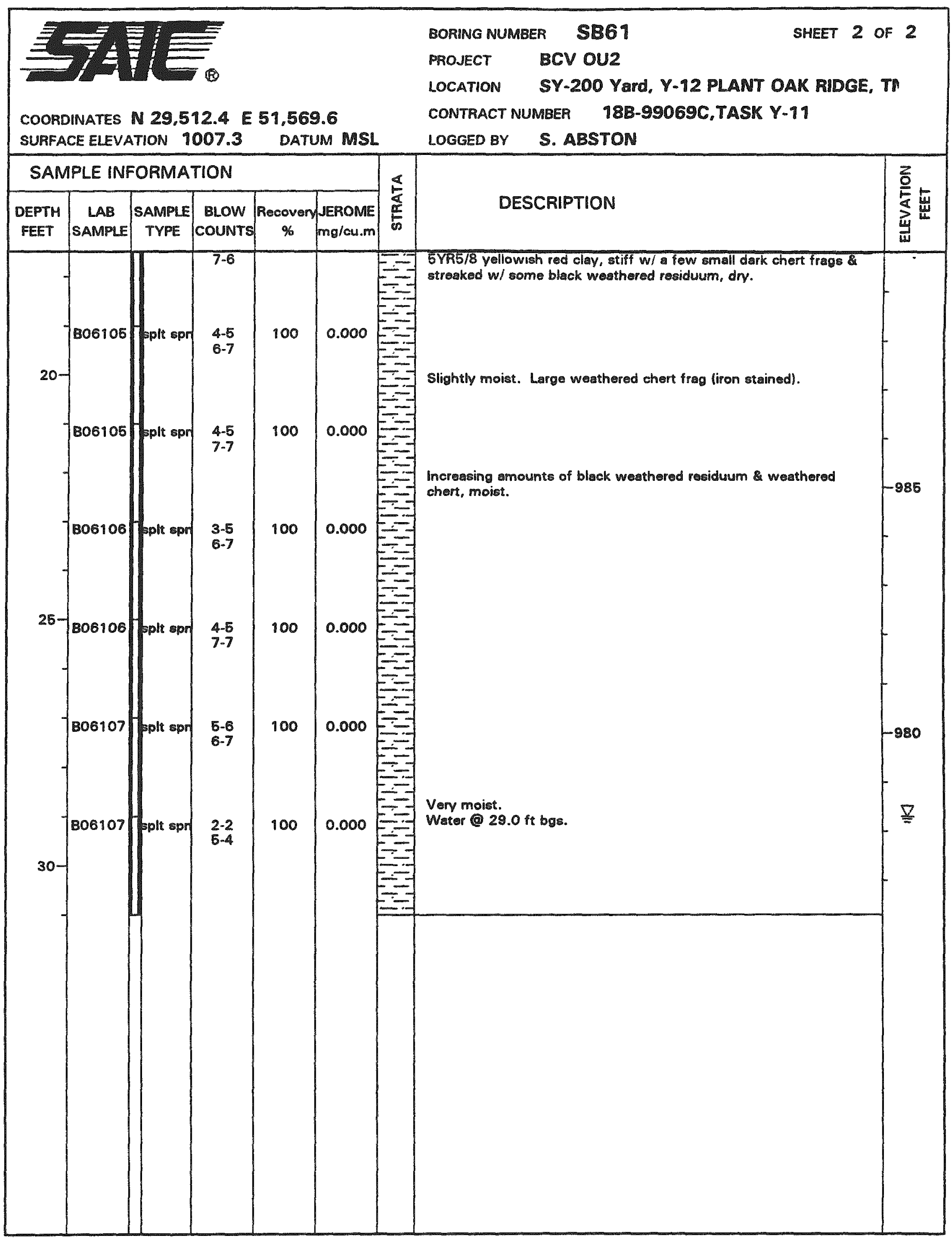




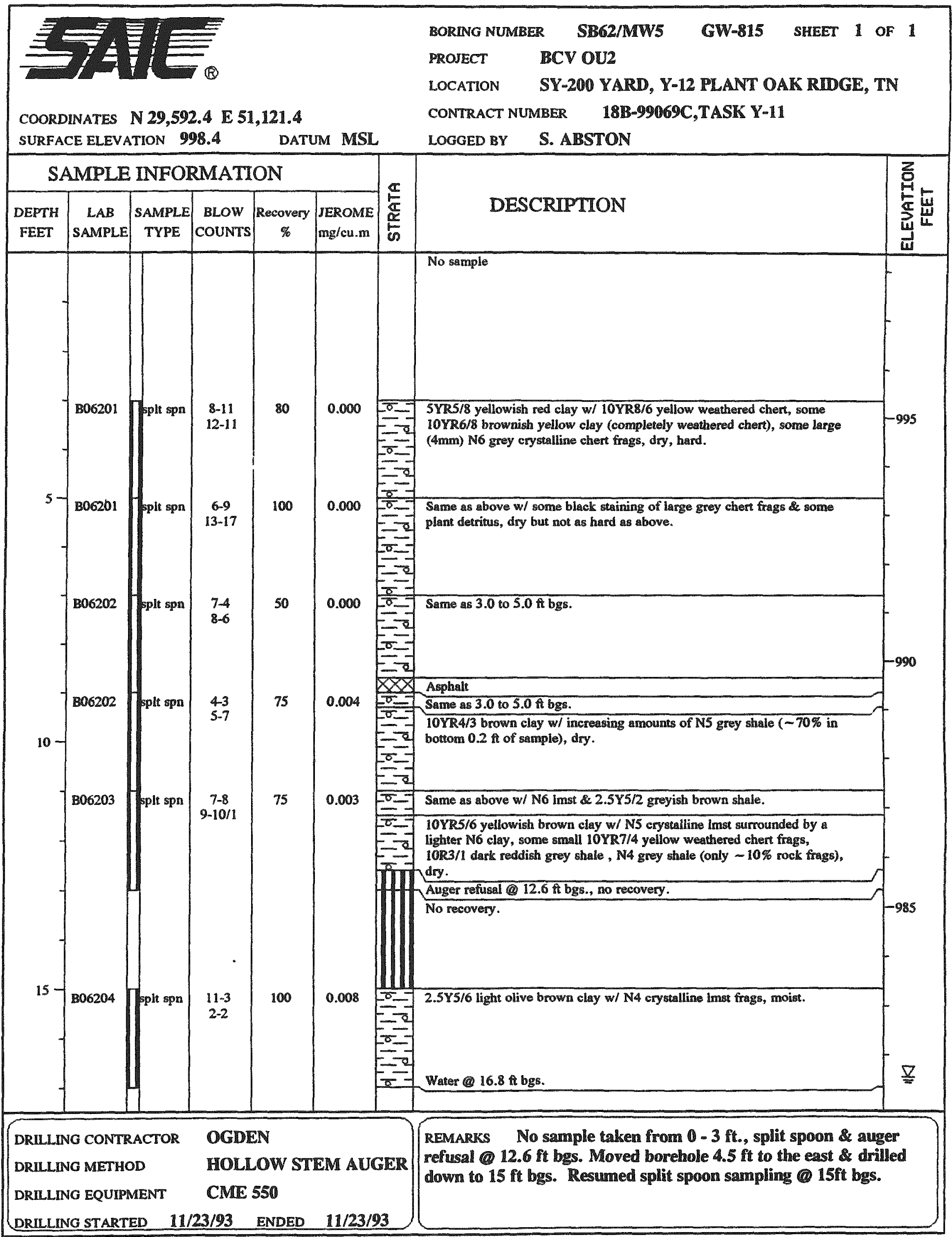




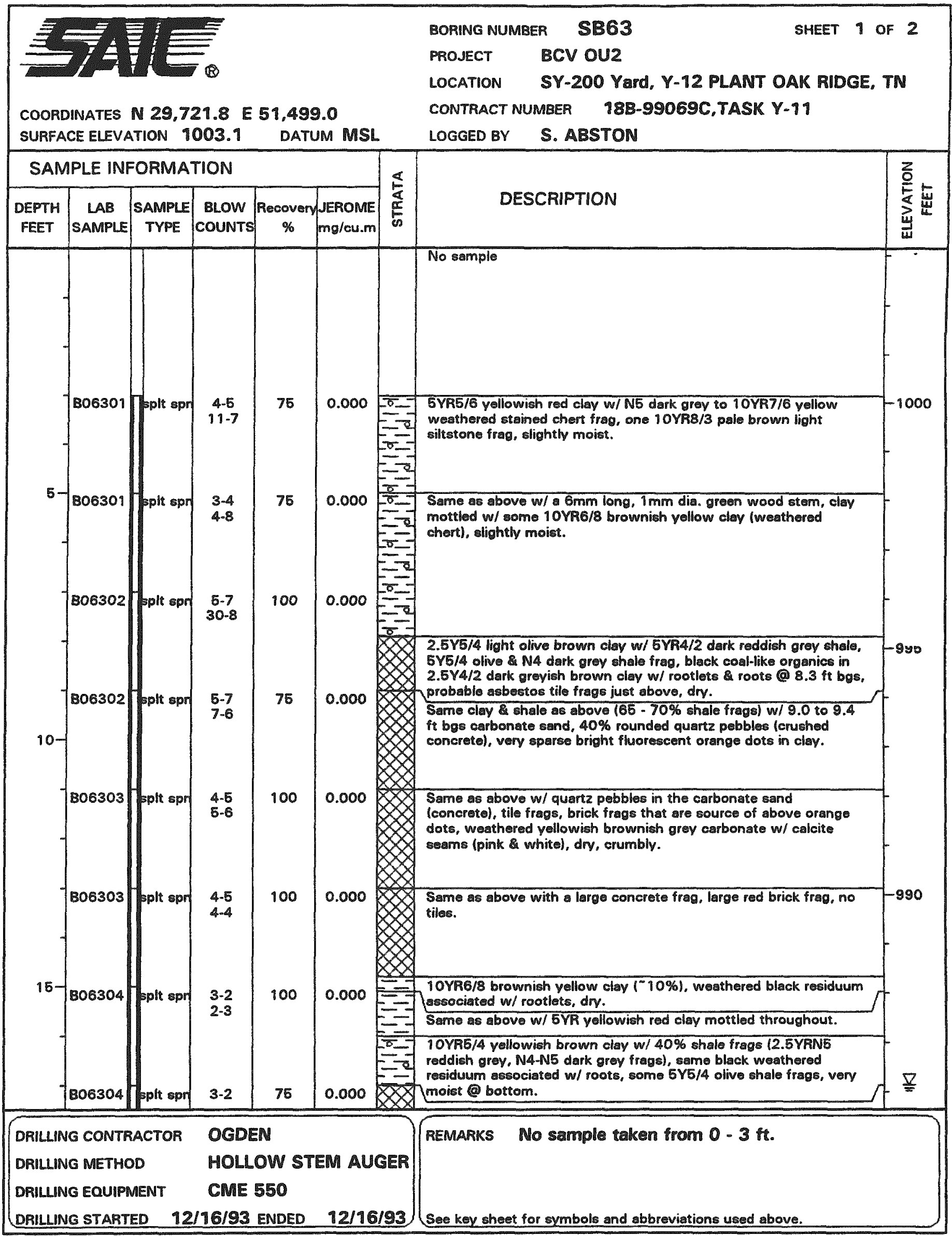




\section{A-101}

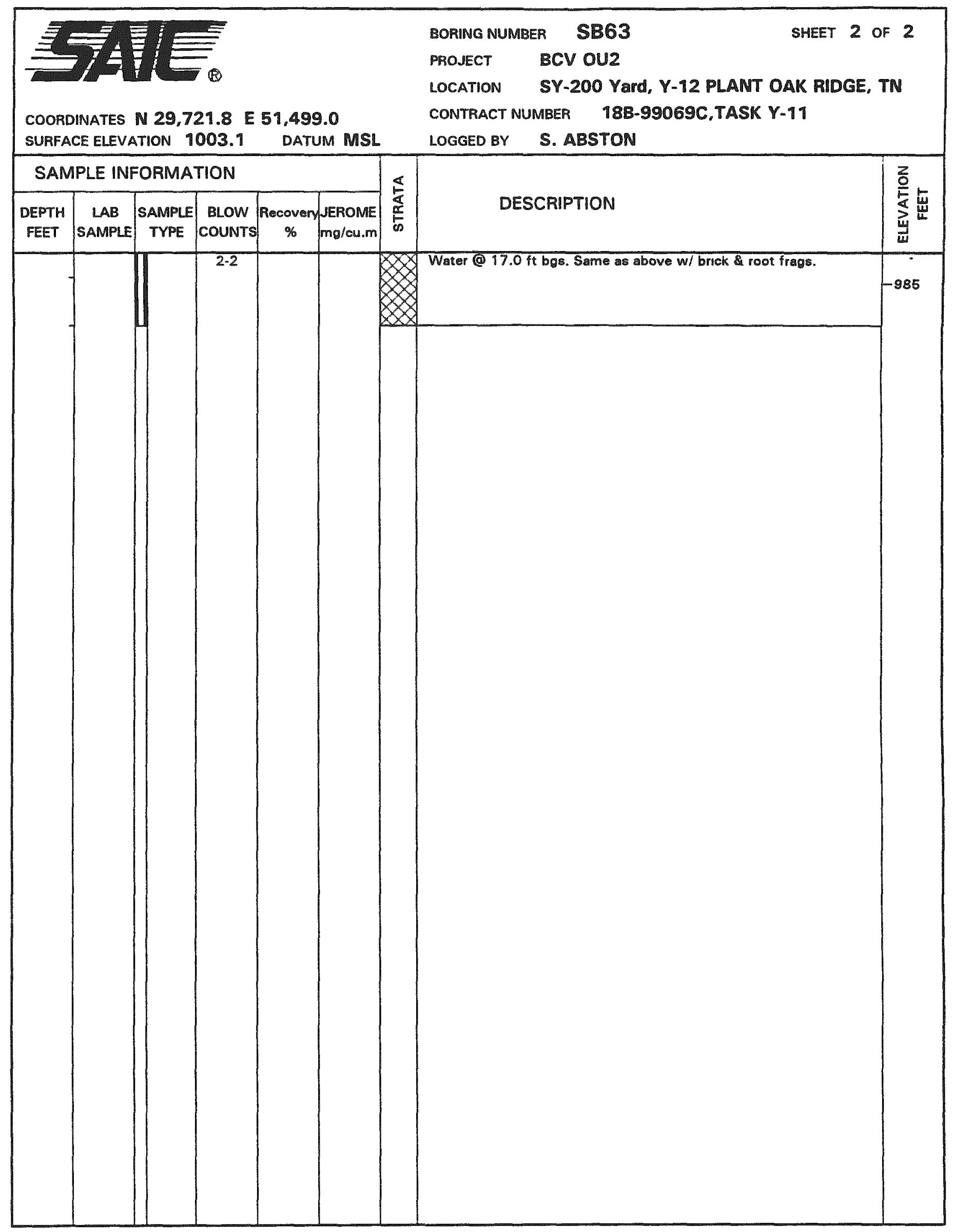




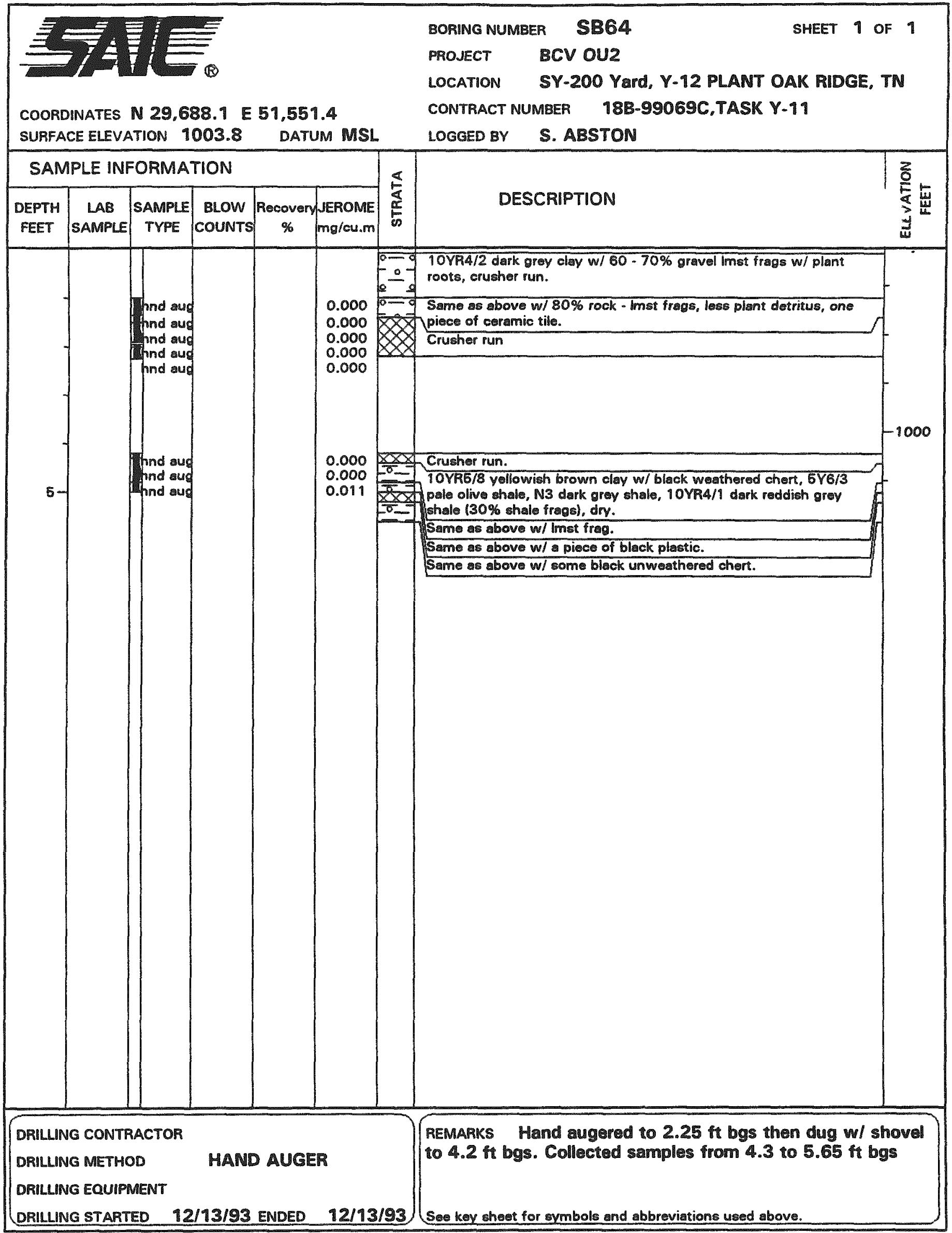




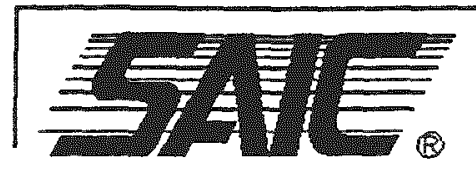

COORDINATES N 29,443.2 E 52,324.6
BORING NUMBER PI/MWI

PROJECT

BCV OU2

LOCATION SY-200 YARD, Y-12 PLANT OAK RIDGE, TN

CONTRACT NUMBER 18B-99069C, TASK Y-11

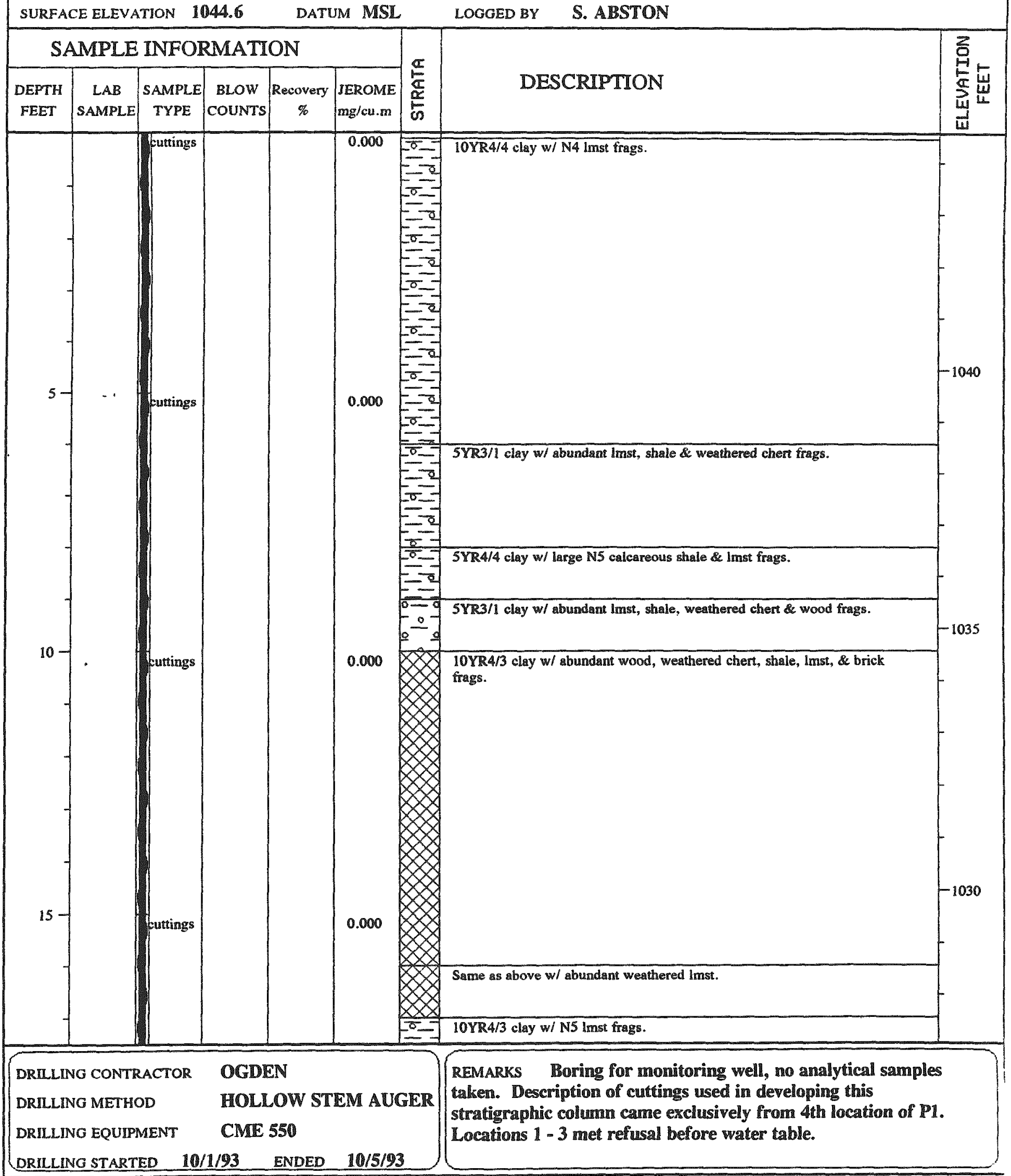




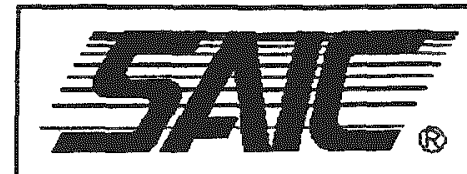

COORDINATES N 29,443.2 E $\mathbf{5 2 , 3 2 4 . 6}$

SURFACE ELEVATION 1044.6

\section{BORING NUMBER}

PROIECT

\section{BCV OU2}

LOCATION SY-200 YARD, Y-12 PLANT OAK RIDGE, TN CONTRACT NUMBER 18B-99069C,TASK Y-11

LOGGED BY S. ABSTON

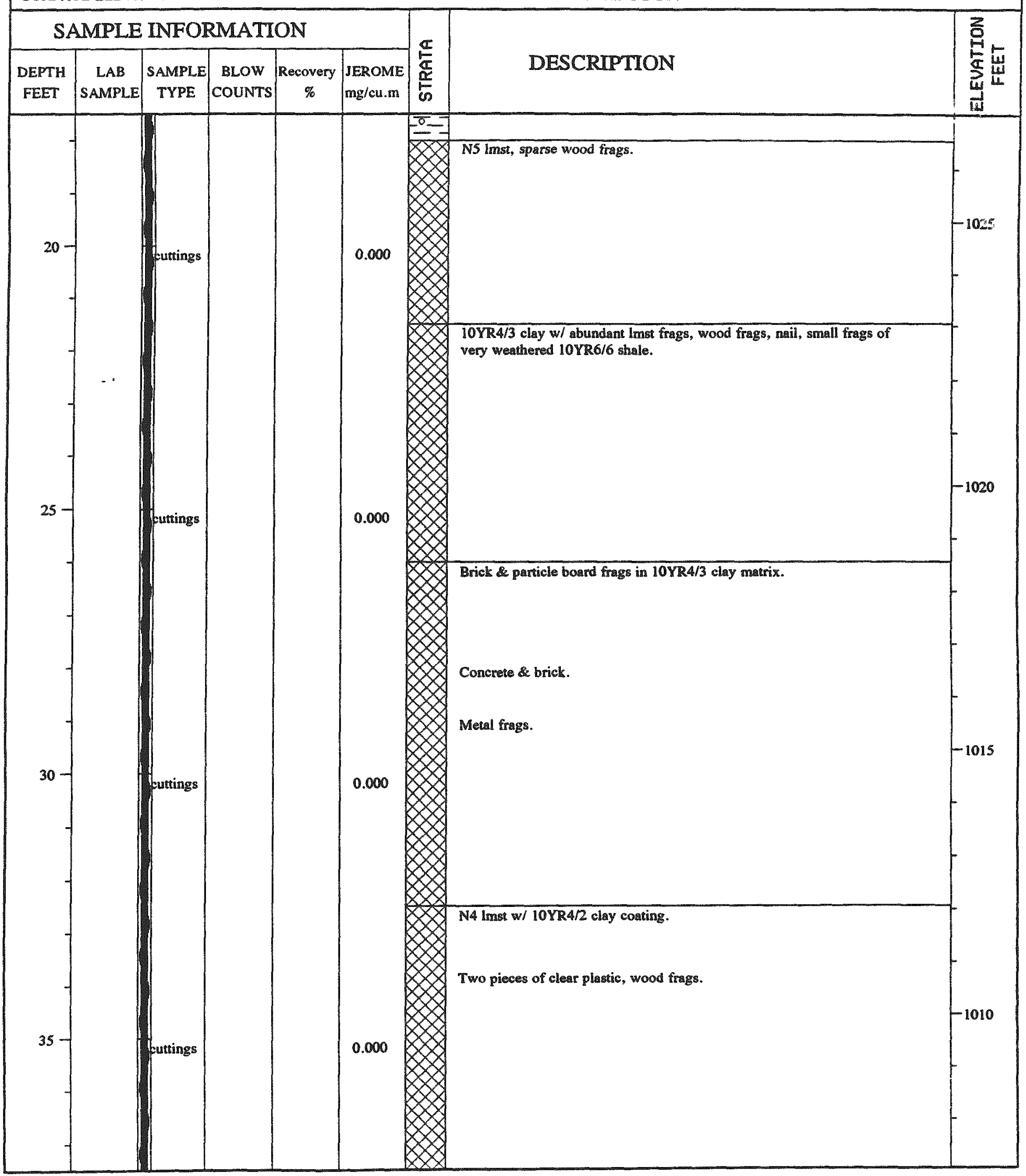




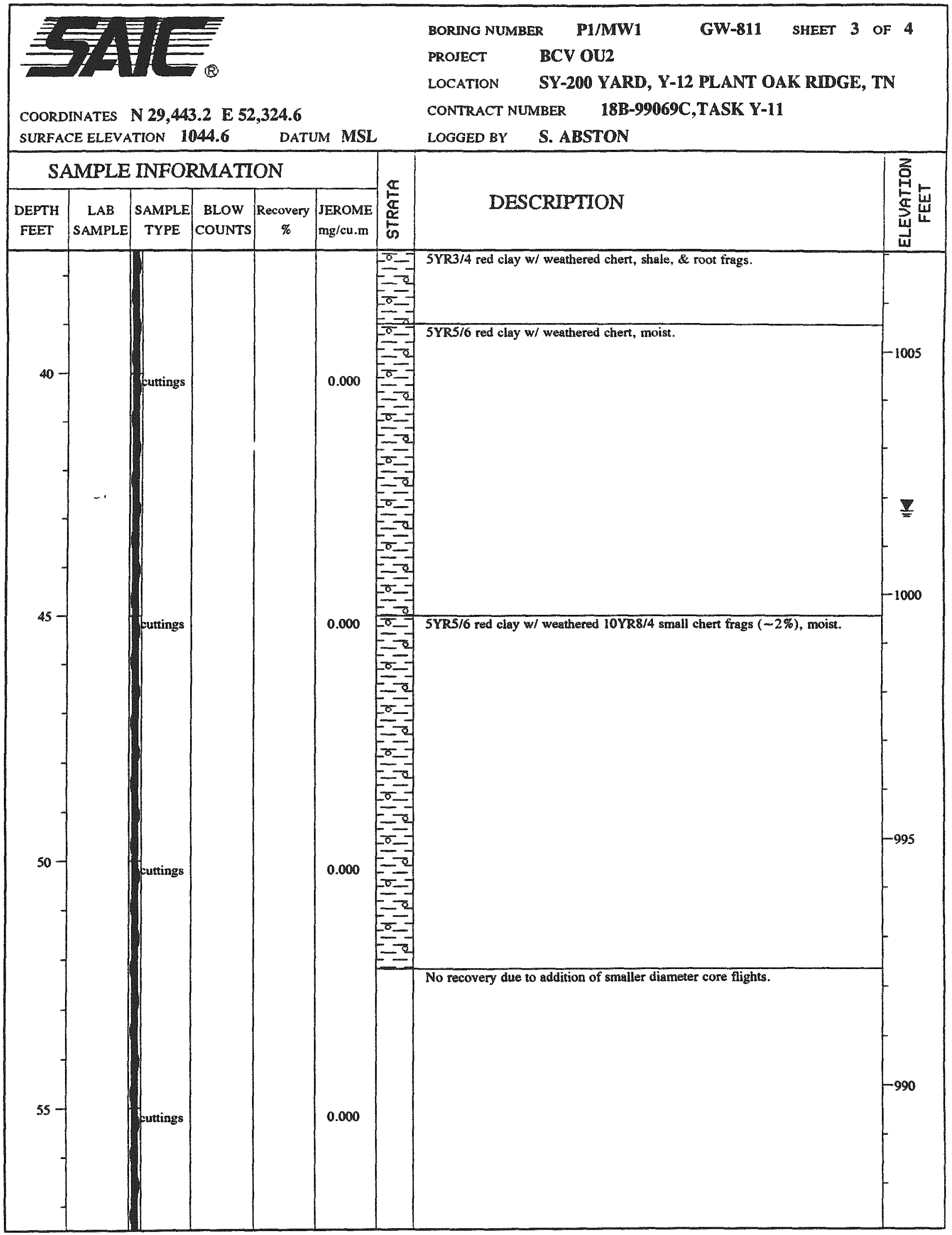




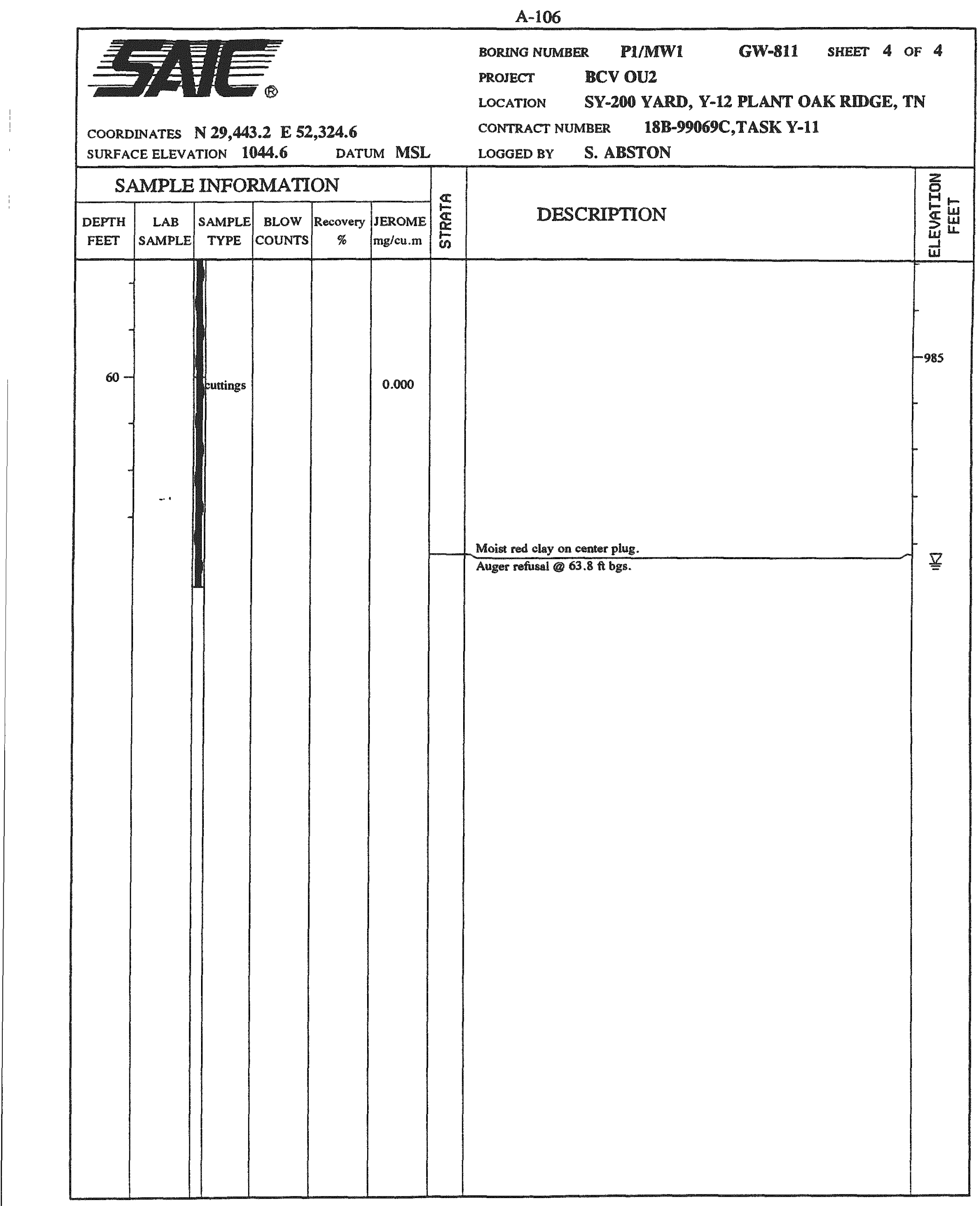




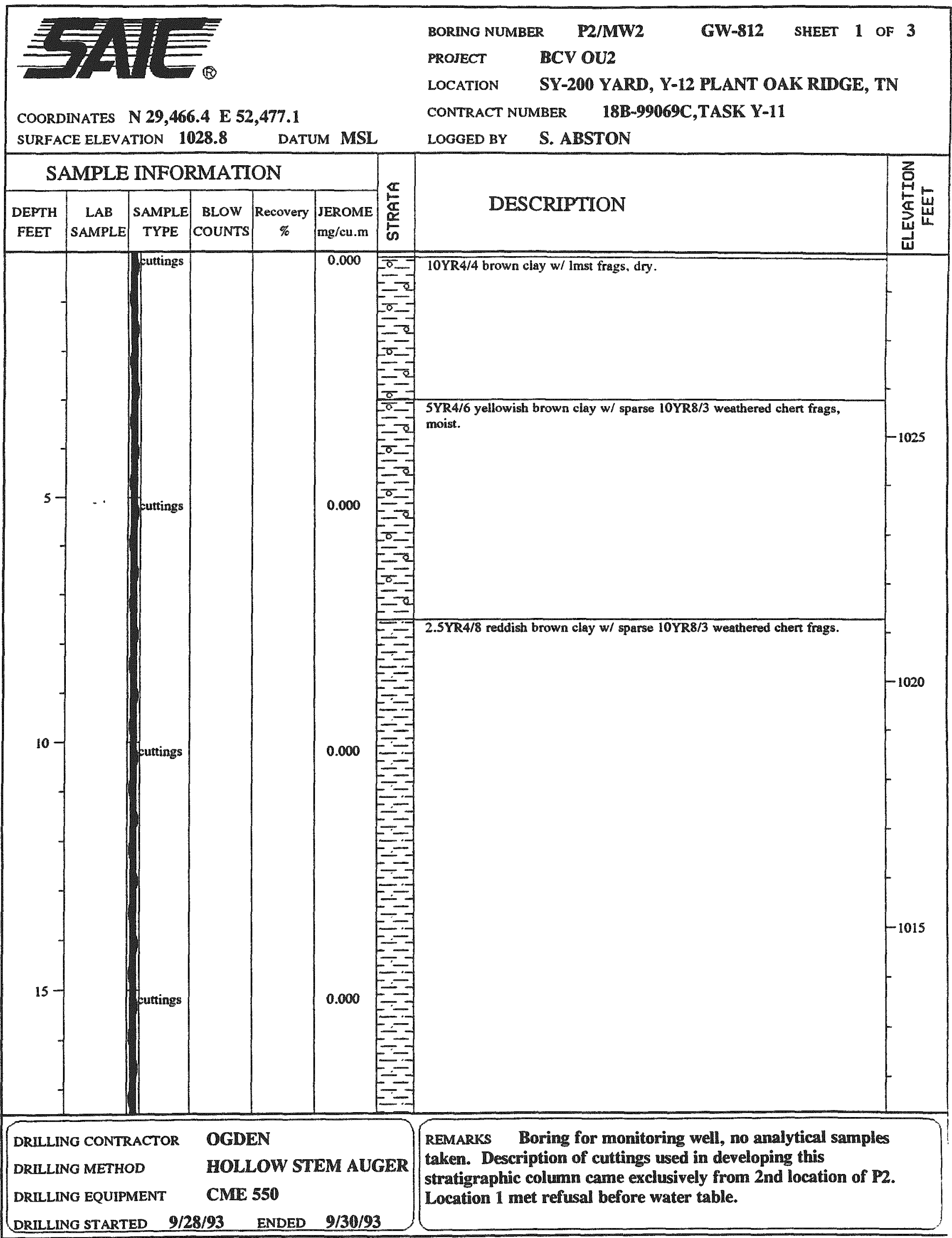




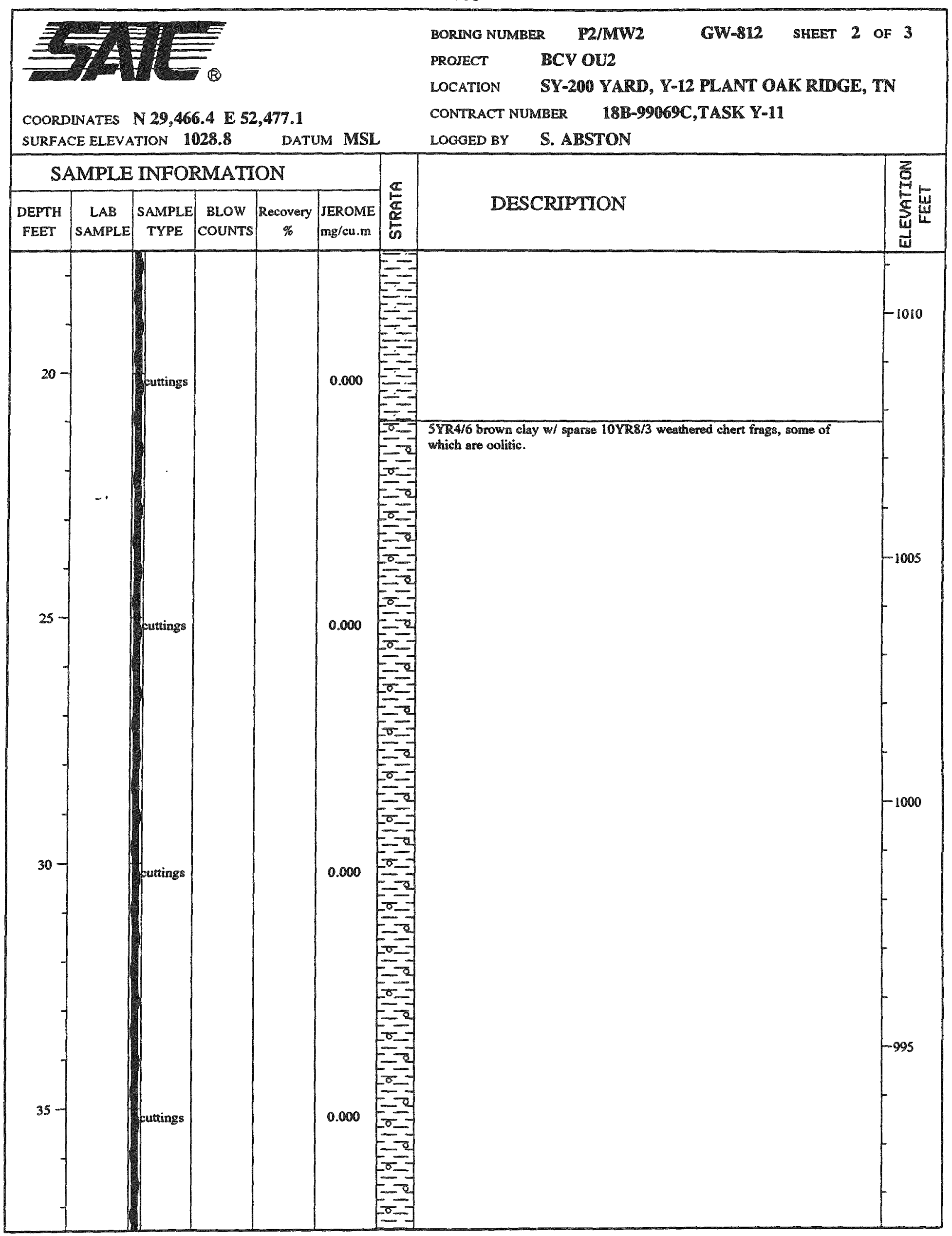




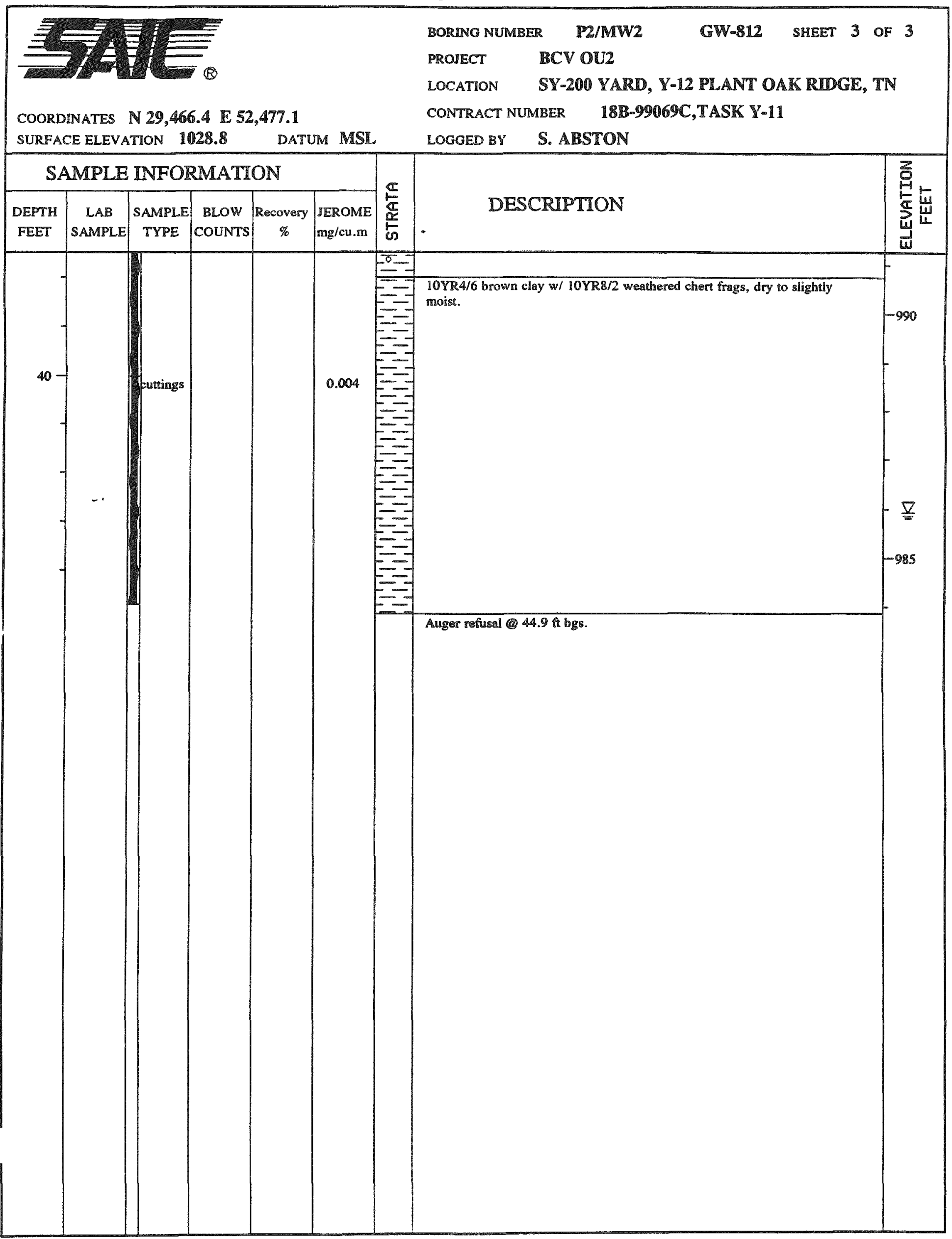




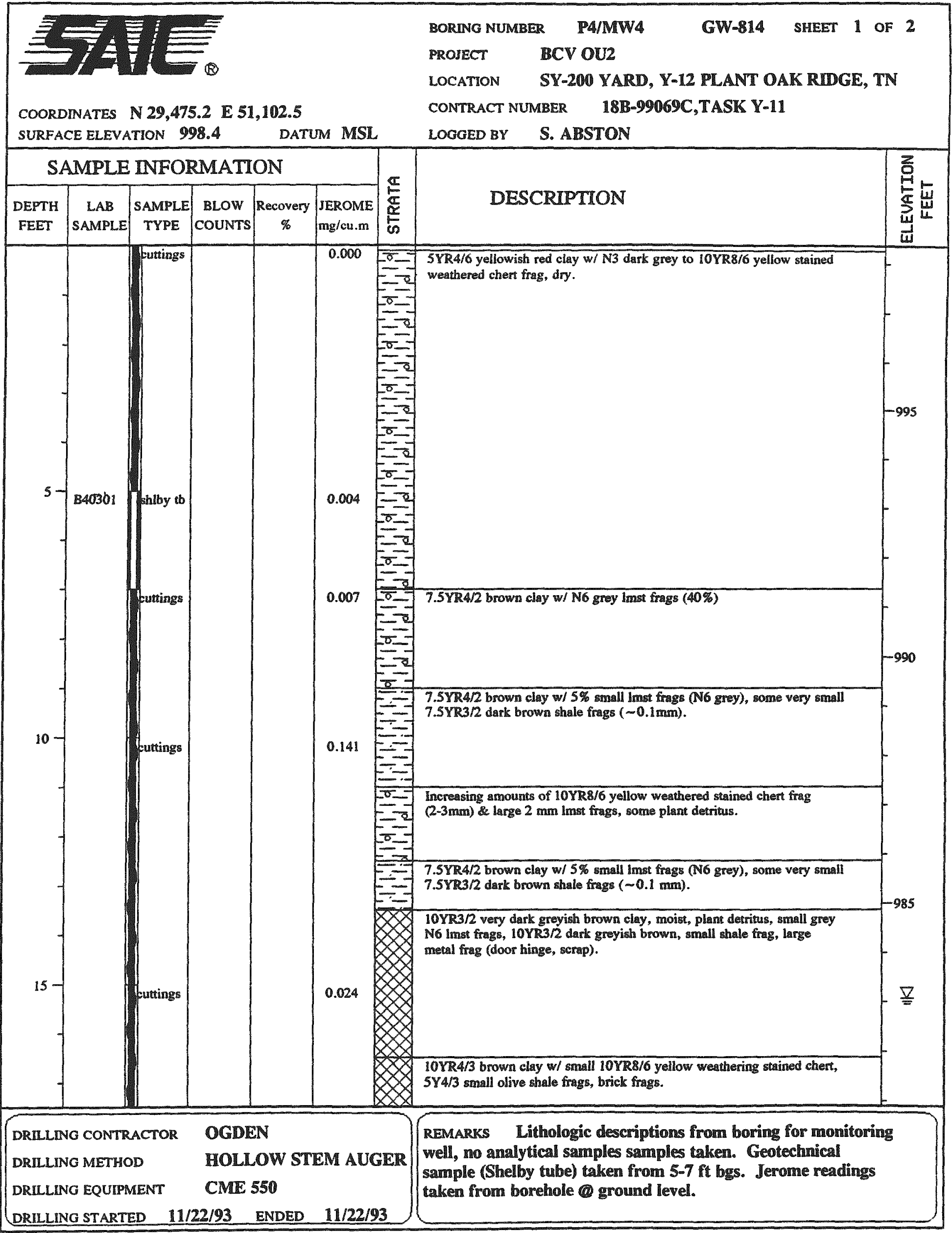


A-111

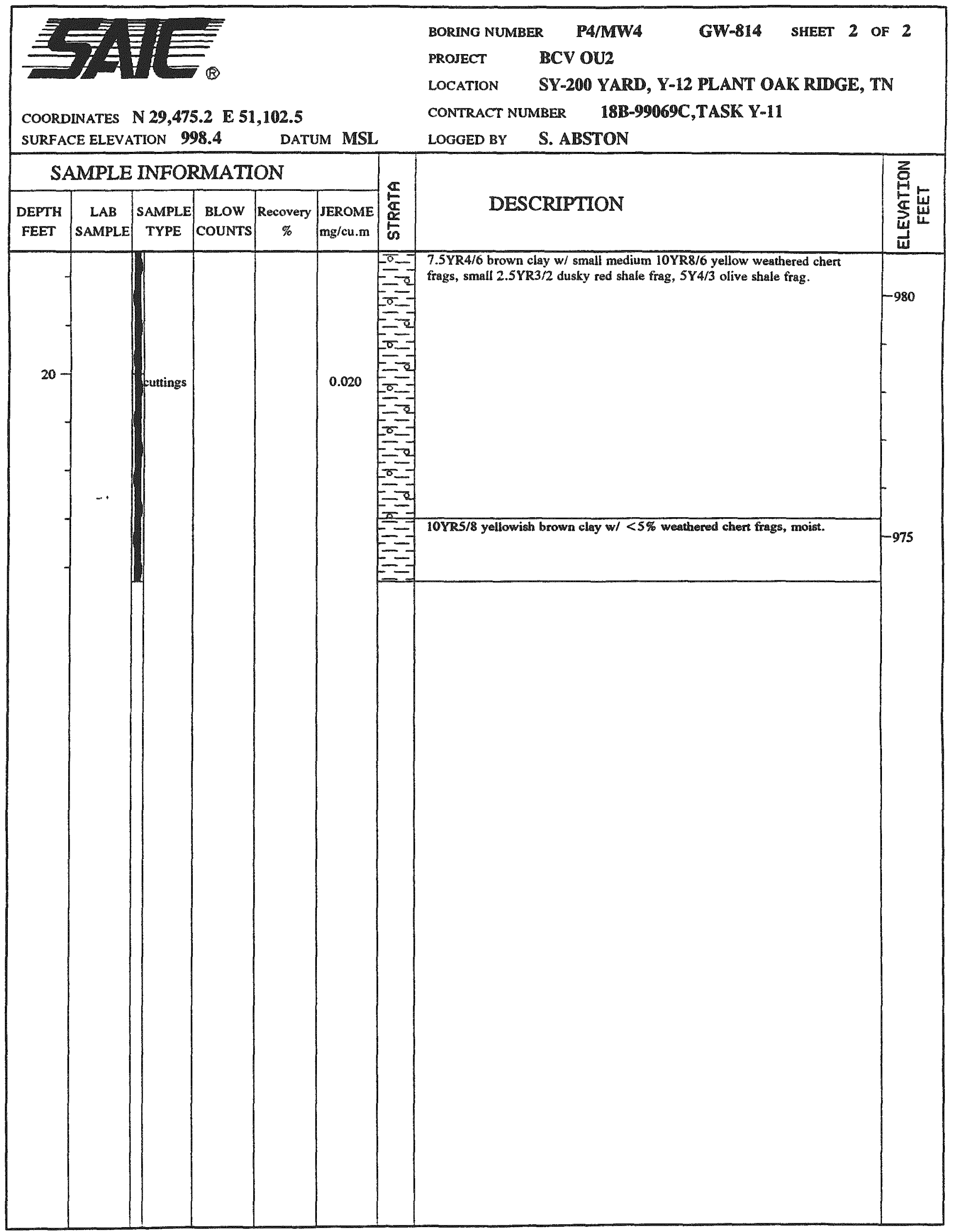


Historical Soil Boring Logs 

A-115

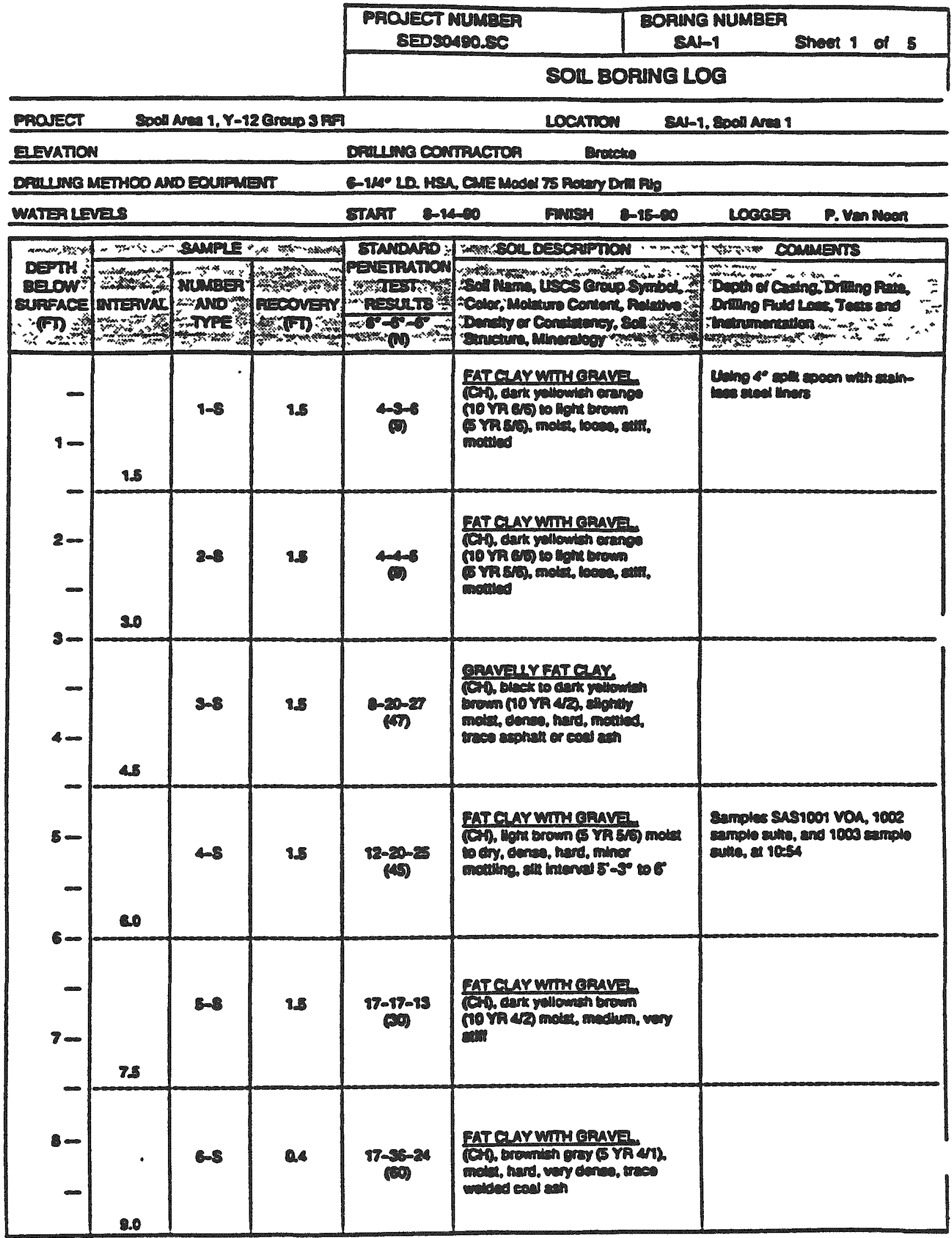


A-116

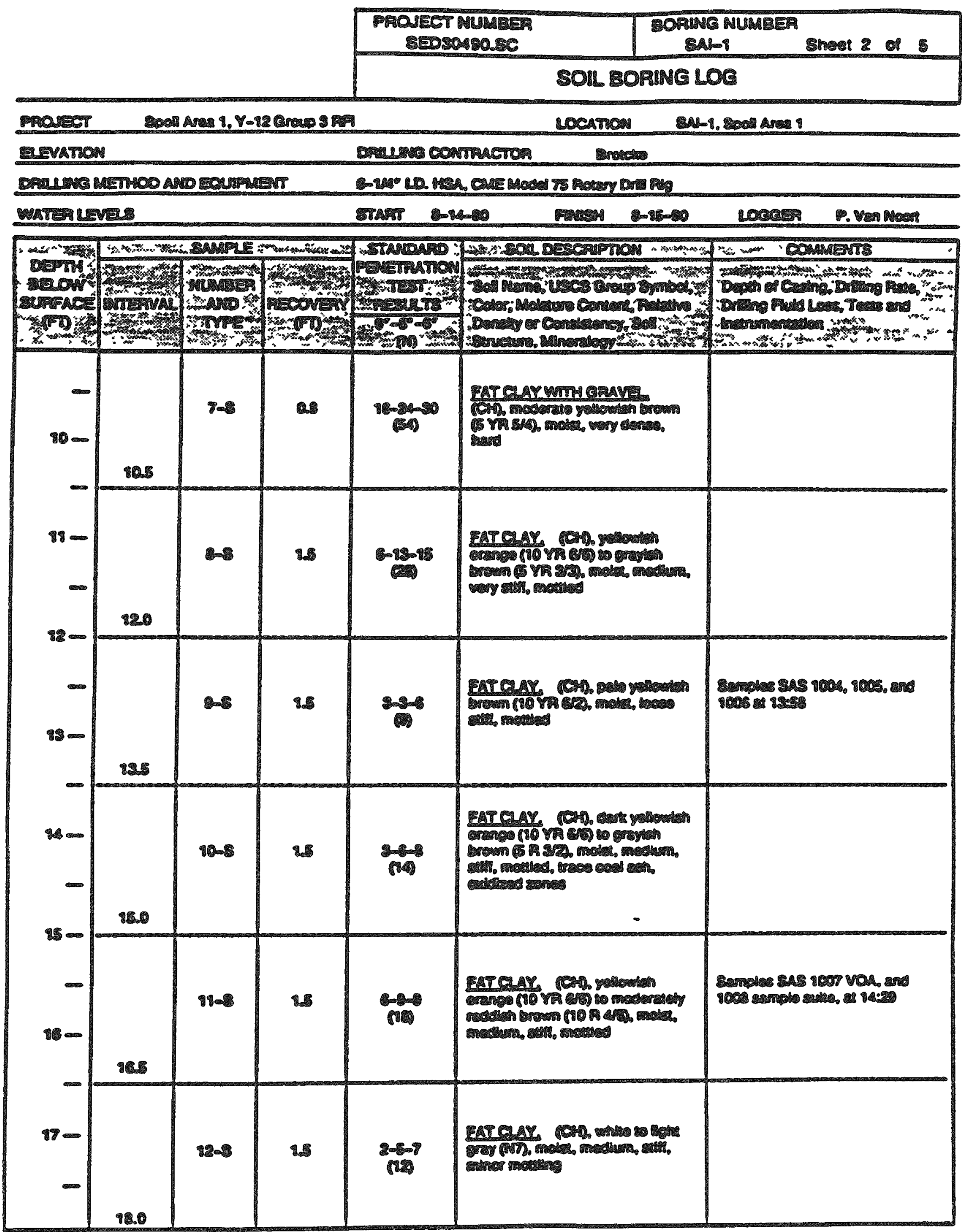




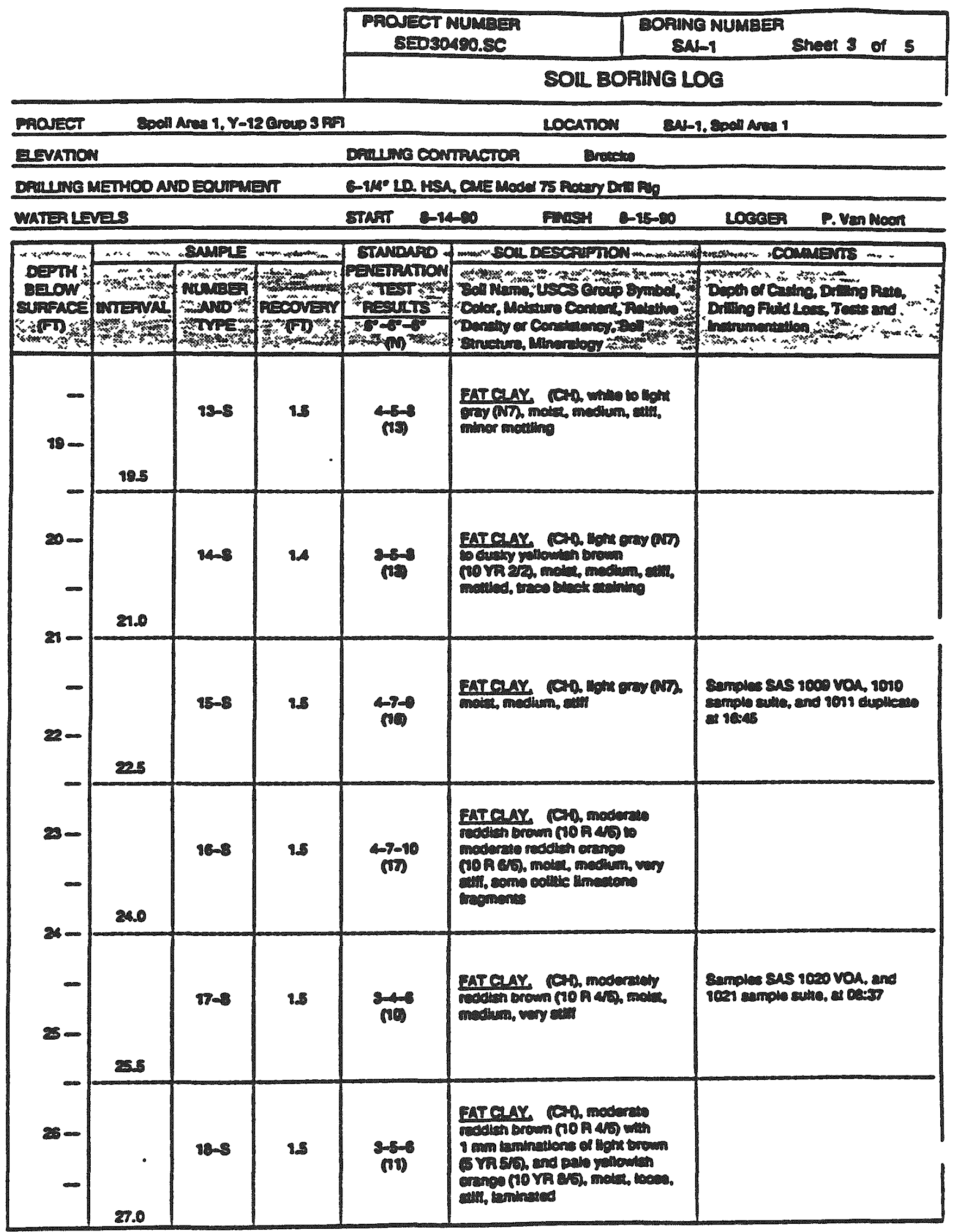




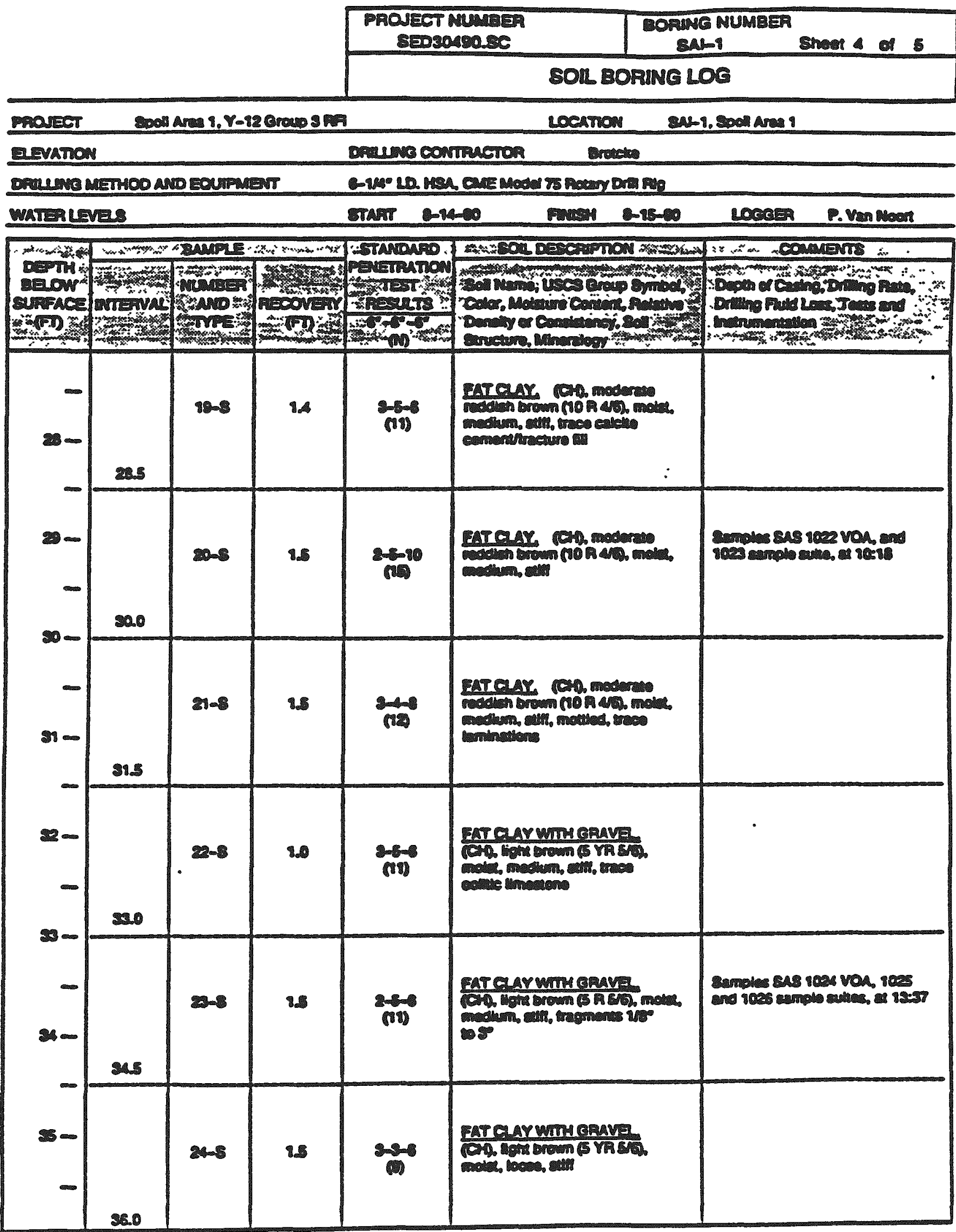


A-119

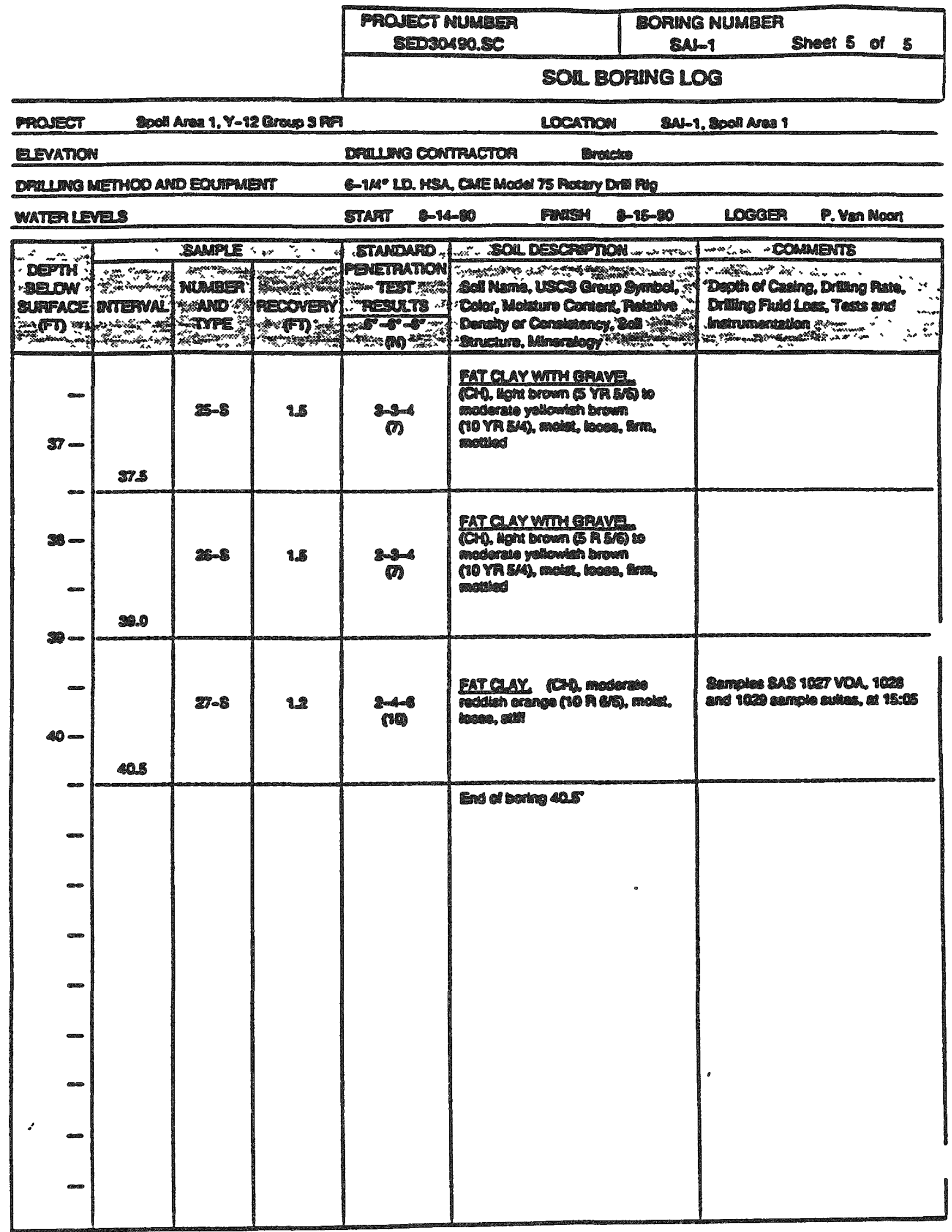




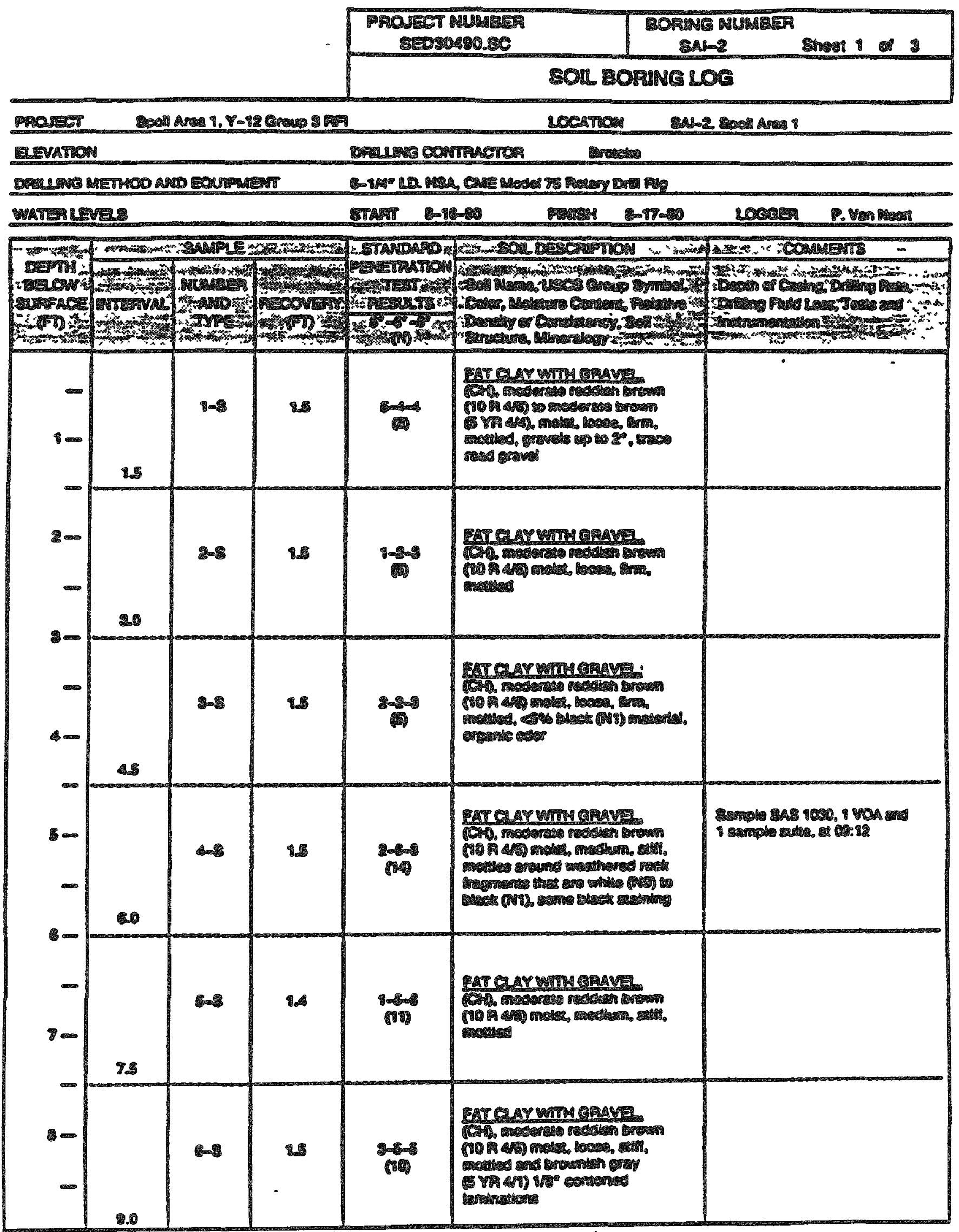




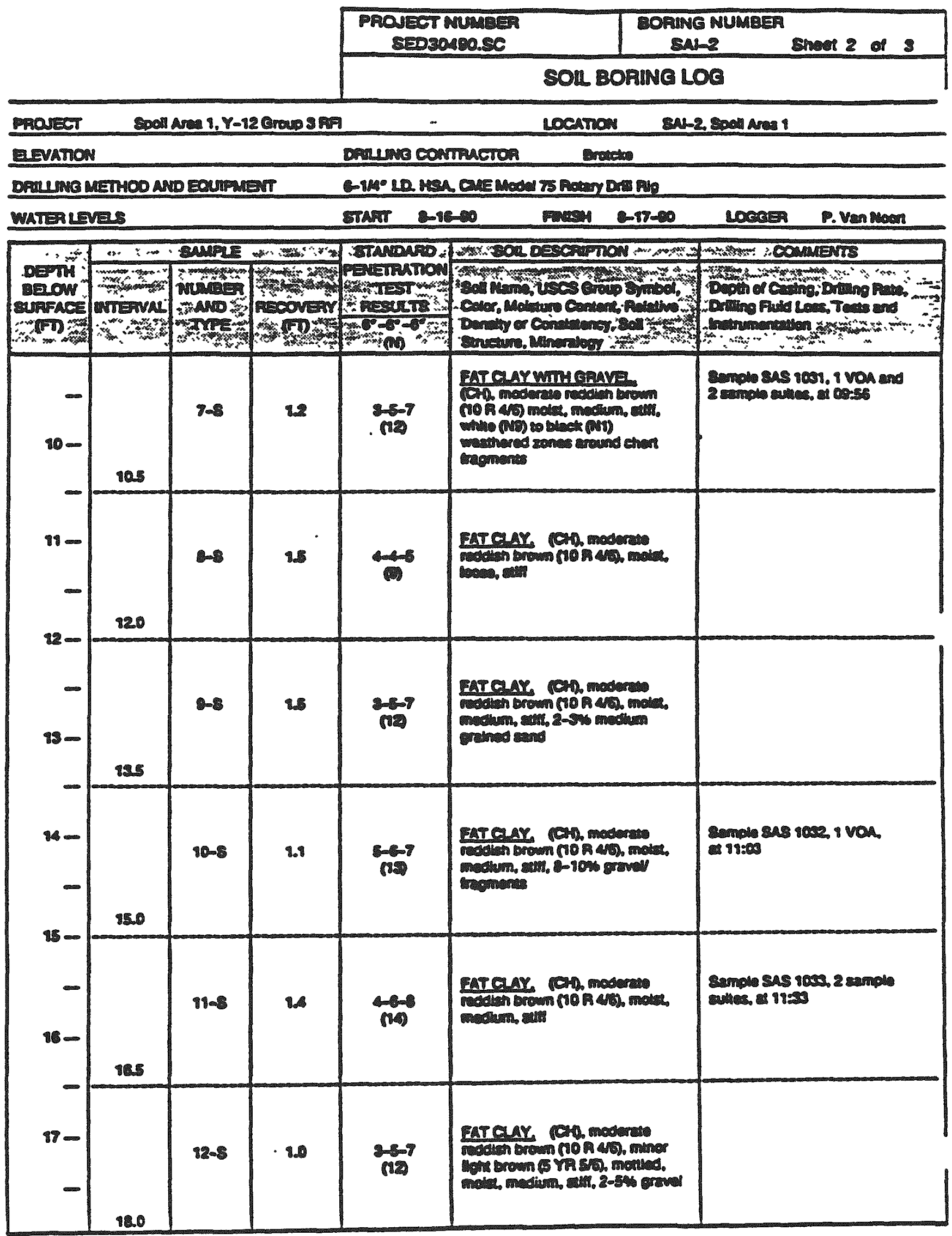


A-122

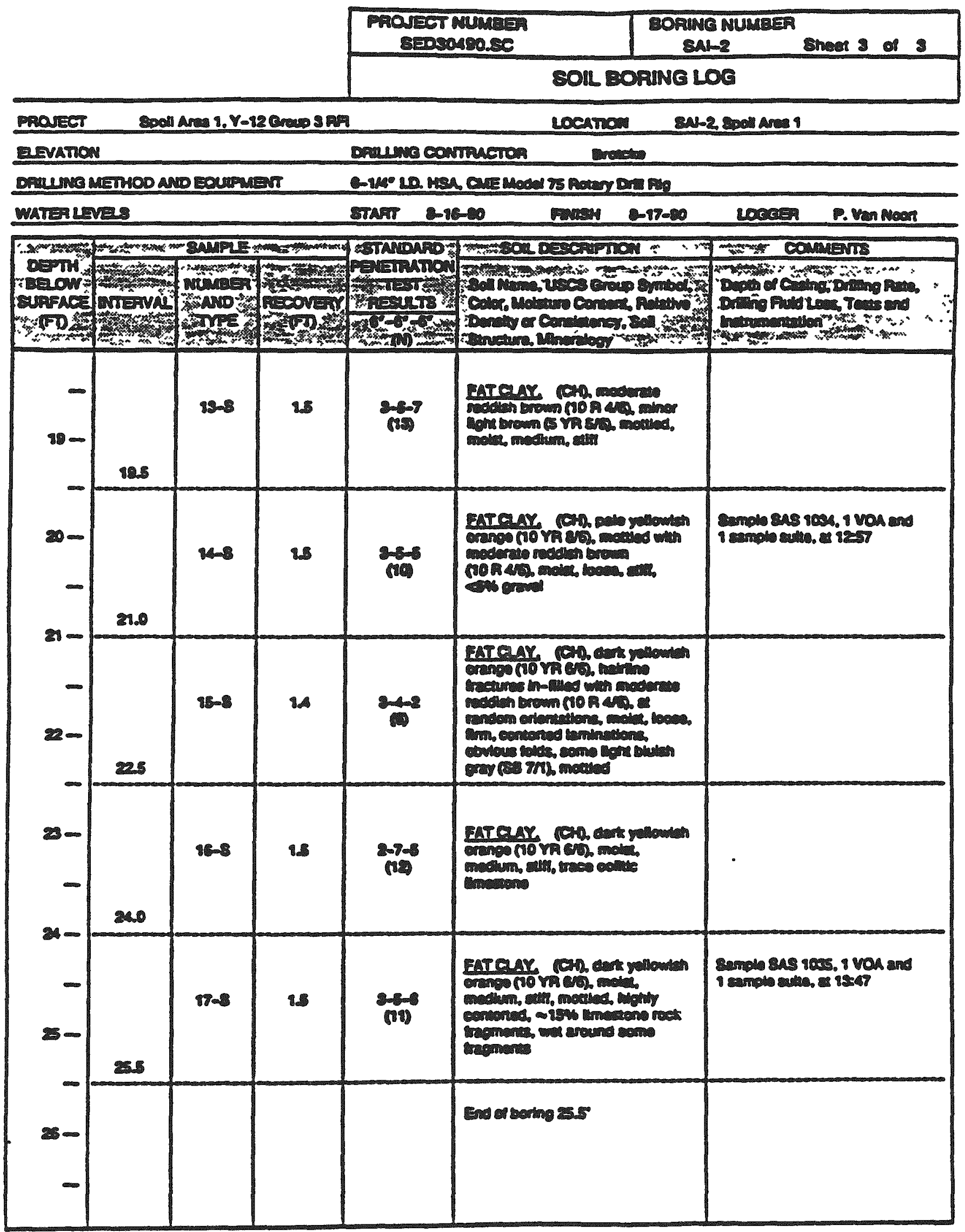


A-123

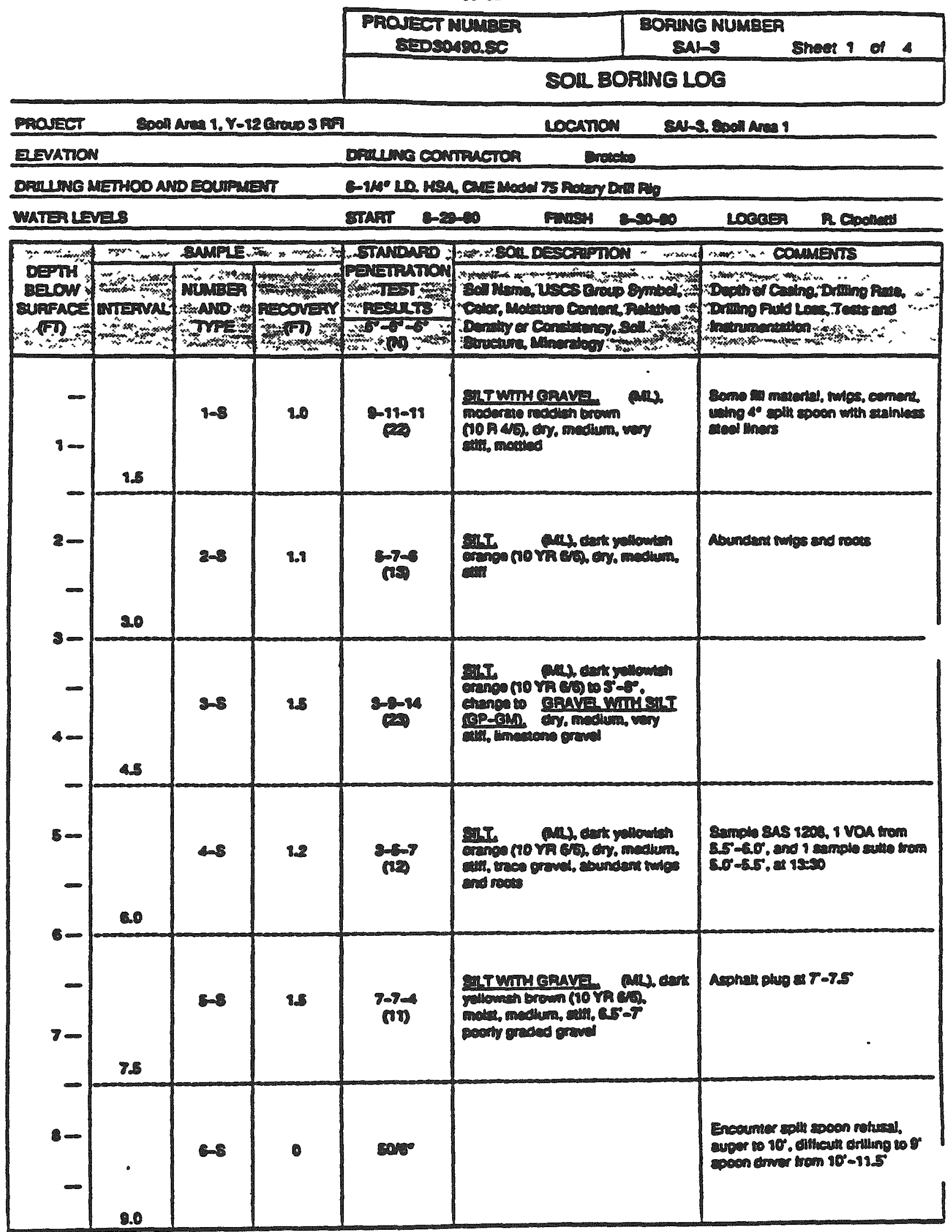


A-124

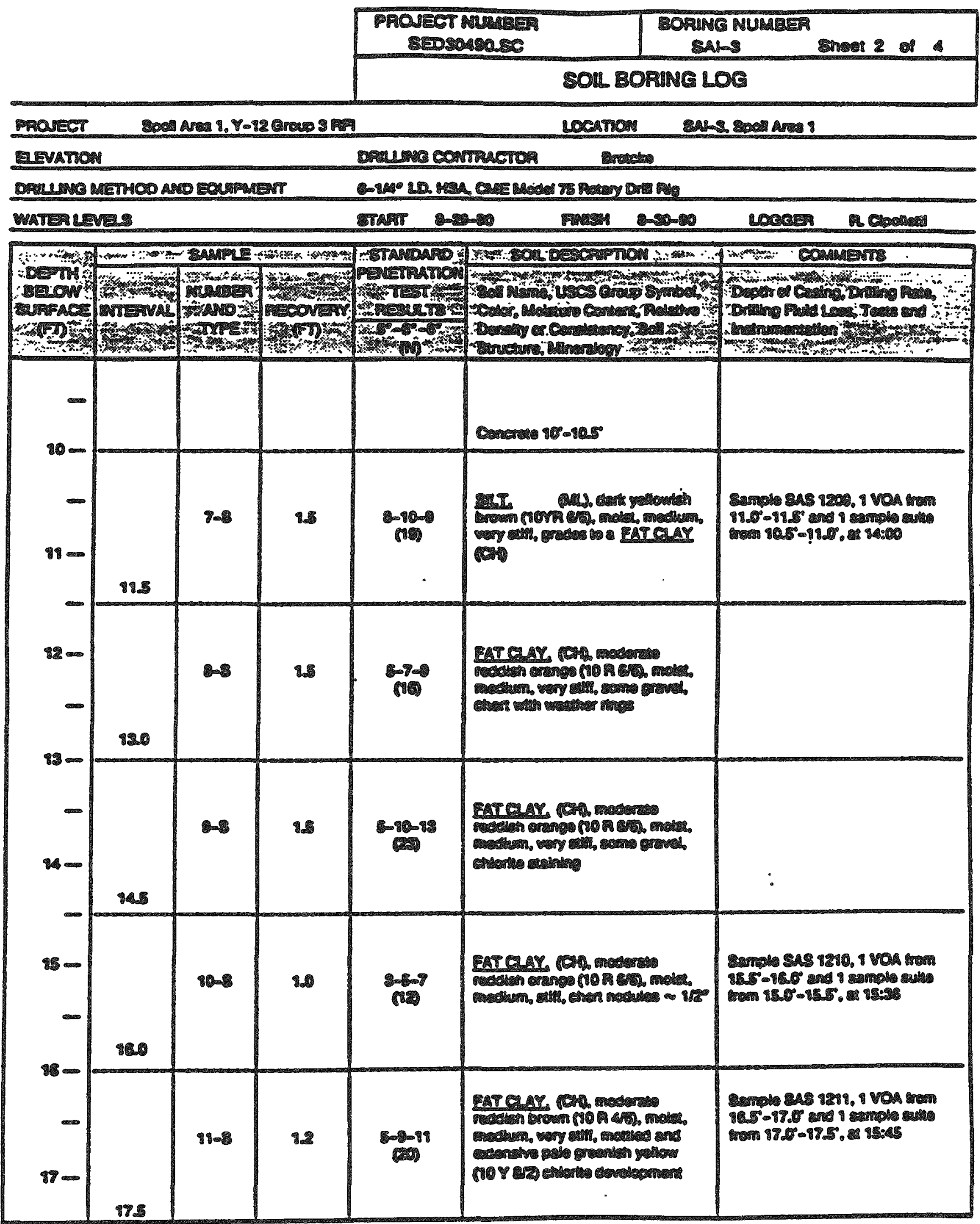




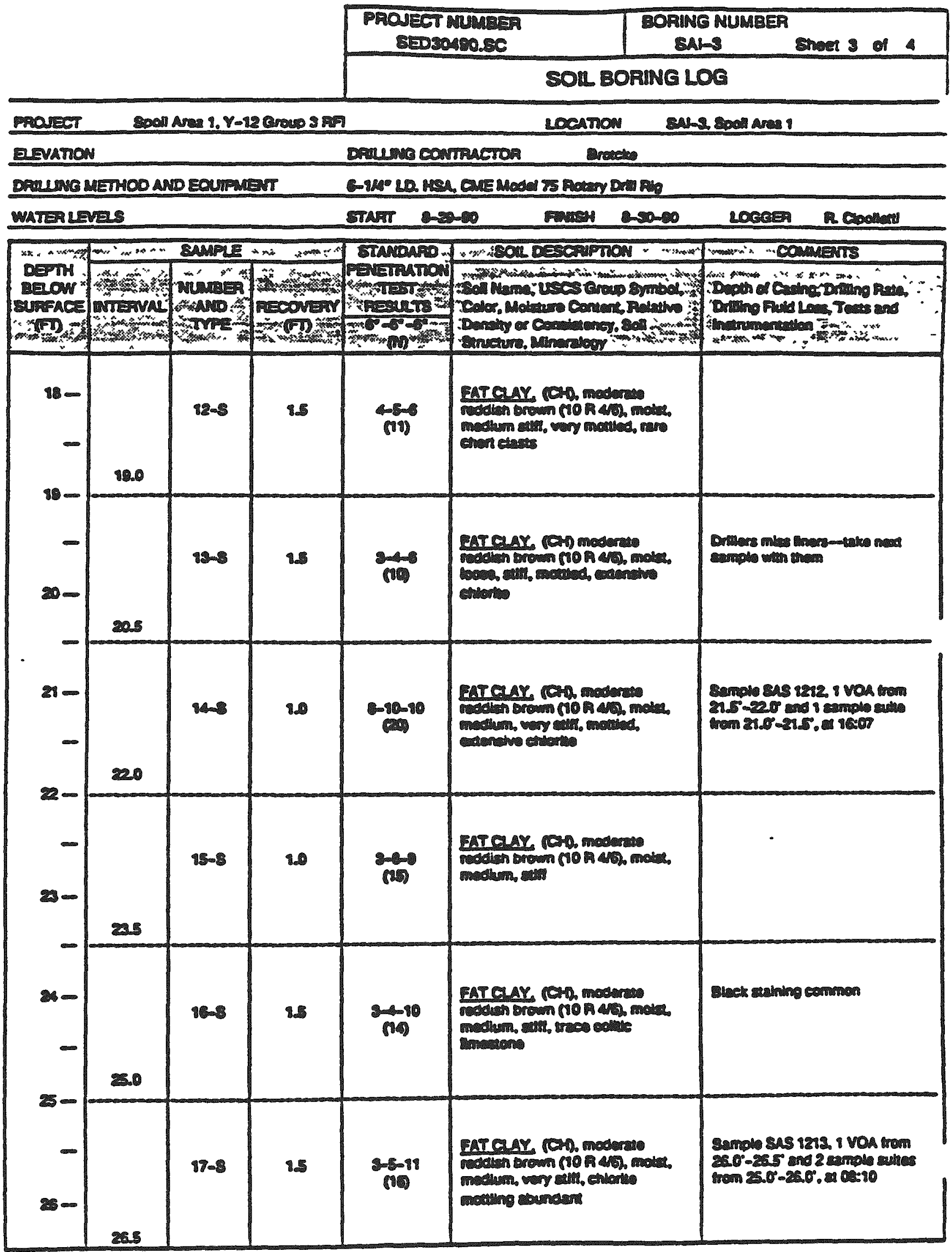


A-126

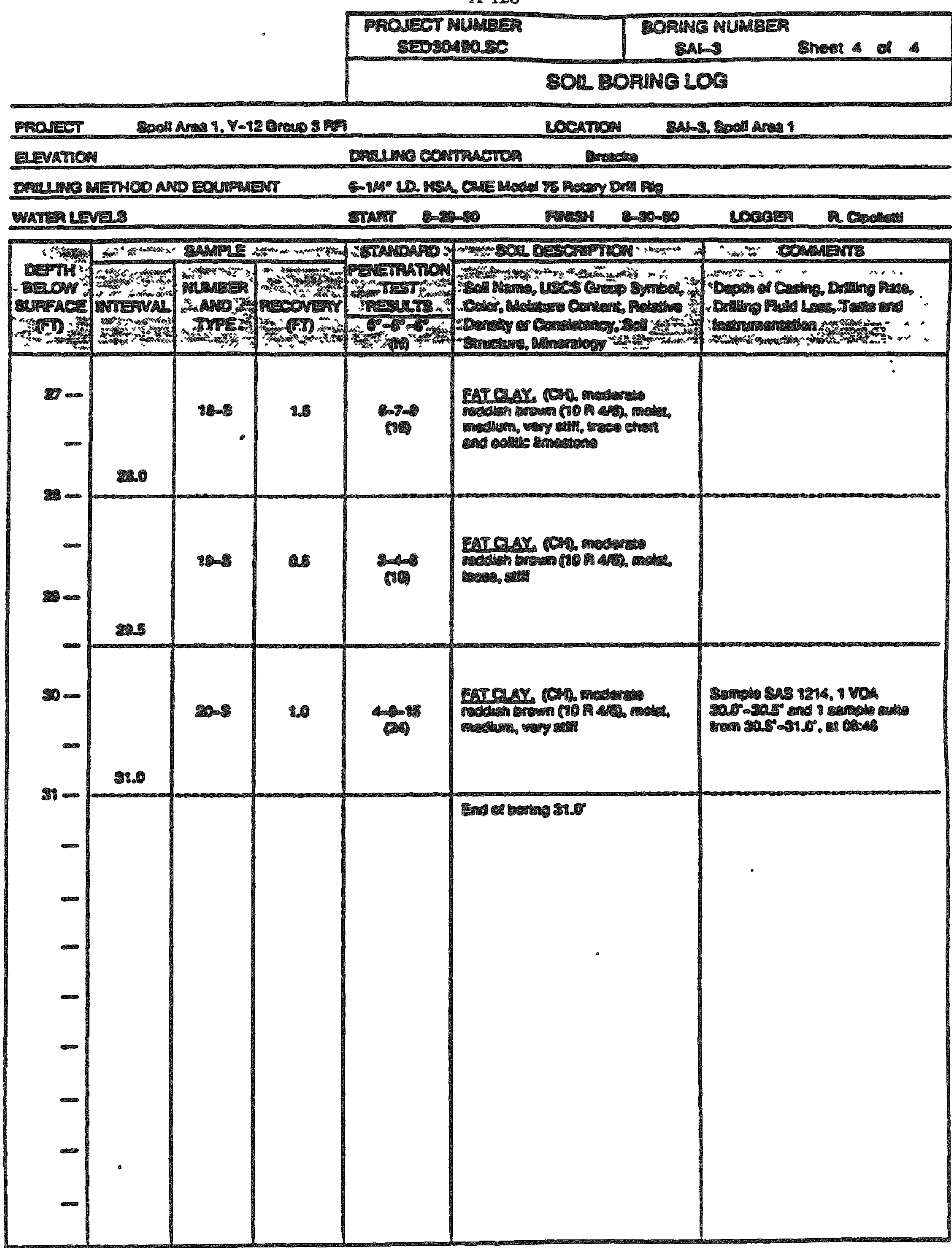




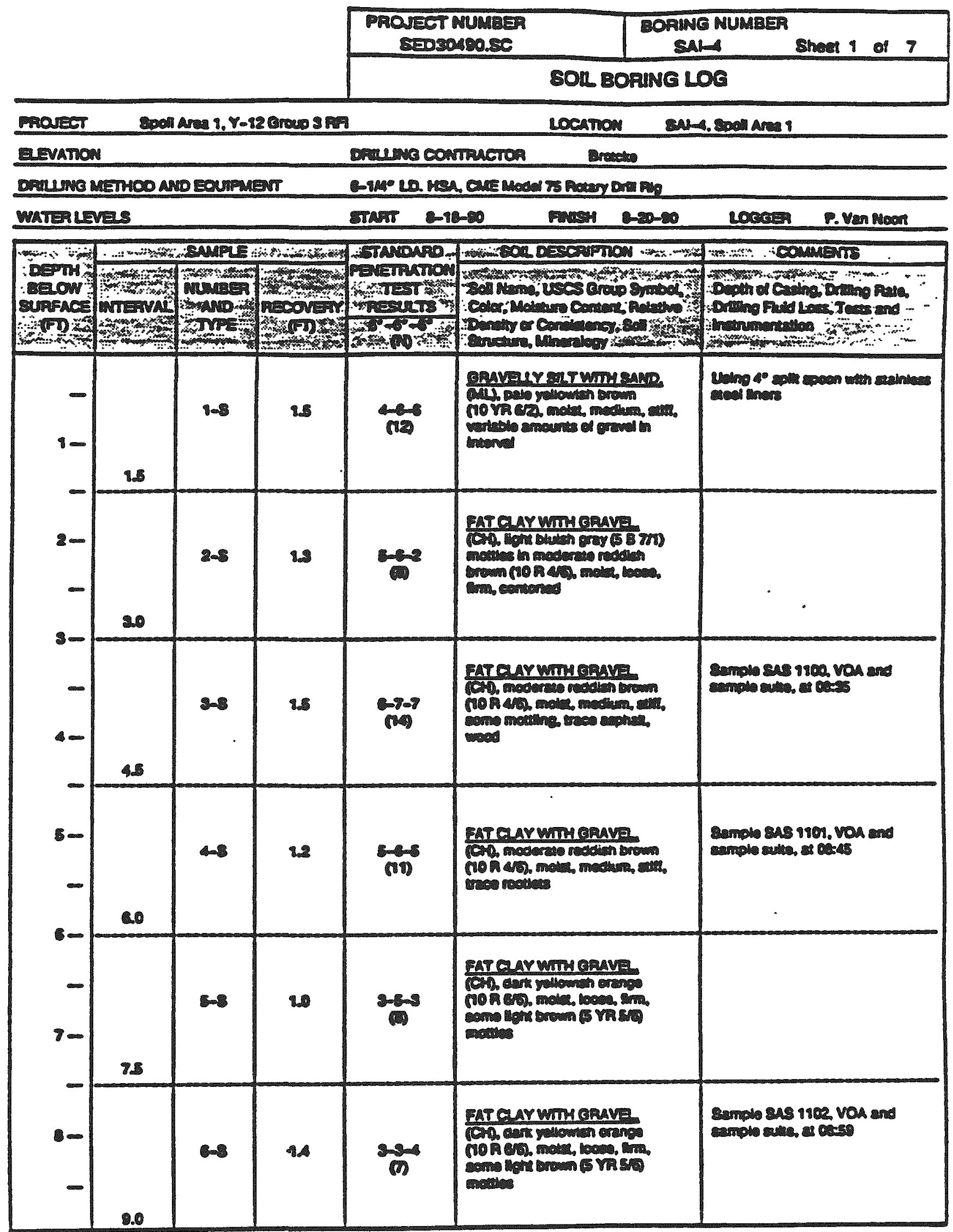


A-128

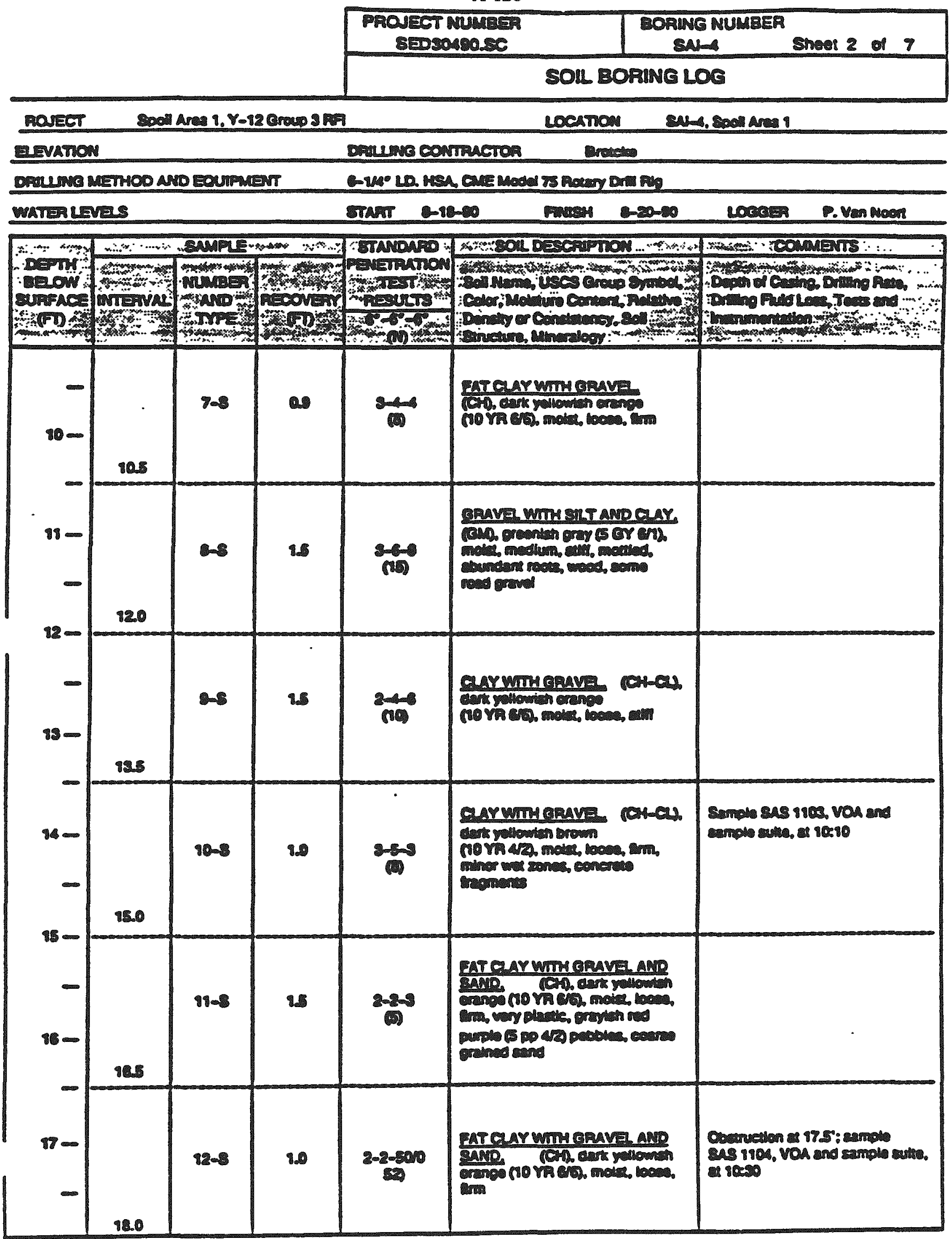


A-129

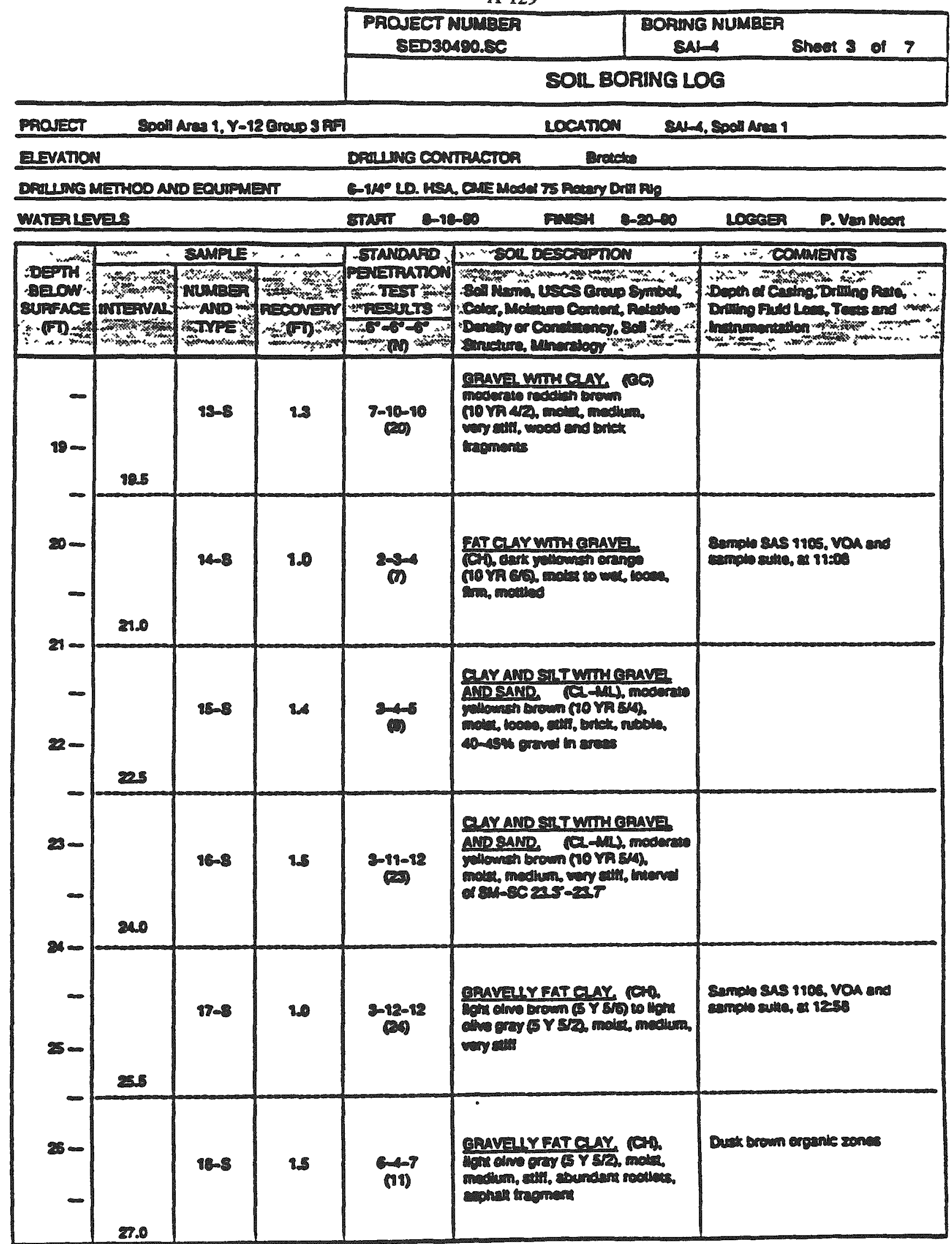




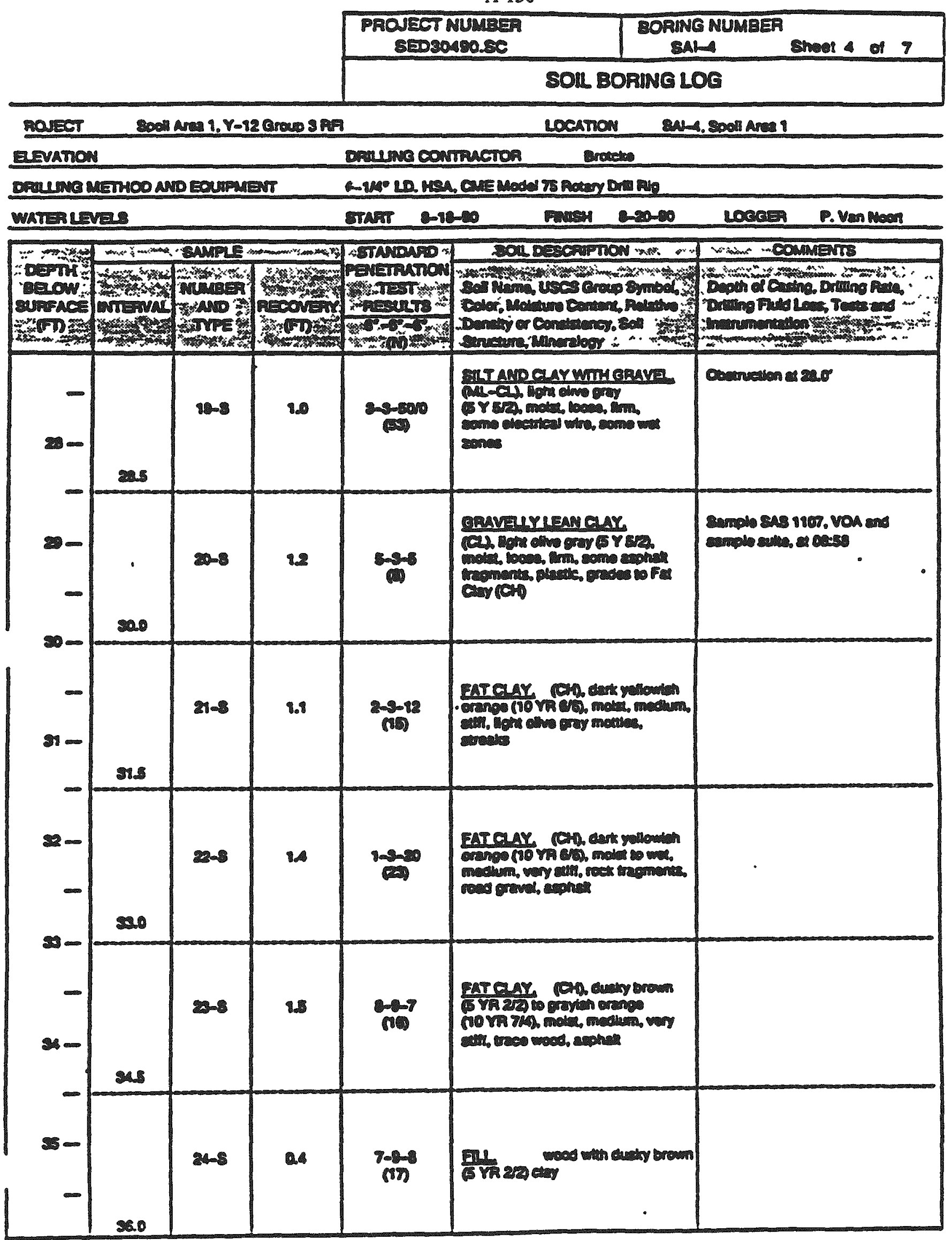


A-131

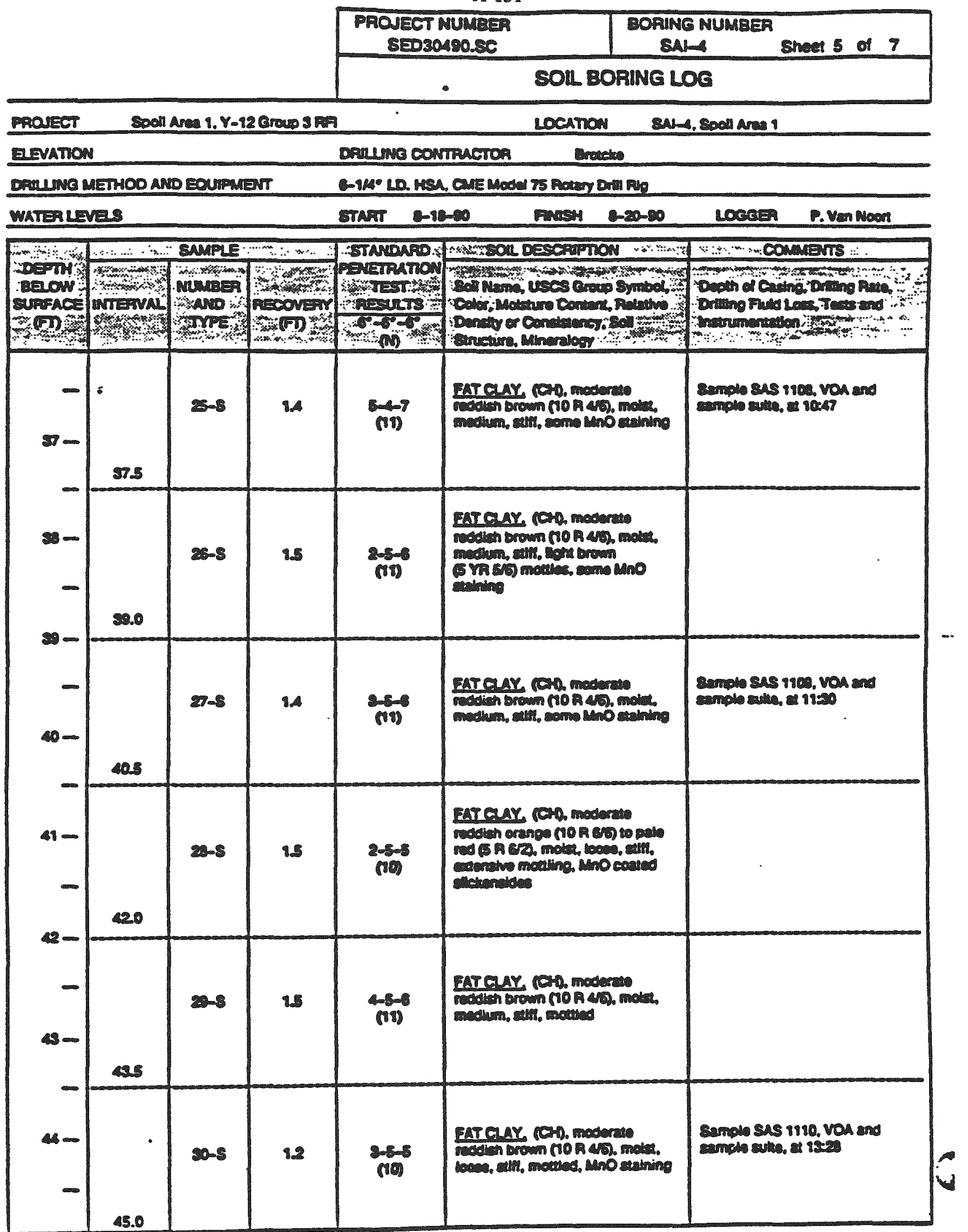


A-132

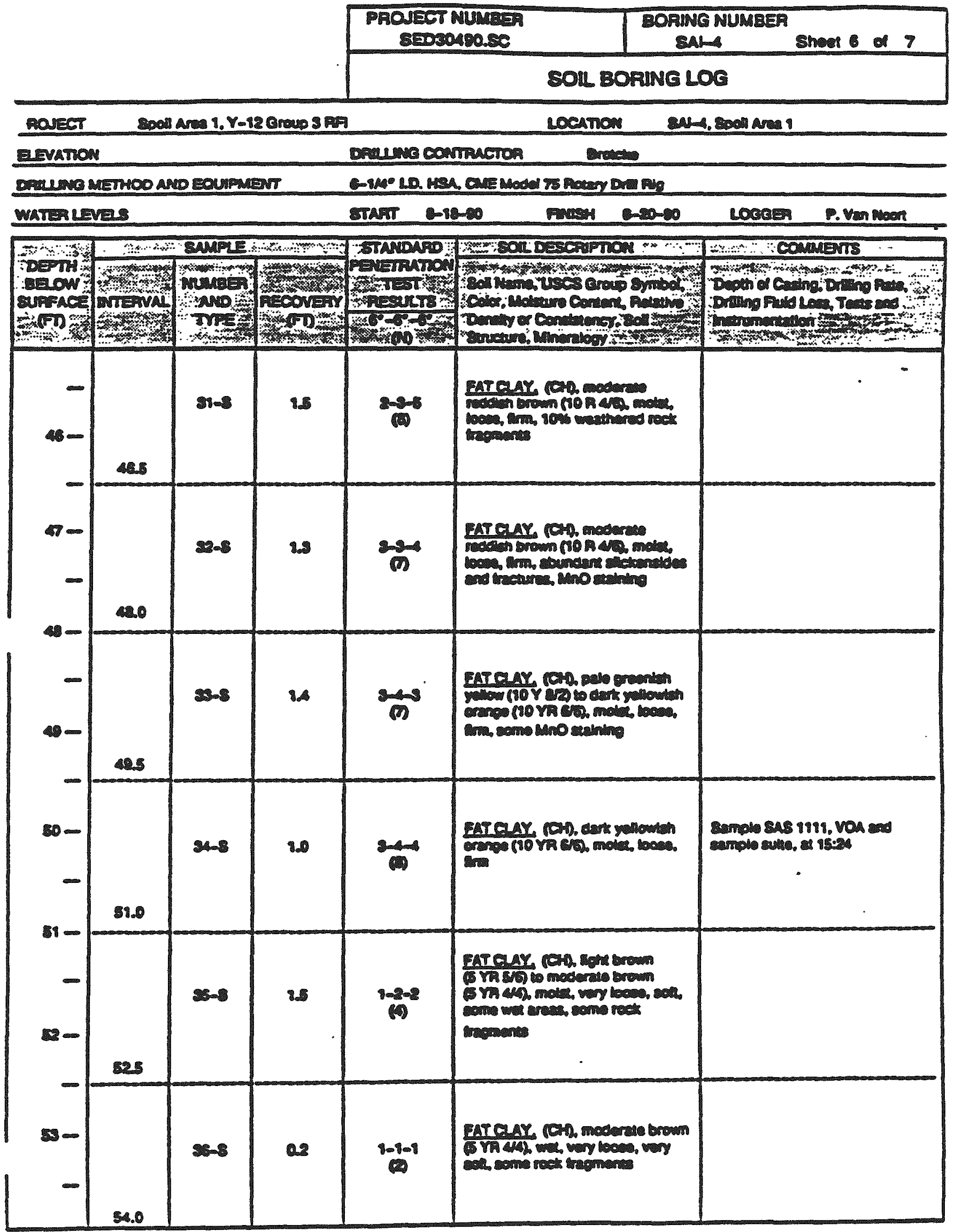


A-133

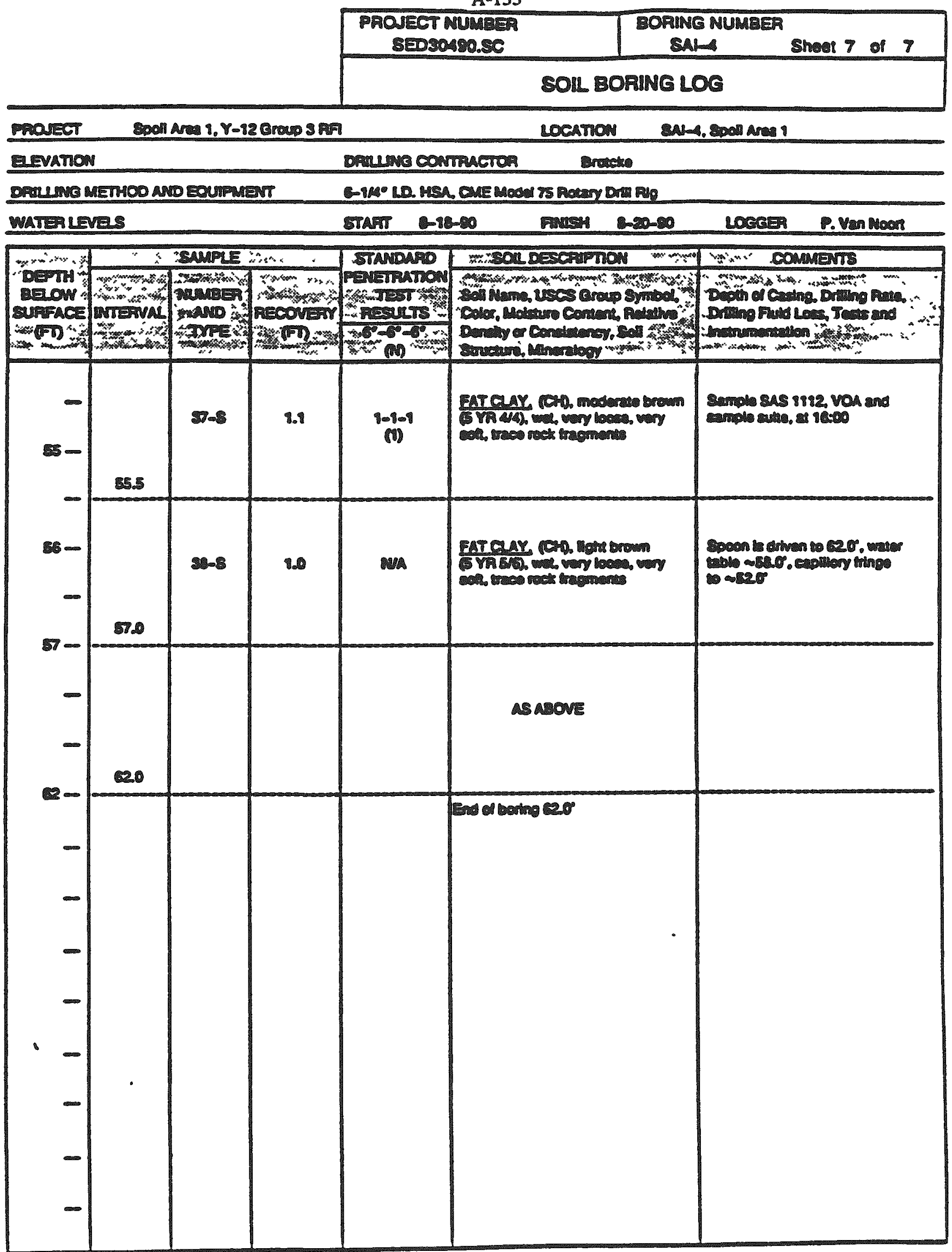


A-134

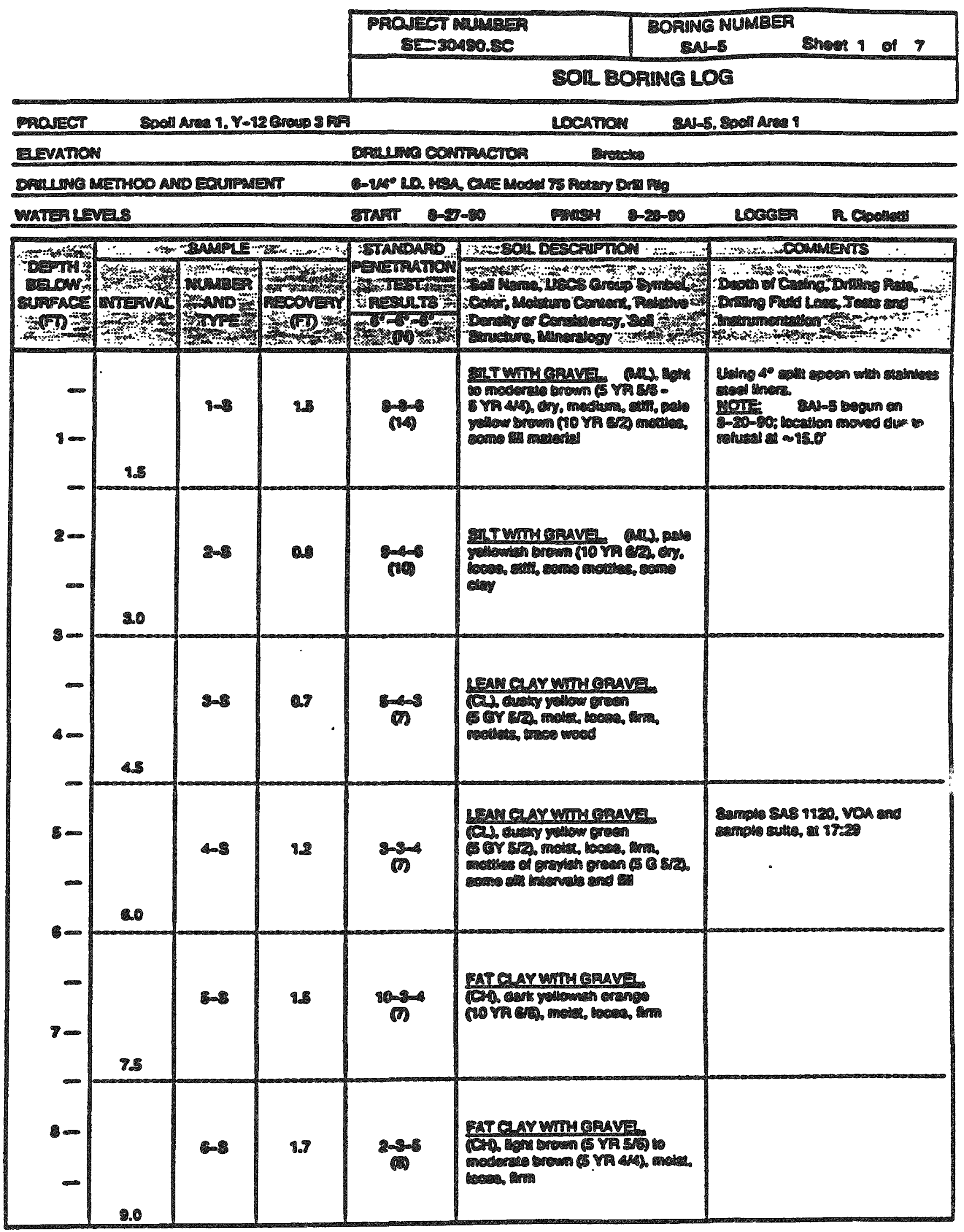




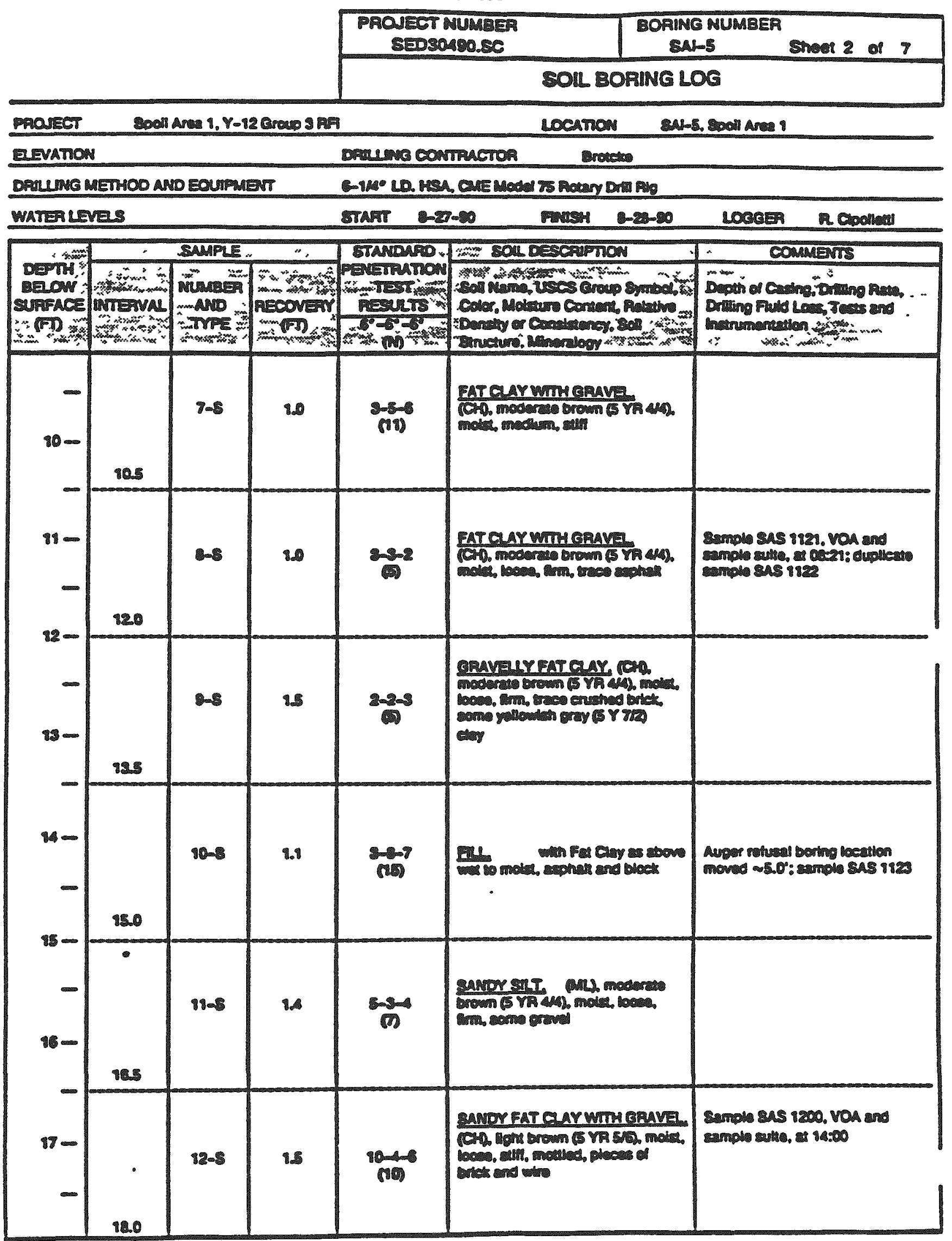




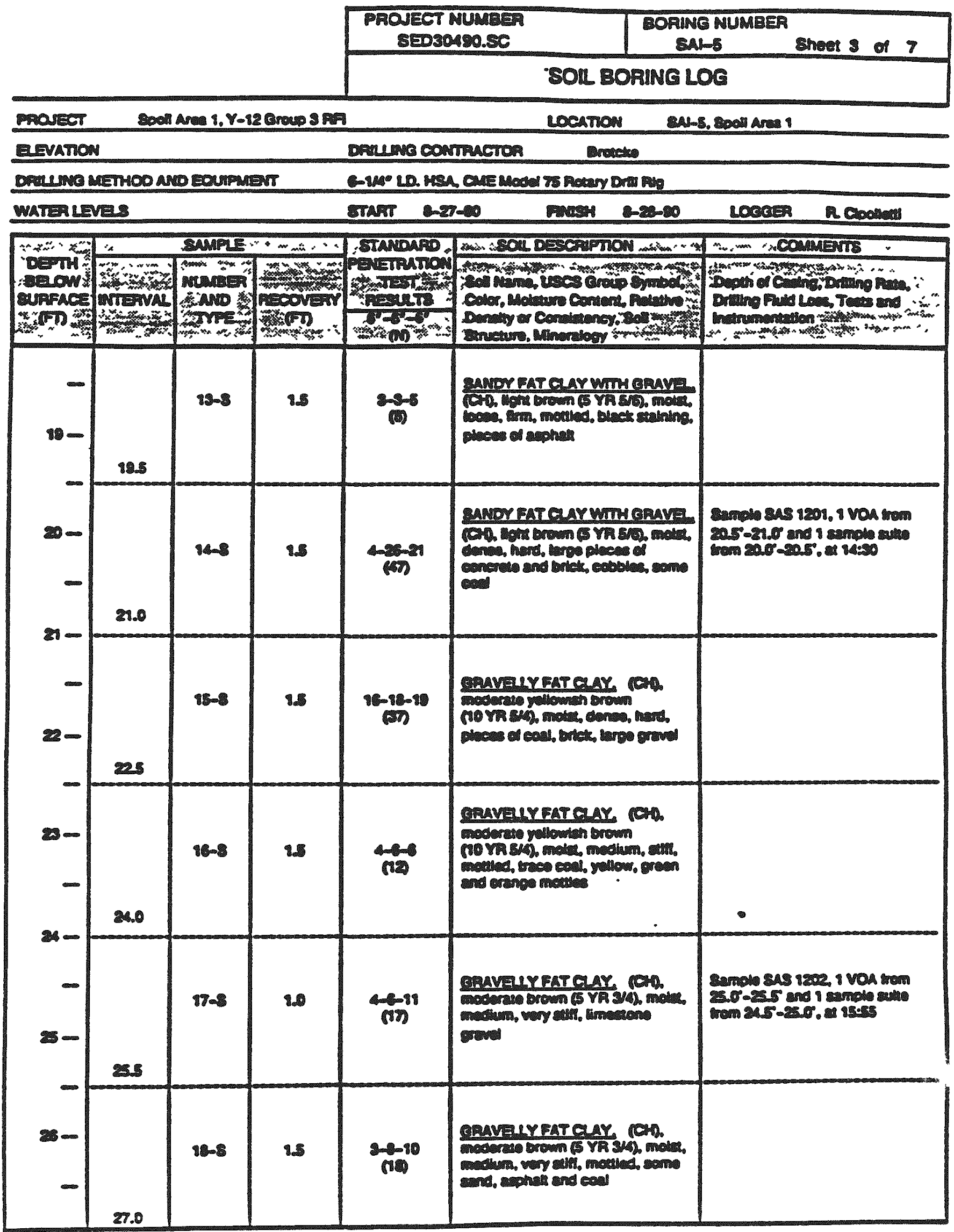




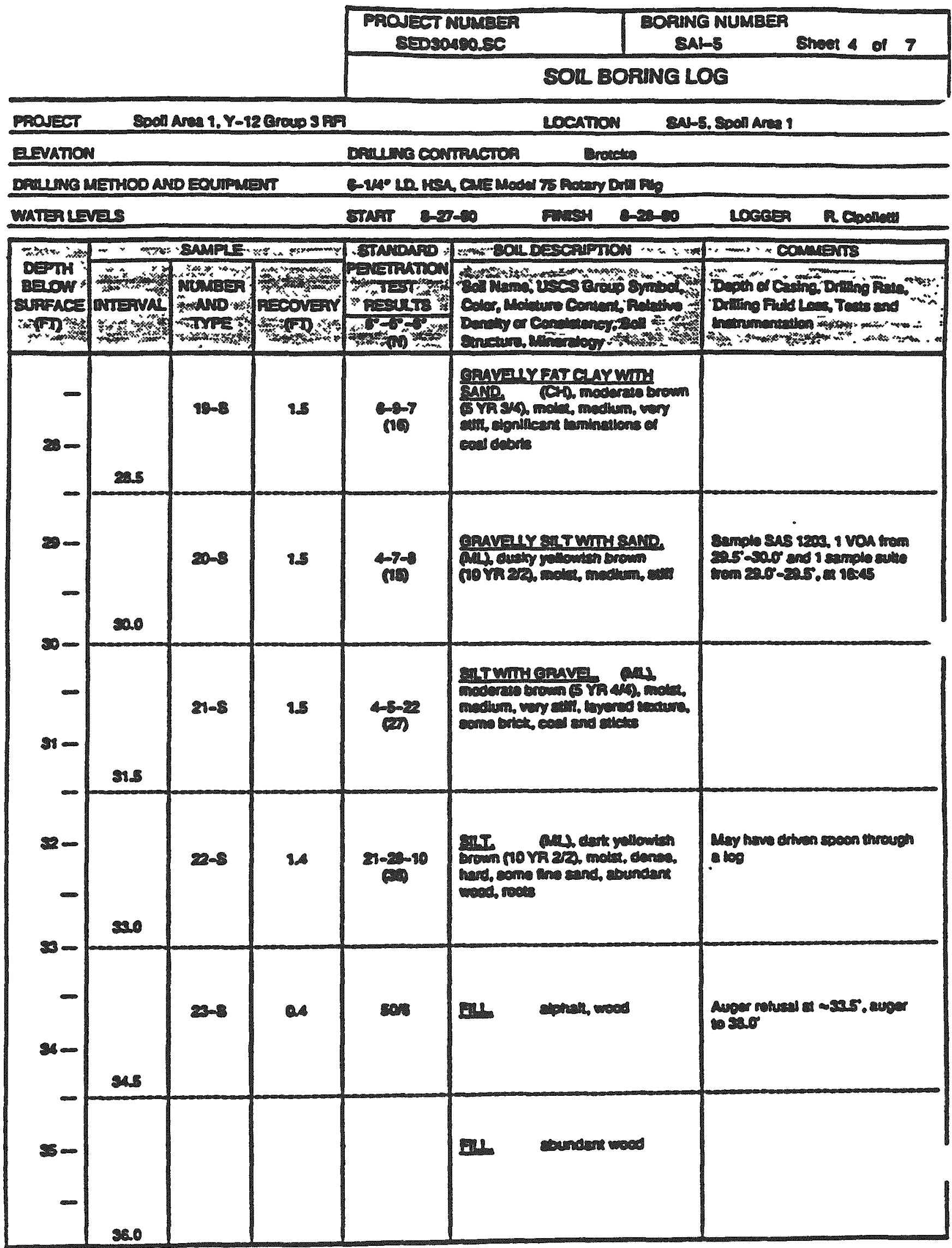


A-138

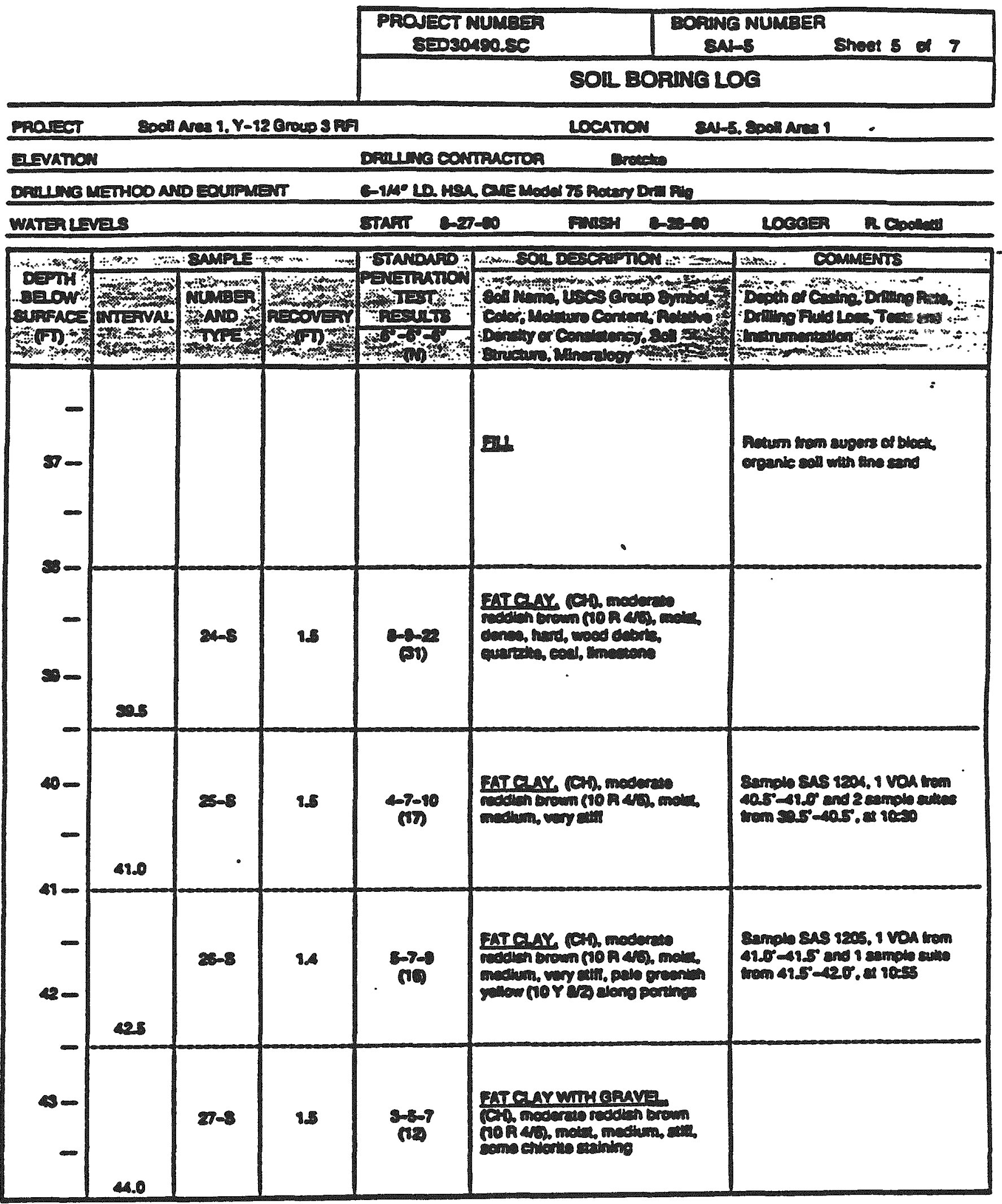


A-139

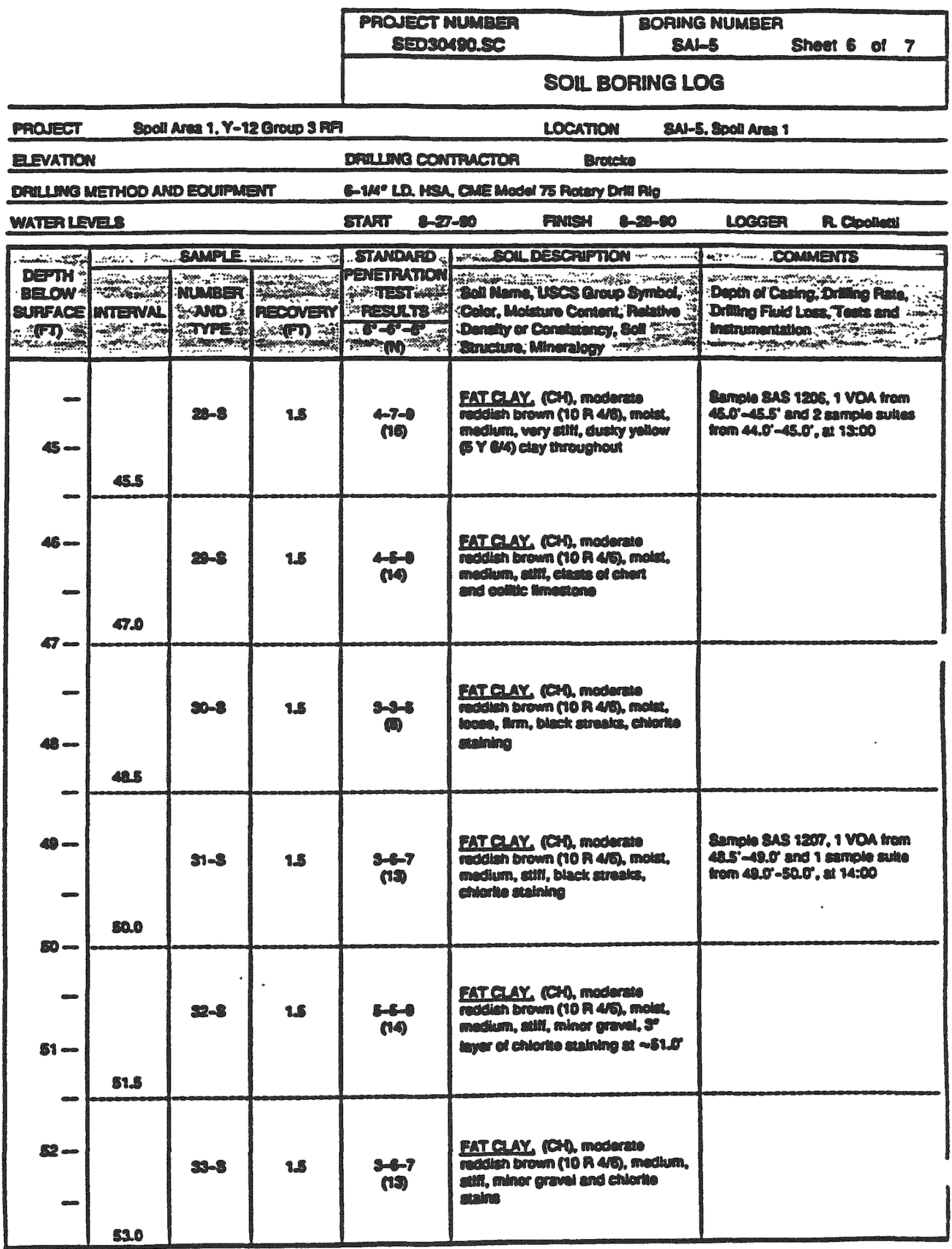


A-140

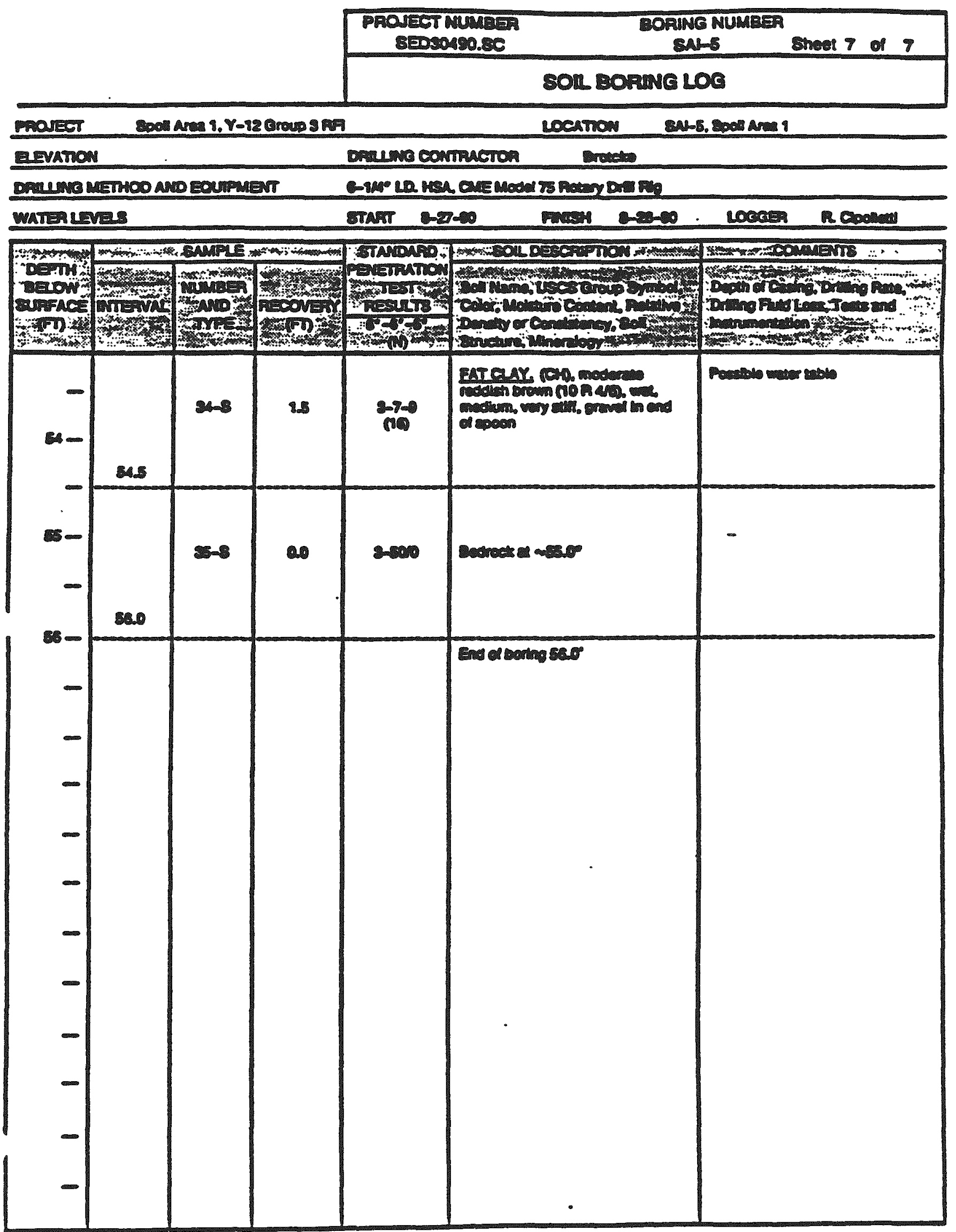




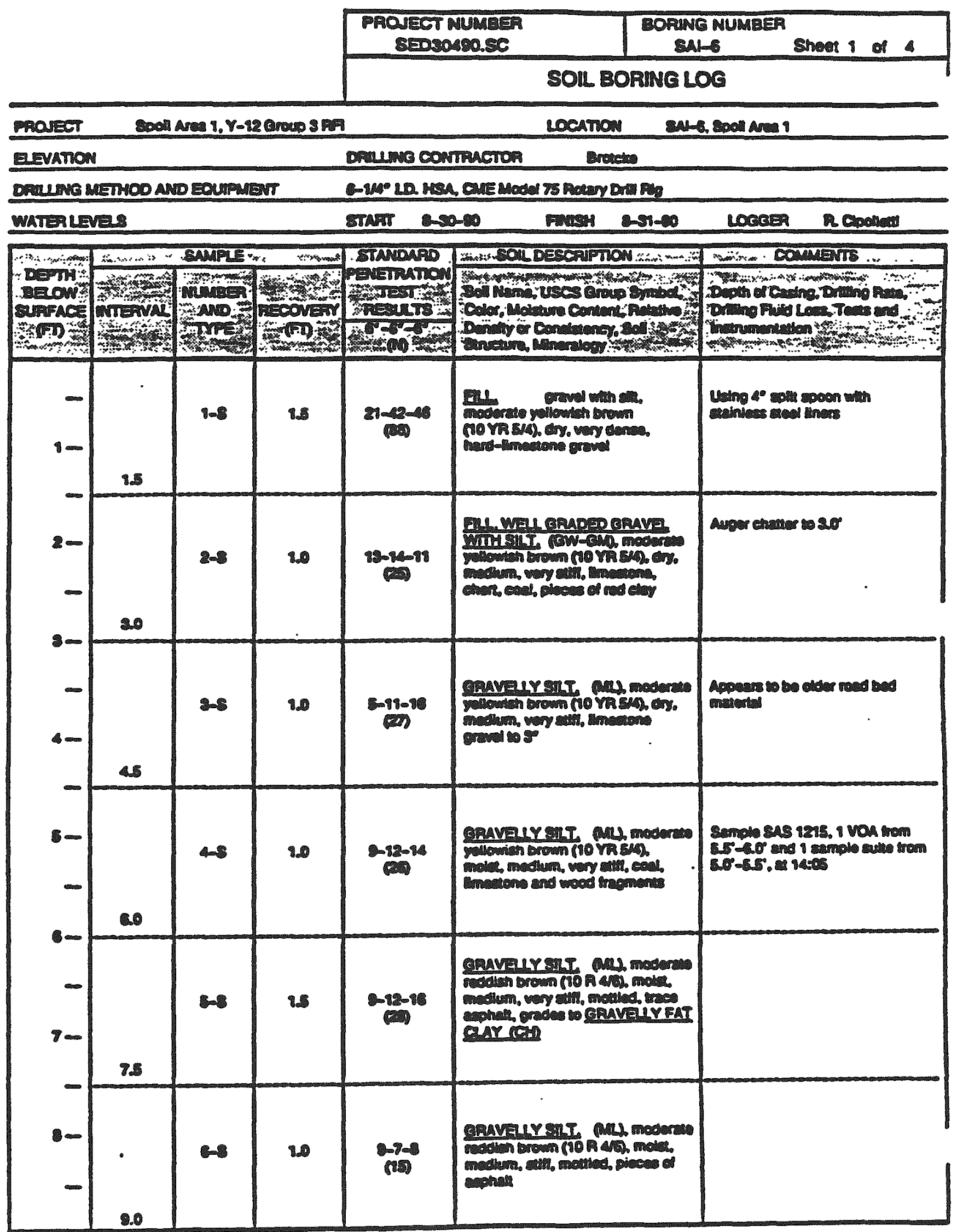


A-142

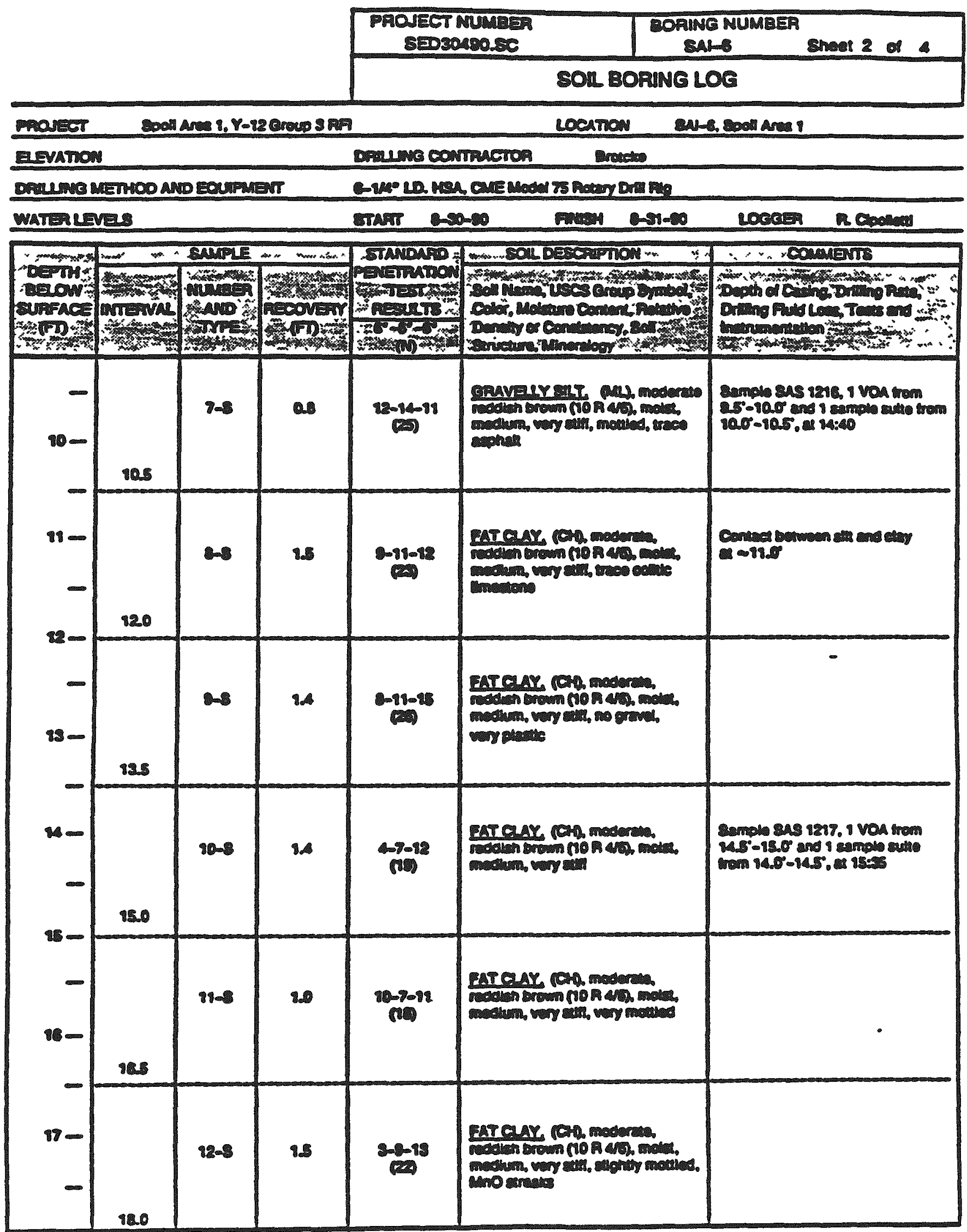


A-143

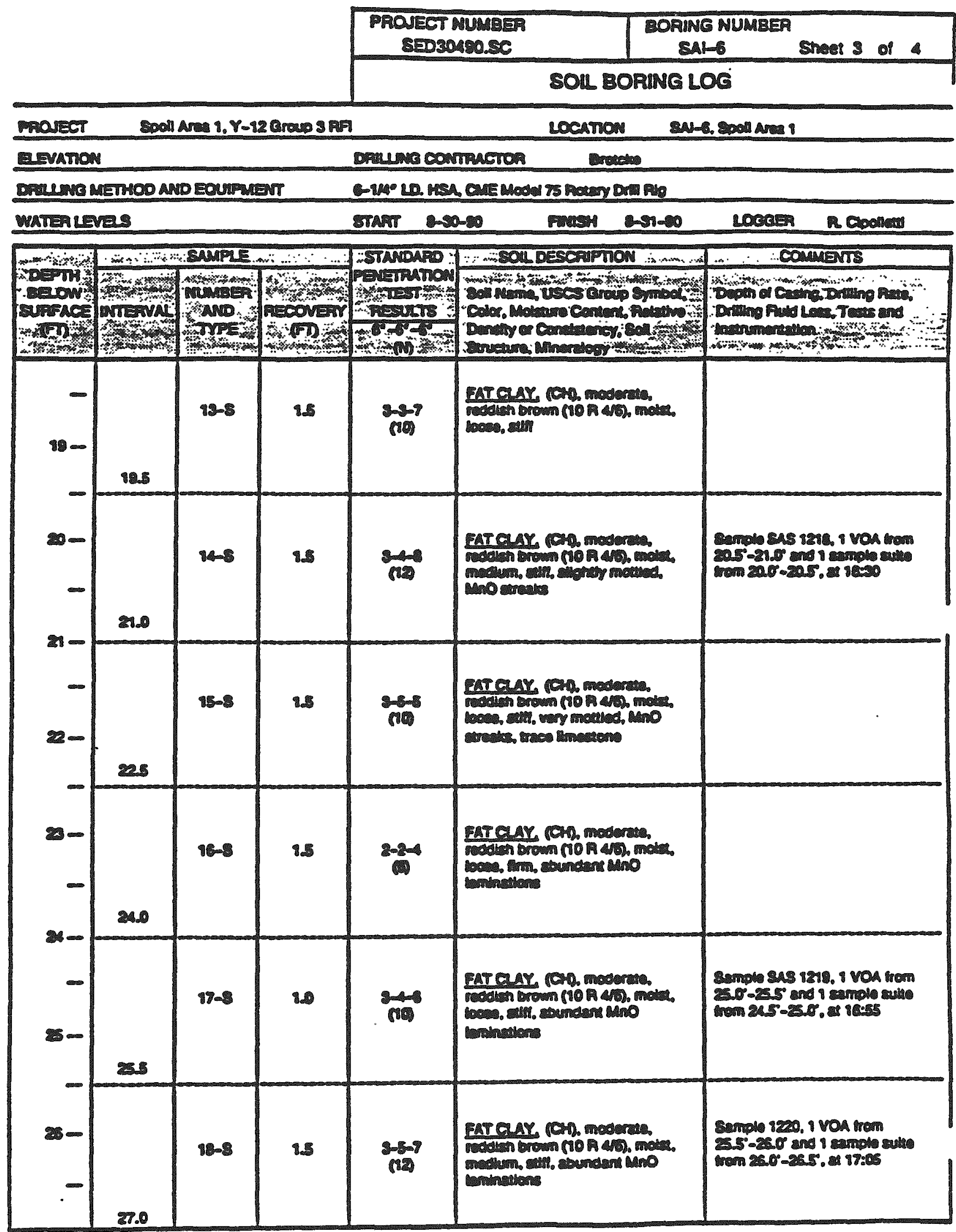




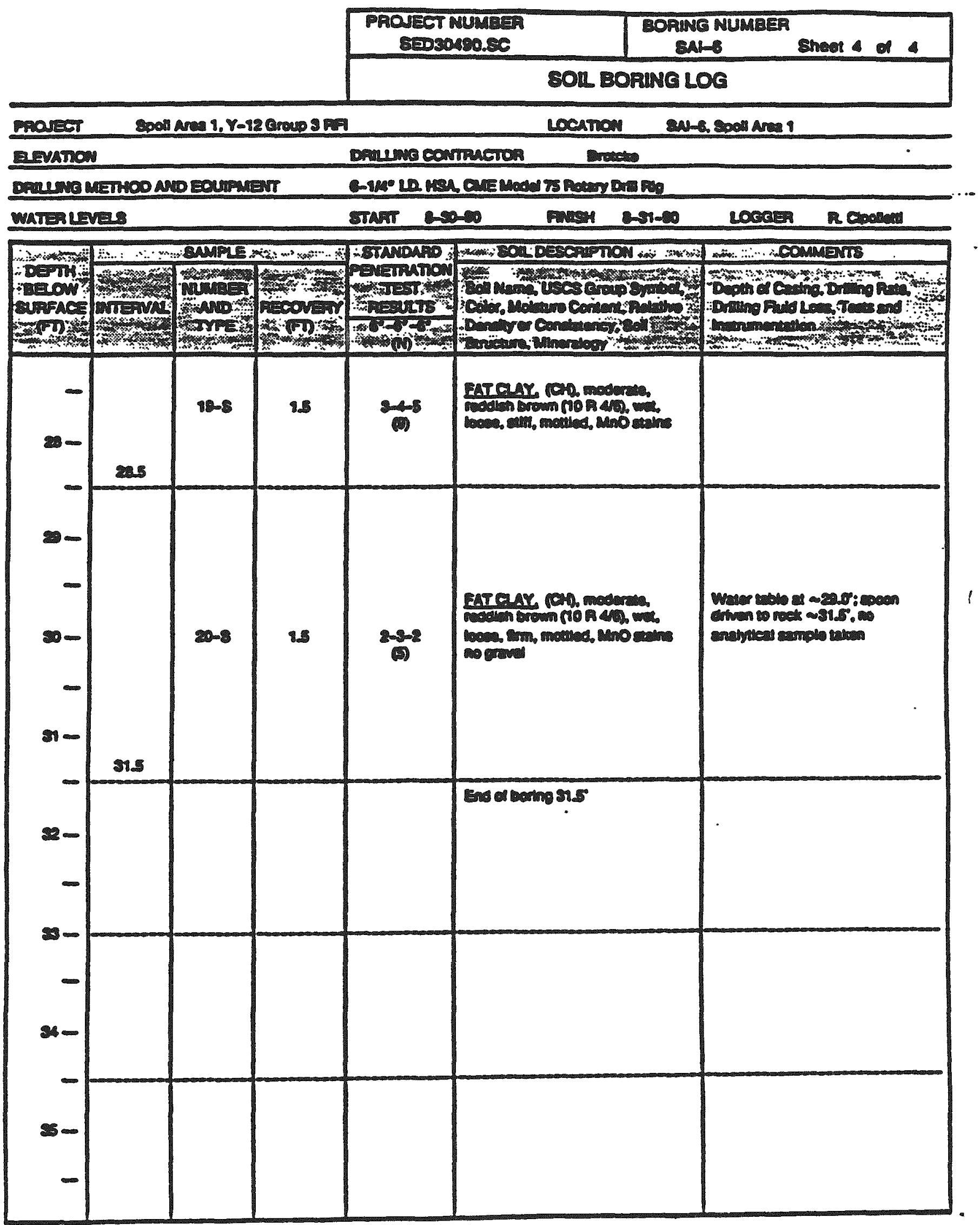


Current Groundwater Monitoring Well Construction Logs and Lithology Logs 

BEAR CREEK VALLEY OU 2

\section{WELL INSTALLATION DIAGRAM}

WELL NO. MW-1/GW-811

Coordinates:

N 29,443.2

E 52,324.6

LOGGED BY: Susan L. Abston

DRILLING DATES

DRILLING COMPANY: Ogden

STARTED:

9-24-93

DRILLER: George Akins HELPER: Bob Snow, Casey Minks

FINISHED: $\quad$ 10-27-93

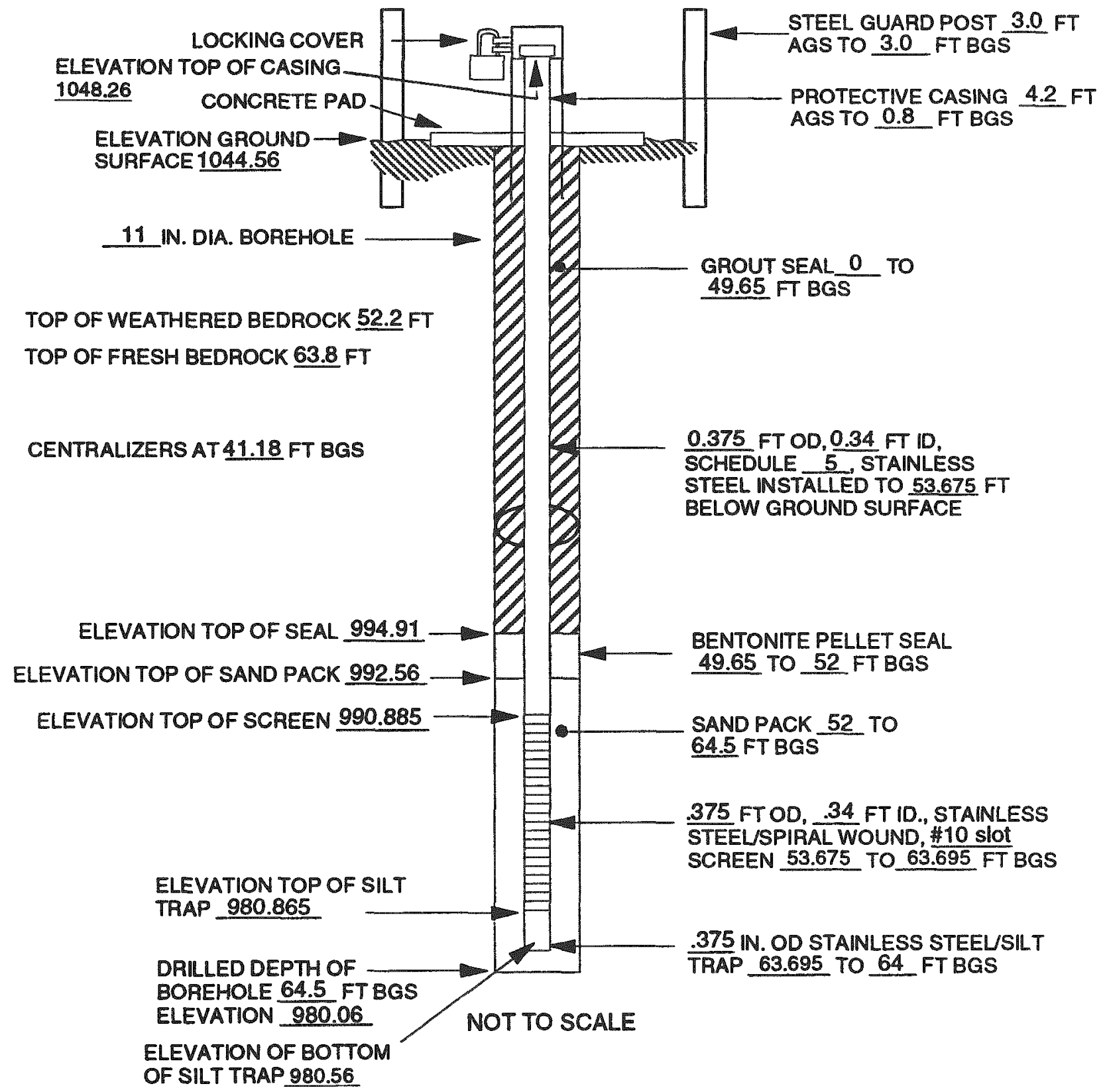


A-148

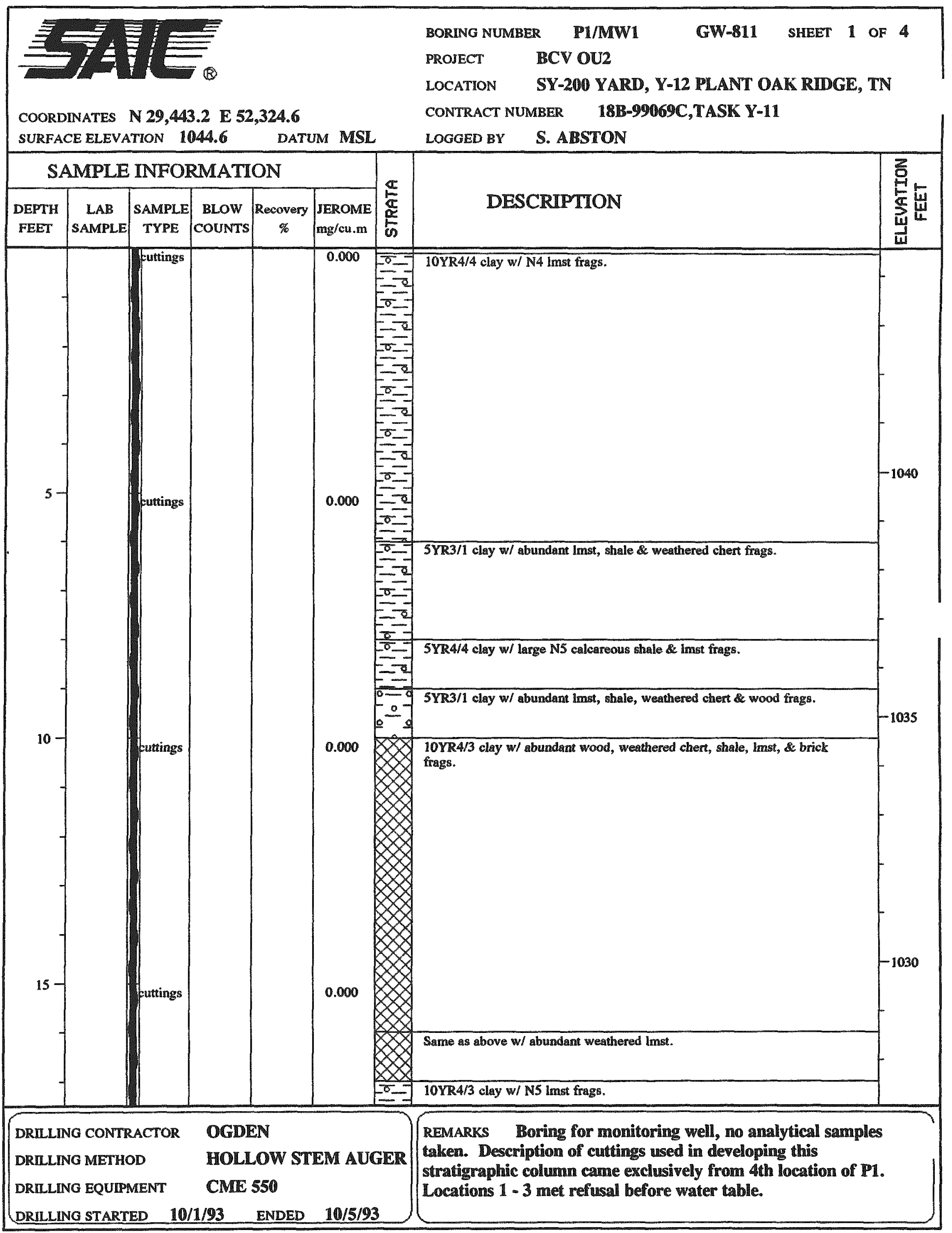


A-149

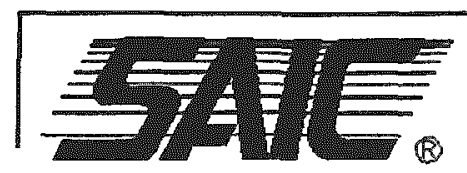

COORDINATES N 29,443.2 E 52,324.6 SURFACE ELEVATION 1044.6

\begin{abstract}
DATUM MSL
\end{abstract}

\section{BORING NUMBER P1/MW1 \\ PROJECT $\quad$ BCV OU2}

LOCATION SY-200 YARD, Y-12 PLANT OAK RIDGE, TN

CONTRACT NUMBER $\quad$ 18B-99069C,TASK Y-11

LOGGED BY S. ABSTON

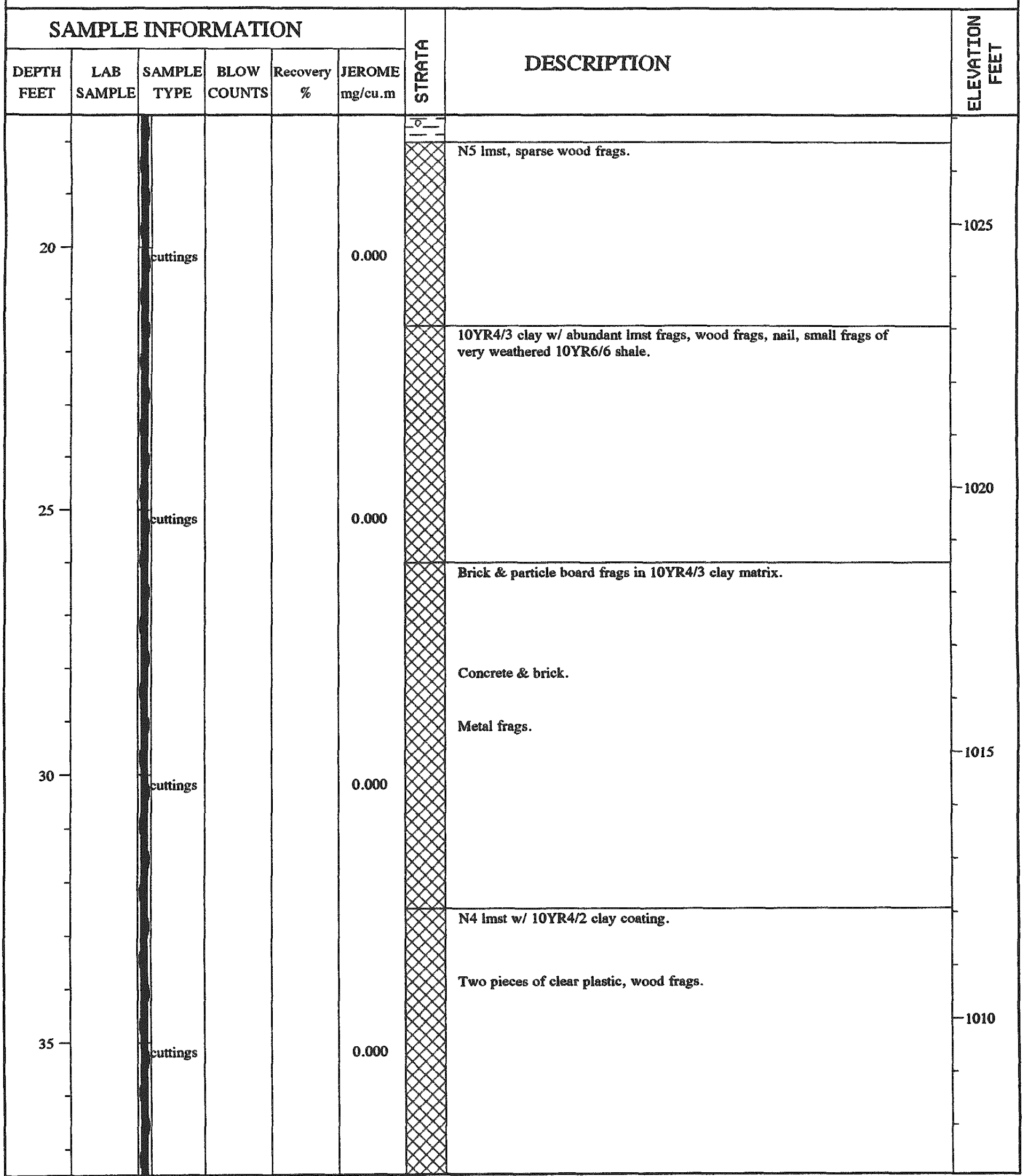


A-150

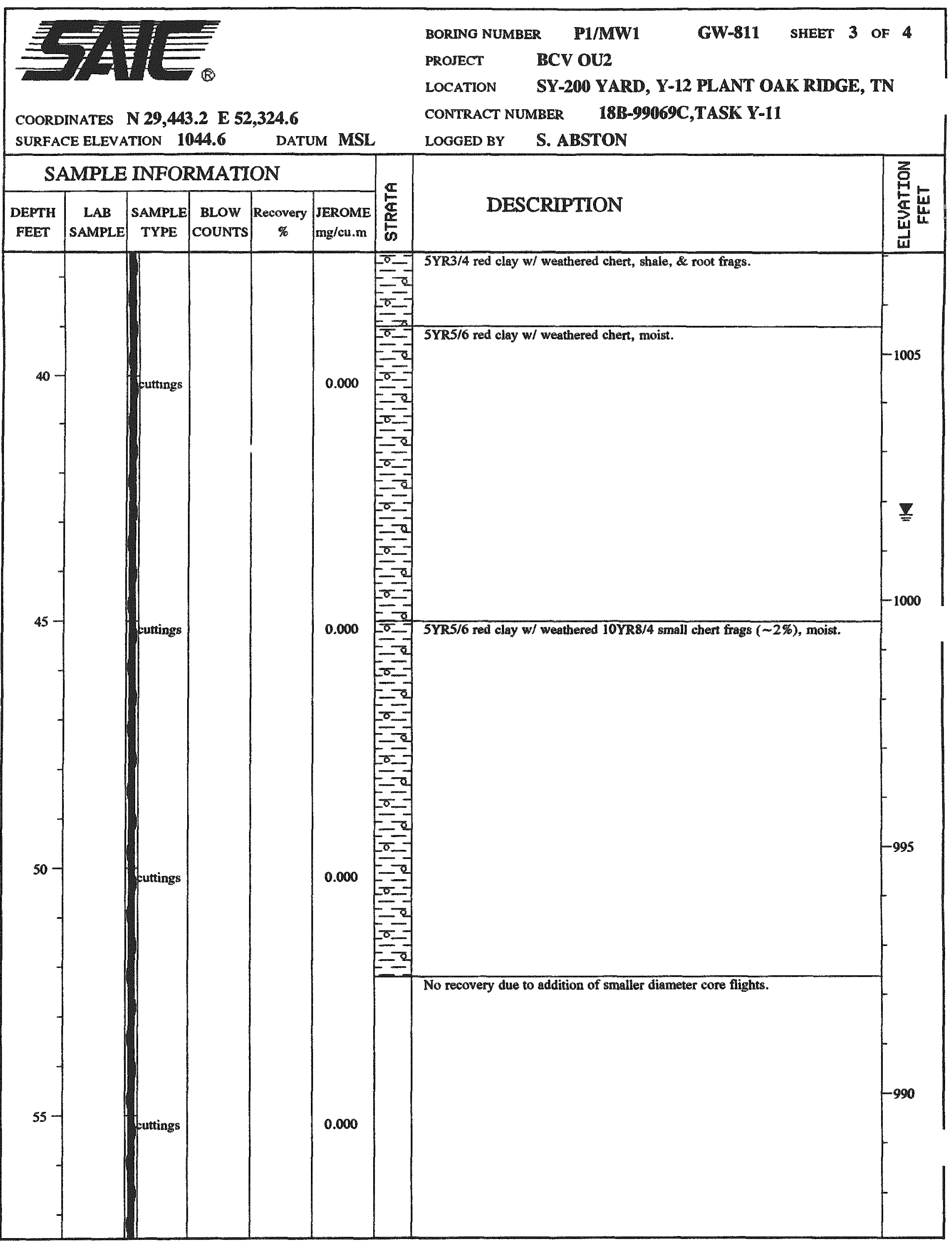




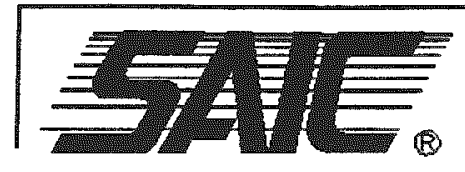

COORDINATES N 29,443.2 E 52,324.6

SURFACE ELEVATION 1044.6

\section{BORING NUMBER}

PROJECT

LOCATION

CONTRACT N

S. ABSTON

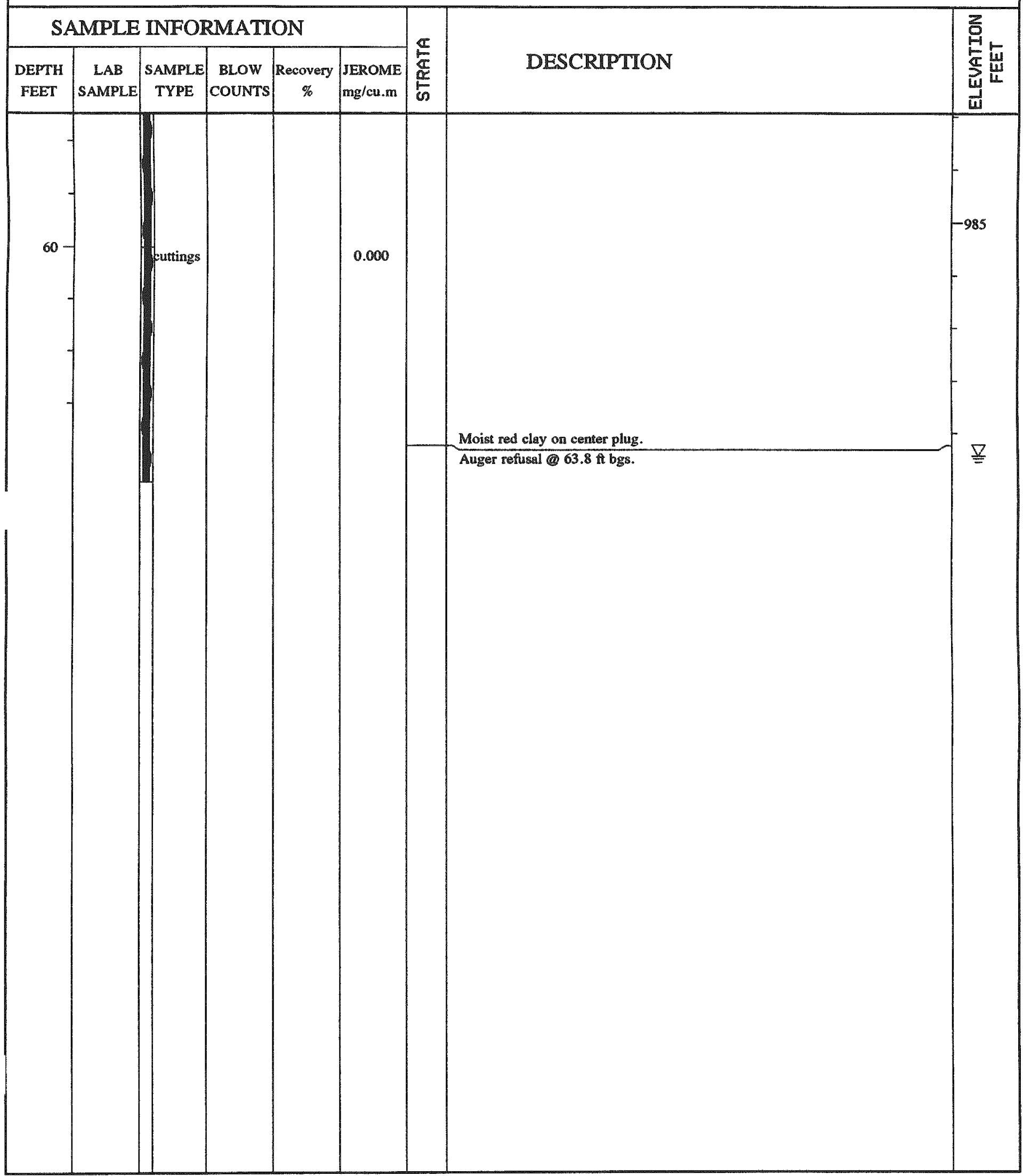




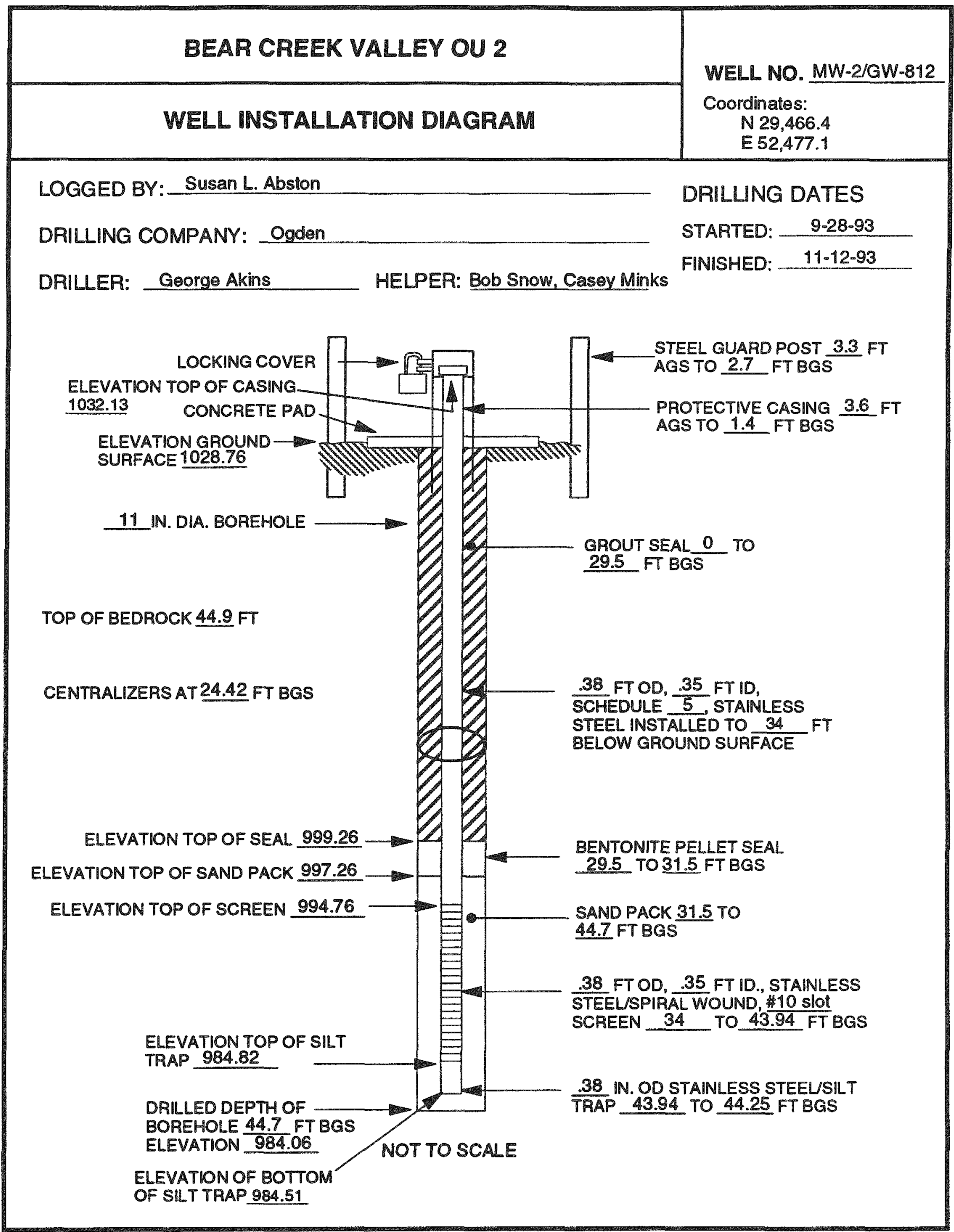


A-153

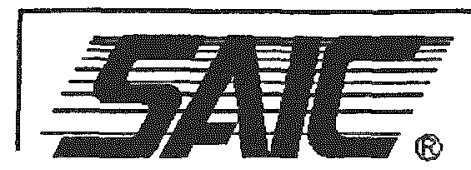

COORDINATES N 29,466.4 E $\mathbf{5 2 , 4 7 7 . 1}$

SURFACE ELEVATION 1028.8
BORING NUMBER P2/MW2

GW-812

SHEET 1 OF 3

PROIECT $\quad$ BCV OU2

LOCATION SY-200 YARD, Y-12 PLANT OAK RIDGE, TN

CONTRACT NUMBER $\quad$ 18B-99069C,TASK Y-11

\begin{tabular}{|c|c|c|c|c|c|c|c|c|}
\hline \multicolumn{6}{|c|}{ SAMPLE INFORMATION } & \multirow[b]{2}{*}{ 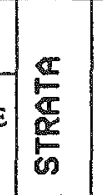 } & \multirow[b]{2}{*}{ DESCRIPTION } & \multirow{2}{*}{ 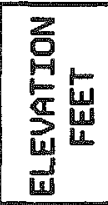 } \\
\hline $\begin{array}{c}\text { DEPTH } \\
\text { FEET }\end{array}$ & $\begin{array}{c}\text { LAB } \\
\text { SAMPLE }\end{array}$ & $\begin{array}{c}\text { SAMPLE } \\
\text { TYPE }\end{array}$ & $\begin{array}{c}\text { BLOW } \\
\text { COUNTS }\end{array}$ & $\left.\right|_{\%} ^{\text {Recovery }}$ & $\begin{array}{l}\text { JEROME } \\
\mathrm{mg} / \mathrm{cu} . \mathrm{m}\end{array}$ & & & \\
\hline $10-$ & & curtings & & & $\begin{array}{l}0.000 \\
0.000 \\
0.000 \\
0.000 \\
\end{array}$ & 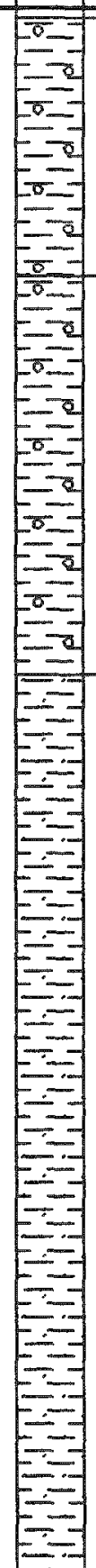 & $\begin{array}{l}\text { 10YR4/4 brown clay w/ Imst frags, dry. } \\
\begin{array}{l}\text { YYR } 4 / 6 \text { yellowish brown clay w/ sparse } 10 \mathrm{YR} / 3 \text { weathered chert frags, } \\
\text { moist. }\end{array} \\
2.5 \mathrm{YR} 4 / 8 \text { reddish brown clay w/ sparse } 10 \mathrm{YR} 8 / 3 \text { weathered chert frags. }\end{array}$ & \begin{tabular}{|l} 
\\
-1025 \\
\\
\\
-1020 \\
\\
\\
\\
\\
\\
\end{tabular} \\
\hline $\begin{array}{l}\text { DRILLII } \\
\text { DRILLII } \\
\text { DRILLII } \\
\text { DRILLI }\end{array}$ & $\begin{array}{l}\text { NG CONTI } \\
\text { NG METH } \\
\text { NG EQUIP } \\
\text { NG START }\end{array}$ & $\begin{array}{l}\text { RACTOR } \\
\text { OD } \\
\text { MENT } \\
\text { ED } \quad 9 / 2\end{array}$ & $\begin{array}{l}\text { OGDH } \\
\text { HOLI } \\
\text { CME } \\
8 / 93\end{array}$ & $\begin{array}{l}\text { EN } \\
\text { LOW STI } \\
550 \\
\text { ENDED }\end{array}$ & $\begin{array}{l}\text { EM AUC } \\
9 / 30 / 9\end{array}$ & IGER & $\begin{array}{l}\text { REMARks Boring for monitoring well, no analytical sa } \\
\text { taken. Deseription of cuttings used in developing this } \\
\text { stratigraphic column came exclusively from } 2 \text { 2nd location } \\
\text { Location } 1 \text { met refusal before water table. }\end{array}$ & \\
\hline
\end{tabular}


A-154

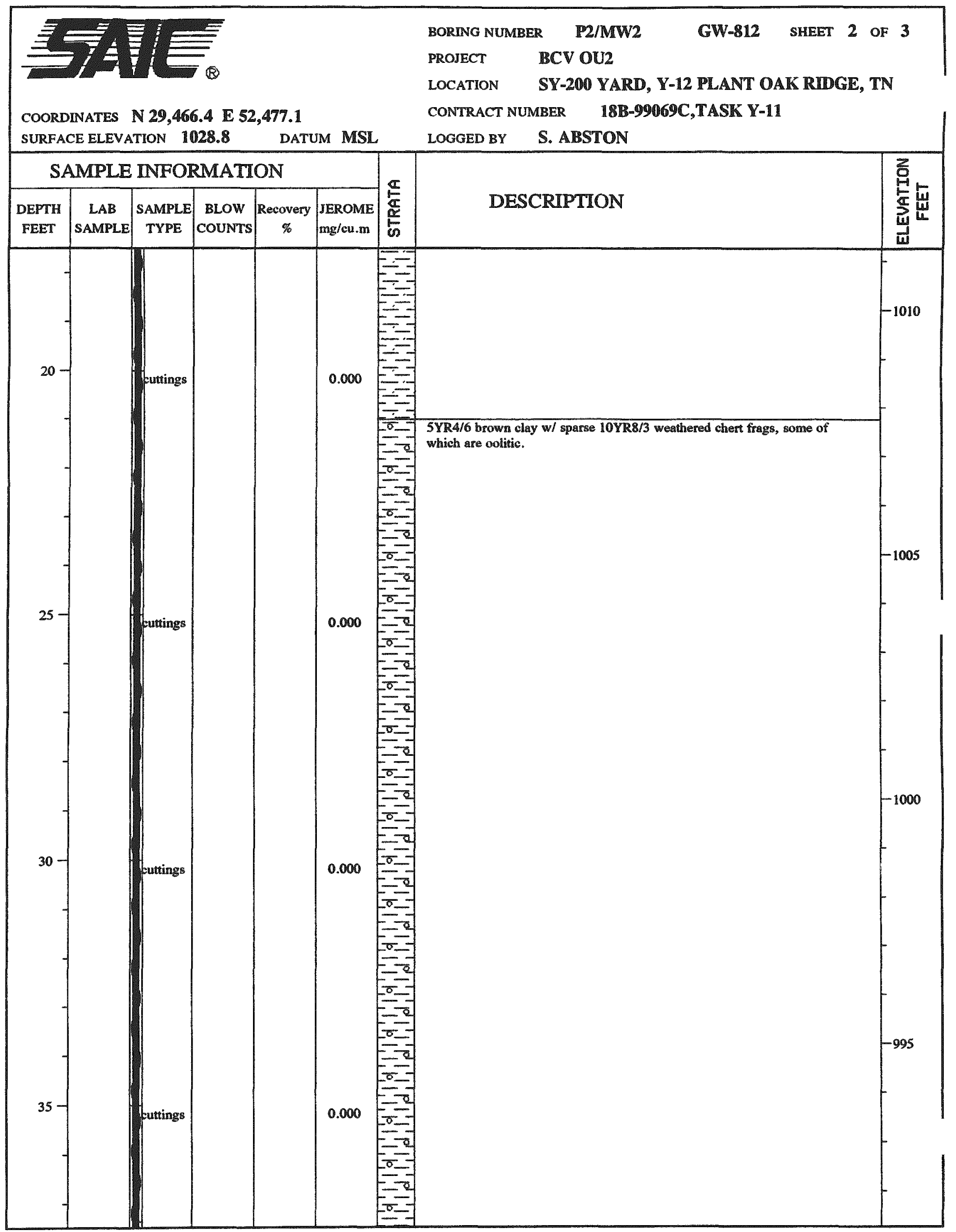




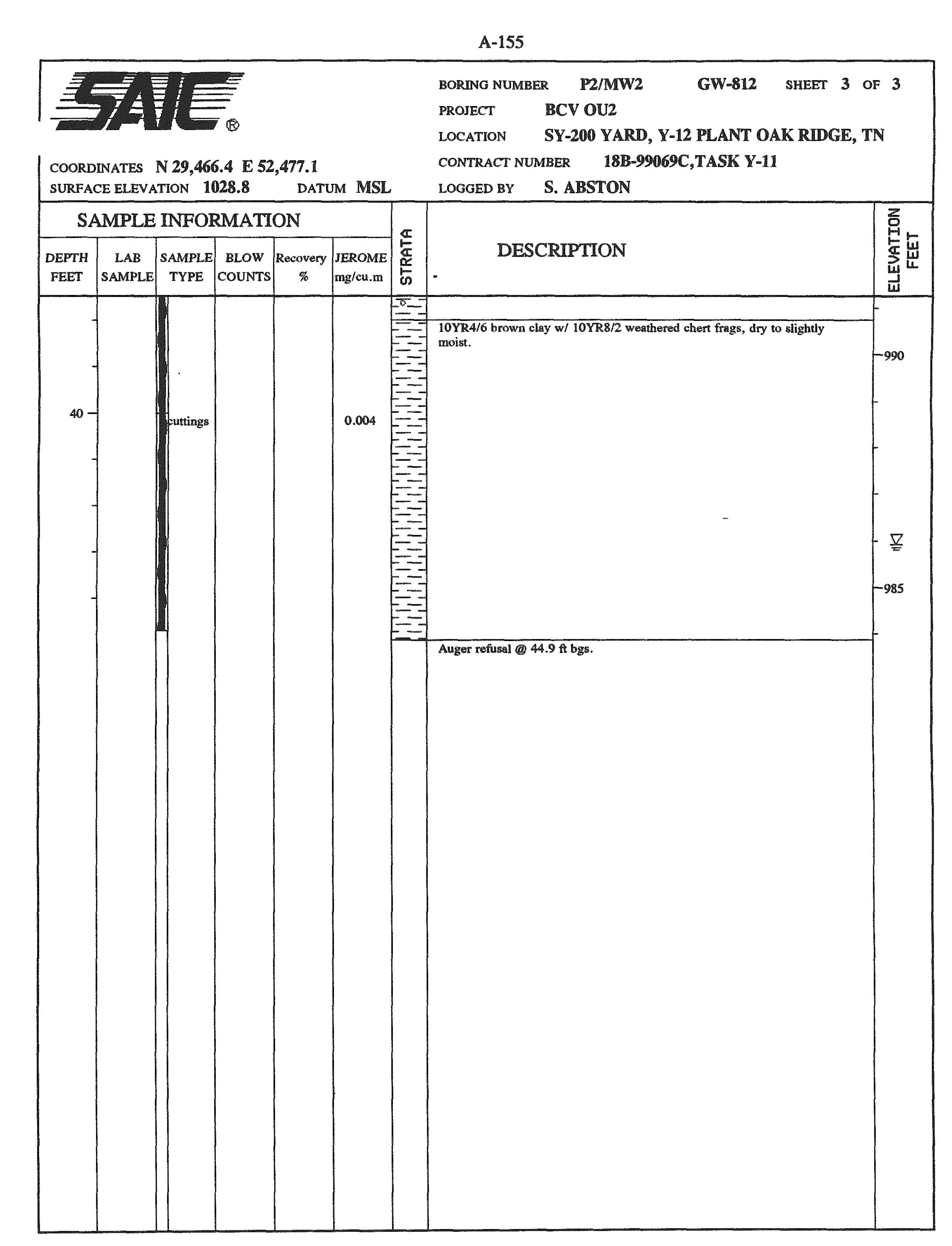




\section{BEAR CREEK VALLEY OU 2}

\section{WELL INSTALLATION DIAGRAM}

WELL NO. MW-3/GW-813

Coordinates:

N 29,590.1

E $51,474.3$

LOGGED BY: Susan L. Absion

DRILLING COMPANY: Ogden

DRILLER: George Akins

HELPER: Bob Snow

\section{DRILLING DATES}

STARTED: 11-18-93

FINISHED: $12-30-93$

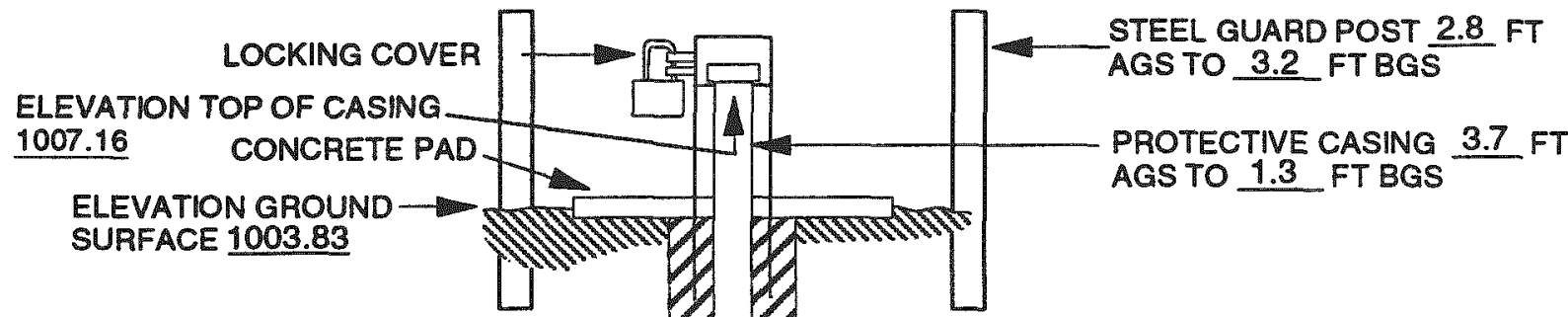

10-1/4 IN. DIA. BOREHOLE

GROUT SEAL 0 TO

8.3 FTBGS
.37 FT OD, 32 FT ID,

SCHEDULE 5 , STAINLESS

STEEL INSTALLED TO 12.46 FT

BELOW GROUND SUAFACE

ELEVATION TOP OF SEAL 995.53 ELEVATION TOP OF SAND PACK 992.48 ELEVATION TOP OF SCREEN 991.37

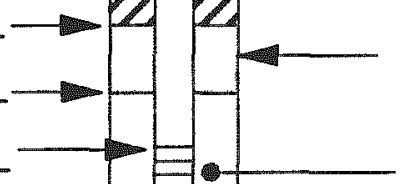

BENTONITE PELLET SEAL 8.3 TO 11.35 FT BGS

SAND PACK 11.35 TO 25.51 FT BGS

.37 FT OD, 32 FT ID., STAINLESS STEELSPIRAL WOUND, \#10 Slot SCREEN 12.46 TO 22.51 FT BGS

ELEVATION TOP OF SILT TRAP 981.32

DRILLED DEPTH OF BOREHOLE 25.51 FT BGS ELEVATION 978.32

ELEVATION OF BOTTOM OF SILT TRAP 979.32
.37 IN. OD STANLESS STEEUSLT TRAP 22.51 TO 24.51 FTBGS 


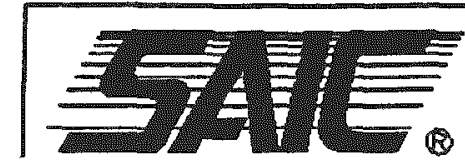

COORDINATES N 29,590.1 E 51,474.3

\begin{tabular}{|c|c|c|c|c|c|}
\hline & & rION & 9 & DATU & UM \\
\hline \multicolumn{6}{|c|}{ SAMPLE INFORMATION } \\
\hline $\begin{array}{c}\text { DEPTH } \\
\text { FEET }\end{array}$ & $\begin{array}{c}\text { LAB } \\
\text { SAMPLE }\end{array}$ & $\begin{array}{c}\text { SAMPLE } \\
\text { TYPE }\end{array}$ & $\begin{array}{l}\text { BLOW } \\
\text { COUNTS }\end{array}$ & $\begin{array}{c}\text { Recovery } \\
\%\end{array}$ & $\begin{array}{l}\text { JEROM } \\
\mathrm{mg} / \mathrm{cu} .\end{array}$ \\
\hline
\end{tabular}

BORING NUMBER SR41/MW3 GW-813 SHEET 1 OF 2

PROIECT BCV OU2

LOCATION SY-200 YARD, Y-12 PLANT OAK RIDGE, TN

CONTRACT NUMBER 18B-99069C, TASK Y-11

LOGGED BY S. ABSTON

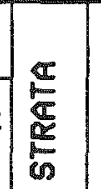

Ton

2

(3)


A-158

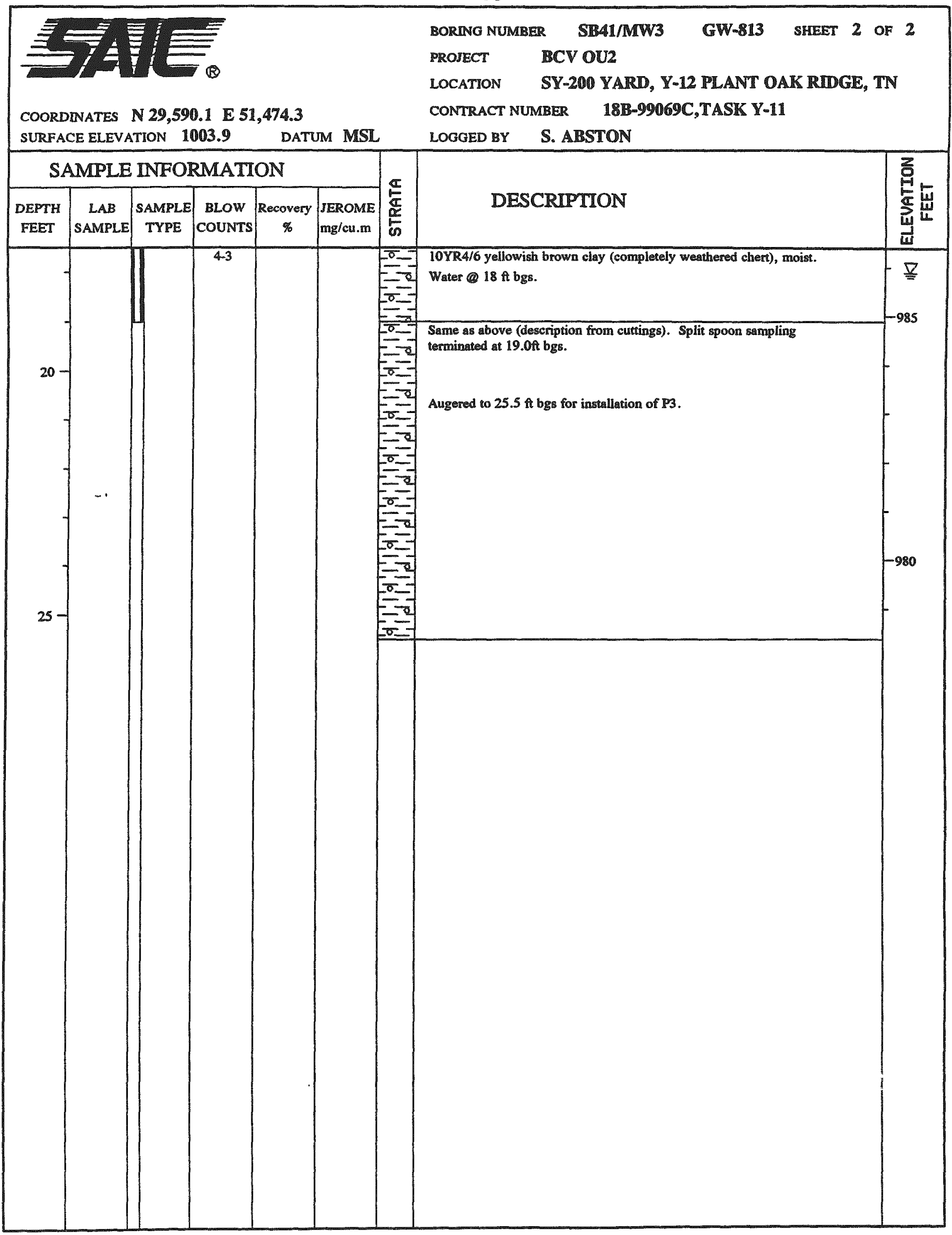


BEAR CREEK VALLEY OU 2

\section{WELL INSTALLATION DIAGRAM}

WELL NO. MW-4/GW-814

Coordinates:

N 29,475.2

E 51,102.5

LOGGED BY: Susan L. Abston

DRILLING DATES

DRILLING COMPANY: Ogden

STARTED: $11-22-93$

FINISHED: $12-30-93$

DRILLER: George Akins

HELPER: Bob Snow

STEEL GUARD POST 2.9 FT

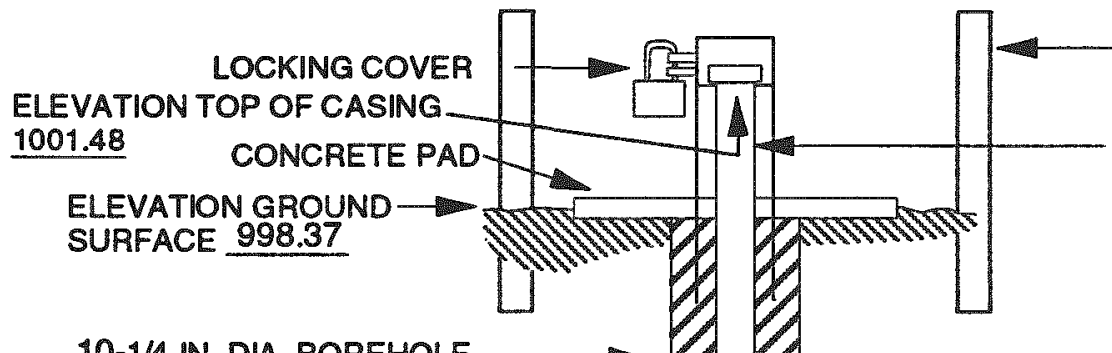

AGSTO 3.1 FTBGS

PROTECTIVE CASING 3.4 FT

AGS TO 1.6 FT BGS

GROUT SEAL 0 TO

10-1/4 IN. DIA. BOREHOLE

6.8 FT BGS

.37 FTOD, .33 FT ID,

SCHEDULE 5 , STAINLESS

STEEL INSTALLED TO 11.23 FT

BELOW GROUND SURFACE

ELEVATION TOP OF SEAL 991.57

ELEVATION TOP OF SAND PACK 989.4

ELEVATION TOP OF SCREEN 987.14

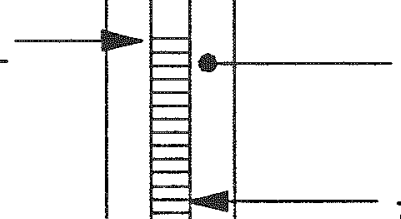

BENTONITE PELLET SEAL

6.8 TO 8.93 FT BGS

SAND PACK 8.93 TO

$\underline{24.3}$ FTBGS

.37 FT OD, .33 FT ID., STAINLESS

STEEL/SPIRAL WOUND, \#10 Slot

SCREEN 11.23 TO 21.33 FT BGS

ELEVATION TOP OF SILT

TRAP 977.04

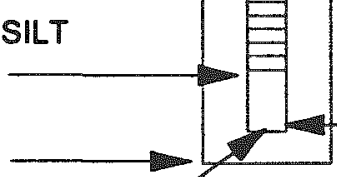

.37 IN. OD STAINLESS STEELSILT

DRILLED DEPTH OF

BOREHOLE 24.3 FT BGS

ELEVATION 974.07

\section{NOT TO SCALE}

ELEVATION OF BOTTOM

OF SILT TRAP 975.07 
A-160

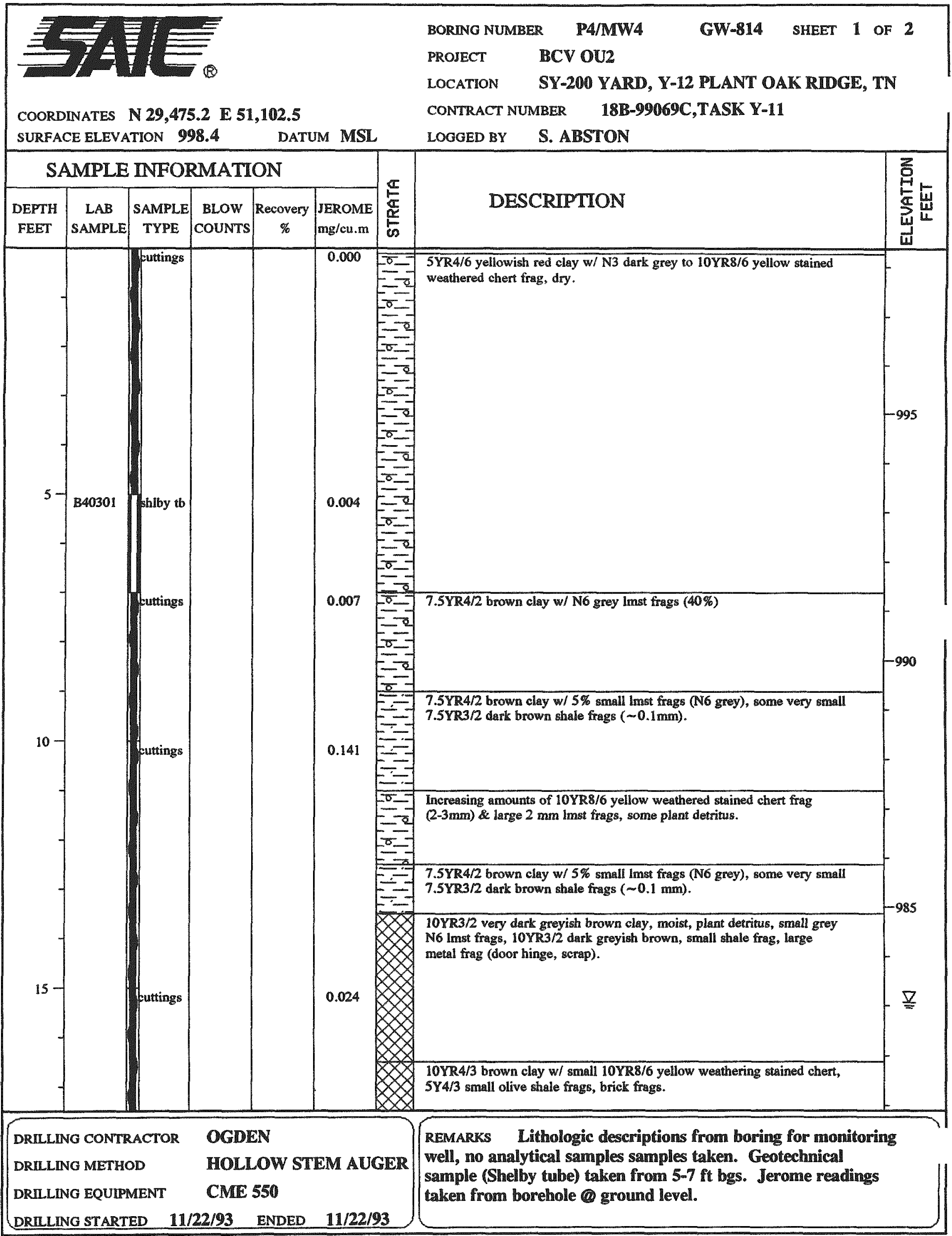


A-161

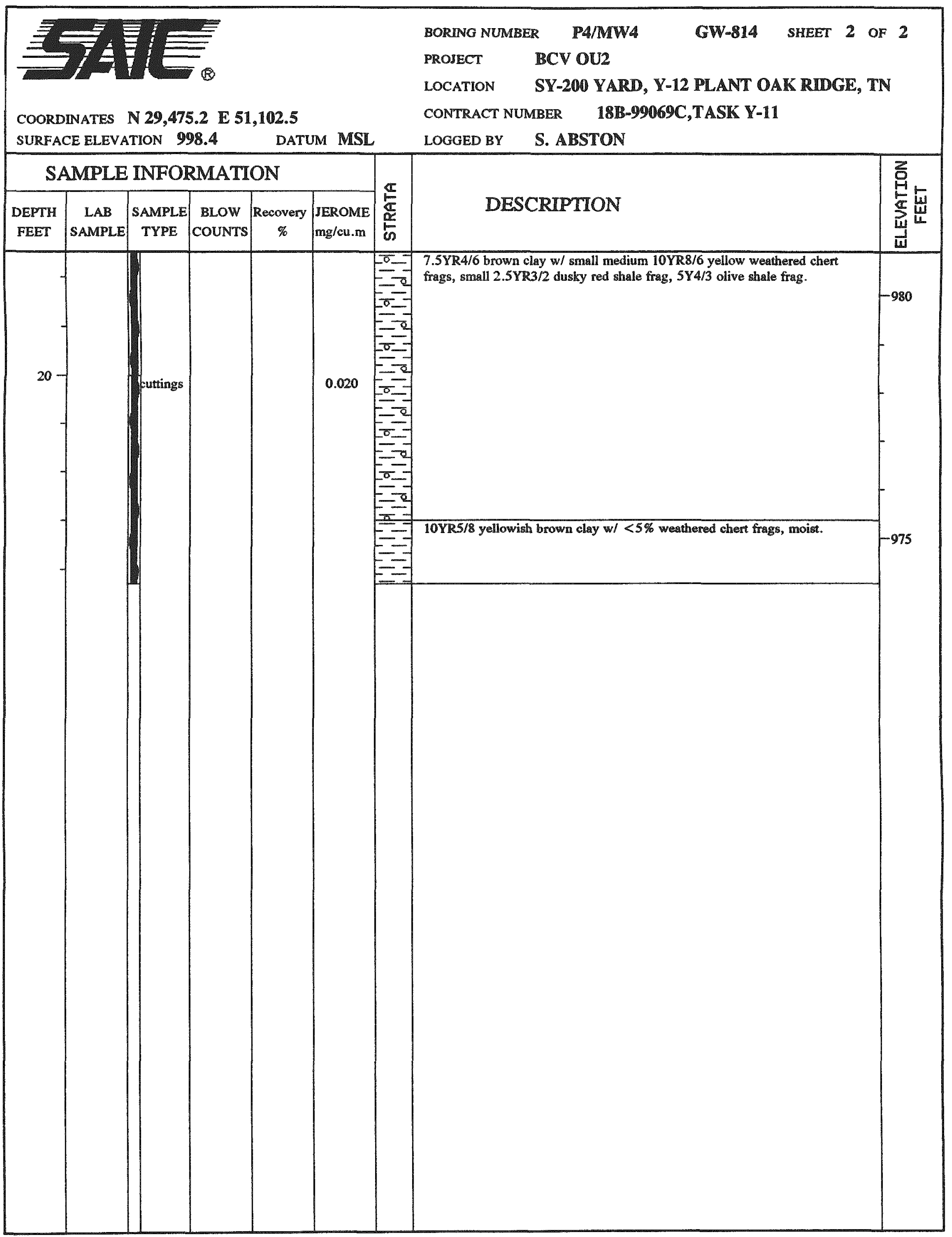


OANL-DWG 6OM-1368"

\section{BEAR CREEK VALLEY OU 2}

\section{WELL INSTALLATION DIAGRAM}

WELL NO. MW.5/GW-315

Coordinates:

N 29,592.4

E 51,121.4

LOGGED BY: Susan L. Abston

DRILLING COMPANY: Ogden

DRILLER: George Akins

HELPER: Bob Snow

\section{DRILLING DATES}

STARTED: $11-23-93$

FINISHED: $\quad 12-30-93$

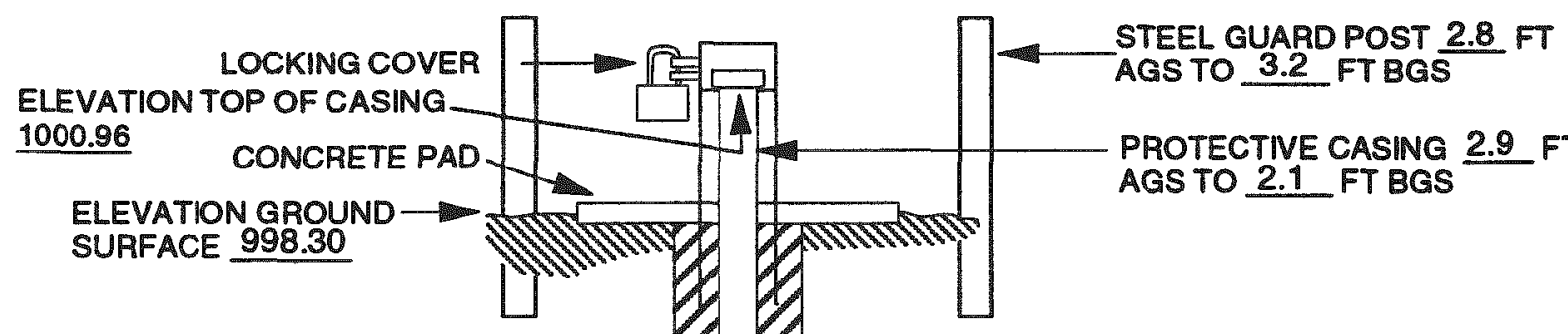

10-1/4 IN. DIA. BOREHOLE

$$
\begin{aligned}
& \text { GROUT SEAL O TO } \\
& 2.9 \text { FT BGS }
\end{aligned}
$$

.375 FT OD. 34 FT ID,

SCHEDULE 5 STANLESS

STEEL INSTALLED TO $\mathbf{8 . 5 3}$ FT

BELOW GROUND SURFACE

BENTONITE PELLET SEAL

2.9 TO 6.4 FTBGS

ELEVATION TOP OF SAND PACK 991.90

ELEVATION TOP OF SEAL 995.40

ELEVATION TOP OF SCREEN 989.77

SAND PACK 6.4 TO

21.1 FTBGS

.38 FTOD, .34 FTID., STAINLESS

STEELSPIRAL WOUND, 10 SlOt SCREEN $\mathbf{8 . 5 3}$ TO 18.52 FT BGS

ELEVATION TOP OF SILT TRAP 979.78

DRILLED DEPTH OF BOREHOLE 21.1 FT BGS ELEVATION 977.2

ELEVATION OF BOTTOM

.38 IN. OD STAINLESS STEELSILT TRAP 18.52 TO 20.6 FTBGS

OF SILT TRAP 977.70 


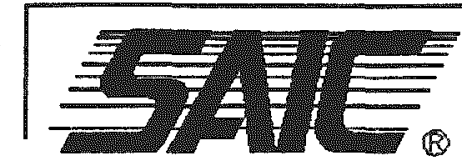

COOROINATES N 29,592.4 E 51, 121.4 SURFACE ELEVATION 998.4
BORING NUMBER

PROJECT

LOCATION

BCV OU2

CONTRACT NUMBER $\quad$ 18B-99069C,TASK Y-11

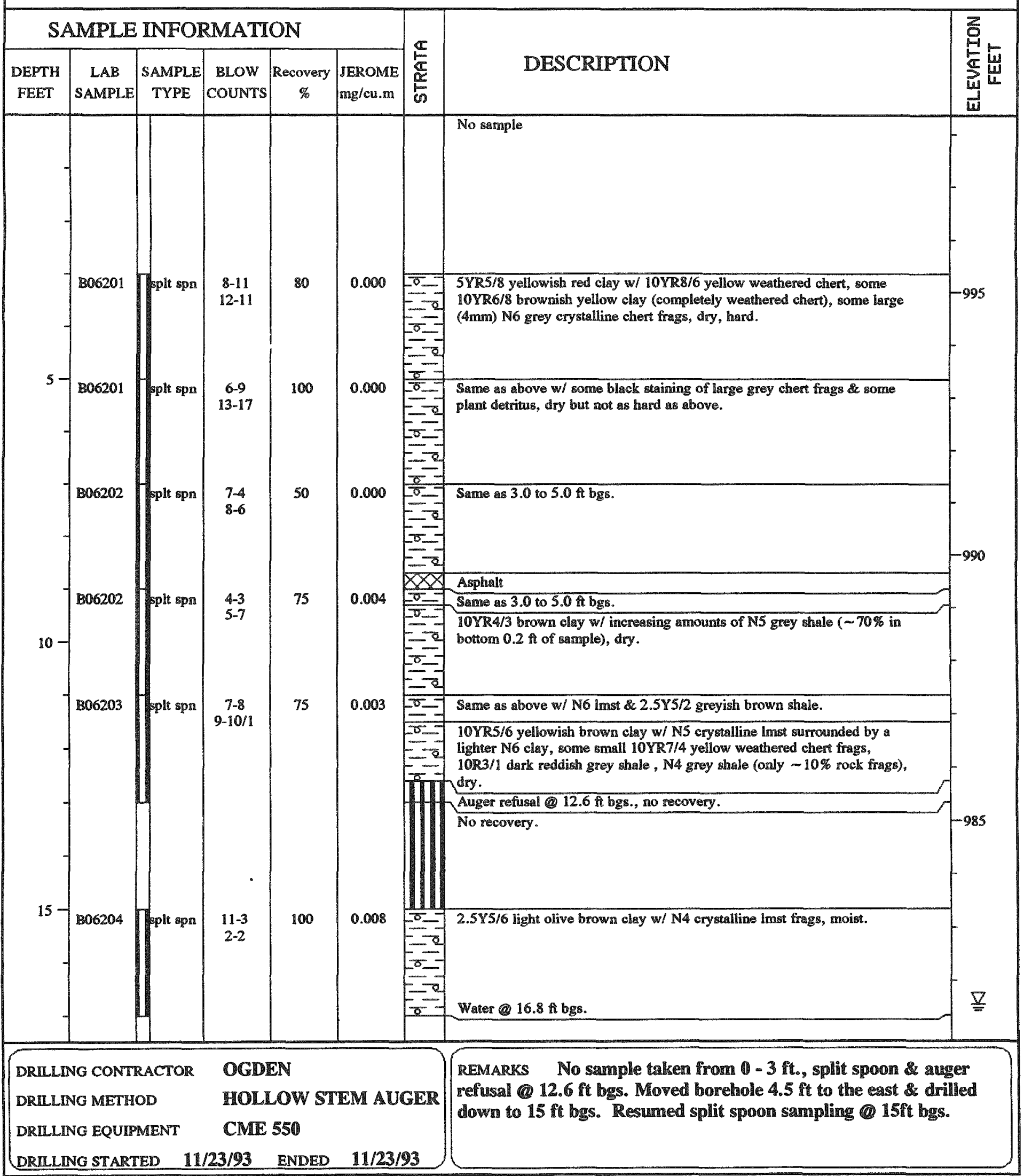



Historical Groundwater Monitoring Well

Construction Logs and Lithology Logs 



\begin{tabular}{|c|c|c|c|c|}
\hline \multirow{2}{*}{$\begin{array}{l}1 \text { GERAGHTY } \\
\text { \& MILLR, INC. } \\
\text { \& Gound-Water Consuntants }\end{array}$} & \multirow{2}{*}{\multicolumn{2}{|c|}{$\begin{array}{c}\text { WELL CONSTRUCTON } \\
\text { DIAGRAM }\end{array}$}} & \multicolumn{2}{|c|}{ BORING NO. G W-306 } \\
\hline & & & & Site Characterization \\
\hline $\begin{array}{l}\text { LOCATHON } \\
\begin{array}{c}Y-12 \text { Plant, } \\
\text { Rust SDoil Area }\end{array}\end{array}$ & $\begin{array}{c}\text { Y.12 PLANT COOADINATES } \\
\text { NORTH } 29,345.98 \\
\text { EAST } 49.655 .17 \\
\end{array}$ & $\begin{array}{r}\text { SURFACE EIEVATION } \\
989.44 \mathrm{ft} \text { ms }\end{array}$ & & $\begin{array}{c}\text { TOP OF CASING ELEVATION } \\
991.78 \mathrm{ft} \text { ms! }\end{array}$ \\
\hline $\begin{array}{l}\text { GEOLOGIST } \\
\text { d. Walker }\end{array}$ & $\begin{array}{l}\text { OALUNG CONTRATOA } \\
\text { Joe Samples Drilling }\end{array}$ & $\begin{array}{r}\text { DRILUNG METHOO } \\
\text { Air ROtary }\end{array}$ & & $\begin{array}{l}\text { ALG TYPE } \\
\text { Chicago Preumatic }\end{array}$ \\
\hline $\begin{array}{c}\text { DEVELOPMENT METHOD } \\
\text { SWab }\end{array}$ & $\begin{array}{l}\text { VOLUME EVACUATED } \\
164 \text { gallons }\end{array}$ & $\begin{array}{r}\text { DATE WEU COMPLETE } \\
09-25.87\end{array}$ & & $\begin{array}{l}\text { CORMATION MONTOREO } \\
\text { Maynardville Limestone }\end{array}$ \\
\hline
\end{tabular}

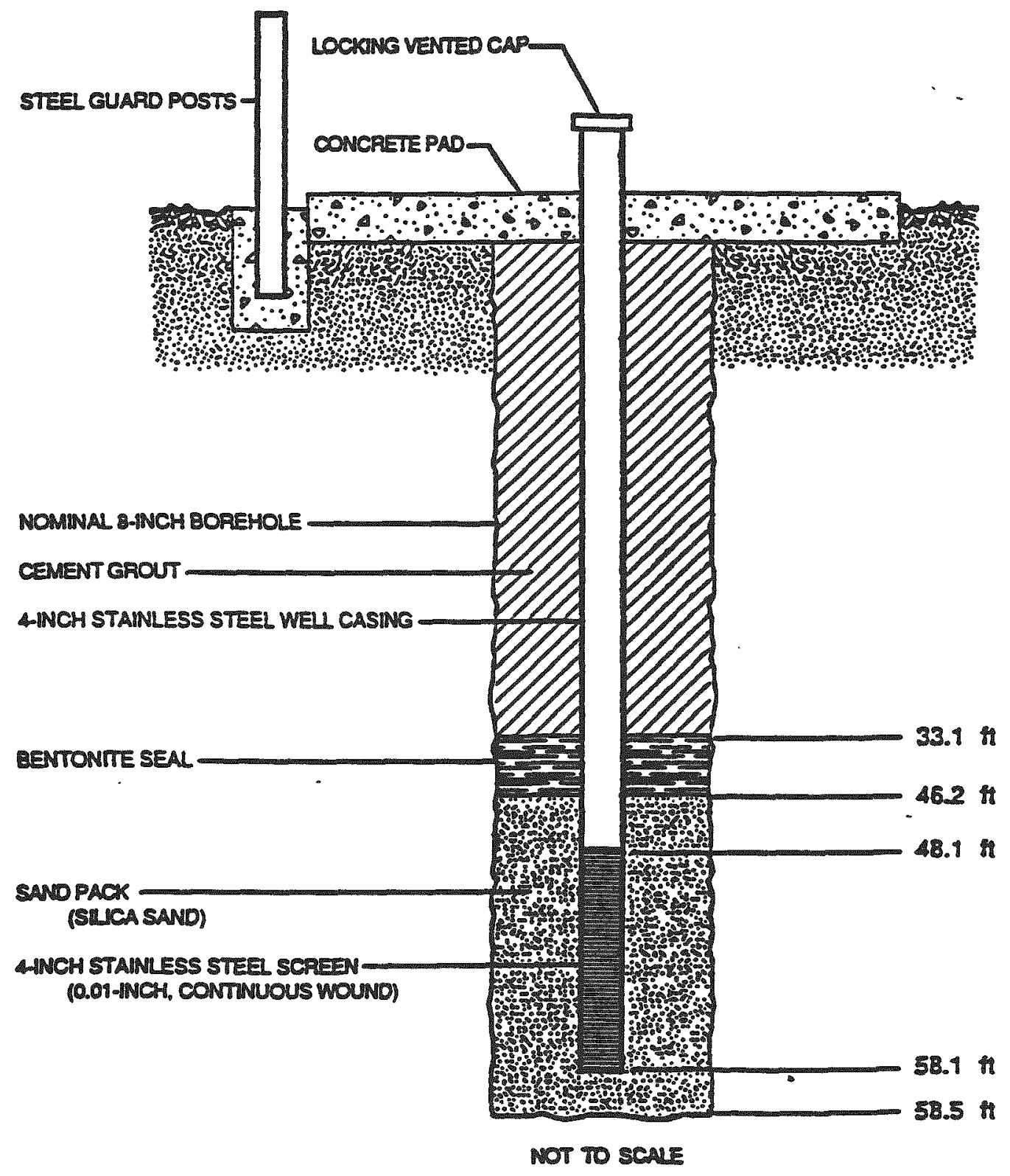

NOTE: ML DEPTH MEASUREMENTS N FEET DEI DW GROUND SUPFACE 


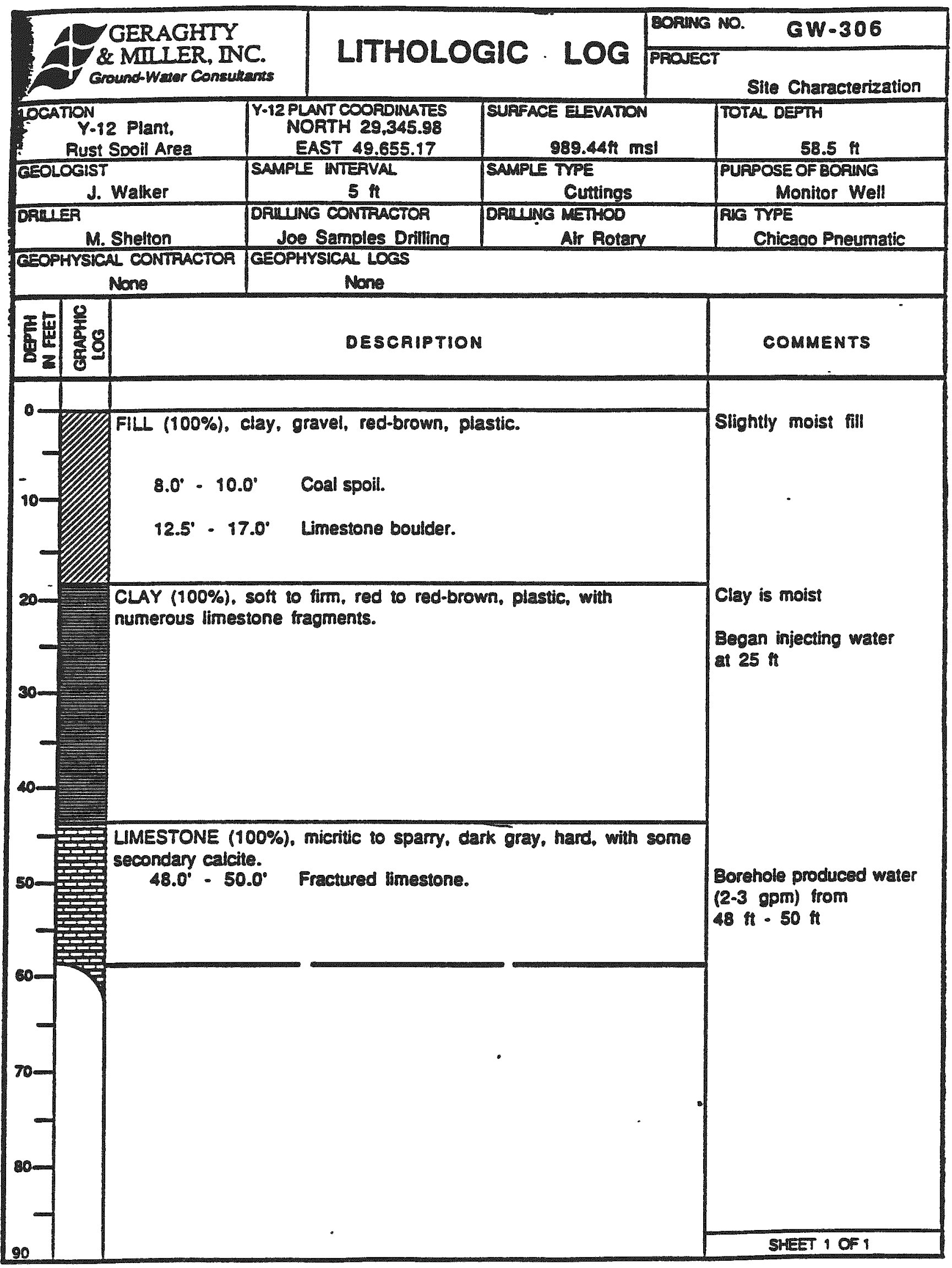




\begin{tabular}{|c|c|c|c|c|}
\hline \multirow{2}{*}{$\begin{array}{l}\text { GERAGHTY } \\
\text { \& MILLER, INC. } \\
\text { \& cound.Warer Consunanss }\end{array}$} & \multirow{2}{*}{\multicolumn{2}{|c|}{$\begin{array}{c}\text { WELL CONSTRUCTION } \\
\text { DIAGRAM }\end{array}$}} & \multirow{2}{*}{\multicolumn{2}{|c|}{ 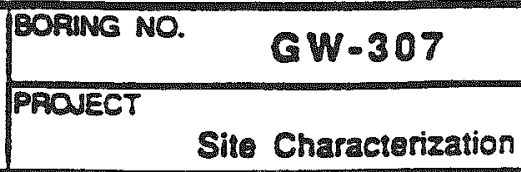 }} \\
\hline & & & & \\
\hline $\begin{array}{l}\text { OCATION } \\
\text { Y-12 Plans, } \\
\text { Rust Sooil Area }\end{array}$ & $\begin{array}{l}\text {-12 PLANT COORDINATES } \\
\text { NORTH } 29.250 .41 \\
\text { EAST } 49.520 .99\end{array}$ & $\begin{array}{r}\text { SURFACE ELEVATION } \\
991.01 \mathrm{Hms}\end{array}$ & & $\begin{array}{c}\text { TOP OF CASING ELEVATION } \\
993.14 \mathrm{msl}\end{array}$ \\
\hline $\begin{array}{c}\text { GEOLOGIST } \\
\text { T. Phillios }\end{array}$ & $\begin{array}{l}\text { DRULNG CONTRACTOA } \\
\text { Soils and Materials Eng. }\end{array}$ & $\begin{array}{c}\text { DRULNG METHOD } \\
\text { Auger }\end{array}$ & & $\begin{array}{l}\text { RIG TYPE } \\
\text { CME - } 45\end{array}$ \\
\hline $\begin{array}{c}\text { EVELOPMENT METHOD } \\
\text { SWab }\end{array}$ & $\begin{array}{r}\text { VOLUME EVACUATED } \\
125 \text { gallons }\end{array}$ & $\begin{array}{r}\text { DATE WEL COMPLEI } \\
07.15 .87\end{array}$ & & $\begin{array}{l}\text { FORMATON MONTORED } \\
\text { Unconsolidated Zone }\end{array}$ \\
\hline
\end{tabular}

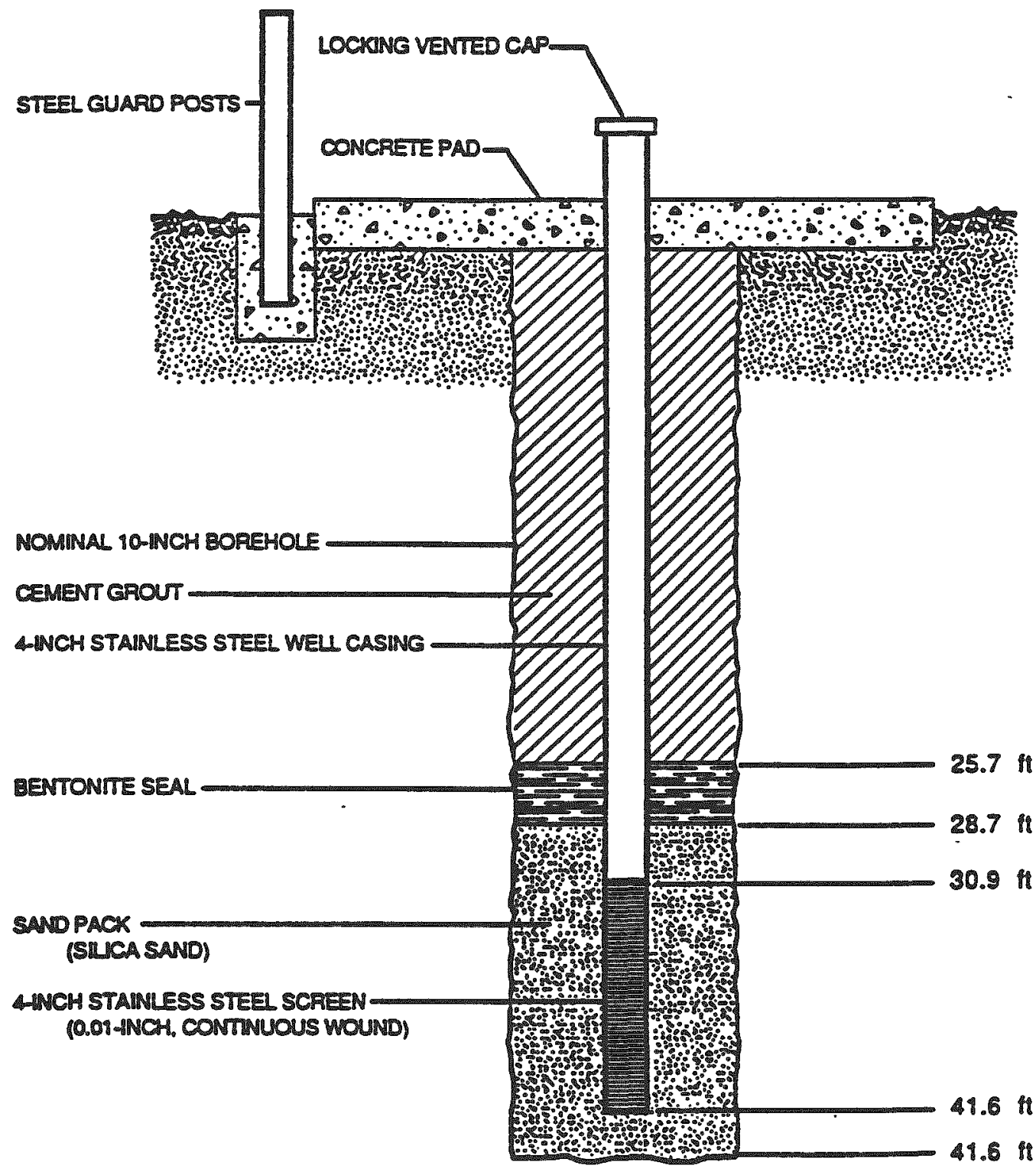

NOT TO SCAE 


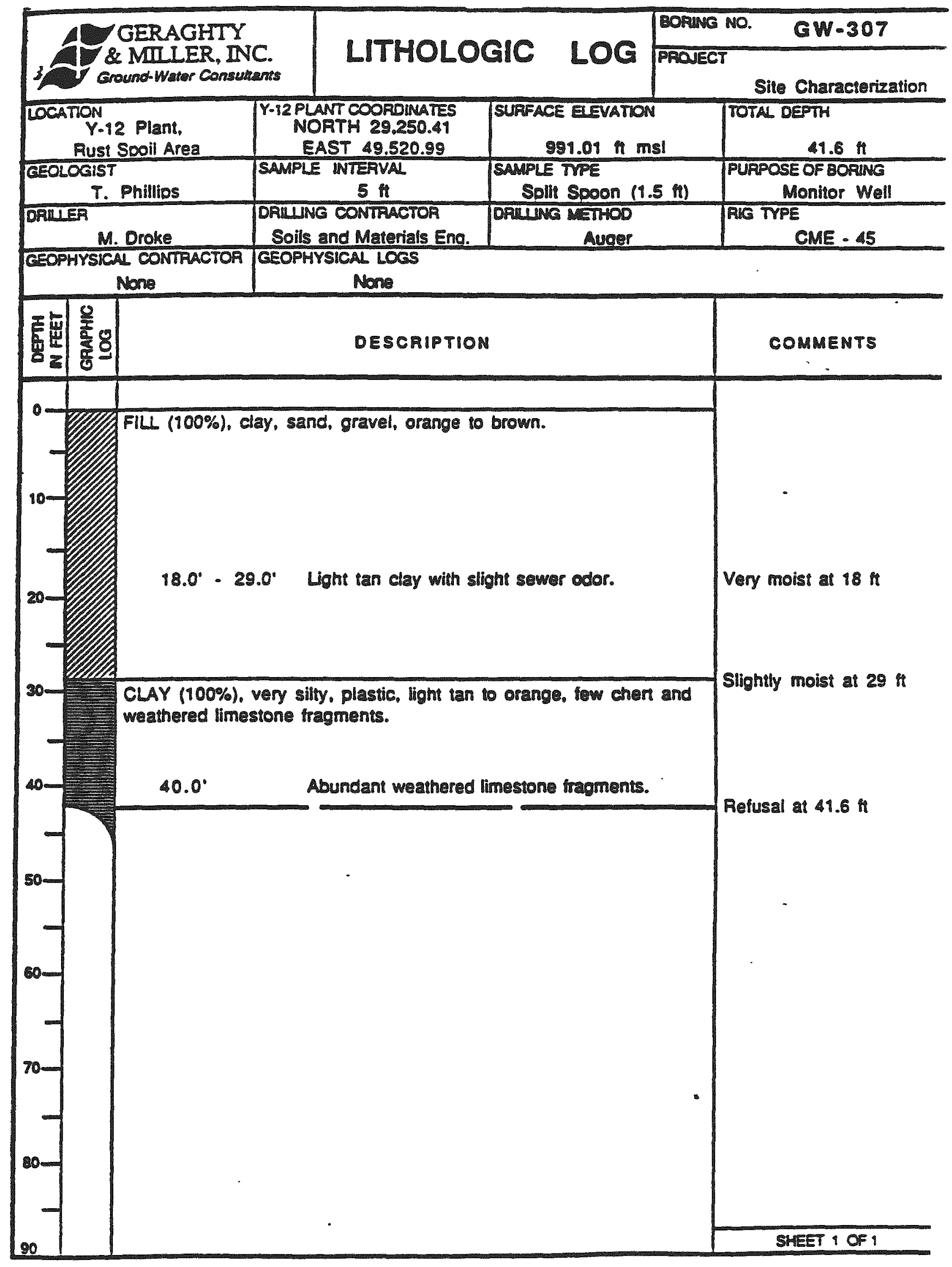




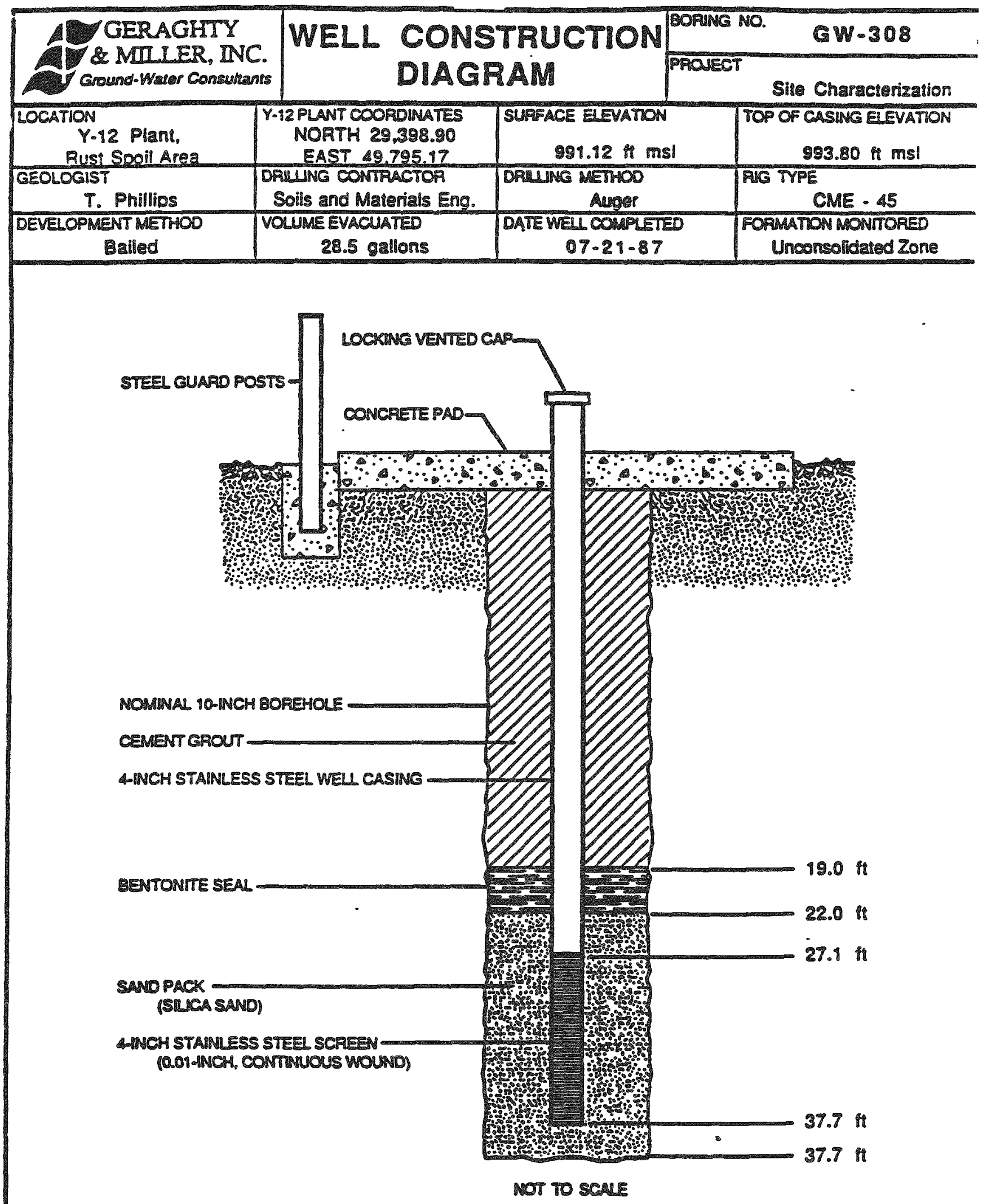

NOTE: AL DEPTH MEASUREMENTS WEET BELOW GPONO SUAFACE 


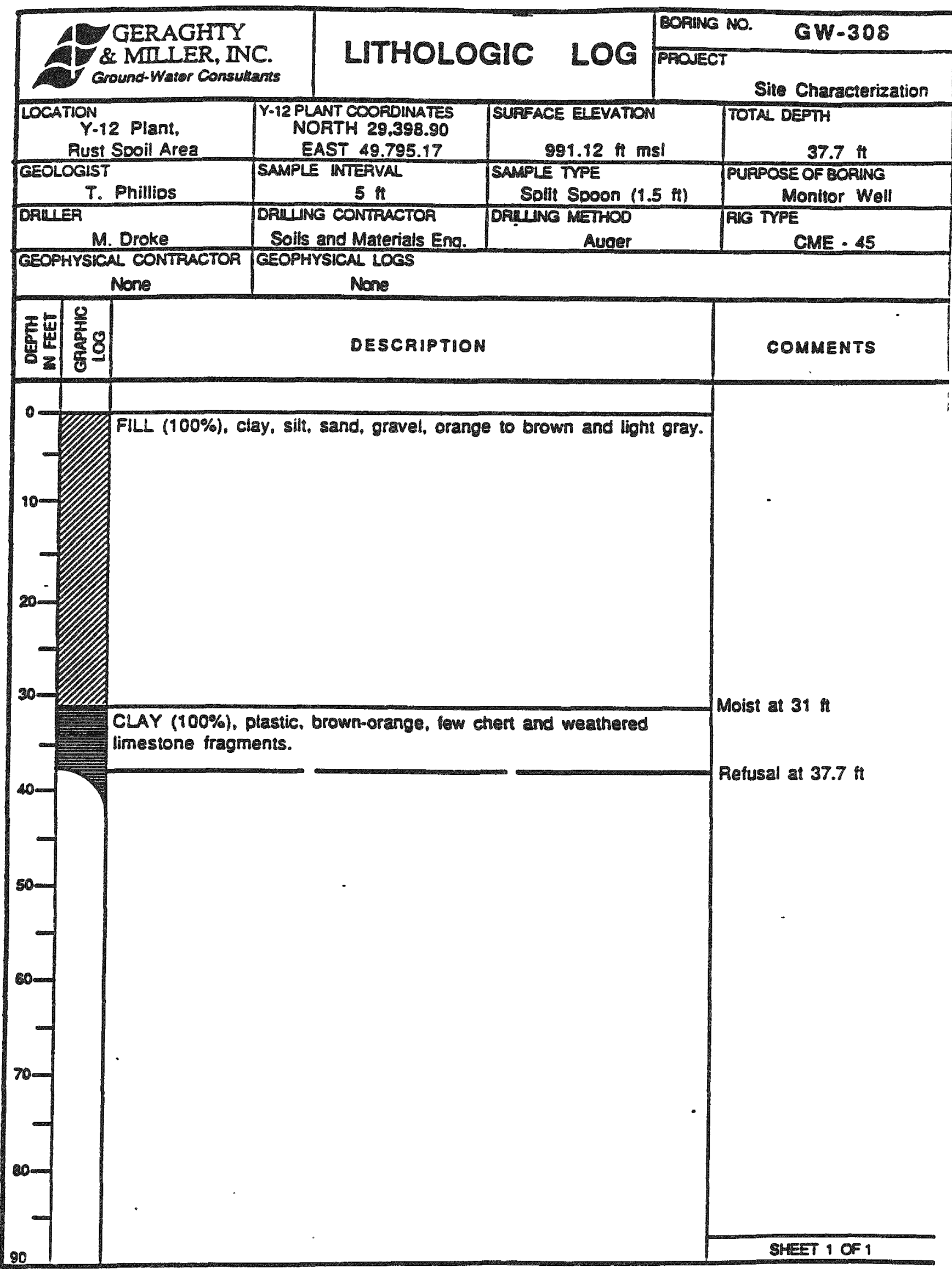




\begin{tabular}{|c|c|c|c|c|}
\hline \multirow{2}{*}{$\begin{array}{c}\text { GERAGHIY } \\
\text { \& MULILR, INC. } \\
\text { Ground-Water Consustants }\end{array}$} & \multirow{2}{*}{\multicolumn{2}{|c|}{$\begin{array}{c}\text { WELL CONSTRUCTION } \\
\text { DIAGRAM }\end{array}$}} & \multirow{2}{*}{\multicolumn{2}{|c|}{\begin{tabular}{|l} 
BORUNG NO. \\
PRONECT \\
Site Characterizatior
\end{tabular}}} \\
\hline & & & & \\
\hline $\begin{array}{l}\text { OCATION } \\
\text { Y-12 Plant. } \\
\text { Rust Spoil Area }\end{array}$ & $\begin{array}{c}\text { Y.12 PLANT COORDIMTES } \\
\text { NORTH } 29.529 .87 \\
\text { EAST } 50.176 .33\end{array}$ & $\begin{array}{r}\text { SURFACE ELEVATONO } \\
985.77 \mathrm{~m} \mathrm{~m}\end{array}$ & & $\begin{array}{c}\text { TOP OF CASING ELEVATIOI } \\
988.17 \mathrm{~m} \mathrm{ms!}\end{array}$ \\
\hline $\begin{array}{r}\text { GEOLOGIST } \\
\text { A. MoComb }\end{array}$ & $\begin{array}{l}\text { DALLUNG CONTRACTOA } \\
\text { Joe Samples Drilling }\end{array}$ & $\begin{array}{r}\text { DRILUMG METHOO } \\
\text { Air Fotary }\end{array}$ & & $\begin{array}{l}\text { गIG TYPE } \\
\text { Chicago Pneumatic }\end{array}$ \\
\hline $\begin{array}{c}\text { DEVELOPMENT MENHOD } \\
\text { Swab }\end{array}$ & $\begin{array}{l}\text { VOLUME EVACUATED } \\
120 \text { gallons }\end{array}$ & $\begin{array}{r}\text { DATE WEII COMPLI] } \\
09-24-87\end{array}$ & & $\begin{array}{l}\text { ORMATONONONTORED } \\
\text { Maynardville Limeston }\end{array}$ \\
\hline
\end{tabular}

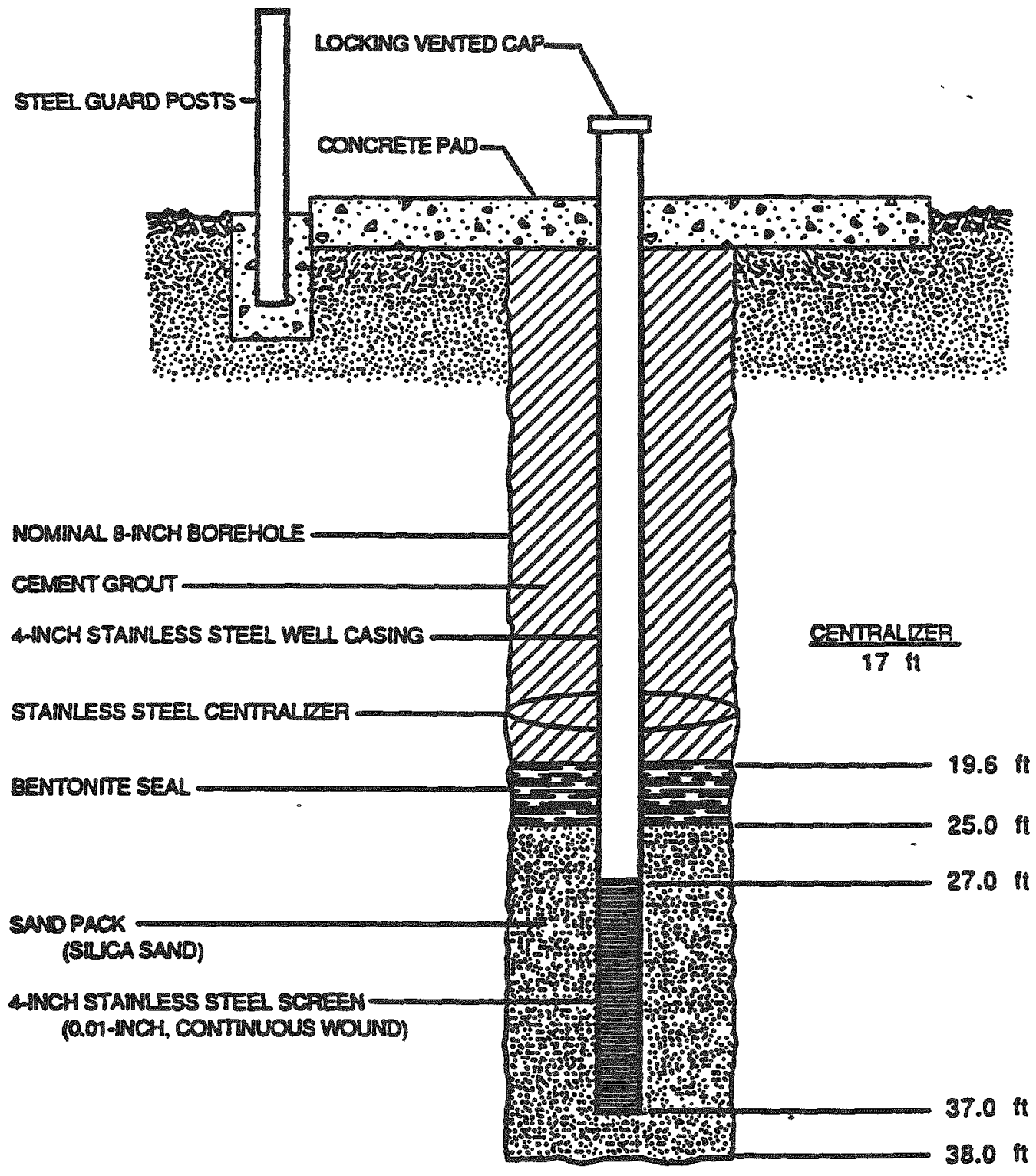

NOT TO SCALE

NOTE: AUL DEPTH MEASUAEMENTS N FEET BELOW GROUNO SURFACE 


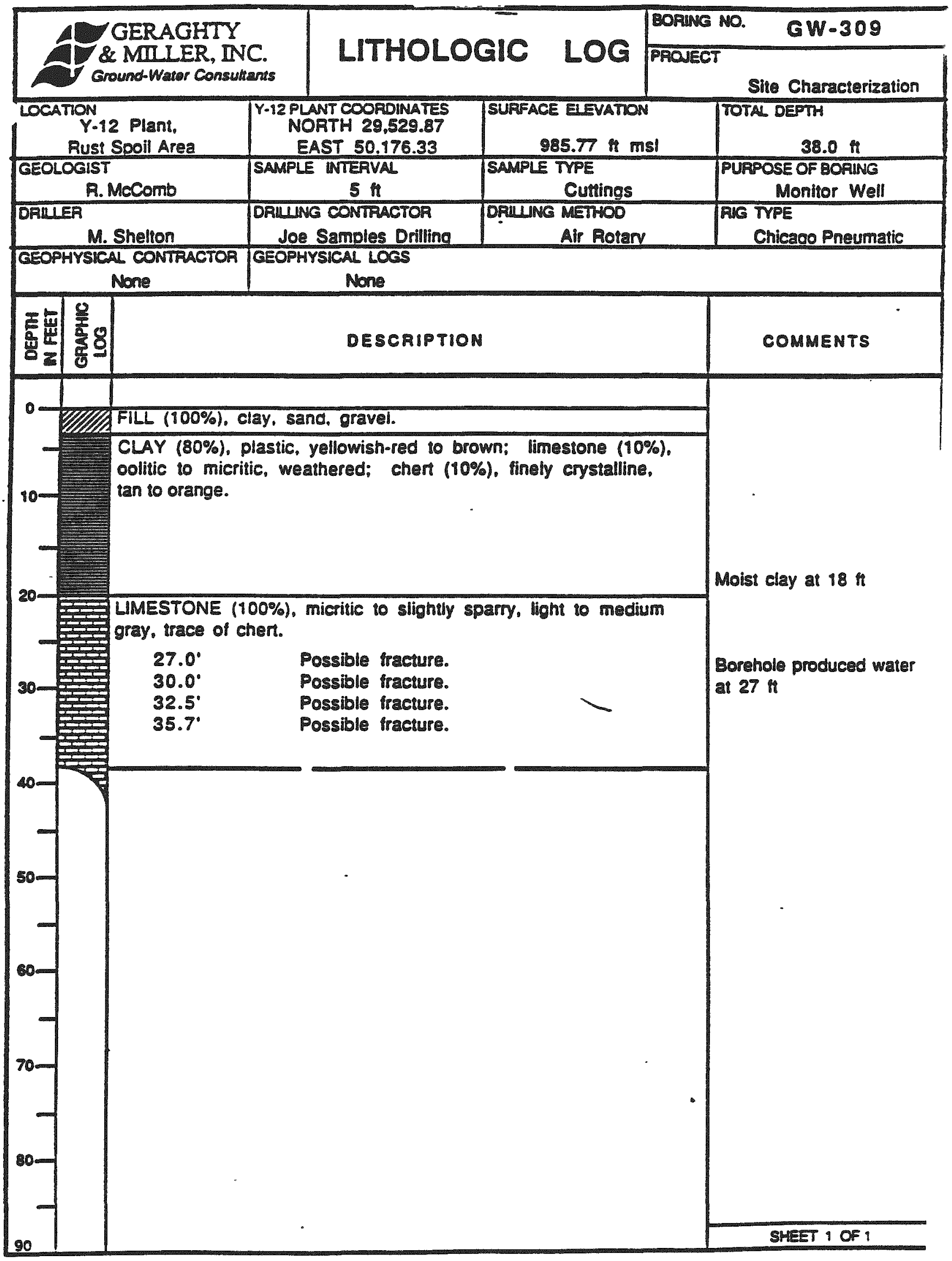




\begin{tabular}{|c|c|c|c|c|}
\hline \multirow{2}{*}{$\begin{array}{l}\text { 1GERAGHTY } \\
\text { \& MLLLR, INC. } \\
\text { Ground-Water Consuttants }\end{array}$} & \multirow{2}{*}{\multicolumn{2}{|c|}{$\begin{array}{c}\text { WELL CONSTRUCTION } \\
\text { DIAGRAM }\end{array}$}} & \multirow{2}{*}{\multicolumn{2}{|c|}{ 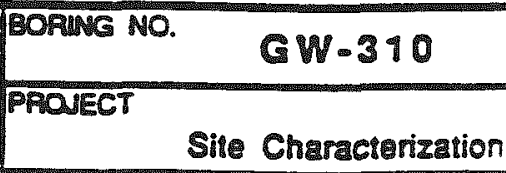 }} \\
\hline & & & & \\
\hline $\begin{array}{c}\text { LOCATION } \\
\text { Y.12 Plant. } \\
\text { Rust Spoil Area } \\
\end{array}$ & $\begin{array}{c}\text { Y.12 PLANT COORDINATES } \\
\text { NORTH } 29.437 .19 \\
\text { EAST } 50.497 .34 \\
\end{array}$ & $\begin{array}{r}\text { SURFACE ELEYATOON } \\
992.40 \mathrm{~ms}\end{array}$ & & $\begin{array}{c}\text { TOP OF CASING ELEVATION } \\
995.35 \text { it msl }\end{array}$ \\
\hline $\begin{array}{l}\text { GEOLOGIST } \\
\text { T. Phillios }\end{array}$ & $\begin{array}{l}\text { DALUNG CONTRACTOA } \\
\text { Soils and Materials Eng. }\end{array}$ & $\begin{array}{r}\text { DRLLANG METHOO } \\
\text { AUgOr }\end{array}$ & & $\begin{array}{l}\text { RIG TMPE } \\
\text { CME - } 45\end{array}$ \\
\hline $\begin{array}{c}\text { DEVELOPMENT METHOD } \\
\text { Hand Pump }\end{array}$ & $\begin{array}{l}\text { VOLUME EVACUATED } \\
158 \text { gallons }\end{array}$ & $\begin{array}{r}\text { DATE WEU COMPLTI } \\
07.23 .87\end{array}$ & & $\begin{array}{l}\text { FOPMATION MONTORED } \\
\text { Unconsolidated Zone }\end{array}$ \\
\hline
\end{tabular}

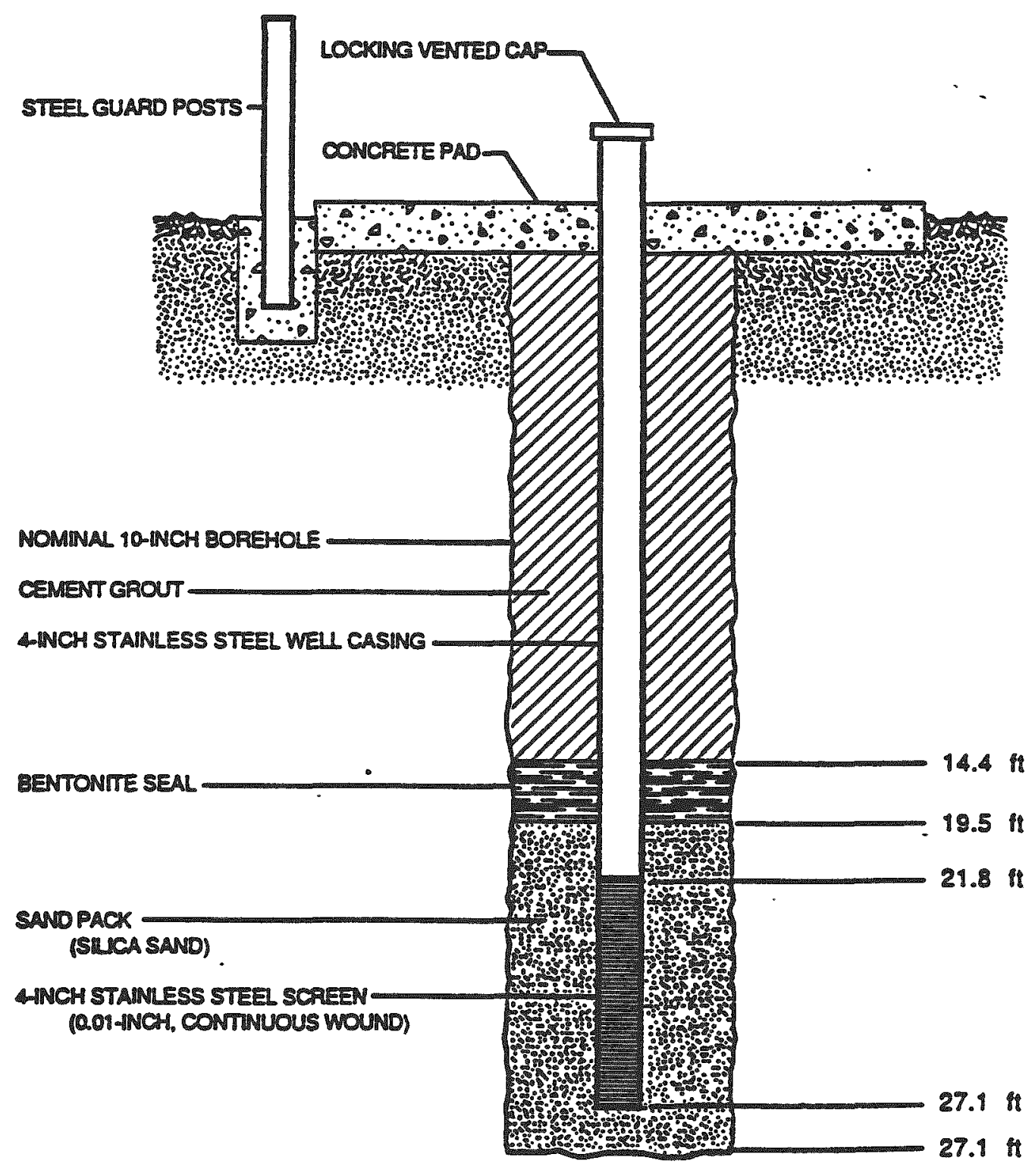

NOT TO SCAL 


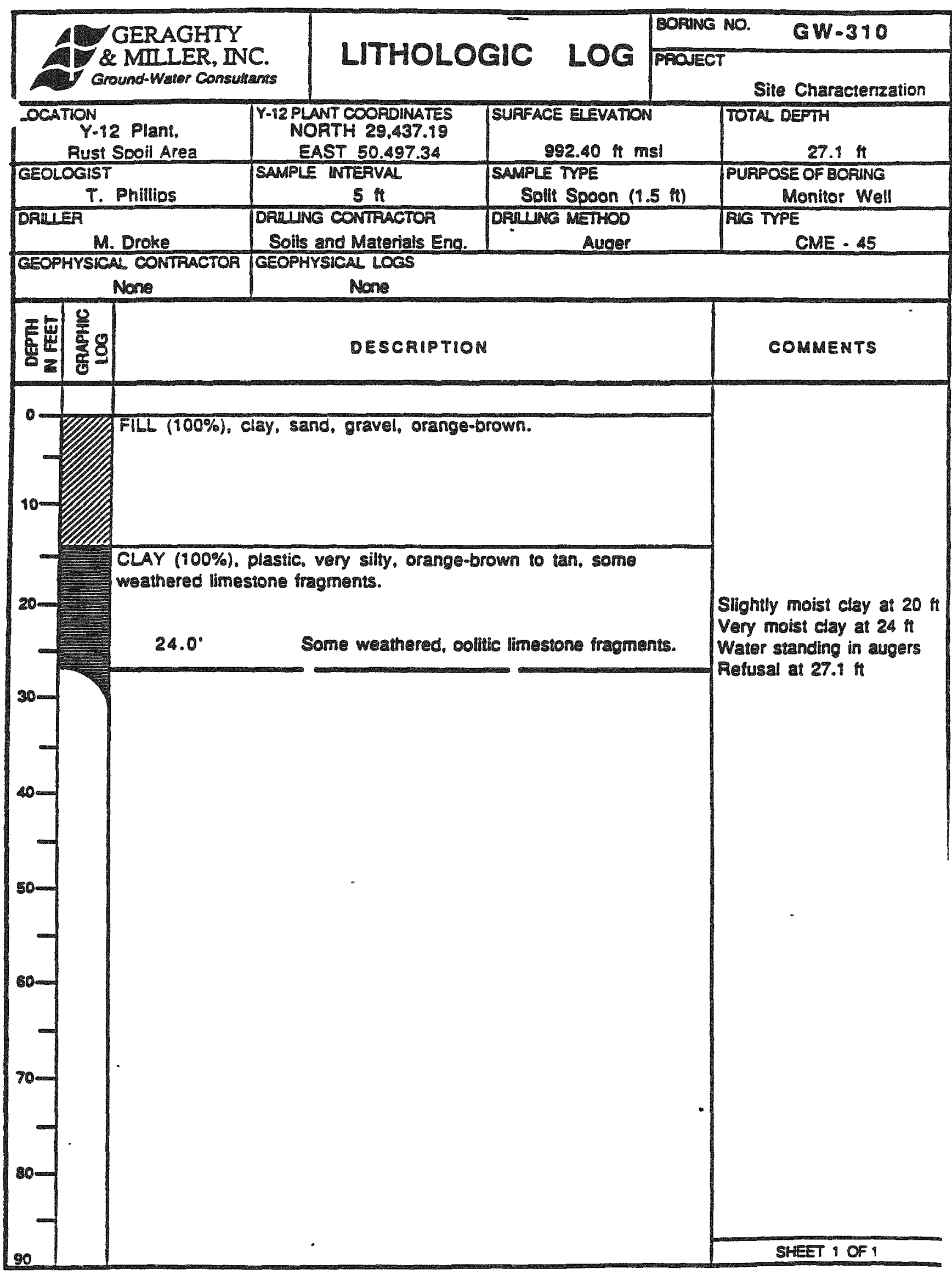




\begin{tabular}{|c|c|c|c|c|}
\hline \multirow{2}{*}{$\begin{array}{l}\text { TERAGHIT } \\
\text { \& MULER, INC. } \\
\text { Groundwarer Consuttents }\end{array}$} & \multirow{2}{*}{\multicolumn{2}{|c|}{$\begin{array}{c}\text { WELL CONSTRUCTION } \\
\text { DIAGAM }\end{array}$}} & \multirow{2}{*}{\multicolumn{2}{|c|}{\begin{tabular}{|l} 
BORING No. GW-311 \\
PRONECT
\end{tabular}}} \\
\hline & & & & \\
\hline $\begin{array}{l}\text { LOCATION } \\
\text { Y.12 Plant. } \\
\text { Rusis Sooil Area }\end{array}$ & $\begin{array}{c}\text { Y.12 PLANT COOADINATES } \\
\text { NORTH } 29.266 .88 \\
\text { EAST } 50.125 .50\end{array}$ & $\begin{array}{r}\text { SUAFACE ELEVATION } \\
296.43 \mathrm{~ms}\end{array}$ & & $\begin{array}{c}\text { TOP OF CASING ELEVATION } \\
899.35 \mathrm{ft} \mathrm{msl}\end{array}$ \\
\hline $\begin{array}{c}\text { GEOLOGIST } \\
\text { T. Phillios }\end{array}$ & $\begin{array}{l}\text { DAULING CONTACTOR } \\
\text { Soils and Materials Eng. }\end{array}$ & $\begin{array}{c}\text { DPRING METHOO } \\
\text { Auger }\end{array}$ & & $\begin{array}{l}\text { ALG TPE } \\
\text { CME - } 45\end{array}$ \\
\hline $\begin{array}{c}\text { DEVELOPMENT METHOO } \\
\text { Bailed }\end{array}$ & $\begin{array}{l}\text { VOLUME EVACUATED } \\
16 \text { gallonS }\end{array}$ & \begin{tabular}{|} 
TATE WEL COMPLETE \\
$0 \%-15.87$
\end{tabular} & & $\begin{array}{l}\text { ROFMATON MONTORED } \\
\text { Unconsolidated Zone }\end{array}$ \\
\hline
\end{tabular}

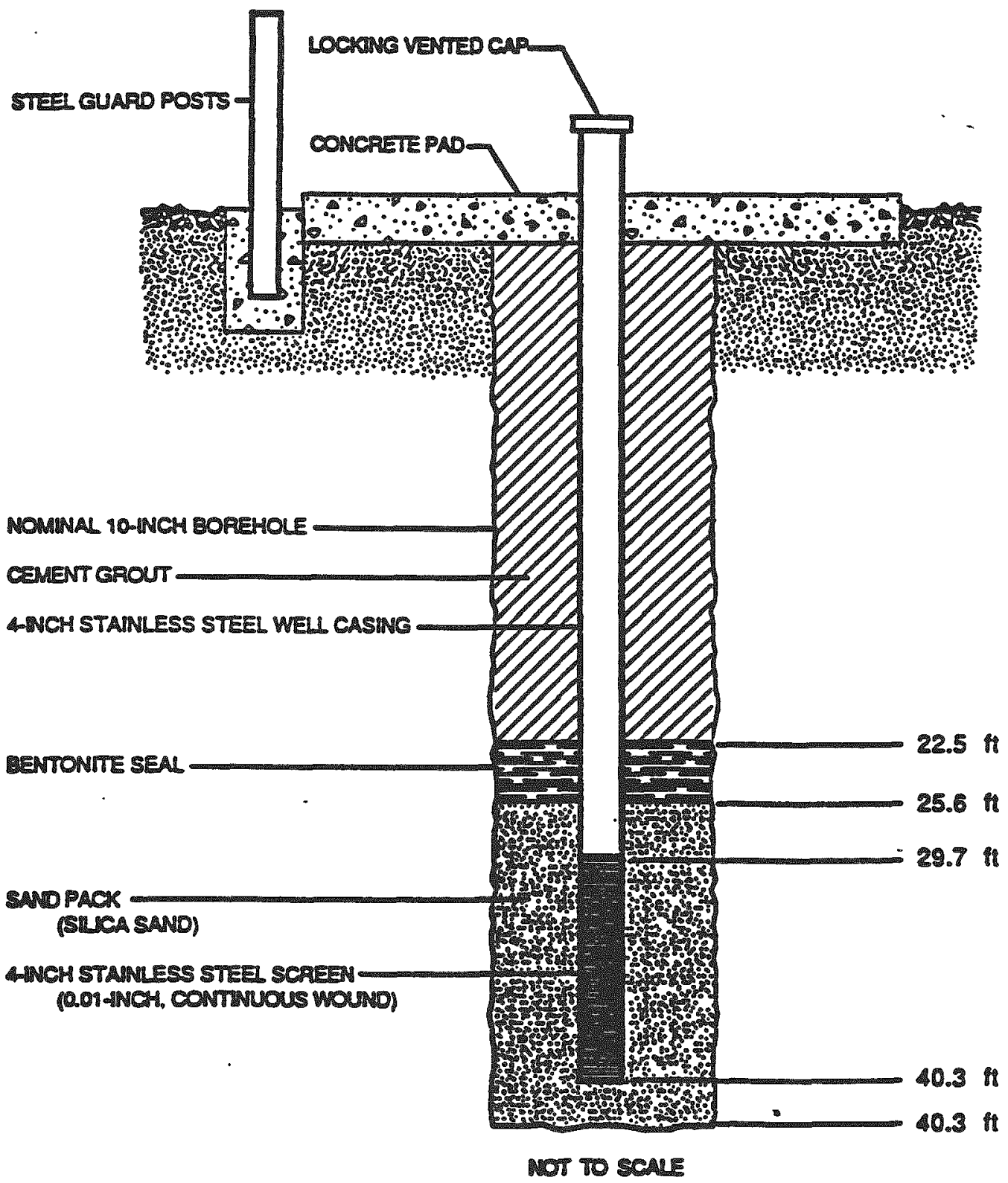

NOTE: ALL DEPTH MEASUAEMENTS N FEET BELOW GROUND SURFACE 


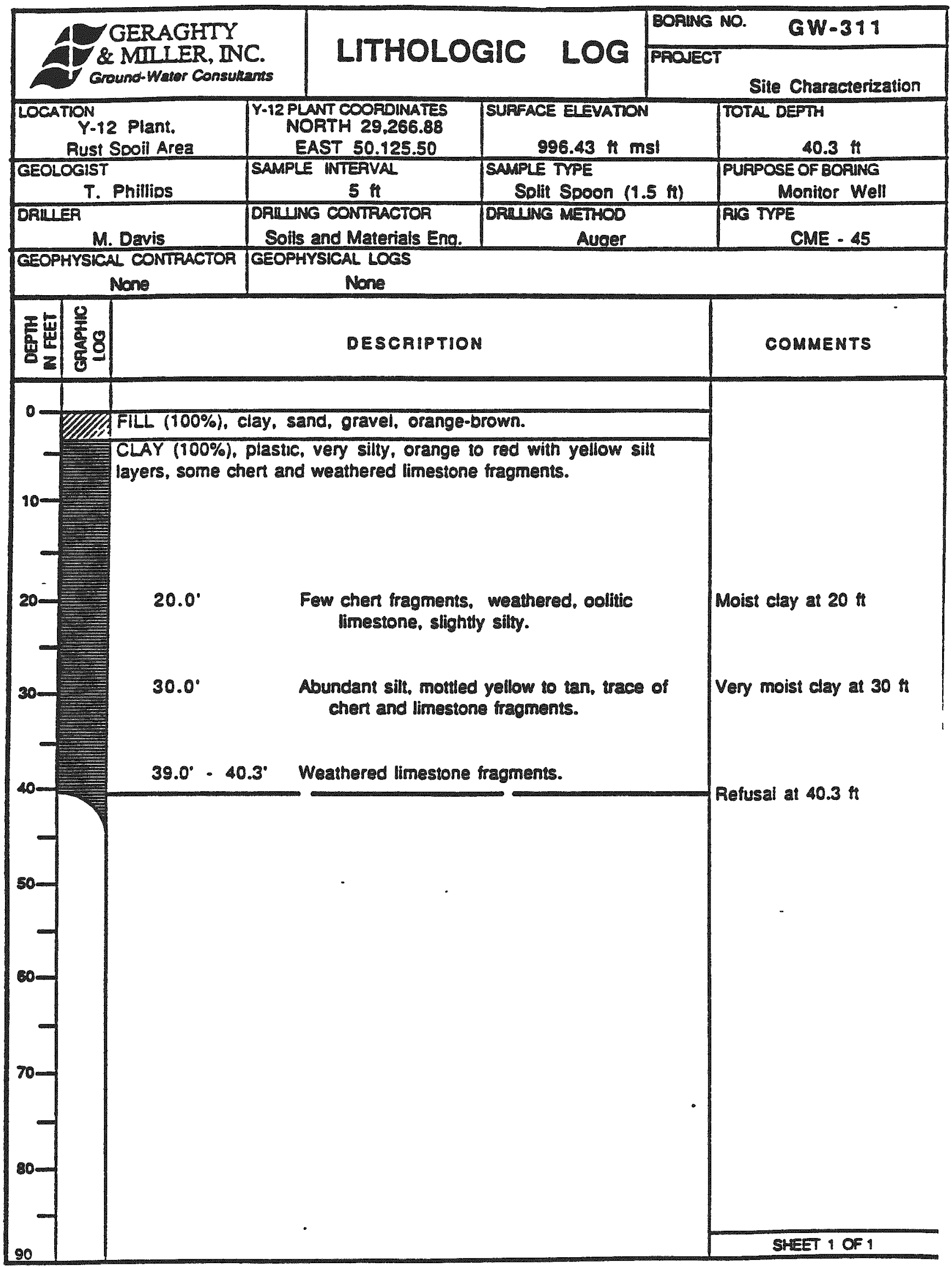




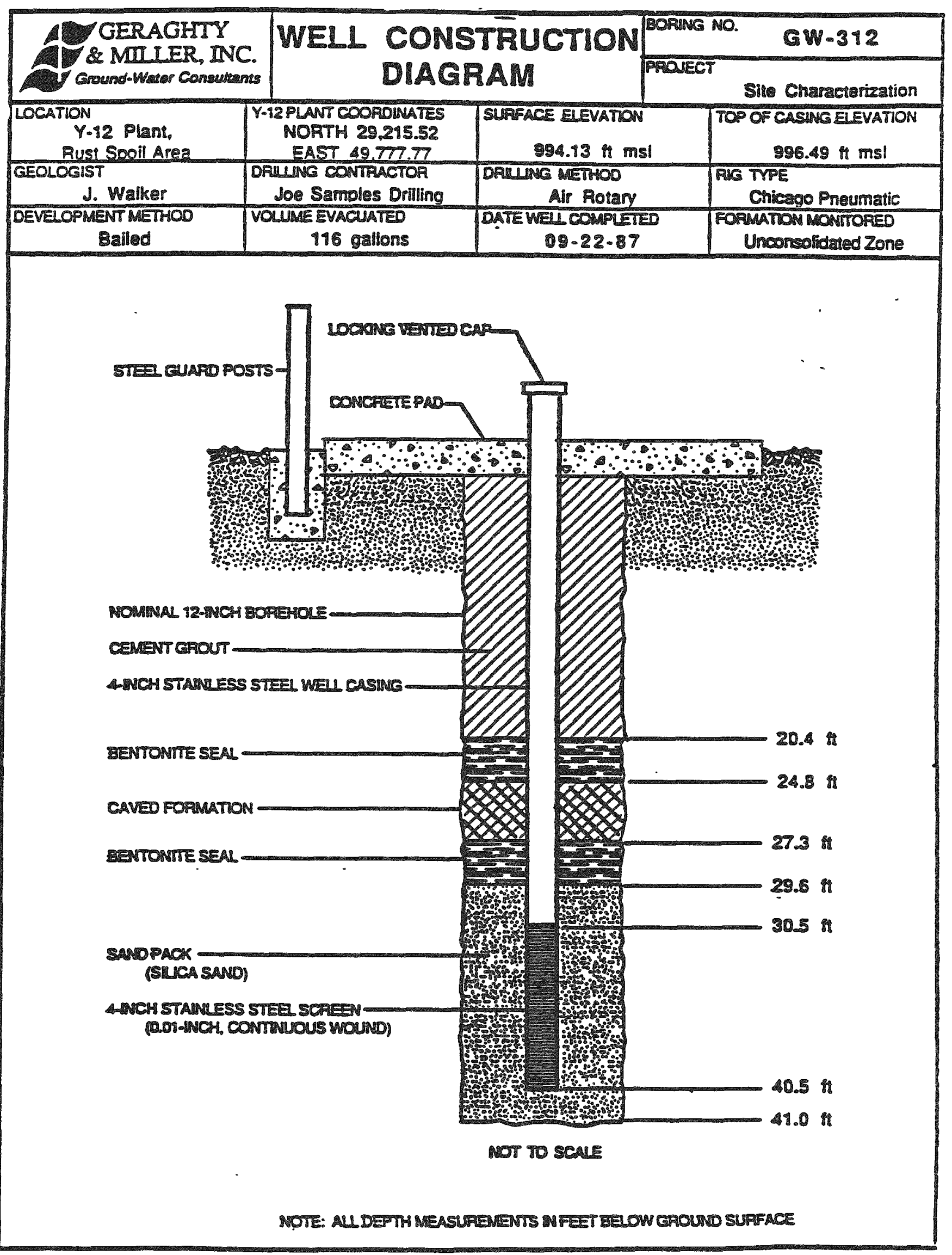




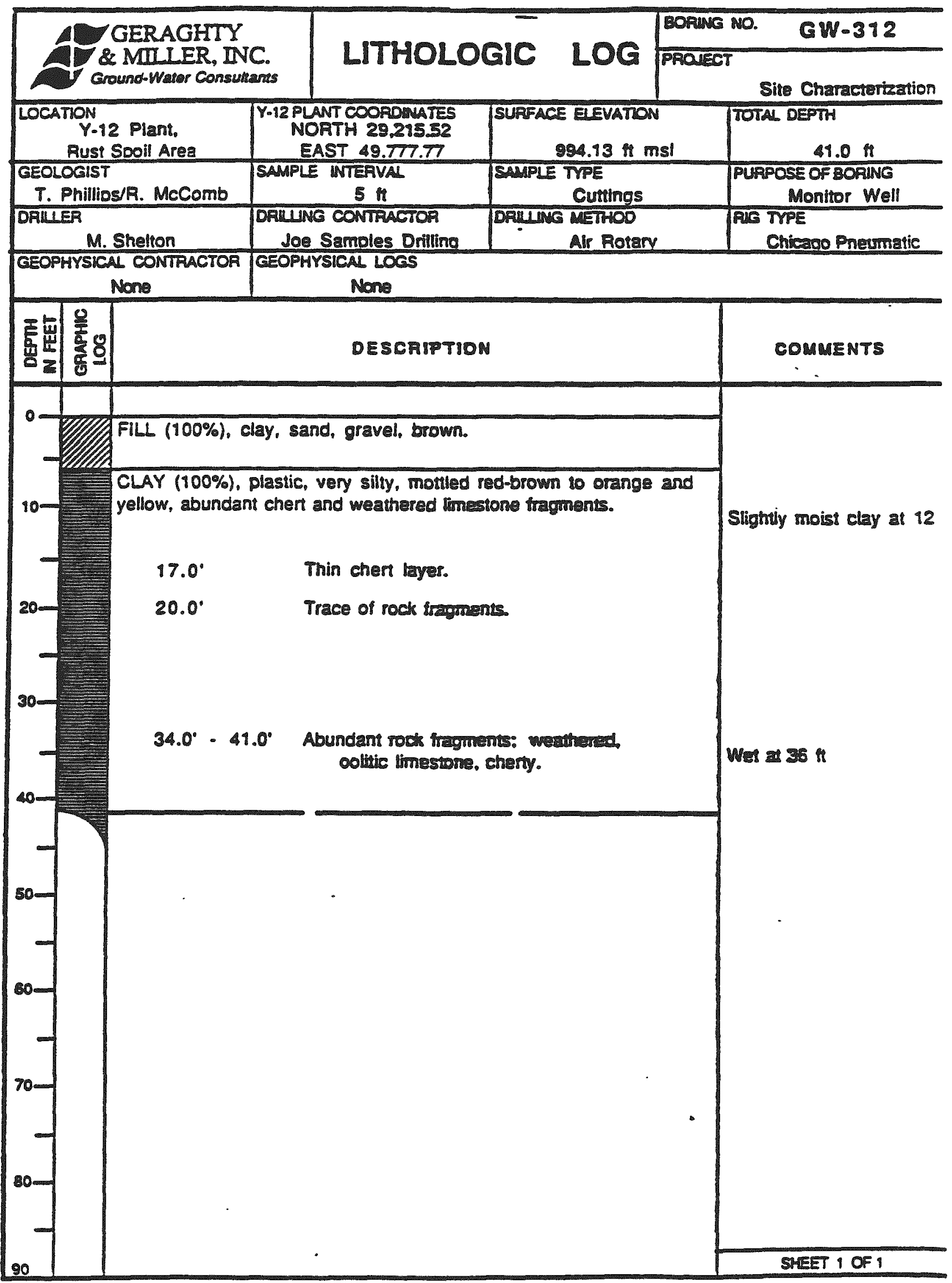




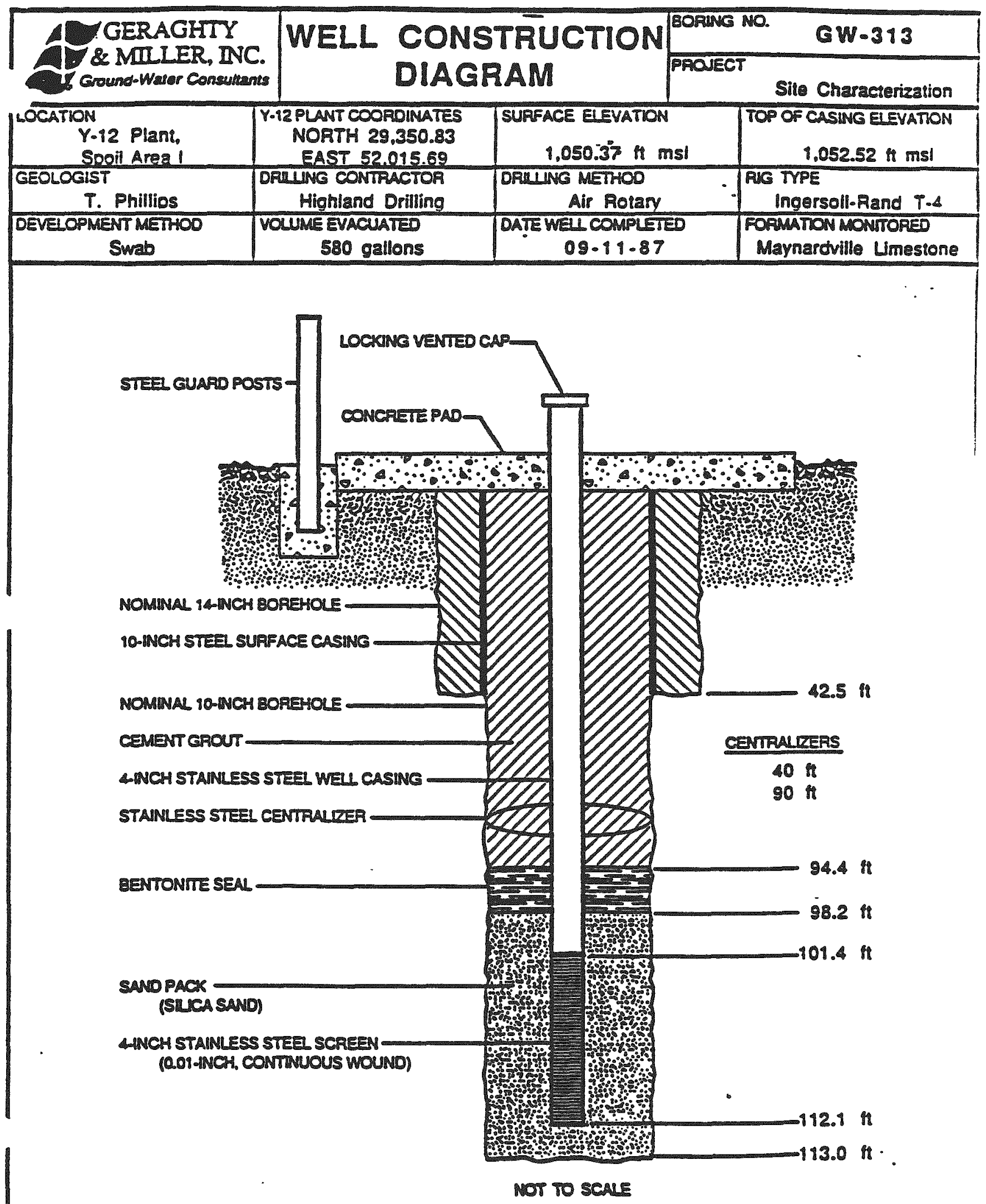

NOTE: ALL DEPTH MEASUAEMENTS IN FEET BELOW GROUNO SURFACE 


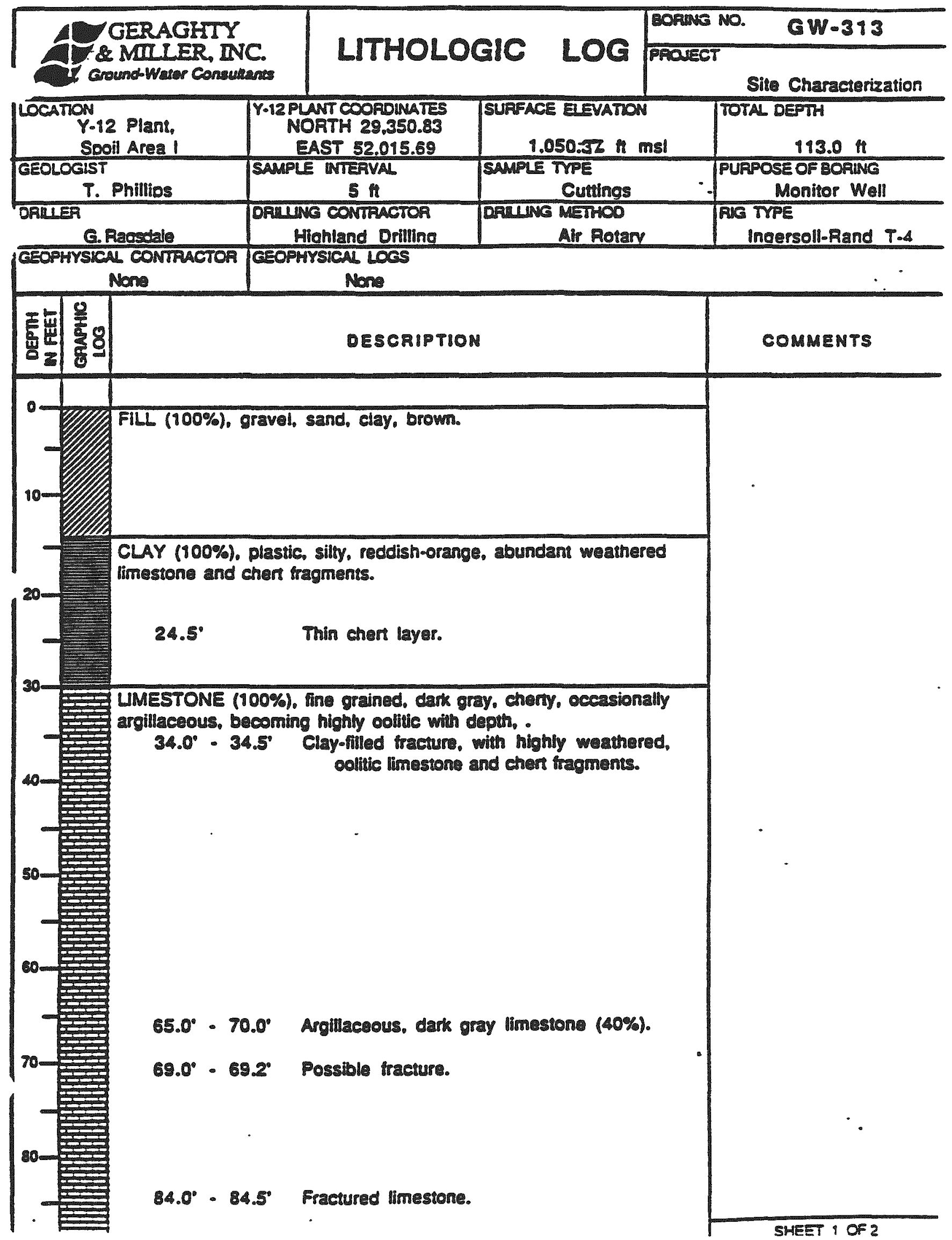


A-183

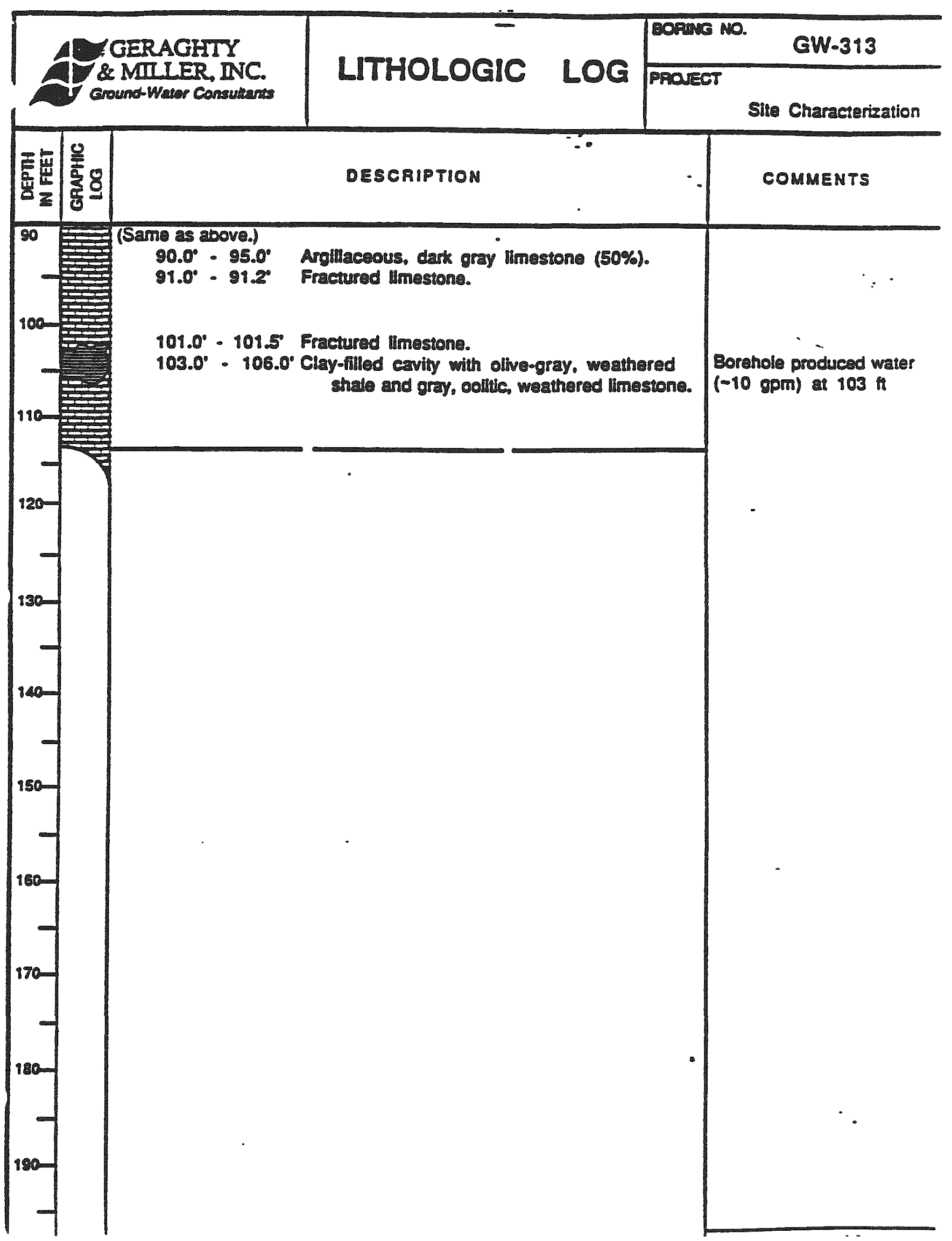




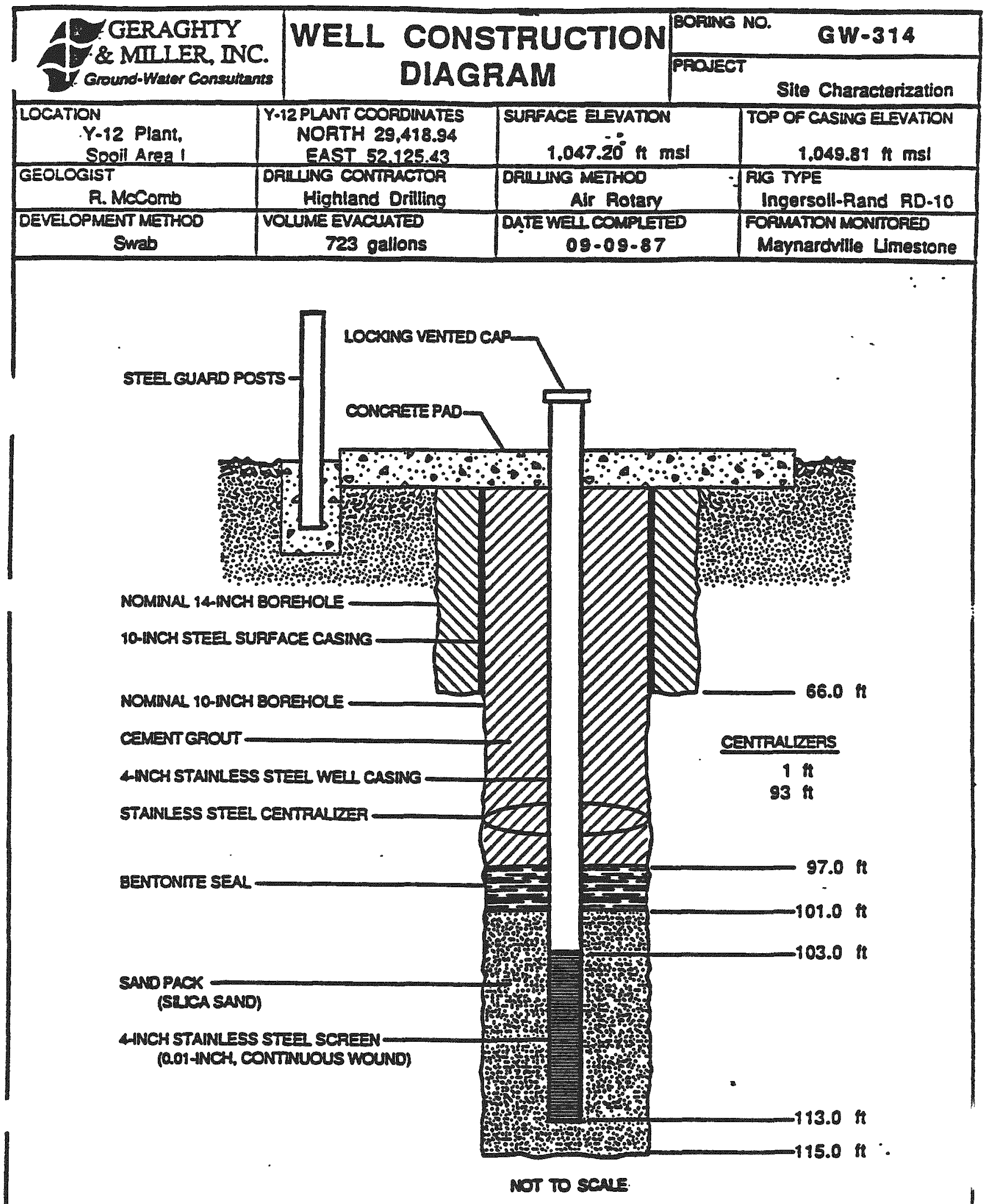

NOTE: ALL DEPTH MEASUREMENTS N FEET BELOW GROUND SUPFACE 


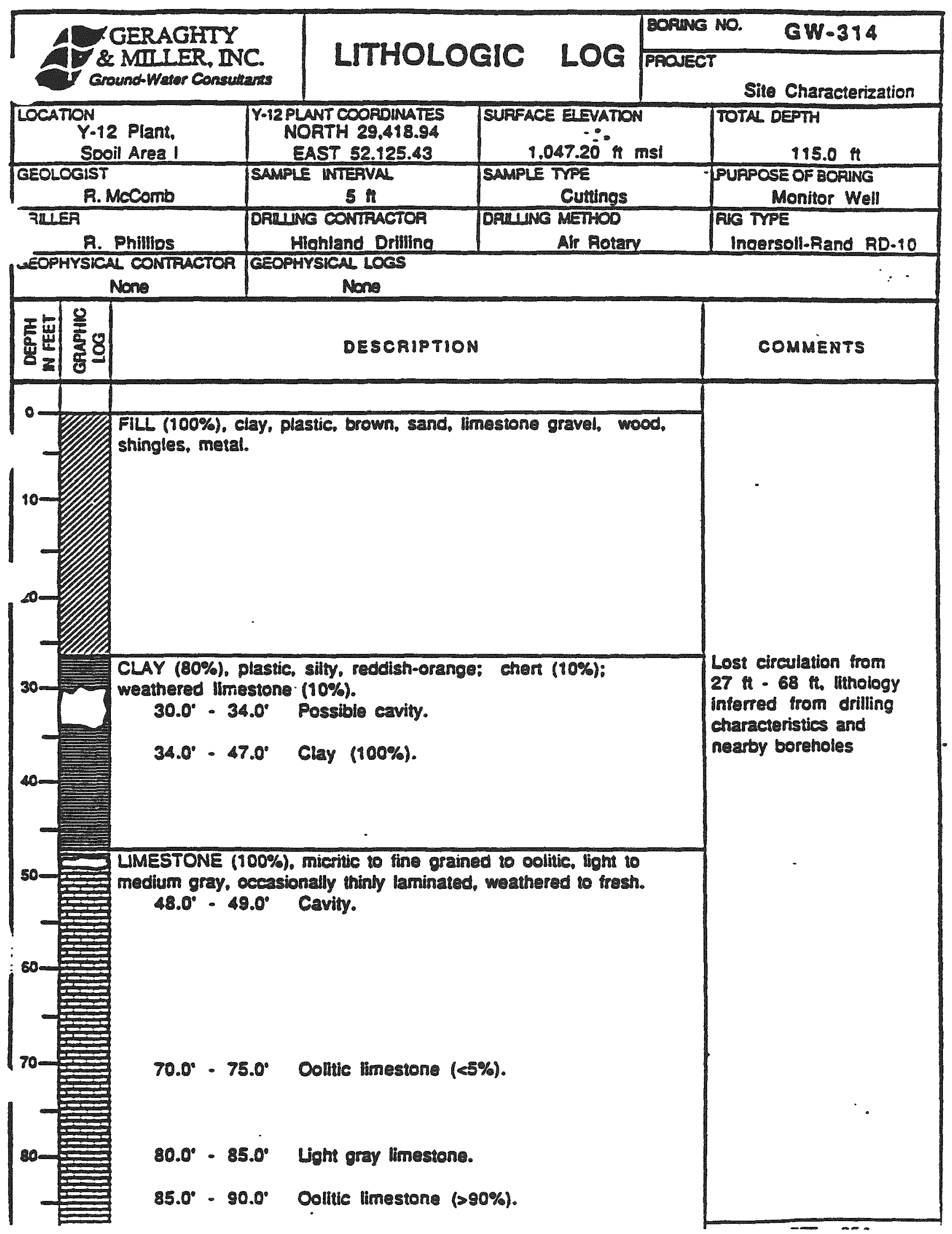




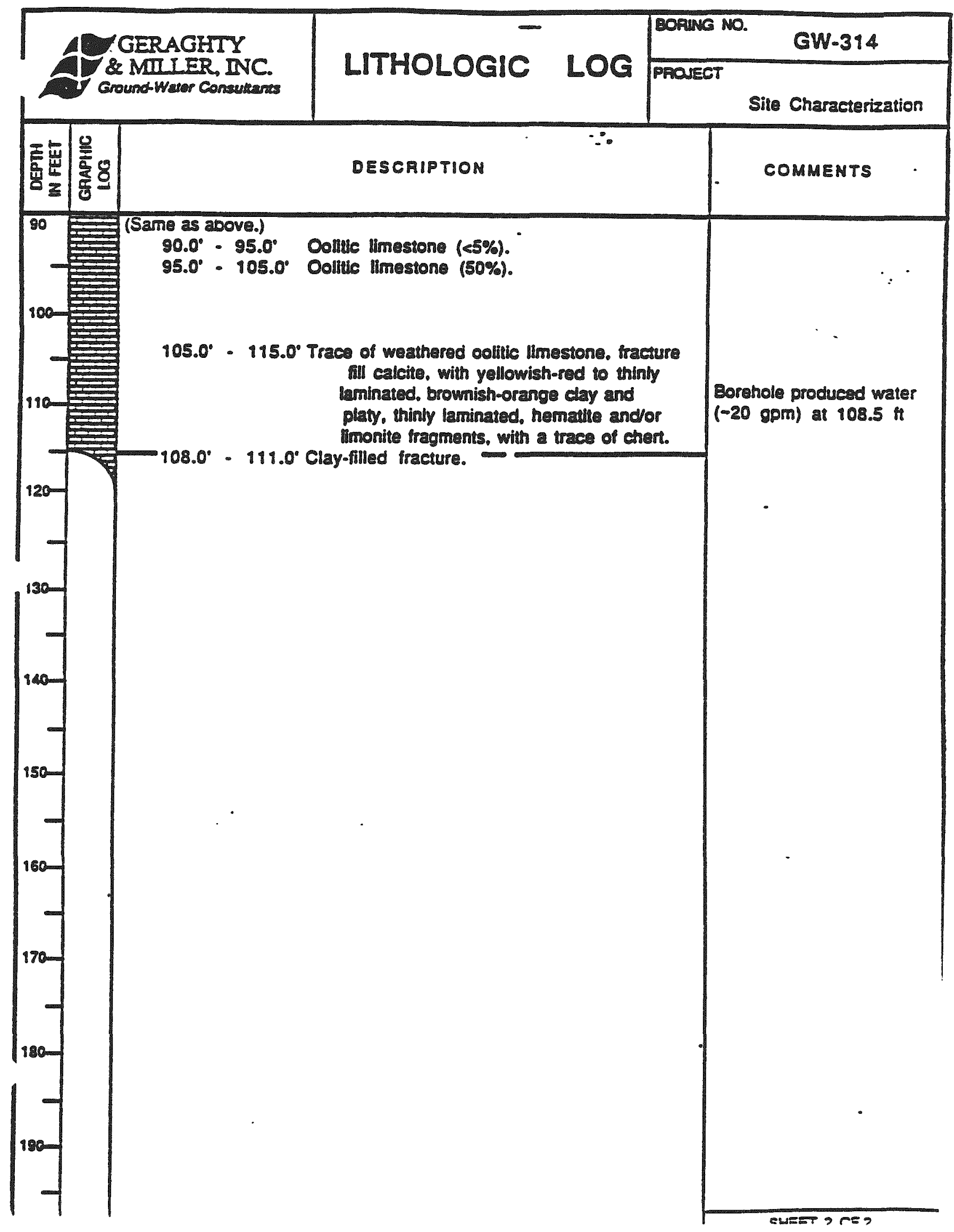




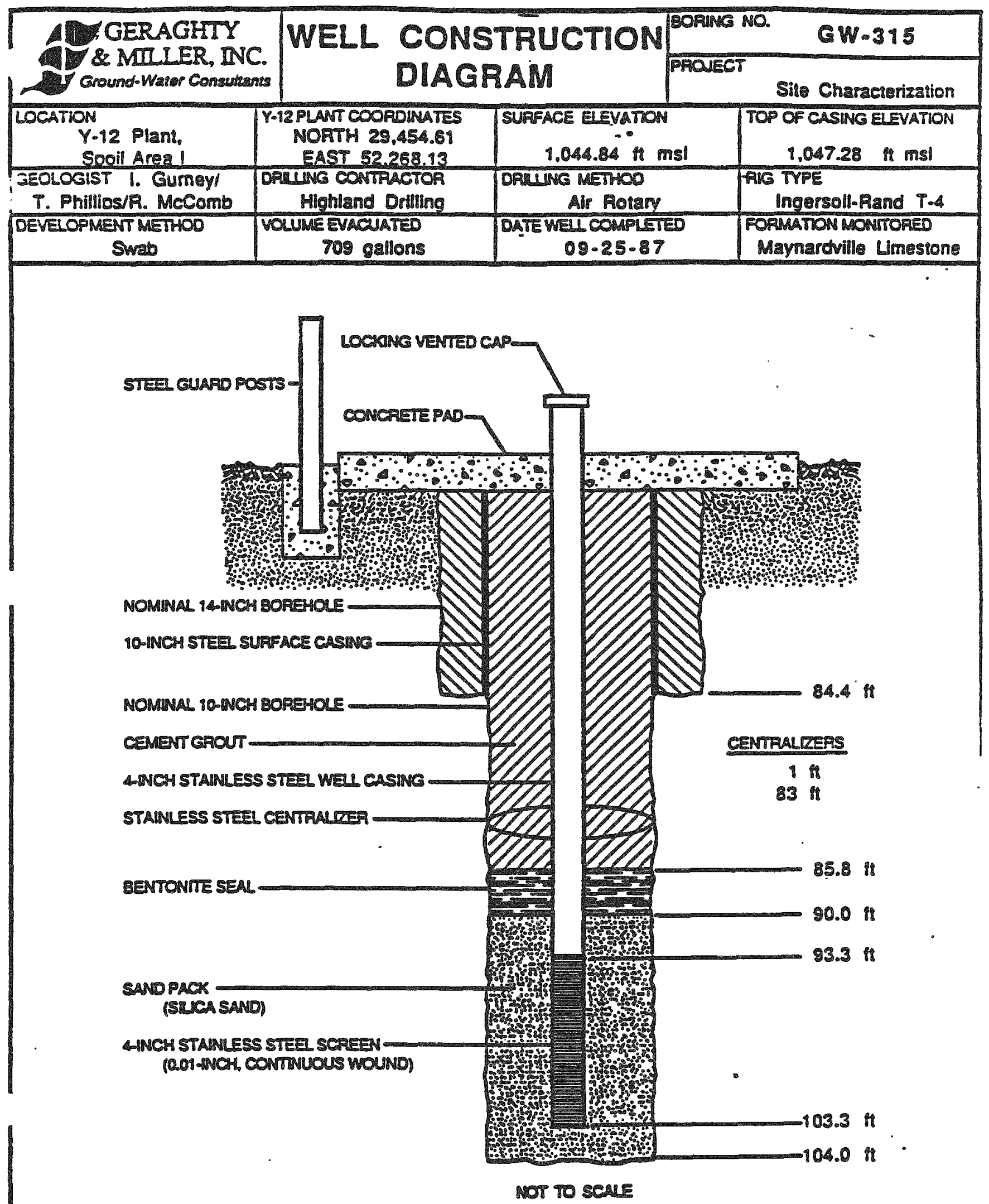

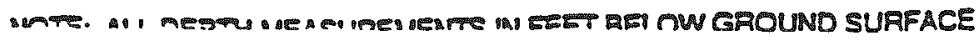




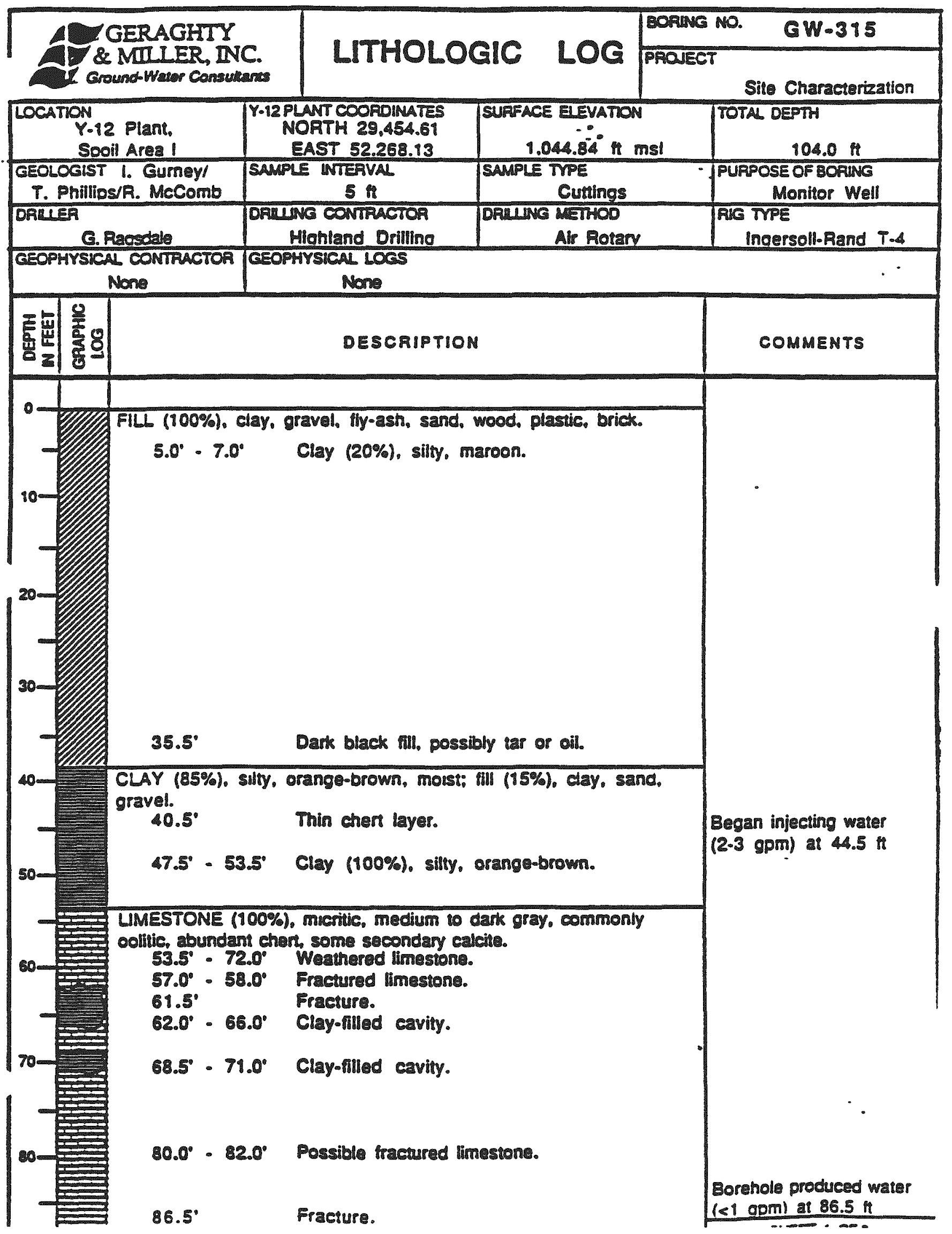




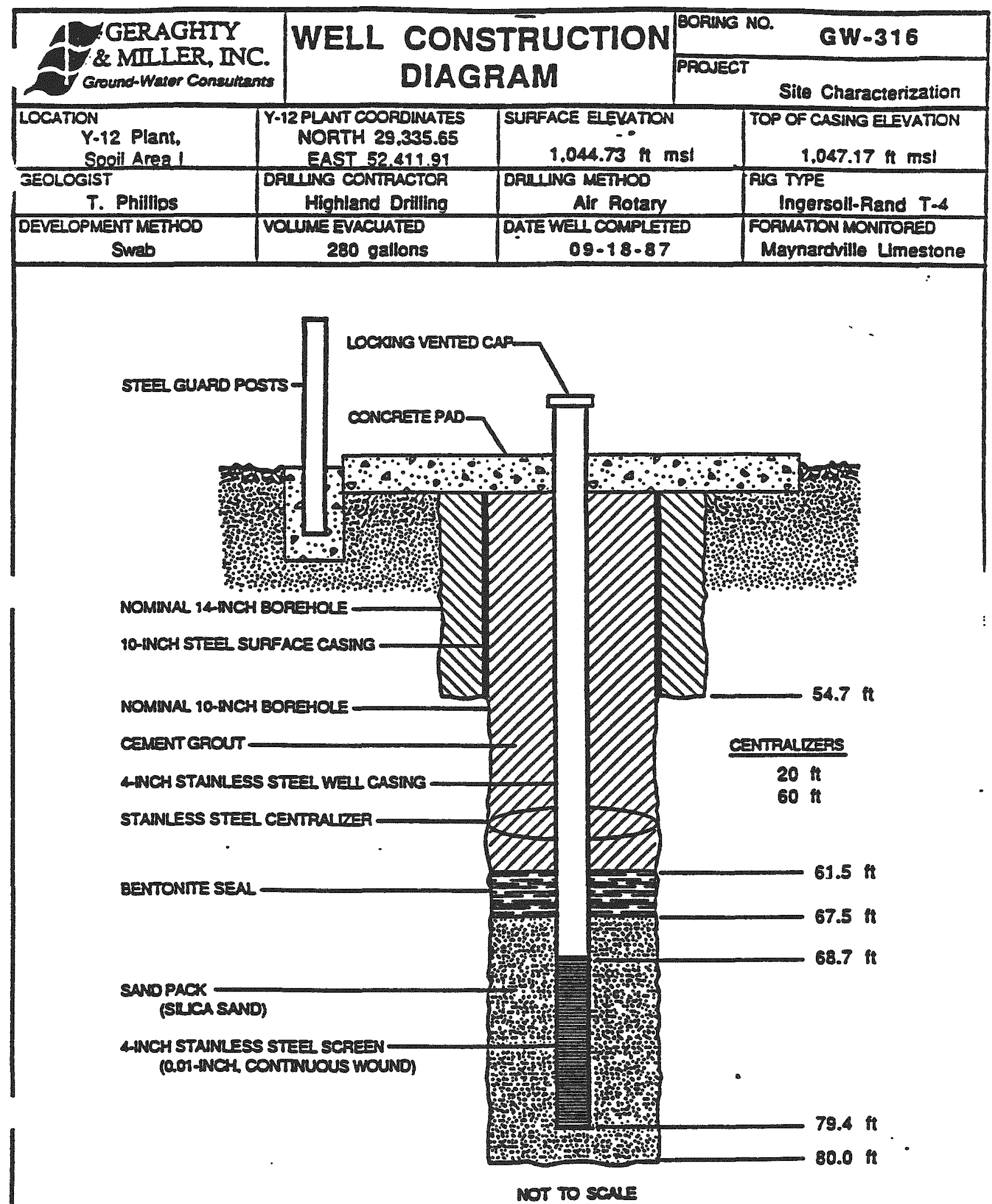




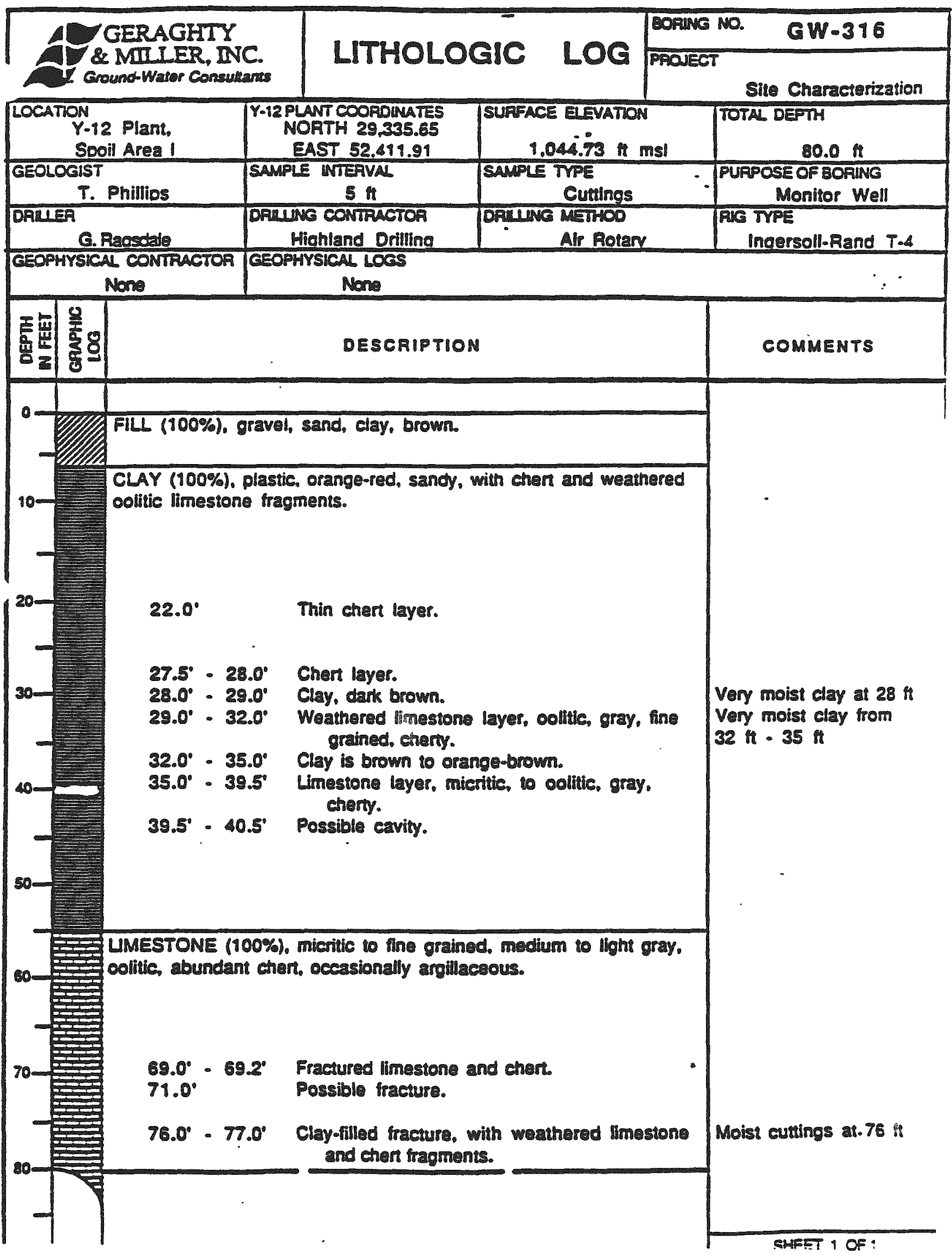




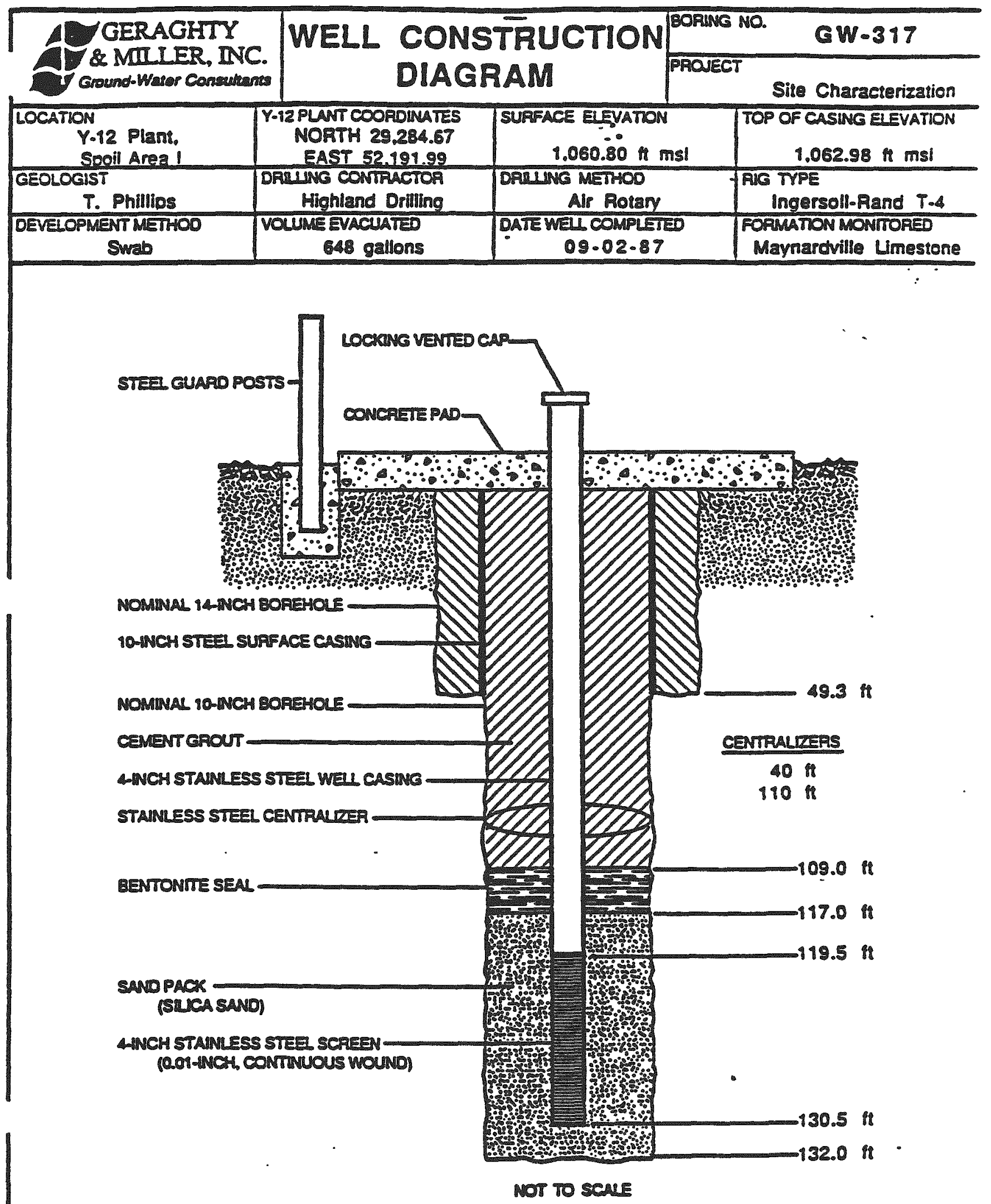

NOTE. AI DFPTH MF ASUAEMENTS WFET BELOW GROUNO SURFACE 


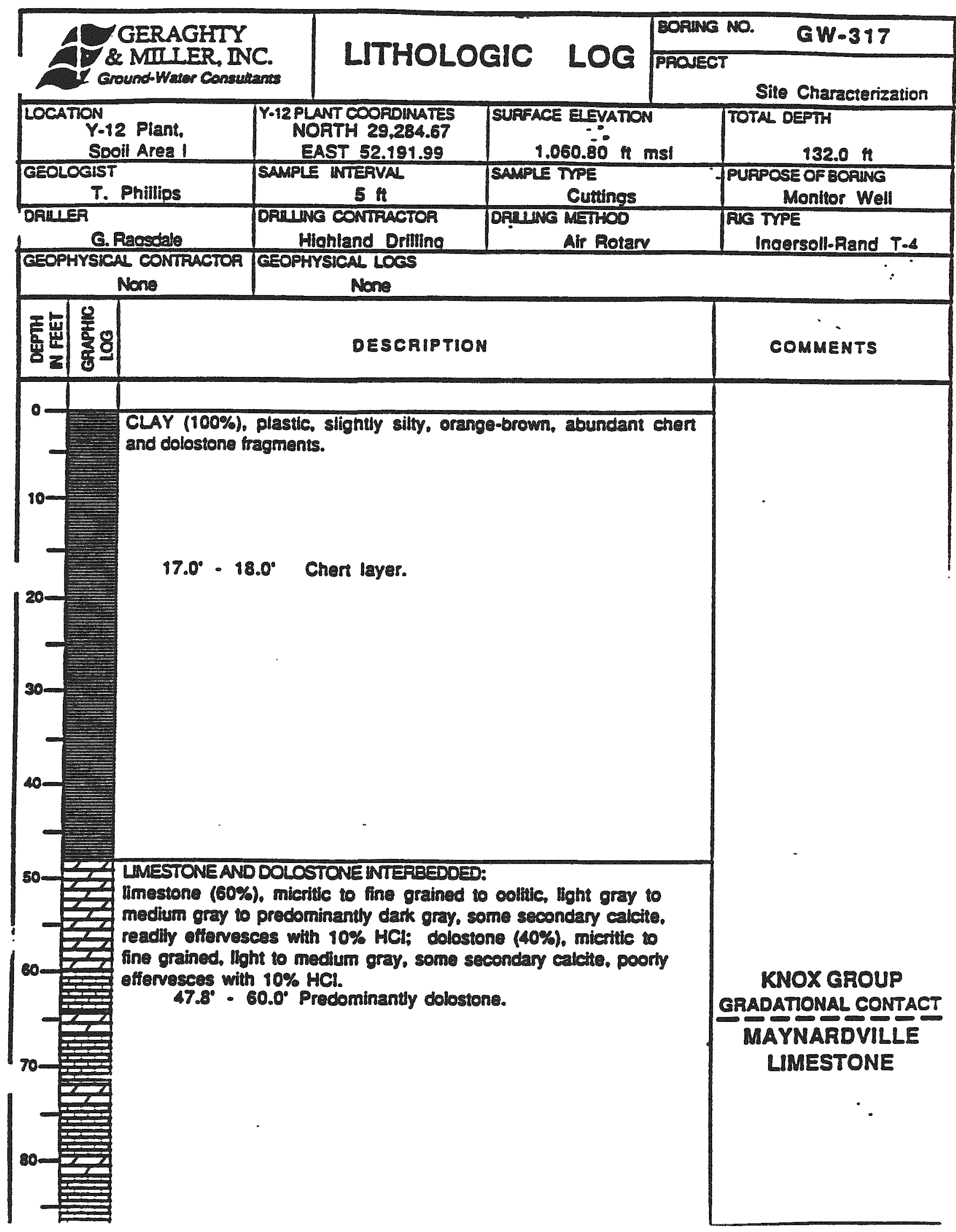




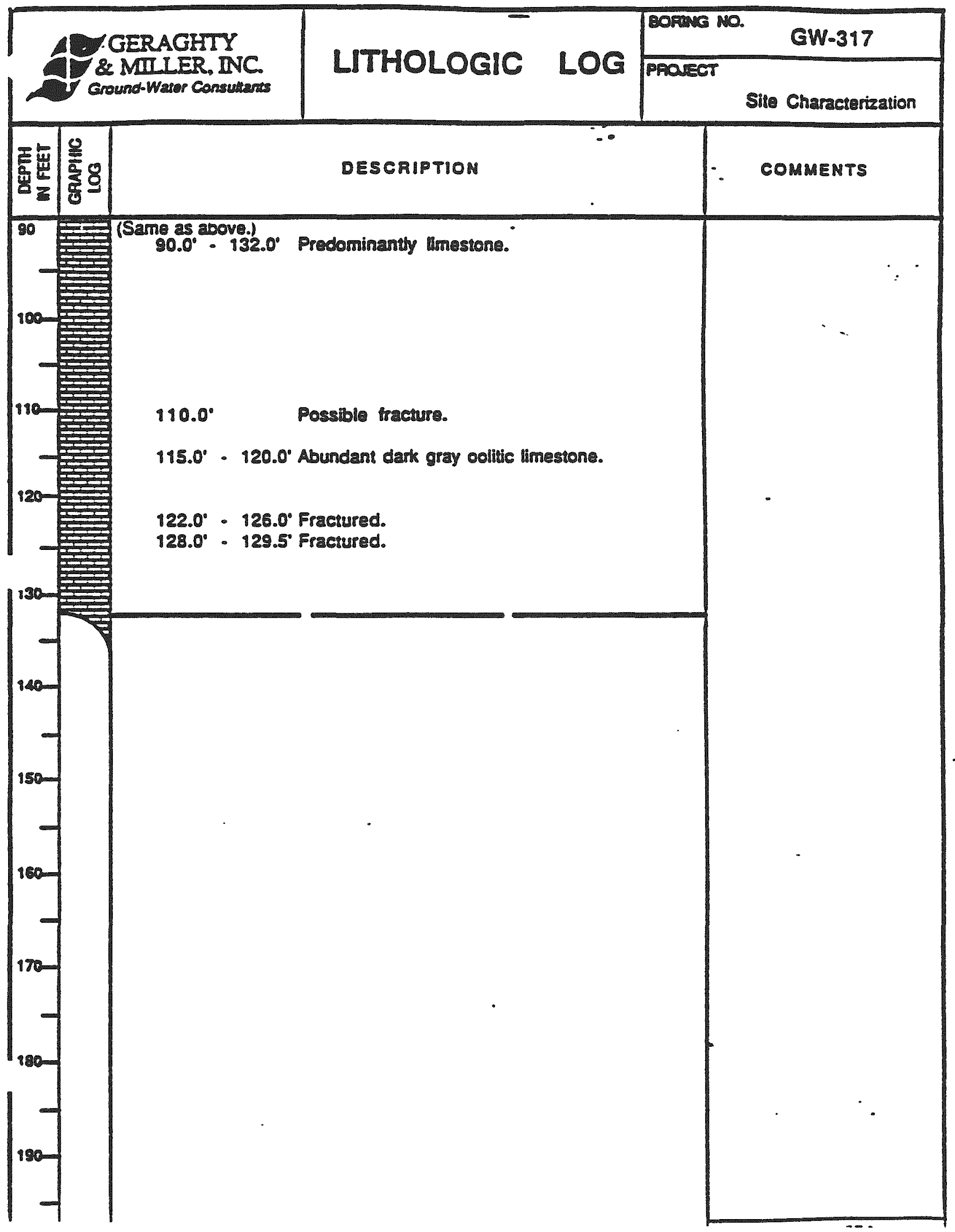




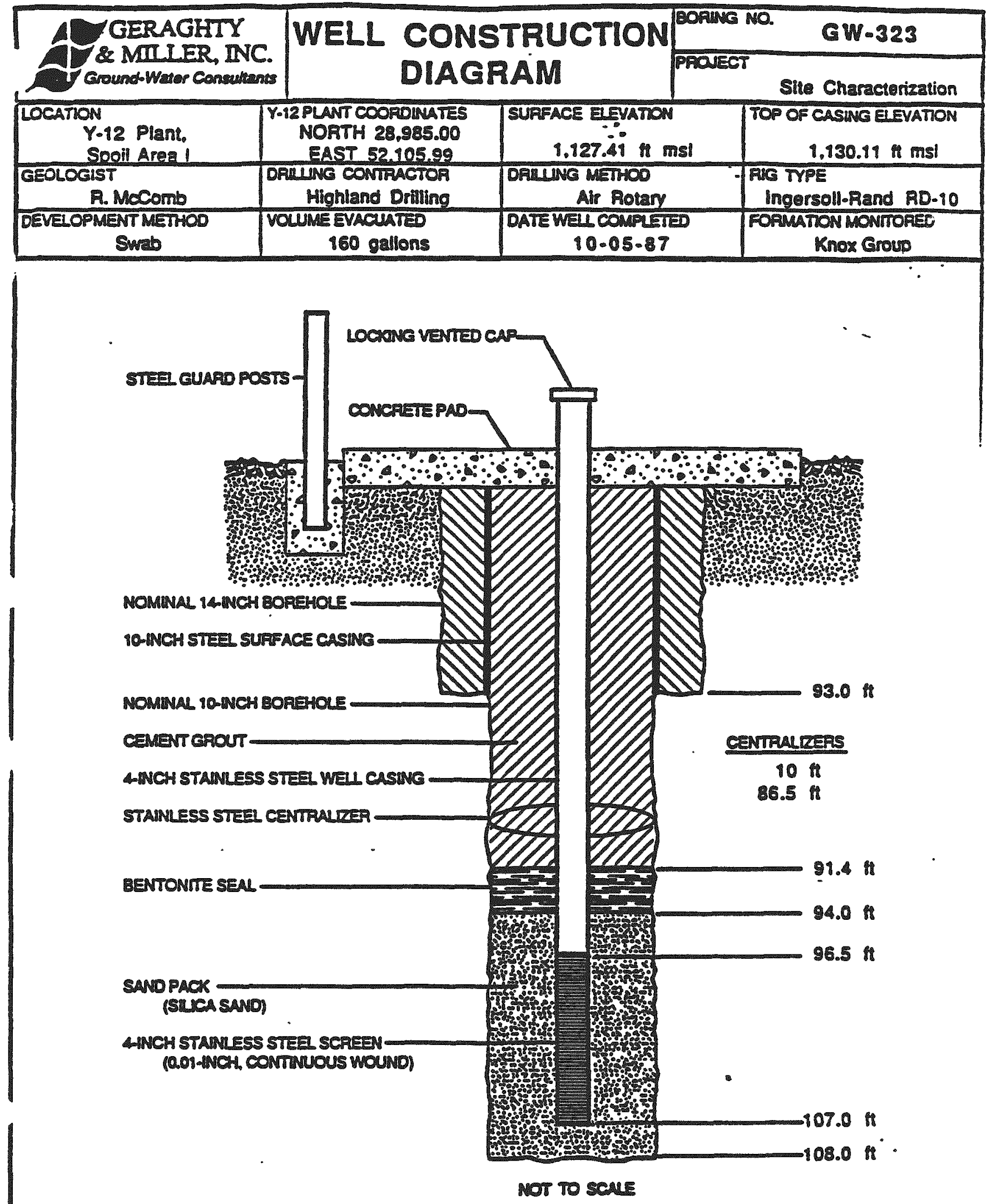

MOTE. AI OFPTH MFASUREMENTS NFET BELW GAOUNO SURFACE 


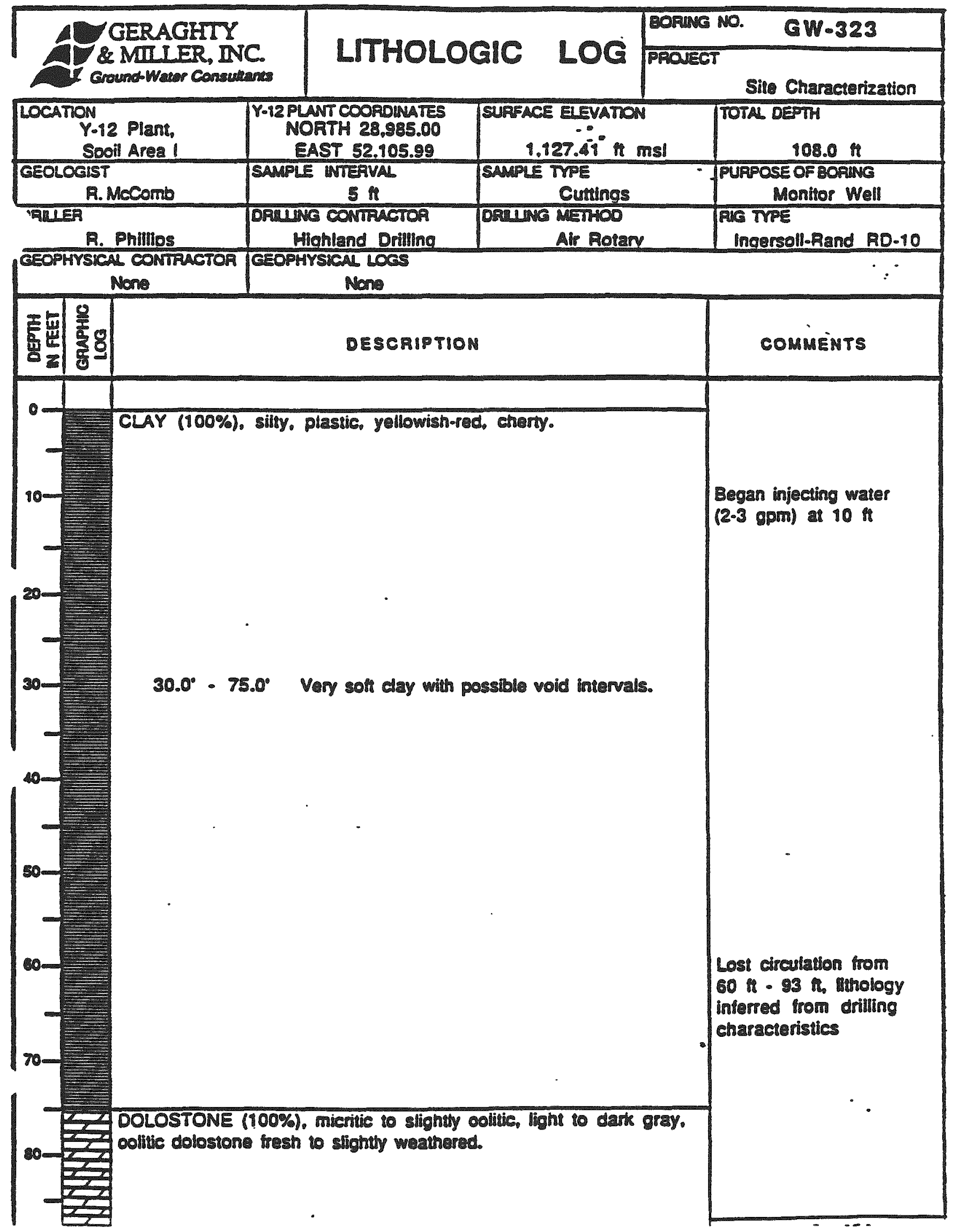




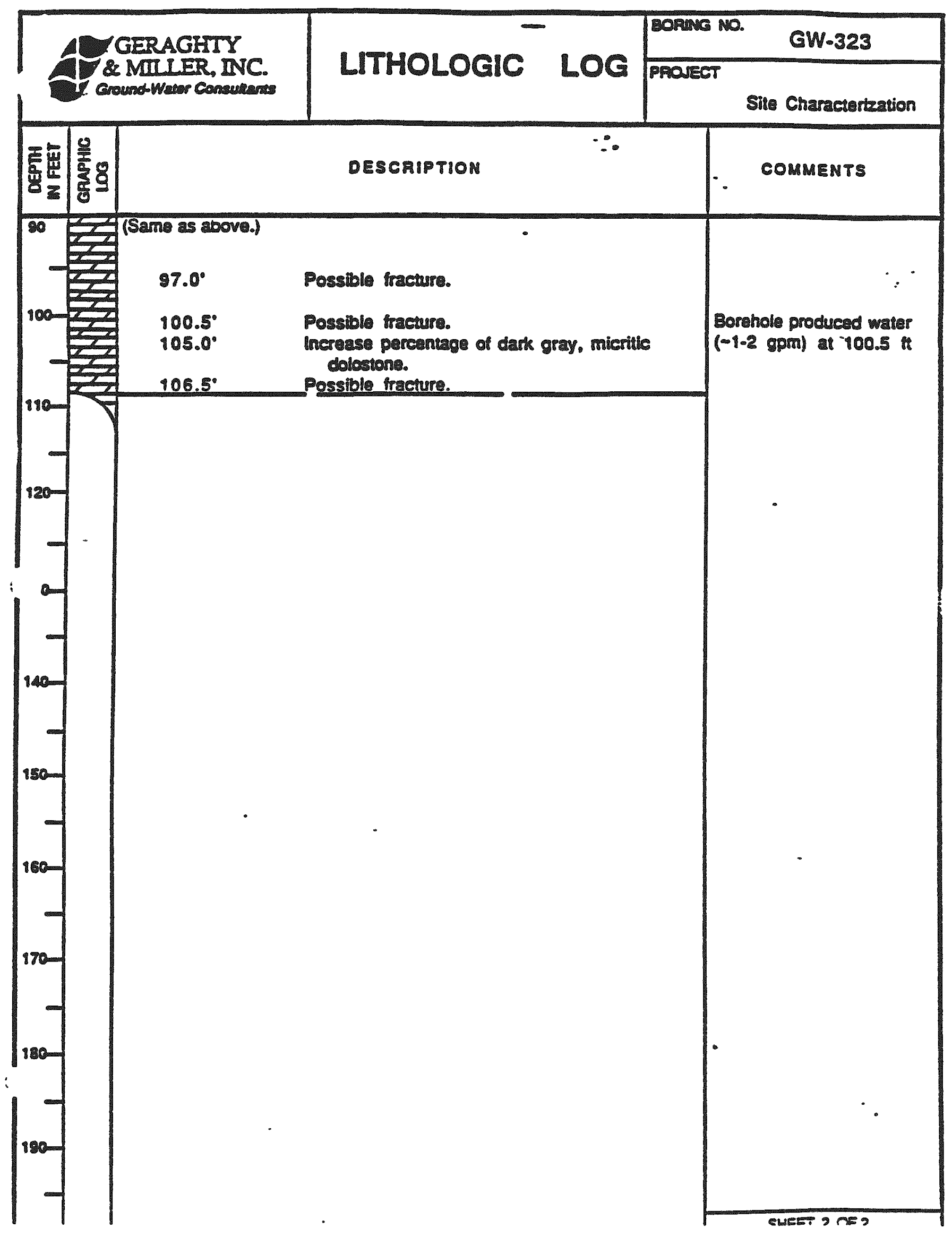


Field Change Orders 


.


Site - Bear Creek Valley Operable Unit 2

\begin{tabular}{|c|c|c|c|c|c|c|}
\hline FCO No. & BASELINE CHANGE & DATE ISSUED & DESCRIPTION OF CHANGE & ORIGNATOR & STATUS & $\begin{array}{l}\text { DATE ACTION } \\
\text { REOUIAED }\end{array}$ \\
\hline BCV-001 & $\begin{array}{l}\text { MINOR } \\
\text { O MAJOR } \\
\text { OTHER }\end{array}$ & 9790 & 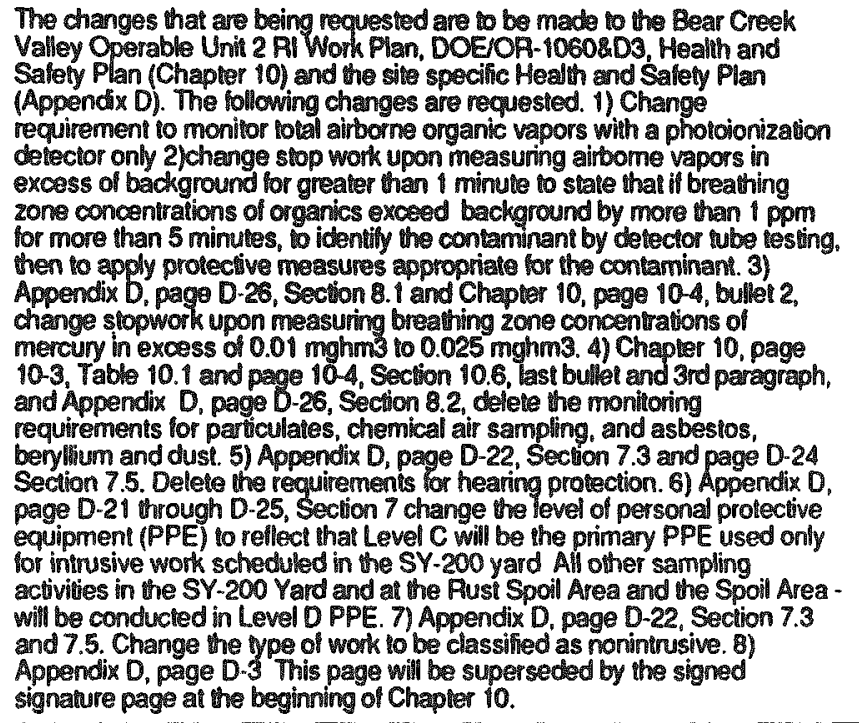 & C. Allison Bailey & COMPLETE & \\
\hline BCV-002 & $\begin{array}{l}\text { MINOR } \\
\text { OMAJOR } \\
\text { O OTHER }\end{array}$ & 9/14/93 & $\begin{array}{l}\text { 1. Change frequency of equipment rinsate blank to one blank per piece of } \\
\text { equipment, per week, per } 20 \text { sample. } 2 \text { ) Change description and lrequency } \\
\text { of field blanks to include } 3 \text { types of field blanks as follows: a. one field blank } \\
\text { per lot ty of ASTM II water b. one field blank of deconlarnination source } \\
\text { water evey } 2 \text { weeks per water soupce c. pour water field blank of ASTMII } \\
\text { (water exposed during sampling - collect al } 5 \% \text { frequency.) }\end{array}$ & Tami Barrent & COMPLETE & \\
\hline BCV-Do3 & $\begin{array}{l}\text { MINOR } \\
\text { O MAJOR } \\
\text { O OTHER }\end{array}$ & $9 / 15193$ & $\begin{array}{l}\text { Use SAIC data validation procedure TP-DM-300-7 rather than MMES } \\
\text { procedure. }\end{array}$ & Dan Land & COMPLETE & \\
\hline BCV-004 & $\begin{array}{l}\text { MINOR } \\
\text { O MAJOR } \\
\text { OTHER }\end{array}$ & $9 / 15 / 93$ & $\begin{array}{l}\text { Sample coding system has been modtiled. See attached for description. } \\
\text { System will be included in logbooks. }\end{array}$ & Dan Land & COMPLETE & \\
\hline BCV-005 & $\begin{array}{l}\text { MINOR } \\
\text { O MAJOR } \\
\text { O OTHER }\end{array}$ & $9 / 15 / 23$ & $\begin{array}{l}\text { Data receipt and tracking will be done using SAlC procedures: } \\
\text { TP-DM-300-5, TP-DM-300-6. TP-DM-300-9.all data management will be } \\
\text { done using SEIMS. }\end{array}$ & Dan Land & COMPLETE & \\
\hline BCV-006 & $\begin{array}{l}\text { MINOR } \\
\text { O MANOR } \\
\text { O OTHER }\end{array}$ & $9 / 15 / 93$ & $\begin{array}{l}\text { All data will be loaded into SEIMS not VAX SAS databases. Data entry will } \\
\text { be perfomed using TP.DM-300-2. }\end{array}$ & Dan Land & COMPLETE & \\
\hline BCV.007 & $\begin{array}{l}\text { MINOR } \\
\text { O MAJOR } \\
\text { O OTHER }\end{array}$ & $915 / 93$ & $\begin{array}{l}\text { Process of documenting database changes will ba done using database } \\
\text { change request form. Programmatic changes will have program listing and } \\
\text { output attached lo form. Form is attached. }\end{array}$ & Dan Land & COMPLETE & \\
\hline
\end{tabular}




\section{Site - Bear Creek Valley Operable Unit 2}

\begin{tabular}{|c|c|c|c|c|c|c|}
\hline FCONO. & BASELINE CHANGE & DATE ISSUED & DESCRIPTION OF CHANGE & ORIGINATOR & STATUS & $\begin{array}{l}\text { DATEACTION } \\
\text { REQUIRED } \\
\end{array}$ \\
\hline BCV-008 & $\begin{array}{l}\text { - MINOR } \\
\text { O MAJOR } \\
\text { O OTHER }\end{array}$ & $10 / 2293$ & 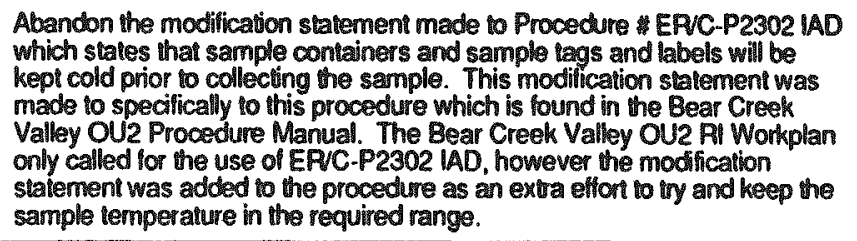 & Allison Bailey & COMPLETE & \\
\hline BCV-009 & $\begin{array}{l}\text { - MINOR } \\
\text { g MAJOR } \\
\text { O OTHER }\end{array}$ & $9 / 1493$ & $\begin{array}{l}\text { In the Bear Creek Valley RI Work Plan, DOE,OR-1060 and D3 page } 7-5 \text { of } \\
\text { the Field Sampling Plan, change the second sentence on the first paragaraph } \\
\text { to state that split spoon soil samples will be collected every iwo foot inslead } \\
\text { of every five foot. }\end{array}$ & C. Allison Bailey & COMPLETE & \\
\hline BCV-010 & $\begin{array}{l}\text { OMNOR } \\
\text { O MAJOR } \\
\text { O OTHER }\end{array}$ & 9/16/93 & $\begin{array}{l}\text { The Operable Unit } 2 \text { Remedial Investigation Work Plan calls for the use of } \\
2 \text { split spoons however due lo need of sample volume increase at times a } 3 \\
\text { in split spoon will be used as needed. }\end{array}$ & Susan Absion & COMPLETE & \\
\hline BCV.011 & $\begin{array}{l}\text { : MINOR } \\
\text { O MAJOR } \\
\text { O OTHER }\end{array}$ & $9 / 16 / 93$ & $\begin{array}{l}\text { If unable to collect enough soil sample to fill sample jars the sample } \\
\text { interval will not be collected and sent for analysis. }\end{array}$ & Susan Absion & COMPLETE & \\
\hline BCV-012 & $\begin{array}{l}\text { - MINOR } \\
\text { O MAJOR } \\
\text { O OTHER }\end{array}$ & 9/15/93 & $\begin{array}{l}\text { Move the sampling locations in the Rust Spoil Area (ASA) lor the Bear } \\
\text { Creek Valley Operable Unit } 2 \text { Remedial investigation activities so that the } \\
\text { locations transect the center of the site. }\end{array}$ & c. Allison Bailey & COMPLETE & \\
\hline BCV-013 & $\begin{array}{l}\text { - MINOR } \\
\text { OMAJOR } \\
\text { O OTHER }\end{array}$ & 9/22/93 & $\begin{array}{l}\text { Asbestos and Radionuclide samples are not required to be cooled and } \\
\text { temperalure blanks and doecks should not be required. }\end{array}$ & C. Allison Bailey & COMPLETE & \\
\hline BCV-014 & $\begin{array}{l}\text { MINOR } \\
\text { O MAJOR } \\
\text { O OTHER }\end{array}$ & $9 / 27 / 93$ & 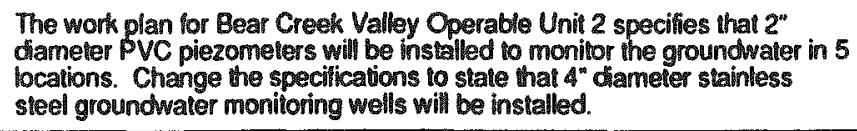 & c. Allison Bailey & COMPLETE & \\
\hline BCV-015 & $\begin{array}{l}\text { O MINOR } \\
\text { OMAJOR } \\
\text { O OTHER }\end{array}$ & $10 / 19 / 93$ & 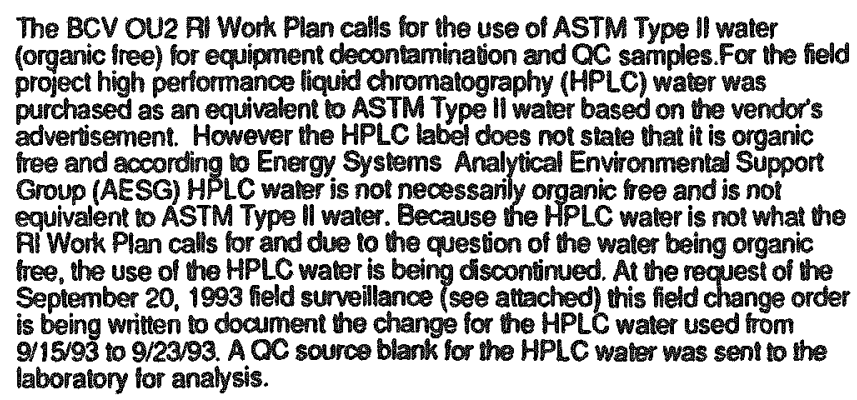 & Allison Bailey & COMPLETE & \\
\hline BCV-016 & $\begin{array}{l}\text { - MINOR } \\
\text { O MAJOR } \\
\text { O OTHER }\end{array}$ & 7903 & $\begin{array}{l}\text { This FCO is being requesed to replace the use of ASTM Type II water for } \\
\text { the trip blanks, field blanks and decon of equipment with the use of ASTM } \\
\text { Type I waler for the Bear Creek Valley Operable Unit } 2 \text { Project. }\end{array}$ & & COMPLETE & \\
\hline
\end{tabular}




\section{Site - Bear Creek Valley Operable Unit 2}

\begin{tabular}{|c|c|c|c|c|c|c|}
\hline FCONO. & BASELINE CHANGE & DATE ISSUED & DESCAIPTION OF CHANGE & ORIGINATOR & STATUS & $\begin{array}{l}\text { DATE ACTION } \\
\text { REOUIRED } \\
\end{array}$ \\
\hline BCV-017 & $\begin{array}{l}\text { - MINOR } \\
\text { O MAJOR } \\
\text { O OTHER }\end{array}$ & $11 / 3 / 93$ & 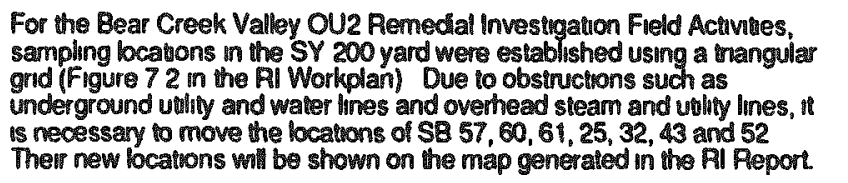 & C Allson Balley & COMPLETE & \\
\hline BCV-018 & $\begin{array}{l}\text { - MINOR } \\
\text { O MAJOR } \\
\text { O OTHER }\end{array}$ & $11 / 3,93$ & 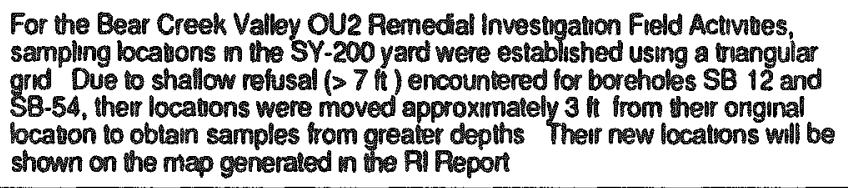 & C Allison Balley & COMPLETE & \\
\hline BCV-019 & $\begin{array}{l}\text { MINOR } \\
\text { MAJOR } \\
\text { O OTHER }\end{array}$ & $11 / 3 / 93$ & $\begin{array}{l}\text { In the Waste Managernent Plan for the Bear Creek Valley OU2 PI, it states } \\
\text { that sol cuttungs will be returned to the borehole from which they came and } \\
\text { thal the top } 3 \text { to } 5 \text { leet of the borehole will be grouted In the SY } 200 \text { Yard it } \\
\text { has been recommended by Sleve Walker Energy Systems Technical } \\
\text { Lead that boreholes SB-55, } 565758,59,60 \& 61 \text { be grouted from the } \\
\text { bottom up because the bonngs are located in the road If the entre depth of } \\
\text { each borehole is not grouled it may eventually cause a weak or soft spot in } \\
\text { the road The sol cutings will be spread in the SY } 200 \text { yard }\end{array}$ & C Allison Bailey & COMPLETE & \\
\hline BCV.020 & $\begin{array}{l}\text { MINOR } \\
\text { OMAJOR } \\
\text { O OTHER }\end{array}$ & $11 / 393$ & $\begin{array}{l}\text { Move the proposed locations for pezometers P1 and P2 in Spoll Area } 1 \\
\text { for the Bear Creek Valley OU2 Remedial Investigation (see Figure } 7 \text { in } \\
\text { the Ril Work Plan) Their new locatons wil be shown on the map } \\
\text { generated in the AI Report }\end{array}$ & C Allson Banley & COMPLETE & \\
\hline BCV-021 & $\begin{array}{l}\text { MINOR } \\
\text { O MAJOR } \\
\text { O OTHER }\end{array}$ & $11 / 3 / 93$ & 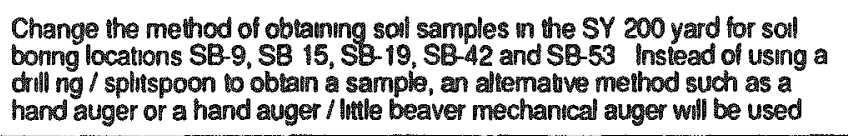 & C Allson Banley & COMPLETE & \\
\hline BCV-022 & $\begin{array}{l}\text { - MINOR } \\
\text { O MAJOR } \\
\text { O OTHER }\end{array}$ & $11 / 5 / 93$ & $\begin{array}{l}\text { Change the estumated yolume of waste water given in the Waste } \\
\text { Management Plan for Bear Creek Valley OU2 PI (Y/ER 121, September } \\
\text { 1993) from } 300 \text { gallons to } 6,500 \text { gallons This increase is due lo the } \\
\text { increase in the use of water for equipment decontamination More waler is } \\
\text { needed than anucipated due to the cohesive nature of the day and the } \\
\text { increase in number and depth of boreholes }\end{array}$ & Alison Balley & COMPLETE & \\
\hline BGV-023 & $\begin{array}{l}\text { O MINOR } \\
\text { O MAJOR } \\
\text { O OTHER }\end{array}$ & $11 / 1593$ & 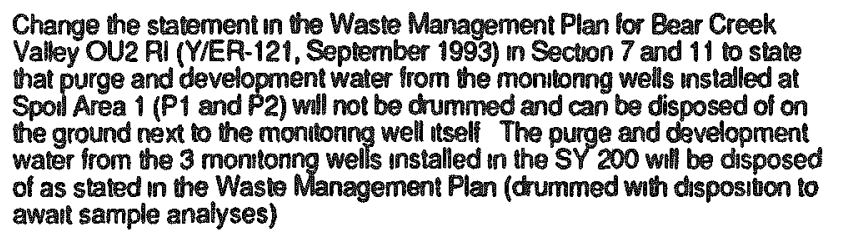 & C Allison Bailey & COMPLETE & \\
\hline
\end{tabular}




\section{Site - Bear Creek Valley Operable Unit 2}

\begin{tabular}{|c|c|c|c|c|c|c|}
\hline FCONO. & BASELINE CHANGE & DATE ISSUED & DESCRIPTION OF CHANGE & ORIGMATOR & STATUS & $\begin{array}{l}\text { DATE ACTION } \\
\text { REQUIRED } \\
\end{array}$ \\
\hline BCV-024 & $\begin{array}{l}\text { O MINOR } \\
\text { O MAJOR } \\
\text { O OTHER }\end{array}$ & $11 / 593$ & 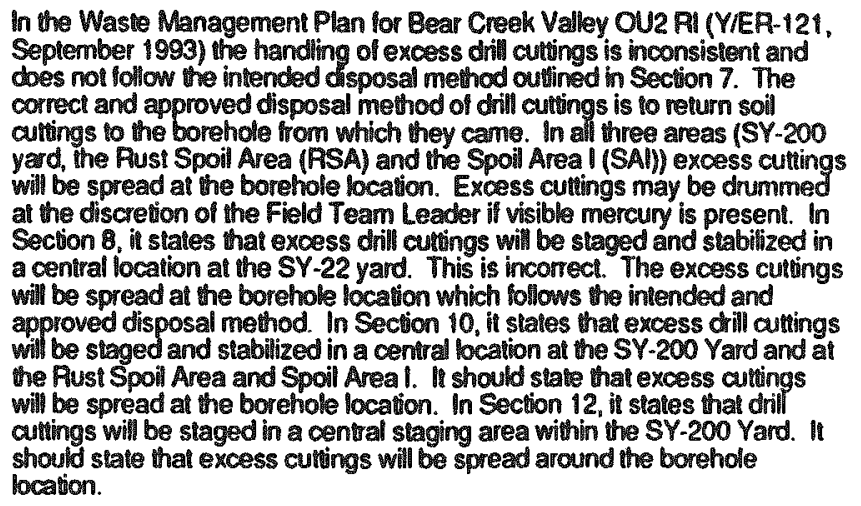 & C. Allison Bailey & COMPLETE & . \\
\hline BCV-025 & $\begin{array}{l}\text { O MINOR } \\
\text { O MAJOR } \\
\text { O OTHER }\end{array}$ & $11 / 23 / 93$ & $\begin{array}{l}\text { For the BCV OU2 PI field activities, discontinue the use of centralizers } \\
\text { when installing monitoring wells in the SY-200 yard. }\end{array}$ & C Allison Bailey & COMPLETE & \\
\hline BCV-026 & $\begin{array}{l}\text { - MINOR } \\
\text { O MAJOR } \\
\text { O OTHER }\end{array}$ & $11 / 2393$ & $\begin{array}{l}\text { Move the location of boring } 18 \text { approximately } 6 \text { feet lo the west and boring } \\
41 \text { approximately } 6 \text { leet north from their oiginal location on the sampling grid } \\
\text { in the SY-200 Yard. Their new locations will be show on the map in the Pil } \\
\text { Report. }\end{array}$ & C. Allison Bailey & COMPLETE & \\
\hline BCV-027 & $\begin{array}{l}\text { MINOR } \\
\text { O MAJOR } \\
\text { O OTHER }\end{array}$ & $11 / 2393$ & $\begin{array}{l}\text { Move the location of the monitoring well located bewween borings } 10 \text { and } 11 \\
\text { in the SY-200 Yard approximately } 10.5 \text { feet east of the originallocation. } \\
\text { shown on the sampling grid in the RIWP. The new location will be shown } \\
\text { on the are map in the fl report. }\end{array}$ & C Allison Bailey & COMPLETE & \\
\hline BCV-028 & $\begin{array}{l}\text { MINOR } \\
\text { M MAJOR } \\
\text { O OTHER }\end{array}$ & $11 / 2393$ & $\begin{array}{l}\text { Move the location of the monitoring well located between boring } 54 \text { and } 55 \\
\text { approximalely } 6 \text { feet northeast of whe original location shown the sampling } \\
\text { grid for the St-200 Yard. The new localion will be shown the area map in } \\
\text { the RI Report. }\end{array}$ & C. Allison Bailey & COMPLETE & \\
\hline BCV-029 & $\begin{array}{l}\text { - MINOR } \\
\text { O MAJOR } \\
\text { O OTHER }\end{array}$ & $12 / 293$ & $\begin{array}{l}\text { Move the location of boring no. } 31 \text { 10 fest north, no. } 23 \text { 10 feet east, no. } 46 \\
15 \text { feet southeast, and no. } 50 \text { is feet southwest from their original location } \\
\text { on the sampling grid in the } 5 Y \text {-200 Yard. Their new locations will be shown } \\
\text { on the map in the Ril report. }\end{array}$ & C. Allison Bailey & COMPLETE & \\
\hline
\end{tabular}




\section{Site - Bear Creek Valley Operable Unit 2}

\begin{tabular}{|c|c|c|c|c|c|c|}
\hline FCONO. & BASELINE CMANGE & DATE ISSUED & DESCRIPTION OF CHANGE & ORIGINATOR & STATUS & $\begin{array}{l}\text { DATE ACTION } \\
\text { REOUIRED }\end{array}$ \\
\hline BCV-030 & $\begin{array}{l}\text { MINOR } \\
\text { O MAJOR } \\
\text { O OTHER }\end{array}$ & $12 / 293$ & 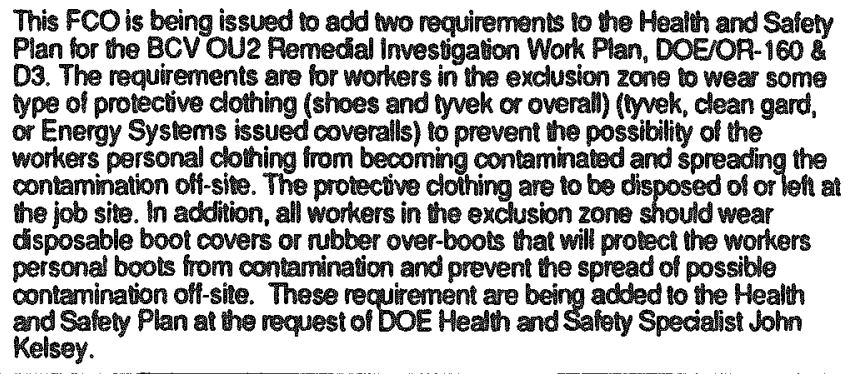 & C. Alison Bailey & COMPLETE & \\
\hline 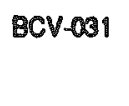 & $\begin{array}{l}\text { MNNOR } \\
\text { O MAJOR } \\
\text { O OTHER }\end{array}$ & $12 / 1490$ & $\begin{array}{l}\text { Add the use of a disposable tellon scoop to collect sediment samples to } \\
\text { procedure ESP-304-1 (sediment collection procedure). }\end{array}$ & C Allison Bailey & COMPLETE & \\
\hline BCV.032 & $\begin{array}{l}\text { - MNOR } \\
\text { MANOR } \\
\text { O OTHER }\end{array}$ & $12 / 1493$ & $\begin{array}{l}\text { Add the use of a disposable laboratory deaned glass grab jar to collect } \\
\text { surtace water samples to procedure ESP.301-1 (collecting surface water } \\
\text { samples). }\end{array}$ & C Allison Bailey & COMPLETE & \\
\hline
\end{tabular}




$$
\text { A-204 }
$$

Table A.1. Well development activities

\begin{tabular}{lcccrr}
\hline $\begin{array}{c}\text { Monitoring } \\
\text { well \# }\end{array}$ & $\begin{array}{c}\text { Start } \\
\text { date/time }\end{array}$ & $\begin{array}{c}\text { Completion } \\
\text { date/time }\end{array}$ & $\begin{array}{c}\text { One well } \\
\text { volume }\end{array}$ & $\begin{array}{c}\text { Three well } \\
\text { volumes }\end{array}$ & $\begin{array}{c}\text { Groundwater } \\
\text { removed }\end{array}$ \\
\hline MW-1 & $12-20-93 / 1131$ & $12-28-93 / 1335$ & $8.1 \mathrm{gal}$ & $24.3 \mathrm{gal}$ & $56.5 \mathrm{gal}$ \\
MW-2 & $12-20-93 / 1515$ & $12-28-93 / 1520$ & $15.2 \mathrm{gal}$ & $45.6 \mathrm{gal}$ & $90 \mathrm{gal}$ \\
MW-3 & $12-27-93 / 1600$ & $12-27-93 / 1646$ & $6.48 \mathrm{gal}$ & $19.4 \mathrm{gal}$ & $49 \mathrm{gal}$ \\
MW-4 & $12-22-93 / 1531$ & $12-28-93 / 0858$ & $6.8 \mathrm{gal}$ & $20.4 \mathrm{gal}$ & $55 \mathrm{gal}$ \\
MW-5 & $12-27-93 / 1345$ & $12-27-93 / 1507$ & $3.62 \mathrm{gal}$ & $10.9 \mathrm{gal}$ & $12 \mathrm{gal}$ \\
\hline
\end{tabular}




\section{A-205}

Table A.2. Well purge activities

\begin{tabular}{lcccccc}
\hline $\begin{array}{c}\text { Monitoring } \\
\text { well \# }\end{array}$ & $\begin{array}{c}\text { Start } \\
\text { date/time }\end{array}$ & $\begin{array}{c}\text { Completion } \\
\text { date/time }\end{array}$ & $\begin{array}{c}\text { One well } \\
\text { volume }\end{array}$ & $\begin{array}{c}\text { Three well } \\
\text { volumes }\end{array}$ & $\begin{array}{c}\text { Well } \\
\text { purged } \\
\text { dry Y/N }\end{array}$ & $\begin{array}{c}\text { Groundwater } \\
\text { removed }\end{array}$ \\
\hline MW-1 & $12-29-93 / 1358$ & $12-29-93 / 1409$ & $6.51 \mathrm{gal}$ & $19.53 \mathrm{gal}$ & $\mathrm{Y}$ & $13.9 \mathrm{gal}$ \\
MW-2 & $12-30-93 / 0855$ & $12-30-93 / 0910$ & $3.95 \mathrm{gal}$ & $11.85 \mathrm{gal}$ & $\mathrm{N}$ & $12 \mathrm{gal}$ \\
MW-3 & $12-29-93 / 1052$ & $12-29-93 / 1114$ & $6.79 \mathrm{gal}$ & $20.37 \mathrm{gal}$ & $\mathrm{N}$ & $35 \mathrm{gal}$ \\
MW-4 & $12-29-93 / 0940$ & $12-29-93 / 0952$ & $6.74 \mathrm{gal}$ & $20.22 \mathrm{gal}$ & $\mathrm{Y}$ & $9 \mathrm{gal}$ \\
MW-5 & $12-29-93 / 1013$ & $12-29-93 / 1024$ & $3.69 \mathrm{gal}$ & $11.07 \mathrm{gal}$ & $\mathrm{Y}$ & $5.5 \mathrm{gal}$ \\
\hline
\end{tabular}



SA-1 Figures 



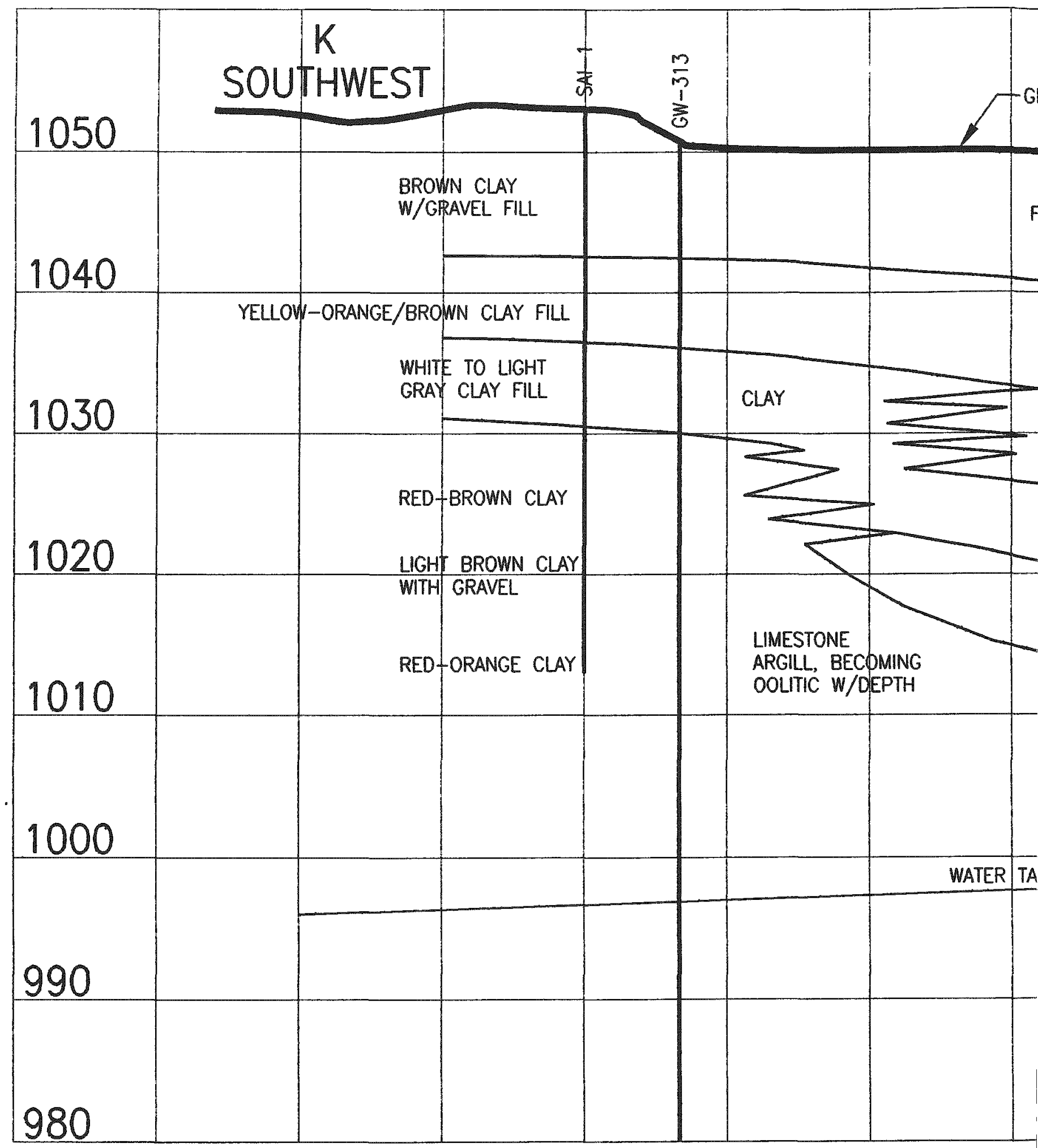




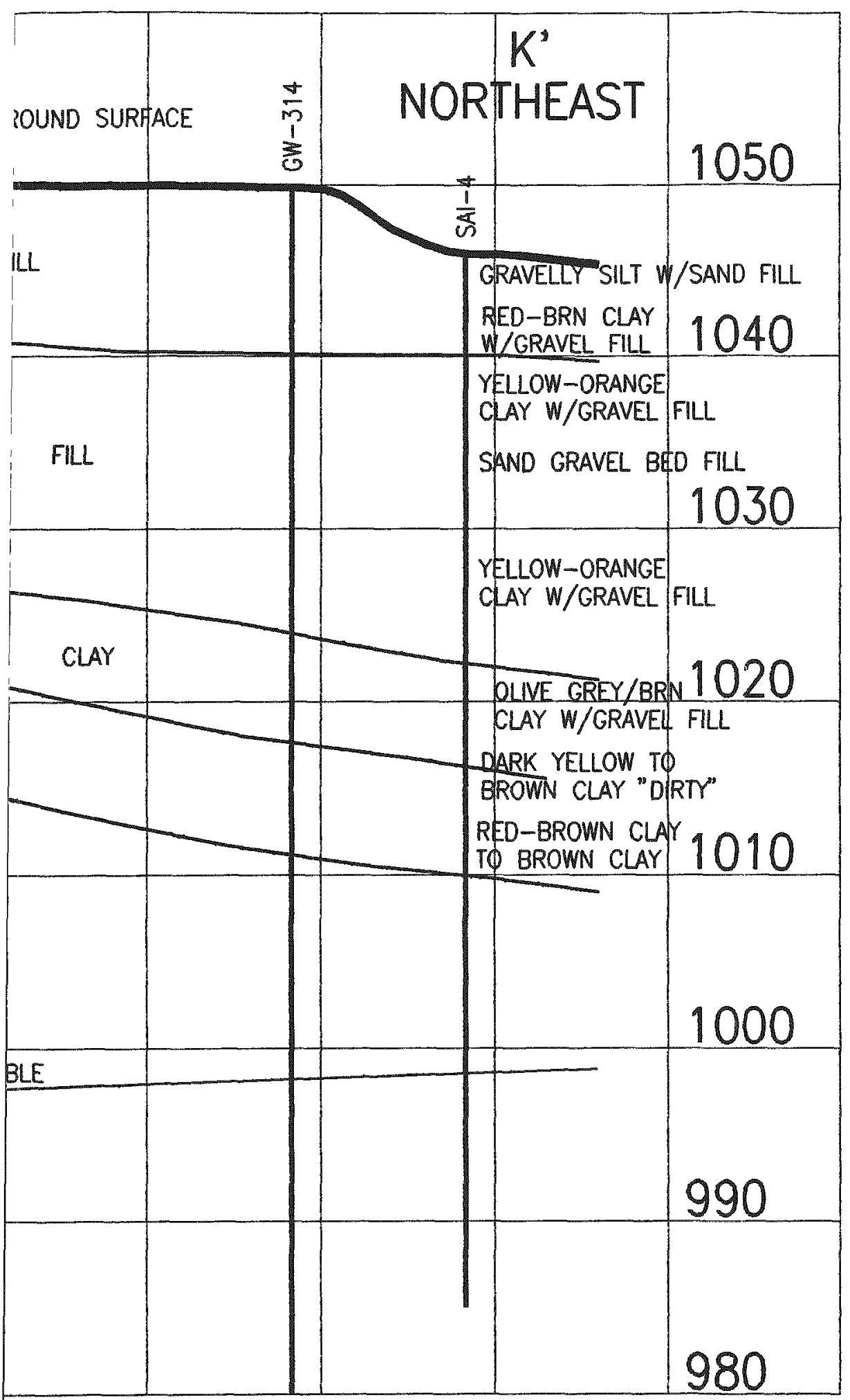

LEGEND:

GW. MONITORING WELL SB SOLLBORINO

$\begin{array}{llllll}0 & 2 & 4 & 6 & 8 & 10\end{array}$ VERTICAL SCALE: $1^{\prime \prime}=10^{\circ}$

$\begin{array}{llll}0 & 10 & 20 & 30\end{array}$

HORIZONTAL SCALE: $1^{\prime \prime}=30^{\circ}$

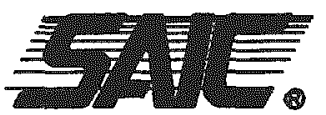

Science Applications International Corporation

BEAR CREEK VALLEY OU2 SPOIL AREA 1 PROFILE $K-K$ ' SOIL LITHOLOGY
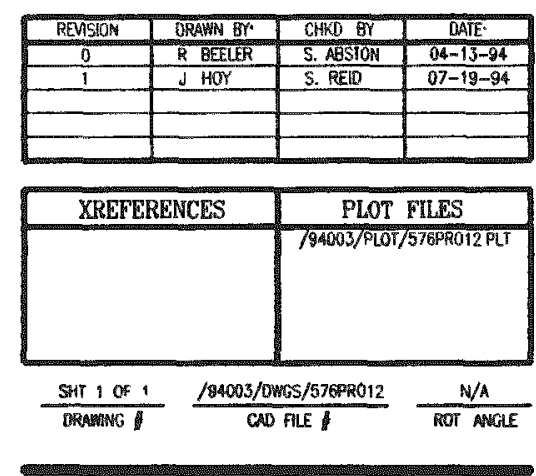


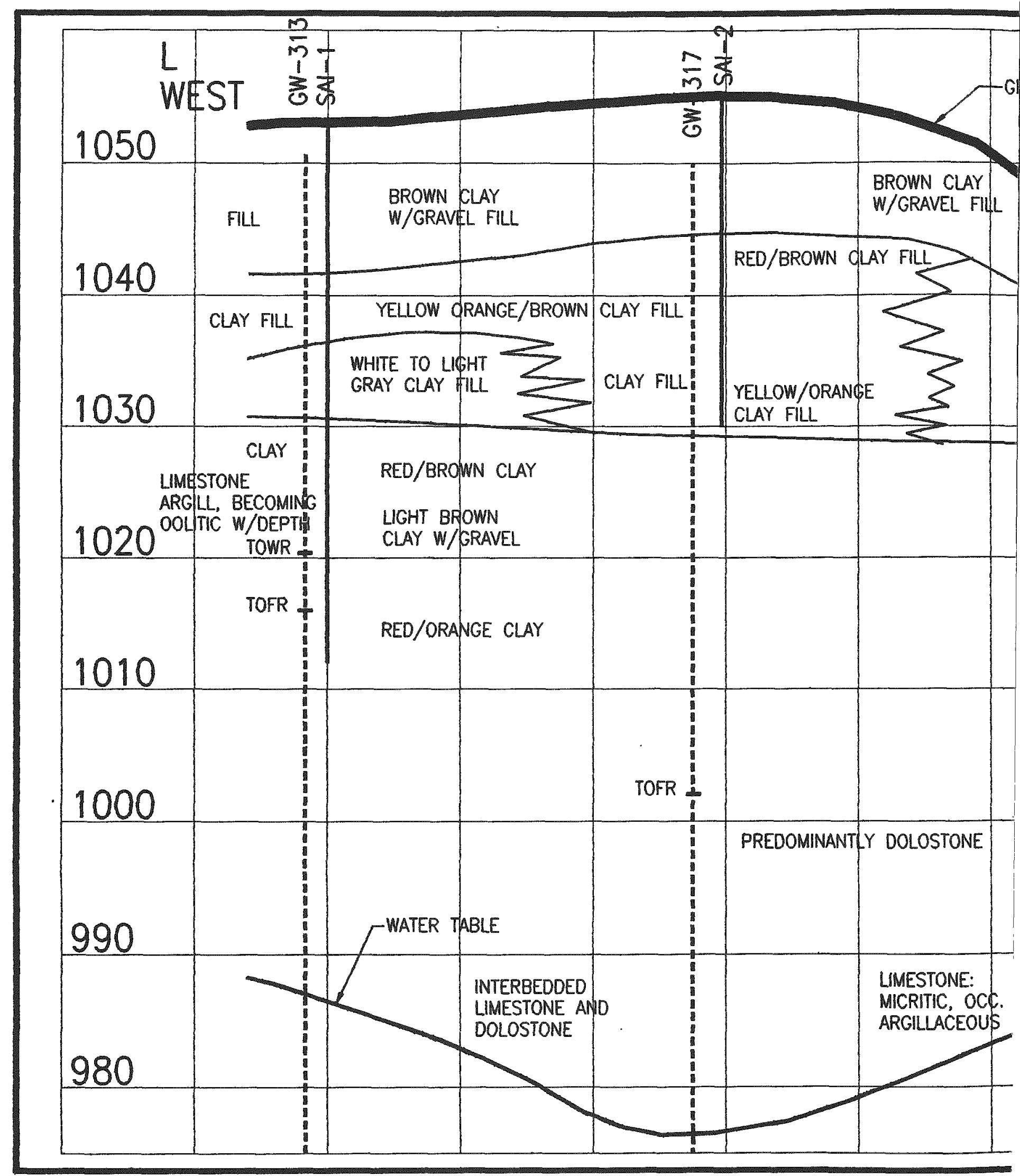




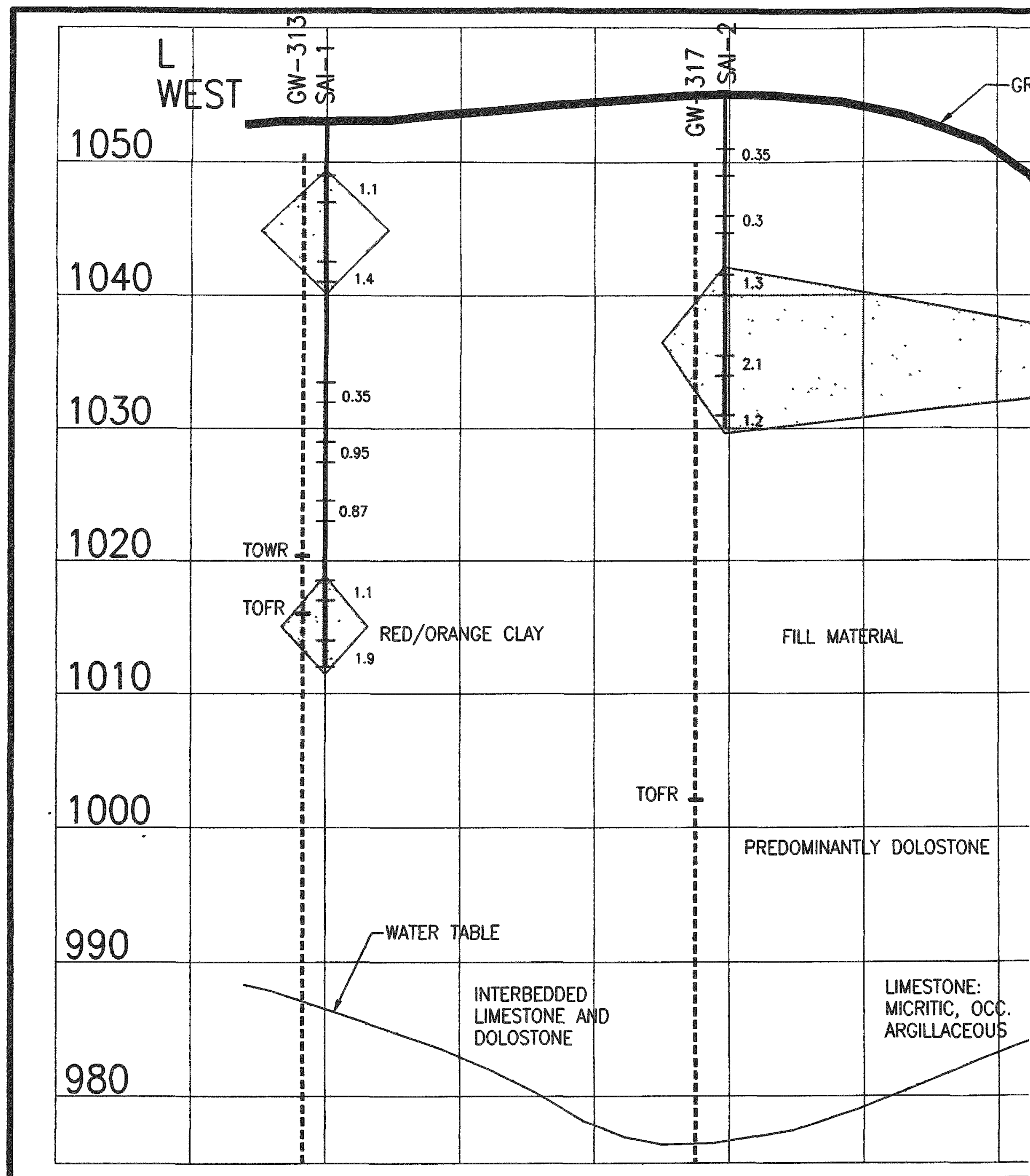




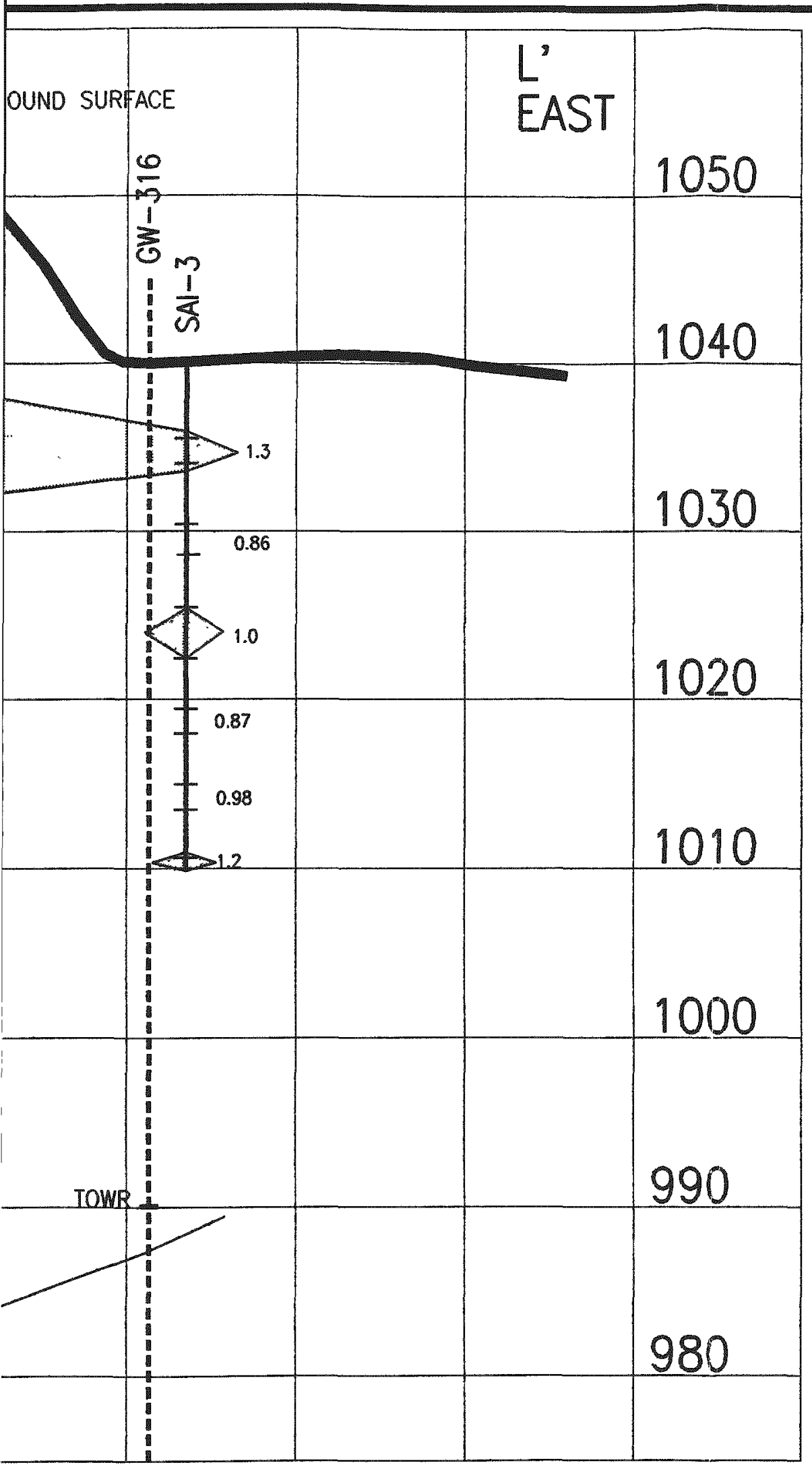

LEGEND:

GW....................MONITORING WELL SB ....................... SOILBORING

i

H......................... PROJECTED WELLS

Q...> 1.007 ppm (95\% UCB BACKGROUND)

$\begin{array}{llllll}0 & 2 & 4 & 6 & 8 & 10\end{array}$ 20

VERTICAL SCALE: $1^{\prime \prime}=10^{\circ}$

0

$30 \quad 60$

HORIZONTAL SCALE: $1^{\prime \prime}=60^{\prime}$

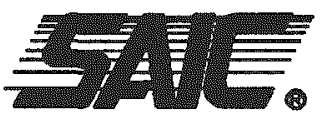

Science Applications International Corporation

BEAR CREEK VALLEY OU2

SPOIL AREA 1, PROFILE L-L'

BERYLLIUM CONCENTRATIONS
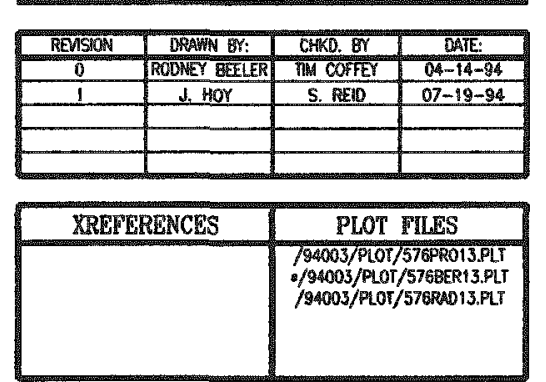

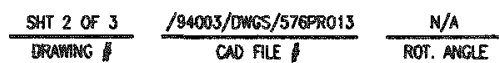




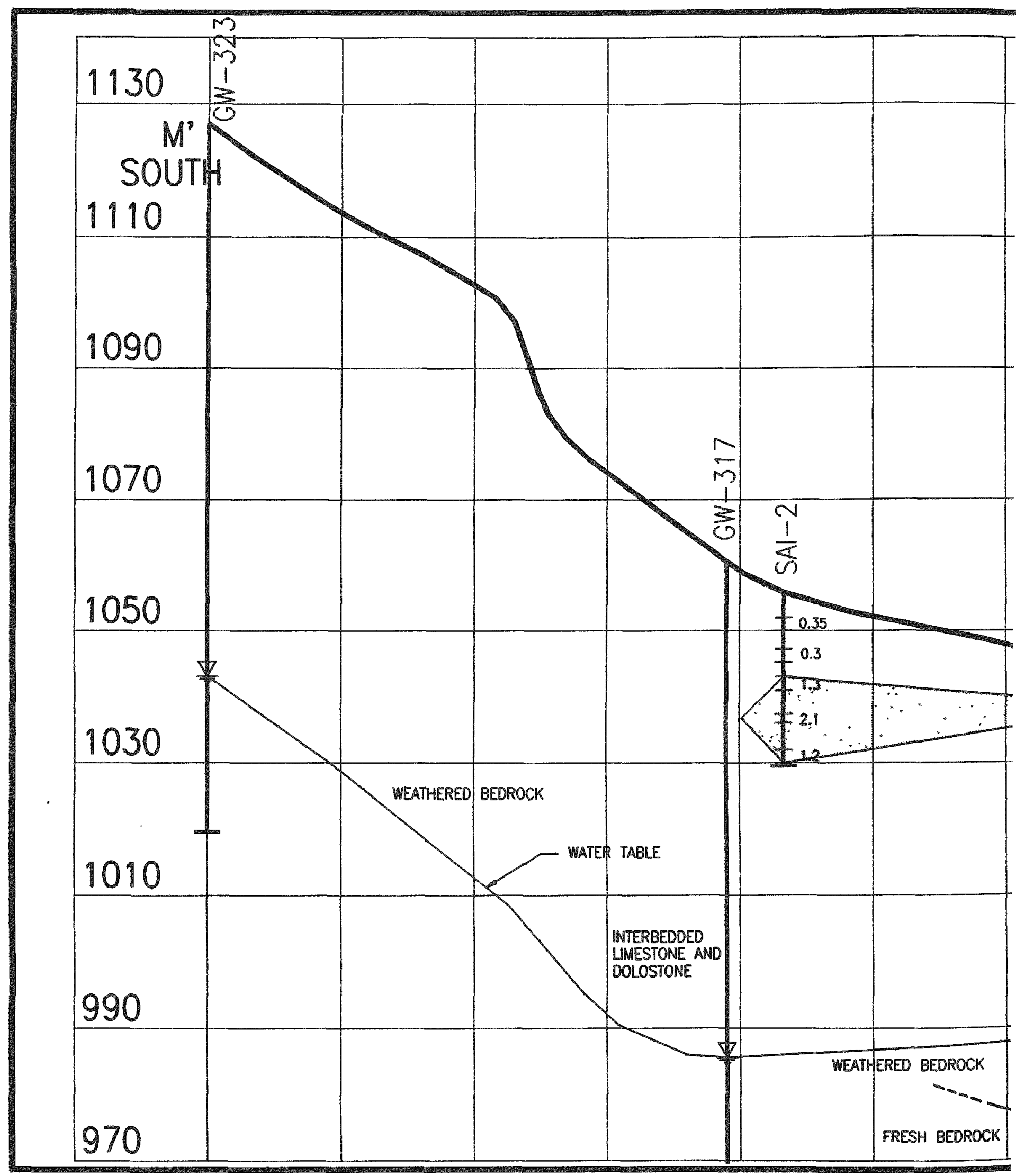




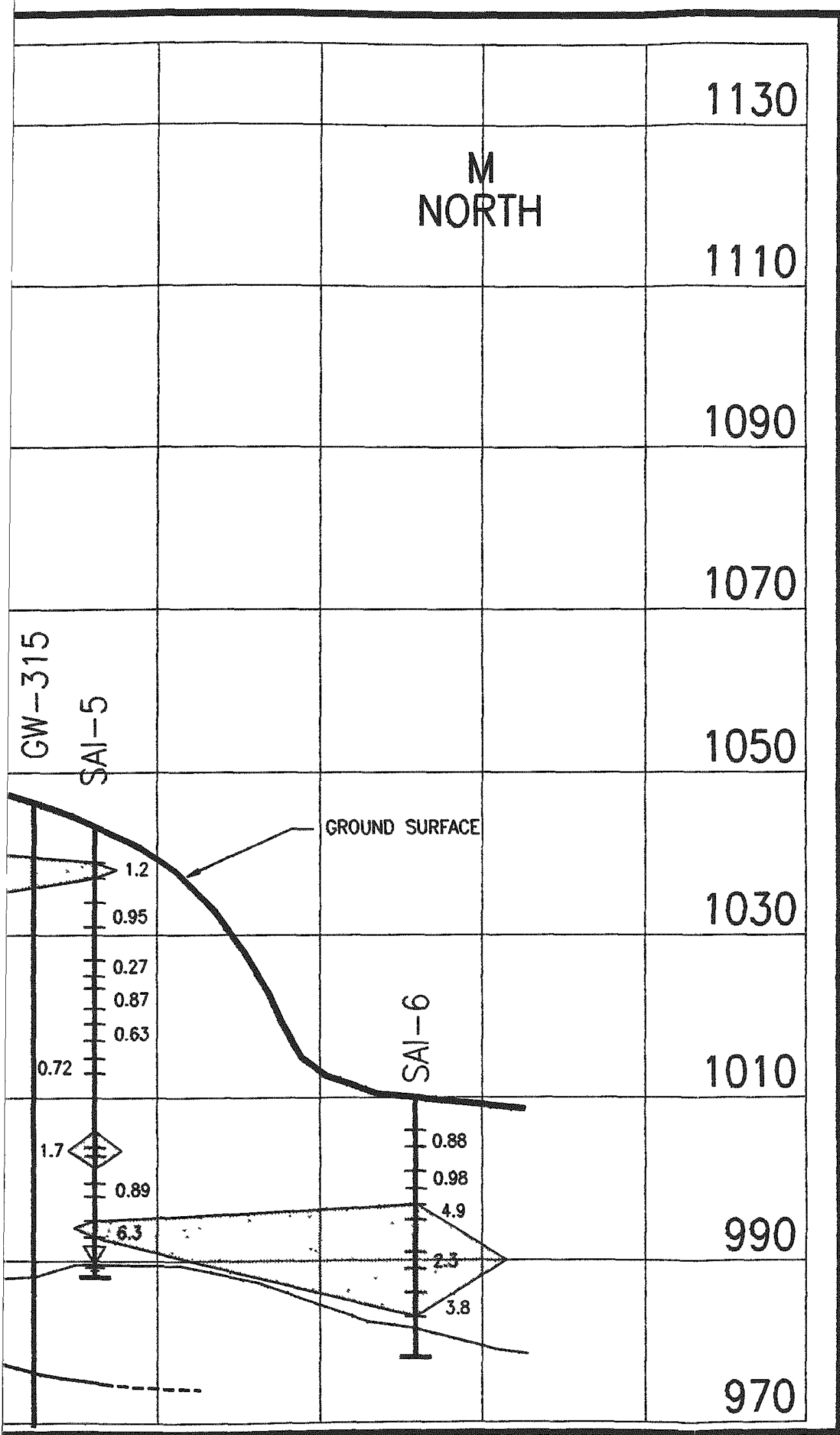

LEGEND:

GW...................MONITORING WELL

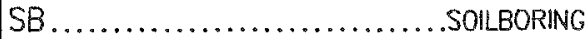

ZGROUNDWATER ENCOUNTERED IN BOREHOLE $\underline{1}$.

GROUNDWATER TABLE

P...> 1.007 PPM (95\% UCB BACKGROUND)

\begin{tabular}{lll}
$0 \quad 5 \quad 10 \quad 20$ \\
\hline
\end{tabular}

VERTICAL SCALE: $1^{\prime \prime}=20^{\circ}$

$\begin{array}{llll}0 & 40 & 80 & 160\end{array}$

HORIZONTAL SCALE: $1^{\prime \prime}=80^{\circ}$

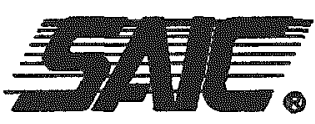

Science Applications International Corporation

BEAR CREEK VALLEY OU2 SPOIL AREA 1 PROFILE M-M' BERYLLIUM CONCENTRATIONS
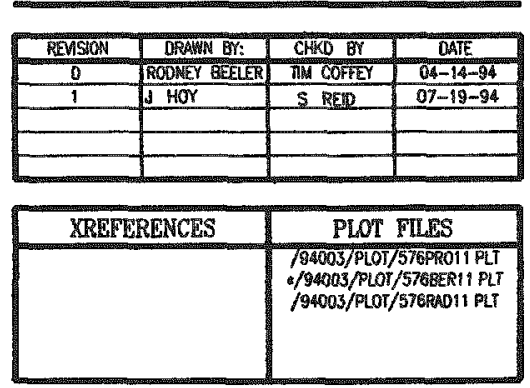

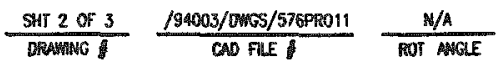

40 60 
$$
\text { . }
$$ 


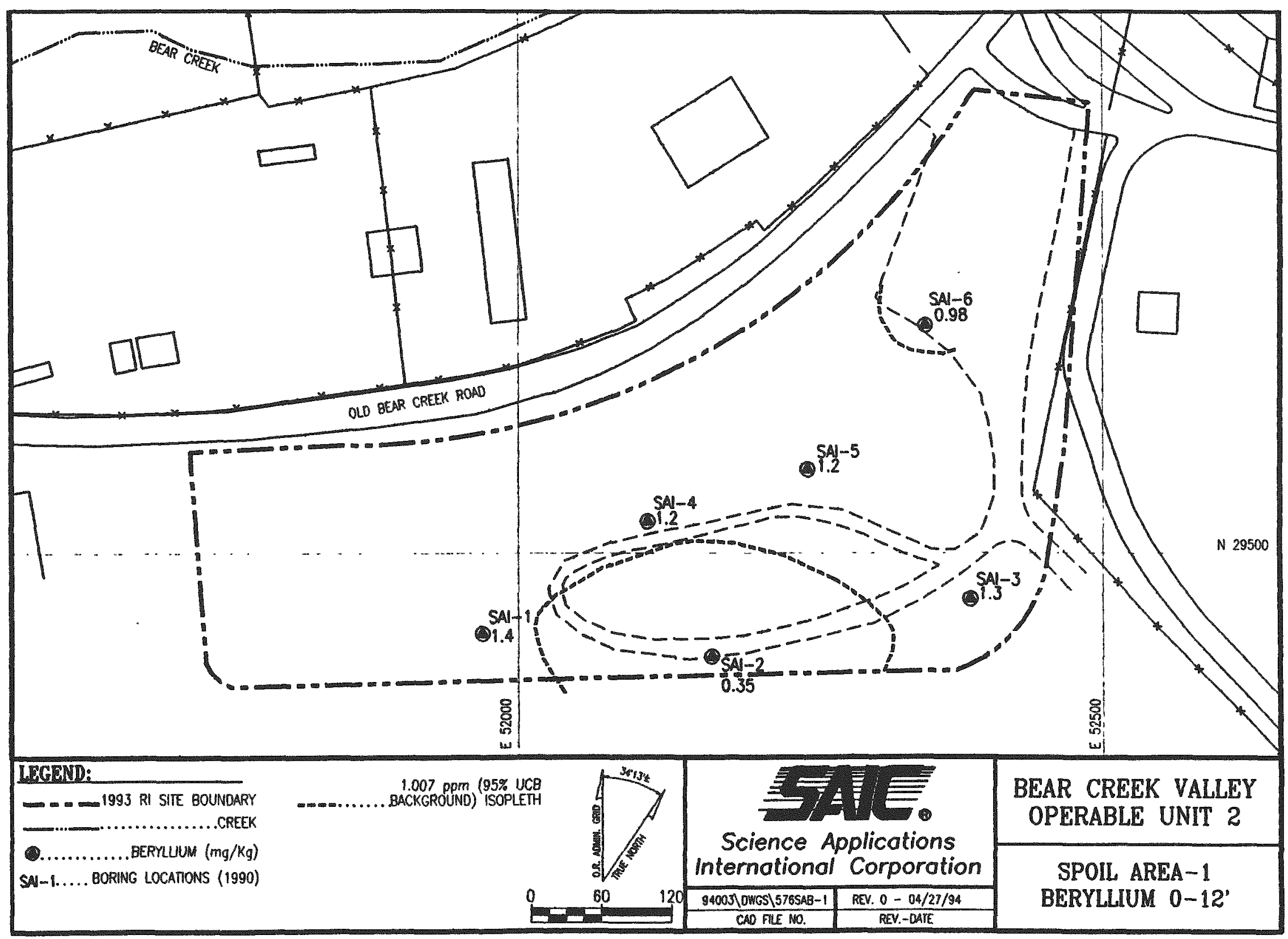




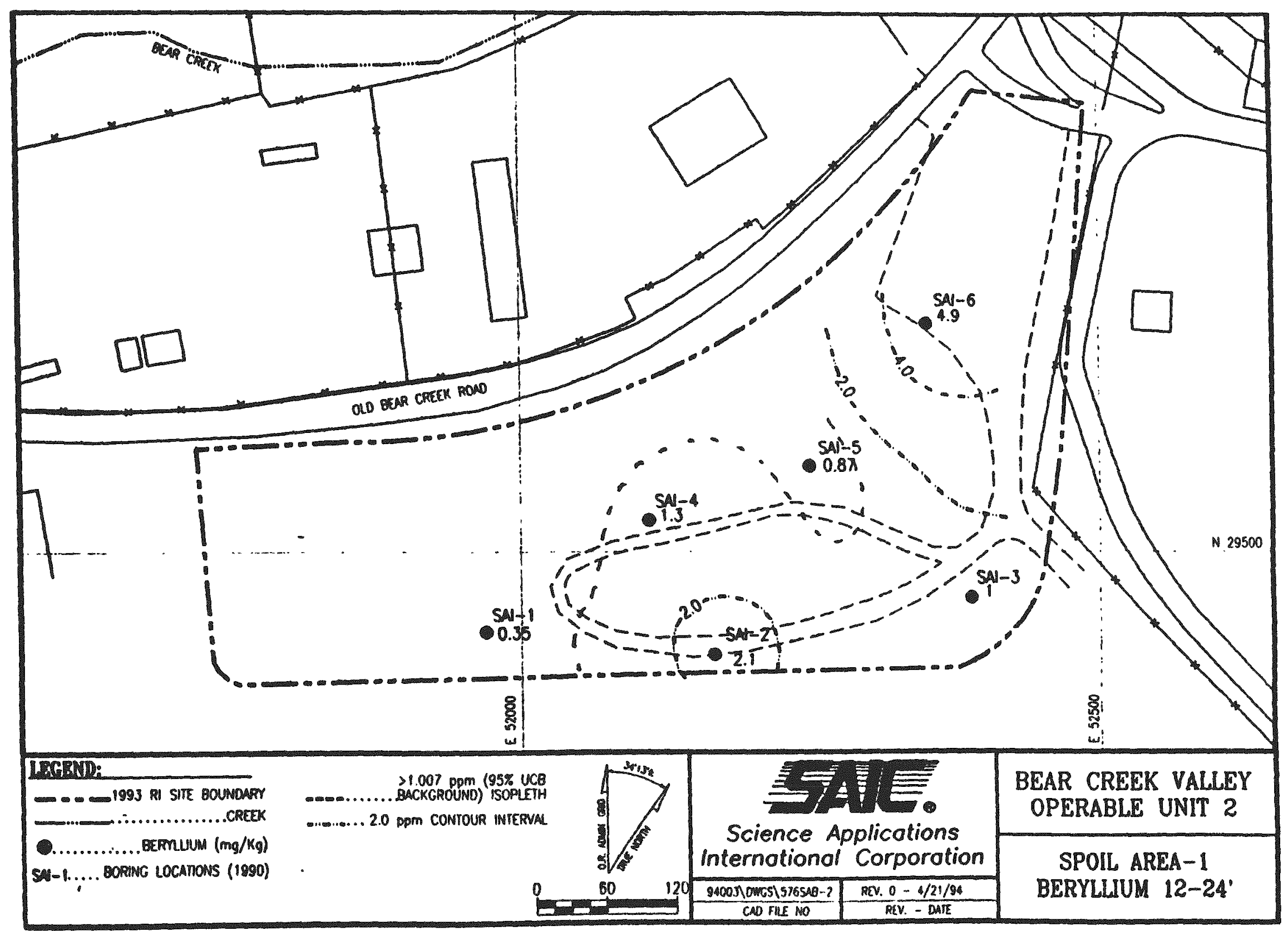




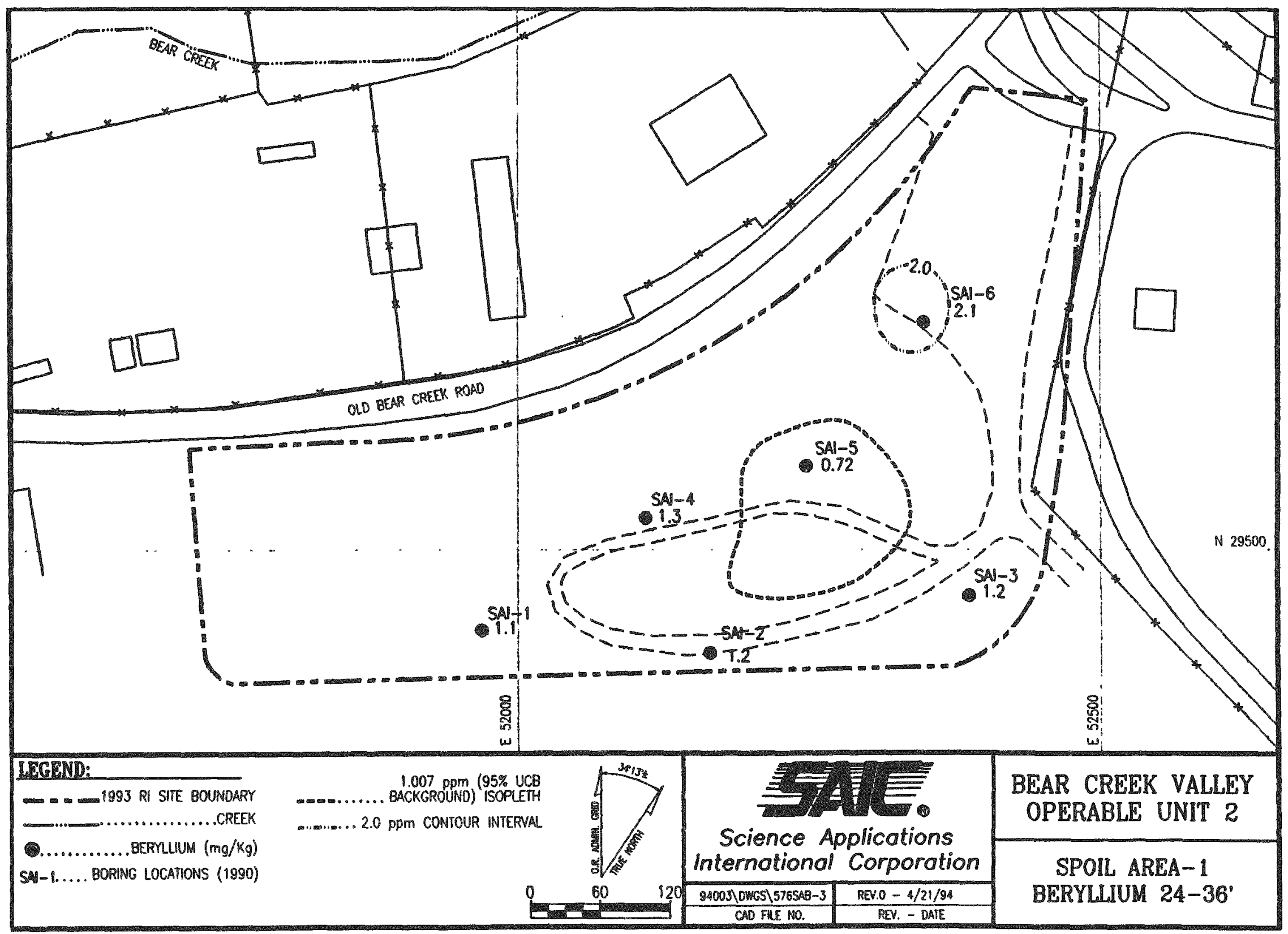




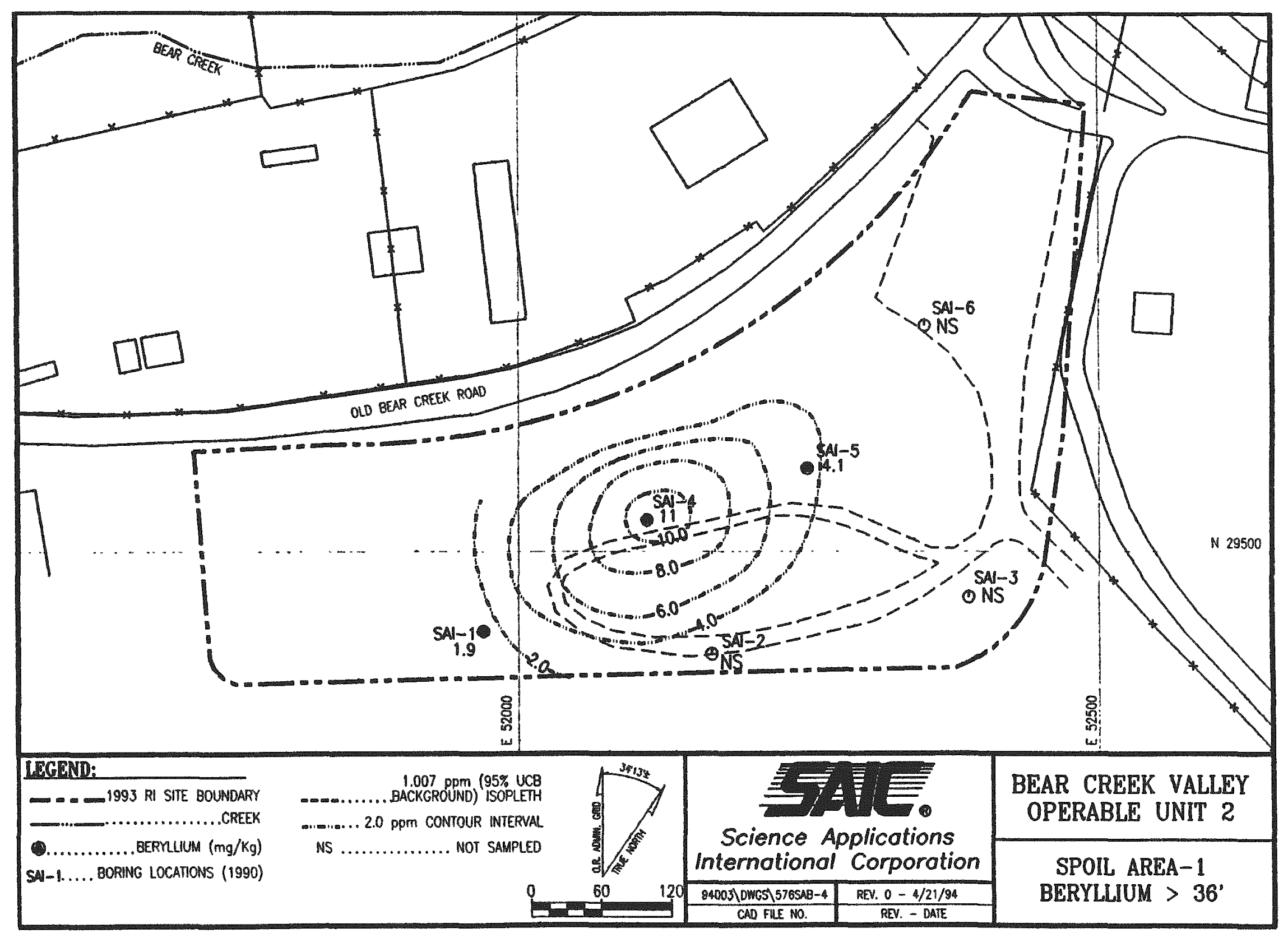




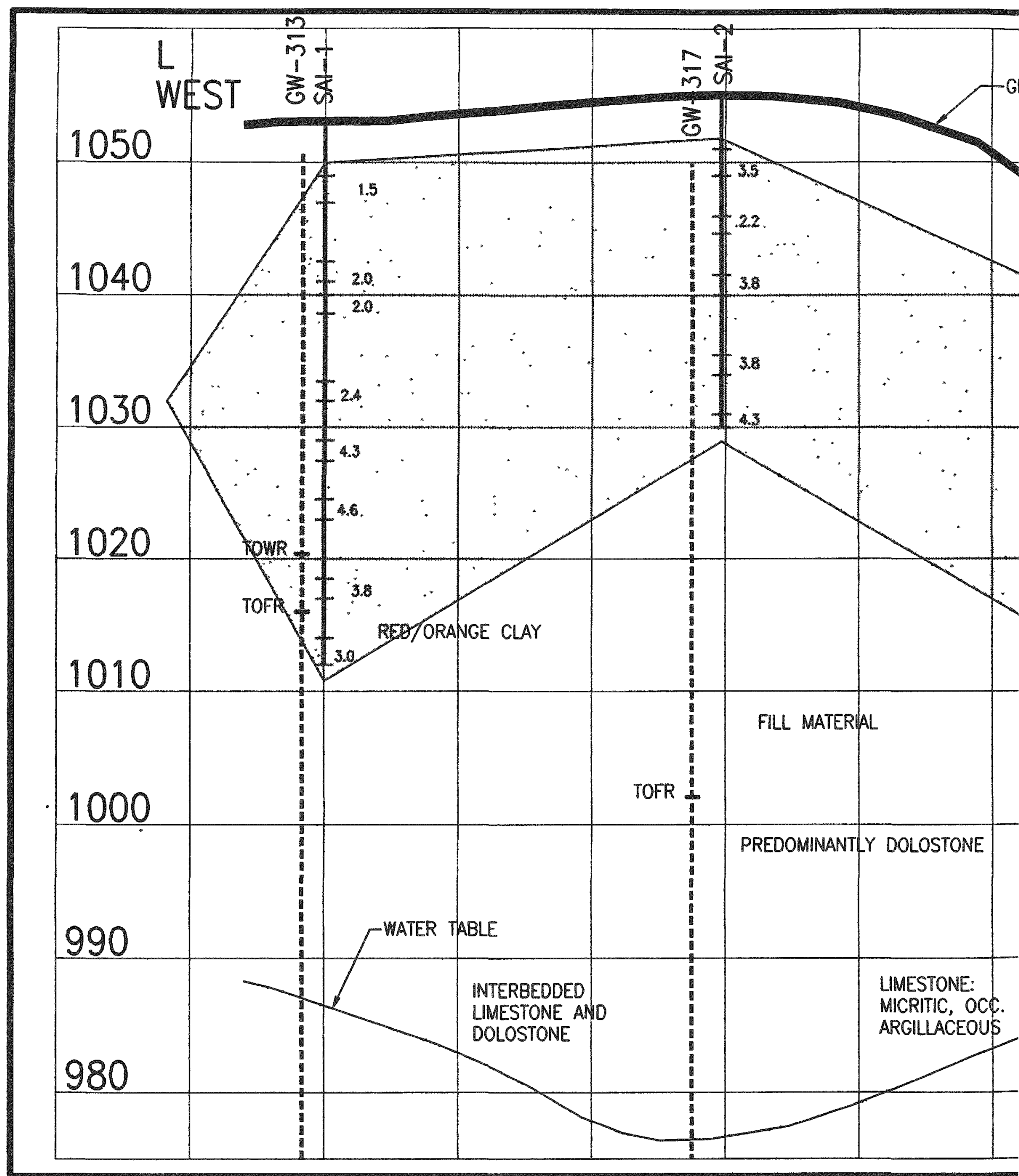




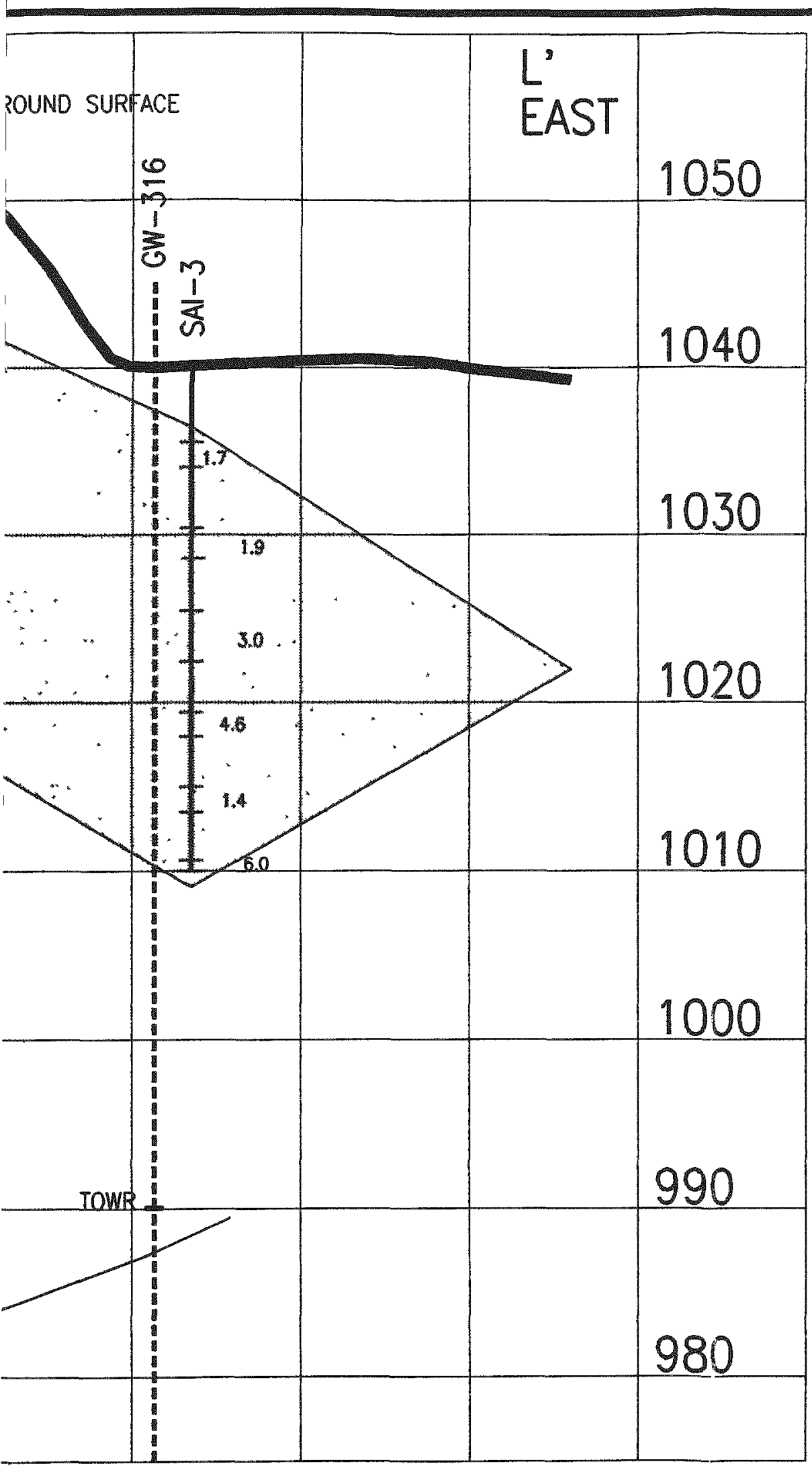

LEGEND:

GW.

MONITORING WELL

SB $\ldots \ldots \ldots \ldots \ldots \ldots \ldots \ldots \ldots$ SOLLBORING

I

PROJECTED WEI

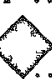

$\begin{array}{llllll}0 & 2 & 4 & 6 & 8 & 10\end{array}$ 20 VERTICAL SCALE: $1^{\prime \prime}=10^{\prime}$

0 30 60

HORIZONTAL SCALE $\cdot 1^{\prime \prime}=60^{\circ}$

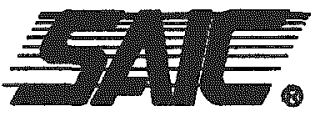

Science Applications International Corporation

BEAR CREEK VALLEY OU2 SPOIL AREA 1, PROFILE L-L' RADIUM CONCENTRATIONS
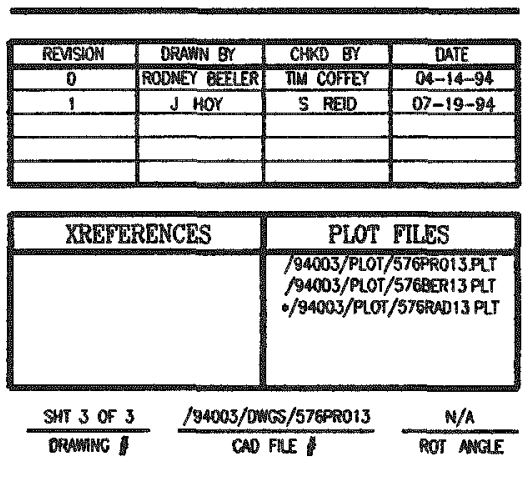


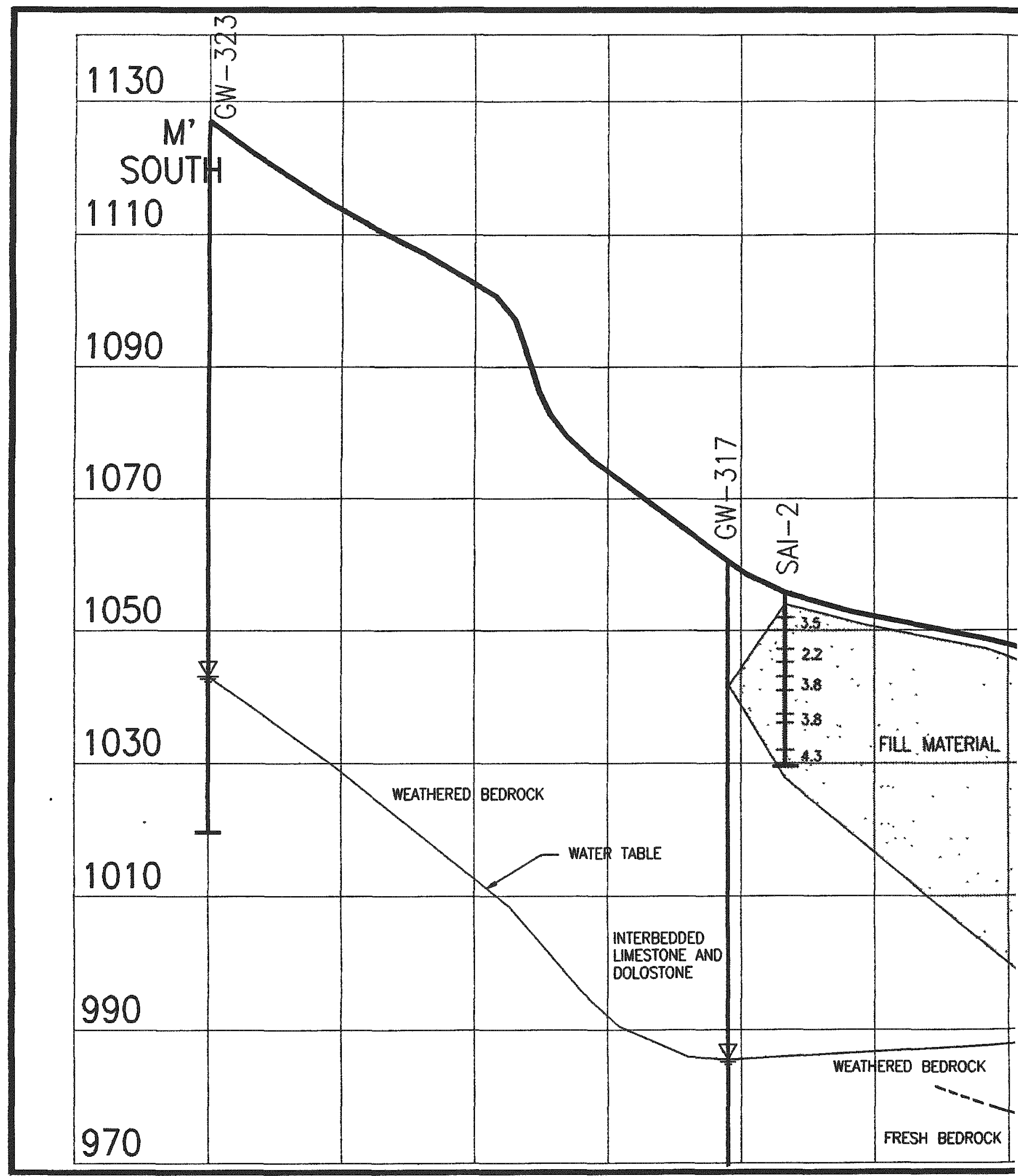




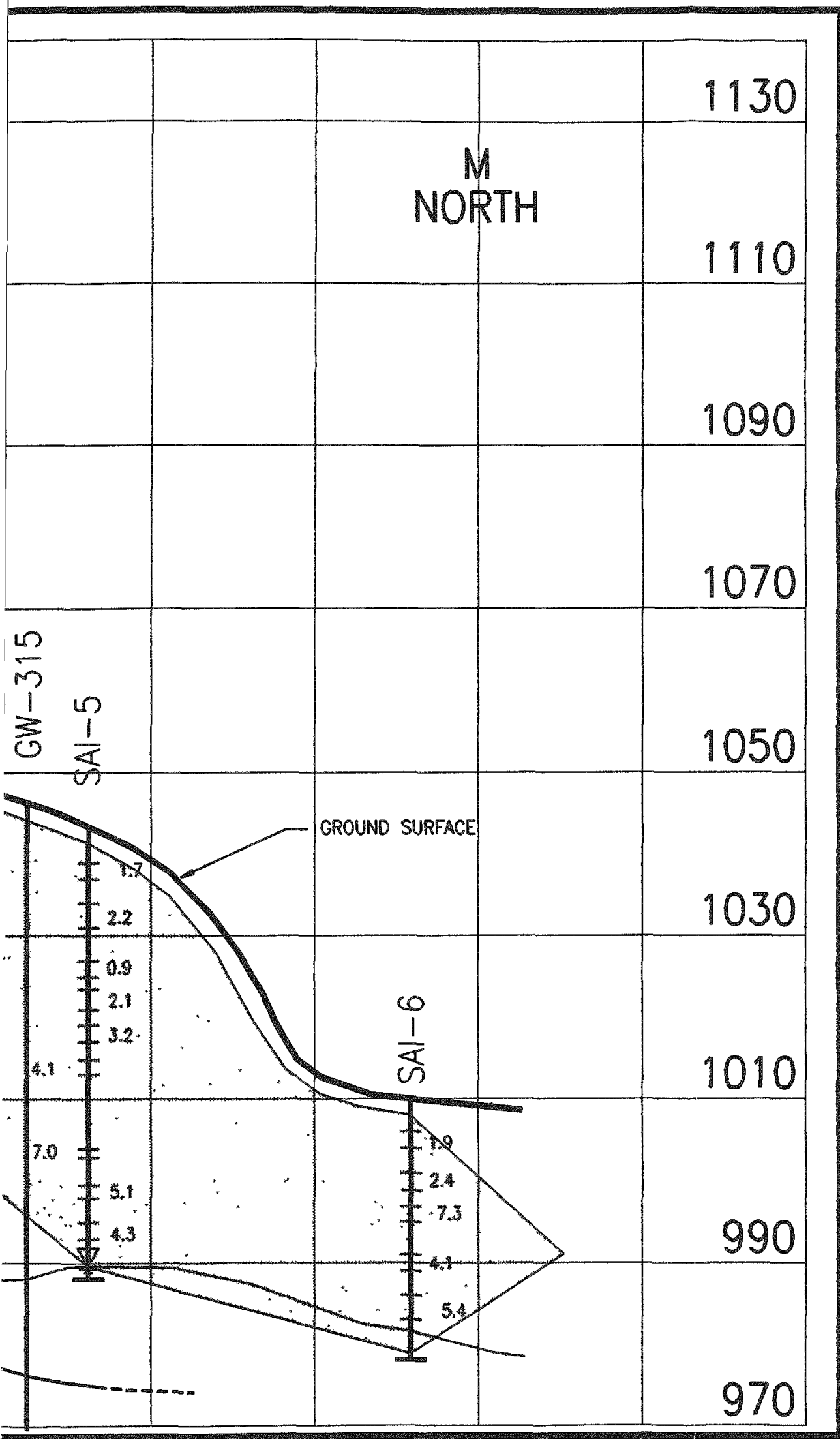

LEGEND:

GW .................. MONITORING WELL

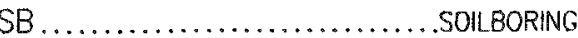

Z GROUNDWATER ENCOUNTERED IN BOREHOLE

T..................GROUNDWATER TABLE

3

$>69 \times 10^{-3} \mathrm{pCl} / \mathrm{g}$ PRG

$\begin{array}{lllll}0 & 5 & 10 & 20 & 40\end{array}$

VERTICAL SCALE: $1^{\prime \prime}=20^{\circ}$

$\begin{array}{llll}0 & 40 & 80 & 160\end{array}$

HORIZONTAL SCALE: $1^{\prime \prime}=80^{\circ}$

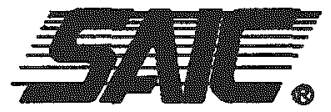

Science Applications International Corporation

BEAR CREEK VALLEY OU2 SPOIL AREA 1 PROFILE M-M' RADIUM CONCENTRATIONS
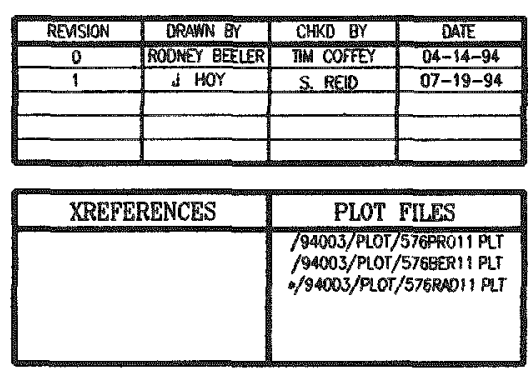

$\frac{\text { SHT } 3 \text { OF } 3}{\text { DRANG I }} \frac{/ 94003 / \text { OWOS } / 576 P R O 11}{\text { CAD FLE }} \frac{\text { N/A }}{\text { ROT ANGEE }}$ 
$$
\text { . }
$$ 


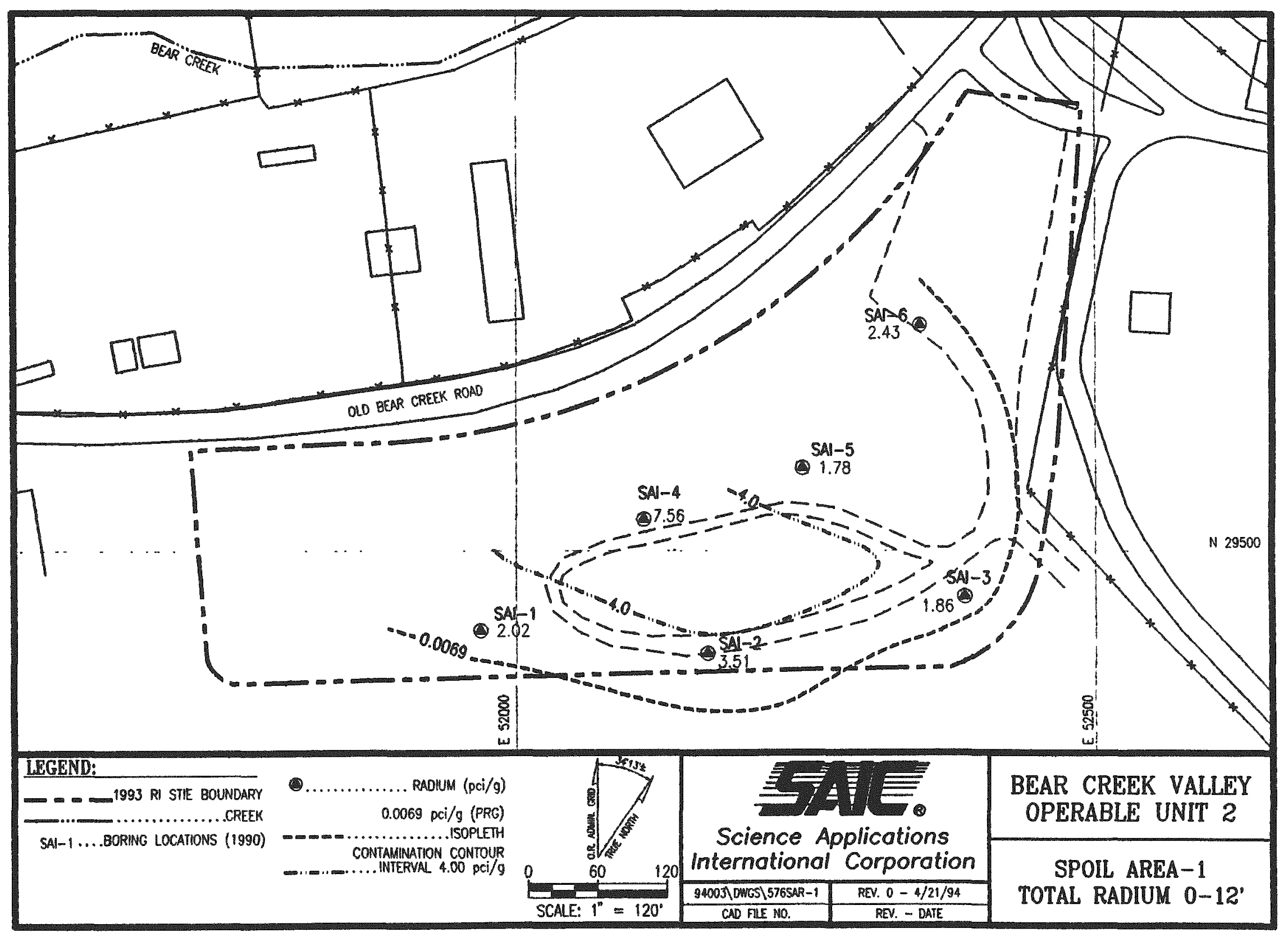




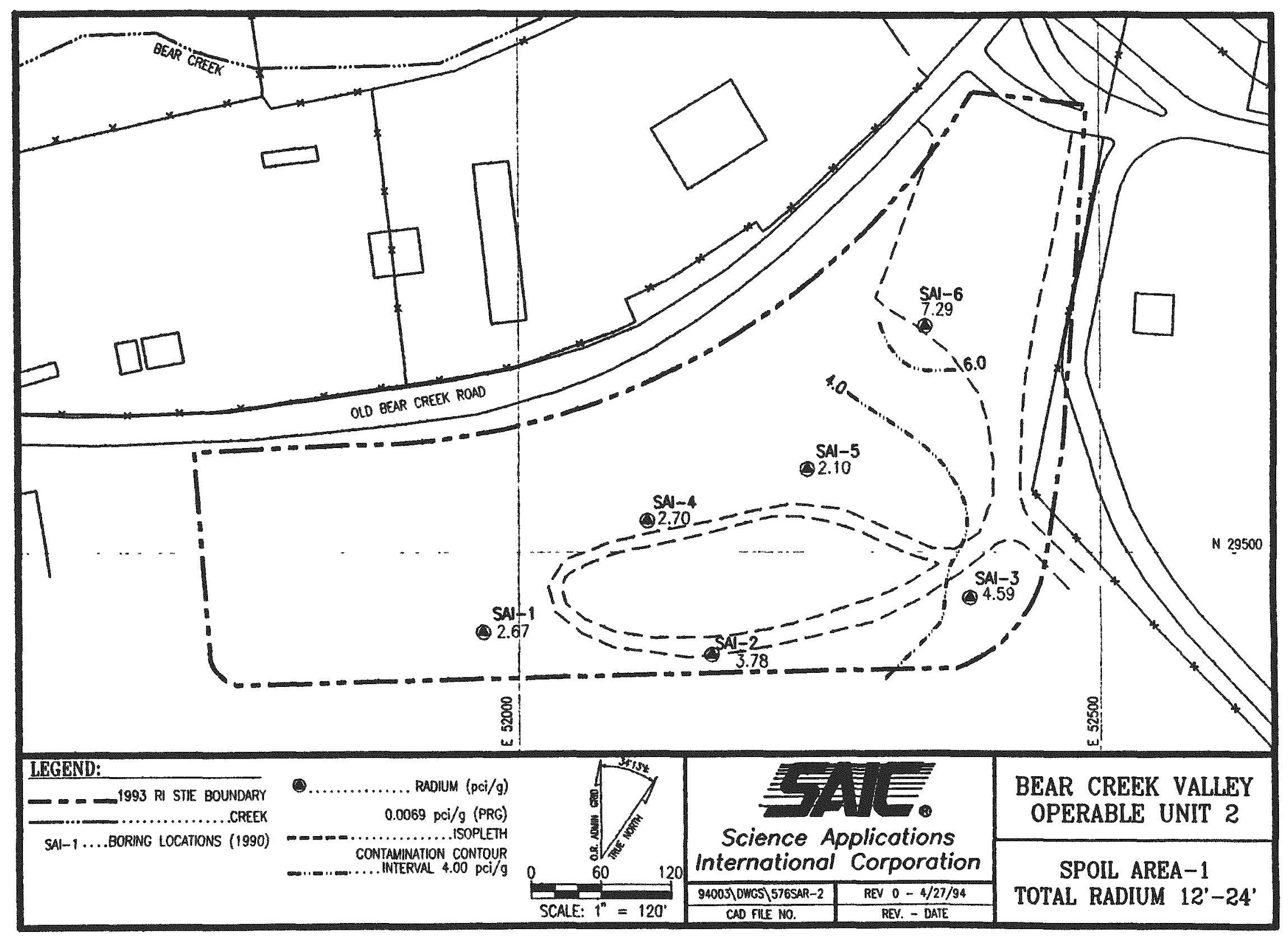




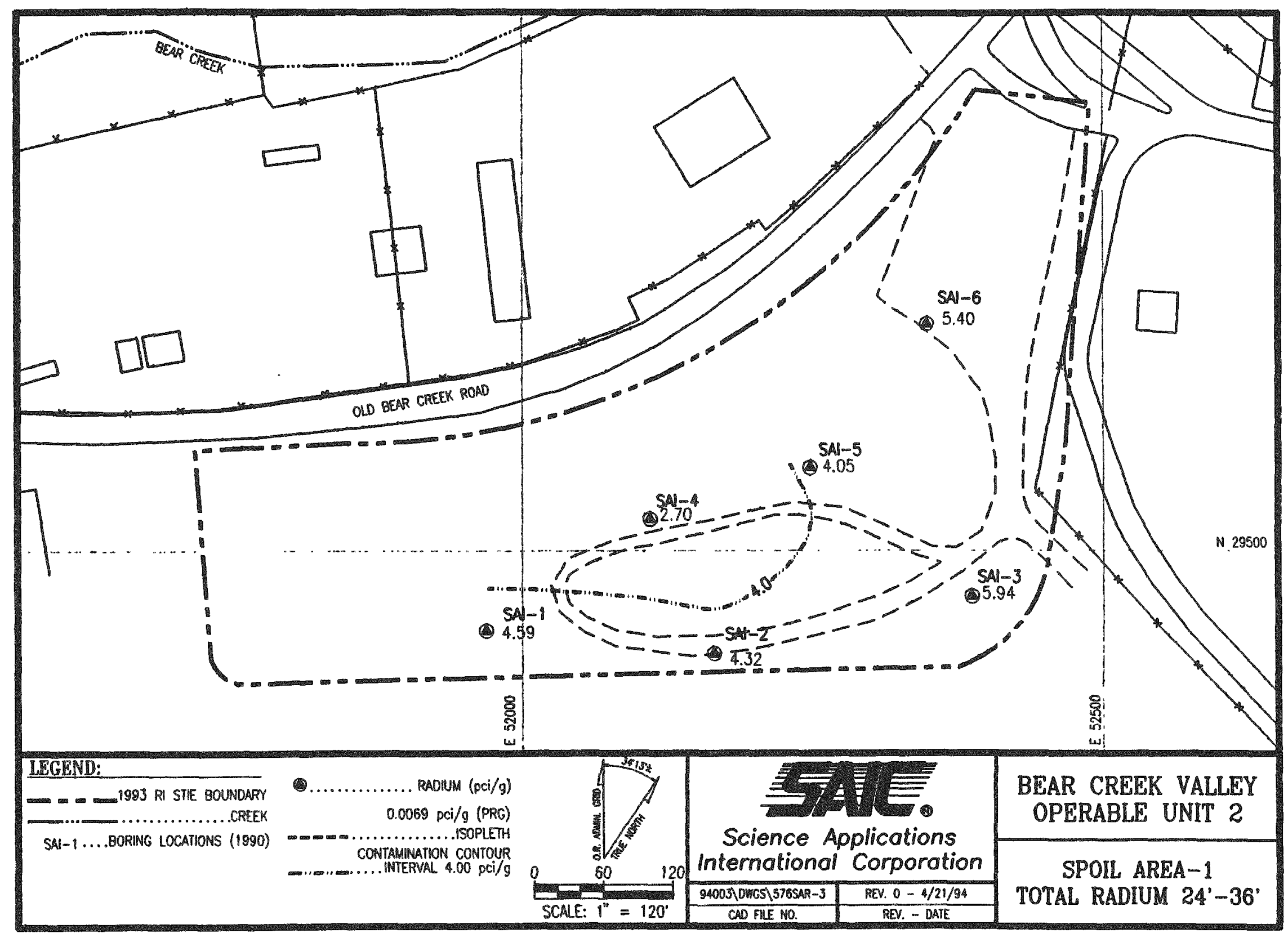




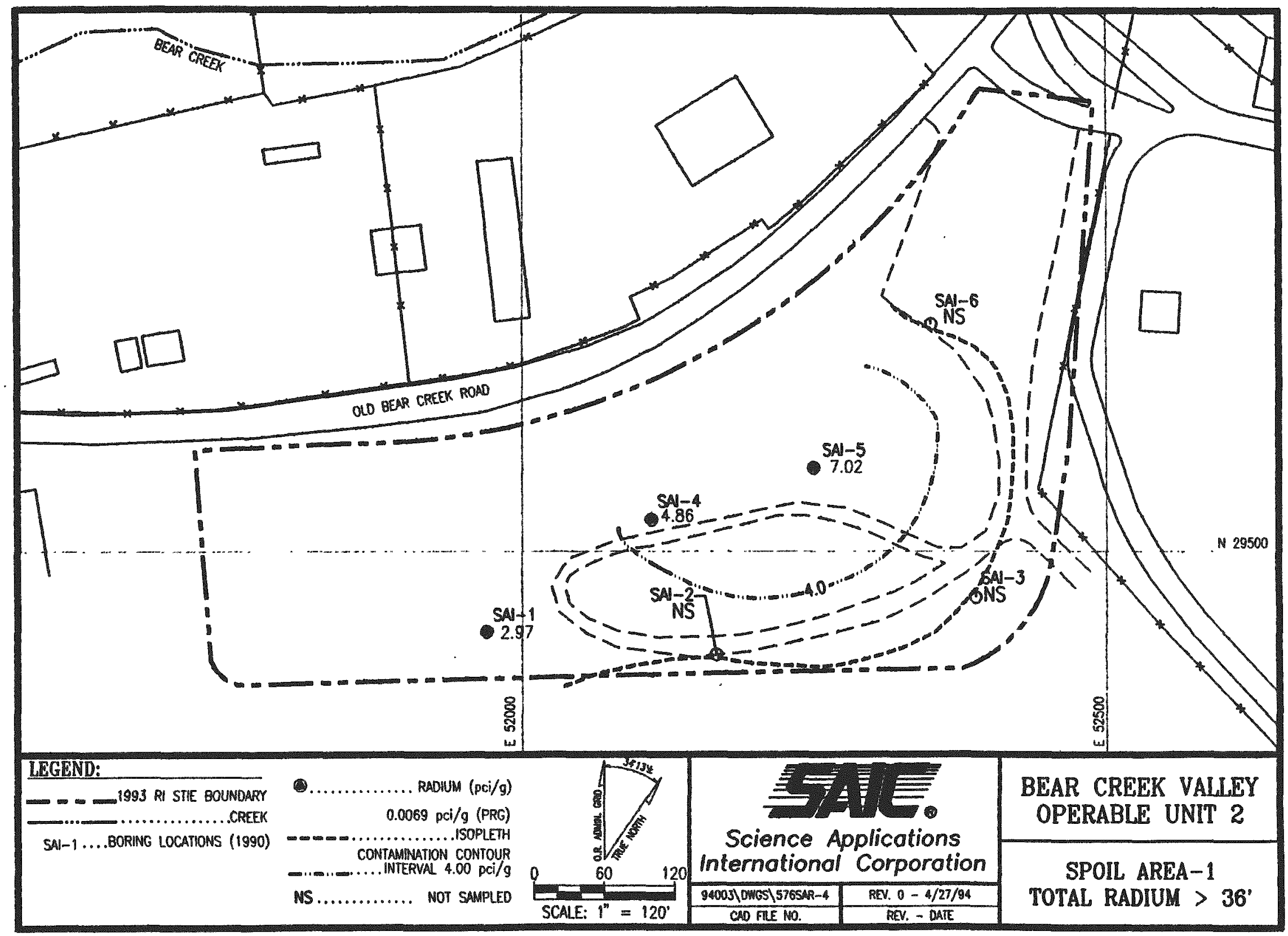


SY-200 Yard Figures

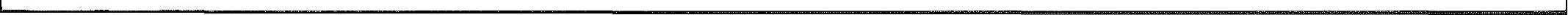




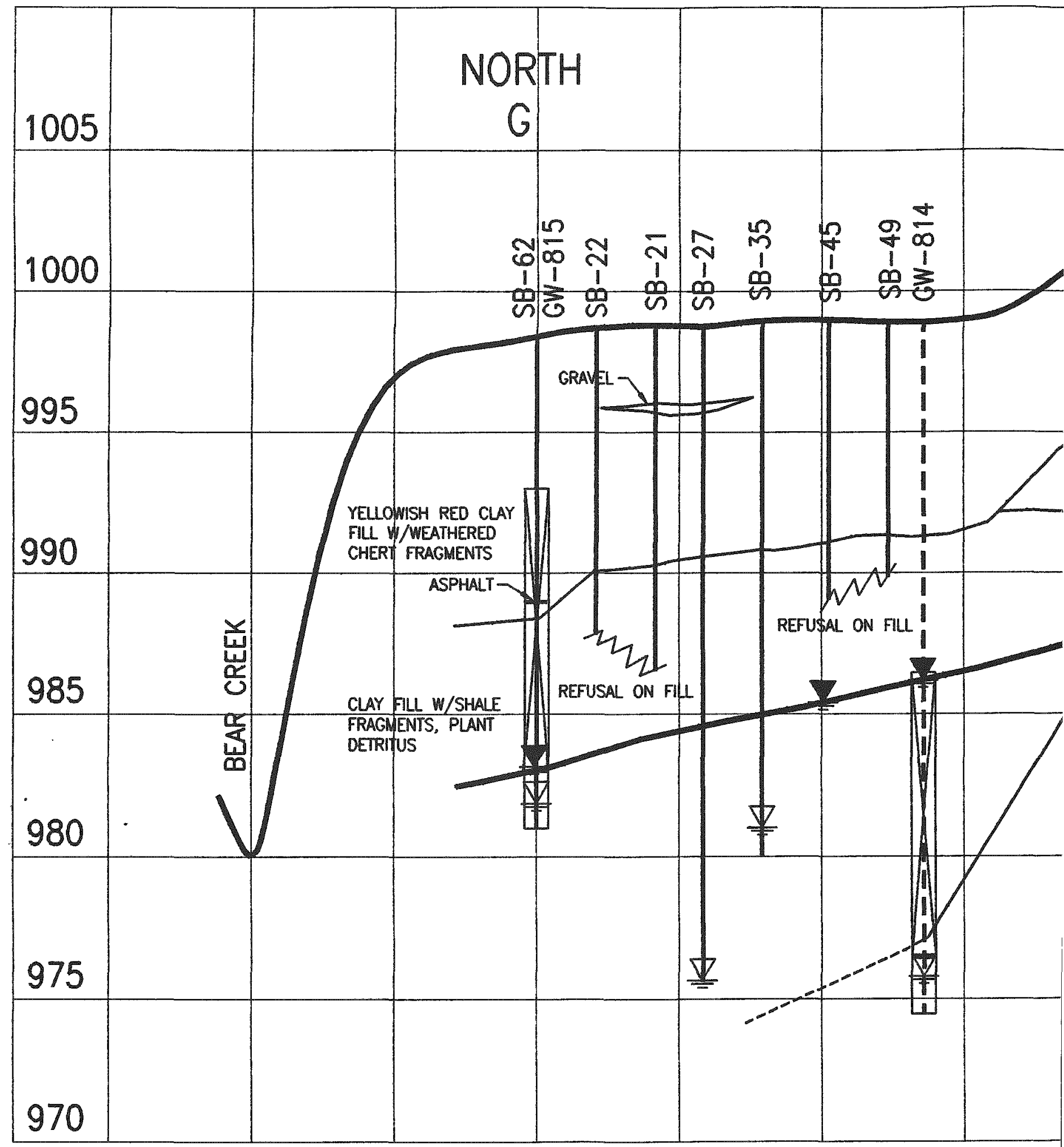




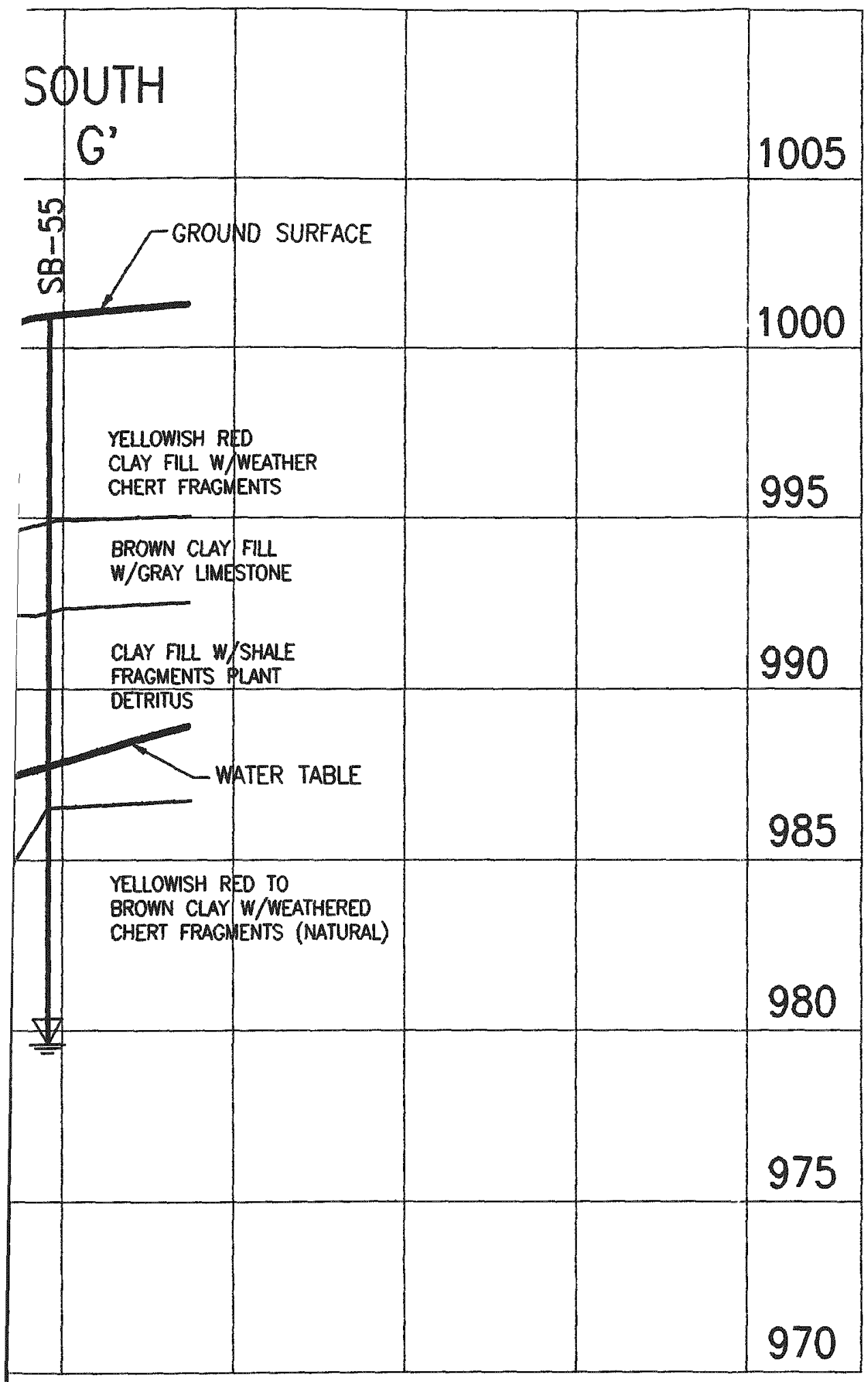

LEGEND:

GW.

..GROUNDWATER WELL

SB ........................ SOLLBORING

${ }_{\text {VGROUNDWATER ENCOUNTERED IN BOREHOLE }}$

I....................GROUNDWATER TABLE

PROJECTED MONITORING WELL SCREENED INTERVAL

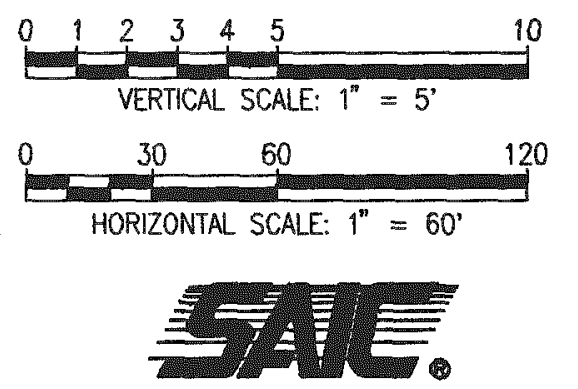

Science Applications International Corporation

BEAR CREEK VALLEY OU2 SY-200 SITE, PROFILE G-G' SOIL LITHOLOGY
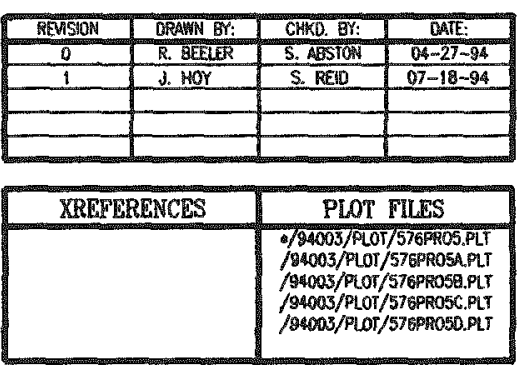

SHI 1 OF 5 /94003/0WOS/576PROS N/A

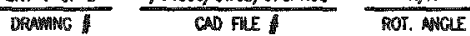




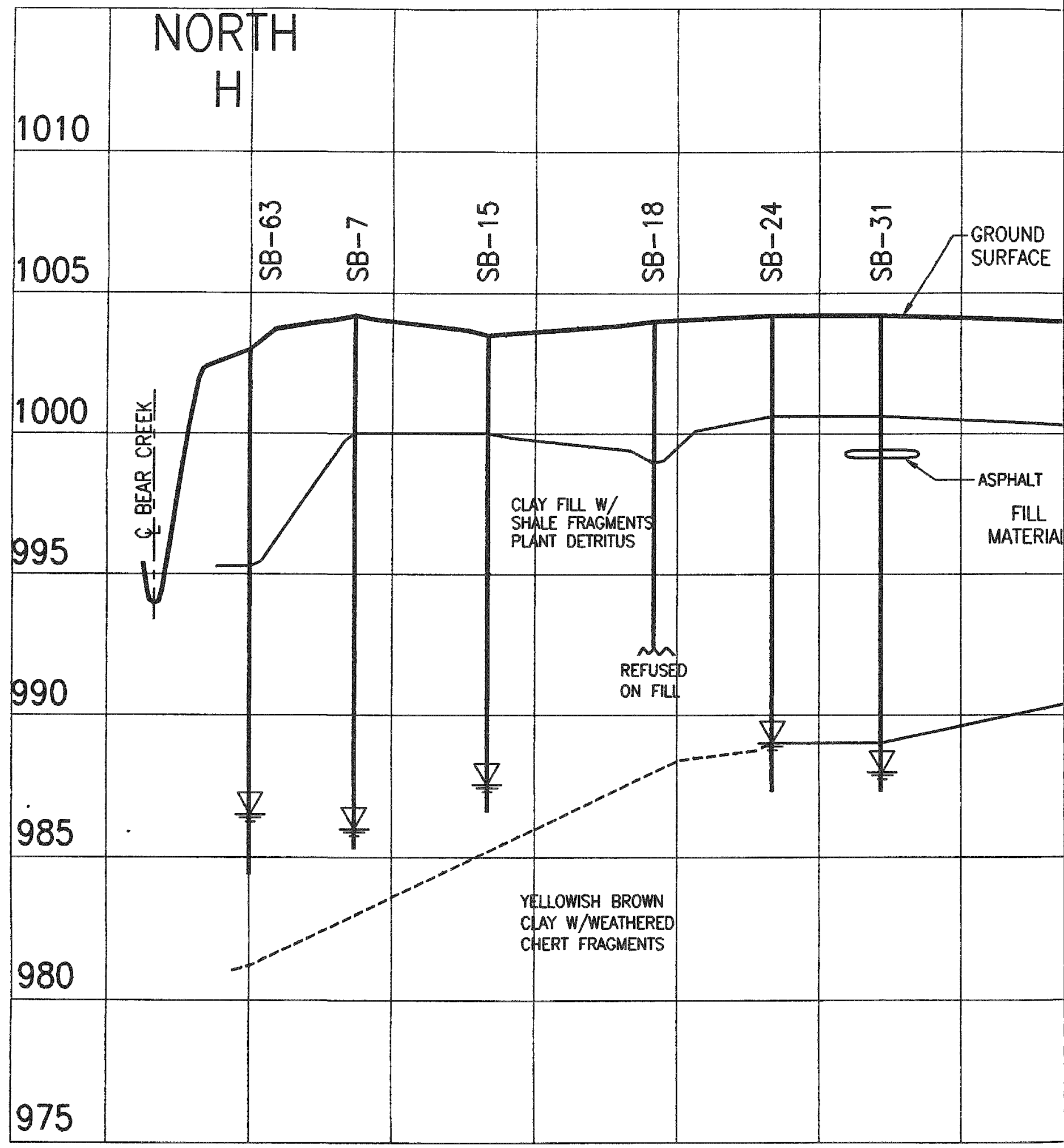




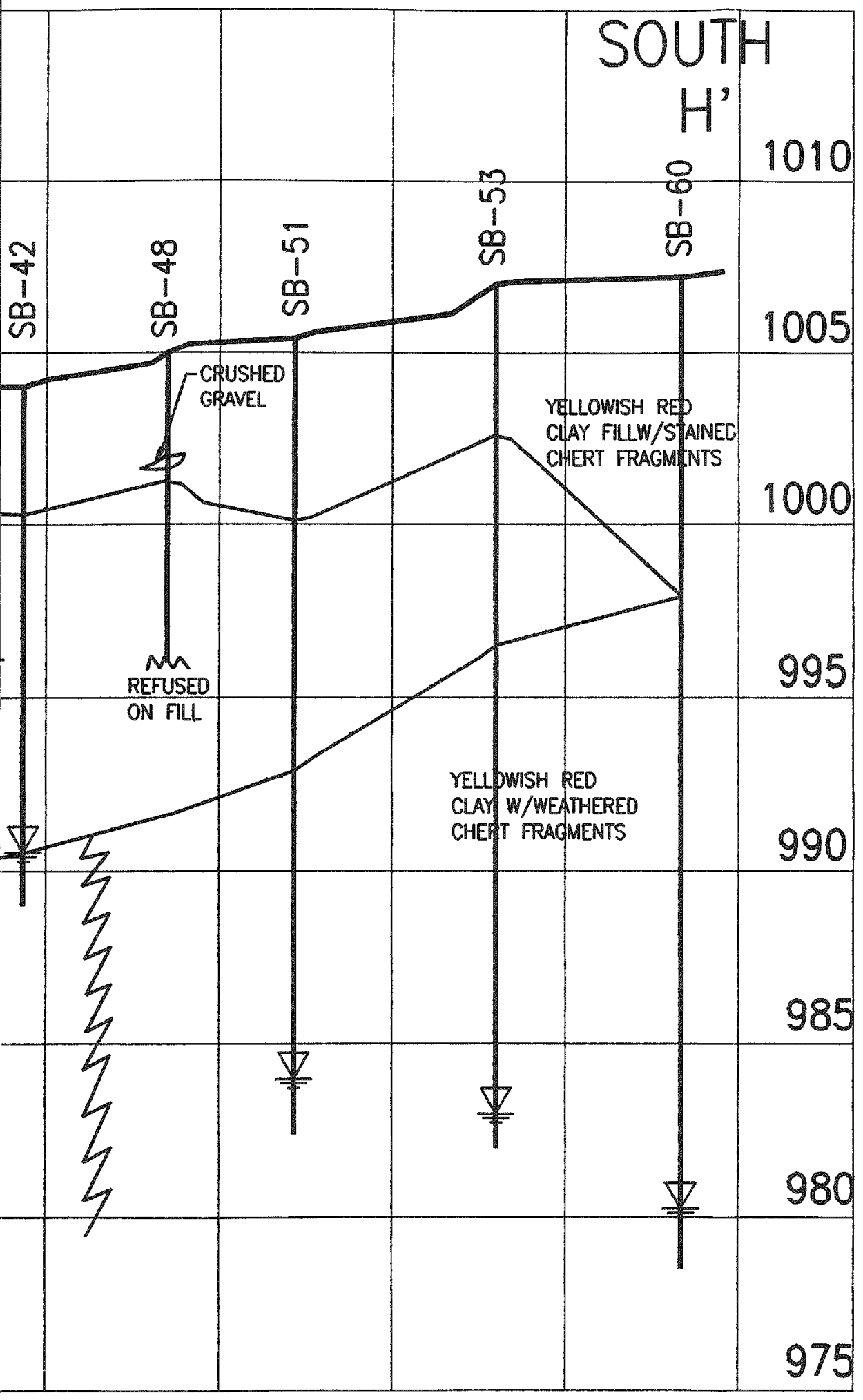

LEGEND:

GW.................... MONITORING WELL SB .......................... SOLBORING

要GROUNDWATER ENCOUNTERED IN BOREHOLE

$\begin{array}{llllll}0 & 1 & 2 & 3 & 4 & 5\end{array}$ 10 VERTICAL SCALE: $1^{\prime \prime}=5^{\prime}$

$\begin{array}{llll}0 & 10 & 20 & 30\end{array}$ 60

HORIZONTAL SCALE: $1^{\prime \prime}=30^{\circ}$

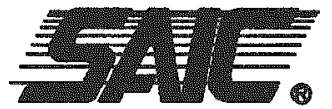

Science Applications International Corporation

BEAR CREEK VALLEY OU2 SY-200 YARD, PROFILE H-H' SOIL LITHOLOGY

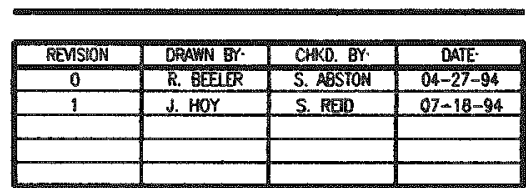

\begin{tabular}{|c|c|}
\hline XREFERENCES & PLOT FLES \\
\hline & 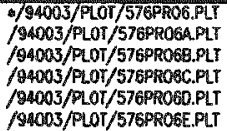 \\
\hline
\end{tabular}




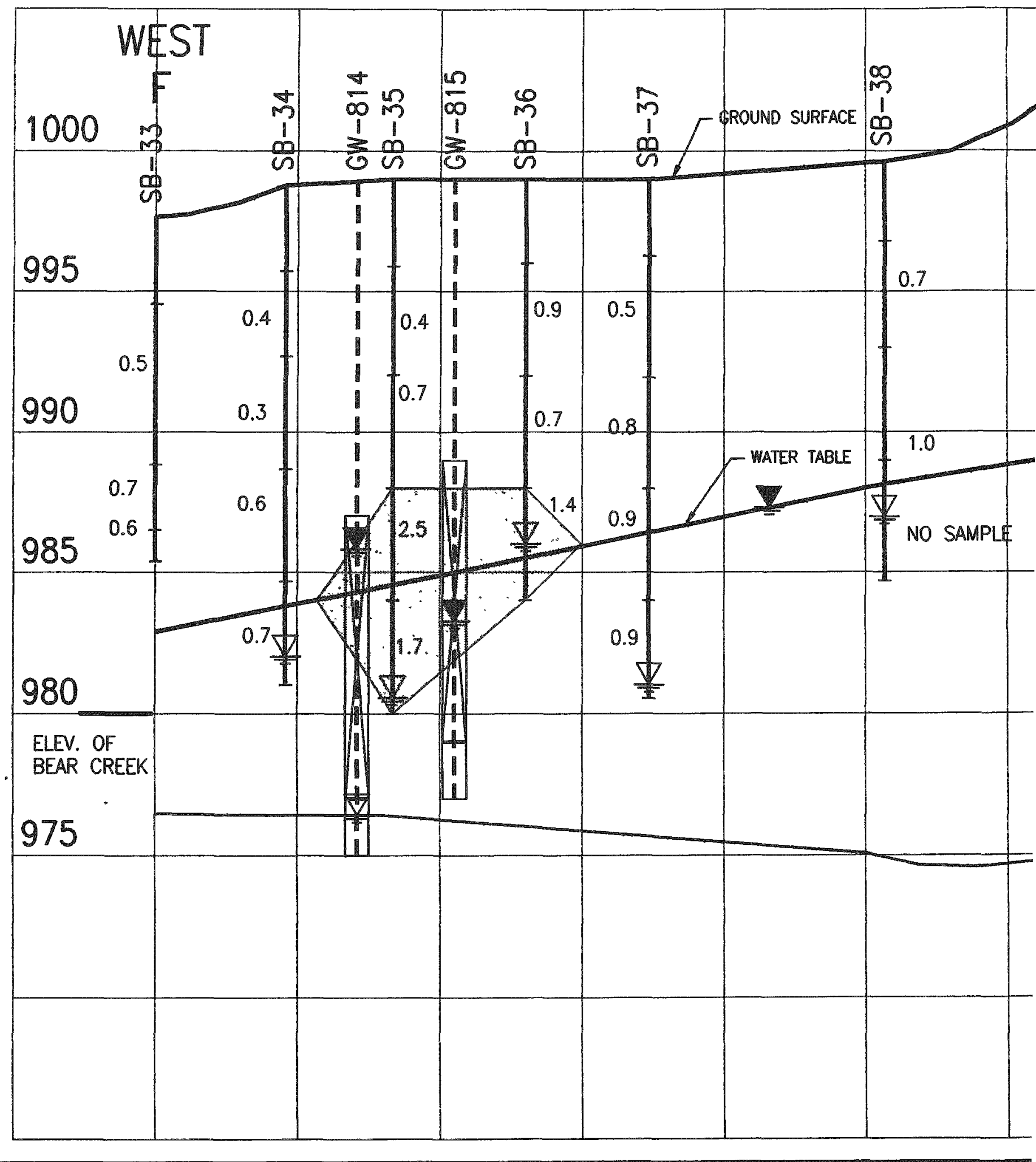




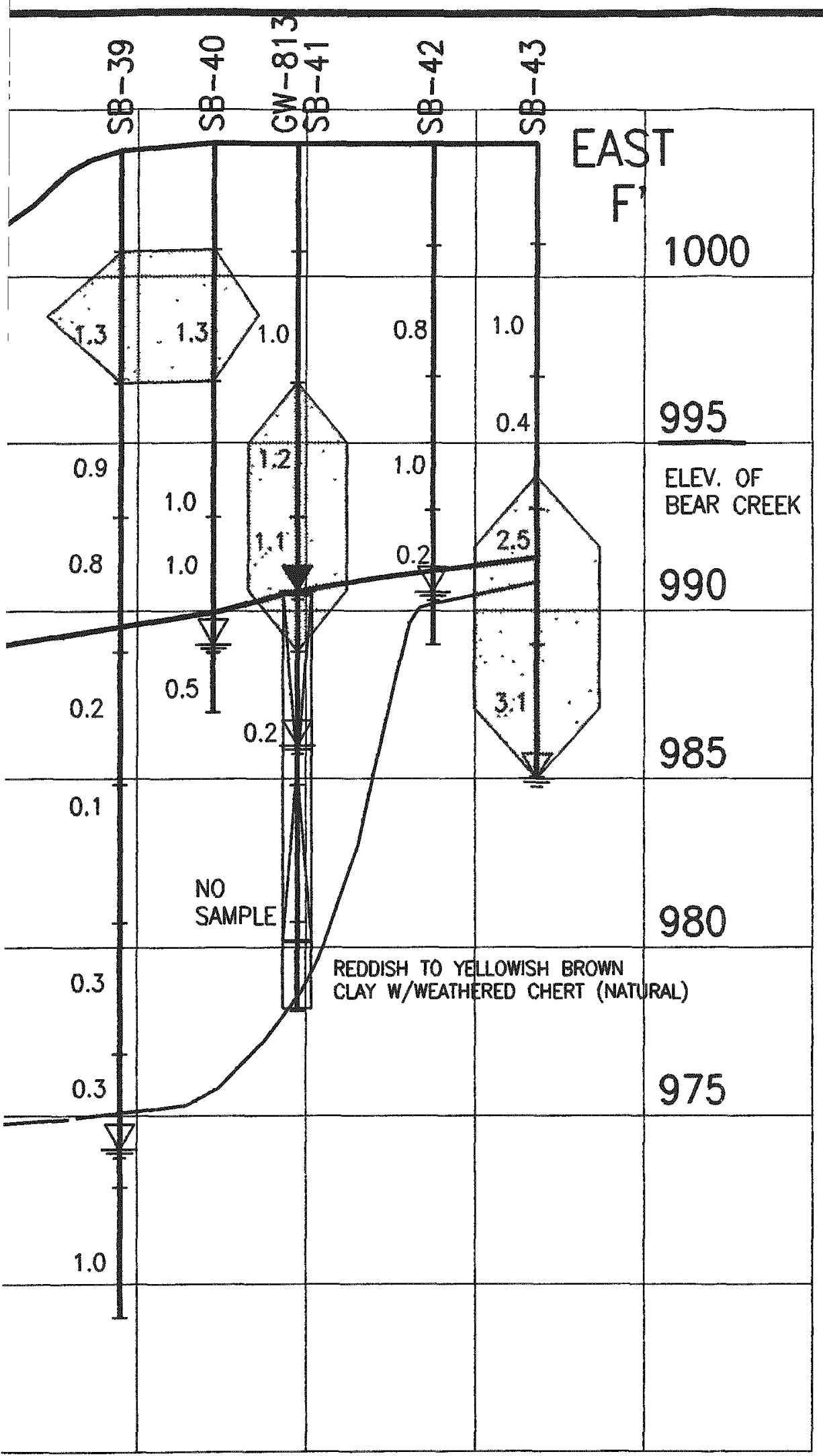

LEGEND:

GW.................... MONITORING WELL

SB..........................SOLBORING DGROUNDWATER ENCOUNTERED IN BOREHOLE W................. GROUNDWATER TABLE

i .PROJECTED WELLS

由.

8

SCREENED INTERVAL

$>1.007$ PPM BERYLLIUM (95\% UCB BACKGROUND)

$\begin{array}{lllll}0 & 1 & 2 & 3 & 4\end{array}$

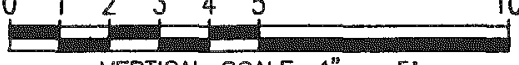

VERTICAL SCALE: $1^{\prime \prime}=5^{\circ}$

$\begin{array}{rrrr}0 & 30 & 60 & 120\end{array}$

HORIZONTAL SCALE: $1^{\prime \prime}=60^{\circ}$

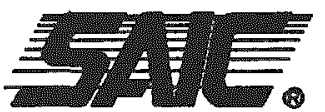

Science Applications International Corporation

BEAR CREEK VALLEY OU2 SY-200 SITE PROFILE F-F' BERYLLUUM CONCENTRATIONS
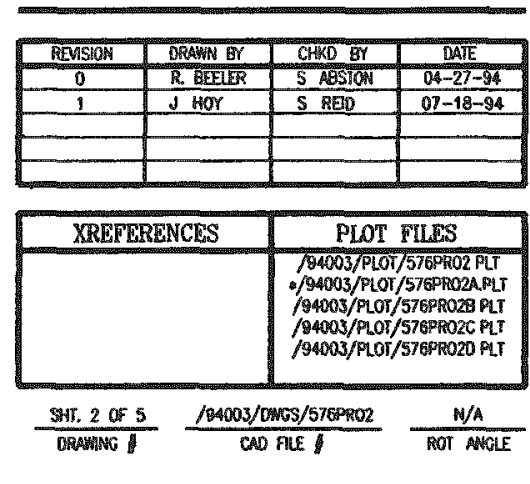


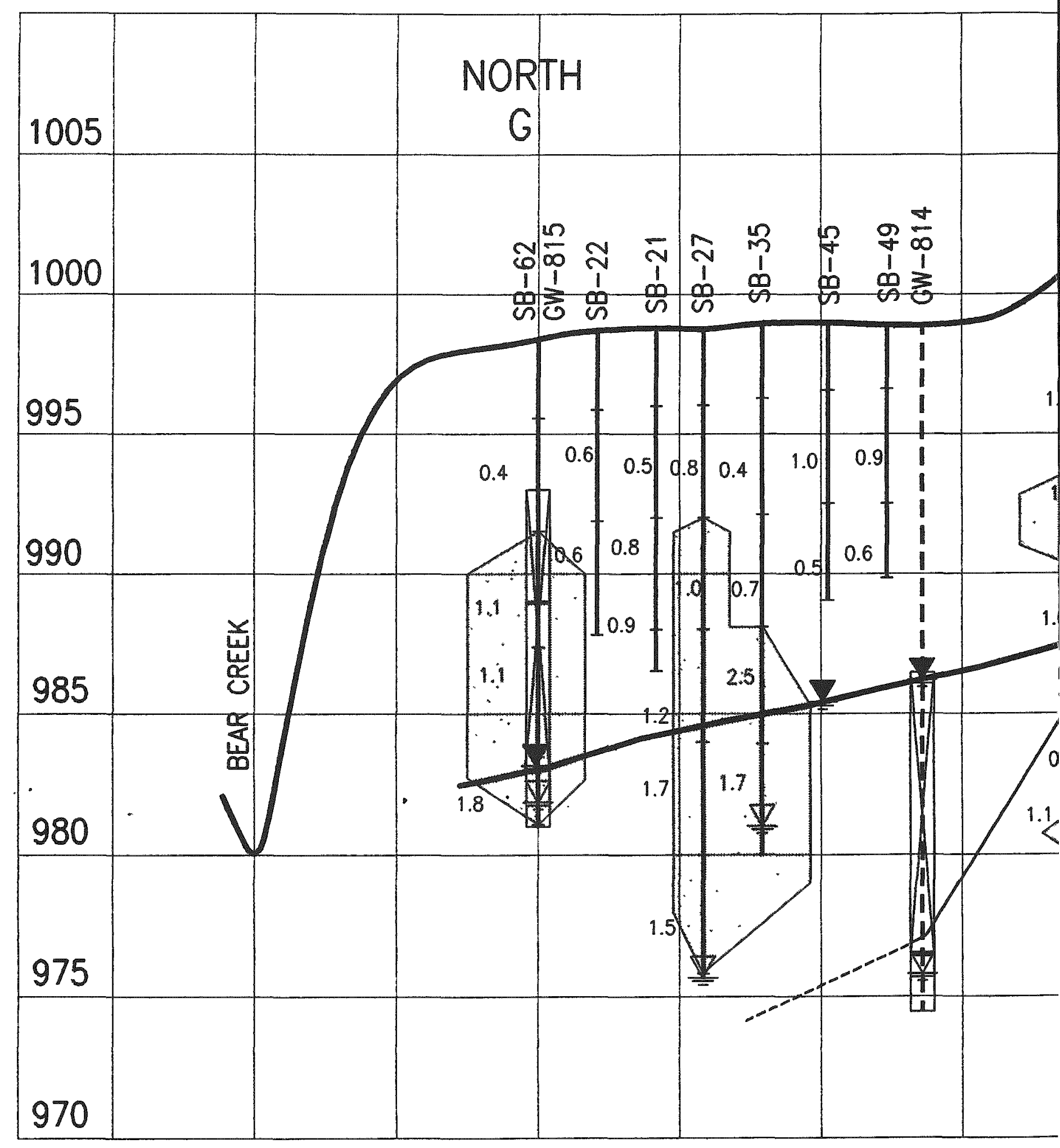




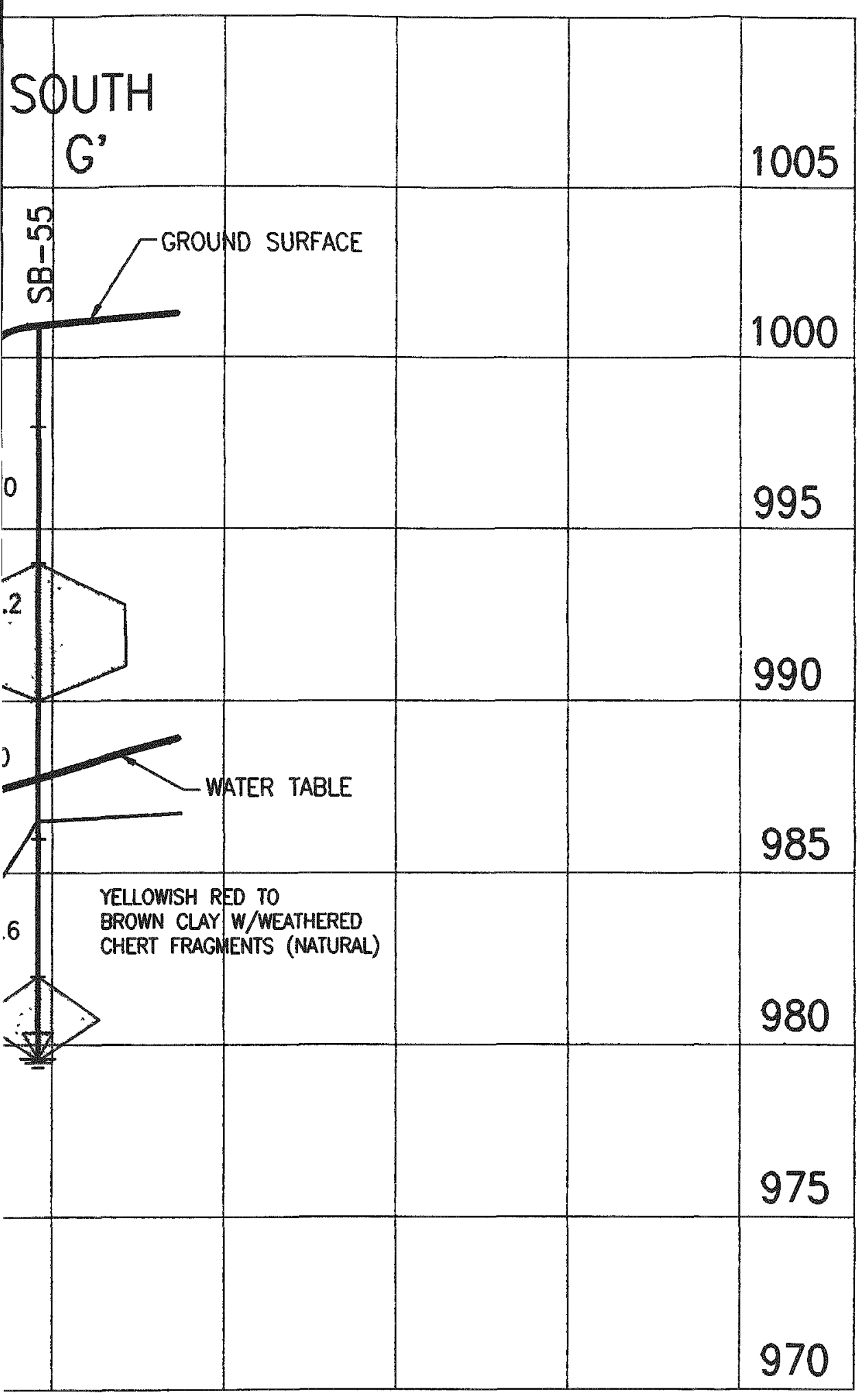

\section{LEGEND:}

GW..... MONITORING WELL

$\mathrm{SB}$ SOILBORING

$\nabla_{\text {GROUNDWATER ENCOUNTERED IN BOREHOLE }}$ II. .GROUNOWATER TABLE

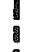

.............. PROJECTED MONITORING WELL

W

.SCREENED INTERVAL

\%

$>1.007$ PPM BERYLLUM (95\% UCB BACKGROUND)

\section{$\begin{array}{llllll}0 & 1 & 2 & 3 & 4 & 5\end{array}$ 10 VERTICAL SCALE: $1^{\prime \prime}=5^{\prime}$}

${ }_{\text {HORIZONTAL SCALE: } 1^{\prime \prime}=60^{\circ}}^{30 \quad 60}$

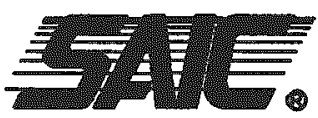

Science Applications International Corporation

BEAR CREEK VALLEY OU2

SY-200 SITE, PROFILE G-G' BERYLLIUM CONCENTRATIONS
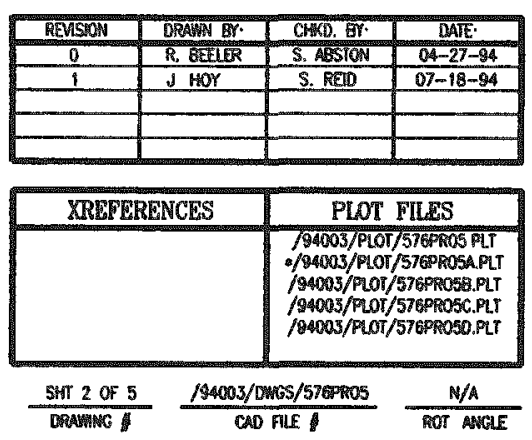
$$
\text { . }
$$ 


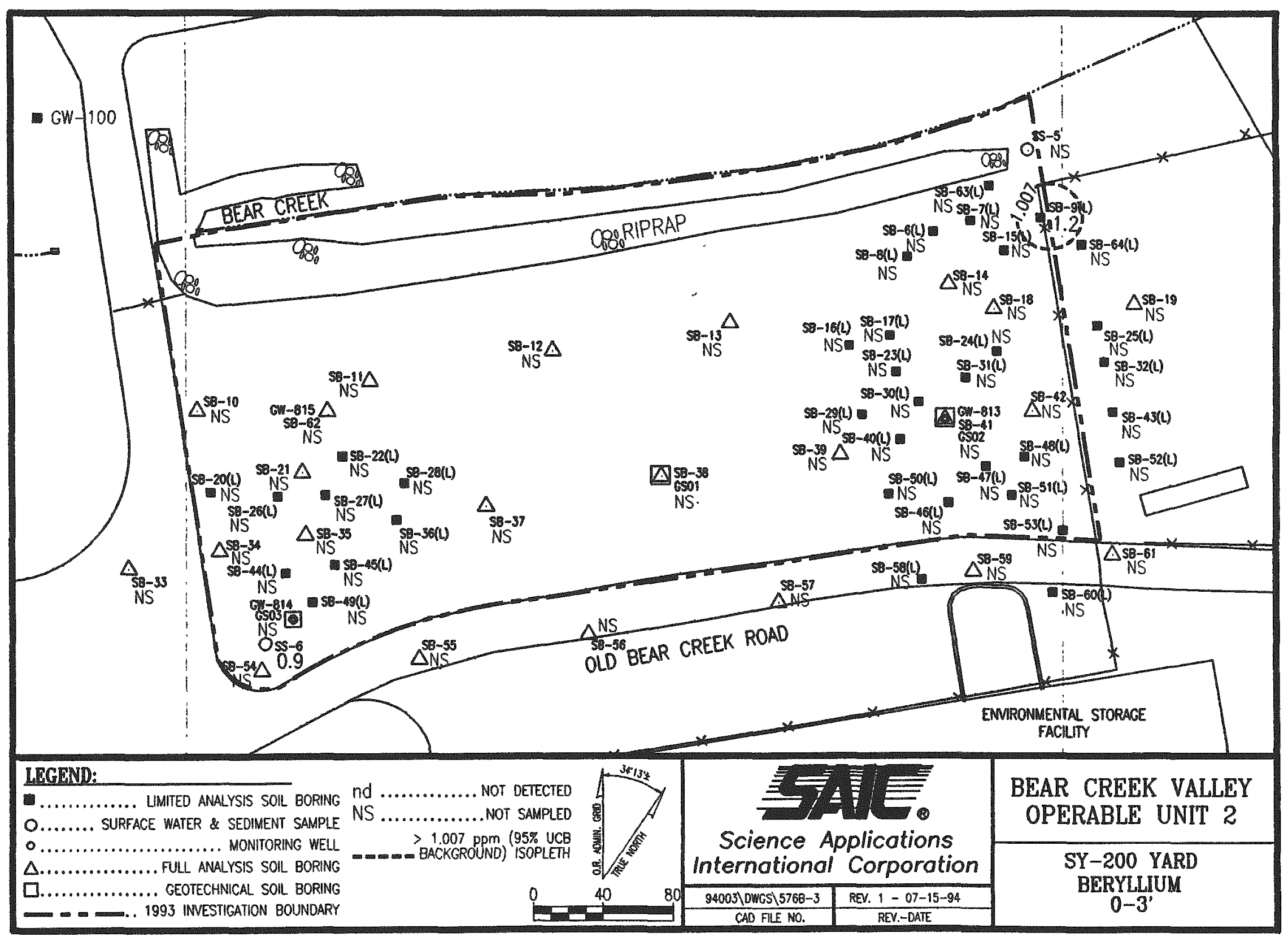




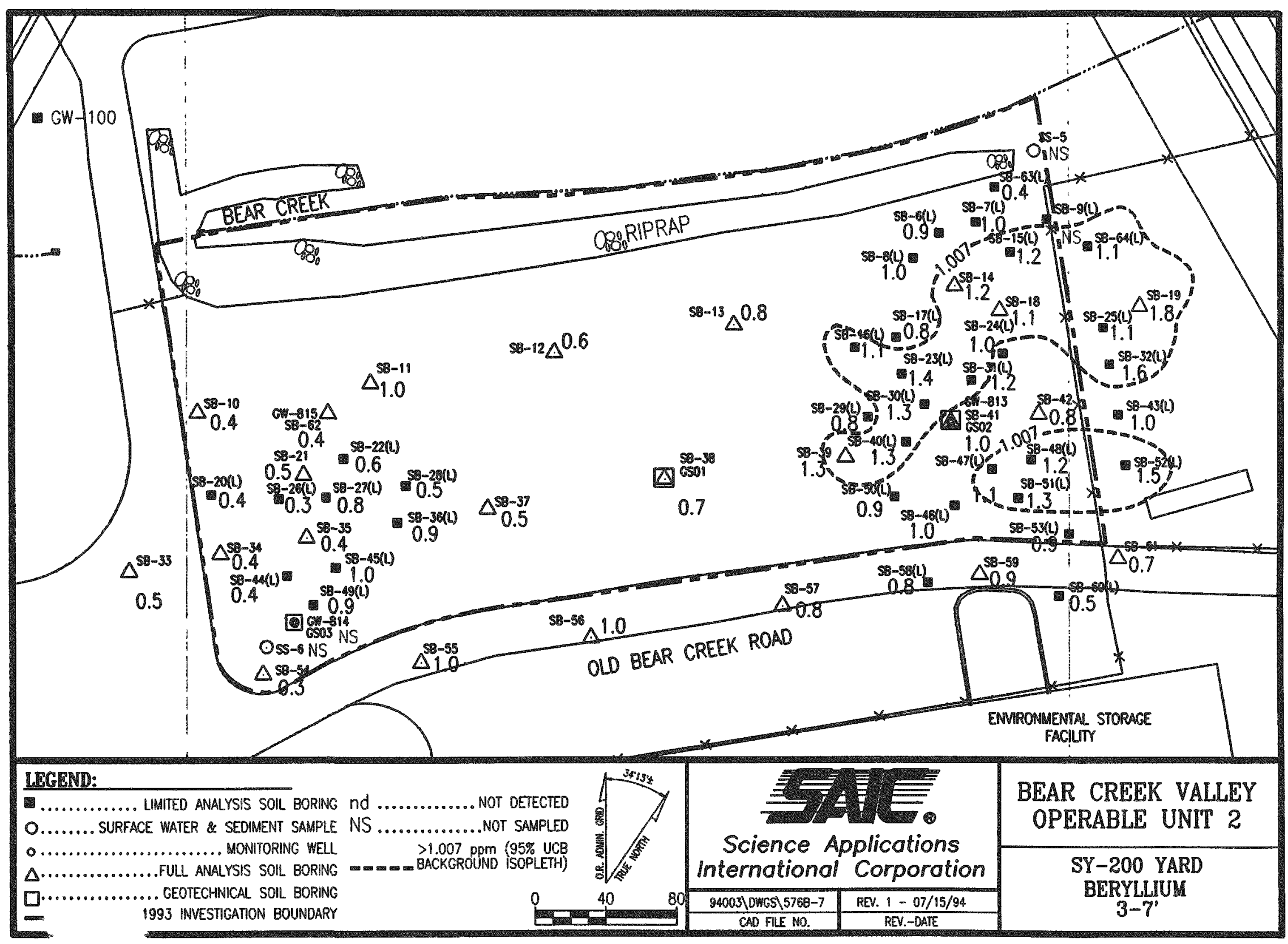




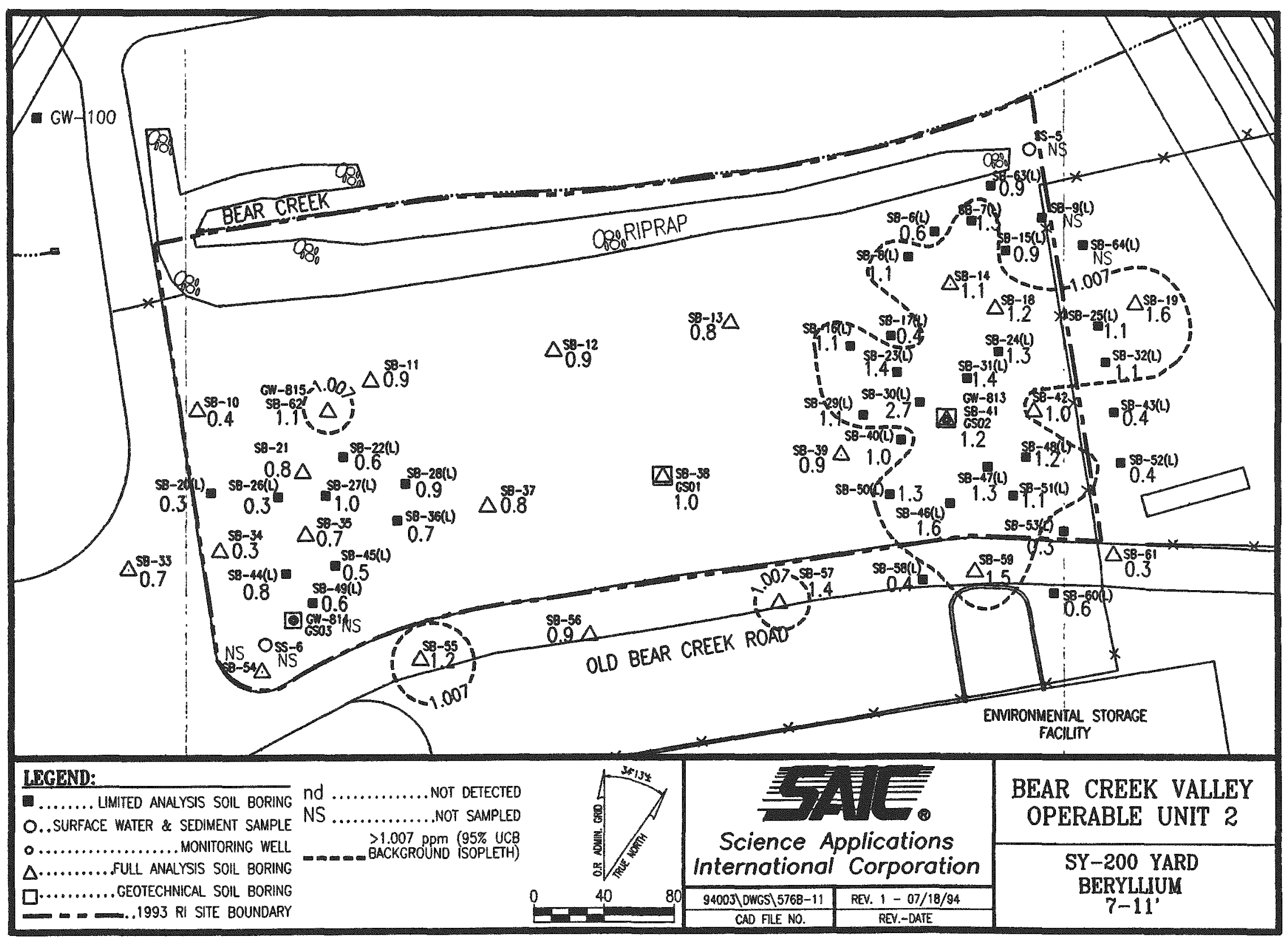




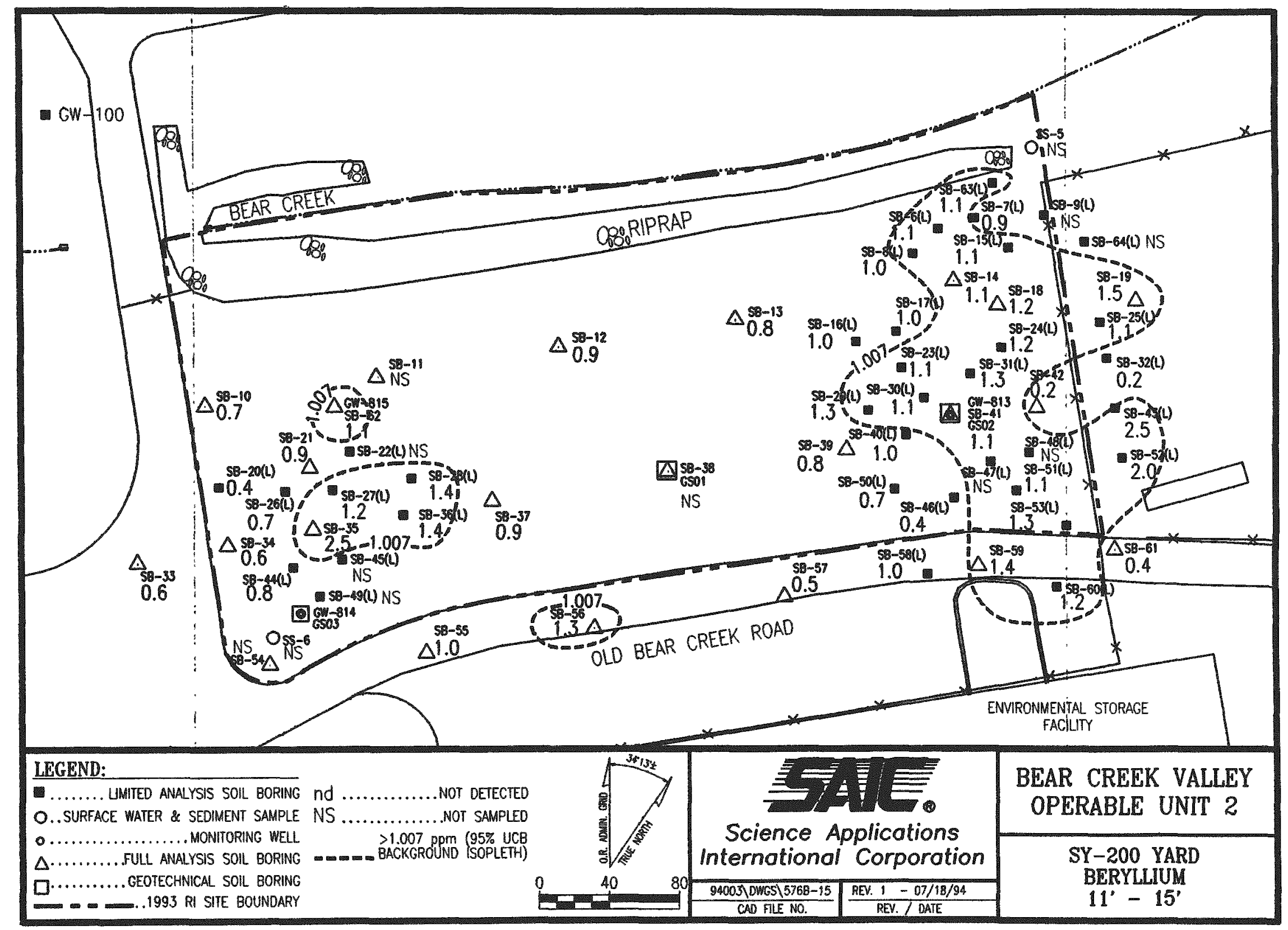




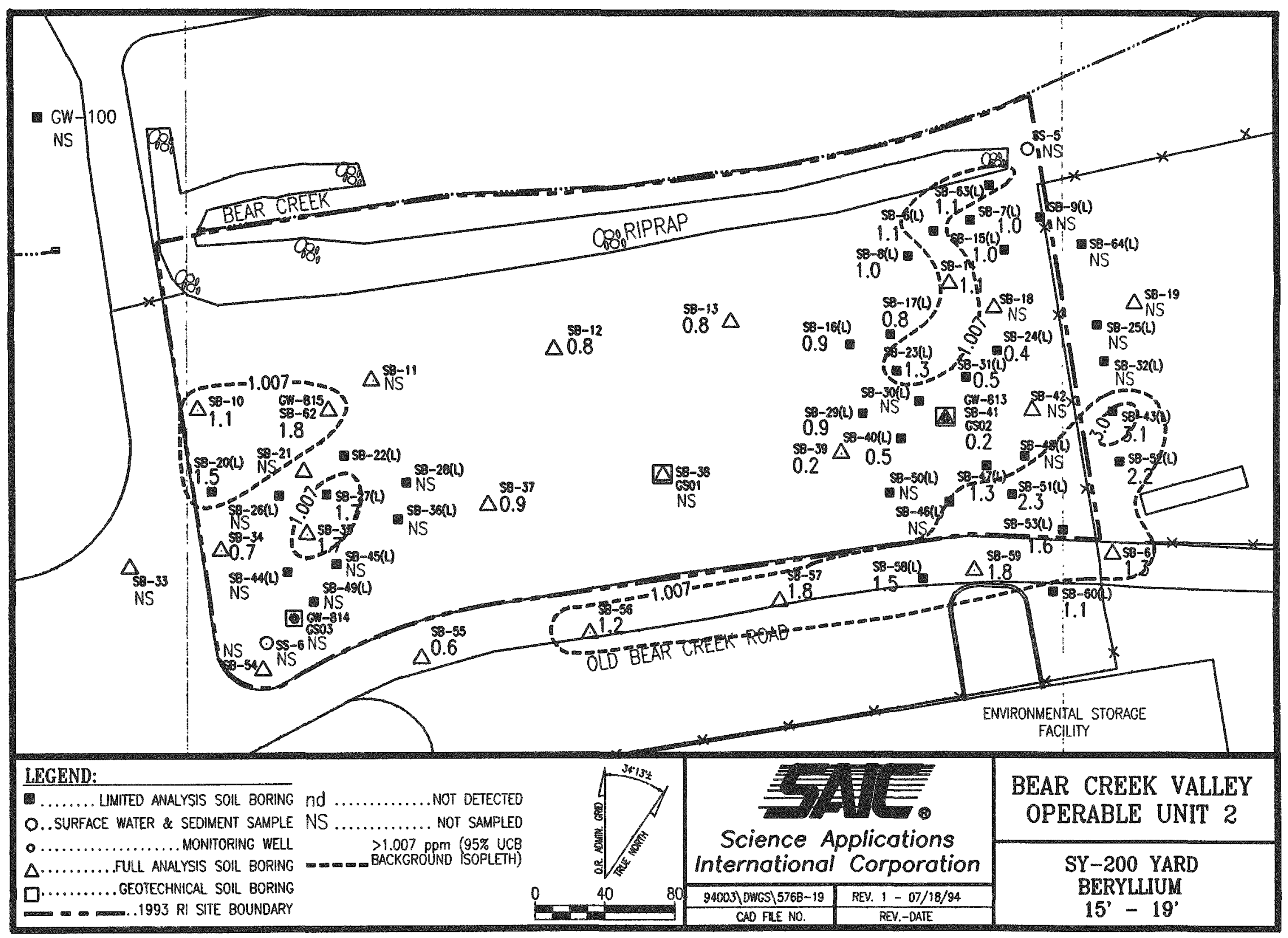




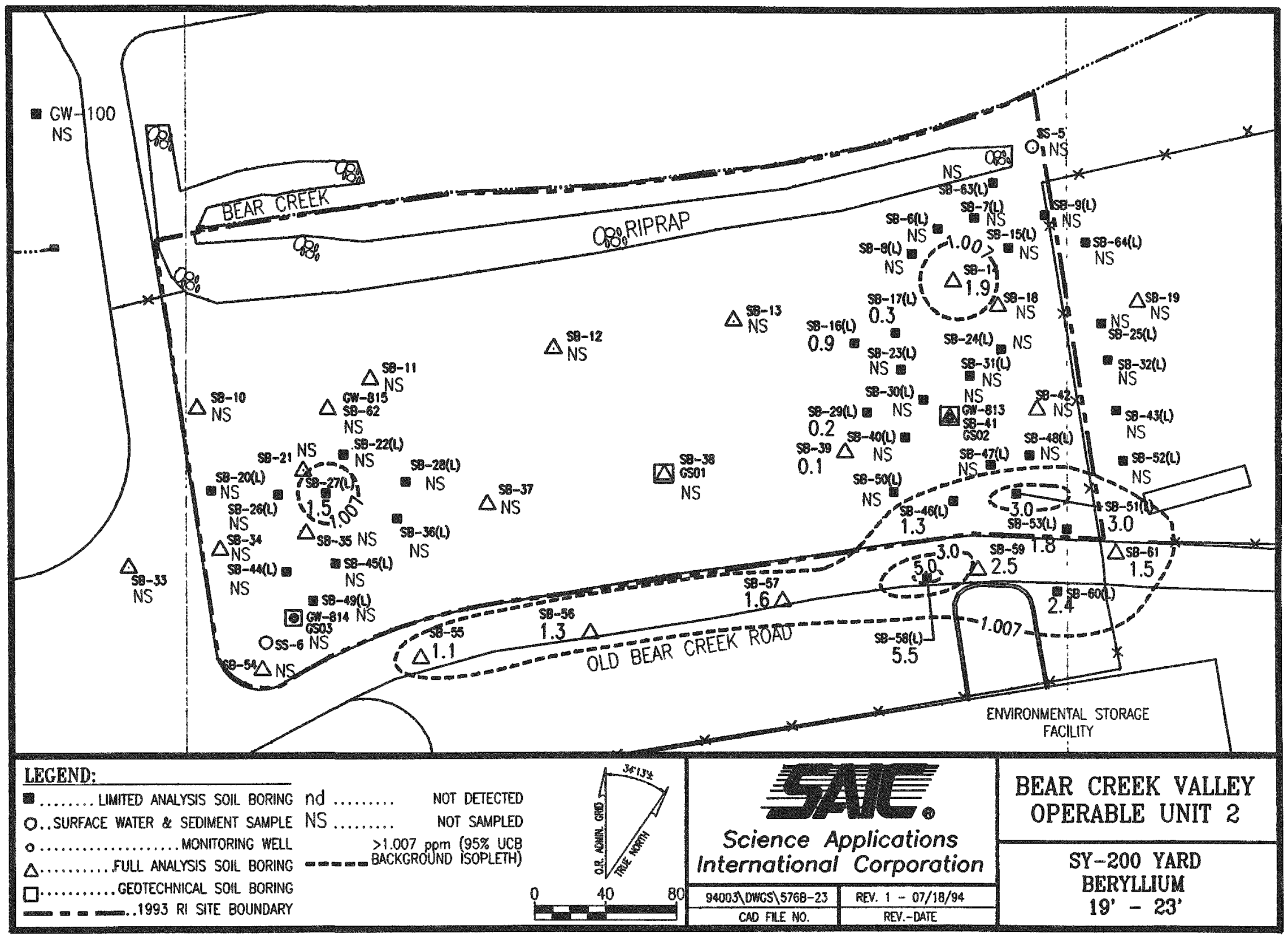




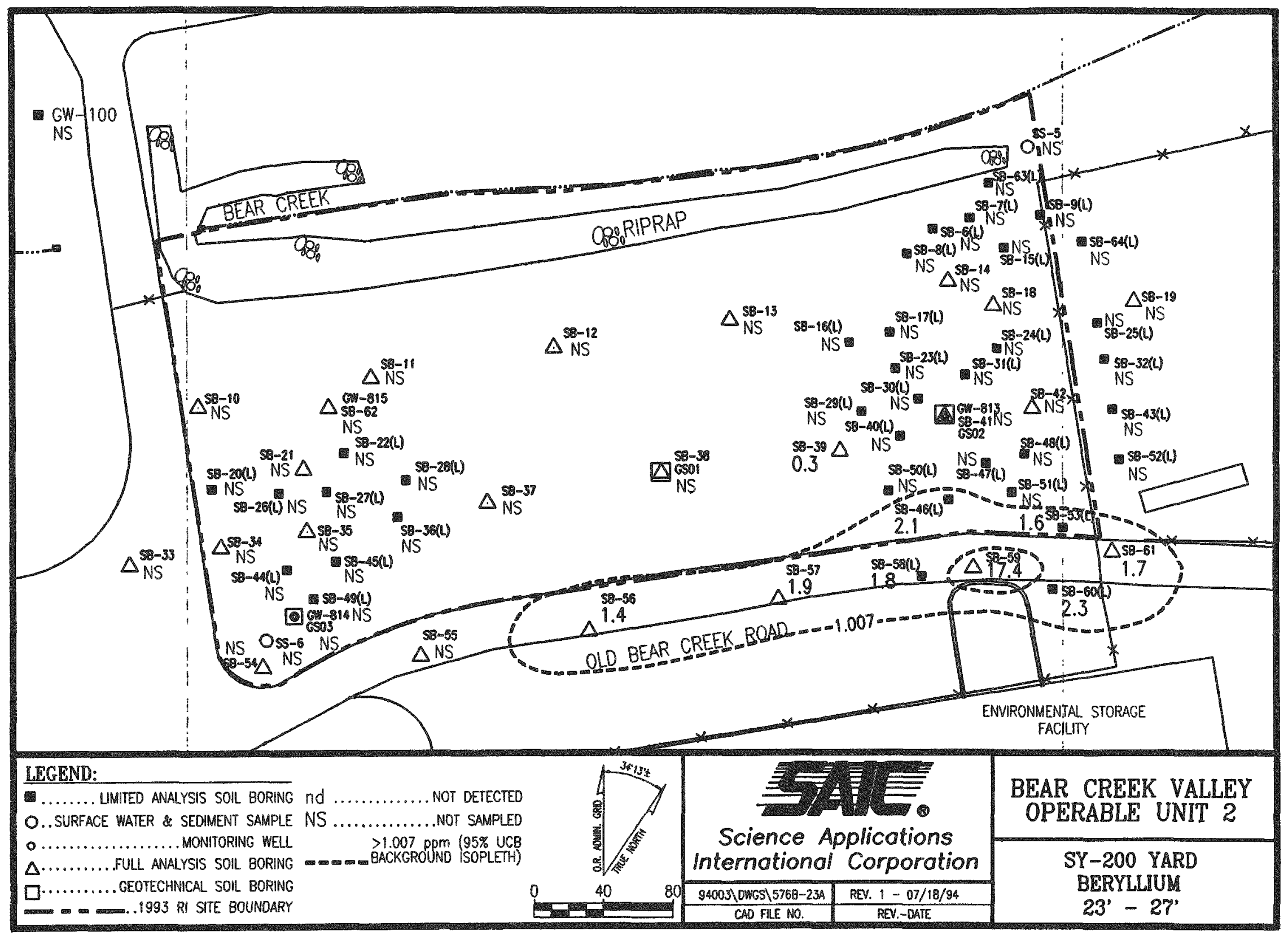




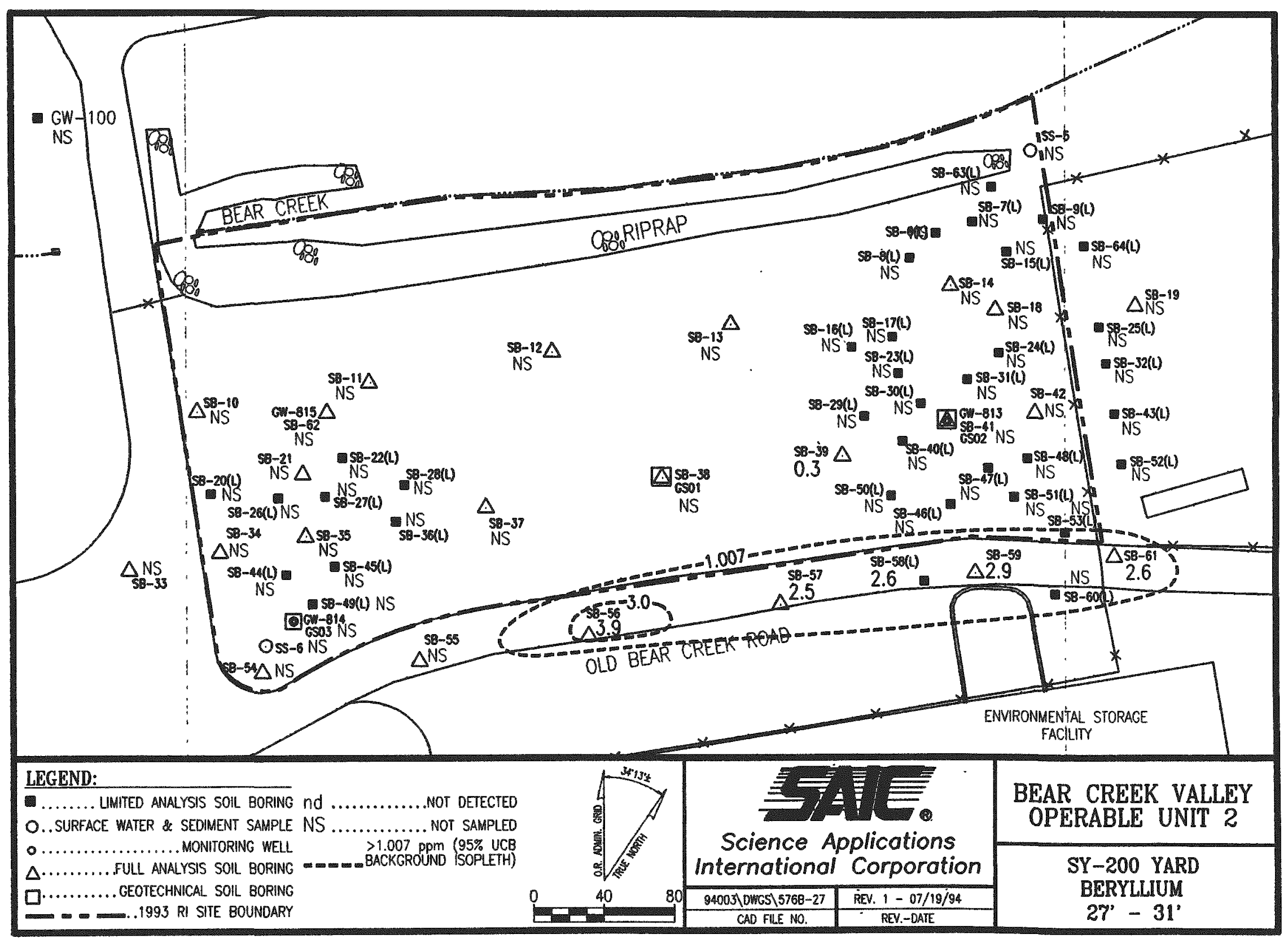




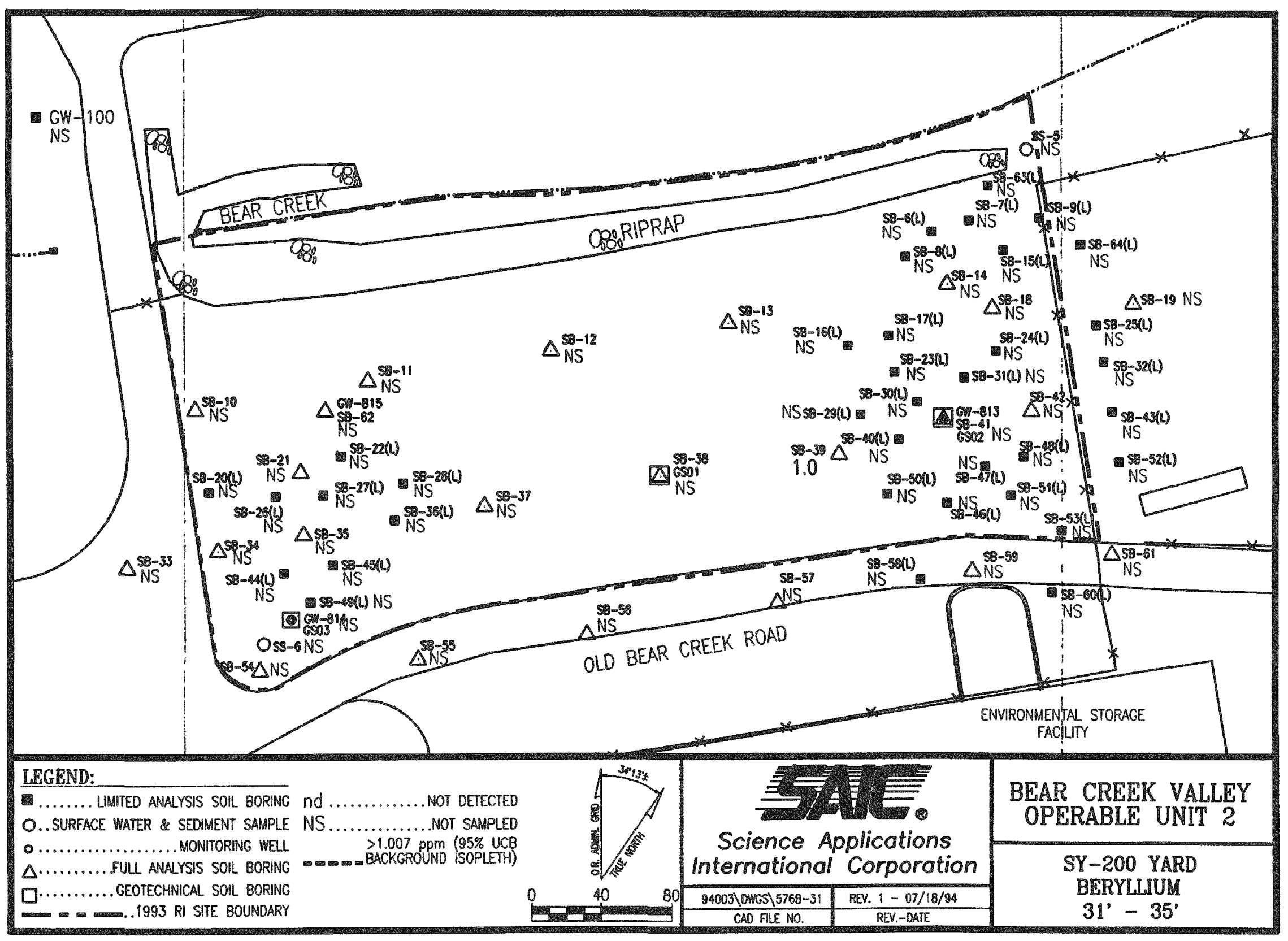




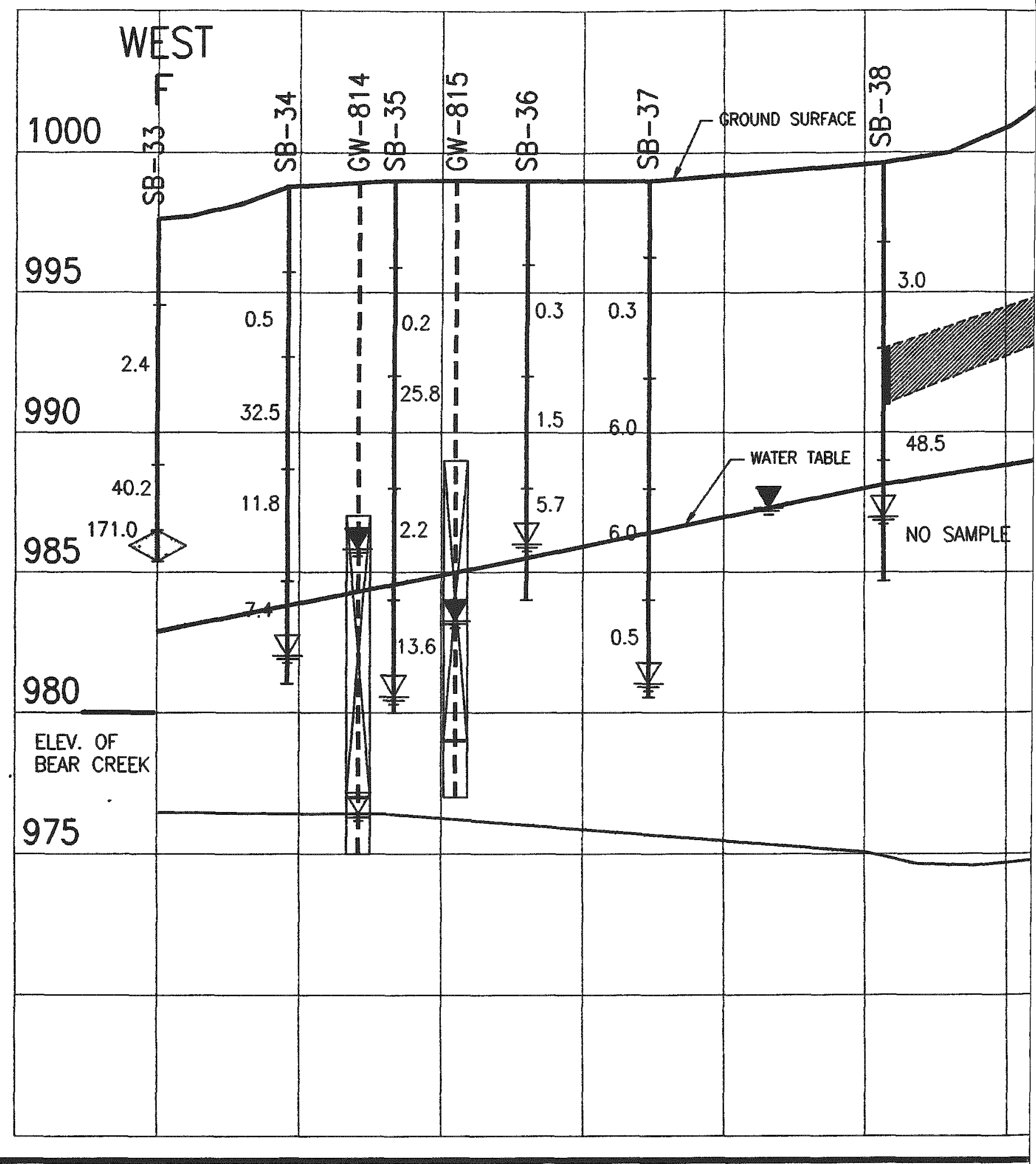




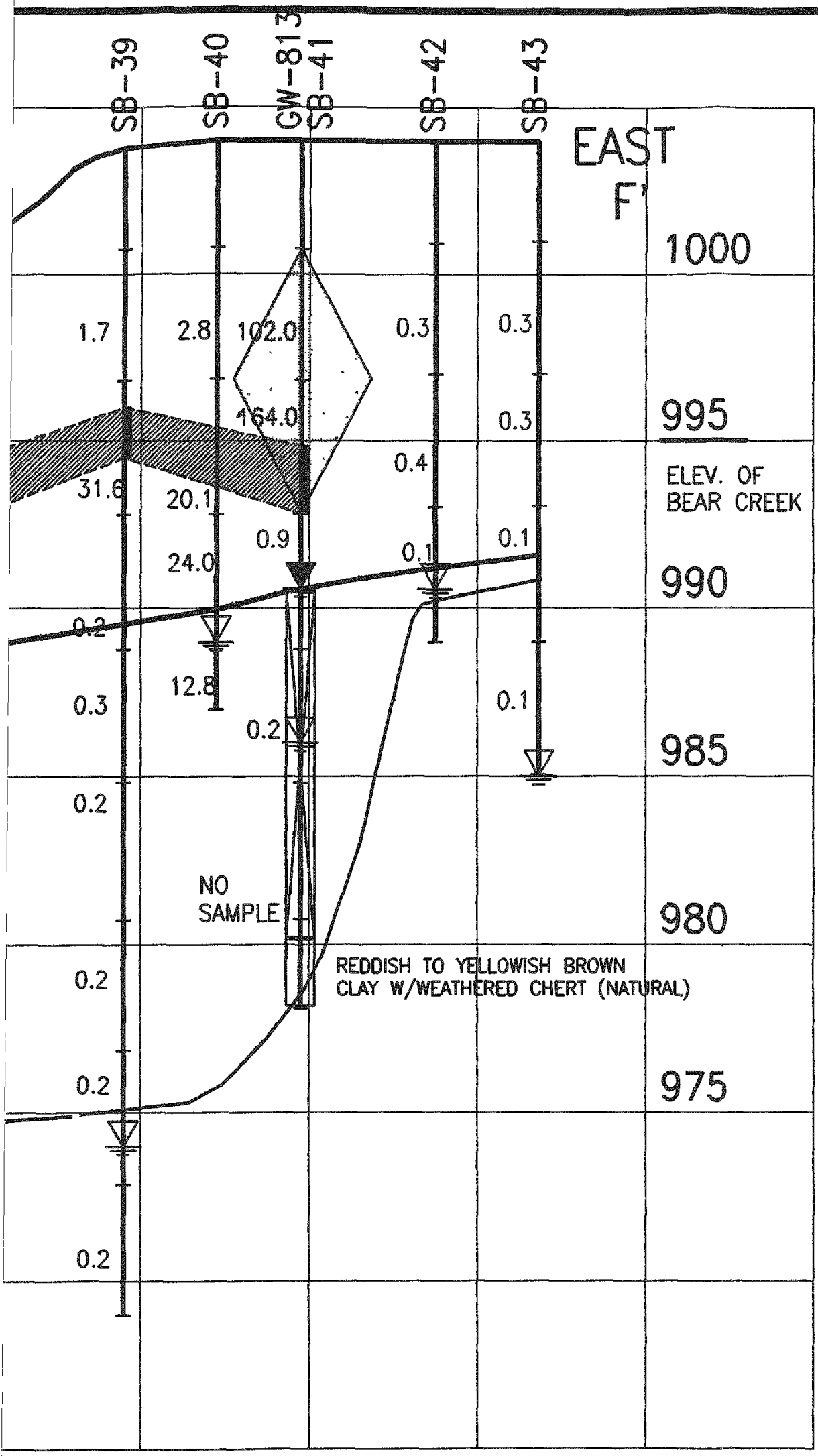

LEGEND:

GW.

SB....................... SOLBORING

ZGROUNOWATER ENCOUNTERED IN BOREHOLE

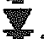

GROUNDWATER TABLE

(1)

PRONECTED WELLS

H.

SCREENED INTERVAL.

2 ANALYTICAL DATA>82 PPM (PRG) MERCURY

.

VISIBLE MERCURY

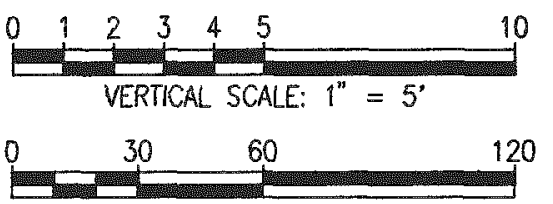

HORIZONTAL SCALE: $1^{\prime \prime}=60^{\prime}$

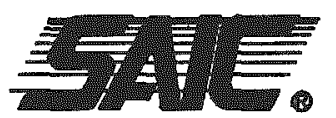

Science Applications International Corporation

BEAR CREEK VALLEY OU2 SY-200 SITE PROFILE F-F" MERCURY CONCENTRATIONS
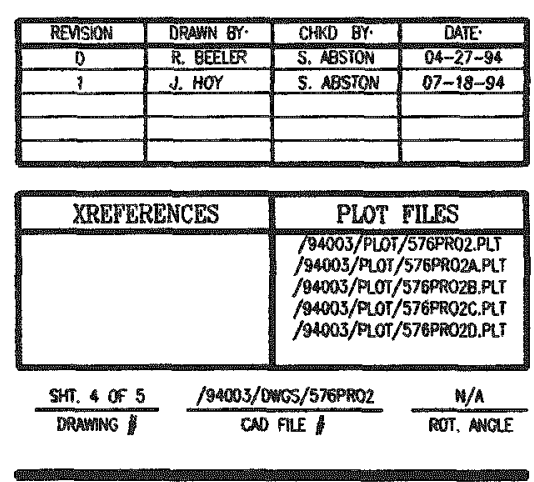


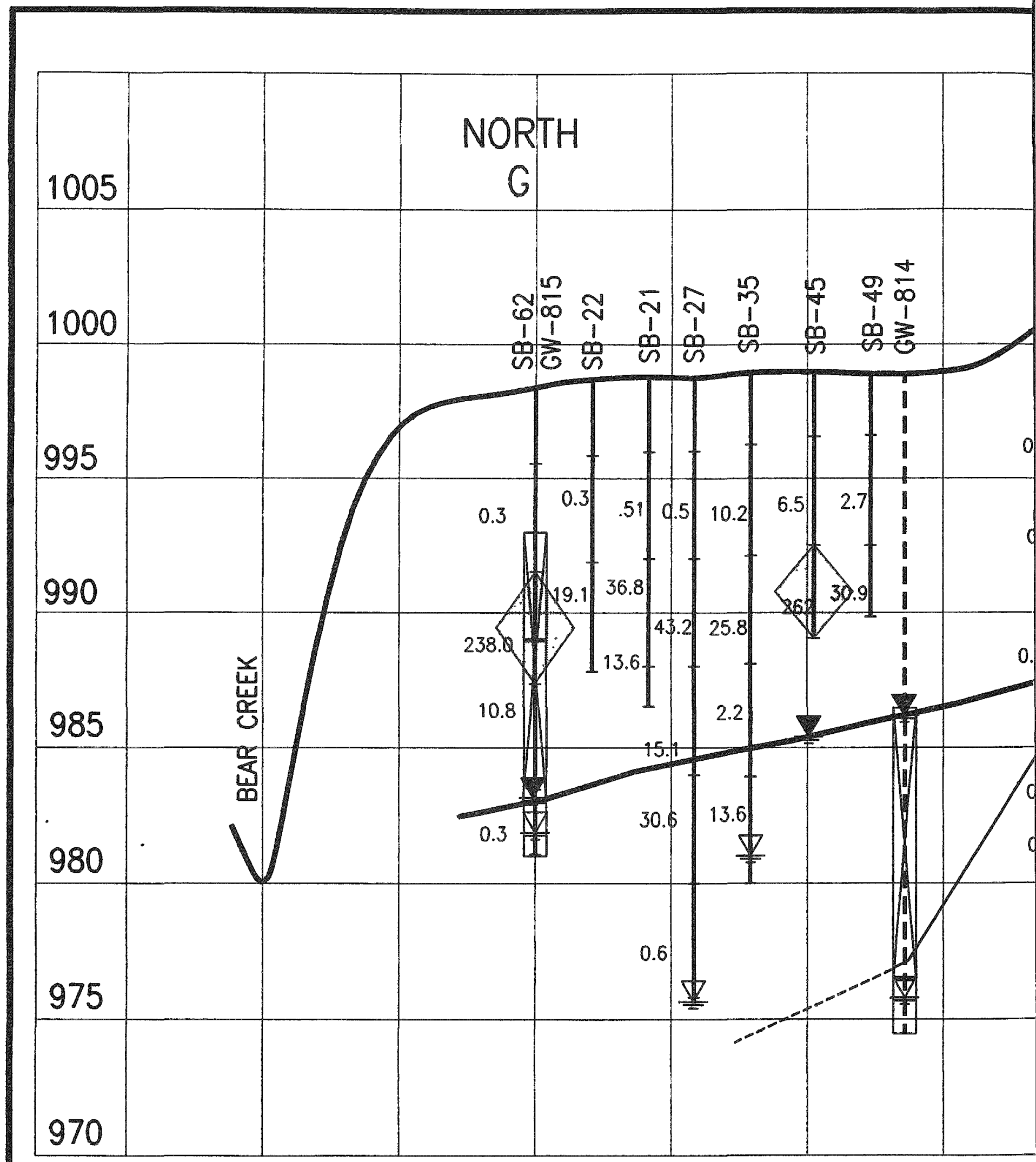




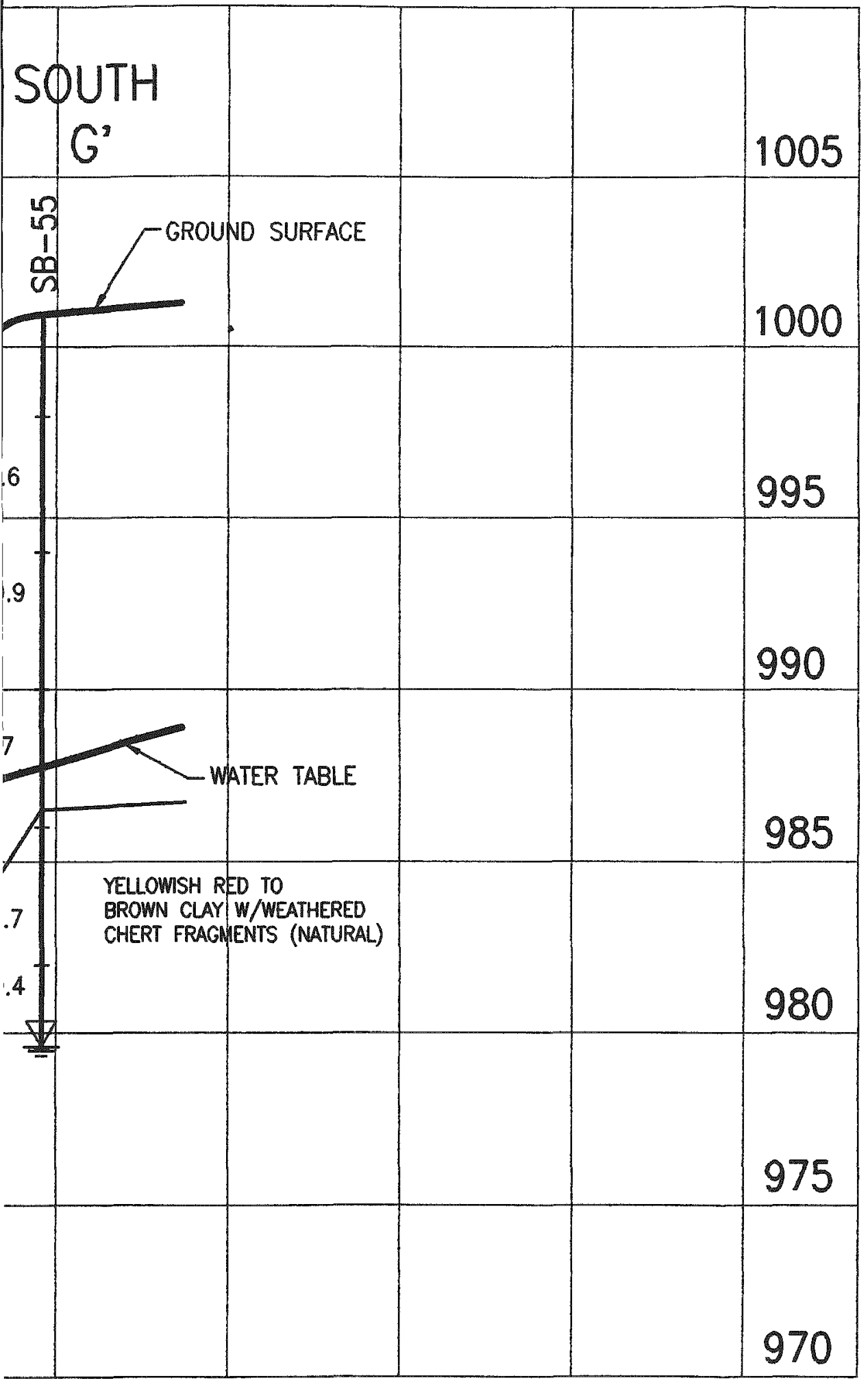

LEGEND:

GW.......

GROUNDWATER WELL

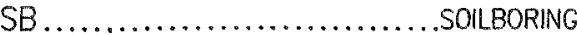

Z GROUNDWATER ENCOUNTERED IN BOREHOLE I $\ldots . . . . . . \ldots \ldots . . .$. GROUNDWATER TABLE

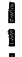

I........... PROJECTED MONITORING WELL 1.

SCREENED INTERVAL

A

ANALYTICAL DATA>82 PPM (PRG) MERCURY

$\begin{array}{llllll}0 & 1 & 2 & 3 & 4 & 5\end{array}$ 10 VERTICAL SCALE: $1^{\prime \prime}=5^{\circ}$

0

30

60

HORIZONTAL SCALE: $1^{\prime \prime}=60^{\circ}$

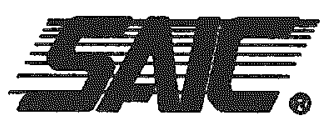

Science Applications International Corporation

BEAR CREEK VALLEY OU2 SY-200 SITE, PROFILE G-G' MERCURY CONCENTRATIONS
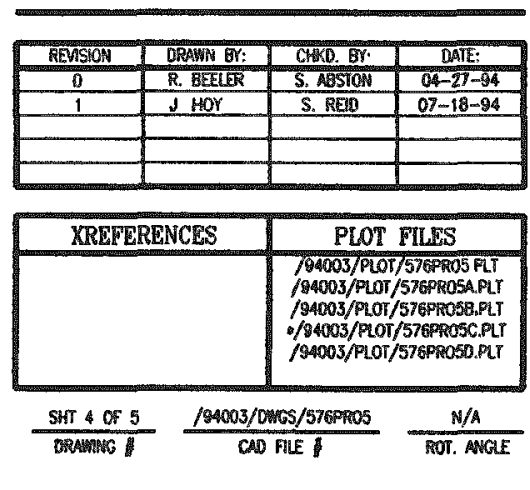


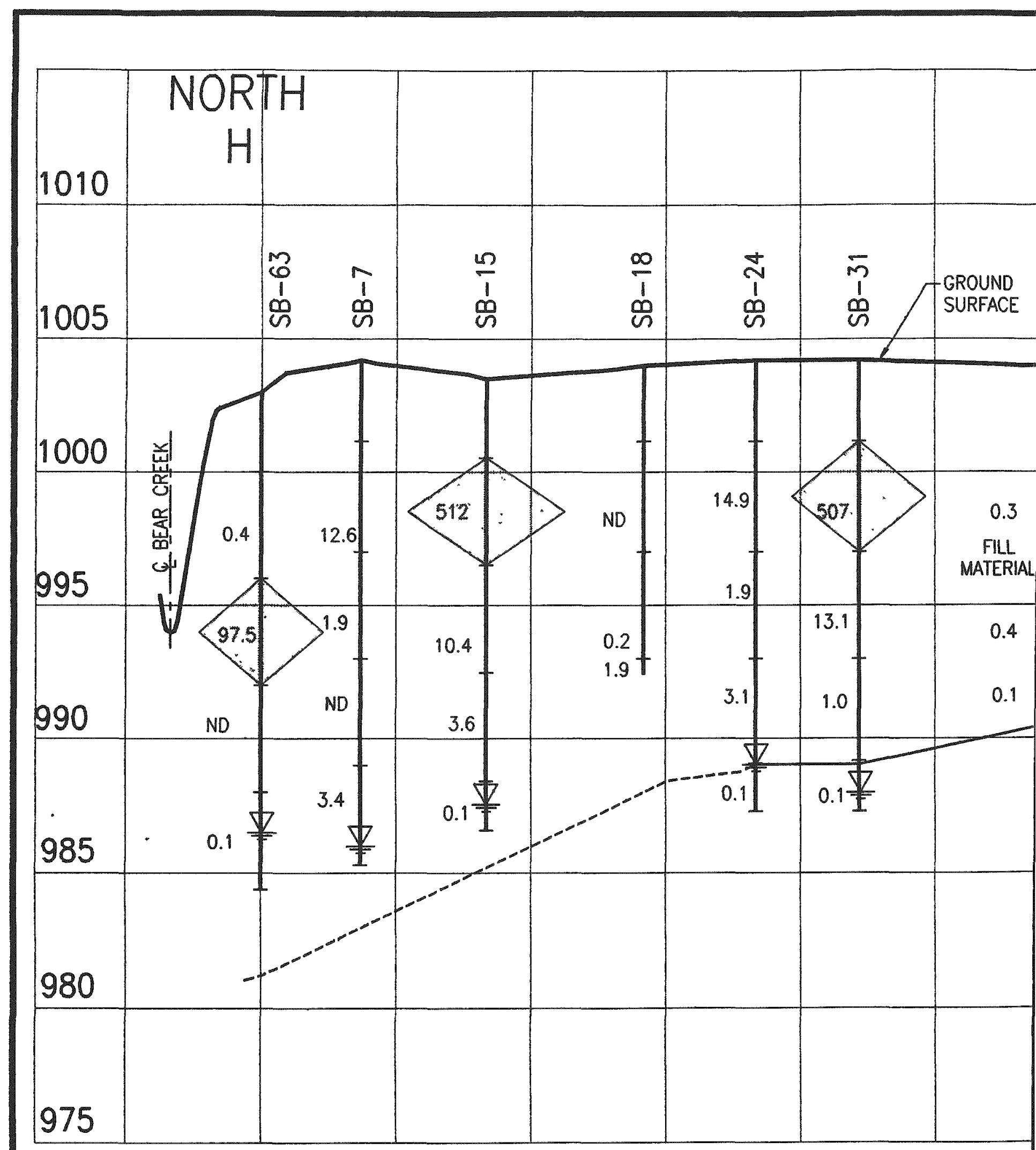




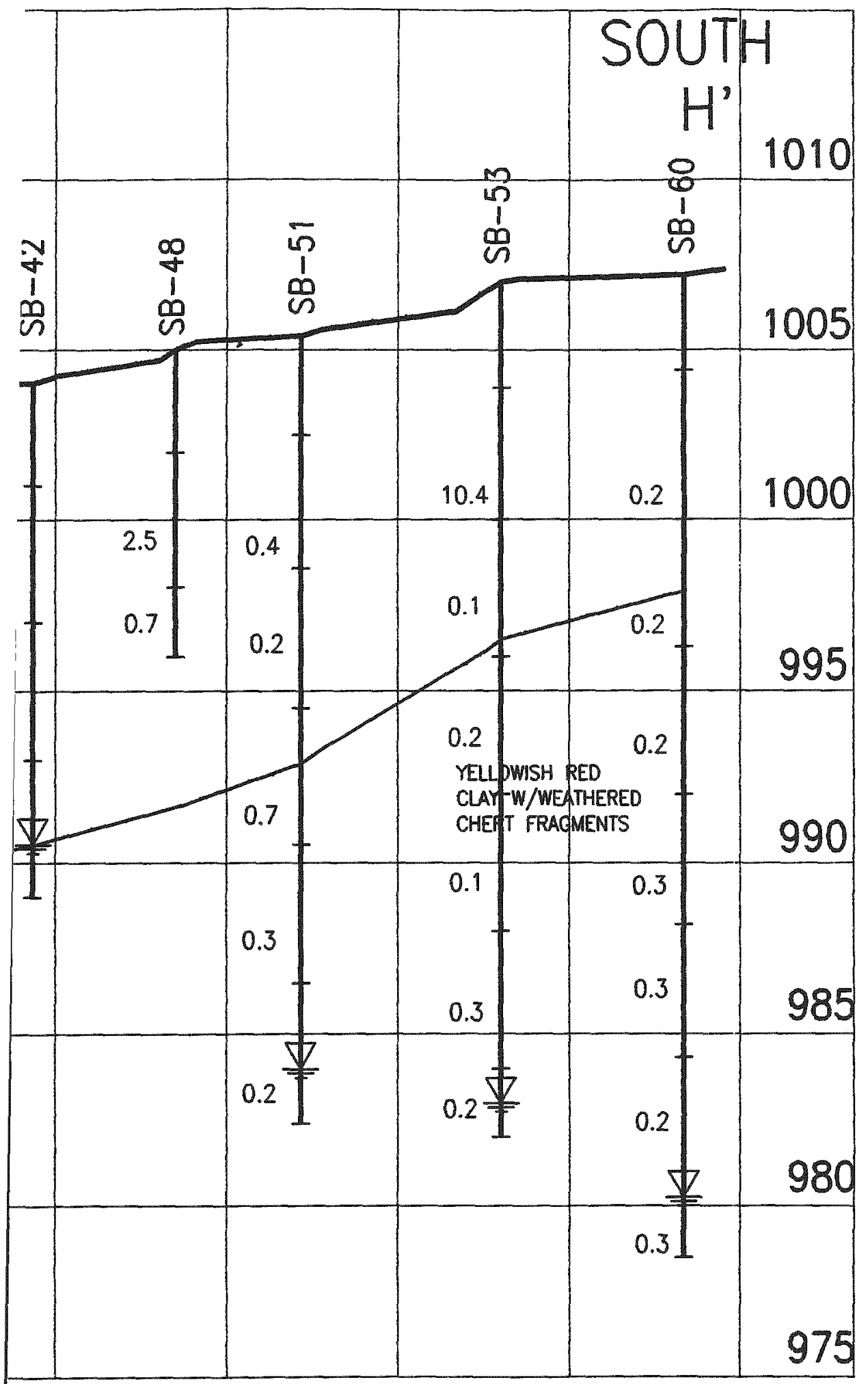

LEGEND:

GW...................MONITORING WELL SB .......................... SOILBORING

ZGROUNDWATER ENCOUNTERED IN BOREHOLE 1 analytical DATA>82 PPM (PRG) MERGURY . VISIBLE MERCURY 10

2345 VERTICAL SCALE: $1^{\prime \prime}=5^{\prime}$

$\begin{array}{llll}0 & 10 & 20 & 30\end{array}$ 60 HORIZONTAL SCALE: $1^{\prime \prime}=30^{\circ}$

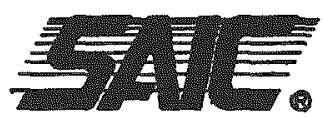

Science Applications International Corporation

BEAR CREEK VALLEY OU2 SY-200 YARD, PROFILE H-H' MERCURY CONCENTRATIONS

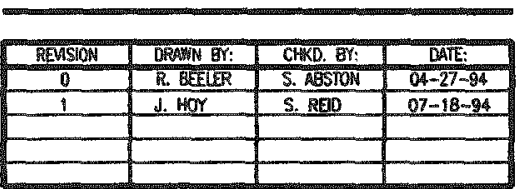

\begin{tabular}{|c|c|}
\hline XPRPERENCES & PLOT FLLS \\
\hline & 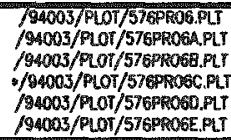 \\
\hline
\end{tabular}

SWI. 4 of 6 /94003/CWGS/576PRO6 
$$
\text { . }
$$ 


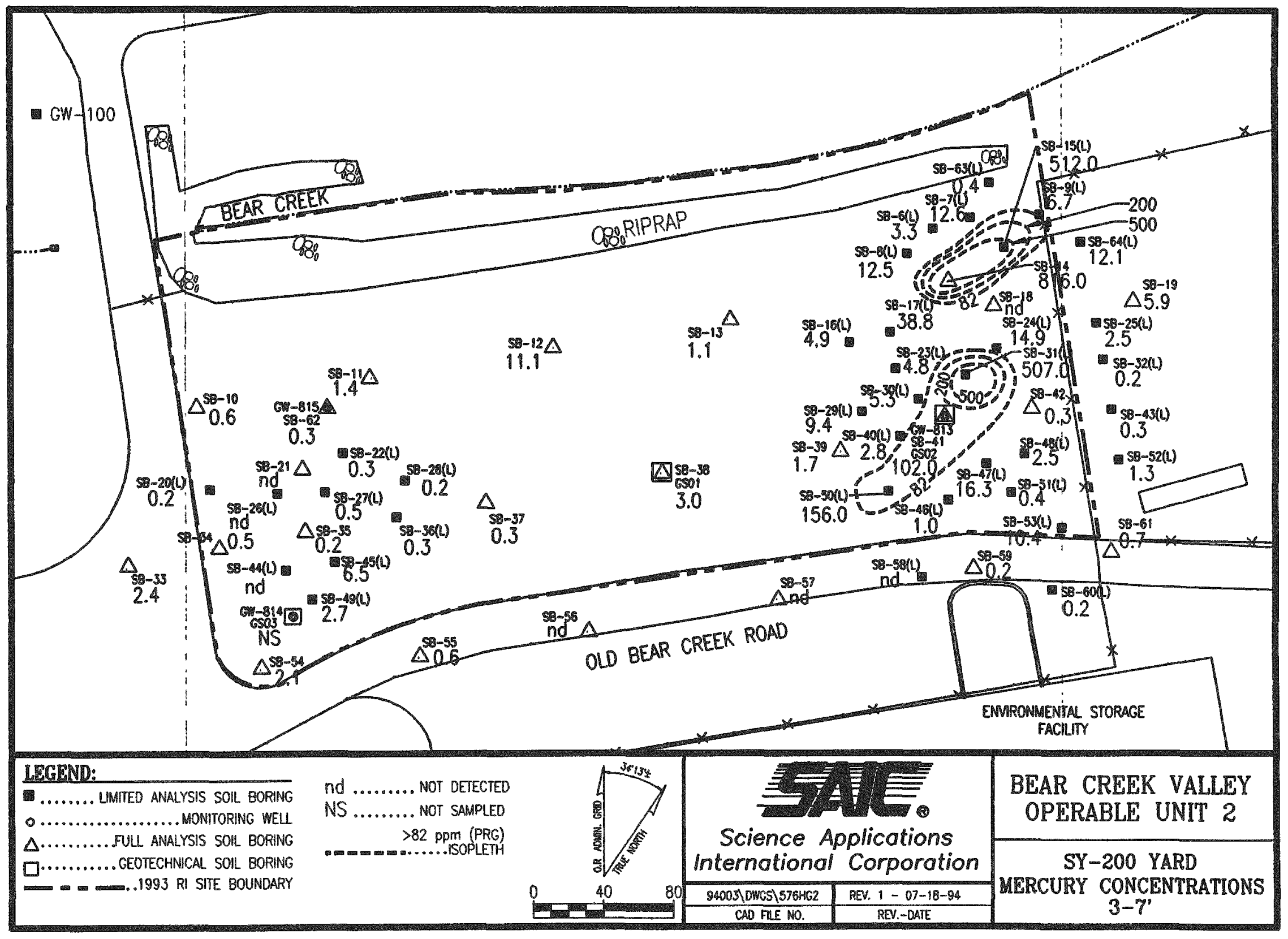




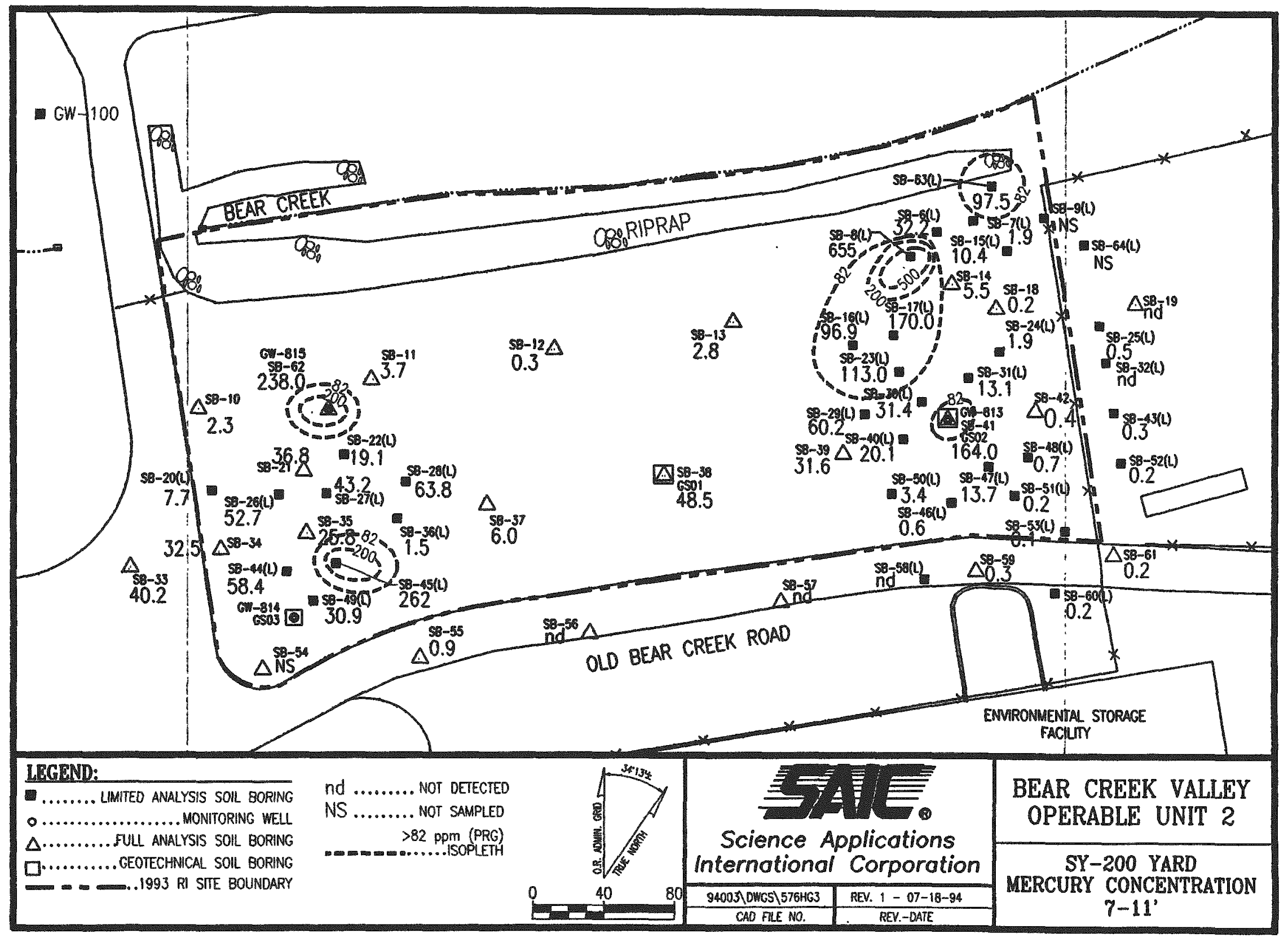

峁 


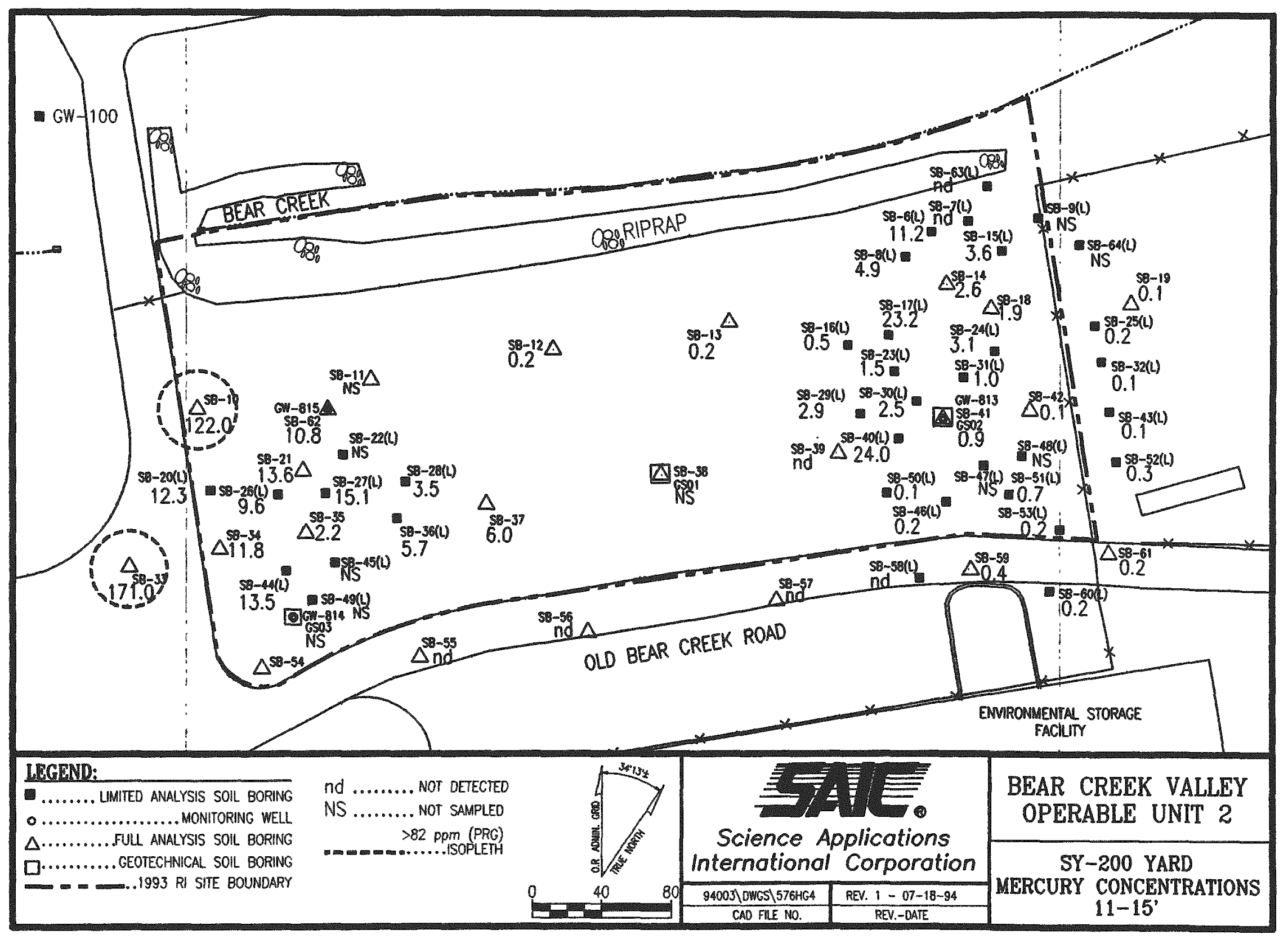




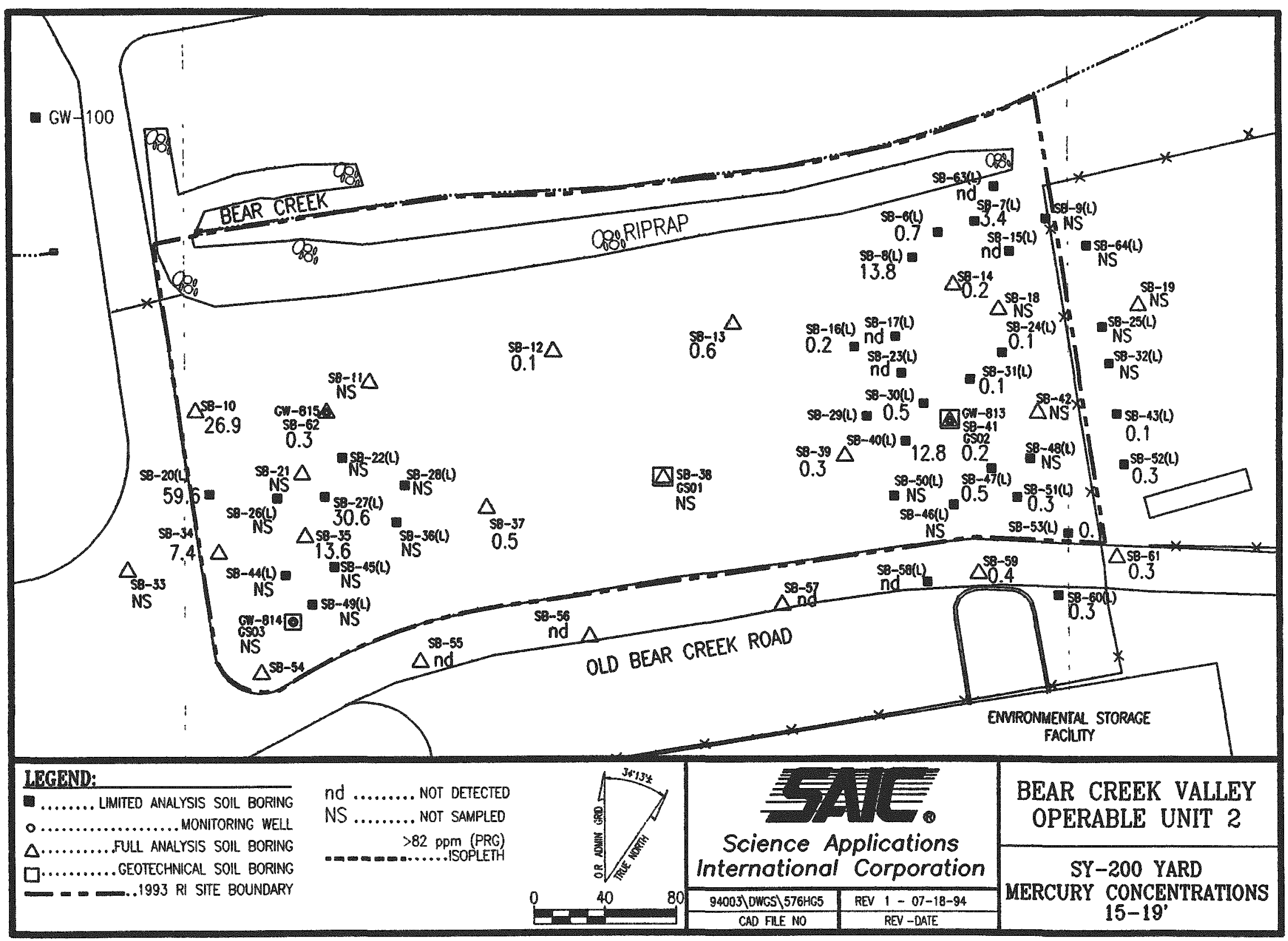




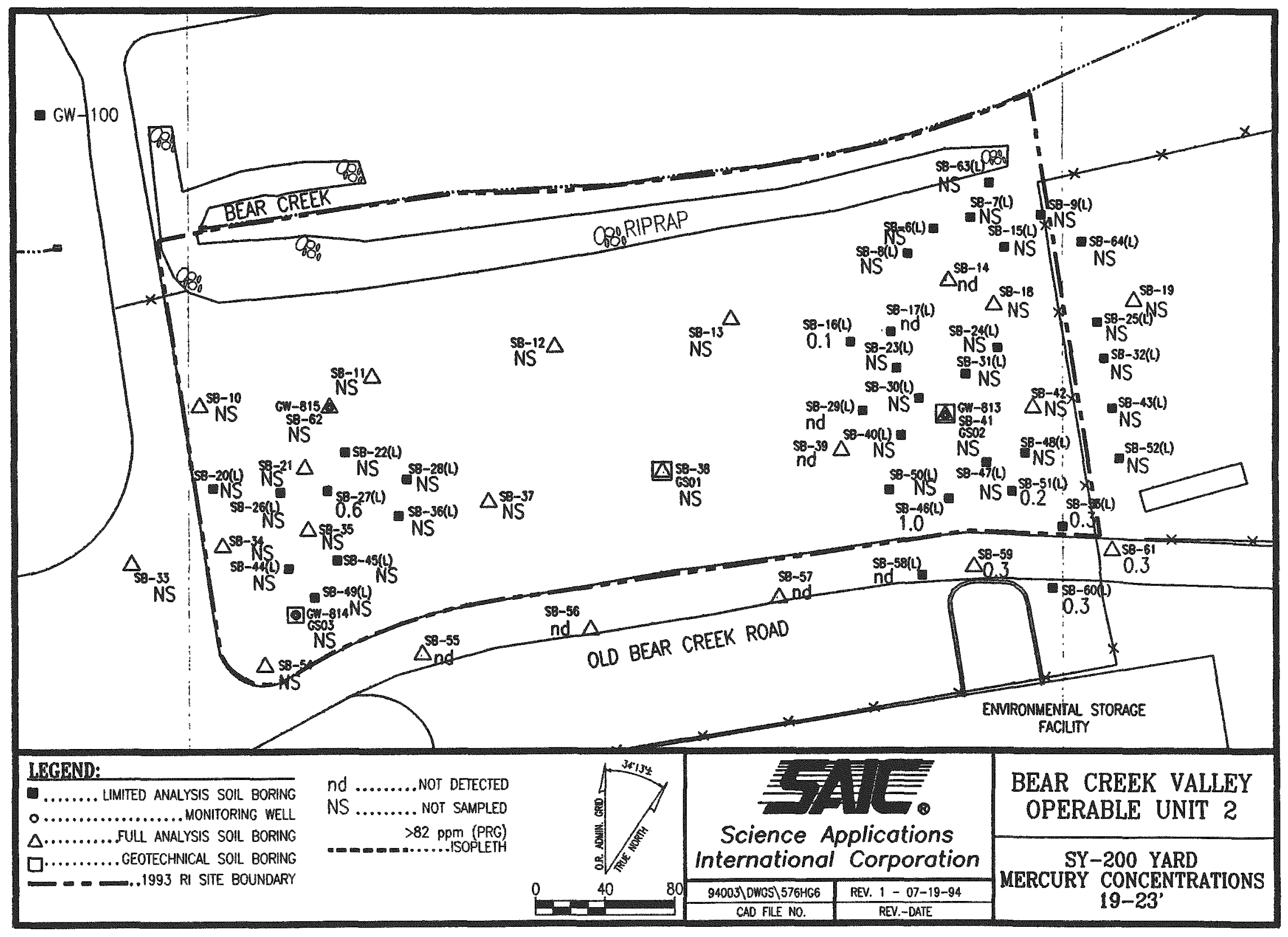




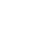




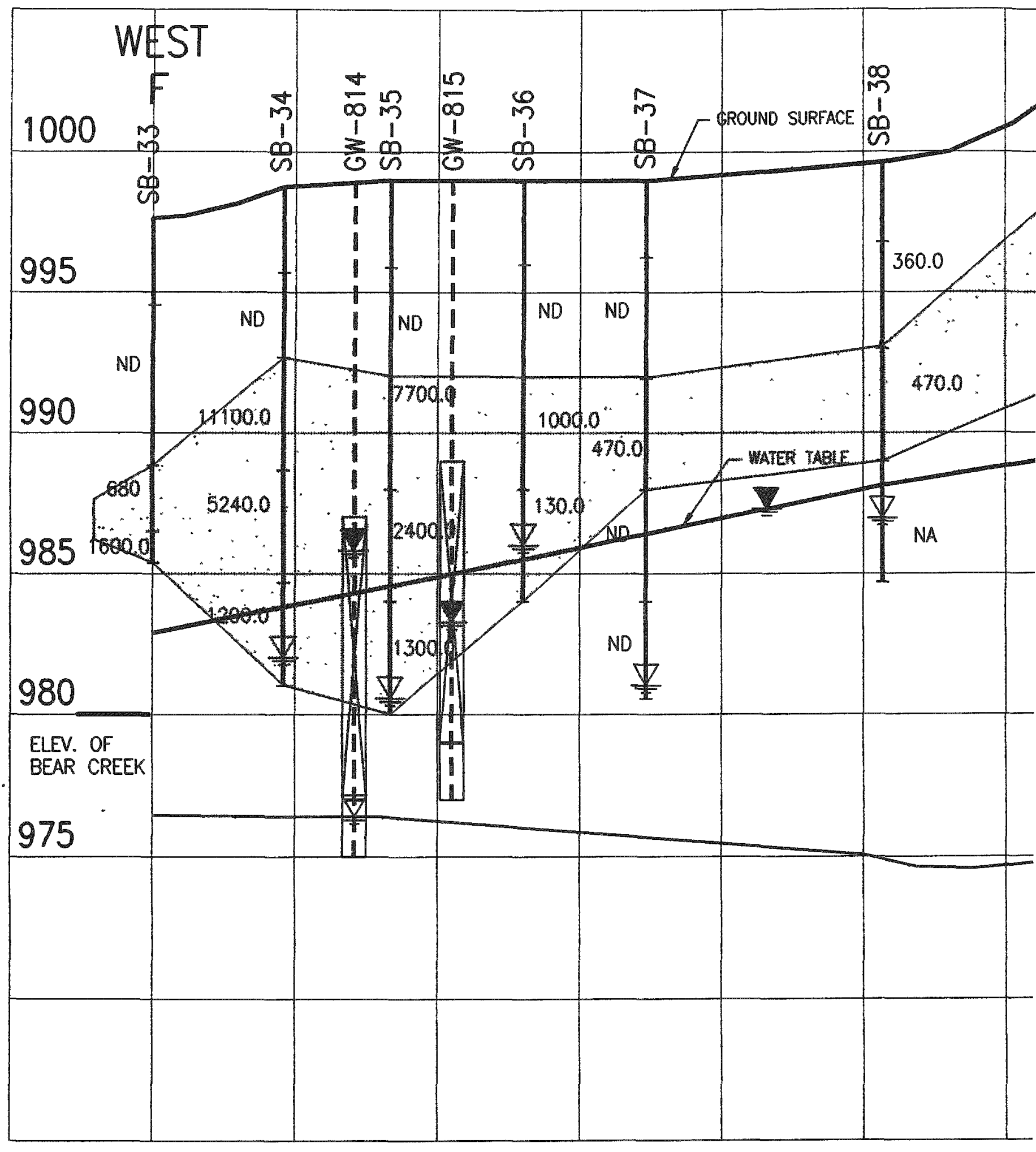




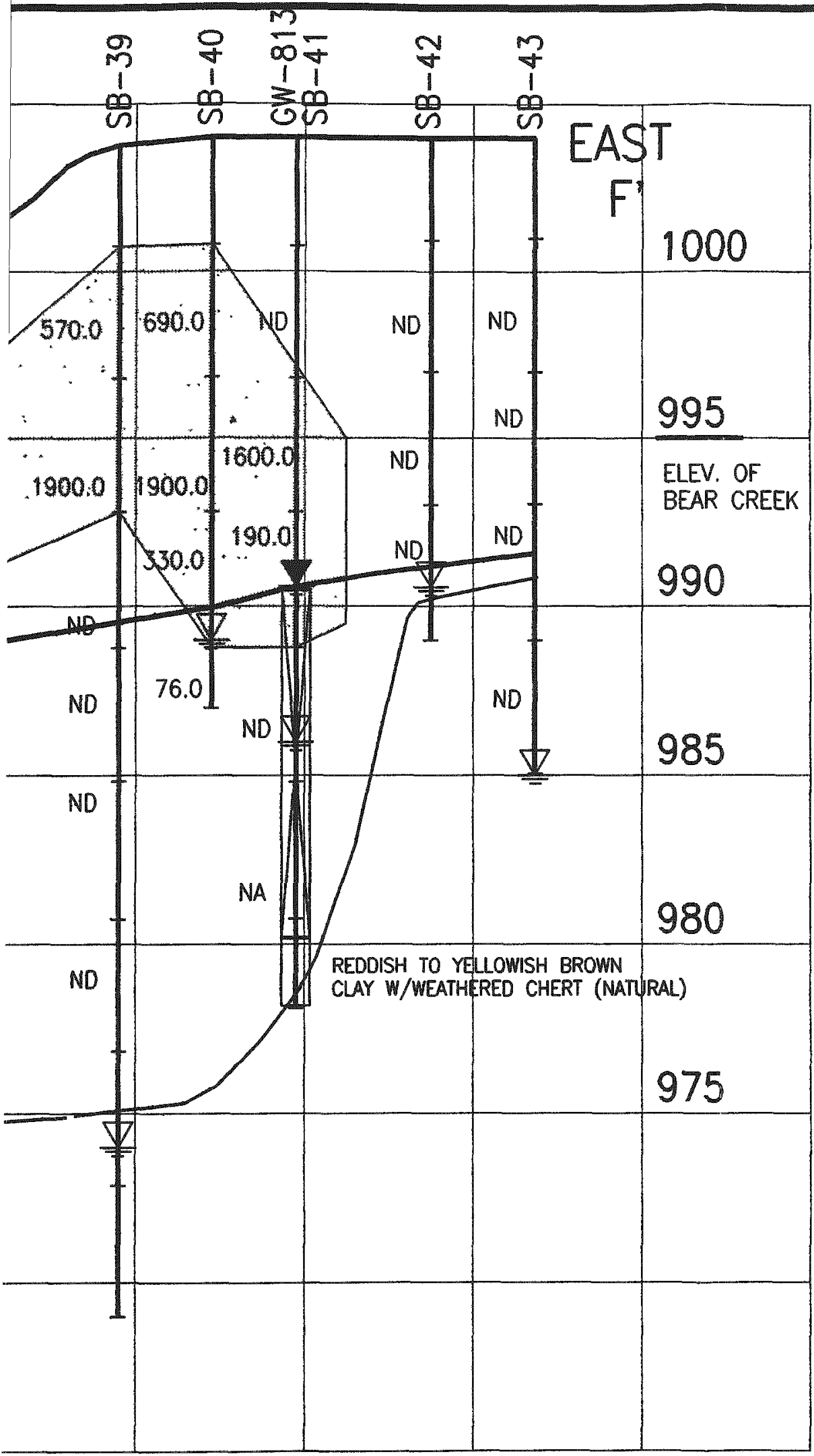

LEGEND:

GW.

.GROUNDWATER WELL SB ........................ SOIBORING ZGROUNDWATER ENCOUNTERED IN BOREHOLE ................... GROUNOWATER TABLE i

PROJECTED WELLS

SCREENED INTERVAL

$>83$ PPB (PRG) COMBINED AROCLOR $1254 \& 1260$ ND. ................... NOT DETECTED NA NOT ANALYZED

\section{$\begin{array}{lllllll}0 & 1 & 2 & 3 & 4 & 5 & 10\end{array}$ VERTICAL SCALE: $1^{n}=5^{\prime}$}

0

$30 \quad 60$ 120

HORIZONTAL SCALE: $1^{n}=60^{\circ}$

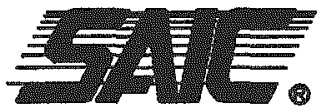

Science Applications International Corporation

BEAR CREEK VALLEY OU2 SY-200 SITE PROFILE F-F' AROCLOR 1254 \& 1260 CONCEN.
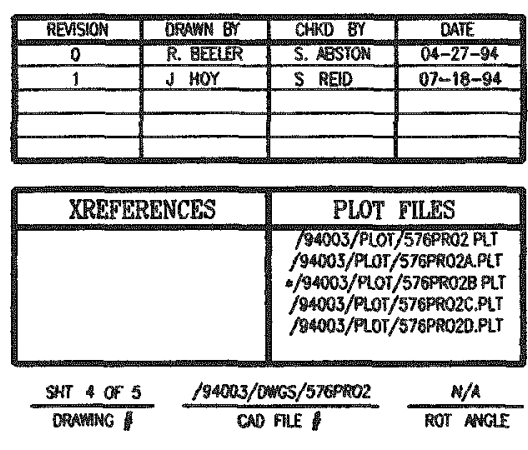


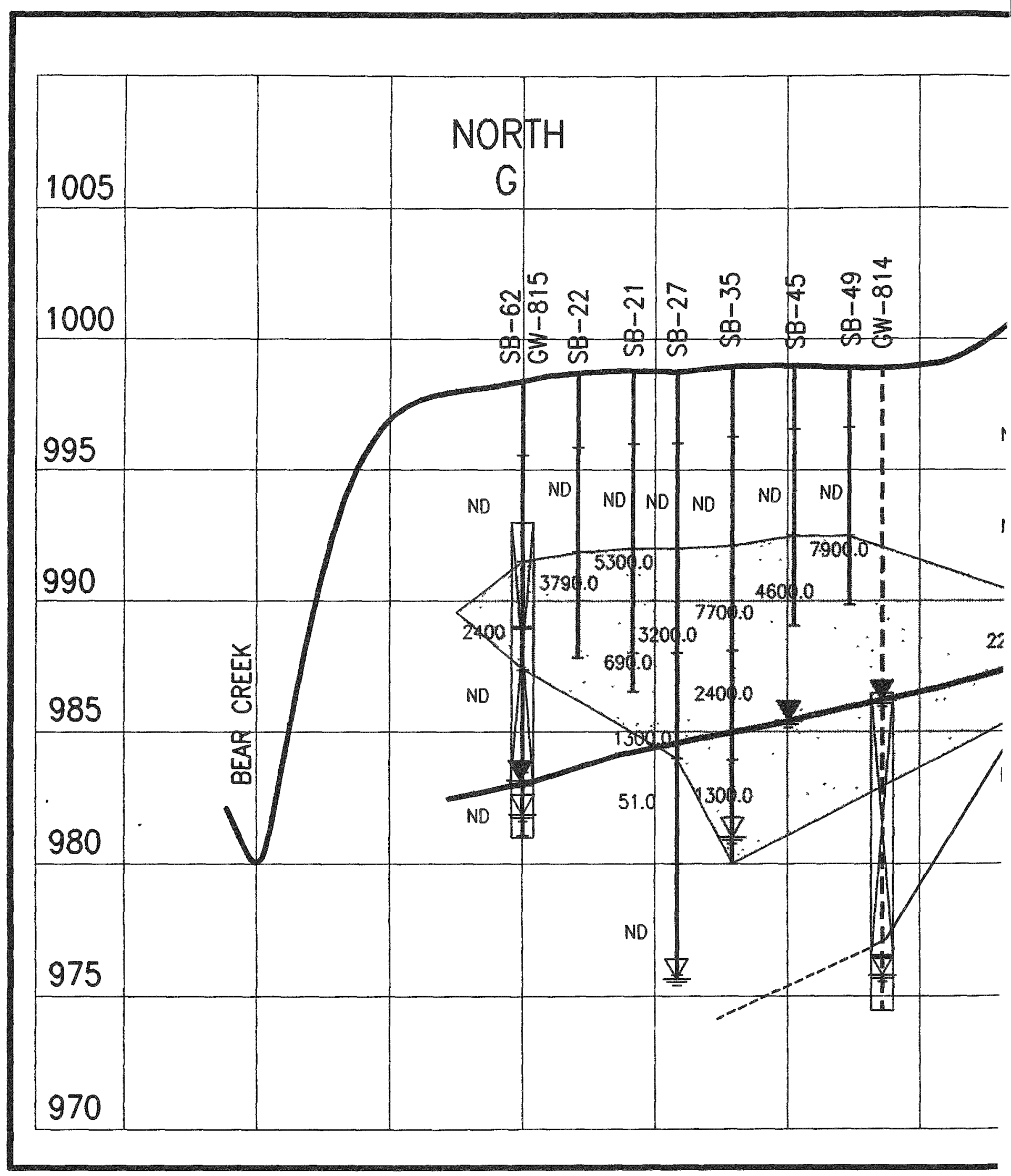




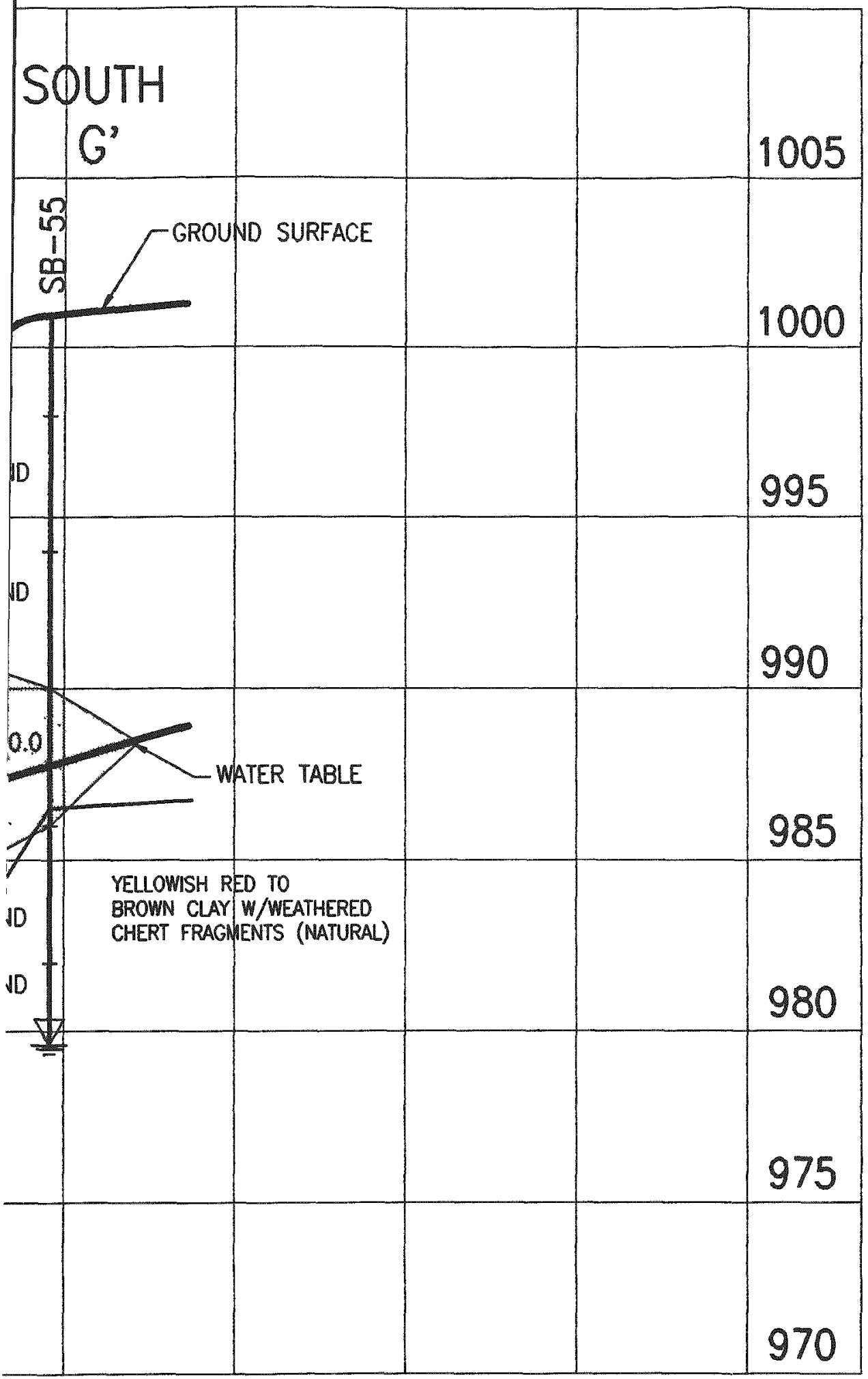

LEGEND:

GW GROUNOWATER WELL

SB ......................... SOILBORING ZGROUNDWATER ENCOUNTERED IN BOREHOLE I. GROUNDWATER TABLE

PROJECTED MONITORING WELL n. SCREENED INTERVAL

$>83$ PPB (PRG) (AROCLOR $1254 \& 1260$ ) NO ........................NOT OETECTED NA $\ldots \ldots \ldots \ldots \ldots \ldots \ldots \ldots$ NOT ANALYZED

012345 10

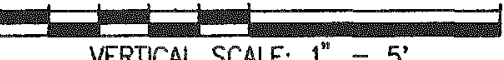

0 30 60 120

HORIZONTAL SCALE: $1^{\prime \prime}=60^{\circ}$

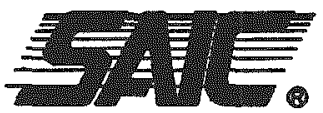

Science Applications International Corporation

BEAR CREEK VALLEY OU2

SY-200 SITE, PROFILE G-G' AROCLOR 1254 \& 1260 CONCEN.
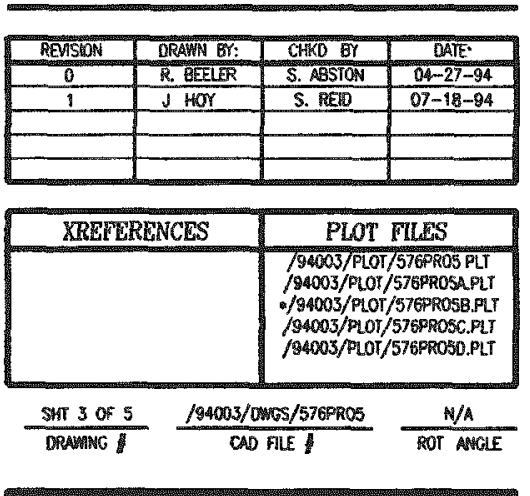


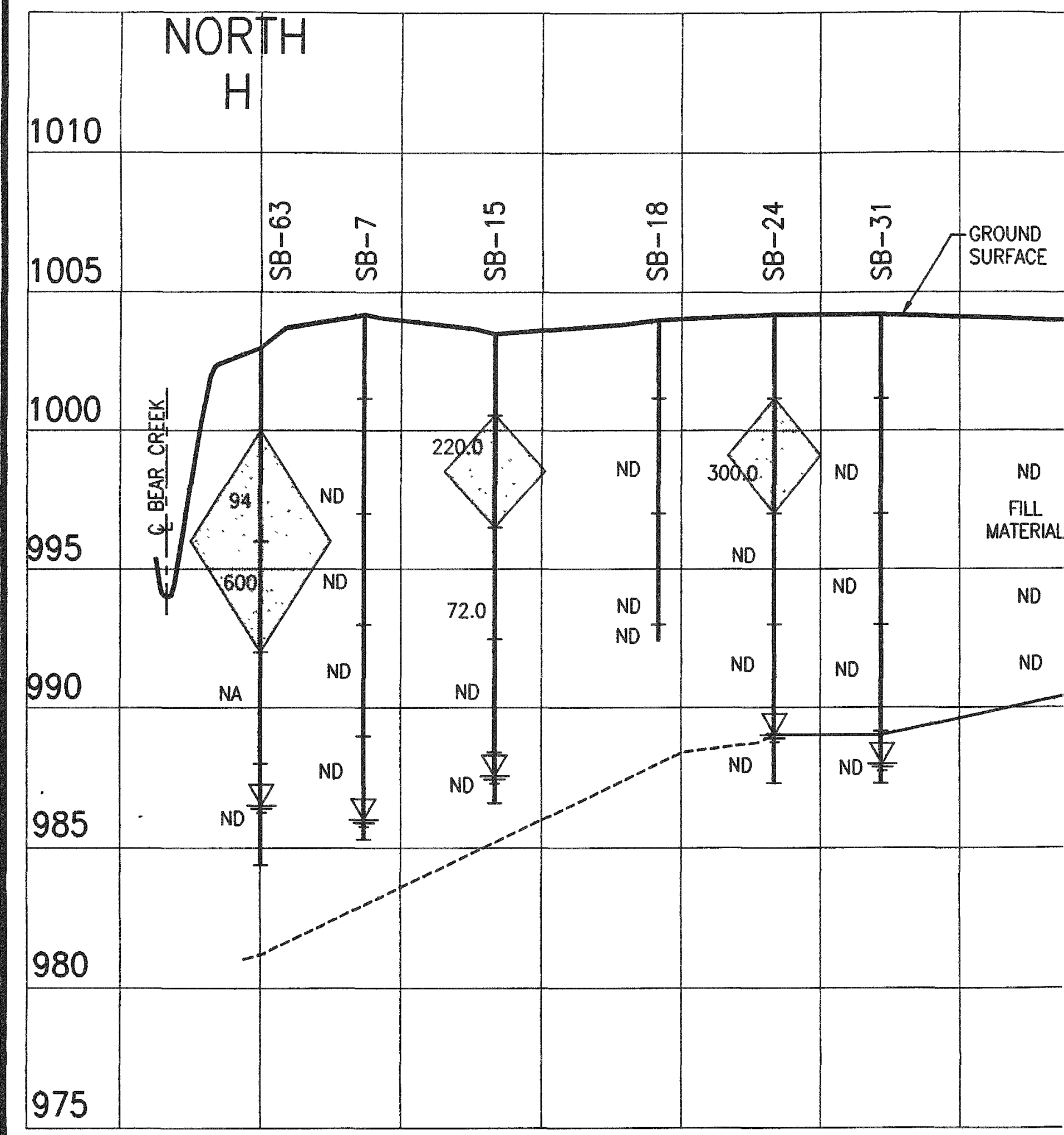




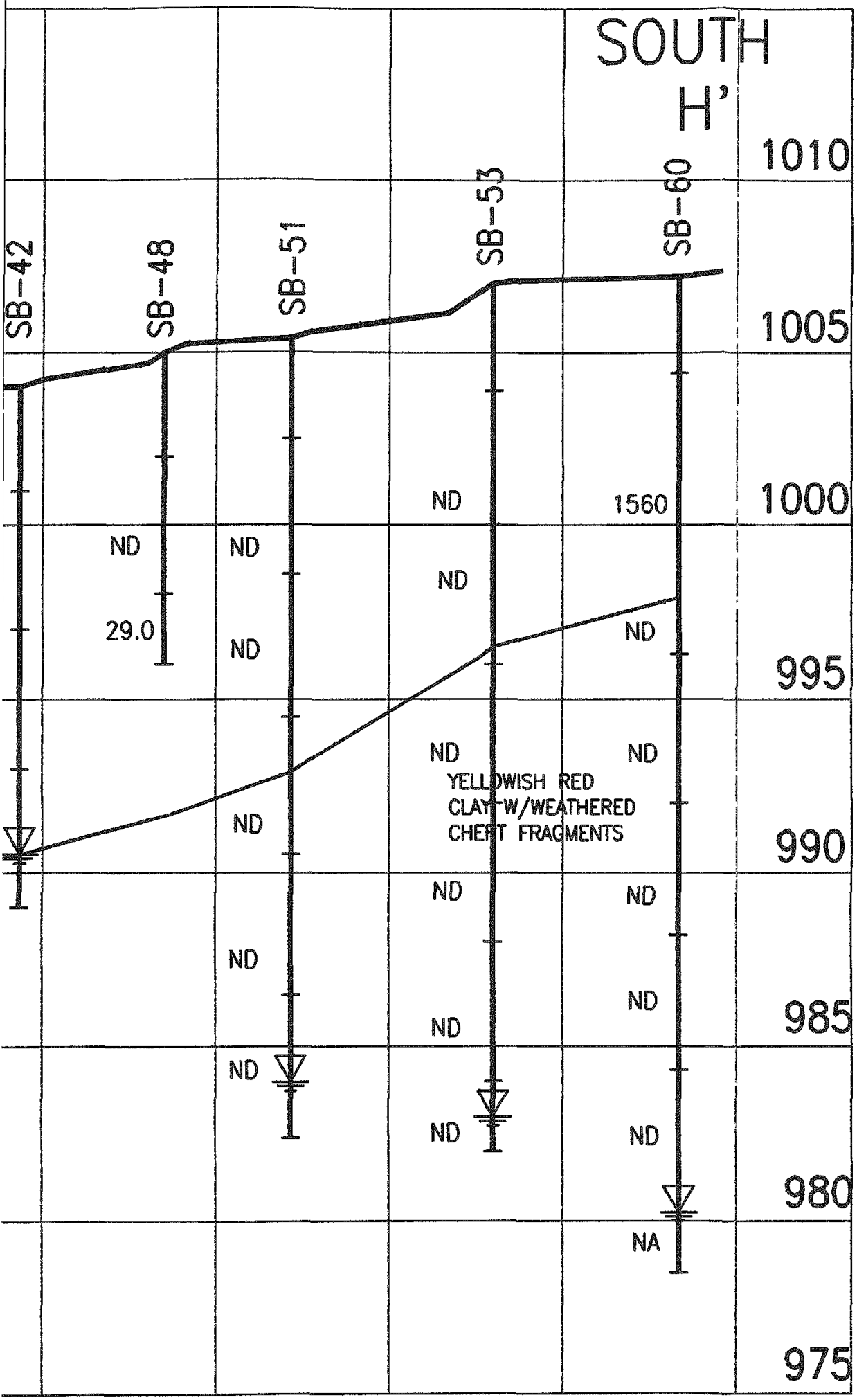

\section{LEGEND:}

GW...................MONITORING WELL

SB ........................ SOILBORING

УGROUNDWATER ENCOUNTERED IN BOREHOLE

$>83$ PPB (PRG) COMBINED

PCB'S (AROCLOR $1254 \& 1260$ )

ND....................... NOT DETECTED

NA $\ldots \ldots \ldots \ldots \ldots \ldots \ldots$ NOT ANALYZED

$\begin{array}{lllllll}0 & 1 & 2 & 3 & 4 & 5\end{array}$

VERTICAL SCALE: $1^{\prime \prime}=5^{\prime}$

$\begin{array}{lllll}0 & 10 & 20 & 30 & 60\end{array}$

HORIZONTAL SCALE: $1^{\prime \prime}=30^{\circ}$

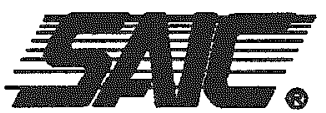

Science Applications International Corporation

BEAR CREEK VALLEY OU2

SY-200 YARD, PROFILE H-H' AROCLOR $1254 \& 1260$ CONCEN.
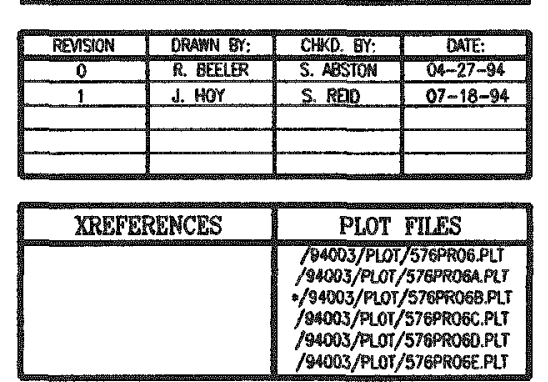

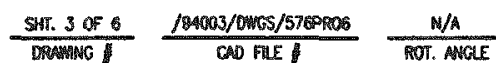


$$
\text { . }
$$ 


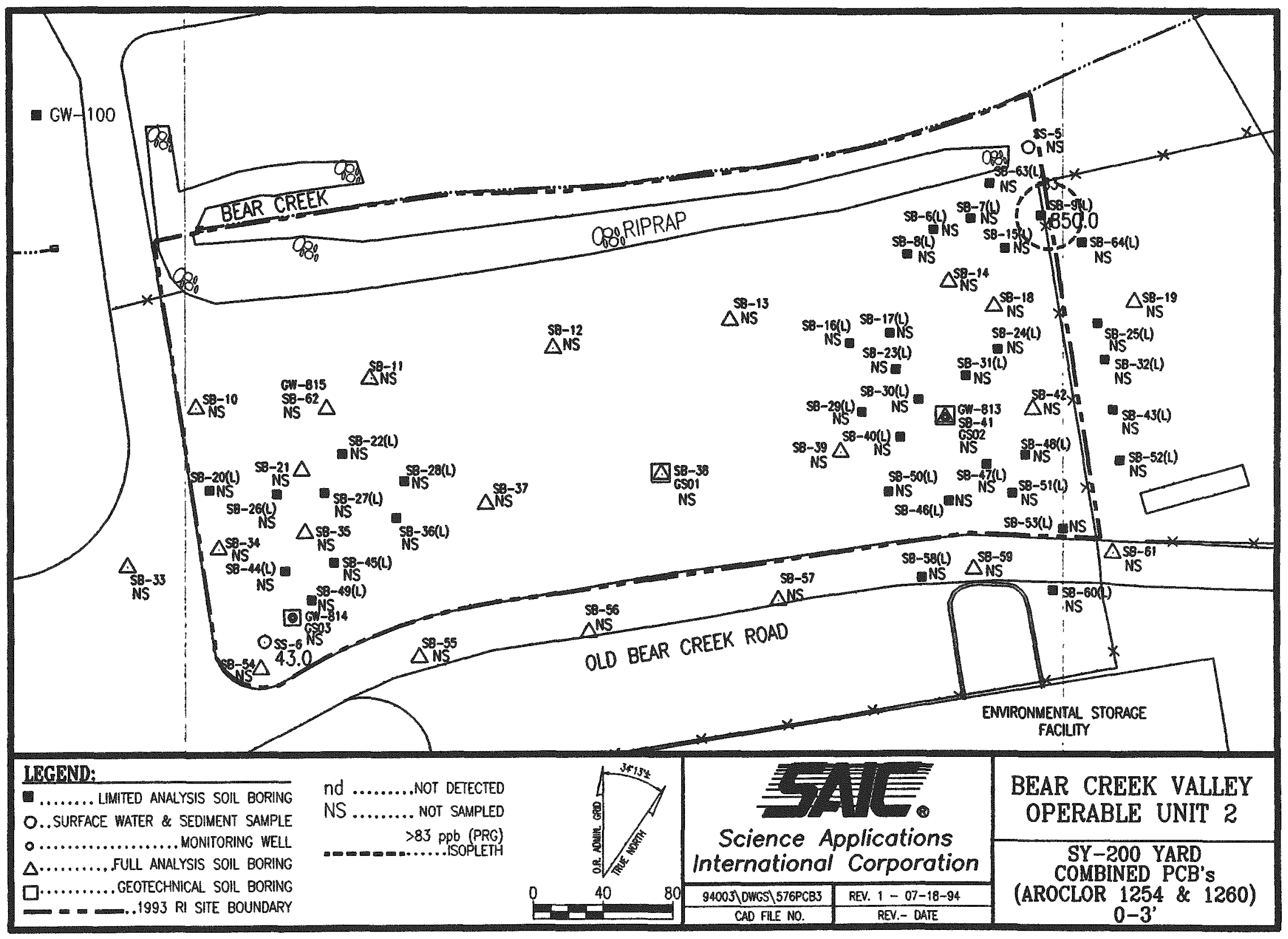




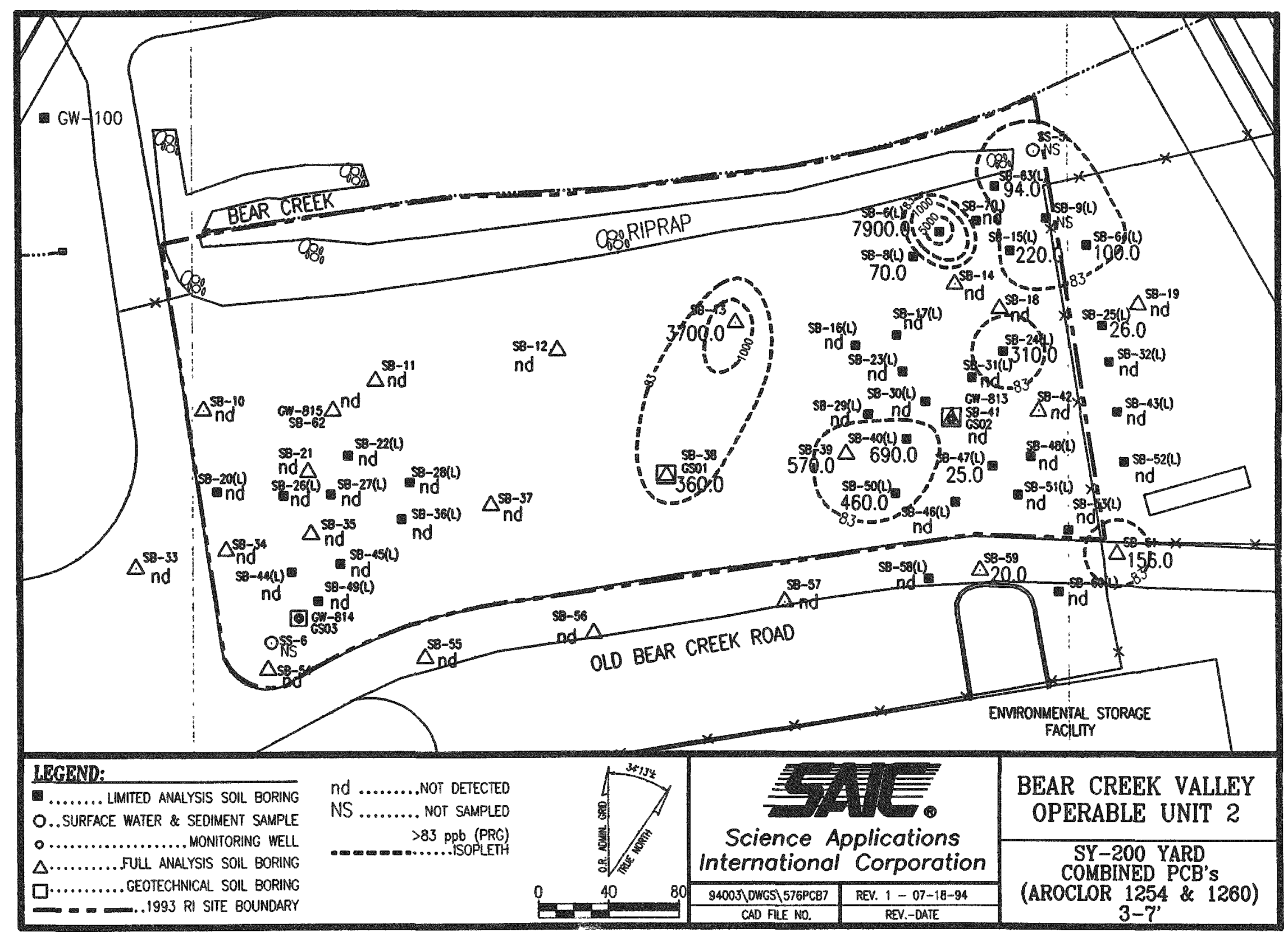




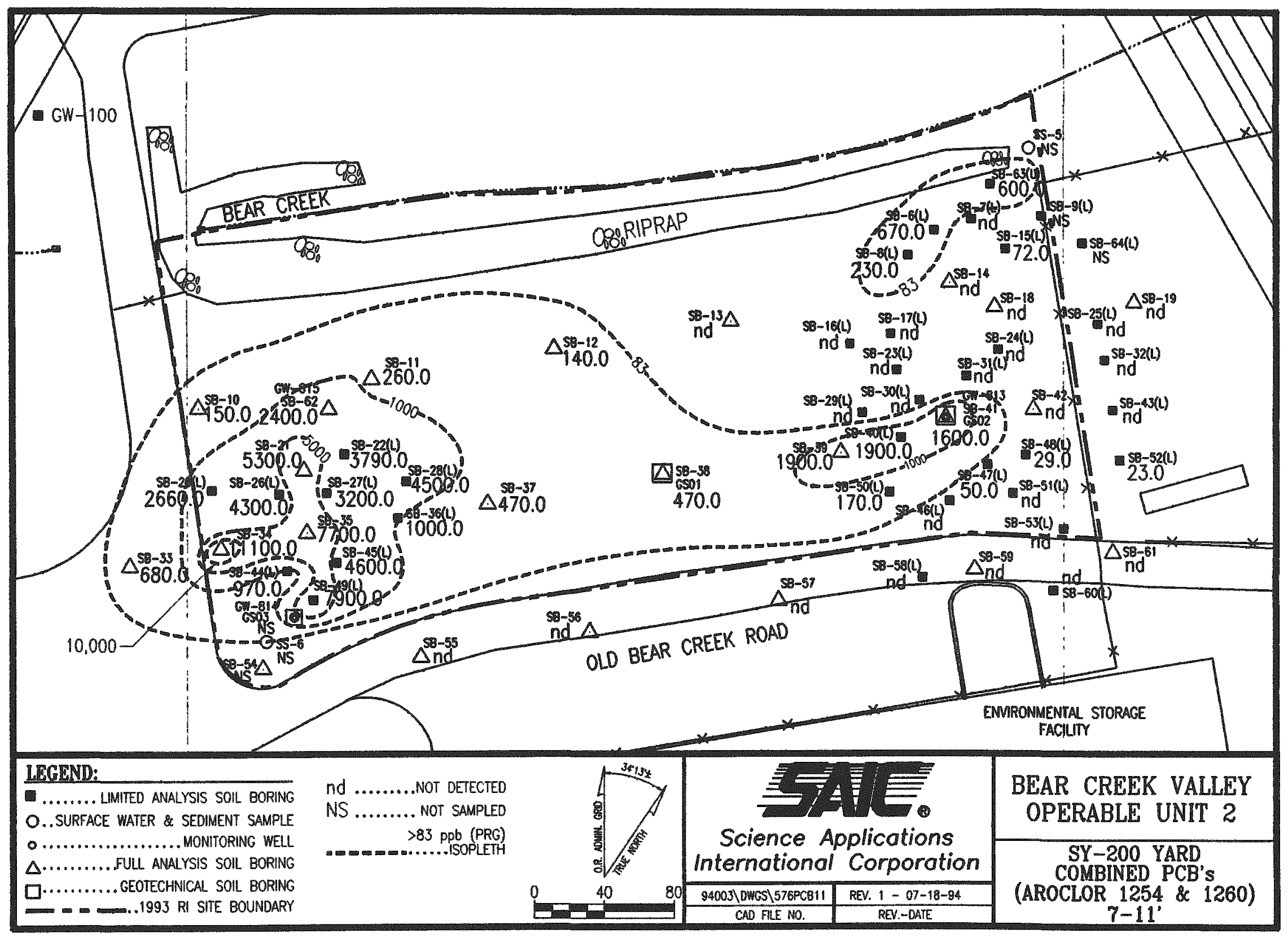




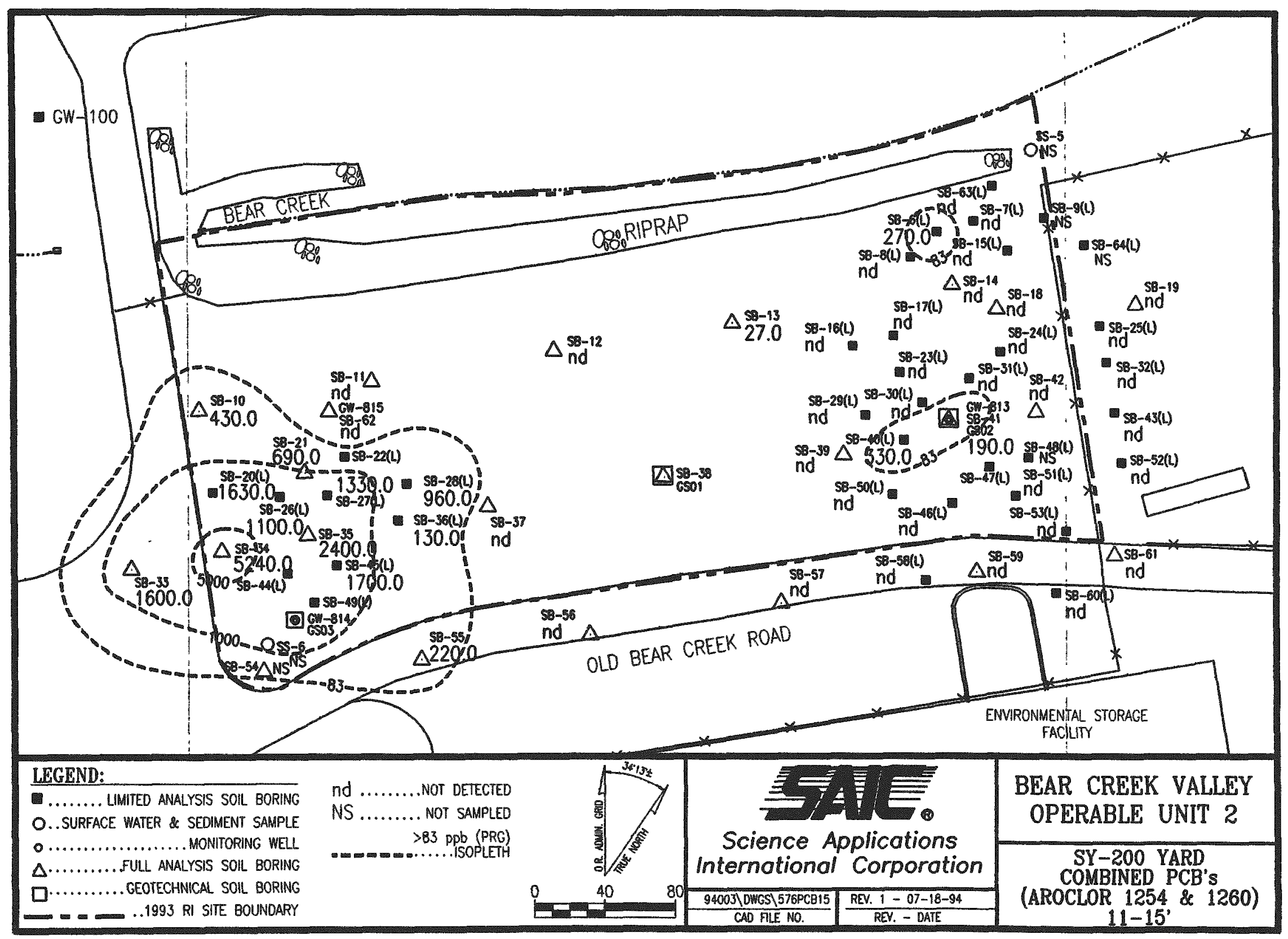




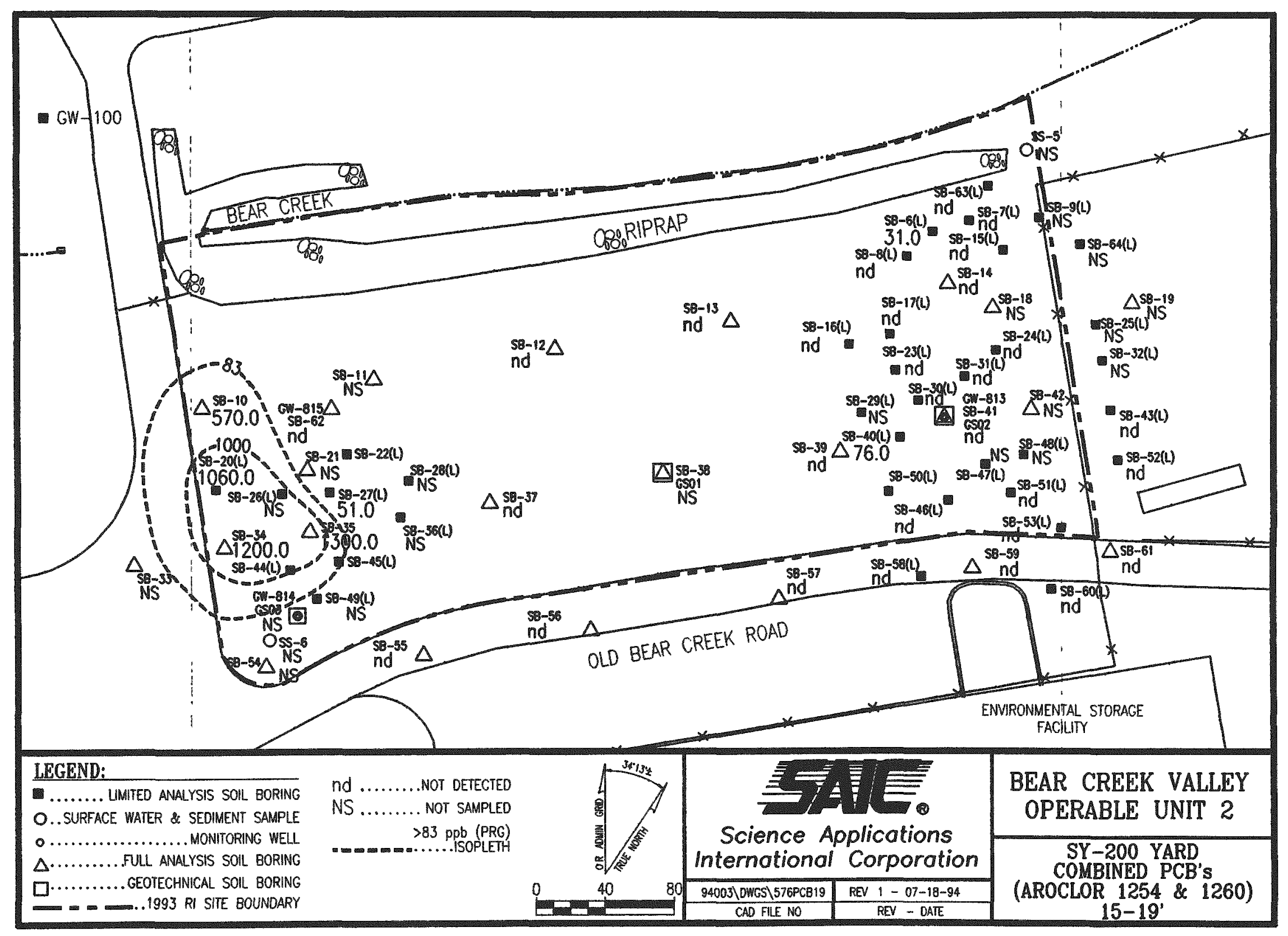




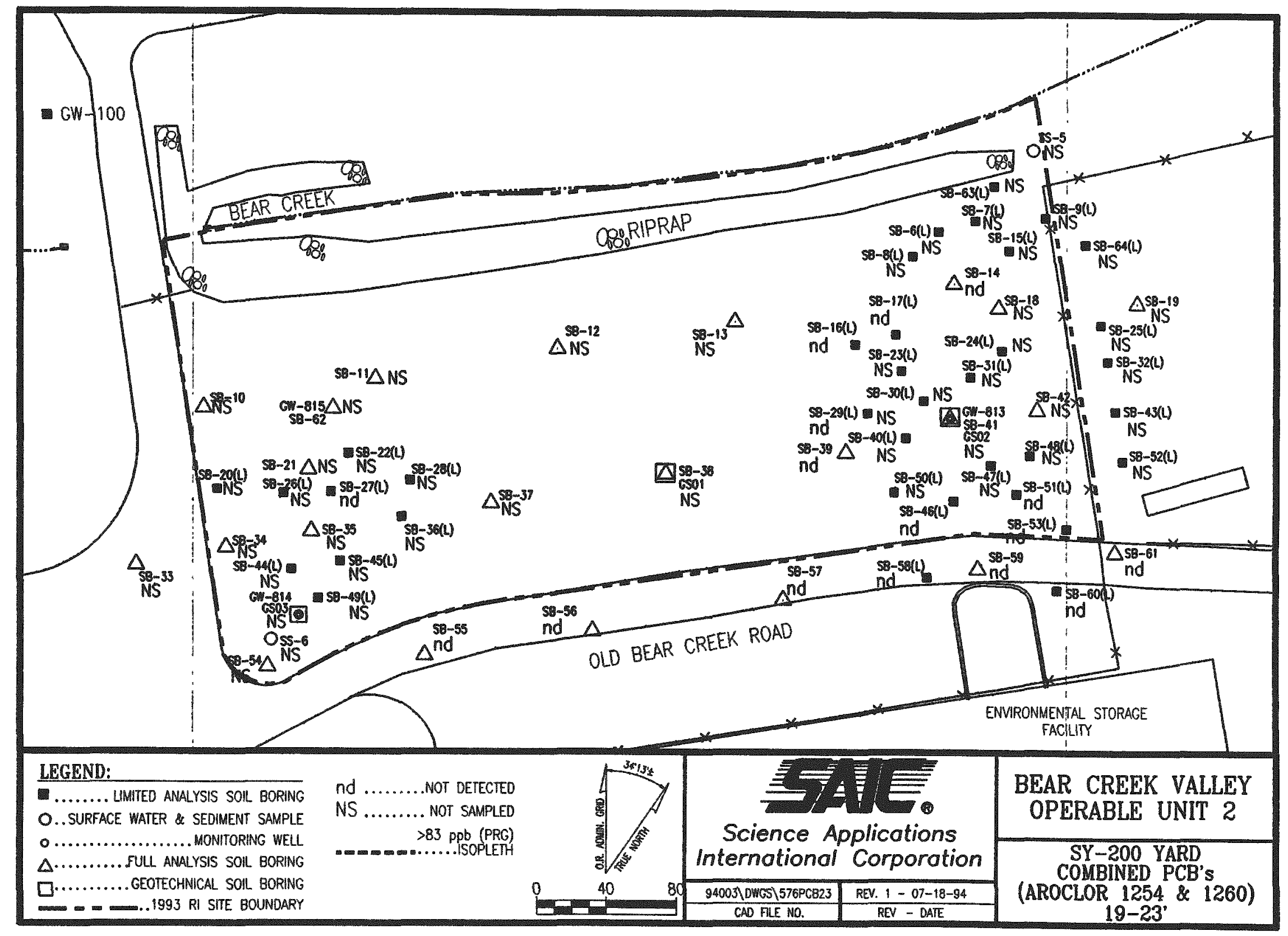




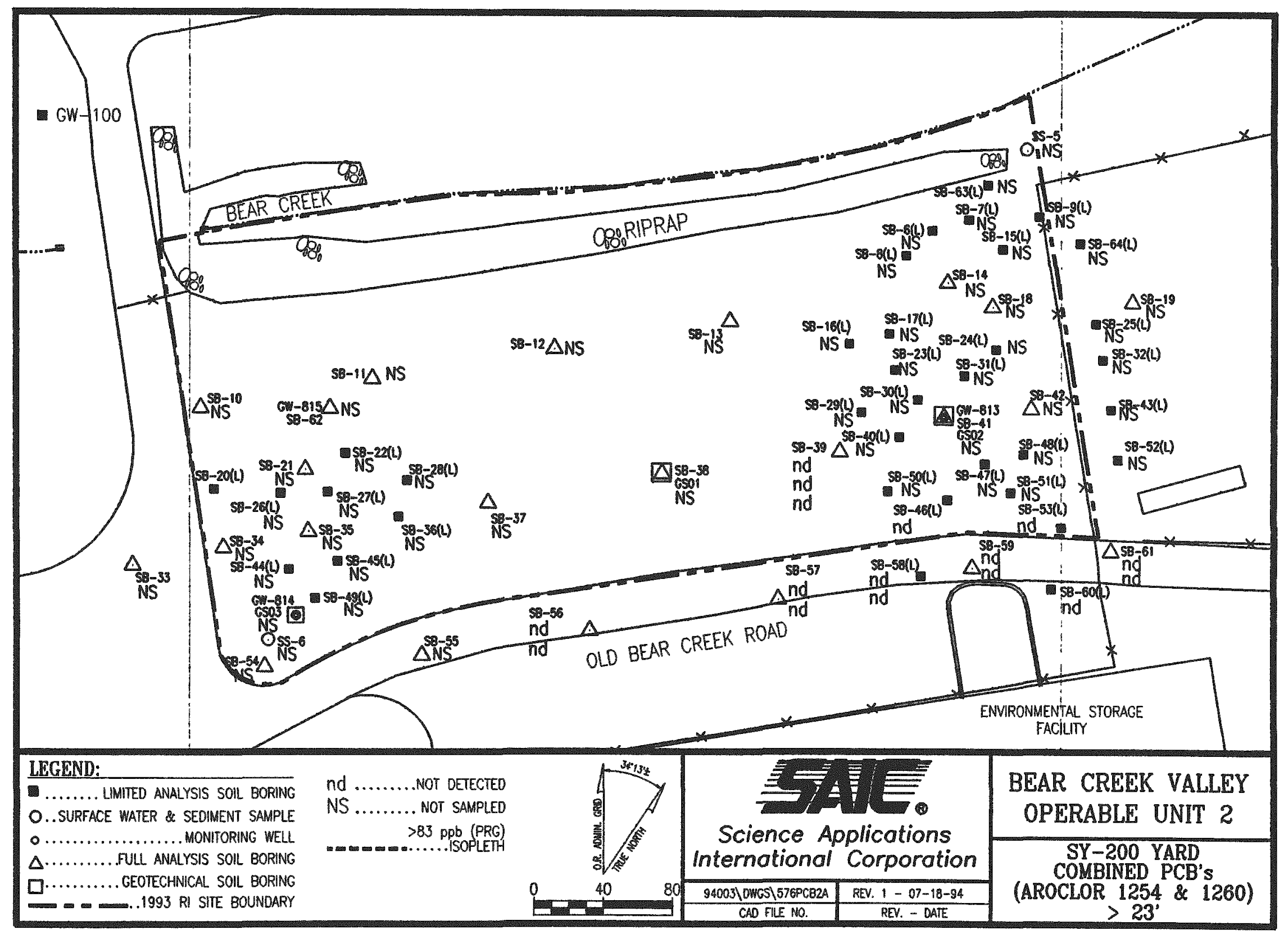




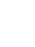




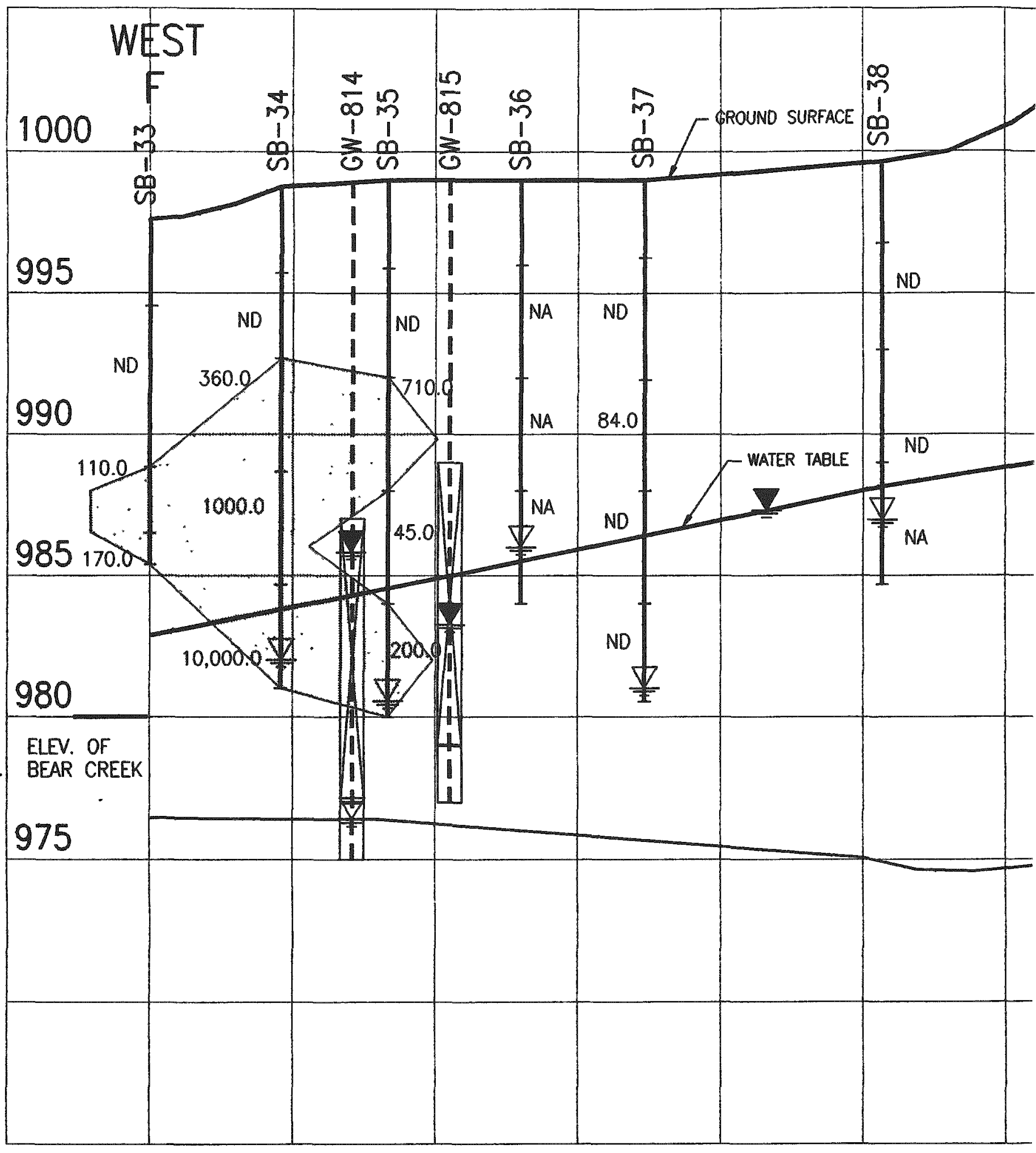




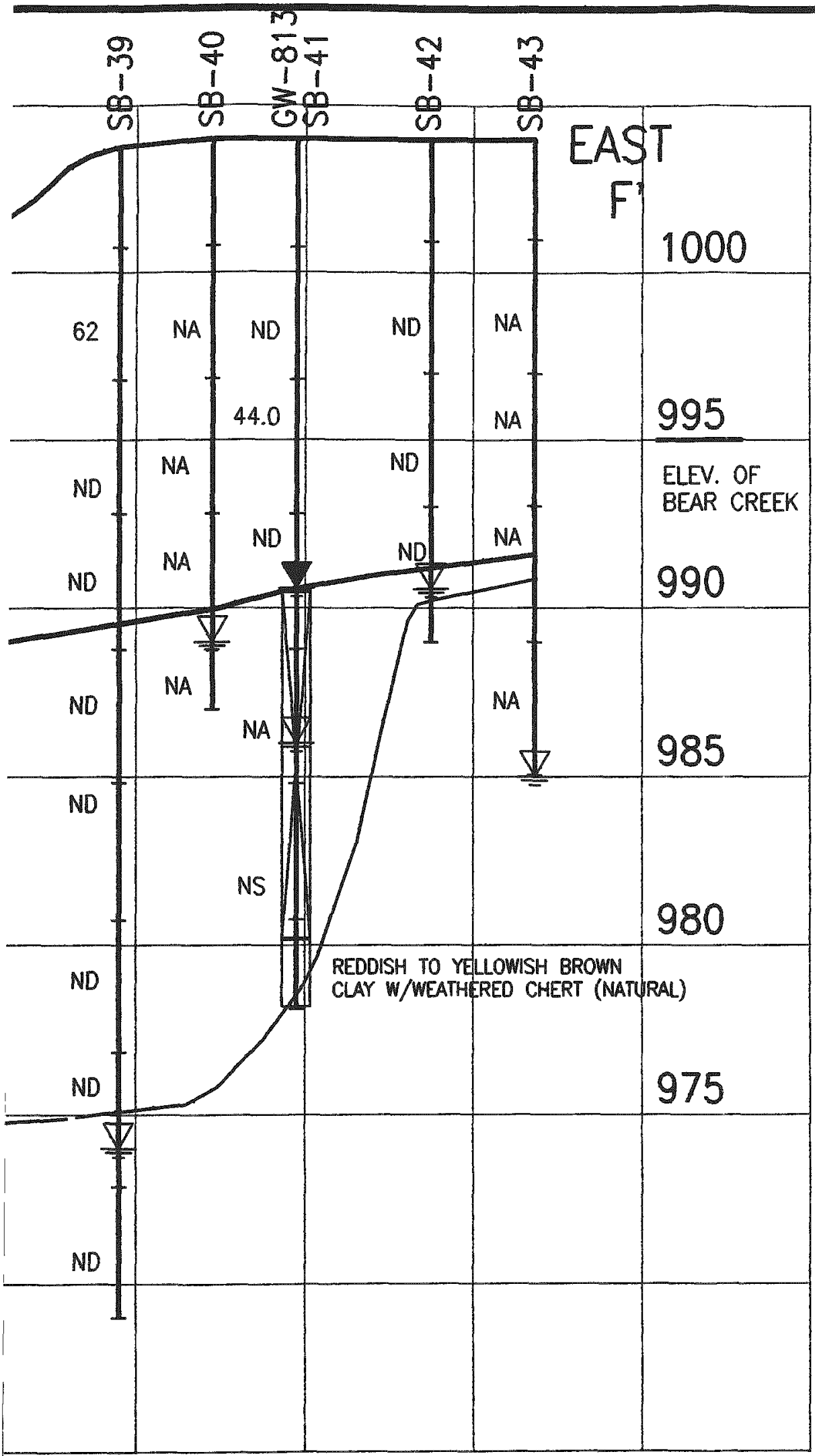

LEGEND:

GW.

.GROUNDWATER WELL

SB $\ldots \ldots \ldots \ldots \ldots \ldots \ldots \ldots \ldots$ SOLBORING

ZGROUNDWATER ENCOUNTERED IN BOREHOLE

I .................. GROUNOWATER TABLE

i

PROJECTED WELLS

W

SCREENED INTERVAL

Q....> 88 PPB (PRG) BENZO (A) PYRENE ND .................... NOT DETECTED

NA $\ldots \ldots \ldots \ldots \ldots \ldots \ldots \ldots$ NOT ANALYZED

NS.................... NOT SAMPLED

0

$\begin{array}{llllll}0 & 1 & 2 & 3 & 4 & 5\end{array}$

VERTICAL SCALE: $7^{n}=5^{\circ}$

0

30

60 120

2

HORIZONTAL SCALE: $1^{\prime \prime}=60^{\circ}$

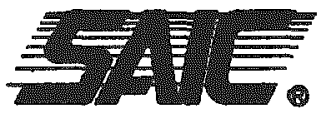

Science Applications International Corporation

BEAR CREEK VALLEY OU2 SY-200 SITE PROFILE F-F" BENZO (A) PYRENE CONCEN.
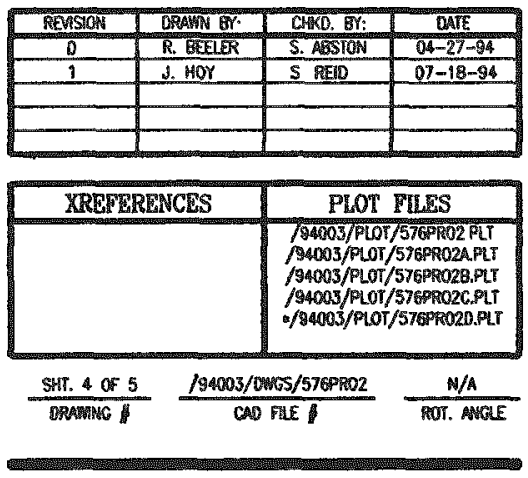


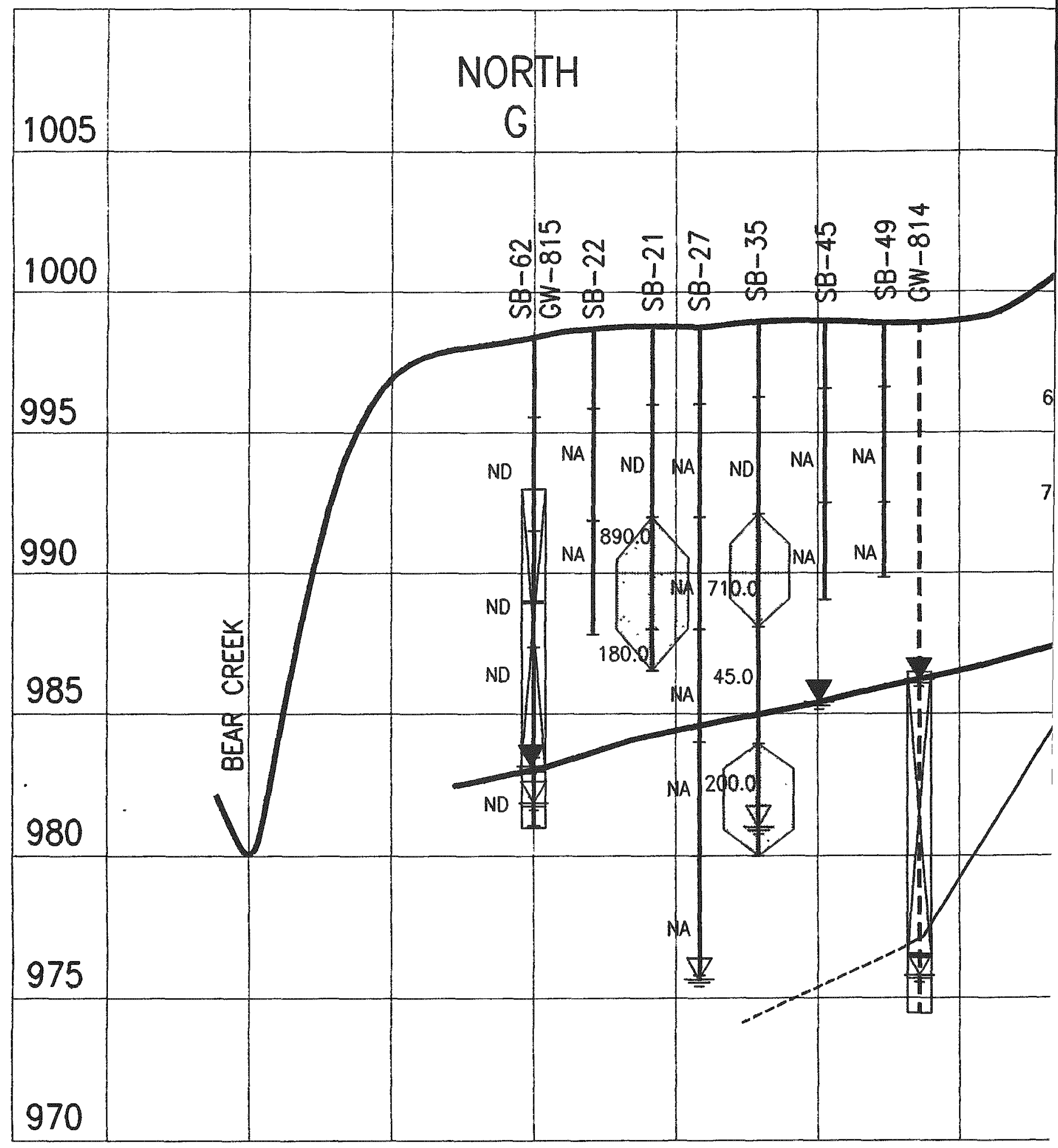




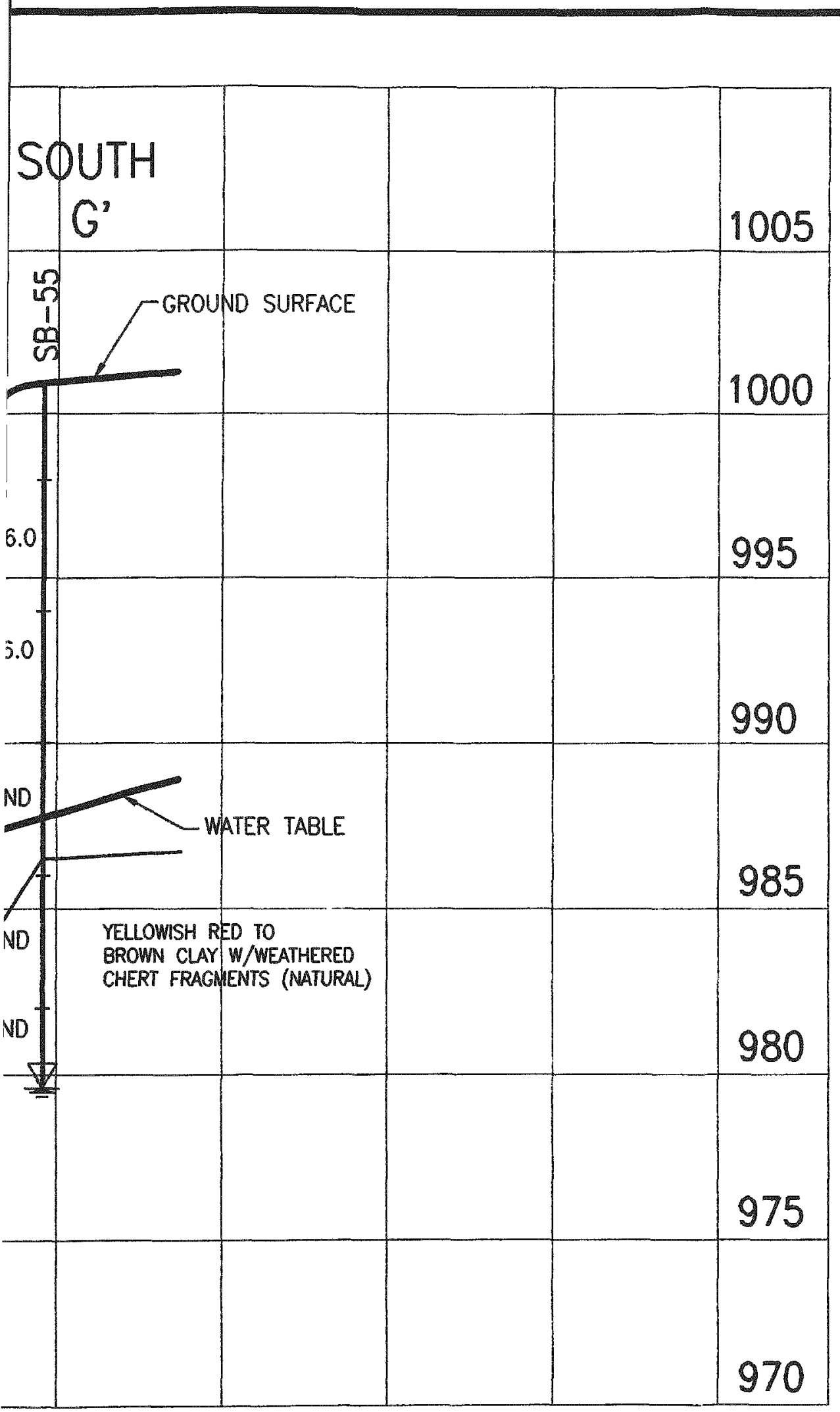

\section{LEGEND:}

GW..................GROUNDWATER WELL

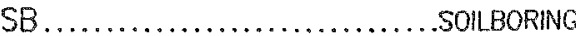
ZGROUNDWATER ENCOUNTERED IN BOREHOLE Viv . GROUNDWATER TABLE PROJECTED MONITORING WELL SCREENED INTERVAL ....> 88 PPB (PRG) BENZO (A) PYRENE ND $\ldots \ldots \ldots \ldots \ldots \ldots \ldots \ldots \ldots$ NOT DETECTED NA $\ldots \ldots \ldots \ldots$ NOT ANALYZED

$\begin{array}{lllllll}0 & 1 & 2 & 3 & 4 & 5 & 10\end{array}$ VERTICAL SCALE: $1^{\prime \prime}=5^{\prime}$ $\begin{array}{lrr}0 & 30 & 60\end{array}$ $\begin{array}{rrr}0 \quad 30 & 60 & 120\end{array}$ $\begin{array}{lrr}0 & 30 & 60\end{array}$ HORIZONTAL SCALE: $9^{\prime \prime}=60^{\prime}$

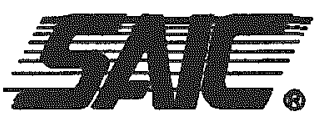

Science Applications International Corporation

BEAR CREEK VALLEY OU2 SY-200 SITE, PROFILE G-G' BENZO (A) PYRENE CONCEN.
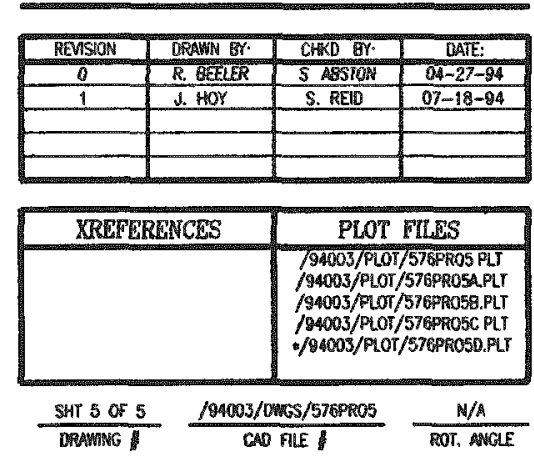


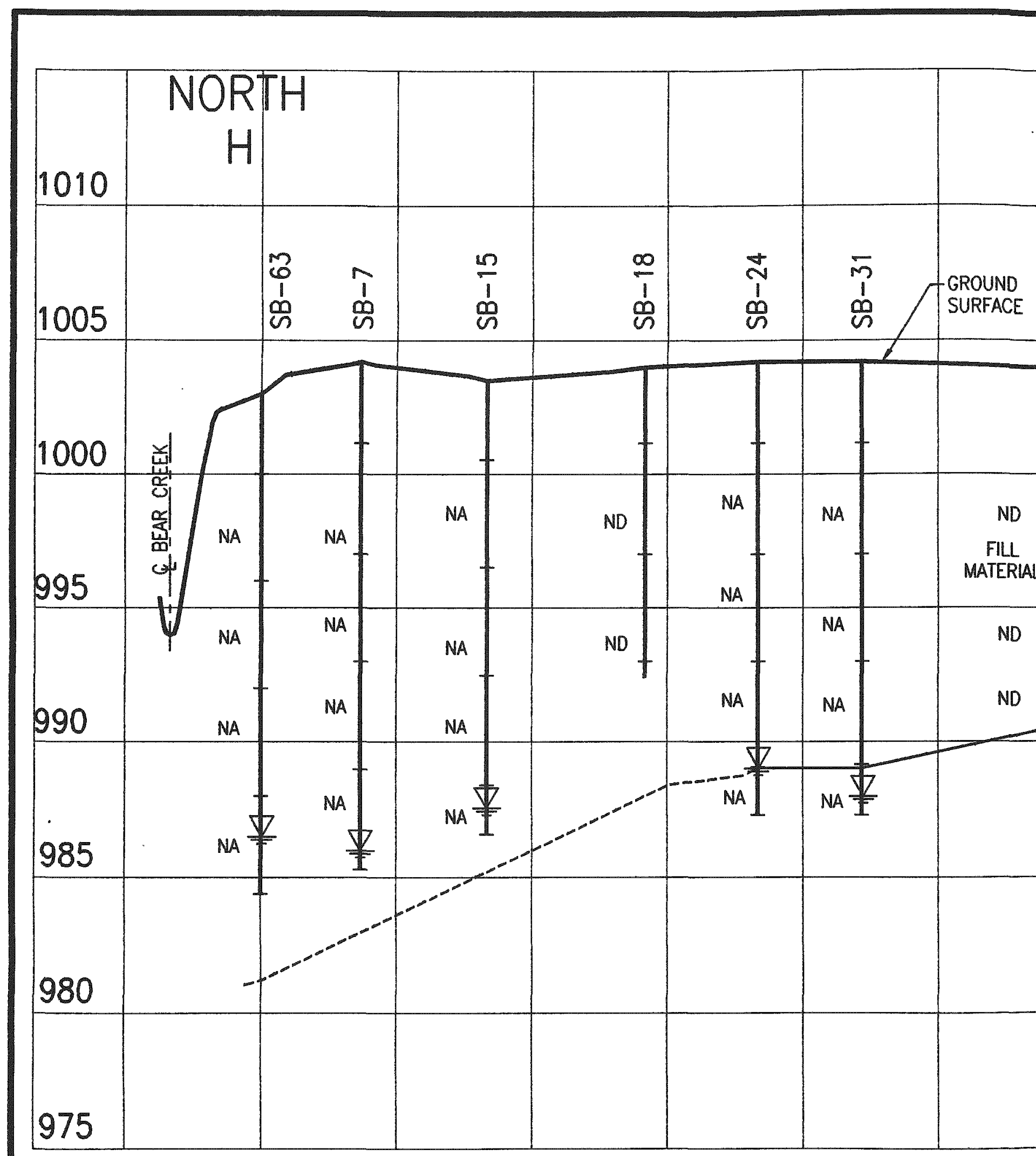




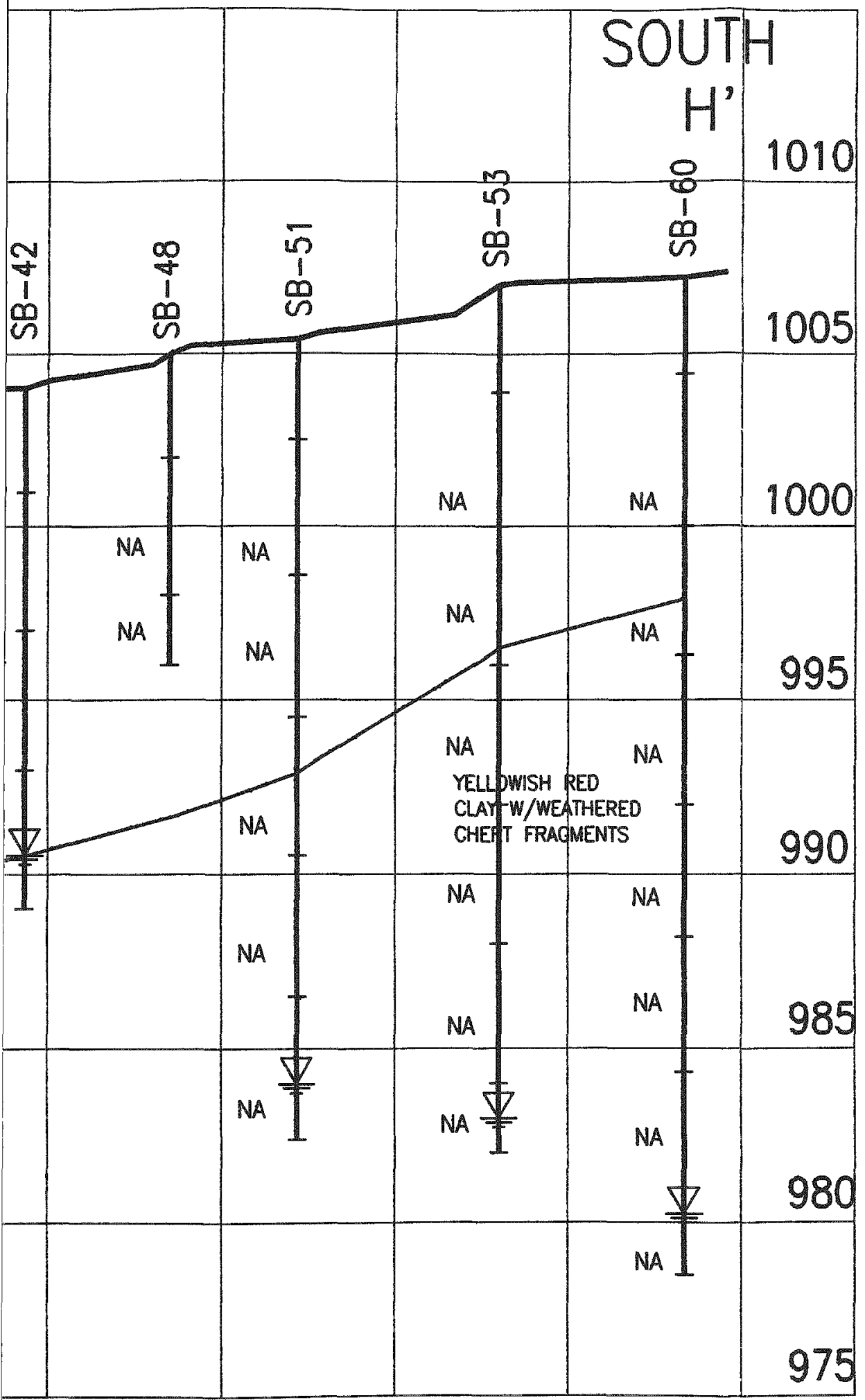

LEGEND:

GW................... MONITORING WELL

SB $\ldots \ldots \ldots \ldots \ldots \ldots \ldots \ldots$ SOILBORING

忞GROUNDWATER ENCOUNTERED IN BOREHOLE $\checkmark \ldots . .>88$ PPB (PRG) BENZO (A) PYRENE ND ....................... NOT DETECTED NA $\ldots . . . \ldots \ldots \ldots . . . . . . . . .$. NOT ANALYZED

$\begin{array}{llllll}0 & 1 & 2 & 3 & 4 & 5\end{array}$ 10 VERTICAL SCALE: $1^{\prime \prime}=5^{\prime}$

$\begin{array}{llll}0 & 10 & 20 & 30\end{array}$ 60

HORIZONTAL SCALE: $1^{\prime \prime}=30^{\circ}$

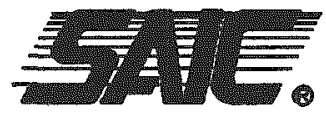

Science Applications International Corporation

BEAR CREEK VALLEY OU2 SY-200 YARD, PROFILE H-H' BENZO (A) PYRENE CONCEN.
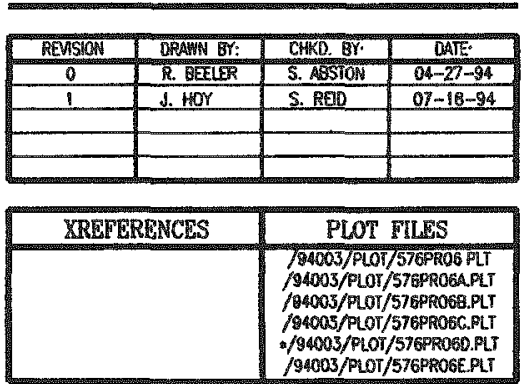

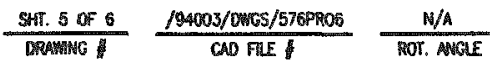


$$
\text { . }
$$ 


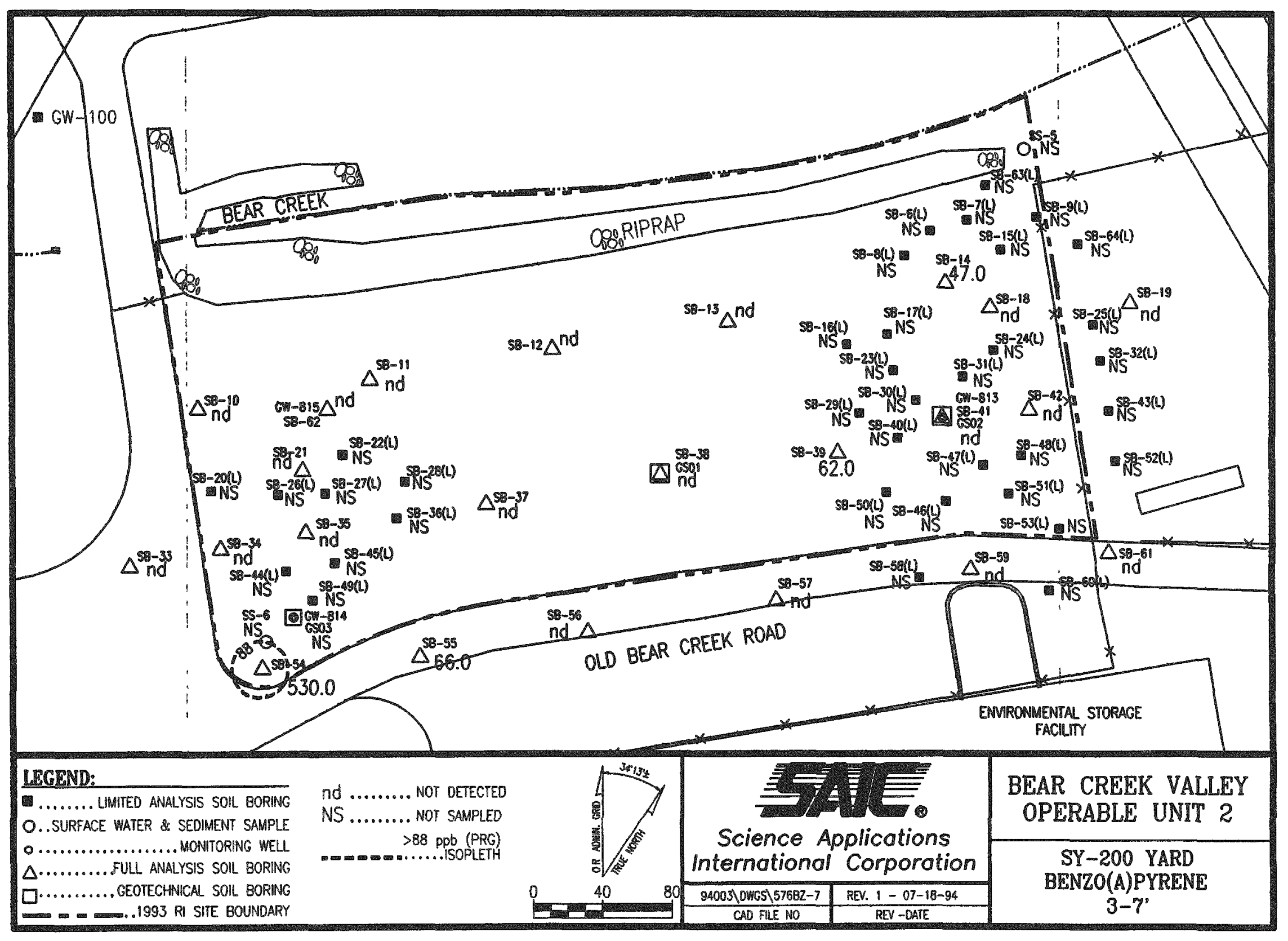




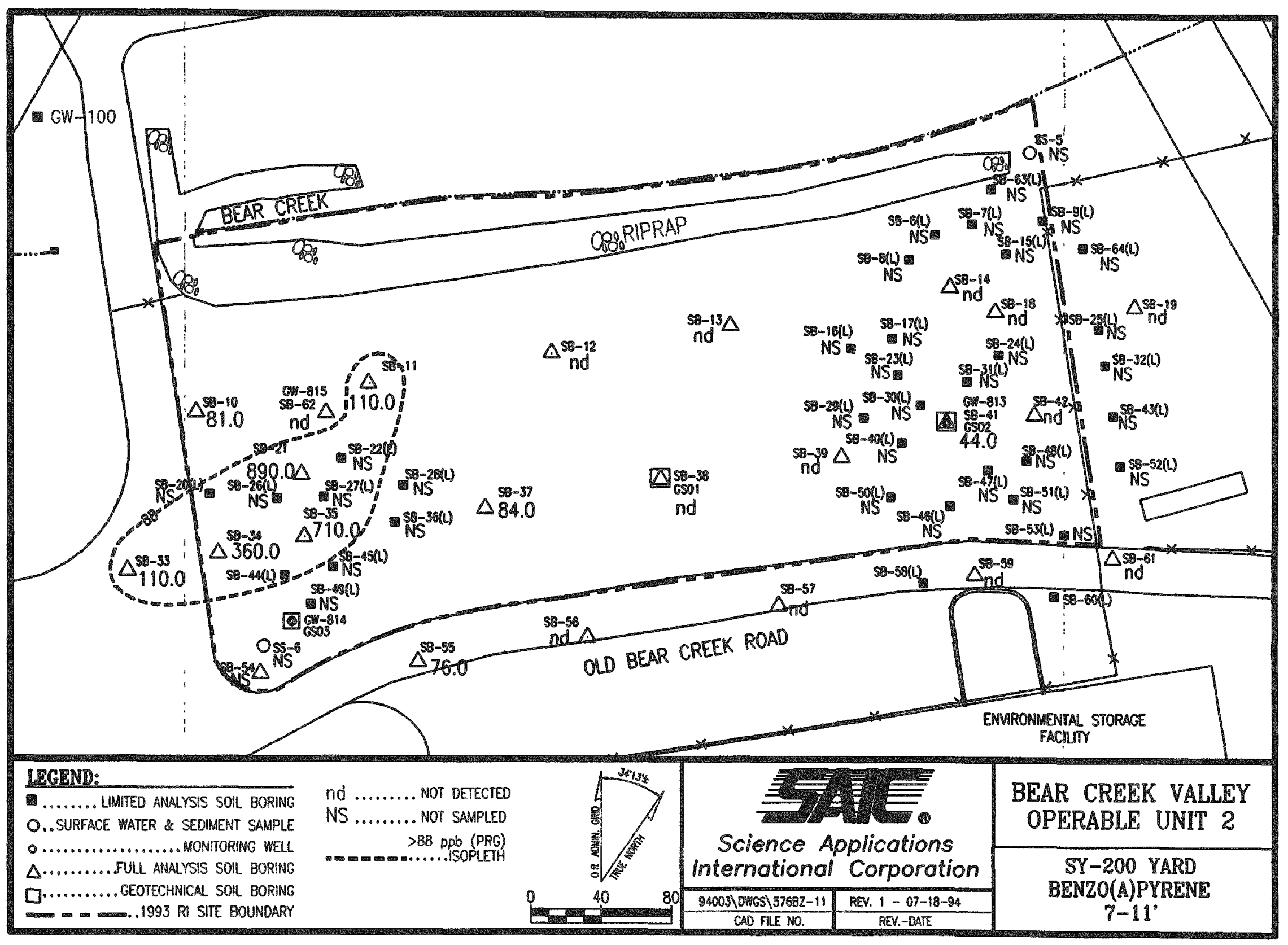




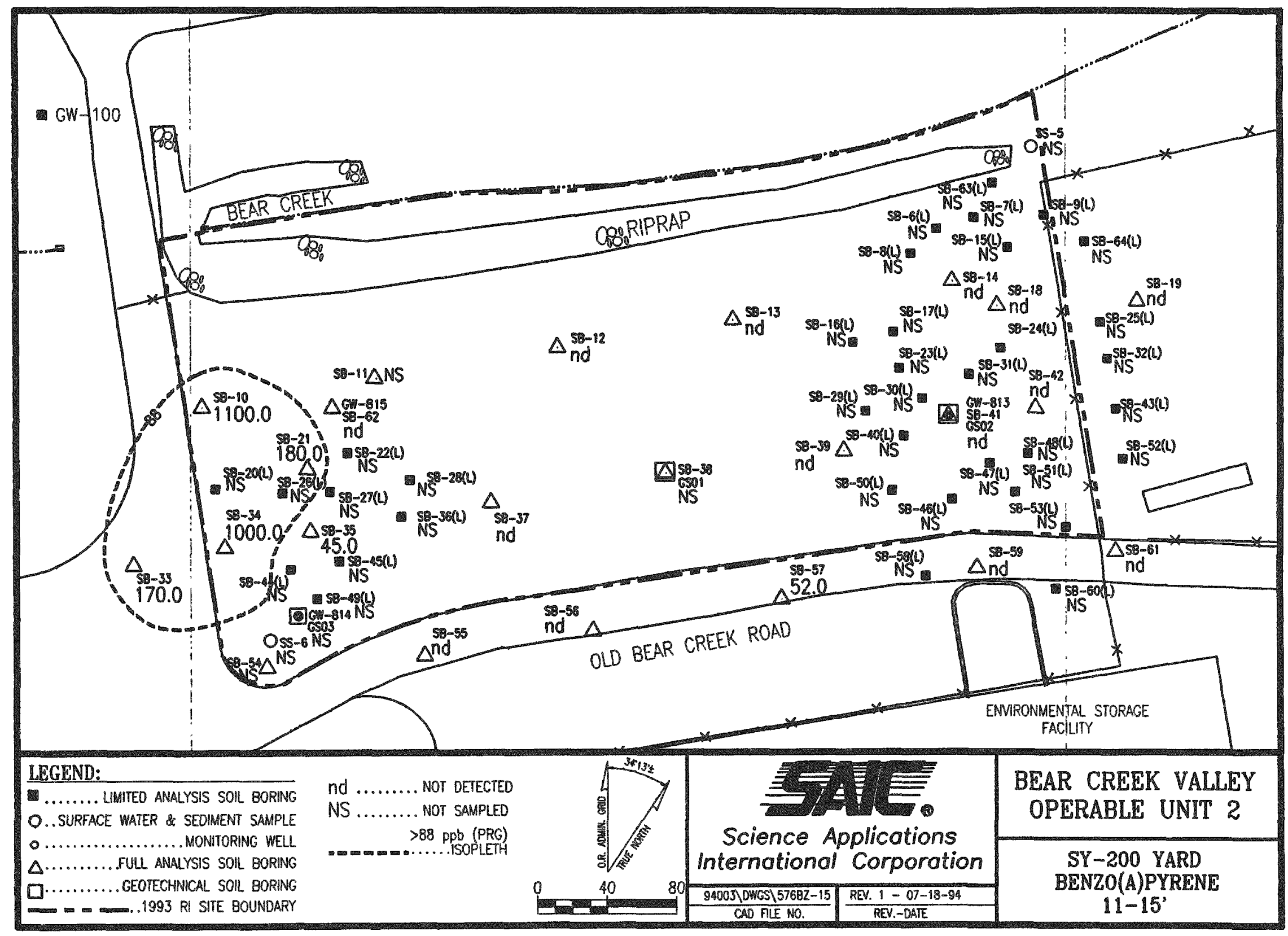




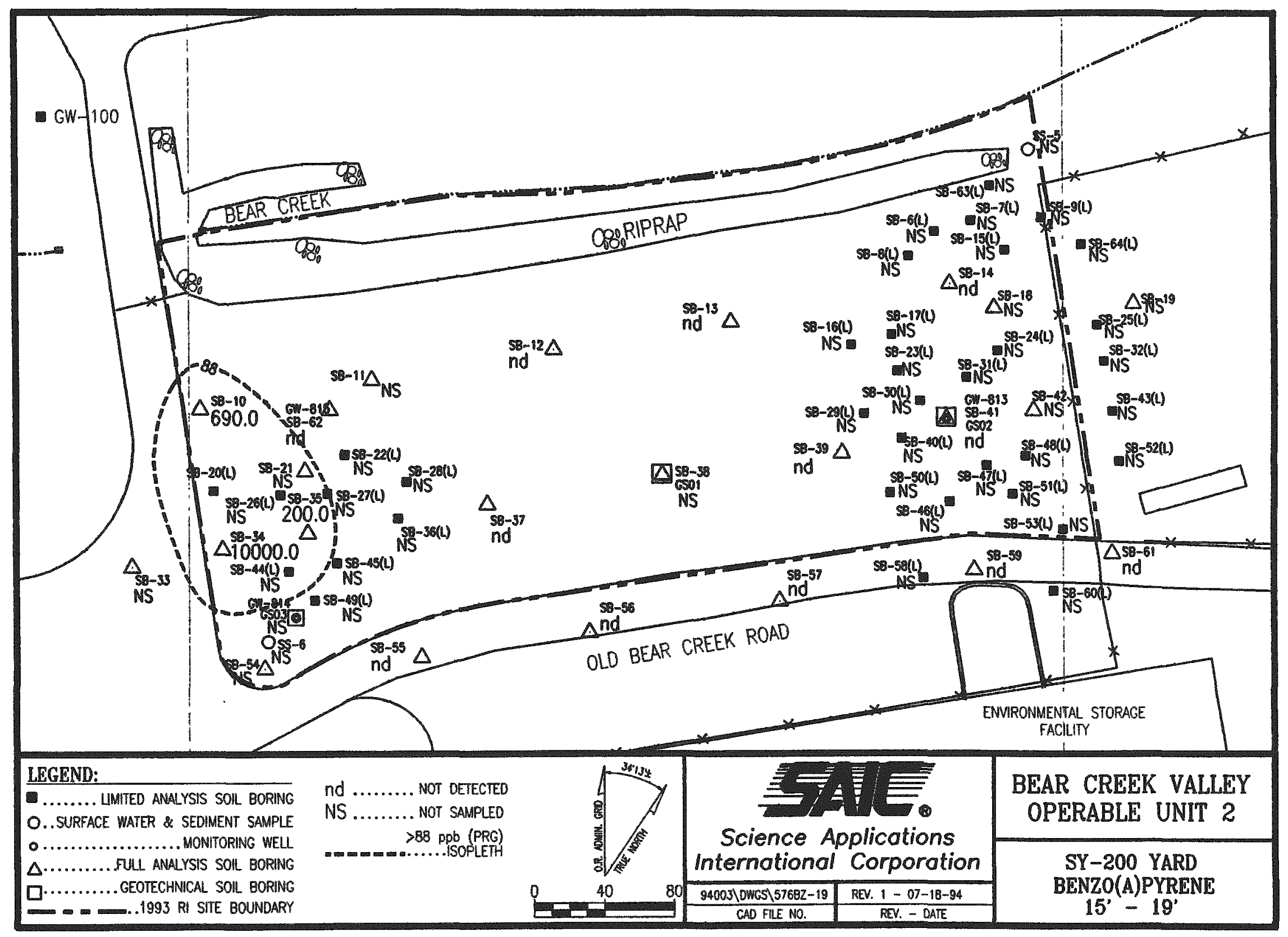




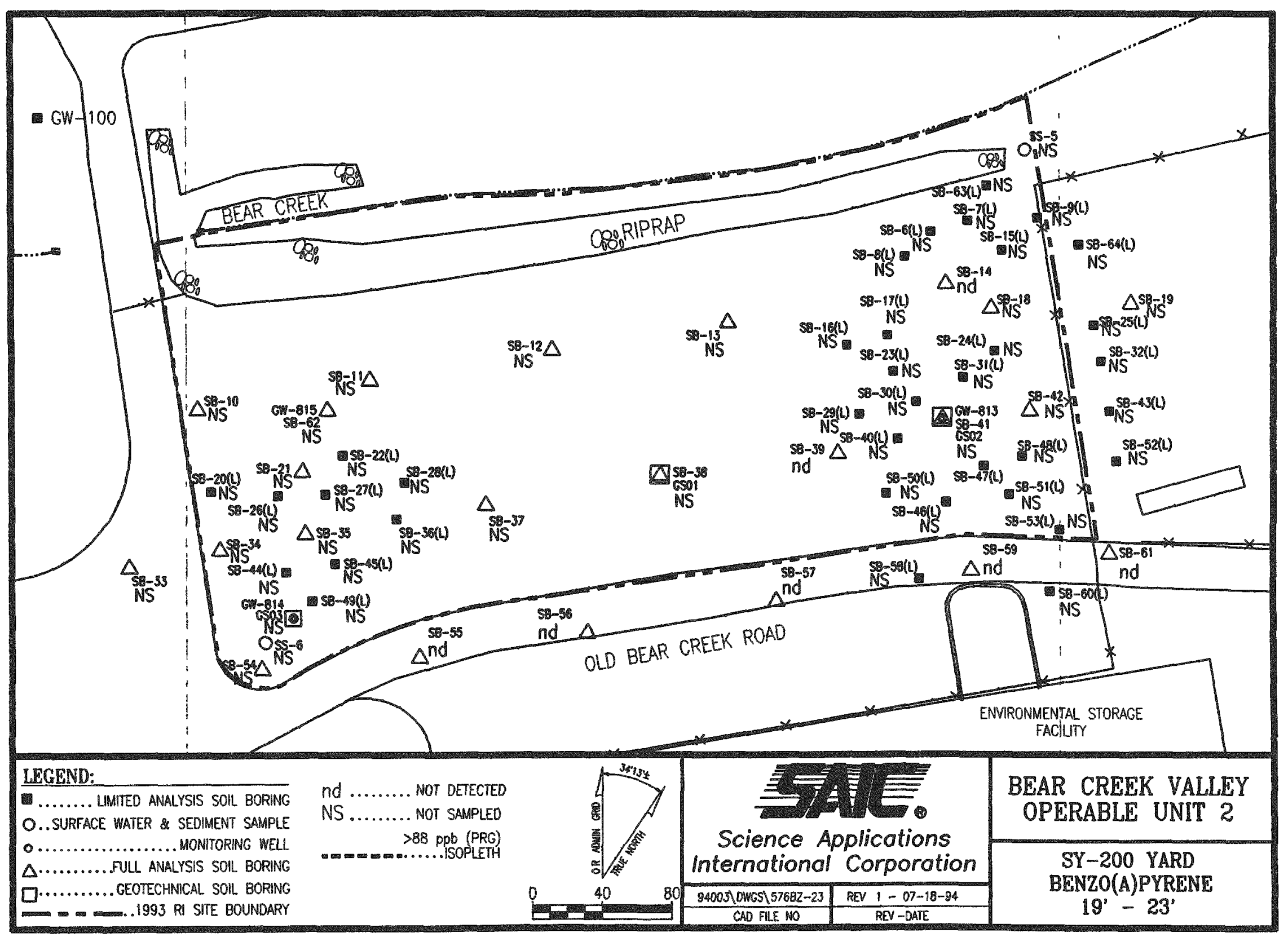





\section{RSA Figures}





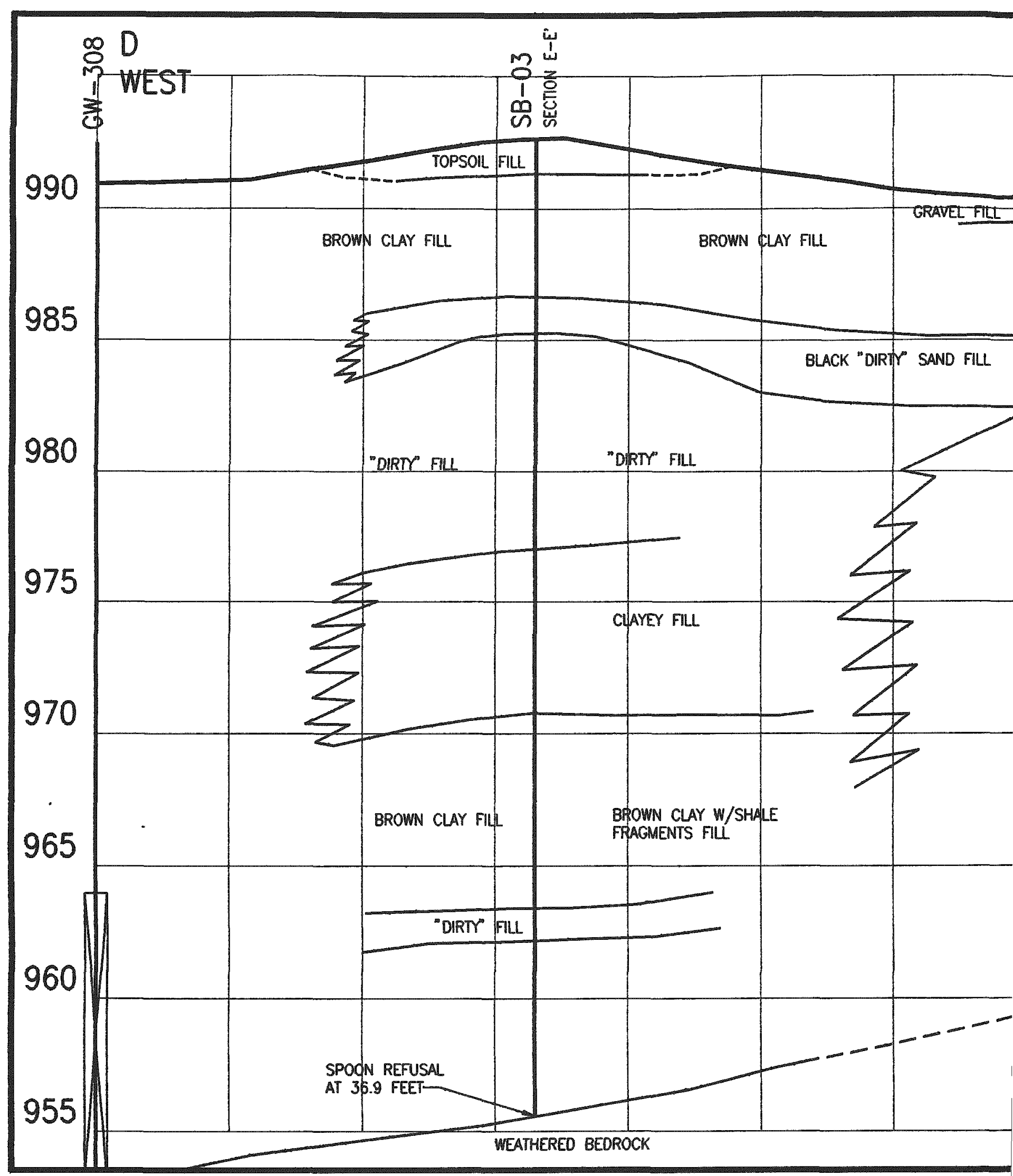




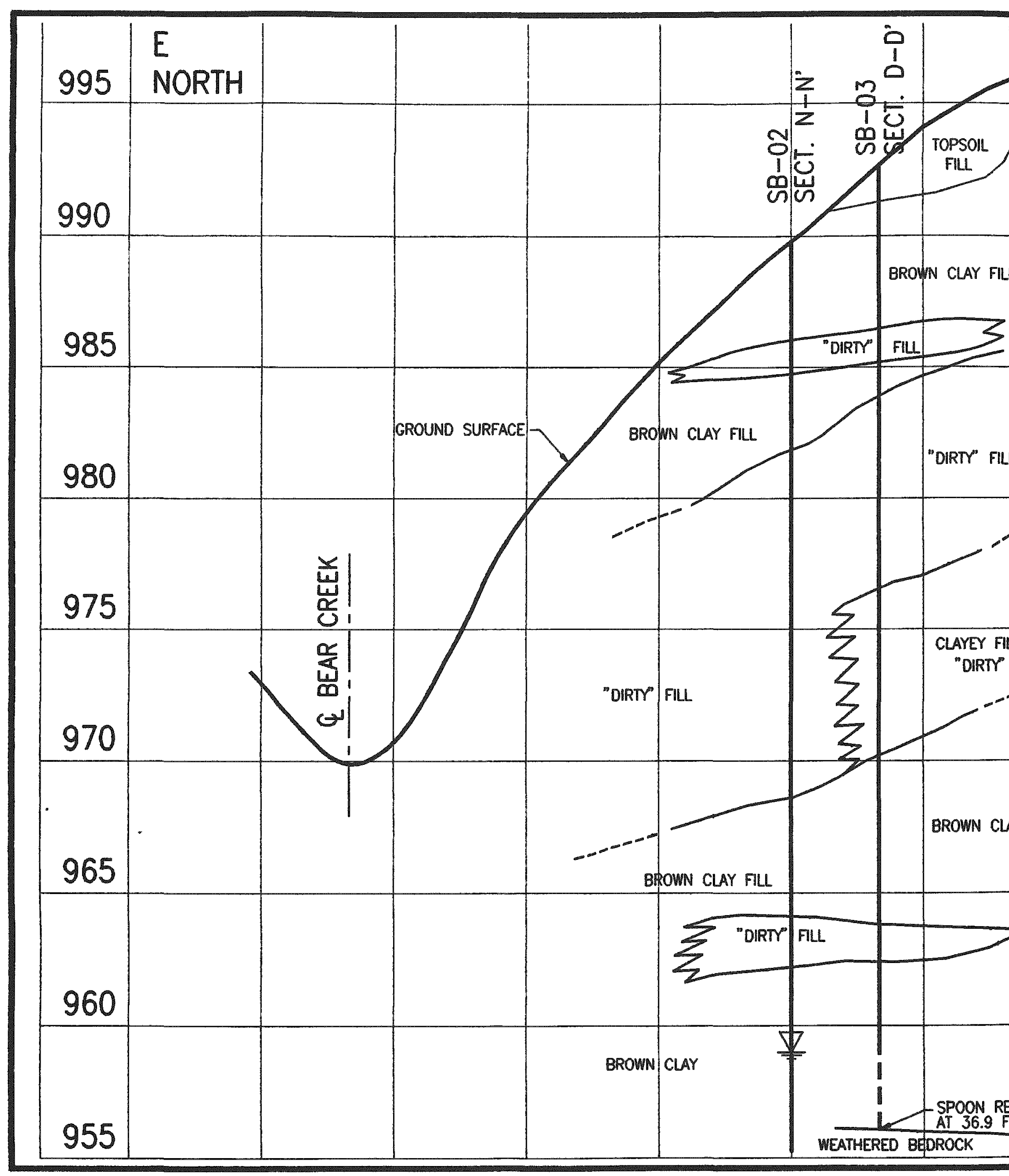




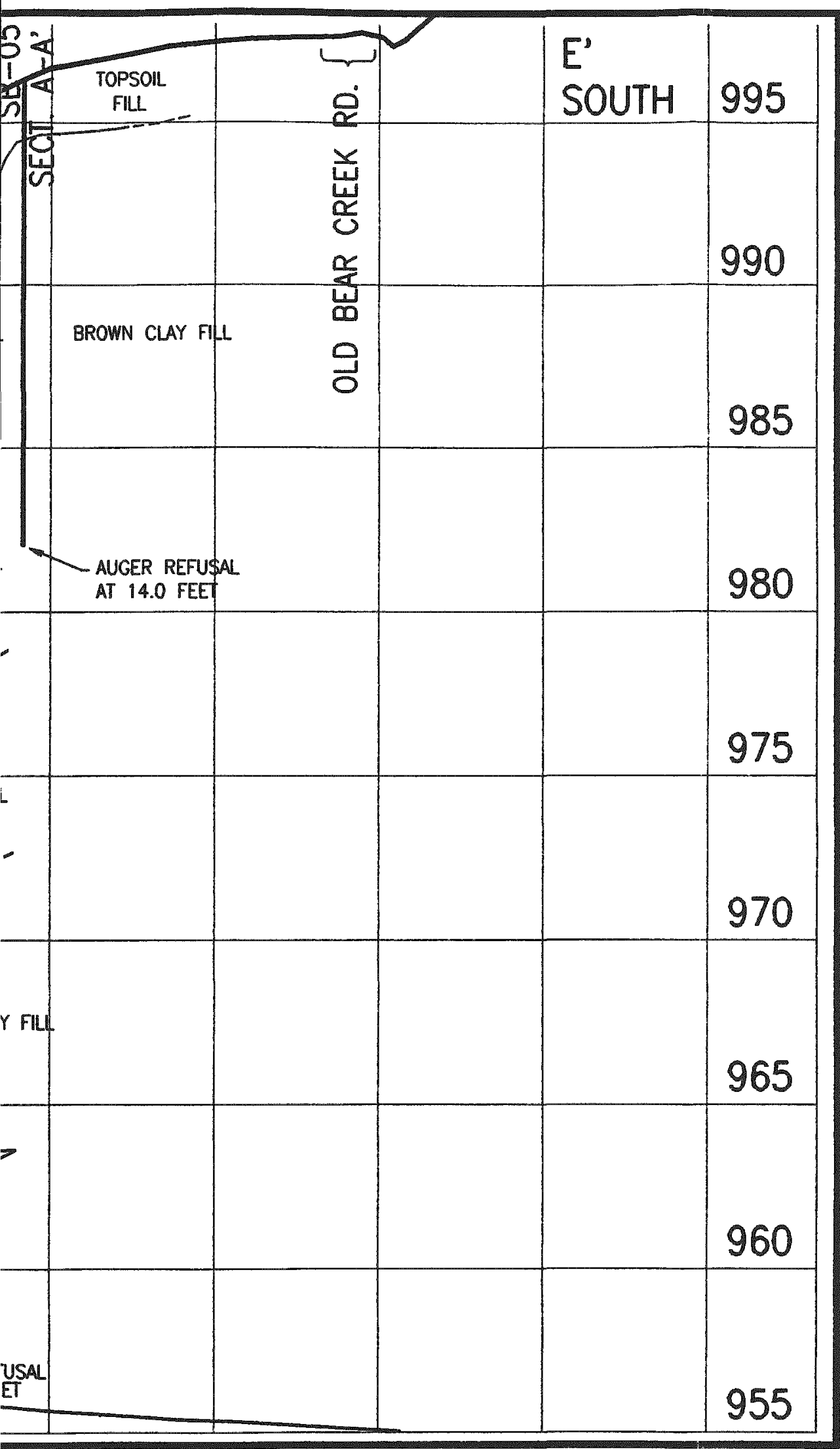

LEGEND:

SB......................... SOILBORING

$\underline{\nabla}$ GROUNDWATER ENCOUNTERED IN BOREHOLE

NOTES:

1.) "DIRTY" FILL INDICATES OBVIOUS FILL MATERIAL INCLUDES WOOD FRAGMENTS, PIECES OF BRICK \& CONCRETE BLOCK WIRE, ETC.
10

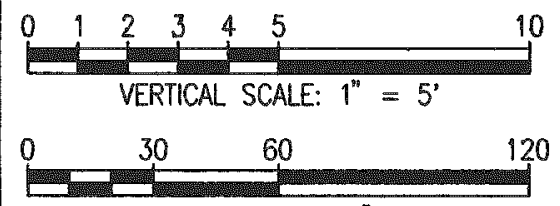

HORIZONTAL SCALE: $1^{\prime \prime}=60^{\circ}$

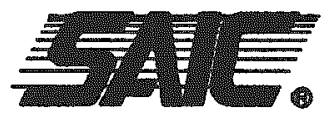

Science Applications International Corporation

BEAR CREEK VALLEY OU2 RUST SPOIL AREA PROFILE E-E' SOIL LITHOLOGY

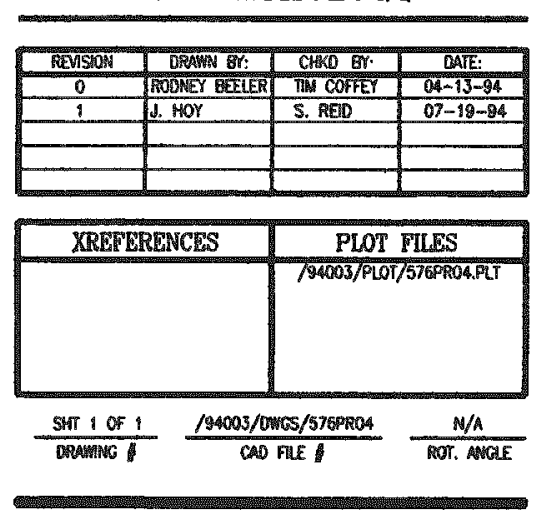




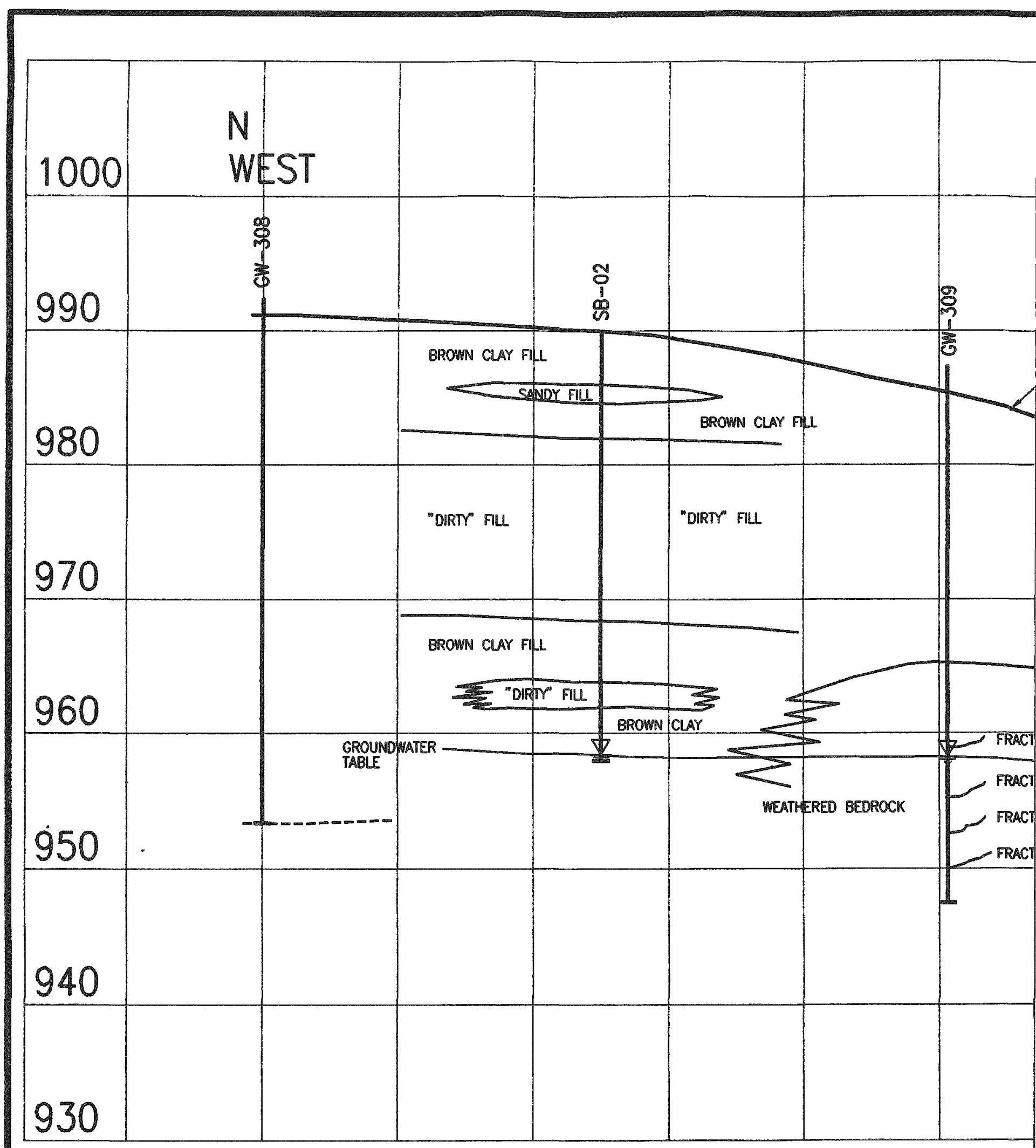




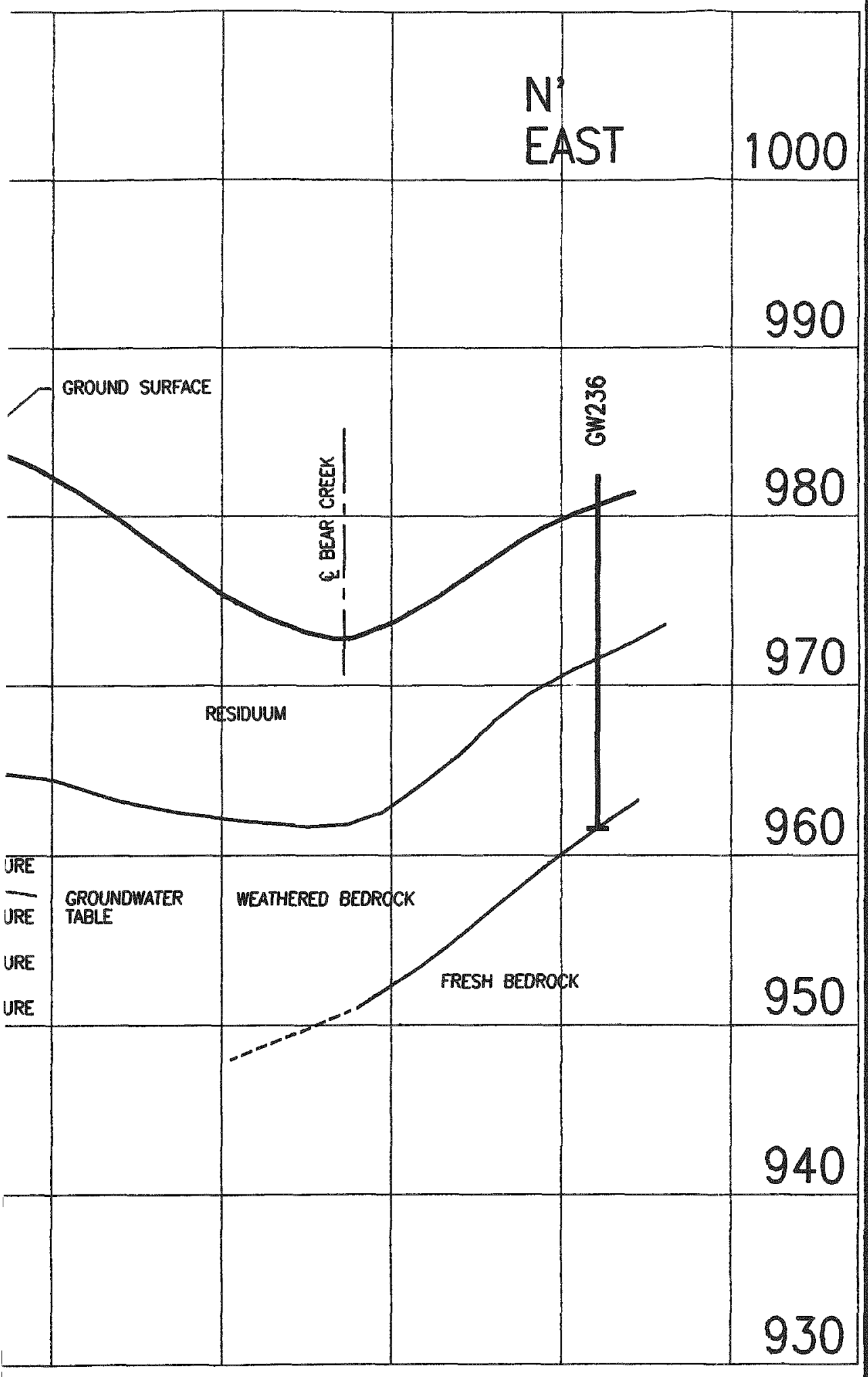

LEGEND:

GW.

MONITORING WELL

SB

SOLLOORING

GROUNDWATER

\$...........ENCOUNTERED IN BOREHOLE

SCREENED INTERVAL

I.................. TOTAL DEPTH OF HOLE
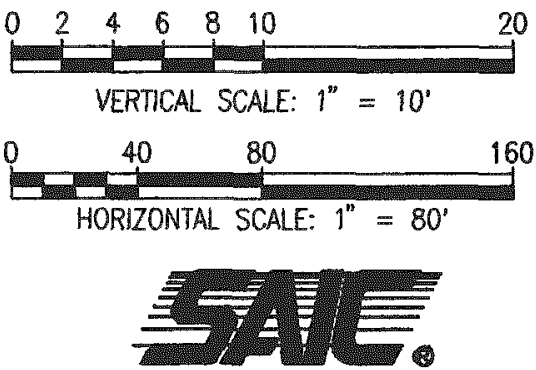

Science Applications International Corporation

BEAR CREEK VALLEY OU2 RUST SPOIL AREA PROFILE N-N' SOIL LITHOLOGY
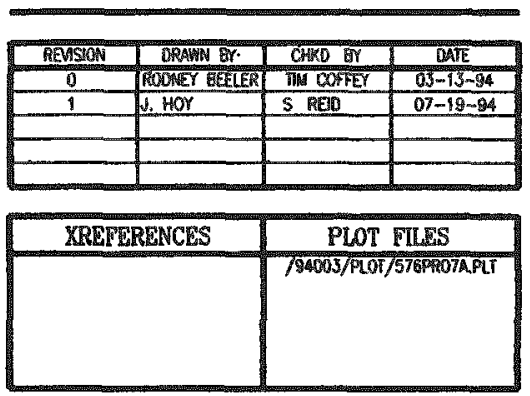

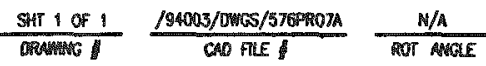




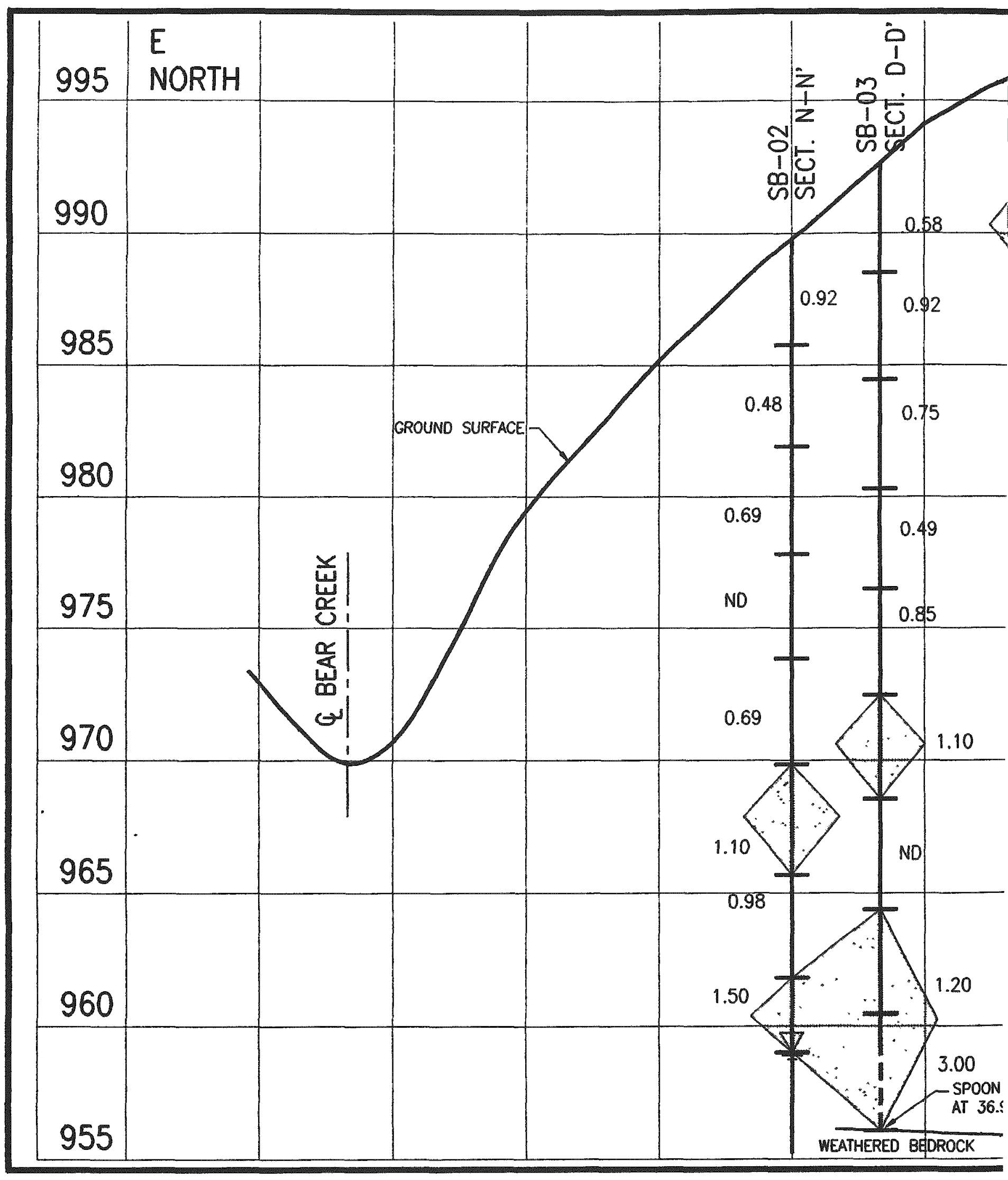




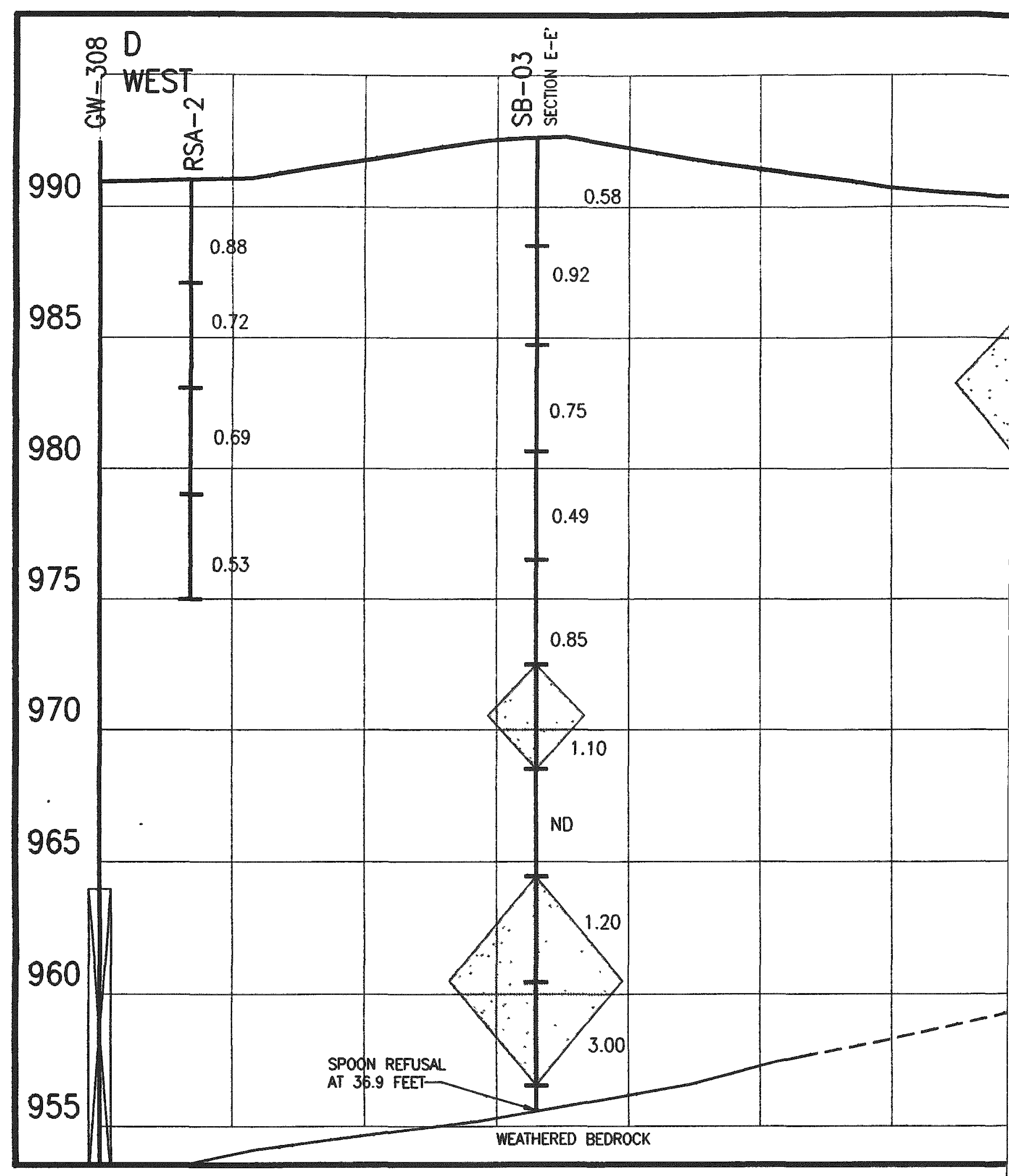


$$
\text { . }
$$ 


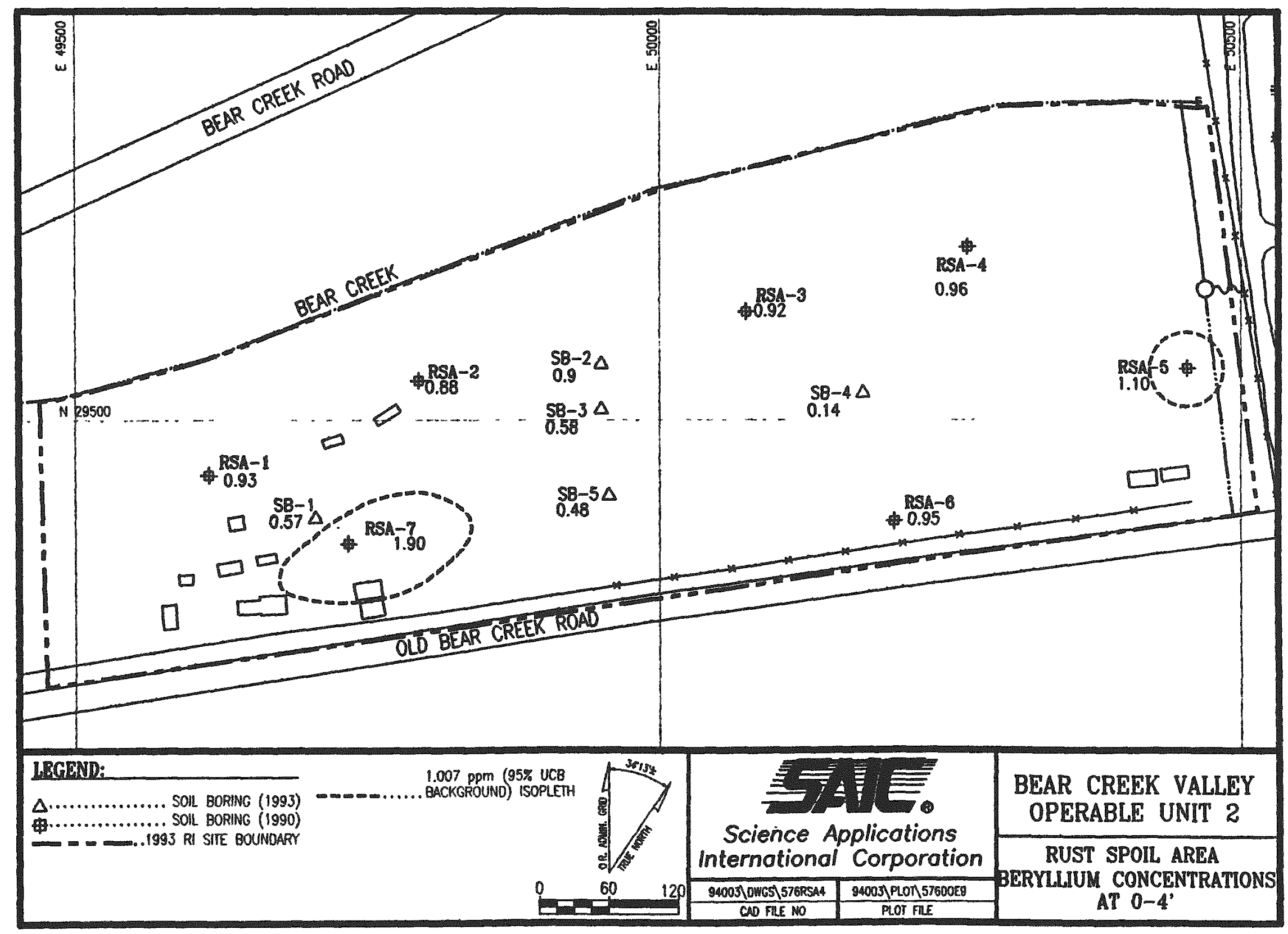




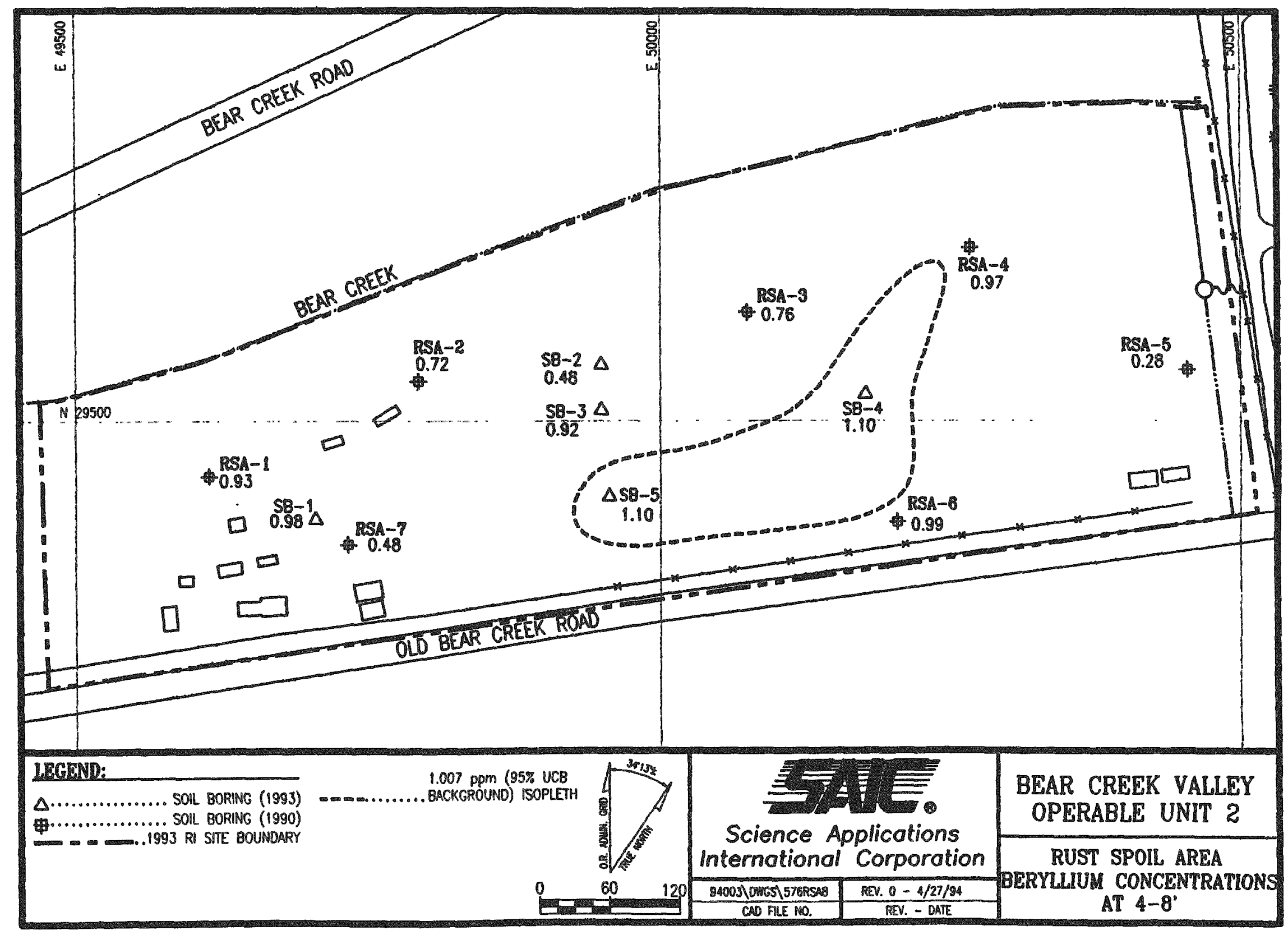




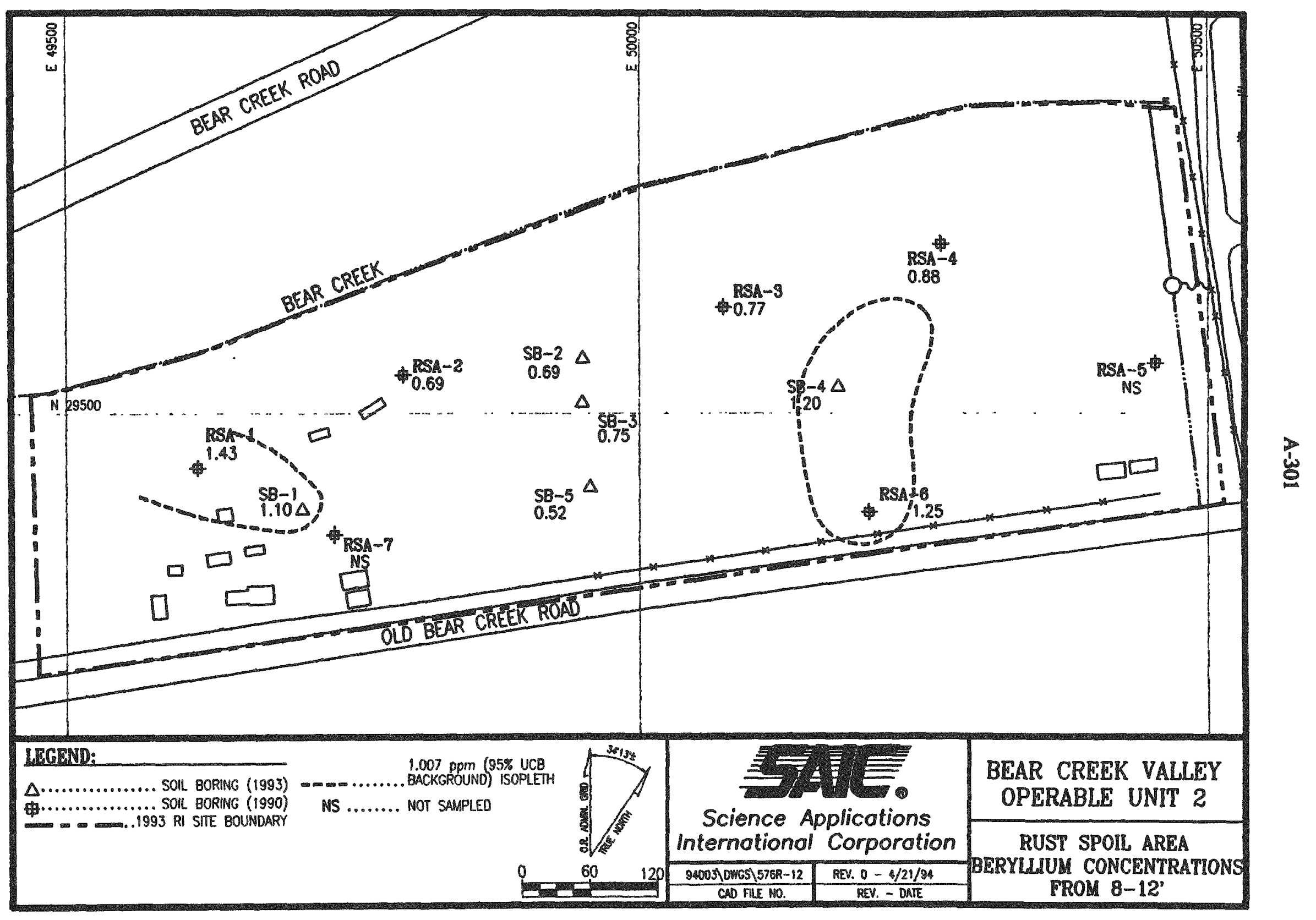




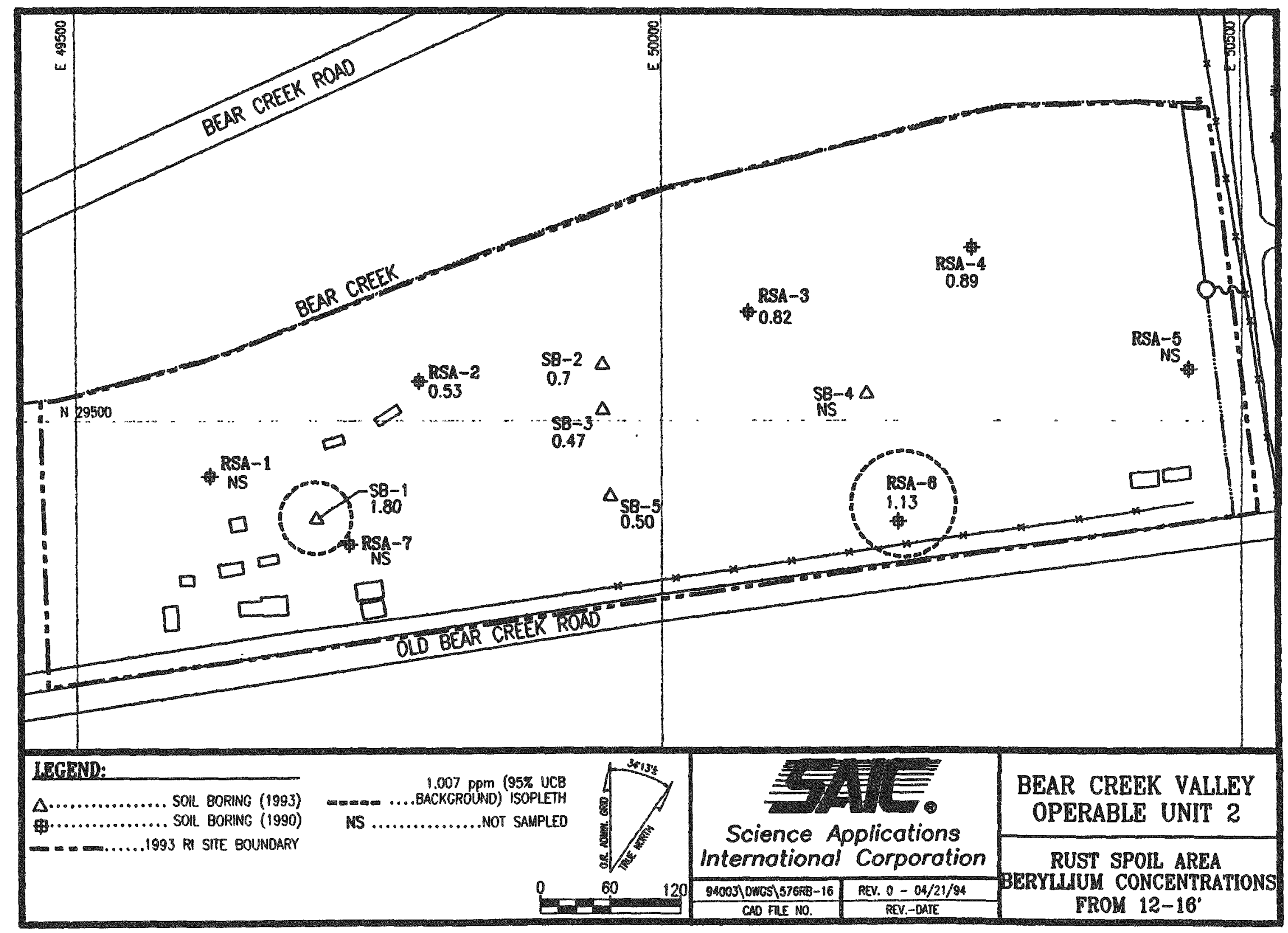




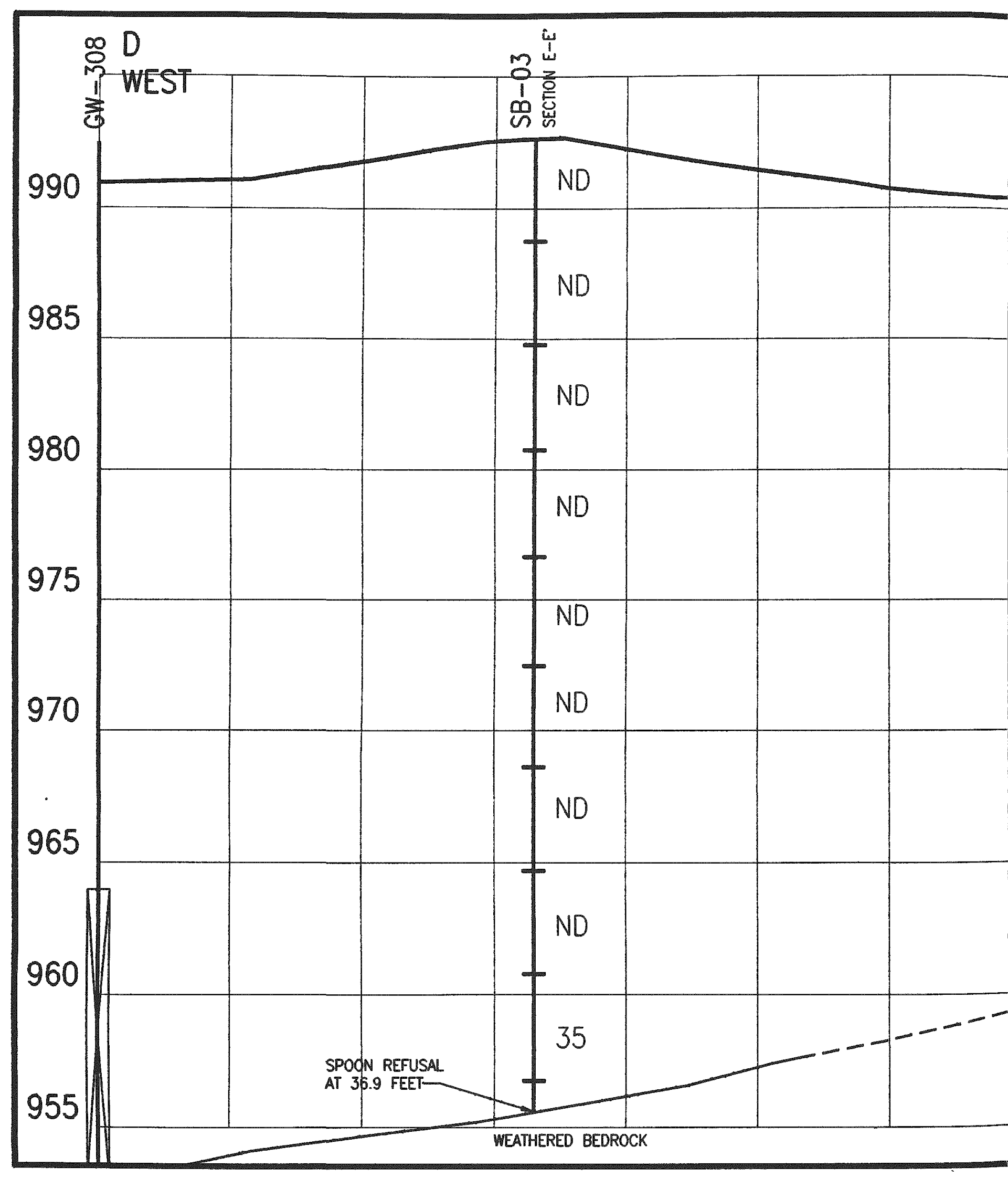




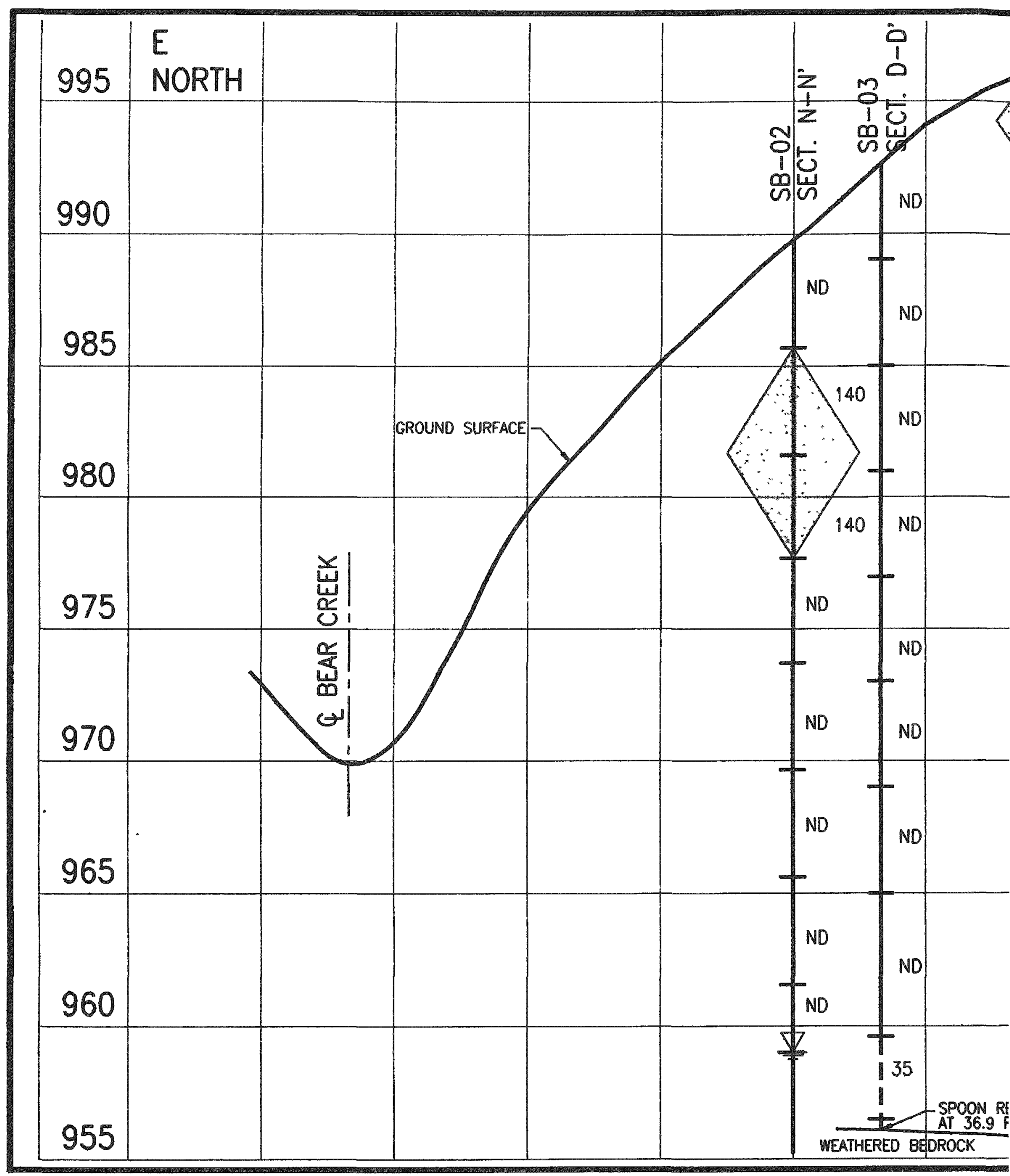




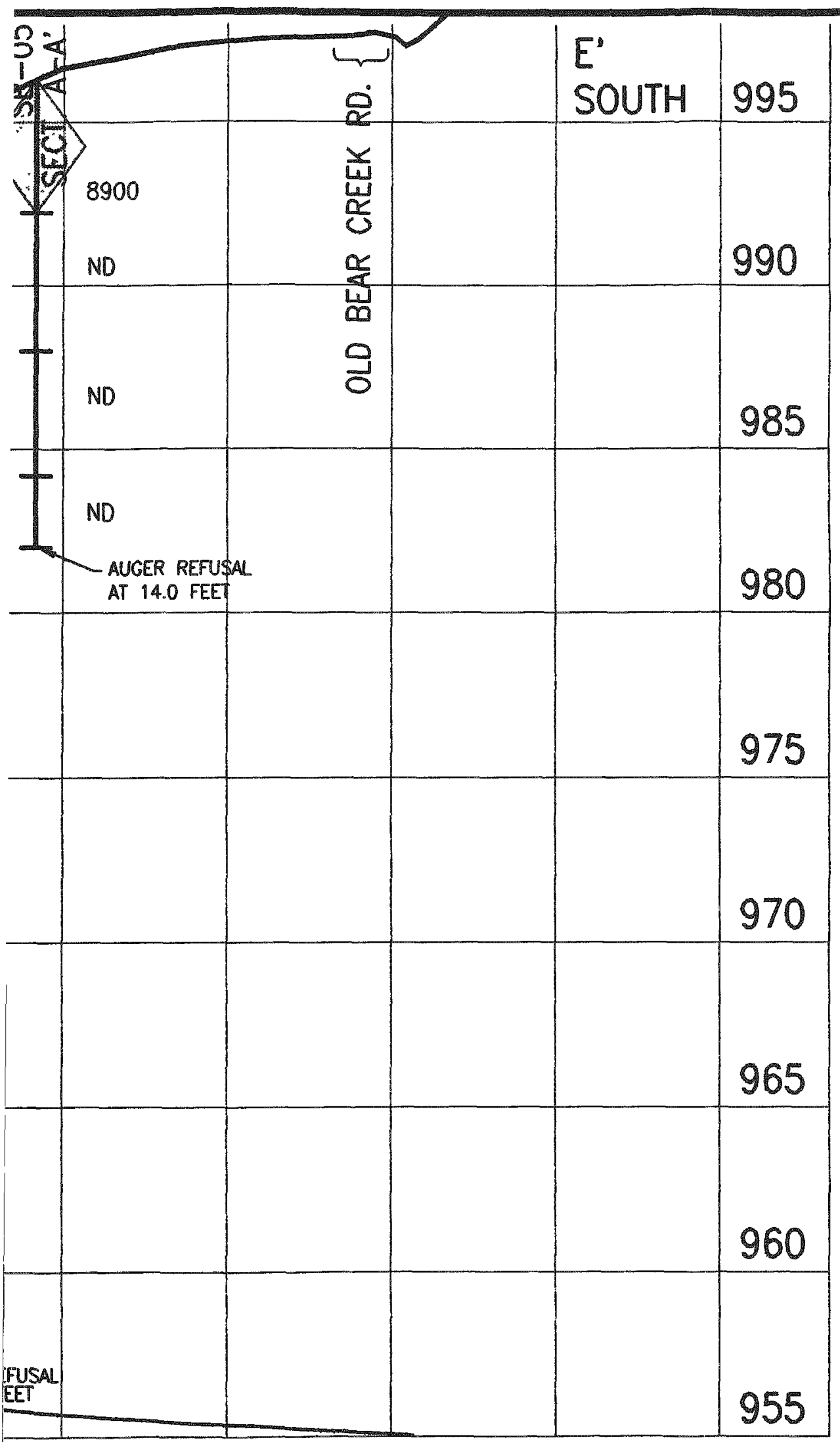

LEGEND:

GW $\ldots \ldots \ldots \ldots \ldots \ldots \ldots$ MONITORING WEU

SB $\ldots \ldots \ldots \ldots \ldots \ldots \ldots \ldots . . . \ldots$ SOLLBORING

NS $\ldots \ldots \ldots \ldots \ldots \ldots \ldots \ldots$ NOT SAMPLED

ND......................NOT DETECTED

要GROUNDWATER ENCOUNTERED IN BOREHOLE

Q $>83 \mathrm{ppb}$ (PRG) (AROCLOR 1254\& 1260)

$0 \quad 123 \quad 4 \quad 5$

10

VERTICAL SCALE: $1 "=5$

0

30

60

120

HORIZONTAL SCALE: $1^{\prime \prime}=60^{\circ}$

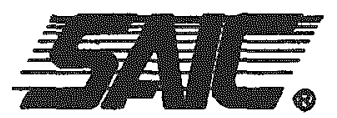

Science Applications International Corporation

BEAR CREEK VALLEY OU2 RUST SPOIL AREA, PROFILE E-E' TOTAL AROCLOR CONCEN.
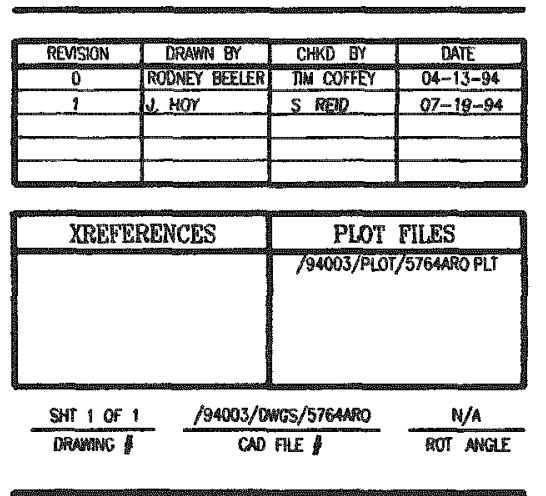
$$
\text { . }
$$ 


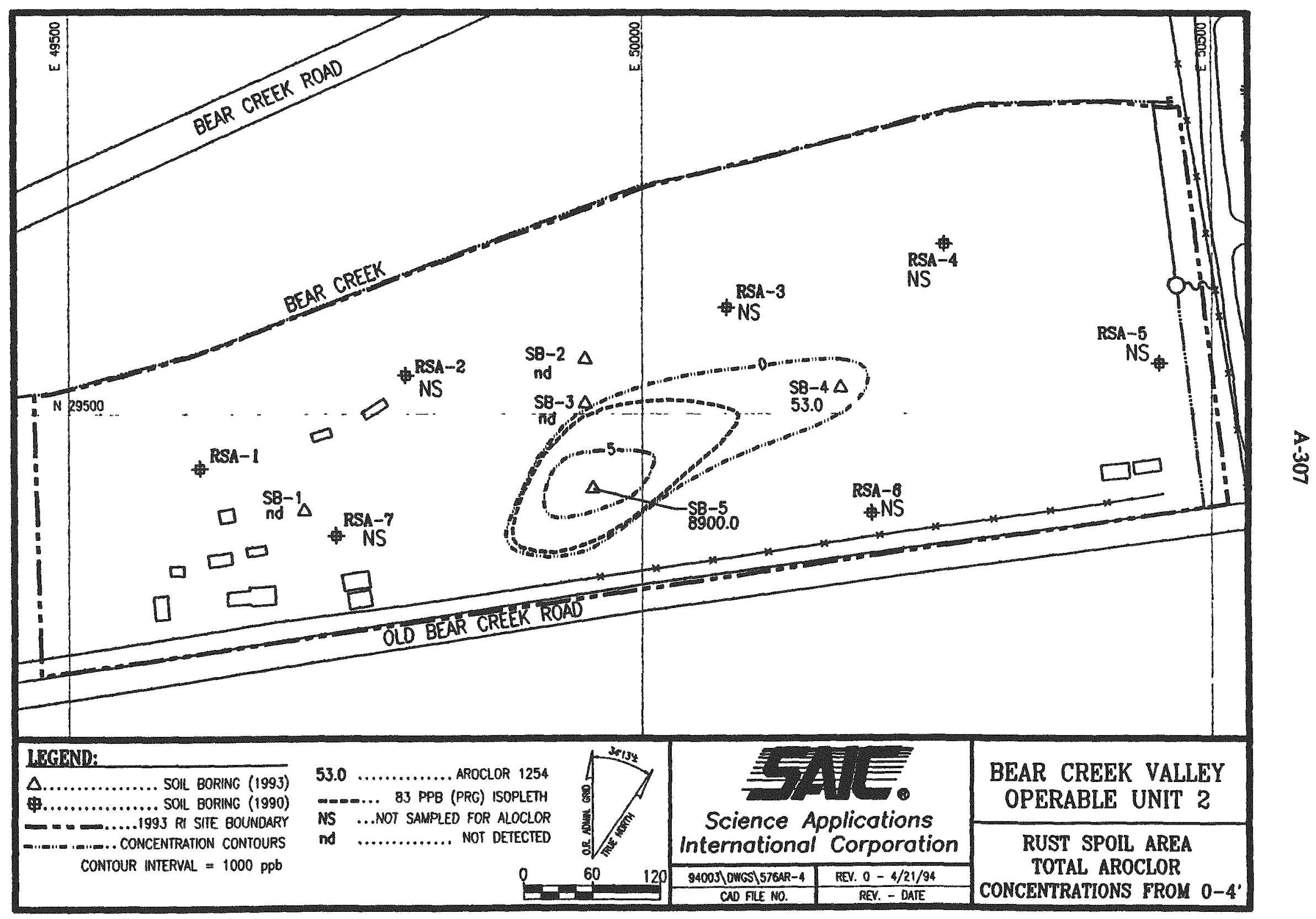




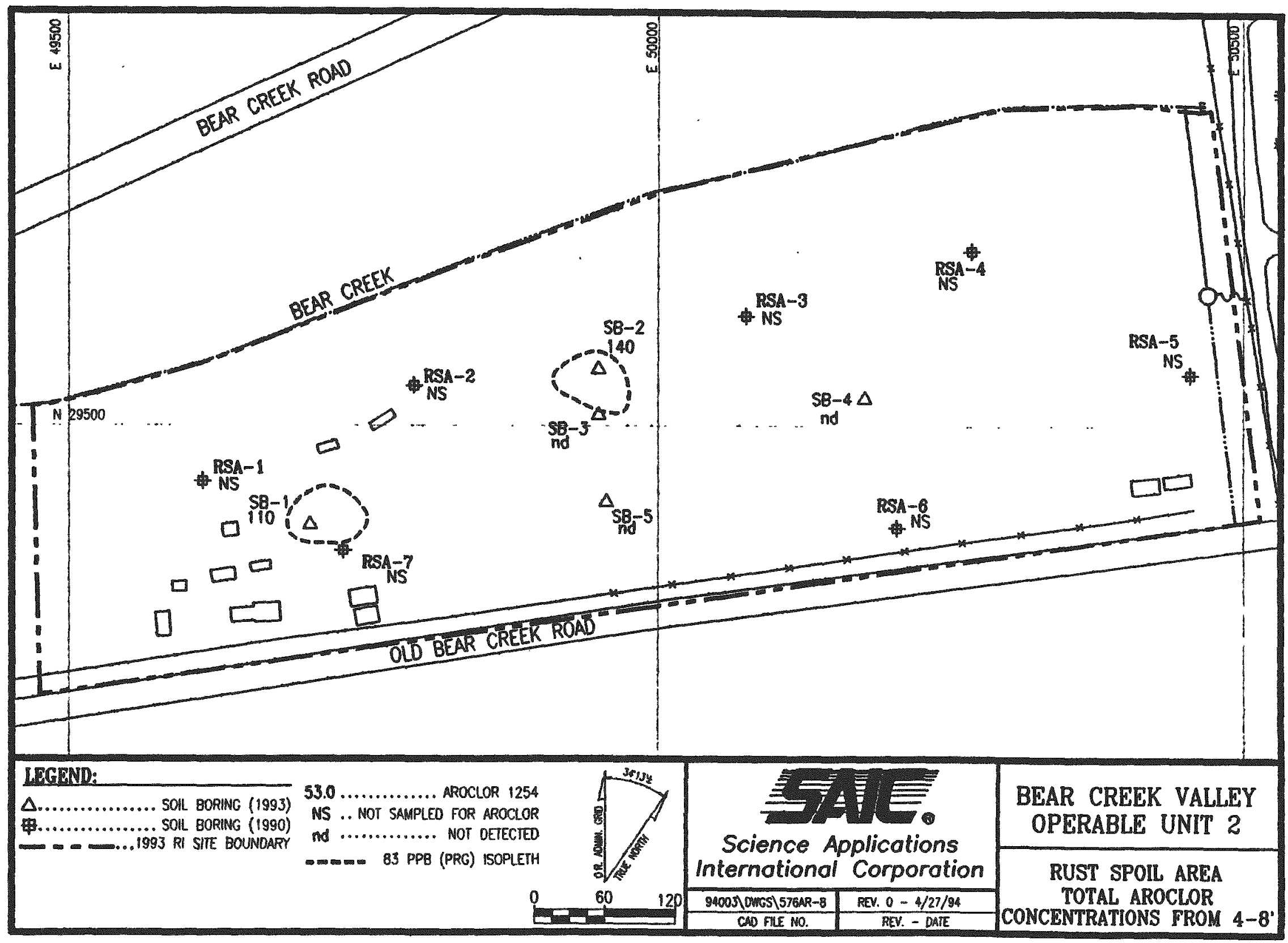

这 


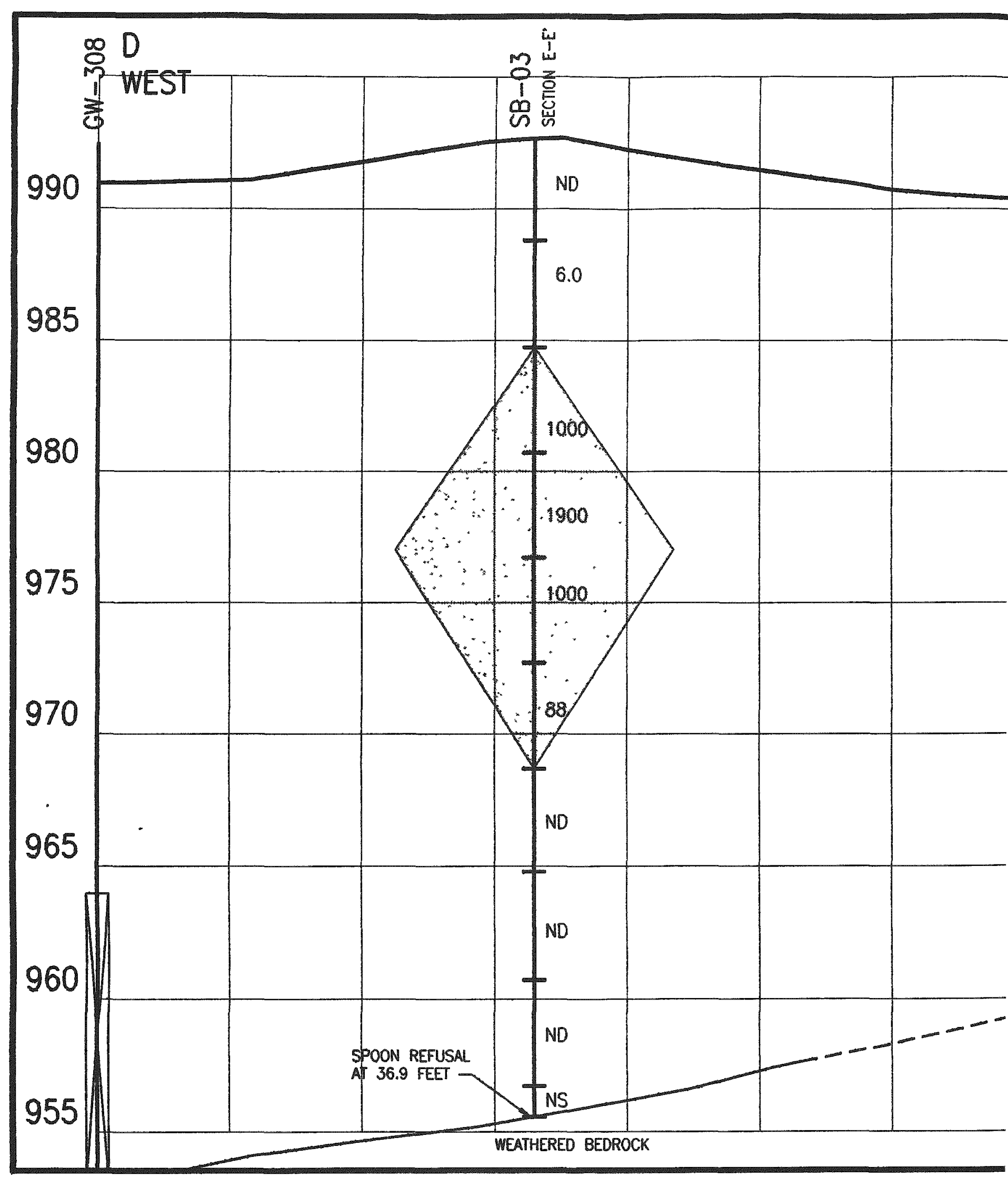




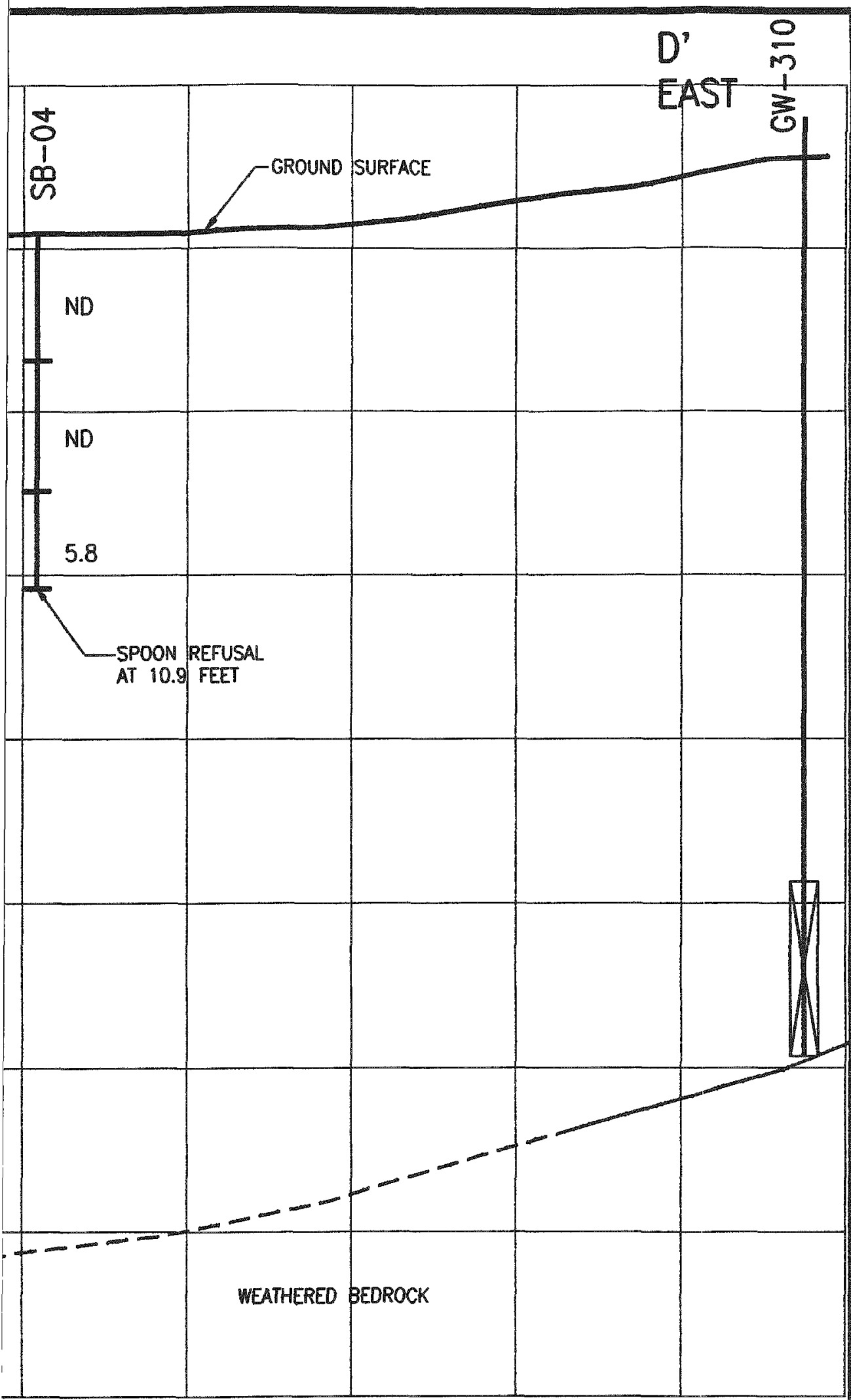

LEGEND:

GW.

MONITORING WELL

SB........................ SOLBORING

NS ............. NOT SAMPLED

ND. ......................NOT DETECTED

H

SCREENED INTERVAL



) BENZO (A) PYRENE

00142345

10

VERTICAL SCALE: $1^{\prime \prime}=5^{\circ}$

0

30

60

120

HORIZONTAL SCALE: $1^{\prime \prime}=60^{\circ}$

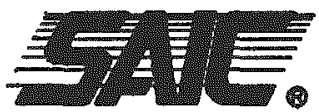

Science Applications International Corporation

BEAR CREEK VALLEY OU2 RUST SPOIL AREA, PROFILE D-D' BENZO (A) PYRENE CONCEN.
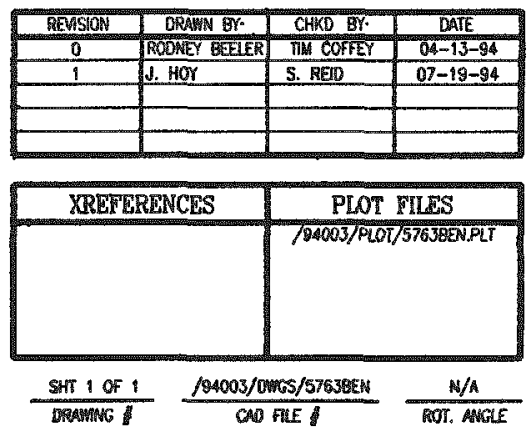


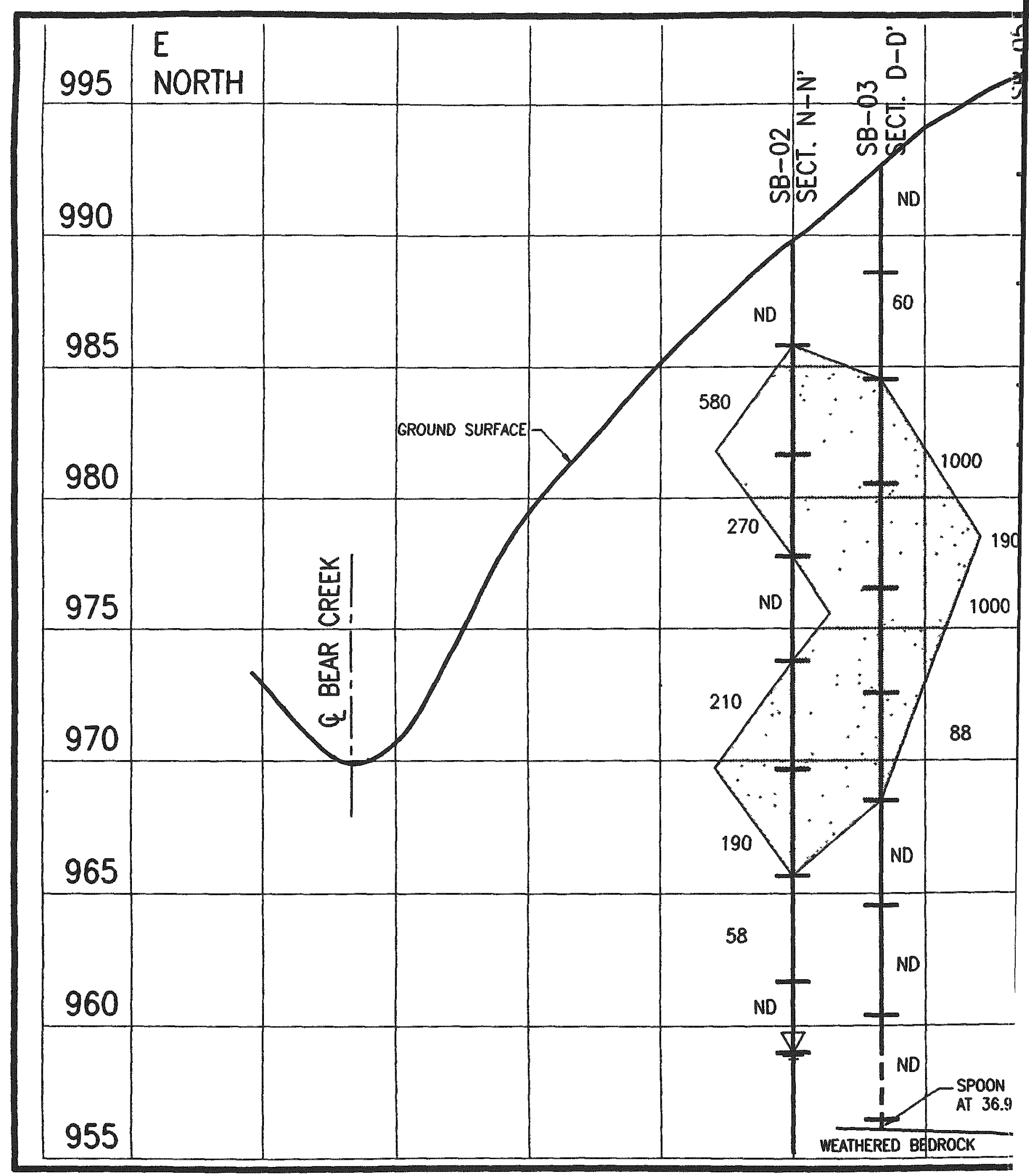




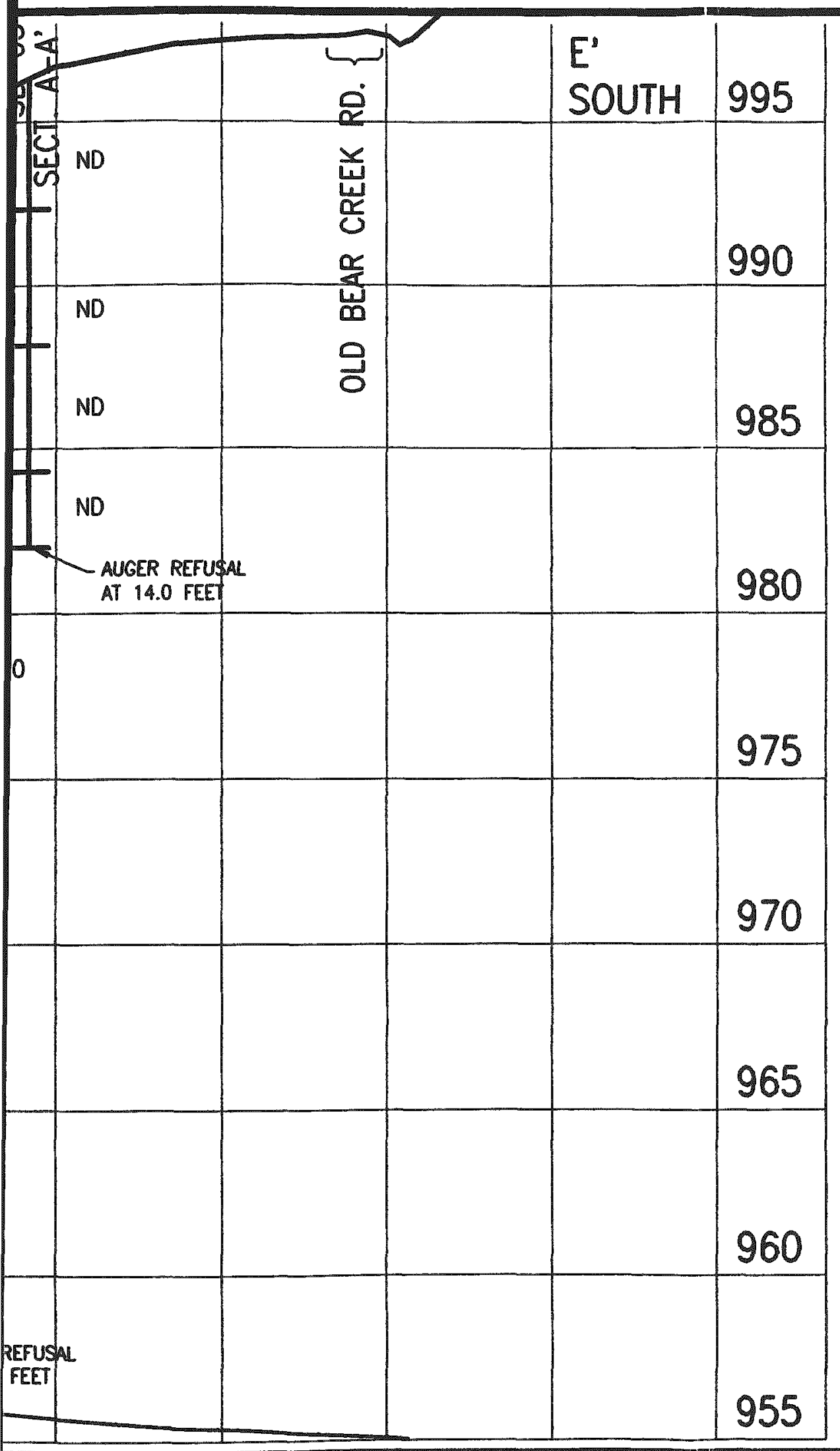

LEGEND:

MW.....................MONITORING WEL

SB $\ldots \ldots \ldots \ldots \ldots \ldots \ldots \ldots \ldots$ SOLLBORNG

NS $\ldots \ldots \ldots \ldots \ldots \ldots \ldots \ldots \ldots$ NOT SAMPLED

ND ........................NOT DETECTED

$\nabla$ GROUNDWATER ENCOUNTERED IN BOREHOLE a. $>88 \mathrm{ppb}$ (PRG) BENZO (A) PYRENE

$\begin{array}{llllll}0 & 1 & 2 & 3 & 4 & 5\end{array}$ 10 VERTICAL SCALE: $i^{\prime \prime}=5^{\prime}$

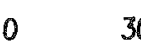

60 120

HORIZONTAL SCALE: $1^{n}=60^{\circ}$

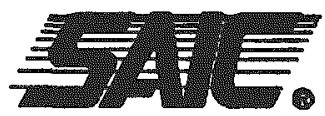

Science Applications International Corporation

BEAR CREEK VALLEY OU2 RUST SPOIL AREA, SECTION E-E' BENZO (A) PYRENE CONCEN.
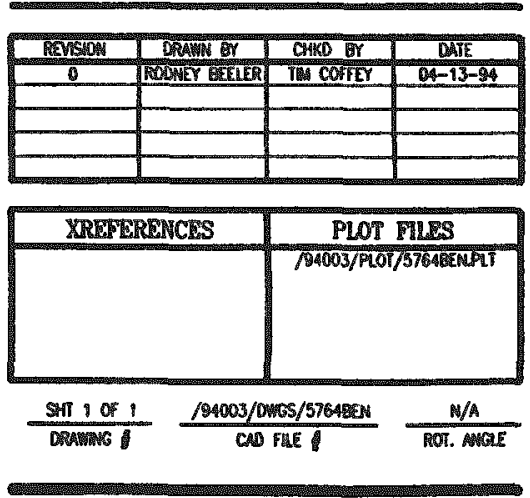


\section{APPENDIX B}

Analytical Data 


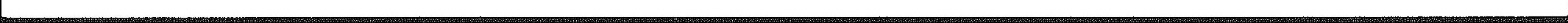


Detects 
TABLE 1. BC-OU2 1993 RI Field Activities Data - Sumamary Of Defected Contamimants For The Rust Spoil Area

Sampling Media: Soil

\begin{tabular}{|c|c|c|c|c|c|c|c|c|}
\hline Analysis & Parameter & Cas Number & $\begin{array}{c}\text { Detection } \\
\text { Lirnit Range }\end{array}$ & $\begin{array}{c}\text { Number of } \\
\text { Deterts }\end{array}$ & $\begin{array}{c}\text { Minimum } \\
\text { Detect }\end{array}$ & $\begin{array}{l}\text { Mean } \\
\text { Result }\end{array}$ & $\begin{array}{c}\text { Maximum } \\
\text { Detect }\end{array}$ & $\begin{array}{c}\text { Standard } \\
\text { Deviation }\end{array}$ \\
\hline \multirow[t]{6}{*}{ Metals MG/KG (AAS) } & Antimony & $7440-36-0$ & $0.22-0.34$ & $4 / 18$ & 0.23 & 0.172 & 0.43 & 0.0906 \\
\hline & Arsenic & $7440-38-2$ & $1.2-1.2$ & 26127 & 2.2 & 6.86 & 18.2 & 4.27 \\
\hline & Cyanide & $57-12-5$ & $0.51-0.63$ & $1 / 19$ & 24.6 & 1.57 & 24.6 & 5.58 \\
\hline & Lead & $7439-92-1$ & & $27 / 27$ & 9.1 & 35.9 & 79.3 & 17.7 \\
\hline & Selenium & $7782-49-2$ & $0.24-1.4$ & $2 / 16$ & 1.2 & 0.35 & 1.9 & 0.505 \\
\hline & Thallium & $7440-28-0$ & $0.13-0.68$ & $8 / 27$ & 0.12 & 0.166 & 0.44 & 0.0889 \\
\hline Metals MG/KG & Mercury & $7439-97-6$ & $0.06-0.06$ & $24 / 27$ & 0.06 & 2.41 & 30.9 & 6.21 \\
\hline \multirow[t]{16}{*}{ Metals MG/KG (ICP) } & Aluminum & $7429-90-5$ & & $27 / 27$ & 5950 & 13600 & 20200 & 4150 \\
\hline & Barium & $7440-39-3$ & & $27 / 27$ & 19 & 148 & 1090 & 194 \\
\hline & Beryllium & $7440-41-7$ & & $27 / 27$ & 0.14 & 0.948 & 3 & 0.558 \\
\hline & Cadmium & $7440-43-9$ & $0.11-0.19$ & $4 / 27$ & 0.36 & 0.164 & 0.95 & 0.234 \\
\hline & Calcium & $7440-70-2$ & & $27 / 27$ & 888 & 39000 & 160000 & 39300 \\
\hline & Chromium & $7440-47-3$ & & $27 / 27$ & 16.7 & 30.3 & 113 & 18.6 \\
\hline & Cobalt & $7440-48-4$ & & $27 / 27$ & 4.6 & 15 & 55.2 & 12.4 \\
\hline & Copper & $7440-50-8$ & & $27 / 27$ & 5.4 & 37.9 & 295 & 56.6 \\
\hline & Iron & $7439-89-6$ & & $27 / 27$ & 11600 & 27000 & 52900 & 12000 \\
\hline & Magnesium & $7439-95-4$ & & $27 / 27$ & 552 & 6530 & 34000 & 7470 \\
\hline & Manganese & $7439-96-5$ & & $27 / 27$ & 153 & 1040 & 7600 & 1810 \\
\hline & Nicke! & $7440-02-0$ & & $27 / 27$ & 7.8 & 35.4 & 311 & 59.9 \\
\hline & Polassium & $7440-09.7$ & & $27 / 27$ & 592 & 1810 & 3060 & 736 \\
\hline & Silver & $7440-22-4$ & $0.38-0.51$ & $24 / 27$ & 0.51 & 1.34 & 3.4 & 0.877 \\
\hline & Sodium & $7440-23-5$ & $66.1-106$ & $21 / 27$ & 28.7 & 98.5 & 242 & 55.9 \\
\hline & Vanadium & $7440-62-2$ & & $27 / 27$ & 10.3 & 28.5 & 76.7 & 13.9 \\
\hline
\end{tabular}




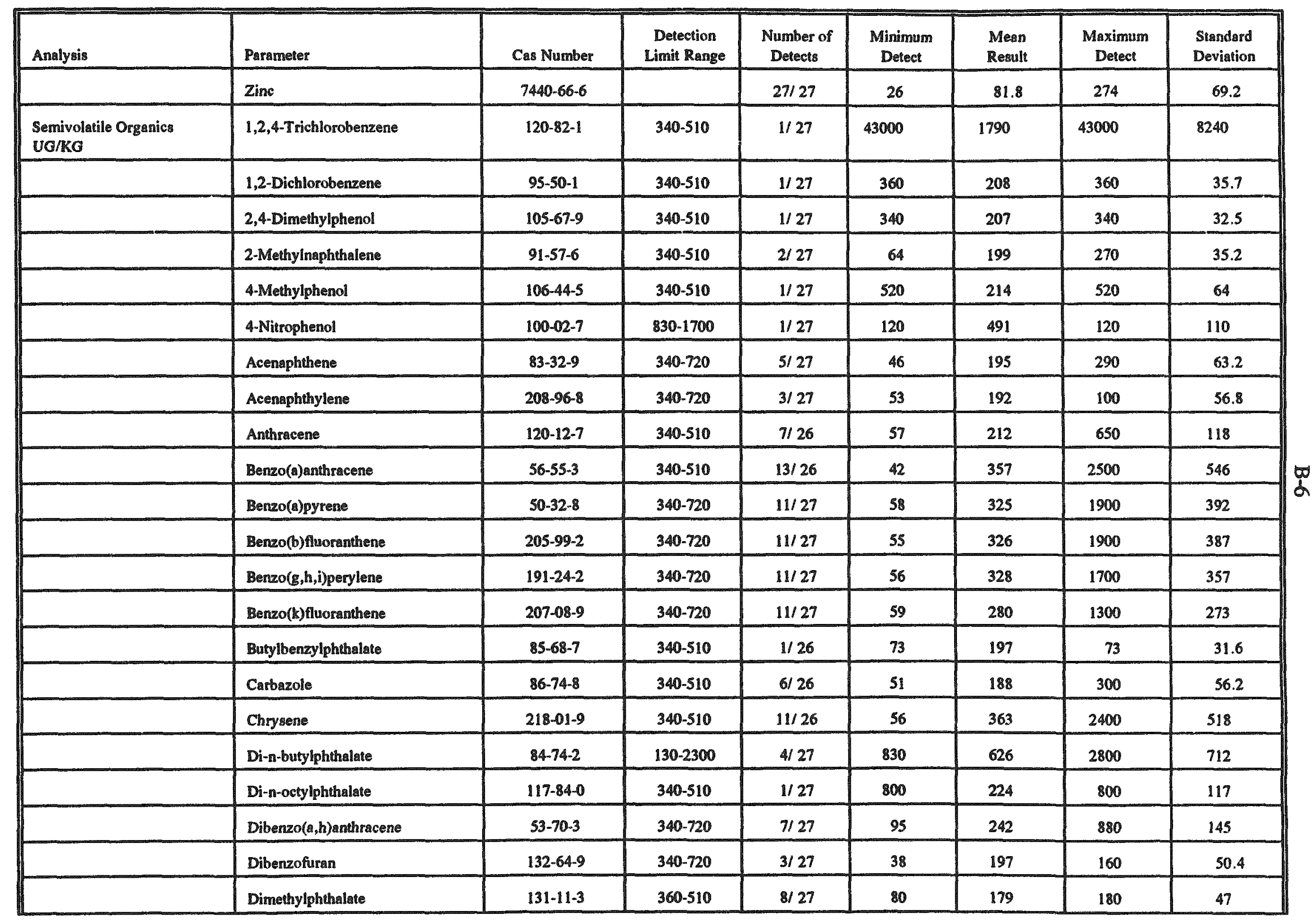




\begin{tabular}{|c|c|c|c|c|c|c|c|c|}
\hline Analysis & Parameter & Cas Number & $\begin{array}{c}\text { Detection } \\
\text { Limit Range } \\
\end{array}$ & $\begin{array}{c}\text { Number of } \\
\text { Detects }\end{array}$ & $\begin{array}{c}\text { Minimum } \\
\text { Detect }\end{array}$ & $\begin{array}{l}\text { Mean } \\
\text { Result } \\
\end{array}$ & $\begin{array}{c}\text { Maximum } \\
\text { Detect }\end{array}$ & $\begin{array}{c}\text { Standard } \\
\text { Deviation } \\
\end{array}$ \\
\hline & Fluoranthene & $206-44-0$ & $340-510$ & $15 / 26$ & 45 & 519 & 3300 & 813 \\
\hline & Fluorene & 86-73-7 & $340-720$ & $5 / 27$ & 55 & 198 & 290 & 60.5 \\
\hline & Indeno( $1,2,3-c, d)$ pyrene & $193-39-5$ & $340-720$ & $11 / 27$ & 45 & 334 & 2000 & 407 \\
\hline & Naphthalene & 91-20-3 & $340-510$ & $4 / 27$ & 72 & 190 & 210 & 38.2 \\
\hline & Pentachlorophenol & $87-86-5$ & $830-1200$ & $1 / 26$ & 47 & 474 & 47 & 97.5 \\
\hline & Phenanthrene & $85.01-8$ & $340-510$ & $14 / 27$ & 44 & 363 & 1900 & 443 \\
\hline & Phenol & $108-95-2$ & $340-510$ & $1 / 27$ & 2500 & 287 & 2500 & 443 \\
\hline & Pyrene & $129-00-0$ & $340-510$ & $16 / 26$ & 39 & 621 & 4600 & 1080 \\
\hline & bis(2-Ethylhexyl)phthalate & $117-81-7$ & $340-510$ & $9 / 27$ & 43 & 1680 & 41000 & 7860 \\
\hline \multirow[t]{14}{*}{ Volatile Organics UGKKG } & 1,1,1-Trichloroethane & $71-55-6$ & $5-1500$ & $1 / 28$ & 170 & 36.3 & 170 & 143 \\
\hline & $\begin{array}{l}\text { 1,1,2-Trichloro- } \\
\text { 1,2,2-Trifluoroethane }\end{array}$ & $76-13-1$ & $10-1500$ & $9 / 28$ & 27 & 1190 & 32000 & 6040 \\
\hline & 1, 1-Dichloroethane & $75-34-3$ & $5-1500$ & $2 / 28$ & 10 & 53.3 & 640 & 182 \\
\hline & 1,1-Dichloroethene & $75-35-4$ & $5-1500$ & $1 / 28$ & 51 & 32 & 51 & 141 \\
\hline & 1,2-Dichloroethane & $107-06-2$ & $5-1500$ & $1 / 28$ & 3 & 30.3 & 3 & 141 \\
\hline & 1,2-Dichloroethene & $540-59-0$ & $5-12$ & 7128 & 4 & 3990 & 110000 & 20800 \\
\hline & 2-Butanone & $78-93-3$ & $10-1500$ & 7228 & 5 & 47.7 & 270 & 146 \\
\hline & 2-Hexanone & $591-78-6$ & $10-1500$ & $1 / 27$ & 11 & 34.9 & 11 & 143 \\
\hline & 4-Methyl-2-pentanone & $108-10-1$ & $10-1500$ & $2 / 27$ & 5 & 42.3 & 210 & 147 \\
\hline & Acetone & $67-64-1$ & $23-1500$ & $15 / 28$ & 14 & 277 & 2500 & 489 \\
\hline & Benzene & $71-43-2$ & $5-1500$ & $1 / 28$ & 10 & 30.5 & 10 & 141 \\
\hline & Carbon Disulfide & $75-15-0$ & $5-1500$ & 7128 & 2 & 31.7 & 19 & 141 \\
\hline & Chlorobenzene & $108-90-7$ & $5-1500$ & $1 / 26$ & 7 & 32.1 & 7 & 146 \\
\hline & Chloroethane & $75-00-3$ & $10-1500$ & $1 / 28$ & 21 & 34.3 & 21 & 140 \\
\hline
\end{tabular}




\begin{tabular}{|c|c|c|c|c|c|c|c|c|}
\hline Analysis & Parameter & Cas Number & $\begin{array}{l}\text { Detection } \\
\text { Limit Range }\end{array}$ & $\begin{array}{l}\text { Number of } \\
\text { Detects }\end{array}$ & $\begin{array}{l}\text { Minimum } \\
\text { Detect }\end{array}$ & $\begin{array}{l}\text { Mean } \\
\text { Result }\end{array}$ & $\begin{array}{c}\text { Maximum } \\
\text { Defect }\end{array}$ & $\begin{array}{l}\text { Standard } \\
\text { Deviation }\end{array}$ \\
\hline & Chloroform & $67-66-3$ & $5-1500$ & $1 / 28$ & 79 & 33 & 79 & 141 \\
\hline & Ethylbenzene & $100-41-4$ & $5-28$ & $4 / 27$ & 5 & 22.7 & 280 & 67.4 \\
\hline & Methylene Chloride & $75-09-2$ & $5-1500$ & $15 / 28$ & 8 & 2130 & 58000 & 11000 \\
\hline & Tetrachloroethene & $127-18-4$ & $5-12$ & $8 / 28$ & 3 & 15300 & 400000 & 75500 \\
\hline & Toluene & $108-88-3$ & $5-28$ & $8 / 28$ & 1 & 44.4 & 750 & 152 \\
\hline & Trichloroethene & $79-01-6$ & $5-28$ & $5 / 28$ & 4 & 3600 & 100000 & 18900 \\
\hline & Xylene, Total & $1330-20-7$ & 5-1500 & $3 / 27$ & 9 & 49.2 & 460 & 165 \\
\hline \multirow[t]{5}{*}{ Pesticide/PCB UO/KO } & Aroclor-1254 & $11097-69-1$ & $36-220$ & $6 / 21$ & 35 & 470 & 8900 & 1930 \\
\hline & Aroclor-1260 & $11096-82-5$ & $34-360$ & $1 / 20$ & 50 & 42.9 & 50 & 45.1 \\
\hline & Endosulfan I & $959-98-8$ & $1.8-18$ & $1 / 20$ & 0.65 & 2.06 & 0.65 & 2.28 \\
\hline & Heptachlor & $76-44-8$ & $1.8-18$ & $5 / 22$ & 0.63 & 1.93 & 0.98 & 2.2 \\
\hline & p,p'-Methoxychlor & $72-43-5$ & $18-180$ & $1 / 20$ & 1.5 & 20.3 & 1.5 & 22.9 \\
\hline \multirow[t]{5}{*}{ Radionuelides PCI/O } & Gross Alpha & $14127-62-9$ & & $18 / 18$ & 7.29 & 12.1 & 17.5 & 2.9 \\
\hline & Gross Beta & $12587-47-2$ & & $27 / 27$ & 19.1 & 30.5 & 55.7 & 9.47 \\
\hline & U-234 & $13966-29-5$ & & $27 / 27$ & 0.961 & 2.34 & 7.91 & 1.5 \\
\hline & U.235 & $15117-96-1$ & & $27 / 27$ & 0.003 & 0.148 & 0.806 & 0.161 \\
\hline & U.238 & $7440-61-1$ & & $27 / 27$ & 0.702 & 1.89 & 4.3 & 0.922 \\
\hline
\end{tabular}

Raw dat available through $Y-12$ OREIS

Data summary does not include $Q A / Q C$ sample results

Grand mean and standard deviation include nondetects set to $1 / 2$ of the reporting limit

Results < counting uncertainty were considered as nondetects for radionuclides 
TABLE 1. BC-OU2 1993 RI F'eld Activities Data - Summary Of Detected Contaminants For The SY-200 Yard Sampling Media: Soil

\begin{tabular}{|c|c|c|c|c|c|c|c|c|}
\hline Analysis & Parameter & Cas Number & $\begin{array}{l}\text { Detection } \\
\text { Limit Range }\end{array}$ & $\begin{array}{c}\text { Number of } \\
\text { Deteet }\end{array}$ & $\begin{array}{l}\text { Minimum } \\
\text { Detect }\end{array}$ & $\begin{array}{l}\text { Mean } \\
\text { Result }\end{array}$ & $\begin{array}{l}\text { Maximum } \\
\text { Detect }\end{array}$ & $\begin{array}{l}\text { Standard } \\
\text { Deviation }\end{array}$ \\
\hline \multirow[t]{6}{*}{ Metals MO/KO (AAS) } & Antimony & $7440-36-0$ & $0.21-0.87$ & $76 / 108$ & 0.2 & 0.355 & 0.92 & 0.165 \\
\hline & Arsenic & $7440-38-2$ & $0.19-3$ & $218 / 230$ & 0.54 & 12.6 & 69.2 & 12.5 \\
\hline & Cyanide & $57-12-5$ & $0.52-0.77$ & $2 / 98$ & 0.6 & 0.325 & 1.6 & 0.135 \\
\hline & Lead & $7439-92-1$ & & $39 / 39$ & 2.6 & 36.8 & 100 & 24.9 \\
\hline & Selenium & $7782-49-2$ & $0.23-2.5$ & $46 / 129$ & 0.34 & 0.967 & 12.9 & 1.35 \\
\hline & Thallium & $7440-28-0$ & $0.15-2.8$ & $64 / 232$ & 0.15 & 0.314 & 1.4 & 0.294 \\
\hline Metals MO/KG & Mercury & $7439-97-6$ & $0.05-1.4$ & $187 / 231$ & 0.05 & 20.9 & 816 & 78.5 \\
\hline \multirow[t]{16}{*}{ Metals MG/KO (ICP) } & Aluminum & $7429-90-5$ & & $232 / 232$ & 6000 & 17600 & 40100 & 6170 \\
\hline & Barium & $7440-39-3$ & & $232 / 232$ & 8.5 & 75.9 & 317 & 59.2 \\
\hline & Beryllium & $7440-41-7$ & $0.25-0.25$ & $231 / 232$ & 0.08 & 1.17 & 17.4 & 1.26 \\
\hline & Cadmium & $7440-43-9$ & $0.11-0.76$ & $23 / 214$ & 0.12 & 0.135 & 3.1 & 0.275 \\
\hline & Calcium & $7440-70-2$ & $20.2-77.7$ & $227 / 232$ & 52.9 & 9990 & 120000 & 15000 \\
\hline & Chromium & $7440-47-3$ & & $232 / 232$ & 12.1 & 27.4 & 64.8 & 6.29 \\
\hline & Cobalt & $7440-48-4$ & $7.3-13.5$ & $228 / 232$ & 2.7 & 21.6 & 336 & 34.3 \\
\hline & Copper & $7440-50-8$ & & $232 / 232$ & 5 & 29.4 & 245 & 20.9 \\
\hline & Iron & $7439-89-6$ & & $232 / 232$ & 8540 & 30300 & 102000 & 12600 \\
\hline & Lead & $7439-92-1$ & & $193 / 193$ & 9.6 & 56.7 & 435 & 61.2 \\
\hline & Magnesium & $7439-95-4$ & & $232 / 232$ & 231 & 2630 & 17400 & 2680 \\
\hline & Manganese & $7439-96-5$ & & $232 / 232$ & 88.5 & 733 & 5070 & 559 \\
\hline & Nickel & $7440-02-0$ & & $232 / 232$ & 4.1 & 24.7 & 122 & 13.7 \\
\hline & Potassium & $7440-09-7$ & & $232 / 232$ & 296 & 1540 & 4890 & 935 \\
\hline & Silver & $7440-22-4$ & $0.24-1.8$ & $153 / 232$ & 0.35 & 0.963 & 3.3 & 0.733 \\
\hline & Sodium & $7440-23-5$ & $19.6-144$ & $119 / 232$ & 20 & 47.1 & 120 & 25.6 \\
\hline
\end{tabular}




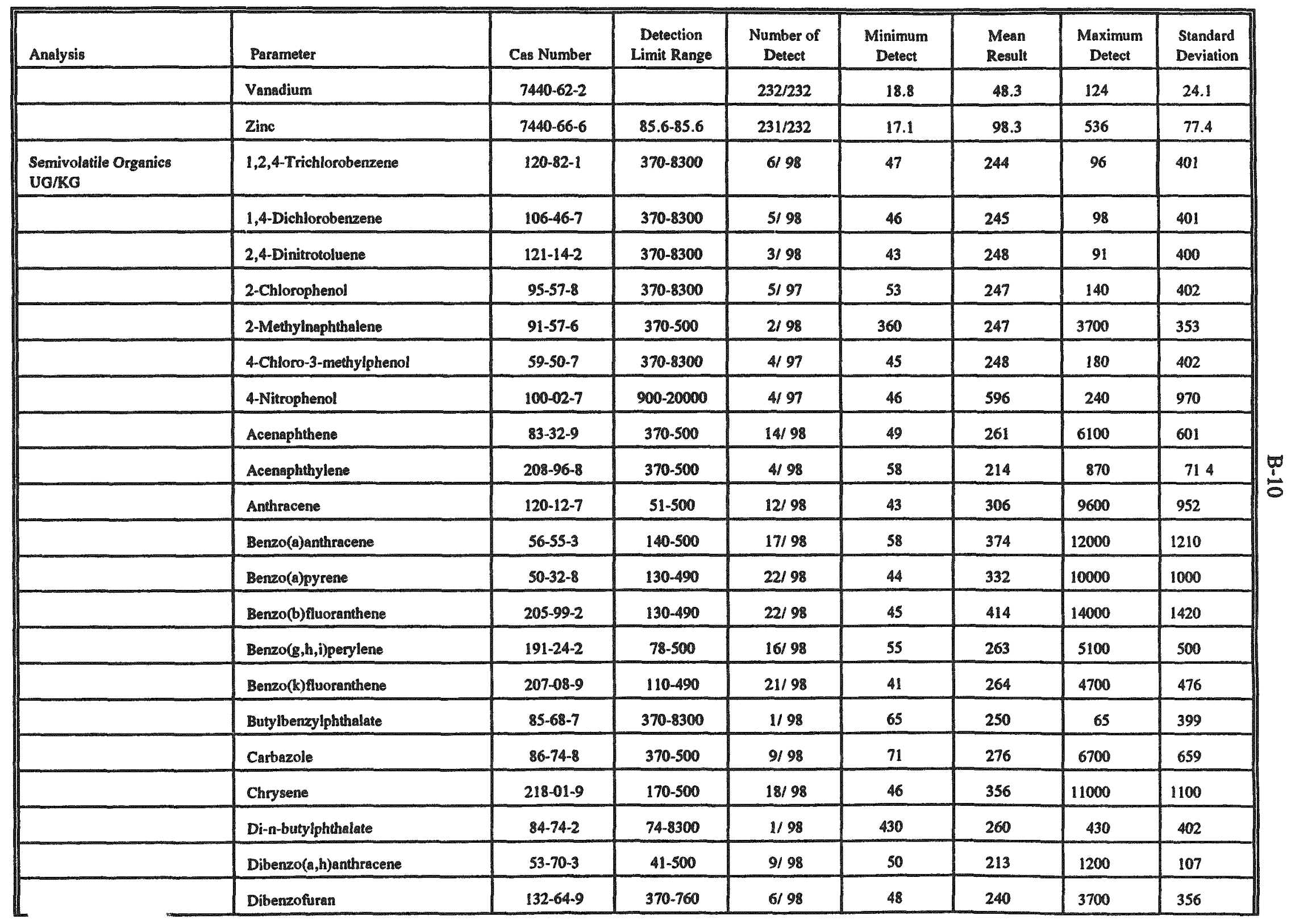




\begin{tabular}{|c|c|c|c|c|c|c|c|c|}
\hline Anallysis & Parameter & Cas Number & $\begin{array}{c}\text { Detection } \\
\text { Limit Range }\end{array}$ & $\begin{array}{l}\text { Number of } \\
\text { Detect }\end{array}$ & $\begin{array}{l}\text { Minimum } \\
\text { Detect }\end{array}$ & $\begin{array}{l}\text { Mean } \\
\text { Resuit }\end{array}$ & $\begin{array}{l}\text { Maximum } \\
\text { Detect }\end{array}$ & $\begin{array}{l}\text { Standard } \\
\text { Deviation }\end{array}$ \\
\hline & Diethylphthalate & $84-66-2$ & $370-8300$ & $1 / 98$ & 43 & 250 & 43 & 399 \\
\hline & Dimethylghthwlate & $131-11-3$ & $370-8300$ & $1 / 98$ & 48 & 250 & 48 & 399 \\
\hline & Fluoranthene & $206-44-0$ & $71-490$ & $30 / 98$ & 41 & 539 & 24000 & 2430 \\
\hline & Fluorene & $86-73-7$ & $370-500$ & $10 / 98$ & 58 & 289 & 8000 & 792 \\
\hline & Indeno(1,2,3-c,d)pyrene & $193-39-5$ & $74-500$ & $16 / 98$ & 47 & 274 & 5700 & 562 \\
\hline & N-Nitroso-di-n-propylamine & $621-64-7$ & $370-8300$ & $2 / 98$ & 56 & 249 & 85 & 399 \\
\hline & Naphthalene & $91-20-3$ & $370-500$ & $5 / 98$ & 45 & 363 & 14000 & 1400 \\
\hline & Pentachlorophenol & $87-86-5$ & $900-20000$ & $5 / 97$ & 62 & 593 & 300 & 971 \\
\hline & Phenanthrene & $85-01-8$ & 56.500 & $20 / 98$ & 48 & 597 & 30000 & 3030 \\
\hline & Phenol & $108-95-2$ & $370-8300$ & $2 / 97$ & 58 & 250 & 62 & 401 \\
\hline & Pyrene & $129-00-0$ & $67-490$ & $33 / 98$ & 44 & 517 & 22000 & 2230 \\
\hline & bis(2-Ethylhexyl)phthallate & $117-81-7$ & $370-8300$ & $24 / 98$ & 41 & 227 & 590 & 408 \\
\hline \multirow[t]{10}{*}{ Volatile Organics UG/RG } & $\begin{array}{l}\text { 1,1,2-Trichloro- } \\
\text { 1,2,2-Trifluoroethane }\end{array}$ & $76-13-1$ & $1-15$ & $17 / 98$ & 1 & 8.47 & 180 & 7.8 \\
\hline & 2-Butanone & $78-93-3$ & $11-1600$ & $4 / 98$ & 5 & 14.9 & 28 & 80.2 \\
\hline & 4-Methyl-2-pentanone & $108-10-1$ & $6-1600$ & $9 / 98$ & 3 & 14.8 & 25 & 80.2 \\
\hline & Acetone & $67-64-1$ & $12-1600$ & 62198 & 14 & 157 & 2100 & 280 \\
\hline & Benzene & $71-43-2$ & $6-1600$ & $3 / 98$ & 1 & 11.4 & 6 & 80.5 \\
\hline & Carbon Disulfide & $75-15-0$ & $6-1600$ & $1 / 98$ & 2 & 11.4 & 2 & 80.5 \\
\hline & Ethylbenzene & $100-41-4$ & $6-1600$ & $2 / 98$ & 4 & 11.5 & 11 & 80.5 \\
\hline & Methylene Chloride & $75-09-2$ & $6-64$ & $48 / 98$ & 6 & 32 & 470 & 59.5 \\
\hline & Tolusene & $108-88-3$ & $6-1600$ & $4 / 98$ & 1 & 11.3 & 2 & 80.5 \\
\hline & Xylene, Total & $1330-20-7$ & $6-1600$ & $2 / 98$ & 23 & 11.8 & 27 & 80.5 \\
\hline Pesticide/PCB UG/KG & $4,4^{\circ}-\mathrm{DDE}$ & $72-55-9$ & $3.3-390$ & $1 / 98$ & 4.1 & 7.03 & 4.1 & 22.6 \\
\hline
\end{tabular}




\begin{tabular}{|c|c|c|c|c|c|c|c|c|}
\hline Analysis & Parameter & Cas Number & $\begin{array}{c}\text { Detection } \\
\text { Limit Range } \\
\end{array}$ & $\begin{array}{c}\text { Number of } \\
\text { Detect }\end{array}$ & $\begin{array}{c}\text { Minimum } \\
\text { Detect }\end{array}$ & $\begin{array}{l}\text { Mean } \\
\text { Result }\end{array}$ & $\begin{array}{c}\text { Maximum } \\
\text { Detect }\end{array}$ & $\begin{array}{l}\text { Standard } \\
\text { Deviation }\end{array}$ \\
\hline & Aroclor-1254 & $11097-69-1$ & $32-3700$ & $19 / 231$ & 43 & 102 & 2400 & 302 \\
\hline & Aroclor-1260 & $11096-82-5$ & $11-4100$ & $66 / 231$ & 20 & 453 & 10000 & 1240 \\
\hline & Endrin & $72-20-8$ & $3.3-390$ & $2 / 98$ & 1.2 & 7 & 1.9 & 22.6 \\
\hline & p, $8^{\circ}$-Methoxychlor & $72-43-5$ & 4.4-2000 & 2198 & 9.2 & 35.9 & 41 & 116 \\
\hline \multirow[t]{5}{*}{ Radionuclides PCI/O } & Gross Alph & $14127-62-9$ & & $185 / 185$ & 0 & 14.8 & 55 & 6.87 \\
\hline & Gross Beta & $12587-47-2$ & & $232 / 232$ & 8.74 & 28.3 & 91.3 & 10.1 \\
\hline & U-234 & $13966-29-5$ & & $232 / 232$ & 0.129 & 2.07 & 11.2 & 1.51 \\
\hline & U-235 & $15117-96-1$ & & $126 / 126$ & 0 & 0.115 & 0.667 & 0.118 \\
\hline & U-238 & $7440-61-1$ & & $232 / 232$ & 0.0572 & 1.95 & 13.3 & 1.48 \\
\hline \multirow[t]{2}{*}{ Miscellaneous \% } & Amosite & - & 0.0 & $5 / 98$ & 1 & 0.051 & 1 & 0.221 \\
\hline & Chrysotile & - & $0-0$ & $4 / 98$ & 1 & 0.0408 & 1 & 0.199 \\
\hline
\end{tabular}

Data Source : Bear Creek Valley OU2 1993 RI held activities validated dato

Raw data available through $1-12$ OREIS

Data does not include QA/QC

Grand mean and standard deviation include nondetects set to $1 / 2$ of the reporting limit

Results $<$ counting uncertainty were considered as mondetects for radionuclides 
TABLE 1. BC-OU2 1993 RI Field Activities Data - Summary Of Detected Contaminants For The Rust Spoil Area Sampling Media: Strean Sediments

\begin{tabular}{|c|c|c|c|c|c|c|c|c|}
\hline Analysis & Parameter & Cas Number & $\begin{array}{c}\text { Detection } \\
\text { Limit Range }\end{array}$ & $\begin{array}{c}\text { Number of } \\
\text { Detects }\end{array}$ & $\begin{array}{c}\text { Minimum } \\
\text { Detect }\end{array}$ & $\begin{array}{l}\text { Mean } \\
\text { Result }\end{array}$ & $\begin{array}{l}\text { Maximumn } \\
\text { Detect }\end{array}$ & $\begin{array}{l}\text { Standard } \\
\text { Deviation }\end{array}$ \\
\hline \multirow[t]{3}{*}{ Metals MG/KG (AAS) } & Antimony & $7440-360$ & $0.28-0.28$ & $2 / 3$ & 0.23 & 0.237 & 0.34 & 0.1 \\
\hline & Arsenic & $7440-38-2$ & & $3 / 3$ & 31.7 & 38.8 & 50.3 & 10.1 \\
\hline & Thallium & $7440-28-0$ & $0.3-0.3$ & $2 / 3$ & 0.33 & 0.293 & 0.4 & 0.129 \\
\hline Metals Mo/Ka & Mercury & $7439-97-6$ & & $3 / 3$ & 0.17 & 0.233 & 0.29 & 0.0603 \\
\hline \multirow[t]{18}{*}{ Metals MG/KO (ICP) } & Aluminum & $7429-90-5$ & & $3 / 3$ & 8240 & 13700 & 17900 & 4940 \\
\hline & Barium & $7440-39-3$ & & $3 / 3$ & 25.7 & 39 & 47.9 & 11.7 \\
\hline & Beryllium & $7440-41-7$ & & $3 / 3$ & 0.53 & 0.777 & 0.93 & 0.216 \\
\hline & Cadmium & $7440-43-9$ & $0.12-0.13$ & $1 / 3$ & 3.5 & 1.21 & 3.5 & 1.98 \\
\hline & Caleium & $7440-70-2$ & & $3 / 3$ & 1510 & 30700 & 87100 & 48800 \\
\hline & Chromium & $7440-47-3$ & & $3 / 3$ & 16.5 & 20.3 & 23.9 & 3.71 \\
\hline & Cobalt & $7440-48-4$ & & $3 / 3$ & 19.7 & 47.8 & 103 & 47.8 \\
\hline & Copper & $7440-50-8$ & & $3 / 3$ & 25.1 & 65.5 & 131 & 57.2 \\
\hline & Iron & $7439-89.6$ & & $3 / 3$ & 19400 & 27900 & 32500 & 7370 \\
\hline & Lead & $7439-92-1$ & & $3 / 3$ & 66.4 & 88.2 & 114 & 24 \\
\hline & Magnesium & $7439-95-4$ & & $3 / 3$ & 639 & 1030 & 1350 & 360 \\
\hline & Manganese & $7439-96-5$ & & $3 / 3$ & 737 & 2550 & 6060 & 3040 \\
\hline & Nickel & $7440-02-0$ & & $3 / 3$ & 21.8 & 66.6 & 147 & 69.8 \\
\hline & Potassium & $7440-09-7$ & & $3 / 3$ & 412 & 625 & 829 & 209 \\
\hline & Silver & $7440-22-4$ & $0.63-0.63$ & $2 / 3$ & 0.58 & 0.698 & 1.2 & 0.454 \\
\hline & Sodium & $7440-23-5$ & $30.5-35.3$ & $1 / 3$ & 116 & 49.6 & 116 & 57.5 \\
\hline & Vanadium & $7440-62-2$ & & $3 / 3$ & 44.9 & 65.3 & 75.9 & 17.6 \\
\hline & Zinc & $7440-66-6$ & & $3 / 3$ & 123 & 207 & 302 & 90.1 \\
\hline
\end{tabular}




\begin{tabular}{|c|c|c|c|c|c|c|c|c|}
\hline Analysis & Parameter & Cas Number & $\begin{array}{c}\text { Detection } \\
\text { Limit Range } \\
\end{array}$ & $\begin{array}{c}\text { Number of } \\
\text { Detects }\end{array}$ & $\begin{array}{c}\text { Minimum } \\
\text { Detect }\end{array}$ & $\begin{array}{l}\text { Mean } \\
\text { Result }\end{array}$ & $\begin{array}{c}\text { Maximum } \\
\text { Detect }\end{array}$ & $\begin{array}{l}\text { Standard } \\
\text { Deviation }\end{array}$ \\
\hline \multirow{3}{*}{$\begin{array}{l}\text { Semivolatile Organics } \\
\text { UG/KG }\end{array}$} & Fluoranthene & $206-44-0$ & $600-600$ & 213 & 49 & 138 & 65 & 141 \\
\hline & Naphthalene & $91-20-3$ & $480-510$ & $1 / 3$ & 64 & 186 & 64 & 106 \\
\hline & Pyrene & $129-00-0$ & $480-600$ & $1 / 3$ & 52 & 197 & 52 & 129 \\
\hline $\begin{array}{l}\text { Petroleum Hydrocarbons } \\
\text { MG/KO }\end{array}$ & Total Petroleun Hydrocarbons & TPH & $16.9-21.6$ & 113 & 60.6 & 26.6 & 60.6 & 29.5 \\
\hline Volatile Organics UG/KG & Tetrachloroethene & $127-18-4$ & $7-8$ & $1 / 3$ & 7 & 4.83 & 7 & 1.89 \\
\hline Pesticide/PCB UG/KG & Aroclor- 1260 & $11096-82-5$ & $49-49$ & $2 / 3$ & 21 & 28.8 & 41 & 10.7 \\
\hline \multirow[t]{5}{*}{ Radionuclides PCH/O } & Gross Alpha & $14127-62-9$ & & $3 / 3$ & 6.08 & 17.1 & 23.3 & 9.56 \\
\hline & Gross Befa & $12587-47-2$ & & $3 / 3$ & 20.6 & 25.8 & 29.1 & 4.58 \\
\hline & $U-234$ & 13966-29-5 & & $3 / 3$ & 2.56 & 3.05 & 3.86 & 0.709 \\
\hline & U-235 & $15117-96-1$ & & $3 / 3$ & 0.183 & 0.274 & 0.397 & 0.111 \\
\hline & U-238 & $7440-61-1$ & & $3 / 3$ & 2.57 & 3.15 & 3.95 & 0.716 \\
\hline
\end{tabular}

Raw data available through Y-12 OREIS

Data does not include QA/QC

Grand mean and standard deviation include nondetects set to $1 / 2$ of the reporting limil

Results $<$ counting uncertainty were considered as nondetects for radionuclides 
TABLE 1. FC-OU2 1993 RI Field Activities Data - Summary Of Detected Contaminants For The Spoil Area 1 Sampling Media: Stream Sediments

\begin{tabular}{|c|c|c|c|c|c|c|c|c|}
\hline Analysis & Parameter & Cas Number & $\begin{array}{c}\text { Detection } \\
\text { Limit Range }\end{array}$ & $\begin{array}{c}\text { Number of } \\
\text { Detects }\end{array}$ & $\begin{array}{l}\text { Minimum } \\
\text { Detect }\end{array}$ & $\begin{array}{l}\text { Mean } \\
\text { Result }\end{array}$ & $\begin{array}{c}\text { Maximum } \\
\text { Detect }\end{array}$ & $\begin{array}{l}\text { Standard } \\
\text { Deviation }\end{array}$ \\
\hline \multirow[t]{4}{*}{ Metals MO/KG (AAS) } & Antimony & $7440-36-0$ & & $4 / 4$ & 0.24 & 0.295 & 0.32 & 0.0379 \\
\hline & Arsenic & $7440-38-2$ & & 414 & 15.6 & 25.5 & 42.4 & 11.8 \\
\hline & Selenium & $7782-49-2$ & $0.24-0.31$ & 214 & 2 & 1.12 & 2.2 & 1.14 \\
\hline & Thallium & $7440-28-0$ & $0.21-0.24$ & $1 / 4$ & 0.32 & 0.164 & 0.32 & 0.104 \\
\hline Metals MOKKO & Mercury & $7439-97.6$ & & 414 & 0.1 & 0.23 & 0.52 & 0.197 \\
\hline \multirow[t]{17}{*}{ Metals MG/KG (ICP) } & Aluminum & $7429-90-5$ & & 414 & 6840 & 10500 & 14100 & 3220 \\
\hline & Rarium & $7440-39-3$ & & $4 / 4$ & 17.9 & 40.1 & 73.1 & 25.2 \\
\hline & Beryllium & $7440-41-7$ & & 414 & 0.29 & 0.478 & 0.71 & 0.174 \\
\hline & Calcium & $7440-70-2$ & & 414 & 958 & 27100 & 62600 & 28300 \\
\hline & Chromium & $7440-47-3$ & & $4 / 4$ & 29.1 & 37.4 & 48.8 & 9.11 \\
\hline & Cobalt & $7440-48-4$ & & 414 & 2.4 & 6.65 & 10 & 3.15 \\
\hline & Copper & $7440-50-8$ & & 414 & 10.3 & 13.1 & 14.8 & 2.04 \\
\hline & Iron & $7439-89-6$ & & 414 & 15500 & 24300 & 33300 & 7290 \\
\hline & Lead & $7439-92-1$ & & 414 & 18.3 & 29.1 & 38 & 8.96 \\
\hline & Magnesium & $7439-95-4$ & & $4 / 4$ & 487 & 2420 & 4950 & 1920 \\
\hline & Manganese & $7439-96-5$ & & 414 & 55.8 & 483 & 932 & 366 \\
\hline & Nickel & $7440-02-0$ & & 414 & 5.8 & 11.3 & 19.4 & 5.86 \\
\hline & Potassium & $7440-09-7$ & & 414 & 319 & 417 & 557 & 103 \\
\hline & Silver & $7440-22-4$ & $0.46-0.52$ & $1 / 4$ & 0.69 & 0.358 & 0.69 & 0.222 \\
\hline & Sodium & $7440-23-5$ & $33.3-65.8$ & $1 / 4$ & 76.5 & 36 & 76.5 & 28 \\
\hline & Vanadium & $7440-62-2$ & & $4 / 4$ & 35.2 & 51.5 & 58.4 & 11 \\
\hline & Zine & $7440-66-6$ & & $4 / 4$ & 43.1 & 60.9 & 70.4 & 12.7 \\
\hline
\end{tabular}




\begin{tabular}{|c|c|c|c|c|c|c|c|c|}
\hline Analysis & Parameter & Cas Number & $\begin{array}{c}\text { Detection } \\
\text { Limit Range } \\
\end{array}$ & $\begin{array}{c}\text { Number of } \\
\text { Detects }\end{array}$ & $\begin{array}{c}\text { Minimum } \\
\text { Detect }\end{array}$ & $\begin{array}{l}\text { Mean } \\
\text { Result }\end{array}$ & $\begin{array}{c}\text { Maximum } \\
\text { Detect }\end{array}$ & $\begin{array}{l}\text { Standard } \\
\text { Deviation }\end{array}$ \\
\hline $\begin{array}{l}\text { Semivolatile Organics } \\
\text { UG/KG }\end{array}$ & Naphthalene & $91-20-3$ & $430-500$ & $1 / 4$ & 53 & 186 & 53 & 89.7 \\
\hline \multirow[t]{2}{*}{ Volatile Organics UG/KO } & 4-Methyl-2-pentanone & $108-10-1$ & & $4 / 4$ & 4 & 8.25 & 130 & 4.03 \\
\hline & Acetone & $67-64-1$ & $13-27$ & $1 / 4$ & 51 & 19.5 & 51 & 21.2 \\
\hline Pesticide/PCB UG/KG & Aroclor-1260 & $11096-82-5$ & $44-50$ & $1 / 4$ & 31 & 25.3 & 31 & 4.03 \\
\hline \multirow[t]{5}{*}{ Radionuclides PCV/O } & Gross Alph & $14127-62-9$ & & 414 & 8.17 & 14.8 & 20.3 & 5.78 \\
\hline & Gross Bets & $12587-47.2$ & & 414 & 8.68 & 15.1 & 21.1 & 5.93 \\
\hline & $\mathrm{U}-234$ & $13966-29-5$ & & $4 / 4$ & 1.71 & 2.14 & 2.93 & 0.54 \\
\hline & U-235 & $15117-96-1$ & & $4 ! 4$ & 0.107 & 0.253 & 0.431 & 0.145 \\
\hline & U-238 & $7440-61-1$ & & $4 / 4$ & 1.43 & 1.78 & 2.03 & 0.262 \\
\hline
\end{tabular}

Data Source : Bear Creek Valley OU2 1993 RI field activities validated data Raw data available through Y-12 OREIS

Data does not include QA/QC

Orand mean and standard deviation include nondetects set to $1 / 2$ of the reporting limit

Results $<$ counting uncertainty were considered nondetect for radionuclides 
TABLE 1. BC-OU2 1993 RI Field Activities Data - Summary Of Detected Contaminants For The SY-200 Yard Sampling Media: Stream Sediments

\begin{tabular}{|c|c|c|c|c|c|c|c|c|}
\hline Analysis & Parmmeter & Cas Number & $\begin{array}{c}\text { Detection } \\
\text { Limit Range }\end{array}$ & $\begin{array}{c}\text { Number of } \\
\text { Detects }\end{array}$ & $\begin{array}{l}\text { Minimum } \\
\text { Detect }\end{array}$ & $\begin{array}{l}\text { Mean } \\
\text { Result }\end{array}$ & $\begin{array}{l}\text { Maximum } \\
\text { Detect }\end{array}$ & $\begin{array}{l}\text { Standard } \\
\text { Deviation }\end{array}$ \\
\hline \multirow[t]{4}{*}{ Metals MG/KO (AAS) } & Antimony & $7440-36-0$ & & $2 / 2$ & 0.26 & 0.375 & 0.49 & 0.163 \\
\hline & Arsenic & $7440-38-2$ & & 2) 2 & 29.1 & 30.3 & 31.5 & 1.7 \\
\hline & Selenium & $7782-49-2$ & $0.35-0.35$ & $1 / 2$ & 3.3 & 1.74 & 3.3 & 2.21 \\
\hline & Thallium & $7440-28-0$ & & $2 / 2$ & 0.37 & 0.405 & 0.44 & 0.0495 \\
\hline Metals MG/KG & Mereury & $7439-97-6$ & & $2 / 2$ & 0.23 & 0.865 & 1.5 & 0.898 \\
\hline \multirow[t]{16}{*}{ Metals MO/KO (ICP) } & Aluminum & $7429-90-5$ & & $2 / 2$ & 15600 & 25100 & 34500 & 13400 \\
\hline & Barium & $7440-39-3$ & & $2 / 2$ & 29 & 29.5 & 30 & 0.707 \\
\hline & Beryllium & $7440-41-7$ & & $2 / 2$ & 0.88 & 1.24 & 1.6 & 0.509 \\
\hline & Calcium & $7440-70-2$ & & 212 & 1360 & 7630 & 13900 & 8870 \\
\hline & Chromium & $7440-47-3$ & & 2) 2 & 25.6 & 37.4 & 49.1 & 16.6 \\
\hline & Cobalt & $7440-48-4$ & & $2 / 2$ & 18.7 & 20.3 & 21.9 & 2.26 \\
\hline & Copper & $7440-50-8$ & & 212 & 36.4 & 41.1 & 45.7 & 6.58 \\
\hline & Iron & $7439-89.6$ & & $2 / 2$ & 28700 & 33200 & 37600 & 6290 \\
\hline & Lead & $7439-92-1$ & & $2 / 2$ & 66 & 81.1 & 96.1 & 21.3 \\
\hline & Magnesium & $7439-95-4$ & & $2 / 2$ & 1630 & 1730 & 1820 & 134 \\
\hline & Manganese & $7439-96-5$ & & $2 / 2$ & 406 & 496 & 586 & 127 \\
\hline & Nickel & $7440-02-0$ & & $2 / 2$ & 28.2 & 34.5 & 40.8 & 8.91 \\
\hline & Potassium & $7440-09-7$ & & $2 / 2$ & 930 & 1200 & 1460 & 375 \\
\hline & Silver & $7440-22-4$ & & $2 / 2$ & 0.65 & 0.655 & 0.66 & 0.00707 \\
\hline & Vanadium & $7440-62-2$ & & 212 & 57.5 & 70 & 82.5 & 17.7 \\
\hline & Zine & $7440-66-6$ & & $2 / 2$ & 129 & 150 & 170 & 29 \\
\hline \multirow[t]{2}{*}{ Volatile Organics UO/KG } & 4-Methyl-2-pentanone & $108-10-1$ & & $2 / 2$ & 16 & 40 & 64 & 33.9 \\
\hline & Acetone & $67-64-1$ & $29-29$ & $1 / 2$ & 280 & 147 & 280 & 188 \\
\hline
\end{tabular}




\begin{tabular}{|c|c|c|c|c|c|c|c|c|}
\hline Analysiz & Parameter & Cas Number & $\begin{array}{c}\text { Detection } \\
\text { Limit Range }\end{array}$ & $\begin{array}{c}\text { Number of } \\
\text { Detects }\end{array}$ & $\begin{array}{c}\text { Minimum } \\
\text { Detect }\end{array}$ & $\begin{array}{l}\text { Mean } \\
\text { Result }\end{array}$ & $\begin{array}{c}\text { Maximum } \\
\text { Detect }\end{array}$ & $\begin{array}{l}\text { Standard } \\
\text { Deviation }\end{array}$ \\
\hline Pesticide/PCB UG/KO & Aroclor- 1260 & 11096-82-5 & $49-49$ & $1 / 2$ & 43 & 33.8 & 43 & 13.1 \\
\hline \multirow[t]{5}{*}{ Radionuclides PCI/G } & Gross Alpha & $14127-62-9$ & & $2 / 2$ & 7.73 & 13.3 & 18.8 & 7.83 \\
\hline & Gross Beta & $12587-47-2$ & & $2 / 2$ & 15.2 & 22.8 & 30.3 & 10.7 \\
\hline & U.234 & $13966-29-5$ & & $2 / 2$ & 1.87 & 2.36 & 2.84 & 0.686 \\
\hline & U-235 & $15117-96-1$ & & $2 / 2$ & 0.12 & 0.16 & 0.2 & 0.0566 \\
\hline & U-238 & $7440-61-1$ & & $2 / 2$ & 1.84 & 2.24 & 2.63 & 0.559 \\
\hline
\end{tabular}

Data Source : Bear Creek Valley OU2 1993 RI field activities validated data Raw data available through $\mathrm{Y}-12$ OREIS

Data does not include QA/QC

Grand mean and standard deviation include nondetects set to $1 / 2$ of the reporting limit

Results < counting uncertainty were considered as nondetects for radionuclides 
TABLE 1. BC-OU2 1993 RI Field Activities Data - Summary Of Detected Contaminants For The Rust Spoil Area Sampling Media: Surface Water

\begin{tabular}{|c|c|c|c|c|c|c|c|c|}
\hline Analysis & Parameter & Cas Number & $\begin{array}{c}\text { Detection } \\
\text { Limil Range }\end{array}$ & $\begin{array}{l}\text { Number of } \\
\text { Detects }\end{array}$ & $\begin{array}{c}\text { Minimum } \\
\text { Detect }\end{array}$ & $\begin{array}{l}\text { Mean } \\
\text { Result }\end{array}$ & $\begin{array}{c}\text { Maximum } \\
\text { Detect }\end{array}$ & $\begin{array}{l}\text { Standard } \\
\text { Deviation }\end{array}$ \\
\hline \multirow[t]{4}{*}{ Anions MO/L } & Chloride & & & $3 / 3$ & 3.4 & 17.9 & 46.4 & 24.7 \\
\hline & Fluoride & $16984-48-8$ & & $3 / 3$ & 0.13 & 0.153 & 0.18 & 0.0252 \\
\hline & Nitrate/Nitrite (NO3/NO2-N) & $14797-55-8$ & $0.2-0.2$ & $1 / 3$ & 31 & 10.4 & 31 & 17.8 \\
\hline & Sulfate & & & $3 / 3$ & 8.7 & 35.8 & 75.9 & 35.4 \\
\hline \multirow[t]{4}{*}{ Metals UG/L (AAS) } & Antimony & $7440-36-0$ & $1.8-1.8$ & $1: 3$ & 4.3 & 2.03 & 4.3 & 1.96 \\
\hline & Antimony (Dissolved) & $7440-36-0$ & & $3 / 3$ & 4.9 & 6.1 & 8.2 & 1.82 \\
\hline & Lead & $7439-92-1$ & & $3 / 3$ & 1.6 & 60.9 & 172 & 96.3 \\
\hline & Lead (Dissolved) & $7439-92-1$ & & $3 / 3$ & 2 & 6.9 & 16.5 & 8.31 \\
\hline \multirow[t]{15}{*}{ Metals UG/L (ICP) } & Aluminum & $7429-90-5$ & $140-140$ & $2 / 3$ & 3050 & 9870 & 26500 & 14500 \\
\hline & Barium & $7440-39-3$ & & $3 / 3$ & 34 & 97.4 & 173 & 70.3 \\
\hline & Barium (Dissolved) & $7440-39-3$ & & $3 / 3$ & 22 & 46.6 & 88.1 & 36.1 \\
\hline & Caleium & $7440-70-2$ & & $3 / 3$ & 37600 & 92500 & 79000 & 75800 \\
\hline & Calcium (Dissolved) & $7440-70-2$ & & $3 / 3$ & 36900 & 89600 & 188000 & 85300 \\
\hline & Chromium & $7440-47-3$ & $2.5-3.9$ & $1 / 3$ & 29.2 & 10.8 & 29.2 & 15.9 \\
\hline & Cobalt & $7440-48-4$ & $2.9-2.9$ & $1 / 3$ & 46.7 & 16.5 & 46.7 & 26.1 \\
\hline & Copper (Dissolved) & $7440-50-8$ & $5-5$ & $2 / 3$ & 7.6 & 6.4 & 9.1 & 3.46 \\
\hline & Iron & $7439-89-6$ & $166-166$ & $2 / 3$ & 5410 & 16000 & 42600 & 23200 \\
\hline & Magnesium & $7439-95-4$ & & $3 / 3$ & 4040 & 10000 & 16800 & 6410 \\
\hline & Magnesium (Dissolved) & $7439-95-4$ & & $3 / 3$ & 3800 & 8610 & 17700 & 7870 \\
\hline & Manganese & $7439-96-5$ & 15.1-15.1 & $2 / 3$ & 79.8 & 582 & 1660 & 934 \\
\hline & Potassium & $7440-09-7$ & & $3 / 3$ & 2640 & 3560 & 4660 & 1020 \\
\hline & Potassium (Dissolved) & $7440-09-7$ & & $3 / 3$ & 2130 & 2710 & 3560 & 754 \\
\hline & Sodium & $7440-23-5$ & & $3 / 3$ & 852 & 5800 & 15200 & 8140 \\
\hline
\end{tabular}




\begin{tabular}{|c|c|c|c|c|c|c|c|c|}
\hline Analysis & Parameter & Cas Number & $\begin{array}{l}\text { Detection } \\
\text { Limit Range }\end{array}$ & $\begin{array}{l}\text { Number of } \\
\text { Detects }\end{array}$ & $\begin{array}{l}\text { Minimum } \\
\text { Detect }\end{array}$ & $\begin{array}{l}\text { Mean } \\
\text { Result }\end{array}$ & $\begin{array}{l}\text { Maximum } \\
\text { Detect }\end{array}$ & $\begin{array}{l}\text { Standard } \\
\text { Deviation }\end{array}$ \\
\hline & Sodium (Dissolved) & $7440-23-5$ & & $3 / 3$ & 883 & 5900 & 15900 & 8660 \\
\hline & Vanadium & $7440-62-2$ & $2.4-14.4$ & $1 / 3$ & 116 & 41.5 & 116 & 64.6 \\
\hline & Zine & $7440-66-6$ & $6.6-52.8$ & $1 / 3$ & 673 & 234 & 673 & 380 \\
\hline \multirow[t]{2}{*}{ Semivolatile Organics UG/L } & Butylbenzylphthalate & $85-68-7$ & $10-10$ & $1 / 3$ & 2 & 4 & 2 & 1.73 \\
\hline & Di-n-butylphthalate & $84-74-2$ & $10-10$ & $1 / 3$ & 2 & 4 & 2 & 1.73 \\
\hline \multirow[t]{3}{*}{ Volatile Organies UG/L } & 2-Butanone & $78-93-3$ & $10-10$ & $1 / 3$ & 3 & 4.33 & 3 & 1.15 \\
\hline & Methylene Chloride & $75-09-2$ & $5-5$ & $1 / 3$ & 5 & 3.33 & 5 & 1.44 \\
\hline & Tetrachloroethene & $127-18-4$ & $5-5$ & $1 / 3$ & 1 & 2 & 1 & 0.866 \\
\hline \multirow[t]{5}{*}{ Radionuclides PCI/L } & Gross Alph & $14127-62-9$ & 55-55 & 213 & 6.34 & 28.7 & 24.9 & 24.6 \\
\hline & Gross Beta & $12587-47-2$ & & 66 & 6.08 & 35.5 & 68 & 31.1 \\
\hline & $\mathrm{U}-234$ & $13966-29-5$ & & 616 & 2.6 & 7.33 & 14.6 & 5.49 \\
\hline & U.235 & $15117.96-1$ & & 313 & 0.117 & 0.328 & 0.734 & 0.352 \\
\hline & U.238 & $7440-61-1$ & & 616 & 3.19 & 7.9 & 14.5 & 4.8 \\
\hline \multirow[t]{4}{*}{ Physical Propertie MG/L } & Alkalinity & $\cdot$ & & $3 / 3$ & 96.9 & 119 & 141 & 22.1 \\
\hline & Total Dissolved Solids & & & $3 / 3$ & 167 & 353 & 701 & 302 \\
\hline & Total Suspended Solids & & & $3 / 3$ & 43 & 77.3 & 95 & 29.7 \\
\hline & Turbidity & & & $3 / 3$ & 93 & 109 & 139 & 26 \\
\hline
\end{tabular}

Data Source : Bear Creek Valley OU2 1993 RI field activities validated data Raw data available through $Y-12$ OREIS

Data does not include QA/OC

Grand mean and standard deviation include mondetects set to $1 / 2$ of the reporting limit

Results $<$ counting uncertainty were considered so nondetects for radionuclides 
TABLE 1. BC-OU2 1993 RI Field Activities Data - Summary Or Detected Contaminamts For The Spoil Area I Sampling Media: Surface Water

\begin{tabular}{|c|c|c|c|c|c|c|c|c|}
\hline Analysis & Parameter & Cas Number & $\begin{array}{l}\text { Detection } \\
\text { Limit Range }\end{array}$ & $\begin{array}{c}\text { Number of } \\
\text { Detects }\end{array}$ & $\begin{array}{l}\text { Minimum } \\
\text { Detect }\end{array}$ & $\begin{array}{l}\text { Mean } \\
\text { Result }\end{array}$ & $\begin{array}{c}\text { Maximum } \\
\text { Detect }\end{array}$ & $\begin{array}{l}\text { Standard } \\
\text { Deviation }\end{array}$ \\
\hline \multirow[t]{4}{*}{ Anions MG/L } & Chloride & & $1-1$ & $2 / 3$ & 4 & 3.5 & 6 & 2.78 \\
\hline & Fluoride & $16984-48-8$ & & $3 / 3$ & 0.13 & 0.173 & 0.21 & 0.0404 \\
\hline & Nitrate/Nitrite (NO3/NO2-N) & $14797-55.8$ & $0.2-0.2$ & $1 / 3$ & 0.32 & 0.173 & 0.32 & 0.127 \\
\hline & Sulfate & & $5-5$ & $2 / 3$ & 49.3 & 39.1 & 65.4 & 32.7 \\
\hline \multirow[t]{6}{*}{ Metals UO/L (AAS) } & Antimony & $7440-36-0$ & & 313 & 1.9 & 1.97 & 2.1 & 0.115 \\
\hline & Antimony (Dissolved) & $7440-36-0$ & & $3 / 3$ & 2.1 & 5.4 & 10.3 & 4.33 \\
\hline & Arsenic & $7440-38-2$ & $1.6-1.6$ & $2 / 3$ & 2.3 & 2.23 & 3.6 & 1.4 \\
\hline & Lead & $7439-92-1$ & & $3 / 3$ & 1.2 & 4.27 & 6 & 2.66 \\
\hline & Lead (Dissolved) & $7439-92-1$ & & $3 / 3$ & 4.4 & 6.87 & 11.7 & 4.19 \\
\hline & Selenium & $7782-49-2$ & $4.1-4.1$ & $2 / 3$ & 3.3 & 2.92 & 3.4 & 0.752 \\
\hline Metals UG/L & Mercury (Dissolved) & $7439-97-6$ & $0.2-0.2$ & $1 / 3$ & 0.2 & 0.133 & 0.2 & 0.0577 \\
\hline \multirow[t]{12}{*}{ Metals UG/L (ICP) } & Aluminum & $7429-90-5$ & $107-107$ & $2 / 3$ & 533 & 902 & 2120 & 1080 \\
\hline & Barium & $7440-39-3$ & & $3 / 3$ & 31.8 & 38.6 & 43.3 & 6.03 \\
\hline & Barium (Dissolved) & $7440-39-3$ & & $3 / 3$ & 20.7 & 33.6 & 41.4 & 11.3 \\
\hline & Calcium & $7440-70-2$ & & $3 / 3$ & 25500 & 45400 & 60700 & 18000 \\
\hline & Calcium (Dissolved) & $7440-70-2$ & & $3 / 3$ & 25400 & 43100 & 56200 & 15900 \\
\hline & Chromium & $7440-47-3$ & $2.5-5.6$ & $1 / 3$ & 6.1 & 3.38 & 6.1 & 2.48 \\
\hline & Cobalt (Dissolved) & $7440-48-4$ & $2-2$ & $1 / 3$ & 2.6 & 1.53 & 2.6 & 0.924 \\
\hline & Copper & $7440-50-8$ & $4.2-5$ & $1 / 3$ & 6.5 & 3.7 & 6.5 & 2.43 \\
\hline & Copper (Dissolved) & $7440-50-8$ & $5-5$ & 213 & 18.2 & 16.2 & 28 & 12.9 \\
\hline & Iron & $7439-89-6$ & $150-150$ & $2 / 3$ & 822 & 1540 & 3730 & 1930 \\
\hline & Iron (Dissolved) & $7439-89-6$ & $40.1-80.2$ & $1 / 3$ & 233 & 97.7 & 233 & 118 \\
\hline & Magnesium & $7439-95-4$ & & $3 / 3$ & 3700 & 7020 & 9010 & 2890 \\
\hline
\end{tabular}




\begin{tabular}{|c|c|c|c|c|c|c|c|c|}
\hline Analysis & Parameter & Cas Number & $\begin{array}{l}\text { Detection } \\
\text { Limit Range }\end{array}$ & $\begin{array}{l}\text { Number of } \\
\text { Detects }\end{array}$ & $\begin{array}{c}\text { Minimum } \\
\text { Detect }\end{array}$ & $\begin{array}{l}\text { Mean } \\
\text { Result }\end{array}$ & $\begin{array}{c}\text { Maximum } \\
\text { Detect }\end{array}$ & $\begin{array}{l}\text { Standard } \\
\text { Deviation }\end{array}$ \\
\hline & Magnesium (Dissolved) & $7439-95-4$ & & $3 / 3$ & 3520 & 6700 & 8560 & 2770 \\
\hline & Manganese & $7439-96-5$ & $4.5-4.5$ & $2 / 3$ & 15.2 & 16.9 & 33.2 & 15.5 \\
\hline & Potassium & $7440-09-7$ & & $3 / 3$ & 1770 & 2660 & 3120 & 771 \\
\hline & Potassium (Dissolved) & $7440-09-7$ & & $3 / 3$ & 1680 & 2600 & 3130 & 797 \\
\hline & Sodium & $7440-23-5$ & & $3 / 3$ & 879 & 1290 & 1530 & 360 \\
\hline & Sodium (Dissolved) & $7440-23-5$ & & 313 & 842 & 1240 & 1540 & 358 \\
\hline & Vanadium & $7440-62-2$ & $2.4-2.4$ & 213 & 2.9 & 4.6 & 9.7 & 4.5 \\
\hline & Zine & $7440-66-6$ & $19.3-19.3$ & $2 / 3$ & 13 & 23.4 & 47.5 & 21 \\
\hline & Zine (Dissolved) & $7440-66-6$ & $2.3-2.3$ & $2 / 3$ & 32 & 22.3 & 33.7 & 18.3 \\
\hline \multirow{2}{*}{$\begin{array}{l}\text { Semivolatile Organics } \\
\text { UG/L }\end{array}$} & Butylbenzylphthalate & $85-68-7$ & $9-10$ & $1 / 3$ & 1 & 3.5 & 1 & 2.18 \\
\hline & Di-n-butylphthalate & $84-74-2$ & $9-10$ & $1 / 3$ & 1 & 3.5 & 1 & 2.18 \\
\hline Volatile Organics UG/L & 2-Butanone & $78-93-3$ & $10-10$ & $1 / 3$ & 3 & 4.33 & 3 & 1.15 \\
\hline \multirow[t]{5}{*}{ Radionuclides PCI/L } & Gross Alpha & $14127-62-9$ & & $3 / 3$ & 2.73 & 3.82 & 6 & 1.89 \\
\hline & Gross Beta & $12587-47-2$ & & $6 / 6$ & 1.64 & 4.28 & 6.89 & 2.05 \\
\hline & $\mathbf{U}-234$ & $13966-29-5$ & & $6 / 6$ & 1.26 & 3.44 & 6.21 & 1.87 \\
\hline & $\mathrm{U}-235$ & $15117-96-1$ & & $3 / 3$ & $\begin{array}{l}0.0081 \\
3\end{array}$ & 0.133 & 0.277 & 0.136 \\
\hline & U-238 & $7440-61-1$ & & $6 / 6$ & 0.62 & 1.2 & 1.75 & 0.46 \\
\hline \multirow[t]{4}{*}{ Physical Properties MG/L } & Alkalinity & & & $3 / 3$ & 59.4 & 104 & 127 & 38.5 \\
\hline & Total Dissolved Solids & & & $3 / 3$ & 195 & 220 & 269 & 42.2 \\
\hline & Total Suspended Solids & & $10-10$ & $2 / 3$ & 36 & 36 & 67 & 31 \\
\hline & Turbidity & & & $3 / 3$ & 4 & 58.7 & 136 & 68.9 \\
\hline
\end{tabular}


Data does not include QA/QC

Grand mean and standard deviation include nondetects set to $1 / 2$ of the reporting limit

Results < counting uncertainty were considered as nondetects for radionuclides 
TABLE 1. BC-OU2 1993 RI Field Activities Data - Summary Of Detected Contaminants For The SY-200 Yard Sampling Media: Surface Water

\begin{tabular}{|c|c|c|c|c|c|c|c|c|}
\hline Analysis & Parameter & Cas Number & $\begin{array}{c}\text { Detection } \\
\text { Limit Range } \\
\end{array}$ & $\begin{array}{c}\text { Number of } \\
\text { Detects }\end{array}$ & $\begin{array}{c}\text { Minimum } \\
\text { Detect }\end{array}$ & $\begin{array}{c}\text { Mean } \\
\text { Result } \\
\end{array}$ & $\begin{array}{c}\text { Maximum } \\
\text { Detect }\end{array}$ & $\begin{array}{l}\text { Standard } \\
\text { Deviation } \\
\end{array}$ \\
\hline \multirow[t]{4}{*}{ Anions MO/L } & Chloride & & & $2 / 2$ & 2.9 & 5.8 & 8.7 & 4.1 \\
\hline & Fluoride & $16984-48-8$ & & 212 & 0.27 & 0.31 & 0.35 & 0.0566 \\
\hline & Nitrate/Nitrite (NO3/NO2-N) & $14797-55-8$ & & $2 / 2$ & 0.65 & 1.38 & 2.1 & 1.03 \\
\hline & Sulfate & & & $2 / 2$ & 33.7 & 52.9 & 72 & 27.1 \\
\hline \multirow[t]{4}{*}{ Metale UG/L (AAS) } & Antimony & $7440-36-0$ & $1.8-1.8$ & $1 / 2$ & 1.9 & 1.4 & 1.9 & 0.707 \\
\hline & Antimony (Dissolved) & $7440-36-0$ & & $2 / 2$ & 2.4 & 2.6 & 2.8 & 0.283 \\
\hline & Lead & $7439-92-1$ & & $2 / 2$ & 5.4 & 5.55 & 5.7 & 0.212 \\
\hline & Lead (Dissolved) & $7439-92-1$ & & 212 & 2.1 & 12.2 & 22.2 & 14.2 \\
\hline \multirow[t]{15}{*}{ Metals UG/L (ICP) } & Aluminum & $7429-90-5$ & & $2 / 2$ & 749 & 859 & 968 & 155 \\
\hline & Barium & $7440-39-3$ & $10-10$ & $1 / 2$ & 52.2 & 28.6 & 52.2 & 33.4 \\
\hline & Barium (Dissolved) & $7440-39-3$ & & $2 / 2$ & 10.4 & 29.8 & 49.2 & 27.4 \\
\hline & Calcium & $7440-70-2$ & & $2 / 2$ & 30400 & 45500 & 60600 & 21400 \\
\hline & Calcium (Dissolved) & $7440-70-2$ & & $2 / 2$ & 32300 & 45000 & 57600 & 17900 \\
\hline & Copper & $7440-50-8$ & 2.4-2.4 & $1 / 2$ & 6.1 & 3.65 & 6.1 & 3.46 \\
\hline & Copper (Dissolved) & $7440-50-8$ & $5-5$ & $1 / 2$ & 9.1 & 5.8 & 9.1 & 4.67 \\
\hline & Iron & $7439-89-6$ & & $2 / 2$ & 960 & 1240 & 1520 & 396 \\
\hline & Magnesium & $7439-95-4$ & & 2/ 2 & 2320 & 6310 & 10300 & 5640 \\
\hline & Magnesium (Disgolved) & $7439.95-4$ & & $2 / 2$ & 2350 & 6180 & 10000 & 5410 \\
\hline & Manganese & $7439-96-5$ & & $2 / 2$ & 16.7 & 23.3 & 29.8 & 9.26 \\
\hline & Potassium & 7440-09-7 & & $2 / 2$ & 1030 & 2330 & 3630 & 1840 \\
\hline & Potassium (Dissolved) & $7440-09-7$ & & $2 / 2$ & 858 & 2200 & 3540 & 1900 \\
\hline & Sodium & $7440-23-5$ & & $2 / 2$ & 802 & 2530 & 4260 & 2450 \\
\hline & Sodium (Dissolved) & $7440-23-5$ & & $2 / 2$ & 772 & 2480 & 4190 & 2420 \\
\hline
\end{tabular}




\begin{tabular}{|c|c|c|c|c|c|c|c|c|}
\hline Analysis & Parameter & Cas Number & $\begin{array}{l}\text { Detection } \\
\text { Limit Range }\end{array}$ & $\begin{array}{l}\text { Number of } \\
\text { Detects }\end{array}$ & $\begin{array}{l}\text { Minimum } \\
\text { Defect }\end{array}$ & $\begin{array}{l}\text { Mean } \\
\text { Result }\end{array}$ & $\begin{array}{l}\text { Maximum } \\
\text { Detect }\end{array}$ & $\begin{array}{l}\text { Standard } \\
\text { Deviation }\end{array}$ \\
\hline & Vanadium & $7440-62-2$ & $4.1-4.1$ & $1 / 2$ & 3.6 & 2.83 & 3.6 & 1.1 \\
\hline & Zine & $7440-66-6$ & $19.6-19.6$ & $1 / 2$ & 31.3 & 20.6 & 31.3 & 15.2 \\
\hline \multirow{2}{*}{$\begin{array}{l}\text { Semivolatile Organics } \\
\text { UG/L }\end{array}$} & Butylbenzylphthalate & $85-68-7$ & $10 \cdot 10$ & $1 / 2$ & 1 & 3 & 1 & 2.83 \\
\hline & Di-n-butylphthalate & $84-74-2$ & $10-10$ & $1 / 2$ & 1 & 3 & $\mathbb{1}$ & 2.83 \\
\hline Volatile Organics UO/L & Acetone & $67-64-1$ & & $2 / 2$ & 15 & 1060 & 2100 & 1470 \\
\hline \multirow[t]{5}{*}{ Radionuclides PCVL } & Gross Alpha & $14127-62-9$ & & 212 & 5.36 & 13.3 & 21.2 & 11.2 \\
\hline & Gross Beta & $12587-47-2$ & & 414 & 0.0889 & 8.17 & 14.5 & 6.32 \\
\hline & U-234 & $13966-29-5$ & & $4 / 4$ & 0.109 & 5.05 & 9.83 & 5.37 \\
\hline & U.235 & $15117-96-1$ & & $2 / 2$ & 0.0703 & 0.224 & 0.378 & 0.218 \\
\hline & U-238 & $7440-61-1$ & & 414 & 0.0273 & 3.8 & 7.43 & 4.18 \\
\hline \multirow[t]{4}{*}{ Phy sical Properties Ma/L } & Alkolinity & & & $2 / 2$ & 53.4 & 81.7 & 110 & 40 \\
\hline & Tofal Discolved Solids & & & $2 / 2$ & 129 & 206 & 282 & 108 \\
\hline & Total Suspended Solids & & & $2 / 2$ & 28 & 116 & 204 & 124 \\
\hline & Turbidity & & & 2/ 2 & 23 & 136 & 248 & 159 \\
\hline
\end{tabular}

Data Source : Bear Creek Valley OU2 1993 RI field activities validated data Raw data nailable through Y-12 OREIS

Data does not include QA/OC

Grand mean and standard deviation include nondetects set to $1 / 2$ of the reporting limit

Results < counting uncertainty were considered as nondetects for radionuclides 


\begin{tabular}{|c|c|c|c|c|c|c|c|c|}
\hline Analysis & Parameter & Cas Number & $\begin{array}{c}\text { Detection } \\
\text { Limit Range }\end{array}$ & $\begin{array}{l}\text { Detects } \\
\text { Detect }\end{array}$ & $\begin{array}{c}\text { Minimum } \\
\text { Result }\end{array}$ & $\begin{array}{l}\text { Mean } \\
\text { Detect }\end{array}$ & Maximum & $\begin{array}{l}\text { Standard } \\
\text { Deviation }\end{array}$ \\
\hline \multirow[t]{3}{*}{ Anions MG/L } & Chloride & & & $1 / 1$ & 9.8 & 9.8 & 9.8 & . \\
\hline & Fluoride & $16984-48-8$ & & $1 / 1$ & 0.21 & 0.21 & 0.21 & . \\
\hline & Sulfate & & & $1 / 1$ & 122 & 122 & 122 & . \\
\hline \multirow[t]{5}{*}{ Metals UG/L (AAS) } & Antimony & $7440-36-0$ & & $1 / 1$ & 2.5 & 2.5 & 2.5 & . \\
\hline & Antimony (Dissolved) & $7440-36-0$ & & $1 / 1$ & 6.6 & 6.6 & 6.6 & . \\
\hline & Arsenic & $7440-38-2$ & & $1 / 1$ & 13.3 & 13.3 & 13.3 & $\therefore$ \\
\hline & Lead & $7439-92-1$ & & $\| 1$ & 64.3 & 64.3 & 64.3 & . \\
\hline & Lead (Dissolved) & $7439-92-1$ & & $1 / 1$ & 2.7 & 2.7 & 2.7 & . \\
\hline Metals UG/L & Mercury & $7439-97-6$ & & $1 / 1$ & 21.2 & 21.2 & 21.2 & . \\
\hline \multirow[t]{14}{*}{ Metals UO/L (ICP) } & Aluminum & $7429-90-5$ & & $1 / 1$ & 45600 & 45600 & 45600 & . \\
\hline & Barium & $7440-39-3$ & & $1 / 1$ & 1020 & 1020 & 1020 & . \\
\hline & Barium (Dissolved) & $7440-39-3$ & & $1 / 1$ & 189 & 189 & 189 & . \\
\hline & Beryllium & $7440-41-7$ & & $1 / 1$ & 2.6 & 2.6 & 2.6 & . \\
\hline & Calcium & $7440-70-2$ & & $1 / 1$ & 231000 & 231000 & 231000 & . \\
\hline & Calcium (Dissolved) & $7440-70-2$ & & $1 / 1$ & 157000 & 157000 & 157000 & . \\
\hline & Chromium & $7440-47-3$ & & 1/ 1 & 58 & 58 & 58 & . \\
\hline & Coball & $7440-48-4$ & & $1 / 1$ & 27.1 & 27.1 & 27.1 & . \\
\hline & Cobalt (Dissolved) & $7440-48-4$ & & $1 / 1$ & 2.1 & 2.1 & 2.1 & . \\
\hline & Copper & $7440-50-8$ & & $1 / 1$ & 53 & 53 & 53 & . \\
\hline & Iron & $7439-89-6$ & & $1 / 1$ & 90400 & 90400 & 90400 & . \\
\hline & Iron (Dissolved) & $7439-89-6$ & & $1 / 1$ & 7740 & 7740 & 7740 & . \\
\hline & Magnesium & $7439-95-4$ & & $1 / 1$ & 41500 & 41500 & 41500 & . \\
\hline & Magnesium (Dissolved) & $7439-95-4$ & & 1/ 1 & 25600 & 25600 & 25600 & . \\
\hline
\end{tabular}




\begin{tabular}{|c|c|c|c|c|c|c|c|c|}
\hline Analysis & Parameter & Cas Number & $\begin{array}{l}\text { Detection } \\
\text { Limit Range }\end{array}$ & $\begin{array}{l}\text { Detects } \\
\text { Detect }\end{array}$ & $\begin{array}{l}\text { Minimum } \\
\text { Result }\end{array}$ & $\begin{array}{l}\text { Mean } \\
\text { Detect }\end{array}$ & Maximum & $\begin{array}{l}\text { Standard } \\
\text { Deviation }\end{array}$ \\
\hline & Manganese & $7439-96-5$ & & $1 / 1$ & 4670 & 4670 & 4670 & . \\
\hline & Manganese (Dissolved) & $7439-96-5$ & & $1 / 1$ & 2570 & 2570 & 2570 & . \\
\hline & Nickel & $7440-02-0$ & & $1 / 1$ & 54.1 & 54.1 & 54.1 & . \\
\hline & Polassium & $7440-09-7$ & & $1 / 1$ & 16900 & 16900 & 16900 & . \\
\hline & Potassium (Dissolved) & $7440-09-7$ & & $1 / 1$ & 7210 & 7210 & 7210 & . \\
\hline & Sodium & $7440-23-5$ & & 11 & 9890 & 9890 & 9890 & . \\
\hline & Sodium (Dissolved) & $7440-23-5$ & & $1 / 1$ & 9280 & 9280 & 9280 & . \\
\hline & Vanadium & $7440-62-2$ & & $1 / 1$ & 77.2 & 77.2 & 77.2 & . \\
\hline & Zine & $7440-66-6$ & & $1 / 1$ & 245 & 245 & 245 & . \\
\hline & Zinc (Dissolved) & $7440-66-6$ & & $1 / 1$ & 25.7 & 25.7 & 25.7 & . \\
\hline $\begin{array}{l}\text { Sernivolatile Organics } \\
\text { UG/L }\end{array}$ & Acenaphthene & $83-32-9$ & & $1 / 1$ & 2 & 2 & 2 & . \\
\hline \multirow[t]{5}{*}{ Radionuclides PCI/L } & Gross Alpha & $14127-62-9$ & & $1 / 1$ & 0.289 & 0.289 & 0.289 & . \\
\hline & Gross Beta & $12587-47-2$ & & $2 / 2$ & 19.9 & 19.9 & 19.9 & 0 \\
\hline & $\mathrm{U}-234$ & $13966-29-5$ & & $2 / 2$ & 5.19 & 6.14 & 7.09 & 1.34 \\
\hline & บ-235 & $15117-96-1$ & & $1 / 1$ & 0.165 & 0.165 & 0.165 & . \\
\hline & U-238 & $7440-61-1$ & & 212 & 6.6 & 7.07 & 7.53 & 0.658 \\
\hline \multirow[t]{4}{*}{ Physical Properties MG/L } & Alsalinity & & & $1 / 1$ & 508 & 508 & 508 & . \\
\hline & Total Dissolved Solids & & & $1 / 1$ & 672 & 672 & 672 & . \\
\hline & Total Suspended Solids & & & $1 / 1$ & 1000 & 1000 & 1000 & . \\
\hline & Turbidity & & & $1 / 1$ & 7.3 & 7.3 & 7.3 & . \\
\hline
\end{tabular}

Data Source : Bear Creek Valley OU2 1993 RI field activities validated data Raw data available through $Y-12$ OREIS

Data does not include QA/QC

Grand mean and standard deviation include nondetects set to $1 / 2$ of the reporting limit

Results < counting uncertainty were considered as nondetects for radionuclides 
TABLE 1. BC-OU2 1993 RI Field Activities Dats - Summary OP Detected Contanimamts For The Spoil Area 1 Sampling Media: Ground Water

\begin{tabular}{|c|c|c|c|c|c|c|c|c|}
\hline Analysis & Parameter & Cas Number & $\begin{array}{l}\text { Detection } \\
\text { Limit Range }\end{array}$ & $\begin{array}{c}\text { Number of } \\
\text { Detects }\end{array}$ & $\begin{array}{l}\text { Minimum } \\
\text { Detect }\end{array}$ & $\begin{array}{l}\text { Mean } \\
\text { Result }\end{array}$ & $\begin{array}{l}\text { Maximum } \\
\text { Detect }\end{array}$ & $\begin{array}{c}\text { Standard } \\
\text { Deviation }\end{array}$ \\
\hline \multirow[t]{3}{*}{ Anions $\mathrm{MG} / \mathrm{L}$} & Chloride & & & $2 / 2$ & 2.8 & 10.6 & 18.3 & 11 \\
\hline & Fluoride & $16984-48-8$ & $0.1-0.1$ & $1 / 2$ & 0.1 & 0.075 & 0.1 & 0.0354 \\
\hline & Nitrate/Nitrite (NO3/NO2-N) & $14797-55-8$ & $0.2-0.2$ & $1 / 2$ & 2.7 & 1.4 & 2.7 & 1.84 \\
\hline \multirow[t]{5}{*}{ Metais UG/L (AAS) } & Antimony & $7440-36-0$ & $1.8-1.8$ & $1 / 2$ & 1.9 & 1.4 & 1.9 & 0.707 \\
\hline & Antimony (Dissolved) & $7440-36-0$ & & $2 / 2$ & 13.7 & 14.4 & 15 & 0.919 \\
\hline & Arsenic & $7440-38-2$ & & $1 / 1$ & 3.5 & 3.5 & 3.5 & . \\
\hline & Lead & $7439.92-1$ & & $2 / 2$ & 55.9 & 148 & 240 & 130 \\
\hline & Lead (Dissolved) & $7439-92-1$ & & $2 / 2$ & 3.8 & 5.4 & 7 & 2.26 \\
\hline \multirow[t]{2}{*}{ Metals UG/L } & Mercury & $7439-97-6$ & $0.2-0.2$ & $1 / 2$ & 0.73 & 0.415 & 0.73 & 0.445 \\
\hline & Mercury (Dissolved) & $7439-97-6$ & $0.2-0.2$ & $1 / 2$ & 0.26 & 0.18 & 0.26 & 0.113 \\
\hline \multirow[t]{13}{*}{ Metals UG/L (CCP) } & Aluminum & $7429-90-5$ & & $2 / 2$ & 30800 & 62300 & 93700 & 44500 \\
\hline & Aluminum (Dissolved) & $7429-90-5$ & $25.5-25.5$ & $1 / 2$ & 2120 & 1070 & 2120 & 1490 \\
\hline & Barium & $7440-39-3$ & & 212 & 75 & 113 & 150 & 53 \\
\hline & Barium (Dissolved) & $7440-39-3$ & & 212 & 10.4 & 15.6 & 20.7 & 7.28 \\
\hline & Bergllium & $7440-41-7$ & $18.9-18.9$ & $1 / 2$ & 5.9 & 7.68 & 5.9 & 2.51 \\
\hline & Calcium & $7440-70-2$ & & $2 / 2$ & 21400 & 29300 & 37100 & 11100 \\
\hline & Calcium (Dissolved) & $7440-70-2$ & & 212 & 14000 & 24400 & 34800 & 14700 \\
\hline & Chromium & $7440-47-3$ & & 212 & 52.7 & 91.9 & 131 & 35.4 \\
\hline & Cobalt & $7440-48-4$ & & $2 / 2$ & 14.4 & 39 & 63.5 & 34.7 \\
\hline & Cobalt (Dissolved) & $7440-48-4$ & & 212 & 2.6 & 2.6 & 2.6 & 0 \\
\hline & Copper & $7440-50-8$ & & $2 / 2$ & 37.4 & 87.7 & 138 & 71.1 \\
\hline & Copper (Dissolved) & $7440-50-8$ & $5-5$ & $1 / 2$ & 6.6 & 4.55 & 66 & 2.9 \\
\hline & Iron & $7439-89-6$ & & 212 & 40400 & 79200 & 1 & 54900 \\
\hline
\end{tabular}




\begin{tabular}{|c|c|c|c|c|c|c|c|c|}
\hline Analysis & Parameter & Cas Number & $\begin{array}{l}\text { Detection } \\
\text { Limit Range }\end{array}$ & $\begin{array}{c}\text { Number of } \\
\text { Detects }\end{array}$ & $\begin{array}{c}\text { Minimum } \\
\text { Detect }\end{array}$ & $\begin{array}{l}\text { Mean } \\
\text { Result }\end{array}$ & $\begin{array}{c}\text { Maximum } \\
\text { Detect }\end{array}$ & $\begin{array}{l}\text { Siandard } \\
\text { Deviation }\end{array}$ \\
\hline & Iron (Dissolved) & $7439-89-6$ & $33.1-33.1$ & 112 & 3770 & 1890 & 3770 & 2650 \\
\hline & Magnesium & $7439-95-4$ & & $2 / 2$ & 7600 & 8340 & 9080 & 1050 \\
\hline & Magnesium (Dissolved) & $7439-95-4$ & & $2 / 2$ & 1440 & 2370 & 3290 & 1310 \\
\hline & Manganese & $7439-96-5$ & & 2/ 2 & 784 & 2360 & 3930 & 2220 \\
\hline & Manganese (Dissolved) & $7439-96-5$ & & $2 / 2$ & 40.5 & 81.8 & 123 & 58.3 \\
\hline & Nickel & $7440-02-0$ & & $2 / 2$ & 45.5 & 95.3 & 145 & 70.4 \\
\hline & Nickel (Dissolved) & $7440-02-0$ & $4.7-4.7$ & $1 / 2$ & 6.5 & 4.43 & 6.5 & 2.93 \\
\hline & Potassium & $7440-09-7$ & & $2 / 2$ & 12200 & 15300 & 18300 & 4310 \\
\hline & Potagsium (Dissolved) & $7440-09-7$ & & $2 / 2$ & 5300 & 7100 & 8900 & 2550 \\
\hline & Sodium & $7440-23-5$ & & 2/ 2 & 1520 & 2450 & 3380 & 1320 \\
\hline & Sodium (Dissolved) & $7440-23-5$ & & 212 & 1480 & 2570 & 3650 & 1530 \\
\hline & Vanadium & $7440-62-2$ & & $2 / 2$ & 79.1 & 140 & 201 & 86.2 \\
\hline & Vanadium (Dissolved) & $7440-62-2$ & $2.5-2.5$ & $1 / 2$ & 7.7 & 4.48 & 7.7 & 4.56 \\
\hline & Zine & $7440-66-6$ & & $2 / 2$ & 256 & 602 & 947 & 489 \\
\hline & Zinc (Dissolved) & $7440-66-6$ & $2-2$ & $1 / 2$ & 38.2 & 19.6 & 38.2 & 26.3 \\
\hline Semivolatile Organics UG/L & bis(2-Ethylhexyl)phthalate & $117-81-7$ & & $2 / 2$ & 1 & 1.5 & 2 & 0.707 \\
\hline \multirow[t]{6}{*}{ Volatile Organics UO/L } & 1,1,2-Trichloro-1,2,2-Trifluoroethane & $76-13-1$ & $10-10$ & $1 / 2$ & 3 & 4 & 3 & 1.41 \\
\hline & 1,2-Dichloroethene & $540-59-0$ & $5-5$ & $1 / 2$ & 11 & 6.75 & 11 & 6.01 \\
\hline & Acetone & $67-64-1$ & $12-12$ & $1 / 2$ & 81 & 43.5 & 81 & 53 \\
\hline & Carbon Disulfide & $75-15-0$ & $5-5$ & $1 / 2$ & 2 & 2.25 & 2 & 0.354 \\
\hline & Tetrachloroethene & $127-18-4$ & $5-5$ & $1 / 2$ & 14 & 8.25 & 14 & 8.13 \\
\hline & Trichloroethene & $79-01-6$ & $5-5$ & $1 / 2$ & 7 & 4.75 & 7 & 3.18 \\
\hline \multirow[t]{2}{*}{ Radionuclides PCI/L } & Gross Alpha & $14127-62-9$ & $63.1-63.1$ & $1 / 2$ & 30.2 & 46.7 & 30.2 & 23.3 \\
\hline & Gross Beta & $12587-47-2$ & & 414 & 4.6 & 41.9 & 98.9 & 44 \\
\hline
\end{tabular}




\begin{tabular}{|c|c|c|c|c|c|c|c|c|}
\hline Analysis & Parameter & Cas Number & $\begin{array}{c}\text { Detection } \\
\text { Limil Range }\end{array}$ & $\begin{array}{c}\text { Number of } \\
\text { Detects }\end{array}$ & $\begin{array}{c}\text { Minimum } \\
\text { Detect }\end{array}$ & $\begin{array}{l}\text { Mean } \\
\text { Resuli }\end{array}$ & $\begin{array}{c}\text { Maximum } \\
\text { Detect }\end{array}$ & $\begin{array}{l}\text { Standard } \\
\text { Deviation }\end{array}$ \\
\hline & $U-234$ & $13966-29-5$ & & 414 & 0.0146 & 0.998 & 2.51 & 1.18 \\
\hline & U.235 & $15117-96-1$ & & $2 / 2$ & 0.0313 & 0.0567 & 0.082 & 0.0359 \\
\hline & $\mathrm{U}-238$ & $7440-61-1$ & & $4 / 4$ & 0 & 0.698 & 1.7 & 0.796 \\
\hline \multirow[t]{4}{*}{ Physical Properties MO/L } & Alkalinity & & & $2 / 2$ & 53.7 & 78.4 & 103 & 34.9 \\
\hline & Total Dissolved Solids & & & $2 / 2$ & 353 & 392 & 430 & 54.4 \\
\hline & Total Suspended Solids & & & 2.12 & 753 & 1270 & 1780 & 726 \\
\hline & Turbidity & & & 212 & 855 & 2320 & 3780 & 2070 \\
\hline
\end{tabular}

Data Source : Bear Creek Valley oU2 1993 RI field activities validated date

Raw data available through $Y-12$ OREIS

Data does not include $\mathrm{QA} / \mathrm{QC}$

Grand mean and standard deviation include nondetects set to $1 / 2$ of the reporting limit

Results $<$ counting uncertainty were considered as nondetects for radionuclides 
TABLE 1. BC-OU2 1993 RI Field Activities Data - Summary Of Detected Comiaminamts For The SY-200 Yard Sampling Media: Gronnd Water

\begin{tabular}{|c|c|c|c|c|c|c|c|c|}
\hline Analysis & Parameter & Cas Number & $\begin{array}{l}\text { Detection } \\
\text { Limit Range }\end{array}$ & $\begin{array}{c}\text { Number of } \\
\text { Detects }\end{array}$ & $\begin{array}{c}\text { Minimum } \\
\text { Detect }\end{array}$ & $\begin{array}{l}\text { Mean } \\
\text { Result }\end{array}$ & $\begin{array}{c}\text { Maximum } \\
\text { Detect }\end{array}$ & $\begin{array}{l}\text { Standard } \\
\text { Deviation }\end{array}$ \\
\hline \multirow[t]{3}{*}{ Anions MO/L } & Chloride & & $1-1$ & $1 / 3$ & 487 & 163 & 487 & 281 \\
\hline & Fluoride & $16984-48-8$ & $0.1-0.1$ & $2 / 3$ & 0.1 & 0.0933 & 0.13 & 0.0404 \\
\hline & Sulfate & & $5-5$ & $2 / 3$ & 13.8 & 14.2 & 26.4 & 12 \\
\hline \multirow[t]{4}{*}{ Metals UG/L AAS } & Antimony & $7440-36-0$ & $1.8-1.8$ & $1 / 3$ & 2 & 1.27 & 2 & 0.635 \\
\hline & Antimony (Dissolved) & $7440-36-0$ & & $3 / 3$ & 2.9 & 5.47 & 9.3 & 3.38 \\
\hline & Lead & $7439-92-1$ & & $3 / 3$ & 8.3 & 15.9 & 29.4 & 11.7 \\
\hline & Lead (Dissolved) & $7439-92-1$ & & $3 / 3$ & 2.3 & 2.63 & 3.2 & 0.493 \\
\hline \multirow[t]{2}{*}{ Metais UG/L } & Mercury & $7439-97-6$ & $0.2-0.2$ & $2 / 3$ & 0.68 & 0.76 & 1.5 & 0.703 \\
\hline & Mercury (Diswolved) & $7439-97-6$ & & $3 / 3$ & 0.34 & 1.38 & 3.4 & 1.75 \\
\hline \multirow[t]{14}{*}{ Metals UG/L ICP } & Aluminum & $7429-90-5$ & & 3/ 3 & 3130 & 7300 & 14100 & 5940 \\
\hline & Barium & $7440-39-3$ & & $3 / 3$ & 109 & 255 & 526 & 235 \\
\hline & Barium (Dissolved) & $7440-39-3$ & & $3 / 3$ & 88.1 & 251 & 536 & 247 \\
\hline & Calcium & $7440-70-2$ & & $3 / 3$ & 130000 & 211000 & 341000 & 114000 \\
\hline & Calcium (Dissolved) & $7440-70-2$ & & $3 / 3$ & 128000 & 222000 & 367000 & 127000 \\
\hline & Chromium & $7440-47-3$ & & $3 / 3$ & 6.8 & 14.4 & 18.7 & 6.6 \\
\hline & Cobalt & $7440-48-4$ & $6.2-8.2$ & $1 / 3$ & 60.4 & 22.5 & 60.4 & 32.8 \\
\hline & Cobalt (Dissolved) & $7440-48-4$ & $2-2$ & $2 / 3$ & 4.8 & 23.2 & 63.8 & 35.2 \\
\hline & Copper & $7440-50-8$ & $5-5$ & 2/ 3 & 14.6 & 11.2 & 16.5 & 7.59 \\
\hline & Copper (Dissolved) & $7440-50-8$ & $5-5$ & $2 / 3$ & 7.6 & 7.4 & 12.1 & 4.8 \\
\hline & Iron & $7439-89-6$ & & $3 / 3$ & 3770 & 8380 & 14200 & 5320 \\
\hline & Magnesium & $7439-95-4$ & & $3 / 3$ & 17900 & 27600 & 42500 & 13100 \\
\hline & Magnesium (Dissolved) & $7439-95-4$ & & $3 / 3$ & 17300 & 28200 & 44100 & 14100 \\
\hline & Manganese & $7439-96-5$ & & $3 / 3$ & 2070 & 4120 & 5490 & 1810 \\
\hline
\end{tabular}




\begin{tabular}{|c|c|c|c|c|c|c|c|c|}
\hline Analysis & Parameter & Cas Number & $\begin{array}{c}\text { Detection } \\
\text { Limit Range } \\
\end{array}$ & $\begin{array}{c}\text { Number of } \\
\text { Detects }\end{array}$ & $\begin{array}{c}\text { Minimum } \\
\text { Detect }\end{array}$ & $\begin{array}{l}\text { Mean } \\
\text { Resuit }\end{array}$ & $\begin{array}{c}\text { Maximum } \\
\text { Detect }\end{array}$ & $\begin{array}{l}\text { Standard } \\
\text { Deviation }\end{array}$ \\
\hline & Manganese (Dissolved) & $7439-96-5$ & & $3 / 3$ & 2120 & 4130 & 5620 & 1810 \\
\hline & Nickel & $7440-02-0$ & $4.7-4.7$ & 213 & 16.1 & 11.8 & 16.9 & 8.18 \\
\hline & Nickel (Dissolved) & $7440-02-0$ & $4.7-4.7$ & $1 / 3$ & 7.3 & 4 & 7.3 & 2.86 \\
\hline & Potassium & $7440-09.7$ & & $3 / 3$ & 4120 & 7090 & 12900 & 5030 \\
\hline & Potassium (Dissolved) & $7440-09-7$ & & 313 & 3260 & 6180 & 11300 & 4450 \\
\hline & Sodium & $7440-23-5$ & & $3 / 3$ & 7470 & 54000 & 134000 & 69600 \\
\hline & Sodium (Dissolved) & $7440-23-5$ & & 313 & 7570 & 57700 & 144000 & 75100 \\
\hline & Vanadium & $7440-62-2$ & & $3 / 3$ & 7.3 & 15.2 & 26.6 & 10.1 \\
\hline & Zine & $7440-66-6$ & & $3 / 3$ & 34 & 41.1 & 52.6 & 10 \\
\hline \multirow[t]{4}{*}{ Semivolatile Organics UG/L } & Butylbenzylphthalate & $85-68-7$ & $10-10$ & $1 / 3$ & 2 & 4 & 2 & 1.73 \\
\hline & Di-n-butylphthalate & $84-74-2$ & $10-10$ & 213 & 1 & 3 & 3 & 2 \\
\hline & Diethylphthalate & $84-66-2$ & $10-10$ & $1 / 3$ & 2 & 4 & 2 & 1.73 \\
\hline & bis(2-Ethylhexyl)phthalate & $117-81-7$ & & $3 / 3$ & 2 & 2.67 & 4 & 1.15 \\
\hline \multirow[t]{3}{*}{ Volatile Organies UG/L } & Chloroform & $67-66-3$ & $5-5$ & $1 / 3$ & 1 & 2 & 1 & 0.866 \\
\hline & Tetrachloroethene & $127-18-4$ & $5-5$ & $2 / 3$ & 2 & 2.5 & 3 & 0.5 \\
\hline & Trichloroethene & $79-01-6$ & $5-5$ & $1 / 3$ & 2 & 2.33 & 2 & 0.289 \\
\hline \multirow[t]{5}{*}{ Radionuclides PCI/L } & Gross Alpha & $14127-62-9$ & $22-22$ & 213 & 0 & 11.4 & 12.3 & 11 \\
\hline & Gross Beta & $12587-47-2$ & $442-442$ & $5 / 6$ & 17.9 & 156 & 239 & 163 \\
\hline & $\mathrm{U}-234$ & $13966-29-5$ & & 616 & 0.32 & 2.66 & 7.38 & 3.2 \\
\hline & $\mathrm{U}-235$ & $15117-96-1$ & & $3 / 3$ & 0.0495 & 0.281 & 0.428 & 0.203 \\
\hline & U-238 & $7440-61-1$ & & 616 & 0.0534 & 2.99 & 8.69 & 4.38 \\
\hline \multirow[t]{3}{*}{ Physical Properties MG/L } & Alkalinity & & & 313 & 153 & 277 & 516 & 207 \\
\hline & Total Dissolved Solids & & & $3 / 3$ & 310 & 970 & 1920 & 843 \\
\hline & Total Suspended Solids & & & $3 / 3$ & 184 & 1140 & 2730 & 1390 \\
\hline
\end{tabular}




\begin{tabular}{|c|c|c|c|c|c|c|c|c|}
\hline Analysis & Parameter & Cas Number & $\begin{array}{l}\text { Detection } \\
\text { Limit Range }\end{array}$ & $\begin{array}{l}\text { Number of } \\
\text { Detects }\end{array}$ & $\begin{array}{c}\text { Minimum } \\
\text { Detect }\end{array}$ & $\begin{array}{l}\text { Mean } \\
\text { Result }\end{array}$ & $\begin{array}{c}\text { Maximum } \\
\text { Detect }\end{array}$ & $\begin{array}{l}\text { Slandard } \\
\text { Deviation }\end{array}$ \\
\hline & Turbidity & & & $3 / 3$ & 190 & 510 & 780 & 298 \\
\hline
\end{tabular}

Dat Source : Bear Creek Valley OU2 1993 RI field activities validated data

Raw data available through $Y-12$ OREIS

Data does not include QA/QC

Grand mean and standerd deviation include nondetects set to $1 / 2$ of the reporting limit

Results $<$ counting uncertainty were considered as nondetects for radionuclides 
Non-Detects 
- 
TABLE 2. BC.OU2 1993 RI Field Activities Data - Summary Of Parameters Never Detected For The Rust Spoil Area Saupuling Media: Soi

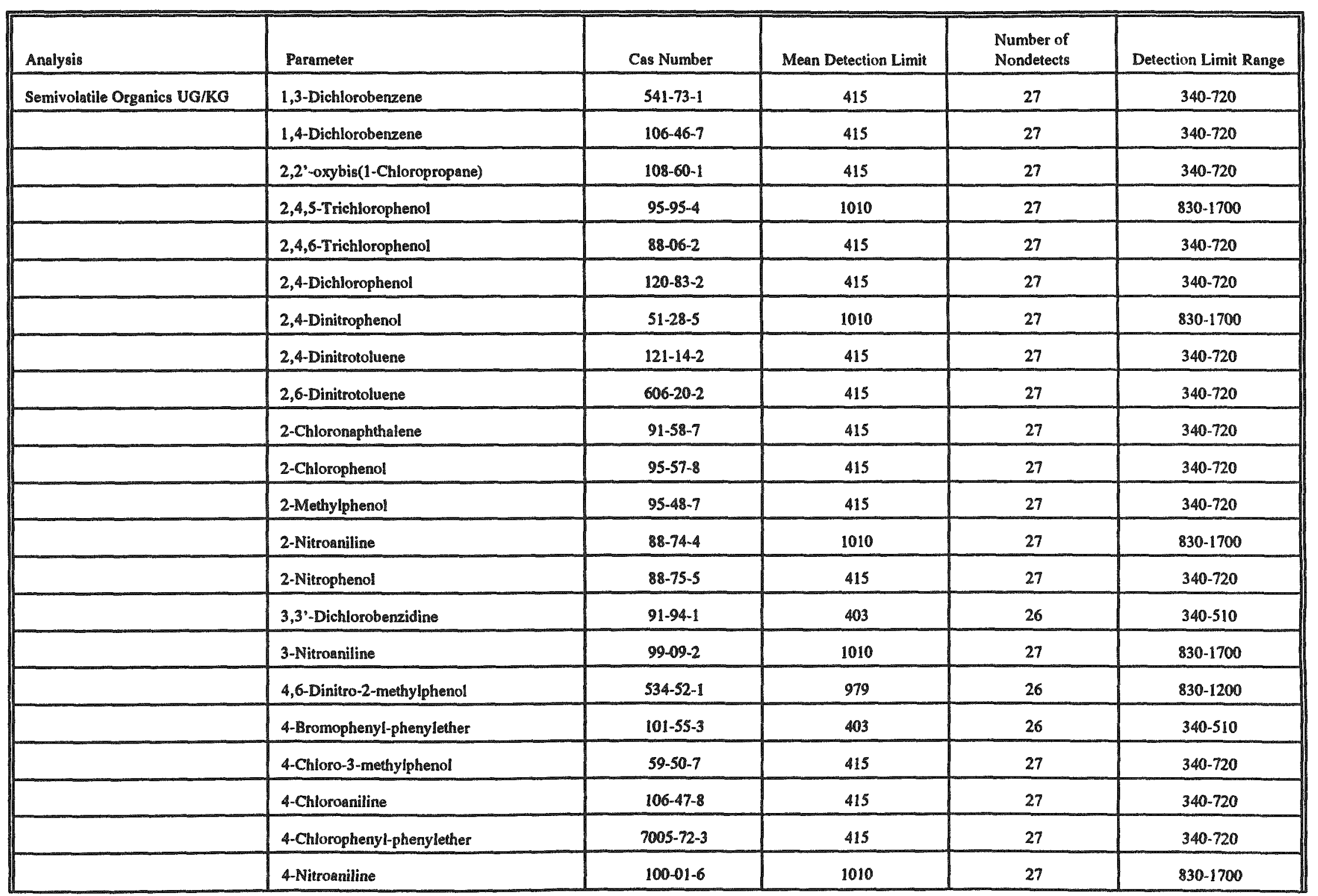




\begin{tabular}{|c|c|c|c|c|c|}
\hline Analysis & Parameter & Cas Number & Mean Detection Limit & $\begin{array}{l}\text { Number of } \\
\text { Nondetects }\end{array}$ & Detection Limit Range \\
\hline & Diethylphthalate & $84-66-2$ & 415 & 27 & $340-720$ \\
\hline & Hexachlorobenzene & $118-74-1$ & 403 & 26 & $340-510$ \\
\hline & Hexachlorobutadiene & $87-68-3$ & 415 & 27 & $340-720$ \\
\hline & Hexachlorocyclopentadiene & $77-47-4$ & 415 & 27 & $340-720$ \\
\hline & Herachloroethane & $67-72-1$ & 415 & 27 & $340-720$ \\
\hline & Isophorone & $78-59-1$ & 415 & 27 & $340-720$ \\
\hline & N-Nitroso-di-n-propylamine & $621-64-7$ & 415 & 27 & $340-720$ \\
\hline & N-Nitrosodiphenylamine & $86-30-6$ & 403 & 26 & $340-510$ \\
\hline & Nitrobenzene & $98-95-3$ & 415 & 27 & $340-720$ \\
\hline & bis(2-Chloroethoxy) methane & $111-91-1$ & 415 & 27 & $340-720$ \\
\hline & bis(2-Chloroethyl)ether & $111-44-4$ & 415 & 27 & $340-720$ \\
\hline \multirow[t]{13}{*}{ Volatile Organics UG/KG } & 1,1,2,2-Tetrachloroethane & $79-34-5$ & 62.6 & 27 & $5 \cdot 1500$ \\
\hline & 1,1,2-Trichloroethane & $79-00-5$ & 60.5 & 28 & $5-1500$ \\
\hline & 1,2-Dichloropropane & $78-87-5$ & 60.5 & 28 & $5-1500$ \\
\hline & Bromodichloromethane & $75-27-4$ & 60.5 & 28 & $5-1500$ \\
\hline & Bromoform & $75-25-2$ & 60.5 & 28 & $5-1500$ \\
\hline & Bromomethane & $74-83-9$ & 67.5 & 28 & $10-1500$ \\
\hline & Carbon Tetrachloride & $56-23-5$ & 60.5 & 28 & $5-1500$ \\
\hline & Chloromethane & $74-87-3$ & 67.5 & 28 & $10-1500$ \\
\hline & Dibromochloromethane & $124-48-1$ & 60.5 & 28 & $5-1500$ \\
\hline & Styrene & $100-42-5$ & 62.6 & 27 & $5-1500$ \\
\hline & Vinyl Chloride & $75-01-4$ & 67.5 & 28 & $10-1500$ \\
\hline & cis-1,3-Dichloropropene & $10061-01-5$ & 60.5 & 28 & $5-1500$ \\
\hline & trans-1,3-Dichloropropene & $10061-02-6$ & 60.5 & 28 & $5-1500$ \\
\hline
\end{tabular}




\begin{tabular}{|c|c|c|c|c|c|}
\hline Analysis & Parameter & Cas Number & Mean Detection Limit & $\begin{array}{l}\text { Number of } \\
\text { Nondetects }\end{array}$ & Detection Limil Range \\
\hline \multirow[t]{23}{*}{ Pesticide/PCB UG/KG } & $4,4^{\prime}-\mathrm{DDD}$ & $72-54-8$ & 8.27 & 20 & $3.4-36$ \\
\hline & 4,4'-DDE & $72-55-9$ & 8.27 & 20 & $3.4-36$ \\
\hline & $4,4^{\prime}-\mathrm{DDT}$ & $50-29-3$ & 8.27 & 20 & $3.4-36$ \\
\hline & Aldrin & $309-00-2$ & 4.16 & 20 & i.8-18 \\
\hline & Aroclor-1016 & $12674-11-2$ & 82.7 & 20 & $34-360$ \\
\hline & Aroclor-1221 & $11104-28-2$ & 167 & 20 & $69-730$ \\
\hline & Aroclor- 1232 & $11141-16-5$ & 82.7 & 20 & $34-360$ \\
\hline & Aroclor- 1242 & $53469-21-9$ & 82.7 & 20 & $34-360$ \\
\hline & Aroclor-1248 & $12672-29-6$ & 82.7 & 20 & $34-360$ \\
\hline & Dieldrin & $60-57.1$ & 8.27 & 20 & $3.4-36$ \\
\hline & Endosulfan II & $33213-65-9$ & 8.27 & 20 & $3.4-36$ \\
\hline & Endosul fan Sulfate & $1031-07-8$ & 8.27 & 20 & $3.4-36$ \\
\hline & Endrin & $72-20-8$ & 8.27 & 20 & $3.4-36$ \\
\hline & Endrin Aldehyde & $7421-93-4$ & 8.27 & 20 & $3.4-36$ \\
\hline & Endrin Ketone & $53494-70-5$ & 8.27 & 20 & $3.4-36$ \\
\hline & Heptachlor Epoxide & $1024-57-3$ & 4.16 & 20 & $1.8-18$ \\
\hline & Toxaphene & $8001-35-2$ & 416 & 20 & $180-1800$ \\
\hline & Ipha-BHC & $319-84-6$ & 4.16 & 20 & $1.8-18$ \\
\hline & Alpha-Chlordane & $5103-71-9$ & 4.16 & 20 & $1.8-18$ \\
\hline & beta-BHC & $319-85-7$ & 4.16 & 20 & $1.8-18$ \\
\hline & delta-BHC & $319-86-8$ & 4.16 & 20 & 1.818 \\
\hline & gamma-BHC (Lindane) & $58-89-9$ & 4.16 & 20 & $1.8-18$ \\
\hline & gamma-Chlordane & $5103-74-2$ & 4.16 & 20 & $1.8-18$ \\
\hline
\end{tabular}


Data Source : Bear Creek Valley OU2 1993 RI field activities validated data

Rav dato available through Y-12 ORE

Data does not include $\mathrm{QA} / \mathrm{QC}$

Results $<$ counting uncertainty were considered as nondetects for radionuclides 
TABLE 2. BC-OU2 1993 RI Field Activities Data - Summary or Parameters Never Detected For The SY-200 Yard

Sampling Media: Soil

\begin{tabular}{|c|c|c|c|c|c|}
\hline Analysis & Parameter & Cas Number & Mean Detection Limit & $\begin{array}{l}\text { Number of } \\
\text { Nondetects }\end{array}$ & $\begin{array}{c}\text { Detection } \\
\text { Limil Range }\end{array}$ \\
\hline \multirow[t]{23}{*}{ Semivolatile Organics UG/KO } & 1,2-Dichlorobenzene & $95-50-1$ & 504 & 98 & $370-8300$ \\
\hline & 1,3-Dichlorobenzene & $541-73-1$ & 504 & 98 & 370.8300 \\
\hline & 2,2'-oxybis(1-Chloropropane) & $108-60-1$ & 504 & 98 & $370-8300$ \\
\hline & 2,4,5-Trichlorophenol & $95-95-4$ & 1220 & 97 & $900-20000$ \\
\hline & 2,4,6-Trichlorophenol & $88-06-2$ & 505 & 97 & $370-8300$ \\
\hline & 2,4-Dichlorophenol & $120-83-2$ & 505 & 97 & $370-8300$ \\
\hline & 2,4-Dimethylphenol & $105-67-9$ & 505 & 97 & $370-8300$ \\
\hline & 2,4-Dinitrophenol & $51-28-5$ & 1220 & 97 & $900-20000$ \\
\hline & 2,6-Dinitrotoluene & $606-20-2$ & 504 & 98 & $370-8300$ \\
\hline & 2-Chloronephthalene & $91-58-7$ & 504 & 98 & $370-8300$ \\
\hline & 2-Methylphenol & $95-48-7$ & 505 & 97 & $370-8300$ \\
\hline & 2-Nitroaniline & $88-74-4$ & 1220 & 98 & $900-20000$ \\
\hline & 2-Nitrophenol & $88-75-5$ & 505 & 97 & $370-8300$ \\
\hline & 3,3'-Dichlorobenzidine & 91-94-1 & 504 & 98 & 370.8300 \\
\hline & 3-Nitroaniline & $99-09-2$ & 1220 & 98 & $900-20000$ \\
\hline & 4,6-Dinitro-2-methylphenol & $534-52-1$ & 1220 & 97 & $900-20000$ \\
\hline & 4-Bromophenyl-phenylether & $101-55-3$ & 504 & 98 & $370-8300$ \\
\hline & 4-Chloroaniline & $106-47-8$ & 504 & 98 & $370-8300$ \\
\hline & 4-Chlorophenyl-phenylether & $7005-72-3$ & 504 & 98 & $370-8300$ \\
\hline & 4-Methylphenol & $106-44-5$ & 505 & 97 & $370-8300$ \\
\hline & 4-Nitroaniline & $100-01-6$ & 1220 & 98 & $900-20000$ \\
\hline & Di-n-octylphthalate & $117-84-0$ & 504 & 98 & $370-8300$ \\
\hline & Hexachlorobenzene & $118-74-1$ & 504 & 98 & $370-8300$ \\
\hline
\end{tabular}




\begin{tabular}{|c|c|c|c|c|c|}
\hline Analysis & Parameter & Cas Number & Mean Detection Limit & $\begin{array}{l}\text { Number of } \\
\text { Nondetects }\end{array}$ & $\begin{array}{c}\text { Detection } \\
\text { Limit Range }\end{array}$ \\
\hline & Hextchlorobutadiene & $87-68-3$ & 504 & 98 & $370-8300$ \\
\hline & Hexachlorocyclopentadiene & $77-47-4$ & 504 & 98 & 370.8300 \\
\hline & Hexachloroethane & $67-72-1$ & 504 & 98 & $370-8300$ \\
\hline & Isophorone & $78-59-1$ & 504 & 98 & $370-8300$ \\
\hline & $\mathbb{N}$-Nitrosodiphenylamine & $86-30-6$ & 504 & 98 & $370-8300$ \\
\hline & Nitrobenzene & $98-95-3$ & 504 & 98 & $370-8300$ \\
\hline & bis(2-Chloroethoxy)methane & $111-91-1$ & 504 & 98 & $370-8300$ \\
\hline & bir(2-Chloroethyl)ether & $111-44-4$ & 504 & 98 & 370.8300 \\
\hline \multirow[t]{16}{*}{ Volatile Organics UO/KG } & 1,1,1-Trichloroethene & 71-55-6 & 22.7 & 98 & $6-1600$ \\
\hline & 1,1,2,2-Tetrachloroethane & $79-34-5$ & 22.7 & 98 & $2-1600$ \\
\hline & 1,1,2-Trichloroethane & $79-00-5$ & 22.7 & 98 & $6-1600$ \\
\hline & 1,1-Dichloroethane & $75-34-3$ & 22.7 & 98 & $6-1600$ \\
\hline & 1,1-Dichloroethene & $75-35-4$ & 22.7 & 98 & $6-1600$ \\
\hline & 1,2-Dichloroethane & $107-06-2$ & 22.7 & 98 & $6-1600$ \\
\hline & 1,2-Dichloroethene & $540-59-0$ & 22.7 & 98 & $6-1600$ \\
\hline & 1,2-Dichloropropane & $78-87-5$ & 22.7 & 98 & $6-1600$ \\
\hline & 2-Hexanone & $591-78-6$ & 29.2 & 98 & 10.1600 \\
\hline & Bromodichloromethane & $75-27-4$ & 22.7 & 98 & $6-1600$ \\
\hline & Bromoform & $75-25-2$ & 22.7 & 98 & $6-1600$ \\
\hline & Bromomethane & 74-83-9 & 29.3 & 98 & $11 \cdot 1600$ \\
\hline & Carbon Tetrachloride & $56-23-5$ & 22.7 & 98 & $6-1600$ \\
\hline & Chlorobenzene & $108-90-7$ & 22.7 & 98 & $6-1600$ \\
\hline & Chloroethane & $75-00-3$ & 29.3 & 98 & $11-1600$ \\
\hline & Chloroform & $67-66-3$ & 22.7 & 98 & $6-1600$ \\
\hline
\end{tabular}




\begin{tabular}{|c|c|c|c|c|c|}
\hline Analysig & Parameter & Cas Number & Mean Detection Limit & $\begin{array}{l}\text { Number of } \\
\text { Nondetects }\end{array}$ & $\begin{array}{c}\text { Detection } \\
\text { Limit Range }\end{array}$ \\
\hline & Chloromethane & $74-87-3$ & 29.3 & 98 & $11-1600$ \\
\hline & Dibromochloromethane & $124-48-1$ & 22.7 & 98 & $6-1600$ \\
\hline & Styrene & $100-42-5$ & 22.7 & 98 & $6-1600$ \\
\hline & Tetrachloroethene & $127-18-4$ & 22.7 & 98 & $3-1600$ \\
\hline & Trichloroethene & $79-01-6$ & 22.7 & 98 & $6-1600$ \\
\hline & Vinyl Chloride & $75-01-4$ & 29.3 & 98 & $11-1600$ \\
\hline & cis-1,3-Dichloropropene & $10061-01-5$ & 22.7 & 98 & $6-1600$ \\
\hline & trans-1,3-Dichloropropene & $10061-02-6$ & 22.7 & 98 & $6-1600$ \\
\hline \multirow[t]{16}{*}{ Pesticide/PCB UG/KG } & $4,4^{\prime}-\mathrm{DDD}$ & $72-54-8$ & 14 & 98 & 3.3-390 \\
\hline & $4,4^{\circ}-\mathrm{DDT}$ & $50-29-3$ & 14 & 98 & $3.3-390$ \\
\hline & Aldrin & $309-00-2$ & 7.15 & 98 & $1.7-200$ \\
\hline & Aroclor-1016 & $12674-11-2$ & 140 & 231 & $32-3900$ \\
\hline & Aroclor-1221 & $11104-28-2$ & 282 & 231 & $65-7900$ \\
\hline & Aroclor- 1232 & $11141-16-5$ & 140 & 231 & $32-3900$ \\
\hline & Aroclor-1242 & $53469-21-9$ & 140 & 231 & $32-3900$ \\
\hline & Aroclor-1248 & $12672-29-6$ & 140 & 231 & $32-3900$ \\
\hline & Dieldrin & $60-57-1$ & 14 & 98 & $0.27-390$ \\
\hline & Endostifan 1 & 959-98-8 & 7.15 & 98 & $1,7-200$ \\
\hline & Endosulfan II & $33213-65-9$ & 14 & 98 & 3.3-390 \\
\hline & Endosulfan Suffate & $1031-07-8$ & 14 & 98 & $3.3-390$ \\
\hline & Endrin Aldehyde & $7421-93-4$ & 14 & 98 & 3.3.390 \\
\hline & Endrin Ketone & $53494-70-5$ & 14 & 98 & $3.3-390$ \\
\hline & Heptachlor & $76-44-8$ & 7.15 & 98 & $1.7-200$ \\
\hline & Heptachlor Epoxide & $1024-57-3$ & 7.15 & 98 & $1.7-200$ \\
\hline
\end{tabular}




\begin{tabular}{|c|c|c|c|c|c|}
\hline Analysis & Parameter & Cas Number & Mean Detection Limit & $\begin{array}{l}\text { Number of } \\
\text { Nondetects }\end{array}$ & $\begin{array}{l}\text { Detection } \\
\text { Limit Range }\end{array}$ \\
\hline & Toxaphene & $8001-35-2$ & 715 & 98 & $170-20000$ \\
\hline & alpha-BHC & $319-84-6$ & 7.15 & 98 & $1.7-200$ \\
\hline & alpha-Chlordane & $5103-71-9$ & 7.15 & 98 & $1.7-200$ \\
\hline & beta-BHC & $319-85-7$ & 7.15 & 98 & $1.7-200$ \\
\hline & deltu-BHC & $319-86-8$ & 7.15 & 98 & $1.7-200$ \\
\hline & gamma-BHC (Lindene) & $58-89-9$ & 7.15 & 98 & $1.7-200$ \\
\hline & gamma-Chlordane & $5103-74-2$ & 7.15 & 98 & $1.7-200$ \\
\hline \multirow[t]{3}{*}{ Miscellaneous \% } & Actinolite/Tremolite & $\infty$ & 0 & 98 & $0-0$ \\
\hline & Anthophyllite & - & 0 & 98 & 0.0 \\
\hline & Crocidolite & - & 0 & 98 & $0-0$ \\
\hline
\end{tabular}

Data Source : Bear Creek Valley ou2 1993 RI field activities validated data

Raw data available through Y-12 OREIS

Data does not include QA/QC

Results $<$ counting uncertainty were considered as nondefects for radionuclides 
TABLE 2. BC-OU2 1993 RI Field Activities Data - Summary Of Parameters Never Detected For The Rust Spoil Area Sampling Media: Streani Sediments

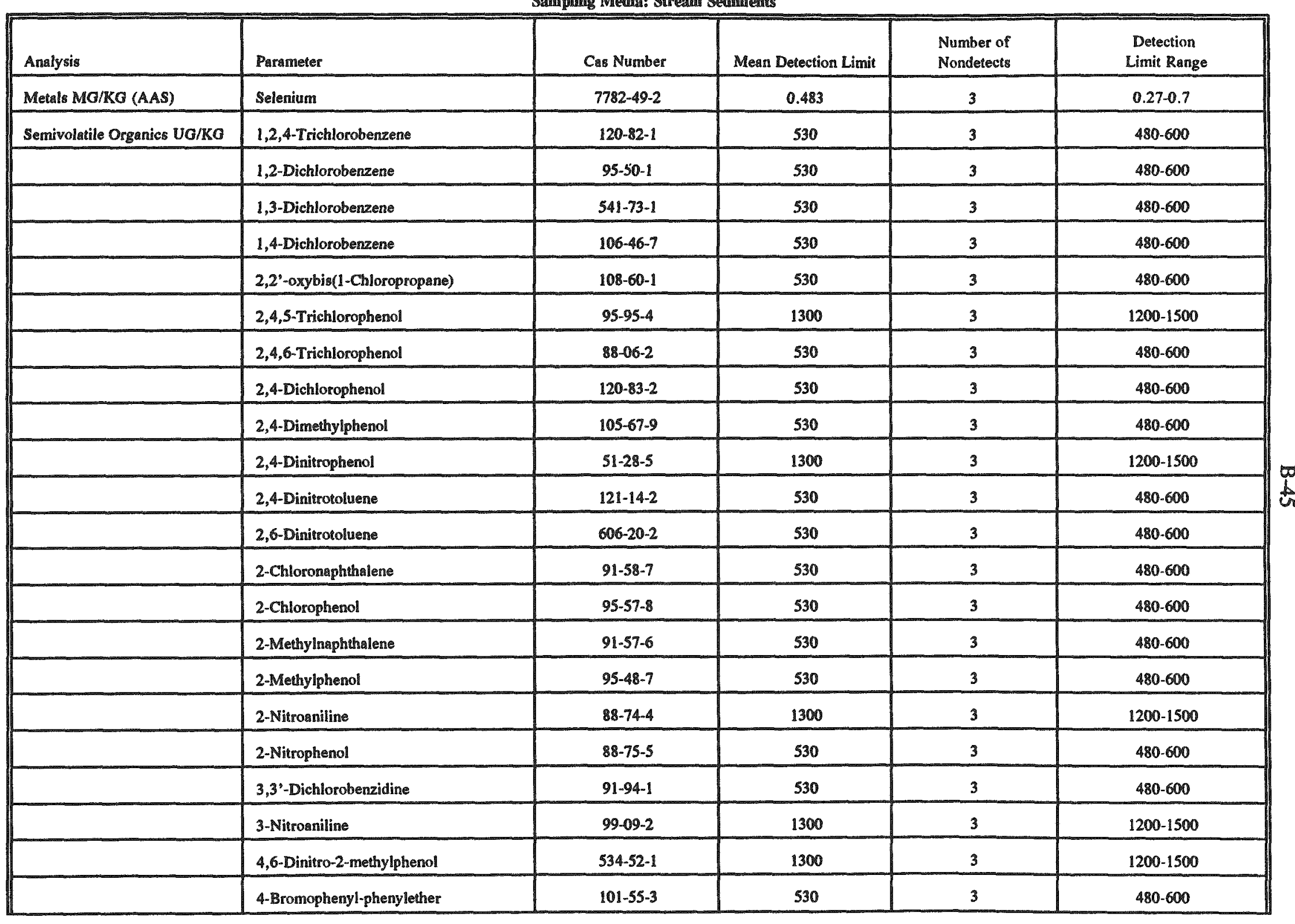




\begin{tabular}{|c|c|c|c|c|c|}
\hline Analysis & Parameter & Cas Number & Mean Detection Limit & $\begin{array}{l}\text { Number of } \\
\text { Nondetects }\end{array}$ & $\begin{array}{l}\text { Detection } \\
\text { Limit Range }\end{array}$ \\
\hline & 4-Chloro-3-methylphenol & $59-50-7$ & 530 & 3 & 480.600 \\
\hline & 4-Chloroaniline & $106-47-8$ & 530 & 3 & $480-600$ \\
\hline & 4-Chlorophenyl-phenylether & $7005-72-3$ & 530 & 3 & $480-600$ \\
\hline & 4-Methylphenol & $106-44-5$ & 530 & 3 & $480-600$ \\
\hline & 4-Nitroaniline & $100-01-6$ & 1300 & 3 & $1200-1500$ \\
\hline & 4-Nitrophenol & $100-02.7$ & 1300 & 3 & $1200-1500$ \\
\hline & Acenaphthene & $83-32-9$ & 530 & 3 & $480-600$ \\
\hline & Acemaphthylene & $208-96-8$ & 530 & 3 & $480-600$ \\
\hline & Anthracene & $120-12-7$ & 530 & 3 & $480-600$ \\
\hline & Benzo(a)anthracene & $56-55-3$ & 530 & 3 & $480-600$ \\
\hline & Benzo(a)pyrene & $50-32-8$ & 530 & 3 & $480-600$ \\
\hline & Benzo(b)fluoranthene & $205-99-2$ & 530 & 3 & $480-600$ \\
\hline & Benzo $(g, h, i)$ perylene & $191-24-2$ & 530 & 3 & $480-600$ \\
\hline & Benzo(k)fluoranthene & $207-08-9$ & 530 & 3 & $480-600$ \\
\hline & Butylbenzylphthalase & $85-68-7$ & 530 & 3 & $480-600$ \\
\hline & Carbazole & $86-74-8$ & 530 & 3 & $480-600$ \\
\hline & Chrysene & $218-01-9$ & 530 & 3 & $480-600$ \\
\hline & Di-n-butylghthalate & $84-74-2$ & 530 & 3 & $480-600$ \\
\hline & Di-n-octylphthalate & $117-84-0$ & 530 & 3 & 480.600 \\
\hline & Dibenzo(a,h)anthracene & $53-70-3$ & 530 & 3 & $480-600$ \\
\hline & Dibenzofuran & $132-64-9$ & 530 & 3 & $480-600$ \\
\hline & Diethylphthalate & $84-66-2$ & 530 & 3 & $480-600$ \\
\hline & Dimethylphthalate & $131-11-3$ & 530 & 3 & $480-600$ \\
\hline & Fluorene & $86-73-7$ & 530 & 3 & $480-600$ \\
\hline
\end{tabular}




\begin{tabular}{|c|c|c|c|c|c|}
\hline Analysis & Parameter & Cas Number & Mean Detection Limit & $\begin{array}{l}\text { Number of } \\
\text { Nondetects }\end{array}$ & $\begin{array}{c}\text { Detection } \\
\text { Limit Range }\end{array}$ \\
\hline & Hexachlorobenzene & $118-74-1$ & 530 & 3 & $480-600$ \\
\hline & Hexachlorobutadiene & $87-68-3$ & 530 & 3 & $480-600$ \\
\hline & Hexachlorocyclopentadiene & $77-47-4$ & 530 & 3 & $480-600$ \\
\hline & Hexachloroethene & $67-72-1$ & 530 & 3 & $480-600$ \\
\hline & Indeno(1,2,3-c,0)pyrene & $193-39-5$ & 530 & 3 & $480-600$ \\
\hline & Isophorone & $78-59-1$ & 530 & 3 & $480-600$ \\
\hline & N-Nitroso-di-n-propylamine & $621-64-7$ & 530 & 3 & $480-600$ \\
\hline & N-Nitrosodiphenylamine & $86-30-6$ & 530 & 3 & 480.600 \\
\hline & Nitrobenzene & $98-95-3$ & 530 & 3 & $480-600$ \\
\hline & Pentachlorophenol & $87-86-5$ & 1300 & 3 & $1200-1500$ \\
\hline & Phenanthrene & $85-01-8$ & 530 & 3 & 480.600 \\
\hline & Phenol & $108-95-2$ & 530 & 3 & $480-600$ \\
\hline & bis(2-Chloroethoxy)methane & $111-91-1$ & 530 & 3 & $480-600$ \\
\hline & bis(2-Chloroethyl)ether & $111-44-4$ & 530 & 3 & $480-600$ \\
\hline & bis(2-Ethylhexyl)phthalate & $117-81-7$ & 530 & 3 & $480 \cdot 600$ \\
\hline \multirow[t]{9}{*}{ Volatile Organics UG/KG } & 1,1,1-Trichloroethane & $71-55-6$ & 8 & 3 & 7.9 \\
\hline & 1,1,2,2-Tetrachloroethane & $79-34-5$ & 8 & 3 & 7.9 \\
\hline & 1,1,2-Trichloro-1,2,2-Trifluoroethane & $76-13-1$ & 16.3 & 3 & $15-18$ \\
\hline & 1,1,2-Trichloroethane & $79-00-5$ & 8 & 3 & $7-9$ \\
\hline & 1,1-Dichloroethane & $75-34-3$ & 8 & 3 & $7-9$ \\
\hline & 1,1-Dichloroethene & $75-35-4$ & 8 & 3 & $7-9$ \\
\hline & 1,2-Dichloroethane & $107-06-2$ & 8 & 3 & $7-9$ \\
\hline & 1,2-Dichloroethene & $540-59-0$ & 8 & 3 & $7-9$ \\
\hline & 1,2-Dichloropropane & $78-87-5$ & 8 & 3 & $7-9$ \\
\hline
\end{tabular}




\begin{tabular}{|c|c|c|c|c|c|}
\hline Analysis & Parameter & Cas Number & Mean Detection Limit & $\begin{array}{l}\text { Number of } \\
\text { Nondetects }\end{array}$ & $\begin{array}{l}\text { Detection } \\
\text { Limit Range }\end{array}$ \\
\hline & 2-Butanone & $78-93-3$ & 16.3 & 3 & $15-18$ \\
\hline & 2-Hexanone & $591-78-6$ & 16.3 & 3 & $15-18$ \\
\hline & 4-Methyl-2-pentanone & $108-10-1$ & 16.3 & 3 & $15-18$ \\
\hline & Acetone & $67-64-1$ & 16.3 & 3 & $15-18$ \\
\hline & Benzene & $71-43-2$ & 8 & 3 & $7-9$ \\
\hline & Bromodichloromethane & $75-27-4$ & 8 & 3 & $7-9$ \\
\hline & Bromoform & $75-25-2$ & 8 & 3 & 7.9 \\
\hline & Bromomethane & $74-83-9$ & 16.3 & 3 & $15-18$ \\
\hline & Carbon Disulfide & $75-15-0$ & 8 & 3 & 7.9 \\
\hline & Carbon Tetrachloride & $56-23-5$ & 8 & 3 & $7-9$ \\
\hline & Chlorobenzene & $108-90-7$ & 8 & 3 & $7-9$ \\
\hline & Chloroethane & $75-00-3$ & 16.3 & 3 & $15-18$ \\
\hline & Chloroform & $67-66-3$ & 8 & 3 & $7-9$ \\
\hline & Chloromethane & $74-87-3$ & 16.3 & 3 & $15-18$ \\
\hline & Dibromochloromethane & $124-48-1$ & 8 & 3 & $7-9$ \\
\hline & Ethylbenzene & $100-41-4$ & 8 & 3 & $7-9$ \\
\hline & Methylene Chloride & $75-09-2$ & 16.3 & 3 & $15-18$ \\
\hline & Styrene & $100-42-5$ & 8 & 3 & 7.9 \\
\hline & Toluene & $108-88-3$ & 8 & 3 & $7-9$ \\
\hline & Trichloroethene & $79-01-6$ & 8 & 3 & $7-9$ \\
\hline & Vinyl Chloride & $75-01-4$ & 16.3 & 3 & 15-18 \\
\hline & Xylene, Total & $1330-20-7$ & 8 & 3 & 7.9 \\
\hline & cis-1,3-Dichloropropene & $10061-01-5$ & 8 & 3 & 7.9 \\
\hline & trans-1,3-Dichloropropene & $10061-02-6$ & 8 & 3 & $7-9$ \\
\hline
\end{tabular}




\begin{tabular}{|c|c|c|c|c|c|}
\hline Andlygis & Parameter & Cas Number & Mean Detection Limit & $\begin{array}{l}\text { Number of } \\
\text { Nondetects }\end{array}$ & $\begin{array}{l}\text { Detection } \\
\text { Limit Range } \\
\end{array}$ \\
\hline \multirow{24}{*}{ Pesticide/PCB UG/KG } & $4,4^{\circ}-\mathrm{DDD}$ & $72-54-8$ & 5.4 & 3 & $4.9-6.2$ \\
\hline & $4,4^{\prime}-\mathrm{DDE}$ & $72-55-9$ & 5.4 & 3 & $4.9-6.2$ \\
\hline & 4,4'-DDT & 50-29-3 & 5.4 & 3 & $4.9-6.2$ \\
\hline & Aldrin & $309-00-2$ & 2.7 & 3 & $2.5-3.1$ \\
\hline & Aroclor-1016 & $12674-111-2$ & 54 & 3 & $49-62$ \\
\hline & Aroclor-1221 & $11104-28-2$ & 106 & 3 & $99-120$ \\
\hline & Aroclor-1232 & $11141-16-5$ & 54 & 3 & $49-62$ \\
\hline & Aroclor-1242 & $53469-21-9$ & 54 & 3 & $49-62$ \\
\hline & Aroclor-1248 & $12672-29-6$ & 54 & 3 & $49-62$ \\
\hline & Aroclor-1254 & $11097-69-1$ & 54 & 3 & 49.62 \\
\hline & Dieldrin & $60-57-1$ & 5.4 & 3 & $4.9-6.2$ \\
\hline & Endosulfan & $959-98-8$ & 2.7 & 3 & $2.5-3.1$ \\
\hline & Endosulfan II & $33213-65-9$ & 5.4 & 3 & $4.9-6.2$ \\
\hline & Endosulfan Sulfate & $1031-07-8$ & 5.4 & 3 & $4.9-6.2$ \\
\hline & Endrin & $72-20-8$ & 5.4 & 3 & $4.9-6.2$ \\
\hline & Endrin Aldehyde & $7421.93-4$ & 5.4 & 3 & $4.9-6.2$ \\
\hline & Endris Ketone & $53494-70-5$ & 5.4 & 3 & $4.9 \cdot 6.2$ \\
\hline & Heptachlor & $76-44 \cdot 8$ & 2.7 & 3 & $2.5-3.1$ \\
\hline & Heptachlor Epoxide & $1024-57-3$ & 2.7 & 3 & 2.5-3.1 \\
\hline & Toxaphene & $2001-35-2$ & 270 & 3 & $250-310$ \\
\hline & alpha-BHC & $319-84-6$ & 2.7 & 3 & $2.5-3.1$ \\
\hline & Alpha-Chlordane & $5103-71-9$ & 2.7 & 3 & $2.5-3.1$ \\
\hline & beta-BHC & $319-85-7$ & 2.7 & 3 & 2.5-3.1 \\
\hline & delta-BHC & $319-86-8$ & 2.7 & 3 & $2.5-3.1$ \\
\hline
\end{tabular}




\begin{tabular}{|c|c|c|c|c|c|}
\hline Analyøis & Parameter & Cas Number & Mean Detection Limit & $\begin{array}{l}\text { Number of } \\
\text { Nondetects }\end{array}$ & $\begin{array}{c}\text { Detection } \\
\text { Limit Range }\end{array}$ \\
\hline & gamma-BHC (Lindane) & $58-89-9$ & 2.7 & 3 & $2.5-3.1$ \\
\hline & gamma-Chlordane & $5103-74-2$ & 2.7 & 3 & $2.5-3.1$ \\
\hline & p, $\mathrm{p}^{9}$-Methorychlor & $72-43-5$ & 27 & 3 & $25-31$ \\
\hline
\end{tabular}

Data Source : Bear Creek Valley OU2 1993 RI field activities validated data

Raw data available through $Y-12$ OREIS

Data does not include QA/QC

Results $<$ counting uncertainty were considered as nondetects for radionuclides 
TABLE 2. BC-OU2 1993 RI Field Activities Data - Summary Of Parameters Never Detected For The Spoil Area 1 Sampling Media: Streama Sechiments

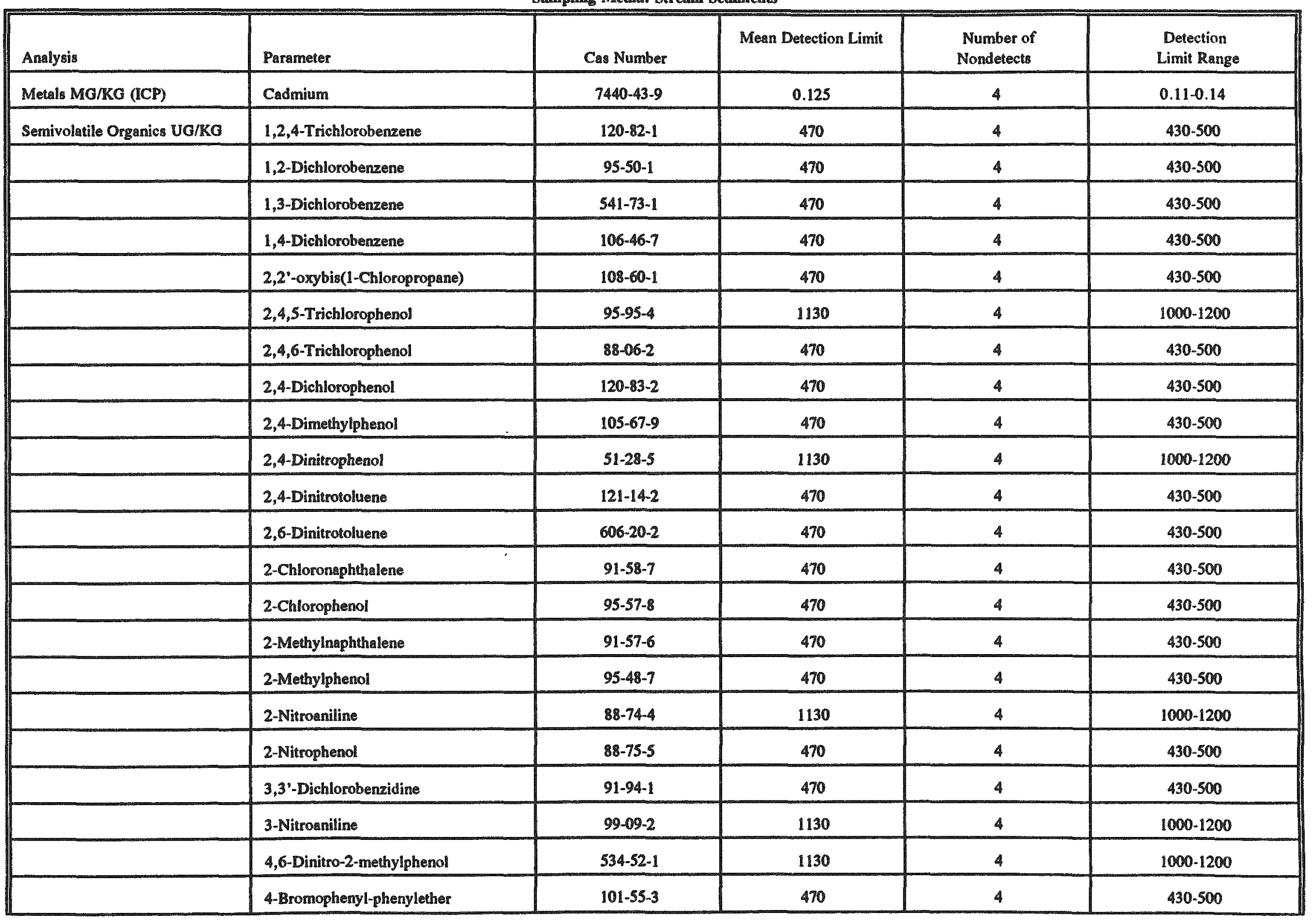




\begin{tabular}{|c|c|c|c|c|c|}
\hline Analysis & Parameter & Cas Number & Mean Detection Limit & $\begin{array}{l}\text { Number of } \\
\text { Nondetects }\end{array}$ & $\begin{array}{l}\text { Detection } \\
\text { Limit Range }\end{array}$ \\
\hline & 4-Chloro-3-methylphenol & $59-50-7$ & 470 & 4 & $430-500$ \\
\hline & 4-Chloroaniline & $106-47-8$ & 470 & 4 & $430-500$ \\
\hline & 4-Chlorophenyl-phenylether & $7005-72-3$ & 470 & 4 & $430-500$ \\
\hline & 4-Methylphenol & $106-44-5$ & 470 & 4 & $430-500$ \\
\hline & 4-Nitroaniline & $100-01-6$ & 1130 & 4 & $1000-1200$ \\
\hline & 4-Nitrophenol & $100-02-7$ & 1130 & 4 & $1000-1200$ \\
\hline & Acenaphthene & $32-9$ & 470 & 4 & $430-500$ \\
\hline & Acenaphthylene & $208-96-8$ & 470 & 4 & $430-500$ \\
\hline & Anthracene & $120-12-7$ & 470 & 4 & $430-500$ \\
\hline & Benzo(a)anthracene & $56-55-3$ & 470 & 4 & $430-500$ \\
\hline & Benzo(a)pyrene & $50-32-8$ & 470 & 4 & $430-500$ \\
\hline & Benzo(b)fluoranthene & 205-99-2 & 470 & 4 & $430-500$ \\
\hline & Benzo(g,h,i)perylene & $191-24-2$ & 470 & 4 & $430-500$ \\
\hline & Benzo(k) fluoranthene & $207-08-9$ & 470 & 4 & $430-500$ \\
\hline & Butylbenzylphthalate & $85-68-7$ & 470 & 4 & $430-500$ \\
\hline & Carbazole & $86-74-8$ & 470 & 4 & $430-500$ \\
\hline & Chrysene & 218-01-9 & 470 & 4 & $430-500$ \\
\hline & Di-n-butylphthalate & $84-74-2$ & 470 & 4 & $430-500$ \\
\hline & Di-n-octylphthalate & $117-84-0$ & 470 & 4 & $430-500$ \\
\hline & Dibenzo $(a, h)$ anthracene & $53-70-3$ & 470 & 4 & $430-500$ \\
\hline & Dibenzofuran & $132-64-9$ & 470 & 4 & $430-500$ \\
\hline & Diethylphthalate & $84-66.2$ & 470 & 4 & $430-500$ \\
\hline & Dimethylphthalate & $131-11-3$ & 470 & 4 & $430-500$ \\
\hline & Fluoranthene & $206-44-0$ & 470 & 4 & $430-500$ \\
\hline
\end{tabular}




\begin{tabular}{|c|c|c|c|c|c|}
\hline Analysis & Parameter & Cas Number & Mean Detection Limit & $\begin{array}{l}\text { Number of } \\
\text { Nondetects }\end{array}$ & $\begin{array}{l}\text { Detection } \\
\text { Limit Range }\end{array}$ \\
\hline & Fluorene & $86-73-7$ & 470 & 4 & $430-500$ \\
\hline & Hexachlorobenzene & $118-74-1$ & 470 & 4 & 430.500 \\
\hline & Hexachlorobutadiene & $87-68-3$ & 470 & 4 & 430.500 \\
\hline & Hexachlorocyclopentadiene & $77-47-4$ & 470 & 4 & $430-500$ \\
\hline & Hexachloroethane & $67-72-1$ & 470 & 4 & $430-500$ \\
\hline & Indeno $(1,2,3-c, d)$ pyrene & $193-39-5$ & 470 & 4 & $430-500$ \\
\hline & Isophorone & $78-59-1$ & 470 & 4 & $430-500$ \\
\hline & N-Nitroso-di-n-propylamine & $621-64-7$ & 470 & 4 & $430-500$ \\
\hline & N-Nitrosodiphenylamine & $86-30-6$ & 470 & 4 & $430-500$ \\
\hline & Nitrobenzene & $98-95-3$ & 470 & 4 & $430-500$ \\
\hline & Pentachlorophenol & $87-86-5$ & 1130 & 4 & $1000-1200$ \\
\hline & Phenanthrene & $85-01-8$ & 470 & 4 & $430-500$ \\
\hline & Phenol & $108-95-2$ & 470 & 4 & $430-500$ \\
\hline & Pyrene & $129-00-0$ & 470 & 4 & $430-500$ \\
\hline & bis(2-Chloroethoxy)methane & $111-91-1$ & 470 & 4 & $430-500$ \\
\hline & bis(2-Chloroethyl)ether & $111-44-4$ & 470 & 4 & $430-500$ \\
\hline & bis(2-Ethylhexyl)phthalate & $117-81-7$ & 470 & 4 & $430-500$ \\
\hline Volatile-OrganicaUG/KO & 1,1,1-Trichloroethane & $71.55-6$ & 7.5 & 4 & 7.8 \\
\hline & 1,1,2,2-Tetrachloroethane & $79-34-5$ & 7.5 & 4 & 7.8 \\
\hline & 1,1,2-Trichloro-1,2,2-Trifluoroethane & $76-13-1$ & 14.5 & 4 & $13-16$ \\
\hline & 1,1,2-Trichloroethane & $79-00-5$ & 7.5 & 4 & 7.8 \\
\hline & 1,1-Dichloroethane & $75-34-3$ & 7.5 & 4 & 7.8 \\
\hline & 1,1-Dichloroethene & $75-35-4$ & 7.5 & 4 & 7.8 \\
\hline & 1,2-Dichloroethane & $107-06-2$ & 7.5 & 4 & $7-8$ \\
\hline
\end{tabular}




\begin{tabular}{|c|c|c|c|c|c|}
\hline Analysis & Parameter & Cas Number & Mean Detection Limit & $\begin{array}{l}\text { Number of } \\
\text { Nondetects }\end{array}$ & $\begin{array}{l}\text { Detection } \\
\text { Limit Range }\end{array}$ \\
\hline & 1,2-Dichloroethene & $540-59-0$ & 7.5 & 4 & 7.8 \\
\hline & 1,2-Dichloropropane & $78-87-5$ & 7.5 & 4 & $7-8$ \\
\hline & 2-Butanone & $78-93-3$ & 14.5 & 4 & $13-16$ \\
\hline & 2-Hexanone & $591-78-6$ & 14.5 & 4 & $13-16$ \\
\hline & Benzene & $71-43-2$ & 7.5 & 4 & $7-8$ \\
\hline & Bromodichloromethane & $75-27-4$ & 7.5 & 4 & $7-8$ \\
\hline & Bromoform & $75-25-2$ & 7.5 & 4 & $7-8$ \\
\hline & Bromomethane & $74-83-9$ & 14.5 & 4 & $13-16$ \\
\hline & Carbon Disulingle & $75-15-0$ & 7.5 & 4 & $7-8$ \\
\hline & Carbon Tetrachloride & $56-23-5$ & 7.5 & 4 & 7.8 \\
\hline & Chlorobenzene & $108-90-7$ & 7.5 & 4 & 7.8 \\
\hline & Chloroethane & $75-00-3$ & 14.5 & 4 & $13-16$ \\
\hline & Chloroform & $67-66-3$ & 7.5 & 4 & $7-8$ \\
\hline & Chloromethane & $74-87-3$ & 14.5 & 4 & $13-16$ \\
\hline & Dibromochloromethane & $124-48-1$ & 7.5 & 4 & 7.8 \\
\hline & Ethylbenzene & $100-41-4$ & 7.5 & 4 & 7.8 \\
\hline & Methylene Chloride & $75-09-2$ & 16.3 & 4 & $13-23$ \\
\hline & Styrene & $100-42-5$ & 7.5 & 4 & $7-8$ \\
\hline & Tetrachloroethene & $127-18-4$ & 7.5 & 4 & $7-8$ \\
\hline & Toluene & $108-88.3$ & 7.5 & 4 & 7.8 \\
\hline & Trichloroethene & $79-01-6$ & 7.5 & 4 & $7-8$ \\
\hline & Vinyl Chloride & $75-01-4$ & 14.5 & 4 & $13-16$ \\
\hline & Xylene, Tosal & 1330-20-7 & 7.5 & 4 & 7.8 \\
\hline & cir-1,3-Dichloropropene & $10061-01-5$ & 7.5 & 4 & $7-8$ \\
\hline
\end{tabular}




\begin{tabular}{|c|c|c|c|c|c|}
\hline Analysis & Parameter & Cas Number & Mean Detection Limit & $\begin{array}{l}\text { Number of } \\
\text { Nondetects }\end{array}$ & $\begin{array}{l}\text { Detection } \\
\text { Limit Range }\end{array}$ \\
\hline & trans-1,3-Dichloropropene & $10061-02-6$ & 7.5 & 4 & $7-8$ \\
\hline \multirow[t]{23}{*}{ Pesticide/PCB UG/RG } & $4,4^{\prime}-\mathrm{DDD}$ & $72-54-8$ & 5.13 & 4 & $4.4-6.5$ \\
\hline & $4,4^{\prime}-\mathrm{DDE}$ & $72.55-9$ & 5.13 & 4 & 4.4-6.5 \\
\hline & 4,4'-DDT & $50-29-3$ & 5.13 & 4 & $4.4-6.5$ \\
\hline & Aldrin & $309-00-2$ & 2.58 & 4 & $2.2-3.3$ \\
\hline & Aroclor-1016 & $12674-11-2$ & 51.3 & 4 & $44-65$ \\
\hline & Aroclor- 1221 & $11104-28-2$ & 102 & 4 & $88-130$ \\
\hline & Aroclor-1232 & $11141-16-5$ & 51.3 & 4 & 44.65 \\
\hline & Aroclor- 1242 & $53469-21-9$ & 51.3 & 4 & $44-65$ \\
\hline & Aroclor-1248 & $12672-29-6$ & 51.3 & 4 & $44-65$ \\
\hline & Aroclor-1254 & $11097-69-1$ & 51.3 & 4 & $44-65$ \\
\hline & Dieldrin & $60-57-1$ & 5.13 & 4 & $4.4-6.5$ \\
\hline & Endosulfan $\mathbb{1}$ & $959.98-8$ & 2.58 & 4 & $2.2-3.3$ \\
\hline & Endosulfan II & $33213-65-9$ & 5.13 & 4 & $4.4-6.5$ \\
\hline & Endosulfan Sulfate & $1031-07-8$ & 5.13 & 4 & $4.4-6.5$ \\
\hline & Endrin & $72-20-8$ & 5.13 & 4 & $4.4-6.5$ \\
\hline & Endrin Aldehyde & $7421-93-4$ & 5.13 & 4 & $4.4-6.5$ \\
\hline & Endrin Ketone & $53494-70-5$ & 5.13 & 4 & $4.4-6.5$ \\
\hline & Heptachlor & $76-44-8$ & 2.58 & 4 & $2.2-3.3$ \\
\hline & Heptachlor Epoxide & $1024-57-3$ & 2.58 & 4 & $2.2-3.3$ \\
\hline & Toxaphene & $8001-35-2$ & 258 & 4 & $220-330$ \\
\hline & alpha-BHC & $319-84-6$ & 2.58 & 4 & $2.2-3.3$ \\
\hline & alphs-Chlordane & $5103-71-9$ & 2.58 & 4 & $2.2-3.3$ \\
\hline & beta-BHC & $319-85-7$ & 2.58 & 4 & $2.2-3.3$ \\
\hline
\end{tabular}




\begin{tabular}{|l|l|c|c|c|c||}
\hline \hline Analysis & Parameter & Cas Number & Mean Detection Limit & $\begin{array}{c}\text { Number of } \\
\text { Nondetects }\end{array}$ & $\begin{array}{c}\text { Detection } \\
\text { Limit Range }\end{array}$ \\
\hline & delta-BHC & $319-86-8$ & 2.58 & 4 & $2.2-3.3$ \\
\hline & gamma-BHC (Lindane) & $58-89-9$ & 2.58 & 4 & $2.2-3.3$ \\
\hline & gamma-Chlordane & $5103-74-2$ & 2.58 & 4 & $2.2-3.3$ \\
\hline & p,p'-Methoxychlor & $72-43-5$ & 25.8 & 4 & $22-33$ \\
\hline
\end{tabular}

Data Source : Bear Creek Valley OU2 1993 RI field activities validated data

Raw data available through Y-12 OREIS

Dat does not include QA/OC

Results $<$ counting uncertainty were considered nondetects for radionuclides 
TABLE 2. BC-OU2 1993 RI Field Activities Data - Sumamary Of Parameters Never Detected For The SY-200 Yard Sampling Media: Stream Sediments

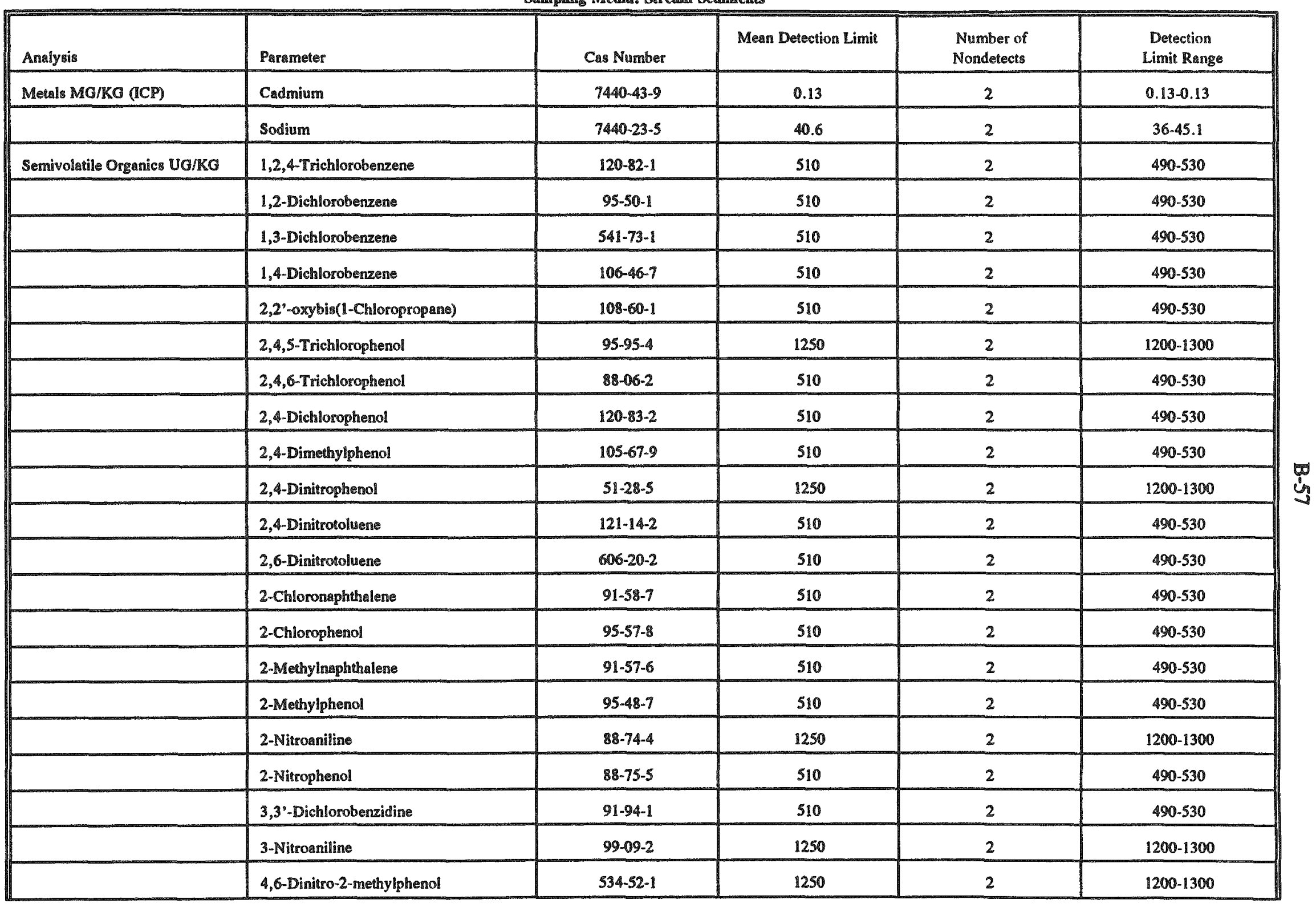




\begin{tabular}{|c|c|c|c|c|c|}
\hline Andlysis & Parameter & Cas Number & Mean Detection Limit & $\begin{array}{l}\text { Number of } \\
\text { Nondetects }\end{array}$ & $\begin{array}{l}\text { Detection } \\
\text { Limit Range }\end{array}$ \\
\hline & 4-Bromophenyl-phenylether & $101-55-3$ & 510 & 2 & $490-530$ \\
\hline & 4-Chloro-3-methylphenol & $59-50-7$ & 510 & 2 & $490-530$ \\
\hline & 4-Chloroaniline & $106-47-8$ & 510 & 2 & $490-530$ \\
\hline & 4-Chlorophenyl-phenylether & $7005-72-3$ & 510 & 2 & $490-530$ \\
\hline & 4-Methylphenol & $106-44-5$ & 510 & 2 & $490-530$ \\
\hline & 4-Nitroaniline & $100-01-6$ & 1250 & 2 & $1200-1300$ \\
\hline & 4-Nitrophenol & $100-02-7$ & 1250 & 2 & $1200-1300$ \\
\hline & Acenaphthene & $83-32-9$ & 510 & 2 & $490-530$ \\
\hline & Acenaphthylene & $208-96-8$ & 510 & 2 & $490-530$ \\
\hline & Anthracene & $120-12-7$ & 510 & 2 & $490-530$ \\
\hline & Benzo(ø)anthracene & $56-55-3$ & 510 & 2 & $490-530$ \\
\hline & Benzo(a)pyrene & $50-32-8$ & 510 & 2 & $490-530$ \\
\hline & Benzo(b)fluoranthene & $205-99-2$ & 510 & 2 & $490-530$ \\
\hline & Benzo $(\mathrm{g}, \mathrm{h}, \mathrm{i})$ perylene & $191-24-2$ & 510 & 2 & $490-530$ \\
\hline & Benzo(k)fluoranthene & 207-08-9 & 510 & 2 & $490-530$ \\
\hline & Butylbenzy lphthalate & $85-68-7$ & 510 & 2 & $490-530$ \\
\hline & Carbazole & $86-74-8$ & 510 & 2 & $490-530$ \\
\hline & Chrysene & $218-01-9$ & 510 & 2 & $490-530$ \\
\hline & Di-n-butylphthalate & $84-74-2$ & 510 & 2 & $490-530$ \\
\hline & Di-n-octylphthalate & $117-840$ & 510 & 2 & $490-530$ \\
\hline & Dibenzo(a,h)anthracene & 53-70-3 & 510 & 2 & $490-530$ \\
\hline & Dibenzofuran & $132-64-9$ & 510 & 2 & $490-530$ \\
\hline & Diethylphthalate & $84-66-2$ & 510 & 2 & $490-530$ \\
\hline & Dimethylphthalate & $131-11-3$ & 510 & 2 & $490-530$ \\
\hline
\end{tabular}




\begin{tabular}{|c|c|c|c|c|c|}
\hline Analysis & Parameter & Cas Number & Mean Detection Limit & $\begin{array}{l}\text { Number of } \\
\text { Nondetects }\end{array}$ & $\begin{array}{l}\text { Detection } \\
\text { Limit Range }\end{array}$ \\
\hline & Fluoranthene & $206-44-0$ & 510 & 2 & 490.530 \\
\hline & Fluorene & $86-73-7$ & 510 & 2 & $490-530$ \\
\hline & Hexachlorobenzene & $118-74-1$ & 510 & 2 & $490-530$ \\
\hline & Hexachlorobutadiene & $87-68-3$ & 510 & 2 & $490-530$ \\
\hline & Hexachlorocyclopentadiene & $77-47-4$ & 510 & 2 & $490-530$ \\
\hline & Hexachloroethane & $67-72-1$ & 510 & 2 & 490.530 \\
\hline & Indeno(1,2,3-c,d)pyrene & 193-39-5 & 510 & 2 & $490-530$ \\
\hline & Isophorone & $78-59-1$ & 510 & 2 & $490-530$ \\
\hline & N-Nitroso-di-n-propylamine & $621-64-7$ & 510 & 2 & $490-530$ \\
\hline & N-Nitrosodiphenylamine & $86-30-6$ & 510 & 2 & $490-530$ \\
\hline & Naphthalene & $91-20-3$ & 510 & 2 & $490-530$ \\
\hline & Nitrobenzene & $98-95-3$ & 510 & 2 & 490.530 \\
\hline & Pentachlorophenol & $87-86-5$ & 1250 & 2 & $1200-1300$ \\
\hline & Phenanthrene & $85-01-8$ & 510 & 2 & $490-530$ \\
\hline & Phenol & $108-95-2$ & 510 & 2 & $490-530$ \\
\hline & Pyrene & $129-00-0$ & 510 & 2 & $490-530$ \\
\hline & bis(2-Chloroethoxy)methane & $111-91-1$ & 510 & 2 & $490-530$ \\
\hline & bis(2-Chlorcethyl)ether & $111-44-4$ & 510 & 2 & $490 \cdot 530$ \\
\hline & bis(2-Ethylhexyl)phthalate & $117-81-7$ & 510 & 2 & $490-530$ \\
\hline Volatile Organics UG/KG & 1,1,1-Trichloroethane & $71-55-6$ & 7.5 & 2 & $7-8$ \\
\hline & 1,1,2,2-Tetrachloroethane & $79-34-5$ & 7.5 & 2 & 7.8 \\
\hline & 1,1,2-Trichloro-1,2,2-Trifluoroethane & $76-13-1$ & 15.5 & 2 & $15-16$ \\
\hline & 1,1,2-Trichloroethane & $79-00-5$ & 7.5 & 2 & 7.8 \\
\hline & 1,1-Dichloroethane & $75-34-3$ & 7.5 & 2 & 7.8 \\
\hline
\end{tabular}




\begin{tabular}{|c|c|c|c|c|c|}
\hline Analysis & Parameter & Cas Number & Mean Detection Limit & $\begin{array}{l}\text { Number of } \\
\text { Nondetects }\end{array}$ & $\begin{array}{l}\text { Detection } \\
\text { Limit Range }\end{array}$ \\
\hline & 1,1-Dichloroethene & $75-35-4$ & 7.5 & 2 & $7-8$ \\
\hline & 1,2-Dichloroethane & $107-06-2$ & 7.5 & 2 & 7.8 \\
\hline & 1,2-Dichloroethene & $540-59-0$ & 7.5 & 2 & $7-8$ \\
\hline & 1,2-Dichloropropane & $78-87-5$ & 7.5 & 2 & $7-8$ \\
\hline & 2-Buranone & 78-93-3 & 15.5 & 2 & $15-16$ \\
\hline & 2-Hexanone & $591-78-6$ & 15.5 & 2 & $15-16$ \\
\hline & Benzene & $71-43-2$ & 7.5 & 2 & $7-8$ \\
\hline & Bromodichloromethane & $75-27-4$ & 7.5 & 2 & 7.8 \\
\hline & Bromoform & $75-25-2$ & 7.5 & 2 & $7-8$ \\
\hline & Bromomethane & 74-83-9 & 15.5 & 2 & $15-16$ \\
\hline & Carbon Disulfide & $75-15-0$ & 7.5 & 2 & 7.8 \\
\hline & Carbon Tetrachloride & $56-23-5$ & 7.5 & 2 & $7-8$ \\
\hline & Chlorobenzene & $108-90-7$ & 7.5 & 2 & $7-8$ \\
\hline & Chloroethene & $75-00-3$ & 15.5 & 2 & $15-16$ \\
\hline & Chloroform & $67-66-3$ & 7.5 & 2 & $7-8$ \\
\hline & Chloromethane & $74-87-3$ & 15.5 & 2 & $15-16$ \\
\hline & Dibromochloromethane & $124-48-1$ & 7.5 & 2 & 7.8 \\
\hline & Ethylbenzene & $100-41-4$ & 7.5 & 2 & $7-8$ \\
\hline & Methylene Chloride & $75-09-2$ & 15.5 & 2 & $15-16$ \\
\hline & Styrene & $100-42-5$ & 7.5 & 2 & $7-8$ \\
\hline & Tetrachloroethene & $127-18-4$ & 7.5 & 2 & 7.8 \\
\hline & Toluene & $108-88-3$ & 7.5 & 2 & $7-8$ \\
\hline & Trichloroethene & $79-01-6$ & 7.5 & 2 & $7-8$ \\
\hline & Vinyl Chloride & $75-01-4$ & 15.5 & 2 & $15-16$ \\
\hline
\end{tabular}




\begin{tabular}{|c|c|c|c|c|c|}
\hline Analysis & Parameter & Cas Number & Mean Detection Limit & $\begin{array}{l}\text { Number of } \\
\text { Nondetects }\end{array}$ & $\begin{array}{l}\text { Detection } \\
\text { Limit Range }\end{array}$ \\
\hline & Xylene, Total & $1330-20-7$ & 7.5 & 2 & $7-8$ \\
\hline & cis-1,3-Dichloropropene & $10061-01-5$ & 7.5 & 2 & 7.8 \\
\hline & trans-1,3-Dichloropropene & $10061-02-6$ & 7.5 & 2 & $7-8$ \\
\hline \multirow[t]{21}{*}{ Pesticide/PCB UG/KG } & $4,4^{\prime}-\mathrm{DDD}$ & $72-54-8$ & 5.1 & 2 & $4.9-5.3$ \\
\hline & $4,4^{\circ}-\mathrm{DDE}$ & $72-55-9$ & 5.1 & 2 & $4.9-5.3$ \\
\hline & $4,4^{9}-\mathrm{DDT}$ & $50-29-3$ & 5.1 & 2 & $4.9-5.3$ \\
\hline & Aldrin & $309-00-2$ & 2.55 & 2 & $2.4-2.7$ \\
\hline & Aroclor-1016 & $12674-11-2$ & 51 & 2 & $49-53$ \\
\hline & Aroclor- 1221 & $11104-28-2$ & 104 & 2 & $98-110$ \\
\hline & Aroclor- 1232 & $11141-16-5$ & 51 & 2 & $49-53$ \\
\hline & Aroelor- 1242 & $53469-21-9$ & 51 & 2 & $49-53$ \\
\hline & Aroclor-1248 & $12672-29-6$ & 51 & 2 & $49-53$ \\
\hline & Aroclor- 1254 & $11097-69-1$ & 51 & 2 & $49-53$ \\
\hline & Dieldrin & $60.57-1$ & 5.1 & 2 & 4.9 .5 .3 \\
\hline & Endosulfan 1 & $959-98-8$ & 2.55 & 2 & $2.4-2.7$ \\
\hline & Endosulfan II & $33213-65-9$ & 5.1 & 2 & 4.9 .5 .3 \\
\hline & Endosulfan Sulfate & $1031-07-8$ & 5.1 & 2 & $4.9-5.3$ \\
\hline & Endrin & $72-20-8$ & 5.1 & 2 & $4.9-5.3$ \\
\hline & Endrin Aldehyde & $7421-93-4$ & 5.1 & 2 & $4.9-5.3$ \\
\hline & Endrin Ketone & $53494-70-5$ & 5.1 & 2 & $4.9-5.3$ \\
\hline & Heptachlor & $76-44-8$ & 2.55 & 2 & $2.4-2.7$ \\
\hline & Heptachlor Epoxide & $1024-57-3$ & 2.55 & 2 & $2.4-2.7$ \\
\hline & Toxaphene & $8001-35-2$ & 255 & 2 & $240-270$ \\
\hline & alpha-BHC & $319-84-6$ & 2.55 & 2 & $2.4-2.7$ \\
\hline
\end{tabular}




\begin{tabular}{|c|c|c|c|c|c|}
\hline Analysis & Parameter & Cas Number & Mean Detection Limit & $\begin{array}{l}\text { Number of } \\
\text { Nondetects }\end{array}$ & $\begin{array}{l}\text { Detection } \\
\text { Limit Range }\end{array}$ \\
\hline & alpha-Chlordane & $5103-71-9$ & 2.55 & 2 & $2.4-2.7$ \\
\hline & beta-BHC & $319-85-7$ & 2.55 & 2 & $2.4-2.7$ \\
\hline & delta-BHC & $319-86-8$ & 2.55 & 2 & $2.4-2.7$ \\
\hline & gamma-BHC (Lindane) & $58-89-9$ & 2.55 & 2 & $2.4-2.7$ \\
\hline & gamma-Chlordane & $5103-74-2$ & 2.55 & 2 & $2.4-2.7$ \\
\hline & p,p-Methoxychlor & $72-43-5$ & 25.5 & 2 & $24-27$ \\
\hline
\end{tabular}

Datu Source : Bear Creek Valley OU2 1993 RI field activities validated data

Raw data avilable through Y-12 OREIS

Date does not include QA/QC

Results < counting uncertainty were considered as nondetects for radionuclides 
TABLE 2. BC-OU2 1993 RI Field Activities Data - Summary Of Parameters Never Detected For The Rust Spoil Area Sampling Media: Surface Water

\begin{tabular}{|c|c|c|c|c|c|}
\hline Andlysis & Parameter & Cas Number & $\begin{array}{l}\text { Mean Detection } \\
\text { Limit } \\
\end{array}$ & $\begin{array}{l}\text { Number of } \\
\text { Nondefects }\end{array}$ & $\begin{array}{c}\text { Detection } \\
\text { Limit Range }\end{array}$ \\
\hline \multirow[t]{7}{*}{ Metals UG/L (AAS) } & Arsenic & $7440-38-2$ & 4.9 & 3 & 1.8-10.4 \\
\hline & Arsenic (Dissolved) & $7440-38-2$ & 2.2 & 3 & $2.2-2.2$ \\
\hline & Cyanide & $57-12-5$ & 10 & 3 & $10-10$ \\
\hline & Selenium & $7782-49-2$ & 4.27 & 3 & $4.1-4.6$ \\
\hline & Selenium (Dissolved) & $7782-49-2$ & 2.7 & 3 & $2.7-2.7$ \\
\hline & Thallium & $7440-28-0$ & 1.3 & 3 & $1.3-1.3$ \\
\hline & Thallium (Dissolved) & $7440-28-0$ & 1.8 & 3 & $1.8-1.8$ \\
\hline \multirow[t]{2}{*}{ Metals UG/L } & Mercury & $7439-97-6$ & 0.2 & 3 & $0.2-0.2$ \\
\hline & Mercury (Dissolved) & $7439-97-6$ & 0.2 & 3 & $0.2-0.2$ \\
\hline \multirow[t]{14}{*}{ Metals UG/L (ICP) } & Aluminum (Dissolved) & $7429-90-5$ & 22.9 & 3 & $16-26.7$ \\
\hline & Beryllium & $7440-41-7$ & 0.733 & 3 & $0.3-1.6$ \\
\hline & Beryllium (Dissolved) & $7440-41-7$ & 0.1 & 3 & $0.1-0.1$ \\
\hline & Cadmium & $7440-43-9$ & 1.7 & 3 & $1.7-1.7$ \\
\hline & Cadmium (Dissolved) & $7440-43-9$ & 1 & 3 & $1-1$ \\
\hline & Chromium (Dissolved) & $7440-47-3$ & 5.6 & 3 & 5.6 .5 .6 \\
\hline & Cobalt (Dissolved) & $7440-48-4$ & 2 & 3 & $2-2$ \\
\hline & Copper & $7440.50-8$ & 26.3 & 3 & $3.2-69.1$ \\
\hline & Iron (Dissolved) & $7439-89-6$ & 58.8 & 3 & $29.7-80.2$ \\
\hline & Manganese (Dissolved) & $7439-96-5$ & 3.7 & 3 & $2.1-6.1$ \\
\hline & Nickel & $7440-02-0$ & 17 & 3 & $4.4-40.9$ \\
\hline & Nickel (Dissolved) & $7440-02-0$ & 4.7 & 3 & $4.7-4.7$ \\
\hline & Silver & $7440-22-4$ & 2.1 & 3 & $2.1-2.1$ \\
\hline & Silver (Dissolved) & $7440-22-4$ & 4.1 & 3 & $4.1-4.1$ \\
\hline
\end{tabular}




\begin{tabular}{|c|c|c|c|c|c|}
\hline Analysis & Parameter & Cas Number & $\begin{array}{l}\text { Mean Detection } \\
\text { Limit }\end{array}$ & $\begin{array}{l}\text { Number of } \\
\text { Nondetects }\end{array}$ & $\begin{array}{l}\text { Detection } \\
\text { Limit Range }\end{array}$ \\
\hline & Vanadium (Dissolved) & $7440-62-2$ & 2.5 & 3 & $2.5-2.5$ \\
\hline & Zine (Dissolved) & $7440-66-6$ & 4.63 & 3 & $2-7$ \\
\hline \multirow[t]{22}{*}{ Semivolatile Organics UG/L } & 1,2,4-Trichlorobenzene & $120-82-1$ & 10 & 3 & $10-10$ \\
\hline & 1,2-Dichlorobenzene & $95-50-1$ & 10 & 3 & $10-10$ \\
\hline & 1,3-Dichlorobenzene & $541-73-1$ & 10 & 3 & $10-10$ \\
\hline & 1,4-Dichlorobenzene & $106-46-7$ & 10 & 3 & $10-10$ \\
\hline & $2,2^{\prime}$-oxybis(1-Chloropropane) & $108-60-1$ & 10 & 3 & $10-10$ \\
\hline & 2,4,5-Trichlorophenol & $95-95-4$ & 25 & 3 & $25-25$ \\
\hline & 2,4,6-Trichlorophenol & $88-06-2$ & 10 & 3 & $10-10$ \\
\hline & 2,4-Dichlorophenol & $120-83-2$ & 10 & 3 & $10 \cdot 10$ \\
\hline & 2,4-Dimethylphenol & $105-67-9$ & 10 & 3 & $10-10$ \\
\hline & 2,4-Dinitrophenol & $51-28-5$ & 25 & 3 & $25-25$ \\
\hline & 2,4-Dinitrotoluene & $121-14-2$ & 10 & 3 & $10-10$ \\
\hline & 2,6-Dinitrotolusene & $606-20-2$ & 10 & 3 & $10-10$ \\
\hline & 2-Chloronaphthalene & 91-58-7 & 10 & 3 & $10-10$ \\
\hline & 2-Chlorophenol & $95-57-8$ & 10 & 3 & $10-10$ \\
\hline & 2-Methylnaphthalene & $91-57-6$ & 10 & 3 & $10-10$ \\
\hline & 2-Methylphenol & $95-48-7$ & 10 & 3 & $10-10$ \\
\hline & 2-Nitroaniline & $88-74-4$ & 25 & 3 & $25-25$ \\
\hline & 2-Nitrophenol & $88-75-5$ & 10 & 3 & $10-10$ \\
\hline & 3,3'-Dichlorobenzidine & 91-94-1 & 10 & 3 & $10-10$ \\
\hline & 3-Nitroaniline & $99-09-2$ & 25 & 3 & $25-25$ \\
\hline & 4,6-Dinitro-2-methylphenol & $534-52-1$ & 25 & 3 & $25-25$ \\
\hline & 4-Bromophenyl-phenylether & $101-53-3$ & 10 & 3 & $10 \cdot 10$ \\
\hline
\end{tabular}




\begin{tabular}{|c|c|c|c|c|c|}
\hline Analysis & Parameter & Cas Number & $\begin{array}{c}\text { Mean Detection } \\
\text { Limit }\end{array}$ & $\begin{array}{l}\text { Number of } \\
\text { Nondetects }\end{array}$ & $\begin{array}{c}\text { Detection } \\
\text { Limit Range }\end{array}$ \\
\hline & 4-Chloro-3-methylphenol & $59-50-7$ & 10 & 3 & $10-10$ \\
\hline & 4-Chloroaniline & $106-47-8$ & 10 & 3 & $10-10$ \\
\hline & 4-Chlorophenyl-phenylether & $7005-72-3$ & 10 & 3 & $10-10$ \\
\hline & 4-Methylphenol & $106-44-5$ & 10 & 3 & $10-10$ \\
\hline & 4-Nitroaniline & $100-01-6$ & 25 & 3 & $25-25$ \\
\hline & 4-Nitrophenol & $100-02.7$ & 25 & 3 & $25-25$ \\
\hline & Acenaphthene & $83-32-9$ & 10 & 3 & $10-10$ \\
\hline & Acenaphthylene & $208-96-8$ & 10 & 3 & $10-10$ \\
\hline & Anthracene & $120-12-7$ & 10 & 3 & $10-10$ \\
\hline & Benzo(a)anthracene & $56-55-3$ & 10 & 3 & $10-10$ \\
\hline & Benzo(a)pyrene & $30-32-8$ & 10 & 3 & $10-10$ \\
\hline & Benzo(b)fluorenthene & $205-99-2$ & 10 & 3 & $10-10$ \\
\hline & Benzo $(g, h, i)$ perylene & 191-24-2 & 10 & 3 & $10-10$ \\
\hline & Benzo(k)fuoranthene & $207-08-9$ & 10 & 3 & $10-10$ \\
\hline & Carbazole & $86-74-8$ & 10 & 3 & $10-10$ \\
\hline & Chrysene & $218-01-9$ & 10 & 3 & $10-10$ \\
\hline & Di-n-octylphthalate & $117-84-0$ & 10 & 3 & $10-10$ \\
\hline & Dibenzo $(a, h) a n t h r a c e n e$ & $53-70-3$ & 10 & 3 & $10-10$ \\
\hline & Dibenzofuran & $132-64-9$ & 10 & 3 & $10 \cdot 10$ \\
\hline & Diethylphthalate & $84-66-2$ & 10 & 3 & $10-10$ \\
\hline & Dimethylphthalate & $131-11-3$ & 10 & 3 & $10-10$ \\
\hline & Fluoranthene & $206-44-0$ & 10 & 3 & $10-10$ \\
\hline & Fluorene & 86-73-7 & 10 & 3 & $10-10$ \\
\hline & Hexachlorobenzene & $118-74-1$ & 10 & 3 & $10-10$ \\
\hline
\end{tabular}




\begin{tabular}{|c|c|c|c|c|c|}
\hline Analysis & Parameter & Cas Number & $\begin{array}{l}\text { Mean Detection } \\
\text { Limit }\end{array}$ & $\begin{array}{l}\text { Number of } \\
\text { Nondetects }\end{array}$ & $\begin{array}{c}\text { Detection } \\
\text { Limit Range }\end{array}$ \\
\hline & Hexachlorobutadiene & $87-68-3$ & 10 & 3 & $10-10$ \\
\hline & Hexachlorocyclopentadiene & $77-47-4$ & 10 & 3 & $10-10$ \\
\hline & Hexachloroethane & $67-72-1$ & 10 & 3 & $10-10$ \\
\hline & Indeno $(1,2,3-\varepsilon, d)$ pyrene & $193-39-5$ & 10 & 3 & $10-10$ \\
\hline & Isophorone & $78.59-1$ & 10 & 3 & $10-10$ \\
\hline & N-Nitroso-di-n-propylamine & $621-64-7$ & 10 & 3 & $10-10$ \\
\hline & N-Nitrosodiphenylamine & $86-30-6$ & 10 & 3 & $10-10$ \\
\hline & Naphthalene & $91-20-3$ & 10 & 3 & $10-10$ \\
\hline & Nitrobenzene & $98-95-3$ & 10 & 3 & $10-10$ \\
\hline & Pentachlorophenol & $87-86-5$ & 25 & 3 & $25-25$ \\
\hline & Phenanthrene & $85-01.8$ & 10 & 3 & $10-10$ \\
\hline & Phenol & $108-95-2$ & 10 & 3 & $10-10$ \\
\hline & Pyrene & $129-00-0$ & 10 & 3 & $10-10$ \\
\hline & bis(2-Chloroethoxy)methane & $111-91-1$ & 10 & 3 & $10 \cdot 10$ \\
\hline & bis(2-Chloroethyl)ether & $111-44-4$ & 10 & 3 & $10-10$ \\
\hline & bis(2-Ethylhexyl)phthalate & $117-81-7$ & 10 & 3 & $10 \cdot 10$ \\
\hline Volatile Organics UG/L & 1,1,1-Trichloroethane & $71-55-6$ & 5 & 3 & $5-5$ \\
\hline & 1,1,2,2-Tetrechloroethane & $79-34-5$ & 5 & 3 & $5-5$ \\
\hline & 1,1,2-Trichloro-1,2,2-Trifluoroethane & $76-13-1$ & 10 & 3 & $10-10$ \\
\hline & 1,1,2-Trichloroethane & $79-00-5$ & 5 & 3 & $5-5$ \\
\hline & 1,1-Dichloroethane & $75-34-3$ & 5 & 3 & $5-5$ \\
\hline & 1,1-Dichloroethene & $75-35-4$ & 5 & 3 & $5-5$ \\
\hline & 1,2-Dichloroethane & 107-06-2 & 5 & 3 & $5-5$ \\
\hline & 1,2-Dichloroethene & $540-59-0$ & 5 & 3 & $5-5$ \\
\hline
\end{tabular}




\begin{tabular}{|c|c|c|c|c|c|}
\hline Analysis & Parameter & Cas Number & $\begin{array}{l}\text { Mean Detection } \\
\text { Limit }\end{array}$ & $\begin{array}{l}\text { Number of } \\
\text { Nondetects }\end{array}$ & $\begin{array}{c}\text { Detection } \\
\text { Limit Range }\end{array}$ \\
\hline & 1,2-Dichloropropane & $78-87-5$ & 5 & 3 & $5-5$ \\
\hline & 2-Hexanone & $591-78-6$ & 10 & 3 & $10-10$ \\
\hline & 4-Methyl-2-pentanone & $108-10-1$ & 10 & 3 & $10-10$ \\
\hline & Acetone & $67-64-1$ & 10 & 3 & $10-10$ \\
\hline & Benzene & $71-43-2$ & 5 & 3 & $5-5$ \\
\hline & Bromodichloromethane & $75-27-4$ & 5 & 3 & $5-5$ \\
\hline & Bromoform & $75-25-2$ & 5 & 3 & $5-5$ \\
\hline & Bromomethane & $74-83-9$ & 10 & 3 & $10 \cdot 10$ \\
\hline & Carbon Disulfide & $75-15-0$ & 5 & 3 & 5.5 \\
\hline & Carbon Telrachloride & $56-23-5$ & 5 & 3 & 5.5 \\
\hline & Chlorobenzene & $108-90-7$ & 5 & 3 & $5 \cdot 5$ \\
\hline & Chloroethane & $75-00-3$ & 10 & 3 & $10 \cdot 10$ \\
\hline & Chloroform & $67-66-3$ & 5 & 3 & $5-5$ \\
\hline & Chloromethane & $74-87-3$ & 10 & 3 & $10-10$ \\
\hline & Dibromochloromethane & $124-48-1$ & 5 & 3 & $5-5$ \\
\hline & Ethylbenzene & $100-41-4$ & 5 & 3 & $5-5$ \\
\hline & Styrene & $100-42-5$ & 5 & 3 & $5-5$ \\
\hline & Toluene & $108-88-3$ & 5 & 3 & 5.5 \\
\hline & Trichloroethene & $79-01-6$ & 5 & 3 & $5-5$ \\
\hline & Vinyl Chloride & $75-01-4$ & 10 & 3 & $10-10$ \\
\hline & Xylene, Total & $1330-20-7$ & 5 & 3 & $5-5$ \\
\hline & cig-1,3-Dichloropropene & $10061-01-5$ & 5 & 3 & $5-5$ \\
\hline & trans-1,3-Dichloropropene & $10061-02-6$ & 5 & 3 & $5-5$ \\
\hline Pesticide/PCB UG/L & $4,4^{\circ}-\mathrm{DDD}$ & $72-54-8$ & 0.0997 & 3 & $0.099-0.1$ \\
\hline
\end{tabular}




\begin{tabular}{|c|c|c|c|c|c|}
\hline Analysis & Parameter & Cas Number & $\begin{array}{l}\text { Mean Detection } \\
\text { Limil }\end{array}$ & $\begin{array}{l}\text { Number of } \\
\text { Nondetects }\end{array}$ & $\begin{array}{c}\text { Detection } \\
\text { Limit Range }\end{array}$ \\
\hline & $4,4^{\prime} \cdot \mathrm{DDE}$ & $72-55-9$ & 0.0997 & 3 & $0.099-0.1$ \\
\hline & 4,4'-DDT & $50-29-3$ & 0.0997 & 3 & $0.099-0.1$ \\
\hline & Aldrin & $309-00-2$ & 0.0503 & 3 & $0.05-0.051$ \\
\hline & Aroclor-1016 & $12674-11-2$ & 0.997 & 3 & 0.99 .1 \\
\hline & Aroclor-1221 & $11104-28-2$ & 2 & 3 & $2-2$ \\
\hline & Aroclor- 1232 & $11141-16-5$ & $0: 997$ & 3 & $0.99-1$ \\
\hline & Aroclor-1242 & $53469-21-9$ & 0.997 & 3 & $0.99-1$ \\
\hline & Aroclor-1248 & $12672-29-6$ & 0.997 & 3 & $0.99-1$ \\
\hline & Aroclor-1254 & $11097-69-1$ & 0.997 & 3 & $0.99-1$ \\
\hline & Aroclor-1260 & $11096-82-5$ & 0.997 & 3 & $0.99-1$ \\
\hline & Dieldrin & $60-57-1$ & 0.0997 & 3 & $0.099-0.1$ \\
\hline & Endosulfan I & $959-98-8$ & 0.0503 & 3 & $0.05-0.051$ \\
\hline & Endosulfan II & $33213-65-9$ & 0.0997 & 3 & $0.099-0.1$ \\
\hline & Endosulfan Sulfate & $1031-07-8$ & 0.0997 & 3 & $0.099-0.1$ \\
\hline & Endrin & $72-20-8$ & 0.0997 & 3 & $0.099-0.1$ \\
\hline & Endrin Aldehyde & $7421-93-4$ & 0.0997 & 3 & $0.099-0.1$ \\
\hline & Endrin Ketone & $53494-70-5$ & 0.0997 & 3 & $0.099-0.1$ \\
\hline & Heptachlor & $76-44-8$ & 0.0503 & 3 & $0.05-0.051$ \\
\hline & Heptachlor Epoxide & $1024-57-3$ & 0.0503 & 3 & $0.05-0.051$ \\
\hline & Toxaphene & $8001-35-2$ & 5.03 & 3 & $5-5.1$ \\
\hline & alpha-BHC & $319-84-6$ & 0.0503 & 3 & $0.05-0.051$ \\
\hline & alpha-Chlordane & $5103-71-9$ & 0.0503 & 3 & $0.05-0.051$ \\
\hline & beta-BHC & 319-85-7 & 0.0503 & 3 & $0.05-0.051$ \\
\hline & delta-BHC & $319-86-8$ & 0.0503 & 3 & $0.05-0.051$ \\
\hline
\end{tabular}




\begin{tabular}{|c|c|c|c|c|c|}
\hline Analysis & Parameter & Cas Number & $\begin{array}{l}\text { Mean Detection } \\
\text { Limil } \\
\end{array}$ & $\begin{array}{l}\text { Number of } \\
\text { Nondeteets }\end{array}$ & $\begin{array}{c}\text { Detection } \\
\text { Limit Range }\end{array}$ \\
\hline & gamma-BHC (Lindane) & $58-89-9$ & 0.0503 & 3 & $0.05-0.051$ \\
\hline & gamma-Chlordane & $5103-74-2$ & 0.0503 & 3 & $0.05-0.051$ \\
\hline & p,p'-Methoxychlor & $72-43-5$ & 0.503 & 3 & $0.5-0.51$ \\
\hline
\end{tabular}

Data Source : Bear Creek Valley oU2 1993 RI field activities validated data Raw dat available through Y-12 OREIS

Dato does not include QA/OC

Results < counting uncertainty were considered as nondetects for radionuclides 
TABLE 2. BC-OU2 1993 RI Field Activities Data - Summary Of Parameters Never Detected For The Spoil Area 1 Sampling Media: Surface water

\begin{tabular}{|c|c|c|c|c|c|}
\hline Analysis & Parameter & Cas Number & Mean Detection Limit & $\begin{array}{l}\text { Number of } \\
\text { Nondetects }\end{array}$ & $\begin{array}{l}\text { Detection } \\
\text { Limit Range }\end{array}$ \\
\hline \multirow[t]{5}{*}{ Metals UG/L (AAS) } & Arsenic (Dissolved) & $7440-38-2$ & 2.2 & 3 & $2.2-2.2$ \\
\hline & Cyanide & $57-12-5$ & 10 & 3 & $10-10$ \\
\hline & Selenium (Dissolved) & $7782-49-2$ & 2.7 & 3 & $2.7-2.7$ \\
\hline & Thallium & $7440-28-0$ & 1.3 & 3 & $1.3-1.3$ \\
\hline & Thallium (Dissolved) & $7440-28-0$ & 1.8 & 3 & 1.81 .8 \\
\hline Metals UG/L & Mercury & $7439-97-6$ & 0.2 & 3 & $0.2-0.2$ \\
\hline \multirow[t]{13}{*}{ Metsis UG/L (ICP) } & Aluminum (Dissolved) & $7429-90-5$ & 42 & 3 & $29.7-62.4$ \\
\hline & Beryllium & $7440-41-7$ & 0.3 & 1 & $0.3-0.3$ \\
\hline & Beryllium (Dissolved) & $7440-41-7$ & 0.1 & 3 & $0.1-0.1$ \\
\hline & Cadmium & $7440-43-9$ & 1.23 & 3 & $1-1.7$ \\
\hline & Cadmium (Dissolved) & $7440-43-9$ & 1.03 & 3 & $1-1.1$ \\
\hline & Chromium (Dissolved) & $7440-47-3$ & 5.6 & 3 & $5.6-5.6$ \\
\hline & Cobalt & $7440-48-4$ & 2.3 & 3 & $2-2.9$ \\
\hline & Manganese (Dissolved) & $7439-96-5$ & 3.27 & 3 & $2.1-4.8$ \\
\hline & Nickel & $7440-02-0$ & 4.6 & 3 & 4.4-4.7 \\
\hline & Nickel (Dissolved) & $7440-02-0$ & 4.7 & 3 & $4.7-4.7$ \\
\hline & Silver & $7440-22-4$ & 3.43 & 3 & $2.1-4.1$ \\
\hline & Silver (Dissolved) & $7440-22-4$ & 4.1 & 3 & $4.1-4.1$ \\
\hline & Vanadium (Dissolved) & $7440-62-2$ & 2.5 & 3 & $2.5-2.5$ \\
\hline \multirow[t]{4}{*}{ Semivolatile Organics UG/L } & 1,2,4-Trichlorobenzene & $120-82-1$ & 9.67 & 3 & 9.10 \\
\hline & 1,2-Dichlorobenzene & $95-50-1$ & 9.67 & 3 & $9-10$ \\
\hline & 1,3-Dichlorobenzene & $541-73-1$ & 9.67 & 3 & $9-10$ \\
\hline & 1,4-Dichlorobenzene & $106-46-7$ & 9.67 & 3 & $9 \cdot 10$ \\
\hline
\end{tabular}




\begin{tabular}{|c|c|c|c|c|c|}
\hline Analysis & Parameter & Cas Number & Mean Detection Limit & $\begin{array}{l}\text { Number of } \\
\text { Nondelects }\end{array}$ & $\begin{array}{l}\text { Detection } \\
\text { Limit Range }\end{array}$ \\
\hline & $2,2^{\circ}$-axybis(1-Chloropropane) & $108-60-1$ & 9.67 & 3 & $9-10$ \\
\hline & 2,4,5-Trichlorophenol & $95-95-4$ & 24.3 & 3 & $23-25$ \\
\hline & 2,4,6-Trichlorophenol & $88-06-2$ & 9.67 & 3 & $9-10$ \\
\hline & 2,4-Dichlorophenol & $120-83-2$ & 9.67 & 3 & $9-10$ \\
\hline & 2,4-Dimethylphenol & $105-67-9$ & 9.67 & 3 & $9-10$ \\
\hline & 2,4-Dinitrophenol & $51-28-5$ & 24.3 & 3 & $23-25$ \\
\hline & 2,4-Dinitrotoluene & $121-14-2$ & 9.67 & 3 & $9-10$ \\
\hline & 2,6-Dinitrotoluene & $606-20-2$ & 9.67 & 3 & $9-10$ \\
\hline & 2-Chloronaphthalene & $91-58-7$ & 9.67 & 3 & $9-10$ \\
\hline & 2-Chlorophenol & $95-57-8$ & 9.67 & 3 & $9-10$ \\
\hline & 2-Methylnaphthaiene & $91-57-6$ & 9.67 & 3 & $9-10$ \\
\hline & 2-Methylphenol & $95-48-7$ & 9.67 & 3 & $9-10$ \\
\hline & 2-Nitroaniline & $88-74-4$ & 24.3 & 3 & $23-25$ \\
\hline & 2-Nitrophenol & $88-75-5$ & 9.67 & 3 & $9-10$ \\
\hline & 3,3'-Dichlorobenzidine & $91-94-1$ & 9.67 & 3 & $9-10$ \\
\hline & 3-Nitroaniline & $99-09-2$ & 24.3 & 3 & $23-25$ \\
\hline & 4,6-Dinitro-2-methylphenol & $534-52-1$ & 24.3 & 3 & $23-25$ \\
\hline & 4-Bromophenyl-phenylether & $101-55-3$ & 9.67 & 3 & $9-10$ \\
\hline & 4-Chloro-3-methyiphenol & $59-50.7$ & 9.67 & 3 & $9-10$ \\
\hline & 4-Chloroaniline & $106-47-8$ & 9.67 & 3 & $9-10$ \\
\hline & 4-Chlorophenyl-phenylether & $7005-72-3$ & 9.67 & 3 & 9.10 \\
\hline & 4-Methylphenol & $106-44-5$ & 9.67 & 3 & $9-10$ \\
\hline & 4-Nitroaniline & $100-01-6$ & 24.3 & 3 & $23-25$ \\
\hline & 4-Nitrophenol & $100-02-7$ & 24.3 & 3 & $23-25$ \\
\hline
\end{tabular}




\begin{tabular}{|c|c|c|c|c|c|}
\hline Analygis & Parameter & Cas Number & Mean Detection Limit & $\begin{array}{l}\text { Number of } \\
\text { Nondetects }\end{array}$ & $\begin{array}{c}\text { Detection } \\
\text { Limit Range } \\
\end{array}$ \\
\hline & Acenaphthene & $83-32-9$ & 9.5 & 2 & $9-10$ \\
\hline & Acenaphthylene & $208-96-8$ & 9.67 & 3 & $9 \cdot 10$ \\
\hline & Anthracene & $120-12-7$ & 9.67 & 3 & $9-10$ \\
\hline & Benzo(@)anthracene & $56-55-3$ & 9.67 & 3 & $9-10$ \\
\hline & Benzo(a)pyrene & $50-32-8$ & 9.67 & 3 & $9-10$ \\
\hline & Benzo(b) fluoranthene & $205-99-2$ & 9.67 & 3 & $9-10$ \\
\hline & Benzo(g,h,i)perylene & $191-24-2$ & 9.67 & 3 & $9-10$ \\
\hline & Benzo(k) fluoranthene & $207-08-9$ & 9.67 & 3 & $9-10$ \\
\hline & Carbazole & $86-74-8$ & 9.67 & 3 & $9-10$ \\
\hline & Chrysene & $218-01-9$ & 9.67 & 3 & $9-10$ \\
\hline & Di-n-octylphthalate & 117.840 & 9.67 & 3 & $9-10$ \\
\hline & Dibenzo(a,h)anthracene & $53-70-3$ & 9.67 & 3 & $9-10$ \\
\hline & Dibenzofuran & $132-64-9$ & 9.67 & 3 & 9.10 \\
\hline & Diethylphthalate & $84-66-2$ & 9.67 & 3 & $9-10$ \\
\hline & Dimethylphthalate & $131-11-3$ & 9.67 & 3 & 9.10 \\
\hline & Fluoranthene & 206-44-0 & 9.67 & 3 & 9.10 \\
\hline & Fluorene & $86-73-7$ & 9.67 & 3 & $9-10$ \\
\hline & Hexachlorobenzene & $118-74-1$ & 9.67 & 3 & $9-10$ \\
\hline & Hexachlorobutadiene & $87-68-3$ & 9.67 & 3 & $9-10$ \\
\hline & Hexachlorocyclopentadiene & $77-47-4$ & 9.67 & 3 & $9-10$ \\
\hline & Hexachloroethane & $67-72-1$ & 9.67 & 3 & $9-10$ \\
\hline & Indeno(1,2,3-c,d)pyrene & $193-39-5$ & 9.67 & 3 & $9-10$ \\
\hline & Isophorone & $78-59-1$ & 9.67 & 3 & $9-10$ \\
\hline & N-Nitroso-di-n-propylamine & $621-64-7$ & 9.67 & 3 & $9-10$ \\
\hline
\end{tabular}




\begin{tabular}{|c|c|c|c|c|c|}
\hline Analysis & Parameter & Cas Number & Mean Detection Limit & $\begin{array}{l}\text { Number of } \\
\text { Nondetects }\end{array}$ & $\begin{array}{l}\text { Detection } \\
\text { Limit Range }\end{array}$ \\
\hline & N-Nitrosodiphenylamine & $86-30-6$ & 9.67 & 3 & $9-10$ \\
\hline & Naphthalene & $91-20-3$ & 9.67 & 3 & $9-10$ \\
\hline & Nitrobenzene & 98-95-3 & 9.67 & 3 & $9-10$ \\
\hline & Pentachlorophenol & $87-86-5$ & 24.3 & 3 & 23-25 \\
\hline & Phenanthrene & $85-01-8$ & 9.67 & 3 & $9-10$ \\
\hline & Phenol & $108-95-2$ & 9.67 & 3 & $9-10$ \\
\hline & Pyrene & $129-00-0$ & 9.5 & 2 & $9-10$ \\
\hline & bir(2-Chloroethoxy)methane & $111-91-1$ & 9.67 & 3 & $9-10$ \\
\hline & bis(2-Chloroethyl)ether & $111-44-4$ & 9.67 & 3 & $9-10$ \\
\hline & bis(2-Ethylhexyl)phthalate & $117-81-7$ & 9.67 & 3 & $9-10$ \\
\hline \multirow[t]{14}{*}{ Volatile Organics Ua/L } & 1,1,1-Trichloroethane & $71-55-6$ & 5 & 3 & $5-5$ \\
\hline & 1,1,2,2-Tetrachloroethane & $79-34-5$ & 5 & 3 & 5.5 \\
\hline & 1,1,2-Trichloro-1,2,2-Trifluoroethane & $76-13-1$ & 10 & 3 & $10-10$ \\
\hline & 1,1,2-Trichloroethane & $79-00-5$ & 5 & 3 & $5-5$ \\
\hline & 1,1-Dichloroethane & $75-34-3$ & 5 & 3 & $5-5$ \\
\hline & 1,1-Dichloroethene & $75-35-4$ & 5 & 3 & $5-5$ \\
\hline & 1,2-Dichlorvethane & $107-06-2$ & 5 & 3 & $5-5$ \\
\hline & 1,2-Dichloroethene & $540-59-0$ & 5 & 3 & $5-5$ \\
\hline & 1,2-Dichloropropane & $78-87-5$ & 5 & 3 & $5-5$ \\
\hline & 2-Hexanone & $591-78-6$ & 10 & 3 & $10-10$ \\
\hline & 4-Methyl-2-pentanone & $108-10-1$ & 10 & 3 & $10-10$ \\
\hline & Acetone & $67-64-1$ & 8.33 & 3 & $5-10$ \\
\hline & Benzene & $71-43-2$ & 5 & 3 & $5-5$ \\
\hline & Bromodichloromethane & $75-27-4$ & 5 & 3 & $5-5$ \\
\hline
\end{tabular}




\begin{tabular}{|c|c|c|c|c|c|}
\hline Analysis & Perameter & Cas Number & Mean Detection Limit & $\begin{array}{l}\text { Number of } \\
\text { Nondetects }\end{array}$ & $\begin{array}{l}\text { Detection } \\
\text { Limit Range }\end{array}$ \\
\hline & Bromoform & $75-25-2$ & 5 & 3 & $5-5$ \\
\hline & Bromomethane & $74-83-9$ & 10 & 3 & $10-10$ \\
\hline & Carbon Disulfide & $75-15-0$ & 5 & 3 & $5-5$ \\
\hline & Carbon Tetrachloride & $56-23-5$ & 5 & 3 & $5-5$ \\
\hline & Chlorobenzene & $108-90-7$ & 5 & 3 & $5-5$ \\
\hline & Chloroethane & $75-00-3$ & 10 & 3 & $10-10$ \\
\hline & Chloroform & $67-66-3$ & 5 & 3 & $5-5$ \\
\hline & Chloromethane & $74-87-3$ & 10 & 3 & $10-10$ \\
\hline & Dibromochloromethane & $124-48-1$ & 5 & 3 & $5-5$ \\
\hline & Ethylbenzene & $100-41-4$ & 5 & 3 & 5.5 \\
\hline & Methylene Chloride & $75-09-2$ & 8.33 & 3 & $5-10$ \\
\hline & Styrene & $100-42-5$ & 5 & 3 & $5-5$ \\
\hline • & Tetrachloroethene & $127-18-4$ & 5 & 3 & $5-5$ \\
\hline & Toluene & $108-88-3$ & 5 & 3 & $5-5$ \\
\hline & Trichloroethene & $79-01-6$ & 5 & 3 & $5-5$ \\
\hline & Vinyl Chloride & $75-01-4$ & 10 & 3 & $10-10$ \\
\hline & Xylene, Total & $1330-20-7$ & 5 & 3 & $5-5$ \\
\hline & cis-1,3-Dichloropropene & $10061-01-5$ & 5 & 3 & $5-5$ \\
\hline & trans-1,3-Dichloropropene & $10061-02-6$ & 5 & 3 & $5-5$ \\
\hline Pesticide/PCB UG/L & $4,4^{\circ}-\mathrm{DDD}$ & $72-54-8$ & 0.1 & 3 & $0.1-0.1$ \\
\hline & 4,4'-DDE & $72-55-9$ & 0.1 & 3 & $0.1-0.1$ \\
\hline & $4,4^{\prime}-\mathrm{DDT}$ & $50-29-3$ & 0.1 & 3 & $0.1-0.1$ \\
\hline & Aldrin & $309-00-2$ & 0.0507 & 3 & $0.05-0.051$ \\
\hline & Aroclor-1016 & $12674-11-2$ & 1 & 3 & $1-1$ \\
\hline
\end{tabular}




\begin{tabular}{|c|c|c|c|c|c|}
\hline Analysis & Parameter & Cas Number & Mean Detection Limit & $\begin{array}{l}\text { Number of } \\
\text { Nondetects }\end{array}$ & $\begin{array}{l}\text { Detection } \\
\text { Limil Range }\end{array}$ \\
\hline & Aroclor-1221 & $11104-28-2$ & 2 & 3 & $2-2$ \\
\hline & Aroclor-1232 & $11141-16-5$ & 1 & 3 & $1-1$ \\
\hline & Aroclor -1242 & $53469-21-9$ & 1 & 3 & $1-1$ \\
\hline & Aroclor-1248 & $12672-29-6$ & 1 & 3 & $1-1$ \\
\hline & Aroclor-1254 & $11097-69-1$ & 1 & 3 & $1-1$ \\
\hline & Aroclor-1260 & $11096-82-5$ & 1 & 3 & $1-1$ \\
\hline & Dieldrin & $60-57-1$ & 0.1 & 3 & $0.1-0.1$ \\
\hline & Endosulfan I & 959-98-8 & 0.0507 & 3 & $0.05-0.051$ \\
\hline & Ẽndosulfan II & $33213-65-9$ & 0.1 & 3 & $0.1-0.1$ \\
\hline & Endosulfan Sulfate & $1031-07-8$ & 0.1 & 3 & $0.1-0.1$ \\
\hline & Endrin & $72-20-8$ & 0.1 & 3 & $0.1-0.1$ \\
\hline & Endrin Aldehyde & $7421-93-4$ & 0.1 & 3 & $0.1-0.1$ \\
\hline & Endrin Ketone & $53494-70-5$ & 0.1 & 3 & $0.1-0.1$ \\
\hline & Heptachlor & $76-44-8$ & 0.0507 & 3 & $0.05-0.051$ \\
\hline & Heptachlor Epoxide & $1024-57-3$ & 0.0507 & 3 & $0.05-0.051$ \\
\hline & Toxaphene & $8001-35-2$ & 5.07 & 3 & $5-5.1$ \\
\hline & alpha-BHC & $319-84-6$ & 0.0507 & 3 & $0.05-0.051$ \\
\hline & alpha-Chlordane & $5103-71-9$ & 0.0507 & 3 & $0.05-0.051$ \\
\hline & beta-BHC & $319-85.7$ & 0.0507 & 3 & $0.05-0.051$ \\
\hline & delta-BHC & $319-86-8$ & 0.0507 & 3 & $0.05-0.051$ \\
\hline & gamma-BHC (Lindane) & $58-89-9$ & 0.0507 & 3 & $0.05-0.051$ \\
\hline & gamma-Chlordane & $5103-74-2$ & 0.0507 & 3 & $0.05-0.051$ \\
\hline & p,p'-Methosychlor & $72-43-5$ & 0.507 & 3 & $0.5-0.51$ \\
\hline
\end{tabular}


Raw data mailable through Y-12 OREIS

Data does nol include QA/QC

Results $<$ counting uncertainty were considered as nondetects for radionuclides 
TABLE 2. BC-OU2 1993 RI Fleld Activities Data - Summary Of Parameters Never Detected For The SY-204 Yard Sampling Media: Surface Water

\begin{tabular}{|c|c|c|c|c|c|}
\hline Analysis & Parameter & Cas Number & Mean Detection Limit & $\begin{array}{l}\text { Number of } \\
\text { Nondetects }\end{array}$ & $\begin{array}{c}\text { Detection } \\
\text { Limit Range }\end{array}$ \\
\hline \multirow[t]{7}{*}{ Metals UG/L (AAS) } & Arsenic & $7440-38-2$ & 1.9 & 2 & $1.6-2.2$ \\
\hline & Arsenic (Dissolved) & $7440-38-2$ & 2.2 & 2 & $2.2-2.2$ \\
\hline & Cyanide & $57-12-5$ & 10 & 2 & $10-10$ \\
\hline & Selenitum & $7782-49-2$ & 4.1 & 1 & $4.1-4.1$ \\
\hline & Selenium (Disgolved) & $7782-49-2$ & 2.7 & 2 & $2.7-2.7$ \\
\hline & Thallium & $7440-28-0$ & 1.3 & 2 & $1.3-1.3$ \\
\hline & Thallium (Dissolved) & $7440-28-0$ & 1.8 & 2 & $1.8-1.8$ \\
\hline \multirow[t]{2}{*}{ Metals UG/L } & Mercury & $7439-97-6$ & 0.2 & 2 & $0.2-0.2$ \\
\hline & Mercury (Dissolved) & $7439-97-6$ & 0.2 & 2 & $0.2-0.2$ \\
\hline \multirow[t]{14}{*}{ Metals UG/L (ICP) } & Aluminum (Dissolved) & $7429-90-5$ & 23.2 & 2 & $16-30.3$ \\
\hline & Beryllium & $7440-41-7$ & 0.3 & 1 & $0.3-0.3$ \\
\hline & Beryllium (Dissolved) & $7440-41-7$ & 0.1 & 2 & $0.1-0.1$ \\
\hline & Cadmiun & $7440-43-9$ & 1.35 & 2 & $1-1.7$ \\
\hline & Cadmium (Dissolved) & $7440-43-9$ & $\mathbb{1}$ & 2 & $1-1$ \\
\hline & Chromium & $7440-47-3$ & 4.05 & 2 & $2.5-5.6$ \\
\hline & Chromium (Dissolved) & $7440-47-3$ & 5.6 & 2 & $5.6-5.6$ \\
\hline & Cobalt & $7440-48-4$ & 2.45 & 2 & 2.2 .9 \\
\hline & Cobalt (Dissolved) & $7440-48-4$ & 2 & 2 & $2-2$ \\
\hline & Iron (Dissolved) & $7439.89-6$ & 28.9 & 2 & $25.1-32.6$ \\
\hline & Manganese (Dissolved) & $7439-96-5$ & 3.45 & 2 & $1.9-5$ \\
\hline & Nickel & $7440-02-0$ & 4.55 & 2 & 4.4 .4 .7 \\
\hline & Nickel (Dissolved) & $7440-02-0$ & 4.7 & 2 & $4.7 \times 4.7$ \\
\hline & Silver & $7440-22-4$ & 3.1 & 2 & $2.1-4.1$ \\
\hline
\end{tabular}




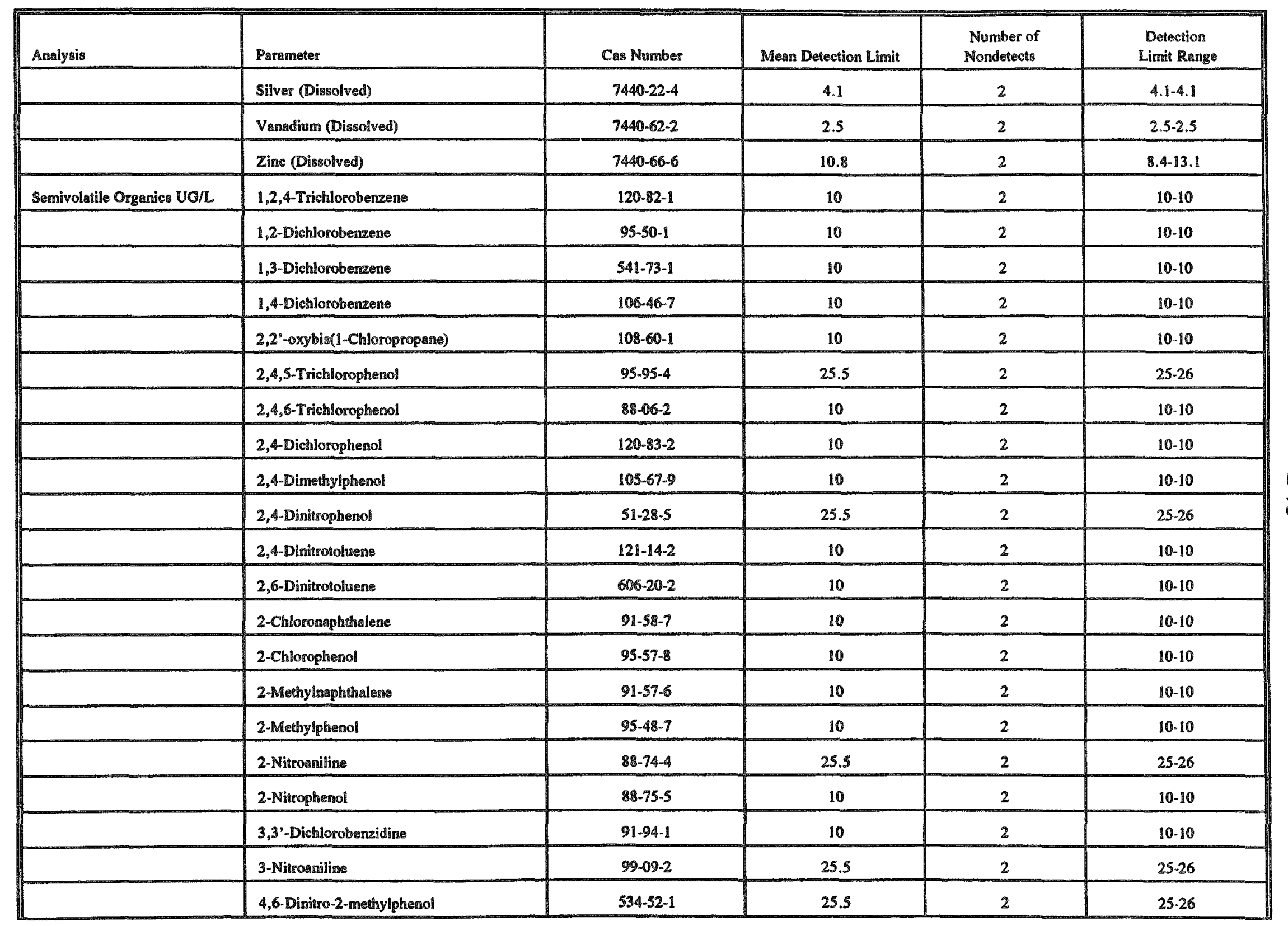




\begin{tabular}{|c|c|c|c|c|c|}
\hline Analysis & Parameter & Cas Number & Mean Detection Limit & $\begin{array}{l}\text { Number of } \\
\text { Nondetects }\end{array}$ & $\begin{array}{c}\text { Detection } \\
\text { Limit Range }\end{array}$ \\
\hline & 4-Bromophenyl-phenylether & $101-55-3$ & 10 & 2 & $10-10$ \\
\hline & 4-Chloro-3-methylphenol & $59-50-7$ & 10 & 2 & $10-10$ \\
\hline & 4-Chloroaniline & $106-47-8$ & 10 & 2 & $10-10$ \\
\hline & 4-Chlorophenyl-phenylether & $7005-72-3$ & 10 & 2 & $10 \cdot 10$ \\
\hline & 4-Methylphenol & $106-44-5$ & 10 & 2 & $10-10$ \\
\hline & 4-Nitroaniline & $100-01-6$ & 25.5 & 2 & $25-26$ \\
\hline & 4-Nitrophenol & $100-02-7$ & 25.5 & 2 & $25-26$ \\
\hline & Acenaphthene & $83-32-9$ & 10 & 2 & $10-10$ \\
\hline & Acenaphthylene & $208-96-8$ & 10 & 2 & $10-10$ \\
\hline & Anthracene & $120-12-7$ & 10 & 2 & $10-10$ \\
\hline & Benzo(a)anthracene & $56-55-3$ & 10 & 2 & $10-10$ \\
\hline & Benzo(a)pyrene & $50-32-8$ & 10 & 1 & $10-10$ \\
\hline & Benzo(b)fluoranthene & $205-99-2$ & 10 & 1 & $10-10$ \\
\hline & Benzo(g,h,i)perylene & $191-24-2$ & 10 & 1 & $10-10$ \\
\hline & Benzo(k)fluoranthene & $207-08-9$ & 10 & 1 & $10-10$ \\
\hline & Carbazole & $66-74-8$ & 10 & 2 & $10-10$ \\
\hline & Chrygene & $218-01-9$ & 10 & 2 & $10-10$ \\
\hline & Di-m-octylphthalate & $117-84-0$ & 10 & 1 & $10-10$ \\
\hline & Dibenzo(a,h)anthracene & $53-70-3$ & 10 & 1 & $10-10$ \\
\hline & Dibenzofuran & $132-64-9$ & 10 & 2 & $10-10$ \\
\hline & Diethylphthalate & $84-66-2$ & 10 & 2 & $10-10$ \\
\hline & Dimethylphthalate & $131-11-3$ & 10 & 2 & $10-10$ \\
\hline & Fluoranthene & $206-44-0$ & 10 & 2 & $10 \cdot 10$ \\
\hline & Fluorene & $86-73-7$ & 10 & 2 & $10-10$ \\
\hline
\end{tabular}




\begin{tabular}{|c|c|c|c|c|c|}
\hline Analysis & Parameter & Cas Number & Mean Detection Limit & $\begin{array}{l}\text { Number of } \\
\text { Nondetects }\end{array}$ & $\begin{array}{c}\text { Detection } \\
\text { Limit Range }\end{array}$ \\
\hline & Hexachlorobenzene & $118-74-1$ & 10 & 2 & $10-10$ \\
\hline & Hexachlorobutadiene & $87-68-3$ & 10 & 2 & $10-10$ \\
\hline & Hexachlorocyclopentadiene & $77-47-4$ & 10 & 2 & $10-10$ \\
\hline & Hexachloroethane & $67-72-1$ & 10 & 2 & $10 \cdot 10$ \\
\hline & Indeno(1,2,3-c,d)pyrene & $193-39-5$ & 10 & 1 & $10-10$ \\
\hline & Isophorone & $78-59-1$ & 10 & 2 & $10 \cdot 10$ \\
\hline & N-Nitroso-di-n-propylamine & $621-64-7$ & 10 & 2 & $10-10$ \\
\hline & N-Nitrosodiphenylamine & $86-30-6$ & 10 & 2 & $10-10$ \\
\hline & Naphthalene & $91-20-3$ & 10 & 2 & $10-10$ \\
\hline & Nitrobenzene & 98-95-3 & 10 & 2 & $10-10$ \\
\hline & Pentachlorophenol & $87-86-5$ & 25.5 & 2 & $25-26$ \\
\hline & Phenanthrene & $85-01-8$ & 10 & 2 & $10-10$ \\
\hline & Phenol & $108-95-2$ & 10 & 2 & $10-10$ \\
\hline & Pyrene & 129-00-0 & 10 & 2 & $10-10$ \\
\hline & bis(2-Chloroethoxy)methane & $111-91-1$ & 10 & 2 & $10-10$ \\
\hline & bis(2-Chloroethyl)ether & $111-44-4$ & 10 & 2 & $10-10$ \\
\hline & bis(2-Ethylhexyl)phthalate & $117-81-7$ & 10 & 2 & $10-10$ \\
\hline Volatile Organics UG/L & 1,1,1-Trichloroethane & $71-55-6$ & 5 & 2 & $5-5$ \\
\hline & 1,1,2,2-Tetrachloroethane & 79-34-5 & 5 & 2 & $5-5$ \\
\hline & 1,1,2-Trichloro-1,2,2-Trifluoroethane & $76-13-1$ & 10 & 2 & $10-10$ \\
\hline & 1,1,2-Trichloroethane & $79-00-5$ & 5 & 2 & $5-5$ \\
\hline & 1,1-Dichloroethane & $75-34-3$ & 5 & 2 & $5-5$ \\
\hline & 1,1-Dichloroethene & $75-35-4$ & 5 & 2 & $5-5$ \\
\hline & 1,2-Dichloroethane & $107-06-2$ & 5 & 2 & $5-5$ \\
\hline
\end{tabular}




\begin{tabular}{|c|c|c|c|c|c|}
\hline Analysis & Parameter & Cas Number & Mean Detection Limit & $\begin{array}{l}\text { Number of } \\
\text { Nondetects }\end{array}$ & $\begin{array}{l}\text { Detection } \\
\text { Limil Range }\end{array}$ \\
\hline & 1,2-Dichloroethene & $540-59-0$ & 5 & 2 & $5-5$ \\
\hline & 1,2-Dichloropropane & 78.87 .5 & 5 & 2 & $5-5$ \\
\hline & 2-Butanone & $78-93-3$ & 10 & 2 & $10-10$ \\
\hline & 2-Hexanone & $591-78-6$ & 10 & 2 & $10-10$ \\
\hline & 4-Methyl-2-pentanone & $108-10-1$ & 10 & 2 & $10-10$ \\
\hline & Benzene & $71-43-2$ & 5 & 2 & $5-5$ \\
\hline & Bromodichloromethane & $75-27-4$ & 5 & 2 & $5-5$ \\
\hline & Bromoform & $75-25-2$ & 5 & 2 & $5-5$ \\
\hline & Bromomethme & $74-83-9$ & 10 & 2 & $10-10$ \\
\hline & Carbon Disulfide & $75-15-0$ & 5 & 2 & $5-5$ \\
\hline & Carbon Tetrachloride & $56-23-5$ & 5 & 2 & $5-5$ \\
\hline & Chlorobenzene & $108-90-7$ & 5 & 2 & $5-5$ \\
\hline & Chloroethane & $75-00-3$ & 10 & 2 & $10-10$ \\
\hline & Chloroform & $67-66-3$ & 5 & 2 & 5.5 \\
\hline & Chloromethane & $74-87-3$ & 10 & 2 & $10-10$ \\
\hline & Dibromochloromethane & $124-48-1$ & 5 & 2 & $5-5$ \\
\hline & Ethylbenzene & $100-41-4$ & 5 & 2 & $5-5$ \\
\hline & Melhylene Chloride & $75-09-2$ & 7.5 & 2 & $5-10$ \\
\hline & Styrene & $100-42-5$ & 5 & 2 & $5-5$ \\
\hline & Tetrachloroethene & $127-18-4$ & 5 & 2 & $5-5$ \\
\hline & Toluene & $108-88-3$ & 5 & 2 & 5.5 \\
\hline & Trichloroethene & $79-01-6$ & 5 & 2 & $5-5$ \\
\hline & Vinyl Chloride & $75-01-4$ & 10 & 2 & $10-10$ \\
\hline & Xylene, Total & $1330-20-7$ & 5 & 2 & $5-5$ \\
\hline
\end{tabular}




\begin{tabular}{|c|c|c|c|c|c|}
\hline Analysis & Parameter & Cas Number & Mean Detection Limit & $\begin{array}{l}\text { Number of } \\
\text { Nondetects }\end{array}$ & $\begin{array}{l}\text { Detection } \\
\text { Limit Range }\end{array}$ \\
\hline & cis-1,3-Dichloropropene & $10061-01-5$ & 5 & 2 & $5-5$ \\
\hline & trans-1,3-Dichloropropene & $10061-02-6$ & 5 & 2 & $5-5$ \\
\hline \multirow[t]{22}{*}{ Pesticide/PCB UG/L } & 4, $4^{\prime}-\mathrm{DDD}$ & $72-54-8$ & 0.103 & 2 & $0.096-0.11$ \\
\hline & $4,4^{\prime}-\mathrm{DDE}$ & $72-55-9$ & 0.103 & 2 & $0.096-0.1 \mathrm{i}$ \\
\hline & $4,4^{\prime}-\mathrm{DDT}$ & $50-29-3$ & 0.103 & 2 & $0.096-0.11$ \\
\hline & Aldrin & $309-00-2$ & 0.0505 & 2 & $0.048-0.053$ \\
\hline & Aroclor-1016 & $12674-11-2$ & 1.03 & 2 & $0.96 \cdot 1.1$ \\
\hline & Aroclor-1221 & $11104-28-2$ & 2 & 2 & $1.9-2.1$ \\
\hline & Aroclor-1232 & $11141-16 \cdot 5$ & 1.03 & 2 & $0.96-1.1$ \\
\hline & Aroclor -1242 & $53469-21-9$ & 1.03 & 2 & $0.96-1.1$ \\
\hline & Aroclor-1248 & $12672-29-6$ & 1.03 & 2 & $0.96-1.1$ \\
\hline & Aroclor-1254 & $11097-69-1$ & 1.03 & 2 & $0.96-1.1$ \\
\hline & Aroclor- 1260 & $11096-82-5$ & 1.03 & 2 & $0.96-1.1$ \\
\hline & Dieldrin & $60-57-1$ & 0.103 & 2 & $0.096-0.11$ \\
\hline & Endosulfan I & $959-98-8$ & 0.0505 & 2 & - $\quad 0.048-0.053$ \\
\hline & Endosulfan II & $33213-65-9$ & 0.103 & 2 & $0.096-0.11$ \\
\hline & Endosuifan Sulfate & $1031-07-8$ & 0.103 & 2 & $0.096-0.11$ \\
\hline & Endrin & $72-20-8$ & 0.103 & 2 & $0.096-0.11$ \\
\hline & Endrin Aldehyde & $7421-93-4$ & 0.103 & 2 & $0.096-0.11$ \\
\hline & Endrin Ketone & $53494-70-5$ & 0.103 & 2 & $0.096-0.11$ \\
\hline & Heptachlor & $76-44-8$ & 0.0505 & 2 & $0.048-0.053$ \\
\hline & Heptachlor Epoxide & 1024-57-3 & 0.0505 & 2 & $0.048-0.053$ \\
\hline & Toxaphene & $8001-35-2$ & 5.05 & 2 & $4.8-5.3$ \\
\hline & alpha-BHC & $319-84-6$ & 0.0505 & 2 & $0.048-0.053$ \\
\hline
\end{tabular}




\begin{tabular}{|c|c|c|c|c|c|}
\hline Analysig & Parameter & Côs Number & Mean Detection Limit & $\begin{array}{l}\text { Number of } \\
\text { Nondetects }\end{array}$ & $\begin{array}{l}\text { Detection } \\
\text { Limit Range }\end{array}$ \\
\hline & alpha-Chlordane & $5103-71-9$ & 0.0505 & 2 & $0.048-0.053$ \\
\hline & bets-BHC & $319-85-7$ & 0.0505 & 2 & $0.048-0.053$ \\
\hline & delta-BHC & $319-86-8$ & 0.0505 & 2 & $0.048-0.053$ \\
\hline & gamma-BHC (Lindane) & $58-89-9$ & 0.0505 & 2 & $0.048-0.053$ \\
\hline & gamma-Chlordane & $5103-74-2$ & 0.0505 & 2 & $0.048-0.053$ \\
\hline & $p, p^{9}$-Methoxychlor & $72-43-5$ & 0.505 & 2 & $0.48-0.53$ \\
\hline
\end{tabular}

Data Source : Bear Creek Valley OU2 1993 RI field activities validated data

Raw data available through $Y-12$ OREIS

Data does not include QA/QC

Results $<$ counting uncertainty were considered as nondetects for radionuclides 
TARLE 2. BC-OU2 1993 RI Field Activities Datu - Sumanary Or Parameters Never Detected For The Spoil Aren 1 Sampling Media: Spring/Seep Water

\begin{tabular}{|c|c|c|c|c|c|}
\hline Analysis & Parameter & Cas Number & Mean Detection Limit & $\begin{array}{l}\text { Number of } \\
\text { Nondetects }\end{array}$ & $\begin{array}{c}\text { Detection } \\
\text { Limit Range }\end{array}$ \\
\hline Anions MG/L & Nitrate/Nitrite (NO3/NO2-N) & $14797-55-8$ & 0.2 & 1 & 0.2 .0 .2 \\
\hline \multirow[t]{5}{*}{ Metals UG/L (AAS) } & Arsenic (Dissolved) & $7440-38-2$ & 2.2 & 1 & $2.2-2.2$ \\
\hline & Cyanide & $57-12-5$ & 10 & 1 & $10-10$ \\
\hline & Selenium (Dissolved) & $7782-49-2$ & 2.7 & 1 & $2.7-2.7$ \\
\hline & Thallium & $7440-28-0$ & 1.3 & 1 & $1.3-1.3$ \\
\hline & Thallium (Dissolved) & $7440-28-0$ & 1.8 & 1 & $1.8-1.8$ \\
\hline Metals Uo/L & Mercury (Dissolved) & $7439-97-6$ & 0.2 & 1 & $0.2-0.2$ \\
\hline \multirow[t]{10}{*}{ Metals UG/L (ICP) } & Aluminum (Dissolved) & $7429-90-5$ & 16 & 1 & $16-16$ \\
\hline & Beryllium (Dissolved) & $7440-41-7$ & 0.1 & 1 & $0.1-0.1$ \\
\hline & Cadmium & $7440-43-9$ & 1 & 1 & 1.1 \\
\hline & Cadmium (Dissolved) & $7440-43-9$ & 1 & 1 & $1-1$ \\
\hline & Chromium (Dissolved) & $7440-47-3$ & 5.6 & 1 & $5.6-5.6$ \\
\hline & Copper (Dissolved) & $7440-50-8$ & 5 & 1 & $5-5$ \\
\hline & Nickel (Dissolved) & $7440-02.0$ & 4.7 & 1 & $4.7-4.7$ \\
\hline & Silver & $7440-22-4$ & 4.1 & 1 & $4.1-4.1$ \\
\hline & Silver (Dissolved) & $7440-22-4$ & 4.1 & 1 & $4.1-4.1$ \\
\hline & Vanadium (Dissolved) & $7440-62-2$ & 2.5 & 1 & $2.5-2.5$ \\
\hline \multirow[t]{6}{*}{ Semivolatile Organics UG/L } & 1,2,4-Trichlorobenzene & $120-82-1$ & 10 & 1 & $10-10$ \\
\hline & 1,2-Dichlorobenzene & $95-50-1$ & 10 & 1 & $10-10$ \\
\hline & 1,3-Dichlorobenzene & $541-73-1$ & 10 & 1 & $10-10$ \\
\hline & 1,4-Dichlorobenzene & $106-46-7$ & 10 & 1 & $10-10$ \\
\hline & 2,2'-oxybis(1-Chloropropane) & $108-60-1$ & 10 & 1 & $10-10$ \\
\hline & 2,4,5-Trichlorophenol & $95-95-4$ & 26 & 1 & $26-26$ \\
\hline
\end{tabular}




\begin{tabular}{|c|c|c|c|c|c|}
\hline Analysis & Parameter & Cas Number & Mean Detection Limit & $\begin{array}{l}\text { Number of } \\
\text { Nondetects }\end{array}$ & $\begin{array}{l}\text { Detection } \\
\text { Limit Range }\end{array}$ \\
\hline & 2,4,6-Trichlorophenol & $88-06-2$ & 10 & 1 & $10-10$ \\
\hline & 2,4-Dichlorophenol & $120-83-2$ & 10 & 1 & $10 \cdot 10$ \\
\hline & 2,4-Dimethylphenol & $105-67-9$ & 10 & 1 & $10 \cdot 10$ \\
\hline & 2,4-Dinitrophenol & $51-28-5$ & 26 & 1 & $26-26$ \\
\hline & 2,4-Dinitrotoluene & $121-14-2$ & 10 & 1 & $10-10$ \\
\hline & 2,6-Dinitrotoluene & $606-20-2$ & 10 & 1 & $10-10$ \\
\hline & 2-Chloronaphthalene & $91-58-7$ & 10 & 1 & $10-10$ \\
\hline & 2-Chlorophenol & $95-57-8$ & 10 & 1 & $10-10$ \\
\hline & 2-Methylnaphthalene & $91-57-6$ & 10 & 1 & $10-10$ \\
\hline & 2-Methylphenol & $95-48-7$ & 10 & 1 & $10-10$ \\
\hline & 2-Nitroaniline & $88-74-4$ & 26 & 1 & $26-26$ \\
\hline & 2-Nitrophenol & 88-75-5 & 10 & 1 & $10-10$ \\
\hline & 3,3'-Dichlorobenzidine & $91-94-1$ & 10 & 1 & $10-10$ \\
\hline & 3-Nitroaniline & $99-09-2$ & 26 & 1 & $26-26$ \\
\hline & 4,6-Dinitro-2-methylphenol & $534-52-1$ & 26 & 1 & $26-26$ \\
\hline & 4-Bromophenyl-phenylether & $101-55-3$ & 10 & 1 & $10-10$ \\
\hline & 4-Chloro-3-methylphenol & $59-50-7$ & 10 & 1 & $10-10$ \\
\hline & 4-Chloroaniline & $106-47-8$ & 10 & 1 & $10-10$ \\
\hline & 4-Chloropheny!-phenylether & $7005-72-3$ & 10 & 1 & $10-10$ \\
\hline & 4-Methylphenol & $106-44-5$ & 10 & 1 & $10-10$ \\
\hline & 4-Nitroaniline & $100-01-6$ & 26 & 1 & $26-26$ \\
\hline & 4-Nitrophenol & $100-02-7$ & 26 & 1 & $26-26$ \\
\hline & Acenaphthylene & 208-96-8 & 10 & 1 & $10-10$ \\
\hline & Anthracene & $120-12-7$ & 10 & 1 & $10-10$ \\
\hline
\end{tabular}




\begin{tabular}{|c|c|c|c|c|c|}
\hline Andysis . & Parameter & Cas Number & Mean Detection Limit & $\begin{array}{l}\text { Number of } \\
\text { Nondetects }\end{array}$ & $\begin{array}{c}\text { Detection } \\
\text { Limit Range }\end{array}$ \\
\hline & Benzo(a)anthracene & $56-55-3$ & 10 & 1 & $10-10$ \\
\hline & Benzo(a)pyrene & $50-32-8$ & 10 & 1 & $10-10$ \\
\hline & Benzo(b)fluoranthene & $205-99-2$ & 10 & 1 & $10-10$ \\
\hline & Benzo(g,h,i)perylene & $191-24-2$ & 10 & 1 & $10-10$ \\
\hline & Benzo(k)fluoranthene & $207-089$ & 10 & 1 & $10-10$ \\
\hline & Butylbenzy lphthalate & $85-68-7$ & 10 & 1 & $10-10$ \\
\hline & Carbazole & $86-74-8$ & 10 & 1 & $10 \cdot 10$ \\
\hline & Chrysene & 218-01-9 & 10 & 1 & $10-10$ \\
\hline & Di-n-butylphthalate & $84-74-2$ & 10 & 1 & $10 \cdot 10$ \\
\hline & Di-n-octylphthalate & $117-84-0$ & 10 & 1 & $10 \cdot 10$ \\
\hline & Dibenzo $(\mathrm{a}, \mathrm{h})$ anthracene & $53.70-3$ & 10 & 1 & $10-10$ \\
\hline & Dibenzofuran & $132-64-9$ & 10 & 1 & $10-10$ \\
\hline & Diethylphthalate & $84-66-2$ & 10 & 1 & $10-10$ \\
\hline & Dimethylphthalate & $131-11-3$ & 10 & 1 & $10 \cdot 10$ \\
\hline & Fluoranthene & $206.44-0$ & 10 & 1 & $10-10$ \\
\hline & Fluorene & 86-73-7 & 10 & 1 & $10-10$ \\
\hline & Hexachlorobenzene & 118-74-1 & 10 & 1 & $10-10$ \\
\hline & Hexachlorobutadiene & $87-68.3$ & 10 & 1 & $10-10$ \\
\hline & Hexachlorocyclopentadiene & $77-47-4$ & 10 & 1 & $10-10$ \\
\hline & Hexachloroethane & $67-72-1$ & 10 & 1 & $10-10$ \\
\hline & Indeno $(1,2,3-c, d)$ pyrene & $193-39-5$ & 10 & 1 & $10-10$ \\
\hline & Isophorone & $78.59-1$ & 10 & 1 & $10-10$ \\
\hline & N-Nitroso-di-n-propylamine & $621-64-7$ & 10 & 1 & $10-10$ \\
\hline & N-Nitrosodiphenylamine & $86-30-6$ & 10 & 1 & $10-10$ \\
\hline
\end{tabular}




\begin{tabular}{|c|c|c|c|c|c|}
\hline Analysia & Parameter & Cas Number & Mean Detection Limit & $\begin{array}{l}\text { Number of } \\
\text { Nondetects }\end{array}$ & $\begin{array}{l}\text { Detection } \\
\text { Limit Range }\end{array}$ \\
\hline & Naphthalene & $91-20-3$ & 10 & 1 & $10-10$ \\
\hline & Nitrobenzene & $98-95-3$ & 10 & 1 & $10 \cdot 10$ \\
\hline & Pentachlorophenol & $87-86-5$ & 26 & 1 & $26-26$ \\
\hline & Phenanthrene & $85-01-8$ & 10 & 1 & $10 \cdot 10$ \\
\hline & Phenol & $108-95.2$ & 10 & $\mathbb{1}$ & $10-10$ \\
\hline & Pyrene & $129-00-0$ & 10 & 1 & $10-10$ \\
\hline & bis(2-Chloroethoxy)methane & $111-91-1$ & 10 & 1 & $10-10$ \\
\hline & bis(2-Chloroethyl)ether & $111-44-4$ & 10 & 1 & $10-10$ \\
\hline & bis(2-Ethylhexyl)phthalate & $117-81-7$ & 10 & 1 & $10-10$ \\
\hline \multirow[t]{15}{*}{ Volatile Organies Ua/L } & 1,1,1-Trichloroethane & $71-55-6$ & 5 & 1 & $5-5$ \\
\hline & 1,1,2,2-Tetrachloroethane & $79-34-5$ & 5 & 1 & 5.5 \\
\hline & 1,1,2-Trichloro-1,2,2-Trifluoroethane & $76-13-1$ & 10 & 1 & $10-10$ \\
\hline & 1,1,2-Trichloroethane & $79-00-5$ & 5 & 1 & $5-5$ \\
\hline & 1,1-Dichloroethane & $75-34-3$ & 5 & 1 & $5-5$ \\
\hline & 1,1-Dichloroethene & $75-35-4$ & 5 & 1 & $5-5$ \\
\hline & 1,2-Dichloroethane & $107-06-2$ & 5 & 1 & $5-5$ \\
\hline & 1,2-Dichloroethene & $540-59-0$ & 5 & 1 & $5-5$ \\
\hline & 1,2-Dichloropropane & $78-87-5$ & 5 & 1 & $5-5$ \\
\hline & 2-Butanone & $78-93-3$ & 10 & 1 & $10-10$ \\
\hline & 2-Hexanone & $591-78-6$ & 10 & 1 & $10-10$ \\
\hline & 4-Methyl-2-pentanone & $108-10-1$ & 10 & 1 & $10-10$ \\
\hline & Acetone & $67-64-1$ & 5 & 1 & $5-5$ \\
\hline & Benzene & $71-43-2$ & 5 & 1 & $5-5$ \\
\hline & Bromodichloromethane & $75-27-4$ & 5 & 1 & 5.5 \\
\hline
\end{tabular}




\begin{tabular}{|c|c|c|c|c|c|}
\hline Analysis & Parameter & Cas Number & Mean Detection Limit & $\begin{array}{l}\text { Number of } \\
\text { Nondetects }\end{array}$ & $\begin{array}{l}\text { Detection } \\
\text { Limit Range }\end{array}$ \\
\hline & Bromoform & $75-25-2$ & 5 & 1 & $5-5$ \\
\hline & Bromomethane & $74-83-9$ & 10 & 1 & $10-10$ \\
\hline & Carbon Disulfide & $75-15-0$ & 5 & 1 & $5-5$ \\
\hline & Carbon Tetrachloride & $56-23-5$ & 5 & 1 & $5-5$ \\
\hline & Chlorobenzene & $108-90-7$ & 5 & 1 & $5-5$ \\
\hline & Chloroethane & $75-00-3$ & 10 & 1 & $10-10$ \\
\hline & Chloroform & $67-66-3$ & 5 & 1 & $5-5$ \\
\hline & Chloromethane & $74-87-3$ & 10 & 1 & $10 \cdot 10$ \\
\hline & Dibromochloromethane & $124-48-1$ & 5 & 1 & $5-5$ \\
\hline & Ethylbenzene & $100-41-4$ & 5 & 1 & $5-5$ \\
\hline & Methylene Chloride & $75.09-2$ & 10 & 1 & $10-10$ \\
\hline & Styrene & $100-42-5$ & 5 & 1 & 5-5 \\
\hline & Tetrachloroethene & $127-18-4$ & 5 & 1 & 5.5 \\
\hline & Toluene & 108-88-3 & 5 & 1 & $5-5$ \\
\hline & Trichloroethene & $79-01-6$ & 5 & 1 & $5-5$ \\
\hline & Vinyl Chloride & $75-01-4$ & 10 & 1 & $10-10$ \\
\hline & Xylene, Total & $1330-20-7$ & 5 & 1 & $5-5$ \\
\hline & cis-1,3-Dichloropropene & $10061-01-5$ & 5 & 1 & $5-5$ \\
\hline & trans-1,3-Dichloropropene & $10061-02-6$ & 5 & 1 & $5-5$ \\
\hline \multirow[t]{5}{*}{ Pesticide/PCB UG/L } & 4, $4^{\prime}-\mathrm{DDD}$ & $72-54-8$ & 0.11 & 1 & $0.11-0.11$ \\
\hline & 4,4'-DDE & $72-55-9$ & 0.11 & 1 & $0.11-0.11$ \\
\hline & $4,4^{\prime}-\mathrm{DDT}$ & $50-29-3$ & 0.11 & 1 & $0.11-0.11$ \\
\hline & Aldrin & $309-\infty 0-2$ & 0.054 & 1 & $0.054-0.054$ \\
\hline & Aroclor-1016 & $11-2$ & 1.1 & 1 & $1.1-1.1$ \\
\hline
\end{tabular}




\begin{tabular}{|c|c|c|c|c|c|}
\hline Analysis & Parameter & Cas Number & Mean Detection Limit & $\begin{array}{l}\text { Number of } \\
\text { Nondetects }\end{array}$ & $\begin{array}{c}\text { Detection } \\
\text { Limit Range }\end{array}$ \\
\hline & Aroclor-1221 & $11104-28-2$ & 2.2 & 1 & $2.2-2.2$ \\
\hline & Aroclor- 1232 & $11141-16-5$ & 1.1 & 1 & $1.1-1.1$ \\
\hline & Aroclor-1242 & $53469-21-9$ & 1.1 & 1 & $1.1-1.1$ \\
\hline & Aroclor-1248 & $12672-29-6$ & 1.1 & 1 & $1.1-1.1$ \\
\hline & Aroclor-1254 & $11097-69-1$ & 1.1 & 1 & $1.1-1.1$ \\
\hline & Aroclor- 1260 & $11096-82-5$ & 1.1 & 1 & $1.1-1.1$ \\
\hline & Dieldrin & $60-57-1$ & 0.11 & 1 & $0.11-0.11$ \\
\hline & Endosulfan I & $959-98-8$ & 0.054 & 1 & $0.054-0.054$ \\
\hline & Endosulfan II & $33213-65-9$ & 0.11 & 1 & $0.11-0.11$ \\
\hline & Endosulfan Sulfate & $1031-07-8$ & 0.11 & 1 & $0.11-0.11$ \\
\hline & Endrin & $72-20-8$ & 0.11 & 1 & $0.11-0.11$ \\
\hline & Endrin Aldehyde & $7421-93-4$ & 0.11 & 1 & $0.11-0.11$ \\
\hline & Endrin Retone & $53494-70-5$ & 0.11 & 1 & $0.11-0.11$ \\
\hline & Heptachlor & $76-44-8$ & 0.054 & 1 & $0.054-0.054$ \\
\hline & Heptachlor Epoxide & $1024-57-3$ & 0.054 & 1 & $0.054-0.054$ \\
\hline & Toxaphene & $8001.35-2$ & 5.4 & 1 & $5.4-5.4$ \\
\hline & alpha-BHC & $319-84-6$ & 0.054 & 1 & $0.054-0.054$ \\
\hline & alpha-Chlordane & $5103-71-9$ & 0.054 & 1 & $0.054-0.054$ \\
\hline & beta-BHC & $319-85-7$ & 0.054 & 1 & $0.054-0.054$ \\
\hline & delta-BHC & $319-86-8$ & 0.054 & 1 & $0.054-0.054$ \\
\hline & gamm-BHC (Lindane) & $58.89-9$ & 0.054 & 1 & $0.054-0.054$ \\
\hline & gamma-Chlordane & $3103-74-2$ & 0.054 & 1 & $0.054-0.054$ \\
\hline & p,p'-Methoxychlor & $72-43-5$ & 0.54 & 1 & $0.54-0.54$ \\
\hline
\end{tabular}


Data Source : Bear Creek Valley OU2 1993 RI field activities validared data

Raw data available through $Y-12$ OREIS

Data does not include QA/QC

Results $<$ counting uncertainty were considered as nondetects for radionuclides 
TABLE 2. BC-OU2 1993 RI Fiedd Activities Data - Summary Of Parameters Never Detected For The Spoil Area 1

Sampling Media: Ground Water

\begin{tabular}{|c|c|c|c|c|c|}
\hline Analysis & Parameter & Cas Number & Mesn Detection Limit & $\begin{array}{l}\text { Number of } \\
\text { Nondetects }\end{array}$ & $\begin{array}{c}\text { Detection } \\
\text { Limit Range }\end{array}$ \\
\hline Anions $\mathrm{MG} / \mathrm{L}$ & Sulfate & & 27.5 & 2 & $5-50$ \\
\hline \multirow[t]{5}{*}{ Metals UO/L (AAS) } & Arsenic (Dissolved) & $7440-38-2$ & 2.2 & 2 & $2.2-2.2$ \\
\hline & Cyanide & $57-12-5$ & 10 & 2 & $10-10$ \\
\hline & Selenium (Dissolved) & $7782-49-2$ & 2.7 & 2 & $2.7-2.7$ \\
\hline & Thallium & $7440-28-0$ & 1.3 & 2 & $1.3-1.3$ \\
\hline & Thallium (Dissolved) & $7440-28-0$ & 1.8 & 2 & $1.8-1.8$ \\
\hline \multirow[t]{6}{*}{ Metals UG/L (ICP) } & Beryllium (Dissolved) & $7440-41-7$ & 0.1 & 2 & $0.1-0.1$ \\
\hline & Cadmium & $7440-43-9$ & 1 & 2 & $1-1$ \\
\hline & Cadmium (Dissolved) & $7440-43-9$ & 1.1 & 2 & $1-1.2$ \\
\hline & Chromium (Dissolved) & $7440-47-3$ & 5.6 & 2 & $5.6-5.6$ \\
\hline & Silver & $7440-22-4$ & 4.1 & 2 & $4.1-4.1$ \\
\hline & Silver (Dissolved) & $7440-22-4$ & 4.1 & 2 & $4.1-4.1$ \\
\hline \multirow[t]{10}{*}{ Sernivoletile Organics UG/L } & 1,2,4-Trichlorobenzene & $120-82-1$ & 10.5 & 2 & $10-11$ \\
\hline & 1,2-Dichlorobenzene & $95-50-1$ & 10.5 & 2 & $10-11$ \\
\hline & 1,3-Dichlorobenzene & $541-73-1$ & 10.5 & 2 & $10-11$ \\
\hline & 1,4-Dichlorobenzene & $106-46-7$ & 10.5 & 2 & $10-11$ \\
\hline & 2,2'-oxybis(1-Chloropropane) & $108-60-1$ & 10.5 & 2 & $10-11$ \\
\hline & 2,4,5-Trichlotophenol & $95-95-4$ & 26.5 & 2 & $26-27$ \\
\hline & 2,4,6-Trichlorophenol & $88-06-2$ & 10.5 & 2 & $10-11$ \\
\hline & 2,4-Dichlorophenol & $120-83-2$ & 10.5 & 2 & $10-11$ \\
\hline & 2,4-Dimethylphenol & $105-67-9$ & 10.5 & 2 & $10-11$ \\
\hline & 2,4-Dinitrophenol & 51.28 .5 & 26.5 & 2 & $26-27$ \\
\hline
\end{tabular}




\begin{tabular}{|c|c|c|c|c|c|}
\hline Analysis & Parameter & Cas Number & Mean Detection Limil & $\begin{array}{l}\text { Number of } \\
\text { Nondetects }\end{array}$ & $\begin{array}{l}\text { Detection } \\
\text { Limit Range }\end{array}$ \\
\hline & 2,4-Dinitrotoluene & $121-14-2$ & 10.5 & 2 & $10-11$ \\
\hline & 2,6-Dinitrotoluene & $606-20-2$ & 10.5 & 2 & $10-11$ \\
\hline & 2-Chloronaphthalene & $91-58-7$ & 10.5 & 2 & $10-11$ \\
\hline & 2-Chlorophenol & $95-57-8$ & 10.5 & 2 & 10.11 \\
\hline & 2-Methylnaphthalene & $91-57-6$ & 10.5 & 2 & $10-11$ \\
\hline & 2-Methylphenol & $95-48-7$ & 10.5 & 2 & $10-11$ \\
\hline & 2-Nitroaniline & $88-74-4$ & 26.5 & 2 & $26-27$ \\
\hline & 2-Nitrophenol & $88-75-5$ & 10.5 & 2 & $10-11$ \\
\hline & 3,3'-Dichlorobenzidine & $91-94-1$ & 10.5 & 2 & $10-11$ \\
\hline & 3-Nitroaniline & $99-09-2$ & 26.5 & 2 & $26-27$ \\
\hline & 4,6-Dinitro-2-methylphenol & $534-52-1$ & 26.5 & 2 & $26-27$ \\
\hline & 4-Bromophenyl-phenylether & $101-55-3$ & 10.5 & 2 & $10-11$ \\
\hline & 4-Chloro-3-methylphenol & $59-50-7$ & 10.5 & 2 & $10-11$ \\
\hline & 4-Chloroaniline & $106-47 \cdot 8$ & 10.5 & 2 & $10-11$ \\
\hline & 4-Chlorophenyl-phenylether & $7005-72-3$ & 10.5 & 2 & $10-11$ \\
\hline & 4-Methylphenol & $106-44-5$ & 10.5 & 2 & $10-11$ \\
\hline & 4-Nitronniline & $100-01-6$ & 26.5 & 2 & $26-27$ \\
\hline & 4-Nitrophenol & $100-02-7$ & 26.5 & 2 & $26-27$ \\
\hline & Acenaphthene & $83-32-9$ & 10.5 & 2 & $10-11$ \\
\hline & Acenaphthylene & $208-96-8$ & 10.5 & 2 & $10-11$ \\
\hline & Anthracene & $120-12-7$ & 10.5 & 2 & $10-11$ \\
\hline & Benzo(a)anthracene & $56-55-3$ & 10.5 & 2 & $10-11$ \\
\hline & Benzo(a)pyrene & $50-32-8$ & 10.5 & 2 & $10-11$ \\
\hline & Benzo(b)fluoranthene & $205-99-2$ & 10.5 & 2 & $10-11$ \\
\hline
\end{tabular}




\begin{tabular}{|c|c|c|c|c|c|}
\hline Analy isis & Parameter & Cas Number & Mean Detection Limit & $\begin{array}{l}\text { Number of } \\
\text { Nondetects }\end{array}$ & $\begin{array}{l}\text { Detection } \\
\text { Limil Range }\end{array}$ \\
\hline & $\operatorname{Benzo}(g, h, i)$ perylene & $191-24-2$ & 10.5 & 2 & $10-11$ \\
\hline & Benzo(k)fluoranthene & $207.08-9$ & 10.5 & 2 & $10-11$ \\
\hline & Butylbenzylphthalate & $85-68-7$ & 10.5 & 2 & $10-11$ \\
\hline & Carbazole & $86-74-8$ & 10.5 & 2 & $10-11$ \\
\hline & Chrysene & $218-01-9$ & 10.5 & 2 & $10-11$ \\
\hline & Di-a-butylphthalate & $84-74-2$ & 10.5 & 2 & $10-11$ \\
\hline & Di-m-octylphthalate & $117-84-0$ & 10.5 & 2 & $10-11$ \\
\hline & Dibenzo(a,h)anthracene & $53-70-3$ & 10.5 & 2 & $10-11$ \\
\hline & Dibenzofuran & $132-64-9$ & 10.5 & 2 & $10-11$ \\
\hline & Diethylphthalate & $84-66-2$ & 10.5 & 2 & $10-11$ \\
\hline & Dimethylphthalate & $131-11-3$ & 10.5 & 2 & $10-11$ \\
\hline & Fuoranthene & $206-44-0$ & 10.5 & 2 & $10-11$ \\
\hline & Fluorene & $86-73-7$ & 10.5 & 2 & $10-11$ \\
\hline & Hexachlorobenzene & $118-74-1$ & 10.5 & 2 & $10-11$ \\
\hline & Hexachlorobutadiene & $87-68-3$ & 10.5 & 2 & $10-11$ \\
\hline & Hexachlorocyclopentadiene & $77-47-4$ & 10.5 & 2 & $10-11$ \\
\hline & Hexachloroethane & $67-72-1$ & 10.5 & 2 & $10-11$ \\
\hline & Indeno( $1,2,3-c, d)$ pyrene & 193-39-5 & 10.5 & 2 & $10-11$ \\
\hline & Isophorone & $78-59-1$ & 10.5 & 2 & $10-11$ \\
\hline & N-Nitroso-di-n-propylamine & $621-64-7$ & 10.5 & 2 & $10-11$ \\
\hline & N-Nitrosodiphenylmmine & $86-30-6$ & 10.5 & 2 & $10-11$ \\
\hline & Naphthalene & $91-20-3$ & 10.5 & 2 & 10.11 \\
\hline & Nitrobenzene & 98-95-3 & 10.5 & 2 & $10-11$ \\
\hline & Pentachlorophenol & $87-86-5$ & 26.5 & 2 & $26-27$ \\
\hline
\end{tabular}




\begin{tabular}{|c|c|c|c|c|c|}
\hline Analysis & Parameter & Cas Number & Mean Detection Limit & $\begin{array}{l}\text { Number of } \\
\text { Nondetects }\end{array}$ & $\begin{array}{l}\text { Detection } \\
\text { Limit Range }\end{array}$ \\
\hline & Phenanthrene & $85-01-8$ & 10.5 & 2 & $10-11$ \\
\hline & Phenol & $108-95-2$ & 10.5 & 2 & $10-11$ \\
\hline & Pyrene & $129-00-0$ & 10.5 & 2 & $10-11$ \\
\hline & bis(2-Chloroethoxy)methane & $111-91-1$ & 10.5 & 2 & $10-11$ \\
\hline & bis(2-Chloroethyl)ether & $111-44-4$ & 10.5 & 2 & $10-11$ \\
\hline \multirow[t]{19}{*}{ Volatile Organics UG/L } & 1,1,1-Trichloroethane & $71-55-6$ & 5 & 2 & $5-5$ \\
\hline & 1,1,2,2-Tetrachloroethane & $79-34-5$ & 5 & 2 & $5-5$ \\
\hline & 1,1,2-Trichloroethane & $79-00-5$ & 5 & 2 & $5-5$ \\
\hline & 1,1-Dichloroethane & $75-34-3$ & 5 & 2 & $5-5$ \\
\hline & 1,1-Dichloroethene & $75-35-4$ & 5 & 2 & $5-5$ \\
\hline & 1,2-Dichloroethane & $107-06-2$ & 5 & 2 & $5-5$ \\
\hline & 1,2-Dichloropropane & $78-87-5$ & 5 & 2 & $5-5$ \\
\hline & 2-Butanone & $78-93-3$ & 7.5 & 2 & $5-10$ \\
\hline & 2-Hexanone & $591-78-6$ & 10 & 2 & $10-10$ \\
\hline & 4-Methyl-2-pentanone & $108-10-1$ & 10 & 2 & $10-10$ \\
\hline & Benzene & $71-43-2$ & 5 & 2 & $5-5$ \\
\hline & Bromodichloromethane & $75-27-4$ & 5 & 2 & $5-5$ \\
\hline & Bromoform & $75-25-2$ & 5 & 2 & $5-5$ \\
\hline & Bromomethane & $74-83-9$ & 10 & 2 & $10-10$ \\
\hline & Carbon Tetrachloride & $56-23-5$ & 5 & 2 & 5.5 \\
\hline & Chlorobenzene & $108-90-7$ & 5 & 2 & $5-5$ \\
\hline & Chloroethane & $75-00-3$ & 10 & 2 & $10-10$ \\
\hline & Chloroform & $67-66-3$ & 5 & 2 & $5-5$ \\
\hline & Chloromethane & $74-87-3$ & 10 & 2 & $10-10$ \\
\hline
\end{tabular}




\begin{tabular}{|c|c|c|c|c|c|}
\hline Analy tis & Parameter & Can Number & Mean Detection Limit & $\begin{array}{l}\text { Number of } \\
\text { Nondetects }\end{array}$ & $\begin{array}{c}\text { Detection } \\
\text { Limit Range }\end{array}$ \\
\hline & Dibromochloromethane & $124-48-1$ & 5 & 2 & $5-5$ \\
\hline & Ethylbenzene & $100-41-4$ & 5 & 2 & $5-5$ \\
\hline & Methylene Chloride & $75-09-2$ & 6 & 2 & $5-7$ \\
\hline & Styrene & $100-42-5$ & 5 & 2 & $5-5$ \\
\hline & Toluene & $108-88-3$ & 5 & 2 & $5-5$ \\
\hline & Vinyl Chloride & $75-01-4$ & 10 & 2 & $10-10$ \\
\hline & Xylene, Total & $1330-20-7$ & 5 & 2 & $5-5$ \\
\hline & eis-1,3-Dichloropropene & $10061-01-5$ & 5 & 2 & $5-5$ \\
\hline & Irans-1,3-Dichloropropene & $10061-02-6$ & 5 & 2 & $5-5$ \\
\hline \multirow[t]{15}{*}{ Pesticide/PCB UO/L } & $4,4^{\prime}-\mathrm{DDD}$ & $72-54-8$ & 0.105 & 2 & $0.1-0.11$ \\
\hline & 4,4'-DDE & $72-55-9$ & 0.105 & 2 & $0.1-0.11$ \\
\hline & 4, $4^{\prime}-\mathrm{DDT}$ & $50-29-3$ & 0.105 & 2 & $0.1-0.11$ \\
\hline & Aldrin & $309-00-2$ & 0.0525 & 2 & $0.051-0.054$ \\
\hline & Aroclor-1016 & $12674-11-2$ & 1.05 & 2 & $1-1.1$ \\
\hline & Aroclor-1221 & $11104-28-2$ & 2.1 & 2 & $2-2.2$ \\
\hline & Aroclor-1232 & 11141-16-5 & 1.05 & 2 & $1-1.1$ \\
\hline & Aroclor-1242 & $53469-21-9$ & 1.05 & 2 & $1-1.1$ \\
\hline & Aroclor-1248 & 12672-29-6 & 1.05 & 2 & $1-1.1$ \\
\hline & Aroclor-1254 & $11097-69-1$ & 1.05 & 2 & $1-1.1$ \\
\hline & Aroclor-1260 & $11096-82-5$ & 1.05 & 2 & $1-1.1$ \\
\hline & Dieldrin & $60.57-1$ & 0.105 & 2 & $0.1-0.11$ \\
\hline & Endosulfan I & 959-98-8 & 0.0525 & 2 & $0.051-0.054$ \\
\hline & Endosulfan II & $33213-65-9$ & 0.105 & 2 & $0.1-0.11$ \\
\hline & Endosulfan Sulfate & $1031-07-8$ & 0.105 & 2 & $0.1-0.11$ \\
\hline
\end{tabular}




\begin{tabular}{|c|c|c|c|c|c|}
\hline Analysis & Parameter & Cas Number & Mean Detection Limit & $\begin{array}{l}\text { Number of } \\
\text { Nondetects }\end{array}$ & $\begin{array}{c}\text { Defection } \\
\text { Limit Range }\end{array}$ \\
\hline & Endrin & $72-20-8$ & 0.105 & 2 & $0.1-0.11$ \\
\hline & Endrin Aldehyde & $7421-93-4$ & 0.105 & 2 & $0.1-0.11$ \\
\hline & Endrin Ketone & $53494-70-5$ & 0.105 & 2 & $0.1-0.11$ \\
\hline & Heptachlor & $76-44-8$ & 0.0525 & 2 & $0.051-0.054$ \\
\hline & Heptachlor Epoxide & $1024-57-3$ & 0.0525 & 2 & $0.051-0.054$ \\
\hline & Toxaphene & $8001-35-2$ & 5.25 & 2 & $5.1-5.4$ \\
\hline & alpha-BHC & $319-84-6$ & 0.0525 & 2 & $0.051-0.054$ \\
\hline & alpha-Chlordme & $5103-71-9$ & 0.0525 & 2 & $0.051-0.054$ \\
\hline & beta-BHC & $319-85-7$ & 0.0525 & 2 & $0.051-0.054$ \\
\hline & delta-BHC & $319-86-8$ & 0.0525 & 2 & $0.051-0.054$ \\
\hline & gamma-BHC (Lindane) & $58-89-9$ & 0.0525 & 2 & $0.051-0.054$ \\
\hline & gamma-Chlordane & $5103-74-2$ & 0.0525 & 2 & $0.051-0.054$ \\
\hline & p,p'-Methoxychlor & $72-43-5$ & 0.525 & 2 & $0.51-0.54$ \\
\hline
\end{tabular}

Data Source : Bear Creek Valley OU2 1993 RI field activities validated data

Raw data available through $Y-12$ OREIS

Data does not include QA/QC

Results < counting uncertainty were considered as nondetects for radionuclides 
TABLE 2. BC-OU2 1993 RI Field Activities Data - Summary Of Parameters Never Detected For The SY-2M0 Yard Sampling Media: Groand Water

\begin{tabular}{|c|c|c|c|c|c|}
\hline Analysis & Parameter & Cas Number & Mean Detection Limit & $\begin{array}{l}\text { Numer of } \\
\text { Nondetects }\end{array}$ & $\begin{array}{c}\text { Detection } \\
\text { Limit Range }\end{array}$ \\
\hline Anions $\mathrm{MG} / \mathrm{L}$ & Nitrate/Nitrite (NO3/NO2-N) & $14797-55-8$ & 0.2 & 3 & $0.2-0.2$ \\
\hline \multirow[t]{5}{*}{ Metals UG/L (AAS) } & Arsenic (Dissolved) & $7440-38-2$ & 2.2 & 3 & $2.2-2.2$ \\
\hline & Cyanide & $57-12-5$ & 10 & 3 & $10-10$ \\
\hline & Selenium (Dissolved) & $7782-49-2$ & 2.7 & 3 & $2.7-2.7$ \\
\hline & Thellium & $7440-28-0$ & 1.3 & 3 & $1.3-1.3$ \\
\hline & Thallium (Dissolved) & $7440-28-0$ & 1.8 & 3 & $1.8-1.8$ \\
\hline \multirow[t]{11}{*}{ Metals UG/L (ICP) } & Aluminum (Dissolved) & $7429-90-5$ & 24.2 & 3 & $16.9-37.9$ \\
\hline & Beryllium & $7440-41-7$ & 0.1 & 3 & $0.1-0.1$ \\
\hline & Beryllium (Dissolved) & $7440-41-7$ & 0.1 & 3 & $0.1-0.1$ \\
\hline & Cadmium & $7440-43-9$ & 1 & 3 & $1-1$ \\
\hline & Cadmium (Dissolved) & $7440-43-9$ & 1 & 3 & $1-1$ \\
\hline & Chromium (Dissolved) & $7440-47-3$ & 5.6 & 3 & $5.6-5.6$ \\
\hline & Iron (Dissolved) & $7439-89-6$ & 79.5 & 3 & $23.8-179$ \\
\hline & Silver & $7440-22-4$ & 4.1 & 3 & $4.1-4.1$ \\
\hline & Silver (Dissolved) & $7440-22-4$ & 4.1 & 3 & $4.1 \cdot 4.1$ \\
\hline & Vanadium (Dissolved) & $7440-62-2$ & 2.5 & 3 & $2.5-2.5$ \\
\hline & Zine (Dissolved) & $7440-66-6$ & 15.9 & 3 & $14.8-16.6$ \\
\hline \multirow[t]{6}{*}{ Semivolatile Organics UG/L } & 1,2,4-Trichlorobenzene & $120-82-1$ & 10.3 & 3 & $10-11$ \\
\hline & 1,2-Dichlorobenzene & $95-50-1$ & 10.3 & 3 & $10-11$ \\
\hline & 1,3-Dichlorobenzene & $541-73-1$ & 10.3 & 3 & $10-11$ \\
\hline & 1,4-Dichlorobenzene & $106-46-7$ & 10.3 & 3 & $10-11$ \\
\hline & 2,2'-oxybis(1-Chloropropane) & $108-60-1$ & 10.3 & 3 & $10-11$ \\
\hline & 2,4,5-Trichlorophenol & $95-95-4$ & 26.3 & 3 & $25-28$ \\
\hline
\end{tabular}




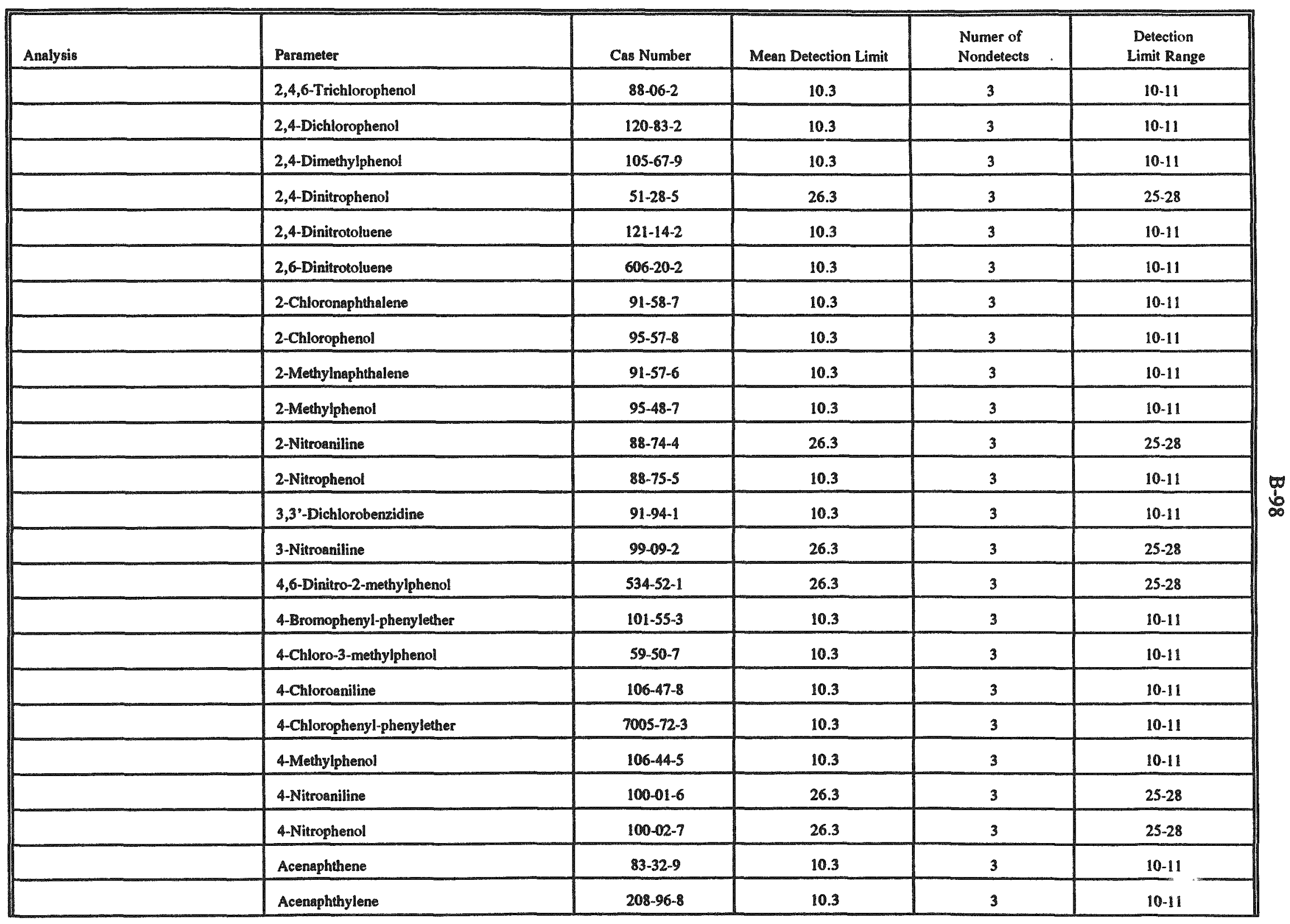




\begin{tabular}{|c|c|c|c|c|c|}
\hline Analysis & Parameter & Cas Number & Mean Detection Limit & $\begin{array}{l}\text { Numer of } \\
\text { Nondetects }\end{array}$ & $\begin{array}{l}\text { Detection } \\
\text { Limit Range }\end{array}$ \\
\hline & Anthracene & $120-12-7$ & 10.3 & 3 & $10-11$ \\
\hline & Benzo(a)anthracene & $56-55-3$ & 10.3 & 3 & $10-11$ \\
\hline & Benzo(a)pyrene & $50-32-8$ & 10.3 & 3 & $10-11$ \\
\hline & Benzo(b)fluoranthene & $205-99-2$ & 10.3 & 3 & $10-11$ \\
\hline & Benzo $(g, h, i)$ perylene & $191-24-2$ & 10.3 & 3 & $10-11$ \\
\hline & Benzo(k) fluoranthene & $207-0 \%-9$ & 10.3 & 3 & $10-11$ \\
\hline & Carbazole & $86-74-8$ & 10.3 & 3 & $10-11$ \\
\hline & Chrysene & $218-01-9$ & 10.3 & 3 & $10-11$ \\
\hline & Di-n-octylphthalate & $117-84-0$ & 10.3 & 3 & $10-11$ \\
\hline & Dibenzo(a,h)anthracene & $53-70-3$ & 10.3 & 3 & $10-11$ \\
\hline & Dibenzofuran & $132-64-9$ & 10.3 & 3 & $10-11$ \\
\hline & Dimethylphthalate & $131-11-3$ & 10.3 & 3 & $10-11$ \\
\hline & Fluoranthene & $206-44-0$ & 10.3 & 3 & $10-11$ \\
\hline & Fluorene & 86-73-7 & 10.3 & 3 & $10-11$ \\
\hline & Hexachlorobenzene & $118-74-1$ & 10.3 & 3 & $10-11$ \\
\hline & Hexachlorobutadiene & $87-68-3$ & 10.3 & 3 & $10-11$ \\
\hline & Hexachlorocyclopentadiene & $77-47-4$ & 10.3 & 3 & $10-11$ \\
\hline & Hexachloroethane & $67-72-1$ & 10.3 & 3 & $10-11$ \\
\hline & Indeno $(1,2,3-c, d)$ pyrene & 193-39-5 & 10.3 & 3 & $10-11$ \\
\hline & Isophorone & $78-59-1$ & 10.3 & 3 & $10-11$ \\
\hline & N-Nitroso-di-n-propylamine & $621-64-7$ & 10.3 & 3 & $10-11$ \\
\hline & N-Nirrosodiphenylamine & $86-30-6$ & 10.3 & 3 & $10-11$ \\
\hline & Naphthalene & $91-20-3$ & 10.3 & 3 & $10-11$ \\
\hline & Nitrobenzene & $98-95-3$ & 10.3 & 3 & $10-11$ \\
\hline
\end{tabular}




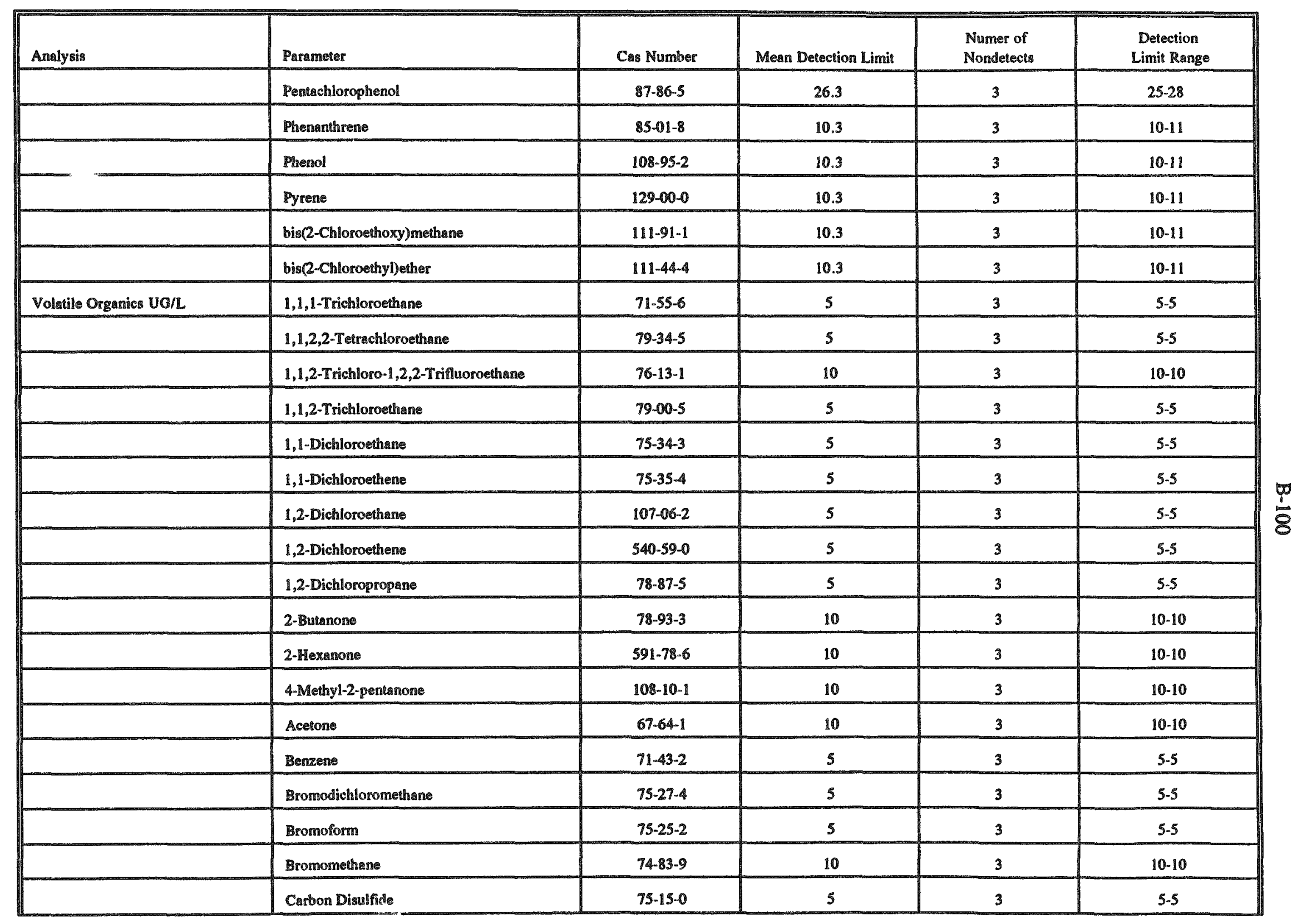




\begin{tabular}{|c|c|c|c|c|c|}
\hline Analysis & Parameter & Cas Number & Mean Defection Limit & $\begin{array}{l}\text { Numer of } \\
\text { Nondetects }\end{array}$ & $\begin{array}{l}\text { Detection } \\
\text { Limit Range }\end{array}$ \\
\hline & Carbon Tetrachloride & $56-23-5$ & 5 & 3 & $5-5$ \\
\hline & Chlorobenzene & $108-90-7$ & 5 & 3 & $5-5$ \\
\hline & Chloroethane & $75-00-3$ & 10 & 3 & $10-10$ \\
\hline & Chloromethane & $74-87-3$ & 10 & 3 & $10-10$ \\
\hline & Dibromochloromethane & $124-48-1$ & 5 & 3 & $5-5$ \\
\hline & Ethylbenzene & $100-41-4$ & 5 & 3 & $5-5$ \\
\hline & Methylene Chloride & $75-09-2$ & 5 & 3 & $5-5$ \\
\hline & Styrene & $100-42-5$ & 5 & 3 & $5-5$ \\
\hline & Toluene & $108-88-3$ & 5 & 3 & $5-5$ \\
\hline & Vinyl Chloride & $75-01-4$ & 10 & 3 & $10 \cdot 10$ \\
\hline & Xylene, Total & $1330-20-7$ & 5 & 3 & $5-5$ \\
\hline & cis-1,3-Dichloropropene & $10061-01-5$ & 5 & 3 & $5-5$ \\
\hline & trans-1,3-Dichloropropene & $10061-02-6$ & 5 & 3 & $5-5$ \\
\hline \multirow[t]{11}{*}{ Pesticide/PCB UG/L } & $4,4^{\circ}-\mathrm{DDD}$ & $72-54-8$ & 0.109 & 3 & $0.098-0.12$ \\
\hline & $4,4^{\prime}-\mathrm{DDE}$ & $72-55-9$ & 0.109 & 3 & $0.098-0.12$ \\
\hline & 4,4'-DDT & 50.29-3 & 0.109 & 3 & $0.098-0.12$ \\
\hline & Aldrin & $309-00-2$ & 0.054 & 3 & $0.049-0.06$ \\
\hline & Aroclor-1016 & $12674-11.2$ & 1.09 & 3 & $0.98-1.2$ \\
\hline & Aroclor-1221 & $11104-28-2$ & 2.17 & 3 & $2-2.4$ \\
\hline & Aroclor-1232 & $11141-16-5$ & 1.09 & 3 & $0.98-1.2$ \\
\hline & Aroclor-1242 & $53469-21.9$ & 1.09 & 3 & $0.98-1.2$ \\
\hline & Aroclor-1248 & $12672-29-6$ & 1.09 & 3 & $0.98-1.2$ \\
\hline & Aroclor-1254 & $11097-69.1$ & 1.09 & 3 & $0.98-1.2$ \\
\hline & Aroclor-1260 & $11096-82-5$ & 1.09 & 3 & $0.98-1.2$ \\
\hline
\end{tabular}




\begin{tabular}{|c|c|c|c|c|c|}
\hline Analysis & Parameter & Cas Number & Mean Detection Limit & $\begin{array}{l}\text { Numer of } \\
\text { Nondetects }\end{array}$ & $\begin{array}{c}\text { Detection } \\
\text { Limit Range }\end{array}$ \\
\hline & Dieldrin & $60-57-1$ & 0.109 & 3 & $0.098-0.12$ \\
\hline & Endosulfan I & $959-98-8$ & 0.054 & 3 & $0.049-0.06$ \\
\hline & Endosulfan II & $33213-65-9$ & 0.109 & 3 & $0.098-0.12$ \\
\hline & Endosulfan Sulfate & $1031-07-8$ & 0.109 & 3 & $0.098-0.12$ \\
\hline & Endrin & $72-20-8$ & 0.109 & 3 & $0.098-0.12$ \\
\hline . & Endrin Aldehyde & $7421-93-4$ & 0.109 & 3 & $0.098-0.12$ \\
\hline & Endrin Ketone & $53494-70-5$ & 0.109 & 3 & $0.098-0.12$ \\
\hline & Heptachlor & $76-44$ & 0.054 & 3 & $0.049-0.06$ \\
\hline & Heptachlor Epoxide & $1024-57-3$ & 0.054 & 3 & $0.049-0.06$ \\
\hline & Toxaphene & $8001-35-2$ & 5.4 & 3 & $4.9-6$ \\
\hline & alpha-BHC & $319-84-6$ & 0.054 & 3 & $0.049-0.06$ \\
\hline & alpha-Chlordane & $5103-71-9$ & 0.054 & 3 & $0.049-0.06$ \\
\hline & beta-BHC & $319-85-7$ & 0.054 & 3 & $0.049-0.06$ \\
\hline & delta-BHC & $319-86.8$ & 0.054 & 3 & $0.049-0.06$ \\
\hline & gamma-BHC (Lindane) & $58-89-9$ & 0.054 & 3 & $0.049-0.06$ \\
\hline & gamma-Chlordane & $5103-74-2$ & 0.054 & 3 & $0.049 \cdot 0.06$ \\
\hline & p,p'-Methoxychlor & $72-43-5$ & 0.54 & 3 & $0.49-0.6$ \\
\hline
\end{tabular}

Data Source : Bear Creek Valley OU2 1993 RI field activities validated dat

Raw data available through $Y-12$ OREIS

Data does not inelude QA/QC.

Results $<$ counting uncertainty were considered as nondetects for radionuclides 


\section{APPENDIX C}

Human Health Risk Assessment Data 



\section{C-3}

Table C.12. Summary of tentatively identified compounds found in the BCV OU2 soil data

\begin{tabular}{lccc}
\hline & $\begin{array}{c}\text { Number of } \\
\text { TICs } \\
\text { identified }\end{array}$ & $\begin{array}{c}\text { Number of } \\
\text { analytes on } \\
\text { target analyte list }\end{array}$ & $\begin{array}{c}\text { Ratio of } \\
\text { number of TICs } \\
\text { to number on TAL } \\
\text { (as a percentage) }\end{array}$ \\
\hline $\begin{array}{l}\text { Rust Spoil Area } \\
\text { (RSA) } \\
\text { (historical data) }\end{array}$ & 1 & 34 & 2.9 \\
$\begin{array}{l}\text { Rust Spoil Area } \\
\text { (RSA) }\end{array}$ & 23 & & 18.3 \\
$\begin{array}{l}\text { (current data) } \\
\text { SY-200 Area } \\
\text { (SY-200) } \\
\text { (current data) }\end{array}$ & 42 & 126 & 33.3 \\
$\begin{array}{l}\text { Spoil Area 1 } \\
\text { (SA-1) } \\
\text { (historical data) }\end{array}$ & 43 & 126 & \\
\hline
\end{tabular}

- TICs = Tentatively Identified Compounds

- $\mathrm{TAL}=$ Target Analyte List 


\section{$\mathrm{C}-4$}

Table C.1b. Tentatively identified compounds found in the BCV OU2 RSA soil data

\begin{tabular}{|c|c|c|c|c|c|}
\hline $\begin{array}{l}\text { Tentatively } \\
\text { identified } \\
\text { compound }\end{array}$ & $\begin{array}{c}\text { Number of } \\
\text { samples } \\
\text { where TICs } \\
\text { were found }\end{array}$ & $\begin{array}{l}\text { Number of } \\
\text { TICs } \\
\text { found in } \\
\text { samples }\end{array}$ & $\begin{array}{l}\text { Mean } \\
\text { TIC } \\
\text { conc. } \\
\text { (mg/kg) }\end{array}$ & $\begin{array}{l}\text { Minimum } \\
\text { TIC } \\
\text { conc. } \\
\text { (mg/kg) }\end{array}$ & $\begin{array}{l}\text { Maximum } \\
\text { TIC } \\
\text { conc. } \\
\text { (mg/kg) }\end{array}$ \\
\hline \multicolumn{6}{|c|}{ RSA: Current Data } \\
\hline $\begin{array}{l}\text { 2-FLUORO-4- } \\
\text { NITROPHENOL }\end{array}$ & 1 & 1 & 8.1E-02 & 8.1E-02 & $8.1 E-02$ \\
\hline 2-HEPTANONE & 1 & 1 & $1.2 \mathrm{E}-02$ & $1.2 \mathrm{E}-02$ & $1.2 \mathrm{E}-02$ \\
\hline 2-NONANONE & 1 & 1 & $2.1 \mathrm{E}-02$ & $2.1 \mathrm{E}-02$ & $2.1 E-02$ \\
\hline $\begin{array}{l}\text { 2-PHENYL- } \\
\text { NAPHTHALENE }\end{array}$ & 2 & 2 & $2.2 \mathrm{E}-01$ & $2.2 \mathrm{E}-01$ & $2.2 E-01$ \\
\hline $\begin{array}{l}\text { BUTANE,1,1'- } \\
\text { [OXYBIS }(2,1-E T H\end{array}$ & 1 & 1 & $4.9 \mathrm{E}+\infty$ & $4.9 \mathrm{E}+00$ & $4.9 \mathrm{E}+00$ \\
\hline $\begin{array}{l}\text { CYCLOPENTA(DEF)- } \\
\text { PHENANTHRENON }\end{array}$ & 1 & 1 & $1.8 \mathrm{E}-01$ & $1.8 E-01$ & $1.8 \mathrm{E}-01$ \\
\hline $\begin{array}{l}\text { DIMETHYLDIETHYL } \\
\text { PHENOL ISOMER }\end{array}$ & 1 & 1 & $1.8 \mathrm{E}+\infty$ & $1.8 \mathrm{E}+\infty 0$ & $1.8 \mathrm{E}+\infty$ \\
\hline $\begin{array}{l}\text { ETHANONE,1- } \\
\text { PHENYL. }\end{array}$ & 1 & 1 & $1.1 \mathrm{E}-01$ & $1.1 E-01$ & $1.1 \mathrm{E}-01$ \\
\hline NAPHTHALENE & 1 & 1 & $1.7 \mathrm{E}-02$ & $1.7 \mathrm{E}-02$ & $1.7 \mathrm{E}-02$ \\
\hline $\begin{array}{l}\text { PHOSPHORIC ACID, } \\
\text { TRIPHENYL E }\end{array}$ & 2 & 2 & $3.7 \mathrm{E}+00$ & $2.3 E+00$ & $5.1 E+00$ \\
\hline $\begin{array}{l}\text { PROPANOIC ACID, } \\
\text { 2-METHY, } 2 \text {. }\end{array}$ & 1 & 1 & $6.9 \mathrm{E}-02$ & $6.9 \mathrm{E}-02$ & $6.9 \mathrm{E}-02$ \\
\hline $\begin{array}{l}\text { SUBSTITUTED } \\
\text { BENZENE }\end{array}$ & 1 & 1 & $3.9 \mathrm{E}-02$ & $3.9 \mathrm{E}-02$ & $3.9 \mathrm{E}-02$ \\
\hline UNKNOWN & 23 & 80 & $4.59 \mathrm{E}-01$ & $6.0 \mathrm{E}-03$ & $11.0 \mathrm{E}+\infty 0$ \\
\hline UNKNOWN ALKANE & 1 & 1 & $4.9 \mathrm{E}-02$ & $4.9 \mathrm{E}-02$ & $4.9 \mathrm{E}-02$ \\
\hline $\begin{array}{l}\text { UNKNOWN } \\
\text { BENZOFLUORENE } \\
\text { ISOMER }\end{array}$ & 1 & 2 & 2.35E-01 & $1.9 \mathrm{E}-01$ & $2.8 \mathrm{E}-01$ \\
\hline $\begin{array}{l}\text { UNKNOWN } \\
\text { CARBOXYLIC ACID }\end{array}$ & 1 & 1 & $3.3 \mathrm{E}-01$ & $3.3 \mathrm{E}-01$ & $3.3 \mathrm{E}-01$ \\
\hline $\begin{array}{l}\text { UNRNOWN CYCLIC } \\
\text { HYDROCARBON }\end{array}$ & 4 & 7 & 1.82E-01 & $9.0 \mathrm{E}-03$ & $3.6 \mathrm{E}-01$ \\
\hline $\begin{array}{l}\text { UNKNOWN } \\
\text { CYCLOHEXANE } \\
\text { ISOMER }\end{array}$ & 1 & 1 & $8.7 \mathrm{E}-02$ & 8.7E-02 & $8.7 \mathrm{E}-02$ \\
\hline $\begin{array}{l}\text { UNKNOWN ESTER } \\
\text { HEXANEDIOIC ACID }\end{array}$ & 2 & 2 & $3.05 E-01$ & $2.3 \mathrm{E}-01$ & $3.8 \mathrm{E}-01$ \\
\hline $\begin{array}{l}\text { UNKNOWN } \\
\text { HYDROCARBON }\end{array}$ & 17 & 76 & $3.58 \mathrm{E}-01$ & $4.0 \mathrm{E}-02$ & $3.7 \mathrm{E}+00$ \\
\hline $\begin{array}{l}\text { UNKNOWN } \\
\text { METHYLPHENAN- } \\
\text { THRENE }\end{array}$ & 1 & 1 & $1.8 \mathrm{E}-01$ & $1.8 \mathrm{E}-01$ & $1.8 \mathrm{E}-01$ \\
\hline UNKNOWN PAH & 6 & 18 & $5.51 E-01$ & $7.2 E-02$ & $3.4 E+00$ \\
\hline
\end{tabular}




\section{C-5}

Table C.1b. (continued)

\begin{tabular}{lccccc}
\hline $\begin{array}{l}\text { Tentatively } \\
\text { identified } \\
\text { compound }\end{array}$ & $\begin{array}{c}\text { Number of } \\
\text { samples } \\
\text { where TICs } \\
\text { were found }\end{array}$ & $\begin{array}{c}\text { Number of } \\
\text { TICs } \\
\text { found in } \\
\text { samples }\end{array}$ & $\begin{array}{c}\text { Mean } \\
\text { TIC } \\
\text { conc. } \\
\text { (mg/kg) }\end{array}$ & $\begin{array}{c}\text { Minimum } \\
\text { TIC } \\
\text { conc. } \\
\text { (mg/kg) }\end{array}$ & $\begin{array}{c}\text { Maximum } \\
\text { TIC } \\
\text { conc. } \\
\text { (mg/kg) }\end{array}$ \\
\hline UNKNOWN PNA & 5 & 8 & $1.26 \mathrm{E}-01$ & $6.9 \mathrm{E}-02$ & $2.2 \mathrm{E}-01$
\end{tabular}

RSA: Historical Data

2-PROPANOL

8

8

- $\mathrm{TICS}=$ Tentatively Identified Compounds 


$$
\text { C.6 }
$$

Table C.1C. Tentatively identified compounds found in the BCV OU2 current SY-200 soil data

\begin{tabular}{|c|c|c|c|c|c|}
\hline $\begin{array}{l}\text { Tentatively } \\
\text { identified } \\
\text { compound }\end{array}$ & $\begin{array}{r}\text { Number of } \\
\text { samples } \\
\text { where TICs } \\
\text { were found }\end{array}$ & $\begin{array}{r}\text { Number of } \\
\text { TICs } \\
\text { found in } \\
\text { samples }\end{array}$ & $\begin{array}{r}\text { Mean } \\
\text { TIC } \\
\text { conc. } \\
(\mathrm{mg} / \mathrm{kg})\end{array}$ & $\begin{array}{r}\text { Minimum } \\
\text { TIC } \\
\text { conc. } \\
\text { (mg/kg) }\end{array}$ & $\begin{array}{r}\text { Maximum } \\
T I C \\
\text { conc. } \\
(\mathrm{mg} / \mathrm{kg})\end{array}$ \\
\hline \multicolumn{6}{|c|}{$S Y-200$} \\
\hline $\begin{array}{l}\text { ALDOL } \\
\text { CONDENSATION } \\
\text { PRODUCT }\end{array}$ & 11 & 11 & 2127.3 & 1700.0 & 2900.0 \\
\hline $\begin{array}{l}\text { BENZENE, } \\
\text { PENTACHLORO- }\end{array}$ & 1 & 1 & 120.0 & 120.0 & 120.0 \\
\hline $\begin{array}{l}\text { DECAHYORO- } \\
\text { NAPHTHALENE } \\
\text { ISOMER }\end{array}$ & 1 & 1 & 8.9 & 8.9 & 8.9 \\
\hline $\begin{array}{l}\text { DHETHYL } \\
\text { NAPHTHALENE } \\
\text { ISOMER }\end{array}$ & 1 & 1 & 150.0 & 150.0 & 150.0 \\
\hline $\begin{array}{l}\text { ETHYLMETHYL- } \\
\text { BENZENE ISOMER }\end{array}$ & 1 & 1 & 17.0 & 17.0 & 17.0 \\
\hline $\begin{array}{l}\text { HEXADECANOIC } \\
\text { ACID ESTER }\end{array}$ & 1 & 1 & 170.0 & 170.0 & 170.0 \\
\hline $\begin{array}{l}\text { HEXANEDIOIC ACID } \\
\text { ESTER }\end{array}$ & 1 & 1 & 82.0 & 82.0 & 82.0 \\
\hline $\begin{array}{l}\text { METHYL } \\
\text { ANTHRACENE } \\
\text { ISOMER }\end{array}$ & 2 & 3 & 2613.3 & 240.0 & 4200.0 \\
\hline $\begin{array}{l}\text { METHYL } \\
\text { PHENANTHRENE } \\
\text { ISOMER }\end{array}$ & 1 & 1 & 2500.0 & 2500.0 & 2500.0 \\
\hline $\begin{array}{l}\text { METHYL PYRENE } \\
\text { ISOMER }\end{array}$ & 3 & 5 & 3826.0 & 120.0 & 8400.0 \\
\hline $\begin{array}{l}\text { METHYLETHYL } \\
\text { BENZENE ISOMER }\end{array}$ & 1 & 1 & 320.0 & 320.0 & 320.0 \\
\hline $\begin{array}{l}\text { METHYLPROPYL- } \\
\text { BENZENE ISOMER }\end{array}$ & 1 & 1 & 21.0 & 21.0 & 21.0 \\
\hline NAPHTHALENE & 2 & 2 & 168.5 & 17.0 & 320.0 \\
\hline $\begin{array}{l}\text { OCTADECANOIC } \\
\text { ACID ESTER }\end{array}$ & 1 & 1 & 250.0 & 250.0 & 250.0 \\
\hline PCB ISOMER & 1 & 6 & 295.0 & 190.0 & 460.0 \\
\hline $\begin{array}{l}\text { PROPANOIC ACID } \\
\text { ISOMER }\end{array}$ & 2 & 2 & 405.0 & 400.0 & 410.0 \\
\hline $\begin{array}{l}\text { PROPANOIC ACID, } \\
\text { 2-METHYL, 1- }\end{array}$ & 17 & 17 & 267.6 & 80.0 & 420.0 \\
\hline $\begin{array}{l}\text { SUBSTITUTED } \\
\text { BENZENE }\end{array}$ & 5 & 8 & 71.8 & 8.0 & 230.0 \\
\hline $\begin{array}{l}\text { SUBSTITUTED } \\
\text { PHENANTHRENE }\end{array}$ & 1 & 1 & 8400.0 & 8400.0 & 8400.0 \\
\hline SULFUR COMPOUND & 1 & 1 & 2200.0 & 2200.0 & 2200.0 \\
\hline
\end{tabular}


Table C.1c (continued)

\begin{tabular}{|c|c|c|c|c|c|}
\hline $\begin{array}{l}\text { Tentatively } \\
\text { identified } \\
\text { compound }\end{array}$ & $\begin{array}{r}\text { Number of } \\
\text { samples } \\
\text { where TICs }^{\mathrm{a}} \\
\text { were found }\end{array}$ & $\begin{array}{r}\text { Number of } \\
\text { TICs } \\
\text { found in } \\
\text { samples }\end{array}$ & $\begin{array}{r}\text { Mean } \\
\text { TIC } \\
\text { conc. } \\
(\mathrm{mg} / \mathrm{kg})\end{array}$ & $\begin{array}{r}\text { Minimum } \\
T \mathrm{TC} \\
\text { conc. } \\
(\mathrm{mg} / \mathrm{kg})\end{array}$ & $\begin{array}{r}\text { Maximum } \\
\text { TIC } \\
\text { conc. } \\
(\mathrm{mg} / \mathrm{kg})\end{array}$ \\
\hline $\begin{array}{l}\text { UNKNOWN } \\
\text { TRIMETHYL- } \\
\text { NAPHTHALENE } \\
\text { ISOMER }\end{array}$ & 1 & 1 & 490.0 & 490.0 & 490.0 \\
\hline UNKNOWN & 49 & 102 & 259.7 & 6.0 & 4100.0 \\
\hline UNKNOWN ACID & 1 & 3 & 120.0 & 80.0 & 200.0 \\
\hline $\begin{array}{l}\text { UNINNOWN } \\
\text { ALCOHOL }\end{array}$ & 1 & 1 & 80.0 & 80.0 & 80.0 \\
\hline UNKNOWN ALKANE & 9 & 23 & 329.3 & 80.0 & 1300.0 \\
\hline $\begin{array}{l}\text { UNKNOWN } \\
\text { AROCLOR }\end{array}$ & 3 & 11 & 145.0 & 78.0 & 240.0 \\
\hline $\begin{array}{l}\text { UNKAOWN } \\
\text { AROMATIC }\end{array}$ & 1 & 1 & 150.0 & 150.0 & 150.0 \\
\hline $\begin{array}{l}\text { UNKNOWN } \\
\text { CARBOXYLIC ACID }\end{array}$ & 4 & 4 & 132.5 & 120.0 & 160.0 \\
\hline $\begin{array}{l}\text { UNKNOWN } \\
\text { COMPOUND }\end{array}$ & 2 & 2 & 1520.0 & 540.0 & 2500.0 \\
\hline $\begin{array}{l}\text { UNKNOWN CYCLIC } \\
\text { HYDROCARBON }\end{array}$ & 5 & 6 & 356.8 & 7.0 & 730.0 \\
\hline $\begin{array}{l}\text { UNLNOWN ESTER } \\
\text { CARBOXYLIC ACID }\end{array}$ & 3 & 3 & 106.0 & 78.0 & 150.0 \\
\hline $\begin{array}{l}\text { UNKNOWN ESTER } \\
\text { HEXANEDIOIC ACID }\end{array}$ & 6 & 6 & 930.0 & 120.0 & 4000.0 \\
\hline $\begin{array}{l}\text { UNKNOWN } \\
\text { HYDROCARBON }\end{array}$ & 49 & 220 & 234.4 & 6.0 & 2400.0 \\
\hline UNKNOWN KETONE & 6 & 7 & 155.4 & 83.0 & 280.0 \\
\hline UNKNOWN PAH & 3 & 11 & 114.3 & 78.0 & 280.0 \\
\hline UNIKNOWN PCB & 5 & 24 & 416.2 & 120.0 & 1200.0 \\
\hline $\begin{array}{l}\text { UNKNOWN } \\
\text { PHTHALATE }\end{array}$ & 1 & 1 & 82.0 & 82.0 & 82.0 \\
\hline $\begin{array}{l}\text { UNKNOWN } \\
\text { PHTHALATE } \\
\text { ESTER }\end{array}$ & 2 & 2 & 175.0 & 160.0 & 190.0 \\
\hline UNKNOWN PNA & 1 & 2 & 78.0 & 78.0 & 78.0 \\
\hline $\begin{array}{l}\text { UNKNOWN } \\
\text { POLYNUCLEAR } \\
\text { ACID } \\
\text { COMPOUND }\end{array}$ & 1 & 1 & 340.0 & 340.0 & 340.0 \\
\hline
\end{tabular}




\section{C-8}

Table C.1c (continued)

\begin{tabular}{lccrrr}
\hline $\begin{array}{l}\text { Tentatively } \\
\text { identified } \\
\text { compound }\end{array}$ & $\begin{array}{r}\text { Number of } \\
\text { samples } \\
\text { where TICs } \\
\text { were found }\end{array}$ & $\begin{array}{r}\text { Number of } \\
\text { TICs } \\
\text { found in } \\
\text { samples }\end{array}$ & $\begin{array}{r}\text { Mean } \\
\text { TIC } \\
\text { conc. } \\
\text { (mg/kg) }\end{array}$ & $\begin{array}{r}\text { Minimum } \\
\text { TIC } \\
\text { conc. } \\
(\text { mgg/kg) }\end{array}$ & $\begin{array}{r}\text { Maximum } \\
\text { TIC } \\
\text { conc. }\end{array}$ \\
\hline $\begin{array}{l}\text { UNKNOWN } \\
\text { POLYNUCLEAR } \\
\text { AROMATC }\end{array}$ & 7 & 38 & 1595.8 & 170.0 & 13000.0 \\
UNIKNOWN SILICONE & & & & & \\
\hline
\end{tabular}

- TICs = Tentatively Identified Compounds 
C-9

Table C.1d. Tentatively identified compounds found in the BCV OU2 historical SA-1 soil data

\begin{tabular}{|c|c|c|c|c|c|}
\hline $\begin{array}{l}\text { Tentatively } \\
\text { identified } \\
\text { compound }\end{array}$ & $\begin{array}{l}\text { Number of } \\
\text { samples } \\
\text { where TICs } \\
\text { were found }\end{array}$ & $\begin{array}{l}\text { Number } \\
\text { of TICs } \\
\text { found in } \\
\text { samples }\end{array}$ & $\begin{array}{c}\text { Mean } \\
\text { TIC } \\
\text { conc. } \\
\text { (mgkg) }\end{array}$ & $\begin{array}{l}\text { Minimum } \\
\text { TIC } \\
\text { conc. } \\
\text { (mg/kg) }\end{array}$ & $\begin{array}{c}\text { Maximum } \\
\text { TIC } \\
\text { conc. } \\
\text { (mg/kg) }\end{array}$ \\
\hline 1-HEXANOL, 2-ETHYL- & 2 & 2 & $1.21 \mathrm{E}+00$ & $3.10 \mathrm{E}-01$ & $2.10 \mathrm{E}+00$ \\
\hline 11H-BENZO(A)FLUORENE & 1 & 2 & $6.50 \mathrm{E}-01$ & $4.80 \mathrm{E}-01$ & $8.20 \mathrm{E}-01$ \\
\hline 2-PROPENOIC ACID, 2-METHYL- & 1 & 1 & $4.90 \mathrm{E}-01$ & $4.90 \mathrm{E}-01$ & $4.90 \mathrm{E}-01$ \\
\hline 3-FLUORANTHENAMINE & 1 & 1 & $4.90 \mathrm{E}-01$ & $4.90 \mathrm{E}-01$ & $4.90 \mathrm{E}-01$ \\
\hline 3-HEPTANONE, 2,4-DIMETHYL- & 1 & 1 & $1.90 \mathrm{E}+00$ & $1.90 \mathrm{E}+\infty 0$ & $1.90 E+00$ \\
\hline $\begin{array}{l}\text { 4H-CYCLOPENTA (DEF) } \\
\text { PHENANTHRE }\end{array}$ & 1 & 1 & $6.80 \mathrm{E}-01$ & $6.80 \mathrm{E}-01$ & $6.80 \mathrm{E}-01$ \\
\hline 7H-BENZ(DE)ANTHRACEN-7-ONE & 1 & 1 & $4.90 \mathrm{E}-01$ & $4.90 \mathrm{E}-01$ & $4.90 \mathrm{E}-01$ \\
\hline 9,10-ANTHRACENEDIONE & 1 & 1 & $4.70 \mathrm{E}-01$ & $4.70 \mathrm{E}-01$ & $4.70 \mathrm{E}-01$ \\
\hline 9-OCTADECENAMIDE, $(Z)$ & 4 & 4 & $3.45 E-01$ & $2.20 \mathrm{E}-01$ & $4.90 \mathrm{E}-01$ \\
\hline ACETIC ACID, ETHYL ESTER & 1 & 1 & $4.00 \mathrm{E}-02$ & $4.00 \mathrm{E}-02$ & $4.00 \mathrm{E}-02$ \\
\hline BENZ(A)ANTHRACENE, 8-METHYL- & 1 & 1 & $4.90 \mathrm{E}-01$ & $4.90 \mathrm{E}-01$ & $4.90 \mathrm{E}-01$ \\
\hline BENZENEACETIC ACID & 1 & 1 & $1.90 \mathrm{E}-01$ & $1.90 \mathrm{E}-01$ & $1.90 \mathrm{E}-01$ \\
\hline $\begin{array}{l}\text { BENZO(B)NAPHTHO(2,1-D)- } \\
\text { THIOPHENE }\end{array}$ & 1 & 1 & $7.30 \mathrm{E}-01$ & $7.30 \mathrm{E}-01$ & $7.30 \mathrm{E}-01$ \\
\hline BENZO(C)PHENANTHRENE & 1 & 1 & $5.70 \mathrm{E}-01$ & $5.70 \mathrm{E}-01$ & $5.70 \mathrm{E}-01$ \\
\hline BENZO(DFLUORANTHENE & 2 & 2 & $1.20 E+00$ & $1.10 \mathrm{E}+\infty$ & $1.30 \mathrm{E}+\infty 0$ \\
\hline BUTANE, 2-METHYL- & 3 & 3 & $9.73 \mathrm{E}-03$ & $7.20 E-03$ & $1.10 E-02$ \\
\hline CYCLOHEPTANE, METHYL- & 1 & 1 & $7.00 \mathrm{E}-03$ & $7.00 \mathrm{E}-03$ & $7.00 \mathrm{E}-03$ \\
\hline CYCLOHEXANE, 1,1,2-TRIMETHYL- & 1 & 1 & $8.00 \mathrm{E}-03$ & 8.00E-03 & 8.00E-03 \\
\hline CYCLOHEXANE, 1,2-DIMETHYL-TR & 1 & 1 & $8.70 E-03$ & $8.70 \mathrm{E}-03$ & $8.70 \mathrm{E}-03$ \\
\hline CYCLOHEXANE, 1,3-DIMETHYL-,TR & 1 & 1 & $6.60 \mathrm{E}-03$ & $6.60 \mathrm{E}-03$ & $6.60 \mathrm{E}-03$ \\
\hline CYCLOHEXANE, ETHYL- & 1 & 1 & $1.00 \mathrm{E}-02$ & $1.00 \mathrm{E}-02$ & $1.00 \mathrm{E}-02$ \\
\hline CYCLOOCTANE, 1,4-DIMETHYL-TR & 1 & 1 & $1.80 \mathrm{E}-02$ & $1.80 \mathrm{E}-02$ & $1.80 \mathrm{E}-02$ \\
\hline $\begin{array}{l}\text { D-GALACTITOL } \\
\text { 2-(ACETILMETHYL }\end{array}$ & 1 & 1 & $7.80 \mathrm{E}-01$ & $7.80 \mathrm{E}-01$ & $7.80 \mathrm{E}-01$ \\
\hline DECANE & 1 & 1 & $1.30 \mathrm{E}-02$ & $1.30 \mathrm{E}-02$ & $1.30 \mathrm{E}-02$ \\
\hline DECANOIC ACID, ALKYL ESTER & 1 & 3 & $4.10 E-01$ & $2.50 \mathrm{E}-01$ & $6.70 \mathrm{E}-01$ \\
\hline DODECANOIC ACID, ALKYL ESTER & 1 & 1 & $2.20 \mathrm{E}-01$ & $2.20 \mathrm{E}-01$ & $2.20 \mathrm{E}-01$ \\
\hline HEPTANE, 3-METHYLENE- & 1 & 1 & $4.00 \mathrm{E}-01$ & 4.00E-01 & $4.00 \mathrm{E}-01$ \\
\hline HEXADECANOICACID & 12 & 12 & $3.01 \mathrm{E}-01$ & $1.50 \mathrm{E}-01$ & $6.80 \mathrm{E}-01$ \\
\hline HEXANEDIOIC ACID, BIS(2-METHYL & 1 & 1 & $200 \mathrm{E}-01$ & $2.00 \mathrm{E}-01$ & $2.00 \mathrm{E}-01$ \\
\hline HEXANEDIOIC ACID, DIOCTYL ES & 11 & 11 & $6.90 \mathrm{E}+00$ & $2.50 \mathrm{E}-01$ & $2.00 \mathrm{E}+01$ \\
\hline $\begin{array}{l}\text { HEXANEDIOIC ACID, } \\
\text { MONO(2-ETHYL }\end{array}$ & 1 & 1 & $1.90 \mathrm{E}-01$ & $1.90 \mathrm{E}-01$ & $1.90 \mathrm{E}-01$ \\
\hline
\end{tabular}




\section{C-10}

Table C.1d. (comtimued)

\begin{tabular}{lccccc}
\hline $\begin{array}{l}\text { Tentatively } \\
\text { identified } \\
\text { compound }\end{array}$ & $\begin{array}{c}\text { Number of } \\
\text { samples } \\
\text { where TICs } \\
\text { were found }\end{array}$ & $\begin{array}{c}\text { Number } \\
\text { of TICs } \\
\text { found in } \\
\text { samples }\end{array}$ & $\begin{array}{c}\text { Mean } \\
\text { TIC } \\
\text { conc. } \\
\text { (mg/kg) }\end{array}$ & $\begin{array}{c}\text { Minimum } \\
\text { TIC } \\
\text { conc. } \\
\text { (mg/kg) }\end{array}$ & $\begin{array}{c}\text { Maximum } \\
\text { TIC } \\
\text { conc. } \\
\text { (mg/kg) }\end{array}$ \\
\hline $\begin{array}{l}\text { METHYL PHENANTHRENE } \\
\text { NONANE }\end{array}$ & 1 & 1 & $5.80 \mathrm{E}-01$ & $5.80 \mathrm{E}-01$ & $5.80 \mathrm{E}-01$ \\
NOT IDENTIFIED & 1 & 1 & $8.70 \mathrm{E}-03$ & $8.70 \mathrm{E}-03$ & $8.70 \mathrm{E}-03$ \\
PENTANAL & 1 & 1 & $3.20 \mathrm{E}-01$ & $3.20 \mathrm{E}-01$ & $3.20 \mathrm{E}-01$ \\
PENTANE (ACN) (DOT) & 1 & 1 & $8.00 \mathrm{E}-03$ & $8.90 \mathrm{E}-03$ & $8.90 \mathrm{E}-03$ \\
PHTHALIC ANHYDRIDE & 1 & 1 & $7.80 \mathrm{E}-03$ & $7.80 \mathrm{E}-03$ & $7.80 \mathrm{E}-03$ \\
PROPANOIC ACID, 2-METHYL-, 1- & 2 & 2 & $2.85 \mathrm{E}-01$ & $2.40 \mathrm{E}-01$ & $3.30 \mathrm{E}-01$ \\
TRICHLOROFLUOROMETHANE & 3 & 3 & $3.20 \mathrm{E}-01$ & $2.80 \mathrm{E}-01$ & $3.90 \mathrm{E}-01$ \\
UNKNOWN & 2 & 2 & $1.95 \mathrm{E}-03$ & $1.90 \mathrm{E}-03$ & $2.00 \mathrm{E}-03$ \\
\hline
\end{tabular}

- TICS = Tentatively Identified Compounds 
Table C.2. Summary statistics for BCV OU2 soils

\begin{tabular}{|c|c|c|c|c|c|c|c|c|}
\hline Analyte & $\begin{array}{l}\text { Frequency } \\
\text { of } \\
\text { detect }\end{array}$ & $\begin{array}{l}\text { Minimurn } \\
\text { detected } \\
\text { concentration }\end{array}$ & $\begin{array}{l}\text { Maximum } \\
\text { detected } \\
\text { concentration }\end{array}$ & Distribution & $\begin{array}{l}\text { Mean } \\
\text { concentration }\end{array}$ & $\begin{array}{l}\text { Upper } 95 \% \\
\text { confidence } \\
\text { limit on mean }\end{array}$ & $\begin{array}{l}\text { Maximum } \\
\text { nondetected } \\
\text { concentration }\end{array}$ & $\begin{array}{l}\text { Units } \\
\text { of } \\
\text { measure }\end{array}$ \\
\hline \multicolumn{9}{|c|}{ RSA } \\
\hline ALUMINUM & $23 / 23$ & $5.95 \mathrm{E}+03$ & $3.00 \mathrm{E}+04$ & $\mathbf{N}$ & $1.79 \mathrm{E}+04$ & $2.02 \mathrm{E}+04$ & & $\mathrm{mg} / \mathrm{kg}$ \\
\hline ANTIMONY & $3 / 6$ & $2.30 \mathrm{E}-01$ & $3.60 \mathrm{E}-01$ & L & $2.65 \mathrm{E}-01$ & $3.18 \mathrm{E}-01$ & & $\mathrm{mg} / \mathrm{kg}$ \\
\hline BERYLLIUM & $23 / 23$ & $1.40 \mathrm{E}-01$ & $2.10 \mathrm{E}+00$ & N & $9.11 \mathrm{E}-01$ & $1.05 \mathrm{E}+00$ & & $\mathrm{mg} / \mathrm{kg}$ \\
\hline CADMIUM & $16 / 23$ & $3.60 \mathrm{E}-01$ & $5.70 \mathrm{E}+00$ & L & $2.29 \mathrm{E}+\infty 0$ & $4.93 E+00$ & & $\mathrm{mg} / \mathrm{kg}$ \\
\hline CALCIUM & $21 / 23$ & $3.20 \mathrm{E}+02$ & $1.60 \mathrm{E}+05$ & L & $3.44 E+04$ & $6.22 E+04$ & & $\mathrm{mg} / \mathrm{kg}$ \\
\hline CHROMIUM & $23 / 23$ & $1.67 E+01$ & $1.13 \mathrm{E}+02$ & L & $3.14 E+01$ & $3.64 \mathrm{E}+01$ & & $\mathrm{mg} / \mathrm{kg}$ \\
\hline FLUORENE & $2 / 11$ & $7.10 \mathrm{E}-02$ & $8.30 \mathrm{E}-02$ & $\mathbf{N}$ & $3.52 \mathrm{E}-01$ & $4.46 \mathrm{E}-01$ & & $\mathrm{mg} / \mathrm{kg}$ \\
\hline IRON & $23 / 23$ & $1.16 \mathrm{E}+04$ & $4.60 \mathrm{E}+04$ & N & $2.77 \mathrm{E}+04$ & $3.11 E+04$ & & $\mathrm{mg} / \mathrm{kg}$ \\
\hline LEAD & $23 / 23$ & $1.11 \mathrm{E}+01$ & $1.50 \mathrm{E}+02$ & L & $4.18 E+01$ & $5.05 \mathrm{E}+01$ & & $\mathrm{mg} / \mathrm{kg}$ \\
\hline MAGNESIUM & $19 / 23$ & $8.70 \mathrm{E}+02$ & $3.40 \mathrm{E}+04$ & L & $6.03 E+03$ & $9.67 \mathrm{E}+03$ & & $\mathrm{mg} / \mathrm{kg}$ \\
\hline MANGANESE & $23 / 23$ & $1.53 \mathrm{E}+02$ & $2.80 \mathrm{E}+03$ & L & $6.97 \mathrm{E}+02$ & $8.99 \mathrm{E}+02$ & & $\mathrm{mg} / \mathrm{kg}$ \\
\hline MERCURY & $14 / 23$ & $6.00 \mathrm{E}-02$ & $7.90 \mathrm{E}+00$ & L & $1.12 \mathrm{E}+00$ & $2.00 E+00$ & & $\mathrm{mg} / \mathrm{kg}$ \\
\hline NICKEL & $23 / 23$ & $1.25 \mathrm{E}+01$ & $3.11 E+02$ & L & $3.48 E+01$ & $4.61 E+01$ & & $\mathrm{mg} / \mathrm{kg}$ \\
\hline NIOBIUM & $0 / 12$ & & & NT & 8.43E-01 & & $9.30 \mathrm{E}-01$ & $\mathrm{mg} / \mathrm{kg}$ \\
\hline PHOSPHORUS & $12 / 12$ & $2.40 E+02$ & $1.60 E+03$ & L & $6.57 \mathrm{E}+02$ & $9.78 E+02$ & & $\mathrm{mg} / \mathrm{kg}$ \\
\hline
\end{tabular}


Table C.2 (continued)

\begin{tabular}{|c|c|c|c|c|c|c|c|c|}
\hline Analyte & $\begin{array}{l}\text { Frequency } \\
\text { of } \\
\text { detect }\end{array}$ & $\begin{array}{l}\text { Minimum } \\
\text { detected } \\
\text { concentration }\end{array}$ & $\begin{array}{l}\text { Maximum } \\
\text { detected } \\
\text { concentration }\end{array}$ & Distribution & $\begin{array}{l}\text { Mean } \\
\text { concentration }\end{array}$ & $\begin{array}{l}\text { Upper } 95 \% \\
\text { confidence } \\
\text { limit on mean }\end{array}$ & $\begin{array}{l}\text { Maximum } \\
\text { nondetected } \\
\text { concentration }^{2}\end{array}$ & $\begin{array}{l}\text { Units } \\
\text { of } \\
\text { measure }\end{array}$ \\
\hline POTASSIUM & $23 / 23$ & $6.80 \mathrm{E}+02$ & $3.40 \mathrm{E}+03$ & $\mathbf{L}$ & $1.82 E+03$ & $2.15 E+03$ & & $\mathrm{mg} / \mathrm{kg}$ \\
\hline SELENIUM & $1 / 18$ & $1.20 \mathrm{E}+00$ & $1.20 \mathrm{E}+00$ & $\mathbb{L}$ & $5.58 \mathrm{E}-01$ & $6.69 \mathrm{E}-01$ & & $\mathrm{mg} / \mathrm{kg}$ \\
\hline SILVER & $8 / 23$ & $5.30 \mathrm{E}-01$ & $1.90 \mathrm{E}+00$ & $\mathrm{~L}$ & $7.01 \mathrm{E}-01$ & $1.00 E+00$ & & $\mathrm{mg} / \mathrm{kg}$ \\
\hline SODIUM & $12 / 23$ & $2.70 \mathrm{E}+01$ & $7.90 E+02$ & $\mathrm{~L}$ & $8.97 E+01$ & $1.31 E+02$ & & $\mathrm{mg} / \mathrm{kg}$ \\
\hline THALLIUM & $4 / 11$ & $1.20 \mathrm{E}-01$ & $1.80 \mathrm{E}-01$ & $\mathbf{N}$ & $2.24 \mathrm{E}-01$ & $2.57 \mathrm{E}-01$ & & $\mathrm{mg} / \mathrm{kg}$ \\
\hline THORIUM, TOTAL & $2 / 12$ & $2.90 \mathrm{E}+01$ & $3.00 E+01$ & $\mathbb{L}$ & $2.00 \mathrm{E}+01$ & $2.83 E+01$ & & $\mathrm{mg} / \mathrm{kg}$ \\
\hline URANIUM, TOTAL & $12 / 12$ & $3.80 \mathrm{E}+00$ & $1.20 \mathrm{E}+01$ & L & $6.08 \mathrm{E}+00$ & $7.36 \mathrm{E}+00$ & & $\mathrm{mg} / \mathrm{kg}$ \\
\hline VANADIUM & $23 / 23$ & $1.03 E+01$ & $7.67 E+01$ & $\mathbf{L}$ & $3.64 \mathrm{E}+01$ & $4.39 \mathrm{E}+01$ & & $\mathrm{mg} / \mathrm{kg}$ \\
\hline ZINC & $19 / 23$ & $4.60 E+01$ & $2.74 E+02$ & $\mathbf{N}$ & $1.01 E+02$ & $1.28 E+02$ & & $\mathrm{mg} / \mathrm{kg}$ \\
\hline \multicolumn{9}{|l|}{ Radionuclides } \\
\hline PCT URANIUM-235 & $1 / 1$ & $5.00 \mathrm{E}-01$ & $5.00 \mathrm{E}-01$ & NT & $5.00 \mathrm{E}-01$ & & & WT\% \\
\hline PCT URANIUM-238 & $1 / 1$ & $9.95 E+01$ & $9.95 \mathrm{E}+01$ & NT & $9.95 E+01$ & & & WT\% \\
\hline THORIUM-232 & $2 / 12$ & $3.17 E+00$ & $3.28 \mathrm{E}+00$ & $\mathbb{L}$ & $2.19 \mathrm{E}+\infty 0$ & $3.09 \mathrm{E}+00$ & & $\mathrm{pCi} / \mathrm{g}$ \\
\hline URANIUM-234 & $23 / 23$ & $1.04 E+00$ & $7.91 E+\infty$ & $\mathbf{L}$ & $2.33 \mathrm{E}+\infty$ & $2.81 E+\infty$ & & $\mathrm{pCi} / \mathrm{g}$ \\
\hline URANIUM-235 & $23 / 23$ & $1.18 \mathrm{E}-02$ & $4.03 \mathrm{E}-01$ & $\mathbf{L}$ & $1.17 \mathrm{E}-01$ & $1.59 \mathrm{E}-01$ & & $\mathrm{pCi} / \mathrm{s}$ \\
\hline URANIUM-238 & $23 / 23$ & $1.01 E+00$ & $4.30 \mathrm{E}+00$ & $\mathbb{L}$ & $2.14 \mathrm{E}+00$ & $2.52 E+00$ & & $\mathrm{pCl} / \mathrm{g}$ \\
\hline \multicolumn{9}{|l|}{ Organics } \\
\hline 1,1,1-TRICHLOROETHANE & $1 / 23$ & $1.70 \mathrm{E}-01$ & $1.70 \mathrm{E}-01$ & $\mathbb{L}$ & $3.43 \mathrm{E} \cdot 02$ & $6.15 \mathbb{E}-02$ & & $\mathrm{mg} / \mathrm{kg}$ \\
\hline 1,1,2,2-TETRACHLOROETHANE & $0 / 23$ & & & NT & $7.71 \mathrm{E}-02$ & & $1.50 \mathrm{E}+\infty$ & $\mathrm{mg} / \mathrm{kg}$ \\
\hline
\end{tabular}


Table C.2 (continued)

\begin{tabular}{|c|c|c|c|c|c|c|c|c|}
\hline Analyne & $\begin{array}{l}\text { Frequency } \\
\text { of } \\
\text { detect }\end{array}$ & $\begin{array}{l}\text { Minimum } \\
\text { detected } \\
\text { concentration }\end{array}$ & $\begin{array}{l}\text { Maximum } \\
\text { delected } \\
\text { concentration }\end{array}$ & Distribution & $\begin{array}{l}\text { Mean } \\
\text { concentration }\end{array}$ & $\begin{array}{l}\text { Upper } 95 \% \\
\text { confidence } \\
\text { limit on mean }\end{array}$ & $\begin{array}{l}\text { Maximum } \\
\text { nondetected } \\
\text { concentration }\end{array}$ & $\begin{array}{l}\text { Units } \\
\text { of } \\
\text { measure }\end{array}$ \\
\hline $\begin{array}{l}\text { 1,1,2-TRICHLORO-1,2,2- } \\
\text { TRIFLUOROETHANE }\end{array}$ & $4 / 11$ & $3.70 \mathrm{E}-02$ & $3.20 E+01$ & L & $2.91 \mathrm{E}-02$ & 4.64E-01 & & $\mathrm{mg} / \mathrm{kg}$ \\
\hline 1,1,2-TRICHLOROETHANE & $0 / 23$ & & & NT & $7.71 \mathrm{E}-02$ & & $1.50 \mathrm{E}+00$ & $\mathrm{mg} / \mathrm{kg}$ \\
\hline 1,1-DICHLOROETHANE & $2 / 23$ & $1.00 \mathrm{E}-02$ & $6.40 \mathrm{E}-01$ & L & $2.26 \mathrm{E}-04$ & 3.82E-02 & & $\mathrm{mg} / \mathrm{kg}$ \\
\hline 1,2-DICHLOROBENZENE & $1 / 11$ & $3.60 \mathrm{E}-01$ & $3.60 \mathrm{E}-01$ & $\mathbb{N}$ & $3.74 \mathrm{E}-01$ & $3.84 \mathrm{E}-01$ & & $\mathrm{mg} / \mathrm{kg}$ \\
\hline 1,2-DICHLOROETHANE & $1 / 23$ & 3.00E-03 & $3.00 \mathrm{E}-03$ & $\mathbf{L}$ & 2.63E-02 & 4.51E-02 & & $\mathrm{mg} / \mathrm{kg}$ \\
\hline 1,2-DICHLOROETHENE (TOTAL) & $4 / 23$ & $3.70 \mathrm{E}-02$ & $1.10 \mathrm{E}+02$ & $\mathbf{L}$ & $3.21 \mathrm{E}-04$ & $7.22 \mathrm{E}-02$ & & $\mathrm{mg} / \mathrm{kg}$ \\
\hline 1,2-DICHLOROPROPANE & $0 / 23$ & & & NT & $7.71 \mathrm{E}-02$ & & $1.50 \mathrm{E}+\infty$ & $\mathrm{mg} / \mathrm{kg}$ \\
\hline 1,3-DICHLOROBENZENE & $0 / 11$ & & & NT & $4.06 \mathrm{E}-01$ & & $7.20 \mathrm{E}-01$ & $\mathrm{mg} / \mathrm{kg}$ \\
\hline 2,4-DICHLOROPHENOL & $0 / 11$ & & & NT & $4.06 \mathrm{E}-01$ & & $7.20 \mathrm{E}-01$ & $\mathrm{mg} / \mathrm{kg}$ \\
\hline 2,4-DIMETHYLPHENOL & $1 / 11$ & 3.40E-01 & $3.40 \mathrm{E}-01$ & $\mathbb{N}$ & 3.72E-01 & 3.83E-01 & & $\mathrm{mg} / \mathrm{kg}$ \\
\hline 2,4-DINITROPHENOL & on1 & & & NT & $9.85 \mathrm{E}-01$ & & $1.70 \mathrm{E}+00$ & $\mathrm{mg} / \mathrm{kg}$ \\
\hline 2,4-DINITROTOLUENE & $0 / 11$ & & & NT & $4.06 \mathrm{E}-01$ & & 7.20E-01 & $\mathrm{mg} / \mathrm{kg}$ \\
\hline 2,6-DINITROTOLUENE & $0 / 11$ & & & NT & $4.06 \mathrm{E}-01$ & & $7.20 \mathrm{E}-01$ & $\mathrm{mg} / \mathrm{kg}$ \\
\hline 2-BUTANONE & $4 / 23$ & $5.00 \mathrm{E}-03$ & $2.70 \mathrm{E}-01$ & L & $8.63 \mathrm{E}-03$ & $2.75 \mathrm{E}-02$ & & $\mathrm{mg} / \mathrm{kg}$ \\
\hline 2-CHLORONAPHTHALENE & $0 / 11$ & & & NT & $4.06 \mathrm{E}-01$ & & $7.20 \mathrm{E}-01$ & $\mathrm{mg} / \mathrm{kg}$ \\
\hline
\end{tabular}


Table C.2 (comtimued)

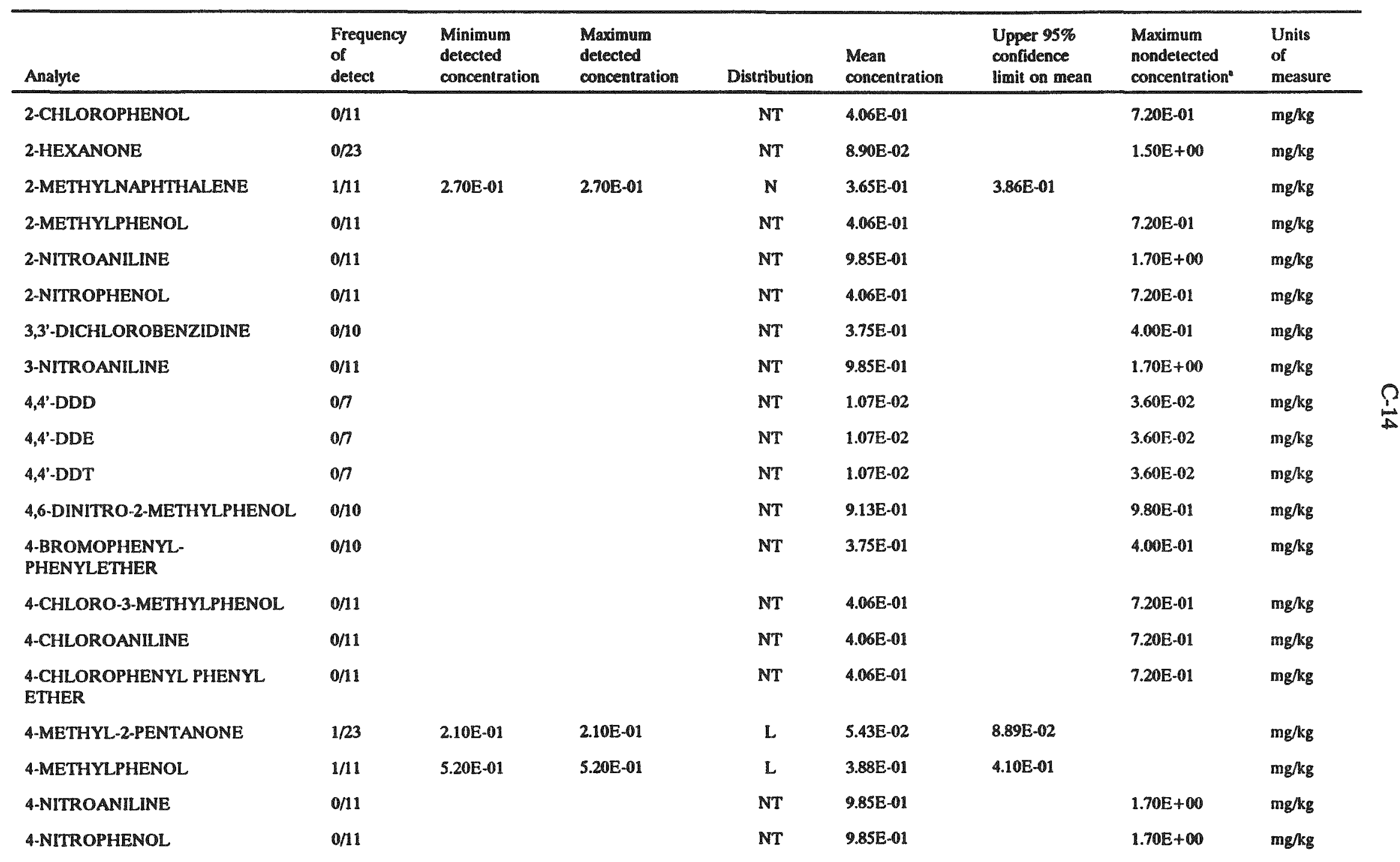


Table C.2 (continued)

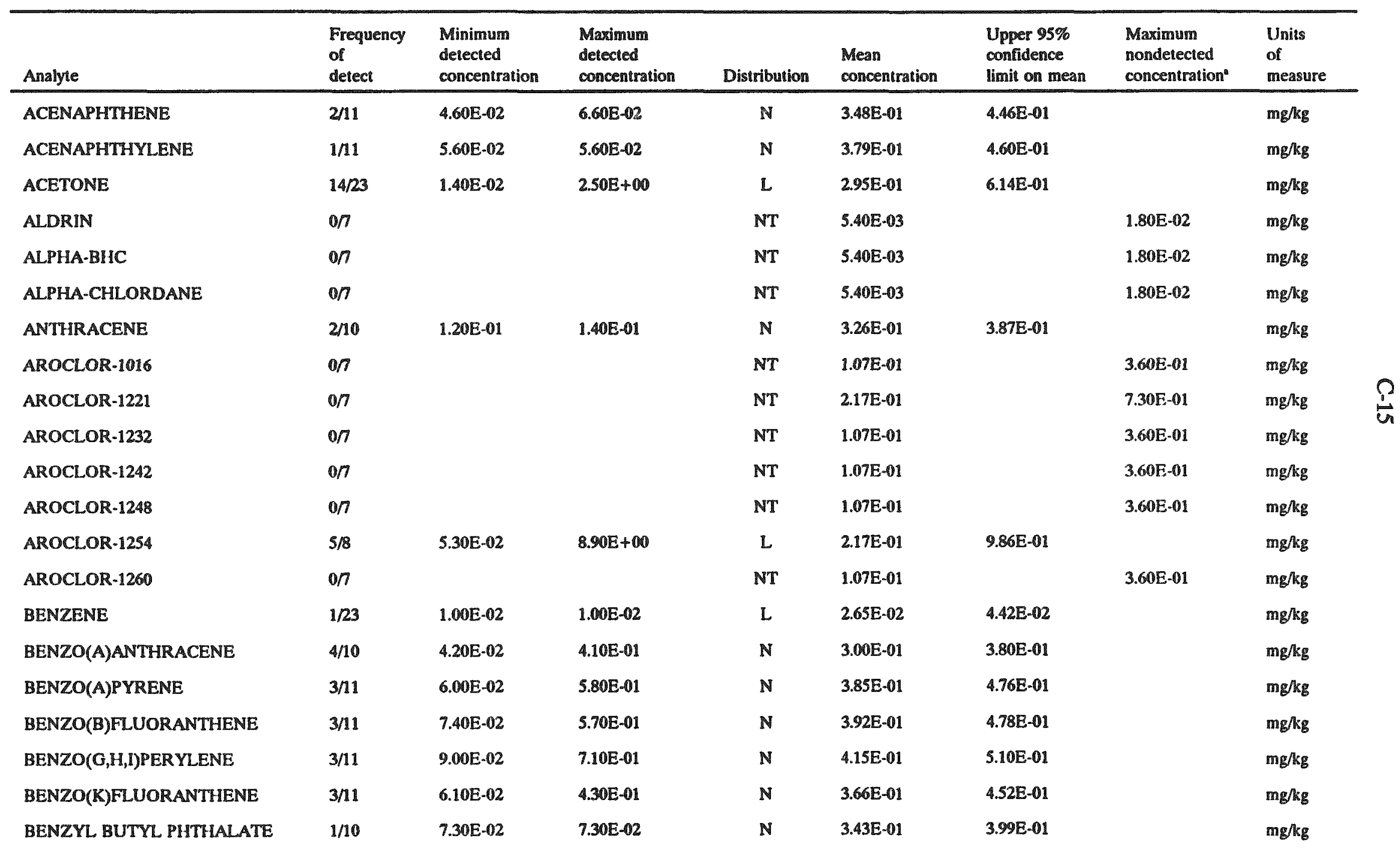


Table C.2. (comtinued)

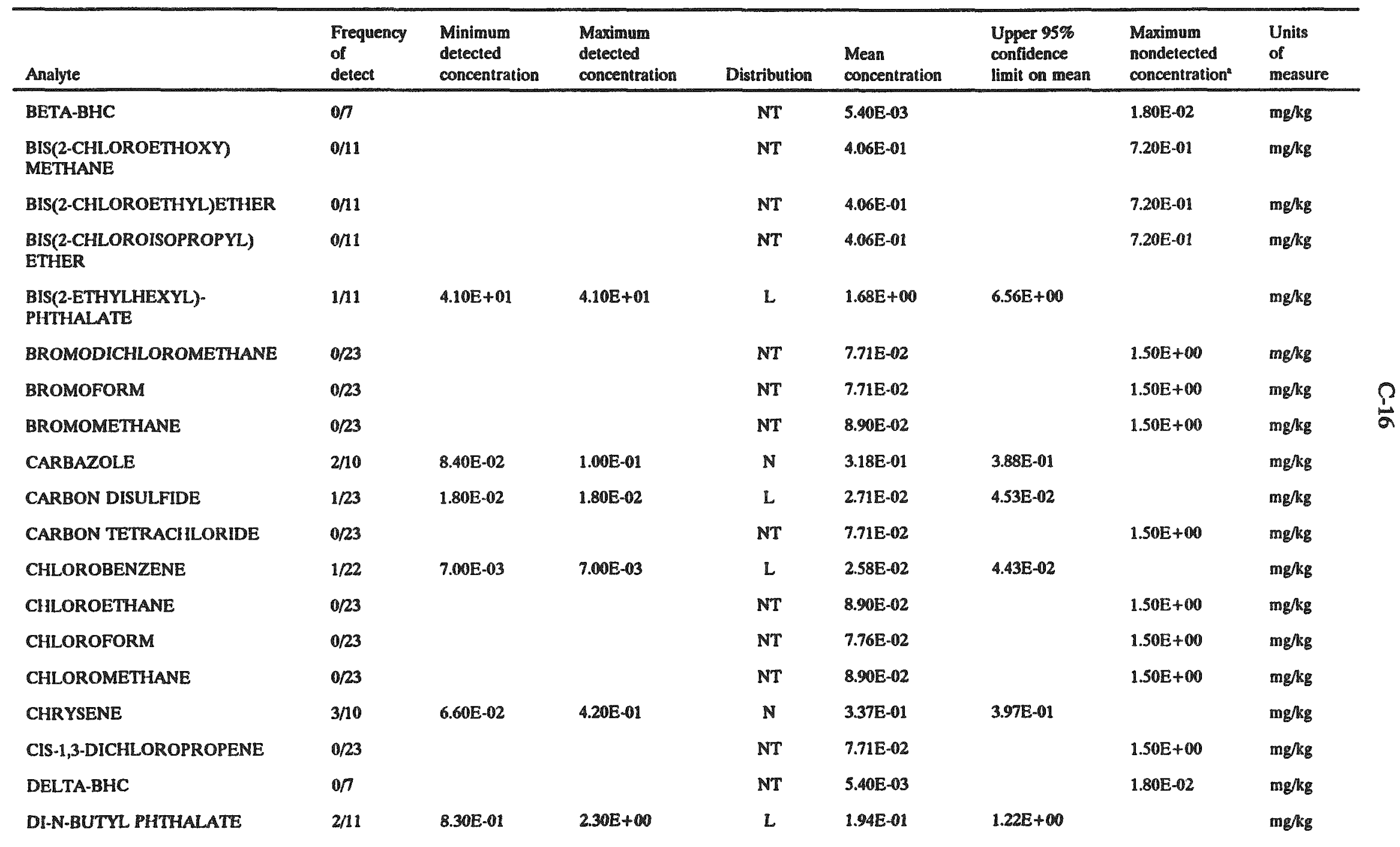


Table C.2. (continued)

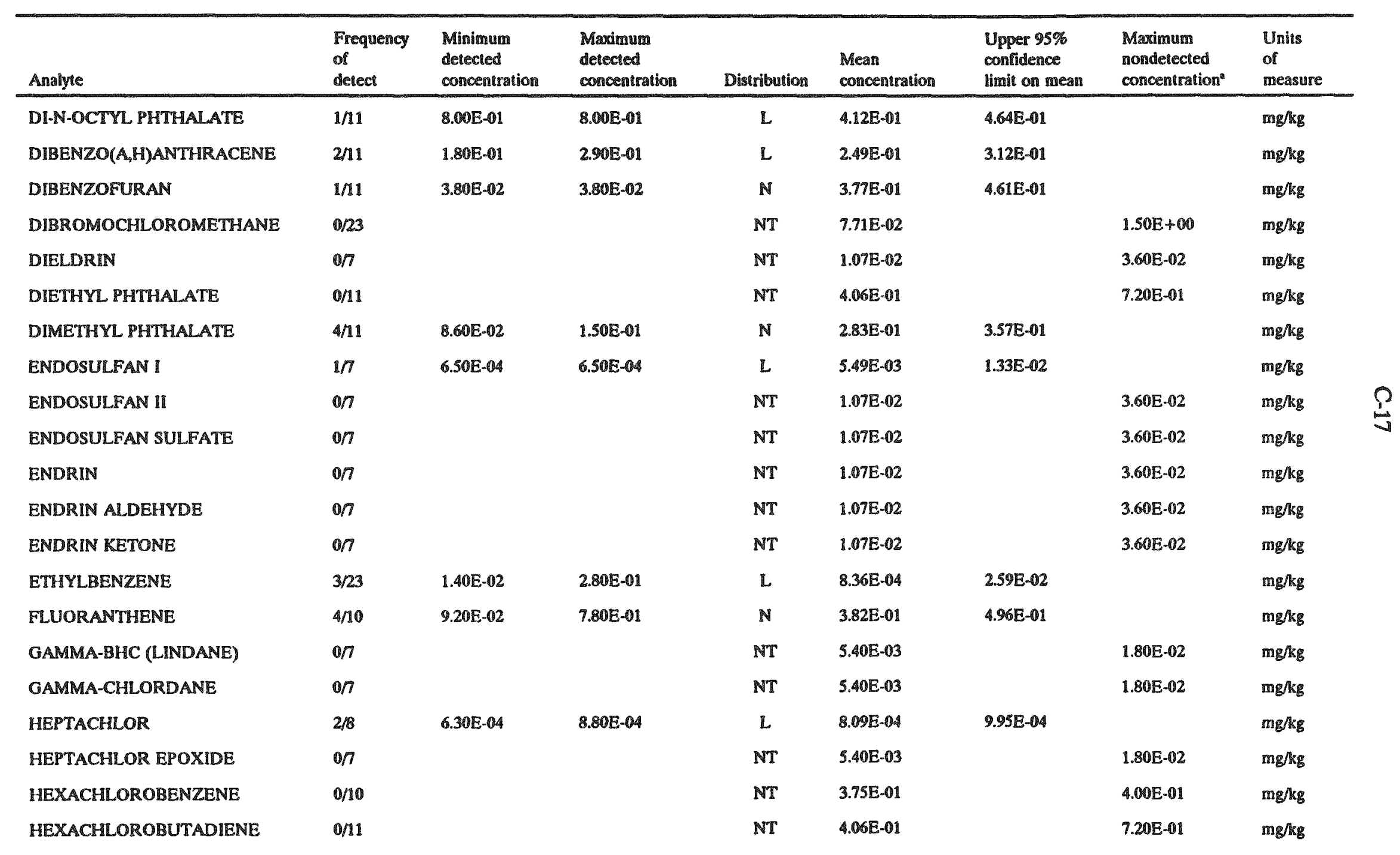


Table C.2 (continued)

\begin{tabular}{|c|c|c|c|c|c|c|c|c|}
\hline Analyte & $\begin{array}{l}\text { Frequency } \\
\text { of } \\
\text { detect }\end{array}$ & $\begin{array}{l}\text { Minimum } \\
\text { detected } \\
\text { concentration }\end{array}$ & $\begin{array}{l}\text { Maximum } \\
\text { detected } \\
\text { concentration }\end{array}$ & Distribution & $\begin{array}{l}\text { Mean } \\
\text { concentration }\end{array}$ & $\begin{array}{l}\text { Upper } 95 \% \\
\text { confidence } \\
\text { limit on mean }\end{array}$ & $\begin{array}{l}\text { Maximum } \\
\text { nondetected } \\
\text { concentration }^{2}\end{array}$ & $\begin{array}{l}\text { Units } \\
\text { of } \\
\text { measure }\end{array}$ \\
\hline $\begin{array}{l}\text { HEXACHLOROCYCLO. } \\
\text { PENTADIENE }\end{array}$ & $0 / 11$ & & & NT & $4.06 \mathrm{E}-01$ & & $7.20 \mathrm{E}-01$ & $\mathrm{mg} / \mathrm{kg}$ \\
\hline HEXACHLOROETHANE & $0 / 11$ & & & NT & $4.06 \mathrm{E}-01$ & & $7.20 \mathrm{E}-01$ & $\mathrm{mg} / \mathrm{kg}$ \\
\hline INDENO(1,2,3-CD)PYRENE & $3 / 11$ & $7.40 \mathrm{E}-02$ & $8.40 \mathrm{E}-01$ & $\mathbf{N}$ & $4.14 \mathrm{E}-01$ & $5.25 \mathrm{E}-01$ & & $\mathrm{mg} / \mathrm{kg}$ \\
\hline ISOPHORONE & $0 / 11$ & & & $\mathbb{N T}$ & $4.06 \mathrm{E}-01$ & & $7.20 \mathrm{E}-01$ & $\mathrm{mg} / \mathrm{kg}$ \\
\hline METHYLENE CHLORIDE & $5 / 23$ & $1.20 \mathrm{E}-02$ & $5.80 \mathrm{E}+01$ & L & $3.74 E-03$ & 4.45E-02 & & $\mathrm{mg} / \mathrm{kg}$ \\
\hline $\begin{array}{l}\text { N-NITROSO-DI-N- } \\
\text { PROPYLAMINE }\end{array}$ & $0 / 11$ & & & NT & $4.06 \mathrm{E}-01$ & & $7.20 \mathrm{E}-01$ & $\mathrm{mg} / \mathrm{kg}$ \\
\hline N-NITROSODIPHENYLAMINE & $0 / 10$ & & & NT & $3.75 \mathrm{E}-01$ & & $4.00 \mathrm{E}-01$ & $\mathrm{mg} / \mathrm{kg}$ \\
\hline NAPHTHALENE & $1 / 11$ & $9.00 \mathrm{E}-02$ & $9.00 \mathrm{E}-02$ & $\mathbf{N}$ & $3.49 \mathrm{E}-01$ & $3.97 \mathrm{E}-01$ & & $\mathrm{mg} / \mathrm{kg}$ \\
\hline NITROBENZENE & $0 / 11$ & & & NT & $4.06 \mathrm{E}-01$ & & $7.20 \mathrm{E}-01$ & $\mathrm{mg} / \mathrm{kg}$ \\
\hline P,P'-METHOXYCHLOR & $1 / n$ & $1.50 \mathrm{E}-03$ & $1.50 \mathrm{E}-03$ & L & $7.46 \mathrm{E}-02$ & $2.87 \mathrm{E}-01$ & & $\mathrm{mg} / \mathrm{kg}$ \\
\hline PENTACHLOROPHENOL & $1 / 10$ & $4.70 \mathrm{E}-02$ & $4.70 \mathrm{E}-02$ & $\mathbf{N}$ & $8.30 \mathrm{E}-01$ & $9.91 \mathrm{E}-01$ & & $\mathrm{mg} / \mathrm{kg}$ \\
\hline PHENANTHRENE & $5 / 1$ & $6.20 \mathrm{E}-02$ & $9.20 \mathrm{E}-01$ & $\mathbb{N}$ & $3.99 \mathrm{E}-01$ & $5.26 \mathrm{E}-01$ & & $\mathrm{mg} / \mathrm{kg}$ \\
\hline PHENOL & $1 / 11$ & $2.50 \mathrm{E}+00$ & $2.50 \mathrm{E}+\infty 0$ & $L$ & $5.25 \mathrm{E}-01$ & 7.13E-01 & & $\mathrm{mg} / \mathrm{kg}$ \\
\hline PYRENE & $5 / 10$ & $3.90 \mathrm{E} \cdot 02$ & $1.10 \mathrm{E}+00$ & $\mathbf{L}$ & $2.52 \mathrm{E}-01$ & $5.23 \mathrm{E}-01$ & & $\mathrm{mg} / \mathrm{kg}$ \\
\hline STYRENE & $0 / 23$ & & & NT & $7.71 \mathrm{E}-02$ & & $1.50 \mathrm{E}+00$ & $\mathrm{mg} / \mathrm{kg}$ \\
\hline TETRACHLOROETHENE & $5 / 23$ & $7.20 \mathrm{E}-02$ & $4.00 \mathrm{E}+02$ & $\mathbf{L}$ & $9.46 \mathrm{E}-04$ & $1.62 \mathrm{E}-01$ & & $\mathrm{mg} / \mathrm{kg}$ \\
\hline TOLUENE & $4 / 23$ & $3.00 \mathrm{E}-03$ & $7.50 \mathrm{E}-01$ & $L$ & $1.81 \mathrm{E}-03$ & $1.87 \mathrm{E}-02$ & & $\mathrm{mg} / \mathrm{kg}$ \\
\hline TOXAPHENE & on & & & $\mathrm{NT}$ & $5.40 \mathrm{E}-01$ & & $1.80 \mathrm{E}+00$ & $\mathrm{mg} / \mathrm{kg}$ \\
\hline TRANS-1,3-DICUI OROPROPENE & $0 / 23$ & & & NT & $7.71 \mathrm{E}-02$ & & $1.50 \mathrm{E}+\infty 0$ & $\mathrm{mg} / \mathrm{kg}$ \\
\hline TRICHLOROE THENE & $3 / 23$ & $2.20 \mathrm{E}-01$ & $1.00 \mathrm{E}+02$ & $\mathbf{L}$ & $3.17 \mathrm{E}-05$ & $9.70 \mathrm{E}-02$ & & $\mathrm{mg} / \mathrm{kg}$ \\
\hline
\end{tabular}


Table C.2 (contimued)

\begin{tabular}{|c|c|c|c|c|c|c|c|c|}
\hline Analyte & $\begin{array}{l}\text { Frequency } \\
\text { of } \\
\text { detect }\end{array}$ & $\begin{array}{l}\text { Minimum } \\
\text { detected } \\
\text { concentration }\end{array}$ & $\begin{array}{l}\text { Maximum } \\
\text { detected } \\
\text { concentration }\end{array}$ & Distribution & $\begin{array}{l}\text { Mean } \\
\text { concentration }\end{array}$ & $\begin{array}{l}\text { Upper } 95 \% \\
\text { confidence } \\
\text { limit on mean }\end{array}$ & $\begin{array}{l}\text { Maximum } \\
\text { nondetected } \\
\text { concentration }\end{array}$ & $\begin{array}{l}\text { Units } \\
\text { of } \\
\text { measure }\end{array}$ \\
\hline VINYL ACETATE & $0 / 12$ & & & NT & $3.23 \mathrm{E}-02$ & & $7.60 \mathrm{E}-02$ & $\mathrm{mg} / \mathrm{kg}$ \\
\hline VINYL CHLORIDE & $0 / 23$ & & & NT & $8.90 \mathrm{E}-02$ & & $1.50 \mathrm{E}+00$ & $\mathrm{mg} / \mathrm{kg}$ \\
\hline XYLENE (TOTAL) & $2 / 23$ & $2.50 \mathrm{E}-02$ & $4.60 \mathrm{E}-01$ & L & $2.20 \mathrm{E}-04$ & $4.61 \mathrm{E} \cdot 02$ & & $\mathrm{mg} / \mathrm{kg}$ \\
\hline
\end{tabular}

SY-200

Inorganics

\begin{tabular}{|c|c|c|c|c|c|c|}
\hline ALUMINUM & $75 / 89$ & $6.00 E+03$ & $2.66 \mathrm{E}+04$ & $\mathbb{N}$ & $1.65 E+04$ & $1.73 \mathrm{E}+04$ \\
\hline ANTIMONY & $37 / 60$ & $2.20 \mathrm{E}-01$ & $9.20 \mathrm{E}-01$ & $\mathrm{~L}$ & $4.12 \mathrm{E}-01$ & $4.59 \mathrm{E}-01$ \\
\hline ARSENIC & $70 / 89$ & $1.80 \mathrm{E}+\infty 0$ & $6.92 \mathrm{E}+01$ & L & $1.57 \mathrm{E}+01$ & $2.10 \mathrm{E}+01$ \\
\hline BARIUM & $75 / 89$ & $8.50 E+00$ & $2.77 \mathrm{E}+02$ & $\mathbb{N}$ & $7.40 \mathrm{E}+01$ & $8.37 \mathrm{E}+01$ \\
\hline BERYLLIUM & $75 / 89$ & $3.00 \mathrm{E}-01$ & $1.70 \mathrm{E}+00$ & $\mathbb{N}$ & $9.14 \mathrm{E}-01$ & $9.83 E-01$ \\
\hline CADMIUM & $12 / 84$ & $1.20 \mathrm{E}-01$ & $3.10 E+00$ & $\mathbb{N}$ & $2.56 \mathrm{E}-01$ & $3.24 \mathrm{E}-01$ \\
\hline CALCIUM & $75 / 89$ & $3.07 \mathrm{E}+02$ & $5.80 \mathrm{E}+04$ & L & $9.61 \mathrm{E}+03$ & $1.21 \mathrm{E}+04$ \\
\hline CHROMUUM & $75 / 89$ & $1.74 \mathrm{E}+01$ & $4.61 E+01$ & L & $2.91 \mathrm{E}+01$ & $3.01 \mathrm{E}+01$ \\
\hline COBALT & $73 / 89$ & $2.80 \mathrm{E}+00$ & $9.88 \mathrm{E}+01$ & $\mathbb{L}$ & $1.57 \mathrm{E}+01$ & $1.78 \mathrm{E}+01$ \\
\hline COPPER & $75 / 89$ & $7.70 \mathrm{E}+00$ & $6.69 \mathrm{E}+01$ & $\mathbf{L}$ & $3.10 \mathrm{E}+01$ & $3.36 \mathrm{E}+01$ \\
\hline CYANIDE & $0 / 31$ & & & $\mathbb{N T}$ & $6.08 \mathrm{E}-01$ & \\
\hline FLUORENE & $5 / 32$ & $5.80 \mathrm{E}-02$ & $1.30 \mathrm{E}-01$ & $\mathbb{N}$ & $3.74 \mathrm{E}-01$ & 4.12E-01 \\
\hline IRON & $75 / 89$ & $1.47 \mathrm{E}+04$ & $4.28 \mathrm{E}+04$ & $\mathrm{~L}$ & $3.00 \mathrm{E}+04$ & $3.11 \mathrm{E}+04$ \\
\hline LEAD & $75 / 89$ & $1.44 \mathrm{E}+01$ & $3.89 \mathrm{E}+02$ & $\mathbf{L}$ & $4.61 E+01$ & $5.16 \mathrm{E}+01$ \\
\hline MAGNESIUM & $75 / 89$ & $2.31 E+02$ & $1.74 E+04$ & $\mathbf{L}$ & $2.81 E+03$ & $3.39 \mathrm{E}+03$ \\
\hline
\end{tabular}


Table C.2. (continued)

\begin{tabular}{|c|c|c|c|c|c|c|c|c|}
\hline Analyte & $\begin{array}{l}\text { Frequency } \\
\text { of } \\
\text { detect }\end{array}$ & $\begin{array}{l}\text { Minimum } \\
\text { detected } \\
\text { concentration }\end{array}$ & $\begin{array}{l}\text { Maximum } \\
\text { detected } \\
\text { concentration }\end{array}$ & Distribution & $\begin{array}{l}\text { Mean } \\
\text { concentration }\end{array}$ & $\begin{array}{l}\text { Upper } 95 \% \\
\text { confidence } \\
\text { limit on mean }\end{array}$ & $\begin{array}{l}\text { Maximum } \\
\text { nondetected } \\
\text { concentration }^{\mathrm{a}}\end{array}$ & $\begin{array}{l}\text { Units } \\
\text { of } \\
\text { measure }\end{array}$ \\
\hline MANGANESE & $75 / 89$ & $8.85 E+01$ & $3.23 E+03$ & $L$ & $6.32 \mathrm{E}+02$ & $7.32 \mathrm{E}+02$ & & $\mathrm{mg} / \mathrm{kg}$ \\
\hline MERCURY & $73 / 89$ & $9.00 \mathrm{E}-02$ & $8.16 \mathrm{E}+02$ & $\mathbf{L}$ & $1.35 \mathrm{E}+01$ & $2.17 \mathrm{E}+01$ & & $\mathrm{mg} / \mathrm{kg}$ \\
\hline NICKEL & $75 / 89$ & $7.40 E+00$ & $6.01 E+01$ & $\mathbf{L}$ & $2.46 \mathrm{E}+01$ & $2.68 \mathrm{E}+01$ & & $\mathrm{mg} / \mathrm{kg}$ \\
\hline POTASSIUM & $75 / 89$ & $3.42 E+02$ & $3.61 E+03$ & $L$ & $1.64 E+03$ & $1.88 \mathrm{E}+03$ & & $\mathrm{mg} / \mathrm{kg}$ \\
\hline SELENIUM & $15 / 49$ & $3.40 \mathrm{E}-01$ & $4.70 \mathrm{E}+00$ & $\mathbf{L}$ & $4.62 \mathrm{E}-01$ & 8.23E-01 & & $\mathrm{mg} / \mathrm{kg}$ \\
\hline SILVER & $53 / 89$ & $4.60 \mathrm{E}-01$ & $3.00 E+00$ & $L$ & $1.09 \mathrm{E}+00$ & $1.26 \mathrm{E}+00$ & & $\mathrm{mg} / \mathrm{kg}$ \\
\hline SODIUM & $32 / 89$ & $2.30 \mathrm{E}+01$ & $1.08 E+02$ & $\mathbb{N}$ & $5.70 \mathrm{E}+01$ & $6.07 \mathrm{E}+01$ & & $\mathrm{mg} / \mathrm{kg}$ \\
\hline THALLIUM & $21 / 89$ & $1.80 \mathrm{E}-01$ & $1.20 \mathrm{E}+\mathrm{m}$ & L & $2.55 \mathrm{E}-01$ & $2.90 \mathrm{E}-01$ & & $\mathrm{mg} / \mathrm{kg}$ \\
\hline VANADIUM & $75 / 89$ & $2.31 \mathrm{E}+01$ & $9.25 E+01$ & $\mathbb{L}$ & $5.35 \mathrm{E}+01$ & $5.83 \mathrm{E}+01$ & & $\mathrm{mg} / \mathrm{kg}$ \\
\hline ZINC & $74 / 89$ & $3.64 E+01$ & $5.36 \mathrm{E}+02$ & $L$ & $1.04 E+02$ & $1.16 \mathrm{E}+02$ & & $\mathrm{mg} / \mathrm{kg}$ \\
\hline \multicolumn{9}{|l|}{ Radionuclides } \\
\hline URANIUM-234 & $89 / 89$ & $5.04 \mathrm{E}-01$ & $8.10 \mathrm{E}+00$ & $L$ & $2.27 \mathrm{E}+00$ & $2.53 E+00$ & & $\mathrm{pCi} / \mathrm{g}$ \\
\hline URANIUM-235 & $58 / 58$ & $0.00 \mathrm{E}+00$ & $6.67 \mathrm{E}-01$ & $\mathbf{L}$ & $1.48 \mathrm{E}-01$ & $1.93 E-01$ & & $\mathrm{pCi} / \mathrm{g}$ \\
\hline URANIUM-238 & $89 / 89$ & $5.27 \mathbb{E}-01$ & $1.33 E+01$ & $\mathbb{L}$ & $2.11 E+\infty$ & $2.35 E+00$ & & $\mathrm{pCi} / \mathrm{g}$ \\
\hline \multicolumn{9}{|l|}{ Organics } \\
\hline 1,1,1-TRICHLOROETHANE & $0 / 32$ & & & NT & $6.81 \mathrm{E}-03$ & & $1.40 \mathrm{E}-02$ & $\mathrm{mg} / \mathrm{kg}$ \\
\hline 1,1,2,2-TETRACHLOROETHANE & $0 / 32$ & & & NT & $6.69 \mathrm{E}-03$ & & $1.40 \mathrm{E}-02$ & $\mathrm{mg} / \mathrm{kg}$ \\
\hline $\begin{array}{l}\text { 1,1,2-TRICHLORO-1,2,2- } \\
\text { TRIFLUOROETHANE }\end{array}$ & $3 / 32$ & $2.00 \mathrm{E}-03$ & $1.40 \mathrm{E}-02$ & $\mathbf{N}$ & $1.22 E-02$ & $1.30 \mathrm{E}-02$ & & $\mathrm{mg} / \mathrm{kg}$ \\
\hline
\end{tabular}


Table C.2. (continued)

\begin{tabular}{|c|c|c|c|c|c|c|c|c|}
\hline Analyte & $\begin{array}{l}\text { Frequency } \\
\text { of } \\
\text { detect }\end{array}$ & $\begin{array}{l}\text { Minimum } \\
\text { detected } \\
\text { concentration }\end{array}$ & $\begin{array}{l}\text { Maximum } \\
\text { detected } \\
\text { concentration }\end{array}$ & Distribution & $\begin{array}{l}\text { Mean } \\
\text { concentration }\end{array}$ & $\begin{array}{l}\text { Upper } 95 \% \\
\text { confidence } \\
\text { limit on mean }\end{array}$ & $\begin{array}{l}\text { Maximum } \\
\text { nondetected } \\
\text { concentration" }\end{array}$ & $\begin{array}{l}\text { Units } \\
\text { of } \\
\text { measure }\end{array}$ \\
\hline 1,1,2-TRICHLOROETHANE & $0 / 32$ & & & NT & $6.81 \mathrm{E}-03$ & & $1.40 \mathrm{E}-02$ & $\mathrm{mg} / \mathrm{kg}$ \\
\hline 1,1-DICHLOROETHENE & 0/32 & & & NT & $6.81 \mathrm{E}-03$ & & $1.40 \mathrm{E}-02$ & $\mathrm{mg} / \mathrm{kg}$ \\
\hline 1,2,4.TRICHLOROBENZENE & $2 / 32$ & $5.70 \mathrm{E}-02$ & $8.40 \mathrm{E}-02$ & $\mathbf{N}$ & $4.02 \mathrm{E}-01$ & $4.30 \mathrm{E}-01$ & & $\mathrm{mg} / \mathrm{kg}$ \\
\hline 1,2-DICHLOROETHENE (TOTAL) & $0 / 32$ & & & NT & $681 \mathrm{E}-03$ & & $1.40 \mathrm{E}-02$ & $\mathrm{mg} / \mathrm{kg}$ \\
\hline 1,2-DICHLOROPROPANE & $0 / 32$ & & & NT & $6.81 \mathrm{E}-03$ & & $1.40 \mathrm{E}-02$ & $\mathrm{mg} / \mathrm{kg}$ \\
\hline 1,3-DICHLOROBENZENE & 0/32 & & & NT & 4.22E-01 & & $5.30 \mathrm{E}-01$ & $\mathrm{mg} / \mathrm{kg}$ \\
\hline 1,4-DICHLOROBENZENE & $1 / 32$ & $5.50 \mathrm{E}-02$ & $5.50 \mathrm{E}-02$ & $\mathbf{N}$ & 4.11E-01 & 4.33E-01 & & $\mathrm{mg} / \mathrm{kg}$ \\
\hline 2,4-DIMETHYLPHENOL & $0 / 32$ & & & NT & $4.22 \mathrm{E}-01$ & & $5.30 \mathrm{E}-01$ & $\mathrm{mg} / \mathrm{kg}$ \\
\hline 2,4-DINITROPHENOL & $0 / 32$ & & & NT & $1.02 E+00$ & & $1.30 \mathrm{E}+00$ & $\mathrm{mg} / \mathrm{kg}$ \\
\hline 2,4-DINITROTOLUENE & $0 / 32$ & & & NT & $4.22 \mathrm{E}-01$ & & $5.30 \mathrm{E}-01$ & $\mathrm{mg} / \mathrm{kg}$ \\
\hline 2,6-DINITROTOLUENE & $0 / 32$ & & & NT & $4.22 \mathrm{E}-01$ & & $5.30 \mathrm{E}-01$ & $\mathrm{mg} / \mathrm{kg}$ \\
\hline 2-BUTANONE & $1 / 32$ & $2.80 \mathrm{E}-02$ & $2.80 \mathrm{E}-02$ & L & $1.41 \mathrm{E}-02$ & $1.51 \mathrm{E}-02$ & & $\mathrm{mg} / \mathrm{kg}$ \\
\hline 2-CHLORONAPHTHALENE & $0 / 32$ & & & NT & $4.22 \mathrm{E}-01$ & & $5.30 \mathrm{E}-01$ & $\mathrm{mg} / \mathrm{kg}$ \\
\hline 2.CHLOROPHENOL & $1 / 32$ & $6.50 \mathrm{E}-02$ & $6.50 \mathrm{E}-02$ & $\mathbf{N}$ & $4.12 \mathrm{E}-01$ & 4.33E-01 & & $\mathrm{mg} / \mathrm{kg}$ \\
\hline 2-HEXANONE & $0 / 32$ & & & NT & $1.36 \mathrm{E}-02$ & & $2.80 \mathrm{E} \cdot 02$ & $\mathrm{mg} / \mathrm{kg}$ \\
\hline
\end{tabular}


Table C.2. (continuod)

\begin{tabular}{|c|c|c|c|c|c|c|c|c|}
\hline Analyte & $\begin{array}{l}\text { Frequency } \\
\text { of } \\
\text { detect } \\
\end{array}$ & $\begin{array}{l}\text { Minimum } \\
\text { detected } \\
\text { concentration } \\
\end{array}$ & $\begin{array}{l}\text { Maximurn } \\
\text { detected } \\
\text { concentration }\end{array}$ & Distribution & $\begin{array}{l}\text { Mean } \\
\text { concentration }\end{array}$ & $\begin{array}{l}\text { Upper } 95 \% \\
\text { confidence } \\
\text { limit on mean }\end{array}$ & $\begin{array}{l}\text { Maximum } \\
\text { nondelected } \\
\text { concentration }\end{array}$ & $\begin{array}{l}\text { Units } \\
\text { of } \\
\text { measure }\end{array}$ \\
\hline 2-METHYLNAPHTHALENE & $0 / 32$ & & & NT & 4.22E-01 & & $5.30 \mathrm{E}-01$ & $\mathrm{mg} / \mathrm{kg}$ \\
\hline 2-METHYLPHENOL & $0 / 32$ & & & NT & 4.22E-01 & & $5.30 \mathrm{E}-01$ & $\mathrm{mg} / \mathrm{kg}$ \\
\hline 2-NITROANILINE & $0 / 32$ & & & NT & $1.02 \mathrm{E}+00$ & & $1.30 \mathrm{E}+00$ & $\mathrm{mg} / \mathrm{kg}$ \\
\hline 2-NITROPHENOL & 1/32 & & & NT & $4.22 \mathrm{E}-01$ & & $5.30 \mathrm{E}-01$ & $\mathrm{mg} / \mathrm{kg}$ \\
\hline 3,3'-DICHLOROBENZIDINE & $0 / 32$ & & & NT & $4.22 \mathrm{E}-01$ & & $5.30 \mathrm{E}-01$ & $\mathrm{mg} / \mathrm{kg}$ \\
\hline 3-NITROANILINE & $0 / 32$ & & & NT & $1.02 \mathrm{E}+00$ & & $1.30 \mathrm{E}+00$ & $\mathrm{mg} / \mathrm{kg}$ \\
\hline $4,4^{\prime}-\mathrm{DDD}$ & $0 / 32$ & & & NT & 2.82E-02 & & $3.90 \mathrm{E}-01$ & $\mathrm{mg} / \mathrm{kg}$ \\
\hline 4, $4^{\circ}-\mathrm{DDE}$ & $0 / 32$ & & & NT & 2.82E-02 & & $3.90 \mathrm{E}-01$ & $\mathrm{mg} / \mathrm{kg}$ \\
\hline $4,4^{\circ}-\mathrm{DDT}$ & $0 / 32$ & & & NT & $2.82 \mathrm{E}-02$ & & $3.90 \mathrm{E}-01$ & $\mathrm{mg} / \mathrm{kg}$ \\
\hline 4,6-DINITRO-2-METHYLPHENOL & $0 / 32$ & & & NT & $1.02 \mathrm{E}+00$ & & $1.30 \mathrm{E}+00$ & $\mathrm{mg} / \mathrm{kg}$ \\
\hline $\begin{array}{l}\text { 4-BROMOPHENYL- } \\
\text { PHENYLETHER }\end{array}$ & $0 / 32$ & & & NT & $4.22 \mathrm{E}-01$ & & $5.30 \mathrm{E}-01$ & $\mathrm{mg} / \mathrm{kg}$ \\
\hline 4-CHLORO-3-METHYLPHENOL & $1 / 32$ & $4.50 \mathrm{E}-02$ & $4.50 \mathrm{E}-02$ & $\mathbf{N}$ & 4.11E-01 & $4.34 \mathrm{E}-01$ & & $\mathrm{mg} / \mathrm{kg}$ \\
\hline 4.CHLOROANILINE & $0 / 32$ & & & NT & 4.22E- -01 & & $5.30 \mathrm{E}-01$ & $\mathrm{mg} / \mathrm{kg}$ \\
\hline $\begin{array}{l}\text { 4-CHLOROPHENYL PHENYL } \\
\text { ETHER }\end{array}$ & $0 / 32$ & & & NT & $4.22 \mathrm{E}-01$ & & $5.30 \mathrm{E}-01$ & $\mathrm{mg} / \mathrm{kg}$ \\
\hline 4-METHYL-2-PENTANONE & $3 / 32$ & $3.00 \mathrm{E}-03$ & $1.60 \mathrm{E}-02$ & $\mathbf{N}$ & $1.28 \mathrm{E}-02$ & $1.42 \mathrm{E}-02$ & & $\mathrm{mg} / \mathrm{kg}$ \\
\hline 4-METHYLPHENOL & $0 / 32$ & & & NT & $4.22 \mathrm{E}-01$ & & $5.30 \mathrm{E}-01$ & $\mathrm{mg} / \mathrm{kg}$ \\
\hline 4-NITROANILINE & $0 / 32$ & & & NT & $1.02 \mathrm{E}+00$ & & $1.30 \mathrm{E}+00$ & $\mathrm{mg} / \mathrm{kg}$ \\
\hline 4-NITROPHENOL & $1 / 32$ & $4.60 \mathrm{E}-02$ & $4.60 \mathrm{E}-02$ & $\mathbf{N}$ & $9.92 \mathrm{E}-01$ & $1.05 \mathrm{E}+00$ & & $\mathrm{mg} / \mathrm{kg}$ \\
\hline ACENAPHTHENE & $5 / 32$ & $6.70 \mathrm{E}-02$ & $1.30 \mathrm{E}-01$ & $\mathbf{N}$ & $3.75 \mathrm{E}-01$ & 4.13E-01 & & $\mathrm{mg} / \mathrm{kg}$ \\
\hline ACENAPHTHYLENE & $0 / 32$ & & & NT & 4.22E- -01 & & $5.30 \mathrm{E}-01$ & $\mathrm{mg} / \mathrm{kg}$ \\
\hline
\end{tabular}


Table C.2 (contimued)

\begin{tabular}{|c|c|c|c|c|c|c|c|c|}
\hline Analyte & $\begin{array}{l}\text { Frequency } \\
\text { of } \\
\text { detect }\end{array}$ & $\begin{array}{l}\text { Minimum } \\
\text { detected } \\
\text { concentration }\end{array}$ & $\begin{array}{l}\text { Maximum } \\
\text { detected } \\
\text { concentration }\end{array}$ & Distribution & $\begin{array}{l}\text { Mean } \\
\text { concentration }\end{array}$ & $\begin{array}{l}\text { Upper } 95 \% \\
\text { confidence } \\
\text { limit on mean }\end{array}$ & $\begin{array}{l}\text { Maximum } \\
\text { nondetected } \\
\text { concentration }^{\mathrm{a}}\end{array}$ & $\begin{array}{l}\text { Units } \\
\text { of } \\
\text { measure }\end{array}$ \\
\hline ACETONE & $15 / 32$ & $1.40 \mathrm{E}-02$ & $9.40 \mathrm{E}-01$ & $L$ & $5.84 \mathrm{E}-02$ & $1.14 \mathrm{E}-01$ & & $\mathrm{mg} / \mathrm{kg}$ \\
\hline ALDRIN & $0 / 32$ & & & NT & $1.43 E-02$ & & $2.00 \mathrm{E}-01$ & $\mathrm{mg} / \mathrm{kg}$ \\
\hline ALPHA-BHC & $0 / 32$ & & & NT & $1.43 E-02$ & & $2.00 \mathrm{E}-01$ & $\mathrm{mg} / \mathrm{kg}$ \\
\hline ALPHA-CHLORDANE & $0 / 32$ & & & NT & $1.43 \mathrm{E}-02$ & & $2.00 \mathrm{E}-01$ & $\mathrm{mg} / \mathrm{kg}$ \\
\hline ANTHRACENE & $4 / 32$ & $1.20 \mathrm{E}-01$ & $2.40 \mathrm{E}-01$ & $\mathbb{N}$ & $3.96 \mathrm{E}-01$ & $4.22 \mathrm{E}-01$ & & $\mathrm{mg} / \mathrm{kg}$ \\
\hline AROCLOR-1016 & $0 / 88$ & & & NT & $2.26 \mathrm{E}-01$ & & $3.90 E+00$ & $\mathrm{mg} / \mathrm{kg}$ \\
\hline AROCLOR-1221 & $0 / 88$ & & & $\mathbf{N T}$ & $4.58 \mathrm{E}-01$ & & $7.90 \mathrm{E}+00$ & $\mathrm{mg} / \mathrm{kg}$ \\
\hline AROCLOR-1232 & $0 / 88$ & & & $\mathrm{NT}$ & $2.26 \mathrm{E}-01$ & & $3.90 \mathrm{E}+00$ & $\mathrm{mg} / \mathrm{kg}$ \\
\hline AROCLOR-1242 & $0 / 88$ & & & NT & $2.26 \mathrm{E}-01$ & & $3.90 \mathrm{E}+\infty 0$ & $\mathrm{mg} / \mathrm{kg}$ \\
\hline AROCLOR-1248 & $0 / 88$ & & & NT & $2.26 \mathbb{E}-01$ & & $3.90 \mathrm{E}+00$ & $\mathrm{mg} / \mathrm{kg}$ \\
\hline AROCLOR-1254 & $9 / 88$ & $3.10 \mathrm{E}-01$ & $1.90 \mathrm{E}+00$ & $\mathbf{N}$ & $2.17 E-01$ & $3.09 \mathrm{E}-01$ & & $\mathrm{mg} / \mathrm{kg}$ \\
\hline AROCLOR-1260 & $41 / 88$ & $2.50 \mathrm{E}-02$ & $1.00 \mathrm{E}+01$ & $\mathbb{L}$ & $2.40 \mathrm{E}-01$ & $4.25 \mathrm{E}-01$ & & $\mathrm{mg} / \mathrm{kg}$ \\
\hline BENZENE & $0 / 32$ & & & $\mathrm{NT}$ & $6.81 \mathrm{E}-03$ & & $1.40 \mathrm{E}-02$ & $\mathrm{mg} / \mathrm{kg}$ \\
\hline BENZO(A)ANTHRACENE & $7 / 32$ & $5.80 \mathrm{E}-02$ & $9.90 \mathrm{E}-01$ & $\mathbf{N}$ & 4.37E-01 & 4.97E-01 & & $\mathrm{mg} / \mathrm{kg}$ \\
\hline BENZO(A)PYRENE & $10 / 32$ & $4.40 \mathrm{E}-02$ & $8.90 \mathrm{E}-01$ & $\mathbb{N}$ & $3.78 \mathrm{E}-01$ & $4.34 \mathrm{E}-01$ & & $\mathrm{mg} / \mathrm{kg}$ \\
\hline BENZO(B)FLUORANTHENE & $9 / 32$ & $4.50 \mathrm{E}-02$ & $1,30 E+00$ & $\mathbf{L}$ & $1.89 \mathrm{E}-01$ & $3.23 E-01$ & & $\mathrm{mg} / \mathrm{kg}$ \\
\hline BENZO(G,H,I)PERYLENE & $7 / 32$ & $6.30 \mathrm{E}-02$ & $4.30 \mathrm{E}-01$ & $\mathbb{N}$ & $3.78 \mathrm{E}-01$ & $4.15 \mathrm{E}-01$ & & $\mathrm{mg} / \mathrm{kg}$ \\
\hline BENZO(K)FLUORANTHENE & $10 / 32$ & $4.10 \mathrm{E}-02$ & $4.10 \mathrm{E}-01$ & $\mathbb{N}$ & $3.39 \mathrm{E}-01$ & $3.85 \mathrm{E}-01$ & & $\mathrm{mg} / \mathrm{kg}$ \\
\hline BENZYL BUTYL PHTHALATE & $0 / 32$ & & & NT & $4.22 \mathrm{E}-01$ & & $5.30 \mathrm{E}-01$ & $\mathrm{mg} / \mathrm{kg}$ \\
\hline BETA-BHC & $0 / 32$ & & & NT & $1.43 \mathrm{E}-02$ & & $2.00 \mathrm{E}-01$ & $\mathrm{mg} / \mathrm{kg}$ \\
\hline
\end{tabular}


Table C.2 (contimued)

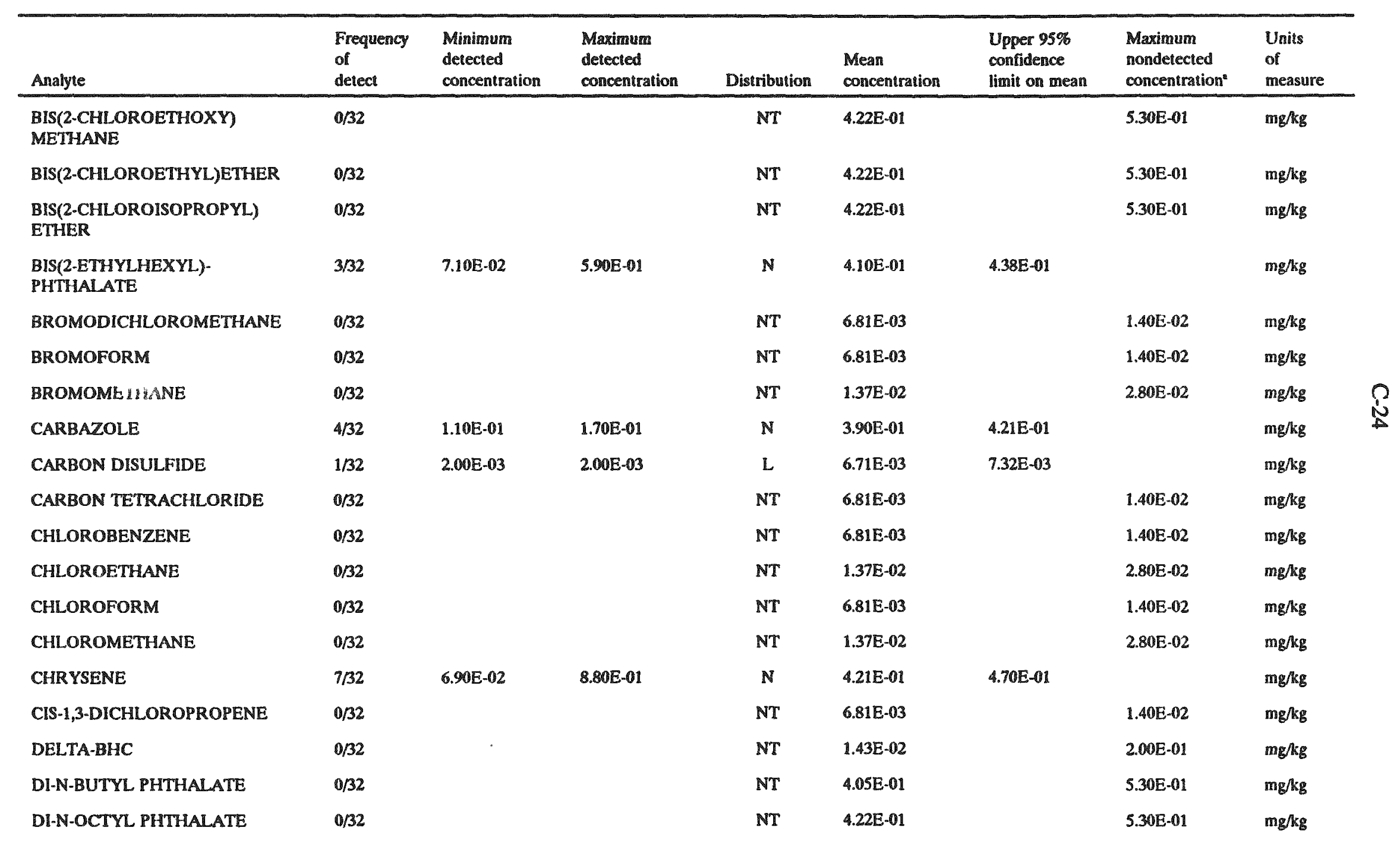


Table C.2 (continued)

\begin{tabular}{|c|c|c|c|c|c|c|c|c|}
\hline Analyte & $\begin{array}{l}\text { Frequency } \\
\text { of } \\
\text { detect }\end{array}$ & $\begin{array}{l}\text { Minimum } \\
\text { detected } \\
\text { concentration }\end{array}$ & $\begin{array}{l}\text { Maximum } \\
\text { detected } \\
\text { concentration }\end{array}$ & Distribution & $\begin{array}{l}\text { Mean } \\
\text { concentration }\end{array}$ & $\begin{array}{l}\text { Upper } 95 \% \\
\text { confidence } \\
\text { limil on mean }\end{array}$ & $\begin{array}{l}\text { Maximum } \\
\text { nondetected } \\
\text { concentration }^{\mathrm{a}}\end{array}$ & $\begin{array}{l}\text { Units } \\
\text { of } \\
\text { measure }\end{array}$ \\
\hline DIBENZO(A,H)ANTHRACENE & $4 / 32$ & $7.70 \mathrm{E}-82$ & $1.20 \mathrm{E}-01$ & $\mathbb{N}$ & $3.74 \mathrm{E}-01$ & $4.13 E-01$ & & $\mathrm{mg} / \mathrm{kg}$ \\
\hline DIBENZOFURAN & $3 / 32$ & $5.00 \mathrm{E}-02$ & $6.40 \mathrm{E}-02$ & $\mathbb{N}$ & $3.90 \mathrm{E}-01$ & $4.24 \mathrm{E}-01$ & & $\mathrm{mg} / \mathrm{kg}$ \\
\hline DIBROMOCHLOROMETHANE & $0 / 32$ & & & NT & $6.81 \mathrm{E}-03$ & & $1.40 \mathrm{E}-02$ & $\mathrm{mg} / \mathrm{kg}$ \\
\hline DIELDRIN & $0 / 32$ & & & NT & $2.82 \mathrm{E}-02$ & & $3.90 \mathrm{E}-01$ & $\mathrm{mg} / \mathrm{kg}$ \\
\hline DIETHYL PHTHALATE & $0 / 32$ & & & NT & $4.22 E-01$ & & $5.30 \mathrm{E}-01$ & $\mathrm{mg} / \mathrm{kg}$ \\
\hline DIMETHYL PHTHALATE & $0 / 32$ & & & $\mathrm{NT}$ & $4.22 E-01$ & & $5.30 \mathrm{E}-01$ & $\mathrm{mg} / \mathrm{kg}$ \\
\hline ENDOSULFAN I & $0 / 32$ & & & $\mathbb{N T}$ & $1.43 \mathrm{E}-02$ & & $2.00 \mathrm{E}-01$ & $\mathrm{mg} / \mathrm{kg}$ \\
\hline ENDOSULFAN II & $0 / 32$ & & & NT & $2.82 \mathrm{E}-02$ & & $3.90 \mathrm{E}-01$ & $\mathrm{mg} / \mathrm{kg}$ \\
\hline ENDOSULFAN SULFATE & $0 / 32$ & & & NT & $2.82 \mathrm{E}-02$ & & $3.90 \mathrm{E}-01$ & $\mathrm{mg} / \mathrm{kg}$ \\
\hline ENDRIN & $0 / 32$ & & & $\mathrm{NT}$ & $2.82 \mathrm{E}-02$ & & $3.90 \mathrm{E}-01$ & $\mathrm{mg} / \mathrm{kg}$ \\
\hline ENDRIN ALDEHYDE & $0 / 32$ & & & NT & $2.82 \mathrm{E}-02$ & & $3.90 \mathrm{E}-01$ & $\mathrm{mg} / \mathrm{kg}$ \\
\hline ENDRIN KETONE & $0 / 32$ & & & NT & $2.82 \mathrm{E}-02$ & & $3.90 \mathrm{E}-01$ & $\mathrm{mg} / \mathrm{kg}$ \\
\hline ETHYLBENZENE & $0 / 32$ & & & NT & $6.81 \mathrm{E}-03$ & & $1.40 \mathrm{E}-02$ & $\mathrm{mg} / \mathrm{kg}$ \\
\hline FLUORANTHENE & $14 / 32$ & $4.40 \mathrm{E}-02$ & $1.40 \mathrm{E}+\infty$ & $\mathrm{L}$ & $2.18 \mathrm{E}-01$ & $3.30 \mathrm{E}-01$ & & $\mathrm{mg} / \mathrm{kg}$ \\
\hline GAMMA-BHC (LINDANE) & $0 / 32$ & & & $\mathrm{NT}$ & $1.43 E-02$ & & $2.00 \mathrm{E}-01$ & $\mathrm{mg} / \mathrm{kg}$ \\
\hline GAMMA-CHLORDANE & $0 / 32$ & & & NT & $1.43 \mathrm{E}-02$ & & $2.00 \mathrm{E}-01$ & $\mathrm{mg} / \mathrm{kg}$ \\
\hline HEPTACHLOR & $0 / 32$ & & & NT & $1.43 \mathrm{E}-02$ & & $2.00 \mathrm{E}-01$ & $\mathrm{mg} / \mathrm{kg}$ \\
\hline HEPTACHLOR EPOXIDE & $0 / 32$ & & & NT & $1.43 \mathrm{E}-02$ & & $2.00 \mathrm{E}-01$ & $\mathrm{mg} / \mathrm{kg}$ \\
\hline HEXACHLOROBENZENE & $0 / 32$ & & & NT & $4.22 \mathrm{E}-01$ & & $5.30 \mathrm{E}-01$ & $\mathrm{mg} / \mathrm{kg}$ \\
\hline HEXACHLOROBUTAOIENE & $0 / 32$ & & & $\mathrm{NT}$ & $4.22 \mathrm{E}-01$ & & $5.30 \mathrm{E}-01$ & $\mathrm{mg} / \mathrm{kg}$ \\
\hline
\end{tabular}


Table C.2. (continued)

\begin{tabular}{|c|c|c|c|c|c|c|c|c|}
\hline Analyte & $\begin{array}{l}\text { Frequency } \\
\text { of } \\
\text { detect }\end{array}$ & $\begin{array}{l}\text { Minimum } \\
\text { detected } \\
\text { concentration }\end{array}$ & $\begin{array}{l}\text { Maximum } \\
\text { detected } \\
\text { concentration }\end{array}$ & Distribution & $\begin{array}{l}\text { Mean } \\
\text { concentration }\end{array}$ & $\begin{array}{l}\text { Upper } 95 \% \\
\text { confidence } \\
\text { limit on mean }\end{array}$ & $\begin{array}{l}\text { Maximum } \\
\text { nondetected } \\
\text { concentration }\end{array}$ & $\begin{array}{l}\text { Units } \\
\text { of } \\
\text { measure }\end{array}$ \\
\hline $\begin{array}{l}\text { HEXACHLOROCYCLO- } \\
\text { PENTADIENE }\end{array}$ & $0 / 32$ & & & NT & 4.22E-01 & & $5.30 \mathrm{E}-01$ & $\mathrm{mg} / \mathrm{kg}$ \\
\hline HEXACHLOROETHANE & $0 / 32$ & & & NT & $4.22 \mathrm{E}-01$ & & $5.30 \mathrm{E}-01$ & $\mathrm{mg} / \mathrm{kg}$ \\
\hline INDENO(1,2,3-CD)PYRENE & $7 / 32$ & $4.90 \mathrm{E}-02$ & $5.30 \mathrm{E}-01$ & $\mathbf{N}$ & 3.87E-01 & 4.27E-01 & & $\mathrm{mg} / \mathrm{kg}$ \\
\hline ISOPHORONE & $0 / 32$ & & & NT & 4.22E-01 & & $5.30 \mathrm{E}-01$ & $\mathrm{mg} / \mathrm{kg}$ \\
\hline METHYLENE CHLORIDE & $11 / 32$ & $9.00 \mathrm{E}-03$ & $8.80 \mathrm{E}-02$ & L & $1.10 \mathrm{E}-02$ & 2.08E-02 & & $\mathrm{mg} / \mathrm{kg}$ \\
\hline $\begin{array}{l}\text { N-NITROSO-DI-N- } \\
\text { PROPYLAMINE }\end{array}$ & $0 / 32$ & & & NT & $4.22 \mathrm{E}-01$ & & $5.30 \mathrm{E}-01$ & $\mathrm{mg} / \mathrm{kg}$ \\
\hline N-NITROSODIPHENYLAMINE & $0 / 32$ & & & NT & 4.22E- -01 & & $5.30 \mathrm{E}-01$ & $\mathrm{mg} / \mathrm{kg}$ \\
\hline NAPHTHALENE & $2 / 32$ & $4.50 \mathrm{E}-02$ & $7.10 \mathrm{E}-02$ & $\mathbf{N}$ & 4.01E-01 & $4.30 \mathrm{E}-01$ & & $\mathrm{mg} / \mathrm{kg}$ \\
\hline NITROBENZENE & $0 / 32$ & & & NT & 4.22E-01 & & $5.30 \mathrm{E}-01$ & $\mathrm{mg} / \mathrm{kg}$ \\
\hline P,P'-METHOXYCHLOR & $0 / 32$ & & & $\mathrm{NT}$ & $1.43 \mathrm{E}-01$ & & $2.00 \mathrm{E}+00$ & $\mathrm{mg} / \mathrm{kg}$ \\
\hline PENTACHLOROPHENOL & $1 / 32$ & $6.50 \mathrm{E}-02$ & $6.50 \mathrm{E}-02$ & $\mathbf{N}$ & 9.93E-01 & $1.05 \mathrm{E}+00$ & & $\mathrm{mg} / \mathrm{kg}$ \\
\hline PHENANTHRENE & $9 / 32$ & $5.50 \mathrm{E}-02$ & $1.20 \mathrm{E}+00$ & L & $2.24 \mathrm{E}-01$ & $3.49 \mathrm{E}-01$ & & $\mathrm{mg} / \mathrm{kg}$ \\
\hline PHENOL & $1 / 32$ & $6.20 \mathrm{E}-02$ & $6.20 \mathrm{E}-02$ & $\mathbf{N}$ & 4.12E-01 & 4.33E-01 & & $\mathrm{mg} / \mathrm{kg}$ \\
\hline PYRENE & $14 / 32$ & 4.60E-02 & $1.40 \mathrm{E}+00$ & L & 2.19E-01 & 3.30E-01 & & $\mathrm{mg} / \mathrm{kg}$ \\
\hline STYRENE & $0 / 32$ & & & NT & $6.81 \mathrm{E}-03$ & & $1.40 \mathrm{E}-02$ & $\mathrm{mg} / \mathrm{kg}$ \\
\hline TETRACHLOROETHENE & $0 / 32$ & & & NT & $6.72 \mathrm{E}-03$ & & $1.40 \mathrm{E}-02$ & $\mathrm{mg} / \mathrm{kg}$ \\
\hline TOLUENE & $2 / 32$ & $1.00 \mathrm{E}-03$ & $1.00 \mathrm{E}-03$ & $\mathbf{N}$ & $6.50 \mathrm{E}-03$ & $7,20 \mathrm{E}-03$ & & $\mathrm{mg} / \mathrm{kg}$ \\
\hline TOXAPHENE & $0 / 32$ & & & NT & $1.43 E+00$ & & $2.00 \mathrm{E}+01$ & $\mathrm{mg} / \mathrm{kg}$ \\
\hline TRANS-1,3-DICHLOROPROPENE & $0 / 32$ & & & NT & $6.81 \mathrm{E}-03$ & & $1.40 \mathrm{E}-02$ & $\mathrm{mg} / \mathrm{kg}$ \\
\hline TRICHLOROETHENE & $\$ \beta 2$ & & & NT & $6.81 \mathrm{E}-03$ & & $1.40 \mathrm{E}-02$ & $\mathrm{mg} / \mathrm{kg}$ \\
\hline
\end{tabular}


Table C.2. (continued)

\begin{tabular}{|c|c|c|c|c|c|c|c|c|}
\hline Analyte & $\begin{array}{l}\text { Frequency } \\
\text { of } \\
\text { detect }\end{array}$ & $\begin{array}{l}\text { Minimum } \\
\text { detected } \\
\text { concentration }\end{array}$ & $\begin{array}{l}\text { Maximum } \\
\text { detected } \\
\text { concentration }\end{array}$ & Distribution & $\begin{array}{l}\text { Mean } \\
\text { concentration }\end{array}$ & $\begin{array}{l}\text { Upper } 95 \% \\
\text { confidence } \\
\text { limit on mean }\end{array}$ & $\begin{array}{l}\text { Maximum } \\
\text { nondetected } \\
\text { concentration }^{\mathrm{a}}\end{array}$ & $\begin{array}{l}\text { Units } \\
\text { of } \\
\text { measure }\end{array}$ \\
\hline VINYL CHLORIDE & $0 / 32$ & & & $\mathrm{NT}$ & $1.37 \mathrm{E}-02$ & & $2.80 \mathrm{E}-02$ & $\mathrm{mg} / \mathrm{kg}$ \\
\hline XYLENE (TOTAL) & $0 / 32$ & & & $\mathrm{NT}$ & $6.81 E-03$ & & $1.40 \mathrm{E}-02$ & $\mathrm{mg} / \mathrm{kg}$ \\
\hline
\end{tabular}

SA. 1

$\begin{array}{llll}\text { InOIganics } & 11 / 12 & 1.37 \mathrm{E}+04 & 3.18 \mathrm{E}+04 \\ \text { ALUMINUM } & 0 / 8 & & \\ \text { ANTIMONY } & 11 / 12 & 2.30 \mathrm{E}+00 & 2.34 \mathrm{E}+01 \\ \text { ARSENIC } & 12 / 12 & 3.60 \mathrm{E}+01 & 3.85 \mathrm{E}+02 \\ \text { BARIUM } & 12 / 12 & 3.00 \mathrm{E}-01 & 1.50 \mathrm{E}+00 \\ \text { BERYLLIUM } & 12 / 12 & 1.20 \mathrm{E}+00 & 4.10 \mathrm{E}+00 \\ \text { CADMIUM } & 12 / 12 & 7.84 \mathrm{E}+01 & 9.40 \mathrm{E}+04 \\ \text { CALCIUM } & 12 / 12 & 2.45 \mathrm{E}+01 & 4.27 \mathrm{E}+01 \\ \text { CHROMIUM } & 12 / 12 & 9.30 \mathrm{E}+00 & 2.32 \mathrm{E}+01 \\ \text { COBALT } & 12 / 12 & 1.61 \mathrm{E}+01 & 8.59 \mathrm{E}+01 \\ \text { COPPER } & 1 / 12 & 1.10 \mathrm{E}+00 & 1.10 \mathrm{E}+00 \\ \text { CYANIDE } & 2 / 12 & 1.90 \mathrm{E} \cdot 01 & 2.30 \mathrm{E}-01 \\ \text { FLUORENE } & 11 / 12 & 1.70 \mathrm{E}+04 & 3.69 \mathrm{E}+04 \\ \text { IRON } & 12 / 12 & 1.15 \mathrm{E}+01 & 1.10 \mathrm{E}+02 \\ \text { LEAD } & 11 / 12 & 5.32 \mathrm{E}+02 & 2.05 \mathrm{E}+04 \\ \text { MAGNESIUM } & 12 / 12 & 3.71 \mathrm{E}+02 & 2.03 \mathrm{E}+03 \\ \text { MANGANESE } & & & \end{array}$

\begin{tabular}{|c|c|c|c|c|}
\hline $\mathbb{N}$ & $2.17 \mathrm{E}+04$ & $2.62 \mathrm{E}+04$ & & $\mathrm{mg} / \mathrm{kg}$ \\
\hline $\mathrm{NT}$ & $1.15 E+01$ & & $1.20 \mathrm{E}+01$ & $\mathrm{mg} / \mathrm{kg}$ \\
\hline L & $8.37 \mathbb{E}+00$ & $1.27 \mathrm{E}+01$ & & $\mathrm{mg} / \mathrm{kg}$ \\
\hline $\mathbb{N}$ & $1.79 \mathrm{E}+02$ & $2.34 \mathrm{E}+02$ & & $\mathrm{mg} / \mathrm{kg}$ \\
\hline $\mathbf{N}$ & $1.00 E+00$ & $1.20 \mathrm{E}+\infty$ & & $\mathrm{mg} / \mathrm{kg}$ \\
\hline $\mathbf{N}$ & $2.82 E+00$ & $3.31 E+00$ & & $\mathrm{mg} / \mathrm{kg}$ \\
\hline L & $6.00 E+04$ & $5.42 \mathrm{E}+05$ & & $\mathrm{mg} / \mathrm{kg}$ \\
\hline L & $3.25 \mathrm{E}+01$ & $3.59 \mathrm{E}+01$ & & $\mathrm{mg} / \mathrm{kg}$ \\
\hline $\mathbf{N}$ & $1.65 \mathrm{E}+01$ & $1.86 \mathrm{E}+01$ & & $\mathrm{mg} / \mathrm{kg}$ \\
\hline $\mathbb{L}$ & $2.77 E+01$ & $3.44 \mathrm{E}+01$ & & $\mathrm{mg} / \mathrm{kg}$ \\
\hline $\mathbf{N}$ & $7.83 \mathrm{E}-01$ & $9.97 \mathrm{E}-01$ & & $\mathrm{mg} / \mathrm{kg}$ \\
\hline L & $2.19 E-01$ & $2.47 E-01$ & & $\mathrm{mg} / \mathrm{kg}$ \\
\hline $\mathbf{N}$ & $2.69 E+04$ & $3.21 E+04$ & & $\mathrm{mg} / \mathrm{kg}$ \\
\hline L & $3.34 E+01$ & $4.69 E+01$ & & $\mathrm{mg} / \mathrm{kg}$ \\
\hline $\mathbf{N}$ & $5.89 \mathbb{E}+03$ & $8.77 E+03$ & & $\mathrm{mg} / \mathrm{kg}$ \\
\hline L & $9.40 E+02$ & $1.20 E+03$ & & $\mathrm{mg} / \mathrm{kg}$ \\
\hline
\end{tabular}


Table C.2. (continued)

\begin{tabular}{|c|c|c|c|c|c|c|c|c|}
\hline Analyte & $\begin{array}{l}\text { Frequency } \\
\text { of } \\
\text { detect }\end{array}$ & $\begin{array}{l}\text { Minimum } \\
\text { detected } \\
\text { concentration }\end{array}$ & $\begin{array}{l}\text { Maximum } \\
\text { detected } \\
\text { concentration }\end{array}$ & Distribution & $\begin{array}{l}\text { Mean } \\
\text { concentration }\end{array}$ & $\begin{array}{l}\text { Upper } 95 \% \\
\text { confidence } \\
\text { limit on mean }\end{array}$ & $\begin{array}{l}\text { Maximum } \\
\text { nondetected } \\
\text { concentration }\end{array}$ & $\begin{array}{l}\text { Units } \\
\text { of } \\
\text { measure }\end{array}$ \\
\hline MERCURY & $8 / 12$ & $6.00 E-02$ & $2.60 \mathrm{E}+00$ & $\mathbf{L}$ & $3.63 \mathrm{E}-01$ & $9.65 \mathrm{E}-01$ & & $\mathrm{mg} / \mathrm{kg}$ \\
\hline NICKEL & $12 / 12$ & $1.24 \mathrm{E}+01$ & $5.10 E+01$ & $\mathbf{N}$ & $2.88 \mathrm{E}+01$ & $3.51 E+01$ & & $\mathrm{mg} / \mathrm{kg}$ \\
\hline NIOBIUM & $0 / 12$ & & & NT & $1.62 \mathrm{E}+\infty 0$ & & $1.70 \mathrm{E}+00$ & $\mathrm{mg} / \mathrm{kg}$ \\
\hline PHOSPHORUS & $8 / 12$ & $1.07 \mathrm{E}+02$ & $4.47 \mathrm{E}+02$ & $\mathbf{N}$ & $1.91 \mathrm{E}+02$ & $2.67 \mathrm{E}+02$ & & $\mathrm{mg} / \mathrm{kg}$ \\
\hline POTASSIUM & $11 / 12$ & $4.11 E+02$ & $5.43 E+03$ & $\mathbf{N}$ & $2.91 \mathrm{E}+03$ & $3.88 \mathrm{E}+03$ & & $\mathrm{mg} / \mathrm{kg}$ \\
\hline SULVER & $0 / 12$ & & & NT & $1.38 \mathrm{E}+00$ & & $1.50 \mathrm{E}+00$ & $\mathrm{mg} / \mathrm{kg}$ \\
\hline SODIUM & $12 / 12$ & $9.23 E+0$ & $2.06 \mathrm{E}+02$ & $\mathbf{N}$ & $1.48 \mathrm{E}+02$ & $1.65 \mathrm{E}+02$ & & $\mathrm{mg} / \mathrm{kg}$ \\
\hline URANIUM, TOTAL & $12 / 12$ & $2.00 \mathrm{E}+00$ & $6.89 \mathrm{E}+01$ & $\mathbb{L}$ & $8.55 \mathrm{E}+00$ & $1.53 \mathrm{E}+01$ & & $\mathrm{mg} / \mathrm{kg}$ \\
\hline VANADIUM & $12 / 12$ & $1.77 \mathrm{E}+01$ & $6.54 \mathrm{E}+01$ & $\mathbb{L}$ & $3.57 \mathrm{E}+01$ & $4.31 \mathrm{E}+01$ & & $\mathrm{mg} / \mathrm{kg}$ \\
\hline ZINC & $12 / 12$ & $5.18 \mathrm{E}+01$ & $4.38 \mathrm{E}+02$ & $\mathbb{L}$ & $9.44 \mathrm{E}+01$ & $1.26 \mathrm{E}+02$ & & $\mathrm{mg} / \mathrm{kg}$ \\
\hline \multicolumn{9}{|l|}{ Radionuclides } \\
\hline PCT URANIUM-235 & $3 / 3$ & $4.00 \mathrm{E}-01$ & $1.00 \mathrm{E}+00$ & $\mathbb{L}$ & $6.98 \mathrm{E}-01$ & $1.10 E+00$ & & WT\% \\
\hline RADIUM-226 & $12 / 12$ & $1.49 \mathrm{E}+00$ & $7.57 \mathrm{E}+00$ & $\mathbf{L}$ & $2.59 \mathrm{E}+00$ & $3.22 \mathrm{E}+00$ & & $\mathrm{pCi} / \mathrm{g}$ \\
\hline URANIUM-234 & $12 / 12$ & $6.74 \mathrm{E}-01$ & $2.32 \mathrm{E}+01$ & $\mathbb{L}$ & $2.88 \mathrm{E}+00$ & $5.15 E+00$ & & $\mathrm{pCi} / \mathrm{g}$ \\
\hline URANIUM-235 & $12 / 12$ & $3.07 \mathrm{E}-02$ & $1.06 \mathrm{E}+00$ & $\mathbb{L}$ & $1.31 \mathrm{E}-01$ & $2.35 \mathrm{E}-01$ & & $\mathrm{pCi} / \mathrm{g}$ \\
\hline URANIUM-238 & $12 / 12$ & $6.67 \mathrm{E}-01$ & $2.30 \mathrm{E}+01$ & $\mathbf{L}$ & $2.85 \mathrm{E}+\infty$ & $5.11 E+00$ & & $\mathrm{pCi} / \mathrm{g}$ \\
\hline \multicolumn{9}{|l|}{ Organics } \\
\hline 1,1,1-TRICHLOROETHANE & $0 / 12$ & & & NT & $6.08 \mathrm{E}-03$ & & $7.00 \mathrm{E}-03$ & $\mathrm{mg} / \mathrm{kg}$ \\
\hline 1,1,2,2-TETRACHLOROETHANE & $0 / 12$ & & & $\mathbb{N T}$ & $6.08 \mathrm{E}-03$ & & $7.00 \mathrm{E}-03$ & $\mathrm{mg} / \mathrm{kg}$ \\
\hline
\end{tabular}


Table C.2 (continued)

\begin{tabular}{|c|c|c|c|c|c|c|c|c|}
\hline Analyte & $\begin{array}{l}\text { Frequency } \\
\text { of } \\
\text { detect }\end{array}$ & $\begin{array}{l}\text { Minimum } \\
\text { delected } \\
\text { concentration }\end{array}$ & $\begin{array}{l}\text { Maximum } \\
\text { detected } \\
\text { concentration }\end{array}$ & Distribution & $\begin{array}{l}\text { Mean } \\
\text { concentration }\end{array}$ & $\begin{array}{l}\text { Upper } 95 \% \\
\text { confidence } \\
\text { limit on mean }\end{array}$ & $\begin{array}{l}\text { Maximum } \\
\text { nondetected } \\
\text { concentration }\end{array}$ & $\begin{array}{l}\text { Units } \\
\text { of } \\
\text { measure }\end{array}$ \\
\hline 1,1,2-TRICHLOROETHANE & $0 / 12$ & & & NT & $6.08 \mathrm{E}-03$ & & $7.00 \mathrm{E}-03$ & $\mathrm{mg} / \mathrm{kg}$ \\
\hline 1,1-DICHLOROETHANE & $0 / 12$ & & & NT & $6.08 \mathrm{E}-03$ & & $7.00 \mathrm{E}-03$ & $\mathrm{mg} / \mathrm{kg}$ \\
\hline 1,1-DICHLOROETHENE & $0 / 12$ & & & NT & $6.08 \mathrm{E}-03$ & & $7.00 \mathrm{E}-03$ & $\mathrm{mg} / \mathrm{kg}$ \\
\hline 1,2,4-TRICHLOROBENZENE & $0 / 12$ & & & NT & $8.78 \mathrm{E}-01$ & & $1.80 \mathrm{E}+\infty 0$ & $\mathrm{mg} / \mathrm{kg}$ \\
\hline 1,2-DICHLOROBENZENE & $0 / 12$ & & & NT & $8.78 E-01$ & & $1.80 \mathrm{E}+00$ & $\mathrm{mg} / \mathrm{kg}$ \\
\hline 1,2-DICHLOROETHANE & $0 / 12$ & & & NT & $6.08 \mathrm{E}-03$ & & $7.00 \mathrm{E}-03$ & $\mathrm{mg} / \mathrm{kg}$ \\
\hline 1,2-DICHLOROETHENE (TOTAL) & $2 / 12$ & $2.00 \mathrm{E}-03$ & $2.00 \mathrm{E}-03$ & $\mathbb{N}$ & $5.42 \mathrm{E}-03$ & $6.26 \mathrm{E}-03$ & & $\mathrm{mg} / \mathrm{kg}$ \\
\hline 1,2-DICHLOROPROPANE & $0 / 12$ & & & $\mathbb{N T}$ & $6.08 \mathrm{E}-03$ & & $7.00 \mathrm{E}-03$ & $\mathrm{mg} / \mathrm{kg}$ \\
\hline 1,3-DICHLOROBENZENE & $0 / 12$ & & & NT & $8.78 \mathrm{E}-01$ & & $1.80 E+00$ & $m g / k g$ \\
\hline 1,4-DICHLOROBENZENE & $0 / 12$ & & & NT & $8.78 \mathrm{E}-01$ & & $1.80 \mathrm{E}+00$ & $\mathrm{mg} / \mathrm{kg}$ \\
\hline 2,4,5-TRICHLOROPHENOL & $0 / 12$ & & & $\mathbf{N T}$ & $4.26 \mathrm{E}+00$ & & $8.70 \mathrm{E}+00$ & $\mathrm{mg} / \mathrm{hg}$ \\
\hline 2,4,6-TRICHLOROPHENOL & $0 / 12$ & & & $\mathbb{N T}$ & $8.78 \mathrm{E}-01$ & & $1.80 \mathrm{E}+00$ & $\mathrm{mg} / \mathrm{kg}$ \\
\hline 2,4-DICHLOROPHENOL & $0 / 12$ & & & NT & $8.78 \mathrm{E}-01$ & & $1.80 \mathrm{E}+00$ & $\mathrm{mg} / \mathrm{kg}$ \\
\hline 2,4-DIMETHYLPHENOL & $0 / 12$ & & & NT & $8.78 \mathrm{E}-01$ & & $1.80 \mathrm{E}+\infty 0$ & $\mathrm{mg} / \mathrm{kg}$ \\
\hline 2,4-DINITROPHENOL & $0 / 12$ & & & $\mathbb{N T}$ & $4.26 \mathrm{E}+00$ & & $8.70 \mathrm{E}+00$ & $\mathrm{mg} / \mathrm{kg}$ \\
\hline 2,4-DINITROTOLUENE & $0 / 12$ & & & NT & 8.78E-01 & & $1.80 \mathrm{E}+\infty$ & $\mathrm{mg} / \mathrm{kg}$ \\
\hline 2,6-DINITROTOLUENE & $0 / 12$ & & & NT & $8.78 \mathrm{E}-01$ & & $1.80 \mathrm{E}+00$ & $\mathrm{mg} / \mathrm{kg}$ \\
\hline 2-BUTANONE & $0 / 11$ & & & $\mathrm{NT}$ & $1.23 \mathrm{E}-02$ & & $1.30 \mathrm{E}-02$ & $\mathrm{mg} / \mathrm{kg}$ \\
\hline 2.CHLORONAPHTHALENE & $0 / 12$ & & & $\mathbf{N T}$ & $8.78 \mathrm{E}-01$ & & $1.80 \mathrm{E}+00$ & $\mathrm{mg} / \mathrm{kg}$ \\
\hline 2.CHLOROPHENOL & $0 / 12$ & & & $\mathrm{NT}$ & $8.78 \mathrm{E}-01$ & & $1.80 \mathrm{E}+00$ & $\mathrm{mg} / \mathrm{kg}$ \\
\hline 2.HEXANONE & $0 / 12$ & & & $\mathrm{NT}$ & $1.23 \mathrm{E}-02$ & & $1.30 \mathrm{E}-02$ & $\mathrm{mg} / \mathrm{kg}$ \\
\hline
\end{tabular}


Table C.2 (continued)

\begin{tabular}{|c|c|c|c|c|c|c|c|c|}
\hline Analyte & $\begin{array}{l}\text { Frequency } \\
\text { of } \\
\text { detect }\end{array}$ & $\begin{array}{l}\text { Minimum } \\
\text { detected } \\
\text { concentration }\end{array}$ & $\begin{array}{l}\text { Maximum } \\
\text { detected } \\
\text { concentration }\end{array}$ & Distribution & $\begin{array}{l}\text { Mean } \\
\text { concentration }\end{array}$ & $\begin{array}{l}\text { Upper } 95 \% \\
\text { confidence } \\
\text { limit on mean }\end{array}$ & $\begin{array}{l}\text { Maximun } \\
\text { nondetected } \\
\text { concentration }\end{array}$ & $\begin{array}{l}\text { Units } \\
\text { of } \\
\text { neasure }\end{array}$ \\
\hline 2-METHYLNAPHTHALENE & $0 / 12$ & & & NT & $8.78 \mathrm{E} \cdot 01$ & & $1.80 \mathrm{E}+00$ & $\mathrm{mg} / \mathrm{kg}$ \\
\hline 2-METHYLPHENOL & $0 / 12$ & & & NT & $8.78 E-01$ & & $1.80 \mathrm{E}+00$ & $\mathrm{mg} / \mathrm{kg}$ \\
\hline 2-NITROANILINE & $0 / 12$ & & & $\mathbf{N T}$ & $4.26 \mathrm{E}+00$ & & $8.70 \mathrm{E}+00$ & $\mathrm{mg} / \mathrm{kg}$ \\
\hline 2-NITROPHENOL & $0 / 12$ & & & NT & $8.78 E-01$ & & $1.80 \mathrm{E}+00$ & $\mathrm{mg} / \mathrm{kg}$ \\
\hline 3,3'-DICHLOROBENZIDINE & $0 / 12$ & & & $\mathbf{N T}$ & $1.76 \mathrm{E}+00$ & & $3.60 \mathrm{E}+\infty 0$ & $\mathrm{mg} / \mathrm{kg}$ \\
\hline 3-NITROANILINE & $0 / 12$ & & & NT & $4.26 \mathrm{E}+00$ & & $8.70 \mathrm{E}+00$ & $\mathrm{mg} / \mathrm{kg}$ \\
\hline 4,6-DINITRO-2-METHYLPHENOL & $0 / 12$ & & & NT & $4.26 \mathrm{E}+00$ & & $8.70 \mathrm{E}+00$ & $\mathrm{mg} / \mathrm{kg}$ \\
\hline $\begin{array}{l}\text { 4-BROMOPHENYL PHENYL } \\
\text { ETHER }\end{array}$ & $0 / 12$ & & & NT & $8.78 \mathrm{E}-01$ & & $1.80 \mathrm{E}+00$ & $\mathrm{mg} / \mathrm{kg}$ \\
\hline 4-CHLORO-3-METHYLPHENOL & $0 / 12$ & & & $\mathbb{N T}$ & $8.78 \mathrm{E}-01$ & & $1.80 \mathrm{E}+00$ & $\mathrm{mg} / \mathrm{kg}$ \\
\hline 4-CHLOROANILINE & $0 / 12$ & & & NT & $8.78 \mathrm{E}-01$ & & $1.80 \mathrm{E}+00$ & $\mathrm{mg} / \mathrm{kg}$ \\
\hline $\begin{array}{l}\text { 4-CHLOROPHENYL PHENYL } \\
\text { ETHER }\end{array}$ & $0 / 12$ & & & $\mathrm{NT}$ & $8.78 \mathrm{E}-01$ & & $1.80 \mathrm{E}+00$ & $\mathrm{mg} / \mathrm{kg}$ \\
\hline 4-METHYL-2-PENTANONE & $1 / 12$ & $1.20 \mathrm{E}-02$ & $1.20 \mathrm{E}-02$ & $\mathbf{N}$ & $1.23 \mathrm{E}-02$ & $1.25 \mathrm{E}-02$ & & $\mathrm{mg} / \mathrm{kg}$ \\
\hline 4-METHYLPHENOL & $0 / 12$ & & & $\mathrm{NT}$ & $8.78 \mathrm{E}-01$ & & $1.80 \mathrm{E}+00$ & $\mathrm{mg} / \mathrm{kg}$ \\
\hline 4-NITROANILINE & $0 / 12$ & & & NT & $4.26 \mathrm{E}+100$ & & $8.70 \mathrm{E}+00$ & $\mathrm{mg} / \mathrm{kg}$ \\
\hline 4-NITROPHENOL & $0 / 12$ & & & $\mathrm{NT}$ & $4.26 E+\infty 0$ & & $8.70 \mathrm{E}+00$ & $\mathrm{mg} / \mathrm{kg}$ \\
\hline ACENAPHTHENE & $1 / 12$ & $1.10 \mathrm{E}-01$ & $1.10 \mathrm{E}-01$ & $L$ & $8.02 E-01$ & $1.24 \mathrm{E}+00$ & & $\mathrm{mg} / \mathrm{kg}$ \\
\hline ACENAPHTHYLENE & $0 / 12$ & & & NT & $8.78 \mathrm{E}-01$ & & $1.80 \mathrm{E}+\infty 0$ & $\mathrm{mg} / \mathrm{kg}$ \\
\hline ACETONE & $2 / 12$ & $4.50 \mathrm{E}-02$ & $2.20 \mathrm{E}-01$ & $\mathbf{L}$ & $2.81 \mathrm{E}-03$ & $7.01 \mathrm{E}-02$ & & $\mathrm{mg} / \mathrm{kg}$ \\
\hline ANTHRACENE & $2 / 12$ & $2.00 \mathrm{E}-01$ & $3.20 \mathrm{E}-01$ & $\mathrm{~L}$ & $2.76 \mathrm{E}-01$ & $3.46 \mathrm{E}-01$ & & $\mathrm{mg} / \mathrm{kg}$ \\
\hline BENZENE & $0 / 12$ & & & NT & $6.08 \mathrm{E}-03$ & & $7.00 \mathrm{E}-03$ & $\mathrm{mg} / \mathrm{kg}$ \\
\hline
\end{tabular}


Table C2. (continued)

\begin{tabular}{|c|c|c|c|c|c|c|c|c|}
\hline Analyte & $\begin{array}{l}\text { Frequency } \\
\text { of } \\
\text { detect }\end{array}$ & $\begin{array}{l}\text { Minimum } \\
\text { delected } \\
\text { concentration }\end{array}$ & $\begin{array}{l}\text { Maximum } \\
\text { detected } \\
\text { concentration }\end{array}$ & Distribution & $\begin{array}{l}\text { Mean } \\
\text { concentration }\end{array}$ & $\begin{array}{l}\text { Upper } 95 \% \\
\text { confidence } \\
\text { limit on mean }\end{array}$ & $\begin{array}{l}\text { Maximum } \\
\text { nondetected } \\
\text { concentration }\end{array}$ & $\begin{array}{l}\text { Units } \\
\text { of } \\
\text { measure }\end{array}$ \\
\hline BENZO(A)ANTHRACENE & $5 / 12$ & $2.00 \mathrm{E}-01$ & $6.10 \mathrm{E}-01$ & $\mathbf{L}$ & $3.26 \mathrm{E}-01$ & $4.16 \mathrm{E}-01$ & & $\mathrm{mg} / \mathrm{gg}$ \\
\hline BENZO(A)PYRENE & $5 / 12$ & 1.50E-01 & $4.80 \mathrm{E}-01$ & $\mathbf{L}$ & $2.82 \mathrm{E}-01$ & $3.61 \mathrm{E}-01$ & & $\mathrm{mg} / \mathrm{kg}$ \\
\hline BENZO(B)FLUORANTHENE & $4 / 12$ & $1.60 E-01$ & $4.20 \mathrm{E}-01$ & $\mathbf{L}$ & $3.03 \mathrm{E}-01$ & $3.76 \mathrm{E}-01$ & & $\mathrm{mg} / \mathrm{kg}$ \\
\hline BENZO(G,H,I)PERYLENE & $3 / 2$ & $1.20 \mathrm{E}-01$ & $2.80 \mathrm{E}-01$ & $\mathbf{L}$ & $2.39 \mathrm{E}-01$ & $3.23 \mathrm{E}-01$ & & $\mathrm{mg} / \mathrm{kg}$ \\
\hline BENZO(K)FLUORANTHENE & $5 / 12$ & $2.00 \mathrm{E}-01$ & $5.80 \mathrm{E}-01$ & $L$ & 3.32E-01 & $4.10 \mathrm{E}-01$ & & $\mathrm{mg} / \mathrm{kg}$ \\
\hline BENZOIC ACID & $1 / 12$ & $5.50 \mathrm{E}-02$ & $5.50 E-02$ & $\mathbf{N}$ & $4.10 E+00$ & $5.63 E+00$ & & $\mathrm{mg} / \mathrm{kg}$ \\
\hline BENZYL ALCOHOL & $0 / 12$ & & & NT & $8.78 \mathrm{E}-01$ & & $1.80 \mathrm{E}+00$ & $\mathrm{mg} / \mathrm{kg}$ \\
\hline BENZYL BUTYL PHTHALATE & on2 & & & $\mathrm{NT}$ & $8.78 \mathrm{E}-01$ & & $1.80 \mathrm{E}+00$ & $\mathrm{mg} / \mathrm{kg}$ \\
\hline $\begin{array}{l}\text { BIS(2-CHLOROETHOXY) } \\
\text { METHANE }\end{array}$ & $0 / 12$ & & & NT & $8.78 \mathrm{E}-01$ & & $1.80 \mathrm{E}+00$ & $\mathrm{mg} / \mathrm{kg}$ \\
\hline BIS(2-CHLOROETHYL)ETHER & $0 / 12$ & & & NT & $8.78 \mathrm{E}-\mathrm{m}$ & & $1.80 \mathrm{E}+00$ & $\mathrm{mg} / \mathrm{kg}$ \\
\hline $\begin{array}{l}\text { BIS(2-CHLOROISOPROPYL) } \\
\text { ETHER }\end{array}$ & $0 / 12$ & & & NT & $8.78 \mathrm{E}-01$ & & $1.80 \mathrm{E}+00$ & $\mathrm{mg} / \mathrm{kg}$ \\
\hline $\begin{array}{l}\text { BIS(2-ETHYLHEXYL)- } \\
\text { PHTHALATE }\end{array}$ & $0 / 12$ & & & NT & $1.00 \mathrm{E}+00$ & & $1.80 \mathrm{E}+00$ & $\mathrm{mg} / \mathrm{kg}$ \\
\hline BROMODICHLOROMETHANE & $0 / 12$ & & & $\mathrm{NT}$ & $6.08 \mathrm{E}-03$ & & $7.00 \mathrm{E} 03$ & $\mathrm{mg} / \mathrm{kg}$ \\
\hline BROMOFORM & $0 / 12$ & & & NT & $6.08 \mathrm{E}-03$ & & $7.00 \mathrm{E}-03$ & $\mathrm{mg} / \mathrm{kg}$ \\
\hline BROMOMETHANE & $0 / 12$ & & & NT & $1.23 \mathrm{E}-02$ & & $1.30 \mathrm{E}-02$ & $\mathrm{mg} / \mathrm{kg}$ \\
\hline CARBON DISULFIDE & $0 / 12$ & & & NT & $6.08 \mathrm{E}-03$ & & $7.00 \mathrm{E}-03$ & $\mathrm{mg} / \mathrm{kg}$ \\
\hline CARBON TETRACHLORIDE & $0 / 12$ & & & NT & $6.08 \mathrm{E}-03$ & & $7.00 \mathrm{E}-03$ & $\mathrm{mg} / \mathrm{kg}$ \\
\hline CHLOROBENZENE & $0 / 12$ & & & NT & $6.08 \mathrm{E}-03$ & & $7.00 \mathrm{E}-03$ & $\mathrm{mg} / \mathrm{kg}$ \\
\hline CHLOROETHANE & $0 / 12$ & & & NT & $1.23 \mathrm{E}-02$ & & $1.30 \mathrm{E} \cdot 02$ & $\mathrm{mg} / \mathrm{kg}$ \\
\hline
\end{tabular}


Table C.2 (contimued)

\begin{tabular}{|c|c|c|c|c|c|c|c|c|}
\hline Analyte & $\begin{array}{l}\text { Frequency } \\
\text { of } \\
\text { detect }\end{array}$ & $\begin{array}{l}\text { Minimum } \\
\text { delected } \\
\text { concentration }\end{array}$ & $\begin{array}{l}\text { Maximum } \\
\text { detected } \\
\text { concentration }\end{array}$ & Distribution & $\begin{array}{l}\text { Mean } \\
\text { concentration }\end{array}$ & $\begin{array}{l}\text { Upper } 95 \% \\
\text { confidence } \\
\text { limit on mean }\end{array}$ & $\begin{array}{l}\text { Maximum } \\
\text { nondetected } \\
\text { concentration" }\end{array}$ & $\begin{array}{l}\text { Units } \\
\text { of } \\
\text { neasure }\end{array}$ \\
\hline CHLOROFORM & $0 / 12$ & & & NT & $6.17 \mathrm{E}-03$ & & $7.00 \mathrm{E}-03$ & $\mathrm{mg} / \mathrm{kg}$ \\
\hline CHLOROMETHANE & $0 / 12$ & & & $\mathrm{NT}$ & $1.23 E-02$ & & $1.30 \mathrm{E}-02$ & $\mathrm{mg} / \mathrm{kg}$ \\
\hline CHRYSENE & $5 / 12$ & $2.70 \mathrm{E}-01$ & $5.70 \mathrm{E}-01$ & $\mathrm{~L}$ & $3.70 \mathrm{E}-01$ & 4.45E-01 & & $\mathrm{mg} / \mathrm{kg}$ \\
\hline CIS-1,3-DICHLOROPROPENE & $0 / 12$ & & & NT & $6.08 \mathrm{E}-03$ & & $7.00 \mathrm{E}-03$ & $\mathrm{mg} / \mathrm{kg}$ \\
\hline DI-N-BUTYL PHTHALATE & $0 / 12$ & & & NT & B.78E-01 & & $1.80 \mathrm{E}+00$ & $\mathrm{mg} / \mathrm{kg}$ \\
\hline DI-N-OCTYL PHTHALATE & $0 / 12$ & & & NT & $8.78 \mathrm{E}-01$ & & $1.80 \mathrm{E}+00$ & $\mathrm{mg} / \mathrm{kg}$ \\
\hline DIBENZO(A,H)ANTHRACENE & $0 / 12$ & & & NT & $8.78 \mathrm{E}-01$ & & $1.80 \mathrm{E}+\infty$ & $\mathrm{mg} / \mathrm{kg}$ \\
\hline DIBENZOFURAN & $1 / 12$ & $1.70 \mathrm{E}-01$ & $1.70 \mathrm{E}-01$ & $L$ & $7.85 \mathrm{E}-01$ & $1.15 \mathrm{E}+\infty 0$ & & $\mathrm{mg} / \mathrm{kg}$ \\
\hline DIBROMOCHLOROMETHANE & $0 / 12$ & & & NT & $6.08 \mathrm{E}-03$ & & $7.00 \mathrm{E}-03$ & $\mathrm{mg} / \mathrm{kg}$ \\
\hline DIETHYL PHTHALATE & $1 / 12$ & $7.70 \mathrm{E}-02$ & $7.70 \mathrm{E}-02$ & $\mathbf{L}$ & $9.46 \mathrm{E}-01$ & $1.58 \mathrm{E}+00$ & & $\mathrm{mg} / \mathrm{kg}$ \\
\hline DIMETHYL PHTHALATE & $1 / 12$ & $6.40 \mathrm{E} \cdot 02$ & $6.40 \mathrm{E}-02$ & $\mathbb{N}$ & $8.45 \mathrm{E}-01$ & $1.16 \mathrm{E}+00$ & & $\mathrm{mg} / \mathrm{kg}$ \\
\hline ETHYLBENZENE & $0 / 12$ & & & $\mathbf{N T}$ & $6.08 \mathrm{E}-03$ & & $7.00 \mathrm{E}-03$ & $\mathrm{mg} / \mathrm{kg}$ \\
\hline FLUORANTHENE & $5 / 12$ & 4.10E-01 & $1.40 \mathrm{E}+00$ & $\mathbb{L}$ & $5.24 \mathrm{E}-01$ & $8.09 \mathrm{E}-01$ & & $\mathrm{mg} / \mathrm{kg}$ \\
\hline HEXACHLOROBENZENE & $0 / 12$ & & & NT & $8.78 \mathrm{E}-01$ & & $1.80 \mathrm{E}+\infty 0$ & $\mathrm{mg} / \mathrm{kg}$ \\
\hline HEXACHLOROBUTADIENE & $0 / 12$ & & & $\mathbb{N T}$ & $8.78 \mathrm{E}-01$ & & $1.80 E+\infty 0$ & $\mathrm{mg} / \mathrm{kg}$ \\
\hline $\begin{array}{l}\text { HEXACILOROCYCLO. } \\
\text { PENTADIENE }\end{array}$ & $0 / 12$ & & & $\mathrm{NT}$ & $8.78 \mathrm{E}-01$ & & $1.80 \mathrm{E}+00$ & $\mathrm{mg} / \mathrm{kg}$ \\
\hline HEXACHLOROETHANE & $0 / 12$ & & & NT & $8.78 \mathrm{E}-01$ & & $1.80 \mathrm{E}+\infty$ & $\mathrm{mg} / \mathrm{kg}$ \\
\hline INDENO(1,2,3-CD)PYRENE & $3 / 12$ & $9.90 \mathrm{E}-02$ & $2.60 \mathrm{E}-01$ & $\mathrm{~L}$ & $2.07 \mathbb{E}-01$ & $2.92 \mathrm{E}-01$ & & $\mathrm{mg} / \mathrm{kg}$ \\
\hline ISOPHORONE & $0 / 12$ & & & NT & $8.78 \mathrm{E}-01$ & & $1.80 \mathrm{E}+\infty 0$ & $\mathrm{mg} / \mathrm{kg}$ \\
\hline METHYLENE CHLORIDE & $2 / 12$ & $2.00 \mathrm{E}-03$ & 2.00E-03 & $\mathrm{L}$ & $7.39 \mathrm{E}-03$ & $1.02 \mathrm{E}-02$ & & $\mathrm{mg} / \mathrm{kg}$ \\
\hline
\end{tabular}


Table C2 (continued)

\begin{tabular}{|c|c|c|c|c|c|c|c|c|}
\hline Analyte & $\begin{array}{l}\text { Frequency } \\
\text { of } \\
\text { detect }\end{array}$ & $\begin{array}{l}\text { Minimum } \\
\text { detected } \\
\text { concentration }\end{array}$ & $\begin{array}{l}\text { Maximum } \\
\text { detected } \\
\text { concentration }\end{array}$ & Distribution & $\begin{array}{l}\text { Mean } \\
\text { concentration }\end{array}$ & $\begin{array}{l}\text { Upper } 95 \% \\
\text { confidence } \\
\text { limit on mean }\end{array}$ & $\begin{array}{l}\text { Maximum } \\
\text { nondetected } \\
\text { concentration }\end{array}$ & $\begin{array}{l}\text { Units } \\
\text { of } \\
\text { measure }\end{array}$ \\
\hline $\begin{array}{l}\text { N-NITROSO-DI-N- } \\
\text { PROPYLAMINE }\end{array}$ & $0 / 12$ & & & NT & $8.78 \mathrm{E}-01$ & & $1.80 \mathrm{E}+00$ & $\mathrm{mg} / \mathrm{kg}$ \\
\hline N-NITROSODIPHENYLAMINE & $0 / 12$ & & & NT & $8.78 \mathrm{E}-01$ & & $1.80 E+00$ & $\mathrm{mg} / \mathrm{kg}$ \\
\hline NAPHTHALENE & $1 / 12$ & $1.50 \mathrm{E}-01$ & $1.50 \mathrm{E}-01$ & L & $7.89 \mathrm{E}-01$ & $1.17 \mathrm{E}+00$ & & $\mathrm{mg} / \mathrm{kg}$ \\
\hline NITROBENZENE & $0 / 12$ & & & NT & $8.78 \mathrm{E}-01$ & & $1.80 \mathrm{E}+00$ & $\mathrm{mg} / \mathrm{kg}$ \\
\hline PENTACHLOROPHENOL & $0 / 12$ & & & NT & $4.26 E+00$ & & $8.70 \mathrm{E}+00$ & $\mathrm{mg} / \mathrm{kg}$ \\
\hline PHENANTHRENE & $5 / 12$ & $1.90 \mathrm{E}-01$ & $1.10 \mathrm{E}+\infty$ & $\mathbb{L}$ & 4.03E-01 & $6.29 \mathrm{E}-01$ & & $\mathrm{mg} / \mathrm{kg}$ \\
\hline PHENOL & $0 / 12$ & & & $\mathbb{N T}$ & $8.78 \mathrm{E}-01$ & & $1.80 \mathrm{E}+00$ & $\mathrm{mg} / \mathrm{kg}$ \\
\hline PYRENE & $5 / 12$ & $3.80 \mathrm{E}-01$ & $1.00 \mathrm{E}+00$ & $\mathbb{L}$ & $4.82 \mathrm{E}-01$ & $6.76 \mathrm{E}-01$ & & $\mathrm{mg} / \mathrm{kg}$ \\
\hline STYRENE & $0 / 12$ & & & $\mathrm{NT}$ & $6.08 \mathrm{E}-03$ & & $7.00 \mathrm{E}-03$ & $\mathrm{mg} / \mathrm{kg}$ \\
\hline TETRACHLOROETHENE & $2 / 12$ & $1.00 \mathrm{E}-03$ & $4.00 \mathrm{E}-03$ & $\mathbf{N}$ & $5.50 \mathrm{E}-03$ & $6.31 \mathrm{E}-03$ & & $m g / k g$ \\
\hline TOLUENE & $1 / 12$ & $3.00 \mathrm{E}-03$ & $3.00 \mathrm{E}-03$ & $\mathbb{N}$ & $5.83 \mathrm{E}-03$ & $6.32 \mathrm{E}-03$ & & $\mathrm{mg} / \mathrm{kg}$ \\
\hline TRANS-1,3-DICHLOROPROPENE & $0 / 12$ & & & NT & $6.08 \mathrm{E}-03$ & & $7.00 \mathrm{E}-03$ & $\mathrm{mg} / \mathrm{kg}$ \\
\hline TRICHLOROETHENE & $0 / 12$ & & & $\mathbb{N T}$ & $6.08 \mathrm{E}-03$ & & $7.00 \mathrm{E}-03$ & $\mathrm{mg} / \mathrm{kg}$ \\
\hline VINYL ACETATE & $0 / 12$ & & & $\mathbb{N T}$ & $1.23 \mathrm{E}-02$ & & $1.30 \mathrm{E}-02$ & $\mathrm{mg} / \mathrm{kg}$ \\
\hline VINYL CHLORIDE & $0 / 12$ & & & NT & $1.23 \mathrm{E}-02$ & & $1.30 \mathrm{E}-02$ & $\mathrm{mg} / \mathrm{kg}$ \\
\hline XYLENE (TOTAL) & $1 / 12$ & $2.00 \mathrm{E}-03$ & $2.00 \mathrm{E}-03$ & $\mathbf{N}$ & $5.75 \mathrm{E}-03$ & $6.38 \mathrm{E}-03$ & & $\mathrm{mg} / \mathrm{kg}$ \\
\hline
\end{tabular}

$\mathrm{N}=$ Normal Distribution; $\mathbb{L}=$ Lognormal Distribution; $\mathrm{NT}=$ No Test performed (analyte was not detected or insufficient data were available to perform test).

- The maximum nondetected concentration is reported only for those analytes which were not detected in any sample. 
C-34

Table C3. Comparison of BCV OU2 soil data with BSCP data"

\begin{tabular}{|c|c|c|c|c|c|c|c|}
\hline Analyte & $\begin{array}{l}\text { Freq. } \\
\text { of } \\
\text { detect }\end{array}$ & $\begin{array}{l}\text { BCV OU2 } \\
\text { median } \\
\text { conc. }\end{array}$ & $\begin{array}{l}\text { BCV OU2 } \\
\text { UCB95 } \\
\text { cone. }\end{array}$ & $\begin{array}{l}\text { BSCP } \\
\text { raedian } \\
\text { conc. }\end{array}$ & $\begin{array}{l}\text { BSCP } \\
\text { UCB9S } \\
\text { conc. }\end{array}$ & $\begin{array}{l}\text { Is BCV OU2 } \\
\text { UCB95 less } \\
\text { than BSCP } \\
\text { UCB95 ? }\end{array}$ & $\begin{array}{l}\text { Units } \\
\text { of } \\
\text { neasure }\end{array}$ \\
\hline \multicolumn{8}{|c|}{ RSA } \\
\hline \multicolumn{8}{|l|}{ Inorganics } \\
\hline ALUMINUM & $23 / 23$ & $1.90 \mathrm{E}+04$ & $1.93 \mathrm{E}+04$ & $1.78 \mathrm{E}+04$ & $2.02 E+04$ & - & $\mathrm{mg} / \mathrm{kg}$ \\
\hline ARSENIC & $23 / 23$ & $6.70 E+00$ & $9.16 \mathrm{E}+00$ & $6.84 \mathrm{E}+01$ & $1.08 E+02$ & 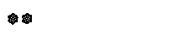 & $\mathrm{mg} / \mathrm{kg}$ \\
\hline BARIUM & $23 / 23$ & $1.00 \mathrm{E}+02$ & $1.06 \mathrm{E}+02$ & $1.16 E+01$ & $1.60 \mathrm{E}+01$ & & mg/kg \\
\hline BERYLLIUM & $23 / 23$ & $9.20 \mathrm{E}-01$ & $9.88 E-01$ & $7.53 \mathrm{E}-01$ & $9.60 \mathrm{E}-01$ & & $\mathrm{mg} / \mathrm{kg}$ \\
\hline CALCIUM & $21 / 23$ & $2.20 E+04$ & $2.87 \mathrm{E}+04$ & $1.72 \mathrm{E}+02$ & $2.69 \mathrm{E}+02$ & & $\mathrm{mg} / \mathrm{kg}$ \\
\hline CHROMIUM & $23 / 23$ & $2.70 E+01$ & $3.34 \mathrm{E}+01$ & $2.898+01$ & $3.45 E+01$ & - & $\mathrm{mg} / \mathrm{gg}$ \\
\hline COBALT & $23 / 23$ & $1.20 \mathrm{E}+01$ & $1.46 \mathrm{E}+01$ & $1.51 E+00$ & $3.54 E+00$ & & mofkg \\
\hline COPPER & $23 / 23$ & $2.90 \mathrm{E}+01$ & $4.25 E+01$ & $3.08 \mathrm{E}+01$ & $3.83 E+01$ & & $\mathrm{mg} / \mathrm{kg}$ \\
\hline IRON & $23 / 23$ & $274 E+04$ & $2.97 \mathrm{E}+04$ & $4.12 E+04$ & $4.55 E+04$ & $\infty$ & mgkgs \\
\hline LEAD & $23 / 23$ & $3.80 E+01$ & $4.40 E+01$ & $3.39 E+01$ & $5.12 \mathrm{E}+01$ & - & mg/kg \\
\hline MAGNESIUM & 19/23 & $3.70 \mathrm{E}+03$ & $5.34 E+03$ & $4.51 E+02$ & $5.39 E+02$ & & $\mathrm{mg} / \mathrm{kg}$ \\
\hline MANGANESE & $23 / 23$ & $6.20 \mathrm{E}+02$ & $7.08 \mathrm{E}+02$ & $9.25 E+01$ & $1.86 \mathrm{E}+02$ & & mg/sg \\
\hline MERCURY & $14 / 23$ & $1.00 \mathrm{E}+00$ & $1.45 E+\infty 0$ & $2.48 E-01$ & $2.93 E-01$ & & $\mathrm{mg} / \mathrm{kg}$ \\
\hline NICKEL & $23 / 23$ & $230 E+01$ & $3.46 \mathrm{E}+01$ & $1.58 E+01$ & $2.02 E+01$ & & mg/kg \\
\hline POTASSIUM & $23 / 23$ & $1.70 E+03$ & $1.92 E+03$ & $7.98 E+02$ & $9.27 \mathrm{E}+02$ & & $\mathrm{mg} / \mathrm{kg}$ \\
\hline SELENIUM & $1 / 18$ & $5.25 \mathrm{E}-01$ & $6.06 \mathrm{E}-01$ & 8.80E-01 & $1.12 E+00$ & 2 & $\mathrm{mg} / \mathrm{gg}$ \\
\hline SODIUM & $12 / 23$ & $1.01 E+02$ & $2.45 E+02$ & $3.59 \mathrm{E}+02$ & $3.89 \mathrm{E}+02$ & - & mofrgeg \\
\hline VANADIUM & $23 / 23$ & $3.10 \mathrm{E}+01$ & $3.83 E+01$ & $7.83 E+01$ & $8.74 \mathrm{E}+01$ & - & $\operatorname{mg} / k g$ \\
\hline ZINC & $19 / 23$ & $8.00 E+01$ & $1.02 E+02$ & $1.29 E+02$ & $1.73 E+02$ & $\%$ & $\mathrm{mg} / \mathrm{kg}$ \\
\hline \multicolumn{8}{|l|}{ Radionuclides } \\
\hline THORIUM-232 & $2 / 12$ & $2.62 E+\infty$ & $2.80 \mathrm{E}+00$ & $1.25 E+\infty 0$ & $1.71 E+\infty 0$ & & $\mathrm{pCi} / \mathrm{g}$ \\
\hline URANIUM-234 & $23 / 23$ & $1.68 \mathrm{E}+\infty 0$ & $2.46 \mathrm{E}+00$ & $1.91 E+\infty 0$ & $2.29 \mathrm{E}+\infty 0$ & & $\mathrm{pCi} / \mathrm{g}$ \\
\hline URANIUM-235 & $23 / 23$ & $8.91 E-02$ & $1.14 \mathrm{E}-01$ & $1.95 E-01$ & $3.59 E-01$ & 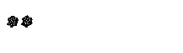 & $\mathrm{pCi} / \mathrm{g}$ \\
\hline URANIUM-238 & $23 / 23$ & $1.67 \mathrm{E}+00$ & $2.28 \mathrm{E}+\infty 0$ & $2.10 E+00$ & $2.52 \mathrm{E}+00$ & $\cdot$ & $\mathrm{pCi} / \mathrm{g}$ \\
\hline
\end{tabular}

$\$ Y-200$

Inorganics

$\begin{array}{llllllll}\text { ALUMINUM } & 75 / 89 & 1.66 \mathrm{E}+04 & 1.67 \mathrm{E}+04 & 1.78 \mathrm{E}+04 & 2.02 \mathrm{E}+04 & \mathrm{mg} / \mathrm{kg} \\ \text { ARSENIC } & 70 / 89 & 8.90 \mathrm{E}+00 & 1.15 \mathrm{E}+01 & 6.84 \mathrm{E}+01 & 1.08 \mathrm{E}+02 & \text { mg/kg } \\ \text { BARIUM } & 75 / 89 & 6.51 \mathrm{E}+01 & 6.04 \mathrm{E}+01 & 1.16 \mathrm{E}+01 & 1.60 \mathrm{E}+01 & \mathrm{mg} / \mathrm{kg} \\ \text { BERYLLIUM } & 75 / 89 & 9.40 \mathrm{E}-01 & 8.99 \mathrm{E}-01 & 7.53 \mathrm{E}-01 & 9.60 \mathrm{E}-01 & \mathrm{mg} / \mathrm{kg}\end{array}$




\section{C-35}

Table C3. (continued)

\begin{tabular}{|c|c|c|c|c|c|c|c|}
\hline Analyle & $\begin{array}{l}\text { Freq. } \\
\text { of } \\
\text { detect }\end{array}$ & $\begin{array}{l}\text { BCV OU2 } \\
\text { median } \\
\text { conc. }\end{array}$ & $\begin{array}{l}\text { BCV OU2 } \\
\text { UCB95 } \\
\text { conc. }\end{array}$ & $\begin{array}{l}\text { BSCP } \\
\text { median } \\
\text { conc. }\end{array}$ & $\begin{array}{l}\text { DSCP } \\
\text { UCB95 } \\
\text { conc. }\end{array}$ & $\begin{array}{l}\text { Is BCV OU2 } \\
\text { UCB95 less } \\
\text { than BSCP } \\
\text { UCB95 ?b }\end{array}$ & $\begin{array}{l}\text { Units } \\
\text { of } \\
\text { neasure }\end{array}$ \\
\hline CALCIUM & $75 / 89$ & $7.25 \mathrm{E}+03$ & $7.58 \mathrm{E}+03$ & $1.72 \mathrm{E}+02$ & $2.69 E+02$ & & $\mathrm{mg} / \mathrm{kg}$ \\
\hline CHROMIUM & $75 / 89$ & $2.73 E+01$ & $2.81 \mathrm{E}+01$ & $2.89 E+01$ & $3.45 E+01$ & $\Rightarrow$ & mog/sg \\
\hline COBALT & $73 / 89$ & $1.52 \mathrm{E}+01$ & $1.41 E+01$ & $1.51 E+00$ & $3.54 E+\infty 0$ & & $\mathrm{mg} / \mathrm{kg}$ \\
\hline COPPER & $75 / 89$ & $2.51 \mathrm{E}+01$ & $2.84 E+01$ & $3.08 \mathrm{E}+01$ & $3.83 \mathrm{E}+01$ & $\pitchfork$ & $\mathrm{mg} / \mathrm{kg}$ \\
\hline IRON & $75 / 89$ & $269 E+04$ & $2.87 E+104$ & $4.12 \mathrm{E}+04$ & $4.55 E+04$ & $\rightarrow$ & $\mathrm{mg} / \mathrm{sg}$ \\
\hline LEAD & $75 / 89$ & $3.47 E+01$ & $4.12 E+01$ & $3.39 E+01$ & $5.12 \mathrm{E}+01$ & - & mg/kg \\
\hline MAGNESIUM & $75 / 89$ & $254 \mathrm{E}+03$ & $2.38 E+03$ & $4.51 E+02$ & $5.39 E+02$ & & $\mathrm{mg} / \mathrm{kg}$ \\
\hline MANGANESE & $75 / 89$ & $5.53 E+02$ & $5.46 \mathrm{E}+02$ & $9.25 \mathrm{E}+01$ & $1.86 \mathrm{E}+02$ & & mg/kg \\
\hline MERCURY & $73 / 89$ & $5.30 E+\infty$ & $9.09 E+00$ & $2.48 E-01$ & $2.93 \mathrm{E}=01$ & & $\mathbf{m g} / \mathrm{kg}$ \\
\hline NICKEL & $75 / 89$ & $2.39 E+01$ & $2.30 \mathrm{E}+01$ & $1.58 \mathrm{E}+01$ & $2.02 E+01$ & & mog/kg \\
\hline POTASSIUM & $75 / 89$ & $1.46 \mathrm{E}+03$ & $1.46 \mathrm{E}+03$ & $7.98 \mathrm{E}+02$ & $9.27 \mathrm{E}+02$ & & $m g / k g$ \\
\hline SELENIUM & $15 / 49$ & $5.00 \mathrm{E}-01$ & $8.76 E-01$ & $8.80 \mathrm{E}-01$ & $1.12 E+00$ & 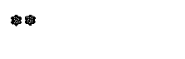 & meths \\
\hline SODIUM & $32 / 89$ & $5.80 \mathrm{E}+01$ & $5.68 \mathbb{E}+01$ & $3.59 E+02$ & $3.89 E+02$ & * & mengs \\
\hline VANADIUM & $75 / 89$ & $4.57 E+01$ & $4.85 E+01$ & $7.83 \mathrm{E}+01$ & $8.74 E+01$ & 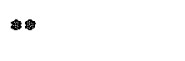 & mg/s: \\
\hline ZINC & $74 / 89$ & $8.20 \mathrm{E}+01$ & $9.31 E+01$ & $1.29 \mathrm{E}+02$ & $1.73 E+02$ & - & $\mathrm{mg} / \mathrm{ks}$ \\
\hline \multicolumn{8}{|l|}{ Radionuclides } \\
\hline URANIUM-234 & $89 / 89$ & $1.93 E+\infty$ & $2.14 E+00$ & $1.91 E+00$ & $2.29 \mathrm{E}+00$ & - & $\mathrm{pCi} / \mathrm{g}$ \\
\hline URANIUM-235 & $58 / 58$ & $1.06 \mathrm{E}-01$ & $1.12 \mathrm{E}-01$ & $1.95 \mathrm{E}-01$ & $3.59 \mathrm{E}-01$ & 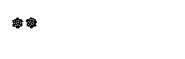 & $\mathrm{pCi} / \mathrm{s}$ \\
\hline URANIUM-238 & $89 / 89$ & $1.86 E+00$ & $1.99 E+00$ & $2.10 E+\infty 0$ & $2.52 E+\infty 0$ & 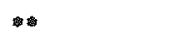 & $\mathrm{pCi} / \mathrm{s}$ \\
\hline
\end{tabular}

SA-1

Inorganics

$\begin{array}{lllllll}\text { ALUMINUM } & 11 / 12 & 2.43 \mathrm{E}+04 & 4.06 \mathrm{E}+04 & 1.78 \mathrm{E}+04 & 2.02 \mathrm{E}+04 & \mathrm{mg} / \mathrm{kg} \\ \text { ARSENIC } & 11 / 12 & 4.95 \mathrm{E}+00 & 8.72 \mathrm{E}+00 & 6.84 \mathrm{E}+01 & 1.08 \mathrm{E}+02 & \mathrm{mg} / \mathrm{kg} \\ \text { BARIUM } & 12 / 2 & 1.64 \mathrm{E}+02 & 2.14 \mathrm{E}+02 & 1.16 \mathrm{E}+01 & 1.60 \mathrm{E}+01 & \mathrm{mg} / \mathrm{kg} \\ \text { BERYLLIUM } & 12 / 2 & 1.04 \mathrm{E}+00 & 1.19 \mathrm{E}+00 & 7.53 \mathrm{E}-01 & 9.60 \mathrm{E}-01 & \mathrm{mg} / \mathrm{kg} \\ \text { CALCIUM } & 12 / 12 & 5.87 \mathrm{E}+03 & 1.14 \mathrm{E}+04 & 1.72 \mathrm{E}+02 & 2.69 \mathrm{E}+02 & \mathrm{mg} / \mathrm{kg} \\ \text { CHROMIUM } & 12 / 12 & 3.22 \mathrm{E}+01 & 3.54 \mathrm{E}+01 & 2.89 \mathrm{E}+01 & 3.45 \mathrm{E}+01 & \mathrm{mg} / \mathrm{kg} \\ \text { COBALT } & 12 / 2 & 1.62 \mathrm{E}+01 & 1.83 \mathrm{E}+01 & 1.51 \mathrm{E}+00 & 3.54 \mathrm{E}+00 & \mathrm{mg} / \mathrm{kg} \\ \text { COPPER } & 12 / 12 & 2.34 \mathrm{E}+01 & 3.16 \mathrm{E}+01 & 3.08 \mathrm{E}+01 & 3.83 \mathrm{E}+01 & \mathrm{mg} / \mathrm{kg} \\ \text { IRON } & 11 / 12 & 2.89 \mathrm{E}+04 & 5.71 \mathrm{E}+04 & 4.12 \mathrm{E}+04 & 4.55 \mathrm{E}+04 & \mathrm{mg} / \mathrm{kg} \\ \text { LEAD } & 12 / 12 & 2.64 \mathrm{E}+01 & 3.78 \mathrm{E}+01 & 3.39 \mathrm{E}+01 & 5.12 \mathrm{E}+01 & \mathrm{mg} / \mathrm{kg}\end{array}$


C-36

Table C.3. (continued)

\begin{tabular}{|c|c|c|c|c|c|c|c|}
\hline Analyte & $\begin{array}{l}\text { Freq. } \\
\text { of } \\
\text { detect }\end{array}$ & $\begin{array}{l}\text { BCV OU2 } \\
\text { median } \\
\text { cone. }\end{array}$ & $\begin{array}{l}\text { BCV OU2 } \\
\text { UCB95 } \\
\text { conc. }\end{array}$ & $\begin{array}{l}\text { BSCP } \\
\text { median } \\
\text { cone. }\end{array}$ & $\begin{array}{l}\text { BSCP } \\
\text { UCB9S } \\
\text { conc. }\end{array}$ & $\begin{array}{l}\text { Is BCV OU2 } \\
\text { UCB95 less } \\
\text { than BSCP } \\
\text { UCB95 ? }\end{array}$ & $\begin{array}{l}\text { Units } \\
\text { of } \\
\text { measure }\end{array}$ \\
\hline MAGNESIUM & $11 / 12$ & $6.17 E+03$ & $9.16 \mathrm{E}+03$ & $4.51 E+02$ & $5.39 E+02$ & & mglkg \\
\hline MANGANESE & $12 / 12$ & $8.16 \mathrm{E}+02$ & $1.07 \mathrm{E}+03$ & $9.25 E+01$ & $1.86 \mathrm{E}+02$ & & $\mathrm{mg} / \mathrm{kg}$ \\
\hline MERCURY & $8 / 12$ & $1.80 \mathrm{E}-01$ & 4.45E-01 & $2.48 \mathrm{E}-01$ & $2.93 \mathrm{E}-01$ & & $\mathrm{mg} / \mathrm{kg}$ \\
\hline NICKEL & $12 / 12$ & $2.93 E+01$ & $3.34 \mathrm{E}+01$ & $1.58 \mathrm{E}+01$ & $2.02 \mathrm{E}+01$ & & $\mathrm{mg} / \mathrm{gg}$ \\
\hline POTASSIUM & $11 / 12$ & $3.39 \mathrm{E}+03$ & $3.57 E+03$ & $7.98 E+02$ & $9.27 \mathrm{E}+02$ & & mg/kg \\
\hline SODIUM & $12 / 12$ & $1.53 E+02$ & $1.64 E+02$ & $3.59 E+02$ & $3.89 \mathrm{E}+02$ & $*$ & mglkg \\
\hline VANADIUM & $12 / 12$ & $3.08 \mathrm{E}+01$ & $4.04 E+01$ & $7.83 E+01$ & $8.74 \mathbb{E}+01$ & \% & $\operatorname{mg} / \mathrm{kg}$ \\
\hline ZINC & $12 / 12$ & $7.65 \mathrm{E}+01$ & $1.08 \mathrm{E}+02$ & $1.29 \mathrm{E}+02$ & $1.73 E+02$ & 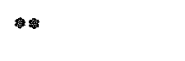 & $\operatorname{mg} / \mathrm{kg}$ \\
\hline \multicolumn{8}{|l|}{ Radionuclides } \\
\hline RADIUM-226 & $12 / 12$ & $2.09 E+00$ & $2.95 E+00$ & $1.61 E+\infty$ & $2.11 E+\infty 0$ & & $\mathrm{pCi} / \mathrm{s}$ \\
\hline URANIUM-234 & $12 / 12$ & $1.40 E+00$ & $2.92 E+100$ & $1.91 E+00$ & $229 E+00$ & & $\mathrm{pCi} / \mathrm{s}$ \\
\hline URANIUM-235 & $12 / 12$ & $6.38 \mathrm{E}-02$ & $1.33 E-01$ & $1.95 \mathrm{E}-01$ & $3.59 \mathrm{E}-01$ & 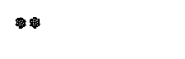 & $\mathrm{pCi} / \mathrm{s}$ \\
\hline URANIUM-238 & $12 / 12$ & $1.39 E+00$ & $289 \mathrm{E}+\infty 0$ & $210 E+00$ & $252 E+00$ & & pCi/g \\
\hline
\end{tabular}

UCB95 = Upper 95\% Confidence Bound on Median Concentration

a Background Soil Characterization Project (BSCP) (Energy Systems 1993c); Copper Ridge horizon C.

- If the BCV OU2 analyte UCB95 concentration is less than the BSCP UCB95 concentration (represented by and/or **), that analyte is considered to be background; a single asterisk (") is shown if the BCV OU2 UCB95 is $<$ BSCP UCB95, and a double asterisk (**) is shown if the BCV OU2 UCB95 is < BSCP median concentration. 
Table C.42. Toxicity screening for BCV OU2 detected inorganic and organic analytes in soil (carcinogenic and noncarcinogenic, ingestion and inhalation)

\begin{tabular}{|c|c|c|c|c|c|c|c|c|c|c|c|c|c|c|}
\hline Analyse & $\begin{array}{l}\text { Maxinum } \\
\text { detected } \\
\text { conc.; } \\
\text { mg/kg }\end{array}$ & $\begin{array}{l}\text { Oral } \\
\text { RfD; } \\
\text { mg/kg-d }\end{array}$ & $\begin{array}{l}\text { Inmal. } \\
\text { RIDD: } \\
\text { mg/kg-d }\end{array}$ & $\begin{array}{l}\text { Oral } \\
\text { SF; } \\
\text { lig-d/mg }\end{array}$ & $\begin{array}{l}\text { Inhal. } \\
\mathrm{SF} ; \\
\mathrm{kg}-\mathrm{d} / \mathrm{mg}\end{array}$ & $\begin{array}{l}\text { Noncarc. } \\
\text { oral } \\
\text { foxicity } \\
\text { score }\end{array}$ & $\begin{array}{l}\text { \% of } \\
\text { Total } \\
\text { monc. } \\
\text { oral } \\
\text { score }\end{array}$ & $\begin{array}{l}\text { Noncarc. } \\
\text { in inal. } \\
\text { toxicity } \\
\text { score }\end{array}$ & $\begin{array}{l}\text { \% of } \\
\text { Total } \\
\text { nonc. } \\
\text { inhal. } \\
\text { score }\end{array}$ & $\begin{array}{l}\text { Carcin. } \\
\text { oral } \\
\text { toxicity } \\
\text { score }\end{array}$ & $\begin{array}{l}\text { \% of } \\
\text { Total } \\
\text { carc. } \\
\text { oral } \\
\text { score }\end{array}$ & $\begin{array}{l}\text { Carcin. } \\
\text { inhal. } \\
\text { loxicity } \\
\text { score }\end{array}$ & $\begin{array}{l}\% \text { of } \\
\text { Total } \\
\text { carc. } \\
\text { inhal. } \\
\text { score }\end{array}$ & $\begin{array}{l}\text { Final } \\
\text { decision }\end{array}$ \\
\hline & & & & & & RSA & & & & & & & & \\
\hline \multicolumn{15}{|l|}{ Inorganics } \\
\hline ANTIMONY & $3.60 \mathrm{E}-01$ & 4.0E-04 & & & & $9.00 E+02$ & 0.66 & & & & & & & DROP \\
\hline BARIUM & $1.86 \mathrm{E}+02$ & $7.0 \mathrm{E}-02$ & $1.4 E-04$ & & & $266 E+03$ & 1.94 & $1.30 E+06$ & 0.66 & & & & & \\
\hline BERYLLIUM & $210 E+\infty 00$ & $5.0 \mathrm{E}-03$ & & $4.3 E+\infty$ & $8.4 E+00$ & $4.20 \mathrm{E}+02$ & 0.31 & & & $9.03 E+00$ & 10.46 & $1.76 \mathrm{E}+01$ & 33.62 & \\
\hline CADMUUM & $5.70 \mathrm{E}+00$ & $1.0 \mathrm{E}-03$ & & & $6.1 E+00$ & $5.70 E+03$ & 4.16 & & & & & $3.48 \mathrm{E}+01$ & 66.26 & \\
\hline CYANIDE & $246 \mathrm{E}+01$ & $20 \mathrm{E}-02$ & & & & $1.23 E+03$ & 0.90 & & & & & & & DROP \\
\hline FLUORENE & $8.30 \mathrm{E}-02$ & $4.0 E-02$ & & & & $208 E+\infty 0$ & 0.00 & & & & & & & DROP \\
\hline MANGANESE & $280 E+03$ & $1.4 \mathrm{E}-01$ & 1.4E-05 & & & $200 E+04$ & 14.60 & $1.96 \mathrm{E}+08$ & 99.29 & & & & & \\
\hline MERCURY & $7.90 E+10$ & $3.0 \mathrm{E}-04$ & $8.6 \mathrm{E}-05$ & & & $263 \mathrm{E}+04$ & 19.22 & $9.22 \mathrm{E}+04$ & 0.05 & & & & & \\
\hline NICKEL & $3.11 E+02$ & $20 \mathrm{E}-02$ & & & & $1.56 \mathrm{E}+04$ & 11.35 & & & & & & & \\
\hline SILVER & $1.90 E+00$ & $5.0 \mathrm{E}-03$ & & & & $3.80 E+02$ & 0.28 & & & & & & & DROP \\
\hline URANIUM, TOTAL & $1.20 \mathrm{E}+01$ & $3.0 \mathrm{E}-03$ & & & & $4.00 E+03$ & 292 & & & & & & & \\
\hline \multicolumn{15}{|l|}{ Organics } \\
\hline $\begin{array}{l}\text { 1,1,2.TRICHLORO. } \\
\text { 1,2,2.TRIFLUORO. } \\
\text { ETHANE }\end{array}$ & $3.20 \mathrm{E}+01$ & $3.0 \mathrm{E}+01$ & $8.6 \mathrm{E}+\infty$ & & & $1.07 E+\infty 0$ & 0.00 & $3.73 E+00$ & 0.00 & & & & & DROP \\
\hline $\begin{array}{l}\text { 1,1-DICHLORO- } \\
\text { ETHANE }\end{array}$ & $6.40 \mathrm{E}-01$ & $1.0 \mathrm{E}-01$ & $1.4 \mathrm{E}-01$ & & & $5.40 \mathrm{E}+00$ & 0.00 & $4.48 E+00$ & 0.00 & & & & & DROP \\
\hline $\begin{array}{l}\text { 1,1-DICHLORO- } \\
\text { ETHENE }\end{array}$ & $5.10 \mathrm{E}-02$ & $9.0 \mathrm{E}-03$ & & $6.0 \mathrm{E}-01$ & $1.2 E+00$ & $5.67 E+00$ & 0.00 & & & $3.06 \mathrm{E}-02$ & 0.04 & $6.12 \mathrm{E}-02$ & 0.12 & DROP \\
\hline $\begin{array}{l}\text { 1,24-TRICHLORO. } \\
\text { BENZENE }\end{array}$ & $4.30 \mathrm{E}+01$ & $1.0 \mathrm{E}-02$ & 5.7E-02 & & & $4.30 \mathrm{E}+03$ & 3.14 & $7.53 E+02$ & 0.00 & & & & & \\
\hline
\end{tabular}


Table C.4a. (continued)

\begin{tabular}{|c|c|c|c|c|c|c|c|c|c|c|c|c|c|c|}
\hline Analyre & $\begin{array}{l}\text { Maximum } \\
\text { delected } \\
\text { conc.; } \\
\text { mg/kg }\end{array}$ & $\begin{array}{l}\text { Oral } \\
\mathbb{R P D} ; \\
\mathrm{mg} / \mathrm{kg}-\mathrm{d}\end{array}$ & $\begin{array}{l}\text { Inhal, } \\
\text { RfD } \\
\text { mg/kg-d }\end{array}$ & $\begin{array}{l}\text { Oral } \\
\mathrm{SP} ; \\
\mathrm{lg}-\mathrm{d} / \mathrm{mg}\end{array}$ & $\begin{array}{l}\text { Inhal. } \\
\text { SF; } \\
\text { Ig-d/mag }\end{array}$ & $\begin{array}{l}\text { Noncarc. } \\
\text { oral } \\
\text { toxicily } \\
\text { score }\end{array}$ & $\begin{array}{l}\text { \% of } \\
\text { Total } \\
\text { mone. } \\
\text { oral } \\
\text { score }\end{array}$ & $\begin{array}{l}\text { Noncarc. } \\
\text { inhal. } \\
\text { toxicity } \\
\text { score }\end{array}$ & $\begin{array}{l}\text { \% of } \\
\text { Total } \\
\text { none. } \\
\text { inhal. } \\
\text { score }\end{array}$ & $\begin{array}{l}\text { Carcin. } \\
\text { oral } \\
\text { toxicity } \\
\text { score }\end{array}$ & $\begin{array}{l}\% \text { of } \\
\text { Total } \\
\text { carc. } \\
\text { oral } \\
\text { score }\end{array}$ & $\begin{array}{l}\text { Carcin. } \\
\text { inhal. } \\
\text { toxicity } \\
\text { score }\end{array}$ & $\begin{array}{l}\% \text { of } \\
\text { Total } \\
\text { carc. } \\
\text { inhal. } \\
\text { score }\end{array}$ & $\begin{array}{l}\text { Final } \\
\text { decisione }^{\circ}\end{array}$ \\
\hline $\begin{array}{l}\text { 1,2-DICHLORO- } \\
\text { BENZENE }\end{array}$ & $3.60 \mathrm{E}-01$ & $9.0 \mathrm{E}-02$ & $5.7 \mathrm{E}-02$ & & & $4.00 \mathrm{E}+\infty 0$ & 0.00 & $6.30 \mathrm{E}+00$ & 0.00 & & & & & DROP \\
\hline $\begin{array}{l}\text { 1,2-DICHLORO- } \\
\text { ETHANE }\end{array}$ & $3.00 E-03$ & & & $9.1 \mathrm{E}-02$ & $9.1 \mathrm{E}-12$ & & & & & $273 \mathrm{E}-04$ & 0.00 & $273 \mathrm{E}-04$ & 000 & DROP \\
\hline $\begin{array}{l}\text { 1,2-DICHLORO- } \\
\text { ETHENE (TOTAL) }\end{array}$ & $1.10 \mathrm{E}+02$ & $9.0 \mathrm{E}-03$ & & & & $1.22 E+04$ & 8.92 & & & & & & & \\
\hline $\begin{array}{l}\text { 24-DIMETHYL- } \\
\text { PHENOL }\end{array}$ & $3.40 \mathrm{E}-01$ & $20 \mathrm{E}-02$ & & & & $1.70 \mathrm{E}+01$ & 001 & & & & & & & DROP \\
\hline 2-BUTANONE & $270 \mathrm{E}-01$ & $6.0 \mathrm{E}-01$ & $29 \mathrm{E}-01$ & & & $4.50 \mathrm{E}-01$ & 0.00 & 9.45E-01 & 0.00 & & & & & DROP \\
\hline $\begin{array}{l}\text { M-METHYL-2- } \\
\text { PENTANONE }\end{array}$ & $210 E-01$ & $8.0 E-02$ & $23 \mathrm{E}-02$ & & & $263 E+00$ & 0.00 & $9.19 E+\infty$ & 0.00 & & & & & DROP \\
\hline 4-METHYLPHENOL & $5.20 E-01$ & $5.0 \mathrm{E}-03$ & & & & $1.04 E+02$ & 0.08 & & & & & & & DROP \\
\hline ACENAPHTHENE & $6.60 \mathrm{E}-02$ & $6.0 \mathrm{E}-02$ & & & & $1.10 \mathrm{E}+00$ & 0.00 & & & & & & & DROP \\
\hline ACETONE & $250 E+\infty 0$ & $1.0 \mathrm{E}-01$ & & & & $250 E+01$ & 0.02 & & & & & & & DROP \\
\hline ANTHRACENE & $1.40 \mathrm{E}-01$ & $3.0 \mathrm{E}-01$ & & & & 4.67E-01 & 0.00 & & & & & & & DROP \\
\hline AROCLOR-1254 & $8.90 \mathrm{E}+00$ & & & $7.7 \mathrm{E}+\infty$ & & & & & & $6.85 \mathrm{E}+01$ & 79.39 & & & \\
\hline BENZENE & $1.00 \mathrm{E}-02$ & & & $2.9 \mathrm{E}-02$ & $29 \mathrm{E}-02$ & & & & & 290E-04 & 0.00 & $290 \mathrm{E}-04$ & 0.00 & DROP \\
\hline $\begin{array}{l}\text { BENZO(A)- } \\
\text { ANTHRACENE }\end{array}$ & $410 E-01$ & & & 7.3E-01 & & & & & & $299 \mathrm{E}-01$ & 0.35 & & & DROP \\
\hline BENZO(A)PYRENE & $5.80 \mathrm{E}-01$ & & & $7.3 E+\infty 0$ & & & & & & $423 E+00$ & 4.90 & & & \\
\hline $\begin{array}{l}\text { BENZO(B)- } \\
\text { FLUORANTHENE }\end{array}$ & $5.70 \mathrm{E}-01$ & & & 7.3E-01 & & & & & & $4.16 \mathrm{E}-01$ & 0.48 & & & DROP \\
\hline $\begin{array}{l}\text { BENZO(K)- } \\
\text { PLUORANTHENE }\end{array}$ & $4.30 \mathrm{E}-01$ & & & $7.3 \mathrm{E}-02$ & & & & & & $3.14 \mathrm{E}-02$ & 0.04 & & & DROP \\
\hline
\end{tabular}


Table C.42 (continued)

\begin{tabular}{|c|c|c|c|c|c|c|c|c|c|c|c|c|c|c|}
\hline Amslyte & $\begin{array}{l}\text { Maximu m } \\
\text { detected } \\
\text { conc; } \\
\text { mg/kg }\end{array}$ & 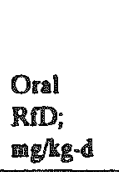 & $\begin{array}{l}\text { Imhal. } \\
\text { RmD; } \\
\text { mg/kg-d }\end{array}$ & $\begin{array}{l}\text { Oral } \\
\text { SF; } \\
\text { LE-d/mg }\end{array}$ & 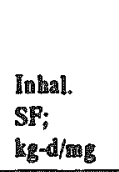 & $\begin{array}{l}\text { Noncare. } \\
\text { oral } \\
\text { toxicity } \\
\text { core }\end{array}$ & $\begin{array}{l}\text { \% of } \\
\text { Total } \\
\text { mone. } \\
\text { oral } \\
\text { score }\end{array}$ & $\begin{array}{l}\text { Noncarc. } \\
\text { inhal. } \\
\text { loxicity } \\
\text { score }\end{array}$ & $\begin{array}{l}\% \text { of } \\
\text { Total } \\
\text { monc. } \\
\text { inhal. } \\
\text { scose }\end{array}$ & $\begin{array}{l}\text { Carcis. } \\
\text { oral } \\
\text { toxicily } \\
\text { score }\end{array}$ & $\begin{array}{l}\% \text { of } \\
\text { Total } \\
\text { carc. } \\
\text { oral } \\
\text { score }\end{array}$ & $\begin{array}{l}\text { Carcin. } \\
\text { inhal. } \\
\text { toricity } \\
\text { score }\end{array}$ & $\begin{array}{l}\% \text { of } \\
\text { Total } \\
\text { carc. } \\
\text { inhal. } \\
\text { score }\end{array}$ & $\begin{array}{l}\text { Final } \\
\text { decision }\end{array}$ \\
\hline $\begin{array}{l}\text { BENZYL BUTYL } \\
\text { PHTHALATE }\end{array}$ & $7.30 \mathrm{E}-02$ & $20 \mathrm{E}-01$ & & & & $3.65 \mathrm{E}-01$ & 0.00 & & & & & & & DROP \\
\hline $\begin{array}{l}\text { BIS(2-ETHYL- } \\
\text { HEXYL)- } \\
\text { PHTHALATE }\end{array}$ & $4.10 \mathrm{E}+01$ & $20 E-02$ & & $1.4 E-02$ & & $20 S E+03$ & 1.50 & & & $5.74 \mathrm{E}-01$ & 0.66 & & & \\
\hline CARBAZOLE & $1.00 \mathrm{E}-01$ & & & $20 \mathrm{E}-02$ & & & & & & $200 \mathrm{E}-03$ & 0.00 & & & DROP \\
\hline CARBON DISULFIDE & $1.80 \mathrm{E}-02$ & $1.0 \mathrm{E}-01$ & $29 E-03$ & & & $1.80 \mathrm{E}-01$ & 0.00 & $6.30 \mathrm{E}+00$ & 0.00 & & & & & DROP \\
\hline CHLOROBENZENE & $3.00 \mathrm{E}-03$ & $20 \mathrm{E}-02$ & $5.7 \mathrm{E}-03$ & & & $3.50 \mathrm{E}-01$ & 0.00 & $1.23 E+\infty$ & 0.00 & & & & & DROP \\
\hline CHRYSENE & $4.20 \mathrm{E}-01$ & & & $7.3 \mathrm{E}-03$ & & & & & & $3.07 \mathbb{E}-03$ & 0.00 & & & DROP \\
\hline $\begin{array}{l}\text { DI-N.BUTYL } \\
\text { PHTLALATE }\end{array}$ & $2.30 E+00$ & $1.0 \mathrm{E}-01$ & & & & $230 \mathrm{E}+01$ & 0.02 & & & & & & & DROP \\
\hline $\begin{array}{l}\text { DI-N-OCTYL } \\
\text { PHTHALATE }\end{array}$ & $8.00 \mathrm{E}-01$ & $20 \mathrm{E}-02$ & & & & $4.00 \mathrm{E}+01$ & 0.03 & & & & & & & DROP \\
\hline $\begin{array}{l}\text { DIBENZO(A,H)- } \\
\text { ANTHRACENE }\end{array}$ & $290 \mathrm{E}-01$ & & & $7.3 E+\infty$ & & & & & & $212 \mathbb{E}+00$ & 2.45 & & & \\
\hline $\begin{array}{l}\text { DIMETHYL } \\
\text { PHTHALATE }\end{array}$ & $1.50 \mathrm{E}-01$ & $1.0 E+01$ & & & & $1.50 E-02$ & 0.00 & & & & & & & DROP \\
\hline ETHYLBENZENE & $280 \mathrm{E}-01$ & $1.0 \mathrm{E}-01$ & $2.9 \mathrm{E}-01$ & & & $2.80 E+00$ & 0.00 & $9.80 \mathrm{E}-01$ & 0.00 & & & & & DROF \\
\hline FLUORANTHENE & $7.80 \mathrm{E}-01$ & $4.0 \mathrm{E}-02$ & & & & $1.95 E+01$ & 0.01 & & & & & & & DROP \\
\hline HEPTACHLOR & $8.80 \mathrm{E}-04$ & $5.0 \mathrm{E}-04$ & & $4.5 E+\infty$ & $4.5 E+\infty$ & $1.76 E+\infty$ & 0.00 & & & $3.96 \mathrm{E}-03$ & 0.00 & $3.96 \mathrm{E}-03$ & 0.01 & DROP \\
\hline $\begin{array}{l}\text { INDENO }(1,2,3-C D) \text { - } \\
\text { PYRENE }\end{array}$ & $8.40 \mathrm{E}-01$ & & & $7.3 \mathrm{E}-01$ & & & & & & $6.13 \mathrm{E}-01$ & 0.71 & & & DROP \\
\hline $\begin{array}{l}\text { METHYLENE } \\
\text { CHLORIDE }\end{array}$ & $5.80 \mathrm{E}+01$ & $6.0 \mathrm{E}-02$ & $8.6 \mathrm{E}-01$ & 7.5E-03 & & $9.67 \mathrm{E}+02$ & 0.71 & $6.77 \mathrm{E}+01$ & 0.00 & 4.35E-01 & 0.50 & & & DROP \\
\hline $\begin{array}{l}\text { P,P'- } \\
\text { METHOXYCHLOR }\end{array}$ & $1.50 \mathrm{E}-03$ & $5.0 \mathrm{E}-03$ & & & & $3.00 \mathrm{E}-01$ & 0.00 & & & & & & & DROP \\
\hline
\end{tabular}


Table C42 (continued)

\begin{tabular}{|c|c|c|c|c|c|c|c|c|c|c|c|c|c|c|}
\hline Amalyte & $\begin{array}{l}\text { Maximum } \\
\text { detected } \\
\text { conc.; } \\
\text { mg/kg }\end{array}$ & $\begin{array}{l}\text { Oral } \\
\text { RfD; } \\
\text { ang/kg-d }\end{array}$ & $\begin{array}{l}\text { Inhal. } \\
\text { RfD'; } \\
\text { gafled }\end{array}$ & $\begin{array}{l}\text { Oral } \\
\text { SF; } \\
\text { lg-d/mg }\end{array}$ & $\begin{array}{l}\text { Inhal. } \\
\mathrm{SP}^{2} \\
\text { \&ged/mg }\end{array}$ & $\begin{array}{l}\text { Nomcarc. } \\
\text { oral } \\
\text { toxicify } \\
\text { score }\end{array}$ & $\begin{array}{l}\% \text { of } \\
\text { Total } \\
\text { monc. } \\
\text { oral } \\
\text { gcore }\end{array}$ & $\begin{array}{l}\text { Nomcape. } \\
\text { in mal. } \\
\text { loxicity } \\
\text { score }\end{array}$ & $\begin{array}{l}\% \text { of } \\
\text { Total } \\
\text { mone. } \\
\text { inhal. } \\
\text { score }\end{array}$ & $\begin{array}{l}\text { Carciu. } \\
\text { oral } \\
\text { toxicity } \\
\text { score }\end{array}$ & $\begin{array}{l}\% \text { of } \\
\text { Total } \\
\text { care. } \\
\text { oral } \\
\text { score }\end{array}$ & $\begin{array}{l}\text { Carcin. } \\
\text { inhlal. } \\
\text { losicity } \\
\text { score }\end{array}$ & $\begin{array}{l}\% \text { of } \\
\text { Total } \\
\text { carc. } \\
\text { in hal. } \\
\text { score }\end{array}$ & $\begin{array}{l}\text { Final } \\
\text { decisione }^{\circ}\end{array}$ \\
\hline $\begin{array}{l}\text { PENTACHLORO- } \\
\text { PHENOL }\end{array}$ & $4.70 \mathrm{E}-02$ & $3.0 \mathrm{E}-02$ & & $1.2 \mathrm{E}-01$ & & $1.57 E+\infty$ & 0.00 & & & $5.64 \mathrm{E}-03$ & 0.01 & & & DROP \\
\hline PHENOL & $2.50 E+00$ & $6.0 \mathrm{E}-01$ & & & & $4.17 E+\infty$ & 0.00 & & & & & & & DROP \\
\hline PYRENE & $1.10 \mathrm{E}+00$ & $3.0 \mathrm{E}-02$ & & & & $3.67 E+01$ & 0.03 & & & & & & & DROP \\
\hline $\begin{array}{l}\text { TETRACHLORO- } \\
\text { ETHENE }\end{array}$ & $4.00 \mathrm{E}+02$ & $1.0 \mathrm{E}-02$ & & & & $4,00 E+04$ & 29.19 & & & & & & & \\
\hline TOLUENE & $7.50 \mathrm{E}-01$ & $20 \mathrm{E}-01$ & $1.1 E-01$ & & & $3.75 E+00$ & 0.00 & $6.56 \mathrm{E}+10$ & 0.00 & & & & & DROP \\
\hline XYLENE (TOTAL) & $9.60 \mathrm{E}-01$ & $20 \mathrm{E}+00$ & & & & $230 \mathrm{E}-01$ & 0.00 & & & & & & & DROP \\
\hline Toxicity Score Totals & & & & & & $1.37 E+05$ & 100.0 & $1.97 \mathrm{E}+0$ & 100.0 & $8.63 E+01$ & 100.0 & $5.25 \mathrm{E}+01$ & 100.0 & \\
\hline & & & & & & $\$ S Y-200$ & & & & & & & & \\
\hline \multicolumn{15}{|l|}{ Inorganics } \\
\hline ANTIMONY & $9.20 \mathrm{E}-01$ & $4.0 \mathrm{E}-04$ & & & & $230 E+03$ & 0.08 & & & & & & & DROP \\
\hline BARIUM & $277 \mathrm{E}+02$ & $7.0 \mathrm{E}-02$ & $1.4 \mathrm{E}-04$ & & & $3.96 \mathrm{E}+03$ & 0.14 & $1.94 E+06$ & 0.82 & & & & & DROP \\
\hline CADMUUM & $3.10 E+\infty$ & 1.0E-03 & & & $6.1 E+00$ & $3.10 \mathrm{E}+03$ & 0.11 & & & & & $1.89 E+01$ & 100.0 & \\
\hline FLUORENE & $1.30 \mathrm{E}-01$ & $4.0 \mathrm{E}-02$ & & & & $3.25 E+\infty$ & 0.00 & & & & & & & DROP \\
\hline MANGANESE & $3.23 E+03$ & 1.4E-01 & 1.4E-0S & & & $2.31 E+04$ & 0.84 & $226 \mathbb{E}+08$ & 95.18 & & & & & \\
\hline MERCURY & $8.16 \mathrm{E}+02$ & $3.0 \mathrm{E}-04$ & $8.6 \mathrm{E}-05$ & & & $272 E+06$ & 98.69 & $9.52 E+06$ & 4.01 & & & & & \\
\hline NICKEL & $6.01 \mathrm{E}+01$ & $20 \mathrm{E}-02$ & & & & $3.01 E+03$ & 0.11 & & & & & & & DROP \\
\hline SILVER & $3.00 \mathrm{E}+00$ & $5.0 \mathrm{E}-03$ & & & & $6.00 E+02$ & 0.02 & & & & & & & DROP \\
\hline
\end{tabular}


Table C.4a. (continued)

\begin{tabular}{|c|c|c|c|c|c|c|c|c|c|c|c|c|c|c|}
\hline Analyte & $\begin{array}{l}\text { Masimura } \\
\text { detected } \\
\text { conc:; } \\
\text { w/lgeg }\end{array}$ & $\begin{array}{l}\text { Oral } \\
\text { Rm; } \\
\text { mg/kg-d }\end{array}$ & $\begin{array}{l}\text { Inhal. } \\
\mathrm{ROD}^{4} \\
\text { Iagkg-d }\end{array}$ & $\begin{array}{l}\text { Oral } \\
\text { SF; } \\
\text { kg-d/mg }\end{array}$ & $\begin{array}{l}\text { Imhal. } \\
\text { SF; } \\
\text { Ig-d/mg }\end{array}$ & $\begin{array}{l}\text { Noncarc. } \\
\text { oral } \\
\text { toxicity } \\
\text { score }\end{array}$ & $\begin{array}{l}\% \text { of } \\
\text { Total } \\
\text { nose. } \\
\text { oral } \\
\text { scoose }\end{array}$ & $\begin{array}{l}\text { Nomcarc. } \\
\text { inbogl. } \\
\text { toxicily } \\
\text { score }\end{array}$ & $\begin{array}{l}\% \text { of } \\
\text { Total } \\
\text { nonc. } \\
\text { inlusl. } \\
\text { score }\end{array}$ & $\begin{array}{l}\text { Carcia. } \\
\text { oral } \\
\text { toxicily } \\
\text { score }\end{array}$ & $\begin{array}{l}\% \text { of } \\
\text { Total } \\
\text { carc. } \\
\text { oral } \\
\text { score }\end{array}$ & $\begin{array}{l}\text { Carcin. } \\
\text { inmal. } \\
\text { toxicity } \\
\text { score }\end{array}$ & $\begin{array}{l}\% \text { of } \\
\text { Total } \\
\text { carc. } \\
\text { in hal. } \\
\text { score }\end{array}$ & $\begin{array}{l}\text { Final } \\
\text { decisione }^{e}\end{array}$ \\
\hline \multicolumn{15}{|l|}{ Orgarics } \\
\hline $\begin{array}{l}\text { 1,1,2-TRICHLORO- } \\
\text { 1,22-TRIRLUORO- } \\
\text { ETHANE }\end{array}$ & $1.40 \mathrm{E}-02$ & $3.0 \mathrm{E}+01$ & $8.6 \mathrm{E}+00$ & & & 4.67E-04 & 0.00 & 1.63E-03 & 0.00 & & & & & DROP \\
\hline $\begin{array}{l}\text { 1,24-TRICHLORO- } \\
\text { BENZENE }\end{array}$ & 8.40E-02 & $1.0 \mathrm{E}-02$ & $5.7 \mathrm{E}-02$ & & & $8.40 E+00$ & 0.00 & $1.47 E+\infty 0$ & 0.00 & & & & & DROP \\
\hline $\begin{array}{l}\text { 1.4-DICHLORO- } \\
\text { BENZENE }\end{array}$ & 5.50E-02 & & $23 E-01$ & $24 E-02$ & & & & 241E-01 & 0.00 & $1.32 E-03$ & 0.00 & & & DROP \\
\hline 2-BUTANONE & 280E-02 & 6.0E-01 & 29E-0I & & & 4.67E-02 & 0.00 & 9.80E.02 & 0.00 & & & & & DROP \\
\hline 2.CHLOROPHENOL & $6.50 \mathrm{E} .02$ & $5.0 \mathrm{E}-03$ & & & & $1.30 \mathrm{E}+01$ & 0.00 & & & & & & & DROP \\
\hline $\begin{array}{l}\text { 4-METHYL-2- } \\
\text { PENTANONE }\end{array}$ & $1.60 E-02$ & $8.0 \mathrm{E}-02$ & $23 E-02$ & & & $200 \mathrm{E}-01$ & 0.00 & 7.00E-01 & 0.00 & & & & & DROP \\
\hline ACENAPHTHENE & $1.30 \mathrm{E}-01$ & $6.0 \mathrm{E}-02$ & & & & $217 E+00$ & 0.00 & & & & & & & DROP \\
\hline ACETONE & $9.40 \mathrm{E}-01$ & $1.0 \mathrm{E}-01$ & & & & $9.40 \mathrm{E}+00$ & 0.00 & & & & & & & DROP \\
\hline ANTHRACENE & $240 \mathrm{E}-01$ & $3.0 \mathrm{E}-01$ & & & & $8.00 \mathrm{E}-01$ & 0.00 & & & & & & & DROP \\
\hline AROCLOR-1254 & $1.90 \mathrm{E}+\infty$ & & & $7.7 \mathrm{E}+\infty$ & & & & & & $1.46 \mathrm{E}+01$ & 14.47 & & & \\
\hline AROCLOR-1260 & $1.00 E+01$ & & & $7.7 \mathrm{E}+\infty$ & & & & & & $7.70 E+01$ & 76.15 & & & \\
\hline $\begin{array}{l}\text { BENZO(A)- } \\
\text { ANTHRACENE }\end{array}$ & $9.90 \mathrm{E}-01$ & & & $7.3 E-01$ & & & & & & $7.23 \mathrm{E}-01$ & 0.71 & & & DROP \\
\hline BENZO(A)PYRENE & $8.90 \mathrm{E} .01$ & & & $7.3 E+\infty$ & & & & & & $6.50 \mathrm{E}+00$ & 6.43 & & & \\
\hline $\begin{array}{l}\text { BENZO(B)- } \\
\text { FLUORANTIENE }\end{array}$ & $1.30 E+00$ & & & 7.3E-01 & & & & & & 2.49E-01 & 0.94 & & & DROP \\
\hline $\begin{array}{l}\text { BENZO(K). } \\
\text { PLUORANTHENE }\end{array}$ & $4.10 \mathrm{E}-01$ & & & 7.3E-02 & & & & & & $299 \mathrm{E}-02$ & 0.03 & & & DROP \\
\hline
\end{tabular}


Table C.42. (continued)

\begin{tabular}{|c|c|c|c|c|c|c|c|c|c|c|c|c|c|c|}
\hline Analiyte & $\begin{array}{l}\text { Marimum } \\
\text { detected } \\
\text { conc.; } \\
\text { molkg }\end{array}$ & $\begin{array}{l}\text { Oral } \\
\text { RiD; } \\
\text { Rigig-d }\end{array}$ & $\begin{array}{l}\text { Inhal. } \\
\text { RiD'; } \\
\text { meflikg-d }\end{array}$ & $\begin{array}{l}\text { Oral } \\
\text { SF; } \\
\text { lg-d/mg }\end{array}$ & $\begin{array}{l}\text { Inhal. } \\
\text { SF; } \\
\text { ig-d/mg }\end{array}$ & $\begin{array}{l}\text { Nowcarc. } \\
\text { oral } \\
\text { ioxicity } \\
\text { score }\end{array}$ & $\begin{array}{l}\% \text { of } \\
\text { Total } \\
\text { mone. } \\
\text { oral } \\
\text { scose }\end{array}$ & $\begin{array}{l}\text { Noncarc. } \\
\text { ish phl. } \\
\text { loxicity } \\
\text { score }\end{array}$ & $\begin{array}{l}\text { \% of } \\
\text { Total } \\
\text { mone. } \\
\text { inhal. } \\
\text { score }\end{array}$ & $\begin{array}{l}\text { Carcin. } \\
\text { oral } \\
\text { toxicity } \\
\text { score }\end{array}$ & $\begin{array}{l}\text { \% of } \\
\text { Total } \\
\text { care. } \\
\text { oral } \\
\text { score }\end{array}$ & $\begin{array}{l}\text { Carcia. } \\
\text { inhal. } \\
\text { loricity } \\
\text { score }\end{array}$ & $\begin{array}{l}\text { \% of } \\
\text { Total } \\
\text { carc. } \\
\text { in } \\
\text { score }\end{array}$ & $\begin{array}{l}\text { Final } \\
\text { decision }\end{array}$ \\
\hline $\begin{array}{l}\text { BIS(2-ETHYL- } \\
\text { HEXYL)- } \\
\text { PHTHALATE }\end{array}$ & $5.90 \mathrm{E}-01$ & $20 \mathrm{E}-02$ & & 1.4E-02 & & $295 E+01$ & 0.00 & & & $8.26 \mathrm{E}-03$ & 0.01 & & & DROP \\
\hline CARBAZOLE & $1.70 E-01$ & & & $20 \mathrm{E}-02$ & & & & & & $3.40 \mathrm{E}-03$ & 0.00 & & & DROP \\
\hline CARBON DISULFIDE & $200 E-03$ & 1.0E-01 & 29E-03 & & & $200 E-02$ & 0.00 & $7.00 \mathrm{E}-01$ & 0.00 & & & & & DROP \\
\hline CHRYSENE & $8.80 \mathrm{E}-01$ & & & $7.3 \mathrm{E}-03$ & & & & & & $6.42 E-03$ & 0.01 & & & DROP \\
\hline $\begin{array}{l}\text { DIBENZO(A,H)- } \\
\text { ANTHRACENE }\end{array}$ & $1.20 \mathrm{E}-01$ & & & $7.3 E+\infty 0$ & & & & & & $8.76 \mathrm{E}-0 \mathrm{I}$ & 0.87 & & & DROP \\
\hline FLUORANTHENE & $1.40 \mathrm{E}+\infty 0$ & $4.0 \mathrm{E}-02$ & & & & $3.50 \mathrm{E}+01$ & 0.00 & & & & & & & DROP \\
\hline $\begin{array}{l}\text { INDENO(1,23-CD)- } \\
\text { PYRENE }\end{array}$ & $5.30 E-01$ & & & $7.3 E-01$ & & & & & & $3.87 \mathrm{E}-01$ & 0.38 & & & DROP \\
\hline $\begin{array}{l}\text { METHYLENE } \\
\text { CHLORIDE }\end{array}$ & $8.80 \mathrm{E}-02$ & $6.0 \mathrm{E}-02$ & $8.6 \mathrm{E}-01$ & 7.SE-03 & & $1.47 E+00$ & 0.00 & $1.03 E-01$ & 0.00 & $6.60 \mathrm{E}-04$ & 0.00 & & & DROP \\
\hline $\begin{array}{l}\text { PENTACHLORO- } \\
\text { PHENOL }\end{array}$ & $6.50 \mathrm{E}-02$ & $3.0 \mathrm{E}-02$ & & $1.2 \mathrm{E}-01$ & & $217 E+00$ & 0.00 & & & $7.80 \mathrm{E}-03$ & 0.01 & & & DROP \\
\hline PHENOL & ธ.20E- -02 & $6.0 \mathrm{E}-01$ & & & & $1.03 E-01$ & 0.00 & & & & & & & DROP \\
\hline PYRENE & $1.40 E+00$ & $3.0 E-02$ & & & & $4.67 E+01$ & 0.00 & & & & & & & DROP \\
\hline TOLUENE & $1.00 \mathrm{E}-03$ & $20 \mathrm{E}-01$ & $1.1 \mathrm{E}-01$ & & & $5.00 E-03$ & 0.00 & $8.75 \mathrm{E}-03$ & 0.00 & & & & & DROP \\
\hline \multirow[t]{2}{*}{ Toxicity Score Totals } & & & & & & $276 E+06$ & 100.0 & $238 E+08$ & 100.0 & $1.01 E+02$ & 100.0 & $1.89 \mathrm{E}+01$ & 100.0 & \\
\hline & & & & & & SA-1 & & & & & & . & & \\
\hline \multicolumn{15}{|l|}{ Imorganics } \\
\hline BARIUM & $3.85 \mathrm{E}+02$ & $7.0 \mathrm{E}-02$ & $1.4 \mathrm{E}-0 \mathrm{~A}$ & & & $5.50 \mathrm{E}+03$ & 9.37 & $270 E+06$ & 1.86 & & & & & \\
\hline
\end{tabular}


Table C.4a (continued)

\begin{tabular}{|c|c|c|c|c|c|c|c|c|c|c|c|c|c|c|}
\hline Analye & $\begin{array}{l}\text { Maxionin } \\
\text { detected } \\
\text { conc.; } \\
\text { mg/ks }\end{array}$ & $\begin{array}{l}\text { Oral } \\
\mathbb{R r D} ; \\
\text { a } \\
\end{array}$ & $\begin{array}{l}\text { Imhal. } \\
\text { RfD'; } \\
\text { mglisg-d }\end{array}$ & $\begin{array}{l}\text { Oral } \\
\mathrm{SF} ; \\
\mathrm{sg}-\mathrm{d} / \mathrm{mg}\end{array}$ & $\begin{array}{l}\text { Imhal. } \\
\text { SF; } \\
\text { ig-d/mg }\end{array}$ & $\begin{array}{l}\text { Noncanc. } \\
\text { oral } \\
\text { toxicity } \\
\text { score }\end{array}$ & $\begin{array}{l}\text { \% of } \\
\text { Total } \\
\text { mome. } \\
\text { oral } \\
\text { score }\end{array}$ & $\begin{array}{l}\text { Noneare. } \\
\text { inhal. } \\
\text { toxicity } \\
\text { ecore }\end{array}$ & $\begin{array}{l}\text { \% of } \\
\text { Total } \\
\text { indas. } \\
\text { intal. } \\
\text { score }\end{array}$ & $\begin{array}{l}\text { Carcin. } \\
\text { ordil } \\
\text { tomicity } \\
\text { score }\end{array}$ & $\begin{array}{l}\text { \% of } \\
\text { Total } \\
\text { care. } \\
\text { oral } \\
\text { score }\end{array}$ & $\begin{array}{l}\text { Carcin. } \\
\text { inhal. } \\
\text { toxicity } \\
\text { score }\end{array}$ & $\begin{array}{l}\text { \% of } \\
\text { Total } \\
\text { carc. } \\
\text { inhal. } \\
\text { score }\end{array}$ & $\begin{array}{l}\text { Final } \\
\text { decision }^{c}\end{array}$ \\
\hline BERYLLUM & $1.50 \mathrm{E}+00$ & $5.0 \mathrm{E}-03$ & & $4.3 E+\infty$ & $8.4 \mathbb{E}+\infty 0$ & $3.00 \mathrm{E}+02$ & 0.51 & & & $6.45 E+00$ & 58.95 & $1.26 \mathrm{E}+01$ & 33.50 & \\
\hline CADMIUM & $4.10 \mathrm{E}+00$ & $1.0 E-03$ & & & $6.1 \mathrm{E}+\infty 0$ & $4.10 E+03$ & 6.98 & & & & & $2.50 \mathrm{E}+01$ & 66.50 & \\
\hline CYANIDE & $1.10 \mathrm{E}+00$ & $20 \mathrm{E}-02$ & & & & $5.50 E+01$ & 0.09 & & & & & & & DROP \\
\hline FLUORENE & $230 \mathrm{E}-01$ & $4.0 \mathrm{E}-02$ & & & & $5.75 E+00$ & 0.01 & & & & & & & DROP \\
\hline MANGANESE & $203 E+03$ & $1.4 E-01$ & 1.4E-05 & & & $1.45 E+04$ & 24.69 & $1.42 E+08$ & 98.12 & & & & & \\
\hline MERCURY & $260 E+00$ & $3.0 \mathrm{E}-04$ & 8.6E-05 & & & $8.67 \mathrm{E}+03$ & 14.76 & $3.03 E+04$ & 0.02 & & & & & \\
\hline NICKEL & $3.10 \mathrm{E}+01$ & $20 \mathrm{E}-02$ & & & & $255 E+03$ & 4.34 & & & & & & & \\
\hline URANIUM, TOTAL & $6.89 \mathrm{E}+01$ & $3.0 \mathrm{E}-03$ & & & & $230 E+04$ & 39.11 & & & & & & & \\
\hline \multicolumn{15}{|l|}{ Organic } \\
\hline $\begin{array}{l}\text { 1,2-DICHLORO- } \\
\text { ETHENE (TOTAL) }\end{array}$ & $200 \mathrm{E}-03$ & $9.0 \mathrm{E}-03$ & & & & 2.22E-01 & 0.00 & & & & & & & DROP \\
\hline $\begin{array}{l}\text { 4-METHYL-2- } \\
\text { PENTANONE }\end{array}$ & $1.20 \mathrm{E}-02$ & $8.0 \mathrm{E}-02$ & $23 \mathrm{E}-02$ & & & $1.50 \mathrm{E}-01$ & 0.00 & $5.25 \mathrm{E}-01$ & 0.00 & & & & & DROP \\
\hline ACENAPHTHENE & $1.10 \mathrm{E}-01$ & $6.0 \mathrm{E}-02$ & & & & $1.83 E+00$ & 0.00 & & & & & & & DROP \\
\hline ACETONE & $220 E-01$ & $1.0 E-01$ & & & & $220 E+\infty 0$ & 0.00 & & & & & & & DROP \\
\hline ANTHRACENE & $3.20 \mathrm{E}-01$ & $3.0 \mathrm{E}-01$ & & & & $1.07 \mathrm{E}+00$ & 0.00 & & & & & & & DROP \\
\hline $\begin{array}{l}\text { BENZO(A)- } \\
\text { ANTHRACENE }\end{array}$ & 6.10E-01 & & & $7.3 \mathrm{E}-01$ & & & & & & $4.45 \mathrm{E}-01$ & 4.07 & & & \\
\hline BENZO(A)PYRENE & $4.80 \mathrm{E}-01$ & & & $7.3 E+\infty 0$ & & & & & & $3.50 \mathbb{E}+\infty 0$ & 32.02 & & & \\
\hline $\begin{array}{l}\text { BENZO(B)- } \\
\text { FLUORANTHENE }\end{array}$ & $4.20 \mathrm{E}-01$ & & & $7.3 E-01$ & & & & & & 3.07E-01 & 280 & & & \\
\hline $\begin{array}{l}\text { BENZO(K)- } \\
\text { FLUORANTHENE }\end{array}$ & $5.80 \mathrm{E}-01$ & & & $7.3 \mathrm{E}-02$ & & & & & & 4.23E-02 & 0.39 & & & DROP \\
\hline
\end{tabular}


Table C42. (continued)

\begin{tabular}{|c|c|c|c|c|c|c|c|c|c|c|c|c|c|c|}
\hline Amalyte & $\begin{array}{l}\text { Maximumat } \\
\text { detected } \\
\text { conc.; } \\
\text { me/kg }\end{array}$ & $\begin{array}{l}\text { Oral } \\
\text { RrD; } \\
\text { mg//gg-d }\end{array}$ & $\begin{array}{l}\text { Inhal. } \\
\mathbf{R T}^{\mathbf{3}} \text {; } \\
\text { me/kg-d }\end{array}$ & $\begin{array}{l}\text { Oral } \\
\text { SP; } \\
\mathrm{kg}-\mathrm{d} / \mathrm{mg}\end{array}$ & $\begin{array}{l}\text { Inhal. } \\
\text { SF; } \\
\text { lgg-d/mg }\end{array}$ & $\begin{array}{l}\text { Noncarc. } \\
\text { oral } \\
\text { foricity } \\
\text { score }\end{array}$ & $\begin{array}{l}\text { \%of of } \\
\text { Total } \\
\text { none. } \\
\text { oral } \\
\text { score }\end{array}$ & $\begin{array}{l}\text { Noncare. } \\
\text { inhal. } \\
\text { foxicity } \\
\text { score }\end{array}$ & $\begin{array}{l}\text { \%o of } \\
\text { Total } \\
\text { nonc. } \\
\text { inhal. } \\
\text { scose }\end{array}$ & $\begin{array}{l}\text { Carcili. } \\
\text { oral } \\
\text { foxicity } \\
\text { score }\end{array}$ & $\begin{array}{l}\text { \%of of } \\
\text { Total } \\
\text { carc. } \\
\text { oral } \\
\text { score }\end{array}$ & $\begin{array}{l}\text { Carcin. } \\
\text { inhal. } \\
\text { toxicity } \\
\text { score }\end{array}$ & $\begin{array}{l}\% \text { of } \\
\text { Total } \\
\text { carc. } \\
\text { inthal. } \\
\text { score }\end{array}$ & $\begin{array}{l}\text { Fimal } \\
\text { decision: }\end{array}$ \\
\hline BENZOIC ACID & $5.50 \mathrm{E}-02$ & $4.0 \mathrm{E}+00$ & & & & $1.38 \mathrm{E}-02$ & 0.00 & & & & & & & DROP \\
\hline CHRYSENE & $5.70 \mathrm{E}-01$ & & & $7.3 E-03$ & & & & & & $4.16 \mathrm{E}-03$ & 0.04 & & & DROP \\
\hline $\begin{array}{l}\text { DIETHYL } \\
\text { PHTHALATE }\end{array}$ & $7.70 \mathrm{E} \cdot 02$ & $8.0 \mathrm{E}-01$ & & & & $9.63 E-02$ & 0.00 & & & & & & & DROP \\
\hline $\begin{array}{l}\text { DIMETHYL } \\
\text { PHTHALATE }\end{array}$ & $6.40 \mathrm{E}-02$ & $1.0 \mathrm{E}+01$ & & & & $6.40 \mathrm{E}-03$ & 0.00 & & & & & & & DROP \\
\hline FLUORANTHENE & $1.40 E+00$ & $4.0 \mathrm{E}-02$ & & & & $3.50 \mathrm{E}+01$ & 0.06 & & & & & & & DROP \\
\hline $\begin{array}{l}\text { INDENO }(1,2,3-C D) \\
\text { PYRENE }\end{array}$ & $260 E-01$ & & & $7.3 \mathrm{E}-01$ & & & & & & $1.90 \mathrm{E}-0 \mathrm{1}$ & 1.73 & & & \\
\hline $\begin{array}{l}\text { METHYLENE } \\
\text { CHLORIDE }\end{array}$ & $2.00 \mathrm{E} .03$ & $6.0 \mathrm{E}-02$ & $8.6 \mathrm{E}-01$ & $7.5 E-03$ & & 3.33E-02 & 0.00 & 233E-03 & 0.00 & $1.50 \mathrm{E}-05$ & 0.00 & & & DROP \\
\hline PYRENE & $1.00 E+00$ & $3.0 \mathrm{E}-02$ & & & & $3.33 E+01$ & 0.06 & & & & & & & DROP \\
\hline $\begin{array}{l}\text { TETRACHLORO- } \\
\text { ETHENE }\end{array}$ & $4.00 \mathrm{E}-03$ & $1.0 \mathrm{E} \cdot 02$ & & & & $4.00 \mathrm{E}-01$ & 0.00 & & & & & & & DROP \\
\hline TOLUENE & $3.00 \mathrm{E}-03$ & $20 \mathrm{E}-01$ & $1.1 E-01$ & & & $1.50 \mathrm{E}-02$ & 0.00 & 263E-02 & 0.00 & & & & & DROP \\
\hline XYLENE (TOTAL) & $200 \mathrm{E}-03$ & $20 \mathrm{E}+00$ & & & & $1.00 \mathrm{E}-03$ & 0.00 & & & & & & & DROP \\
\hline Toxiciry Score Totals & & & & & & $5.87 E+04$ & 100.0 & $1.45 \mathrm{E}+08$ & 100.0 & $1.09 \mathrm{E}+01$ & 100.0 & $3.76 \mathrm{E}+01$ & 100.0 & \\
\hline
\end{tabular}

- Because the speciation of chromium is unknown, it was not included in this toxicity screening.

- RfCs were converted to units of mg/kg-d (i.e., RTD units) by using the inhalation rate and body weight of an adult [i.e., $\left.R f C \times 20 \mathrm{~m}^{3} / \mathrm{d} \times(1 / 70 \mathrm{~kg})=\operatorname{RfD}\right)(\mathrm{EPA} 1989 \mathrm{a})$.

-A decision to eliminate (designated by "DROP" in this table) analytes based on toxicity screening can be made when all existing pathway scores are less than $1 \%$ of the total score (for each and all existing pathways) (EPA 1989a). For example, if an analyte toxicity score was less than $1 \%$ for noncarcinogenic oral and inhalation pathways, but greater than $1 \%$ for the carcinogenic oral pathway, then that analyte could not be eliminated from the COPC list based on this toxicity screening test (EPA 1989a). 
Table C.4b. Toxicity screening for BCV OU2 detected radionuclides in soil (carcinogenic, ingestion, inhalation, and external exposure)

\begin{tabular}{|c|c|c|c|c|c|c|c|c|c|c|c|}
\hline Analyie & $\begin{array}{l}\text { Maximum } \\
\text { detected } \\
\text { conc. } \\
\text { (pCi/g) }\end{array}$ & $\begin{array}{l}\text { Ingestion } \\
\mathrm{SF} \\
(1 / \mathrm{pCi})\end{array}$ & $\begin{array}{l}\text { Inhalation } \\
\text { SF } \\
\text { (1/pCi) }\end{array}$ & $\begin{array}{l}\text { External } \\
\text { exposure } \\
\text { SF } \\
(\mathrm{g} / \mathrm{pCi}-\mathrm{y})\end{array}$ & $\begin{array}{l}\text { Ingestion } \\
\text { toxicity } \\
\text { score }\end{array}$ & $\begin{array}{l}\% \text { of Total } \\
\text { Ingestion } \\
\text { loxicity } \\
\text { score }\end{array}$ & $\begin{array}{l}\text { Inhalation } \\
\text { toxicity } \\
\text { score }\end{array}$ & $\begin{array}{l}\text { \% of Total } \\
\text { inhalation } \\
\text { toxicity } \\
\text { score }\end{array}$ & $\begin{array}{l}\text { External } \\
\text { exposure } \\
\text { toxicity } \\
\text { score }\end{array}$ & $\begin{array}{l}\text { \% of Total } \\
\text { ext. exp. } \\
\text { toxicity } \\
\text { score }\end{array}$ & $\begin{array}{l}\text { Final } \\
\text { decision }^{2}\end{array}$ \\
\hline & & & & RSA & & & & & & & \\
\hline \multicolumn{12}{|l|}{ Radionuclides } \\
\hline THORIUM-232 & $3.28 \mathrm{E}+00$ & $1.2 \mathrm{E}-11$ & $2.8 \mathrm{E}-08$ & $2.6 \mathrm{E}-11$ & $3.94 E-11$ & 23.72 & $9.18 \mathrm{E}-08$ & 30.87 & $8.53 E-11$ & 26.43 & \\
\hline URANIUM-234 & $7.91 \mathrm{E}+00$ & $1.6 \mathrm{E}-11$ & $2.6 \mathrm{E}-08$ & $3.0 \mathrm{E}-11$ & $1.27 \mathrm{E}-10$ & 76.28 & $2.06 \mathrm{E}-07$ & 69.13 & $2.37 \mathrm{E}-10$ & 73.57 & \\
\hline \multirow[t]{2}{*}{ Toxicity Score Totals } & & & & & $1.66 \mathrm{E}-10$ & 100.0 & $2.97 \mathrm{E}-07$ & 100.0 & $3.23 E-10$ & 100.0 & \\
\hline & & & & SA-1 & & & & & & & \\
\hline \multicolumn{12}{|l|}{ Radionuclides } \\
\hline RADIUM-226 & $7.57 \mathrm{E}+00$ & $1.2 \mathrm{E}-10$ & $3.0 \mathrm{E}-09$ & $6.0 \mathrm{E}-06$ & $9.08 \mathrm{E}-10$ & 47.22 & $2.27 E-08$ & 1.25 & $4.54 \mathrm{E}-05$ & 98.21 & \\
\hline URANIUM-234 & $2.32 E+01$ & $1.6 \mathrm{E}-11$ & $2.6 \mathrm{E}-08$ & $3.0 \mathrm{E}-11$ & $3.71 E-10$ & 19.31 & $6.03 E-07$ & 33.12 & $6.96 \mathrm{E}-10$ & 0.00 & \\
\hline URANIUM-238 & $2.30 E+01$ & $2.8 \mathrm{E}-11$ & $5.2 \mathrm{E}-08$ & $3.6 \mathrm{E}-08$ & $6.44 \mathrm{E}-10$ & 33.47 & $1.20 \mathrm{E}-06$ & 65.63 & $8.28 \mathrm{E}-07$ & 1.79 & \\
\hline Toxicity Score Totals & & & & & $1.92 \mathrm{E}-09$ & 100.0 & $1.82 \mathrm{E}-06$ & 100.0 & 4.62E-05 & 100.0 & \\
\hline
\end{tabular}

- A decision to eliminate (designated by "DROP" in this table) analytes based on toxicity screening can be made when all existing pathway scores are less than $1 \%$ of the total score (for each and all existing pathways) (EPA 1989a); note, no radionuclides were eliminated using this test in this BRA. 
Table C.5. Comparison of maximum detected conoentrations for BCV OU2 soil with PRGs (for analytes dropped because of toxicity screeming)

\begin{tabular}{|c|c|c|c|c|c|c|}
\hline Analyte & $\begin{array}{l}\text { Frequency } \\
\text { of } \\
\text { detect }\end{array}$ & $\begin{array}{l}\text { Maximum } \\
\text { detected } \\
\text { concentration }\end{array}$ & $\begin{array}{l}\text { Residential } \\
\text { PRG for } \\
\text { HQ }=1.0\end{array}$ & $\begin{array}{l}\text { Residential } \\
\text { PRG for } \\
\text { risk = 1.0E-04 }\end{array}$ & $\begin{array}{l}\text { Residential } \\
\text { PRG for } \\
\text { risk }=1.0 \mathrm{E}-06\end{array}$ & $\begin{array}{l}\text { Units } \\
\text { of } \\
\text { measure }\end{array}$ \\
\hline & & RSA & & & & \\
\hline \multicolumn{7}{|l|}{ Inorganics } \\
\hline ANTIMONY & $3 / 6$ & $3.60 \mathrm{E}-01$ & $1.1 E+02$ & & & $\mathrm{mg} / \mathrm{kg}$ \\
\hline CYANIDE & $1 / 20$ & $2.46 \mathrm{E}+01$ & $5.5 \mathrm{E}+03$ & & & $\mathrm{mg} / \mathrm{kg}$ \\
\hline FLUORENE & $2 / 11$ & $8.30 \mathrm{E}-02$ & $1.1 E+04$ & & & $\mathrm{mg} / \mathrm{kg}$ \\
\hline SILVER & $8 / 23$ & $1.90 \mathrm{E}+00$ & $1.4 \mathbb{E}+03$ & & & $\mathrm{mg} / \mathrm{kg}$ \\
\hline \multicolumn{7}{|l|}{ Organics } \\
\hline $\begin{array}{l}\text { 1,1,2-TRICHLORO- } \\
\text { 1,2,2-TRIFLUOROETHANE }\end{array}$ & $4 / 11$ & $3.20 E+01$ & $8.2 E+06$ & & & $\mathrm{mg} / \mathrm{kg}$ \\
\hline 1,1-DICHLOROETHANE & $2 / 23$ & $6.40 \mathrm{E}-01$ & $2.7 E+04$ & & & $\mathrm{mg} / \mathrm{kg}$ \\
\hline 1,1-DICHLOROETHENE & $1 / 23$ & $5.10 \mathrm{E}-02$ & $2.5 \mathbb{E}+03$ & $1.1 \mathbb{E}+02$ & $1.1 \mathrm{E}+\infty$ & $m g / k g$ \\
\hline 1,2-DICHLOROBENZENE & $1 / 11$ & $3.60 \mathrm{E}-01$ & $2.5 E+04$ & & & $\mathrm{mg} / \mathrm{kg}$ \\
\hline 1,2-DICHLOROETHANE & $1 / 23$ & $3.00 \mathrm{E}-03$ & & $7.0 \mathrm{E}+02$ & $7.0 \mathrm{E}+\infty$ & $\mathrm{mg} / \mathrm{kg}$ \\
\hline 2,4-DIMETHYLPHENOL & $1 / 11$ & $3.40 \mathbb{E}-01$ & $5.5 \mathbb{E}+03$ & & & $\mathrm{mg} / \mathrm{kg}$ \\
\hline 2-BUTANONE & $4 / 23$ & $2.70 \mathrm{E}-01$ & $1.6 \mathrm{E}+05$ & & & $\mathrm{mg} / \mathrm{kg}$ \\
\hline 4-METHYL-2-PENTANONE & $1 / 23$ & $2.10 \mathrm{E}-01$ & $1.4 \mathrm{E}+04$ & & & $\mathrm{mg} / \mathrm{kg}$ \\
\hline 4-METHYLPHENOL & $1 / 11$ & $5.20 \mathbb{E}-01$ & $1.4 E+03$ & & & $\mathrm{mg} / \mathrm{kg}$ \\
\hline ACENAPHTHENE & $2 / 11$ & $6.60 \mathrm{E}-02$ & $1.6 \mathrm{E}+04$ & & & $\mathrm{mg} / \mathrm{kg}$ \\
\hline ACETONE & $14 / 23$ & $2.50 \mathrm{E}+00$ & $2.7 \mathrm{E}+04$ & & & $\mathrm{mg} / \mathrm{kg}$ \\
\hline
\end{tabular}


Table C.5. (continued)

\begin{tabular}{|c|c|c|c|c|c|c|}
\hline Analyte & $\begin{array}{l}\text { Frequency } \\
\text { of } \\
\text { detect }\end{array}$ & $\begin{array}{l}\text { Maximum } \\
\text { detected } \\
\text { concentration }\end{array}$ & $\begin{array}{l}\text { Residential } \\
\text { PRG for } \\
\text { HQ }=1.0\end{array}$ & $\begin{array}{l}\text { Residential } \\
\text { PRG for } \\
\text { risk }=1.0 \mathrm{E}-04\end{array}$ & $\begin{array}{l}\text { Residential } \\
\text { PRG for } \\
\text { risk = } 1.0 \mathrm{E}-06\end{array}$ & $\begin{array}{l}\text { Units } \\
\text { of } \\
\text { measure }\end{array}$ \\
\hline ANTHRACENE & $2 / 10$ & $1.40 \mathrm{E}-01$ & $8.2 E+04$ & & & $\mathrm{mg} / \mathrm{kg}$ \\
\hline BENZENE & $1 / 23$ & $1.00 \mathbb{E}-02$ & & $2.2 \mathrm{E}+03$ & $2.2 \mathrm{E}+01$ & $\mathrm{mg} / \mathrm{kg}$ \\
\hline BENZO(A)ANTHRACENE & $4 / 10$ & 4.10E-01 & & $8.8 \mathrm{E}+01$ & $8.8 \mathrm{E}-01$ & $\mathrm{mg} / \mathrm{kg}$ \\
\hline BENZO(B)FLUORANTHENE & $3 / 11$ & $5.70 \mathrm{E}-01$ & & $8.8 \mathrm{E}+01$ & 8.8E-01 & $\mathrm{mg} / \mathrm{kg}$ \\
\hline BENZYL BUTY PHTHALATE & $1 / 10$ & $7.30 \mathrm{E}-02$ & $5.5 \mathrm{E}+04$ & & & $\mathrm{mg} / \mathrm{kg}$ \\
\hline CARBAZOLE & $2 / 10$ & $1.00 \mathrm{E}-01$ & & $3.2 \mathrm{E}+03$ & $3.2 \mathrm{E}+01$ & $\mathrm{mg} / \mathrm{kg}$ \\
\hline CARBON DISULFIDE & $1 / 23$ & $1.80 \mathrm{E}-02$ & $2.7 \mathrm{E}+04$ & & & $\mathrm{mg} / \mathrm{kg}$ \\
\hline CHLOROBENZENE & $1 / 22$ & $7.00 \mathrm{E}-03$ & $5.5 \mathrm{E}+03$ & & & $\mathrm{mg} / \mathrm{kg}$ \\
\hline DIMETHYL PHTHALATE & $4 / 11$ & $1.50 \mathrm{E}-01$ & $2.7 \mathrm{E}+06$ & & & $\mathrm{mg} / \mathrm{kg}$ \\
\hline ETHYLBENZENE & $3 / 23$ & $2.80 \mathrm{E}-01$ & $2.7 \mathrm{E}+04$ & & & $\mathrm{mg} / \mathrm{kg}$ \\
\hline FLUORANTHENE & $4 / 10$ & $7.80 \mathrm{E}-01$ & $1.1 \mathrm{E}+04$ & & & $\mathrm{mg} / \mathrm{kg}$ \\
\hline HEPTACHLOR & $2 / 8$ & $8.80 \mathrm{E}-04$ & $1.4 \mathrm{E}+02$ & $1.4 \mathbb{E}+01$ & $1.4 \mathbb{E}-01$ & $\mathrm{mg} / \mathrm{kg}$ \\
\hline INDENO(1,2,3-CD)PYRENE & $3 / 11$ & $8.40 \mathrm{E}-01$ & & $8.8 E+01$ & $8.8 \mathrm{E}-01$ & $\mathrm{mg} / \mathrm{kg}$ \\
\hline METHYLENE CHLORIDE & $5 / 23$ & $5.80 \mathrm{E}+01$ & $1.6 \mathrm{E}+04$ & $8.5 \mathrm{E}+03$ & $8.5 \mathrm{E}+01$ & $\mathrm{mg} / \mathrm{kg}$ \\
\hline P,P'-METHOXYCHLOR & $1 / 7$ & $1.50 \mathrm{E}-03$ & $1.4 E+03$ & & & $\mathrm{mg} / \mathrm{kg}$ \\
\hline PENTACHLOROPHENOL & $1 / 10$ & $4.70 \mathrm{E}-02$ & $8.2 \mathrm{E}+03$ & $5.3 \mathrm{E}+02$ & $5.3 E+\infty 0$ & $\mathrm{mg} / \mathrm{kg}$ \\
\hline
\end{tabular}


Table C.5. (continued)

\begin{tabular}{|c|c|c|c|c|c|c|}
\hline Analyte & $\begin{array}{l}\text { Frequency } \\
\text { of } \\
\text { detect }\end{array}$ & $\begin{array}{l}\text { Maximum } \\
\text { detected } \\
\text { concentration }\end{array}$ & $\begin{array}{l}\text { Residential } \\
\text { PRG for } \\
\text { HQ }=1.0\end{array}$ & $\begin{array}{l}\text { Residential } \\
\text { PRG for } \\
\text { risk = } 1.0 \mathrm{E}-04\end{array}$ & $\begin{array}{l}\text { Residential } \\
\text { PRG for } \\
\text { risk }=1.0 \mathrm{E}-06\end{array}$ & $\begin{array}{l}\text { Units } \\
\text { of } \\
\text { measure }\end{array}$ \\
\hline PHENOL & $1 / 11$ & $2.50 \mathrm{E}+00$ & $1.6 \mathrm{E}+05$ & & & $\mathrm{mg} / \mathrm{kg}$ \\
\hline PYRENE & $5 / 10$ & $1.10 \mathrm{E}+\infty$ & $8.2 E+03$ & & & $\mathrm{mg} / \mathrm{kg}$ \\
\hline TOLUENE & $4 / 23$ & $7.50 \mathrm{E}-01$ & $5.5 \mathrm{E}+04$ & & & $\mathrm{mg} / \mathrm{kg}$ \\
\hline XYLENE (TOTAL) & $2 / 23$ & $4.60 \mathrm{E}-01$ & $5.5 \mathrm{E}+05$ & & & $\mathrm{mg} / \mathrm{kg}$ \\
\hline \multicolumn{7}{|c|}{ SY-200 } \\
\hline \multicolumn{7}{|l|}{ Inorganics } \\
\hline ANTIMONY & $37 / 60$ & $9.20 \mathrm{E}-01$ & $1.1 \mathrm{E}+02$ & & & $\mathrm{mg} / \mathrm{kg}$ \\
\hline BARIUM & $75 / 89$ & $2.77 E+02$ & $1.9 \mathrm{E}+04$ & & & $\mathrm{mg} / \mathrm{kg}$ \\
\hline \multicolumn{7}{|l|}{ Organics } \\
\hline $\begin{array}{l}\text { 1,1,2-TRICHLORO- } \\
\text { 1,2,2-TRIFLUOROETHANE }\end{array}$ & $3 / 32$ & $1.40 \mathrm{E}-02$ & $8.2 E+06$ & & & $\mathrm{mg} / \mathrm{kg}$ \\
\hline 1,2,4-TRICHLOROBENZENE & $2 / 32$ & $8.40 \mathrm{E}-02$ & $2.7 \mathrm{E}+03$ & & & $\mathrm{mg} / \mathrm{kg}$ \\
\hline 1,4-DICHLOROBENZENE & $1 / 32$ & $5.50 \mathrm{E}-02$ & & $2.7 E+03$ & 2.7E+01 & $\mathrm{mg} / \mathrm{kg}$ \\
\hline 2-BUTANONE & $1 / 32$ & $2.80 \mathrm{E}-02$ & $1.6 \mathrm{E}+05$ & & & $\mathrm{mg} / \mathrm{kg}$ \\
\hline 2-CHLOROPHENOL & $1 / 32$ & $6.50 \mathrm{E}-02$ & $1.4 E+03$ & & & $\mathrm{mg} / \mathrm{kg}$ \\
\hline
\end{tabular}


Table C5. (continued)

\begin{tabular}{|c|c|c|c|c|c|c|}
\hline Analyte & $\begin{array}{l}\text { Frequency } \\
\text { of } \\
\text { detect }\end{array}$ & $\begin{array}{l}\text { Maximum } \\
\text { detected } \\
\text { concentration }\end{array}$ & $\begin{array}{l}\text { Residential } \\
\text { PRG for } \\
\mathrm{HQ}=1.0\end{array}$ & $\begin{array}{l}\text { Residential } \\
\text { PRG for } \\
\text { risk = 1.0E-04 }\end{array}$ & $\begin{array}{l}\text { Residential } \\
\text { PRG for } \\
\text { risk = } 1.0 \mathrm{E}-06\end{array}$ & $\begin{array}{l}\text { Units } \\
\text { of } \\
\text { measure } \\
\end{array}$ \\
\hline 4-METHYYL-2-PENTANONE & $3 / 32$ & $1.60 \mathrm{E}-02$ & $1.4 \mathrm{E}+04$ & & & $\mathrm{mg} / \mathrm{kg}$ \\
\hline ACENAPHTHENE & $5 / 32$ & $1.30 \mathrm{E}-01$ & $1.6 \mathrm{E}+04$ & & & $\mathrm{mg} / \mathrm{kg}$ \\
\hline ACETONE & $15 / 32$ & $9.40 \mathrm{E}-01$ & $2.7 \mathrm{E}+04$ & & & $\mathrm{mg} / \mathrm{kg}$ \\
\hline ANTHRACENE & $4 / 32$ & $2.40 E-01$ & $8.2 \mathrm{E}+04$ & & & mg/kg \\
\hline BENZO(A)ANTHRACENE* & $7 / 32$ & $9.90 \mathrm{E}-01^{*}$ & & $8.8 \mathrm{E}+01$ & $8.8 \mathrm{E}-01$ & $\mathrm{mg} / \mathrm{kg}$ \\
\hline BENZO(B)FLUORANTHENE & $9 / 32$ & $1.30 \mathrm{E}+00^{*}$ & & $8.8 \mathrm{E}+01$ & $8.8 \mathrm{E}-01$ & $\mathrm{mg} / \mathrm{kg}$ \\
\hline BENZO(K)FLUORANTHENE & $10 / 32$ & 4.10E-01 & & $8.8 \mathrm{E}+02$ & $8.8 E+00$ & $\mathrm{mg} / \mathrm{kg}$ \\
\hline BIS(2-ETHYLHEXYL)PHTHALATE & $3 / 32$ & $5.90 \mathrm{E}-01$ & $5.5 E+03$ & $4.6 \mathrm{E}+03$ & $4.6 \mathrm{E}+01$ & $\mathrm{mg} / \mathrm{kg}$ \\
\hline CARBAZOLE & $4 / 32$ & $1.70 \mathrm{E}-01$ & & $3.2 E+03$ & $3.2 E+01$ & $\mathrm{mg} / \mathrm{kg}$ \\
\hline CARBON DISULFIDE & $1 / 32$ & $2.00 \mathrm{E}-03$ & $2.7 \mathrm{E}+04$ & & & $\mathrm{mg} / \mathrm{kg}$ \\
\hline CHRYSENE & $7 / 32$ & $8.80 \mathrm{E}-01$ & & $8.8 \mathrm{E}+03$ & $8.8 E+01$ & $\mathrm{mg} / \mathrm{kg}$ \\
\hline DIBENZO(A,H)ANTHRACENE & $4 / 32$ & $1.20 \mathrm{E}-01$ & & $8.8 \mathrm{E}+00$ & $8.8 \mathrm{E}-02$ & $\mathrm{mg} / \mathrm{kg}$ \\
\hline FLUORANTHENE & $14 / 32$ & $1.40 \mathrm{E}+00$ & $1.1 \mathrm{E}+04$ & & & $\mathrm{mg} / \mathrm{kg}$ \\
\hline INDENO(1,2,3-CD)PYRENE & $7 / 32$ & $5.30 \mathrm{E}-01$ & & $8.8 \mathrm{E}+01$ & $8.8 \mathrm{E}-01$ & $\mathrm{mg} / \mathrm{kg}$ \\
\hline METHYLENE CHLORIDE & $11 / 32$ & $8.80 \mathrm{E}-02$ & $1.6 \mathrm{E}+04$ & $8.5 E+03$ & $8.5 E+01$ & $\mathrm{mg} / \mathrm{kg}$ \\
\hline PENTACHLOROPHENOL & $1 / 32$ & $6.50 \mathrm{E}-02$ & $8.2 E+03$ & $5.3 \mathrm{E}+02$ & $5.3 \mathrm{E}+00$ & $\mathrm{mg} / \mathrm{kg}$ \\
\hline PHENOL & $1 / 32$ & $6.20 \mathrm{E}-02$ & $1.6 \mathrm{E}+05$ & & & $\mathrm{mg} / \mathrm{kg}$ \\
\hline PYRENE & $14 / 32$ & $1.40 \mathrm{E}+00$ & $8.2 E+03$ & & & $\mathrm{mg} / \mathrm{kg}$ \\
\hline TOLUENE & $2 / 32$ & $1.00 \mathrm{E}-03$ & $5.5 E+04$ & & & $\mathrm{mg} / \mathrm{kg}$ \\
\hline
\end{tabular}


Table C.5. (continued)

\begin{tabular}{|c|c|c|c|c|c|c|}
\hline Analyte & $\begin{array}{l}\text { Frequency } \\
\text { of } \\
\text { detect }\end{array}$ & $\begin{array}{l}\text { Maximum } \\
\text { detected } \\
\text { concentration }\end{array}$ & $\begin{array}{l}\text { Residential } \\
\text { PRG for } \\
\text { HQ = } 1.0\end{array}$ & $\begin{array}{l}\text { Residential } \\
\text { PRG for } \\
\text { risk }=1.0 \mathrm{E}-04\end{array}$ & $\begin{array}{l}\text { Residential } \\
\text { PRG for } \\
\text { risk = 1.0E-06 }\end{array}$ & $\begin{array}{l}\text { Units } \\
\text { of } \\
\text { measure }\end{array}$ \\
\hline \multicolumn{7}{|c|}{ SA-1 } \\
\hline \multicolumn{7}{|l|}{ Inorganics } \\
\hline CYANIDE & $1 / 12$ & $1.10 \mathrm{E}+00$ & $5.5 \mathrm{E}+03$ & & & $\mathrm{mg} / \mathrm{kg}$ \\
\hline FLUORENE & $2 / 12$ & $2.30 \mathrm{E}-01$ & $1.1 \mathrm{E}+04$ & & & $\mathrm{mg} / \mathrm{kg}$ \\
\hline \multicolumn{7}{|l|}{ Organics } \\
\hline 1,2-DICHLOROETHENE (TOTAL) & $2 / 12$ & $2.00 \mathrm{E}-03$ & $2.5 E+03$ & & & $\mathrm{mg} / \mathrm{kg}$ \\
\hline 4-METHYL-2-PENTANONE & $1 / 12$ & $1.20 \mathrm{E}-02$ & $1.4 \mathrm{E}+04$ & & & $\mathrm{mg} / \mathrm{kg}$ \\
\hline ACENAPHTHENE & $1 / 12$ & $1.10 \mathrm{E} .01$ & $1.6 \mathbb{E}+04$ & & & $\mathrm{mg} / \mathrm{kg}$ \\
\hline ACETONE & $2 / 12$ & $2.20 \mathbb{E}-01$ & 2.7E+04 & & & $\mathrm{mg} / \mathrm{kg}$ \\
\hline ANTHRACENE & $2 / 12$ & $3.20 \mathrm{E}-01$ & $8.2 E+04$ & & & $\mathrm{mg} / \mathrm{kg}$ \\
\hline BENZO(K)FLUORANTHENE & $5 / 12$ & $5.80 \mathrm{E}-01$ & & $8.8 \mathrm{E}+02$ & $8.8 \mathrm{E}+00$ & $\mathrm{mg} / \mathrm{kg}$ \\
\hline BENZOIC ACID & $1 / 12$ & $5.50 \mathrm{E}-02$ & $1.1 \mathrm{E}+06$ & & & $\mathrm{mg} / \mathrm{kg}$ \\
\hline CHRYSENE & $5 / 12$ & $5.70 \mathrm{E}-01$ & & $8.8 \mathrm{E}+03$ & $8.8 \mathrm{E}+01$ & $\mathrm{mg} / \mathrm{kg}$ \\
\hline DIETHYL PHTHALATE & $1 / 12$ & $7.70 \mathrm{E}-02$ & $2.2 \mathrm{E}+05$ & & & $\mathrm{mg} / \mathrm{kg}$ \\
\hline DIMETHYL PHTHALATE & $1 / 12$ & $6.40 \mathrm{E}-02$ & $2.7 \mathrm{E}+\infty 6$ & & & $\mathrm{mg} / \mathrm{kg}$ \\
\hline FLUORANTHENE & $5 / 12$ & $1.40 \mathrm{E}+00$ & $1.1 \mathrm{E}+04$ & & & $\mathrm{mg} / \mathrm{kg}$ \\
\hline METHYLENE CHLORIDE & $2 / 12$ & $2.00 \mathrm{E}-03$ & $1.6 \mathrm{E}+04$ & $8.5 E+03$ & $8.5 E+01$ & $\mathrm{mg} / \mathrm{kg}$ \\
\hline PYRENE & $5 / 12$ & $1.00 E+00$ & $8.2 E+03$ & & & $\mathrm{mg} / \mathrm{kg}$ \\
\hline TETRACHLOROETHENE & $2 / 12$ & $4.00 \mathrm{E}-03$ & $2.7 \mathrm{E}+03$ & & & $\mathrm{mg} / \mathrm{kg}$ \\
\hline
\end{tabular}


Table C.5. (continued)

\begin{tabular}{|c|c|c|c|c|c|c|}
\hline Analyte & $\begin{array}{l}\text { Frequency } \\
\text { of } \\
\text { detect }\end{array}$ & $\begin{array}{l}\text { Maximum } \\
\text { detected } \\
\text { concentration }\end{array}$ & $\begin{array}{l}\text { Residential } \\
\text { PRG for } \\
H O=1.0\end{array}$ & $\begin{array}{l}\text { Residential } \\
\text { PRG for } \\
\text { risk = } 1.0 \mathrm{E}-04\end{array}$ & $\begin{array}{l}\text { Residential } \\
\text { PRG for } \\
\text { risk }=1.0 \mathrm{E}-06\end{array}$ & $\begin{array}{l}\text { Units } \\
\text { of } \\
\text { measure }\end{array}$ \\
\hline TOLUENE & $1 / 12$ & $3.00 \mathrm{E}-03$ & $5.5 \mathrm{E}+04$ & & & $\mathrm{mg} / \mathrm{kg}$ \\
\hline XYLENE (TOTAL) & $1 / 12$ & $2.00 \mathrm{E}-03$ & $5.5 E+05$ & & & $\mathrm{mg} / \mathrm{kg}$ \\
\hline
\end{tabular}

- Analytes with a maximum detected concentration greater than the residential ingestion of soil PRG (for risk $=1.0 \mathrm{E}-06$ ) are marked with an asterisk ( ${ }^{*}$ ); note, no other analytes listed in this table (i.e., analytes dropped from the toxicity screening test) have maximum detected concentrations that give a rish $\geq 1.0 \mathrm{E}-06$ and/or an HQ $\geq 1.0$. - Preliminary remediation goals (PRGs), for the residential land use scenario (ingestion of soil pathway), calculated for target risks of $1.0 \mathrm{E}-04$ and $1.0 \mathrm{E}-06$ for carcinogens, and a target hazard quotient of 1.0 for noncarcinogens. 
C-52

Table C.6. Amalytes in BCV OU2 soils eliminated from COPC list

\begin{tabular}{|c|c|c|}
\hline Analyte & $\begin{array}{c}\text { Frequency } \\
\text { of detect }\end{array}$ & $\begin{array}{l}\text { Reason analyte is } \\
\text { not on COPC list }\end{array}$ \\
\hline \multicolumn{3}{|c|}{ RSA } \\
\hline \multicolumn{3}{|l|}{ Inorganics } \\
\hline ALUMINUM & $23 / 23$ & $\mathbb{B}$ \\
\hline ANTIMONY & $3 / 6$ & $\mathbf{T}$ \\
\hline ARSENIC & $23 / 23$ & $B$ \\
\hline CALCIUM & $21 / 23$ & $\mathbb{E}$ \\
\hline CHROMIUM & $23 / 23$ & $\mathbf{B}$ \\
\hline COPPER & $23 / 23$ & $\mathbf{E}$ \\
\hline CYANIDE & $1 / 20$ & $\mathbf{T}$ \\
\hline FLUORENE & $2 / 11$ & $\mathrm{~T}$ \\
\hline IRON & $23 / 23$ & $\mathbf{B}, \mathbf{E}$ \\
\hline LEAD & $23 / 23$ & B \\
\hline MAGNESIUM & $19 / 23$ & $\mathbf{E}$ \\
\hline PHOSPHORUS & $12 / 12$ & $\mathbf{E}$ \\
\hline POTASSIUM & $23 / 23$ & E \\
\hline SELENIUM & $1 / 18$ & B \\
\hline SILVER & $8 / 23$ & $\mathbf{T}$ \\
\hline SODIUM & $12 / 23$ & $\mathrm{~B}, \mathrm{E}$ \\
\hline VANADIUM & $23 / 23$ & B \\
\hline ZINC & $19 / 23$ & B \\
\hline \multicolumn{3}{|l|}{ Radionuclides } \\
\hline URANIUM-235 & $23 / 23$ & B \\
\hline URANIUM-238 & $23 / 23$ & B \\
\hline \multicolumn{3}{|l|}{ Organics } \\
\hline $\begin{array}{l}\text { 1,1,2-TRICHLORO- } \\
\text { 1,2,2-TRIFLUOROETHANE }\end{array}$ & $4 / 11$ & $T$ \\
\hline 1,1-DICHILOROETHANE & $2 / 23$ & $\mathbf{T}$ \\
\hline 1,1-DICHLOROETHENE & $1 / 23$ & $\mathrm{~T}$ \\
\hline 1,2-DICHLOROBENZENE & $1 / 11$ & $\mathbf{T}$ \\
\hline 1,2-DICHLOROETHANE & $1 / 23$ & $\mathbf{T}$ \\
\hline 2,4-DIMETHYLPHENOL & $1 / 11$ & $\mathbf{T}$ \\
\hline
\end{tabular}


C-53

Table C.6. (continued)

\begin{tabular}{|c|c|c|}
\hline Analyte & $\begin{array}{l}\text { Frequency } \\
\text { of detect }\end{array}$ & $\begin{array}{l}\text { Reason analyte is } \\
\text { not on COPC list }\end{array}$ \\
\hline 2-BUTANONE & $4 / 23$ & $\mathrm{~T}$ \\
\hline 4-METHYL-2-PENTANONE & $1 / 23$ & $\mathrm{~T}$ \\
\hline 4-METHYLPHENOL & $1 / 11$ & $\mathbf{T}$ \\
\hline ACENAPHTHENE & $2 / 11$ & $\mathrm{~T}$ \\
\hline ACETONE & $14 / 23$ & $T$ \\
\hline ANTHRACENE & $2 / 10$ & $T$ \\
\hline BENZENE & $1 / 23$ & $\mathrm{~T}$ \\
\hline BENZO(A)ANTHRACENE & $4 / 10$ & $\mathbf{T}$ \\
\hline BENZO(B)FLUORANTHENE & $3 / 11$ & $\mathbf{T}$ \\
\hline BENZO(K)FLUORANTHENE & $3 / 11$ & $\mathbf{T}$ \\
\hline BENZYL BUTYL PHTHALATE & $1 / 10$ & $\mathrm{~T}$ \\
\hline CARBAZOLE & $2 / 10$ & $\mathbf{T}$ \\
\hline CARBON DISULFIDE & $1 / 23$ & $\mathrm{~T}$ \\
\hline CHLOROBENZENE & $1 / 22$ & $\mathbf{T}$ \\
\hline CHRYSENE & $3 / 10$ & $\mathbf{T}$ \\
\hline DI-N-BUTYL PHTHALATE & $2 / 11$ & $\mathbf{T}$ \\
\hline DI-N-OCTYL PHTHALATE & $1 / 11$ & $\mathbf{T}$ \\
\hline DIMETHYL PHTHALATE & $4 / 11$ & $\mathbf{T}$ \\
\hline ETHYLBENZENE & $3 / 23$ & $\mathrm{~T}$ \\
\hline FLUORANTHENE & $4 / 10$ & $\mathrm{~T}$ \\
\hline HEPTACHLOR & $2 / 8$ & $\mathrm{~T}$ \\
\hline INDENO(1,2,3-CD)PYRENE & $3 / 11$ & $\mathrm{~T}$ \\
\hline METHYLENE CHLORIDE & $5 / 23$ & $\mathbf{T}$ \\
\hline P,P'-METHOXYCHLOR & $1 / 7$ & $\mathrm{~T}$ \\
\hline PENTACHLOROPHENOL & $1 / 10$ & $\mathbf{T}$ \\
\hline PHENOL & $1 / 11$ & $\mathrm{~T}$ \\
\hline PYRENE & $5 / 10$ & $\mathbf{T}$ \\
\hline TOLUENE & $4 / 23$ & $\mathrm{~T}$ \\
\hline XYLENE (TOTAL) & $2 / 23$ & $\mathrm{~T}$ \\
\hline
\end{tabular}


C-54

Table C.6. (continued)

\begin{tabular}{|c|c|c|}
\hline Analyte & $\begin{array}{l}\text { Frequency } \\
\text { of detect }\end{array}$ & $\begin{array}{l}\text { Reason analyte is } \\
\text { not on COPC list }\end{array}$ \\
\hline ALUMINUM & $75 / 89$ & B \\
\hline ANTIMONY & $37 / 60$ & T \\
\hline ARSENIC & $70 / 89$ & $\mathbf{B}$ \\
\hline BARIUM & $75 / 89$ & $\mathrm{~T}$ \\
\hline BERYLLIUM & $75 / 89$ & B \\
\hline CALCIUM & $75 / 89$ & E \\
\hline CHROMIUM & $75 / 89$ & B \\
\hline COPPER & $75 / 89$ & $\mathbf{B}, \mathbf{E}$ \\
\hline FLUORENE & $5 / 32$ & $\mathbf{T}$ \\
\hline IRON & $75 / 89$ & $\mathrm{~B}, \mathrm{E}$ \\
\hline LEAD & $75 / 89$ & B \\
\hline MAGNESIUM & $75 / 89$ & $\mathbf{E}$ \\
\hline NICKEL & $75 / 89$ & $\mathrm{~T}$ \\
\hline POTASSIUM & $75 / 89$ & $\mathbf{E}$ \\
\hline SELENIUM & $15 / 49$ & B \\
\hline SILVER & $53 / 89$ & $\mathrm{~T}$ \\
\hline SODIUM & $32 / 89$ & $\mathbf{B}, \mathbf{E}$ \\
\hline VANADIUM & $75 / 89$ & B \\
\hline ZINC & $74 / 89$ & B \\
\hline \multicolumn{3}{|l|}{ Radionuclides } \\
\hline URANIUM-234 & $89 / 89$ & B \\
\hline URANIUM-235 & $58 / 58$ & B \\
\hline URANIUM-238 & $89 / 89$ & B \\
\hline \multicolumn{3}{|l|}{ Organics } \\
\hline $\begin{array}{l}\text { 1,1,2-TRICHLORO- } \\
\text { 1,2,2-TRIFLUOROETHANE }\end{array}$ & $3 / 32$ & $\mathrm{~T}$ \\
\hline 1,2,4-TRICHLOROBENZENE & $2 / 32$ & $T$ \\
\hline 1,4-DICHLOROBENZENE & $1 / 32$ & $\mathbf{T}$ \\
\hline 2-BUTANONE & $1 / 32$ & $\mathbf{T}$ \\
\hline 2-CHLOROPHENOL & $1 / 32$ & $\mathrm{~T}$ \\
\hline 4-METHYL-2-PENTANONE & $3 / 32$ & $\mathbf{T}$ \\
\hline
\end{tabular}


C-55

Table C.6. (continued)

\begin{tabular}{lcc}
\hline Analyte & $\begin{array}{c}\text { Frequency } \\
\text { of detect }\end{array}$ & $\begin{array}{c}\text { Reason analyte is } \\
\text { not on COPC } \text { list }^{\mathrm{a}}\end{array}$ \\
\hline ACENAPHTHENE & $5 / 32$ & $\mathrm{~T}$ \\
ACETONE & $15 / 32$ & $\mathrm{~T}$ \\
ANTHRACENE & $4 / 32$ & $\mathrm{~T}$ \\
BENZO(K)FLUORANTHENE & $10 / 32$ & $\mathrm{~T}$ \\
BIS(2-ETHYLHEXYL)PHTHALATE & $3 / 32$ & $\mathrm{~T}$ \\
CARBAZOLE & $4 / 32$ & $\mathrm{~T}$ \\
CARBON DISULFIDE & $1 / 32$ & $\mathrm{~T}$ \\
CHRYSENE & $7 / 32$ & $\mathrm{~T}$ \\
FLUORANTHENE & $14 / 32$ & $\mathrm{~T}$ \\
INDENO(1,2,3-CD)PYRENE & $7 / 32$ & $\mathrm{~T}$ \\
METHYLENE CHLORIDE & $11 / 32$ & $\mathrm{~T}$ \\
PENTACHLOROPHENOL & $1 / 32$ & $\mathrm{~T}$ \\
PHENOL & $1 / 32$ & $\mathrm{~T}$ \\
PYRENE & $14 / 32$ & $\mathrm{~T}$ \\
TOLUENE & $2 / 32$ & $\mathrm{~T}$
\end{tabular}

SA-1

Inorganics

ARSENIC

$11 / 12$

B

CALCIUM

$12 / 12$

E

COPPER

$12 / 12$

$\mathrm{B}, \mathrm{E}$

CYANIDE

$1 / 12$

T

FLUORENE

2/12

T

IRON

$11 / 12$

E

LEAD

$12 / 12$

B

MAGNESIUM

$11 / 12$

E

PHOSPHORUS

$8 / 12$

E

POTASSIUM

$11 / 12$

E

SODIUM

$12 / 12$

$\mathrm{B}, \mathrm{E}$

VANADIUM

$12 / 12$

$B$

ZINC

$12 / 12$

B 
C-56

Table C.6. (continued)

\begin{tabular}{lcc}
\hline Analyte & $\begin{array}{c}\text { Frequency } \\
\text { of detect }\end{array}$ & $\begin{array}{c}\text { Reason analyte is } \\
\text { not on COPC list }\end{array}$ \\
\hline Radionuclides & & $\mathrm{B}$ \\
URANIUM-235 & $12 / 12$ & \\
Organics & & \\
1,2-DICHLOROETHENE (TOTAL) & & $\mathrm{T}$ \\
4-METHYL-2-PENTANONE & $2 / 12$ & $\mathrm{~T}$ \\
ACENAPHTHENE & $1 / 12$ & $\mathrm{~T}$ \\
ACETONE & $1 / 12$ & $\mathrm{~T}$ \\
ANTHRACENE & $2 / 12$ & $\mathrm{~T}$ \\
BENZO(K)FLUORANTHENE & $2 / 12$ & $\mathrm{~T}$ \\
BENZOIC ACID & $5 / 12$ & $\mathrm{~T}$ \\
CHRYSENE & $1 / 12$ & $\mathrm{~T}$ \\
DIETHYL PHTHALATE & $5 / 12$ & $\mathrm{~T}$ \\
DIMETHYL PHTHALATE & $1 / 12$ & $\mathrm{~T}$ \\
FLUORANTHENE & $1 / 12$ & $\mathrm{~T}$ \\
METHYLENE CHLORIDE & $5 / 12$ & $\mathrm{~T}$ \\
PYRENE & $2 / 12$ & $\mathrm{~T}$ \\
TETRACHLOROETHENE & $5 / 12$ & $\mathrm{~T}$ \\
TOLUENE & $2 / 12$ & $\mathrm{~T}$ \\
XYLENE (TOTAL) & $1 / 12$ & $1 / 12$ \\
\hline
\end{tabular}

- B = Based on the comparison of BCV OU2 soil data with data from the Background Soil Characterization Project (Energy Systems 1993c); E = Essential nutrient (EPA 1989a; Dunne 1990); $\mathbf{T}=$ Based on toxicity screening (EPA 1989a). 
Table C.7. Comparison of BCV OU2 soil mondetocted analyte concentrations with residential PRGs

\begin{tabular}{|c|c|c|c|c|c|c|c|c|}
\hline Analyte & $\begin{array}{l}\text { Frequency } \\
\text { of detect }\end{array}$ & $\begin{array}{l}\text { Mastimum } \\
\text { nondetected } \\
\text { concentration }\end{array}$ & $\begin{array}{l}\text { Residential } \\
\text { PRG for } \\
H Q=1.0\end{array}$ & $\begin{array}{l}\text { Residential } \\
\text { PRG for } \\
\text { risk = 1.0E-04 }\end{array}$ & $\begin{array}{l}\text { Residential } \\
\text { PRG for } \\
\text { risk = } 1.0 \mathrm{E}-06\end{array}$ & $\begin{array}{l}\text { Is max. } \\
\text { conc. }> \\
\mathrm{PRG} \text { at } \\
\mathrm{HI}=1.0 \%\end{array}$ & $\begin{array}{l}\text { Is max. } \\
\text { conc. }> \\
\text { PRG al } \\
\text { risk }=1.0 \mathrm{E}-06 ?\end{array}$ & $\begin{array}{l}\text { Units } \\
\text { of } \\
\text { measure }\end{array}$ \\
\hline \multicolumn{9}{|c|}{ RSA } \\
\hline \multicolumn{9}{|l|}{ Organics } \\
\hline AROCLOR-1016 & on & $3.60 \mathrm{E}-01$ & $1.9 \mathrm{E}+01$ & $8.3 E+00$ & $8.3 E-02$ & & YES & $\mathrm{mg} / \mathrm{kg}$ \\
\hline AROCLOR-1221 & on & $7.30 \mathrm{E}-01$ & & $8.3 E+00$ & $8.3 E-02$ & & YES & $\mathrm{mg} / \mathrm{kg}$ \\
\hline AROCLOR-1232 & on & $3.60 \mathrm{E}-01$ & & $8.3 E+00$ & $8.3 \mathrm{E}-02$ & & YES & $\mathrm{mg} / \mathrm{kg}$ \\
\hline AROCLOR-1242 & or & $3.60 \mathrm{E}-01$ & & $8.3 \mathrm{E}+00$ & 8.3E.02 & & YES & mg/kg \\
\hline AROCLOR-1248 & on & $3.60 \mathrm{E}-01$ & & $8.3 E+00$ & $8.3 \mathrm{E}-02$ & & YES & $\mathrm{mg} / \mathrm{kg}$ \\
\hline AROCLOR-1260 & or & $3.60 \mathrm{E}-01$ & & $8.38+\infty$ & $8.3 E-02$ & & YES & $\mathrm{mg} / \mathrm{kg}$ \\
\hline BIS(2.CHLOROETHYL)ETHER & $0 / 1$ & $7.20 \mathrm{E}-01$ & & $5.8 \mathrm{E}+01$ & $5.8 \mathrm{E}-01$ & & YES & mg/kg \\
\hline N-NITROSO-DI-N-PROPYLAMINE & $0 / 11$ & $7.20 E-01$ & & $9.1 \mathbb{E}+\infty$ & $9.1 \mathrm{E}-02$ & & YES & $\mathrm{mg} / \mathrm{kg}$ \\
\hline TOXAPHENE & on & $1.80 \mathrm{E}+00$ & & $5.8 E+01$ & $5.8 \mathrm{E}-01$ & & YES & $\mathrm{mg} / \mathrm{kg}$ \\
\hline VINYL CHLORIDE & ons & $1.50 E+00$ & & $3.4 E+01$ & $3.4 E-01$ & & YES & $\mathrm{mg} / \mathrm{kg}$ \\
\hline
\end{tabular}

SY-200

\section{Organics}

ALDRIN

ALPHA-BHC

AROCLOR-1016

AROCLOR-1221

AROCLOR-1232

AROCLOR-1242

$\begin{array}{lllll}0 / 32 & 2.00 \mathrm{E}-01 & 8.2 \mathrm{E}+00 & 3.8 \mathrm{E}+00 & 3.8 \mathrm{E}-02 \\ 0 / 32 & 2.00 \mathrm{E}-01 & & 1.0 \mathrm{E}+01 & 1.0 \mathrm{E}-01 \\ 0 / 88 & 3.90 \mathrm{E}+00 & 1.9 \mathrm{E}+01 & 8.3 \mathrm{E}+00 & 8.3 \mathrm{E}-02 \\ 0 / 88 & 7.90 \mathrm{E}+00 & & 8.3 \mathrm{E}+00 & 8.3 \mathrm{E}-02 \\ 0 / 88 & 3.90 \mathrm{E}+\infty & & 8.3 \mathrm{E}+\infty & 8.3 \mathrm{E}-02 \\ 0 / 88 & 3.90 \mathrm{E}+00 & & 8.3 \mathrm{E}+\infty & 8.3 \mathrm{E}-02\end{array}$

$\begin{array}{cc}\text { YES } & \mathrm{mg} / \mathrm{kg} \\ \text { YES } & \mathrm{mg} / \mathrm{kg} \\ \text { YES } & \mathrm{mg} / \mathrm{kg} \\ \text { YES } & \mathrm{mg} / \mathrm{kg} \\ \text { YES } & \mathrm{mg} / \mathrm{kg} \\ \text { YES } & \mathrm{mg} / \mathrm{kg}\end{array}$


Table C.7. (continued)

\begin{tabular}{|c|c|c|c|c|c|c|c|c|}
\hline Analyte & $\begin{array}{l}\text { Frequency } \\
\text { of detect }\end{array}$ & $\begin{array}{l}\text { Maximum } \\
\text { nondetected } \\
\text { concentration }\end{array}$ & $\begin{array}{l}\text { Residential } \\
\text { PRG for } \\
H Q=1.0\end{array}$ & $\begin{array}{l}\text { Residential } \\
\text { PRG for } \\
\text { risk = 1.0B-04 }\end{array}$ & $\begin{array}{l}\text { Residential } \\
\text { PRG for } \\
\text { risk = } 1.0 \mathrm{E}-06\end{array}$ & $\begin{array}{l}\text { Is max. } \\
\text { cone. } \\
\text { PRG al } \\
\text { HI }=1.0 \%\end{array}$ & $\begin{array}{l}\text { Is max. } \\
\text { conc. }> \\
\text { PRG at } \\
\text { risk }=1.0 \mathrm{E}-06 ?\end{array}$ & $\begin{array}{l}\text { Units } \\
\text { of } \\
\text { measure }\end{array}$ \\
\hline AROCLOR-1248 & $0 / 88$ & $3.90 \mathrm{E}+00$ & & $8.3 \mathrm{E}+00$ & $8.3 E-02$ & & YES & $\mathrm{mg} / \mathrm{kg}$ \\
\hline DIELDRIN & $0 / 32$ & $3.90 \mathrm{E}-01$ & $1.4 \mathrm{E}+01$ & $4.0 \mathrm{E}+00$ & $4.0 \mathrm{E}-02$ & & YES & $\mathrm{mg} / \mathrm{kg}$ \\
\hline HEPTACHLOR & $0 / 32$ & $2.00 E-01$ & $1.4 \mathrm{E}+02$ & $1.4 E+01$ & $1.4 \mathrm{E}-01$ & & YES & mg/kg \\
\hline HEPTACHLOR EPOXIDE & $0 / 32$ & $2.00 E-01$ & $3.6 \mathrm{E}+00$ & $7.0 \mathrm{E}+00$ & $7.0 \mathrm{E}-02$ & & YES & $\mathrm{mg} / \mathrm{kg}$ \\
\hline HEXACHLOROBENZENE & $0 / 32$ & $5.30 \mathrm{E}-01$ & $2.2 E+02$ & $4.0 \mathrm{E}+01$ & $4.0 E-01$ & & YES & $\mathrm{mg} / \mathrm{kg}$ \\
\hline N-NITROSO-DI-N-PROPYLAMINE & $0 / 32$ & $5.30 \mathrm{E}-01$ & & $9.1 E+\infty$ & $9.1 E-02$ & & YES & $\mathrm{mg} / \mathrm{kg}$ \\
\hline TOXAPHENE & $0 / 32$ & $2.00 \mathrm{E}+01$ & & $5.8 \mathrm{E}+01$ & $5.8 \mathrm{E}-01$ & & YES & $\mathrm{mg} / \mathrm{kg}$ \\
\hline \multicolumn{9}{|c|}{ SA-1 } \\
\hline \multicolumn{9}{|l|}{ Organics } \\
\hline 2,4-DINITROTOLUENE & $0 / 2$ & $1.80 \mathrm{E}+00$ & $5.5 E+02$ & $9.4 E+01$ & $9.4 \mathrm{E}-01$ & & YES & $\mathrm{mg} / \mathrm{kg}$ \\
\hline 2,6-DINITROTOLUENE & $0 / 12$ & $1.80 \mathrm{E}+00$ & $2.7 E+02$ & $9.4 E+01$ & $9.4 E-01$ & & YES & $\mathrm{mg} / \mathrm{kg}$ \\
\hline 3,3'-DICHLOROBENZIDINE & $0 / 12$ & $3.60 \mathrm{E}+00$ & & $1.4 \mathrm{E}+02$ & $1.4 \mathrm{E}+\infty$ & & YES & $\mathrm{mg} / \mathrm{kg}$ \\
\hline BIS(2.CHLOROETHYL)ETHER & $0 / 12$ & $1.80 \mathrm{E}+00$ & & $5.8 \mathrm{E}+01$ & $5.8 \mathrm{E}-01$ & & YES & $\mathrm{mg} / \mathrm{kg}$ \\
\hline DIBENZO(A,H)ANTHRACENE & $0 / 12$ & $1.80 \mathrm{E}+\infty$ & & $8.8 \mathrm{E}+00$ & $8.8 \mathrm{E}-02$ & & YES & $\mathrm{mg} / \mathrm{kg}$ \\
\hline MEXACHLOROBENZENE & O/2 & $1.80 \mathrm{E}+00$ & $2.2 E+02$ & $4.0 E+01$ & $4.0 \mathrm{E}-01$ & & YES & $\mathrm{mg} / \mathrm{kg}$ \\
\hline N-NITROSO-DI-N-PROPYLAMINE & on2 & $1.80 \mathrm{E}+00$ & & $9.1 E+\infty$ & $9.1 E-02$ & & YES & $\mathrm{mg} / \mathrm{kg}$ \\
\hline PENTACHLOROPHENOL & on2 & $8.70 \mathrm{E}+00$ & $8.2 E+03$ & $5.3 \mathrm{E}+02$ & $5.3 \mathrm{E}+\infty$ & & YES & mg/kg \\
\hline
\end{tabular}

- Preliminary remediation goals (PRGs), for the residential land use scenario (ingestion of soil pathway), calculated for a target risk of 1.0E-04 and 1.0E-06 for carcinogens, and a target hazard quotient of 1.0 for noncarcinogens.

Only analytes whose maximum nondetected concentration is greater than the PRG (based on $\mathrm{HQ}=1.0$ and/or risk $=1.0 \mathrm{E}-06$ ) are reported in this table. 
Table C.7. (comtinued)

- Note, no analyte reported in this table has a maximum nondetected concentration greater than the PRG for an $H Q=1.0$. 
Table C.8. Comparison of maximum detected concentrations for BCV OU2 soil COPCs with residential PRGs

\begin{tabular}{|c|c|c|c|c|c|c|c|c|c|c|}
\hline Analyte & $\begin{array}{l}\text { Freq. } \\
\text { of } \\
\text { detect }\end{array}$ & $\begin{array}{l}\text { Maximum } \\
\text { detected } \\
\text { conc. }\end{array}$ & $\begin{array}{l}\text { Residential } \\
\text { PRG for } \\
H Q=1.0\end{array}$ & $\begin{array}{l}\text { Residential } \\
\text { PRG for } \\
\text { risk = 1.0E-04 }\end{array}$ & $\begin{array}{l}\text { Residential } \\
\text { PRG for } \\
\text { risk = } 1.0 \mathrm{E}-06\end{array}$ & $\begin{array}{l}\text { Is max. } \\
\text { conc. }> \\
\text { PRG for } \\
H O=1.0 \text { ? }\end{array}$ & $\begin{array}{l}\text { Is max. } \\
\text { conc. }> \\
\text { PRG for } \\
\text { risk }= \\
1.0 \mathrm{E}-06 ?^{\circ}\end{array}$ & $\begin{array}{l}\text { Units } \\
\text { of } \\
\text { measure }\end{array}$ & $\begin{array}{l}\text { Depth } \\
\text { where } \\
\text { maximum } \\
\text { conc. was } \\
\text { delected }\end{array}$ & $\begin{array}{l}\text { Borehole } \\
\text { where } \\
\text { maximum } \\
\text { conc. was } \\
\text { detected }\end{array}$ \\
\hline \multicolumn{11}{|c|}{ RSA } \\
\hline \multicolumn{11}{|l|}{ Inorganics } \\
\hline BARIUM & $23 / 23$ & $1.86 \mathrm{E}+02$ & $1.9 \mathrm{E}+04$ & & & & & $\mathrm{mg} / \mathrm{kg}$ & $4-8$ & SB04 \\
\hline BERYLLIUM & $23 / 23$ & $2.10 \mathrm{E}+100$ & $1.4 \mathrm{E}+03$ & $1.5 \mathrm{E}+01$ & 1.5E-01 & & YES & $\mathrm{mg} / \mathrm{kg}$ & 0.5 & $106-007$ \\
\hline CADMIUM & $16 / 23$ & $5.70 E+00$ & $2.7 E+02$ & & & & & $\mathrm{mg} / \mathrm{kg}$ & 0.5 & $106-001$ \\
\hline MANGANESE & $23 / 23$ & $2.80 \mathrm{E}+03$ & $3.8 \mathrm{E}+04$ & & & & & $\mathrm{mg} / \mathrm{kg}$ & 0.5 & $106-006$ \\
\hline MERCURY & $14 / 23$ & $7.90 \mathrm{E}+00$ & $8.2 \mathrm{E}+01$ & & & & & $\mathrm{mg} / \mathrm{kg}$ & $4-8$ & \$B02 \\
\hline NICKEL & $23 / 23$ & $3.11 E+02$ & $5.5 \mathbb{E}+03$ & & & & & $\mathrm{mg} / \mathrm{kg}$ & $0-4$ & SB05 \\
\hline $\begin{array}{l}\text { URANIUM } \\
\text { (SOLUBLE SALTS) }\end{array}$ & $12 / 12$ & $1.20 E+01$ & $8.2 \mathrm{E}+02$ & & & & & $\mathrm{mg} / \mathrm{kg}$ & $5-10$ & $106-003$ \\
\hline \multicolumn{11}{|l|}{ Radionuclides } \\
\hline THORIUM-232 & $2 / 12$ & $3.28 \mathrm{E}+00$ & & $6.4 E+03$ & $6.4 \mathrm{E}+01$ & & & $\mathrm{pCi} / \mathrm{g}$ & $5-10$ & $106-006$ \\
\hline URANIUM-234 & $23 / 23$ & $7.91 \mathrm{E}+00$ & & $4.8 \mathrm{E}+03$ & $4.8 \mathrm{E}+01$ & & & $\mathrm{pCi} / \mathrm{g}$ & $4-8$ & $\mathrm{SBO2}$ \\
\hline \multicolumn{11}{|l|}{ Organics } \\
\hline $\begin{array}{l}\text { 1,2,4-TRICHLORO- } \\
\text { BENZENE }\end{array}$ & $1 / 11$ & $4.30 \mathrm{E}+01$ & $2.7 E+03$ & & & & & $\mathrm{mg} / \mathrm{kg}$ & 0.4 & SBOS \\
\hline $\begin{array}{l}\text { 1,2-DICHLOROETHENE } \\
\text { (TOTAL) }\end{array}$ & $4 / 23$ & $1.10 \mathrm{E}+02$ & $2.5 \mathrm{E}+03$ & & & & & $\mathrm{mg} / \mathrm{kg}$ & $0-4$ & \$B05 \\
\hline AROCLOR-1254 & $5 / 8$ & $8.90 \mathrm{E}+00$ & & $8.3 E+00$ & $8.3 \mathrm{E}-02$ & & YES & $\mathrm{mg} / \mathrm{kg}$ & $0-4$ & SB05 \\
\hline BENZO(A) $)^{2}$ YRENE & $3 / 11$ & $5.80 \mathrm{E}-01$ & & $8.8 \mathrm{E}+00$ & $8.8 \mathrm{E}-02$ & & YES & $\mathrm{mg} / \mathrm{kg}$ & 4.8 & SB02 \\
\hline
\end{tabular}


Table C.8. (continued)

\begin{tabular}{|c|c|c|c|c|c|c|c|c|c|c|}
\hline Analyte & $\begin{array}{l}\text { Freq. } \\
\text { of } \\
\text { detect }\end{array}$ & $\begin{array}{l}\text { Masimum } \\
\text { detected } \\
\text { conc. }\end{array}$ & $\begin{array}{l}\text { Residential } \\
\text { PRG for } \\
\mathrm{HQ}=\mathbf{1 . 0}\end{array}$ & $\begin{array}{l}\text { Residemtial } \\
\text { PRG for } \\
\text { risk }=1.0 \mathrm{E}-04\end{array}$ & $\begin{array}{l}\text { Residential } \\
\text { PRG for } \\
\text { rish = 1.0E-06 }\end{array}$ & $\begin{array}{l}\text { Is mas. } \\
\text { conc. }> \\
\text { PRG for } \\
H Q=1.0 ?\end{array}$ & $\begin{array}{l}\text { Is max. } \\
\text { conc. }> \\
\text { PRG for } \\
\text { risk }= \\
1.0 \mathrm{E}-06 ?^{8}\end{array}$ & $\begin{array}{l}\text { Units } \\
\text { of } \\
\text { measure }\end{array}$ & $\begin{array}{l}\text { Depth } \\
\text { where } \\
\text { maximum } \\
\text { conc. was } \\
\text { detected }\end{array}$ & $\begin{array}{l}\text { Borehole } \\
\text { where } \\
\text { maximum } \\
\text { conc. was } \\
\text { detected }\end{array}$ \\
\hline $\begin{array}{l}\text { BIS(2-ETHYLHEXYL)- } \\
\text { PHTHALATE }\end{array}$ & $\mathbb{1} / 1$ & $4.10 E+01$ & $5.5 \mathrm{E}+03$ & $4.6 E+03$ & $4.6 \mathrm{E}+01$ & & & $\mathrm{mg} / \mathrm{kg}$ & $0-4$ & SB05 \\
\hline $\begin{array}{l}\text { DIBENZO(A,H)- } \\
\text { ANTIIRACENE }\end{array}$ & $2 / 11$ & 2.90E-01 & & $8.8 E+00$ & $8.8 \mathrm{E}-02$ & & YES & $\mathrm{mg} / \mathrm{kg}$ & $4-8$ & SBO2 \\
\hline TETRACHLOROETHENE & $5 / 23$ & $4.00 E+02$ & $2.7 E+03$ & & & & & $m g / k g$ & $0-4$ & SBOS \\
\hline
\end{tabular}

SY-200

Inorganics

$\begin{array}{llll}\text { CADMIUM } & 12184 & 3.10 \mathrm{E}+00 & 2.7 \mathrm{E}+02 \\ \text { MANGANESE } & 75 / 89 & 3.23 \mathrm{E}+03 & 3.8 \mathrm{E}+04 \\ \text { MERCURY } & 73 / 89 & 8.16 \mathrm{E}+02 & 8.2 \mathrm{E}+01\end{array}$

YES

$\begin{array}{lcc}\mathrm{mg} / \mathrm{kg} & 7-11 & \mathrm{SB} 27 \\ \mathrm{mg} / \mathrm{kg} & 7-11 & \mathrm{SB} 50 \\ \mathrm{mg} / \mathrm{kg} & 3-7 & \mathrm{SB} 14\end{array}$

\section{Organics}

\section{AROCLOR-1254}

$\begin{array}{ll}9 / 88 & 1.90 \mathrm{E}+00 \\ 41 / 88 & 1.00 \mathrm{E}+01 \\ 7 / 32 & 9.90 \mathrm{E}-01 \\ 10 / 32 & 8.90 \mathrm{E}-01 \\ 9 / 32 & 1.30 \mathrm{E}+00 \\ & \\ 4 / 32 & 1.20 \mathrm{E}-01\end{array}$

$\begin{array}{ll}8.3 \mathrm{E}+00 & 8.3 \mathrm{E}-02 \\ 8.3 \mathrm{E}+00 & 8.3 \mathrm{E}-02 \\ 8.8 \mathrm{E}+01 & 8.8 \mathrm{E}-01 \\ 8.8 \mathrm{E}+00 & 8.8 \mathrm{E}-02 \\ 8.8 \mathrm{E}+01 & 8.8 \mathrm{E}-01 \\ 8.8 \mathrm{E}+00 & 8.8 \mathrm{E}-02\end{array}$

YES

$\mathrm{mg} / \mathrm{kg} \quad 3-7$

SB06

AROCLOR-1260 BENZO(A)ANTHRACENE

BENZO(A)PYRENE

BENZO(B)-

FLUORANTHENE

$4 / 32 \quad 1.20 \mathrm{E}-01$

$8.8 \mathrm{E}+00$

$8.8 \mathrm{E}-02$

$\begin{array}{llll}\text { YES }^{*} \mathrm{mg} / \mathrm{kg} & 7-11 & \end{array}$

YES mg/kg 7-11 SB35

YES $\mathrm{mg} / \mathrm{kg} \quad 7-11 \quad$ SB21

$\begin{array}{llll}\text { YES } & \mathrm{mg} / \mathrm{kg} & 3.6 & \mathrm{SB} 54\end{array}$

ANTHRACENE 
Table C.8. (continued)

\begin{tabular}{|c|c|c|c|c|c|c|c|c|c|c|}
\hline Analyte & $\begin{array}{l}\text { Freq. } \\
\text { of } \\
\text { detect }\end{array}$ & $\begin{array}{l}\text { Maximum } \\
\text { detected } \\
\text { conc. }\end{array}$ & $\begin{array}{l}\text { Residential } \\
\text { PRG for } \\
H Q=1.0\end{array}$ & $\begin{array}{l}\text { Residential } \\
\text { PRG for } \\
\text { risk = } 1.0 \mathrm{E}-04\end{array}$ & $\begin{array}{l}\text { Residential } \\
\text { PRG for } \\
\text { risk = 1.0E-06 }\end{array}$ & $\begin{array}{l}\text { Is max. } \\
\text { conc. }> \\
\text { PRG for } \\
H Q=1.0 ?\end{array}$ & $\begin{array}{l}\text { Is max. } \\
\text { conc. }> \\
\text { PRG for } \\
\text { risk }= \\
1.0 \mathrm{E}-06 ?^{\circ}\end{array}$ & $\begin{array}{l}\text { Units } \\
\text { of } \\
\text { measure }\end{array}$ & $\begin{array}{l}\text { Depth } \\
\text { where } \\
\text { maximum } \\
\text { conc. was } \\
\text { detected }\end{array}$ & $\begin{array}{l}\text { Borehole } \\
\text { where } \\
\text { maximum } \\
\text { conc. was } \\
\text { detected }\end{array}$ \\
\hline \multicolumn{11}{|c|}{ SA-1 } \\
\hline \multicolumn{11}{|l|}{ Inorganics } \\
\hline BARIUM & $12 / 12$ & $3.85 \mathrm{E}+02$ & $1.9 \mathrm{E}+04$ & & & & & $\mathrm{mg} / \mathrm{kg}$ & $4.5-7.5$ & SA-1.4 \\
\hline BERYLLIUM & $12 / 12$ & $1.50 \mathrm{E}+00$ & $1.4 E+03$ & $1.5 \mathrm{E}+01$ & $1.5 \mathrm{E}-01$ & & YES & $\mathrm{mg} / \mathrm{kg}$ & $4.5-7.5$ & SA-1.4 \\
\hline CADMIUM & $12 / 12$ & $4.10 E+00$ & $2.7 \mathrm{E}+02$ & & & & & $\mathrm{mg} / \mathrm{kg}$ & $9-10.5$ & SA-1.4 \\
\hline CHROMIUM VI & $12 / 12$ & $4.27 \mathrm{E}+01$ & $1.4 \mathrm{E}+03$ & & & & & $\mathrm{mg} / \mathrm{kg}$ & 9.10 .5 & SA-1.2 \\
\hline MANGANESE & $12 / 12$ & $2.03 E+03$ & $3.8 E+04$ & & & & & mg/kg & $4.5-6$ & SA-1.1 \\
\hline MERCURY & $8 / 12$ & $2.60 \mathrm{E}+00$ & $8.2 \mathrm{E}+01$ & & & & & $\mathrm{mg} / \mathrm{kg}$ & $4.5-6$ & SA-1.3 \\
\hline NICKEL & $12 / 12$ & $5.10 \mathrm{E}+01$ & $5.5 E+03$ & & & & & $\mathrm{mg} / \mathrm{kg}$ & $4.5-6$ & SA-1.1 \\
\hline $\begin{array}{l}\text { URANIUM } \\
\text { (SOLUBLE SALTS) }\end{array}$ & $12 / 12$ & $6.89 \mathrm{E}+01$ & $8.2 E+02$ & & & & & $m g / k g$ & $9-10.5$ & SA-1.6 \\
\hline \multicolumn{11}{|l|}{ Radionuclides } \\
\hline RADIUM-226 & $12 / 12$ & $7.57 \mathrm{E}+00$ & & $6.9 \mathrm{E}-01$ & $6.9 \mathrm{E}-03$ & & YES & $\mathrm{pCi} / \mathrm{s}$ & $9-10.5$ & SA-1.4 \\
\hline URANIUM-234 & $12 / 12$ & $2.32 \mathrm{E}+01$ & & $4.8 \mathrm{E}+03$ & $4.8 \mathrm{E}+01$ & & & $\mathrm{pCi} / \mathrm{g}$ & $9-10.5$ & SA-1.6 \\
\hline URANIUM-238 & $12 / 12$ & $2.30 \mathrm{E}+01$ & & $1.1 E+02$ & $1.1 E+\infty$ & & YES & $\mathrm{pCi} / \mathrm{g}$ & $9-10.5$ & SA-1.6 \\
\hline \multicolumn{11}{|l|}{ Organics } \\
\hline BENZO(A)ANTHRACENE & $5 / 12$ & $6.10 \mathrm{E}-01$ & & $8.8 \mathrm{E}+01$ & $8.8 \mathrm{E}-01$ & & & $\mathrm{mg} / \mathrm{kg}$ & $9-12$ & SA-1.5 \\
\hline BENZO(A)PYRENE & $5 / 12$ & $4.80 \mathrm{E}-01$ & & $8.8 \mathrm{E}+00$ & $8.8 \mathrm{E}-02$ & & YES & $\mathrm{mg} / \mathrm{kg}$ & $9 \cdot 12$ & SA-1.5 \\
\hline
\end{tabular}


Table C8. (contimued)

\begin{tabular}{|c|c|c|c|c|c|c|c|c|c|c|}
\hline Analyte & $\begin{array}{l}\text { Freq. } \\
\text { of } \\
\text { detect } \\
\end{array}$ & $\begin{array}{l}\text { Maximum } \\
\text { detected } \\
\text { conc. }\end{array}$ & $\begin{array}{l}\text { Residential } \\
\text { PRG for } \\
H Q=1.0\end{array}$ & $\begin{array}{l}\text { Residential } \\
\text { PRG for } \\
\text { risk = } 1.0 \mathrm{E}-04\end{array}$ & $\begin{array}{l}\text { Residential } \\
\text { PRG for } \\
\text { risk = 1.0E-06 }\end{array}$ & $\begin{array}{l}\text { Is max. } \\
\text { conc. }> \\
P R G \text { for } \\
\mathrm{HQ}=1.0 \text { ? }\end{array}$ & $\begin{array}{l}\text { Is max. } \\
\text { conc. }> \\
\text { PRG for } \\
\text { risk }= \\
1.0 \mathrm{E}-06 \text { P }^{\circ}\end{array}$ & $\begin{array}{l}\text { Units } \\
\text { of } \\
\text { measure } \\
\end{array}$ & $\begin{array}{l}\text { Depth } \\
\text { where } \\
\text { maximum } \\
\text { conc. was } \\
\text { detected }\end{array}$ & $\begin{array}{l}\text { Borehole } \\
\text { where } \\
\text { maximum } \\
\text { conc. was } \\
\text { detected }\end{array}$ \\
\hline $\begin{array}{l}\text { BENZO(B)- } \\
\text { FLUORANTHENE }\end{array}$ & $4 / 12$ & $4.20 \mathrm{E}-01$ & & $8.8 \mathrm{E}+01$ & $8.8 \mathrm{E}-01$ & & & $\mathrm{mg} / \mathrm{kg}$ & $9-12$ & SA-1.5 \\
\hline INDENO(1,2,3-CD)PYRENE & $3 / 12$ & $2.60 \mathrm{E}-01$ & & $8.8 E+01$ & $8.8 \mathrm{E}-01$ & & & $\mathrm{mg} / \mathrm{kg}$ & $9-12$ & SA-1.5 \\
\hline
\end{tabular}

- Preliminary remediation goals (PRGs), for the residential land use scenario (ingestion of soil pathway), calculated for a target risk of $1.0 \mathrm{E}-04$ and $1.0 \mathrm{E}-06$ for carcinogens, and a target hazard quotient of 1.0 for noncarcinogens.

An asterisk ( ) implies the maximum detected concentration is above the PRG at a risk $=1.0 \mathrm{E}-04$. 
Table C9. Summary statistics for detected analytes in the BCV OU2 groundwater

\begin{tabular}{|c|c|c|c|c|c|c|c|}
\hline Analyte & $\begin{array}{l}\text { Frequency } \\
\text { of detect }\end{array}$ & $\begin{array}{l}\text { Minimum } \\
\text { detected conc. }\end{array}$ & $\begin{array}{l}\text { Maximum } \\
\text { detected conc. }\end{array}$ & Distribution & $\begin{array}{l}\text { Mean } \\
\text { concentration }\end{array}$ & $\begin{array}{l}\text { UCL.95 } \\
\text { concentration }^{\mathrm{a}}\end{array}$ & $\begin{array}{l}\text { Units of } \\
\text { measure }\end{array}$ \\
\hline & & & RSA & & & & \\
\hline \multicolumn{8}{|l|}{ Inorganics } \\
\hline ALUMINUM & $101 / 108$ & $2.00 \mathrm{E}-02$ & $1.00 \mathrm{E}+01$ & $\mathbf{L}$ & $2.06 \mathrm{E}+00$ & $3.99 \mathrm{E}+00$ & $\mathrm{mg} / \mathrm{L}$ \\
\hline ANTIMONY & $3 / 108$ & $7.10 \mathrm{E}-02$ & $2.00 \mathrm{E}-01$ & $\mathbf{L}$ & $2.68 \mathrm{E}-02$ & $2.80 \mathrm{E}-02$ & $\mathrm{mg} / \mathrm{h}$ \\
\hline ARSENIC & $8 / 82$ & $5.20 \mathrm{E}-02$ & $8.50 \mathrm{E}-02$ & $\mathbf{L}$ & $2.87 \mathrm{E}-02$ & $3.05 \mathrm{E}-02$ & $\mathrm{mg} / \mathrm{L}$ \\
\hline BARIUM & $108 / 108$ & $8.50 \mathrm{E}-03$ & $2.30 \mathrm{E}-01$ & $\mathbb{L}$ & $5.88 \mathrm{E}-02$ & $6.53 \mathrm{E}-02$ & $\mathrm{mg} / \mathrm{L}$ \\
\hline BER YLLIUM & $17 / 108$ & $3.00 \mathrm{E}-04$ & $1.30 \mathrm{E}-03$ & $\mathbb{L}$ & $2.03 \mathrm{E}-04$ & $2.21 \mathrm{E}-04$ & $\mathrm{mg} / \mathrm{L}$ \\
\hline BORON & $92 / 108$ & $4.70 E-03$ & $1.20 \mathrm{E}+00$ & $L$ & $1.28 \mathrm{E}-01$ & $2.10 \mathrm{E}-01$ & $\mathrm{mg} / \mathrm{L}$ \\
\hline CADMIUM & $15 / 108$ & $3.10 \mathrm{E}-03$ & $2.10 \mathrm{E} \cdot 02$ & $\mathbf{L}$ & $1.93 E-03$ & $2.09 \mathrm{E}-03$ & $\mathrm{mg} / \mathrm{L}$ \\
\hline CALCIUM & $108 / 108$ & $7.10 \mathrm{E}+01$ & $3.90 \mathrm{E}+02$ & $\mathbb{L}$ & $1.62 \mathrm{E}+02$ & $1.73 E+02$ & $\mathrm{mg} / \mathrm{L}$ \\
\hline CHLORIDE & $107 / 108$ & $1.00 \mathrm{E}+00$ & $6.80 \mathrm{E}+01$ & $L$ & $3.15 \mathrm{E}+01$ & $4.48 \mathrm{E}+01$ & $\mathrm{mg} / \mathrm{h}$ \\
\hline CHROMUM & $42 / 108$ & $1.00 \mathrm{E}-02$ & $1.50 \mathrm{E}-01$ & $\mathbb{L}$ & $1.32 \mathrm{E}-02$ & $1.59 \mathrm{E}-02$ & $\mathrm{mg} / \mathrm{L}$ \\
\hline COBALT & $3 / 108$ & $5.20 \mathrm{E}-03$ & $6.30 \mathrm{E}-03$ & $L$ & $2.53 E-03$ & $2.60 \mathrm{E}-03$ & $\mathrm{mg} / \mathrm{L}$ \\
\hline IRON & $105 / 108$ & $1.10 \mathrm{E}-02$ & $2.00 \mathrm{E}+01$ & L & $1.70 \mathrm{E}+00$ & $\quad 3.87 \mathrm{E}+00$ & $\mathrm{mg} / \mathrm{h}$ \\
\hline LITHIUM & $9 / 26$ & $1.00 \mathrm{E}-03$ & $5.00 \mathrm{E}-02$ & L & $5.94 E-03$ & $1.63 \mathrm{E}-02$ & $\mathrm{mg} / \mathrm{L}$ \\
\hline MANGANESE & $105 / 108$ & $1.00 \mathrm{E}-03$ & $3.80 \mathrm{E}+00$ & $L$ & $1.16 \mathrm{E}+00$ & $3.29 \mathrm{E}+00$ & $\mathrm{mg} / \mathrm{L}$ \\
\hline NIOBIUM & $6 / 26$ & $7.10 \mathrm{E}-03$ & $1.60 \mathrm{E}-02$ & $\mathbb{L}$ & $5.11 E-03$ & $6.34 \mathrm{E}-03$ & $\mathrm{mg} / \mathrm{L}$ \\
\hline NITRATE & $107 / 108$ & $2.00 \mathrm{E}-01$ & $7.20 \mathrm{E}+01$ & $\mathbf{L}$ & $2.59 \mathrm{E}+01$ & $4.38 \mathrm{E}+01$ & $\mathrm{mg} / \mathrm{L}$ \\
\hline POTASSIUM & $108 / 108$ & $1.20 \mathrm{E}+00$ & $1.50 \mathrm{E}+02$ & $\mathbf{L}$ & $6.53 E+00$ & $7.82 E+00$ & $\mathrm{mg} / \mathrm{L}$ \\
\hline SODIUM & $108 / 108$ & $1.60 \mathrm{E}+00$ & $7.50 \mathrm{E}+01$ & $\mathbf{L}$ & $1.23 E+01$ & $1.43 E+01$ & $\mathrm{mg} / \mathrm{L}$ \\
\hline STRONTIUM & $108 / 108$ & $3.80 \mathrm{E}-02$ & $3.10 \mathrm{E}+00$ & $L$ & $3.56 \mathrm{E}-01$ & $4.15 \mathrm{E}-01$ & $\mathrm{mg} / \mathrm{L}$ \\
\hline SULFATE & $107 / 108$ & $2.00 \mathrm{E}+00$ & $1.65 \mathrm{E}+02$ & L & $7.33 E+01$ & $1.05 \mathrm{E}+02$ & $\mathrm{mg} / \mathrm{L}$ \\
\hline
\end{tabular}


Table C9. (continued)

\begin{tabular}{|c|c|c|c|c|c|c|c|}
\hline Analyte & $\begin{array}{l}\text { Frequency } \\
\text { of detect }\end{array}$ & $\begin{array}{l}\text { Minimum } \\
\text { detected conc. }\end{array}$ & $\begin{array}{l}\text { Maximum } \\
\text { detected conc. }\end{array}$ & Distribution & $\begin{array}{l}\text { Mean } \\
\text { concentration }\end{array}$ & $\begin{array}{l}\text { UCL9S } \\
\text { concentration }^{2}\end{array}$ & $\begin{array}{l}\text { Units of } \\
\text { measure }\end{array}$ \\
\hline URANIUM (SOLUBLE SALTS) & $70 / 108$ & $1.00 \mathrm{E}-03$ & $5.80 \mathrm{E}-02$ & $\mathbf{L}$ & $1.71 \mathrm{E}-03$ & $2.06 \mathrm{E}-03$ & $\mathrm{mg} / \mathrm{L}$ \\
\hline VANADIUM & $13 / 108$ & $5.40 \mathrm{E}-03$ & $1.60 \mathrm{E}-02$ & L & $3.08 \mathrm{E}-03$ & $3.32 \mathrm{E}-03$ & $\mathrm{mg} / \mathrm{L}$ \\
\hline ZIRCONIUM & $4 / 26$ & $1.40 \mathrm{E}-03$ & $1.00 \mathrm{E}-02$ & L & $2.47 \mathrm{E}-03$ & $4.13 \mathrm{E}-03$ & $\mathrm{mg} / \mathrm{L}$ \\
\hline \multicolumn{8}{|l|}{ Radionuclides } \\
\hline CESIUM-137 & $11 / 14$ & $2.26 \mathrm{E}-03$ & $4.94 E+01$ & L & $3.85 \mathrm{E}+01$ & $3.26 \mathrm{E}+04$ & $\mathrm{pCi} / \mathrm{L}$ \\
\hline STRONTIUM-90 & $5 / 14$ & $1.62 \mathrm{E}+00$ & $1.24 \mathrm{E}+02$ & $\mathrm{~L}$ & $1.84 \mathrm{E}+01$ & $1.03 E+02$ & $\mathrm{pCi} / \mathrm{L}$ \\
\hline TECHNETIUM-99 & $6 / 11$ & $1.31 E+02$ & $2.04 E+03$ & L & $5.19 \mathrm{E}+02$ & $2.04 \mathrm{E}+0.3$ & $\mathrm{pCi} / \mathrm{L}$ \\
\hline URANIUM-234 & $70 / 108$ & 3.37E-01 & $1.95 \mathbb{E}+01$ & $\mathrm{~L}$ & $5.77 \mathrm{E}-01$ & $6.95 \mathrm{E}-01$ & $\mathrm{pCi} / \mathrm{L}$ \\
\hline URANIUM-235 & $70 / 108$ & $1.54 \mathrm{E}-02$ & 8.91E-01 & $\mathbb{L}$ & $2.63 \mathrm{E}-02$ & $3.17 \mathrm{E}-02$ & $\mathrm{pCi} / \mathrm{L}$ \\
\hline URANIUM-238 & $70 / 108$ & 3.34E-01 & $1.94 \mathrm{E}+01$ & $\mathbb{L}$ & $5.72 \mathrm{E}-01$ & $6.89 E-01$ & $\mathrm{pCi} / \mathrm{L}$ \\
\hline \multicolumn{8}{|l|}{ Organics } \\
\hline 1,1,1-TRICHLOROETHANE & $24 / 82$ & $1.00 \mathrm{E}-103$ & $4.00 \mathrm{E}-03$ & $L$ & $2.45 \mathrm{E}-03$ & $2.53 \mathrm{E}-03$ & $\mathrm{mg} / \mathrm{L}$ \\
\hline 1,1-DICHLOROETHANE & $15 / 82$ & $1.00 E-03$ & $4.00 E_{-03}$ & $L$ & $2.58 \mathrm{E}-03$ & $2.66 \mathrm{E}-03$ & $\mathrm{mg} / \mathrm{L}$ \\
\hline 1,2-DICHLOROETHENE (TOTAL) & $52 / 82$ & $1.00 \mathrm{E}-03$ & $2.00 \mathrm{E}-01$ & $\mathbb{L}$ & $1.40 \mathrm{E}-02$ & $1.93 E-02$ & $\mathrm{mg} / \mathrm{L}$ \\
\hline 4-METHYL-2-PENTANONE & $10 / 82$ & $1.00 \mathrm{E}-03$ & $8.00 \mathrm{E}-03$ & $\mathbf{L}$ & $4.83 E-03$ & $5.14 E-03$ & $\mathrm{mg} / \mathrm{L}$ \\
\hline ACETONE & $12 / 82$ & $3.00 \mathrm{E}-03$ & $4.00 \mathrm{E}-02$ & $\mathcal{L}$ & $5.92 E-03$ & $6.40 \mathrm{E}-03$ & $\mathrm{mg} / \mathrm{L}$ \\
\hline BROMODICHLOROMETHANE & $1 / 82$ & $7.00 E-04$ & $7.00 \mathrm{E}-04$ & $L$ & $2.49 \mathrm{E}-03$ & $2.55 \mathrm{E}-03$ & $\mathrm{mg} / \mathrm{l}$ \\
\hline CARBON TETRACHLORIDE & $35 / 82$ & $9.00 \mathrm{E}-04$ & $4.00 \mathrm{E}-03$ & $\mathbb{L}$ & $2.55 \mathrm{E}-03$ & $2.73 E-03$ & $\mathrm{mg} / \mathrm{L}$ \\
\hline CHLOROFORM & $54 / 82$ & $5.00 \mathrm{E}-04$ & $6.00 \mathrm{E}-03$ & $\mathbb{L}$ & $2.74 \mathrm{E}-03$ & $3.19 \mathrm{E}-03$ & $\mathrm{mg} / \mathrm{L}$ \\
\hline TRICHLOROETHENE & $82 / 82$ & $3.00 \mathrm{E}-03$ & $1.20 \mathrm{E}-01$ & $\mathbb{L}$ & $4.36 \mathrm{E}-02$ & $5.21 E-02$ & $\mathrm{mg} / \mathrm{L}$ \\
\hline
\end{tabular}


Table C.9. (continued)

\begin{tabular}{|c|c|c|c|c|c|c|c|}
\hline Analyte & $\begin{array}{l}\text { Frequency } \\
\text { of detect }\end{array}$ & $\begin{array}{l}\text { Minimum } \\
\text { detected conc. }\end{array}$ & $\begin{array}{l}\text { Maximum } \\
\text { detected conc. }\end{array}$ & Distribution & $\begin{array}{l}\text { Mean } \\
\text { concentration }\end{array}$ & $\begin{array}{l}\text { UCL95 } \\
\text { concentration" }^{8}\end{array}$ & $\begin{array}{l}\text { Units of } \\
\text { measure }\end{array}$ \\
\hline VINYL ACETATE & $1 / 82$ & $2.00 \mathrm{E}-03$ & $2.00 \mathrm{E}-03$ & $\mathbf{L}$ & $4.97 \mathrm{E}-03$ & $5.07 \mathrm{E}-03$ & $\mathrm{mg} / \mathrm{L}$ \\
\hline \multicolumn{8}{|c|}{$\$ Y-200$} \\
\hline \multicolumn{8}{|l|}{ Inorganics } \\
\hline ALUMINUM & $4 / 4$ & $3.13 \mathrm{E}+00$ & $1.41 E+01$ & $\mathbf{L}$ & $8.52 E+\infty 0$ & $7.28 \mathrm{E}+02$ & $\mathrm{mg} / \mathrm{L}$ \\
\hline ANTIMONY & $1 / 4$ & $2.00 \mathrm{E}-03$ & $2.00 \mathrm{E}-03$ & L & $1.17 \mathrm{E}-03$ & $3.96 \mathrm{E}-03$ & $\mathrm{mg} / \mathrm{L}$ \\
\hline BARIUM & $4 / 4$ & $1.09 \mathrm{E}-01$ & $5.50 \mathrm{E}-01$ & $\mathbf{L}$ & $3.32 E-01$ & $1.39 \mathrm{E}+02$ & $\mathrm{mg} / \mathrm{L}$ \\
\hline CALCIUM & $4 / 4$ & $1.30 \mathrm{E}+02$ & $3.55 \mathrm{E}+02$ & L & $2.47 \mathrm{E}+02$ & $2.60 \mathrm{E}+03$ & $\mathrm{mg} / \mathrm{L}$ \\
\hline CHLORIDE & $2 / 4$ & $4.60 \mathrm{E}+02$ & $4.87 E+02$ & $\mathbb{L}$ & $4.90 \mathrm{E}+02$ & $3.03 E+56$ & $\mathrm{mg} / \mathrm{L}$ \\
\hline CHROMIUM & $4 / 4$ & $6.80 \mathrm{E}-03$ & $1.87 \mathrm{E}-02$ & $\mathbf{L}$ & $1.55 \mathrm{E}-02$ & $1.00 \mathrm{E}-01$ & $\mathrm{mg} / \mathrm{L}$ \\
\hline COBALT & $2 / 4$ & $6.04 \mathrm{E}-02$ & $6.55 \mathrm{E}-02$ & L & $3.61 \mathrm{E}-02$ & $2.01 E+08$ & $\mathrm{mg} / \mathrm{L}$ \\
\hline COPPER & $3 / 4$ & $1.46 \mathrm{E}-02$ & $1.65 \mathrm{E}-02$ & $\mathbf{L}$ & $1.30 \mathrm{E}-02$ & $1.34 E+01$ & $\mathrm{mg} / \mathrm{L}$ \\
\hline CYANIDE & $1 / 4$ & $1.37 \mathrm{E}-02$ & $1.37 \mathrm{E}-02$ & $L$ & $7.06 \mathrm{E}-03$ & $7.08 \mathrm{E}-02$ & $\mathrm{mg} / \mathrm{L}$ \\
\hline FLUORIDE & $2 / 4$ & $1.00 \mathrm{E}-01$ & $1.30 \mathrm{E}-01$ & L & $8.24 \mathrm{E}-02$ & $5.30 \mathrm{E}-01$ & $\mathrm{mg} / \mathrm{L}$ \\
\hline IRON & $4 / 4$ & $3.77 \mathrm{E}+00$ & $1.51 E+01$ & L & $1.02 E+01$ & $3.37 \mathrm{E}+02$ & $\mathrm{mg} / \mathrm{L}$ \\
\hline LEAD & $4 / 4$ & $8.30 \mathrm{E}-03$ & $2.94 E-02$ & L & $1.93 E-02$ & $7.52 \mathrm{E}-01$ & $\mathrm{mg} / \mathrm{L}$ \\
\hline MAGNESIUM & $4 / 4$ & $1.79 \mathrm{E}+01$ & $4.39 \mathrm{E}+01$ & L & $3.17 E+01$ & $1.79 E+02$ & $\mathrm{mg} /$ \\
\hline MANGANESE & $4 / 4$ & $2.07 \mathrm{E}+00$ & $5.67 \mathrm{E}+\infty$ & $\mathbf{N}$ & $4.51 E+00$ & $6.47 \mathrm{E}+00$ & $\mathrm{mg} / \mathrm{L}$ \\
\hline MERCURY & $3 / 4$ & $6.80 \mathrm{E}-04$ & $1.50 \mathrm{E}-03$ & L & $8.64 \mathrm{E}-04$ & $6.80 \mathrm{E}+01$ & $\mathrm{mg} / \mathrm{L}$ \\
\hline NICKEL & $3 / 4$ & $1.49 \mathrm{E}-02$ & $1.69 \mathrm{E}-02$ & $L$ & $1.37 \mathrm{E}-02$ & $2.15 E+01$ & $\mathrm{mg} / \mathrm{L}$ \\
\hline POTASSIUM & $4 / 4$ & $4.12 \mathrm{E}+00$ & $1.29 E+01$ & $L$ & $8.51 E+\infty 0$ & $2.71 E+02$ & $\mathrm{mg} / \mathrm{L}$ \\
\hline SODIUM & $4 / 4$ & $7.47 \mathrm{E}+00$ & $1.40 \mathrm{E}+02$ & $L$ & $8.20 \mathrm{E}+01$ & $1.55 \mathrm{E}+09$ & $\mathrm{mg} / \mathrm{L}$ \\
\hline
\end{tabular}


Table C9. (continued)

\begin{tabular}{|c|c|c|c|c|c|c|c|}
\hline Analyte & $\begin{array}{l}\text { Frequency } \\
\text { of detect }\end{array}$ & $\begin{array}{l}\text { Minimum } \\
\text { detected conc. }\end{array}$ & $\begin{array}{l}\text { Maximum } \\
\text { detected conc. }\end{array}$ & Distribution & $\begin{array}{l}\text { Mean } \\
\text { concentration }\end{array}$ & $\begin{array}{l}\text { UCL95 } \\
\text { concentration" }\end{array}$ & $\begin{array}{c}\text { Units of } \\
\text { measure }\end{array}$ \\
\hline SULFATE & $3 / 4$ & $8.70 \mathrm{E}+00$ & $2.64 \mathrm{E}+01$ & L & $1.34 \mathrm{E}+01$ & $2.80 \mathrm{E}+04$ & $\mathrm{mg} / \mathrm{L}$ \\
\hline VANADIUM & $4 / 4$ & $7.30 \mathrm{E}-03$ & $2.66 \mathrm{E}-02$ & L & $1.82 \mathrm{E}-02$ & $5.45 \mathrm{E}-01$ & $\mathrm{mg} / \mathrm{L}$ \\
\hline ZINC & $4 / 4$ & $3.40 \mathrm{E}-02$ & $5.26 \mathrm{E}-02$ & L & $4.26 \mathrm{E}-02$ & $6.97 \mathrm{E}-02$ & $\mathrm{mg} / \mathrm{L}$ \\
\hline \multicolumn{8}{|l|}{ Radionuclides } \\
\hline URANIUM-234 & $4 / 4$ & 4.47E-01 & $7.38 \mathrm{E}+00$ & L & $2.02 E+\infty 0$ & $5.45 \mathrm{E}+06$ & $\mathrm{pCi} / \mathrm{L}$ \\
\hline URANIUM-235 & $4 / 4$ & $2.20 \mathrm{E}-02$ & $4.29 \mathrm{E}-01$ & L & $2.26 \mathrm{E}-01$ & $8.97 \mathrm{E}+08$ & $\mathrm{pCi} / \mathrm{L}$ \\
\hline URANIUM-238 & $4 / 4$ & $1.19 \mathrm{E}-01$ & $8.58 \mathrm{E}+00$ & L & $1.72 E+00$ & $4.84 E+12$ & $\mathrm{pCi} / \mathrm{L}$ \\
\hline \multicolumn{8}{|l|}{ Organics } \\
\hline BENZYL BUTYL PHTHALATE & $1 / 4$ & $2.00 \mathrm{E}-03$ & $2.00 \mathrm{E}-03$ & L & $4.30 \mathrm{E}-03$ & $2.46 \mathrm{E}-02$ & $\mathrm{mg} / \mathrm{L}$ \\
\hline BIS(2-ETHYLHEXYL)PHTHALAATE & $4 / 4$ & $1.00 \mathrm{E}-03$ & $4.00 \mathrm{E}-03$ & L & $2.25 \mathrm{E}-03$ & $3.01 \mathrm{E}-02$ & $\mathrm{mg} / \mathrm{L}$ \\
\hline CHLOROFORM & $1 / 4$ & $1.00 \mathrm{E}-03$ & $1.00 \mathrm{E}-03$ & $\mathrm{~L}$ & $2.15 \mathrm{E}-03$ & $1.23 \mathrm{E}-02$ & $\mathrm{mg} / \mathrm{L}$ \\
\hline DI-N-BUTYL PHTHALATE & $2 / 4$ & $1.00 \mathrm{E}-03$ & $3.00 \mathrm{E}-03$ & L & $3.62 \mathrm{E}-03$ & $3.79 \mathrm{E}-01$ & $\mathrm{mg} / \mathrm{L}$ \\
\hline DIETHYYL PHTHALATE & $1 / 4$ & $2.00 \mathrm{E}-03$ & $2.00 \mathrm{E}-03$ & L & $4.30 \mathrm{E}-03$ & $2.46 \mathrm{E}-02$ & $\mathrm{mg} / \mathrm{L}$ \\
\hline TETRACHLOROETHENE & $3 / 4$ & $2.00 \mathrm{E}-03$ & $3.00 \mathrm{E}-03$ & L & $2.37 \mathrm{E}-03$ & $3.46 \mathrm{E}-03$ & $\mathrm{mg} / \mathrm{L}$ \\
\hline TRICHLOROETHENE & $1 / 4$ & $2.00 \mathrm{E}-03$ & $2.00 \mathrm{E}-03$ & L & $2.38 \mathrm{E}-03$ & $2.94 \mathrm{E}-03$ & $\mathrm{mg} / \mathrm{L}$ \\
\hline & & & SA-1 & & & & \\
\hline \multicolumn{8}{|l|}{ Inorganics } \\
\hline ALUMINUM & $66 / 85$ & 2.10E-02 & $9.37 E+01$ & L & $1.28 \mathrm{E}-01$ & 2.07E-01 & $\mathrm{mg} / \mathrm{L}$ \\
\hline ANTIMONY & $1 / 85$ & $1.90 \mathrm{E}-03$ & $1.90 \mathrm{E}-03$ & L & $2.58 \mathrm{E}-02$ & $2.84 \mathrm{E}-02$ & $\mathrm{mg} / \mathrm{L}$ \\
\hline
\end{tabular}


Table C9. (comtinued)

\begin{tabular}{|c|c|c|c|c|c|c|c|}
\hline Analyte & $\begin{array}{l}\text { Frequency } \\
\text { of detect }\end{array}$ & $\begin{array}{l}\text { Minimum } \\
\text { detected conc. }\end{array}$ & $\begin{array}{l}\text { Maximum } \\
\text { delected conc. }\end{array}$ & Distribution & $\begin{array}{l}\text { Mean } \\
\text { concentration }\end{array}$ & $\begin{array}{l}\text { UCL95 } \\
\text { concentration" }\end{array}$ & $\begin{array}{l}\text { Units of } \\
\text { measure }\end{array}$ \\
\hline BARIUM & $85 / 85$ & $1.20 \mathrm{E}-02$ & $4.00 \mathrm{E}-01$ & $\mathbf{L}$ & $4.38 \mathrm{E}-02$ & 5.17E-02 & $\mathrm{mg} / \mathrm{L}$ \\
\hline BERYLLIUM & $6 / 85$ & $3.10 \mathrm{E}-04$ & $5.90 \mathrm{E} \cdot 03$ & $\mathbf{L}$ & $2.25 \mathrm{E}-04$ & $2.63 \mathrm{E}-04$ & $\mathrm{mg} / \mathrm{l}$ \\
\hline CADMIUM & $9 / 85$ & $3.00 \mathrm{E}-03$ & $9.60 \mathrm{E}-03$ & $\mathbf{L}$ & $1.81 \mathrm{E}-03$ & $1.98 \mathrm{E}-03$ & $\mathrm{mg} / \mathrm{L}$ \\
\hline CALCIUM & $85 / 85$ & $2.14 E+01$ & $1.20 E+02$ & $\mathbf{L}$ & $8.21 E+01$ & $8.88 \mathrm{E}+01$ & $\mathrm{mg} / \mathrm{L}$ \\
\hline CHLORIDE & $84 / 85$ & $1.00 E+00$ & $1.83 \mathrm{E}+01$ & $\mathrm{~L}$ & $5.28 \mathrm{E}+00$ & $6.36 \mathrm{E}+00$ & $\mathrm{mg} / \mathrm{L}$ \\
\hline COBALT & $2 / 85$ & $1.44 \mathrm{E}-02$ & $6.35 \mathrm{E}-02$ & $\mathbf{L}$ & $2.81 \mathrm{E}-03$ & $3.06 \mathrm{E}-03$ & $\mathrm{mg} / \mathrm{L}$ \\
\hline FLUORIDE & $1 / 2$ & $1.00 \mathrm{E}-01$ & $1.00 \mathrm{E}-01$ & $\mathbf{L}$ & $7.50 \mathrm{E}-02$ & & $\mathrm{mg} / \mathrm{L}$ \\
\hline LEAD & $2 / 6$ & $5.59 \mathrm{E}-02$ & $2.40 \mathrm{E}-01$ & L & $5.82 \mathrm{E}-02$ & $4.73 \mathrm{E}-01$ & $\mathrm{mg} / \mathrm{L}$ \\
\hline MANGANESE & $70 / 85$ & $1.20 \mathrm{E}-03$ & $3.93 E+00$ & L & $2.76 \mathrm{E}-02$ & $5.88 \mathrm{E}-02$ & $\mathrm{mg} / \mathrm{L}$ \\
\hline MERCURY & $1 / 2$ & $7.30 \mathrm{E}-04$ & $7.30 \mathrm{E}-04$ & $\mathrm{~L}$ & $4.15 \mathrm{E}-04$ & & $\mathrm{mg} / \mathrm{L}$ \\
\hline NIOBIUM & $2 / 18$ & $1.10 \mathrm{E}-02$ & $1.70 E-02$ & $\mathbf{L}$ & $4.47 \mathrm{E}-03$ & 5.63E-03 & $\mathrm{mg} / \mathrm{L}$ \\
\hline NITRATE & $81 / 83$ & $1.60 \mathrm{E}-01$ & $3.41 E+01$ & $\mathbf{L}$ & $1.27 \mathrm{E}+01$ & $2.62 \mathrm{E}+01$ & $\mathrm{mg} / \mathrm{L}$ \\
\hline POTASSIUM & $85 / 85$ & $1.10 \mathrm{E}+00$ & $1.83 \mathrm{E}+01$ & L & $3.15 E+00$ & $3.64 E+00$ & $\mathrm{mg} / \mathrm{L}$ \\
\hline STRONTIUM & $83 / 83$ & $2.30 \mathrm{E}-02$ & $2.00 \mathrm{E}-01$ & L & $9.10 \mathrm{E}-02$ & $1.07 \mathrm{E}-01$ & $\mathrm{mg} / \mathrm{L}$ \\
\hline SULFATE & $83 / 85$ & $1.60 \mathrm{E}+00$ & $3.98 \mathrm{E}+01$ & $\mathbf{L}$ & $9.27 E+00$ & $1.19 \mathrm{E}+01$ & $\mathrm{mg} / \mathrm{h}$ \\
\hline VANADIUM & $2 / 85$ & $7.91 E-02$ & $2.01 \mathrm{E}-01$ & $L$ & $3.22 \mathrm{E}-03$ & $3.69 \mathrm{E}-03$ & $\mathrm{mg} / \mathrm{L}$ \\
\hline ZIRCONIUM & $1 / 18$ & $1.70 E-02$ & $1.70 \mathrm{E}-02$ & $\mathbb{L}$ & $2.04 \mathrm{E}-03$ & $4.70 \mathrm{E}-03$ & $\mathrm{mg} / \mathrm{L}$ \\
\hline \multicolumn{8}{|l|}{ Radionuclides } \\
\hline URANIUM-234 & $2 / 2$ & $1.35 E+\infty$ & $2.51 E+\infty$ & $L$ & $1.93 \mathrm{E}+00$ & & $\mathrm{pCi} / \mathrm{L}$ \\
\hline URANIUM-235 & $2 / 2$ & $1.57 \mathrm{E}-02$ & $4.10 \mathrm{E}-02$ & $\mathbf{L}$ & $2.83 \mathrm{E}-02$ & & $\mathrm{pCi} / \mathrm{L}$ \\
\hline URANIUM-238 & $2 / 2$ & $9.73 \mathrm{E}-01$ & $1.70 \mathrm{E}+100$ & $\mathbb{L}$ & $1.34 E+00$ & & $\mathrm{pCi} / \mathrm{L}$ \\
\hline
\end{tabular}


Table C.9. (continued)

\begin{tabular}{|c|c|c|c|c|c|c|c|}
\hline Analyie & $\begin{array}{l}\text { Frequency } \\
\text { of detect }\end{array}$ & $\begin{array}{l}\text { Minimum } \\
\text { detected conc. }\end{array}$ & $\begin{array}{l}\text { Maximum } \\
\text { detecied conc. }\end{array}$ & Distribution & $\begin{array}{l}\text { Mean } \\
\text { concentration }\end{array}$ & $\begin{array}{l}\text { UCL95 } \\
\text { concentration }^{\mathrm{a}}\end{array}$ & $\begin{array}{l}\text { Units of } \\
\text { measure }\end{array}$ \\
\hline \multicolumn{8}{|l|}{ Organics } \\
\hline $\begin{array}{l}\text { 1,1,2-TRICHLORO- } \\
\text { 1,2,2-TRIFLUOROETHANE }\end{array}$ & $1 / 2$ & $3.00 \mathrm{E}-03$ & $3.00 \mathrm{E}-03$ & $\mathbf{L}$ & $4.00 \mathrm{E}-03$ & & $\mathrm{mg} / \mathrm{l}$ \\
\hline 1,2-DICHLOROETIENE (TOTAL) & $43 / 67$ & $2.00 \mathrm{E}-03$ & $1.50 \mathrm{E}-01$ & $\mathbf{L}$ & $1.09 \mathrm{E}-02$ & $1.56 \mathrm{E}-02$ & $\mathrm{mg} / \mathrm{L}$ \\
\hline 4-METHYL-2-PENTANONE & $6 / 67$ & $1.00 \mathrm{E}-03$ & $4.00 \mathrm{E}-03$ & $\mathbf{L}$ & 4.82E-03 & $5.23 \mathrm{E}-03$ & $\mathrm{mg} / \mathrm{l}$ \\
\hline ACETONE & $10 / 67$ & 1.00E-03 & $8.10 E-02$ & $L$ & $5.89 \mathrm{E}-03$ & $6.74 E-03$ & $\mathrm{mg} / \mathrm{L}$ \\
\hline BIS(2-ETHYLHEXYL)PHTHALATE & $2 / 2$ & $1.00 \mathrm{E}-03$ & $2.00 \mathrm{E}-03$ & L & $1.50 \mathrm{E}-03$ & & $\mathrm{mg} / \mathrm{L}$ \\
\hline BROMODICHLOROMETHANE & $7 / 67$ & $1.00 \mathrm{E}-03$ & $4.00 \mathrm{E}-03$ & $\mathbb{L}$ & $2.48 \mathrm{E}-03$ & $2.58 \mathrm{E}-03$ & $\mathrm{mg} / \mathrm{L}$ \\
\hline CARBON DISULFIDE & $1 / 67$ & $2.00 \mathrm{E}-03$ & $2.00 \mathrm{E}-03$ & $\mathbb{L}$ & $2.49 \mathrm{E}-03$ & $2.51 \mathrm{E}-03$ & $\mathrm{mg} / \mathrm{L}$ \\
\hline CHLOROFORM & $10 / 67$ & 7.00E-04 & $1.10 \mathrm{E}-02$ & L & $2.98 \mathrm{E}-03$ & 3.31E-03 & $\mathrm{mg} / \mathrm{l}$ \\
\hline METHYLENE CHLORIDE & $9 / 67$ & $9.00 \mathrm{E}-04$ & $1.70 \mathrm{E}-02$ & $\mathbf{L}$ & $2.87 \mathrm{E}-03$ & $3.18 \mathrm{E}-03$ & $\mathrm{mg} / \mathrm{L}$ \\
\hline TETRACHLOROETHENE & $47 / 67$ & $8.00 \mathrm{E}-04$ & $5.50 \mathrm{E}-02$ & $\mathbf{L}$ & $1.15 \mathrm{E}-02$ & $1.52 \mathrm{E}-02$ & $\mathrm{mg} / \mathrm{L}$ \\
\hline TRICHLOROETHENE & $46 / 67$ & $1.00 \mathrm{E}-03$ & $2.20 \mathrm{E}-02$ & $\mathbf{L}$ & $5.27 \mathrm{E}-03$ & $6.30 \mathrm{E}-03$ & $\mathrm{mg} / \mathrm{L}$ \\
\hline VINYL ACETATE & $1 / 65$ & $2.00 \mathrm{E}-03$ & $2.00 \mathrm{E}-03$ & $\mathbf{L}$ & $4.96 \mathrm{E}-03$ & $5.08 \mathrm{E}-03$ & $\mathrm{mg} / \mathrm{L}$ \\
\hline
\end{tabular}

$L=L$ Lognormal Distribution; $N=$ Normal Distribution; UCL.95 = Upper 95\% confidence limit on the mean concentration.

- The UCL95 concentration was calculated for those analytes which were analyzed in at least three samples. 
Table C.10a. Toxicity screening for BCV OU2 detected inorganic and organic analytes in groundwater (carcinogemic and noncarcimogenic; ingestion)

\begin{tabular}{|c|c|c|c|c|c|c|c|c|}
\hline Analyte & $\begin{array}{l}\text { Maximum } \\
\text { detected } \\
\text { conc:; } \\
\mathrm{mg} / \mathrm{L}\end{array}$ & $\begin{array}{l}\text { Oral } \\
\mathrm{RID} ; \\
\mathrm{mg} / \mathrm{kg}-\mathrm{d}\end{array}$ & $\begin{array}{l}\text { Oral } \\
\mathrm{SF} ; \\
\mathrm{sg}-\mathrm{d} / \mathrm{mg}\end{array}$ & $\begin{array}{l}\text { Noncarc. } \\
\text { oral } \\
\text { toxicity } \\
\text { score }\end{array}$ & $\begin{array}{l}\text { \% of } \\
\text { Total } \\
\text { noncarc. } \\
\text { oral } \\
\text { score }\end{array}$ & $\begin{array}{l}\text { Carcin. } \\
\text { oral } \\
\text { toxicity } \\
\text { score }\end{array}$ & $\begin{array}{l}\% \text { of } \\
\text { Total } \\
\text { carcin. } \\
\text { oral } \\
\text { score }\end{array}$ & $\begin{array}{l}\text { Final } \\
\text { decision }\end{array}$ \\
\hline \multicolumn{9}{|c|}{ RSA } \\
\hline \multicolumn{9}{|l|}{ Inorganics } \\
\hline ANTIMONY & $2.00 \mathrm{E}-01$ & 4.0E-04 & & $5.00 E+02$ & 29.36 & & & \\
\hline ARSENIC & $8.50 \mathrm{E}-02$ & $3.0 \mathrm{E}-04$ & & $2.83 E+02$ & 16.64 & & & \\
\hline BARIUM & $2.30 \mathrm{E}-01$ & $7.0 \mathrm{E}-02$ & & $3.29 \mathrm{E}+00$ & 0.19 & & & DROP \\
\hline BERYLLIUM & $1.30 \mathrm{E}-03$ & $5.0 \mathrm{E}-03$ & $4.3 \mathrm{E}+00$ & $2.60 \mathrm{E}-01$ & 0.02 & $5.59 \mathrm{E}-03$ & 90.31 & \\
\hline BORON & $1.20 \mathrm{E}+00$ & $9.0 \mathrm{E}-02$ & & $1.33 E+01$ & 0.78 & & & DROP \\
\hline CADMIUM & 2.10E-02 & $5.0 \mathrm{E}-04$ & & $4.20 \mathrm{E}+01$ & 2.47 & & & \\
\hline MANGANESE & $3.80 \mathrm{E}+00$ & $5.0 \mathrm{E}-03$ & & $7.60 \mathrm{E}+02$ & 44.62 & & & \\
\hline NITRATE & $7.20 \mathrm{E}+01$ & $1.6 \mathrm{E}+00$ & & $4.50 \mathrm{E}+01$ & 2.64 & & & \\
\hline STRONTIUM & $3.10 \mathrm{E}+\infty \mathrm{O}$ & $6.0 \mathrm{E}-01$ & & $5.17 \mathrm{E}+00$ & 0.30 & & & DROP \\
\hline URANIUM (SOLUBLE SALTS) & $5.80 \mathrm{E}-02$ & $3.0 \mathrm{E}-03$ & & $1.93 E+01$ & 1.14 & & & \\
\hline VANADIUM & $1.60 \mathrm{E}-02$ & $7.0 \mathrm{E}-03$ & & $2.29 \mathrm{E}+00$ & 0.13 & & & DROP \\
\hline \multicolumn{9}{|l|}{ Organics } \\
\hline 1,1-DICHLOROETHANE & $4.00 \mathrm{E}-03$ & $1.0 \mathrm{E}-01$ & & $4.00 \mathrm{E}-02$ & 0.00 & & & DROP \\
\hline 1,2-DICHLOROETHENE (TOTAL) & $2.00 \mathrm{E}-01$ & $9.0 \mathrm{E}-03$ & & $2.22 \mathrm{E}+01$ & 1.30 & & & \\
\hline 4-METHYL-2-PENTANONE & $8.00 \mathrm{E}-03$ & 8.0E-02 & & $1.00 \mathrm{E}-01$ & 0.01 & & & DROP \\
\hline ACETONE & $4.00 \mathrm{E}-02$ & $1.0 \mathrm{E}-01$ & & $4.00 \mathrm{E}-01$ & 0.02 & & & DROP \\
\hline BROMODICHLOROMETHANE & $7.00 \mathrm{E}-04$ & $2.0 \mathrm{E}-02$ & $6.2 \mathrm{E}-02$ & $3.50 \mathrm{E}-02$ & 0.00 & 4.34E-05 & 0.70 & DROP \\
\hline
\end{tabular}


Table C.10a (continued)

\begin{tabular}{|c|c|c|c|c|c|c|c|c|}
\hline Analyte & $\begin{array}{l}\text { Maximum } \\
\text { detected } \\
\text { conc; } \\
\text { mg/L }\end{array}$ & $\begin{array}{l}\text { Oral } \\
\text { RfD; } \\
\text { mg/kg-d }\end{array}$ & $\begin{array}{l}\text { Oral } \\
\mathrm{SF} ; \\
\mathrm{kg}-\mathrm{d} / \mathrm{mg}\end{array}$ & $\begin{array}{l}\text { Noncarc. } \\
\text { oral } \\
\text { toxicity } \\
\text { score }\end{array}$ & $\begin{array}{l}\text { \% of } \\
\text { Total } \\
\text { noncarc. } \\
\text { oral } \\
\text { score }\end{array}$ & $\begin{array}{l}\text { Carcin. } \\
\text { oral } \\
\text { toxicity } \\
\text { score }\end{array}$ & $\begin{array}{l}\% \text { of } \\
\text { Total } \\
\text { carcin. } \\
\text { oral } \\
\text { score }\end{array}$ & $\begin{array}{l}\text { Final } \\
\text { decision }\end{array}$ \\
\hline CARBON TETRACHLORIDE & $4.00 \mathrm{E}-03$ & $7.0 \mathrm{E}-04$ & $1.3 \mathrm{E}-01$ & $5.71 E+\infty 0$ & 0.34 & $5.20 \mathrm{E}-04$ & 8.40 & \\
\hline CHLOROFORM & $6.00 \mathrm{E}-03$ & $1.0 \mathrm{E}-02$ & $6.1 \mathrm{E}-03$ & $6.00 \mathrm{E}-01$ & 0.04 & $3.66 \mathrm{E}-05$ & 0.59 & DROP \\
\hline VINYL ACETATE & $2.00 \mathrm{E}-03$ & $1.0 \mathrm{E}+00$ & & $2.00 \mathrm{E}-03$ & 0.00 & & & DROP \\
\hline Toxicity Score Totals & & & & $1.70 \mathrm{E}+03$ & 100.0 & $6.19 \mathrm{E}-03$ & 100.0 & \\
\hline & & & SY-200 & & & & & \\
\hline \multicolumn{9}{|l|}{ Inorganics } \\
\hline ANTIMONY & $2.00 \mathrm{E}-03$ & $4.0 \mathrm{E}-04$ & & $5.00 E+00$ & 0.43 & & & DROP \\
\hline BARIUM & $5.50 \mathrm{E}-01$ & $7.0 \mathrm{E}-02$ & & $7.86 \mathrm{E}+\infty$ & 0.68 & & & DROP \\
\hline CYANIDE & $1.37 \mathrm{E}-02$ & $2.0 \mathrm{E}-02$ & & $6.85 \mathrm{E}-01$ & 0.06 & & & DROP \\
\hline FLUORIDE & $1.30 \mathrm{E}-01$ & $6.0 \mathrm{E}-02$ & & $2.17 E+00$ & 0.19 & & & DROP \\
\hline MANGANESE & $5.67 E+\infty 0$ & $5.0 \mathrm{E}-03$ & & $1.13 E+03$ & 97.74 & & & \\
\hline MERCURY & $1.50 \mathrm{E}-03$ & $3.0 \mathrm{E}-04$ & & $5.00 \mathrm{E}+00$ & 0.43 & & & DROP \\
\hline NICKEL & $1.69 \mathrm{E}-02$ & $2.0 \mathrm{E}-02$ & & 8.45E-01 & 0.07 & & & DROP \\
\hline VANADIUM & $2.66 \mathrm{E}-02$ & 7.0E-03 & & $3.80 \mathrm{E}+00$ & 0.33 & & & DROP \\
\hline ZINC & $5.26 \mathrm{E}-02$ & $3.0 \mathrm{E}-01$ & & $1.75 E-01$ & 0.02 & & & DROP \\
\hline \multicolumn{9}{|l|}{ Organics } \\
\hline BENZYL BUTYL PHTHALATE & $2.00 E-03$ & $2.0 \mathrm{E}-01$ & & $1.00 \mathrm{E}-02$ & 0.00 & & & DROP \\
\hline
\end{tabular}


Table C.10a. (continued)

\begin{tabular}{|c|c|c|c|c|c|c|c|c|}
\hline Analyte & $\begin{array}{l}\text { Maximum } \\
\text { detected } \\
\text { conc.; } \\
\text { mg/ }\end{array}$ & $\begin{array}{l}\text { Oral } \\
\text { RED; } \\
\text { mg/kg-d }\end{array}$ & $\begin{array}{l}\text { Oral } \\
\text { SF; } \\
\mathrm{kg} \cdot d / \mathrm{d} / \mathrm{mg}\end{array}$ & $\begin{array}{l}\text { Noncarc. } \\
\text { oral } \\
\text { toxicity } \\
\text { score }\end{array}$ & $\begin{array}{l}\text { \% of } \\
\text { Total } \\
\text { noncarc. } \\
\text { oral } \\
\text { score }\end{array}$ & $\begin{array}{l}\text { Carcin. } \\
\text { oral } \\
\text { toxicity } \\
\text { score }\end{array}$ & $\begin{array}{l}\% \text { of } \\
\text { Total } \\
\text { carcin. } \\
\text { oral } \\
\text { score }\end{array}$ & $\begin{array}{l}\text { Final } \\
\text { decision" }\end{array}$ \\
\hline BIS(2-ETHYLHEXYL)PHTHALATE & $4.00 \mathrm{E}-03$ & $2.0 \mathrm{E}-02$ & $1.4 \mathrm{E}-02$ & $2.00 \mathrm{E}-01$ & 0.02 & $5.60 \mathrm{E}-05$ & 90.18 & \\
\hline CHLOROFORM & $1.00 \mathrm{E}-03$ & $1.0 \mathrm{E}-02$ & $6.1 \mathrm{E}-03$ & $1.00 \mathrm{E}-01$ & 0.01 & $6.10 \mathrm{E}-06$ & 9.82 & \\
\hline DI-N-BUTYL PHTHALATE & $3.00 \mathrm{E}-03$ & $1.0 \mathrm{E}-01$ & & $3.00 \mathrm{E}-02$ & 0.00 & & & DROP \\
\hline DIETHYL PHTHALATE & $2.00 \mathrm{E}-03$ & $8.0 \mathrm{E}-01$ & & $2.50 \mathrm{E}-03$ & 0.00 & & & DROP \\
\hline TETRACHLOROETHENE & $3.00 \mathrm{E}-03$ & $1.0 \mathrm{E}-02$ & & $3.00 \mathrm{E}-01$ & 0.03 & & & DROP \\
\hline Toxicity Score Totals & & & & $1.16 \mathrm{E}+03$ & 100.0 & $6.21 \mathrm{E}-05$ & 100.0 & \\
\hline & & & SA-1 & & & & & \\
\hline \multicolumn{9}{|l|}{ Inorganics } \\
\hline ANTIMONY & $1.90 \mathrm{E}-03$ & $4.0 \mathrm{E}-04$ & & $4.75 E+00$ & 0.53 & & & DROP \\
\hline BARIUM & $4.00 \mathrm{E}-01$ & $7.0 \mathrm{E}-02$ & & $5.71 \mathrm{E}+00$ & 0.64 & & & DROP \\
\hline BERYLLIUM & $5.90 \mathrm{E}-03$ & $5.0 \mathrm{E}-03$ & $4.3 E+00$ & $1.18 \mathrm{E}+00$ & 0.13 & 2.54E-02 & 98.18 & \\
\hline CADMIUM & $9.60 \mathrm{E}-03$ & $5.0 \mathrm{E}-04$ & & $1.92 \mathrm{E}+01$ & 2.14 & & & \\
\hline FLUORIDE & $1.00 \mathrm{E}-01$ & $6.0 \mathrm{E}-02$ & & $1.67 E+00$ & 0.19 & & & DROP \\
\hline MANGANESE & $3.93 E+00$ & $5.0 \mathrm{E}-03$ & & $7.86 \mathrm{E}+02$ & 87.72 & & & \\
\hline MERCURY & 7.30E-04 & 3.0E-04 & & $2.43 E+\infty 0$ & 0.27 & & & DROP \\
\hline NITRATE & $3.41 \mathrm{E}+.01$ & $1.6 \mathrm{E}+00$ & & $2.13 \mathrm{E}+01$ & 2.38 & & & \\
\hline STRONTIUM & $2.00 \mathrm{E}-01$ & $6.0 \mathrm{E}-01$ & & $3.33 \mathrm{E}-01$ & 0.04 & & & DROP \\
\hline VANADIUM & 2.01E-01 & $7.0 \mathrm{E}-03$ & & $2.87 E+01$ & 3.20 & & & \\
\hline
\end{tabular}


Table C.10a. (continued)

\begin{tabular}{|c|c|c|c|c|c|c|c|c|}
\hline Analyte & $\begin{array}{l}\text { Maximum } \\
\text { detected } \\
\text { conc; } \\
\text { mg } / \mathrm{L}\end{array}$ & $\begin{array}{l}\text { Oral } \\
\mathrm{RID} ; \\
\mathrm{mg} / \mathrm{kg}-\mathrm{d}\end{array}$ & $\begin{array}{l}\text { Oral } \\
\text { SF; } \\
\text { kg-d/ugg }\end{array}$ & $\begin{array}{l}\text { Noncarc. } \\
\text { oral } \\
\text { toxicity } \\
\text { score }\end{array}$ & $\begin{array}{l}\text { \% of } \\
\text { Total } \\
\text { noncarc. } \\
\text { oral } \\
\text { score }\end{array}$ & $\begin{array}{l}\text { Carcin. } \\
\text { oral } \\
\text { toxicity } \\
\text { score }\end{array}$ & $\begin{array}{l}\text { \% of } \\
\text { Total } \\
\text { carcin. } \\
\text { oral } \\
\text { score }\end{array}$ & $\begin{array}{l}\text { Final } \\
\text { decision }^{\mathrm{b}}\end{array}$ \\
\hline \multicolumn{9}{|l|}{ Organics } \\
\hline $\begin{array}{l}\text { 1,1,2-TRICHLORO- } \\
\text { 1,2,2-TRIFLUOROETHANE }\end{array}$ & $3.00 \mathrm{E}-03$ & $3.0 \mathrm{E}+01$ & & $1.00 \mathrm{E}-04$ & 0.00 & & & DROP \\
\hline 1,2-DICHLOROETHENE (TOTAL) & $1.50 \mathrm{E}-01$ & $9.0 \mathrm{E}-03$ & & $1.67 \mathrm{E}+01$ & 1.86 & & & \\
\hline 4-METHYL-2-PENTANONE & $4.00 \mathrm{E}-03$ & $8.0 \mathrm{E}-02$ & & $5.00 \mathrm{E}-02$ & 0.01 & & & DROP \\
\hline ACETONE & $8.10 \mathrm{E}-02$ & $1.0 \mathrm{E}-01$ & & $8.10 \mathrm{E}-01$ & 0.09 & & & DROP \\
\hline BIS(2-ETHYLHEXYL)PHTHALATE & $2.00 \mathrm{E}-03$ & $2.0 \mathrm{E}-02$ & $1.4 \mathrm{E}-02$ & $1.00 \mathrm{E}-01$ & 0.01 & $2.80 \mathrm{E}-05$ & 0.11 & DROP \\
\hline BROMODICHLOROMETHANE & $4.00 \mathrm{E}-03$ & $2.0 \mathrm{E}-02$ & $6.2 \mathrm{E}-02$ & $2.00 \mathrm{E}-01$ & 0.02 & $2.48 \mathrm{E}-04$ & 0.96 & DROP \\
\hline CARBON DISULEIDE & $2.00 \mathrm{E}-03$ & $1.0 \mathrm{E}-01$ & & $2.00 \mathrm{E}-02$ & 0.00 & & & DROP \\
\hline CHLOROFORM & $1.10 \mathrm{E}-02$ & $1.0 \mathrm{E}-02$ & $6.1 \mathrm{E}-03$ & $1.10 \mathrm{E}+00$ & 0.12 & $6.71 \mathrm{E}-05$ & 0.26 & DROP \\
\hline METHYLENE CHLORIDE & $1.70 \mathrm{E}-02$ & $6.0 \mathrm{E}-02$ & $7.5 \mathrm{E}-03$ & $2.83 \mathrm{E}-01$ & 0.03 & $1.28 \mathrm{E}-04$ & 0.49 & DROP \\
\hline TETRACHLOROETHENE & $5.50 \mathrm{E}-02$ & $1.0 \mathrm{E}-02$ & & $5.50 \mathrm{E}+00$ & 0.61 & & & DROP \\
\hline VINYL ACETATE & $2.00 \mathrm{E}-03$ & $1.0 \mathrm{E}+\infty 0$ & & $2.00 \mathrm{E}-03$ & 0.00 & & & DROP \\
\hline Toxicity Score Totals & & & & $8.96 \mathrm{E}+02$ & 100.0 & $2.58 \mathrm{E}-02$ & 100.0 & \\
\hline
\end{tabular}

"Because the speciation of chromium is unknown, it was not included in this toxicity screening.

"A decision to eliminate (designated by "DROP" in this table) analytes based on toxicity screening can be made when all existing pathway scores are less than $1 \%$ of the total score; in this case, only the ingestion pathway was evaluated (EPA 1989a). For example, if an analyte toxicity score was less than $1 \%$ for noncarcinogenic oral pathway, but greater than $1 \%$ for the carcinogenic oral pathway, then that analyte could not be eliminated from the COPC list based on this toxicity screening test (EPA 1989a). 


\section{C-74}

Table C.10b. Toxicity screening for BCV OU2 detected radionuclides in groundwater (carcinogenic, ingestion)

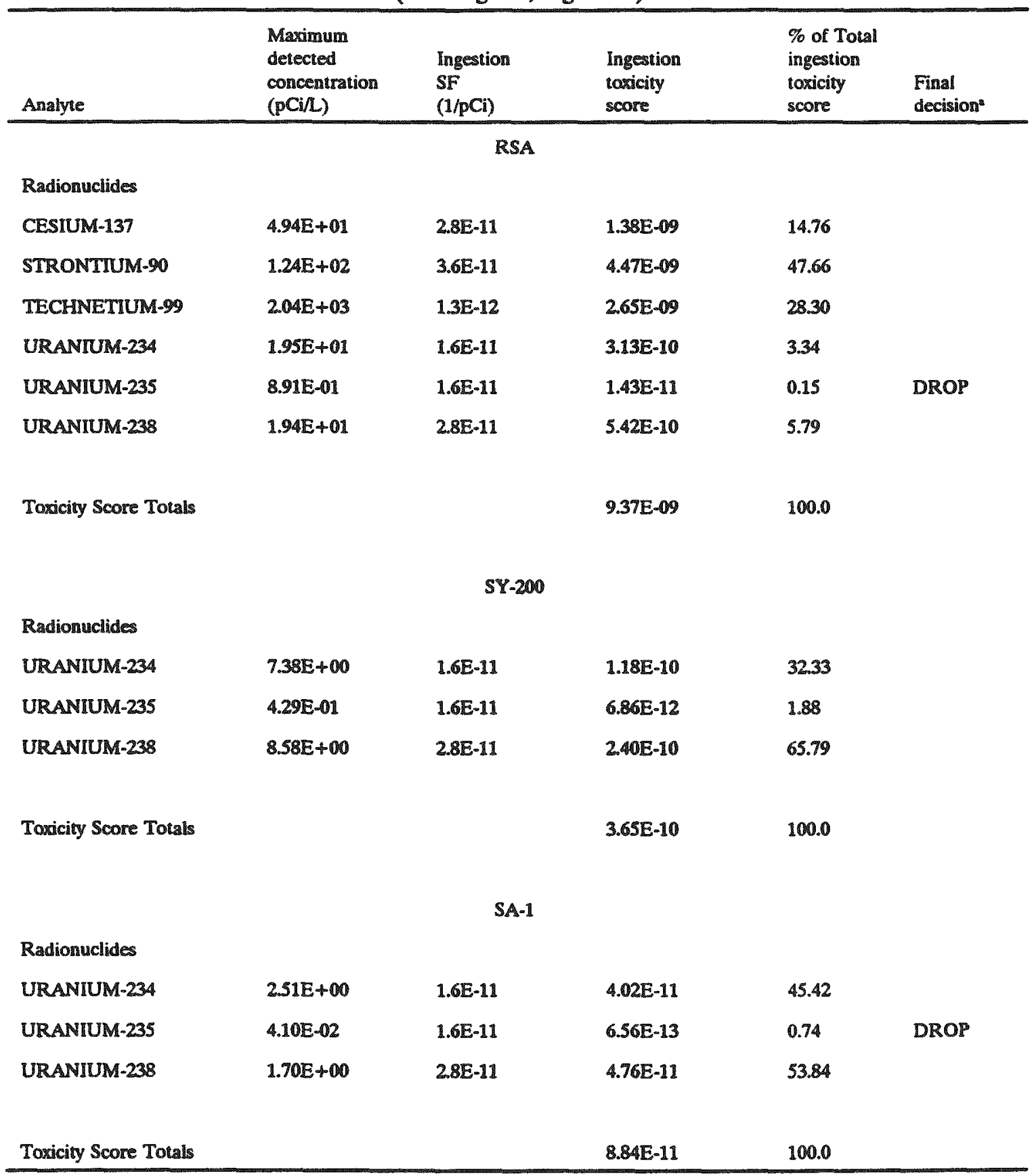

- A decision to eliminate (designated by "DROP" in this table) analytes based on toxicity screening can be made when all exiting pathway scores are less than $1 \%$ of the total score; in this case only the ingestion pathway was evaluated (EPA 1989a). 
Table C.11. Comparison of maximum detectod concentrations for BCV OU2 groundwater with PRGs (for analytes dropped because of toxicity screening)

\begin{tabular}{|c|c|c|c|c|c|c|}
\hline Analyte & $\begin{array}{l}\text { Frequency } \\
\text { of } \\
\text { detect } \\
\end{array}$ & $\begin{array}{l}\text { Maximum } \\
\text { detected } \\
\text { concentration }\end{array}$ & $\begin{array}{l}\text { Residential } \\
\text { PRG for } \\
\text { HQ = } 1.0\end{array}$ & $\begin{array}{l}\text { Residential } \\
\text { PRG for } \\
\text { risk }=1.0 \mathrm{E}-04\end{array}$ & $\begin{array}{l}\text { Residential } \\
\text { PRG for } \\
\text { risk }=1.0 \mathrm{E}-06\end{array}$ & $\begin{array}{l}\text { Units } \\
\text { of } \\
\text { measure }\end{array}$ \\
\hline \multicolumn{7}{|c|}{ RSA } \\
\hline \multicolumn{7}{|l|}{ Inorganics } \\
\hline BARIUM & $108 / 108$ & $2.30 \mathrm{E}-01$ & $2.6 E+00$ & & & $\mathrm{mg} / \mathrm{L}$ \\
\hline BORON & $92 / 108$ & $1.20 \mathrm{E}+00$ & $3.3 E+00$ & & & $\mathrm{mg} / \mathrm{L}$ \\
\hline STRONTIUM & $108 / 108$ & $3.10 \mathrm{E}+00$ & $2.2 \mathrm{E}+01$ & & & $\mathrm{mg} / \mathrm{L}$ \\
\hline VANADIUM & $13 / 108$ & $1.60 \mathrm{E}-02$ & $2.6 \mathrm{E}-01$ & & & $\mathrm{mg} / \mathrm{L}$ \\
\hline \multicolumn{7}{|l|}{ Radionuclides } \\
\hline URANIUM-235 & $70 / 108$ & $8.91 \mathrm{E}-01$ & & $3.0 \mathrm{E}+02$ & $3.0 \mathrm{E}+00$ & $\mathrm{pCi} / \mathrm{L}$ \\
\hline \multicolumn{7}{|l|}{ Organics } \\
\hline 1,1-DICHLOROETHANE & $15 / 82$ & $4.00 \mathrm{E}-03$ & $3.7 \mathrm{E}+00$ & & & $\mathrm{mg} / \mathrm{L}$ \\
\hline 4-METIIYL-2-PENTANONE & $10 / 82$ & $8.00 \mathrm{E}-03$ & $1.8 \mathrm{E}+00$ & & & $\mathrm{mg} / \mathrm{L}$ \\
\hline ACETONE & $12 / 82$ & $4.00 \mathrm{E}-02$ & $3.7 \mathrm{E}+00$ & & & $\mathrm{mg} / \mathrm{L}$ \\
\hline BROMODICHLOROMETHANE & $1 / 82$ & $7.00 \mathrm{E}-04$ & $7.3 \mathrm{E}-01$ & $1.4 \mathrm{E}-01$ & $1.4 \mathrm{E}-03$ & $\mathrm{mg} / \mathrm{L}$ \\
\hline CHLOROFORM & $54 / 82$ & $6.00 \mathrm{E}-03$ & $3.7 \mathrm{E}-01$ & $1.4 \mathrm{E}+00$ & $1.4 \mathrm{E}-02$ & $\mathrm{mg} / \mathrm{L}$ \\
\hline VINYL ACETATE & $1 / 82$ & $2.00 \mathrm{E}-03$ & $3.7 \mathrm{E}+01$ & & & $\mathrm{mg} / \mathrm{L}$ \\
\hline \multicolumn{7}{|c|}{ SY-200 } \\
\hline
\end{tabular}


Table C.11. (continued)

\begin{tabular}{|c|c|c|c|c|c|c|}
\hline Analyte & $\begin{array}{l}\text { Frequency } \\
\text { of } \\
\text { detect }\end{array}$ & $\begin{array}{l}\text { Maximum } \\
\text { detected } \\
\text { concentration }\end{array}$ & $\begin{array}{l}\text { Residential } \\
\text { PRG for } \\
\text { HQ = } 1.0 \\
\end{array}$ & $\begin{array}{l}\text { Residential } \\
\text { PRG for } \\
\text { risk }=1.0 \mathrm{E}-04\end{array}$ & $\begin{array}{l}\text { Residential } \\
\text { PRG for } \\
\text { risk }=1.0 \mathrm{E}-06\end{array}$ & $\begin{array}{l}\text { Units } \\
\text { of } \\
\text { measure }\end{array}$ \\
\hline ANTIMONY & $1 / 4$ & $2.00 \mathrm{E}-03$ & $1.5 \mathrm{E}-02$ & & & $\mathrm{mg} / \mathrm{L}$ \\
\hline BARIUM & $4 / 4$ & $5.50 \mathrm{E}-01$ & $2.6 \mathrm{E}+00$ & & & $\mathrm{mg} / \mathrm{l}$ \\
\hline CYANIDE & $1 / 4$ & $1.37 \mathbb{E}-02$ & 7.3E-01 & & & $\mathrm{mg} / \mathrm{L}$ \\
\hline FLUORIDE & $2 / 4$ & $1.30 \mathrm{E}-01$ & $2.2 \mathrm{E}+00$ & & & $\mathrm{mg} / \mathrm{I}$ \\
\hline MERCURY & $3 / 4$ & $1.50 \mathrm{E}-03$ & $1.1 \mathrm{E}-02$ & & & $\mathrm{mg} / \mathrm{L}$ \\
\hline NICKEL & $3 / 4$ & $1.69 E-02$ & $7.3 \mathrm{E}-01$ & & & $\mathrm{mg} / \mathrm{L}$ \\
\hline VANADIUM & $4 / 4$ & $2.66 \mathbb{E}-02$ & $2.6 \mathrm{E}-01$ & & & $\mathrm{mg} / \mathrm{L}$ \\
\hline ZINC & $4 / 4$ & $5.26 \mathbb{E}-02$ & $1.1 \mathrm{E}+01$ & & & $\mathrm{mg} / \mathrm{l}$ \\
\hline \multicolumn{7}{|l|}{ Organics } \\
\hline BENZYL BUTYL PHTHALATE & $1 / 4$ & $2.00 \mathrm{E}-03$ & $7.3 E+00$ & & & $\mathrm{mg} / \mathrm{L}$ \\
\hline DI-N-BUTYL PHTHALATE & $2 / 4$ & $3.00 \mathrm{E}-03$ & $3.7 \mathrm{E}+00$ & & & $\mathrm{mg} / \mathrm{L}$ \\
\hline DIETHYL PHTHALATE & $1 / 4$ & $2.00 \mathrm{E}-03$ & $2.9 \mathrm{E}+01$ & & & $\mathrm{mg} / \mathrm{L}$ \\
\hline TETRACHLOROETHENE & $3 / 4$ & $3.00 \mathrm{E}-03$ & 3.7E-01 & & & $\mathrm{mg} / \mathrm{L}$ \\
\hline \multicolumn{7}{|c|}{ SA-1 } \\
\hline \multicolumn{7}{|l|}{ Inorganics } \\
\hline ANTIMONY & $1 / 85$ & $1.90 \mathrm{E}-03$ & $1.5 \mathrm{E}-02$ & & & $\mathrm{mg} / \mathrm{L}$ \\
\hline BARIUM & $85 / 85$ & $4.00 \mathrm{E}-01$ & $2.6 \mathbb{E}+00$ & & & $\mathrm{mg} / \mathrm{L}$ \\
\hline FLUORIDE & $1 / 2$ & $1.00 \mathrm{E}-01$ & $2.2 \mathrm{E}+00$ & & & $\mathrm{mg} / \mathrm{L}$ \\
\hline
\end{tabular}


Table C.11. (continued)

\begin{tabular}{|c|c|c|c|c|c|c|}
\hline Analyte & $\begin{array}{l}\text { Frequency } \\
\text { of } \\
\text { detect }\end{array}$ & $\begin{array}{l}\text { Maximum } \\
\text { detected } \\
\text { concentration }\end{array}$ & $\begin{array}{l}\text { Residential } \\
\text { PRG for } \\
\text { HQ = } 1.0\end{array}$ & $\begin{array}{l}\text { Residential } \\
\text { PRG for } \\
\text { risk }=1.0 \mathrm{E}-04\end{array}$ & $\begin{array}{l}\text { Residential } \\
\text { PRG for } \\
\text { risk }=1.0 \mathrm{E}-06\end{array}$ & $\begin{array}{l}\text { Units } \\
\text { of } \\
\text { measure }\end{array}$ \\
\hline MERCURY & $1 / 2$ & $7.30 \mathrm{E}-04$ & $1.1 \mathbb{E}-02$ & & & $\mathrm{mg} / \mathrm{L}$ \\
\hline STRONTIUM & $83 / 83$ & $2.00 \mathrm{E}-01$ & $2.2 E+01$ & & & $\mathrm{mg} / \mathrm{L}$ \\
\hline \multicolumn{7}{|l|}{ Radionuclides } \\
\hline URANIUM-235 & $2 / 2$ & $4.10 \mathrm{E}-02$ & & $3.0 \mathrm{E}+02$ & $3.0 \mathrm{E}+\infty 0$ & $\mathrm{pCi} / \mathrm{L}$ \\
\hline \multicolumn{7}{|l|}{ Organics } \\
\hline $\begin{array}{l}\text { 1,1,2-TRICHLORO- } \\
\text { 1,2,2-TRIFLUOROETHANE }\end{array}$ & $1 / 2$ & $3.00 \mathrm{E}-03$ & $1.1 E+03$ & & & $\mathrm{mg} / \mathrm{L}$ \\
\hline 4-METHYL-2-PENTANONE & $6 / 67$ & $4.00 \mathrm{E}-03$ & $1.8 \mathrm{E}+00$ & & & $\mathrm{mg} / \mathrm{L}$ \\
\hline ACETONE & $10 / 67$ & $8.10 \mathrm{E}-02$ & $3.7 \mathrm{E}+00$ & & & $\mathrm{mg} / \mathrm{L}$ \\
\hline BIS(2-ETIYLHEXYL)PITTHALATE & $2 / 2$ & 2.00E-03 & 7.3E-01 & $6.1 \mathrm{E}-01$ & $6.1 \mathrm{E}-03$ & $\mathrm{mg} / \mathrm{L}$ \\
\hline BROMODICHLOROMETHANE* & $7 / 67$ & $4.00 E-03^{*}$ & $7.3 \mathrm{E}-01$ & $1.4 \mathrm{E}-01$ & $1.4 \mathrm{E}-03$ & $\mathrm{mg} / \mathrm{L}$ \\
\hline CARBON DISULFIDE & $1 / 67$ & $2.00 \mathrm{E}-03$ & $3.7 \mathrm{E}+00$ & & & $\mathrm{mg} / \mathrm{L}$ \\
\hline CHLOROFORM & $10 / 67$ & $1.10 \mathrm{E}-02$ & $3.7 \mathrm{E}-01$ & $1.4 \mathrm{E}+00$ & $1.4 \mathrm{E}-02$ & $\mathrm{mg} / \mathrm{L}$ \\
\hline METHYLENE CHLORIDE* & $9 / 67$ & $1.70 \mathrm{E}-02^{\circ}$ & $2.2 \mathrm{E}+\infty 0$ & $1.1 \mathrm{E}+\infty 0$ & $1.1 \mathrm{E} \cdot 02$ & $\mathrm{mg} / \mathrm{L}$ \\
\hline TETRACHLOROETIENE & $47 / 67$ & $5.50 \mathrm{E}-02$ & 3.7E-01 & & & $\mathrm{mg} / \mathrm{L}$ \\
\hline VINYL ACETATE & $1 / 65$ & $2.00 \mathrm{E}-03$ & $3.7 E+01$ & & & $\mathrm{mg} / \mathrm{L}$ \\
\hline
\end{tabular}

- Analytes with a maximum detected concentration greater than the residential ingestion of groundwater PRG (for risk = 1.0E-06) are marked with an asterisk $(*)$; note, no other analytes listed in this table (i.e., analytes dropped from the toxicity screening test) have maximum detected concentrations that give a risk $\geq 1.0 \mathrm{E}$-06 and/or an $\mathrm{HQ} \geq 1.0$. 
Table C.11. (contimued)

- Preliminary remediation goals (PRGs), for the residential land use scenario (ingestion of groundwater pathway), calculated for target risks of $1.0 \mathrm{E}-04$ and $1.0 \mathrm{E}-06$ for carcinogens, and a target hazard quotient of 1.0 for noncarcinogens. 
Table C.12. Comparison of BCV OU2 groundwater UCL95 analyte concentrations with residential ingestion of groundwater PRGs"

\begin{tabular}{|c|c|c|c|c|c|c|c|}
\hline Analyre & $\begin{array}{l}\text { Frequency } \\
\text { of detect }\end{array}$ & $\begin{array}{l}\text { UCL95 } \\
\text { concentration, }\end{array}$ & $\begin{array}{l}\text { Residential } \\
\mathrm{PRG} \text { for } \\
\mathrm{HQ}=1.0\end{array}$ & $\begin{array}{l}\text { Residential } \\
\text { PRG for } \\
\text { risk = 1.0E-04 }\end{array}$ & $\begin{array}{l}\text { Residential } \\
\text { PRG for } \\
\text { risk = 1.0E-06 }\end{array}$ & $\begin{array}{l}\text { Is UCL9S > } \\
\text { PRG at } \\
\mathrm{HQ}=1.0 ?\end{array}$ & $\begin{array}{l}\text { Is UCL.95 > } \\
\text { PRG at } \\
\text { risk = 1.0E-06? }\end{array}$ \\
\hline & & & RSA & & & & \\
\hline \multicolumn{8}{|l|}{ Inorganics } \\
\hline ANTIMONY & $3 / 108$ & $2.80 \mathrm{E}-02$ & $1.5 \mathrm{E}-02$ & & & YES & \\
\hline ARSENIC & $8 / 82$ & 3.05E-02 & $1.1 E-02$ & & & YES & \\
\hline BERYLLIUM & $17 / 108$ & 2.21E-04 & $1.8 \mathrm{E}-01$ & $2.0 \mathrm{E}-03$ & $2.0 \mathrm{E}-05$ & & YES \\
\hline CADMIUM & $15 / 108$ & $2.09 \mathrm{E}-03$ & $1.8 \mathrm{E}-02$ & & & & \\
\hline CHROMIUM VI & $42 \pi 08$ & $1.59 \mathrm{E}-02$ & $1.8 \mathrm{E}-01$ & & & & \\
\hline MANGANESE & $105 / 108$ & $3.29 \mathrm{E}+\infty$ & $1.8 \mathrm{E}-01$ & & & YES & \\
\hline NITRATE & $107 / 108$ & $4.38 E+01$ & $5.8 \mathrm{E}+01$ & & & & \\
\hline URANIUM (SOLUBLE SALTS) & 70108 & $2.06 \mathrm{E}-03$ & $1.1 \mathrm{E}-01$ & & & & \\
\hline \multicolumn{8}{|l|}{ Radionuclides } \\
\hline CESIUM-137 & $11 / 14$ & $4.94 E+01$ & & $1.7 \mathrm{E}+02$ & $1.7 \mathrm{E}+\infty$ & & YES \\
\hline STRONTIUM-90 & $5 / 14$ & $1.03 E+02$ & & $1.3 E+02$ & $1.3 \mathrm{E}+00$ & & YES \\
\hline TECHNETIUM-99 & $6 / 11$ & $2.04 E+03$ & & $3.7 \mathrm{E}+03$ & $3.7 E+01$ & & YES \\
\hline URANIUM-234 & $70 / 108$ & $6.95 E-01$ & & $3.0 \mathrm{E}+02$ & $3.0 \mathrm{E}+00$ & & \\
\hline URANIUM-238 & $70 / 108$ & $6.89 \mathrm{E}-01$ & & $1.7 \mathrm{E}+02$ & $1.7 \mathrm{E}+\infty$ & & \\
\hline \multicolumn{8}{|l|}{ Organics } \\
\hline 1,2-DICHLOROETHENE (TOTAL) & $52 / 82$ & $1.93 E-02$ & $3.3 \mathrm{E}-01$ & & & & \\
\hline CARBON TETRACHLORIDE & $35 / 82$ & 2.73E-03 & $2.6 \mathrm{E}-02$ & $6.6 \mathrm{E}-02$ & $6.6 \mathrm{E}-04$ & & YES \\
\hline
\end{tabular}


Table C.12 (continued)

\begin{tabular}{|c|c|c|c|c|c|c|c|}
\hline Analyte & $\begin{array}{l}\text { Frequency } \\
\text { of detect }\end{array}$ & $\begin{array}{l}\text { UCL95 } \\
\text { concentration, }\end{array}$ & $\begin{array}{l}\text { Residential } \\
\text { PRG for } \\
H Q=1.0\end{array}$ & $\begin{array}{l}\text { Residential } \\
\text { PRG for } \\
\text { risk = 1.0E-04 }\end{array}$ & $\begin{array}{l}\text { Residential } \\
\text { PRG for } \\
\text { risk = } 1.0 \mathrm{E}-06\end{array}$ & $\begin{array}{l}\text { Is UCL95 > } \\
\text { PRG al } \\
\mathrm{HQ}=1.0 ?\end{array}$ & $\begin{array}{l}\text { Is UCL.95 > } \\
\text { PRG at } \\
\text { risk = 1.0E-06? }\end{array}$ \\
\hline & & & $\$ Y-200$ & & & & \\
\hline \multicolumn{8}{|l|}{ Inorganics } \\
\hline CHROMIUM VI & $4 / 4$ & $1.87 \mathrm{E}-02$ & $1.8 \mathrm{E}-01$ & & & & \\
\hline MANGANESE & $4 / 4$ & $5.67 \mathrm{E}+00$ & $1.8 \mathrm{E}-01$ & & & YES & \\
\hline \multicolumn{8}{|l|}{ Radionuclides } \\
\hline URANIUM-234 & $4 / 4$ & $7.38 \mathrm{E}+00$ & & $3.0 \mathrm{E}+02$ & $3.0 \mathrm{E}+00$ & & YES \\
\hline URANIUM-235 & $4 / 4$ & $4.29 \mathrm{E}-01$ & & $3.0 E+02$ & $3.0 E+\infty$ & & \\
\hline URANIUM-238 & $4 / 4$ & $8.58 \mathrm{E}+00$ & & $1.7 E+02$ & $1.7 E+\infty$ & & YES \\
\hline \multicolumn{8}{|l|}{ Organics } \\
\hline BIS(2-ETHYLHEXY)PHTHALATE & 4/4 & $4.00 \mathrm{E} \cdot 03$ & $7.3 E-01$ & $6.1 E-01$ & $6.1 \mathrm{E}-03$ & & \\
\hline CHLOROFORM & $1 / 4$ & $1.00 \mathrm{E}-03$ & $3.7 \mathrm{E}-01$ & $1.4 E+\infty$ & $1.4 \mathrm{E}-02$ & & \\
\hline & & & SA-1 & & & & \\
\hline \multicolumn{8}{|l|}{ Inotganics } \\
\hline BERYLLIUM & $6 / 85$ & 2.63E-04 & $1.8 \mathrm{E}-01$ & $2.0 \mathrm{E}-03$ & $2.0 \mathrm{E}-05$ & & YES \\
\hline CADMIUM & 9/85 & $1.98 E-03$ & $1.8 \mathrm{E}-02$ & & & & \\
\hline MANGANESE & $70 / 85$ & $5.88 \mathrm{E}-02$ & $1.8 \mathrm{E}-01$ & & & & \\
\hline NITRATE & $81 / 83$ & $2.62 E+01$ & $5.8 \mathrm{E}+01$ & & & & \\
\hline VANADIUM & $2 / 85$ & $3.69 \mathrm{E}-03$ & $2.6 \mathrm{E}-01$ & & & & \\
\hline
\end{tabular}


Table C.12. (continued)

\begin{tabular}{|c|c|c|c|c|c|c|c|}
\hline Analyte & $\begin{array}{l}\text { Frequency } \\
\text { of detect }\end{array}$ & $\begin{array}{l}\text { UCL95 } \\
\text { concentration, }\end{array}$ & $\begin{array}{l}\text { Residential } \\
\text { PRG for } \\
\mathrm{HQ}=1.0\end{array}$ & $\begin{array}{l}\text { Residential } \\
\text { PRG for } \\
\text { risk = 1.0E-04 }\end{array}$ & $\begin{array}{l}\text { Residential } \\
\text { PRG for } \\
\text { risk = 1.0E-06 }\end{array}$ & $\begin{array}{l}\text { Is UCL95 > } \\
\text { PRG at } \\
\mathrm{HQ}=1.0 ?\end{array}$ & $\begin{array}{l}\text { Is UCL95 > } \\
\text { PRG at } \\
\text { risk = 1.0E-06? }\end{array}$ \\
\hline \multicolumn{8}{|l|}{ Radionuclides } \\
\hline URANIUM-234 & $2 / 2$ & $2.51 E+\infty$ & & $3.0 \mathrm{E}+02$ & $3.0 \mathrm{E}+00$ & & \\
\hline URANIUM-238 & $2 / 2$ & $1.70 \mathrm{E}+00$ & & $1.7 \mathrm{E}+02$ & $1.7 \mathrm{E}+\infty$ & & \\
\hline \multicolumn{8}{|l|}{ Organics } \\
\hline 1,2-DICHLOROETHENE (TOTAL) & $43 / 67$ & $1.56 \mathrm{E}-02$ & 3.3E-01 & & & & \\
\hline BROMODICHLOROMETHANE & $7 / 67$ & $2.58 \mathrm{E} \cdot 03$ & $7.3 E-01$ & $1.4 \mathrm{E}-01$ & $1.4 E-03$ & & YES \\
\hline METHYLENE CHLORIDE & $9 / 67$ & $3.18 \mathrm{E}-03$ & $2.2 \mathrm{E}+00$ & $1.1 E+00$ & 1.1E-02 & & \\
\hline
\end{tabular}

PRG = Preliminary remediation goal; HQ $=$ hazard quotient; UCL.95 = Upper $95 \%$ confidence limit on the mean concentration.

- Preliminary remediation goals (PRGs), for the residential land use scenario (ingestion of groundwater pathway), calculated for a target risk of $1.0 \mathrm{E}-04$ and $1.0 \mathrm{E}-06$ for carcinogens, and a target hazard quotient of 1.0 for noncarcinogens.

- The concentration reported in this column is the smallest of two values: the maximum detected concentration versus the upper $95 \%$ confidence limit of the mean concentration.

- Note, no analyte reported in this table has a UCL95 concentration greater than the PRG concentration for a risk of 1.0E-04. 
Table C.13a. On-site resident exposure scenario

\begin{tabular}{|c|c|c|c|c|}
\hline Vari & able & & Value used & Explanation/source \\
\hline \multicolumn{5}{|c|}{ Residential ingestion of soil scenario } \\
\hline \multicolumn{5}{|c|}{ Chronic daily intake $(\mathrm{mg} / \mathrm{kg}-\mathrm{d})=[\mathrm{CS} \times \mathrm{FI} \times \mathrm{EF} / \mathrm{AT}] \times\left[\left(\mathrm{ED}_{\mathrm{c}} \times \mathrm{IR}_{\mathrm{d}} / \mathrm{BW}_{\mathrm{c}}\right)+\left(\mathrm{ED}_{\mathrm{a}} \times \mathbb{R}_{\mathrm{a}} / \mathrm{BW}_{\mathrm{a}}\right)\right]$} \\
\hline \multicolumn{5}{|c|}{ Intake $(\mathrm{pCi})=\mathrm{CS} \times \mathrm{CF} \times \mathrm{EF} \times\left[\left(E_{\mathrm{c}} \times \mathbb{I R}_{\mathrm{c}}\right)+\left(E D_{\mathrm{a}} \times \mathbb{I R}_{\mathrm{a}}\right)\right]$} \\
\hline CS & $=$ & $\begin{array}{l}\text { Concentration in } \\
\text { soil }\end{array}$ & $\begin{array}{l}\text { Chemical-specific (mg/kg; } \\
\mathrm{pCi} / \mathrm{g} \text { ) }\end{array}$ & $\begin{array}{l}\text { Concentration is obtained } \\
\text { from data in Table } 5.1\end{array}$ \\
\hline $\mathbb{I R}_{\mathrm{c}}$ & $=$ & Ingestion rate & $0.0002 \mathrm{~kg} / \mathrm{d}$ & $\begin{array}{l}\text { Child rate (Section 6, } \\
\text { RAGS, EPA 1989a) }\end{array}$ \\
\hline $\mathbb{I R}_{\mathrm{a}}$ & & & $0.0001 \mathrm{~kg} / \mathrm{d}$ & $\begin{array}{l}\text { Adult rate (Section 6, } \\
\text { RAGS, EPA 1989a) }\end{array}$ \\
\hline $\mathrm{CF}$ & $=$ & Conversion factor & $10^{3} \mathrm{~g} / \mathrm{kg}$ & $\begin{array}{l}\text { Necessary to convert to } \\
\text { appropriate units }\end{array}$ \\
\hline FI & $=$ & Fraction ingested & 1 (unitless) & $\begin{array}{l}\text { Maximum value used; } \\
\text { equivalent to } 100 \%\end{array}$ \\
\hline $\mathrm{EF}$ & $=$ & Exposure frequency & 350 d/year & $\begin{array}{l}\text { OSWER Directive (EPA } \\
\text { 1991b) }\end{array}$ \\
\hline $\mathrm{ED}_{\mathrm{c}}$ & & Exposure duration & 6 years & $\begin{array}{l}\text { Two-part (child and adult) } \\
\text { residential exposure for a }\end{array}$ \\
\hline$E D_{\mathrm{a}}$ & & & 24 years & $\begin{array}{l}\text { 30-year duration (OSWER } \\
\text { Directive, EPA 1991b) }\end{array}$ \\
\hline $\mathrm{BW}_{\mathrm{e}}$ & $=$ & Body weight & $15 \mathrm{~kg}$ & $\begin{array}{l}\text { Child (OSWER Directive, } \\
\text { EPA 1991b) }\end{array}$ \\
\hline $\mathrm{BW}_{\mathrm{a}}$ & & & $70 \mathrm{~kg}$ & $\begin{array}{l}\text { Adult (Section 6, RAGS, } \\
\text { EPA 1989a) }\end{array}$ \\
\hline \multirow[t]{2}{*}{ AT } & $=$ & Averaging time & 365 d/year $\times 30$ years & $\begin{array}{l}\text { Averaging time } \\
\text { for noncarcinogens } \\
\text { (EPA 1989a; EPA 1991b) }\end{array}$ \\
\hline & & & $365 \mathrm{~d} /$ year $\times 70$ years & $\begin{array}{l}\text { Averaging time } \\
\text { for carcinogens } \\
\text { (EPA 1989a; EPA 1991b) }\end{array}$ \\
\hline
\end{tabular}


Table C.13b. On-site resident exposure scenario

\begin{tabular}{|c|c|c|c|}
\hline Variable & & Value used & Explanation/source \\
\hline \multicolumn{4}{|c|}{ Residential dermal contact with soil scenario } \\
\hline \multicolumn{4}{|c|}{ Chronic daily intake $(\mathrm{mg} / \mathrm{kg}-\mathrm{d})=[\mathrm{C}$} \\
\hline $\mathrm{CS}=$ & $\begin{array}{l}\text { Concentration in } \\
\text { soil }\end{array}$ & Chemical-specific (mg/kg) & $\begin{array}{l}\text { Concentration is obtained } \\
\text { from data in Table } 5.1\end{array}$ \\
\hline$C F=$ & Conversion factors & $10^{-6} \mathrm{~kg} / \mathrm{mg}$ and $10^{4} \mathrm{~cm}^{2} / \mathrm{m}^{2}$ & $\begin{array}{l}\text { Necessary to convert } \\
\text { to appropriate units }\end{array}$ \\
\hline$S A_{c}=$ & $\begin{array}{l}\text { Available surface } \\
\text { area }\end{array}$ & $0.182 \mathrm{~m}^{2} / \mathrm{d}$ & $\begin{array}{l}\text { Soth Percentile surface area } \\
\text { for head, hands, forearms, } \\
\text { and lower legs; for a child }\end{array}$ \\
\hline$S A_{a}$ & & $0.53 \mathrm{~m}^{2} / \mathrm{d}$ & $\begin{array}{l}\text { and for an adult, } \\
\text { respectively (Dermal } \\
\text { Exposure Assessment, EPA } \\
\text { 1992a) }\end{array}$ \\
\hline $\mathrm{AF}=$ & Adherence factor & $1.0 \mathrm{mg} / \mathrm{cm}^{2}$ & $\begin{array}{l}\text { Adherence factor for soil } \\
\text { (EPA Region IV New } \\
\text { Interim Guidance, 2/11/92) }\end{array}$ \\
\hline $\mathrm{ABS}=$ & Absorption factor & $\begin{array}{l}0.001 \text { (unitless) } \\
0.01 \text { (unitless) }\end{array}$ & $\begin{array}{l}\text { Equivalent to } 0.1 \% \\
\text { for inorganics and } 1.0 \% \text { for } \\
\text { organics (EPA Region IV } \\
\text { New Interim Guidance, } \\
2 / 11 / 92 \text { ) }\end{array}$ \\
\hline$E F=$ & Exposure frequency & 350 d/year & $\begin{array}{l}\text { OSWER Directive (EPA } \\
\text { 1991b) }\end{array}$ \\
\hline $\mathrm{ED}_{\mathrm{c}}=$ & Exposure duration & 6 years & $\begin{array}{l}\text { Two-part (child and adult) } \\
\text { residential exposure } \\
\text { for a } 30 \text {-year duration }\end{array}$ \\
\hline $\mathrm{ED}_{\mathbf{3}}$ & & 24 years & $\begin{array}{l}\text { (OSWER Directive, EPA } \\
\text { 1991b) }\end{array}$ \\
\hline $\mathrm{BW}_{\mathrm{c}}=$ & Body weight & $15 \mathrm{~kg}$ & $\begin{array}{l}\text { Child (OSWER Directive, } \\
\text { EPA 1991b) }\end{array}$ \\
\hline $\mathrm{BW}_{\mathrm{a}}$ & & $70 \mathrm{~kg}$ & $\begin{array}{l}\text { Adult (Section 6, RAGS, } \\
\text { EPA 1989a) }\end{array}$ \\
\hline \multirow[t]{2}{*}{$A T=$} & Averaging time & 365 d/year $\times 30$ years & $\begin{array}{l}\text { Averaging time } \\
\text { for noncarcinogens } \\
\text { (EPA 1989a; EPA 1991b) }\end{array}$ \\
\hline & & 365 d/year $\times 70$ years & $\begin{array}{l}\text { Averaging time } \\
\text { for carcinogens } \\
\text { (EPA 1989a; EPA 1991b) }\end{array}$ \\
\hline
\end{tabular}


Table C.13c. On-site resident exposure scenario

\begin{tabular}{|c|c|c|c|c|}
\hline Varie & able & & Value used & Explanation/source \\
\hline \multicolumn{5}{|c|}{ Residential inhalation of soil scenario } \\
\hline \multicolumn{5}{|c|}{$\begin{aligned} \text { Chronic daily intake }(\mathrm{mg} / \mathrm{kg}-\mathrm{d})= & {[\mathrm{CS} \times \operatorname{IR} \times(1 / \mathrm{PEF}+1 / \mathrm{VF}) \times \mathrm{EF} / \mathrm{AT}] \times } \\
& {\left[\left(\mathrm{ED}_{\mathrm{f}} / \mathrm{BW} \mathrm{W}_{\mathrm{c}}\right)+\left(\mathrm{ED}_{\mathrm{a}} / \mathrm{BW}_{\mathrm{a}}\right)\right] }\end{aligned}$} \\
\hline \multicolumn{5}{|c|}{ Intake $(\mathrm{pCi})=\mathrm{CS} \times \mathrm{CF} \times \mathrm{IR} \times(1 / \mathrm{PEF}+1 / \mathrm{VF}) \times \mathrm{EF} \times\left(\mathrm{ED}_{\mathrm{c}}+\mathrm{ED}_{\mathrm{a}}\right)$} \\
\hline CS & $=$ & $\begin{array}{l}\text { Concentration in } \\
\text { soil }\end{array}$ & $\begin{array}{l}\text { Chemical-specific (mg/kg; } \\
\mathrm{pCi} / \mathrm{g} \text { ) }\end{array}$ & $\begin{array}{l}\text { Concentration is obtained } \\
\text { from data in Table } 5.1\end{array}$ \\
\hline $\mathbb{R}$ & $=$ & Inhalation rate & $20 \mathrm{~m}^{3} / \mathrm{d}$ & $\begin{array}{l}\text { OSWER Directive (EPA } \\
\text { 1991b) }\end{array}$ \\
\hline $\mathrm{CF}$ & $=$ & Conversion factor & $10^{3} \mathrm{~g} / \mathrm{kg}$ & $\begin{array}{l}\text { Necessary to convert to } \\
\text { appropriate units }\end{array}$ \\
\hline PEF & $=$ & $\begin{array}{l}\text { Particulate emission } \\
\text { factor }\end{array}$ & $4.63 \mathrm{E}+09 \mathrm{~m}^{3} / \mathrm{kg}$ & RAGS Part B (EPA 1991a) \\
\hline VF & $=$ & $\begin{array}{l}\text { Volatilization } \\
\text { factor }\end{array}$ & Chemical-specific $\left(\mathrm{m}^{3} / \mathrm{kg}\right)$ & RAGS Part B (EPA 1991a) \\
\hline EF & $=$ & Exposure frequency & $350 \mathrm{~d} /$ year & $\begin{array}{l}\text { OSWER Directive (EPA } \\
\text { 1991b) }\end{array}$ \\
\hline $\mathrm{ED}_{\mathrm{c}}$ & $=$ & Exposure duration & 6 years & $\begin{array}{l}\text { Two-part (child and adult) } \\
\text { residential exposure for a }\end{array}$ \\
\hline $\mathrm{ED}_{\mathrm{a}}$ & & & 24 years & $\begin{array}{l}\text { 30-year duration (OSWER } \\
\text { Directive, EPA 1991b) }\end{array}$ \\
\hline $\mathrm{BW}_{\mathrm{c}}$ & $=$ & Body weight & $15 \mathrm{~kg}$ & $\begin{array}{l}\text { Child (OSWER Directive, } \\
\text { EPA 1991b) }\end{array}$ \\
\hline $\mathrm{BW}_{\mathrm{a}}$ & & & $70 \mathrm{~kg}$ & $\begin{array}{l}\text { Adult (Section 6, RAGS, } \\
\text { EPA 1989a) }\end{array}$ \\
\hline \multirow[t]{2}{*}{$\mathrm{AT}=$} & $=$ & Averaging time & $365 \mathrm{~d} /$ year $\times 30$ years & $\begin{array}{l}\text { Averaging time } \\
\text { for noncarcinogens } \\
\text { (EPA 1989a; EPA 1991b) }\end{array}$ \\
\hline & & & 365 d/year $\times 70$ years & $\begin{array}{l}\text { Averaging time } \\
\text { for carcinogens } \\
\text { (EPA 1989a; EPA 1991b) }\end{array}$ \\
\hline
\end{tabular}




\section{C-85}

Table C.13d. On-site resident exposure scemario

\begin{tabular}{|c|c|c|c|c|}
\hline \multicolumn{3}{|c|}{ Variable } & Value used & \multirow[t]{2}{*}{ Explanation/source } \\
\hline \multicolumn{4}{|c|}{ Residential external exposure scenario } & \\
\hline \multicolumn{5}{|c|}{ Dose $\left(\mathrm{pCi}\right.$-year/g) $=\mathrm{CS} \times(1-\mathrm{Se}) \times \mathrm{Te} \times\left(\mathrm{ED}_{\mathrm{c}}+\mathrm{ED}_{\mathrm{a}}\right) \times \mathrm{EF} \times \mathrm{CF}$} \\
\hline $\operatorname{cs}$ & $=$ & $\begin{array}{l}\text { Concentration in } \\
\text { soil }\end{array}$ & Chemical-specific (pCi/g) & $\begin{array}{l}\text { Concentration is obtained } \\
\text { from data in Table } 5.1\end{array}$ \\
\hline$E D_{c}$ & $=$ & Exposure duration & 6 years & $\begin{array}{l}\text { Two-part (child and adult) } \\
\text { residential exposure }\end{array}$ \\
\hline$E D_{2}$ & & & 24 years & $\begin{array}{l}\text { (OSWER Directive, EPA } \\
\text { 1991b) }\end{array}$ \\
\hline EF & $=$ & Exposure frequency & 350 d/year & $\begin{array}{l}\text { OSWER Directive (EPA } \\
\text { 1991b) }\end{array}$ \\
\hline $\mathrm{CF}$ & $=$ & Conversion factor & $(1 / 365)$ year $/ d$ & $\begin{array}{l}\text { Necessary to convert to } \\
\text { appropriate units. }\end{array}$ \\
\hline Se & $=$ & $\begin{array}{l}\text { Gamma shielding } \\
\text { factor (unitless) }\end{array}$ & 0.2 & $\begin{array}{l}\text { RAGS Part B, EPA 1991a; } \\
\text { sect. 4.1.2 (default value) }\end{array}$ \\
\hline $\mathrm{Te}$ & $=$ & $\begin{array}{l}\text { Gamma exposure } \\
\text { time factor (unitless) }\end{array}$ & 1.0 & $\begin{array}{l}\text { RAGS Part B, EPA 1991a; } \\
\text { sect. } 4.1 .2 \text { (default value, } \\
24 \mathrm{~h} / 24 \mathrm{~h} \text { ) }\end{array}$ \\
\hline
\end{tabular}




\section{C-86}

Table C.13e. On-site resident exposure scenario

\begin{tabular}{|c|c|c|c|c|}
\hline \multicolumn{2}{|c|}{ Variable } & \multicolumn{2}{|r|}{ Value used } & \multirow[t]{2}{*}{ Explanation/source } \\
\hline \multicolumn{4}{|c|}{ Residential ingestion of homegrown fruit scenario } & \\
\hline \multicolumn{5}{|c|}{$\begin{aligned} \text { Chronic daily intake }(\mathrm{mg} / \mathrm{kg}-\mathrm{d})= & {[\mathrm{CV} \times \mathrm{FI} \times \mathrm{IR} \times \mathrm{EF} / \mathrm{AT}] \mathrm{x} } \\
& {\left[\left(\mathrm{ED}_{\mathrm{c}} / \mathrm{BW}_{\mathrm{c}}\right)+\left(\mathrm{ED}_{\mathrm{a}} / \mathrm{BW}_{\mathrm{a}}\right)\right] }\end{aligned}$} \\
\hline \multicolumn{5}{|c|}{ Intake $(p C i)=C V \times C F \times I R \times E F \times\left(E D_{c}+E D_{a}\right)$} \\
\hline $\mathrm{CV}$ & $=$ & $\begin{array}{l}\text { Concentration in } \\
\text { fruit }\end{array}$ & $\begin{array}{l}\text { Chemical-specific } \\
\text { (mg/kg; } \mathrm{pCi} / \mathrm{g} \text { ) }\end{array}$ & $\begin{array}{l}\text { Concentration is obtained } \\
\text { from data in Table C. } 20\end{array}$ \\
\hline $\mathbb{I R}$ & $=$ & Ingestion rate & $0.042 \mathrm{~kg} / \mathrm{d}$ & $\begin{array}{l}\text { OSWER Directive (EPA } \\
\text { 1991b) }\end{array}$ \\
\hline $\mathrm{CF}$ & $=$ & Conversion factor & $10^{3} \mathrm{~g} / \mathrm{kg}$ & $\begin{array}{l}\text { Necessary to convert to } \\
\text { appropriate units }\end{array}$ \\
\hline FI & $=$ & Fraction ingested & 1 (unitless) & $\begin{array}{l}\text { Maximum value used; } \\
\text { equivalent to } 100 \%\end{array}$ \\
\hline $\mathrm{EF}$ & $=$ & Exposure frequency & 350 d/year & $\begin{array}{l}\text { OSWER Directive (EPA } \\
\text { 1991b) }\end{array}$ \\
\hline $\mathrm{ED}_{\mathrm{c}}$ & & Exposure duration & 6 years & $\begin{array}{l}\text { Two-part (child and adult) } \\
\text { residential exposure for a }\end{array}$ \\
\hline $\mathrm{ED}_{\mathrm{a}}$ & & & 24 years & $\begin{array}{l}\text { 30-year duration (OSWER } \\
\text { Directive, EPA 1991b) }\end{array}$ \\
\hline $\mathrm{BW}_{\mathrm{c}}$ & $=$ & Body weight & $15 \mathrm{~kg}$ & $\begin{array}{l}\text { Child (OSWER Directive, } \\
\text { EPA 1991b) }\end{array}$ \\
\hline $\mathrm{BW}_{\mathrm{s}}$ & & & $70 \mathrm{~kg}$ & $\begin{array}{l}\text { Adult (Section 6, RAGS, } \\
\text { EPA 1989a) }\end{array}$ \\
\hline \multirow[t]{2}{*}{$A T=$} & & Averaging time & $365 \mathrm{~d} /$ year $\times 30$ years & $\begin{array}{l}\text { Averaging time } \\
\text { for noncarcinogens } \\
\text { (EPA 1989a; EPA 1991b) }\end{array}$ \\
\hline & & & 365 d/year $\times 70$ years & $\begin{array}{l}\text { Averaging time } \\
\text { for carcinogens } \\
\text { (EPA 1989a; EPA 1991b) }\end{array}$ \\
\hline
\end{tabular}




\section{C-87}

Table C.13f. On-site resident exposure scenario

\begin{tabular}{|c|c|c|c|c|}
\hline Variz & able & & Value used & Explanation/source \\
\hline \multicolumn{5}{|c|}{ Residential ingestion of homegrown vegetables scenario } \\
\hline \multicolumn{5}{|c|}{$\begin{aligned} \text { Chronic daily intake }(\mathrm{mg} / \mathrm{kg}-\mathrm{d})= & {[\mathrm{CV} \times \mathrm{FI} \times \mathrm{IR} \times \mathrm{EF} / \mathrm{AT}] \times } \\
& {\left[\left(\mathrm{ED}_{\mathrm{c}} / \mathrm{BW}_{\mathrm{c}}\right)+\left(\mathrm{ED}_{\mathrm{a}} / \mathrm{BW}_{\mathrm{a}}\right)\right] }\end{aligned}$} \\
\hline \multicolumn{5}{|c|}{ Intake $(p C i)=C V \times C F \times I R \times E F \times\left(E D_{c}+E D_{a}\right)$} \\
\hline $\mathrm{CV}$ & $=$ & $\begin{array}{l}\text { Concentration in } \\
\text { vegetable }\end{array}$ & $\begin{array}{l}\text { Chemical-specific } \\
\text { (mg/kg; pCi/g) }\end{array}$ & $\begin{array}{l}\text { Concentration is obtained } \\
\text { from data in Table C. } 20\end{array}$ \\
\hline $\mathbb{I R}$ & $=$ & Ingestion rate & $0.080 \mathrm{~kg} / \mathrm{d}$ & $\begin{array}{l}\text { OSWER Directive (EPA } \\
\text { 1991b) }\end{array}$ \\
\hline $\mathrm{CF}$ & $=$ & Conversion factor & $10^{3} \mathrm{~g} / \mathrm{kg}$ & $\begin{array}{l}\text { Necessary to convert to } \\
\text { appropriate units }\end{array}$ \\
\hline FI & $=$ & Fraction ingested & 1 (unitless) & $\begin{array}{l}\text { Maximum value used; } \\
\text { equivalent to } 100 \%\end{array}$ \\
\hline EF & $=$ & Exposure frequency & 350 d/year & $\begin{array}{l}\text { OSWER Directive (EPA } \\
\text { 1991b) }\end{array}$ \\
\hline$E D_{c}$ & & Exposure duration & 6 years & $\begin{array}{l}\text { Two-part (child and adult) } \\
\text { residential exposure for a }\end{array}$ \\
\hline $\mathrm{ED}_{\mathrm{a}}$ & & & 24 years & $\begin{array}{l}\text { 30-year duration (OSWER } \\
\text { Directive, EPA 1991b) }\end{array}$ \\
\hline$B W_{c}$ & $=$ & Body weight & $15 \mathrm{~kg}$ & $\begin{array}{l}\text { Child (OSWER Directive, } \\
\text { EPA 1991b) }\end{array}$ \\
\hline$B W_{\mathrm{a}}$ & & & $70 \mathrm{~kg}$ & $\begin{array}{l}\text { Adult (Section 6, RAGS, } \\
\text { EPA 1989a) }\end{array}$ \\
\hline \multirow[t]{2}{*}{$\mathrm{AT}=$} & $=$ & Averaging time & $365 \mathrm{~d} /$ year $\times 30$ years & $\begin{array}{l}\text { Averaging time } \\
\text { for noncarcinogens } \\
\text { (EPA 1989a; EPA 1991b) }\end{array}$ \\
\hline & & & 365 d/year $\times 70$ years & $\begin{array}{l}\text { Averaging time } \\
\text { for carcinogens } \\
\text { (EPA 1989a; EPA 1991b) }\end{array}$ \\
\hline
\end{tabular}


Table C.14a. On-site industrial worker exposure scenario

\begin{tabular}{|c|c|c|c|c|}
\hline \multicolumn{2}{|c|}{ Variable } & \multicolumn{2}{|r|}{ Value used } & \multirow[t]{2}{*}{ Explanation/source } \\
\hline \multicolumn{4}{|c|}{ Industrial worker ingestion of soil scenario } & \\
\hline \multicolumn{5}{|c|}{ Chronic daily intake $(\mathrm{mg} / \mathrm{kg}-\mathrm{d})=(\mathrm{CS} \times \mathrm{FI} \times \mathrm{EF} / \mathrm{AT}) \times\left(\mathrm{ED}_{\mathrm{a}} \times \mathbb{R}_{\mathrm{f}} / \mathrm{BW}_{\mathrm{a}}\right)$} \\
\hline \multicolumn{5}{|c|}{ Intake $(\mathrm{pCi})=\mathrm{CS} \times C F \times E F \times E D_{\mathrm{a}} \times \mathbb{R}_{\mathrm{a}}$} \\
\hline$C S=$ & $=$ & $\begin{array}{l}\text { Concentration in } \\
\text { soil }\end{array}$ & $\begin{array}{l}\text { Chemical-specific (mg/kg; } \\
\text { pCi/g) }\end{array}$ & $\begin{array}{l}\text { Concentration is obtained } \\
\text { from data in Table } 5.1\end{array}$ \\
\hline $\mathbb{I R}_{\mathbf{a}}=$ & $=$ & Ingestion rate & $0.00005 \mathrm{~kg} / \mathrm{d}$ & $\begin{array}{l}\text { Adult industrial rate } \\
\text { (OSWER Directive, EPA } \\
\text { 1991b) }\end{array}$ \\
\hline $\mathrm{CF}=$ & $=$ & Conversion factor & $10^{3} \mathrm{~g} / \mathrm{kg}$ & $\begin{array}{l}\text { Necessary to convert to } \\
\text { appropriate units }\end{array}$ \\
\hline $\mathrm{FI}=$ & $=$ & Fraction ingested & 1 (unitless) & $\begin{array}{l}\text { Maximum value used; } \\
\text { equivalent to } 100 \%\end{array}$ \\
\hline$E F=$ & $=$ & Exposure frequency & 250 d/year & $\begin{array}{l}\text { OSWER Directive (EPA } \\
\text { 1991b) }\end{array}$ \\
\hline$E D_{a}=$ & & Exposure duration & 25 years & $\begin{array}{l}\text { OSWER Directive, EPA } \\
1991 b\end{array}$ \\
\hline $\mathrm{BW}_{\mathrm{a}}$ & $=$ & Body weight & $70 \mathrm{~kg}$ & $\begin{array}{l}\text { Adult (Section 6, RAGS, } \\
\text { EPA 1989a) }\end{array}$ \\
\hline \multirow[t]{2}{*}{$\mathrm{AT}=$} & & Averaging time & 365 d/year $\times 25$ years & $\begin{array}{l}\text { Averaging time } \\
\text { for noncarcinogens } \\
\text { (EPA 1989a; EPA 1991b) }\end{array}$ \\
\hline & & & 365 d/year $\times 70$ years & $\begin{array}{l}\text { Averaging time } \\
\text { for carcinogens } \\
\text { (EPA 1989a; EPA 1991b) }\end{array}$ \\
\hline
\end{tabular}




\section{C-89}

Table C.14b. On-site industrial worker exposure scenario

\begin{tabular}{|c|c|c|c|}
\hline Variable & & Value used & Explanation/source \\
\hline \multicolumn{4}{|c|}{ Industrial worker dermal contact with soil scenario } \\
\hline \multicolumn{2}{|c|}{ Chronic daily intake (mg/kg-d) } & \multicolumn{2}{|c|}{$(C S \times C F \times A F \times A B S \times E F / A T) \times\left(S_{a} \times E D_{a} / B W_{a}\right)$} \\
\hline$C S=$ & $\begin{array}{l}\text { Concentration in } \\
\text { soil }\end{array}$ & Chemical-specific (mg/kg) & $\begin{array}{l}\text { Concentration is obtained } \\
\text { from data in Table } 5.1\end{array}$ \\
\hline$C F=$ & Conversion factors & $10^{-6} \mathrm{~kg} / \mathrm{mg}$ and $10^{4} \mathrm{~cm}^{2} / \mathrm{m}^{2}$ & $\begin{array}{l}\text { Necessary to convert } \\
\text { to appropriate units }\end{array}$ \\
\hline$S A_{1}=$ & $\begin{array}{l}\text { Available surface } \\
\text { area }\end{array}$ & $0.316 \mathrm{~m}^{2} / \mathrm{d}$ & $\begin{array}{l}\text { Average surface area for } \\
\text { head, hands, and forearms } \\
\text { for an adult male (Dermal } \\
\text { Exposure Assessment, EPA } \\
\text { 1992a) }\end{array}$ \\
\hline$A F=$ & Adherence factor & $1.0 \mathrm{mg} / \mathrm{cm}^{2}$ & $\begin{array}{l}\text { Adherence factor for soil } \\
\text { (EPA Region IV New } \\
\text { Interim Guidance, 2/11/92) }\end{array}$ \\
\hline $\mathrm{ABS}=$ & Absorption factor & $\begin{array}{l}0.001 \text { (unitless) } \\
0.01 \text { (unitless) }\end{array}$ & $\begin{array}{l}\text { Equivalent to } 0.1 \% \\
\text { for inorganics and } 1.0 \% \text { for } \\
\text { organics (EPA Region IV } \\
\text { New Interim Guidance, } \\
2 / 11 / 92 \text { ) }\end{array}$ \\
\hline $\mathrm{EF}=$ & Exposure frequency & 250 d/year & $\begin{array}{l}\text { OSWER Directive (EPA } \\
\text { 1991b) }\end{array}$ \\
\hline $\mathrm{ED}_{\mathrm{a}}=$ & Exposure duration & 25 years & $\begin{array}{l}\text { OSWER Directive (EPA } \\
\text { 1991b) }\end{array}$ \\
\hline$B W_{a}=$ & Body weight & $70 \mathrm{~kg}$ & $\begin{array}{l}\text { Adult (Section 6, RAGS, } \\
\text { EPA 1989a) }\end{array}$ \\
\hline \multirow[t]{2}{*}{$\mathbf{A T}=$} & Averaging time & 365 d/year $\times 25$ years & $\begin{array}{l}\text { Averaging time } \\
\text { for noncarcinogens } \\
\text { (EPA 1989a; EPA 1991b) }\end{array}$ \\
\hline & & 365 d/year $\times 70$ years & $\begin{array}{l}\text { Averaging time } \\
\text { for carcinogens } \\
\text { (EPA 1989a; EPA 1991b) }\end{array}$ \\
\hline
\end{tabular}


Table C.14c. On-site industrial worker exposure scenario

\begin{tabular}{|c|c|c|c|c|}
\hline Vari: & able & & Value used & Explanation/source \\
\hline \multicolumn{5}{|c|}{ Industrial worker inhalation of soil scenario } \\
\hline \multicolumn{5}{|c|}{ Chronic daily intake $(\mathrm{mg} / \mathrm{kg}-\mathrm{d})=[\mathrm{CS} \times \mathbb{I R} \times(1 / \mathrm{PEF}+1 / \mathrm{VF}) \times \mathrm{EF} / \mathrm{AT}] \times\left(\mathrm{ED}_{2} / \mathrm{BW}_{\mathrm{a}}\right)$} \\
\hline \multicolumn{5}{|c|}{ Intake $(\mathrm{pCi})=\mathrm{CS} \times \mathrm{CF} \times \mathbb{I R} \times(1 / \mathrm{PEF}+1 / \mathrm{VF}) \times \mathrm{EF} \times \mathrm{ED}_{\mathrm{a}}$} \\
\hline Cs & $=$ & $\begin{array}{l}\text { Concentration in } \\
\text { soil }\end{array}$ & $\begin{array}{l}\text { Chemical-specific (mg/kg; } \\
\mathrm{pCi} / \mathrm{g} \text { ) }\end{array}$ & $\begin{array}{l}\text { Concentration is obtained } \\
\text { from data in Table } 5.1\end{array}$ \\
\hline $\mathbb{R}$ & $=$ & Inhalation rate & $20 \mathrm{~m}^{3} / \mathrm{d}$ & $\begin{array}{l}\text { OSWER Directive (EPA } \\
\text { 1991b) }\end{array}$ \\
\hline CF & $=$ & Conversion factor & $10^{3} \mathrm{~g} / \mathrm{kg}$ & $\begin{array}{l}\text { Necessary to convert to } \\
\text { appropriate units }\end{array}$ \\
\hline PEF & $=$ & $\begin{array}{l}\text { Particulate emission } \\
\text { factor }\end{array}$ & 4.63E $+09 \mathrm{~m}^{3} / \mathrm{kg}$ & RAGS Part B (EPA 1991a) \\
\hline VF & $=$ & $\begin{array}{l}\text { Volatilization } \\
\text { factor }\end{array}$ & Chemical-specific $\left(\mathrm{m}^{3} / \mathrm{kg}\right)$ & RAGS Part B (EPA 1991a) \\
\hline EF & $=$ & Exposure frequency & 250 d/year & $\begin{array}{l}\text { OSWER Directive (EPA } \\
\text { 1991b) }\end{array}$ \\
\hline $\mathrm{ED}_{\mathrm{a}}$ & $=$ & Exposure duration & 25 years & $\begin{array}{l}\text { OSWER Directive (EPA } \\
\text { 1991b) }\end{array}$ \\
\hline $\mathrm{BW}_{\mathrm{a}}$ & $=$ & Body weight & $70 \mathrm{~kg}$ & $\begin{array}{l}\text { Adult (Section 6, RAGS, } \\
\text { EPA 1989a) }\end{array}$ \\
\hline \multirow[t]{2}{*}{ AT } & $=$ & Averaging time & 365 d/year $\times 25$ years & $\begin{array}{l}\text { Averaging time } \\
\text { for noncarcinogens } \\
\text { (EPA 1989a; EPA 1991b) }\end{array}$ \\
\hline & & & 365 d/year $\times 70$ years & $\begin{array}{l}\text { Averaging lime } \\
\text { for carcinogens } \\
\text { (EPA 1989a; EPA 1991b) }\end{array}$ \\
\hline
\end{tabular}




\section{C-91}

Table C.14d. On-site industrial worker exposure scenario

\begin{tabular}{|c|c|c|c|c|}
\hline \multicolumn{3}{|c|}{ Variable } & Value used & Explanation/source \\
\hline \multicolumn{5}{|c|}{ Industrial worker external exposure scenario } \\
\hline \multicolumn{5}{|c|}{ Dose $\left(\mathrm{pCi}-\right.$ year/g) $=\mathrm{CS} \times(1-\mathrm{Se}) \times \mathrm{Te} \times \mathrm{ED}_{\mathrm{a}} \times \mathrm{EF} \times \mathrm{CF}$} \\
\hline $\mathrm{CS}$ & $=$ & $\begin{array}{l}\text { Concentration in } \\
\text { soil }\end{array}$ & Chemical-specific (pCi/g) & $\begin{array}{l}\text { Concentration is obtained } \\
\text { from data in Table } 5.1\end{array}$ \\
\hline $\mathrm{ED}$ & $=$ & Exposure duration & 25 years & $\begin{array}{l}\text { OSWER Directive (EPA } \\
\text { 1991b) }\end{array}$ \\
\hline EF & $=$ & Exposure frequency & 250 d/year & $\begin{array}{l}\text { OSWER Directive (EPA } \\
\text { 1991b) }\end{array}$ \\
\hline $\mathrm{CF}$ & $=$ & Conversion factor & $(1 / 365)$ year/d & $\begin{array}{l}\text { Necessary to convert to } \\
\text { appropriate units. }\end{array}$ \\
\hline Se & $=$ & $\begin{array}{l}\text { Gamma shielding } \\
\text { factor (unitless) }\end{array}$ & 0.2 & $\begin{array}{l}\text { RAGS Part B, EPA 1991a; } \\
\text { sect. } 4.1 .2 \text { (default value) }\end{array}$ \\
\hline $\mathrm{Te}$ & $=$ & $\begin{array}{l}\text { Gamma exposure } \\
\text { time factor (unitless) }\end{array}$ & 0.33 & $\begin{array}{l}\text { RAGS Part B, EPA 1991a; } \\
\text { sect. } 4.1 .2 \text { (default value, } \\
8 \mathrm{~h} / 24 \mathrm{~h} \text { ) }\end{array}$ \\
\hline
\end{tabular}


Table C.15. Chronic daily intakes and doses for BCV OU2 soil COPCs - residential scenario

\begin{tabular}{|c|c|c|c|c|c|c|c|c|}
\hline \multirow[b]{2}{*}{ Analyte } & \multirow[b]{2}{*}{$\begin{array}{l}\text { UCL95 } \\
\text { concentration } \\
\text { (mg/kgi pCi/g) }\end{array}$} & \multicolumn{4}{|c|}{ Carcinogenic effects $(\mathrm{CDI})^{\mathrm{e}}$} & \multicolumn{3}{|c|}{ Noncarcinogenic effects (CDI) } \\
\hline & & $\begin{array}{l}\text { Ingestion } \\
\text { (mg/kg-d; pCi) }\end{array}$ & $\begin{array}{l}\text { Dermal } \\
(\mathrm{mg} / \mathrm{kg}-\mathrm{d})\end{array}$ & $\begin{array}{l}\text { Inhalation } \\
(\mathrm{mg} / \mathrm{kg}-\mathrm{d} ; \mathrm{pCi})\end{array}$ & $\begin{array}{l}\text { External } \\
\text { exposure } \\
\text { (pCi-y/g) } \\
\end{array}$ & $\begin{array}{l}\text { Ingestion } \\
\text { (mg/kg-d) }\end{array}$ & $\begin{array}{l}\text { Dermal } \\
\text { (mg/kg-d) }\end{array}$ & $\begin{array}{l}\text { Inhalation } \\
(\mathrm{mg} / \mathrm{kg}-\mathrm{d})\end{array}$ \\
\hline \multicolumn{9}{|c|}{ RSA } \\
\hline \multicolumn{9}{|l|}{ Inorganics } \\
\hline BARIUM & $1.15 \mathrm{E}+02$ & $1.8 \mathrm{E}-04$ & $4.0 \mathrm{E}-06$ & $5.1 \mathbb{E}-09$ & & $4.2 \mathrm{E}-04$ & $9.4 \mathrm{E}-106$ & $1.2 \mathrm{E}-08$ \\
\hline BERYLLIUM & $1.05 \mathrm{E}+00$ & $1.6 \mathrm{E}-00$ & 3.7E-08 & $4.6 \mathrm{E}-11$ & & $3.8 \mathrm{E}-06$ & $8.6 \mathrm{E}-08$ & $1.1 \mathrm{E}-10$ \\
\hline CADMIUM & $4.93 E+00$ & 7.7E-06 & $1.7 E-07$ & $2.2 \mathrm{E} \cdot 10$ & & $1.8 \mathrm{E}-05$ & $4.0 E-07$ & $5.1 \mathrm{E}-10$ \\
\hline MANGANESE & $8.99 \mathrm{E}+02$ & $1.4 \mathrm{E}-03$ & $3.1 E-05$ & $3.9 \mathrm{E}-08$ & & 3.3E-03 & 7.3E-05 & $9.2 \mathrm{E}-08$ \\
\hline MERCURY & $200 \mathrm{E}+\infty 0$ & $3.1 \mathrm{E}-106$ & $7.0 \mathrm{E}-0 \mathrm{~B}$ & $8.8 \mathrm{E}-11$ & & $7.3 \mathrm{E}-06$ & $1.6 \mathrm{E}-07$ & $2.0 \mathrm{E}-10$ \\
\hline NICKEL & $4.61 E+01$ & $7.2 \mathrm{E}-05$ & $1.6 \mathrm{~B}-06$ & $2.0 \mathrm{E}-09$ & & $1.7 \mathrm{E}-04$ & $3.8 \mathrm{E}-106$ & 4.7E-09 \\
\hline URANIUM (SOLUBLE SALTS) & $7.36 \mathrm{E}+\infty 0$ & $1.2 \mathrm{E}-05$ & $2.6 \mathrm{E}-07$ & $3.2 \mathrm{E}-10$ & & 2.7E-05 & $6.0 \mathrm{E}-07$ & $7.5 \mathrm{E}-10$ \\
\hline \multicolumn{9}{|l|}{ Radionuclides } \\
\hline THORIUM-232 & $3.09 E+\infty$ & $3.9 E+03$ & & $1.4 E-01$ & $7.1 E+01$ & & & \\
\hline URANIUM-234 & $2.81 E+00$ & $3.5 E+03$ & & $1.3 E-01$ & $6.5 E+01$ & & & \\
\hline \multicolumn{9}{|l|}{ Organics } \\
\hline 1,2,4-TRICHLOROBENZENE & $4.31 E+00$ & $6.7 \mathrm{E}-06$ & $1.5 \mathrm{E}-\mathrm{A} 6$ & $1.3 \mathrm{E}-05$ & & $1.6 \mathrm{E}-05$ & $3.5 \mathrm{E}-106$ & $3.1 \mathrm{E}-05$ \\
\hline 1,2-DICHLOROETHENE (TOTAL) & $7.22 \mathrm{E}-02$ & 1.1E-07 & $2.5 \mathrm{E}-08$ & $3.2 E-12$ & & $2.6 \mathrm{E}-07$ & $5.9 \mathrm{E}-08$ & $7.4 \mathrm{E}-12$ \\
\hline AROCLOR-1254 & $9.86 \mathrm{E}-01$ & $1.5 \mathrm{E}-06$ & 3.4E-07 & 4.3E-11 & & $3.6 \mathrm{E}-06$ & $8.0 \mathrm{E}-07$ & $1.0 \mathrm{E}-10$ \\
\hline BENZO(A)PYRENE & $4.76 \mathrm{E}-01$ & $7.4 \mathrm{E}-07$ & $1.7 \mathrm{E}-07$ & $2.1 E-11$ & & $1.7 \mathrm{E}-06$ & $3.9 E-07$ & 4.9E-11 \\
\hline BIS(2-ETHYLHEXYL)PHTHALATE & $6.56 \mathrm{E}+00$ & $1.0 \mathrm{E}-05$ & $2.3 E-06$ & $29 \mathrm{E}-10$ & & 2.4E-05 & $5.3 E-06$ & $6.7 \mathrm{E}-10$ \\
\hline DIBENZO(A,H)ANTHRACENE & $2.90 E-01$ & $4.5 \mathrm{E}-07$ & $1.0 \mathrm{E}-07$ & $1.3 \mathrm{E}-11$ & & $1.1 \mathrm{E}-06$ & 2.4E-07 & $3.0 \mathrm{E}-11$ \\
\hline
\end{tabular}


Table C.15. (continued)

\begin{tabular}{|c|c|c|c|c|c|c|c|c|}
\hline \multirow[b]{2}{*}{ Analyte } & \multirow[b]{2}{*}{$\begin{array}{l}\text { UCL95 } \\
\text { concentration } \\
(\mathrm{mg} / \mathrm{kg} ; \mathrm{pCi} / \mathrm{g})\end{array}$} & \multicolumn{4}{|c|}{ Carcinogenic effects (CDI) } & \multicolumn{3}{|c|}{ Noncarcinogenic effects (CDI) } \\
\hline & & $\begin{array}{l}\text { Ingestion } \\
\text { (mg/kg-d; pCi) }\end{array}$ & $\begin{array}{l}\text { Dermal } \\
\text { (mg/kg-d) }\end{array}$ & $\begin{array}{l}\text { Inhalation } \\
\text { (mg/kg-d; pCi) }\end{array}$ & $\begin{array}{l}\text { Extermal } \\
\text { exposure } \\
\text { (pCi-y/g) }\end{array}$ & $\begin{array}{l}\text { Ingestion } \\
\text { (mg/kg-d) }\end{array}$ & $\begin{array}{l}\text { Dermal } \\
\text { (mg/kg-d) }\end{array}$ & $\begin{array}{l}\text { Inhalation } \\
\text { (mg/kg-d) }\end{array}$ \\
\hline TETRACHLOROETHENE & 1.62E-01 & $2.5 \mathrm{E}-07$ & $5.6 \mathrm{E}-08$ & $7.1 \mathrm{E}-12$ & & $5.9 \mathrm{E}-07$ & $1.3 \mathrm{E}-07$ & $1.7 E-11$ \\
\hline
\end{tabular}

SY-200

Inorganics

CADMIUM

MANGANESE

MERCURY

Organics

\section{AROCLOR-1254}

AROCLOR-1260

BENZO(A)ANTHRACENE

BENZO(A)PYRENE

BENZO(B)FLUORANTHENE

DIBENZO(A,H)ANTHRACENE

$\begin{array}{llll}3.24 \mathrm{E}-01 & 5.1 \mathrm{E}-07 & 1.1 \mathrm{E}-08 & 1.4 \mathrm{E}-11 \\ 7.32 \mathrm{E}+02 & 1.1 \mathrm{E}-03 & 2.6 \mathrm{E}-05 & 3.2 \mathrm{E}-08 \\ 2.17 \mathrm{E}+01 & 3.4 \mathrm{E}-05 & 7.6 \mathrm{E}-07 & 9.5 \mathrm{E}-10\end{array}$

3.09E-01

4.2SE-01

4.97E-01

4.34E-01

$3.23 E-01$

$1.20 \mathrm{E}-01$

$4.8 \mathrm{E}-07$

$6.7 \mathrm{E}-07$

7.8E-07

6.8E-07

5.1E-07

1.9E-07

$\begin{array}{ll}1.1 \mathrm{E}-07 & 1.4 \mathrm{E}-11 \\ 1.5 \mathrm{E}-07 & 1.9 \mathrm{E}-11 \\ 1.7 \mathrm{E}-07 & 2.2 \mathrm{E}-11 \\ 1.5 \mathrm{E}-07 & 1.9 \mathrm{E}-11 \\ 1.1 \mathrm{E}-07 & 1.4 \mathrm{E}-11 \\ 4.2 \mathrm{E}-08 & 5.3 \mathrm{E}-12\end{array}$

SA-1

Inorganics

BARIUM

BERYLLIUM

$\begin{array}{rrrr}2.34 \mathrm{E}+02 & 3.7 \mathrm{E}-04 & 8.1 \mathrm{E}-06 & 1.0 \mathrm{E}-08 \\ 1.20 \mathrm{E}+00 & 1.9 \mathrm{E}-06 & 4.2 \mathrm{E}-08 & 5.3 \mathrm{E}-11\end{array}$

$\begin{array}{lll}1.2 \mathrm{E}-06 & 2.6 \mathrm{E}-08 & 3.3 \mathrm{E}-11 \\ 2.7 \mathrm{E}-03 & 6.0 \mathrm{E}-05 & 7.5 \mathrm{E}-08 \\ 7.9 \mathrm{E}-05 & 1.8 \mathrm{E}-06 & 2.2 \mathrm{E}-09\end{array}$

$\begin{array}{lll}1.1 \mathrm{E}-06 & 2.5 \mathrm{E}-07 & 3.2 \mathrm{E}-11 \\ 1.6 \mathrm{E}-06 & 3.5 \mathrm{E}-07 & 4.4 \mathrm{E}-11 \\ 1.8 \mathrm{E}-06 & 4.0 \mathrm{E}-07 & 5.1 \mathrm{E}-11 \\ 1.6 \mathrm{E}-06 & 3.5 \mathrm{E}-07 & 4.5 \mathrm{E}-11 \\ 1.2 \mathrm{E}-06 & 2.6 \mathrm{E}-07 & 3.3 \mathrm{E}-11 \\ 4.4 \mathrm{E}-07 & 9.8 \mathrm{E}-08 & 1.2 \mathrm{E}-11\end{array}$

$\begin{array}{lll}8.5 E-04 & 1.9 E-05 & 2.4 E-08 \\ 4.4 E-06 & 9.7 E-08 & 1.2 E-10\end{array}$


Table C.15. (continued)

\begin{tabular}{|c|c|c|c|c|c|c|c|c|}
\hline \multirow[b]{2}{*}{ Analyte } & \multirow[b]{2}{*}{$\begin{array}{l}\text { UCL95 } \\
\text { concentralion } \\
\text { (mg/kg; pCi/g) }\end{array}$} & \multicolumn{4}{|c|}{ Carcinogenic effects (CDI) } & \multicolumn{3}{|c|}{ Noncarcinogenic effects $(\mathrm{CDI})^{\mathrm{e}}$} \\
\hline & & $\begin{array}{l}\text { Ingestion } \\
\text { (mg/kg-d; pCi) }\end{array}$ & $\begin{array}{l}\text { Dermal } \\
(\mathrm{mg} / \mathrm{kg}-\mathrm{d})\end{array}$ & $\begin{array}{l}\text { Inhalation } \\
\text { (mg/kg-d; pCi) }\end{array}$ & $\begin{array}{l}\text { Extemal } \\
\text { exposure } \\
(\mathrm{pCi}-\mathrm{y} / \mathrm{g})\end{array}$ & $\begin{array}{l}\text { Ingestion } \\
\text { (mg/kg-d) }\end{array}$ & $\begin{array}{l}\text { Dermal } \\
\text { (mg/kg-d) }\end{array}$ & $\begin{array}{l}\text { Inhalation } \\
\text { (mg/kg-d) }\end{array}$ \\
\hline CADMIUM & $3.31 E+\infty$ & $5.2 \mathrm{E}-16$ & $1.2 \mathrm{E}-07$ & $1.5 \mathrm{E}-10$ & & $1.2 E-05$ & 2.7E-07 & $3.4 \mathrm{E}-10$ \\
\hline CHROMUUM VI & $3.59 \mathrm{E}+01$ & $5.6 \mathrm{E}-05$ & $1.3 \mathrm{E}-06$ & $1.6 \mathrm{E}-09$ & & $1.3 E-04$ & $2.9 \mathrm{E}-06$ & 3.7E-09 \\
\hline MANGANESE & $1.20 \mathrm{E}+03$ & $1.9 E-03$ & $4.2 \mathrm{E}-05$ & $5.3 \mathrm{E}-08$ & & $4.4 \mathrm{E}-03$ & $9.7 \mathrm{E}-05$ & $1.2 \mathrm{E}-07$ \\
\hline MERCURY & $9.65 \mathrm{E}-01$ & $1.5 \mathrm{E}-06$ & $3.4 \mathrm{E}-08$ & $4.2 \mathrm{E}-11$ & & $3.5 \mathrm{E}-06$ & $7.9 \mathrm{E}-08$ & $9.9 \mathrm{E}-11$ \\
\hline NICKEL & $3.51 E+01$ & $5.5 \mathrm{E}-05$ & $1.2 \mathrm{E}-06$ & $1.5 \mathrm{E}-09$ & & $1.3 \mathrm{E}-04$ & $2.9 \mathrm{E}-06$ & $3.6 \mathrm{E}-09$ \\
\hline URANIUM (SOLUBLE SALTS) & $1.53 E+01$ & $2.4 E-05$ & $5.3 \mathrm{E}-07$ & $6.7 \mathrm{E}-10$ & & $5.6 \mathrm{E}-05$ & $1.2 \mathrm{E}-06$ & $1.6 \mathrm{E}-09$ \\
\hline \multicolumn{9}{|l|}{ Radionuclides } \\
\hline RADIUM-226 & $3.22 \mathrm{E}+00$ & $4.1 E+03$ & & $1.5 \mathrm{E}-01$ & $7.4 E+01$ & & & \\
\hline URANIUM-234 & $5.15 E+00$ & $6.5 E+03$ & & $2.3 \mathrm{E}-01$ & $1.2 E+02$ & & & \\
\hline URANIUM-238 & $5.11 \mathrm{E}+00$ & $6.4 E+03$ & & 2.3E-01 & $1.2 E+02$ & & & \\
\hline \multicolumn{9}{|l|}{ Organics } \\
\hline BENZO(A)ANTHRACENE & $4.16 \mathrm{E}-01$ & $6.5 E-07$ & 1.5E-07 & $1.8 \mathrm{E}-11$ & & $1.5 \mathrm{E}-\mathrm{n} 6$ & 3.4E-07 & 4.3E-11 \\
\hline BENZO(A)PYRENE & $3.61 \mathrm{E}-01$ & 5.7E-07 & $1.3 \mathrm{E}-07$ & $1.6 \mathrm{E}-11$ & & $1.3 E-06$ & 2.9E-07 & $3.7 \mathrm{E}-11$ \\
\hline BENZO(B)FLUORANTHENE & $3.76 \mathrm{E}-01$ & $5.9 \mathrm{E}-07$ & $1.3 \mathrm{E}-07$ & $1.7 E-11$ & & $1.4 \mathrm{E}-06$ & $3.1 \mathrm{E}-07$ & $3.9 \mathrm{E}-11$ \\
\hline INDENO(1,2,3-CD)PYRENE & $2.60 \mathrm{E}-01$ & 4.1E-07 & $9.1 \mathrm{E}-08$ & $1.1 \mathrm{E}-11$ & & 9.5E-07 & 2.1E-07 & $2.7 \mathrm{E}-11$ \\
\hline
\end{tabular}

CDI = Chronic Daily Intake

- Carcinogenic and noncarcinogenic CDIs are given for all nonradionuclides, regardless of the EPA class reported in EPA 1994 and EPA 1993 b.

- The soil concentration reported in this column is the smallest of two values: the maximum detected concentration versus the upper $95 \%$ confidence limit on the mean.

The analyte concentrations are in units of $\mathrm{mg} / \mathrm{kg}$ for nonradionuclides and in units of $\mathrm{pCi} / \mathrm{g}$ for radionuclides. 


\section{Table C.15. (continued)}

- For radionuclices, the units for intake (dose) are pCi for the ingestion and inhalation pathways, and the units are pCi-y/g for the external exposure pathway; for nonradionuclides, the units for $\mathrm{CDI}$ are mg/kg-d for all pathways. 
Table C.16. Summary of analyte concentrations in BCV OU2 soils and groundwater (for use in the residential homegrown garden scenario)

\begin{tabular}{|c|c|c|c|c|c|c|}
\hline Analyte & $\begin{array}{l}\text { RSA } \\
\text { soil conc. } \\
\text { (mg/kg; pCi/g) }\end{array}$ & $\begin{array}{l}\text { SY-200 } \\
\text { soil conc. } \\
\text { (mg/kg; pCi/g) }\end{array}$ & $\begin{array}{l}\text { SA-1 } \\
\text { soil conc. } \\
\text { (mg/kg; } \mathrm{pCi} / \mathrm{g} \text { ) }\end{array}$ & $\begin{array}{l}\text { RSA } \\
\text { groundwater conc. } \\
\text { (mg } / \text {; pCi/L) }\end{array}$ & $\begin{array}{l}\text { \$Y-200 } \\
\text { groundwater conc. } \\
\text { (mg/L; pCi/L) }\end{array}$ & $\begin{array}{l}\text { SA-1 } \\
\text { groundwater conc. } \\
\text { (mg/L; pCi/L) }\end{array}$ \\
\hline \multicolumn{7}{|l|}{ Inorganics" } \\
\hline ANTIMONY & & & & $2.80 E-02$ & & \\
\hline ARSENIC & & & & 3.05E-02 & & \\
\hline BARIUM & $1.15 E+02$ & & $2.34 E+02$ & & & \\
\hline BERYLLIUM & $1.05 E+00$ & & $1.20 E+00$ & $2.21 \mathrm{E}-04$ & & $2.63 \mathrm{E}-04$ \\
\hline CADMIUM & $4.93 E+\infty 0$ & $3.24 \mathrm{E}-01$ & $3.31 E+\infty$ & $2.09 \mathrm{E}-03$ & & $1.98 \mathrm{E}-03$ \\
\hline CHROMIUM VI & & & $3.59 E+01$ & $1.59 \mathrm{E}-02$ & $1.87 \mathrm{E}-02$ & \\
\hline MANGANESE & $8.99 E+02$ & $7.32 \mathrm{E}+02$ & $1.20 \mathrm{E}+03$ & $3.29 \mathrm{E}+00$ & $5.67 \mathrm{E}+00$ & $5.88 \mathrm{E}-02$ \\
\hline MERCURY & $2.00 E+00$ & $2.17 E+01$ & $9.65 \mathrm{E}-01$ & & & \\
\hline NICKEL & $4.61 \mathrm{E}+01$ & & $3.51 E+01$ & & & \\
\hline NITRATE & & & & $4.38 \mathrm{E}+01$ & & $2.62 E+01$ \\
\hline URANIUM (SOLUBLE SALTS) & $7.36 E+\infty 00$ & & $1.53 E+01$ & 2.06E-03 & & \\
\hline VANADIUM & & & & & & $3.69 \mathrm{E}-03$ \\
\hline \multicolumn{7}{|l|}{ Radionuclides } \\
\hline CESIUM-137 & & & & $4.94 E+01$ & & \\
\hline RADIUM-226 & & & $3.22 \mathrm{E}+00$ & & & \\
\hline STRONTIUM-90 & & & & $1.03 E+02$ & & \\
\hline TECHNETIUM-99 & & & & $2.04 E+03$ & & \\
\hline THORIUM-232 & $3.09 E+\infty 0$ & & & & & \\
\hline URANIUM-234 & $2.81 E+\infty$ & & $5.15 E+\infty$ & $6.95 \mathrm{E}-01$ & $7.38 E+00$ & $2.51 E+\infty 0$ \\
\hline
\end{tabular}


Table C.16. (continued)

\begin{tabular}{|c|c|c|c|c|c|c|}
\hline Analyre & $\begin{array}{l}\text { RSA } \\
\text { soil conc. } \\
\text { (mg/kg; pCi/g) }\end{array}$ & $\begin{array}{l}\mathrm{SY}-200 \\
\text { soil conc. } \\
\text { (mg/kg; } \mathrm{pCi} / \mathrm{g})\end{array}$ & $\begin{array}{l}\text { SA-1 } \\
\text { soil conc. } \\
\text { (mg/kg; pCi/g) }\end{array}$ & $\begin{array}{l}\text { RSA } \\
\text { groundwater conc. } \\
\text { (mg/L; pCi/L) }\end{array}$ & $\begin{array}{l}\text { SY-200 } \\
\text { groundwater conc. } \\
\text { (mg/L; pCi/L) }\end{array}$ & $\begin{array}{l}\text { SA-1 } \\
\text { groundwater conc. } \\
(\mathrm{mg} / \mathrm{L} ; \mathrm{pCi} / \mathrm{L})\end{array}$ \\
\hline URANIUM-235 & & & & & $4.29 \mathrm{E}-01$ & \\
\hline URANIUM-238 & & & $5.11 \mathrm{E}+\infty$ & $6.89 \mathrm{E} \cdot 01$ & $8.58 \mathrm{E}+00$ & $1.70 \mathrm{E}+\infty 0$ \\
\hline \multicolumn{7}{|l|}{ Organics" } \\
\hline 1,2,4-TRICHLOROBENZENE & $4.31 E+00$ & & & & & \\
\hline 1,2-DICHLOROETHENE (TOTAL) & $7.22 \mathrm{E}-02$ & & & $1.93 \mathrm{E}-02$ & & $1.56 \mathrm{E}-02$ \\
\hline AROCLOR-1254 & $9.86 \mathrm{E}-01$ & $3.09 \mathrm{E}-01$ & & & & \\
\hline AROCLOR-1260 & & $4.25 \mathrm{E}-01$ & & & & \\
\hline BENZO(A)ANTHRACENE & & $4.97 \mathrm{E}-01$ & $4.16 \mathrm{E}-01$ & & & \\
\hline BENZO(A)PYRENE & $4.76 \mathrm{E}-01$ & $4.34 \mathrm{E}-01$ & $3.61 \mathrm{E}-01$ & & & \\
\hline BENZO(B)FLUORANTHENE & & $3.23 \mathrm{E}-01$ & $3.76 \mathrm{E}-01$ & & & \\
\hline BIS(2-ETHYLHEXY)PHTHALATE & $6.56 \mathrm{E}+\infty$ & & & & $4.00 E-03$ & \\
\hline BROMODICHLOROMETHANE & & & & & & $2.58 E-03$ \\
\hline CARBON TETRACHLORIDE & & & & 2.73E-03 & & \\
\hline CHLOROFORM & & & & & $1.00 \mathrm{E}-03$ & \\
\hline DIBENZO(A,H)ANTHRACENE & $2.90 \mathrm{E}-01$ & $1.20 \mathrm{E}-01$ & & & & \\
\hline INDENO(1,2,3-CD)PYRENE & & & $2.60 \mathrm{E}-01$ & & & \\
\hline METHYLENE CHLORIDE & & & & & & $3.18 \mathrm{E}-03$ \\
\hline TETRACHLOROETHENE & $1.62 \mathrm{E}-01$ & & & & & \\
\hline
\end{tabular}

- The soil concentrations for inorganics and organics are in units of $\mathrm{mg} / \mathrm{kg}$, and the groundwater concentrations are in units of mg/L.

- The soil concentrations for radionuclides are in units of $\mathrm{pCi} / \mathrm{g}$, and the groundwater concentrations are in units of $\mathrm{pCi} / \mathrm{L}$. 


\section{C-98}

Table C.17a. Chemical, radionuclide, and plant parameters

\begin{tabular}{lcl}
\hline Chemical and radionuclide independent parameters & Reference \\
\hline $\begin{array}{l}\text { Irrigation rate }(\mathrm{I}) \\
\left(\mathrm{L} / \mathrm{m}^{2} / \text { day) }\right.\end{array}$ & 3.33 & $\begin{array}{l}\text { Whelan } \\
1987\end{array}$ \\
$\begin{array}{l}\text { Length of } \\
\text { growing season }\left(\mathrm{t}_{\mathrm{e}}\right) \\
\text { (days) }\end{array}$ & 60 & $\begin{array}{l}\text { Whelan } \\
1987\end{array}$ \\
$\begin{array}{l}\text { Soil density }(\rho) \\
\left(\mathrm{kg} / \mathrm{m}^{3}\right)\end{array}$ & & \\
& 1500 & $\begin{array}{l}\text { Droppo } \\
1989\end{array}$ \\
$\begin{array}{l}\text { Soil mixing depth }(z) \\
(\mathrm{m})\end{array}$ & & NCRP \\
\hline
\end{tabular}




\section{C-99}

Table C.17b. Chemical, radionuclide, and plant parameters

\begin{tabular}{|c|c|c|c|c|c|c|}
\hline \multirow[b]{2}{*}{ Parameter } & \multicolumn{5}{|c|}{ Plant-fype specific parameters } & \multirow[b]{2}{*}{ Reference } \\
\hline & $\begin{array}{l}\text { Leafy } \\
\text { veg. }\end{array}$ & $\begin{array}{l}\text { Root } \\
\text { veg. }\end{array}$ & Legurmes & $\begin{array}{l}\text { Fruiting } \\
\text { veg. }\end{array}$ & Fruits & \\
\hline $\begin{array}{l}\text { Plant yield }(Y) \\
\left(\mathrm{kg} / \mathrm{m}^{2}\right)\end{array}$ & 2.0 & 0.6 & 0.6 & 0.6 & 0.6 & $\begin{array}{l}\text { NCRP } \\
1985\end{array}$ \\
\hline $\begin{array}{l}\text { Translocation } \\
\text { factor }\left(T_{q}\right)\end{array}$ & 1.0 & 0.1 & 0.1 & 0.1 & 0.1 & $\begin{array}{l}\text { NCRP } \\
1985\end{array}$ \\
\hline $\begin{array}{l}\text { Fraction of } \\
\text { deposition ( })\end{array}$ & 0.2 & 0.2 & 0.2 & 0.2 & 0.2 & $\begin{array}{l}\text { NCRP } \\
1985\end{array}$ \\
\hline
\end{tabular}


Table C.18 Exposure decay constants

\begin{tabular}{|c|c|c|c|c|}
\hline Analyte & $\begin{array}{l}\text { Radioactive } \\
\text { half-life } \\
\text { (yi) }\end{array}$ & $\begin{array}{l}\text { Radioactive } \\
\text { decay constant } \\
(1 / \mathrm{T})\end{array}$ & $\begin{array}{l}\text { Weathering } \\
\text { decay constant } \\
\text { (1/yr) }\end{array}$ & $\begin{array}{l}\text { Weathering and } \\
\text { radioactive decay } \\
\text { constant - } \lambda_{c}(1 / \mathrm{yr})\end{array}$ \\
\hline \multicolumn{5}{|l|}{ Inorgarics (Whelan 1987) } \\
\hline Antimony & $0.00 \mathrm{E}+00$ & $0.00 \mathrm{E}+00$ & $1.81 E+01$ & $1.81 \mathrm{E}+01$ \\
\hline Arsenic & $0.00 \mathrm{E}+00$ & $0.00 \mathrm{E}+00$ & $1.81 E+01$ & $1.81 E+01$ \\
\hline Barium & $0.00 E+00$ & $0.00 \mathrm{E}+00$ & $1.81 \mathrm{E}+01$ & $1.81 \mathrm{E}+01$ \\
\hline Beryllium & $0.00 \mathrm{E}+00$ & $0.00 E+00$ & $1.81 \mathrm{E}+01$ & $1.81 E+01$ \\
\hline Cadmium & $0.00 \mathrm{E}+00$ & $0.00 \mathrm{E}+00$ & $1.81 \mathrm{E}+01$ & $1.81 E+01$ \\
\hline Chromium VI & $0.00 \mathrm{E}+00$ & $0.00 E+00$ & $1.81 E+01$ & $1.81 E+01$ \\
\hline Manganese & $0.00 \mathrm{E}+00$ & $0.00 \mathrm{E}+00$ & $1.81 E+01$ & $1.81 E+01$ \\
\hline Mercury & $0.00 \mathrm{E}+00$ & $0.00 E+00$ & $1.81 E+01$ & $1.81 E+01$ \\
\hline Nickel & $0.00 \mathrm{E}+00$ & $0.00 \mathrm{E}+00$ & $1.81 E+01$ & $1.81 E+01$ \\
\hline Nitrate & $0.00 E+00$ & $0.00 \mathrm{E}+00$ & $1.81 E+01$ & $1.81 E+01$ \\
\hline $\begin{array}{l}\text { Uranium } \\
\text { (soluble salts) }\end{array}$ & $0.00 \mathrm{E}+00$ & $0.00 \mathrm{E}+00$ & $1.81 E+01$ & $1.81 \mathrm{E}+01$ \\
\hline Vanadium & $0.00 E+\infty$ & $0.00 E+\infty$ & $1.81 E+01$ & $1.81 E+01$ \\
\hline \multicolumn{5}{|c|}{ Radionuclides (Radiological Health Handbook, 1970) } \\
\hline Cesium-137 & $3.01 E+01$ & $230 \mathrm{E}-02$ & $1.81 E+01$ & $1.81 E+01$ \\
\hline Radium-226 & $1.60 \mathrm{E}+03$ & 4.33E-04 & $1.81 E+01$ & $1.81 E+01$ \\
\hline Strontium-90 & $2.81 E+01$ & $2.47 \mathrm{E}-02$ & $1.81 E+01$ & $1.81 \mathrm{E}+01$ \\
\hline Technetium-99 & $2.10 \mathrm{E}+05$ & $3.30 \mathrm{E}-06$ & $1.81 \mathrm{E}+01$ & $1.81 \mathrm{E}+01$ \\
\hline Thorium-232 & $1.41 \mathrm{E}+10$ & $4.91 \mathrm{E}-11$ & $1.81 \mathrm{E}+01$ & $1.81 E+01$ \\
\hline Uranium-234 & $2.47 \mathrm{E}+05$ & $2.81 E-06$ & $1.81 \mathrm{E}+01$ & $1.81 \mathrm{E}+01$ \\
\hline Uranium-235 & $7.10 \mathrm{E}+08$ & $9.76 \mathrm{E}-10$ & $1.81 \mathrm{E}+01$ & $1.81 \mathrm{E}+01$ \\
\hline Uranium-238 & $4.15 \mathrm{E}+09$ & $1.54 \mathrm{E}-10$ & $1.81 \mathrm{E}+01$ & $1.81 E+01$ \\
\hline \multicolumn{5}{|l|}{ Onganic (Whelan 1987) } \\
\hline 1,2,4-Tricblorobenzene & $0.00 \mathrm{E}+00$ & $0.00 \mathrm{E}+00$ & $1.81 E+01$ & $1.81 \mathrm{E}+01$ \\
\hline $\begin{array}{l}\text { 1,2-Dichloroethene } \\
\text { (total) }\end{array}$ & $0.00 \mathrm{E}+00$ & $0.00 E+00$ & $1.81 \mathrm{E}+01$ & $1.81 \mathrm{E}+01$ \\
\hline Aroclor-1254 & $0.00 \mathbb{E}+00$ & $0.00 \mathrm{E}+00$ & $1.81 E+01$ & $1.81 \mathrm{E}+01$ \\
\hline Aroclor-1260 & $0.00 \mathrm{E}+00$ & $0.00 \mathrm{E}+00$ & $1.81 E+01$ & $1.81 E+01$ \\
\hline Benzo(a)anthracene & $0.00 E+\infty$ & $0.00 \mathrm{E}+00$ & $1.81 E+01$ & $1.81 E+01$ \\
\hline Benzo(a)pyrene & $0.00 E+00$ & $0.00 \mathrm{E}+00$ & $1.81 E+01$ & $1.81 E+01$ \\
\hline Benzo(b)fluoranthene & $0.00 \mathrm{E}+00$ & $0.00 E+00$ & $1.81 \mathrm{E}+01$ & $1.81 \mathrm{E}+01$ \\
\hline
\end{tabular}




\section{C-101}

Table C.18. (comtinued)

\begin{tabular}{|c|c|c|c|c|}
\hline Analyte & $\begin{array}{l}\text { Radioactive } \\
\text { half-life } \\
\text { (yr) }\end{array}$ & $\begin{array}{l}\text { Radioactive } \\
\text { decay constant } \\
(1 / \mathrm{yr})\end{array}$ & $\begin{array}{l}\text { Weathering } \\
\text { decay constant } \\
(1 / y r)\end{array}$ & $\begin{array}{l}\text { Weathering and } \\
\text { radioactive decay } \\
\text { constant }-\lambda(1 / y r)\end{array}$ \\
\hline $\begin{array}{l}\text { Bis(2-ethylhexyl)- } \\
\text { Phthalate }\end{array}$ & $0.00 \mathrm{E}+00$ & $0.00 \mathrm{E}+00$ & $1.81 E+01$ & $1.81 \mathrm{E}+01$ \\
\hline $\begin{array}{l}\text { Bromodichloro- } \\
\text { methane }\end{array}$ & $0.00 \mathrm{E}+00$ & $0.00 \mathrm{E}+00$ & $1.81 E+01$ & $1.81 \mathrm{E}+01$ \\
\hline Carbon tetrachloride & $0.00 \mathrm{E}+00$ & $0.00 E+00$ & $1.81 \mathrm{E}+01$ & $1.81 \mathrm{E}+01$ \\
\hline Chloroform & $0.00 \mathrm{E}+00$ & $0.00 \mathrm{E}+00$ & $1.81 \mathrm{E}+01$ & $1.81 E+01$ \\
\hline $\begin{array}{l}\text { Dibenzo(a,b)- } \\
\text { anthracene }\end{array}$ & $0.00 E+00$ & $0.00 \mathrm{E}+00$ & $1.81 E+01$ & $1.81 E+01$ \\
\hline $\begin{array}{l}\text { Indeno }(1,2,3-c d) \text { - } \\
\text { pyrene }\end{array}$ & $0.00 \mathrm{E}+\infty$ & $0.00 \mathrm{E}+00$ & $1.81 \mathrm{E}+01$ & $1.81 \mathrm{E}+01$ \\
\hline Methylene chloride & $0.00 \mathrm{E}+\infty 0$ & $0.00 \mathrm{E}+00$ & $1.81 E+01$ & $1.81 \mathrm{E}+01$ \\
\hline Tetrachloroethene & $0.00 E+00$ & $0.00 E+00$ & $1.81 \mathrm{E}+01$ & $1.81 E+01$ \\
\hline
\end{tabular}


Table C.19. Soil-to-plant transfer coefficients

\begin{tabular}{|c|c|c|c|c|c|c|}
\hline Analyte & Leafy vegetables & Root vegelables & Legumes & Fruiting vegetables & Fruits & Reference \\
\hline \multicolumn{7}{|l|}{ Inorganics } \\
\hline Antimony & $1.10 \mathrm{E}-02$ & $1.10 \mathrm{E}-02$ & $1.10 \mathrm{E}-02$ & $1.10 \mathrm{E}-02$ & $1.10 \mathrm{E}-02$ & Strenge 1989 \\
\hline Arsenic & $1.00 \mathrm{E}-02$ & $1.00 \mathrm{E}-02$ & $1.00 E-02$ & $1.00 \mathrm{E}-02$ & $1.00 \mathrm{E}-02$ & Strenge 1989 \\
\hline Barium & $5.00 \mathrm{E}-03$ & $5.00 \mathrm{E}-03$ & $5.00 \mathrm{E}-03$ & $5.00 \mathrm{E}-03$ & $5.00 \mathrm{E}-03$ & Strenge 1989 \\
\hline Beryllium & $4.70 \mathrm{E}-04$ & $4.70 \mathrm{E}-04$ & $4.70 \mathrm{E}-04$ & $4.74 \mathrm{E}-04$ & $4.74 \mathrm{E}-04$ & Strenge 1989 \\
\hline Cadmium & $3.00 \mathrm{E}-01$ & $3.00 \mathrm{E}-01$ & $3.00 \mathrm{E}-01$ & $3.00 \mathrm{E}-01$ & $3.00 \mathrm{E}-01$ & Strenge 1989 \\
\hline Chromium VI & $2.50 \mathrm{E}-04$ & $2.50 \mathrm{E}-04$ & $2.50 E-04$ & $2.50 \mathrm{E} \cdot 04$ & $2.50 \mathrm{E}-04$ & Strenge 1989 \\
\hline Manganese & $3.00 \mathrm{E}-02$ & $3.00 E-02$ & $3.00 \mathrm{E}-02$ & $3.00 \mathrm{E}-02$ & $3.00 \mathrm{E}-02$ & Strenge 1989 \\
\hline Mercury & $3.80 \mathrm{E}-01$ & $3.80 \mathrm{E}-01$ & $3.80 \mathrm{E}-01$ & $3.80 \mathrm{E}-01$ & $3.80 \mathrm{E}-01$ & Strenge 1989 \\
\hline Nickel & $1.90 \mathrm{E}-02$ & $1.90 \mathrm{E}-02$ & $1.90 \mathrm{E}-02$ & $1.90 \mathrm{E}-02$ & $1.90 \mathrm{E}-02$ & Strenge 1989 \\
\hline Nitrate & $0.00 E+00$ & $0.00 \mathrm{E}+00$ & $0.00 \mathrm{E}+00$ & $0.00 \mathrm{E}+00$ & $0.00 \mathrm{E}+00$ & Strenge 1989 \\
\hline $\begin{array}{l}\text { Uranium } \\
\text { (soluble salts) }\end{array}$ & $2.50 \mathrm{E}-03$ & $2.50 \mathrm{E}-03$ & $2.50 \mathrm{E}-03$ & $2.50 \mathrm{E}-03$ & $2.50 \mathrm{E}-03$ & Strenge 1989 \\
\hline Vanadium & $1.30 \mathrm{E}-03$ & $1.30 \mathrm{E}-03$ & $1.30 \mathrm{E}-03$ & $1.30 \mathrm{E}-03$ & $1.30 \mathrm{E}-03$ & Strenge 1989 \\
\hline \multicolumn{7}{|l|}{ Radionuclides } \\
\hline Cesium-137 & $2.00 \mathrm{E}-03$ & $2.00 \mathrm{E}-03$ & $2.00 \mathrm{E}-03$ & $2.0 E-03$ & $2.0 \mathrm{E}-03$ & Strenge 1989 \\
\hline Radium-226 & $1.40 \mathrm{E}-03$ & $1.40 \mathrm{E}-03$ & $1.40 \mathrm{E}-03$ & $1.40 \mathrm{E}-03$ & $1.40 \mathrm{E}-03$ & Strenge 1989 \\
\hline Strontium-90 & $2.00 \mathrm{E}-01$ & $2.00 \mathrm{E}-01$ & $2.00 \mathrm{E}-01$ & $2.00 \mathrm{E}-01$ & $2.00 \mathrm{E}-01$ & Strenge 1989 \\
\hline Technetium-99 & $2.50 \mathrm{E}-01$ & $2.50 \mathrm{E}-01$ & $2.50 \mathrm{E}-01$ & $2.50 \mathrm{E}-01$ & $2.50 \mathrm{E}-01$ & Strenge 1989 \\
\hline Thorium-232 & $4.20 \mathrm{E}-03$ & $4.20 \mathrm{E}-03$ & $4.20 \mathrm{E}-03$ & $4.20 \mathrm{E}-03$ & $4.20 \mathrm{E}-03$ & Strenge 1989 \\
\hline Uranium-234 & $2.50 \mathrm{E}-03$ & $2.50 \mathrm{E}-03$ & $2.50 \mathrm{E}-03$ & $2.50 \mathrm{E}-03$ & $2.50 \mathrm{E}-03$ & Strenge 1989 \\
\hline
\end{tabular}


Table C.19. (continued)

\begin{tabular}{|c|c|c|c|c|c|c|}
\hline Analyte & Leafy vegetables & Root vegetables & Legumes & Fruiting vegetables & Fruits & Reference \\
\hline Uranium-235 & $2.50 \mathrm{E}-03$ & $2.50 \mathrm{E}-03$ & $2.50 \mathrm{E}-03$ & $2.50 \mathrm{E}-03$ & $2.50 E-03$ & Strenge 1989 \\
\hline Uranium-238 & $2.50 \mathrm{E}-03$ & $2.50 \mathrm{E}-03$ & $2.50 \mathrm{E}-03$ & $2.50 \mathrm{E}-03$ & $250 \mathrm{E}-03$ & Strenge 1989 \\
\hline \multicolumn{7}{|l|}{ Organics } \\
\hline 1,2,4-Trichlorobenzene & $3.20 \mathrm{E}-02$ & $3.20 \mathrm{E}-02$ & $3.20 \mathrm{E}-02$ & $3.20 \mathrm{E}-02$ & $3.20 \mathrm{E}-02$ & Strenge 1989 \\
\hline $\begin{array}{l}\text { 1,2-Dichloroethene } \\
\text { (total) }\end{array}$ & $1.40 \mathrm{E}+00$ & $1.40 \mathrm{E}+00$ & $1.40 \mathrm{E}+00$ & $1.40 \mathrm{E}+00$ & $1.40 \mathrm{E}+00$ & Strenge 1989 \\
\hline Aroclor-1254 & $3.10 \mathrm{E}-03$ & $3.10 \mathrm{E}-03$ & $3.10 \mathrm{E}-03$ & $3.10 \mathrm{E}-03$ & $3.10 \mathrm{E}-03$ & Strenge 1989 \\
\hline Aroclor-1260 & $7.10 E-04$ & $7.10 \mathrm{E}-04$ & $7.10 \mathrm{E}-04$ & $7.10 \mathrm{E}-04$ & $7.10 \mathrm{E}-04$ & Strenge 1989 \\
\hline Benzo(a)anthracene & $5.60 \mathrm{E}-03$ & $5.60 \mathrm{E}-03$ & $5.60 \mathrm{E}-03$ & $5.60 \mathrm{E}-03$ & $5.60 \mathrm{E}-03$ & Strenge 1989 \\
\hline Benzo(a)pyrene & $3.00 \mathrm{E}-03$ & $3.00 \mathrm{E}-03$ & $3.00 \mathrm{E}-03$ & $3.00 \mathrm{E}-03$ & $3.00 \mathrm{E}-03$ & Strenge 1989 \\
\hline Benzo(b)tuoranthene & $3.00 \mathrm{E}-03$ & $3.00 \mathrm{E}-03$ & $3.00 E-03$ & $3.00 \mathrm{E}-03$ & $3.00 \mathrm{E}-03$ & Strenge 1989 \\
\hline $\begin{array}{l}\text { Bis(2-ethylhexyl) } \\
\text { Phthalate }\end{array}$ & $2.70 \mathrm{E}-05$ & $2.70 \mathrm{E}-05$ & 2.70E-05 & $2.70 \mathrm{E}-05$ & $2.70 \mathrm{E}-05$ & Strenge 1989 \\
\hline Bromodichloromethane & $4.90 \mathrm{E}-01$ & $4.90 \mathrm{E}-01$ & $4.90 \mathrm{E}-01$ & $4.90 \mathrm{E}-01$ & $4.90 E-01$ & Strenge 1989 \\
\hline Carbon tetrachloride & $2.90 \mathrm{E}-01$ & $2.90 \mathrm{E}-01$ & $2.90 \mathrm{E}-01$ & $2.90 \mathrm{E}-01$ & $2.90 \mathrm{E}-01$ & Strenge 1989 \\
\hline Chloroform & $7.00 \mathrm{E}-01$ & $7.00 \mathrm{E}-01$ & $7.00 \mathrm{E}-01$ & $7.00 \mathrm{E}-01$ & $7.00 \mathrm{E}-01$ & Strenge 1989 \\
\hline Dibenzo(a,h)anthracene & $5.50 \mathrm{E}-03$ & $5.50 \mathrm{E}-03$ & $5.50 E-03$ & $5.50 \mathrm{E}-03$ & $5.50 \mathrm{E}-03$ & Strenge 1989 \\
\hline Indeno(1,2,3-cd)pyrene & $1.70 \mathrm{E}-03$ & $1.70 \mathrm{E}-03$ & $1.70 \mathrm{E}-03$ & $1.70 \mathrm{E}-03$ & $1.70 \mathrm{E}-03$ & Strenge 1989 \\
\hline Methylene chloride & $1.70 \mathrm{E}+00$ & $1.70 \mathrm{E}+00$ & $1.70 \mathrm{E}+00$ & $1.70 \mathrm{E}+00$ & $1.70 \mathrm{E}+00$ & Strenge 1989 \\
\hline Tetrachloroethene & $3.00 \mathrm{E}-01$ & $3.00 \mathrm{E},-01$ & $3.00 \mathrm{E}-01$ & $3.00 \mathrm{E}-01$ & 3.00E-01 & Strenge 1989 \\
\hline
\end{tabular}


Table C.20. Concentrations of BCV OU2 COPCs in vegetables and fruits

\begin{tabular}{|c|c|c|c|c|c|c|c|}
\hline Analyte & $\begin{array}{l}\text { Concentration in } \\
\text { vegetable/fruil } \\
\text { resulting from } \\
\text { unirrigated soil }\end{array}$ & $\begin{array}{l}\text { Concentration } \\
\text { in vegetableffruit } \\
\text { resulting from } \\
\text { irrigated soil }\end{array}$ & $\begin{array}{l}\text { Concentration } \\
\text { in vegetable/fruit } \\
\text { resulting from } \\
\text { direct inrigation }\end{array}$ & $\begin{array}{l}\text { Percent of CV } \\
\text { resulting from } \\
\text { unimigated soil }\end{array}$ & $\begin{array}{l}\text { Percent of CV } \\
\text { resulting from } \\
\text { irrigated soil" }\end{array}$ & $\begin{array}{l}\text { Percent of } \mathrm{CV} \\
\text { resulting from } \\
\text { direct irrigation }\end{array}$ & $\begin{array}{l}\text { Total } \\
\text { vegetable/fruit } \\
\text { concentration } \\
\text { (CV) }\end{array}$ \\
\hline \multicolumn{8}{|c|}{ RSA } \\
\hline \multicolumn{8}{|c|}{ Leafy Vegetables } \\
\hline \multicolumn{8}{|l|}{ Inorganics (mg/kg) } \\
\hline ANTIMONY & $0.00 \mathrm{E}+00$ & 8.61E-05 & $1.76 \mathrm{E}-01$ & $0.00 \%$ & $0.05 \%$ & $99.95 \%$ & $1.76 \mathrm{E}-01$ \\
\hline ARSENIC & $0.00 \mathrm{E}+00$ & $8.53 E-05$ & $1.92 \mathrm{E}-01$ & $0.00 \%$ & $0.04 \%$ & $99.96 \%$ & $1.92 \mathrm{E}-01$ \\
\hline BARIUM & 5.75E-01 & $0.00 \mathrm{E}+00$ & $0.00 \mathrm{E}+00$ & $100.00 \%$ & $0.00 \%$ & $0.00 \%$ & $5.75 E-01$ \\
\hline BERYLLIUM & 4.94E-04 & $2.91 \mathrm{E}-08$ & $1.39 \mathrm{E}-03$ & $26.18 \%$ & $0.00 \%$ & $73.82 \%$ & $1.89 \mathrm{E}-03$ \\
\hline CADMIUM & $1.48 \mathrm{E}+00$ & $1.75 E-04$ & $1.32 E-02$ & $99.11 \%$ & $0.01 \%$ & $0.88 \%$ & $1.49 \mathrm{E}+00$ \\
\hline CHROMIUM VI & $0.00 \mathrm{E}+00$ & $1.11 E-06$ & $1.00 \mathrm{E}-01$ & $0.00 \%$ & $0.00 \%$ & $100.00 \%$ & $1.00 \mathrm{E}-01$ \\
\hline MANGANESE & $2.70 E+01$ & $2.76 \mathrm{E}-02$ & $2.07 E+01$ & $56.53 \%$ & $0.06 \%$ & $43.42 \%$ & $4.77 E+01$ \\
\hline MERCURY & $7.60 \mathrm{E}-01$ & $0.00 \mathrm{E}+00$ & $0.00 \mathrm{E}+00$ & $100.00 \%$ & $0.00 \%$ & $0.00 \%$ & $7.60 \mathrm{E}-01$ \\
\hline NICKEL & $8.76 \mathrm{E}-01$ & $0.00 \mathrm{E}+00$ & $0.00 \mathrm{E}+00$ & $100.00 \%$ & $0.00 \%$ & $0.00 \%$ & $8.76 \mathrm{E}-01$ \\
\hline NITRATE & $0.00 \mathrm{E}+00$ & $0.00 E+00$ & $2.76 \mathrm{E}+02$ & $0.00 \%$ & $0.00 \%$ & $100.00 \%$ & $2.76 \mathrm{E}+02$ \\
\hline URANIUM (SOLUBLE SALTS) & $1.84 \mathrm{E}-02$ & $1.44 \mathrm{E}-06$ & $1.30 \mathrm{E}-02$ & $58.65 \%$ & $0.00 \%$ & $41.34 \%$ & $3.14 \mathrm{E}-02$ \\
\hline \multicolumn{8}{|l|}{ Radionuclides (pCi/g) } \\
\hline CESIUM-137 & $0.00 \mathrm{E}+00$ & $2.76 \mathrm{E}-02$ & $3.11 \mathbb{E}+02$ & $0.00 \%$ & $0.01 \%$ & $99.99 \%$ & $3.11 E+02$ \\
\hline STRONTIUM-90 & $0.00 \mathrm{E}+00$ & $5.76 \mathrm{E}+00$ & $6.48 \mathrm{E}+02$ & $0.00 \%$ & $0.88 \%$ & $99.12 \%$ & $6.54 \mathrm{E}+02$ \\
\hline TECHNETIUM-99 & $0.00 E+00$ & $1.43 E+02$ & $1.28 \mathrm{E}+04$ & $0.00 \%$ & $1.10 \%$ & $98.90 \%$ & $1.30 \mathrm{E}+04$ \\
\hline THORIUM-232 & $1.30 \mathrm{E}-02$ & $0.00 \mathrm{E}+00$ & $0.00 E+00$ & $100.00 \%$ & $0.00 \%$ & $0.00 \%$ & $1.30 E-02$ \\
\hline URANIUM-234 & $7.03 E_{-}-03$ & $4.86 \mathrm{E}-04$ & $4.38 \mathrm{E}+00$ & $0.16 \%$ & $0.01 \%$ & $99.83 \%$ & $4.38 \mathrm{E}+00$ \\
\hline URANIUM-238 & $0.00 \mathrm{~F}+00$ & $4.82 \mathrm{E}-04$ & $4.34 E+\infty 0$ & $0.00 \%$ & $0.01 \%$ & $99.99 \%$ & $4.34 E+00$ \\
\hline
\end{tabular}


Table C.20. (continued)

\begin{tabular}{|c|c|c|c|c|c|c|c|}
\hline Analyte & $\begin{array}{l}\text { Concentration in } \\
\text { vegetable/fruit } \\
\text { resulting from } \\
\text { unirrigated soil }\end{array}$ & $\begin{array}{l}\text { Concentration } \\
\text { in vegetable/fruil } \\
\text { resulting from } \\
\text { irrigated soil }\end{array}$ & $\begin{array}{l}\text { Concentration } \\
\text { in vegetable/fruil } \\
\text { resulting from } \\
\text { direct inrigation }\end{array}$ & $\begin{array}{l}\text { Percent of } \mathrm{CV} \\
\text { resulling from } \\
\text { unirrigated soil }\end{array}$ & $\begin{array}{l}\text { Percent of CV } \\
\text { resulting from } \\
\text { irrigated soil }^{\text {e }}\end{array}$ & $\begin{array}{l}\text { Percent of } \mathrm{CV} \\
\text { resulting from } \\
\text { direct irrigation }\end{array}$ & $\begin{array}{l}\text { Total } \\
\text { vegetable/fruit } \\
\text { concentration } \\
\text { (CV) }\end{array}$ \\
\hline
\end{tabular}

Organics (mg/kg)

\begin{tabular}{|c|c|c|c|c|c|c|c|}
\hline 1,2,4-TRICHLOROBENZENE & $1.38 \mathrm{E}-01$ & $0.00 \mathrm{E}+00$ & $0.00 \mathrm{E}+00$ & $100.00 \%$ & $0.00 \%$ & $0.00 \%$ & $1.38 \mathrm{E}-01$ \\
\hline 1,2-DICILOROETHENE (TOTAL) & $1.01 \mathrm{E}-01$ & $7.56 \mathrm{E}-03$ & $1.22 \mathrm{E}-01$ & $43.92 \%$ & $3.29 \%$ & $52.80 \%$ & $2.30 \mathrm{E}-01$ \\
\hline AROCLOR-1254 & $3.06 \mathrm{E}-03$ & $0.00 \mathrm{E}+00$ & $0.00 \mathrm{E}+00$ & $100.00 \%$ & $0.00 \%$ & $0.00 \%$ & $3.06 \mathrm{E}-03$ \\
\hline BENZO(A)PYRENE & $1.43 \mathrm{E}-03$ & $0.00 E+\infty 0$ & $0.00 \mathrm{E}+00$ & $100.00 \%$ & $0.00 \%$ & $0.00 \%$ & $1.43 E-03$ \\
\hline BIS(2-ETHYLHEXYL)PHTHALATE & $1.77 \mathrm{E}-04$ & $0.00 \mathrm{E}+00$ & $0.00 \mathrm{E}+00$ & $100.00 \%$ & $0.00 \%$ & $0.00 \%$ & 1.77E-04 \\
\hline CARBON TETRACHLORIDE & $0.00 E+00$ & $2.22 \mathrm{E}-04$ & $1.72 E-02$ & $0.00 \%$ & $1.27 \%$ & $98.73 \%$ & $1.74 \mathrm{E}-02$ \\
\hline DIBENZO(A,H)ANTHRACENE & $1.60 \mathrm{E}-03$ & $0.00 F^{\circ}+m$ & $0.00 \mathrm{E}+00$ & $100.00 \%$ & $0.00 \%$ & $0.00 \%$ & $1.60 \mathrm{E}-03$ \\
\hline TETRACHLOROETHENE & $4.86 \mathrm{E}-02$ & $0.00 E+00$ & $0.00 \mathrm{E}+00$ & $100.00 \%$ & $0.00 \%$ & $0.00 \%$ & $4.86 \mathbb{E}-02$ \\
\hline
\end{tabular}

Inorganics ( $\mathrm{mg} / \mathrm{kg}$ )

\begin{tabular}{|c|c|c|c|c|c|c|c|}
\hline ANTIMONY & $0.00 E+\infty 0$ & 8.61E-05 & $0.00 E+00$ & $0.00 \%$ & $100.00 \%$ & $0.00 \%$ & $8.61 \mathrm{E}-05$ \\
\hline ARSENIC & $0.00 \mathrm{E}+00$ & 8.53E-05 & $0.00 E+00$ & $0.00 \%$ & $100.00 \%$ & $0.00 \%$ & 8.53E-05 \\
\hline BARIUM & $5.75 \mathrm{E}-91$ & $0.00 \mathrm{E}+00$ & $0.00 \mathrm{E}+00$ & $100.00 \%$ & $0.00 \%$ & $0.00 \%$ & $5.75 \mathrm{E}-01$ \\
\hline BERYLLIUM & $4.94 \mathrm{E}-04$ & $2.91 E-08$ & $0.00 \mathrm{E}+00$ & $99.99 \%$ & $0.01 \%$ & $0.00 \%$ & $4.94 \mathrm{E}-04$ \\
\hline CADMIUM & $1.48 \mathrm{E}+00$ & $1.75 \mathrm{E}-04$ & $0.00 \mathrm{E}+\infty$ & $99.99 \%$ & $0.01 \%$ & $0.00 \%$ & $1.48 \mathrm{E}+00$ \\
\hline CHROMIUM VI & $0.00 \mathrm{E}+00$ & $1.11 E-06$ & $0.00 \mathrm{E}+00$ & $0.00 \%$ & $100.00 \%$ & $0.00 \%$ & $1.11 \mathrm{E}-06$ \\
\hline MANGANESE & $2.70 E+01$ & $2.76 \mathrm{E}-02$ & $0.00 E+00$ & $99.90 \%$ & $0.10 \%$ & $0.00 \%$ & $2.70 \mathrm{E}+01$ \\
\hline MERCURY & $7.60 \mathrm{E}-01$ & $0.00 \mathrm{E}+00$ & $0.00 E+00$ & $100.00 \%$ & $0.00 \%$ & $0.00 \%$ & $7.60 \mathrm{E}-01$ \\
\hline
\end{tabular}


Table C.20. (continued)

\begin{tabular}{|c|c|c|c|c|c|c|c|}
\hline Analyte & $\begin{array}{l}\text { Concentration in } \\
\text { vegetableffruil } \\
\text { resulting from } \\
\text { unirrigated soil }\end{array}$ & $\begin{array}{l}\text { Concentration } \\
\text { in vegetable/fruit } \\
\text { resulting from } \\
\text { irrigated soil }\end{array}$ & $\begin{array}{l}\text { Concentration } \\
\text { in vegetable/fruit } \\
\text { resulting from } \\
\text { direct irrigation }\end{array}$ & $\begin{array}{l}\text { Percent of } \mathrm{CV} \\
\text { resulting from } \\
\text { unimigated soil }\end{array}$ & $\begin{array}{l}\text { Percent of } \mathrm{CV} \\
\text { resulting from } \\
\text { irrigated soil }\end{array}$ & $\begin{array}{l}\text { Percent of CV } \\
\text { resulting from } \\
\text { direct irrigation }\end{array}$ & $\begin{array}{l}\text { Total } \\
\text { vegetable/fruit } \\
\text { concentration } \\
\text { (CV) }\end{array}$ \\
\hline NICKEL & $8.76 \mathrm{E}-01$ & $0.00 \mathrm{E}+00$ & $0.00 \mathrm{E}+00$ & $100.00 \%$ & $0.00 \%$ & $0.00 \%$ & $8.76 \mathrm{E}-01$ \\
\hline NITRATE & $0.00 \mathrm{E}+00$ & $0.00 \mathrm{E}+\infty$ & $0.00 \mathrm{E}+00$ & NA & NA & NA & $0.00 \mathrm{E}+00$ \\
\hline URANIUM (SOLUBLE SALTS) & $1.84 \mathrm{E}-02$ & $1.44 \mathrm{E}-06$ & $0.00 \mathrm{E}+00$ & $99.99 \%$ & $0.01 \%$ & $0.00 \%$ & $1.84 \mathrm{E}-02$ \\
\hline \multicolumn{8}{|l|}{ Radionuclides ( $\mathrm{pCi} / \mathrm{g}$ ) } \\
\hline CESIUM-137 & $0.00 \mathrm{E}+00$ & $2.76 \mathrm{E}-02$ & $0.00 E+00$ & $0.00 \%$ & $100.00 \%$ & $0.00 \%$ & $2.76 \mathrm{E}-02$ \\
\hline STRONTIUM-90 & $0.00 E+00$ & $5.76 \mathrm{E}+00$ & $0.00 \mathrm{E}+00$ & $0.00 \%$ & $100.00 \%$ & $0.00 \%$ & $5.76 \mathrm{E}+00$ \\
\hline TECHNETIUM-99 & $0.00 \mathrm{E}+00$ & $1.43 E+02$ & $0.00 \mathrm{E}+00$ & $0.00 \%$ & $100.00 \%$ & $0.00 \%$ & $143 \mathrm{E}+02$ \\
\hline THORIUM-232 & $1.30 E-02$ & $0.00 \mathrm{E}+00$ & $0.00 \mathrm{E}+00$ & $100.00 \%$ & $0.00 \%$ & $0.00 \%$ & $1.30 \mathrm{E}-02$ \\
\hline URANIUM-234 & $7.03 \mathrm{E}-03$ & $4.86 \mathrm{E}-04$ & $0.00 E+00$ & $93.53 \%$ & $6.47 \%$ & $0.00 \%$ & $7.51 \mathrm{E}-03$ \\
\hline URANIUM-238 & $0.00 \mathrm{E}+00$ & $4.82 \mathrm{E}-04$ & $0.00 \mathrm{E}+00$ & $0.00 \%$ & $100.00 \%$ & $0.00 \%$ & $4.82 \mathrm{E}-04$ \\
\hline \multicolumn{8}{|l|}{ Organics (mg/mg) } \\
\hline 1,2,4-TRICHLOROBENZENE & $1.38 \mathrm{E}-01$ & $0.00 \mathrm{E}+00$ & $0.00 \mathrm{E}+00$ & $100.00 \%$ & $0.00 \%$ & $0.00 \%$ & 1.38E-01 \\
\hline 1,2-DICHLOROETHENE (TOTAL) & $1.01 \mathrm{E}-01$ & $7.56 \mathrm{E}-03$ & $0.00 \mathrm{E}+00$ & $93,04 \%$ & $6.96 \%$ & $0.00 \%$ & $1.09 \mathrm{E}-01$ \\
\hline AROCLOR-1254 & $3.06 \mathrm{E}-03$ & $0.00 E+00$ & $0.00 \mathrm{E}+00$ & $100.00 \%$ & $0.00 \%$ & $0.00 \%$ & $3.06 \mathrm{E}-03$ \\
\hline BENZO(a)PYRENE & $1.43 \mathrm{E}-03$ & $0.00 \mathrm{E}+00$ & $0.00 E+00$ & $100.00 \%$ & $0.00 \%$ & $0.00 \%$ & $1.43 \mathrm{E}-03$ \\
\hline BIS(2-ETHYLHEXYL)PHTHALATE & $1.77 \mathrm{E}-04$ & $0.00 E+\infty 0$ & $0.00 E+00$ & $100.00 \%$ & $0.00 \%$ & $0.00 \%$ & $1.77 \mathrm{E}-04$ \\
\hline CARBON TETRACHLORIDE & $0.00 \mathrm{E}+00$ & $2.22 E-04$ & $0.00 E+00$ & $0.00 \%$ & $100.00 \%$ & $0.00 \%$ & $2.22 \mathrm{E}-04$ \\
\hline DIBENZO(A,H)ANTHRACENE & $1.60 \mathrm{E}-03$ & $0.00 \mathrm{E}+00$ & $0.00 \mathrm{E}+00$ & $100.00 \%$ & $0.00 \%$ & $0.00 \%$ & 1.60E-03 \\
\hline TETRACHLOROETHENE & $4.86 \mathrm{E}-02$ & $0.00 \mathrm{E}+00$ & $0.00 \mathrm{E}+00$ & $100.00 \%$ & $0.00 \%$ & $0.00 \%$ & $4.86 E \cdot 02$ \\
\hline
\end{tabular}


Table C.20. (continued)

\begin{tabular}{|c|c|c|c|c|c|c|c|}
\hline Analyie & $\begin{array}{l}\text { Concentration in } \\
\text { vegetable/fruit } \\
\text { resulting from } \\
\text { unirrigated soil }\end{array}$ & $\begin{array}{l}\text { Concentration } \\
\text { in vegetable/fruil } \\
\text { resulting from } \\
\text { irrigated soil }\end{array}$ & $\begin{array}{l}\text { Concentration } \\
\text { in vegetable/fruit } \\
\text { resulting from } \\
\text { direct irrigation }\end{array}$ & $\begin{array}{l}\text { Percent of CV } \\
\text { resulting from } \\
\text { unirrigated soil }\end{array}$ & $\begin{array}{l}\text { Percent of CV } \\
\text { resulting from } \\
\text { irrigated soil }^{\mathrm{B}}\end{array}$ & $\begin{array}{l}\text { Percent of CV } \\
\text { resulting from } \\
\text { direct irrigation }\end{array}$ & $\begin{array}{l}\text { Total } \\
\text { vegetable/fruit } \\
\text { concentration }^{b} \\
\text { (CV) }\end{array}$ \\
\hline \multicolumn{8}{|c|}{ Legumes } \\
\hline \multicolumn{8}{|l|}{ Inorganics (mg/kg) } \\
\hline ANTIMONY & $0.00 \mathrm{E}+00$ & $8.61 \mathrm{E}-05$ & $5.87 \mathrm{E}-02$ & $0.00 \%$ & $0.15 \%$ & $99.85 \%$ & $5.88 \mathrm{E}-02$ \\
\hline ARSENIC & $0.00 \mathrm{E}+\infty$ & 8.53E-05 & $6.40 \mathrm{E}-02$ & $0.00 \%$ & $0.13 \%$ & $99.87 \%$ & $6.41 \mathrm{E}-02$ \\
\hline BARIUM & $5.75 \mathrm{E}-01$ & $0.00 \mathrm{E}+00$ & $0.00 \mathrm{E}+00$ & $100.00 \%$ & $0.00 \%$ & $0.00 \%$ & $5.75 \mathrm{E}-01$ \\
\hline BERYLLIUM & 4.94E-04 & 2.91E-08 & $4.64 \mathrm{E}-04$ & $51.55 \%$ & $0.00 \%$ & $48.45 \%$ & $9.57 \mathrm{E}-04$ \\
\hline CADMIUM & $1.48 \mathrm{E}+00$ & $1.75 \mathrm{E}-04$ & $4.39 \mathrm{E}-03$ & $99.69 \%$ & $0.01 \%$ & $0.30 \%$ & $1.48 \mathrm{E}+\infty$ \\
\hline CHROMIUM VI & $0.00 \mathrm{E}+00$ & $1.11 \mathrm{E}-06$ & $3.34 \mathrm{E}-02$ & $0.00 \%$ & $0.00 \%$ & $100.00 \%$ & $3.34 \mathrm{E}-02$ \\
\hline MANGANESE & $2.70 \mathrm{E}+01$ & $2.76 \mathrm{E}-02$ & $6.90 E+00$ & $79.55 \%$ & $0.08 \%$ & $20.37 \%$ & $3.39 \mathrm{E}+01$ \\
\hline MERCURY & $7.60 \mathrm{E}-01$ & $0.00 \mathrm{E}+\infty 0$ & $0.00 \mathrm{E}+\infty 0$ & $100.00 \%$ & $0.00 \%$ & $0.00 \%$ & $7.60 \mathrm{E}-01$ \\
\hline NICKEL & $8.76 \mathrm{E}-01$ & $0.00 \mathrm{E}+\infty$ & $0.00 E+\infty 0$ & $100.00 \%$ & $0.00 \%$ & $0.00 \%$ & $8.76 \mathrm{E}-01$ \\
\hline NITRATE & $0.00 \mathrm{E}+00$ & $0.00 \mathrm{E}+00$ & $9.19 E+01$ & $0.00 \%$ & $0.00 \%$ & $100.00 \%$ & $9.19 E+01$ \\
\hline URANIUM (SOLUBLE SALTS) & $1.84 \mathrm{E}-02$ & $1.44 \mathrm{E}-06$ & $4.32 \mathrm{E}-03$ & $80.97 \%$ & $0.01 \%$ & $19.03 \%$ & $2.27 \mathrm{E}-02$ \\
\hline \multicolumn{8}{|l|}{ Radionuclides (pCi/g) } \\
\hline CESIUM-137 & $0.00 E+00$ & $2.76 \mathrm{E}-02$ & $1.04 E+02$ & $0.00 \%$ & $0.03 \%$ & $99.97 \%$ & $1.04 E+02$ \\
\hline STRONTIUM-90 & $0.00 E+00$ & $5.76 \mathrm{E}+00$ & $2.16 \mathrm{E}+02$ & $0.00 \%$ & $2.60 \%$ & $97.40 \%$ & $2.22 \mathrm{E}+02$ \\
\hline TECHNETIUM-99 & $0.00 \mathrm{E}+00$ & $1.43 \mathrm{E}+02$ & $4.28 \mathrm{E}+03$ & $0.00 \%$ & $3.23 \%$ & $96.77 \%$ & $4.42 E+03$ \\
\hline THORIUM-232 & $1.30 \mathrm{E}-02$ & $0.00 E+00$ & $0.00 \mathrm{E}+00$ & $100.00 \%$ & $0.00 \%$ & $0.00 \%$ & $1.30 \mathrm{E}-02$ \\
\hline URANIUM-234 & $7.03 \mathrm{E}-03$ & $4.86 \mathrm{E}-04$ & $1.46 \mathrm{E}+00$ & $0.48 \%$ & $0.03 \%$ & $99.49 \%$ & $1.47 \mathrm{E}+\infty 0$ \\
\hline
\end{tabular}


Table C.20. (continued)

\begin{tabular}{|c|c|c|c|c|c|c|c|}
\hline Analyte & $\begin{array}{l}\text { Concentration in } \\
\text { vegetable/fruil } \\
\text { resulting from } \\
\text { unirrigated soil }\end{array}$ & $\begin{array}{l}\text { Concentration } \\
\text { in vegetable/fruit } \\
\text { resulting from } \\
\text { irrigated soil }\end{array}$ & $\begin{array}{l}\text { Concentration } \\
\text { in vegetable/fruil } \\
\text { resulting from } \\
\text { direct irrigation }\end{array}$ & $\begin{array}{l}\text { Percent of } \mathrm{CV} \\
\text { resulting from } \\
\text { uninrigated soil }\end{array}$ & $\begin{array}{l}\text { Percent of CV } \\
\text { resulting from } \\
\text { irrigated soil }\end{array}$ & $\begin{array}{l}\text { Percent of } \mathrm{CV} \\
\text { resulting from } \\
\text { direct irrigation }\end{array}$ & $\begin{array}{l}\text { Total } \\
\text { vegetable/fruit } \\
\text { concentration } \\
\text { (CV) }\end{array}$ \\
\hline URANIUM-238 & $0.00 \mathrm{E}+00$ & $4.82 \mathrm{E}-04$ & $1.45 E+00$ & $0.00 \%$ & $0.03 \%$ & $99.97 \%$ & $1.45 \mathrm{E}+\infty 0$ \\
\hline \multicolumn{8}{|l|}{ Organics (mg/kg) } \\
\hline 1,2,4-TRICHLOROBENZENE & $1.38 \mathrm{E}-01$ & $0.00 E+00$ & $0.00 \mathrm{E}+00$ & $100.00 \%$ & $0.00 \%$ & $0.00 \%$ & $1.38 \mathrm{E}-01$ \\
\hline 1,2-DICHLOROETHENE (TOTAL) & 1.01E-01 & $7.56 \mathrm{E}-03$ & $4.05 E-02$ & $67.77 \%$ & $5.07 \%$ & $27.16 \%$ & $1.49 \mathrm{E}-01$ \\
\hline AROCLOR-1254 & $3.06 \mathrm{E}-03$ & $0.00 \mathrm{E}+00$ & $0.00 \mathrm{E}+00$ & $100.00 \%$ & $0.00 \%$ & $0.00 \%$ & $3.06 \mathrm{E}-03$ \\
\hline BENZO(a)PYRENE & $1.43 \mathrm{E}-03$ & $0.00 \mathrm{E}+00$ & $0.00 E+00$ & $100.00 \%$ & $0.00 \%$ & $0.00 \%$ & $1.43 \mathrm{E}-03$ \\
\hline BIS(2-ETHYLHEXYL)PHTHALATE & $1.77 \mathrm{E}-04$ & $0.00 \mathrm{E}+00$ & $0.00 \mathrm{E}+00$ & $100.00 \%$ & $0.00 \%$ & $0.00 \%$ & $1.77 \mathrm{E}-04$ \\
\hline CARBON TETRACHLORIDE & $0.00 \mathrm{E}+00$ & $2.22 \mathrm{E}-04$ & $5.73 E-03$ & $0.00 \%$ & $3.72 \%$ & $96.28 \%$ & $5.95 \mathrm{E}-03$ \\
\hline DIBENZO(A,H)ANTHRACENE & $1.60 \mathrm{E}-03$ & $0.00 \mathrm{E}+00$ & $0.00 \mathrm{E}+00$ & $100.00 \%$ & $0.00 \%$ & $0.00 \%$ & $1.60 \mathrm{E}-03$ \\
\hline TETRACHLOROETHENE & $4.86 \mathrm{E}-02$ & $0.00 \mathrm{E}+00$ & $0.00 \mathrm{E}+00$ & $100.00 \%$ & $0.00 \%$ & $0.00 \%$ & $4.86 \mathrm{E}-02$ \\
\hline \multicolumn{8}{|c|}{ Fruiting Vegelables } \\
\hline \multicolumn{8}{|l|}{ Inorganics (mg/kg) } \\
\hline ANTMMONY & $0.00 E+00$ & $8.61 \mathrm{E}-05$ & $5.87 \mathrm{E}-02$ & $0.00 \%$ & $0.15 \%$ & $99.85 \%$ & $5.88 E-02$ \\
\hline ARSENIC & $0.00 E+00$ & 8.53E-05 & $6.40 \mathrm{E}-02$ & $0.00 \%$ & $0.13 \%$ & $99.87 \%$ & $6.41 \mathrm{E}-02$ \\
\hline BARIUM & $5.75 E-01$ & $0.00 E+00$ & $0.00 E+00$ & $100.00 \%$ & $0.00 \%$ & $0.00 \%$ & $5.75 \mathbb{E}-01$ \\
\hline BERYLLIUM & $4.94 \mathrm{E}-04$ & $2.91 \mathrm{E}-08$ & $4.64 \mathrm{E}-04$ & $51.55 \%$ & $0.00 \%$ & $48.45 \%$ & $9.57 \mathrm{E}-04$ \\
\hline CADMIUM & $1.48 E+00$ & $1.75 \mathrm{E}-04$ & $4.39 E-03$ & $99.69 \%$ & $0.01 \%$ & $0.30 \%$ & $1.48 E+00$ \\
\hline CHROMIUM VI & $0.00 E+00$ & $1.11 E-16$ & $3.34 \mathrm{E}-02$ & $0.00 \%$ & $0.00 \%$ & $100.00 \%$ & $3.34 \mathrm{E}-02$ \\
\hline MANGANESE & $2.70 E+01$ & $2.76 \mathrm{E} \cdot 02$ & $6.90 E+00$ & $79.55 \%$ & $0.08 \%$ & $20.37 \%$ & $3.39 \mathrm{E}+01$ \\
\hline
\end{tabular}


Table C.20. (continued)

\begin{tabular}{|c|c|c|c|c|c|c|c|}
\hline Analyte & $\begin{array}{l}\text { Concentration in } \\
\text { vegetable/truit } \\
\text { resulting from } \\
\text { unirrigated soil }\end{array}$ & $\begin{array}{l}\text { Concentration } \\
\text { in vegetable/fruit } \\
\text { resulting from } \\
\text { irrigated soil }\end{array}$ & $\begin{array}{l}\text { Concentration } \\
\text { in vegetableffruit } \\
\text { resulting from } \\
\text { direct irrigation }\end{array}$ & $\begin{array}{l}\text { Percent of CV } \\
\text { resulting from } \\
\text { unirrigated soil }\end{array}$ & $\begin{array}{l}\text { Percent of } \mathrm{CV} \\
\text { resulting from } \\
\text { irrigated soil" }\end{array}$ & $\begin{array}{l}\text { Percent of } \mathrm{CV} \\
\text { resulting from } \\
\text { direct irrigation }^{\mathbf{a}}\end{array}$ & $\begin{array}{l}\text { Total } \\
\text { vegetable/fruit } \\
\text { concentration } \\
\text { (CV) }\end{array}$ \\
\hline MERCURY & $7.60 \mathrm{E}-01$ & $0.00 \mathrm{E}+00$ & $0.00 \mathrm{E}+00$ & $100.00 \%$ & $0.00 \%$ & $0.00 \%$ & $7.60 \mathrm{E}-01$ \\
\hline NICKEL & $8.76 \mathrm{E}-01$ & $0.00 \mathrm{E}+\infty$ & $0.00 \mathrm{E}+00$ & $100.00 \%$ & $0.00 \%$ & $0.00 \%$ & $8.76 \mathrm{E}-01$ \\
\hline NITRATE & $0.00 \mathrm{E}+00$ & $0.00 \mathrm{E}+00$ & $9.19 \mathrm{E}+01$ & $0.00 \%$ & $0.00 \%$ & $100.00 \%$ & $9.19 \mathrm{E}+01$ \\
\hline URANIUM (SOLUBLE SALTS) & $1.84 \mathrm{E}-02$ & $1.44 \mathrm{E}-06$ & $4.32 \mathrm{E}-03$ & $80.97 \%$ & $0.01 \%$ & $19.03 \%$ & $2.27 \mathrm{E} \cdot 02$ \\
\hline \multicolumn{8}{|l|}{ Radionuclides (pCi/g) } \\
\hline CESIUM-137 & $0.00 \mathrm{E}+00$ & $2.76 \mathrm{E}-02$ & $1.04 E+02$ & $0.00 \%$ & $0.03 \%$ & $99.97 \%$ & $1.04 \mathrm{E}+02$ \\
\hline STRONTIUM-90 & $0.00 \mathrm{E}+\infty 0$ & $5.76 \mathrm{E}+00$ & $2.16 \mathrm{E}+02$ & $0.00 \%$ & $2.60 \%$ & $97.40 \%$ & $2.22 \mathrm{E}+02$ \\
\hline TECHNETIUM-99 & $0.00 \mathrm{E}+00$ & $1.43 E+02$ & $4.28 E+03$ & $0.00 \%$ & $3.23 \%$ & $96.77 \%$ & $4.42 \mathrm{E}+03$ \\
\hline THORIUM-232 & $1.30 E-02$ & $0.00 E+00$ & $0.00 E+00$ & $100.00 \%$ & $0.00 \%$ & $0.00 \%$ & $1.30 \mathrm{E}-02$ \\
\hline URANIUM-234 & $7.03 E-03$ & $4.86 \mathrm{E}-04$ & $1.46 \mathrm{E}+\infty$ & $0.48 \%$ & $0.03 \%$ & $99.49 \%$ & $1.47 \mathrm{E}+\infty 0$ \\
\hline URANIUM-238 & $0.00 \mathrm{E}+00$ & $4.82 \mathrm{E}-04$ & $1.45 E+00$ & $0.00 \%$ & $0.03 \%$ & $99.97 \%$ & $1.45 E+\infty 0$ \\
\hline \multicolumn{8}{|l|}{ Organics (mg/kg) } \\
\hline 1,2,4-TRICHLOROBENZENE & $1.38 \mathrm{E}-01$ & $0.00 \mathrm{E}+00$ & $0.00 \mathrm{E}+00$ & $100.00 \%$ & $0.00 \%$ & $0.00 \%$ & $1.38 \mathrm{E}-01$ \\
\hline 1,2-DICHLOROETHENE (TOTAL) & $1.01 E-01$ & $7.56 \mathrm{E}-03$ & $4.05 \mathrm{E}-02$ & $67.77 \%$ & $5.07 \%$ & $27.16 \%$ & $1.49 \mathrm{E}-01$ \\
\hline AROCLOR-1254 & $3.06 \mathrm{E}-03$ & $0.00 E+00$ & $0.00 \mathrm{E}+00$ & $100.00 \%$ & $0.00 \%$ & $0.00 \%$ & $3.06 \mathrm{E}-03$ \\
\hline BENZO(a)PYRENE & $1.43 E-03$ & $0.00 E+00$ & $0.00 \mathrm{E}+00$ & $100.00 \%$ & $0.00 \%$ & $0.00 \%$ & $1.43 E-03$ \\
\hline BIS(2-ETHYLHEXYL)PHTHALATE & $1.77 E-04$ & $0.00 \mathrm{E}+00$ & $0.00 E+00$ & $100.00 \%$ & $0.00 \%$ & $0.00 \%$ & $1.77 \mathrm{E}-04$ \\
\hline CARBON TETRACHLORIDE & $0.00 E+00$ & $2.22 \mathrm{E}-04$ & $5.73 E-03$ & $0.00 \%$ & $3.72 \%$ & $96.28 \%$ & $5.95 \mathrm{E}-03$ \\
\hline DIBENZO(A,H)ANTHRACENE & $1.60 \mathrm{E}-03$ & $0.00 \mathrm{E}+00$ & $0.00 \mathrm{E}+00$ & $100.00 \%$ & $0.00 \%$ & $0.00 \%$ & $1.60 \mathrm{E}-03$ \\
\hline
\end{tabular}


Table C.20. (continued)

\begin{tabular}{|c|c|c|c|c|c|c|c|}
\hline Analyte & $\begin{array}{l}\text { Concentration in } \\
\text { vegetable/fruit } \\
\text { resulting from } \\
\text { unirrigated soil }\end{array}$ & $\begin{array}{l}\text { Concentration } \\
\text { in vegetable/fruil } \\
\text { resulting from } \\
\text { irrigated soil }\end{array}$ & $\begin{array}{l}\text { Concentration } \\
\text { in vegetablefiruit } \\
\text { resulting from } \\
\text { direct irrigation }\end{array}$ & $\begin{array}{l}\text { Percent of } \mathrm{CV} \\
\text { resulting from } \\
\text { unirrigated soil }\end{array}$ & $\begin{array}{l}\text { Percent of CV } \\
\text { resulting from } \\
\text { irrigated soil }\end{array}$ & $\begin{array}{l}\text { Percent of CV } \\
\text { resulting from } \\
\text { direct irrigation }\end{array}$ & $\begin{array}{l}\text { Total } \\
\text { vegetable/fruit } \\
\text { concentration } \\
\text { (CV) }\end{array}$ \\
\hline TETRACHLOROETHENE & $4.86 \mathrm{E}-02$ & $0.00 \mathrm{E}+\infty$ & $0.00 \mathrm{E}+00$ & $100.00 \%$ & $0.00 \%$ & $0.00 \%$ & $4.86 \mathrm{E}-02$ \\
\hline \multicolumn{8}{|c|}{ Fruits } \\
\hline \multicolumn{8}{|l|}{ Inorganics (mg/kg) } \\
\hline ANTIMONY & $0.00 \mathrm{E}+\infty$ & 8.61E-05 & $5.87 \mathrm{E}-02$ & $0.00 \%$ & $0.15 \%$ & $99.85 \%$ & $5.88 \mathrm{E}-02$ \\
\hline ARSENIC & $0.00 E+00$ & $8.53 \mathrm{E}-05$ & $6.40 \mathrm{E}-02$ & $0.00 \%$ & $0.13 \%$ & $99.87 \%$ & $6.41 \mathrm{E}-02$ \\
\hline BARIUM & 5.75E-01 & $0.00 \mathrm{E}+00$ & $0.00 \mathrm{E}+00$ & $100.00 \%$ & $0.00 \%$ & $0.00 \%$ & $5.75 \mathrm{E}-01$ \\
\hline BERYLLIUM & 4.94E-04 & $2.91 \mathrm{E}-08$ & 4.64E-04 & 51.55\% & $0.00 \%$ & $48.45 \%$ & $9.57 \mathrm{E}-04$ \\
\hline CADMIUM & $1.48 \mathrm{E}+\infty$ & $1.75 E-04$ & $4.39 \mathrm{E}-03$ & $99.69 \%$ & $0.01 \%$ & $0.30 \%$ & $1.48 \mathrm{E}+00$ \\
\hline CHROMIUM VI & $0.00 \mathrm{E}+00$ & $1.11 \mathrm{E}-06$ & $3.34 \mathrm{E}-02$ & $0.00 \%$ & $0.00 \%$ & $100.00 \%$ & 3.34E-02 \\
\hline MANGANESE & $2.70 \mathrm{E}+01$ & $2.76 \mathrm{E}-02$ & $6.90 \mathrm{E}+00$ & $79.55 \%$ & $0.08 \%$ & $20.37 \%$ & $3.39 \mathrm{E}+01$ \\
\hline MERCURY & $7.60 \mathrm{E}-01$ & $0.00 \mathrm{E}+00$ & $0.00 \mathrm{E}+00$ & $100.00 \%$ & $0.00 \%$ & $0.00 \%$ & $7.60 \mathrm{E}-01$ \\
\hline NICKEL & $8.76 \mathrm{E}-01$ & $0.00 \mathrm{E}+\infty$ & $0.00 \mathrm{E}+00$ & $100.00 \%$ & $0.00 \%$ & $0.00 \%$ & $8.76 \mathrm{E}-01$ \\
\hline NITRATE & $0.00 \mathrm{E}+00$ & $0.00 \mathrm{E}+\infty 0$ & $9.19 E+01$ & $0.00 \%$ & $0.00 \%$ & $100.00 \%$ & $9.19 \mathrm{E}+01$ \\
\hline URANIUM (SOLUBLE SALTS) & $1.84 \mathrm{E}-02$ & $1.44 \mathrm{E}-06$ & 4.32E-03 & $80.97 \%$ & $0.01 \%$ & $19.03 \%$ & $2.27 \mathrm{E}-02$ \\
\hline \multicolumn{8}{|l|}{ Radionuclides (pCi/g) } \\
\hline CESIUM-137 & $0.00 \mathrm{E}+00$ & $2.76 \mathrm{E}-02$ & $1.04 \mathrm{E}+02$ & $0.00 \%$ & $0.03 \%$ & $99.97 \%$ & $1.04 \mathbb{E}+02$ \\
\hline STRONTIUM-90 & $0.00 E+00$ & $5.76 \mathrm{E}+00$ & $2.16 \mathrm{E}+02$ & $0.00 \%$ & $2.60 \%$ & $97.40 \%$ & $2.22 \mathrm{E}+02$ \\
\hline TECHNETIUM-99 & $0.00 \mathrm{E}+00$ & $1.43 E+02$ & $4.28 \mathrm{E}+03$ & $0.00 \%$ & $3.23 \%$ & $96.77 \%$ & $4.42 E+03$ \\
\hline THORIUM-232 & $1.30 \mathrm{E}-02$ & $0.00 \mathrm{E}+00$ & $0.00 \mathrm{E}+\infty 0$ & $100.00 \%$ & $0.00 \%$ & $0.00 \%$ & $1.30 \mathrm{E} \cdot 02$ \\
\hline
\end{tabular}


Table C.20. (continued)

\begin{tabular}{|c|c|c|c|c|c|c|c|}
\hline Analyte & $\begin{array}{l}\text { Concentration in } \\
\text { vegetableffruit } \\
\text { resulting from } \\
\text { unirrigated soil }\end{array}$ & $\begin{array}{l}\text { Concentration } \\
\text { in vegetable/fruit } \\
\text { resulting from } \\
\text { irrigated soil }\end{array}$ & $\begin{array}{l}\text { Concentration } \\
\text { in vegetable/fruit } \\
\text { resulting from } \\
\text { direct irrigation }\end{array}$ & $\begin{array}{l}\text { Percent of } \mathrm{CV} \\
\text { resulting from } \\
\text { unirrigated soil }\end{array}$ & $\begin{array}{l}\text { Percent of CV } \\
\text { resulting from } \\
\text { irrigated soil }^{\mathrm{s}}\end{array}$ & $\begin{array}{l}\text { Percent of CV } \\
\text { resulting from } \\
\text { direct irrigation }\end{array}$ & $\begin{array}{l}\text { Total } \\
\text { vegetable/fruit } \\
\text { concentration } \\
\text { (CV) }\end{array}$ \\
\hline URANIUM-234 & $7.03 \mathrm{E}-03$ & $4.86 \mathrm{E}-04$ & $1.46 \mathrm{E}+\infty$ & $0.48 \%$ & $0.03 \%$ & $99.49 \%$ & $1.47 \mathrm{E}+00$ \\
\hline URANIUM-238 & $0.00 E+00$ & $4.82 \mathrm{E}-04$ & $1.45 \mathrm{E}+00$ & $0.00 \%$ & $0.03 \%$ & $99.97 \%$ & $1.45 \mathrm{E}+00$ \\
\hline \multicolumn{8}{|l|}{ Organics (mg/kg) } \\
\hline 1,2,4-TRICHLOROBENZENE & $1.38 \mathrm{E}-01$ & $0.00 \mathrm{E}+00$ & $0.00 E+00$ & $100.00 \%$ & $0.00 \%$ & $0.00 \%$ & $1.38 \mathrm{E}-01$ \\
\hline 1,2-DICHLOROETHENE (TOTAL) & $1.01 \mathrm{E}-01$ & $7.56 \mathrm{E}-03$ & $4.05 E-02$ & $67.77 \%$ & $5.07 \%$ & $27.16 \%$ & $1.49 \mathrm{E}-01$ \\
\hline AROCLOR-1254 & $3.00 \mathrm{E}-03$ & $0.00 \mathrm{E}+00$ & $0.00 E+00$ & $100.00 \%$ & $0.00 \%$ & $0.00 \%$ & $3.06 \mathrm{E}-03$ \\
\hline BENZO(a)PYRENE & $1.43 E-03$ & $0.00 \mathrm{E}+\infty 0$ & $0.00 E+\infty 0$ & $100.00 \%$ & $0.00 \%$ & $0.00 \%$ & $1.43 \mathrm{E}-03$ \\
\hline BIS(2-ETHYLHEXYL)PHTHALATE & $1.77 \mathrm{E}-04$ & $0.00 \mathrm{E}+00$ & $0.00 E+00$ & $100.00 \%$ & $0.00 \%$ & $0.00 \%$ & $1.77 \mathrm{E}-04$ \\
\hline CARBON TETRACHLORIDE & $0.00 E+00$ & $2.22 \mathbb{E}-04$ & $5.73 E-03$ & $0.00 \%$ & $3.72 \%$ & $96.28 \%$ & $5.95 \mathrm{E}-03$ \\
\hline DIBENZO(A,H)ANTHRACENE & $1.60 \mathrm{E}-03$ & $0.00 \mathrm{E}+00$ & $0.00 E+\infty$ & $100.00 \%$ & $0.00 \%$ & $0.00 \%$ & $1.60 \mathrm{E}-03$ \\
\hline TETRACHLOROETHENE & $4.86 \mathrm{E}-02$ & $0.00 \mathrm{E}+00$ & $0.00 \mathrm{E}+00$ & $100.00 \%$ & $0.00 \%$ & $0.00 \%$ & $4.86 \mathrm{E}-02$ \\
\hline \multicolumn{8}{|c|}{$5 Y-200$} \\
\hline \multicolumn{8}{|c|}{ Leafy Vegetables } \\
\hline \multicolumn{8}{|l|}{ Inorganics (mg/kg) } \\
\hline CAOMIUM & $9.72 \mathrm{E}-02$ & $0.00 \mathrm{E}+00$ & $0.00 \mathrm{E}+00$ & $100.00 \%$ & $0.00 \%$ & $0.00 \%$ & $9.72 \mathrm{E}-02$ \\
\hline CHROMUM VI & $0.00 \mathrm{E}+00$ & $1.31 \mathrm{E}-06$ & $1.18 \mathrm{E}-01$ & $0.00 \%$ & $0.00 \%$ & $100.00 \%$ & $1.18 \mathrm{E}-01$ \\
\hline MANGANESE & $2.20 \mathrm{E}+01$ & $4.76 \mathrm{E}-02$ & $3.57 \mathrm{E}+01$ & $38.05 \%$ & $0.08 \%$ & $61.86 \%$ & $5.77 \mathrm{E}+01$ \\
\hline MERCURY & $7.52 \mathrm{E}+\infty$ & $0.00 \mathrm{E}+00$ & $0.00 E+\infty 0$ & $100.00 \%$ & $0.00 \%$ & $0.00 \%$ & $7.52 E+00$ \\
\hline
\end{tabular}


Table C.20. (continued)

\begin{tabular}{|c|c|c|c|c|c|c|c|}
\hline Analyte & $\begin{array}{l}\text { Concentration in } \\
\text { vegetable/fruit } \\
\text { resulting from } \\
\text { unirrigated soil }\end{array}$ & $\begin{array}{l}\text { Concentration } \\
\text { in vegetableffruil } \\
\text { resulting from } \\
\text { irrigated soil }\end{array}$ & $\begin{array}{l}\text { Concentration } \\
\text { in vegetable/fruit } \\
\text { resulting from } \\
\text { direct irrigation }\end{array}$ & $\begin{array}{l}\text { Percent of } \mathrm{CV} \\
\text { resulting from } \\
\text { unirrigated soile }\end{array}$ & $\begin{array}{l}\text { Percent of CV } \\
\text { resulting from } \\
\text { irrigated soil" }\end{array}$ & $\begin{array}{l}\text { Percent of CV } \\
\text { resulting from } \\
\text { direct irrigation }\end{array}$ & $\begin{array}{l}\text { Total } \\
\text { vegetable/f } \\
\text { concentrat } \\
\text { (CV) }\end{array}$ \\
\hline \multicolumn{8}{|l|}{ Radionuclides (pCi/g) } \\
\hline URANIUM-234 & $0.00 E+00$ & $5.16 \mathrm{E}-03$ & $4.65 \mathrm{E}+01$ & $0.00 \%$ & $0.01 \%$ & $99.99 \%$ & 4.65E +01 \\
\hline URANIUM-235 & $0.00 \mathrm{E}+00$ & $3.00 \mathrm{E}-04$ & $2.70 \mathrm{E}+00$ & $0.00 \%$ & $0.01 \%$ & $99.99 \%$ & $2.70 E+00$ \\
\hline URANIUM-238 & $0.00 E+\infty$ & $6.00 \mathrm{E}-03$ & $5.40 \mathrm{E}+01$ & $0.00 \%$ & $0.01 \%$ & $99.99 \%$ & $5.40 E+01$ \\
\hline \multicolumn{8}{|l|}{ Organics (mg/kg) } \\
\hline AROCLOR-1254 & $9.58 \mathrm{E}-04$ & $0.00 E+00$ & $0.00 E+00$ & $100.00 \%$ & $0.00 \%$ & $0.00 \%$ & $9.58 \mathrm{E}-04$ \\
\hline AROCLOR-1260 & $3.02 \mathrm{E}-04$ & $0.00 \mathrm{E}+00$ & $0.00 \mathrm{E}+00$ & $100.00 \%$ & $0.00 \%$ & $0.00 \%$ & $3.02 E-04$ \\
\hline BENZO(A)ANTHRACENE & $2.78 E-03$ & $0.00 \mathrm{E}+00$ & $0.00 E+00$ & $100.00 \%$ & $0.00 \%$ & $0.00 \%$ & $2.78 E-03$ \\
\hline BENZO(A)PYRENE & $1.30 \mathrm{E}-03$ & $0.00 E+00$ & $0.00 \mathrm{E}+00$ & $100.00 \%$ & $0.00 \%$ & $0.00 \%$ & $1.30 \mathrm{E}-03$ \\
\hline BENZO(B)FLUORANTHENE & $9.69 \mathrm{E}-04$ & $0.00 \mathrm{E}+00$ & $0.00 E+00$ & $100.00 \%$ & $0.00 \%$ & $0.00 \%$ & $9.69 \mathrm{E}-04$ \\
\hline BIS(2-ETHYLHEXYL)PHTHALATE & $0.00 \mathrm{E}+00$ & $3.02 E-08$ & $2.52 \mathrm{E}-02$ & $0.00 \%$ & $0.00 \%$ & $100.00 \%$ & $2.52 \mathrm{E}-02$ \\
\hline CHLOROFORM & $0.00 \mathrm{E}+00$ & $1.96 \mathrm{E}-04$ & $6.30 \mathrm{E}-03$ & $0.00 \%$ & $3.02 \%$ & $96.98 \%$ & $6.49 \mathrm{E}-03$ \\
\hline DIBENZO(A,H)ANTHRACENE & $6.60 \mathrm{E}-04$ & $0.00 E+00$ & $0.00 E+00$ & $100.00 \%$ & $0.00 \%$ & $0.00 \%$ & $6.60 \mathrm{E}-04$ \\
\hline \multicolumn{8}{|c|}{ Root Vegetables } \\
\hline \multicolumn{8}{|l|}{ Inorganics (mg/kg) } \\
\hline CADMIUM & $9.72 \mathrm{E}-02$ & $0.00 E+\infty$ & $0.00 E+00$ & $100.00 \%$ & $0.00 \%$ & $0.00 \%$ & $9.72 \mathrm{E}-02$ \\
\hline CHROMIUM VI & $0.00 E+00$ & $1.31 \mathrm{E}-06$ & $0.00 E+00$ & $0.00 \%$ & $100.00 \%$ & $0.00 \%$ & $1.31 \mathrm{E}-06$ \\
\hline MANGANESE & $2.20 \mathrm{E}+01$ & $4.76 \mathrm{E}-02$ & $0.00 E+00$ & $99.78 \%$ & $0.22 \%$ & $0.00 \%$ & $2.20 \mathrm{E}+01$ \\
\hline MERCURY & $7.52 E+\infty 0$ & $0.00 E+00$ & $0.00 E+\infty 0$ & $100.00 \%$ & $0.00 \%$ & $0.00 \%$ & $7.52 E+00$ \\
\hline
\end{tabular}


Table C.20. (oontinued)

\begin{tabular}{|c|c|c|c|c|c|c|c|}
\hline Aralyte & $\begin{array}{l}\text { Concentration in } \\
\text { vegetableffruit } \\
\text { resulting from } \\
\text { unirrigated soil }\end{array}$ & $\begin{array}{l}\text { Concentration } \\
\text { in vegetable/fruil } \\
\text { resulting from } \\
\text { irrigated soil }\end{array}$ & $\begin{array}{l}\text { Concentration } \\
\text { in vegetable/fruit } \\
\text { resulting from } \\
\text { direct irrigation }\end{array}$ & $\begin{array}{l}\text { Percent of } \mathrm{CV} \\
\text { resulting from } \\
\text { unirrigated soil }\end{array}$ & $\begin{array}{l}\text { Percent of CV } \\
\text { resulting from } \\
\text { irrigated soil }\end{array}$ & $\begin{array}{l}\text { Percent of } \mathrm{CV} \\
\text { resulting from } \\
\text { direct irrigation" }\end{array}$ & $\begin{array}{l}\text { Total } \\
\text { vegetable/fruil } \\
\text { concentration } \\
\text { (CV) }\end{array}$ \\
\hline \multicolumn{8}{|l|}{ Radionuclides (pCi/g) } \\
\hline URANIUM-234 & $0.00 \mathrm{E}+00$ & $5.16 \mathrm{E}-03$ & $0.00 \mathrm{E}+00$ & $0.00 \%$ & $100.00 \%$ & $0.00 \%$ & $5.16 \mathrm{E}-03$ \\
\hline URANIUM-235 & $0.00 E+\infty 0$ & $3.00 \mathrm{E}-04$ & $0.00 \mathrm{E}+00$ & $0.00 \%$ & $100.00 \%$ & $0.00 \%$ & $3.00 \mathrm{E}-04$ \\
\hline URANIUM-238 & $0.00 E+\infty 0$ & $6.00 \mathrm{E}-03$ & $0.00 \mathrm{E}+00$ & $0.00 \%$ & $100.00 \%$ & $0.00 \%$ & $6.00 \mathrm{E}-03$ \\
\hline \multicolumn{8}{|l|}{ Organics (mg/kg) } \\
\hline AROCLOR-1254 & $9.58 \mathrm{E}-04$ & $0.00 \mathrm{E}+00$ & $0.00 \mathrm{E}+00$ & $100.00 \%$ & $0.00 \%$ & $0.00 \%$ & $9.58 \mathrm{E}-04$ \\
\hline AROCLOR-1260 & $3.02 \mathrm{E}-04$ & $0.00 E+00$ & $0.00 \mathrm{E}+00$ & $100.00 \%$ & $0.00 \%$ & $0.00 \%$ & $3.02 \mathrm{E}-04$ \\
\hline BENZO(A)ANTHRACENE & $2.78 \mathrm{E}-03$ & $0.00 \mathrm{E}+\infty$ & $0.00 \mathrm{E}+00$ & $100.00 \%$ & $0.00 \%$ & $0.00 \%$ & 2.78E-03 \\
\hline BENZO(A)PYRENE & $1.30 \mathrm{E}-03$ & $0.00 \mathrm{E}+00$ & $0.00 \mathrm{E}+00$ & $100.00 \%$ & $0.00 \%$ & $0.00 \%$ & $1.30 \mathrm{E}-03$ \\
\hline BENZO(B)FLUORANTHENE & $9.69 \mathrm{E}-04$ & $0.00 \mathrm{E}+00$ & $0.00 \mathrm{E}+00$ & $100.00 \%$ & $0.00 \%$ & $0.00 \%$ & $9.69 \mathrm{E}-04$ \\
\hline BIS(2-ETHYLHEXYL)PHTHALATE & $0.00 E+\infty$ & $3.02 \mathrm{E}-08$ & $0.00 E+00$ & $0.00 \%$ & $100.00 \%$ & $0.00 \%$ & $3.02 E-08$ \\
\hline CHLOROFORM & $0.00 \mathrm{E}+\infty 0$ & $1.96 \mathrm{E}-04$ & $0.00 \mathrm{E}+00$ & $0.00 \%$ & $100.00 \%$ & $0.00 \%$ & $1.96 \mathrm{E}-04$ \\
\hline DIBENZO(A,H)ANTHRACENE & $6.60 \mathrm{E}-04$ & $0.00 \mathrm{E}+00$ & $0.00 \mathrm{E}+00$ & $100.00 \%$ & $0.00 \%$ & $0.00 \%$ & $6.60 \mathrm{E}-04$ \\
\hline \multicolumn{8}{|c|}{ Legumes } \\
\hline \multicolumn{8}{|l|}{ Inorganics (mg/kg) } \\
\hline CADMIUM & $9.72 \mathrm{E}-02$ & $0.00 \mathrm{E}+00$ & $0.00 \mathrm{E}+00$ & $100.00 \%$ & $0.00 \%$ & $0.00 \%$ & $9.72 \mathrm{E}-02$ \\
\hline CHROMIUM VI & $0.00 E+00$ & $1.31 \mathrm{E}-06$ & $3.92 E-02$ & $0.00 \%$ & $0.00 \%$ & $100.00 \%$ & $3.92 \mathrm{E}-02$ \\
\hline MANGANESE & $2.20 E+01$ & $4.76 \mathrm{E}-02$ & $1.19 E+01$ & $64.76 \%$ & $0.14 \%$ & $35.10 \%$ & $3.39 \mathrm{E}+01$ \\
\hline MERCURY & $7.52 \mathrm{E}+00$ & $0.00 \mathrm{E}+00$ & $0.00 \mathrm{E}+00$ & $100.00 \%$ & $0.00 \%$ & $0.00 \%$ & $7.52 \mathrm{E}+00$ \\
\hline
\end{tabular}


Table C.20. (continued)

\begin{tabular}{|c|c|c|c|c|c|c|c|}
\hline Analyte & $\begin{array}{l}\text { Concentration in } \\
\text { vegetablefiruit } \\
\text { resulting from } \\
\text { unirrigated soil }\end{array}$ & $\begin{array}{l}\text { Concentration } \\
\text { in vegetableffruil } \\
\text { resulting from } \\
\text { irrigated soil }\end{array}$ & $\begin{array}{l}\text { Concentration } \\
\text { in vegetablefiruit } \\
\text { resulting from } \\
\text { direct irrigation }\end{array}$ & $\begin{array}{l}\text { Percent of } \mathrm{CV} \\
\text { resulting from } \\
\text { unirrigated soil }\end{array}$ & $\begin{array}{l}\text { Percent of } \mathrm{CV} \\
\text { resulting from } \\
\text { irrigated soil }\end{array}$ & $\begin{array}{l}\text { Percent of } \mathrm{CV} \\
\text { resulting from } \\
\text { direct irrigation }\end{array}$ & $\begin{array}{l}\text { Total } \\
\text { vegetable/fruit } \\
\text { concentration } \\
\text { (CV) }\end{array}$ \\
\hline \multicolumn{8}{|l|}{ Radionuclides (pCi/g) } \\
\hline URANIUM-234 & $0.00 E+\infty$ & $5.16 \mathrm{E}-03$ & $1.55 \mathrm{E}+01$ & $0.00 \%$ & $0.03 \%$ & $99.97 \%$ & $1.55 \mathrm{E}+01$ \\
\hline URANIUM-235 & $0.00 \mathrm{E}+00$ & $3.00 \mathrm{E}-04$ & $9.00 \mathrm{E}-01$ & $0.00 \%$ & $0.03 \%$ & $99.97 \%$ & $9.01 \mathrm{E}-01$ \\
\hline URANIUM-238 & $0.00 \mathrm{E}+00$ & $6.00 \mathrm{E}-03$ & $1.80 \mathrm{E}+01$ & $0.00 \%$ & $0.03 \%$ & $99.97 \%$ & $1.80 \mathrm{E}+01$ \\
\hline \multicolumn{8}{|l|}{ Organics (mg/kg) } \\
\hline AROCLOR-1254 & $9.58 E-04$ & $0.00 \mathrm{E}+00$ & $0.00 \mathrm{E}+\infty 0$ & $100.00 \%$ & $0.00 \%$ & $0.00 \%$ & $9.58 \mathrm{E}-04$ \\
\hline AROCLOR-1260 & $3.02 \mathrm{E}-04$ & $0.00 \mathrm{E}+00$ & $0.00 \mathrm{E}+00$ & $100.00 \%$ & $0.00 \%$ & $0.00 \%$ & $3.02 \mathrm{E}-04$ \\
\hline BENZO(A)ANTHRACENE & $2.78 \mathrm{E}-03$ & $0.00 \mathrm{E}+00$ & $0.00 \mathrm{E}+00$ & $100.00 \%$ & $0.00 \%$ & $0.00 \%$ & $2.78 \mathrm{E}-03$ \\
\hline BENZO(A)PYRENE & $1.30 \mathrm{E}-03$ & $0.00 \mathrm{E}+00$ & $0.00 E+00$ & $100.00 \%$ & $0.00 \%$ & $0.00 \%$ & $1.30 \mathrm{E}-03$ \\
\hline BENZO(B)FLUORANTHENE & $9.69 E-04$ & $0.00 \mathrm{E}+00$ & $0.00 \mathrm{E}+00$ & $100.00 \%$ & $0.00 \%$ & $0.00 \%$ & $9.69 \mathrm{E}-04$ \\
\hline BIS(2-ETHYLHEXYL)PHTHALATE & $0.00 \mathrm{E}+\infty 0$ & $3.02 E-08$ & $8.40 \mathrm{E}-03$ & $0.00 \%$ & $0.00 \%$ & $100.00 \%$ & $8.40 \mathrm{E}-03$ \\
\hline CHLOROFORM & $0.00 E+\infty 0$ & $1.96 \mathrm{E}-04$ & $2.10 \mathrm{E}-03$ & $0.00 \%$ & $8.54 \%$ & $91.46 \%$ & $2.29 \mathrm{E}-03$ \\
\hline DIBENZO(A,H)ANTHRACENE & $6.60 \mathrm{E}-04$ & $0.00 \mathrm{E}+00$ & $0.00 \mathrm{E}+00$ & $100.00 \%$ & $0.00 \%$ & $0.00 \%$ & $6.60 \mathrm{E}-04$ \\
\hline \multicolumn{8}{|c|}{ Fruiting Vegetables } \\
\hline \multicolumn{8}{|l|}{ Inorganics (mg/kg) } \\
\hline CADMIUM & $9.72 \mathrm{E}-02$ & $0.00 \mathrm{E}+00$ & $0.00 \mathrm{E}+00$ & $100.00 \%$ & $0.00 \%$ & $0.00 \%$ & $9.72 \mathrm{E}-02$ \\
\hline CHROMIUM VI & $0.00 \mathrm{E}+00$ & $1.31 \mathrm{E}-06$ & $3.92 \mathrm{E}-02$ & $0.00 \%$ & $0.00 \%$ & $100.00 \%$ & $3.92 \mathrm{E}-02$ \\
\hline MANGANESE & $2.20 \mathrm{E}+01$ & $4.76 \mathrm{E}-02$ & $1.19 \mathrm{E}+01$ & $64.76 \%$ & $0.14 \%$ & $35.10 \%$ & $3.39 E+01$ \\
\hline MERCURY & $7.52 \mathrm{E}+00$ & $0.00 \mathrm{E}+00$ & $0.00 E+00$ & $100.00 \%$ & $0.00 \%$ & $0.00 \%$ & $7.52 \mathrm{E}+00$ \\
\hline
\end{tabular}


Table C.20. (comtinued)

\begin{tabular}{|c|c|c|c|c|c|c|c|}
\hline Analyte & $\begin{array}{l}\text { Concentration in } \\
\text { vegetable/fruil } \\
\text { resulting from } \\
\text { unirrigated soil }\end{array}$ & $\begin{array}{l}\text { Concentration } \\
\text { in vegetable/fruil } \\
\text { resulting from } \\
\text { irrigated soil }\end{array}$ & $\begin{array}{l}\text { Concentration } \\
\text { in vegetable/fruil } \\
\text { resulting from } \\
\text { direct irrigation }\end{array}$ & $\begin{array}{l}\text { Percent of } \mathrm{CV} \\
\text { resulling from } \\
\text { unirrigated soil }\end{array}$ & $\begin{array}{l}\text { Percent of CV } \\
\text { resulting from } \\
\text { irrigated soil" }\end{array}$ & $\begin{array}{l}\text { Percent of } \mathrm{CV} \\
\text { resulting from } \\
\text { direct irrigation }\end{array}$ & $\begin{array}{l}\text { Total } \\
\text { vegetable/frui } \\
\text { concentration } \\
\text { (CV) }\end{array}$ \\
\hline \multicolumn{8}{|l|}{ Radionuclides ( $\mathrm{pCi} / \mathrm{g}$ ) } \\
\hline URANIUM-234 & $0.00 E+\infty 0$ & $5.16 \mathrm{E}-03$ & $1.55 \mathrm{E}+01$ & $0.00 \%$ & $0.03 \%$ & $99.97 \%$ & $1.55 \mathrm{E}+01$ \\
\hline URANIUM-235 & $0.00 \mathrm{E}+\infty$ & $3.00 E-04$ & $9.00 \mathrm{E}-01$ & $0.00 \%$ & $0.03 \%$ & $99.97 \%$ & $9.01 \mathrm{E}-01$ \\
\hline URANIUM-238 & $0.00 \mathrm{E}+00$ & $6.00 \mathrm{E}-03$ & $1.80 \mathrm{E}+01$ & $0.00 \%$ & $0.03 \%$ & $99.97 \%$ & $1.80 \mathrm{E}+01$ \\
\hline \multicolumn{8}{|l|}{ Organics (mg/kg) } \\
\hline AROCLOR-1254 & $9.58 \mathrm{E}-04$ & $0.00 \mathrm{E}+\infty$ & $0.00 \mathrm{E}+00$ & $100.00 \%$ & $0.00 \%$ & $0.00 \%$ & $9.58 \mathrm{E}-0.4$ \\
\hline AROCLOR-1260 & $3.02 E-04$ & $0.00 E+\infty$ & $0.00 \mathrm{E}+\infty 0$ & $100.00 \%$ & $0.00 \%$ & $0.00 \%$ & $3.02 \mathrm{E}-04$ \\
\hline BENZO(A)ANTHRACENE & $2.78 \mathrm{E}-03$ & $0.00 E+00$ & $0.00 \mathrm{E}+\infty 0$ & $100.00 \%$ & $0.00 \%$ & $0.00 \%$ & $2.78 \mathrm{E}-03$ \\
\hline BENZO(A)PYRENE & $1.30 \mathrm{E}-03$ & $0.00 E+00$ & $0.00 \mathrm{E}+00$ & $100.00 \%$ & $0.00 \%$ & $0.00 \%$ & $1.30 \mathrm{E}-03$ \\
\hline BENZO(B)FLUORANTHENE & $9.69 \mathrm{E}-04$ & $0.00 E+00$ & $0.00 E+00$ & $100.00 \%$ & $0.00 \%$ & $0.00 \%$ & $9.69 \mathrm{E}-04$ \\
\hline BIS(2-ETHYLHEXYL)PHTHALATE & $0.00 \mathrm{E}+\infty 0$ & $3.02 \mathrm{E}-08$ & $8.40 \mathrm{E}-03$ & $0.00 \%$ & $0.00 \%$ & $100.00 \%$ & $8.40 \mathrm{E}-03$ \\
\hline CHLOROFORM & $0.00 E+\infty$ & $1.96 \mathrm{E}-04$ & $2.10 \mathrm{E}-03$ & $0.00 \%$ & $8.54 \%$ & $91.46 \%$ & $2.29 \mathrm{E}-03$ \\
\hline DIBENZO(A,H)ANTHRACENE & $6.60 \mathrm{E}-04$ & $0.00 E+\infty$ & $0.00 \mathrm{E}+00$ & $100.00 \%$ & $0.00 \%$ & $0.00 \%$ & $6.60 \mathrm{E}-04$ \\
\hline \multicolumn{8}{|c|}{ Fruits } \\
\hline \multicolumn{8}{|l|}{ Inorganics (mg/kg) } \\
\hline CADMIUM & $9.72 \mathrm{E}-02$ & $0.00 \mathrm{E}+00$ & $0.00 E+00$ & $100.00 \%$ & $0.00 \%$ & $0.00 \%$ & $9.72 \mathrm{E}-02$ \\
\hline CHROMIUM VI & $0.00 E+00$ & $1.31 \mathrm{E}-06$ & $3.92 \mathrm{E}-02$ & $0.00 \%$ & $0.00 \%$ & $100.00 \%$ & $3.92 \mathrm{E}-02$ \\
\hline MANGANESE & $2.20 \mathrm{E}+01$ & $4.76 \mathrm{E}-02$ & $1.19 E+01$ & $64.76 \%$ & $0.14 \%$ & $35.10 \%$ & $3.39 \mathrm{E}+01$ \\
\hline MERCURY & $7.52 E+\infty 0$ & $0.00 E+\infty$ & $0.00 E+\infty$ & $100.00 \%$ & $0.00 \%$ & $0.00 \%$ & $7.52 E+00$ \\
\hline
\end{tabular}


Table C.20. (continued)

\begin{tabular}{|c|c|c|c|c|c|c|c|}
\hline Analyle & $\begin{array}{l}\text { Concentration in } \\
\text { vegetable/fruit } \\
\text { resulting from } \\
\text { unirrigaled soil }\end{array}$ & $\begin{array}{l}\text { Concentration } \\
\text { in vegetable/fruil } \\
\text { resulting from } \\
\text { irrigated soil }\end{array}$ & $\begin{array}{l}\text { Concentration } \\
\text { in vegetable/fruit } \\
\text { resulting from } \\
\text { direct irrigation }\end{array}$ & $\begin{array}{l}\text { Percent of } \mathrm{CV} \\
\text { resulting from } \\
\text { unirrigated soil }\end{array}$ & $\begin{array}{l}\text { Percent of CV } \\
\text { resulting from } \\
\text { irrigated soil }\end{array}$ & $\begin{array}{l}\text { Percent of } \mathrm{CV} \\
\text { resulting from } \\
\text { direct irrigation }\end{array}$ & $\begin{array}{l}\text { Total } \\
\text { vegetableffruil } \\
\text { concentration } \\
\text { (CV) }\end{array}$ \\
\hline \multicolumn{8}{|l|}{ Radionuclides (pCi/g) } \\
\hline URANIUM-234 & $0.00 E+00$ & $5.16 \mathrm{E}-03$ & $1.55 \mathrm{E}+01$ & $0.00 \%$ & $0.03 \%$ & $99.97 \%$ & $1.55 E+01$ \\
\hline URANIUM-235 & $0.00 \mathrm{E}+00$ & $3.00 \mathrm{E}-04$ & $9.00 \mathrm{E}-01$ & $0.00 \%$ & $0.03 \%$ & $99.97 \%$ & $9.01 \mathrm{E}-01$ \\
\hline URANIUM-238 & $0.00 \mathrm{E}+00$ & $6.00 \mathrm{E}-03$ & $1.80 \mathrm{E}+01$ & $0.00 \%$ & $0.03 \%$ & $99.97 \%$ & $1.80 \mathrm{E}+01$ \\
\hline \multicolumn{8}{|l|}{ Organics (mg/kg) } \\
\hline AROCLOR-1254 & $9.58 \mathrm{E}-04$ & $0.00 \mathrm{E}+00$ & $0.00 \mathrm{E}+00$ & $100.00 \%$ & $0.00 \%$ & $0.00 \%$ & $9.58 \mathrm{E}-04$ \\
\hline AROCLOR-1260 & $3.02 \mathrm{E}-04$ & $0.00 E+00$ & $0.00 E+00$ & $100.00 \%$ & $0.00 \%$ & $0.00 \%$ & $3.02 \mathrm{E}-04$ \\
\hline BENZO(A)ANTHRACENE & $2.78 \mathrm{E}-03$ & $0.00 \mathrm{E}+\infty 0$ & $0.00 \mathrm{E}+\infty 0$ & $100.00 \%$ & $0.00 \%$ & $0.00 \%$ & $2.78 E-03$ \\
\hline BENZO(A)PYRENE & $1.30 \mathrm{E}-03$ & $0.00 \mathrm{E}+00$ & $0.00 \mathrm{E}+00$ & $100.00 \%$ & $0.00 \%$ & $0.00 \%$ & $1.30 \mathrm{E}-03$ \\
\hline BENZO(B)FLUORANTHENE & $9.69 \mathrm{E} \cdot 04$ & $0.00 E+00$ & $0.00 \mathrm{E}+00$ & $100.00 \%$ & $0.00 \%$ & $0.00 \%$ & $9.69 \mathrm{E}-04$ \\
\hline BIS(2-ETHYLHEXYL)PHTHALATE & $0.00 \mathrm{E}+00$ & $3.02 \mathrm{E}-08$ & $8.40 \mathrm{E}-03$ & $0.00 \%$ & $0.00 \%$ & $100.00 \%$ & $8.40 \mathrm{E}-03$ \\
\hline CHLOROFORM & $0.00 \mathrm{E}+00$ & $1.96 \mathrm{E} \cdot 04$ & $2.10 \mathrm{E}-03$ & $0.00 \%$ & $8.54 \%$ & $91.46 \%$ & $2.29 \mathrm{E}-03$ \\
\hline DIBENZO(A,H)ANTHRACENE & $6.60 \mathrm{E}-04$ & $0.00 \mathrm{E}+00$ & $0.00 \mathrm{E}+00$ & $100.00 \%$ & $0.00 \%$ & $0.00 \%$ & $6.60 \mathrm{E}-04$ \\
\hline
\end{tabular}

SA-1

Leafy Vegetables

Inorganics (mg/kg)

BARIUM

BERYLLIUM

$\begin{array}{lllllll}1.17 \mathrm{E}+00 & 0.00 \mathrm{E}+00 & 0.00 \mathrm{E}+00 & 100.00 \% & 0.00 \% & 0.00 \% & 1.17 \mathrm{E}+00 \\ 5.64 \mathrm{E}-04 & 3.46 \mathrm{E}-08 & 1.66 \mathrm{E}-03 & 25.41 \% & 0.00 \% & 74.59 \% & 2.22 \mathrm{E}-03\end{array}$


Table C.20. (continued)

\begin{tabular}{|c|c|c|c|c|c|c|c|}
\hline Analyte & $\begin{array}{l}\text { Concentration in } \\
\text { vegetable/fruit } \\
\text { resulting from } \\
\text { unirrigated soil }\end{array}$ & $\begin{array}{l}\text { Concentration } \\
\text { in vegetable/fruil } \\
\text { resulting from } \\
\text { irrigated soil }\end{array}$ & $\begin{array}{l}\text { Concentration } \\
\text { in vegetable/fruit } \\
\text { resulting from } \\
\text { direct irrigation }\end{array}$ & $\begin{array}{l}\text { Percent of CV } \\
\text { resulting from } \\
\text { unirrigated soig }\end{array}$ & $\begin{array}{l}\text { Percent of CV } \\
\text { resulting from } \\
\text { irrigated soil }\end{array}$ & $\begin{array}{l}\text { Percent of } \mathrm{CV} \\
\text { resulling from } \\
\text { direct irrigation }\end{array}$ & $\begin{array}{l}\text { Total } \\
\text { vegetable/fruit } \\
\text { concentration } \\
\text { (CV) }\end{array}$ \\
\hline CADMIUM & $9.93 \mathrm{E}-01$ & $1.66 \mathrm{E}-04$ & $1.25 \mathrm{E}-02$ & $98.74 \%$ & $0.02 \%$ & $1.24 \%$ & $1.01 E+\infty 0$ \\
\hline CHROMIUM VI & $8.98 \mathrm{E}-03$ & $0.00 \mathrm{E}+00$ & $0.00 \mathrm{E}+00$ & $100.00 \%$ & $0.00 \%$ & $0.00 \%$ & $8.98 \mathrm{E}-03$ \\
\hline MANGANESE & $3.60 \mathrm{E}+01$ & $4.94 \mathrm{E}-04$ & $3.70 \mathrm{E}-01$ & $98.98 \%$ & $0.00 \%$ & $1.02 \%$ & $3.64 \mathrm{E}+01$ \\
\hline MERCURY & $3.67 \mathrm{E}-01$ & $0.00 E+00$ & $0.00 \mathrm{E}+00$ & $100.00 \%$ & $0.00 \%$ & $0.00 \%$ & $3.67 E-01$ \\
\hline NICKEL & $6.67 \mathrm{E}-01$ & $0.00 \mathrm{E}+00$ & $0.00 E+00$ & $100.00 \%$ & $0.00 \%$ & $0.00 \%$ & $6.67 \mathrm{E}-01$ \\
\hline NITRATE & $0.00 \mathrm{E}+00$ & $0.00 \mathrm{E}+00$ & $1.65 \mathrm{E}+02$ & $0.00 \%$ & $0.00 \%$ & $100.00 \%$ & $1.65 \mathrm{E}+02$ \\
\hline URANIUM (SOLUBLE SALTS) & $3.83 E-02$ & $0.00 \mathrm{E}+00$ & $0.00 \mathrm{E}+00$ & $100.00 \%$ & $0.00 \%$ & $0.00 \%$ & $3.83 \mathbb{E}-02$ \\
\hline VANADIUM & $0.00 \mathrm{E}+00$ & $1.34 \mathrm{E}-06$ & 2.32E- -02 & $0.00 \%$ & $0.01 \%$ & $99.99 \%$ & $2.32 \mathrm{E}-02$ \\
\hline \multicolumn{8}{|l|}{ Radionuclides ( $\mathrm{pCi} / \mathrm{g})$} \\
\hline RADIUM-226 & $4.51 E-03$ & $0.00 E+00$ & $0.00 E+00$ & $100.00 \%$ & $0.00 \%$ & $0.00 \%$ & $4.51 \mathrm{E}-03$ \\
\hline URANIUM-234 & $1.29 \mathrm{E}-02$ & $1.76 \mathrm{E}-03$ & $1.58 \mathrm{E}+01$ & $0.08 \%$ & $0.01 \%$ & $99.91 \%$ & $1.58 \mathrm{E}+01$ \\
\hline URANIUM-238 & $1.28 \mathrm{E}-02$ & $1.19 E-03$ & $1.07 E+01$ & $0.12 \%$ & $0.01 \%$ & $99.87 \%$ & $1.07 \mathrm{E}+01$ \\
\hline \multicolumn{8}{|l|}{ Organies (mg/kg) } \\
\hline 1,2-DICHLOROETHENE (TOTAL) & $0.00 E+00$ & $6.11 E-03$ & $9.82 E-02$ & $0.00 \%$ & $5.86 \%$ & $94.14 \%$ & $1.04 \mathrm{E}-01$ \\
\hline BENZO(A)ANTHRACENE & $2.33 \mathrm{E}-03$ & $0.00 \mathrm{E}+00$ & $0.00 E+00$ & $100.00 \%$ & $0.00 \%$ & $0.00 \%$ & $2.33 E-03$ \\
\hline BENZO(A)PYRENE & $1.08 \mathrm{E}-03$ & $0.00 \mathrm{E}+00$ & $0.00 \mathrm{E}+00$ & $100.00 \%$ & $0.00 \%$ & $0.00 \%$ & $1.08 \mathrm{E}-03$ \\
\hline BENZO(B)FLUORANTHENE & $1.13 \mathrm{E}-03$ & $0.00 \mathrm{E}+00$ & $0.00 \mathrm{E}+00$ & $100.00 \%$ & $0.00 \%$ & $0.00 \%$ & $1.13 E-03$ \\
\hline BROMODICHLOROMETHANE & $0.00 \mathrm{E}+00$ & $3.54 \mathrm{E}-04$ & $1.62 \mathrm{E}-02$ & $0.00 \%$ & $2.13 \%$ & $97.87 \%$ & $1.66 \mathrm{E}-02$ \\
\hline INDENO(1,2,3-CD)PYRENE & $4.42 \mathrm{E}-04$ & $0.00 \mathrm{E}+\infty$ & $0.00 \mathrm{E}+\infty$ & $100.00 \%$ & $0.00 \%$ & $0.00 \%$ & $4.42 \mathrm{E}-0.4$ \\
\hline
\end{tabular}


Table C.20. (continued)

\begin{tabular}{|c|c|c|c|c|c|c|c|}
\hline Analyte & $\begin{array}{l}\text { Concentration in } \\
\text { vegetableffruit } \\
\text { resulting from } \\
\text { unirrigated soil }\end{array}$ & $\begin{array}{l}\text { Concentration } \\
\text { in vegetable/fruit } \\
\text { resulting from } \\
\text { irrigated soil }\end{array}$ & $\begin{array}{l}\text { Concentration } \\
\text { in vegetable/fruit } \\
\text { resulting from } \\
\text { direct irrigation }\end{array}$ & $\begin{array}{l}\text { Percent of CV } \\
\text { resulting from } \\
\text { unirrigated soil" }\end{array}$ & $\begin{array}{l}\text { Percent of CV } \\
\text { resulting from } \\
\text { irrigated soil }\end{array}$ & $\begin{array}{l}\text { Percent of CV } \\
\text { resulting from } \\
\text { direct imrigation }\end{array}$ & $\begin{array}{l}\text { Total } \\
\text { vegetableff } \\
\text { concentrati } \\
\text { (CV) }\end{array}$ \\
\hline \multirow[t]{2}{*}{ METHYLENE CHLORIDE } & $0.00 E+00$ & $1.51 \mathrm{E}-03$ & 2.00E-02 & $0.00 \%$ & $7.02 \%$ & $92.98 \%$ & $2.15 \mathrm{E}-02$ \\
\hline & \multicolumn{6}{|c|}{ Root Vegetables } & \\
\hline \multicolumn{8}{|l|}{ Inorganics (mg/kg) } \\
\hline BARIUM & $1.17 E+\infty$ & $0.00 \mathrm{E}+00$ & $0.00 \mathrm{E}+\infty 0$ & $100.00 \%$ & $0.00 \%$ & $0.00 \%$ & $1.17 E+00$ \\
\hline BERYLLIUM & $3.64 \mathrm{E}-04$ & $3.46 \mathrm{E}-08$ & $0.00 \mathrm{E}+00$ & $99.99 \%$ & $0.01 \%$ & $0.00 \%$ & $5.64 \mathrm{E}-04$ \\
\hline CADMUM & 9.93E-01 & $1.66 \mathrm{E}-04$ & $0.00 E+00$ & $99.98 \%$ & $0.02 \%$ & $0.00 \%$ & $9.93 \mathrm{E}-01$ \\
\hline CHROMIUM VI & $8.98 \mathrm{E}-03$ & $0.00 \mathrm{E}+00$ & $0.00 \mathrm{E}+00$ & $100.00 \%$ & $0.00 \%$ & $0.00 \%$ & $8.98 \mathrm{E}-03$ \\
\hline MANGANESE & $3.60 \mathrm{E}+01$ & 4.94E-04 & $0.00 \mathrm{E}+\infty 0$ & $100.00 \%$ & $0.00 \%$ & $0.00 \%$ & $3.60 \mathrm{E}+01$ \\
\hline MERCURY & $3.67 \mathrm{E}-01$ & $0.00 \mathrm{E}+\infty 0$ & $0.00 \mathrm{E}+\infty 00$ & $100.00 \%$ & $0.00 \%$ & $0.00 \%$ & $3.67 \mathrm{E}-01$ \\
\hline NICKEL & $6.67 \mathrm{E}-01$ & $0.00 \mathrm{E}+00$ & $0.00 E+00$ & $100.00 \%$ & $0.00 \%$ & $0.00 \%$ & $6.67 \mathrm{E}-01$ \\
\hline NITRATE & $0.00 \mathrm{E}+00$ & $0.00 E+\infty$ & $0.00 \mathrm{E}+\infty$ & NA & NA & NA & $0.00 E+00$ \\
\hline URANIUM (SOLUBLE SALTS) & $3.83 \mathrm{E}-02$ & $0.00 \mathrm{E}+00$ & $0.00 \mathrm{E}+00$ & $100.00 \%$ & $0.00 \%$ & $0.00 \%$ & $3.83 \mathrm{E}-02$ \\
\hline VANADIUM & $0.00 \mathrm{E}+00$ & $1.34 \mathrm{E}-06$ & $0.00 \mathrm{E}+00$ & $0.00 \%$ & $100.00 \%$ & $0.00 \%$ & $1.34 \mathrm{E}-06$ \\
\hline \multicolumn{8}{|l|}{ Radionuclides (pCi/g) } \\
\hline RADIUM-226 & 4.51E-03 & $0.00 E+\infty$ & $0.00 E+\infty$ & $100.00 \%$ & $0.00 \%$ & $0.00 \%$ & $4.51 \mathrm{E}-03$ \\
\hline URANIUM-234 & $1.29 \mathrm{E}-02$ & $1.76 \mathrm{E}-03$ & $0.00 E+\infty$ & $88.00 \%$ & $12.00 \%$ & $0.00 \%$ & $1.46 \mathrm{E}-02$ \\
\hline URANIUM-238 & $1.28 \mathrm{E}-02$ & $1.19 \mathrm{E}-03$ & $0.00 E+\infty 0$ & $91.48 \%$ & $8.52 \%$ & $0.00 \%$ & $1.40 \mathrm{E}-02$ \\
\hline \multicolumn{8}{|l|}{ Organics (mg/kg) } \\
\hline 1,2-DICHLOROETHENE (TOTAL) & $0.00 E+00$ & $6.11 \mathrm{E}-03$ & $0.00 E+00$ & $0.00 \%$ & $100.00 \%$ & $0.00 \%$ & $6.11 \mathrm{E}-03$ \\
\hline
\end{tabular}


Table C.20. (conthued)

\begin{tabular}{|c|c|c|c|c|c|c|c|}
\hline Analyte & $\begin{array}{l}\text { Concentration in } \\
\text { vegetableffruit } \\
\text { resulting from } \\
\text { unirrigated soil }\end{array}$ & $\begin{array}{l}\text { Concentration } \\
\text { in vegetable/fruit } \\
\text { resulting from } \\
\text { irrigated soil }\end{array}$ & $\begin{array}{l}\text { Concentration } \\
\text { in vegetable/fruit } \\
\text { resulting from } \\
\text { direct arrigation }\end{array}$ & $\begin{array}{l}\text { Percent of CV } \\
\text { resulting Irom } \\
\text { unimigated soil }\end{array}$ & $\begin{array}{l}\text { Percent of } \mathrm{CV} \\
\text { resulting from } \\
\text { irrigated soil }\end{array}$ & $\begin{array}{l}\text { Percent of CV } \\
\text { resulting from } \\
\text { direct irrigation }\end{array}$ & $\begin{array}{l}\text { Total } \\
\text { vegetable/fruit } \\
\text { concentration } \\
\text { (CV) }\end{array}$ \\
\hline BENZO(A)ANTHRACENE & $2.33 \mathrm{E} \cdot 03$ & $0.00 E+\infty$ & $0.00 \mathrm{E}+00$ & $100.00 \%$ & $0.00 \%$ & $0.00 \%$ & $2.33 \mathrm{E}-03$ \\
\hline BENZO(A)PYRENE & $1.08 \mathrm{E}-03$ & $0.00 E+00$ & $0.00 E+00$ & $100.00 \%$ & $0.00 \%$ & $0.00 \%$ & $1.08 \mathrm{E}-03$ \\
\hline BENZO(B)FLUORANTHENE & $1.13 E \cdot 03$ & $0.00 \mathrm{E}+00$ & $0.00 \mathrm{E}+00$ & $100.00 \%$ & $0.00 \%$ & $0.00 \%$ & 1.13E-03 \\
\hline BROMODICHLOROMETHANE & $0.00 \mathrm{E}+00$ & $3.54 \mathrm{E}-04$ & $0.00 \mathrm{E}+00$ & $0.00 \%$ & $100,00 \%$ & $0.00 \%$ & $3.54 \mathrm{E}-04$ \\
\hline INDENO(1,2,3-CD)PYRENE & 4.42E-0.4 & $0.00 \mathrm{E}+00$ & $0.00 \mathrm{E}+00$ & $100.00 \%$ & $0.00 \%$ & $0.00 \%$ & 4.42E-04 \\
\hline METHYLENE CHLORIDE & $0.00 \mathrm{E}+00$ & $1.51 E-03$ & $0.00 \mathrm{E}+00$ & $0.00 \%$ & $100.00 \%$ & $0.00 \%$ & $1.51 \mathrm{E}-03$ \\
\hline \multicolumn{8}{|c|}{ Legumes } \\
\hline \multicolumn{8}{|l|}{ Inorganies (mg/kg) } \\
\hline BARIUM & $1.17 \mathrm{E}+\infty$ & $0.00 \mathrm{E}+00$ & $0.00 \mathrm{E}+00$ & $100.00 \%$ & $0.00 \%$ & $0.00 \%$ & $1.17 \mathbb{E}+\infty$ \\
\hline BERYLLIUM & $5.64 E-04$ & $3.46 \mathrm{E}-08$ & $5.52 E-04$ & $50.54 \%$ & $0.00 \%$ & $49.46 \%$ & $1.12 E-03$ \\
\hline CADMUM & 9.93E-01 & $1.66 \mathrm{E}-04$ & $4.16 \mathrm{E}-03$ & $99.57 \%$ & $0.02 \%$ & $0.42 \%$ & $9.97 \mathrm{E}-01$ \\
\hline CHROMIUM VI & $8.98 \mathrm{E}-03$ & $0.00 \mathrm{E}+\infty 0$ & $0.00 E+00$ & $100.00 \%$ & $0.00 \%$ & $0,00 \%$ & $8.98 \mathrm{E}-03$ \\
\hline MANGANESE & $3.60 \mathrm{E}+01$ & $4.94 \mathrm{E}-04$ & $1.23 \mathrm{E}-01$ & $99.66 \%$ & $0.00 \%$ & $0.34 \%$ & $3.61 \mathrm{E}+01$ \\
\hline MERCURY & $3.67 E-01$ & $0.00 \mathrm{E}+00$ & $0.00 \mathrm{E}+\infty$ & $100.00 \%$ & $0.00 \%$ & $0.00 \%$ & $3.67 \mathrm{E}-01$ \\
\hline NICKEL & $6.67 \mathrm{E}-01$ & $0.00 \mathrm{E}+00$ & $0.00 \mathrm{E}+00$ & $100.00 \%$ & $0.00 \%$ & $0.00 \%$ & $6.67 \mathrm{E}-01$ \\
\hline NITRATE & $0.00 \mathrm{E}+00$ & $0.00 \mathrm{E}+00$ & $5.50 \mathrm{E}+01$ & $0.00 \%$ & $0.00 \%$ & $100.00 \%$ & $5.50 \mathrm{E}+01$ \\
\hline URANIUM (SOLUBLE SALTS) & $3.83 E-02$ & $0.00 \mathrm{E}+00$ & $0.00 \mathrm{E}+00$ & $100.00 \%$ & $0.00 \%$ & $0.00 \%$ & $3.83 \mathrm{E}-02$ \\
\hline VANADIUM & $0.00 E+\infty 0$ & $1.34 \mathrm{E}-06$ & $7.74 \mathrm{E}-03$ & $0.00 \%$ & $0.02 \%$ & $99.98 \%$ & $7.75 \mathrm{E}-03$ \\
\hline
\end{tabular}

Radionuclides (pCi/g) 
Table C.20. (contimued)

\begin{tabular}{|c|c|c|c|c|c|c|c|}
\hline Analyte & $\begin{array}{l}\text { Concentration in } \\
\text { vegetableffruit } \\
\text { resulting from } \\
\text { unirrigated soil }\end{array}$ & $\begin{array}{l}\text { Concentration } \\
\text { in vegetable/fruit } \\
\text { resulting from } \\
\text { irrigated soil }\end{array}$ & $\begin{array}{l}\text { Concentration } \\
\text { in vegetable/fruit } \\
\text { resulting fron } \\
\text { direet imigation }\end{array}$ & $\begin{array}{l}\text { Percent of } \mathrm{CV} \\
\text { resulting from } \\
\text { unirrigated soil" }\end{array}$ & $\begin{array}{l}\text { Percent of CV } \\
\text { resulting from } \\
\text { irrigated soil" }\end{array}$ & $\begin{array}{l}\text { Percent of CV } \\
\text { resulting from } \\
\text { direct irrigation }\end{array}$ & $\begin{array}{l}\text { Total } \\
\text { vegetable/fr } \\
\text { concentratic } \\
\text { (CV) } \\
\end{array}$ \\
\hline RADIUM-226 & $4.51 \mathrm{E}-03$ & $0.00 \mathrm{E}+00$ & $0.00 \mathrm{E}+00$ & $100.00 \%$ & $0.00 \%$ & $0.00 \%$ & $4.51 \mathrm{E}-03$ \\
\hline URANIUM-234 & $1.29 \mathrm{E}-02$ & $1.76 \mathrm{E}-03$ & $5.27 E+00$ & $0.24 \%$ & $0.03 \%$ & $99.72 \%$ & $5.28 \mathrm{E}+00$ \\
\hline URANIUM-238 & $1.28 \mathrm{E}-02$ & $1.19 \mathrm{E}-03$ & $3.57 \mathrm{E}+00$ & $0.36 \%$ & $0.03 \%$ & $99.61 \%$ & $3.58 \mathrm{E}+00$ \\
\hline \multicolumn{8}{|l|}{ Organics (mg/kg) } \\
\hline 1,2-DICHLOROETHENE (TOTAL) & $0.00 E+00$ & $6.11 \mathrm{E}-03$ & 3.27E-02 & $0.00 \%$ & $15.73 \%$ & $84.27 \%$ & $3.89 \mathrm{E}-02$ \\
\hline BENZO(A)ANTHRACENE & 2.33E-03 & $0.00 \mathrm{E}+00$ & $0.00 E+00$ & $100.00 \%$ & $0.00 \%$ & $0.00 \%$ & $2.33 \mathrm{E}-03$ \\
\hline BENZO(A)PYRENE & $1.08 \mathrm{E}-03$ & $0.00 E+00$ & $0.00 E+00$ & $100.00 \%$ & $0.00 \%$ & $0.00 \%$ & $1.08 \mathrm{E}-03$ \\
\hline BENZO(B)FLUORANTHENE & $1.13 E-03$ & $0.00 \mathrm{E}+00$ & $0.00 \mathrm{E}+00$ & $100.00 \%$ & $0.00 \%$ & $0.00 \%$ & $1.13 \mathrm{E}-03$ \\
\hline BROMODICHLOROMETHANE & $0.00 \mathrm{E}+00$ & $3.54 \mathrm{E}-04$ & $5.41 E-03$ & $0.00 \%$ & $6.13 \%$ & $93.87 \%$ & $5.77 \mathrm{E}-03$ \\
\hline INDENO(1,2,3-CD)PYRENE & 4.42E-04 & $0.00 E+00$ & $0.00 \mathrm{E}+\infty 0$ & $100.00 \%$ & $0.00 \%$ & $0.00 \%$ & $4.42 \mathrm{E}-04$ \\
\hline METHYLENE CHLORIDE & $0.00 \mathrm{E}+00$ & $1.51 \mathrm{E}-03$ & $6.67 \mathrm{E}-03$ & $0.00 \%$ & $18.48 \%$ & $81.52 \%$ & $8.19 \mathrm{E}-03$ \\
\hline \multicolumn{8}{|c|}{ Fruiting Vegetables } \\
\hline \multicolumn{8}{|l|}{ Inorganics (mg/kg) } \\
\hline BARIUM & $1.17 \mathrm{E}+00$ & $0.00 \mathrm{E}+00$ & $0.00 \mathrm{E}+00$ & $100.00 \%$ & $0.00 \%$ & $0.00 \%$ & $1.17 E+\infty$ \\
\hline BERYLLIUM & $5.64 E-04$ & $3.46 \mathrm{E}-08$ & $5.52 \mathrm{E}-04$ & $50.54 \%$ & $0.00 \%$ & $49.46 \%$ & $1.12 \mathrm{E}-03$ \\
\hline CADMIUM & $9.93 E-01$ & $1.66 \mathrm{E}-04$ & $4.16 \mathrm{E}-03$ & $99.57 \%$ & $0.02 \%$ & $0.42 \%$ & 9.97E-01 \\
\hline CHROMIUM VI & $8.98 \mathrm{E}-03$ & $0.00 \mathrm{E}+\infty 0$ & $0.00 \mathrm{E}+00$ & $100.00 \%$ & $0.00 \%$ & $0.00 \%$ & $8.98 \mathrm{E}-03$ \\
\hline MANGANESE & $3.60 \mathrm{E}+01$ & $4.94 \mathrm{E}-04$ & 1.23E-01 & $99.66 \%$ & $0.00 \%$ & $0.34 \%$ & $3.61 \mathrm{E}+01$ \\
\hline MERCURY & $3.67 \mathrm{E}-01$ & $0.00 E+00$ & $0.00 \mathrm{E}+\infty$ & $100.00 \%$ & $0.00 \%$ & $0.00 \%$ & 3.67E-01 \\
\hline
\end{tabular}


Table C.20. (continued)

\begin{tabular}{|c|c|c|c|c|c|c|c|}
\hline Analyne & $\begin{array}{l}\text { Concentration in } \\
\text { vegetableffruit } \\
\text { resulting from } \\
\text { unirriggted soil }\end{array}$ & $\begin{array}{l}\text { Concentration } \\
\text { in vegetable/fruit } \\
\text { resulting from } \\
\text { irrigated soil }\end{array}$ & $\begin{array}{l}\text { Concentration } \\
\text { in vegetableffruit } \\
\text { resulting from } \\
\text { direct irrigation }\end{array}$ & $\begin{array}{l}\text { Percent of CV } \\
\text { resulting Irom } \\
\text { unirrigated soil }\end{array}$ & $\begin{array}{l}\text { Percent of CV } \\
\text { resulting from } \\
\text { irrigated soil }\end{array}$ & $\begin{array}{l}\text { Percent of } \mathrm{CV} \\
\text { resulting from } \\
\text { direct irrigation }\end{array}$ & $\begin{array}{l}\text { Total } \\
\text { vegetable/fruit } \\
\text { concentration } \\
\text { (CV) }\end{array}$ \\
\hline NICKEL & $6.67 \mathrm{E}-01$ & $0.00 \mathrm{E}+00$ & $0.00 \mathrm{E}+\infty 0$ & $100.00 \%$ & $0.00 \%$ & $0.00 \%$ & $6.67 \mathrm{E}-01$ \\
\hline NITRATE & $0.00 \mathrm{E}+00$ & $0.00 \mathrm{E}+00$ & $5.50 \mathrm{E}+01$ & $0.00 \%$ & $0.00 \%$ & $100.00 \%$ & $5.50 E+01$ \\
\hline URANIUM (SOLUBLE SALTS) & $3.83 E-02$ & $0.00 \mathrm{E}+00$ & $0.00 \mathrm{E}+00$ & $100.00 \%$ & $0.00 \%$ & $0.00 \%$ & $3.83 \mathrm{E}-02$ \\
\hline VANADIUM & $0.00 \mathrm{E}+\infty 0$ & 1.34E-06 & $7.74 \mathrm{E}-03$ & $0.00 \%$ & $0.02 \%$ & $99.98 \%$ & $7.75 E-03$ \\
\hline \multicolumn{8}{|l|}{ Radionuclides (pCi/g) } \\
\hline RADIUM-226 & $4.51 \mathbb{E}-03$ & $0.00 \mathrm{E}+00$ & $0.00 E+00$ & $100.00 \%$ & $0.00 \%$ & $0.00 \%$ & $4.51 \mathrm{E}-03$ \\
\hline URANIUM-234 & $1.29 \mathrm{E}-02$ & $1.76 \mathrm{E}-03$ & $5.27 \mathrm{E}+00$ & $0.24 \%$ & $0.03 \%$ & $99.72 \%$ & $5.28 \mathrm{E}+00$ \\
\hline URANIUM-238 & $1.28 \mathrm{E}-02$ & $1.19 \mathrm{E}-03$ & $3.57 \mathrm{E}+00$ & $0.36 \%$ & $0.03 \%$ & $99.61 \%$ & $3.58 \mathrm{E}+00$ \\
\hline \multicolumn{8}{|l|}{ Organics (mg/kg) } \\
\hline 1,2-DICHLOROETHENE (TOTAL) & $0.00 \mathrm{E}+00$ & $6.11 \mathrm{E}-03$ & $3.27 \mathrm{E} \cdot 02$ & $0.00 \%$ & $15.73 \%$ & $84.27 \%$ & $389 E-02$ \\
\hline BENZO(A)ANTHRACENE & $2.33 E-03$ & $0.00 \mathrm{E}+00$ & $0.00 \mathrm{E}+00$ & $100.00 \%$ & $0.00 \%$ & $0.00 \%$ & $2.33 E-03$ \\
\hline BENZO(A)PYRENE & $1.08 \mathrm{E}-03$ & $0.00 \mathrm{E}+00$ & $0.00 \mathrm{E}+\infty 0$ & $100.00 \%$ & $0.00 \%$ & $000 \%$ & $1.08 \mathrm{E}-03$ \\
\hline BENZO(B)FLUORANTHENE & $1.13 E-03$ & $000 \mathrm{E}+00$ & $0.00 \mathrm{E}+\infty$ & $100.00 \%$ & $0.00 \%$ & $0.00 \%$ & 1.13E-03 \\
\hline BROMODICHLOROMETHANE & $0.00 \mathrm{E}+00$ & $3.54 \mathrm{E}-04$ & $5.41 E-03$ & $0.00 \%$ & $6.13 \%$ & $93.87 \%$ & $5.77 \mathrm{E}-03$ \\
\hline INDENO(1,2,3-CD)PYRENE & $4.42 \mathrm{E}-04$ & $0.00 \mathrm{E}+\infty$ & $0.00 \mathrm{E}+\infty$ & $100.00 \%$ & $0.00 \%$ & $0.00 \%$ & 4.42E-04 \\
\hline METHYLENE CHLORIDE & $0.00 \mathrm{E}+00$ & $1.51 \mathrm{E}-03$ & $6.67 \mathrm{E}-03$ & $0.00 \%$ & $18.48 \%$ & $81.52 \%$ & $8.19 \mathrm{E}-03$ \\
\hline \multicolumn{8}{|c|}{ Fruits } \\
\hline \multicolumn{8}{|l|}{ Inorganics (mg/kg) } \\
\hline BARIUM & $1.17 E+\infty$ & $0.00 E+00$ & $0.00 \mathrm{E}+00$ & $100.00 \%$ & $0.00 \%$ & $0.00 \%$ & $1.17 \mathrm{E}+00$ \\
\hline
\end{tabular}


Table C.20. (continued)

\begin{tabular}{|c|c|c|c|c|c|c|c|}
\hline Analyte & $\begin{array}{l}\text { Concentration in } \\
\text { vegetable/fruil } \\
\text { resulting from } \\
\text { unirrigated soil }\end{array}$ & $\begin{array}{l}\text { Concentration } \\
\text { in vegetable/fruit } \\
\text { resulting from } \\
\text { irigated soil }\end{array}$ & $\begin{array}{l}\text { Concentration } \\
\text { in vegetablefruit } \\
\text { resulting from } \\
\text { direct irrigation }\end{array}$ & $\begin{array}{l}\text { Percent of CV } \\
\text { resulting from } \\
\text { unirrigated soil }\end{array}$ & $\begin{array}{l}\text { Percent of CV } \\
\text { resulting from } \\
\text { imigated soil } \\
\end{array}$ & $\begin{array}{l}\text { Percent of } \mathrm{CV} \\
\text { resulting from } \\
\text { direct irrigation }\end{array}$ & $\begin{array}{l}\text { Tolal } \\
\text { vegelable/fruit } \\
\text { concentration } \\
\text { (CV) }\end{array}$ \\
\hline BER YLLIUM & $5.64 \mathrm{E}-04$ & $3.46 \mathrm{E}-08$ & $5.52 \mathrm{E}-04$ & $50.54 \%$ & $0.00 \%$ & $49.46 \%$ & $1.12 \mathrm{E}-03$ \\
\hline CADMIUM & $9.93 \mathrm{E}-01$ & $1.66 \mathrm{E} \cdot 04$ & $4.16 \mathrm{E}-03$ & $99.57 \%$ & $0.02 \%$ & $0.42 \%$ & $9.97 \mathrm{E}-01$ \\
\hline CHROMIUM VI & $8.98 \mathrm{E}-03$ & $0.00 E+00$ & $0.00 \mathrm{E}+00$ & $100.00 \%$ & $0.00 \%$ & $0.00 \%$ & $8.98 \mathrm{E}-03$ \\
\hline MANGANESE & $3.60 E+01$ & $4.94 \mathrm{E}-04$ & $1.23 E-01$ & $99.66 \%$ & $0.00 \%$ & $0.34 \%$ & $3.61 E+01$ \\
\hline MERCURY & $3.67 \mathrm{E}-01$ & $0.00 \mathrm{E}+00$ & $0.00 \mathrm{E}+00$ & $100.00 \%$ & $0.00 \%$ & $0.00 \%$ & $3.67 \mathrm{E}-01$ \\
\hline NICKEL & $6.67 \mathrm{E}-01$ & $0.00 \mathrm{E}+00$ & $0.00 \mathrm{E}+00$ & $100.00 \%$ & $0.00 \%$ & $0.00 \%$ & $6.67 \mathrm{E}-01$ \\
\hline NITRATE & $0.00 \mathrm{E}+00$ & $0.00 E+00$ & $5.50 E+01$ & $0.00 \%$ & $0.00 \%$ & $100.00 \%$ & $5.50 \mathrm{E}+01$ \\
\hline URANIUM (SOLUBLE SALTS) & $3.83 E-02$ & $0.00 E+00$ & $0.00 \mathrm{E}+00$ & $100.00 \%$ & $0.00 \%$ & $0.00 \%$ & $3.83 E-02$ \\
\hline VANADIUM & $0.00 \mathrm{E}+00$ & $1.34 \mathrm{E}-06$ & $7.74 \mathrm{E}-03$ & $0.00 \%$ & $0.02 \%$ & $99.98 \%$ & $7.75 \mathrm{E}-03$ \\
\hline \multicolumn{8}{|l|}{ Radionuclides (pCi/g) } \\
\hline RADIUM-226 & $4.51 E-03$ & $0.00 E+00$ & $0.00 \mathrm{E}+00$ & $100.00 \%$ & $0.00 \%$ & $0.00 \%$ & $4.51 \mathrm{E}-03$ \\
\hline URANIUM-234 & $1.29 \mathrm{E}-02$ & $1.76 \mathrm{E}-\mathrm{n3}$ & $5.27 \mathrm{E}+\infty 0$ & $0.24 \%$ & $0.03 \%$ & $99.72 \%$ & $5.28 \mathrm{E}+00$ \\
\hline URANIUM-238 & $1.28 \mathrm{E}-02$ & $1.19 E-03$ & $3.57 \mathrm{E}+00$ & $0.36 \%$ & $0.03 \%$ & $99.61 \%$ & $3.58 \mathrm{E}+\infty 0$ \\
\hline \multicolumn{8}{|l|}{ Organics (mg/kg) } \\
\hline 1,2-DICHLOROETHENE (TOTAL) & $0.00 \mathrm{E}+\infty 0$ & $6.11 E-03$ & $3.27 \mathrm{E}-02$ & $0.00 \%$ & $15.73 \%$ & $84.27 \%$ & $3.89 E-02$ \\
\hline BENZO(A)ANTHRACENE & $2.33 \mathrm{E}-03$ & $0.00 \mathrm{E}+00$ & $0.00 \mathrm{E}+00$ & $100.00 \%$ & $0.00 \%$ & $0.00 \%$ & $2.33 E-03$ \\
\hline BENZO(A)PYRENE & $1.08 E-03$ & $0.00 \mathrm{E}+00$ & $0.00 \mathrm{E}+00$ & $100.00 \%$ & $0.00 \%$ & $0.00 \%$ & $1.08 \mathrm{E}-03$ \\
\hline BENZO(B)FLUORANTHENE & $1.13 E-03$ & $0.00 \mathrm{E}+00$ & $0.00 \mathrm{E}+00$ & $100.00 \%$ & $0.00 \%$ & $0.00 \%$ & 1.13E-03 \\
\hline BROMODICHLOROMETHANE & $0.00 \mathrm{E}+00$ & $3.54 \mathrm{E}-04$ & $5.41 \mathrm{E}-03$ & $0.00 \%$ & $6.13 \%$ & $93.87 \%$ & $5.77 \mathrm{E}-03$ \\
\hline
\end{tabular}


Table C.20. (comtinued)

\begin{tabular}{|c|c|c|c|c|c|c|c|}
\hline Analyle & $\begin{array}{l}\text { Concentration in } \\
\text { vegetableffruit } \\
\text { resulting from } \\
\text { unirrigated soil }\end{array}$ & $\begin{array}{l}\text { Concentration } \\
\text { in vegetableffruit } \\
\text { resulting from } \\
\text { irrigated soil }\end{array}$ & $\begin{array}{l}\text { Concentration } \\
\text { in vegetablefiruir } \\
\text { resulting from } \\
\text { direct irrigation }\end{array}$ & $\begin{array}{l}\text { Percent of } \mathrm{CV} \\
\text { resulting from } \\
\text { unirrigated soil }\end{array}$ & $\begin{array}{l}\text { Percent of } \mathrm{CV} \\
\text { resulting from } \\
\text { irrigated soil }\end{array}$ & $\begin{array}{l}\text { Percent of CV } \\
\text { resulting from } \\
\text { direct imigation }\end{array}$ & $\begin{array}{l}\text { Total } \\
\text { vegelable/fruil } \\
\text { concentration }^{\text {b }} \\
\text { (CV) }\end{array}$ \\
\hline INDENO(1,2,3-CD)PYRENE & $4.42 E-04$ & $0.00 \mathrm{E}+00$ & $0.00 \mathrm{E}+00$ & $100.00 \%$ & $0.00 \%$ & $0.00 \%$ & $4.42 \mathrm{E}-04$ \\
\hline METIYLENE CHLORIDE & $0.00 E+00$ & $1.51 \mathrm{E}-03$ & $6.67 \mathrm{E}-03$ & $0.00 \%$ & $18.48 \%$ & $81.52 \%$ & $8.19 \mathrm{E}-03$ \\
\hline
\end{tabular}

$\mathrm{CV}=$ Concentration in vegetable/ruit

The relative contributions (from soil $\mathrm{COPCs}$ and groundwater $\mathrm{COPCs}$ ) to the total vegetable/fruit concentration (i.e., percent of $\mathrm{CV}$ ) are shown here.

- The total concentration of a COPC in a vegetable/fruit-type (CV) is the sum of the concentrations from the unirrigated soil, from the irrigated soil, and from direct irrigation with the groundwater; the tolal vegetable/fruit concentrations (CV), for each COPC, were used to evaluate risk/HQ to human health from consumption of the homegrown vegetables and fruit. 
Table C.21. Chronic daily intakes for BCV OU2 COPCs - residential homegrown garden scenario ab

\begin{tabular}{|c|c|c|c|c|c|c|c|c|c|c|c|c|c|c|c|}
\hline \multirow[b]{2}{*}{ Analyte } & \multicolumn{3}{|c|}{ Leafy vegetables } & \multicolumn{3}{|c|}{ Root vegetables } & \multicolumn{3}{|c|}{ Legume vegetables } & \multicolumn{3}{|c|}{ Fruitiag vegetables } & \multicolumn{3}{|c|}{ Pruits } \\
\hline & $\begin{array}{l}\text { Conc: } \\
\text { (mg/kg; } \\
\text { pCi/g) }\end{array}$ & $\begin{array}{l}\text { Ingest. } \\
\text { carcin. } \\
\text { CDI }\end{array}$ & $\begin{array}{l}\text { Ingest. } \\
\text { noncar. } \\
\text { CDI }\end{array}$ & $\begin{array}{l}\text { Conc: } \\
\text { (mig/k; } \\
\text { pCi/g) }\end{array}$ & $\begin{array}{l}\text { Ingest. } \\
\text { carcias. } \\
\text { CDI } \\
\end{array}$ & $\begin{array}{l}\text { Ingest } \\
\text { moncar. } \\
\text { CDI }\end{array}$ & $\begin{array}{l}\text { Conc: } \\
\text { (migks; } \\
\mathrm{pCi} / \mathrm{g})\end{array}$ & $\begin{array}{l}\text { Ingest. } \\
\text { carcim. } \\
\text { CDI }\end{array}$ & $\begin{array}{l}\text { Ingest } \\
\text { noncar. } \\
\text { CDI }\end{array}$ & $\begin{array}{l}\text { Conc. } \\
\text { (mg/kg; } \\
\mathrm{pCi} / \mathrm{g} \text { ) }\end{array}$ & $\begin{array}{l}\text { Ingest } \\
\text { carcis. } \\
\text { CDI }\end{array}$ & $\begin{array}{l}\text { Ingest. } \\
\text { moncar. } \\
\text { CDI }\end{array}$ & $\begin{array}{l}\text { Conc: } \\
\text { (me/kg: } \\
\text { pCi/g) }\end{array}$ & $\begin{array}{l}\text { Ingest. } \\
\text { carcin. } \\
\text { CDI } \\
\end{array}$ & $\begin{array}{l}\text { Ingest } \\
\text { roncar. } \\
\text { CDI } \\
\end{array}$ \\
\hline \multicolumn{16}{|c|}{ RSA } \\
\hline \multicolumn{16}{|l|}{ Inorganics } \\
\hline ANTIMONY & $1.76 \mathrm{E}-01$ & $1.4 E-04$ & $3.3 E-04$ & 8.61E.0S & $7.0 \mathrm{E}-08$ & $1.6 \mathrm{E}-07$ & $5.88 E-02$ & $4.85-05$ & $1.1 \mathrm{E}-04$ & $5.88 E-02$ & $4.8 E-05$ & 1.1E-04 & $5.88 \mathrm{E}-02$ & $2.5 E \cdot 05$ & $5.9 \mathrm{E}-0.5$ \\
\hline ARSENIC & $1.92 \mathrm{E}-01$ & $1.6 \mathrm{E}-0$. & $3.6 \mathrm{E}-0 \mathrm{~A}$ & $8.53 E-05$ & $6.9 \mathrm{E}-08$ & $1.6 \mathrm{E}-07$ & $6.41 E-02$ & $5.2 E-05$ & $1.2 E \cdot 04$ & $6.41 \mathrm{E} .02$ & $5.2 E-05$ & $1.2 E-04$ & $6.41 E-02$ & 27E-05 & 6.4E-05 \\
\hline BARIUM & S.7SE-01 & 4.7E-04 & 1.1E-03 & 5.75E-01 & 4.7E-04 & $1.1 \mathrm{E}-03$ & 5.75E-01 & $4.7 E-04$ & 1.1E.03 & 5.75E-01 & $4.7 E-04$ & $1.1 \mathrm{E}-03$ & $5.75 E-01$ & $25 \mathrm{E}-04$ & $5.7 \mathrm{E}-04$ \\
\hline BERYLUUM & 1.89E-03 & $1.5 \mathrm{E}-06$ & $3.6 \mathrm{E}-06$ & 4.94E-04 & 4.0E-07 & $9.4 \mathrm{E}-07$ & $9.57 \mathrm{E}-04$ & $7.8 \mathrm{E}-07$ & $1.8 \mathrm{E}-06$ & $9.57 \mathrm{E}-04$ & $7.8 \mathrm{E}-07$ & $1.8 \mathrm{E}-66$ & $9.57 \mathrm{E}-04$ & $4.1 \mathrm{E}-07$ & $9.5 E-07$ \\
\hline CADMIUM & $1.49 E+100$ & $1.2 \mathrm{E}-03$ & $28 \mathrm{E}-03$ & $1.48 E+\infty 0$ & 1.2E-03 & $28 \mathrm{E}-03$ & $1.48 E+\infty 0$ & $1.2 \mathrm{E}-03$ & $28 \mathrm{E}-03$ & $1.48 E+00$ & $1.2 E-03$ & $28 E-03$ & $1.48 \mathrm{E}+00$ & 6.3E-04 & 1.5E-03 \\
\hline $\begin{array}{l}\text { CHROMUUM- } \\
\text { VI }\end{array}$ & 1.00E-01 & 8.1E-05 & $1.9 \mathrm{E}-0 \mathrm{~A}$ & 1.11E-06 & $9.0 \mathrm{E}-10$ & $21 E-09$ & $3.34 E-02$ & 27E-05 & $6.3 \mathrm{E}-05$ & 3.34E-02 & $27 E-05$ & 6.3E-05 & $3.34 \mathrm{E}-02$ & $1.4 \mathrm{E}-05$ & 3.3E-05 \\
\hline MANGANESE & $4.77 E+01$ & $3.9 \mathrm{E}-02$ & $9.1 \mathrm{E} .02$ & $270 E+01$ & $22 \mathrm{E}-02$ & $5.1 E-02$ & $3.39 E+01$ & $28 \mathrm{E}-02$ & 6.4E-02 & $3.39 E+01$ & $28 E-02$ & $6.4 E-02$ & $3.39 E+01$ & $1.4 \mathbb{E}-02$ & $3.4 E-02$ \\
\hline MERCURY & $7.60 \mathrm{E}-01$ & $6.2 \mathrm{E}-04$ & 1.4E-03 & $7.60 \mathrm{E}-01$ & $6.2 \mathrm{E} \cdot 04$ & $1.4 \mathbb{E}-03$ & $7.60 \mathrm{E}-01$ & $6.2 E-04$ & 1.4E-03 & 7.606 .01 & $6.26-04$ & I.SE-03 & $7.60 \mathrm{E}-01$ & $3.2 \mathrm{E}-04$ & $7.6 E-04$ \\
\hline NICKEL. & $8.76 \mathrm{E}-01$ & 7.1E-04 & $1.7 \mathrm{E}-03$ & $8.76 \mathrm{E}-01$ & $7.1 \mathrm{E}-04$ & $1.7 \mathrm{E}-03$ & $8.76 \mathrm{E}-01$ & $7.1 \mathrm{E}-0.4$ & $1.7 E-03$ & $8.76 E-01$ & $7.1 \mathrm{E} \cdot 04$ & $1.7 \mathrm{E}-03$ & $8.76 \mathrm{E}-81$ & 3.7E-04 & $8.7 \mathrm{E}-\mathrm{OH}$ \\
\hline NITRATE & $2768+02$ & 22E-01 & $5.2 \mathrm{E}-01$ & $0.00 E+00$ & $0.0 \mathrm{E}+00$ & $0.0 E+60$ & $9.19 E+01$ & $7.5 \mathrm{E}-02$ & 1.7E-01 & $9.19 \mathrm{E}+01$ & $7.5 \mathrm{E}-02$ & $1.7 \mathrm{E}-01$ & $9.19 \mathrm{E}+01$ & $3.9 \mathrm{E}-02$ & $9.2 \mathrm{E}-02$ \\
\hline $\begin{array}{l}\text { URANIUM } \\
\text { (SOLUBLE } \\
\text { SALTS) }\end{array}$ & 3.14E-02 & $26 \mathrm{E}-05$ & $6.0 \mathrm{E}-05$ & 1.84E-02 & 1.5E-0S & $3.5 \mathrm{E}-05$ & $227 \mathrm{E}-12$ & 1.8E-05 & 4.3E-05 & $227 \mathrm{E}-02$ & $1.8 \mathrm{E}-05$ & 4.3E.05 & 227E-02 & $9.7 E-06$ & 23E-05 \\
\hline \multicolumn{16}{|l|}{ Radionuclides } \\
\hline CESIUM-137 & $3.11 E+02$ & $26 E+08$ & & $276 \mathrm{E}-02$ & $23 E+04$ & & $1.04 E+02$ & $8.7 \mathrm{E}+07$ & & $1.04 E+02$ & $8.7 E+07$ & & $1.04 E+02$ & $4.6 \mathrm{E}+07$ & \\
\hline $\begin{array}{l}\text { STRONTIUM } \\
-90\end{array}$ & $6.54 E+02$ & $5.5 E+08$ & & $5.76 E+00$ & $4.8 E+06$ & & $222 E+02$ & $1.9 E+08$ & & $222 \mathrm{E}+02$ & $1.9 \mathrm{E}+08$ & & $222 E+02$ & $9.8 \mathrm{E}+07$ & \\
\hline $\begin{array}{l}\text { TECHNETUUM } \\
.99\end{array}$ & $1.30 \mathrm{E}+04$ & $1.1 \mathrm{E}+10$ & & $1.43 E+02$ & $1.2 \mathrm{E}+08$ & & $4.42 E+03$ & $3.7 \mathbb{E}+\infty 9$ & & $4.42 \mathrm{E}+03$ & $3.7 E+09$ & & $4.42 E+03$ & $1.9 E+09$ & \\
\hline THORUM-232 & 1.30E-02 & $1.1 E+04$ & & $1.30 \mathrm{E}-02$ & $1.1 E+04$ & & $1.30 E-02$ & $1.1 E+04$ & & $1.30 \mathrm{E}-02$ & $1.1 E+04$ & & $1.30 \mathrm{E}-02$ & $5.7 \mathrm{E}+03$ & \\
\hline
\end{tabular}


Table C.21. (contimuod)

\begin{tabular}{|c|c|c|c|c|c|c|c|c|c|c|c|c|c|c|c|}
\hline \multirow[b]{2}{*}{ Analyte } & \multicolumn{3}{|c|}{ Lealy vegetables } & \multicolumn{3}{|c|}{ Root vegetables } & \multicolumn{3}{|c|}{ Legume vegetables } & \multicolumn{3}{|c|}{ Fruiting vegetables } & \multicolumn{3}{|c|}{ Fruits } \\
\hline & $\begin{array}{l}\text { Conc: } \\
\text { (mag/kg; } \\
\text { pCi/g) }\end{array}$ & $\begin{array}{l}\text { Ingest. } \\
\text { carcin. } \\
\text { CDI }\end{array}$ & $\begin{array}{l}\text { Ingest. } \\
\text { noncar. } \\
\text { CDI }\end{array}$ & $\begin{array}{l}\text { Conc: } \\
\text { (mg/tg: } \\
\text { pCi/g) }\end{array}$ & $\begin{array}{l}\text { Ingest. } \\
\text { carcin. } \\
\text { CDI }\end{array}$ & $\begin{array}{l}\text { Ingest. } \\
\text { noncar. } \\
\text { CDI }\end{array}$ & $\begin{array}{l}\text { Conc: } \\
(\mathrm{mg} / \mathrm{kg} \\
\mathrm{pCi/g})\end{array}$ & $\begin{array}{l}\text { Ingest. } \\
\text { carcin. } \\
\text { CDI }\end{array}$ & $\begin{array}{l}\text { Ingest. } \\
\text { noncar. } \\
\mathrm{CDI}\end{array}$ & $\begin{array}{l}\text { Conc: } \\
\text { (mg/kg: } \\
\text { pCi/g) }\end{array}$ & $\begin{array}{l}\text { Ingest. } \\
\text { carcin. } \\
\mathrm{CDI} \\
\end{array}$ & $\begin{array}{l}\text { Ingest } \\
\text { moncar. } \\
\text { CDI } \\
\end{array}$ & $\begin{array}{l}\text { Conc: } \\
\text { (magkg: } \\
\text { pCi/g) }\end{array}$ & $\begin{array}{l}\text { Ingest. } \\
\text { carcin. } \\
\text { CDI }\end{array}$ & $\begin{array}{l}\text { Ingest. } \\
\text { noncar. } \\
\text { CDI } \\
\end{array}$ \\
\hline URANIUM-234 & $4.38 E+00$ & $3.7 \mathrm{E}+06$ & & $7.51 \mathrm{E}-03$ & $6.3 E+03$ & & $1.47 E+00$ & $1.2 \mathrm{E}+06$ & & $1.47 \mathbb{E}+00$ & $1.2 \mathrm{E}+06$ & & $1.47 \mathrm{E}+\infty 0$ & $6.5 \mathrm{E}+05$ & \\
\hline URANIUM-238 & $4.34 E+00$ & $3.6 \mathrm{E}+06$ & & $4.82 \mathrm{E}-04$ & $4.0 \mathrm{E}+02$ & & $1.45 E+00$ & $1.2 E+06$ & & 1.4SE $+\infty$ & $1.2 E+06$ & & $1.45 \mathrm{E}+00$ & $6.4 \mathrm{E}+05$ & \\
\hline \multicolumn{16}{|l|}{ Organics } \\
\hline $\begin{array}{l}\text { 1,2,4-TRI. } \\
\text { CHLORO. } \\
\text { BENZENE }\end{array}$ & 1.38E-01 & $1.1 \mathrm{E}-04$ & 26E.04 & $1.38 \mathrm{E}-01$ & $1.1 E-04$ & 26E-04 & $1.38 \mathrm{E}-01$ & 1.1E-a4 & $26 \mathrm{E}-04$ & $1.38 \mathrm{E}-01$ & $1.1 E-04$ & $26 \mathrm{E} \cdot 09$ & $1.38 E-01$ & $5.9 \mathrm{E}-05$ & 1.4E-04 \\
\hline $\begin{array}{l}\text { 1,2.DI- } \\
\text { CHLORO } \\
\text { ETHENE } \\
\text { (TOTAL) }\end{array}$ & $230 E-01$ & $1.9 E-04$ & 4.4E.04 & $1.09 E-01$ & $8.9 \mathrm{E}-05$ & $21 E-0$ & $1.49 \mathrm{E}-01$ & $1.2 \mathbb{E}-04$ & $28 E-04$ & $1.49 \mathrm{E}-01$ & $1.2 E-04$ & $28 E-04$ & $1.49 \mathbb{E}-01$ & $6.4 E-05$ & $1.5 E-94$ \\
\hline $\begin{array}{l}\text { AROCLOR } \\
-1254\end{array}$ & $3.06 \mathrm{E}-03$ & $25 E-06$ & $5.8 \mathrm{E}-06$ & $3.06 \mathrm{E}-03$ & $25 E$ & $5.8 E \propto 6$ & $3.00 \mathrm{E}-03$ & 25E-06 & 5.8E-06 & $3.06 \mathrm{E}-03$ & 25E-06 & $5.8 E-06$ & $3.06 \mathrm{E}-03$ & $1.3 \mathrm{E}-06$ & $3.1 \mathrm{E}-06$ \\
\hline $\begin{array}{l}\text { BENZO(A)- } \\
\text { PYRENE }\end{array}$ & $1.43 E-03$ & $1.2 \mathrm{E}-\%$ & $27 E-06$ & $1.43 \mathrm{E} .03$ & $1.2 \mathrm{E}-06$ & $27 E-06$ & $1.43 E-03$ & $1.2 \mathrm{E}-06$ & $27 \mathrm{E}-06$ & $1.43 E-03$ & $1.2 E-06$ & 27E-06 & $1.43 E-03$ & $6.1 \mathrm{E}-07$ & $1.4 \mathrm{E}-06$ \\
\hline $\begin{array}{l}\text { BIS(2-ETHYL } \\
\text { HEXYL) } \\
\text { PHTHALATE }\end{array}$ & $1.77 E \cdot 04$ & 1.4E-07 & 3.4E-07 & $1.77 \mathrm{E}-04$ & $1.4 E-07$ & $3.4 E-07$ & $1.77 \mathrm{EM}$ & $1.4 \mathrm{E}-07$ & 3.4E-07 & $1.77 \mathrm{E}-04$ & $1.4 E-07$ & $3.4 E-07$ & $1.77 \mathbb{E}-04$ & $7.6 \mathrm{E}-08$ & $1.8 E-07$ \\
\hline $\begin{array}{l}\text { CARBON } \\
\text { TETRA- } \\
\text { CHLORIDE }\end{array}$ & $1.74 E-02$ & $1.4 \mathrm{E}-05$ & 3.3E-05 & $222 \mathrm{E}-04$ & $1.8 \mathrm{E}-07$ & 4.2E-07 & 5.95E-03 & 4.8E-06 & $1.1 \mathrm{E}-05$ & 5.95E-03 & $4.8 E .06$ & $1.1 \mathrm{E}-05$ & $5.95 \mathbb{E}-03$ & 25E-A6 & $5.9 \mathrm{E}-06$ \\
\hline $\begin{array}{l}\text { DIBENZO- } \\
\text { (A,H)AN- } \\
\text { THRACENE }\end{array}$ & $1.60 \mathrm{E}-03$ & $1.3 \mathrm{E}-06$ & $3.0 \mathrm{E}-06$ & $1.60 \mathrm{E}-03$ & $1.3 \mathrm{E} \cdot 06$ & $3.0 \mathrm{E}-06$ & $1.60 \mathrm{E}-03$ & 1.3E-06 & $3.0 \mathrm{E}-06$ & $1.60 \mathrm{E}-03$ & 1.3E-06 & $3.0 \mathrm{E}-06$ & $1.60 \mathrm{E}-03$ & $6.8 \mathrm{E}-07$ & $1.6 \mathrm{E}-06$ \\
\hline $\begin{array}{l}\text { TETRA- } \\
\text { CHLORO- } \\
\text { ETHENE }\end{array}$ & $4.86 \mathrm{E}-02$ & 4.0E-05 & $9.2 \mathrm{E}-05$ & $4.86 E-12$ & 4.0E-0S & $9.2 \mathrm{E}-05$ & 4.86E-02 & $4.0 \mathrm{E}-05$ & $9.2 \mathrm{E}-05$ & $4.86 E-02$ & 4.0E-05 & $9.2 \mathrm{E}-05$ & $4.86 \mathrm{E}-02$ & $21 \mathrm{E}-05$ & 4.8E-05 \\
\hline
\end{tabular}


Table C.21. (continued)

\begin{tabular}{|c|c|c|c|c|c|c|c|c|c|c|c|c|c|c|c|}
\hline \multirow[b]{2}{*}{ Analyile } & \multicolumn{3}{|c|}{ Leafy vegetables } & \multicolumn{3}{|c|}{ Root vegetables } & \multicolumn{3}{|c|}{ Legume vegetables } & \multicolumn{3}{|c|}{ Pruiting vegetables } & \multicolumn{3}{|c|}{ Pruils } \\
\hline & 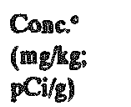 & $\begin{array}{l}\text { Ingest } \\
\text { carcim. } \\
\text { CDI }\end{array}$ & $\begin{array}{l}\text { Ingest. } \\
\text { noncar. } \\
\text { CDI }\end{array}$ & $\begin{array}{l}\text { Conc: } \\
\text { (mg/kg; } \\
\text { pCi/g) }\end{array}$ & $\begin{array}{l}\text { Ingest } \\
\text { carcin. } \\
\text { CDI }\end{array}$ & $\begin{array}{l}\text { Ingesh } \\
\text { moncar. } \\
\text { COI }\end{array}$ & $\begin{array}{l}\text { Conc: } \\
\text { (mellig; } \\
\text { pCi/g) }\end{array}$ & $\begin{array}{l}\text { Ingest. } \\
\text { carcia. } \\
\text { COI }\end{array}$ & $\begin{array}{l}\text { Ingest. } \\
\text { moncar. } \\
\text { CDI }\end{array}$ & $\begin{array}{l}\text { Conc. } \\
\text { (meglép; } \\
\text { pCirg) }\end{array}$ & $\begin{array}{l}\text { Ingest } \\
\text { carcia. } \\
\text { CDI }\end{array}$ & $\begin{array}{l}\text { Ingest } \\
\text { noncar. } \\
\text { CDI }\end{array}$ & $\begin{array}{l}\text { Conc. } \\
\text { (mg/sig: } \\
\text { pCi/g) }\end{array}$ & $\begin{array}{l}\text { Ingest. } \\
\text { carcin. } \\
\text { CDI }\end{array}$ & $\begin{array}{l}\text { Ingest. } \\
\text { nomear. } \\
\text { CDI }\end{array}$ \\
\hline \multicolumn{16}{|c|}{$\$ Y-200$} \\
\hline \multicolumn{16}{|l|}{ Inorganics } \\
\hline CADMIUM & $9.72 \mathbb{E}-02$ & $7.9 \mathrm{E}-05$ & $1.8 \mathrm{E}-04$ & $9.72 \mathrm{E}-02$ & $7.9 \mathrm{E}-05$ & $1.8 E-04$ & $9.72 E-02$ & $7.9 \mathrm{E}-05$ & $1.8 \mathrm{E}-04$ & $9.72 \mathrm{E} .02$ & $7.9 \mathrm{E}-05$ & $1.8 \mathrm{E}-04$ & $9.72 \mathrm{E}-02$ & 4.2E-05 & 9.7E-05 \\
\hline $\begin{array}{l}\text { CHROMIUM- } \\
\text { VI }\end{array}$ & $1.18 E-01$ & $9.6 \mathrm{E}-05$ & $22 E-04$ & $1.31 E-06$ & $1.1 \mathrm{E}-09$ & 25E.09 & $3.92 E-02$ & $3.2 E-05$ & $7.4 \mathrm{E}-05$ & $3.92 \mathrm{E}-02$ & $3.2 \mathrm{E} .05$ & 7.4E-05 & $3.92 \mathrm{E} .02$ & $1.7 \mathrm{E}-05$ & $3.9 \mathrm{E}-0 \mathrm{~S}$ \\
\hline MANGANESE & $5.77 \mathrm{E}+01$ & 9.7E-02 & 1.1E-01 & $220 \mathrm{E}+01$ & $1.8 \mathrm{E}-02$ & $4.2 E-02$ & $3.39 \mathrm{E}+01$ & $28 E-02$ & 6.4E- -02 & $3.39 E+01$ & $28 \mathrm{E}-02$ & $6.4 \mathbb{E}-02$ & $3.39 \mathrm{E}+01$ & $1.4 \mathrm{E}-02$ & 3.4E-02 \\
\hline MERCURY & $7.52 E+00$ & $6.1 \mathrm{E}-03$ & $1.4 E-02$ & $7.52 E+\infty$ & $6.1 \mathrm{E}-03$ & $1.4 E-02$ & $7.52 E+\infty$ & $6.1 E-03$ & $1.4 E-02$ & $7.52 E+\infty$ & $6.1 E-03$ & $1.4 \mathrm{E}-02$ & $7.52 E+00$ & $3.2 \mathrm{E}-03$ & $7.5 E-03$ \\
\hline \multicolumn{16}{|l|}{ Radionaclides } \\
\hline URANIUM-234 & $4.65 E+01$ & $3.9 E+07$ & & $5.16 E-03$ & $4.3 E+03$ & & $1.55 E+01$ & $1.3 E+07$ & & $1.55 E+01$ & $1.3 E+07$ & & $1.55 E+01$ & $6.8 E+06$ & \\
\hline URANIUM.235 & $270 E+\infty 0$ & $23 E+6$ & & $3.00 E-04$ & $25 E+02$ & & .01E01 & $7.6 \mathbb{E}+05$ & & $2.01 E-01$ & $7.6 \mathrm{E}+05$ & & $9.01 E-01$ & $4.0 \mathrm{E}+05$ & \\
\hline URANIUM-238 & $5.40 E+01$ & $4.5 \mathrm{E}+07$ & & $6.00 \mathrm{E}-03$ & $5.0 \mathrm{E}+03$ & & $1.80 E+01$ & $1.5 E+07$ & & $1.80 \mathrm{E}+01$ & $1.5 E+07$ & & $1.80 \mathrm{E}+01$ & $7.9 E+06$ & \\
\hline \multicolumn{16}{|l|}{ Organics } \\
\hline $\begin{array}{l}\text { AROCLOR } \\
.1254\end{array}$ & $9.58 E-04$ & $7.8 \mathrm{E}-07$ & 1.8E-06 & $9.58 \mathrm{E}-04$ & $7.8 \mathrm{E}-07$ & $1.8 E-\infty 6$ & 9.58E-04 & $7.8 E-07$ & $1.8 \mathrm{E}-106$ & $9.58 \mathrm{E}-0 \mathrm{~A}$ & $7.8 \mathrm{E}-07$ & $1.8 \mathrm{E}-06$ & $9.58 \mathrm{E}-04$ & 4.1E.07 & $9.6 \mathrm{E}-07$ \\
\hline $\begin{array}{l}\text { AROCLOR } \\
-1260\end{array}$ & $3.02 \mathrm{E}-04$ & 25E-07 & $5.7 E-07$ & $3.02 \mathrm{E}-04$ & $25 \mathrm{E}-07$ & $5.7 \mathrm{E}-07$ & $3.02 E-04$ & $25 \mathrm{E}-07$ & $5.7 \mathrm{E}-07$ & $3.02 E-04$ & 25E-07 & $5.7 \mathrm{E}-07$ & 3.02E-04 & $1.3 E-07$ & $3.0 \mathrm{E}-07$ \\
\hline $\begin{array}{l}\text { BENZO(A)- } \\
\text { ANTHRA- } \\
\text { CENE }\end{array}$ & $278 \mathrm{E}-03$ & $23 E-06$ & $5.3 E-06$ & $278 \mathrm{E}-03$ & $23 E-06$ & $5.3 E-06$ & $278 \mathrm{E}-03$ & 23E-96 & S.3E-06 & $278 \mathrm{E}-03$ & $23 E-06$ & $5.3 \mathrm{E}-06$ & $278 \mathrm{E}-03$ & $1.2 \mathrm{E}-06$ & 28E-06 \\
\hline $\begin{array}{l}\text { BENZO(A)- } \\
\text { PYRENE }\end{array}$ & 1.30E-03 & $1.1 \mathrm{E} .06$ & 25E- & $1.30 \mathrm{E}-03$ & $1.1 \mathrm{E}-06$ & 25E-06 & $1.30 E-03$ & 1.1E-06 & 25E-06 & $1.30 \mathbb{E}-03$ & 1.1E- 18 & 25E-06 & $1.30 \mathrm{E}-03$ & $5.6 \mathrm{E}-07$ & $1.3 E-06$ \\
\hline
\end{tabular}


Table C.21. (continued)

\begin{tabular}{|c|c|c|c|c|c|c|c|c|c|c|c|c|c|c|c|}
\hline \multirow[b]{2}{*}{ A amalyte } & \multicolumn{3}{|c|}{ Leafy vegetables } & \multicolumn{3}{|c|}{ Root vegetables } & \multicolumn{3}{|c|}{ Legume vegetsbles } & \multicolumn{3}{|c|}{ Fruiting vegetables } & \multicolumn{3}{|c|}{ Fruils } \\
\hline & $\begin{array}{l}\text { Conc. } \\
\text { (mg/kg; } \\
\mathrm{pCi/g)}\end{array}$ & $\begin{array}{l}\text { lagest. } \\
\text { carcin. } \\
\text { CDI } \\
\end{array}$ & $\begin{array}{l}\text { Ingest. } \\
\text { nomcar. } \\
\text { CDI }\end{array}$ & $\begin{array}{l}\text { Conc: } \\
\text { (mg/kg: } \\
\text { pCi/g) }\end{array}$ & $\begin{array}{l}\text { Ingest. } \\
\text { carcin. } \\
\text { CDI } \\
\end{array}$ & $\begin{array}{l}\text { Ingest } \\
\text { nomear. } \\
\text { CDI } \\
\end{array}$ & $\begin{array}{l}\text { Conc. } \\
\text { (mglks; } \\
\mathrm{pCi} / \mathrm{g} \text { ) }\end{array}$ & $\begin{array}{l}\text { Ingese } \\
\text { carcis. } \\
\text { CDI } \\
\end{array}$ & $\begin{array}{l}\text { Ingest. } \\
\text { noncar. } \\
\text { CDI } \\
\end{array}$ & $\begin{array}{l}\text { Conc: } \\
\text { (mglkg: } \\
\text { pCi/g) }\end{array}$ & $\begin{array}{l}\text { Ingest } \\
\text { carcila. } \\
\text { CDI } \\
\end{array}$ & $\begin{array}{l}\text { Ingest. } \\
\text { noncar. } \\
\text { CDI } \\
\end{array}$ & $\begin{array}{l}\text { Conc. } \\
(\mathrm{mg} / \mathrm{kg} \\
\mathrm{pCi} / \mathrm{g})\end{array}$ & $\begin{array}{l}\text { Ingest. } \\
\text { carcin. } \\
\text { CDI } \\
\end{array}$ & $\begin{array}{l}\text { Ingest } \\
\text { noncar. } \\
\text { CDI }\end{array}$ \\
\hline $\begin{array}{l}\text { BENZO(B). } \\
\text { FLUOR- } \\
\text { ANTHENE }\end{array}$ & $9.69 E-04$ & $7.9 \mathrm{E}-07$ & $1.8 E-06$ & $9.69 \mathrm{E}-04$ & $7.9 \mathrm{E}-07$ & $1.8 \mathrm{E}-06$ & $9.69 \mathrm{E}-04$ & $7.9 \mathrm{E}-07$ & $1.8 E-06$ & $9.69 \mathrm{E}-04$ & $7.9 \mathrm{E}-07$ & 1.8E-6 & $9.69 \mathrm{E}-04$ & $4.1 \mathrm{E}-07$ & $9.7 \mathrm{E}-07$ \\
\hline $\begin{array}{l}\text { BIS(2-ETHYL- } \\
\text { HEXYL) } \\
\text { PHTHALATE }\end{array}$ & $252 \mathrm{E}-02$ & 21E-05 & 4.5E-05 & 3.02E-08 & $25 \mathrm{E}-11$ & $5.7 \mathrm{E}-11$ & $8.40 \mathrm{E}-03$ & $6.8 \mathrm{E}-06$ & $1.6 \mathbb{E}-05$ & $8.40 \mathrm{E}-03$ & $6.8 \mathrm{E}-06$ & $1.6 \mathrm{E}-05$ & $8.40 \mathrm{E}-03$ & $3.6 \mathrm{E}-06$ & 8.4E-06 \\
\hline $\begin{array}{l}\text { CHLORO- } \\
\text { FORM }\end{array}$ & $6.49 \mathrm{E}-03$ & $5.3 E-06$ & $1.2 \mathrm{E}-05$ & $1.96 \mathrm{E}-04$ & $1.6 \mathrm{E}-07$ & $3.7 \mathbb{E}-07$ & $229 \mathrm{E}-03$ & $1.9 \mathrm{E}-06$ & $4.3 \mathrm{E}-06$ & $229 \mathrm{E}-03$ & $1.9 \mathrm{E}-06$ & 4.3E-06 & $229 \mathrm{E}-03$ & $9.8 \mathrm{E}-07$ & 23E-06 \\
\hline $\begin{array}{l}\text { DIBENZO- } \\
\text { (A,H)AN. } \\
\text { THRACENE }\end{array}$ & $6.60 \mathrm{E}-04$ & $5.4 E-07$ & $1.3 E-06$ & $6.60 \mathrm{E}-04$ & $5.4 E-07$ & $1.3 \mathrm{E}-06$ & $6.60 \mathrm{E}-04$ & $5.4 \mathrm{E}-07$ & $1.3 \mathrm{E}-06$ & $6.60 \mathrm{E}-04$ & $5.4 E-07$ & $1.3 E-06$ & $6.60 \mathrm{E}-04$ & $28 \mathrm{E}-07$ & $6.6 \mathrm{E}-07$ \\
\hline
\end{tabular}

\begin{tabular}{|c|c|c|c|c|c|c|c|c|c|c|c|c|c|c|c|}
\hline BARUM & $1.17 E+\infty$ & $9.5 E-04$ & $2.2 \mathrm{E} .03$ & $1.17 E+\infty$ & $9.5 \mathrm{E}-04$ & $22 \mathrm{E}-03$ & $1.17 \mathbb{E}+\infty$ & 9.5E 04 & $22 \mathrm{E}-03$ & $1.17 E+00$ & $9.5 E-04$ & $22 E-03$ & $1.17 E+\infty$ & $5.0 \mathrm{E}-04$ & $1.2 \mathrm{E}-03$ \\
\hline BERYLLIUM & 220E-03 & $1.8 E-06$ & $4.2 E .06$ & 5.6AE-OA & $4.6 \mathrm{E}-07$ & $1.1 \mathrm{E}-06$ & $1.12 \mathrm{E}-03$ & 9.1E-07 & $21 E-06$ & $1.12 \mathbb{E}-03$ & $9.1 E-07$ & $21 E \oplus 6$ & $1.12 E .03$ & $4.8 \mathrm{E}-07$ & 1.1E.06 \\
\hline CADMIUM & $1.01 E+\infty 0$ & $8.2 E-04$ & $1.9 \mathrm{E} .03$ & $9.93 \mathrm{E}-01$ & $8.1 E-04$ & 1.9E-03 & $9.97 \mathbb{E}-01$ & $8.1 \mathrm{E}-04$ & $1.9 \mathrm{E}-03$ & $9.97 \mathrm{E}-01$ & $8.1 \mathrm{E}-04$ & $1.9 \mathrm{E}-03$ & $9.97 \mathrm{E}-01$ & 4.3E-04 & 9.9E-04 \\
\hline $\begin{array}{l}\text { CHROMIUM- } \\
\mathrm{V} \mathrm{l}\end{array}$ & $8.98 E-03$ & $7.3 \mathrm{E}-06$ & 1.7E-05 & $8.98 \mathrm{E}-03$ & $7.3 E-06$ & $1.7 \mathbb{E}-05$ & $8.98 \mathrm{E}-03$ & $7.3 \mathrm{E}-06$ & $1.7 E-05$ & $8.98 E-03$ & 7.3E-106 & 1.7E.05 & $8.98 \mathrm{E}-03$ & $3.8 E-06$ & $9.0 \mathrm{E}-06$ \\
\hline MANGANESE & $3.64 E+01$ & $3.0 \mathrm{E}-02$ & $6.9 \mathrm{E}-02$ & $3.60 E+01$ & $2.9 E-02$ & $6.8 \mathrm{E}-02$ & $3.61 E+01$ & $29 \mathrm{E}-02$ & $6.9 \mathrm{E}-02$ & $3.61 E+01$ & $29 \mathrm{E}-02$ & $6.9 E-02$ & $3.61 E+01$ & $1.5 E-02$ & $3.6 \mathrm{E}-02$ \\
\hline MERCURY & $3.67 \mathrm{E}-01$ & $3.0 \mathrm{E}-04$ & $7.0 \mathrm{E}-04$ & 3.67E-01 & $3.0 \mathrm{E}-04$ & 7.0E-04 & $3.67 \mathrm{E}-01$ & $3.0 \mathrm{E}-04$ & $7.0 \mathrm{E}-04$ & $3.67 \mathrm{E}-01$ & $3.0 \mathrm{E}-\mathrm{PA}$ & $7.0 \mathrm{E}-04$ & $3.67 \mathrm{E}-01$ & $1.6 \mathrm{E}-04$ & 3.7E-04 \\
\hline NICKEL & $6.67 \mathrm{E}-01$ & $5.4 E-.94$ & $1.3 \mathrm{E}-03$ & $6.67 \mathrm{E}-01$ & $5.4 \mathrm{E}-04$ & $1.3 E-03$ & $6.67 E-01$ & 5.4E-MA & $1.3 \mathrm{E}-03$ & $6.67 \mathrm{E}-01$ & $5.4 E-04$ & $1.3 \mathrm{E}-03$ & $6.67 \mathrm{E}-01$ & $29 \mathrm{E}-04$ & $6.7 \mathbb{E}-04$ \\
\hline NITRATE & $1.65 E+02$ & 1.3E-01 & $3.1 \mathrm{E}-01$ & $0.00 \mathrm{E}+00$ & $0.0 E+00$ & $0.0 E+\infty$ & $5.50 E+01$ & 4.5E-02 & $1.0 \mathrm{E}-01$ & $5.50 \mathrm{E}+01$ & 4.5E-02 & $1.0 \mathrm{E}-01$ & $5.50 \mathrm{E}+01$ & 24E-02 & $5.5 E-02$ \\
\hline $\begin{array}{l}\text { URANIUM } \\
\text { (SOLUBLE } \\
\text { SALTS) }\end{array}$ & $3.83 E-02$ & 3.1E-05 & $7.3 \mathrm{E}-05$ & $3.83 \mathrm{E}-02$ & $3.1 \mathrm{E}-05$ & 7.3E-0S & $3.83 \mathrm{E}-02$ & $3.1 \mathrm{E}-05$ & $7.3 \mathrm{E}-05$ & $3.83 \mathrm{E}-02$ & $3.1 \mathrm{E}-05$ & $7.3 \mathrm{E}-05$ & $3.83 \mathrm{E}-02$ & $1.6 \mathrm{E}-05$ & $3.8 E-05$ \\
\hline
\end{tabular}


Table C.21. (continued)

\begin{tabular}{|c|c|c|c|c|c|c|c|c|c|c|c|c|c|c|c|}
\hline \multirow[b]{2}{*}{ Analytes } & \multicolumn{3}{|c|}{ Leafy vegetables } & \multicolumn{3}{|c|}{ Root vegetables } & \multicolumn{3}{|c|}{ Legume vegetables } & \multicolumn{3}{|c|}{ Fruiting vegetables } & \multicolumn{3}{|c|}{ Fruits } \\
\hline & $\begin{array}{l}\text { Conc: } \\
\text { (mg/kg: } \\
\text { pCi/g) }\end{array}$ & $\begin{array}{l}\text { Ingest. } \\
\text { carcin. } \\
\text { CDI }\end{array}$ & $\begin{array}{l}\text { Ingest. } \\
\text { momcar. } \\
\text { CDI }\end{array}$ & $\begin{array}{l}\text { Conc: } \\
\text { (mg/kg; } \\
\text { pCi/g) }\end{array}$ & $\begin{array}{l}\text { Ingest. } \\
\text { carcin. } \\
\text { CDI }\end{array}$ & $\begin{array}{l}\text { Ingest. } \\
\text { monear. } \\
\text { CDI }\end{array}$ & $\begin{array}{l}\text { Conc: } \\
\text { (mglig; } \\
\text { pCi/g) }\end{array}$ & $\begin{array}{l}\text { Ingest. } \\
\text { carcia. } \\
\text { CDI }\end{array}$ & $\begin{array}{l}\text { Ingest. } \\
\text { moncar. } \\
\mathrm{CDI}\end{array}$ & $\begin{array}{l}\text { Conc: } \\
\text { (mg/kg; } \\
\text { pCi/g) }\end{array}$ & $\begin{array}{l}\text { Ingest } \\
\text { carein. } \\
\text { CDI }\end{array}$ & $\begin{array}{l}\text { Ingest } \\
\text { nonear. } \\
\text { CDI }\end{array}$ & $\begin{array}{l}\text { Conc: } \\
\text { (mg/kg; } \\
\text { pCi/g) }\end{array}$ & $\begin{array}{l}\text { Ingest. } \\
\text { carcin. } \\
\text { CDI } \\
\end{array}$ & $\begin{array}{l}\text { Ingest } \\
\text { noncar. } \\
\text { CDI } \\
\end{array}$ \\
\hline VANADIUM & $232 \mathbb{E}-02$ & $1.9 \mathrm{E}-05$ & 4.4E-05 & $1.34 \mathrm{E}-66$ & $1.1 \mathrm{E}-09$ & 25E-A9 & $7.75 \mathbb{E}-03$ & $6.3 \mathrm{E}-96$ & 1.5E-05 & $7.75 E-03$ & $6.3 \mathrm{E}-06$ & $1.5 \mathrm{E}-05$ & $7.75 \mathrm{E}-03$ & $3.3 \mathrm{E}-06$ & $7.7 E-06$ \\
\hline \multicolumn{16}{|l|}{ Radionuclides } \\
\hline RADIUM-226 & $4.51 E-03$ & $3.8 \mathrm{E}+03$ & & 4.51E-03 & $3.8 \mathrm{E}+03$ & & $4.51 E-03$ & $3.8 E+03$ & & $4.51 \mathrm{E}-03$ & $3.8 \mathrm{E}+03$ & & $4.51 \mathrm{E}-03$ & $20 \mathrm{E}+03$ & \\
\hline URANIUM-234 & $1.58 E+01$ & $1.3 E+07$ & & $1.46 \mathrm{E}-02$ & $1.2 E+04$ & & $5.28 E+00$ & $4.4 E+06$ & & $5.28 E+00$ & $4.4 \mathrm{E}+06$ & & $5.28 E+00$ & $23 E+06$ & \\
\hline URANIUM-238 & $1.07 E+01$ & $9.0 \mathrm{E}+06$ & & $1.40 \mathrm{E}-02$ & $1.2 \mathrm{E}+04$ & & $3.58 \mathrm{E}+00$ & $3.0 E+06$ & & $3.58 \mathrm{E}+00$ & $3.0 E+06$ & & $3.58 \mathrm{E}+00$ & $1.6 \mathrm{E}+06$ & \\
\hline \multicolumn{16}{|l|}{ Organics } \\
\hline $\begin{array}{l}\text { 1,2-DI- } \\
\text { CHLORO- } \\
\text { ETHENE } \\
\text { (TOTAL) }\end{array}$ & $1.04 \mathrm{E}-01$ & $8.5 E-05$ & $20 E-94$ & $6.11 \mathrm{E}-03$ & $5.0 \mathrm{E}-06$ & $1.2 E-05$ & 3.805 .02 & $3.2 E-05$ & $7.4 E-85$ & $3.89 \mathrm{E}-02$ & $3.2 E-05$ & 7.AE.05 & $3.89 \mathrm{E}-02$ & $1.7 \mathrm{E}-05$ & $3.9 E-05$ \\
\hline $\begin{array}{l}\text { BENZO(A)- } \\
\text { ANTHRA- } \\
\text { CENE }\end{array}$ & 233E-03 & $1.9 E-06$ & A.4E-O6 & 233E-03 & $1.9 \mathrm{E}-06$ & 4.4E- -06 & 233E-03 & $1.9 E-06$ & 4.4E-06 & $233 E-03$ & $1.9 \mathbb{E}-\infty 8$ & 4.4E-06 & 233E-03 & $1.0 \mathrm{E}-06$ & 23E-06 \\
\hline $\begin{array}{l}\text { BENZO(A)- } \\
\text { PYRENE }\end{array}$ & $1.08 \mathrm{E}-03$ & 8.8E-07 & $21 E-06$ & $1.08 \mathrm{E} .03$ & 8.8E-07 & $21 E-106$ & $1.08 \mathrm{E}-03$ & $8.8 E-07$ & 21E-06 & $1.08 \mathrm{E}-03$ & 8.8E-07 & 21E-06 & $1.08 \mathrm{E}-03$ & $4.6 E-07$ & $1.1 \mathrm{E}-06$ \\
\hline $\begin{array}{l}\text { BENZO(B)- } \\
\text { FLUOR- } \\
\text { ANTHENE }\end{array}$ & $1.13 E-03$ & $9.2 \mathrm{E}-07$ & $21 \mathrm{E}-06$ & $1.13 E-03$ & $9.2 \mathrm{E}-07$ & 21E- & $1.13 E-03$ & 9.2E-07 & 21E- & $1.13 E-03$ & $9.2 \mathrm{E}-07$ & $21 E-06$ & 1.13E-03 & $4.8 \mathrm{E}-07$ & $1.1 \mathrm{E}-06$ \\
\hline $\begin{array}{l}\text { BROMO. } \\
\text { DICHLORO- } \\
\text { METHANE }\end{array}$ & $1.66 E-02$ & 1.4E-05 & $3.2 \mathrm{E}-05$ & $3.54 \mathrm{E}-04$ & $29 E-07$ & $6.7 \mathrm{E}-07$ & $5.77 \mathrm{E}-03$ & $4.7 E-06$ & 1.1E-0S & $5.77 E-03$ & 4.7E-06 & I.1E-05 & $5.77 \mathrm{E}-03$ & $25 \mathrm{E}-06$ & $5.8 E-06$ \\
\hline $\begin{array}{l}\text { INDENO(1,23- } \\
\text { CD)PYRENE }\end{array}$ & 4.42E-04 & 3.6E-07 & 8.4E-07 & 4.42E-04 & $3.6 \mathrm{E}-07$ & $8.4 E-07$ & 4.42E-04 & $3,6 \mathrm{E}-07$ & 8.4E-07 & 4.42E-04 & $3.6 \mathrm{E}-07$ & 8.4E-07 & $4.42 \mathrm{E}-04$ & $1.9 \mathrm{E}-07$ & 4.4E-07 \\
\hline $\begin{array}{l}\text { METHYLENE } \\
\text { CHLORIDE }\end{array}$ & 215E-02 & $1.8 \mathrm{E}-05$ & 4.1E-05 & $1.51 E-03$ & 1.2E-06 & $29 \mathrm{E}-06$ & 8.19E-03 & $6.7 E-06$ & $1.6 E-05$ & $8.19 E-03$ & $6.7 E-\infty$ & $1.6 \mathrm{E}-05$ & 8.19E-03 & $3.5 E-06$ & $8.2 E-06$ \\
\hline
\end{tabular}




\section{Table C.21. (oontinued)}

CDI = Chronic Daily Intake

- Carcinogenic and noncarcinogenic CDis are given for all nonradionuclides, regardless of the EPA class reported in EPA 1994 and EPA 1993 .

- For the ingestion of vegetables/fruits pathway, the units for CDIs are $\mathrm{pCi}$ for radionuclides and $\mathrm{mg} / \mathrm{kg}-\mathrm{d}$ for nonradionuclides.

- The values reported in this column are the concentrations on each analyte in vegetables/fruits ( $\mathrm{CV}$ ); units of $\mathrm{mg} / \mathrm{kg}$ are given for nonradınuclides and units of $\mathrm{pCl} / \mathrm{g}$ are given for radionuclides. 
Table C.22 Chronic daily intakes and doses for BCV OU2 soil COPCs - industrial scenario

\begin{tabular}{|c|c|c|c|c|c|c|c|c|}
\hline \multirow[b]{2}{*}{ Analye } & \multirow[b]{2}{*}{$\begin{array}{l}\text { UCL95 } \\
\text { concentration } \\
\text { (mg/kg; pCi/g) }\end{array}$} & \multicolumn{4}{|c|}{ Carcinogenic effects (CDI) } & \multicolumn{3}{|c|}{ Noncarcinogenic effects (CDI) } \\
\hline & & $\begin{array}{l}\text { Ingestion } \\
\text { (mg/kg-d; } \\
\text { pCi) }\end{array}$ & $\begin{array}{l}\text { Dermal } \\
(\mathrm{mg} / \mathrm{hg}-\mathrm{d}) \\
\end{array}$ & $\begin{array}{l}\text { Inhalation } \\
\text { (mg/kg-d; } \\
\text { pCi) }\end{array}$ & $\begin{array}{l}\text { External } \\
\text { exposure } \\
\text { (pCi-y/g) } \\
\end{array}$ & $\begin{array}{l}\text { Ingestion } \\
(\mathrm{mg} / \mathrm{kg}-\mathrm{d})\end{array}$ & $\begin{array}{l}\text { Dermal } \\
(\mathbf{m g} / \mathrm{kg}-\mathrm{d}) \\
\end{array}$ & $\begin{array}{l}\text { Inhalation } \\
(\mathrm{mg} / \mathrm{kg}-\mathrm{d})\end{array}$ \\
\hline \multicolumn{9}{|c|}{ RSA } \\
\hline \multicolumn{9}{|l|}{ Inorganics } \\
\hline BARIUM & $1.15 E+02$ & 2.0E-05 & $1.3 \mathrm{E}-06$ & $1.7 \mathrm{E}-69$ & & $5.6 \mathbb{E}-05$ & $3.6 \mathrm{E}-\infty$ & $4.9 \mathrm{E}-09$ \\
\hline BERYLLIUM & $1.05 \mathrm{E}+00$ & $1.8 \mathrm{E}-07$ & $1.2 \mathrm{E}-08$ & $1.6 \mathrm{E}-11$ & & $5.1 \mathrm{E}-07$ & 3.3E-08 & $4.4 \mathrm{E}-11$ \\
\hline CADMUM & $4.93 E+00$ & $8.6 \mathrm{E}-07$ & $5.4 \mathrm{E}-08$ & $7.4 \mathrm{E}-11$ & & $2.4 \mathrm{E}-06$ & $1.5 \mathrm{E}-07$ & $2.1 E-10$ \\
\hline MANGANESE & $8.99 E+02$ & $1.6 \mathrm{E}-04$ & $9.9 \mathrm{E}-06$ & $1.4 E-68$ & & $4.4 \mathrm{E}-04$ & $2.8 \mathrm{E}-05$ & $3.8 \mathrm{E}-08$ \\
\hline MERCURY & $2.00 E+\infty 0$ & $3.5 \mathrm{E}-07$ & $2.2 \mathrm{E}-08$ & $3.0 E-11$ & & $9.8 \mathrm{E}-07$ & $6.2 \mathrm{E}-08$ & $8.4 E-11$ \\
\hline NICKEL & $4.61 E+01$ & 8.1E-106 & S.1E-07 & $7.0 \mathrm{E}-10$ & & 2.3E-05 & $1.4 E-06$ & $2.0 \mathrm{E}-09$ \\
\hline URANIUM (SOLUBLE SALTS) & $7.36 E+\infty 0$ & $1.3 \mathrm{E}-06$ & 8.1E-08 & $1.1 E-10$ & & $3.6 \mathrm{E}-06$ & $2.3 \mathrm{E}-07$ & 3.1E-10 \\
\hline \multicolumn{9}{|l|}{ Radionuclides } \\
\hline THORIUM-232 & $3.09 E+\infty 0$ & $9.7 \mathrm{E}+02$ & & 8.3E-02 & $1.4 E+01$ & & & \\
\hline URANIUM-234 & $2.81 E+00$ & $8.8 \mathrm{E}+02$ & & 7.6E-02 & $1.3 E+01$ & & & \\
\hline \multicolumn{9}{|l|}{ Organics } \\
\hline 1,2,4-TRICHLOROBENZENE & $4.31 E+\infty 0$ & $7.5 \mathrm{E}-07$ & $4.8 \mathrm{E}-07$ & $4.5 E-06$ & & $2.1 \mathrm{E}-106$ & $1.3 \mathrm{E}-06$ & $1.3 E-05$ \\
\hline 1,2-DICHLOROETHENE (TOTAL) & $7.22 \mathrm{E}-02$ & $1.3 E-08$ & $8.0 E-09$ & $1.1 \mathrm{E}-12$ & & $3.5 E-08$ & $2.2 \mathrm{E}-08$ & 3.1E-12 \\
\hline AROCLOR-1254 & $9.86 \mathrm{E}-01$ & $1.7 E-07$ & $1.1 \mathrm{E}-07$ & $1.5 E-11$ & & $4.8 \mathrm{E}-07$ & $3.0 \mathrm{E}-07$ & $4.2 \mathrm{E}-11$ \\
\hline BENZO(A)PYRENE & $4.76 \mathrm{E}-01$ & $8.3 \mathrm{E}-08$ & $5.3 E-08$ & $7.2 \mathrm{E}-12$ & & 2.3E-07 & $1.5 \mathrm{E}-07$ & $2.0 \mathrm{E}-11$ \\
\hline BIS(2-ETHYLHEXYL)PHTHALATE & $6.56 \mathrm{E}+00$ & $1.1 \mathrm{E}-06$ & $7.2 \mathrm{E}-97$ & 9.9E-11 & & $3.2 \mathrm{E}-06$ & $2.0 \mathrm{E}-06$ & $2.8 \mathrm{E}-10$ \\
\hline DIBENZO(A,H)ANTHRACENE & $2.90 \mathrm{E}-01$ & $5.1 \mathrm{E}-08$ & $3.2 E-08$ & $4.4 E-12$ & & $1.4 \mathrm{E}-07$ & $9.0 \mathrm{E}-08$ & $1.2 \mathrm{E}-11$ \\
\hline
\end{tabular}


Table C.22 (continued)

\begin{tabular}{|c|c|c|c|c|c|c|c|c|}
\hline \multirow[b]{2}{*}{ Analyee } & \multirow[b]{2}{*}{$\begin{array}{l}\text { UCL95 } \\
\text { concentration } \\
\text { (mg/kg; } \mathrm{pCi} / \mathrm{g})\end{array}$} & \multicolumn{4}{|c|}{ Carcinogenic effects (COI) } & \multicolumn{3}{|c|}{ Noncarcinogenic effects (CDI) ${ }^{c}$} \\
\hline & & $\begin{array}{l}\text { Ingestion } \\
(\mathrm{mg} / \mathrm{kg}-\mathrm{d} ; \\
\mathrm{pCi})\end{array}$ & $\begin{array}{l}\text { Dermal } \\
(m g / k g \cdot d)\end{array}$ & $\begin{array}{l}\text { Inhalation } \\
\text { (mg/ng-d; } \\
\text { pCi) }\end{array}$ & $\begin{array}{l}\text { Extermal } \\
\text { exposure } \\
\text { (pCi-y/g) }\end{array}$ & $\begin{array}{l}\text { Ingestion } \\
\text { (mg/gg-d) }\end{array}$ & $\begin{array}{l}\text { Dermal } \\
(m g / k g-d)\end{array}$ & $\begin{array}{l}\text { Inhalation } \\
\left(\mathrm{mg} / \mathrm{kg}^{-1}\right)\end{array}$ \\
\hline TETRACHLOROETHENE & $1.62 \mathrm{E}-01$ & $2.8 \mathrm{E}-08$ & $1.8 \mathrm{E}-08$ & $2.4 \mathrm{E}-12$ & & $7.9 \mathrm{E}-08$ & $5.0 \mathrm{E}-08$ & $6.8 \mathrm{E}-12$ \\
\hline
\end{tabular}

$S Y-200$

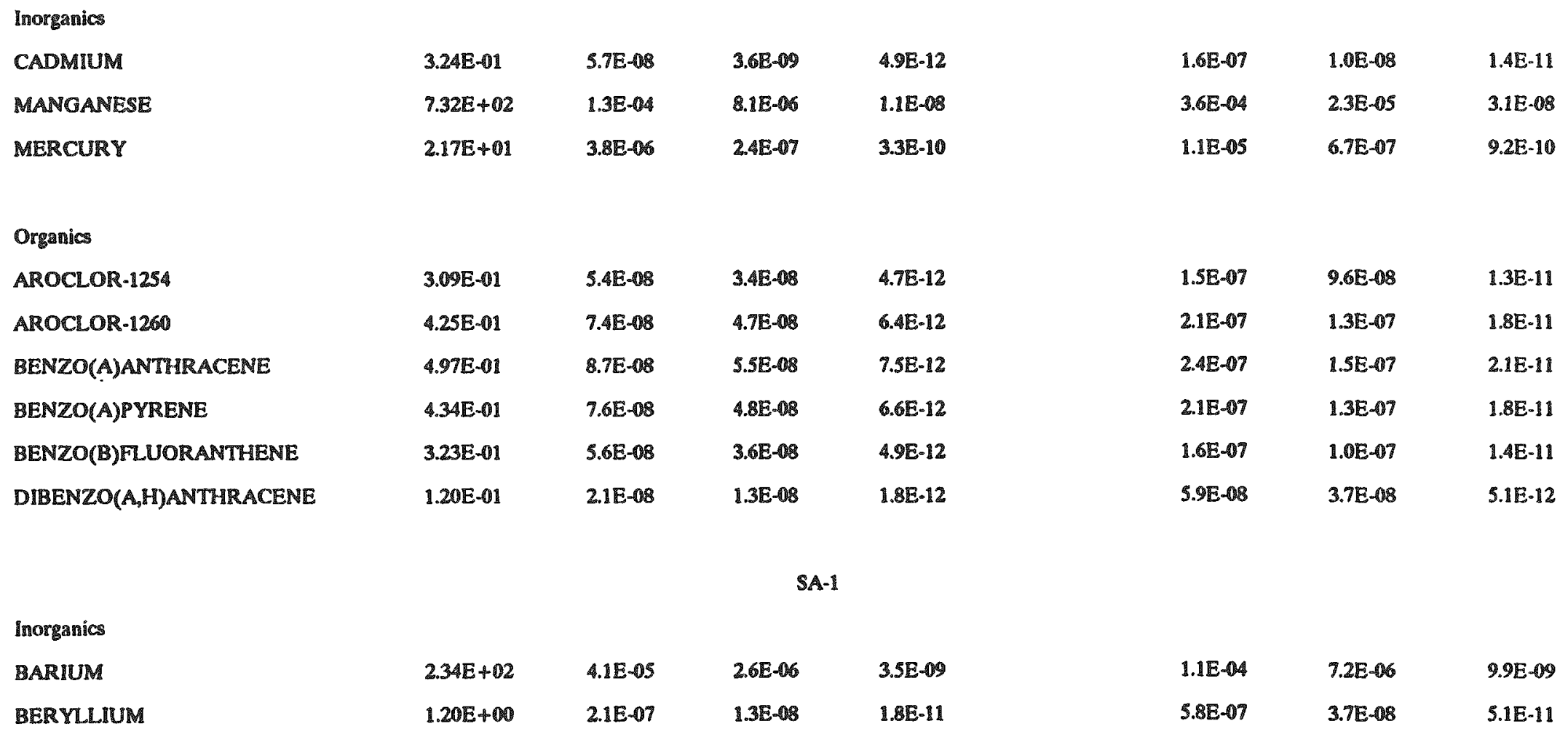


Table C.22. (continued)

\begin{tabular}{|c|c|c|c|c|c|c|c|c|}
\hline \multirow[b]{2}{*}{ Analyte" } & \multirow[b]{2}{*}{$\begin{array}{l}\text { UCL95' } \\
\text { concentration } \\
\text { (mg/kg; PCi/g) }\end{array}$} & \multicolumn{4}{|c|}{ Carcinogenic effects $(\mathrm{CDI})^{\circ}$} & \multicolumn{3}{|c|}{ Noncarcinogenic effects $(\mathrm{CDI})^{\mathrm{c}}$} \\
\hline & & $\begin{array}{l}\text { Ingestion } \\
\text { (mg/kg-d; } \\
\text { pCi) }\end{array}$ & $\begin{array}{l}\text { Dermal } \\
(\mathrm{mg} / \mathrm{kg}-\mathrm{d}) \\
\end{array}$ & $\begin{array}{l}\text { Inhalation } \\
\text { (mg/kg-d; } \\
\text { pCi) } \\
\end{array}$ & $\begin{array}{l}\text { External } \\
\text { exposure } \\
(\mathrm{pCi}-\mathrm{y} / \mathrm{g}) \\
\end{array}$ & $\begin{array}{l}\text { Ingestion } \\
\text { (mg/kg-d) }\end{array}$ & $\begin{array}{l}\text { Dermal } \\
\text { (mg/kg-d) }\end{array}$ & $\begin{array}{l}\text { Inhalation } \\
(\mathrm{mg} / \mathrm{kg}-\mathrm{d})\end{array}$ \\
\hline CADMIUM & $3.31 \mathrm{E}+00$ & $5.8 \mathrm{E}-07$ & $3.7 \mathrm{E}-08$ & $5.0 \mathrm{E}-11$ & & $1.6 \mathrm{E}-06$ & $1.0 \mathrm{E}-07$ & $1.4 \mathrm{E}-10$ \\
\hline CHROMIUM VI & $3.59 \mathrm{E}+01$ & $6.3 \mathrm{E}-06$ & 4.0B-07 & $5.4 \mathrm{E}-10$ & & $1.8 \mathrm{E}-05$ & $1.1 \mathrm{E}-06$ & $1.5 \mathrm{E}-09$ \\
\hline MANGANESE & $1.20 E+03$ & 2.1E-04 & $1.3 \mathrm{E}-05$ & $1.8 \mathrm{E} \cdot 08$ & & $5.9 \mathrm{E}-04$ & 3.7E-05 & $5.1 \mathrm{E}-08$ \\
\hline MERCURY & 9.65E-01 & $1.7 \mathrm{E}-07$ & $1.1 \mathrm{E}-08$ & $1.5 \mathrm{E}-11$ & & 4.7E-07 & $3.0 \mathrm{E}-08$ & $4.1 \mathrm{E}-11$ \\
\hline NICKEL & $3.51 \mathrm{E}+01$ & $6.1 \mathrm{E}-06$ & $3.9 \mathrm{E}-07$ & $5.3 \mathrm{E}-10$ & & 1.7E-05 & $1.1 \mathrm{E}-06$ & $1.5 \mathrm{E}-09$ \\
\hline URANIUM (SOLUBLE SALTS) & $1.53 E+01$ & $2.7 \mathrm{E}-06$ & $1.7 \mathrm{E}-07$ & $2.3 \mathrm{E}-10$ & & 7.5E-06 & $4.7 \mathrm{E}-07$ & $6.5 \mathrm{E}-10$ \\
\hline \multicolumn{9}{|l|}{ Radionuclides } \\
\hline RADIUM-226 & $3.22 E+\infty 00$ & $1.0 E+03$ & & $8.7 \mathrm{E}-02$ & $1.5 E+01$ & & & \\
\hline URANIUM-234 & $5.15 E+\infty 0$ & $1.6 \mathrm{E}+03$ & & $1.4 \mathbb{E}-01$ & $2.4 E+01$ & & & \\
\hline URANIUM-238 & $5.11 E+00$ & $1.6 \mathrm{E}+03$ & & $1.4 \mathrm{E}-01$ & $2.3 E+01$ & & & \\
\hline \multicolumn{9}{|l|}{ Organics } \\
\hline BENZO(A)ANTHRACENE & 4.16E-01 & $7.3 E-08$ & $4.6 \mathrm{E}-08$ & $6.3 E-12$ & & $2.0 E-07$ & $1.3 E-07$ & $1.8 \mathrm{E}-11$ \\
\hline BENZO(A)PYRENE & $3.61 \mathrm{E}-01$ & $6.3 E-08$ & $4.0 \mathrm{E}-08$ & $5.4 E-12$ & & $1.8 E-07$ & $1.1 \mathrm{E}-07$ & $1.5 \mathrm{E}-11$ \\
\hline BENZO(B)FLUORANTHENE & $3.76 \mathrm{E}-01$ & $6.6 \mathrm{E}-08$ & $4.2 E-08$ & $5.7 \mathrm{E}-12$ & & $1.8 \mathrm{E}-07$ & $1.2 \mathrm{E}-07$ & $1.6 \mathrm{E}-11$ \\
\hline INDENO(1,2,3-CD)PYRENE & $2.60 \mathrm{E}-01$ & $4.5 \mathrm{E}-08$ & $2.9 \mathrm{E}-08$ & $3.9 \mathrm{E}-12$ & & $1.3 \mathrm{E}-07$ & $8.0 \mathrm{E}-08$ & $1.1 \mathrm{E}-11$ \\
\hline
\end{tabular}

CDI = Chronic Daily Intake

- Carcinogenic and noncarcinogenic CDIs are given for all nonradionuclides, regardless of the EPA class reported in EPA 1994 and EPA 19936.

- The soil concentration reported in this column is the smallest of two values: the maximum detected concentration versus the upper $95 \%$ confidence limit on the mean.

The analyte concentrations are in units of $\mathrm{mg} / \mathrm{kg}$ for nonradionuclides and in units of $\mathrm{pCi} / \mathrm{g}$ for radionuclides. 


\section{Table C.22. (continued)}

- For radionuclides, the units for intake (dose) are pCi for the ingestion and inhalation pathways, and the units are pCi-y/g for the external exposure pathway; for nonradionuclides, the units for $\mathrm{CDI}$ are $\mathrm{mg} / \mathrm{kg}-\mathrm{d}$ for all pathways. 
Table C.23. Toxicity information for the BCV OU2 moncarcinogenic contaminants of potential concern

\begin{tabular}{|c|c|c|c|c|c|c|c|c|c|}
\hline Chemical & $\begin{array}{l}\text { Chronic } \\
\text { oral } \\
\mathrm{RfD}^{\mathrm{a}, \mathrm{b}} \\
(\mathrm{mg} / \mathrm{kg}-\mathrm{d})\end{array}$ & $\begin{array}{c}\text { Confidence } \\
\text { level }\end{array}$ & $\begin{array}{c}\% \mathrm{GI} \\
\text { absorp. }\end{array}$ & $\begin{array}{l}\% \mathrm{GI} \\
\text { source }\end{array}$ & $\begin{array}{l}\text { Chronic } \\
\text { oral RfD } \\
\text { absorbed } \\
\text { (mg/kg-d) }\end{array}$ & $\begin{array}{l}\text { Chronic } \\
\text { inhale. } \\
\text { RrD }^{\text {ab,e }} \\
\text { (mg/kg-d) }\end{array}$ & $\begin{array}{l}\text { RfD basis } \\
\text { (vehicle) }^{f}\end{array}$ & Critical effect ${ }^{\AA}$ & $\begin{array}{l}\text { Uncertainty } \\
\text { factor } \\
\text { Modifying } \\
\text { factor }\end{array}$ \\
\hline \multicolumn{10}{|l|}{ Inorganics } \\
\hline Antimony & $4.0 \mathrm{E}-04$ & Low & $\geq 15$ & $\begin{array}{l}\text { Friberg } \\
1986\end{array}$ & $6.0 \mathrm{E}-05$ & NA & oral & $\begin{array}{l}\text { gastrointestinal } \\
\text { disorders }\end{array}$ & $\begin{array}{l}\mathrm{UF}=1000 \\
\mathrm{MF}=1\end{array}$ \\
\hline Arsenic & $3.0 \mathrm{E}-04$ & Medium & $>90$ & ATSDR & $3.0 \mathrm{E}-04$ & $\mathbb{N A}$ & oral & $\begin{array}{l}\text { CNS, cardiovas- } \\
\text { cular system, skin }\end{array}$ & $\begin{array}{l}U F=3 \\
M F=1\end{array}$ \\
\hline Barium & $7.0 \mathrm{E}-02^{\mathrm{a}}$ & Medium & 10 & $\begin{array}{l}\text { Owen } \\
1990\end{array}$ & $7.0 \mathrm{E}-03$ & $1.4 \mathbb{E}-04$ & $\begin{array}{l}\text { oral } \\
\text { water }\end{array}$ & $\begin{array}{l}\text { higher mortality } \\
\text { rate from } \\
\text { vascular diseases }\end{array}$ & $\begin{array}{l}U F=3 \\
M F=1\end{array}$ \\
\hline Beryllium & $5.0 \mathrm{E}-03^{\circ}$ & Low & 5 & $\mathbf{N A}$ & $2.5 \mathrm{E}-04$ & $\mathbf{N A}$ & oral & reduced weight & $\begin{array}{l}U F=100 \\
M F=1\end{array}$ \\
\hline $\begin{array}{l}\text { Cadmium } \\
\text { (diet) }\end{array}$ & $1.00 \mathrm{E}-03^{\circ}$ & High & 6 & $\begin{array}{l}\text { Owen } \\
1990\end{array}$ & $6.0 \mathrm{E}-05$ & NA & oral & $\begin{array}{l}\text { renal toxicity } \\
\text { osteomalacia } \\
\text { osteoporosis }\end{array}$ & $\begin{array}{l}U F=10 \\
M F=1\end{array}$ \\
\hline $\begin{array}{l}\text { Cadmium } \\
\text { (water) }\end{array}$ & $5.0 \mathrm{E}-04$ & High & 6 & $\begin{array}{l}\text { Owen } \\
1990\end{array}$ & $3.0 \mathrm{E}-05$ & NA & oral & $\begin{array}{l}\text { renal toxicity } \\
\text { osteomalacia } \\
\text { osteoporosis }\end{array}$ & $\begin{array}{l}U F=10 \\
M F=1\end{array}$ \\
\hline Chromium VI & $5.0 \mathrm{E}-03^{\mathrm{a}}$ & Low & 10.6 & ATSDR & 5.3E-04 & NA & $\begin{array}{l}\text { oral } \\
\text { water }\end{array}$ & $\begin{array}{l}\text { hepatotoxicity, } \\
\text { nephrotoxicity, } \\
\text { dermatitis }\end{array}$ & $\begin{array}{l}\mathrm{UF}=500 \\
\mathrm{MF}=\mathrm{NA}\end{array}$ \\
\hline $\begin{array}{l}\text { Manganese } \\
\text { (diet) }\end{array}$ & $1.4 \mathrm{E}-01^{\mathrm{a}}$ & Medium & 5 & $\begin{array}{l}\text { ATSDR } \\
1990 a\end{array}$ & $7.0 \mathrm{E}-03$ & $1.4 \mathrm{E}-05$ & oral & $\begin{array}{l}\text { lethargy, tremors } \\
\text { mental disturb- } \\
\text { ance, muscle } \\
\text { tonus, CNS }\end{array}$ & $\begin{array}{l}U F=1 \\
M \Gamma=1\end{array}$ \\
\hline
\end{tabular}


Table C.23. (continued)

\begin{tabular}{|c|c|c|c|c|c|c|c|c|c|}
\hline Chemical & $\begin{array}{c}\text { Chronic } \\
\text { oral } \\
\text { RID }^{\mathrm{a} .8} \\
\text { (mg/kg-d) }\end{array}$ & $\begin{array}{c}\text { Confidence } \\
\text { level }\end{array}$ & $\begin{array}{l}\text { \%GI } \\
\text { absorp. }\end{array}$ & $\begin{array}{l}\% \text { GI } \\
\text { source }\end{array}$ & $\begin{array}{l}\text { Chronic } \\
\text { oral RID } \\
\text { absorbed } \\
\text { (mg/kg-d) }\end{array}$ & $\begin{array}{l}\text { Chronic } \\
\text { inhale. } \\
\text { RfD }^{a, b, o} \\
\text { (mg/kg-d) }\end{array}$ & $\begin{array}{l}\text { RfD basis } \\
\text { (vehicle) }\end{array}$ & Critical effect ${ }^{\mathbb{f}}$ & $\begin{array}{l}\text { Uncertainty } \\
\text { factor, } \\
\text { Modifying } \\
\text { factor }\end{array}$ \\
\hline $\begin{array}{l}\text { Manganese } \\
\text { (water) }\end{array}$ & $5.0 \mathrm{E}-03$ & Medium & 5 & $\begin{array}{l}\text { ATSDR } \\
1990 \mathrm{a}\end{array}$ & $2.5 \mathrm{E}-04$ & $1.4 \mathrm{E}-05$ & oral & $\begin{array}{l}\text { lethargy, tremors } \\
\text { mental disturb- } \\
\text { ance, muscle } \\
\text { tonus, CNS }\end{array}$ & $\begin{array}{l}\mathrm{UF}=1 \\
\mathrm{MF}=1\end{array}$ \\
\hline Mercury & $3.0 \mathrm{E}-04^{\mathrm{b}}$ & NA & NA & NA & NA & $8.6 \mathrm{E}-05$ & oral & $\begin{array}{l}\text { CNS, kidney, } \\
\text { gastrointestinal } \\
\text { tract toxicity }\end{array}$ & $\begin{array}{l}\mathrm{UF}=1000 \\
\mathrm{MF}=\mathrm{NA}\end{array}$ \\
\hline Mercury (salts) & $3.0 \mathrm{E}-04^{\mathrm{b}}$ & Medium & $<15$ & $\begin{array}{l}\text { Amdur } \\
1991\end{array}$ & 4.5E-05 & NA & oral & $\begin{array}{l}\text { CNS, kidney, } \\
\text { gastrointestinal } \\
\text { tract toxicity }\end{array}$ & $\begin{array}{l}U F=10 \\
M F=1\end{array}$ \\
\hline Nickel & $2.0 \mathrm{E}-02^{\circ}$ & NA & NA & NA & NA & NA & oral & reduced weight & $\begin{array}{l}U F=100 \\
M F=3^{i}\end{array}$ \\
\hline Nickel (salts) ${ }^{3}$ & $2.0 \mathrm{E}-02$ & Medium & 5 & $\begin{array}{l}\text { Owen } \\
1990\end{array}$ & $1.0 \mathrm{E}-03$ & NA & oral & reduced weight & $\begin{array}{l}U F=100 \\
M F=3\end{array}$ \\
\hline Nitrate & $1.6 \mathrm{E}+00$ & High & NA & NA & $1.6 \mathrm{E}+00$ & NA & oral & $\begin{array}{l}\text { methemoglo- } \\
\text { binemia }\end{array}$ & $\begin{array}{l}U F=1 \\
M F=1\end{array}$ \\
\hline $\begin{array}{l}\text { Uranium } \\
\text { (soluble salts) }\end{array}$ & $3.0 \mathrm{E}-03$ & NA & 5 & ATSDR & $1.5 \mathrm{E}-04$ & NA & NA & kidney & NA \\
\hline Vanadium & 7.0E-03 & NA & 2.6 & $\begin{array}{l}\text { EPA } \\
1987\end{array}$ & $1.8 \mathrm{E}-04$ & NA & oral & $\begin{array}{l}\text { gastrointestinal } \\
\text { system, kidney, } \\
\text { renal function }\end{array}$ & $\begin{array}{l}U F=100 \\
M F=N A\end{array}$ \\
\hline
\end{tabular}


Table C.23. (contimued)

\begin{tabular}{|c|c|c|c|c|c|c|c|c|c|}
\hline Chemical & $\begin{array}{c}\text { Chronic } \\
\text { oral } \\
\text { RTD }^{\text {s, }} \\
\text { (mg/kg-d) }\end{array}$ & $\begin{array}{c}\text { Confidence } \\
\text { level }\end{array}$ & $\begin{array}{l}\text { \%GI } \\
\text { absorp. }\end{array}$ & $\begin{array}{l}\text { \%Gl } \\
\text { source }\end{array}$ & $\begin{array}{l}\text { Chronic } \\
\text { oral RID } \\
\text { absorbed, } \\
\text { (mg/kg-d) }\end{array}$ & $\begin{array}{c}\text { Chronic } \\
\text { inhale. } \\
\text { RTD }^{\mathrm{s}, \mathrm{b}, \mathrm{e}} \\
\text { (mg/kg-d) }\end{array}$ & $\begin{array}{l}\text { RfD basis } \\
\text { (vehicle) }^{f}\end{array}$ & Critical effect ${ }^{8}$ & $\begin{array}{r}\text { Uncertal } \\
\text { factor } \\
\text { Modifyi } \\
\text { factor }\end{array}$ \\
\hline \multicolumn{10}{|l|}{ Organics } \\
\hline Arocior-1254 & NA & NA & 95 & $\begin{array}{l}\text { Owen } \\
1990\end{array}$ & NA & NA & NA & NA & NA \\
\hline Aroclor- 1260 & NA & NA & 90 & ATSDR & NA & NA & NA & NA & NA \\
\hline Benzo(a)anthracene & NA & NA & 100 & $\begin{array}{l}\text { ATSDR; } \\
\text { EPA } \\
1982\end{array}$ & NA & NA & NA & NA & NA \\
\hline Benzo(a)pyrene & NA & NA & 85 & $\begin{array}{l}\text { ATSDR; } \\
\text { EPA } \\
1982\end{array}$ & NA & NA & NA & NA & NA \\
\hline Benzo(b)fluoranthene & NA & NA & 100 & $\begin{array}{l}\text { EPA } \\
1982\end{array}$ & NA & NA & NA & NA & NA \\
\hline $\begin{array}{l}\text { bis(2-Ethyl- } \\
\text { hexyl)Phthalate }\end{array}$ & $2.0 \mathrm{E}-02$ & Medium & 100 & $\begin{array}{l}\text { EPA } \\
1980\end{array}$ & $2.0 \mathrm{E}-02$ & $\mathbf{N A}$ & oral & $\begin{array}{l}\text { increased liver } \\
\text { and kidney } \\
\text { weight in animals }\end{array}$ & $\begin{array}{l}\mathrm{UF}=1000 \\
\mathrm{MF}=1\end{array}$ \\
\hline Bromodichloromethane & $2.0 \mathrm{E}-02$ & NA & 90 & ATSDR & $2.0 \mathrm{E}-02$ & NA & oral & $\begin{array}{l}\text { kidney, cyto- } \\
\text { megaly }\end{array}$ & $\begin{array}{l}\mathrm{UF}=1000 \\
\mathrm{MF}=\mathrm{NA}\end{array}$ \\
\hline Carbon Tetrachloride & $7.0 \mathrm{E}-04$ & Medium & 85 & ATSDR & $7.0 \mathrm{E} \cdot 04$ & NA & oral & $\begin{array}{l}\text { gastrointestinal, } \\
\text { liver, kidney, } \\
\text { CNS }\end{array}$ & $\begin{array}{l}\mathrm{UF}=1000 \\
\mathrm{MF}=\mathrm{NA}\end{array}$ \\
\hline Chloroform & $1.0 \mathrm{E}-02$ & Medium & 98 & ATSDR & $1.0 \mathrm{E}-02$ & NA & oral & $\begin{array}{l}\text { CNS, kidney, } \\
\text { organ damage, } \\
\text { liver }\end{array}$ & $\begin{array}{l}U F-1000 \\
M F=N A\end{array}$ \\
\hline
\end{tabular}


Table C.23. (contimued)

\begin{tabular}{|c|c|c|c|c|c|c|c|c|c|}
\hline Chemical & $\begin{array}{c}\text { Chronic } \\
\text { oral } \\
\text { RfD }^{\mathrm{ab}} \\
\text { (mg/kg-d) }\end{array}$ & $\begin{array}{c}\text { Confidence } \\
\text { levela }\end{array}$ & $\begin{array}{c}\% G I \\
\text { absorp. }\end{array}$ & $\begin{array}{l}\text { \%GI } \\
\text { source }\end{array}$ & $\begin{array}{l}\text { Chronic } \\
\text { oral RfD } \\
\text { absorbed } \\
\text { (mg/kg-d) }\end{array}$ & $\begin{array}{l}\text { Chronic } \\
\text { inhale. } \\
\text { RfO } \\
\text { (mg/kg-d) }\end{array}$ & $\begin{array}{l}\text { RfD basis } \\
\text { (vehicle) }^{t}\end{array}$ & Critical effect & $\begin{array}{l}\text { Uncertainty } \\
\text { factor }{ }^{2, f} \\
\text { Modifying } \\
\text { factor }\end{array}$ \\
\hline Dibenzo(a,h)anthracene & NA & NA & $>90$ & ATSDR & NA & NA & NA & NA & NA \\
\hline 1,2-Dichloroethene & $9.0 \mathrm{E}-03$ & NA & NA & NA & $9.0 \mathrm{E}-03$ & NA & oral & liver & $\begin{array}{l}\mathrm{UF}=3000 \\
\mathrm{MF}=\mathrm{NA}\end{array}$ \\
\hline Indeno(1,2,3-cd)pyrene & $\mathbf{N A}$ & NA & 100 & $\begin{array}{l}\text { EPA } \\
1982\end{array}$ & NA & NA & NA & NA & NA \\
\hline Methylene Chloride & $6.0 \mathrm{E}-02$ & Medium & NA & NA & $6.0 \mathrm{E}-02$ & $8.5 \mathrm{E}-01$ & oral & $\begin{array}{l}\text { impaired liver } \\
\text { function }\end{array}$ & $\begin{array}{l}U F-100 \\
M F=N A\end{array}$ \\
\hline Tetrachloroethene & $1.0 \mathrm{E}-02$ & Medium & 100 & ATSDR & $1.0 \mathrm{E}-02$ & NA & oral & $\begin{array}{l}\text { organ damage, } \\
\text { central nervous } \\
\text { system effects }\end{array}$ & $\begin{array}{l}U F=1000 \\
M F=N A\end{array}$ \\
\hline 1,2,4-Trichlorobenzene & $1.0 \mathrm{E}-02$ & NA & 42 & $\begin{array}{l}\text { EPA } \\
1977\end{array}$ & $4.2 \mathrm{E}-03$ & $5.7 \mathrm{E}-02$ & oral & $\begin{array}{l}\text { increased weight, } \\
\text { liver, porphyria }\end{array}$ & $\begin{array}{l}U F=1000 \\
\text { MF-NA }\end{array}$ \\
\hline
\end{tabular}

ATSDR $=$ Agency for Toxic Substances and Disease Registry, Public Health Services (1987-1990); NA $=$ No data available or data inconclusive; GI $=$ Gastrointestinal (\%GI = percent gastrointestinal absorption); $\mathrm{RfD}=$ Reference dose; $\mathrm{CNS}=$ Central nervous system.

- Based on Integrated Risk Information System (IRIS) (EPA 1994).

b Based on Health Effects Assessment Summary Tables (HEAST) (EPA 1993b).

- The absorbed RfD = (RFD X \%GI absorption); the absorbed RID is used for dermal pathway calculations. RID absorbed $=$ RID (i.e, $\%$ GI $=100)$ when the $\% \mathrm{GI}$ absorption value is unknown (NA), and when the \%GI is greater than 80 .

The chronic oral absorbed RfD is used for the dermal contact pathway calculations (RfD oral absorbed = RfD oral $\times$ \%GI).

- RfCs were converted to units of mg/kg-d (i.e., RfD units) by using the inhalation rate and body weight of an adult [i.e., RfC $\left.\mathrm{x} 20 \mathrm{~m}^{3} / \mathrm{d} \times(1 / 70 \mathrm{~kg})=\mathrm{RFD}\right](\mathrm{EPA} 1989 \mathrm{a})$.

' BEIAS (Biomedical and Environmental Information Analysis Section) Toxicity Profiles (BEIAS 1993).

- The chronic oral RfD for nickel salts and mercury salts were assigned the same RfD values as nickel and mercury metals, respectively; for dermal calculations, the chronic oral absorbed RIDs for mercury salts and nickel salts were used (instead of metals), because the \%GI values were available for these salts and were not available for the metals. 
Table C.24. Toxicity information for the BCV OU2 carcinogenic contaminants of potential concem

\begin{tabular}{|c|c|c|c|c|c|c|c|c|}
\hline Chemical & $\begin{array}{l}\text { Oral } \\
\text { slope } \\
\text { factor } \\
\text { (kg-day/mg) }\end{array}$ & $\begin{array}{l}\text { \%GI } \\
\text { absorp. }\end{array}$ & $\begin{array}{l}\text { \%GI } \\
\text { source }\end{array}$ & $\begin{array}{l}\text { Oral slope } \\
\text { factor } \\
\text { absorbed } \\
\text { (kg-day/mg) }\end{array}$ & $\mathrm{TEF}^{c}$ & $\begin{array}{l}\text { Inhalation } \\
\text { slope } \\
\text { factor } \\
\text { (kg-day/mg) }\end{array}$ & EPA class ${ }^{d}$ & $\begin{array}{l}\text { Type } \\
\text { of } \\
\text { cancer }\end{array}$ \\
\hline \multicolumn{9}{|l|}{ Inorganics } \\
\hline Antimony & $\mathbf{N A}$ & $\geq 15$ & Friberg 1986 & NA & NA & NA & $\mathbb{N A}$ & NA \\
\hline Arsenic & NA & $>90$ & ATSDR & NA & NA & $5.0 \mathrm{E}+01$ & A & $\begin{array}{l}\text { skin, liver, bladder, } \\
\text { respiratory, } \\
\text { gastrointestinal }\end{array}$ \\
\hline Barium & NA & 10 & Owen 1990 & NA & NA & $\mathbf{N A}$ & NA & $\mathbf{N A}$ \\
\hline Beryllium & $4.3 E+00$ & 5 & NA & $8.6 \mathrm{E}+01$ & NA & $8.4 \mathrm{E}+00$ & B2 & $\begin{array}{l}\text { breast, bone, } \\
\text { uterus, lung }\end{array}$ \\
\hline Cadmium (diet) & $\mathbb{N A}$ & 6 & Owen 1990 & $\mathbf{N A}$ & $\mathbf{N A}$ & $6.1 \mathbb{E}+\infty 0$ & $B 1$ & $\begin{array}{l}\text { respiratory } \\
\text { tract, lung }\end{array}$ \\
\hline Cadmium (water) & $\mathbf{N A}$ & 6 & Owen 1990 & $\mathbf{N A}$ & $\mathbb{N A}$ & $6.1 E+00$ & B1 & $\begin{array}{l}\text { respiratory } \\
\text { tract, lung }\end{array}$ \\
\hline Chromium VI & NA & 10.6 & ATSDR & NA & $\mathbf{N A}$ & $4.1 E+01$ & $\mathbf{A}$ & $\begin{array}{l}\text { tumors, } \\
\text { lung }\end{array}$ \\
\hline Manganese (diet) & NA & 5 & $\begin{array}{l}\text { ATSDR } \\
1990 a\end{array}$ & $\mathbf{N A}$ & $\mathbf{N A}$ & NA & $\mathrm{D}$ & NA \\
\hline Manganese (water) & NA & 5 & $\begin{array}{l}\text { ATSDR } \\
19900\end{array}$ & NA & $\mathbb{N A}$ & $\mathbf{N A}$ & D & NA \\
\hline Mercury & NA & NA & NA & $\mathbf{N A}$ & $\mathbf{N A}$ & NA & D & NA \\
\hline Mercury (salts) & $\mathbf{N A}$ & $<15$ & $\begin{array}{l}\text { Amdur } \\
1991\end{array}$ & $\mathbf{N A}$ & NA & NA & D & $\mathbf{N A}$ \\
\hline Nickel & NA & NA & NA & $\mathbf{N A}$ & NA & NA & $\mathbb{N A}$ & NA \\
\hline Nickel (salts) & $\mathbf{N A}$ & 5 & Owen 1990 & NA & NA & $\mathbf{N A}$ & NA & NA \\
\hline Nitrate & NA & $\mathbb{N A}$ & NA & $\mathbf{N A}$ & NA & NA & NA & NA \\
\hline $\begin{array}{l}\text { Uranium } \\
\text { (soluble saits) }\end{array}$ & NA & 5 & ATSDR & NA & NA & NA & NA & NA \\
\hline
\end{tabular}


Table C.24. (contimued)

\begin{tabular}{|c|c|c|c|c|c|c|c|c|}
\hline Chemical & $\begin{array}{l}\text { Oral } \\
\text { slope } \\
\text { factor } \\
\left(\mathrm{kg}-\mathrm{d}^{n} / \mathrm{mg}\right) \\
\end{array}$ & $\begin{array}{l}\text { \%GI } \\
\text { absorp. }\end{array}$ & $\begin{array}{l}\text { \%GI } \\
\text { source }\end{array}$ & $\begin{array}{l}\text { Oral slope } \\
\text { factor } \\
\text { absorbed } \\
\text { (kg-day/mg) }\end{array}$ & $\operatorname{TEF}^{\mathrm{r}}$ & $\begin{array}{l}\text { Inhalation } \\
\text { slope } \\
\text { factor } \\
\text { (kg-day/mg) }\end{array}$ & EPA class & $\begin{array}{l}\text { Type } \\
\text { of } \\
\text { cancer }\end{array}$ \\
\hline Vanadium & NA & 2.6 & EPA 1987 & NA & NA & NA & NA & NA \\
\hline \multicolumn{9}{|l|}{ Organics } \\
\hline Aroclor-1254 & $7.7 \mathrm{E}+00$ & 95 & Owen 1990 & $7.7 \mathrm{E}+00$ & NA & NA & B2 & \multirow{3}{*}{$\begin{array}{l}\text { liver, biliary tract, } \\
\text { gall bladder } \\
\text { liver, biliary tract, } \\
\text { gall bladder } \\
\text { tumors }\end{array}$} \\
\hline Aroclor-1260 & $7.7 \mathrm{E}+00$ & 90 & ATSDR & $7.7 E+00$ & NA & NA & B2 & \\
\hline Benzo(a)anthracene & $7.3 \mathrm{E}-01$ & 100 & $\begin{array}{l}\text { ATSDR; } \\
\text { EPA } 1982\end{array}$ & 7.3E-01 & 0.1 & NA & B2 & \\
\hline Benzo(a)pyrene & $7.3 \mathbb{E}+00$ & 85 & $\begin{array}{l}\text { ATSDR; } \\
\text { EPA } 1982\end{array}$ & $7.3 E+00$ & 1.0 & NA & B2 & stomach tumors \\
\hline Benzo(b)fluoranthene & $7.3 \mathbb{E}-01$ & 100 & EPA 1982 & 7.3E-01 & 0.1 & NA & B2 & tumors \\
\hline $\begin{array}{l}\text { bis(2-Ethylhexyl)- } \\
\text { Phthalate }\end{array}$ & $1.4 \mathrm{E}-02$ & 100 & EPA 1980 & $1.40 \mathrm{E}-02$ & NA & NA & B2 & NA \\
\hline Bromodichloromethane & $6.2 \mathrm{E}-02$ & 90 & ATSDR & $6.2 \mathrm{E}-02$ & NA & NA & B2 & \multirow{2}{*}{$\begin{array}{l}\text { kidney, intestine, } \\
\text { and liver tumors } \\
\text { liver, tumors }\end{array}$} \\
\hline Carbon Tetrachloride & $1.3 \mathrm{E}-01$ & 85 & ATSDR & $1.3 \mathrm{E}-01$ & NA & $5.3 \mathrm{E}-02$ & B2 & \\
\hline Chloroform & $6.1 \mathrm{E}-03$ & 98 & ATSDR & $6.1 \mathrm{E}-03$ & NA & $8.1 \mathrm{E}-02$ & B2 & $\begin{array}{l}\text { colon, recium, } \\
\text { bladder }\end{array}$ \\
\hline Dibenzo( $a, h)$ anthracene & $7.3 E+00$ & $>90$ & ATSDR & $7.3 E+00$ & 1.0 & NA & B2 & tumors \\
\hline 1,2-Dichloroethene & NA & NA & NA & NA & NA & NA & D & NA \\
\hline Indeno(1,2,3-cd)pyrene & $7.3 \mathrm{E}-01$ & 100 & EPA 1982 & $7.3 \mathrm{E}-01$ & 0.1 & NA & B2 & tumors \\
\hline
\end{tabular}


Table C.2A. (contimued)

\begin{tabular}{|c|c|c|c|c|c|c|c|c|}
\hline Chemical & $\begin{array}{l}\text { Oral } \\
\text { slope } \\
\text { factor } \\
\text { (kg-day/mg) }\end{array}$ & $\begin{array}{l}\text { \%GI } \\
\text { absorp. }\end{array}$ & $\begin{array}{l}\text { \%GI } \\
\text { source }\end{array}$ & $\begin{array}{l}\text { Oral slope } \\
\text { factor } \\
\text { absorbed } \\
\text { (kg-day/mg) }\end{array}$ & TEF & $\begin{array}{l}\text { Inhalation } \\
\text { slope } \\
\text { factor } \\
\text { (kg-day/mg) }\end{array}$ & EPA class & $\begin{array}{l}\text { Type } \\
\text { of } \\
\text { cancer }\end{array}$ \\
\hline Methylene Chloride & $7.5 \mathrm{E}-03$ & NA & NA & $7.5 \mathrm{E}-03$ & NA & NA & B2 & $\begin{array}{l}\text { NA (hepatocellular } \\
\text { and alveolar } \\
\text { neoplasms in mice) }\end{array}$ \\
\hline Tetrachloroethene & NA & 100 & ATSDR & NA & NA & NA & B2 & liver, kidney \\
\hline 1,2,4-Trichlorobenzene & NA & 42 & EPA 1977 & NA & NA & NA & $\mathrm{D}$ & NA \\
\hline
\end{tabular}

NA $=$ No data available or data inconclusive.

TEF = Toxicity equivalency factor.

- Based on Integrated Risk Information System (IRIS) (EPA 1994) or Health Effects Assessment Summary Tables (HEAST), (EPA 1993b).

- The absorbed oral slope factor is used for the dermal contact pathway calculations; the absorbed oral SF $=(\mathrm{SF} / \% \mathrm{GI})$.

- The oral SFs for these PAHs are derived by multiplying the benzo(a)pyrene oral SF $(7.3 \mathrm{E}+00)$ by the chemical specific toxicity equivalency factor (TEF)

(EPA 1993a).

EPA Weight of Evidence Classification System for Carcinogenicity-used to characterize the extent to which available data indicate that an agent is a human carcinogen: $\mathrm{A}=$ human carcinogen; $\mathrm{B} 1$ or $\mathbf{B 2}=$ probable human carcinogen ( $\mathrm{B} 1$ indicates that limited data on humans are available and $\mathrm{B} 2$ indicates

sufficient evidence in animals and inadequate or no evidence in humans); $C=$ possible human carcinogen; $D=$ not classifiable as to human carcinogenicity;

$\mathbf{E}=$ evidence of noncarcinogenicity for humans.

- For these aroclors, the oral SF $(7.7 \mathrm{E}+00)$ for polychlorinated biphenyls was used. 
Table C.25. Toxicity information for exposure to the BCV OU2 radionuclide contaminants of potential concern

\begin{tabular}{|c|c|c|c|c|c|c|}
\hline Chemical & $\begin{array}{c}\text { External exposure } \\
\text { slope factor }{ }^{a, b} \\
\text { (g/pCi-y) }\end{array}$ & $\begin{array}{l}\text { Oral } \\
\text { slope factor, } \\
\left((\mathrm{pCi})^{-1}\right)\end{array}$ & $\begin{array}{l}\text { Inhalation } \\
\text { slope factor } \\
\left((p C i)^{-1}\right)\end{array}$ & $\begin{array}{l}\text { ICRP } \\
\text { lung } \\
\text { class }\end{array}$ & $\begin{array}{c}\text { EPA } \\
\text { Class }^{d}\end{array}$ & $\begin{array}{l}\text { Type } \\
\text { of } \\
\text { Cancer }\end{array}$ \\
\hline \multicolumn{7}{|l|}{ Radionuclides } \\
\hline Cesium-137 & $2.0 \mathrm{E}-06$ & $2.8 \mathrm{E}-11$ & $1.9 \mathrm{E}-11$ & $D$ & $\mathbf{A}$ & various \\
\hline Radium-226 & $6.0 \mathrm{E}-06$ & $1.2 \mathrm{E}-10$ & $3.0 \mathrm{E}-09^{\circ}$ & $w$ & $\mathbf{A}$ & various \\
\hline Strontium-90 & $0.0 \mathrm{E}+00$ & $3.6 \mathrm{E}-11$ & $6.2 \mathrm{E}-11$ & $D$ & A & various \\
\hline Technetium-99 & $6.0 \mathrm{E}-13$ & $1.3 \mathrm{E}-12$ & $8.3 \mathrm{E}-12$ & W & $\mathbf{A}$ & various \\
\hline Thorium-232 & 2.6E-11 & $1.2 \mathrm{E}-11$ & $2.8 \mathrm{E}-08$ & $Y$ & $\mathbf{A}$ & various \\
\hline Uranium-234 & $3.0 \mathrm{E}-11$ & $1.6 \mathrm{E}-11$ & $2.6 \mathrm{E}-08$ & $\mathbf{Y}$ & $\mathbf{A}$ & various \\
\hline Uranium-235 & $2.4 \mathrm{E}-07$ & $1.6 E-11$ & $2.5 \mathrm{E}-08$ & $\mathbf{Y}$ & A & various \\
\hline Uranium-238 & $3.6 \mathrm{E}-08$ & $2.8 \mathrm{E}-11$ & $5.2 \mathrm{E}-08$ & $\mathbf{Y}$ & $\mathbf{A}$ & various \\
\hline
\end{tabular}

- Based on Health Effects Assessment Summary Tables (HEAST) (EPA 1993b).

- The radionuclide slope factors include contributions from daughter products.

- Lung clearance classification recommended by the International Commission on Radiological Protection (ICRP): $Y=$ year; $W=$ week; $D=$ day; $G=$ gas.

- EPA Weight of Evidence Classification System for Carcinogenicity-used to characterize the extent to which available data indicate that an agent is a human carcinogen: $\mathrm{A}=$ human carcinogen; $\mathbf{B} 1$ or $\mathbf{B 2}$ = probable human carcinogen (B1 indicates that limited data on humans are available and $\mathrm{B} 2$ indicates sufficient evidence in animals and inadequate or no evidence in humans); $C=$ possible human carcinogen; $D=$ not classifiable as to human carcinogenicity; $\mathbb{E}=$ evidence of noncarcinogeniciry for humans.

- EPA guidance suggests using the inhalation SF for radon-222 (7.7E-12/pCi) when evaluating the inhalation of volatiles pathway for radium-226 (RAGS Part B; EPA 1991a); for the inhalation of dust pathway, the radium-226 inhalation SF (3.0E-09/pCi) was used in this BRA. 
Table C.26. General uncertainty factors in risk assessment

\begin{tabular}{|c|c|c|}
\hline Uncertainty factor & Effect of uncentainty & Comment \\
\hline Use of cancer slope factors & May overestimate risks & $\begin{array}{l}\text { Slopes are upper } 95 \text { th percent confidence limits derived from the linearized } \\
\text { model; considered unlikely to underestimate true risk }\end{array}$ \\
\hline $\begin{array}{l}\text { Risks/doses within an exposure route assumed to be } \\
\text { additive }\end{array}$ & May over- or underestimate risks & Does not account for synergism or antagonism \\
\hline Toxicity values derived primarily from animal studies & May over- or underestimate risks & $\begin{array}{l}\text { Extrapolation from animal to humans may induce error due to differences in } \\
\text { pharmacokinetics, larget organs, and population variability }\end{array}$ \\
\hline $\begin{array}{l}\text { Toxicity values derived primarily from high doses; } \\
\text { most exposures are at low doses }\end{array}$ & May over- or underestimate risks & $\begin{array}{l}\text { Assumes linearity at low doses; tends to have conservative exposure } \\
\text { assumptions }\end{array}$ \\
\hline Toxicity values & May over- or underestimate risks & $\begin{array}{l}\text { Not all values represent the same degree of certainty; all are subject to change } \\
\text { as new evidence becomes available }\end{array}$ \\
\hline Effect of absorption & May over-or underestimate risks & $\begin{array}{l}\text { The assumption that absorption is equivalent across speries is implicit in the } \\
\text { derivation of the critical toxicity values; absorption may actually vary with } \\
\text { species and age }\end{array}$ \\
\hline $\begin{array}{l}\text { Effect of applying critical toxicity values to soil } \\
\text { exposures }\end{array}$ & May overestimate risks & $\begin{array}{l}\text { Assumes bioavailability of contaminants sorbed onto soils is the same as } \\
\text { detected in lab studies; contaminants detected in studies may be more } \\
\text { bioavailable }\end{array}$ \\
\hline Exposures assumed constant over time & May over- or underestimate risks & $\begin{array}{l}\text { Does not account for environmental fate, transport, or transfer that may alter } \\
\text { concentration }\end{array}$ \\
\hline Metal analysis for total metals only & May overestimate risks & $\begin{array}{l}\text { Did not distinguish between valences or speciation; assumed the metal was } \\
\text { present in its most toxic form }\end{array}$ \\
\hline Not all chemicals at the site have toxicity values & May underestimate risks & These chemicals are not addressed quantitatively \\
\hline Exposure assumptions & May over- or underestimate risks & $\begin{array}{l}\text { Assumptions regarding media intake, population characteristics, and exposure } \\
\text { patterns may not characterize exposures }\end{array}$ \\
\hline
\end{tabular}




\section{C-143}

Table C.27a. Evaluation of the high concentration zone for mercury in the SY-200 area: Soil data for mercury

\begin{tabular}{lllclc}
\hline $\begin{array}{l}\text { Sample } \\
\text { depth }\end{array}$ & $\begin{array}{l}\text { Borehole } \\
\text { number }\end{array}$ & $\begin{array}{l}\text { Sample } \\
\text { ID }\end{array}$ & Detected? & $\begin{array}{l}\text { Mercury } \\
\text { conc. }\end{array}$ & $\begin{array}{l}\text { Units } \\
\text { of measure }\end{array}$ \\
\hline $3-6.3^{\prime}$ & SB41 & B04101 & NO & 102.0 & $\mathrm{mg} / \mathrm{kg}$ \\
$3-7^{\prime}$ & SB50 & B05001 & YES & 156.0 & $\mathrm{mg} / \mathrm{kg}$ \\
$3-7^{\prime}$ & SB31 & B03101 & YES & 507.0 & $\mathrm{mg} / \mathrm{kg}$ \\
$3-77^{\prime}$ & SB15 & B01501 & YES & 512.0 & $\mathrm{mg} / \mathrm{kg}$ \\
$3-77^{\prime}$ & SB14 & B01401 & YES & 816.0 & $\mathrm{mg} / \mathrm{kg}$ \\
$7-11^{\prime}$ & SB16 & B01602 & YES & 96.9 & $\mathrm{mg} / \mathrm{kg}$ \\
$7-11^{\prime}$ & SB63 & B06302 & YES & 97.5 & $\mathrm{mg} / \mathrm{kg}$ \\
$7-11^{\prime}$ & SB23 & B02302 & YES & 113.0 & $\mathrm{mg} / \mathrm{kg}$ \\
$7-11^{\prime}$ & SB40 & B04002 & YES & 138.0 & $\mathrm{mg} / \mathrm{kg}$ \\
$7-11^{\prime}$ & SB41 & B04102 & NO & 164.0 & $\mathrm{mg} / \mathrm{kg}$ \\
$7-11^{\prime}$ & SB17 & B01702 & NO & 170.0 & $\mathrm{mg} / \mathrm{kg}$ \\
$7-11^{\prime}$ & SB39 & B03902 & YES & 210.0 & $\mathrm{mg} / \mathrm{kg}$ \\
$7-11^{\prime}$ & SB62 & B06202 & YES & 238.0 & $\mathrm{mg} / \mathrm{kg}$ \\
$7-11^{\prime}$ & SB45 & B04502 & YES & 262.0 & $\mathrm{mg} / \mathrm{kg}$ \\
$7-11^{\prime}$ & SB08 & B00802 & YES & 655.0 & $\mathrm{mg} / \mathrm{kg}$ \\
\hline
\end{tabular}




\section{C-144}

Table C.27b. Evaluation of the high concentration zone for mercury in the SY-200 area: Summary statistics for mercury

\begin{tabular}{llllllll}
\hline $\begin{array}{l}\text { Sample } \\
\text { depth }\end{array}$ & $\begin{array}{l}\text { Freq. } \\
\text { of } \\
\text { detect }\end{array}$ & $\begin{array}{l}\text { Minimum } \\
\text { detected } \\
\text { conc. }\end{array}$ & $\begin{array}{l}\text { Maximum } \\
\text { detected } \\
\text { conc. }\end{array}$ & $\begin{array}{l}\text { Distri- } \\
\text { bution }\end{array}$ & $\begin{array}{l}\text { Mean } \\
\text { conc. }\end{array}$ & $\begin{array}{l}\text { UCL95 } \\
\text { conc. }\end{array}$ & $\begin{array}{l}\text { Units } \\
\text { of } \\
\text { measure }\end{array}$ \\
\hline 3.7 & $10 / 16$ & $3.90 \mathrm{E}-01$ & $8.16 \mathrm{E}+02$ & $\mathrm{~L}$ & $2.78 \mathrm{E}+01$ & $9.76 \mathrm{E}+01$ & $\mathrm{mg} / \mathrm{kg}$ \\
$7.11^{\prime}$ & $11 / 16$ & $4.00 \mathrm{E}+00$ & $6.55 \mathrm{E}+02$ & $\mathrm{~L}$ & $6.42 \mathrm{E}+01$ & $1.48 \mathrm{E}+02$ & $\mathrm{mg} / \mathrm{kg}$ \\
$3-11^{\prime}$ & $21 / 32$ & $3.90 \mathrm{E}-01$ & $8.16 \mathrm{E}+02$ & L & $4.38 \mathrm{E}+01$ & $9.41 \mathrm{E}+01$ & $\mathrm{mg} / \mathrm{kg}$ \\
\hline
\end{tabular}

UCL95 = Upper 95\% confidence limit on the mean concentration; $L=$ Lognormal distribution 
Table C.27c. Evaluation of the high concentration zone for mercury in the SY-200 area:

Human health risk/HO evaluation for mercury

\begin{tabular}{|c|c|c|c|c|c|c|c|c|c|c|}
\hline \multirow[b]{2}{*}{$\begin{array}{l}\text { Sample } \\
\text { depth }\end{array}$} & \multirow[b]{2}{*}{$\begin{array}{l}\text { Freq. } \\
\text { of } \\
\text { detect }\end{array}$} & \multirow[b]{2}{*}{$\begin{array}{l}\text { UCL95 } \\
\text { conc. } \\
\text { (mg/kg) }\end{array}$} & \multicolumn{4}{|c|}{ Residential scenario } & \multicolumn{4}{|c|}{ Industrial worker scenario } \\
\hline & & & $\begin{array}{l}\text { Ingestion } \\
\mathrm{HQ} \\
\text { (resident) }\end{array}$ & $\begin{array}{l}\text { Dermal } \\
\mathrm{HQ} \\
\text { (resident) }\end{array}$ & $\begin{array}{l}\text { Inhalation } \\
\mathrm{HO} \\
\text { (resident) }\end{array}$ & $\begin{array}{l}\text { Total } \\
\mathrm{HI}^{\circ} \\
\text { (resident) }\end{array}$ & $\begin{array}{l}\text { Ingestion } \\
\mathrm{HQ} \\
\text { (industrial) }\end{array}$ & $\begin{array}{l}\text { Dermal } \\
\mathrm{HQ} \\
\text { (industrial) }\end{array}$ & $\begin{array}{l}\text { Inhalation } \\
\mathrm{HQ} \\
\text { (industrial) }\end{array}$ & $\begin{array}{l}\text { Total } \\
\mathrm{HI}^{\mathbf{2}} \\
\text { (industrial) }\end{array}$ \\
\hline $3.7^{\prime}$ & $10 / 16$ & $9.76 \mathrm{E}+01$ & $1.2 E+00$ & $1.8 \mathrm{E}-01$ & $1.2 \mathrm{E}-04$ & $1.4 \mathrm{E}+00$ & $1.6 \mathrm{E}-01$ & $6.7 \mathrm{E}-02$ & $4.8 \mathrm{E}-05$ & 2.3E-01 \\
\hline $7-11^{3}$ & $11 / 16$ & $1.48 \mathrm{E}+02$ & $1.8 \mathrm{E}+\infty$ & $2.7 \mathrm{E}-01$ & $1.8 \mathrm{E}-04$ & $2.1 E+00$ & $2.4 \mathrm{E}-01$ & $1.0 \mathrm{E}-01$ & 7.3E-05 & $3.4 \mathrm{E}-01$ \\
\hline $3 \cdot 11^{\prime}$ & $21 / 32$ & $9.41 E+01$ & $1.1 E+\infty$ & $1.7 \mathrm{E}-01$ & $1.1 \mathrm{E}-04$ & $1.3 E+\infty 0$ & $1.5 \mathrm{E}-01$ & $6.5 \mathrm{E}-02$ & $4.6 \mathrm{E}-05$ & $2.2 \mathrm{E}-01$ \\
\hline
\end{tabular}

$\mathrm{HO}=$ Hazard quotient; $\mathrm{HI}=$ Hazard index; UCL95 $=$ UCL95 $=$ Upper $95 \%$ confidence limit on the mean concentration.

- The total $\mathrm{HI}$ is the sum of the HOs from the ingestion, dermal, and inhalation pathways. 


\section{C-146}

Table C.28a. Evaluation of the high concentration zome for Aroclor-1254 in the SY-200 area:

Soil data for Aroclor-1254

\begin{tabular}{lllccc}
\hline $\begin{array}{l}\text { Sample } \\
\text { depth }\end{array}$ & $\begin{array}{l}\text { Borehole } \\
\text { number }\end{array}$ & $\begin{array}{l}\text { Sample } \\
\text { ID }\end{array}$ & Detected? & $\begin{array}{l}\text { Aroclor-1254 } \\
\text { conc. }\end{array}$ & $\begin{array}{l}\text { Units } \\
\text { of measure }\end{array}$ \\
\hline $3-7^{\prime}$ & SB24 & B02401 & NO & 0.4 & $\mathrm{mg} / \mathrm{kg}$ \\
$2-25^{\prime}$ & SB09 & B00901 & YES & 0.5 & $\mathrm{mg} / \mathrm{kg}$ \\
$3-6^{\prime}$ & SB54 & B05401 & NO & 0.8 & $\mathrm{mg} / \mathrm{kg}$ \\
$3-7^{\prime}$ & SB06 & B00601 & YES & 1.9 & $\mathrm{mg} / \mathrm{kg}$ \\
$7-11^{\prime}$ & SB28 & B02802 & NO & 0.2 & $\mathrm{mg} / \mathrm{kg}$ \\
$7-11^{\prime}$ & SB06 & B00602 & YES & 0.3 & $\mathrm{mg} / \mathrm{kg}$ \\
$7-11^{\prime}$ & SB20 & B02002 & YES & 0.3 & $\mathrm{mg} / \mathrm{kg}$ \\
$7-11^{\prime}$ & SB36 & B03602 & NO & 0.4 & $\mathrm{mg} / \mathrm{kg}$ \\
$7-11^{\prime}$ & SB63 & B06302 & NO & 0.5 & $\mathrm{mg} / \mathrm{kg}$ \\
$7-9^{\prime}$ & SB49 & B04902 & YES & 0.6 & $\mathrm{mg} / \mathrm{kg}$ \\
$7-11^{\prime}$ & SB26 & B02602 & NO & 0.8 & $\mathrm{mg} / \mathrm{kg}$ \\
$7-11^{\prime}$ & SB21 & B02102 & NO & 0.8 & $\mathrm{mg} / \mathrm{kg}$ \\
$7-11^{\prime}$ & SB22 & B02202 & YES & 0.9 & $\mathrm{mg} / \mathrm{kg}$ \\
$7-11^{\prime}$ & SB34 & B03402 & YES & 1.1 & $\mathrm{mg} / \mathrm{kg}$ \\
$7-11^{\prime}$ & SB62 & B06202 & YES & 1.3 & $\mathrm{mg} / \mathrm{kg}$ \\
$7-11^{\prime}$ & SB35 & B03502 & YES & 1.8 & $\mathrm{mg} / \mathrm{kg}$ \\
$7-11^{\prime}$ & SB44 & B04402 & NO & 3.7 & $\mathrm{mg} / \mathrm{kg}$ \\
\hline
\end{tabular}


Table C.28b. Evaluation of the high concentration zone for Aroclor-1254 in the SY-200 area: Summary statistics for Aroclor-1254

\begin{tabular}{llllllll}
\hline $\begin{array}{l}\text { Sample } \\
\text { depth }\end{array}$ & $\begin{array}{l}\text { Freq. } \\
\text { of } \\
\text { detect }\end{array}$ & $\begin{array}{l}\text { Minimum } \\
\text { detected } \\
\text { conc. }\end{array}$ & $\begin{array}{l}\text { Maximum } \\
\text { detected } \\
\text { conc. }\end{array}$ & $\begin{array}{l}\text { Distri- } \\
\text { bution }\end{array}$ & $\begin{array}{l}\text { Mean } \\
\text { conc. }\end{array}$ & $\begin{array}{l}\text { UCL95 } \\
\text { conc. }\end{array}$ & $\begin{array}{l}\text { Units } \\
\text { of } \\
\text { measure }\end{array}$ \\
\hline $3-7^{\prime}$ & $0 / 13$ & & & NT & $4.08 \mathrm{E}-02$ & & $\mathrm{mg} / \mathrm{kg}$ \\
$7-11^{\prime}$ & $6 / 12$ & $3.40 \mathrm{E}-01$ & $1.80 \mathrm{E}+00$ & $\mathrm{~L}$ & $6.15 \mathrm{E}-01$ & $1.37 \mathrm{E}+00$ & $\mathrm{mg} / \mathrm{kg}$ \\
$3-11^{\prime}$ & $6 / 25$ & $3.40 \mathrm{E}-01$ & $1.80 \mathrm{E}+00$ & $\mathrm{~L}$ & $3.78 \mathrm{E}-02$ & $2.41 \mathrm{E}-01$ & $\mathrm{mg} / \mathrm{kg}$ \\
\hline
\end{tabular}

UCL95 = Upper $95 \%$ confidence limit on the mean concentration; $I=$ Lognormal distribution; NT = No distribution test performed (all nondetected concentrations). 
Table C.28c Evaluation of the high conoentration zone for Aroclor-1254 in the SY-200 area:

Human health rist//HQ evaluation for Aroclor-1254

\begin{tabular}{|c|c|c|c|c|c|c|c|c|c|c|}
\hline \multirow[b]{2}{*}{$\begin{array}{l}\text { Sample } \\
\text { depth }\end{array}$} & \multirow[b]{2}{*}{$\begin{array}{l}\text { Freq. } \\
\text { of } \\
\text { detect }\end{array}$} & \multirow[b]{2}{*}{$\begin{array}{l}\text { UCL95 } \\
\text { conc. } \\
(\mathrm{mg} / \mathrm{kg})\end{array}$} & \multicolumn{4}{|c|}{ Residential scenario } & \multicolumn{4}{|c|}{ Industrial worker scenario } \\
\hline & & & $\begin{array}{l}\text { Ingestion } \\
\text { risk } \\
\text { (resident) }\end{array}$ & $\begin{array}{l}\text { Dermal } \\
\text { risk } \\
\text { (resident) }\end{array}$ & $\begin{array}{l}\text { Inhalation } \\
\text { risk } \\
\text { (resident) }\end{array}$ & $\begin{array}{l}\text { Total } \\
\text { risk } \\
\text { (resident) }\end{array}$ & $\begin{array}{l}\text { Ingestion } \\
\text { risk } \\
\text { (industrial) }\end{array}$ & $\begin{array}{l}\text { Dermal } \\
\text { risk } \\
\text { (industrial) }\end{array}$ & $\begin{array}{l}\text { Inhalation } \\
\text { risk } \\
\text { (industrial) }\end{array}$ & $\begin{array}{l}\text { Total } \\
\text { risk }^{\text {(industrial) }} \\
\text { (ind }\end{array}$ \\
\hline $3-7^{2}$ & $0 / 13$ & $4.30 \mathrm{E}-02$ & $5.2 \mathrm{E}-07$ & $1.2 \mathrm{E}-07$ & & $6.3 \mathrm{E}-07$ & 5.8E-08 & 3.7E-08 & & $9.4 \mathrm{E}-08$ \\
\hline $7-11^{8}$ & $6 / 12$ & $1.37 \mathrm{E}+00$ & $1.7 \mathrm{E}-05$ & $3.7 \mathrm{E}-06$ & & $2.0 \mathrm{E}-05$ & $1.8 \mathrm{E}-06$ & $1.2 \mathrm{E}-06$ & & $3.0 \mathrm{E}-06$ \\
\hline $3.11^{\prime}$ & $6 / 25$ & $2.41 \mathbb{E}-01$ & $2.9 \mathrm{E}-06$ & $6.5 \mathrm{E}-07$ & & $3.6 \mathrm{E}-06$ & $3.2 \mathrm{E}-07$ & $2.1 \mathrm{E} .07$ & & $5.3 \mathrm{E}-07$ \\
\hline
\end{tabular}

HO = Hazard quotient; UCL95 = Upper $95 \%$ confidence limit on the mean concentration.

- The total risk is the sum of the risks from the ingestion, dermal, and inhalation pathways. 


\section{APPENDIX D}

Ecological Risk Assessment Data 



\section{TOXICITY PROFULES}

\section{Inorganics}

BARIUM (CAS No. 7440-39-3) is a divalent alkaline-earth metal found only in combination with other elements in nature. The most important of these combinations are the peroxide, chloride, sulfate, carbonate, nitrate, and chlorate. The pure metal xloxidizes readily and reacts with water emitting hydrogen; it is chemically similar to calcium. Due to the element's limited ability to form salts with limited solubility in soil and water, it is expected to have a residence time in soils of hundreds of years and is not expected to be very mobile. Acidic conditions, however, will increase the solubility of some barium compounds, facilitating their movement from the soil to the groundwater.

The soluble forms of barium are readily absorbed into the blood from the intestinal tract. The rates of absorptions of a number of barium salts have been measured in rates following oral exposure to small quantities ( $30 \mathrm{mg} / \mathrm{kg}$ body weight). The relative absorption rates were found to be: $\mathrm{BaCl}_{2}>\mathrm{BaSO}_{4}>\mathrm{BaCO}_{3}$. Large doses of $\mathrm{BaSO}_{4}$ do not increase the uptake of this salt because of its low solubility.

Barium absorbed into the bloodstream disappears in about 24 hours. However, it is deposited in the muscles, lungs and bone. Very little is stored in the kidneys, liver, spleen, brain, heart, or hair. It remains in the muscles for about 30 hours, after which the concentration decreases slowly. The half life of barium in bone is estimated to be about 50 days. At low doses, barium acts as a muscle stimulant, and at higher doses it affects the nervous system, eventually leading to paralysis.

The $\mathrm{LD}_{s_{0}}$ for rats is listed as $630 \mathrm{mg} / \mathrm{kg}$ for $\mathrm{BaCO}_{3}, 118 \mathrm{mg} / \mathrm{kg}$ for $\mathrm{BaCl}_{2}$, and $921 \mathrm{mg} / \mathrm{kg}$ for barium acetate. A LOEAL of $5.1 \mathrm{mg} / \mathrm{kg} / \mathrm{day}$ and a NOEAL of $0.51 \mathrm{mg} / \mathrm{kg} / \mathrm{day}$ for a cardiovascular endpoint have been proposed.

No significant clinical signs of subchronic toxicity were observed in studies done on rats. In another series of experiments on chronic toxicity, no adverse clinical effects were observed.

Environmental criteria and standards developed for barium to date are:

Aquatic Water Quality Criteria

aquatic organism, fresh water acute $4400 \mu \mathrm{g} / \mathrm{L}$, chronic $109 \mu \mathrm{g} / \mathrm{L}$

COPPER (CAS No. 7440-50-8): Copper is a reddish, lustrous, ductile, malleable metal with a natural abundance of up to $70 \mathrm{mg} / \mathrm{kg}$ in the earth's crust. Copper exists in the less stable cuprous $(+1)$ and cupric $(+2)$ states. Several processes appear to determine the fate of copper in the aquatic environment including complexation with humic substances, sorption to hydrous metal oxides (iron and manganese), clays, and organic materials, and some bioaccumulation. Copper is not strongly bioaccumulated and does not appear to transfer significantly through aquatic or terrestrial food chains. The pattern of bioaccumulation of copper in plants and animals may exhibit seasonal variations related to biological activities. Bioconcentration factors for fish are in the range of 10 to 100 . 
Copper is an essential element in most organisms and the uptake of copper is homeostatically controlled in many higher organisms. The range between copper deficiency and toxicity is low in those organisms without effective barriers for controlled absorption, such as invertebrates, fish, and ruminants. The intestinal mucosa acts to some extent as a barrier to the absorption of ingested copper. Absorbed copper is initially bound to the albumin and later more firmly bound to a copper binding protein, $\alpha$-ceruloplasmin. Copper interacts with lead to affect developing erythrocytes. The liver plays a primary role in the homeostasis of copper, including excretion.

Acute poisoning from the ingestion of excessive amounts of copper salts (notably copper sulfate) is characterized by vomiting, hematemesis, hypotension, melena, coma, jaundice, and death. Reports of hemolytic anemia as a consequence of copper exposure exist. Copper-induced carcinogenicity has not been demonstrated.

\section{Environmental criteria and standards developed for copper to date are:}

Aquatic Water Quality Criteria

aquatic organism, fresh water

aquatic organism, marine

acute $1.4 \mu \mathrm{g} / \mathrm{L}$, chronic $1.7 \mu \mathrm{g} / \mathrm{L}$

acute $3.2 \mu \mathrm{g} / \mathrm{L}$, chronic $2.0 \mu \mathrm{g} / \mathrm{L}$

LEAD (CAS No. 7439-92-1) is a bluish-white to silvery-gray metal with a natural abundance of about $0.002 \%$ in the earth's crust. Metallic lead and common lead minerals are insoluble in water. However, several industrially produced lead compounds are readily soluble in water. Lead forms insoluble complexes with carbonate or sulfate ions or ferric hydroxide, limiting its mobility. The transport of lead in aqueous systems is influenced by the oxidation states of lead. Lead exists as a divalent cation in unpolluted waters; however, organic complexation is critical to the binding of lead in polluted waters. The biotransformation of lead to a methylated form by microorganisms can mobilize lead in the environment.

Although lead is bioaccumulated from the surrounding environment by a variety of organisms, lead is not significantly bioconcentrated through the food chain. It is thought that total uptake is a factor of exposure concentration. In aquatic biota, lead concentrations are generally higher in algae and benthic organisms and lower in upper trophic level organisms. Typical bioconcentration factors are 725 for algae, 1700 for snails, 500 for insects, and 42 for fish. Body burdens of lead have been shown to correlate well with the feeding habits of rodents, with the highest levels occurring in insectivores, intermediate levels in herbivores, and the lowest levels in granivores. In general, lead concentrations tend to increase with increasing age of the organism, with the highest lead concentrations occurring in the bones.

The absorption of lead is influenced by diet, age, and gender of the organism exposed, and physical parameters, such as the particular lead compound. Lead is known to affect four major target systems: the central nervous system, the peripheral nerves, the kidney, and the hematopoietic system. It is a mutagen, a teratogen, and a carcinogen and can interfere with reproduction, liver, thyroid, and immune system function. The toxic effects of lead are exacerbated in the presence of cadmium, zinc, copper, and mercury.

The absorption of lead from the gastrointestinal tract is regulated to some extent by the mechanism regulating calcium and phosphorus absorption. Once absorbed, lead selectively accumulates in the bone. It is estimated that $90 \%$ of the lead body burden is found in the bone, followed by the arta, liver, kidney, and pancreas. Lead remains loosely bound to the red blood 


\section{D-5}

cells while in circulation. The biliary pathway is the predominant route of excretion of ingested lead followed by milk, hair, and sweat, which are the secondary pathways of lead excretion.

Chronic exposure to inorganic lead has distinct toxicological effects. Acute lead encephalopathy - a characteristic chronic effect of lead exposure-is followed by neurological disorders, such as tremor, cortical atrophy, and convulsions. Lead-induced decreases in hemoglobin biosynthesis causes marked hematological changes such as anemia and basophilic stripping of erythroblasts. Short-term exposure to lead can cause reversible kidney damage, while prolonged exposure to high concentrations may result in progressive kidney damage and renal failure.

Lead and several lead salts are carcinogenic in experimental animals. Lead salts, primarily phosphates and acetates, when administered through ingestion or injection, have been demonstrated by more than 10 investigators to be carcinogenic in rats and mice. The most characteristic cancer response is bilateral renal carcinoma. The lead compounds tested in animals are almost all soluble salts. Metallic lead, lead oxide, and lead tetra alkyls have not been tested adequately.

Lead can be toxic to plants and can adversely affect growth, photosynthesis, mitosis, and water absorption. The effects of lead vary with plant species and do not tend to occur unless soil concentrations are several hundred $\mathrm{mg} \mathrm{Pb} / \mathrm{kg}$. The bioavailability of lead is influenced by a number of physicochemical factors.

In aquatic systems, the toxic response to lead is increased under conditions of elevated temperatures and lower $\mathrm{pH}$, with younger organisms more susceptible than older organisms. Adverse effects on Daphnid reproduction have occurred at exposure levels of $1.0 \mu \mathrm{g} / \mathrm{L}$. Concentrations of $0.5 \mathrm{mg} / \mathrm{L}$ have delayed metamorphosis in tadpoles.

Survival in rats has been reduced at concentrations of $5 \mathrm{mg} / \mathrm{kg}$ body weight. Lead crosses the placenta and can induce abortion, stillbirths, and increased skeletal malformations.

\section{Environmental criteria and standards developed for lead to date are: \\ Maximum Contaminant Level Goal $\quad 0.02 \mathrm{mg} / \mathrm{L}$ Maximum Contaminant Level $\quad 0.05 \mathrm{mg} / \mathrm{L}$. \\ Aquatic Water Quality Criteria \\ aquatic organism, fresh water acute $8.2 \mathrm{E}+1 \mu \mathrm{g} / \mathrm{L}$, chronic $3.2 \mathrm{E}+0 \mu \mathrm{g} / \mathrm{L}$ aquatic organism, marine acute $1.4 \mathrm{E}+2 \mu \mathrm{g} / \mathrm{L}$, chronic $5.6 \mathrm{E}+0 \mu \mathrm{g} / \mathrm{L}$}

MERCURY (CAS No. 7439-97-6): Mercury is a silver-white, heavy, mobile, liquid metal. It is slightly volatile at typical ambient temperatures. Solid mercury is a tin-white, ductile, malleable mass. Mercury has a natural abundance of $-0.5 \mathrm{mg} / \mathrm{kg}$ in the earth's crust.

Chemical speciation is probably the most important variable influencing the ecotoxicology of mercury. Methylmercury is the most hazardous mercury species due to its high stability, its lipid solubility, and its possession of ionic properties that lead to a high ability to penetrate membranes in living organisms. Mercury methylation in ecosystems is influenced by mercury loadings, microbial activity, nutrient content, $\mathrm{pH}$ and redox condition, suspended sediment load, sedimentation rates, and other variables. The synthesis of methylmercury by bacteria from 
inorganic mercury compounds present in water or sediments is the major source in aquatic environments. This process can occur under both aerobic and anaerobic conditions, but seems to favor anaerobic conditions. Other organomercury compounds appear to decompose rapidly in the environment.

In organisms near the top of the food chain, such as carnivorous fishes, almost all mercury accumulated is in the methylated form, primarily as a result of consuming prey containing methylmercury. The acidification of natural bodies of freshwater is statistically associated with elevated concentrations of methylmercury in the edible tissues of predatory fishes.

Mercury binds strongly with sulfhydryl groups, and has many potential target sites during embryogenesis; phenylmercury and methylmercury compounds are among the strongest known inhibitors of cell division. Organomercury compounds, especially methylmercury, cross placental barriers and can enter mammals by way of the respiratory tract, gastrointestinal tract, skin, or mucous membranes. As compared to inorganic mercury compounds, organomercurials are more completely absorbed, are more soluble in organic solvents and lipids, pass more readily through biological membranes, and are slower to be excreted. Biological membranes, including those at the blood-brain interface and placenta, tend to discriminate against ionic and inorganic mercury, but allow relatively easy passage of methylmercury and dissolved mercury vapor.

The absorption of mercury by inhalation and dermal routes is significant; however, the absorption of elemental mercury following ingestion is minimal. Some elemental mercury remains dissolved in blood and in this form it may be transported to the brain. Unlike elemental mercury, mercurial salts seem to selectively accumulate in the brain. Mercury also accumulates in the kidney following inhalation exposure, where it remains bound to sulfhydryl groups. The renal pathway is the primary route of mercury excretion.

Information pertaining to mercury residues in field collections of living organisms is abundant. Certain species of macrophytes strongly influence mercury cycling. Correlations between mercury contents of zooplankton and suspended particulate matter, and of sediments and fish muscle, suggesting a pathway from sediment to fish by way of suspended matter and zooplankton have been demonstrated.

In birds, it is generally acknowledged that mercury concentrations are highest in species that eat fish and other birds. A strong correlation was shown between the food of birds from Minamata, Japan and the mercury content in feathers; the content was highest in fish-eating seabirds and lowest in herbivorous waterfowl. In other studies, residues have been shown to be highest in the kidney and liver; however, total mercury contents were significantly modified by food preference and availability and by migratory patterns. There is an inverse relationship between total mercury and the percent methylmercury in tissues of various avian species - a pattern that seems to hold for all vertebrates for which data are available.

Bird feathers have been used for some time as indicators of mercury loadings in terrestrial and marine environments. The keratin in bird feathers is not easily degradable and mercury is probably associated with the disulfide bonds of keratin. The most probable source of recent elevated residues in feathers of the Finnish sparrowhawk (Accipiter nisus) was from consumption of avian granivores that had become contaminated as a result of eating seeds treated with organomercury compounds. Mercury levels in feathers of nestling Swedish gyrfalcons (Falco 
rusticolus) showed a better correlation with mercury levels in actual food items than with levels based on adult feathers. There have been measurable declines in mercury content of feathers of several species of raptors in Sweden associated with the banning of an alkylmercury-containing pesticide including species of Haliaeetus, Falco, Bubo, Buteo, and Accipiter. A reduction in mercury content in feathers of free-living birds is thought to be sufficient to establish an improved situation.

Among furbearers in the Wisconsin River drainage system, mercury burdens were higher in fish-eating than in herbivorous species - i.e., river otter $>$ mink $>$ raccoon $>$ red fox $>$ muskrat $>$ beaver. In general, fur contained the highest mercury levels, followed by liver, kidney, muscle, and brain, in that order. Mercury levels in piscivorous furbearers collected from the Wisconsin River basin paralleled mercury levels in fish, crayfish, and bottom sediments from that system. Mink and river otter have been shown to accumulate about 10 -fold more than did predatory fishes from the same drainage areas, suggesting that these furbearers can serve as sensitive indicators of mercury contamination, even at very low levels of contamination.

Inorganic mercury compounds have been demonstrated to be toxic to earthworms (Octochaetus pattoni); in 60 days $50 \%$ died at soil mercury levels of $0.79 \mathrm{mg} / \mathrm{kg}$ and $100 \%$ died at $5.0 \mathrm{mg} / \mathrm{kg}$.

Toxic concentrations of mercury salts have ranged from $<0.1 \mu \mathrm{g} / \mathrm{L}$ to $>200 \mu \mathrm{g} / \mathrm{L}$ for representative species of marine and freshwater organisms. The lower concentrations of $<2.0 \mu \mathrm{g} / \mathrm{L}$ recorded were usually associated with early developmental stages, long exposures, and flow-through tests. Organomercury compounds showed the greatest biocidal potential. In general, toxicity was higher at elevated temperatures and in the presence of other metals such as zinc and lead. Signs of acute mercury poisoning in fish include flaring of gill covers, increased frequency of respiratory movements, loss of equilibrium, and sluggishness. Signs of chronic mercury poisoning in fish include emaciation, brain lesions, cataracts, inability to capture food, and various erratic behaviors. Mercury residues in severely poisoned fish that died soon thereafter have been shown to range (in $\mathrm{mg} / \mathrm{kg} \mathrm{fw}$ ) from 26 to 68 in liver, 16 to 20 in brain, and 5 to 7 in whole body.

Mercury toxicity in birds varies with the form of the element, dose, route of administration, species, sex, age, and physiological condition. Organomercury compounds interact with elevated temperatures and pesticides to produce additive or more than additive toxicity, and with selenium to produce less than additive toxicity. Acute oral toxicities of various mercury formulations have ranged between $\sim 2.2$ and $31.0 \mathrm{mg} / \mathrm{kg}$ body weight for most avian species tested, corresponding to -4 to $40 \mathrm{mg} / \mathrm{kg}$ diet. Residues of mercury in experimentally poisoned passerine birds usually exceeded $20 \mathrm{mg} / \mathrm{kg}$ fresh weight and were similar to concentrations reported in wild birds that died of mercury poisoning. Residues were highest in the brain, followed by the liver, kidney, muscle, and carcass (feather data were not available). Liver residues in red-tailed hawks (Buteo jamaicensis) experimentally killed by methylmercury were $\sim 17 \mathrm{mg} / \mathrm{kg}$ dry weight. Experimentally poisoned grey herons (Ardea cinerea) seemed to be unusually resistant to mercury, as residues of 415 to $752 \mathrm{mg}$ mercury $/ \mathrm{kg}$ dry weight of liver were required to produce a lethal dose. Studies with coturnix have found that methylmercury was always more toxic than inorganic mercury and that young birds were usually more sensitive than older birds. Furthermore some birds poisoned by inorganic mercury recovered after treatment was withdrawn, 
but chicks that were fed methylmercury and later developed toxic signs usually died, even if the treated feed was removed.

Chronic exposure to mercury vapor results in disturbances of the central nervous system, kidney, and gastrointestinal tract in mammals. There may be increased irritability, combativeness, defective patterns, ocular disturbances and tremors. Nervous system manifestations can be reversible albeit slowly, in some cases. Embryotoxic and teratogenic effects of methylmercury have been reported in various experimental animals. Mercuric chloride has also been shown to be teratogenic in experimental animals.

Environmental criteria and standards developed for mercury to date are:

Aquatic Water Quality Criteria

aquatic organism, fresh water acute $1.1 \mathrm{E}+1 \mu \mathrm{g} / \mathrm{L}$, chronic $0.2 \mathrm{E}+0 \mu \mathrm{g} / \mathrm{L}$

aquatic organism, marine

acute $1.9 \mathrm{E}+1 \mu \mathrm{g} / \mathrm{L}$, chronic $0.1 \mathrm{E}+0 \mu \mathrm{g} / \mathrm{L}$

NICKEL (CAS No. 7440-02-0) is a lustrous white, hard, ferromagnetic metal. Nickel has a natural abundance of $\sim 0.018 \%$ in the earth's crust. Nickel is a highly mobile metal in aquatic systems due to a high solubility of nickel compounds in water. However, the precipitation of nickel in the presence of sulfide and high $\mathrm{pH}(9$ and above) reduces the mobility of nickel. Sorption and co-precipitation of nickel in the presence of hydrous iron and manganese oxides are processes that could limit nickel mobility in unpolluted water, although the effect of such processes in polluted water is uncertain. Nickel is bioaccumulated in aquatic systems to varying degrees, ranging from factors of 2,000 to 40,000 by algae, 100 by freshwater plants, and 40 in freshwater fish.

Nickel is known to be absorbed through the skin of mammals. Following ingestion, nickel selectively concentrates in the bones of the rat. Nickel levels in the lung appear to increase with age. Nickel has been found in the bile, and its excretion is largely via feces. Fetal malformations have occurred in ducks, mice, rats, and hamsters following administration of various nickel compounds to pregnant dams.

Nickel and several nickel compounds have been studied for their possible carcinogenic and carcinogenic promoting properties. Nickel has been found to induce malignant tumors in many target organs in experimental animals following exposure by several routes. Salts of nickel have been shown to be carcinogenic at the point of application. Mammalian cell transformation data indicate that several nickel compounds are mutagenic and can cause chromosomal alterations.

Some plants selectively accumulate nickel and have been shown to contain up to $10 \mathrm{mg} / \mathrm{kg}$ dry weight in leaves. Acute toxicity concentrations (LC50) in aquatic organisms include $50 \mu \mathrm{g} / \mathrm{L}$ in rainbow trout (Onchorynchus mykiss) and narrow-mouthed toad (Gastrophryne carolinensis) to $11 \mathrm{mg} / \mathrm{L}$ in Fowler's toad (Bufo fowleri). The exposure of the freshwater crustacean, Daphnia straus to 5 to $10 \mu \mathrm{g} / \mathrm{L}$ resulted in population extinction in three generations. Reproduction of most freshwater species is not impaired at nickel concentrations of $<10 \mu \mathrm{g} / \mathrm{L}$.

ZINC (CAS No. 7440-66-6): Zinc is a bluish-white, lustrous metal with a natural abundance of $\sim 0.02 \%$ by weight in the earth's crust. The presence of zinc in both suspended and dissolved forms depends on changes in the chemical constituents of water. Zinc remains predominantly adsorbed to hydrous metal oxides, clay minerals, and organic substances. Under reducing 
conditions, zinc is reduced to zinc sulfide, reducing its mobility in the aqueous environment. The relative mobility of zinc in soil depends on the same factors as in the aqueous environment. Zinc is an essential element for animals and is involved in the function of some enzymes. Absorption is homeostatically regulated in animals, although high exposure concentrations can result in breakdown of the homeostatic mechanism. Zinc does not appear to be bioconcentrated through the food chain. The major receptor for zinc appears to be the sediments.

Zine is ubiquitous and is considered an essential trace element. Normally, the muscle, liver, kidney, and pancreas contain large amounts of zinc, although other organs such as testis and eye are also reported to have high levels. Absorbed zinc is excreted predominantly via the gastrointestinal tract. To a lesser extent, zinc is eliminated via the renal pathway and lactation. Antagonistic interactions between zinc and cadmium are documented for effects on growth, dermal lesions, copper metabolism, circulation, and body temperature. Zinc has been shown to exert protective effects against the toxicity of lead.

Chronic dermal toxicity following exposure to zinc chloride is characteristic. A gray cyanosis, dermatosis, and ulceration of the nasal passages have been observed in situations involving chronic inhalation exposure to zinc chloride.

Environmental criteria and standards developed for zinc to date are:

Aquatic Water Quality Criteria

aquatic organism, fresh water

acute $1.95 \mu \mathrm{g} / \mathrm{L}$, chronic $47 \mu \mathrm{g} / \mathrm{L}$

aquatic organism, marine acute $170 \mu \mathrm{g} / \mathrm{L}$, chronic $58 \mu \mathrm{g} / \mathrm{L}$

\section{Polychlorinated Biphenyls}

PCBs are a class of chemicals of human health and environmental concern. PCBs are extremely stable compounds and are slow to chemically degrade in the environment. PCBs tend to bond tightly to particulate matter, particularly in soils and sediments of lakes, estuaries, and rivers, where they may remain available for resuspension for at least 8 to 15 years. In the water column, the hydrophobic PCBs partition into the more apolar compartments of the ecosystem or are physically adsorbed on particulate matter. High partition coefficients correlate well with PCB biomagnification in fatty tissues of aquatic organisms, and with incorporation into sediments. PCB levels are usually highest in aquatic sediments containing microparticulates and high organic or clay content.

The transfer of PCBs on microparticulate materials and into phytoplankton is well documented, as is partitioning from aqueous solution into algal lipids. In phytoplankton, PCBs have been shown to exert inhibitory effects on photosynthesis and cell motility. In addition to direct toxic effects on algae, accumulated PCBs can be introduced into the aquatic food chain through this route. In the terrestrial system, however, it is the lower chlorinated isomers (which are more water soluble and more volatile) which have been accumulated preferentially in crop plants relative to higher chlorinated isomers.

PCBs are relatively insoluble in water but are freely soluble in nonpolar organic solvents and in biological lipids. Many fish and wildlife species, including salmon, trout, turtles, eagles, herons, fish-eating birds, mink, river otters, and bats, have been found to contain measurable, 
and in some cases potentially harmful, PCB residues, especially in adipose (fatty) tissues. Biological responses to individual isomers or mixtures can vary widely, even among closely related taxonomic species, and are also influenced by the presence of impurities in the PCB formulation, such as polychlorinated dibenzofurans (PCDFs).

Aquatic invertebrates play an important role in the cycling of PCBs within and between ecosystems. Sediment contamination has been implicated as a maji source of PCB contamination. Great Lakes salmon sampled near stations with high flushing and sedimentation rates contained the lowest PCB tissue concentrations. Although diet is a major route of PCB uptake in many species of fish, there are notable exceptions, and considerable interspecies differences exist in the responses of teleosts to PCB loadings. LC50 concentrations for sensitive freshwater and marine organisms has been shown to vary from 0.1 to $10.0 \mu \mathrm{g} / \mathrm{L}$ during exposures of 7 to 38 days. In general, toxicity increases with increasing exposure, and crustaceans and younger developmental stages are the most sensitive biotic groups.

In fish, the primary biochemical effect of PCBs is the induction of hepatic mixed function oxidase (MFO) systems, which increase the organism's capacity to biotransform or to detoxify xenobiotic chemicals and endogenous steroids.

Snapping turtles (Chelydra serpentia) are capable of storing high concentrations of PCBs in fat without any apparent detrimental effects, and may be useful as biological indicators for lipophilic substances, including PCBs.

Residues of PCBs in birds are modified by numerous biotic factors, including fat content, tissue specificity, sex, and developmental stage. Comparative studies have found the highest PCB residues in birds with low fat content and in poor condition on capture.

In mammals, PCBs are readily absorbed through the gut, respiratory system, and skin. Initially, PCBs concentrate in liver, blood, and muscle. Eventually, accumulations are highest in adipose tissue and skin. Among mammals, the mink is especially sensitive to PCBs. Diets containing 6.7 to $8.6 \mathrm{mg}$ Aroclor PCBs/kg fresh weight killed $50 \%$ of the mink in 9 months. Orally administered Aroclor PCBs resulted in LD-50 values of 750 to $4,000 \mathrm{mg} / \mathrm{kg}$ body weight. Signs of PCB poisoning in mink include anorexia, weight loss, lethargy, and unthrifty appearance. Prior to death, dark fecal stools indicative of the presence of blood from the upper gastrointestinal tract (confirmed by necropsy) and enlarged livers have been observed.

\section{Polycyclic Aromatic Hydrocarbons}

PAHs are a class of compounds consisting of substituted and unsubstituted polycyclic rings, and are generated by the incomplete combustion of organic materials. Their chemical, physical, and biological properties vary with their molecular structure, geometry and conformation. The unsubstituted lower molecular weight PAHs exhibit significant acute toxic effects to some organisms but have not been demonstrated to be carcinogenic. The higher molecular weight PAHs, containing 4 to 7 rings, have been shown to be carcinogenic, mutagenic, or teratogenic to a wide variety of organisms, including fish and other aquatic life, amphibians, birds, and mammals. In general, PAHs do not significantly bioconcentrate up food chains despite their high lipid solubility, possibly as a result of the ability of many organisms to metabolize these 
substances. Inter- and intraspecies response to PAH exposure is variable, particularly under conditions of exposure to multiple PAH compounds.

PAHs are rather persistent in the environment. Some PAHs are carcinogenic, causing both systemic tumors and tumors at the site of application. Several PAHs are shown to promote carcinogenesis in experimental animals. Adverse effects on the liver and kidney are often associated with exposure to PAHs.

Very little information on specific PAHs is available. The environmental fate and transport of these compounds are largely inferred from data on benzo[a]pyrene and mixtures of PAHs. The relatively high $\log \mathbb{K}_{\text {ow }}$ of PAHs indicates that they should be strongly adsorbed to carbonaceous sediments and soils and, hence, have a low mobility in the environment. The available information suggests that these compounds can accumulate in the sediment and biota of the aquatic environment, and that absorption is the dominant aquatic transport/fate process. Atmospheric transport of PAHs generally occurs by adsorption onto airborne particulate matter. Some volatile and relatively low molecular weight PAHs may be transported by volatilization.

PAHs are relatively insoluble in water, but the dissolved portion may undergo rapid, direct photolysis. Singlet oxygen is the oxidant, and quinones are the products of these reactions. In the presence of excessive amounts, oxidation by chlorine and ozone may also be relevant fate process.

Although PAHs are rapidly bioaccumulated, their metabolism and elimination is fairly rapid in most organisms. Bioaccumulation, especially in vertebrates, is usually short-term, and therefore is of less importance. However, the available data indicate that higher molecular weight PAHs are metabolized slowly by microbes and more readily by higher animals. Biodegradation probably occurs more slowly in aquatic systems than in soil, and it may be much more important in systems that are chronically affected by PAH contamination.

Data on the health effects of PAHs predominantly pertain to possible carcinogenic effects. However, the application of carcinogenic PAHs on the skin in experimental animals is reported to cause destruction of sebaceous glands (skin), hyperkeratosis, hyperplasia, and ulceration. Many carcinogenic PAHs are immuno-suppressive agents. Toxic manifestations in the liver and kidney are reported in experimental animals exposed to PAHs. Workers exposed to PAHcontaining materials have exhibited chronic dermatitis, hyperkeratosis, and other skin manifestations.

The potential for PAHs to induce malignant transformation dominates the consideration of health hazards resulting form exposure because there often are no overt signs of toxicity until the dose is high enough to produce a high incidence of tumor.

PAHs administered by various routes have been found to produce varying degrees of carcinogenic response depending upon the type of PAH administered and the recipient animal species. The oral, topical, intraperitoneal, intratracheal, and inhalation are the frequently adopted routes for PAH administration. Skin painting (dermal exposure) experiments have revealed potent carcinogenic effects of several PAHs in the mouse. Similarly, high liver and mammary gland cancer incidence has been observed through the oral route of administration. There are several reports on the carcinogenic effects in the lungs of animals exposed to PAHs. 
BENZOIa]PYRENE (CAS No. 50-32-8): Benzo[a]pyrene (B[a]P) constitutes one of the most extensively studied polycyclic aromatic hydrocarbon groups and has been shown to be a local as well as systemic carcinogen by several routes of administration in multiple species. It is a complete carcinogen when applied to the skin of mice, rats and rabbits. Following intratracheal instillation, $B[a] P$ has induced carcinoma of the respiratory tract in syrian hamsters. Injection-site tumors have been produced in mice, rat and guinea pigs following intramuscular or subcutaneous administration of $\mathrm{B}[\mathrm{a}] \mathrm{P}$. Several in vitro, short-term genotoxicity and mutagenicity tests have confirmed the genotoxic and mutagenic properties of B[a]P. There are reports indicating reproductive and fetotoxic potentials of $B[a] P$ in rats. Synergistic interactions of divergent carcinogenic chemicals with B[a]P has been reported extensively in the literature. However, it is important to note that human epidemiologic data is scanty on the carcinogenicity of B[a]P. 
Table D-I

Screening of Ecological COPCs

Spoil Arra-1 Soil (0-10 ft)

\begin{tabular}{|c|c|c|c|c|c|c|c|c|c|}
\hline & Max or UCL & Phytotoxicity & & & & & Soll & & \\
\hline & concentration & screening & Phyto & Grass & Forb & Tree & screening & Soil & Soil \\
\hline Compound name & $(\mathrm{mg} / \mathrm{kg})$ & $(\mathrm{mg} / \mathrm{kg})$ & COPC? & $\mathrm{BO}$ & 80 & $\mathrm{EO}$ & $(\mathrm{mg} / \mathrm{kg})$ & COPC? & $\times 0$ \\
\hline \multicolumn{10}{|l|}{ NORGANCS } \\
\hline Aluminium & 29313 & 10 & yes & 2442.75 & 2442.75 & 146.57 & 1000 & yes & 0.09 \\
\hline Banium & 385 & 500 & no & & & & 1.31 & yes & 0.88 \\
\hline Beryllium & 1.29 & 10 & no & & & & 1.39 & no & \\
\hline Cadmium & 3.63 & 2 & yes & $\mathrm{NC}$ & NC & NC & 20 & no & \\
\hline Calcium & 57800 & no screening level & yes & $N$ & NC & NC & no screening level & yes & MC \\
\hline Cobalis & 20 & 25 & no & & & & 60 & no & \\
\hline Lead & 110 & 50 & yes & 0.24 & 0.44 & 1.10 & 1 & yes & 0.33 \\
\hline Magnesium & 20500 & no screening level & yes & $\mathrm{NC}$ & NC & NC & 25000 & no & \\
\hline Manganese & 2030 & 500 & yes & $\mathrm{NC}$ & NC & $\mathrm{NC}$ & 2.07 & yes & 2.94 \\
\hline Mercury & 2.6 & 0.3 & yes & 0.52 & MC & $M$ & 1 & yes & 0.01 \\
\hline Nickel & 51 & 25 & yes & 0.28 & 0.51 & 1.02 & 25 & yes & 0.01 \\
\hline Phosphorus & 447 & no screening level & yes & $\mathrm{MC}$ & NC & NC & no screening level & yes & $N C$ \\
\hline Potassium & 4753 & no screening level & yes & NC & MC & $\mathrm{MC}$ & no screening level & yes & $\overline{N C}$ \\
\hline Vanadium & 51.2 & 2.5 & yes & MC & NC & NC & 10 & yes & 0.02 \\
\hline Zine & 78.1 & 20 & yes & 0.08 & 0.26 & MC & 10 & yes & 0.02 \\
\hline \multicolumn{10}{|l|}{ ORGAMCS } \\
\hline Acetone & 0.22 & no screening level & yes & NC & MC & NC & 26 & no & \\
\hline Benzo(a) lenthracene & 0.23 & no screening level & yes & $\mathrm{NC}$ & NC & $\mathrm{NC}$ & 180 & no & \\
\hline Benzo(a)pyrens & 0.22 & no screening level & yes & NC & NC & NC & 0.011 & yes & 0.06 \\
\hline Benzo(b)fluoranthene & 0.31 & no screening level & yes & MC & $\mathrm{NC}$ & NC & no screening level & yes & $N C$ \\
\hline Benzo(g,h,i)perylen & 0.12 & no screening level & yes & NC & NC & NC & no screening level & yes & NC \\
\hline Benzo(k) fluoranthene & 0.31 & no screening level & yes & NC & NC & NC & no screening level & yes & $M C$ \\
\hline Chrysene & 0.29 & no screening level & yes & NC & NC & NC & 450 & no & \\
\hline Fluoranthene & 0.56 & no screening level & yes & NC & NC & MC & 125 & no & \\
\hline Indeno $(1,2,3$-cd)pyrene & 0.099 & no screening level & yes & NC & NC & MC & no screening level & yes & NC \\
\hline Methylene Chloride & 0.002 & no screening level & yes & NC & $\mathrm{NC}$ & MC & 15.04 & no & \\
\hline Phenanthrene & 0.43 & no screening level & yes & MC & NC & NC & 10 & no & \\
\hline Pyrene & 0.49 & no screening level & yes & NC & NC & NC & 75 & no & \\
\hline
\end{tabular}


Table D-2

Screening of Ecological COPCs

SY.200 Soil $(0.10 \mathrm{~m})$

\begin{tabular}{|c|c|c|c|c|c|c|c|c|c|}
\hline & Max or UCL & Phytotoxicily & & & & & Soil & & \\
\hline & concentration & screening & Phylo & Grass & Forb & Tree & screening & Soil & Soll \\
\hline Compound name & $(\mathrm{mg} / \mathrm{kg})$ & $(\mathrm{mg} / \mathrm{kg})$ & COPC? & $\mathrm{BO}$ & 80 & 80 & $(\mathrm{mg} / \mathrm{kg})$ & COPC? & 10 \\
\hline \multicolumn{10}{|l|}{ NOAGANCS } \\
\hline Antimony & 0.46 & 5 & no & & & & 0.35 & yes & 0.00 \\
\hline Barium & 87.63 & 500 & no & & & & 1.31 & yes & 0.20 \\
\hline Cadmium & 0.12 & 2 & no & & & & 20 & no & \\
\hline Calcium & 15907 & no screening level & yes & NC & NC & NC & no screening level & yes & MC \\
\hline Cobalt & 19.8 & 25 & no & & & & 60 & no & \\
\hline Lead & 58.8 & 50 & yes & 0.13 & 0.24 & 0.59 & 1 & yes & 0.18 \\
\hline Magnesium & 3217 & no screening level & yes & NC & NC & NC & 25000 & no & \\
\hline Manganese & 729 & 500 & yes & NC & NC & NC & 2.07 & yes & 1.06 \\
\hline Mercury & 69.8 & 0.3 & yes & 13.96 & $N C$ & NC & 1 & yes & 0.21 \\
\hline Nickel & 27.1 & 25 & yes & 0.15 & 0.27 & 0.54 & 25 & yes & 0.00 \\
\hline Potassium & 1498 & no screening level & yes & NC & NC & NC & no screening level & yes & NC \\
\hline Selenium & 1.33 & 1 & yes & 0.13 & 0.05 & NC & 0.065 & yes & 0.06 \\
\hline Siver & 1.51 & no screening level & yes & NC & MC & $\mathrm{NC}$ & 50 & no & \\
\hline Thallium & 0.3 & 1 & no & & & & no screening level & yes & $N$ \\
\hline Zinc & 115.26 & 20 & yes & 0.12 & 0.38 & NC & 10 & yes & 0.03 \\
\hline \multicolumn{10}{|l|}{ ORGANCS } \\
\hline Acenaphthene & 0.12 & no screening level & yes & NC & $\overline{N C}$ & NC & 175 & no & \\
\hline Acetone & 0.209 & no screening level & yes & NC & NC & NC & 26 & no & \\
\hline Aroclor-1254 & 0.062 & 40 & no & & & & 0.17 & no & \\
\hline Aroclop-1260 & 0.423 & 40 & no & & & & 0.17 & yes & 0.01 \\
\hline Benzola)pyrene & 0.265 & no screening level & yes & NC & NC & $\mathrm{NC}$ & 0.011 & yes & 0.07 \\
\hline Benzo(b)fluoranthene & 0.317 & no screening level & yes & $\overline{N C}$ & $\mathrm{NC}$ & NC & no screening level & yes & NC \\
\hline Benzo(k) Muoranthene & 0.259 & no screening level & yes & NC & MC & NC & no screening level & yes & NC \\
\hline Bis(2-ethylhexyl)phthalate & 0.244 & no screening level & yes & NC & $\mathrm{NC}$ & NC & 15 & no & \\
\hline Chrysene & 0.277 & no screening level & yes & NC & NC & NC & 450 & no & \\
\hline Fluoranthene & 0.3 & no screening level & yos & $N$ & NC & NC & 125 & no & \\
\hline Methylene Chloride & 0.051 & no screening level & yes & NC & NC & NC & 15.04 & no & \\
\hline 4-Methyl-2-pentanone & 0.025 & no screaning level & yes & NC & NC & NC & 12.9 & no & \\
\hline Phenanthrene & 0.292 & no screening level & yes & NC & NC & $\mathrm{NC}$ & 10 & no & \\
\hline Pyrene & 0.3096 & no screening level & yes & $\mathrm{NC}$ & NC & NC & 75 & no & \\
\hline
\end{tabular}


Tablo D.3

Screening of Ecological COPCs

Rust Spoil Area Soil (0-10 ft)

\begin{tabular}{|c|c|c|c|c|c|c|c|c|c|}
\hline & Max or UCL & Phyloloxicity & & & & & Soil & & \\
\hline & concentration & screening & Phyto & Grass & Forb & Tree & screening & Soil & Soil \\
\hline Compound name & $(\mathrm{mg} / \mathrm{kg})$ & $(\mathrm{mg} / \mathrm{kg})$ & COPC? & 80 & $B Q$ & 80 & $(\mathrm{mg} / \mathrm{kg})$ & COPC? & $\times 0$ \\
\hline \multicolumn{10}{|l|}{ NORGANICS } \\
\hline Antimony & 0.36 & 5 & no & & & & 0.35 & yes & 0.00 \\
\hline Barium & 115.4 & 500 & no & & & & 1.31 & yes & 0.26 \\
\hline Beryllium & 1.15 & 10 & no & & & & 1.39 & no & \\
\hline Cadmium & 5.7 & 2 & yes & NC & NC & NC & 20 & no & \\
\hline Calcium & 153941 & no screening level & yes & MC & NC & NC & no screening level & yes & NC \\
\hline Chromium (VI) & 37.23 & 2 & yes & 5.03 & 5.48 & $N$ & 6.17 & yes & 0.02 \\
\hline Cobalt & 16.17 & 25 & no & & & & 60 & no & \\
\hline Copper & 57.6 & 40 & yes & 0.96 & 0.29 & $\mathrm{NC}$ & 1 & yes & 0.17 \\
\hline Cyanide & 2.65 & no screening level & yes & NC & NC & NC & 27.8 & no & \\
\hline Lead & 43.65 & 50 & no & & & & 1 & yes & 0.13 \\
\hline Magnesium & 11894 & no screening level & yes & NC & NC & NC & 25000 & no & \\
\hline Manganese & 953 & 500 & yes & MC & NC & NC & 2.07 & yes & 1.38 \\
\hline Mercury & 4.28 & 0.3 & yos & 0.86 & $M$ & MC & 1 & yes & 0.01 \\
\hline Nickel & 47.32 & 25 & yes & 0.26 & 0.47 & 0.95 & 25 & yes & 0.01 \\
\hline Niobium & 0.87 & no scraening level & yes & NC & MC & NC & no screening level & yes & MC \\
\hline Potassium & 2209 & no screening levol & yes & NC & NC & NC & no screening level & yes & NC \\
\hline Silver & 1.1 & no screening level & yos & NC & NC & $\mathrm{NC}$ & 50 & no & \\
\hline Thallium & 0.15 & 1 & no & & & & no screening level & yes & NC \\
\hline Thorium & 25.63 & no screening level & yes & NC & NC & NC & no screening level & yes & NC \\
\hline Zine & 129.5 & 20 & yes & 0.13 & 0.43 & NC & 10 & yes & 0.04 \\
\hline \multicolumn{10}{|l|}{ OAGAMCS } \\
\hline Acenaphthene & 0.066 & no screening level & yes & NC & NC & NC & 175 & no & \\
\hline Acenaphthylene & 0.056 & no screening level & yes & NC & NC & NC & no screening level & yes & NC \\
\hline Acetone & 1.78 & no screening level & yes & NC & MC & MC & 26 & no & \\
\hline Anthracene & 0.14 & no screening level & yes & NC & NC & NC & 1000 & no & \\
\hline Aroclor-1254 & 8.9 & 40 & no & & & & 0.17 & yes & 0.16 \\
\hline Benzene & 0.037 & no screening level & yes & NC & NC & NC & 6.4 & no & \\
\hline Benzo(a)anthracene & 0.249 & no screening level & yes & NC & MC & NC & 180 & no & \\
\hline Benzo(a)pyrene & 0.361 & no screening level & yes & NC & NC & NC & 0.011 & yes & 0.10 \\
\hline Benzo(b)fluoranthene & 0.36 & no screening level & yes & MC & NC & NC & no screening level & yes & NC \\
\hline Benzo $(g, h, i)$ perylene & 0.4 & no screening level & yes & NC & $M$ & NC & no screening level & yes & NC \\
\hline Benzo(k)fuoranthene & 0.308 & no screening level & yes & NC & NC & NC & no screening level & yes & NC \\
\hline Bis (2-ethylhexyl)phthalate & 22.9 & no screening level & yes & NC & NC & MC & 15 & yes & 0.00 \\
\hline Bromodichloromethane & 0.037 & no screening level & yes & NC & NC & NC & 15 & no & \\
\hline Bromolorm & 0.037 & no screening level & yes & NC & $\mathrm{NC}$ & NC & no screening level & yes & $\mathrm{NC}$ \\
\hline Bromomethane & 0.075 & no screening level & yes & NC & NC & NC & no screening level & yes & NC \\
\hline 2. Butanone & 0.112 & no screening level & yes & NC & NC & NC & 3.3 & no & \\
\hline
\end{tabular}


Table D-3 (cont.)

Screening of Ecological COPCs

Rust Spoil Area Soil $(0.10 \mathrm{ft})$

\begin{tabular}{|c|c|c|c|c|c|c|c|c|c|}
\hline & Max or UCL & Phytotoxicity & & & & & Soil & & \\
\hline & concentration & screening & Phyto & Grass & Forb & Tree & screening & Soil & Soll \\
\hline Compound name & $(\mathrm{mg} / \mathrm{kg})$ & $(\mathrm{mg} / \mathrm{kg})$ & COPC? & $\mathrm{BO}$ & $\mathrm{BO}$ & EQ & $(m g / k g)$ & COPC? & $\times 0$ \\
\hline Butyl benzyl phthalate & 0.073 & no screening level & yes & NC & MC & NC & no screening level & yes & NC \\
\hline Carbazole & 0.1 & no screening level & yes & NC & NC & NC & no screening level & yes & NC \\
\hline Carbon Disulfide & 0.037 & no screening level & yes & NC & NC & $\mathrm{NC}$ & no screening level & yes & NC \\
\hline Carbon Tetrachloride & 0.037 & no screening level & yes & NC & NC & MC & 0.183 & no & \\
\hline Chlorobenzene & 0.037 & no screening level & yos & NC & NC & NC & no screening level & yes & NC \\
\hline Chloroethane & 0.075 & no screening level & yes & NC & NC & NC & no screening level & yes & NC \\
\hline Chlorolorm & 0.066 & no screening level & yes & NC & NC & NC & 23 & no & \\
\hline Chloromethane & 0.075 & no screening level & yes & $\mathrm{NC}$ & NC & NC & no screening level & yes & NC \\
\hline Chrysene & 0.295 & no screening level & yes & NC & NC & $\mathrm{NC}$ & 450 & no & \\
\hline 1,3-Dichloropropene & 0.037 & no screening level & yes & NC & NC & MC & no screening level & yes & NC \\
\hline Dibenzo(a,h)anthracene & 0.245 & no screening level & yes & NC & NC & $M$ & 200 & no & \\
\hline Dibenzofuran & 0.038 & no screening level & yes & NC & NC & NC & no screening level & yes & NC \\
\hline Dibromochloromethane & 0.037 & no screening level & yes & NC & NC & NC & 60 & no & \\
\hline Di-n-butyl phthalate & 1.3 & 200 & no & & & & 48.4 & no & \\
\hline 1.2-Dichlorobenzene & 0.23 & no screening level & yes & NC & NC & NC & 14.4 & no & \\
\hline 1,1-Dichloroethane & 0.109 & no screening level & yes & NC & NC & NC & no screening level & yes & NC \\
\hline 1,2-Dichloroethane & 0.037 & no screening level & yes & NC & NC & NC & 1.9 & no & \\
\hline 1.1-Dichloroethylene & 0.051 & no screening level & yes & NC & NC & $\mathrm{MC}$ & 2.31 & no & \\
\hline 1.2-Dichloroethylens & 110 & no screening level & yes & MC & NC & MC & 100.3 & yes & 0.00 \\
\hline 1,2-Dichloropropane & 0.037 & no screening level & yes & NC & $\mathrm{MC}$ & $\mathrm{MC}$ & 50 & no & \\
\hline 2,4-Dimethylphenol & 0.225 & no screening level & yes & $\mathrm{NC}$ & MC & NC & 750 & no & \\
\hline Dimethyl phthalate & 0.15 & no screening level & yes & NC & NC & $\mathrm{NC}$ & no screening level & yes & NC \\
\hline Di-n-octyl phthalate & 0.321 & no screening level & yes & NC & NC & NC & no screening level & yes & NC \\
\hline Endosulfan & 0.007 & no screening level & yes & NC & NC & NC & no screening level & yes & NC \\
\hline Ethylbenzene & 0.062 & no screening level & yes & $\mathrm{NC}$ & NC & NC & no screening level & yes & NC \\
\hline Fluoranthene & 0.461 & no screening level & yes & NC & NC & NC & 125 & no & \\
\hline Fluorene & 0.083 & no screening level & yes & NC & $\mathrm{MC}$ & NC & 125 & no & \\
\hline Indeno $(1,2,3-$ cd)pyrene & 0.408 & no screening level & yes & NC & NC & NC & no screening level & yes & NC \\
\hline Heptachlor & 0.0008 & no screening level & yes & NC & NC & NC & no screening level & yes & NC \\
\hline 2.Hexanone & 0.075 & no screening level & yes & $\mathrm{NC}$ & NC & NC & no screening level & yes & NC \\
\hline Methoxychlor & 0.0015 & no screening level & yes & NC & NC & NC & no screening level & yes & NC \\
\hline Methylene Chloride & 0.109 & no screening level & yes & NC & NC & NC & 15.04 & no & \\
\hline 2-Methyinaphthalene & 0.002 & no screening level & yes & NC & NC & NC & 1630 & no & \\
\hline 4-Methyl-2-pentanone & 0.113 & no screening level & yes & NC & NC & $N C$ & 12.9 & no & \\
\hline 4-Methylphenol & 0.265 & no screening level & yes & NC & MC & NC & no screening level & yes & NC \\
\hline Naphthalene & 0.09 & no screening level & yes & NC & NC & MC & 400 & no & \\
\hline Pentachlorophenol & 0.047 & no screening level & yes & NC & $\mathrm{NC}$ & NC & no screening level & yes & $\mathrm{NC}$ \\
\hline Phenanthrene & 0.599 & no screening level & yes & $\mathrm{NC}$ & $\mathrm{NC}$ & $\mathrm{NC}$ & 10 & no & \\
\hline
\end{tabular}


Table D-3 (cont)

Scresening of Ecological COPCs

Rust Spoil Area Soll $(0-10 \mathrm{t})$

\begin{tabular}{|c|c|c|c|c|c|c|c|c|c|}
\hline & Max or UCL & Phytotoxicity & & & & & Soil & & \\
\hline & concentration & screening & Phyto & Grass & Forb & Tree & screening & Soll & Soll \\
\hline Compound name & $(\mathrm{mg} / \mathrm{kg})$ & $(\mathrm{mg} / \mathrm{kg})$ & COPC? & $\mathrm{BO}$ & 80 & $\mathrm{BQ}$ & $(\mathrm{mg} / \mathrm{kg})$ & COPC? & $\times 0$ \\
\hline Phenol & 0.63 & no screening level & yes & NC & NC & MC & 60 & no & \\
\hline Pyrene & 0.846 & no screening level & yos & $\mathrm{NC}$ & NC & NC & 75 & no & \\
\hline Styrene & 0.037 & no screening level & yos & NC & NC & NC & no screening level & yes & NC \\
\hline 1,1,2,2-Telrachloroethane & 0.037 & no screening level & yes & NC & NC & NC & 10 & no & \\
\hline Tetrachloroethylene & 323 & no screening level & yes & NC & $\mathrm{NC}$ & NC & no screening level & yes & NC \\
\hline Toluene & 0.091 & 200 & no & & & & 57.3 & no & \\
\hline $1,2,4$-Trichlorobenzene & 14.157 & no screening level & yes & NC & $\mathrm{MC}$ & $\mathrm{NC}$ & no screening level & yes & NC \\
\hline Trichlorotrifluoroethane & 32 & no screening level & yos & NC & NC & NC & no screening level & yes & $\mathrm{NC}$ \\
\hline 1,1,1-Trichloroethane & 0.077 & no screening level & yes & MC & NC & $\mathrm{NC}$ & 90 & no & \\
\hline Trichlorosthylene & 2.898 & no screening level & yes & NC & NC & $\mathrm{NC}$ & 193 & no & \\
\hline Vinyl Acelate & 0.038 & no screening level & yes & $\mathrm{NC}$ & NC & NC & no screening level & yes & $N$ \\
\hline Vinyl Chloride & 0.075 & no screening level & yos & NC & NC & $\mathrm{NC}$ & 0.33 & no & \\
\hline Xylenes & 0.104 & no screening level & yes & NC & NC & NC & 1286 & no & \\
\hline
\end{tabular}


Table D.4. Properties of COPCs at BCV OU 2

\begin{tabular}{|c|c|c|c|c|c|c|c|c|}
\hline \multirow[b]{3}{*}{$\begin{array}{c}\text { Potential } \\
\text { ecological COCs }\end{array}$} & \multicolumn{4}{|c|}{ Biological effects } & \multicolumn{4}{|c|}{ Envirommental fate } \\
\hline & \multicolumn{2}{|c|}{ Aq̨uatic } & \multicolumn{2}{|c|}{ Terrestrial } & \multicolumn{2}{|c|}{ Mobility } & \multicolumn{2}{|c|}{ Persiatence } \\
\hline & $\begin{array}{l}\text { Acute } \\
\text { toxicity level } \\
(\mu / L)\end{array}$ & $\begin{array}{c}\text { Chronic } \\
\text { loxicity level } \\
(n g / L)\end{array}$ & $\begin{array}{c}\text { Acute oral } \\
\text { coxicity level } \\
\text { (mg//ag) }\end{array}$ & $\begin{array}{c}\text { Chronic oral } \\
\text { toxicity level } \\
\text { (mg/kg) } \\
\end{array}$ & $\begin{array}{c}\text { Water solubility } \\
\text { (mg/C) }\end{array}$ & $\begin{array}{l}\text { Soil eorption } \\
\left.\mathbb{K}_{\infty}\right) \text { or soil } \\
\text { partitioning } \\
\text { coefficient }\left(\mathbb{K}_{\mathbf{d}}\right) \\
\end{array}$ & $\begin{array}{l}\text { Degradation } \\
\text { half-life (d) } \\
\end{array}$ & $\begin{array}{c}\text { Bioconcentration } \\
\text { factor (BCF) }\end{array}$ \\
\hline \multicolumn{9}{|c|}{ Metals and rere earths } \\
\hline Antimony & $\begin{array}{l}8.8 \mathrm{E}+1 \mathrm{mg} / \mathrm{L} \\
(\mathrm{WQCAQ})^{\circ}\end{array}$ & $\begin{array}{l}3.0 \mathrm{E}+1 \mu \mathrm{g} / \mathrm{L} \\
\mathrm{WQCAO}^{\circ}\end{array}$ & $\begin{array}{l}700 \text { (rats, ord } \\
\text { LDSO) }\end{array}$ & $\begin{array}{l}5 \mathrm{mg} / 2 \text { days for } \\
30-90 \text { days, } \\
\text { abortion } \\
0.35 \mathrm{mg} / \mathrm{hg} \\
\text { bw/day (rat, oral, } \\
\text { LOAEL) }\end{array}$ & $\begin{array}{l}\text { Insoluble as } \\
\text { metal } \\
\text { Oxide slightly } \\
\text { soluble }\end{array}$ & $150^{d}$ & NA & NA \\
\hline Bมี่นท & $50,000^{\circ}$ & 2000 (MCL) & 5 (rabbit, $\left.\mathrm{BaCl}_{2}\right)^{c}$ & $2\left(\mathrm{rabbil}, \mathrm{BaCl}_{2}\right)^{e}$ & $\begin{array}{l}\text { Decomposes } \\
2.46 \mathrm{mg} / \mathrm{h} \\
\mathrm{BaSO}_{4} \text { at } 25^{\circ}\end{array}$ & $60^{4}$ & $\begin{array}{l}\text { Persistent in } \\
\text { swe }\end{array}$ & $4^{e}$ \\
\hline Copper & $\begin{array}{l}5.4 \text { (4d, hardness } \\
\text { of } 40 \mathrm{mg} / \mathrm{L})^{e} \\
250 \text { (larval } \\
\text { fathead minnow, } \\
\text { LD50y } \\
9.2 \mathrm{E}+0 \mu \mathrm{g} / \mathrm{L} \\
\text { (hardness } \\
\text { dependent) } \\
\text { (WQCAO) }\end{array}$ & $\begin{array}{l}3 \text { (fish) }^{e} \\
\text { Fathead minnow } \\
\text { harvae } 123 \\
(28 \text { day) } \\
6.5 E+0 \mu / L \\
\text { (handness } \\
\text { dependent) } \\
\text { (WoCAO) }\end{array}$ & $\begin{array}{l}140 \mathrm{(rat}_{3} \\
\left.\mathrm{CuCl}_{2}\right)^{c} \\
159\left(\mathrm{rabbit}^{2}\right. \\
\left.\mathrm{CuCO}_{3} \mathrm{Cu}_{(\mathrm{H}} \mathrm{O}\right)^{c}\end{array}$ & $\begin{array}{l}1.21 \text { (rat, oral } \\
\text { TDLo } 35 \text { wk, } \\
\text { effects on } \\
\text { feriility) }\end{array}$ & $\begin{array}{l}\text { Insoluble but } \\
\text { pH sensitive } \\
\text { (chloride and } \\
\text { nitrate soluble, } \\
\text { carbongte, } \\
\text { phosphate } \\
\text { insoluble) }\end{array}$ & $\begin{array}{l}\text { High in } \\
\text { organic } \\
\text { matrix }, 140^{d}\end{array}$ & NA & $200(\text { fish) })^{e}$ \\
\hline
\end{tabular}




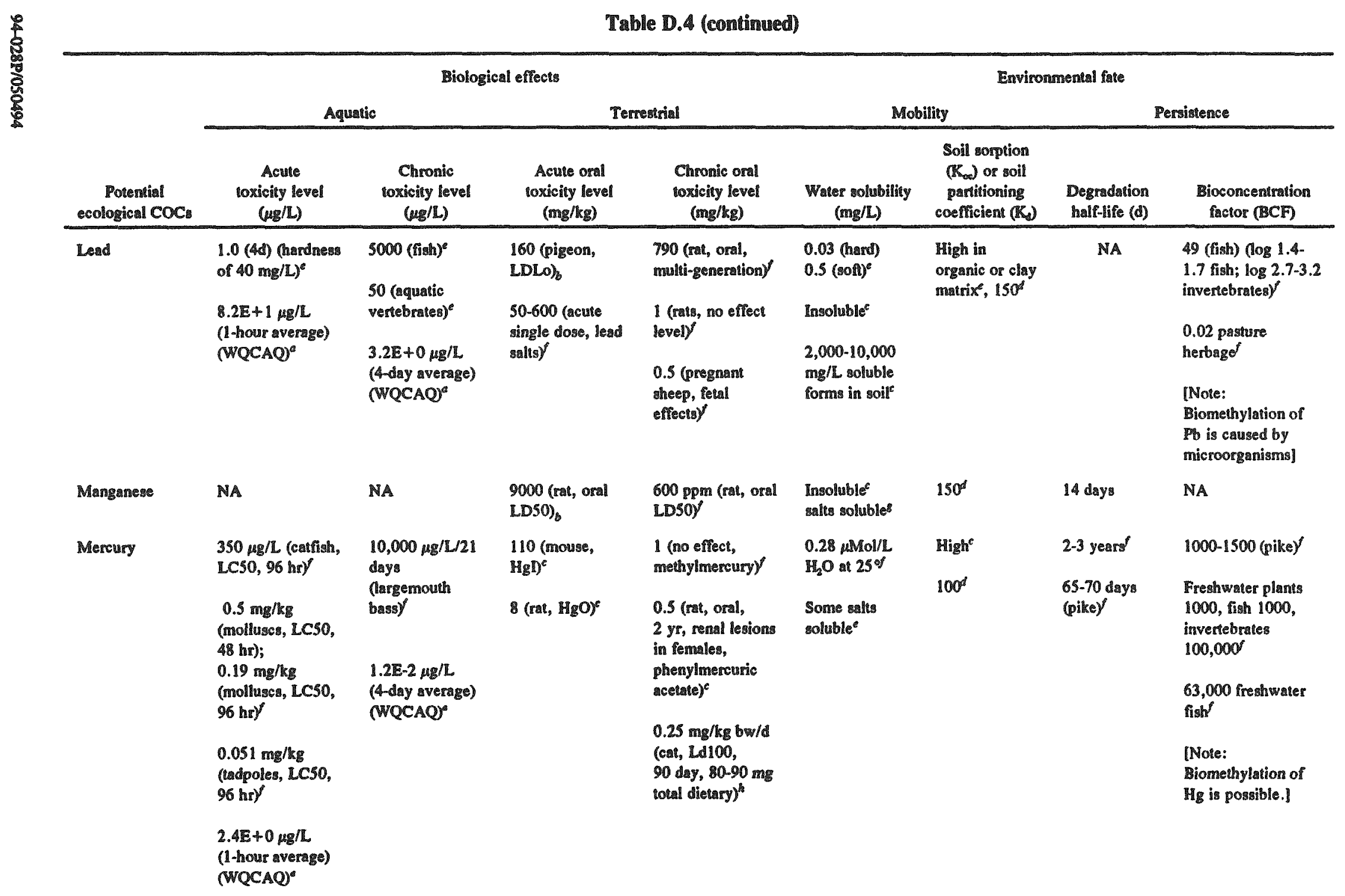


Table D.4 (continued)

\begin{tabular}{|c|c|c|c|c|c|c|c|c|}
\hline \multirow[b]{3}{*}{$\begin{array}{c}\text { Potential } \\
\text { ecological } \mathrm{COC}\end{array}$} & \multicolumn{4}{|c|}{ Biological effects } & \multicolumn{4}{|c|}{ Environmental fate } \\
\hline & \multicolumn{2}{|c|}{ Aquatic } & \multicolumn{2}{|c|}{ Terrestrial } & \multicolumn{2}{|c|}{ Mobility } & \multicolumn{2}{|c|}{ Persistence } \\
\hline & $\begin{array}{l}\text { Acute } \\
\text { toxicily level } \\
\text { (ug/L) }\end{array}$ & $\begin{array}{c}\text { Chronic } \\
\text { toxieity level } \\
\text { (ug/L) }\end{array}$ & $\begin{array}{l}\text { Acute oral } \\
\text { toxicity level } \\
\text { (mg/kg) }\end{array}$ & $\begin{array}{l}\text { Chronic oral } \\
\text { toxicity level } \\
\text { (mg/kg) }\end{array}$ & $\begin{array}{c}\text { Water solubility } \\
(\mathrm{mg} / \mathrm{L})\end{array}$ & $\begin{array}{l}\text { Soil sorption } \\
\left(\mathbb{R}_{\text {ese }}\right) \text { or soil } \\
\text { partitioning } \\
\text { coefficient }\left(\mathbb{K}_{s}\right)\end{array}$ & $\begin{array}{l}\text { Degradation } \\
\text { half-life (d) }\end{array}$ & $\begin{array}{l}\text { Bioconcentration } \\
\text { factor (BCF) }\end{array}$ \\
\hline Nickel & $\begin{array}{l}1.4 \mathrm{E}+3 \mathrm{\mu g} / \mathrm{L} \\
\text { (tolal nickel) } \\
\text { (WQCAQ) }\end{array}$ & $\begin{array}{l}1.6 \mathrm{E}+2 \mu \mathrm{g} / \mathrm{L} \\
\text { (total mickel) } \\
\text { (WQCAO) }\end{array}$ & $\begin{array}{l}5 \text { (guinea pig, } \\
\text { rat, LDLo) }\end{array}$ & 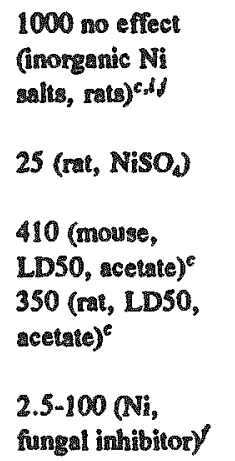 & $\begin{array}{l}\text { Insoluble } \\
\mathrm{NiO} / \mathrm{Ni}^{\mathrm{e}}\end{array}$ & 100 & NA & NA \\
\hline Selemium & 20 (WQCAQ) & 5 (wQCAQr & $\begin{array}{l}6700 \text { (rat, oral } \\
\text { LD50) }\end{array}$ & $\begin{array}{l}134 \text { (moure, oral } \\
\text { TDLo, mulli- } \\
\text { generation)b }\end{array}$ & Insolubles & $110^{4}$ & NA & NA \\
\hline Varadium & NA & NA & $\begin{array}{l}11 \text { (albino mice, } \\
\text { oxides) }\end{array}$ & $\begin{array}{l}10 \text { (rainbow trout, } \\
12 \text { weeks, } \\
\text { minimum toxic } \\
\text { diet cons.y }\end{array}$ & $\begin{array}{l}\text { Insoluble as } \\
\text { metal }\end{array}$ & $55^{8}$ & NA & NA \\
\hline Zins & $\begin{array}{l}49(4 d) \text { (hardness } \\
\text { of } 80 \mathrm{mg} / \mathrm{L})^{e} \text {, } \\
120(W Q C A O)\end{array}$ & $\begin{array}{l}2000 \text { (fish)e }^{e} \\
110 \text { (WQCAQ) }^{\circ}\end{array}$ & $\begin{array}{l}250 \text { (guinea pig, } \\
\text { LDLO, ZnFi) } \\
1190 \text { (rat, oral } \\
\text { LD50, zine } \\
\text { siitrale } \\
\text { hexahydratey }\end{array}$ & $\begin{array}{l}10 \text { (rat, oral, no }^{\circ} \\
\text { effect level) } \\
50 \text { (rat, oral, } \\
\text { reproductive } \\
\text { effects) }\end{array}$ & Insoluble & $52^{d}$ & NA & $\begin{array}{l}47 \text { (fish), } 16,700 \\
\text { (oyster), 85 (clam), } \\
500 \text { (mussely }\end{array}$ \\
\hline
\end{tabular}


Table D.4 (continued)

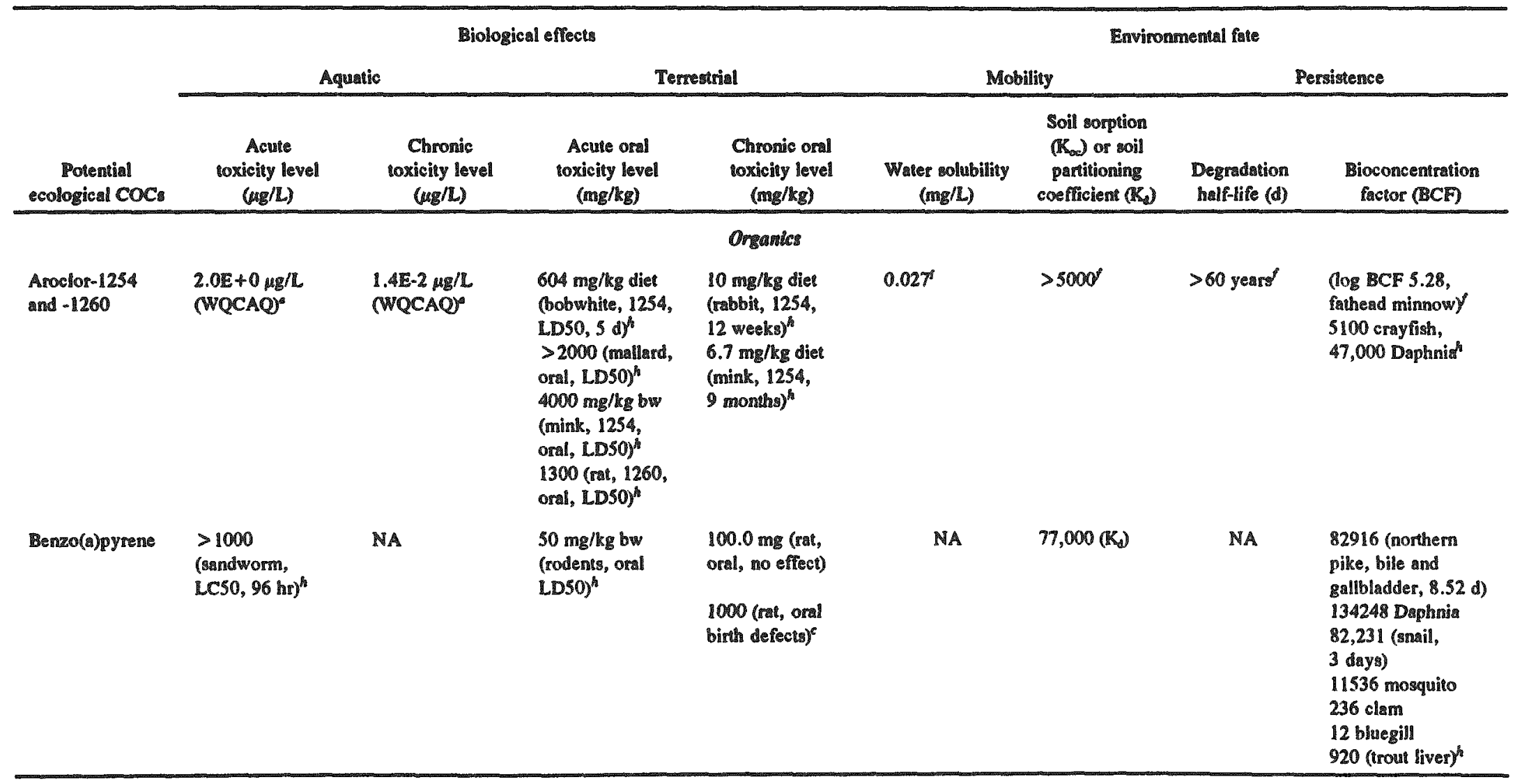


Table D.4 (continued)

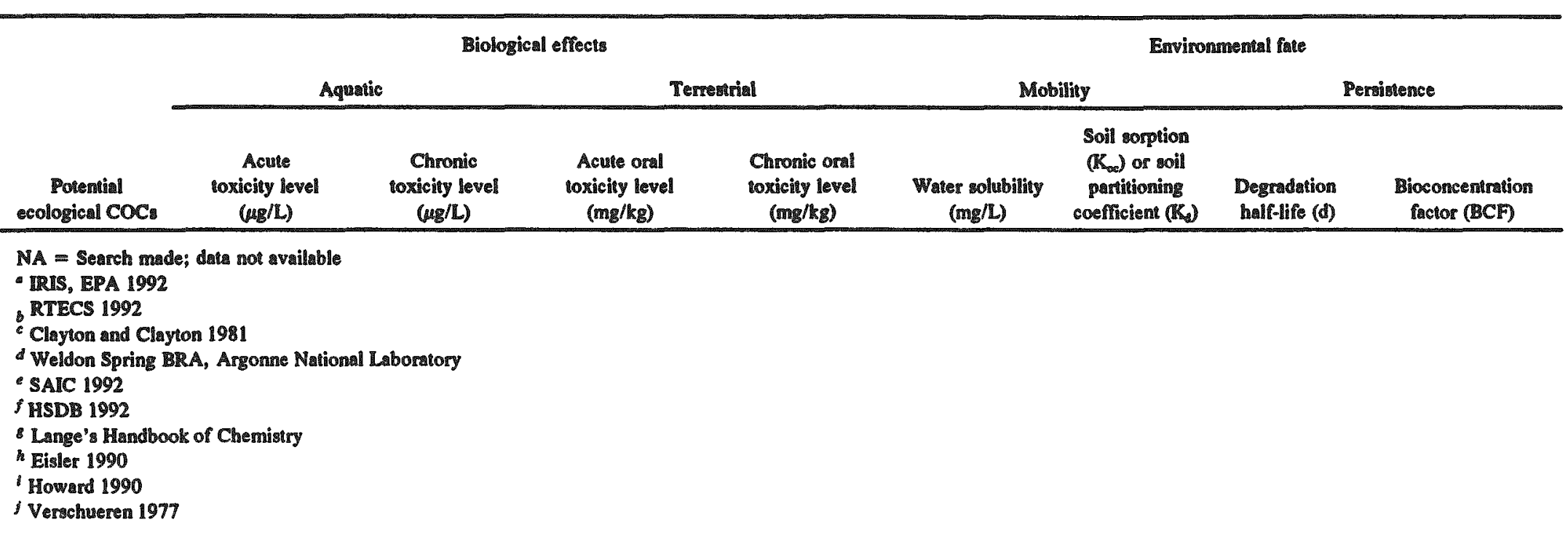


APPENDIX E

Data Quality 
. 


\section{E-3}

Table E.1

Bear Creek Valley OU2 RI FA

Summary of Samples Planned, Collected, and Added

\begin{tabular}{|c|c|c|c|c|}
\hline Station & $\begin{array}{l}\text { Samples } \\
\text { Planned }\end{array}$ & "Samples Added & $\begin{array}{l}\text { Sampies } \\
\text { Collected }\end{array}$ & $\begin{array}{l}\text { \# Samples Nol } \\
\text { Collected }\end{array}$ \\
\hline \multicolumn{5}{|l|}{ Media: Soil } \\
\hline \multicolumn{5}{|c|}{ Location: Rust Spoil Area } \\
\hline SBOI & 5 & 0 & 4 & 1 \\
\hline $\mathrm{SBO2}$ & 5 & 3 & 8 & 0 \\
\hline \$B03 & 4 & 2 & 9 & 0 \\
\hline SB04 & 5 & 0 & 3 & 2 \\
\hline$\$ B 05$ & 5 & 0 & 4 & 1 \\
\hline \multicolumn{5}{|c|}{ Lacation: Storage Yard - 200} \\
\hline SBO6 & 4 & 0 & 4 & 0 \\
\hline \$B07 & 4 & $\overline{0}$ & 4 & 0 \\
\hline \$B08 & 4 & 0 & 4 & 0 \\
\hline \$BO9 & 4 & 0 & 1 & 3 \\
\hline$\$ 810$ & 4 & 0 & 4 & 0 \\
\hline SB11 & 4 & 0 & 2 & 2 \\
\hline \$812 & 4 & 0 & 4 & 0 \\
\hline SB13 & 4 & 0 & 4 & 0 \\
\hline SB14 & 4 & 1 & 5 & 0 \\
\hline SB15 & 4 & 0 & 4 & 0 \\
\hline$\$ 816$ & 4 & 1 & 5 & 0 \\
\hline \$B17 & 4 & 1 & 5 & 0 \\
\hline SB18 & 4 & 0 & 3 & 1 \\
\hline SB19 & 4 & 0 & 3 & 1 \\
\hline SB20 & 4 & 0 & 4 & 0 \\
\hline SB21 & 4 & 0 & 3 & 1 \\
\hline$\overline{\mathrm{SB} 22}$ & 4 & 0 & 2 & 2 \\
\hline 5823 & 4 & 0 & 4 & 0 \\
\hline$\overline{\text { SB24 }}$ & 4 & 0 & 4 & 0 \\
\hline SB25 & 4 & 0 & 3 & 1 \\
\hline \$B26 & 4 & 0 & 3 & 1 \\
\hline SB27 & 4 & 1 & 5 & 0 \\
\hline SB28 & 4 & 0 & 4 & 0 \\
\hline SB29 & 4 & 1 & 5 & 0 \\
\hline SB30 & 4 & 0 & 3 & 1 \\
\hline \$B31 & 4 & 0 & 4 & 0 \\
\hline SB32 & 4 & 0 & 3 & 1 \\
\hline \$B33 & 4 & 0 & 3 & 1 \\
\hline SB34 & 4 & 0 & 4 & 0 \\
\hline SB35 & 4 & 0 & 4 & 0 \\
\hline SB36 & 4 & 0 & 3 & 1 \\
\hline \$B37 & 4 & 0 & 4 & 0 \\
\hline \$B38 & 4 & 0 & 2 & 2 \\
\hline SB39 & 4 & 4 & 8 & 0 \\
\hline$\$ 840$ & 4 & 0 & 4 & 0 \\
\hline SB41 & 4 & 0 & 4 & 0 \\
\hline SB42 & 4 & 0 & 3 & 1 \\
\hline SB43 & 4 & 0 & 4 & 0 \\
\hline SB44 & 4 & $\overline{0}$ & 3 & 1 \\
\hline
\end{tabular}


E-4

\begin{tabular}{|c|c|c|c|c|}
\hline Station & $\begin{array}{l}\text { t Samples } \\
\text { Planned }\end{array}$ & \# Samples Added & $\begin{array}{l}\text { Samples } \\
\text { Collected }\end{array}$ & $\begin{array}{c}\text { \# Samples Not } \\
\text { Collected }\end{array}$ \\
\hline SB45 & 4 & 0 & 2 & 2 \\
\hline SB46 & 4 & 2 & 6 & 0 \\
\hline SB47 & 4 & 0 & 2 & 2 \\
\hline SR48 & 4 & 0 & 2 & 2 \\
\hline SB49 & 4 & 0 & 2 & 2 \\
\hline SB50 & 4 & 0 & 3 & 1 \\
\hline SBS1 & 4 & 1 & 5 & 0 \\
\hline SB52 & 4 & 0 & 4 & 0 \\
\hline SB53 & 4 & 2 & 6 & 0 \\
\hline SB54 & 4 & 0 & 1 & 3 \\
\hline SB55 & 4 & 1 & 5 & 0 \\
\hline SB56 & 4 & 3 & 7 & $\overline{0}$ \\
\hline SB57 & 4 & 3 & 7 & 0 \\
\hline SBS8 & 4 & 3 & 7 & 0 \\
\hline \$B59 & 4 & 3 & 7 & 0 \\
\hline SB60 & 4 & 2 & 6 & 0 \\
\hline \$B61 & 4 & 3 & 7 & 0 \\
\hline SB62 & 0 & 4 & 4 & 0 \\
\hline SB63 & 0 & 4 & 4 & 0 \\
\hline SB64 & 0 & 1 & 1 & 0 \\
\hline \multicolumn{5}{|c|}{ Media: Sediment } \\
\hline \multicolumn{5}{|c|}{ Location: Rust Spoil Area } \\
\hline$\$ \$ 507$ & 1 & 0 & 1 & 0 \\
\hline SS08 & 1 & 0 & 1 & 0 \\
\hline S\$09 & 1 & 0 & 1 & 0 \\
\hline \multicolumn{5}{|l|}{ Spoil Area - 1} \\
\hline SS01 & 1 & 0 & 1 & 0 \\
\hline S\$02 & 1 & 0 & 1 & 0 \\
\hline $\mathrm{SSO3}$ & 1 & 0 & 1 & 0 \\
\hline SSO4 & 1 & $\overline{0}$ & 1 & $\overline{0}$ \\
\hline SSOS & 1 & 0 & 1 & 0 \\
\hline SS06 & 1 & 0 & 1 & 0 \\
\hline \multicolumn{5}{|c|}{ Media: Surface Water } \\
\hline \multicolumn{5}{|c|}{ Location: Rust Spoil Area } \\
\hline SSO1 & 1 & 0 & 0 & 1 \\
\hline $\mathrm{SSO2}$ & 1 & 0 & 1 & 0 \\
\hline SSO3 & 1 & 0 & 1 & 0 \\
\hline SSO4 & 1 & 0 & 1 & 0 \\
\hline SSOS & 1 & 0 & 1 & 0 \\
\hline \$S06 & 1 & 0 & 1 & 0 \\
\hline SS07 & 1 & 0 & 1 & $\overline{0}$ \\
\hline S508 & 1 & 0 & 1 & $\overline{0}$ \\
\hline SS09 & 1 & 0 & 1 & 0 \\
\hline \multicolumn{5}{|c|}{ Media: Groundwater Seep } \\
\hline \multicolumn{5}{|c|}{ Location: Rust Spoil Area } \\
\hline $\mathrm{SOI}$ & 1 & 0 & 1 & $\overline{0}$ \\
\hline $\mathrm{SO2}$ & 1 & 0 & 0 & 1 \\
\hline S03 & 1 & 0 & 0 & 1 \\
\hline \multicolumn{5}{|c|}{ Media: Groundwater } \\
\hline Location: Spoi & & & & \\
\hline
\end{tabular}




\section{E-5}

\begin{tabular}{|c|c|c|c|c|}
\hline Station & $\begin{array}{c}\text { \# Samples } \\
\text { Planned }\end{array}$ & Samples Added & $\begin{array}{c}\text { \# Samples } \\
\text { Collected }\end{array}$ & $\begin{array}{c}\text { \# Samples Nol } \\
\text { Collected }\end{array}$ \\
\hline MW01 & 1 & 0 & 1 & 0 \\
\hline MW02 & 1 & 0 & 1 & 0 \\
\hline Location: Storage Yard - 200 & 1 & 0 & 1 & 0 \\
\hline MW03 & 1 & 0 & 1 & 0 \\
\hline MW04 & 1 & 0 & 1 & 0 \\
\hline MW05 & 1 & \multicolumn{3}{|c|}{} \\
\hline
\end{tabular}


04/07/94

Table E.2

Bear Creek Valley OU2 RI FA

Summary of Rejected Analytes

RUST SPOLL AREA

Environmental Media: Soil

Class: Inorganics

\begin{tabular}{|c|c|c|c|c|c|c|}
\hline \multirow{2}{*}{$\begin{array}{l}\text { CHass: inorganucs } \\
\text { Parrameter }\end{array}$} & \multirow{2}{*}{$\begin{array}{c}\text { Total } \\
\text { Saraphles }\end{array}$} & \multicolumn{2}{|c|}{ Total Rejected } & \multirow[b]{2}{*}{ Reason for Rejection } & \multicolumn{2}{|c|}{ Rejected for Reasom } \\
\hline & & Sanuples & Percent & & Sanuples & Percent \\
\hline Antimony & 284 & 11 & 3.8 & Recovery less than $30 \%$ & 11 & $\overline{3.8}$ \\
\hline Cyanide & 136 & 9 & 6.6 & Recovery less than $30 \%$ & 9 & $\overline{6.6}$ \\
\hline Selenium & 284 & 12 & 4.2 & Recovery less than $30 \%$. & 12 & 4.2 \\
\hline
\end{tabular}

Esivironunental Medis: Soll

Class: Pesticides and PCRs

\begin{tabular}{|c|c|c|c|c|c|c|}
\hline \multirow{2}{*}{$\begin{array}{l}\text { Class: Peseic } \\
\text { Parameter }\end{array}$} & \multirow{2}{*}{$\begin{array}{c}\text { Total } \\
\text { Samples }\end{array}$} & \multicolumn{2}{|c|}{ Total Rejected } & \multirow[b]{2}{*}{ Resoson for Rejection } & \multicolumn{2}{|c|}{ Rejected for Resson } \\
\hline & & Ssumples & Pencent & & Satraptes & Percent \\
\hline \multirow[t]{4}{*}{$4,4^{\circ} \cdot D D D$} & 135 & 7 & 5.1 & $\mathrm{RPD}$ criteria are not met. & T & 0.7 \\
\hline & & & & Surrogate recovery is $<10 \%$. & 3 & 2.2 \\
\hline & & & & Surrogate Recovery: Professional judgement & 3 & 2.2 \\
\hline & & & & was used to flag data. & & \\
\hline \multirow[t]{3}{*}{$4,4^{2}-D D E$} & 135 & 7 & $\overline{5.1}$ & Surrogate recovery is $<10 \%$. & 4 & 2.9 \\
\hline & & & & Surrogate Recovery: Professional judgement & 3 & 2.2 \\
\hline & & & & was used to flag data. & & \\
\hline \multirow[t]{3}{*}{$4,4^{\prime}-\mathrm{DDT}$} & 135 & 7 & 5.1 & RPD criteria are not met. & 1 & 0.7 \\
\hline & & & & Pesticide Cleanup Checks: Professional & 6 & 4.4 \\
\hline & & & & judgement was used to flag data. & & \\
\hline \multirow[t]{3}{*}{ Aldrin } & 135 & 7 & 5.1 & Surrogate recovery is $<10 \%$ & 1 & 0.7 \\
\hline & & & & Pesticide Cleanup Checks: Professional & 6 & 4,4 \\
\hline & & & & judgemant was used to flag data. & & \\
\hline \multirow[t]{3}{*}{ Aroclor-1016 } & 135 & 7 & 5.1 & Surrogate recovery is $<10 \%$ & 4 & 2.9 \\
\hline & & & & Surrogate Recovery: Professional judgement & 3 & 2.2 \\
\hline & & & & was used to flag data. & & \\
\hline \multirow[t]{3}{*}{ Aroclor-1221 } & 135 & 7 & 5.1 & Surrogate recovery is $<10 \%$ & 4 & 2.9 \\
\hline & & & & Surrogate Recovery: Professional judgement & 3 & 2.2 \\
\hline & & & & was used to Dag data. & & \\
\hline \multirow[t]{3}{*}{ Arocior-1232 } & 135 & 7 & 5.1 & Surrogate recovery is $<10 \%$ & 4 & 2.9 \\
\hline & & & & Surrogate Recovery: Professional judgement & 3 & 2.2 \\
\hline & & & & was used to flag data. & & \\
\hline \multirow[t]{3}{*}{ Aroclor-1242 } & 135 & 7 & 5.1 & Surrogate recovery is $<10 \%$. & 4 & 2.9 \\
\hline & & & & Surrogate Recovery: Professional judgement & 3 & 2.2 \\
\hline & & & & was used to flag data. & & \\
\hline \multirow[t]{3}{*}{ Aroclor-1248 } & 135 & 7 & 5.1 & Surrogate recovery is $<10 \%$. & 4 & 2.9 \\
\hline & & & & Surrogare Recovery: Professional judgement & 3 & 2.2 \\
\hline & & & & was used to flag data. & & \\
\hline \multirow[t]{3}{*}{ Aroclor-1254 } & 135 & 6 & 4.4 & Surrogase recovery is $<10 \%$. & 4 & 2.9 \\
\hline & & & & Surrogate Recovery: Professional judgement & 2 & 1.4 \\
\hline & & & & was used to flag data. & & \\
\hline \multirow[t]{3}{*}{ Arocior 1260} & 135 & 7 & 5.1 & Surrogate recovery is $<10 \%$. & 4 & 2.9 \\
\hline & & & & Surrogate Recovery: Professional judgement & 3 & 2.2 \\
\hline & & & & was used to flag data. & & \\
\hline
\end{tabular}




\section{E-7}

04/07/94

Table E.2

Bear Creek Valley OU2 RI FA

Summary of Rejected Analytes

RUST SPOL AREA

\begin{tabular}{|c|c|c|c|c|c|c|}
\hline Dieldrn & 135 & 7 & 5.1 & $\begin{array}{l}\text { RPD criteria are not met. } \\
\text { Pesticide Cleanup Checks: Professional } \\
\text { judgement was used to flag data. }\end{array}$ & $\begin{array}{l}1 \\
6\end{array}$ & $\begin{array}{l}0.7 \\
4.4\end{array}$ \\
\hline Endosulfan I & 135 & 7 & 5.1 & $\begin{array}{l}\text { RPD criteria are not met. } \\
\text { Surrogate recovery is }<10 \% \text {. } \\
\text { Surrogate Recovery: Professional judgement } \\
\text { was used to flag data. }\end{array}$ & $\begin{array}{l}1 \\
3 \\
3\end{array}$ & $\begin{array}{l}0.7 \\
2.2 \\
2.2\end{array}$ \\
\hline Endosulfan II & 135 & 7 & 5.1 & $\begin{array}{l}\text { RPD criteria are not met. } \\
\text { Surrogate recovery is }<10 \% \\
\text { Surrogate Recovery: Professional judgement } \\
\text { was used to flag data. }\end{array}$ & $\begin{array}{l}1 \\
3 \\
3\end{array}$ & $\begin{array}{l}0.7 \\
2.2 \\
2.2\end{array}$ \\
\hline Endosulfan Sulfate & 135 & 7 & 5.1 & $\begin{array}{l}\text { Surrogate recovery is }<10 \% \text {. } \\
\text { Surrogate Recovery: Professional judgement } \\
\text { was used to flag data. }\end{array}$ & $\begin{array}{l}4 \\
3\end{array}$ & $\begin{array}{l}2.9 \\
2.2\end{array}$ \\
\hline Endrin & 135 & 7 & 5.1 & $\begin{array}{l}\text { RPD criteria are not met } \\
\text { Pesticide Cleanup Checks: Professional } \\
\text { judgement was used to flag data. }\end{array}$ & $\begin{array}{l}1 \\
6\end{array}$ & $\begin{array}{l}0.7 \\
4.4\end{array}$ \\
\hline Endrin Aldehyde & 135 & 7 & 5.1 & $\begin{array}{l}\text { Surrogate recovery is }<10 \% \text {. } \\
\text { Surrogate Recovery: Professional judgement } \\
\text { was used to flag data. }\end{array}$ & $\begin{array}{l}4 \\
3\end{array}$ & $\begin{array}{l}2.9 \\
2.2\end{array}$ \\
\hline Endrin Ketone & 135 & 7 & 5.1 & $\begin{array}{l}\text { RPD criteria are not mel. } \\
\text { Surrogate recovery is }<10 \% \text {. } \\
\text { Surrogate Recovery: Professional judgement } \\
\text { was used to flag data. }\end{array}$ & $\begin{array}{l}1 \\
3 \\
3\end{array}$ & $\begin{array}{l}0.7 \\
2.2 \\
2.2\end{array}$ \\
\hline Heptachlor & 135 & 5 & 3.7 & $\begin{array}{l}\text { RPD criteria are not met. } \\
\text { Pesticide Cleanup Checks: Professional } \\
\text { judgement was used to flag data. }\end{array}$ & $\begin{array}{l}1 \\
4\end{array}$ & $\begin{array}{l}0.7 \\
2.9\end{array}$ \\
\hline Heptachlor Epoxide & 135 & 7 & 5.1 & $\begin{array}{l}\text { Surrogate recovery is }<10 \% \\
\text { Surrogate Recovery: Professional judgement } \\
\text { was used to flag data. }\end{array}$ & $\begin{array}{l}4 \\
3\end{array}$ & $\begin{array}{l}2.9 \\
2.2\end{array}$ \\
\hline Toxaphene & 135 & 7 & 5.1 & $\begin{array}{l}\text { Surrogate recovery is }<10 \% \text {. } \\
\text { Surrogate Recovery: Professional judgement } \\
\text { was used to flag data. }\end{array}$ & $\begin{array}{l}4 \\
3\end{array}$ & $\begin{array}{l}2.9 \\
2.2\end{array}$ \\
\hline alpha-BHC & 135 & 7 & 5.1 & $\begin{array}{l}\text { RPD criteria are not met. } \\
\text { \%RSD criteria are not met. } \\
\text { Surrogate recovery is }<10 \% \text {. } \\
\text { Surrogate Recovery: Professional judgement } \\
\text { was used to flag dala. }\end{array}$ & $\begin{array}{l}1 \\
2 \\
3 \\
1\end{array}$ & $\begin{array}{l}0.7 \\
1.4 \\
2.2 \\
0.7\end{array}$ \\
\hline alpha-Chiordane & 135 & 7 & 5.1 & $\begin{array}{l}\text { Surrogate recovery is }<10 \% \text {. } \\
\text { Surrogate Recovery: Professional judgement } \\
\text { was used to flag data. }\end{array}$ & $\begin{array}{l}4 \\
3\end{array}$ & $\begin{array}{l}2.9 \\
2.2\end{array}$ \\
\hline beta-BHC & 135 & 7 & 5.1 & $\begin{array}{l}\text { Surrogate recovery is }<10 \% \text {. } \\
\text { Surrogate Recovery: Professional judgement } \\
\text { was used to flag data. }\end{array}$ & $\begin{array}{l}4 \\
3\end{array}$ & $\begin{array}{l}2.9 \\
2.2\end{array}$ \\
\hline delta-BHC & 135 & 7 & 5.1 & $\begin{array}{l}\text { RPD eriteria are not met. } \\
\text { \%RSD criteria are not met. }\end{array}$ & $\begin{array}{l}7 \\
2\end{array}$ & $\begin{array}{l}0.7 \\
1.4\end{array}$ \\
\hline
\end{tabular}

Report: D004.1

Page 2 
04/07/94

Tale L.2

Bear Creek Valley OU2 RI FA

Summary of Rejected Analytes

RUST SPOTL AREA

\begin{tabular}{|c|c|c|c|c|c|c|}
\hline & & & & $\begin{array}{l}\text { Surrogate recovery is }<10 \% \text {. } \\
\text { Surrogate Recovery: Professional judgement } \\
\text { was used to flag data. }\end{array}$ & $\begin{array}{l}3 \\
1\end{array}$ & $\begin{array}{l}2.2 \\
0.7\end{array}$ \\
\hline gamma-BHC (Lindane) & 135 & 7 & 5.1 & $\begin{array}{l}\text { RPD criteria are not met. } \\
\text { Pesticide Cleanup Checks: Professional } \\
\text { judgement was used to flag data. }\end{array}$ & $\begin{array}{l}1 \\
6\end{array}$ & $\begin{array}{l}0.7 \\
4.4\end{array}$ \\
\hline gamma-Chlordane & 135 & 7 & 5.1 & $\begin{array}{l}\text { Surrogate recovery is }<10 \% \text {. } \\
\text { Surrogate Recovery: Professional judgement } \\
\text { was used to fllag data. }\end{array}$ & $\begin{array}{l}4 \\
3\end{array}$ & $\begin{array}{l}2.9 \\
2.2\end{array}$ \\
\hline p,p-Methoxychior & 135 & 7 & 5.1 & $\begin{array}{l}\text { RPD criteria are not met. } \\
\text { Surrogate recovery is }<10 \% \text {. } \\
\text { Surrogate Recovery: Professional judgement } \\
\text { was used to flag data. }\end{array}$ & $\begin{array}{l}1 \\
3 \\
3\end{array}$ & $\begin{array}{l}0.7 \\
2.2 \\
2.2\end{array}$ \\
\hline
\end{tabular}

Environmental Media: Soll

Class: Semi-volatille Organics

\begin{tabular}{|c|c|c|c|c|c|c|}
\hline \multirow{2}{*}{$\begin{array}{l}\text { Class: Seus: } \\
\text { Parameter }\end{array}$} & \multirow{2}{*}{$\begin{array}{c}\text { Totsal } \\
\text { Samples }\end{array}$} & \multicolumn{2}{|c|}{ Total Rejected } & \multirow[b]{2}{*}{ Reason for Rejection } & \multicolumn{2}{|c|}{ Rejected for Reason } \\
\hline & & Saxpaples & Pereerent & & Samples & Percent \\
\hline 3,3'-Dichlorobenzidine & 136 & 1 & 0.7 & $\begin{array}{l}\text { Extremely low area counts or penfomance } \\
\text { exchibits a major drop off. }\end{array}$ & & 0.7 \\
\hline 4,6-Dinatro-2-methylphen & 136 & 1 & 0.7 & $\begin{array}{l}\text { Extremely low area counts or performance } \\
\text { exhibits a major drop off. }\end{array}$ & & 0.7 \\
\hline 4-Bromophenyl-phenyleth & $\overline{136}$ & $\overline{1}$ & 0.7 & $\begin{array}{l}\text { Extremeiy low ares counes or performance } \\
\text { exhibits a major drop off. }\end{array}$ & & 0.7 \\
\hline Anthracene & 136 & 1 & 0.7 & $\begin{array}{l}\text { Extremely low area counts or performance } \\
\text { exhibiss a major drop off. }\end{array}$ & & 0.7 \\
\hline Benzo(a)anthuracene & 136 & 1 & 0.7 & $\begin{array}{l}\text { Extremely low area counts or performance } \\
\text { exhibits a major drop off. }\end{array}$ & & $\overline{0.7}$ \\
\hline Butylbenzylphthalate & 136 & 1 & 0.7 & $\begin{array}{l}\text { Extremely low area counts or performance } \\
\text { exhibits a major drop off. }\end{array}$ & & 0.7 \\
\hline Carbazole & 136 & 1 & 0.7 & $\begin{array}{l}\text { Extremely low area counts or performance } \\
\text { exhibits a major drop off. }\end{array}$ & & 0.7 \\
\hline Chrysene & 136 & 1 & 0.7 & $\begin{array}{l}\text { Extremely low area counts or performance } \\
\text { exhibits a major drop off. }\end{array}$ & & 0.7 \\
\hline Fluoranthene & 136 & 1 & 0.7 & $\begin{array}{l}\text { Exaremely low area counts or performance } \\
\text { exhibits a major drop off. }\end{array}$ & & 0.7 \\
\hline Hexachlorobenzerse & 136 & 1 & 0.7 & $\begin{array}{l}\text { Extremely low area counts or performance } \\
\text { exhibits a major drop off. }\end{array}$ & & 0.7 \\
\hline N-Nitrosodsphenylamune & 136 & 1 & 0.7 & $\begin{array}{l}\text { Extremely low area counts or performance } \\
\text { exhibits a major drop off. }\end{array}$ & & 0.7 \\
\hline Pentachlorophenol & 136 & 1 & 0.7 & $\begin{array}{l}\text { Extremely low area counts or performance } \\
\text { exhibits a major drop off. }\end{array}$ & & 0.7 \\
\hline Pyrene & 136 & 1 & 0.7 & $\begin{array}{l}\text { Extremely low area counis or performance } \\
\text { shibits a najor drop off. }\end{array}$ & & $\overline{0.7}$ \\
\hline
\end{tabular}


$04 / 07 / 94$

Table $\mathrm{E}_{.2}$

Bear Creek Valley OU2 RII FA

Summary of Rejected Analytes

RUST SPOIL AREA

Environmental Media: Soil

Class: Volatile Organics

\begin{tabular}{|c|c|c|c|c|c|c|}
\hline \multirow{2}{*}{ Parameter } & \multirow{2}{*}{$\begin{array}{l}\text { Total } \\
\text { Samples }\end{array}$} & \multicolumn{2}{|c|}{ Total Rejected } & \multirow[b]{2}{*}{ Reason for Rejection } & \multicolumn{2}{|c|}{ Rejected for Reason } \\
\hline & & Samples & Percent & & Samples & Percent \\
\hline \multirow[t]{2}{*}{ 1,1,2,2-Tetrachloroethane } & 137 & 1 & 0.7 & Extremely low area counts or performance & 1 & 0.7 \\
\hline & & & & exhibits a major drop off. & & \\
\hline \multirow[t]{2}{*}{ 2-Hexanone } & 137 & 1 & 0.7 & Extremely low area counts or performance & 1 & 0.7 \\
\hline & & & & exhibits a major drop off. & & \\
\hline \multirow[t]{2}{*}{ 4-Methyl-2-pentanone } & 137 & 1 & 0.7 & Extremely low area counts or performance & 1 & 0.7 \\
\hline & & & & exhibits a major drop off. & & \\
\hline \multirow[t]{3}{*}{ Chlorobenzene } & 137 & 2 & 1.4 & Initial calibration RRF $<0.05$. & 1 & 0.7 \\
\hline & & & & Extremely low area counis or performance & 1 & 0.7 \\
\hline & & & & exhibits a major drop off. & & \\
\hline \multirow[t]{2}{*}{ Ethylbenzene } & 137 & 1 & 0.7 & Extremely low area counts or performance & 1 & 0.7 \\
\hline & & & & exhibits a major drop off. & & \\
\hline \multirow[t]{2}{*}{ Styrene } & 137 & 1 & 0.7 & Extremely low area counts or performance & $\overline{1}$ & 0.7 \\
\hline & & & & exhibits a major drop off. & & \\
\hline \multirow[t]{2}{*}{ Xylene, Total } & 137 & 1 & 0.7 & Extremely low area counts or performance & $\overline{1}$ & 0.7 \\
\hline & & & & exchibits a major drop off. & & \\
\hline
\end{tabular}

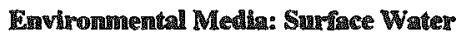

Class: Imor

\begin{tabular}{|c|c|c|c|c|c|c|}
\hline \multirow[b]{2}{*}{ Parameter } & \multirow{2}{*}{$\begin{array}{c}\text { Total } \\
\text { Sagremes }\end{array}$} & \multicolumn{2}{|c|}{ Total Rejected } & \multicolumn{3}{|c|}{ Rejected for Resson } \\
\hline & & Sarringles & Percents & Reagng Rejection & Samaples & Percent \\
\hline Beryllium & 10 & 3 & 30.0 & Blank has a negative value $5 \mathrm{X}$ s the IDL. & 3 & 30.0 \\
\hline
\end{tabular}

Emvinonmental Media: Surfece Water

Chass: Seruip-volatule Organics

\begin{tabular}{|c|c|c|c|c|c|c|}
\hline Parameter & Total & \multicolumn{2}{|c|}{ Totnl Rejected } & \multicolumn{3}{|c|}{ Rejected for Resson } \\
\hline Acenaphthene & 10 & 1 & 10.0 & $\begin{array}{c}\text { MS/MSD or RS/RSD: Professional judgement } \\
\text { was used to llag data. }\end{array}$ & 1 & 10.0 \\
\hline Pyrene & 10 & 1 & 10.0 & $\begin{array}{c}\text { MS/MSD or RS/RSD: Profersional judgement } \\
\text { was used to flag data. }\end{array}$ & 1 & 10.0 \\
\hline
\end{tabular}

Finviromenental Media: Ground

Class: Inorgyantes

\begin{tabular}{|c|c|c|c|c|c|c|}
\hline \multirow[b]{2}{*}{ Paramieter } & \multirow{2}{*}{$\begin{array}{c}\text { Total } \\
\text { Samples }\end{array}$} & \multicolumn{2}{|c|}{ Tos Rejected } & \multirow[b]{2}{*}{ Reasoli Ior Rejection } & \multicolumn{2}{|c|}{ Mejected Por Resson } \\
\hline & & Samples & Pereent & & Samples & Percente \\
\hline Arsenic & 6 & 1 & 16.6 & Recovery less than $30 \%$ & 1 & 16.6 \\
\hline
\end{tabular}


Table E.2

Bear Creek Valley OU2 RI FA

Summary of Rejected Analytes

STORAGE YARD 200

Environmental Media: Soll

Class: Inor留壁ics

\begin{tabular}{|c|c|c|c|c|c|c|}
\hline \multirow{2}{*}{$\begin{array}{l}\text { Class: Horganes } \\
\text { Parameter }\end{array}$} & \multirow{2}{*}{$\begin{array}{c}\text { Total } \\
\text { Saraples }\end{array}$} & \multicolumn{2}{|c|}{ Totsl Rejected } & \multirow[b]{2}{*}{ Reason for Rejection } & \multicolumn{2}{|c|}{ Rejected for Reason } \\
\hline & & Samaples & Percent & & Sagantples & Percent \\
\hline Antimony & 284 & 136 & 47.8 & Recovery less than $30 \%$ & 136 & 47.8 \\
\hline Arsenic & 284 & 2 & 0.7 & Recovery less than $30 \%$. & 2 & 0.7 \\
\hline Cadmium & 284 & 22 & 7.7 & $\begin{array}{l}\text { ICP and Fumace Requirements: Professional } \\
\text { judgement was used to flag dats. }\end{array}$ & 22 & 7.7 \\
\hline Mercury & 284 & 1 & 0.3 & Extraction holding times are grossly exceeded. & 1 & 0.3 \\
\hline Selenium & 284 & 115 & 40.4 & $\begin{array}{l}\text { Post digestion spike recovery is outside } \\
\text { allowable limits. }\end{array}$ & 2 & 0.7 \\
\hline & & & & Recovery less than $30 \%$. & 112 & 39.4 \\
\hline
\end{tabular}

Envimonanental Media: Soil

Chass: Pesticides and PCBs

\begin{tabular}{|lcccccc|} 
Parameter & $\begin{array}{c}\text { Total } \\
\text { Swmples }\end{array}$ & $\begin{array}{c}\text { Total Rejected } \\
\text { Samples }\end{array}$ & Percent & Reasen for Rejection & Rejected for Reason \\
\hline Endrin & 135 & 1 & 0.7 & $\begin{array}{c}\text { Pesticide Cleanup Checks: Professional } \\
\text { judgement was used to flag data. }\end{array}$ & 1 & 0.7 \\
\hline
\end{tabular}

Envirosumental Media: Sol

Clmss: Semi-volatile Organics

\begin{tabular}{|c|c|c|c|c|c|c|}
\hline \multirow{2}{*}{$\begin{array}{l}\text { Clams: Seme } \\
\text { Parranueter }\end{array}$} & \multirow{2}{*}{$\begin{array}{l}\text { Total } \\
\text { Samples }\end{array}$} & \multicolumn{2}{|c|}{ Total Rejected } & \multirow[b]{2}{*}{ Reason for Rejection } & \multicolumn{2}{|c|}{ Rejected for Reasozi } \\
\hline & & Sarapules & Percent & & Samples & Percent \\
\hline 2,4,5-Trichlorophenol & 136 & 1 & 0.7 & Surrogate recovery is $<10 \%$ & $\overline{1}$ & $\overline{0.7}$ \\
\hline 2,4,6-Trichlorophenol & 136 & 1 & 0.7 & Surrogate recovery is $<10 \%$ & 1 & 0.7 \\
\hline 2.4-Dichlorophenol & 136 & 1 & 0.7 & Surrogate recovery is $<10 \%$. & 1 & $\overline{0.7}$ \\
\hline 2.4-Dimethylphenol & 136 & T & $\overline{0.7}$ & Surrogate recovery is $\angle 10 \%$. & 1 & $\overline{0.7}$ \\
\hline 2,4-Dinitrophenol & 136 & 1 & 0.7 & Surrogate recovery is $<10 \%$. & 1 & 0.7 \\
\hline 2-Chlorophenol & 136 & 1 & 0.7 & Surrogate recovery is $<10 \%$. & l & 0.7 \\
\hline 2-Methylphenol & 136 & 1 & 0.7 & Surrogate recovery is $<10 \%$ & 1 & 0.7 \\
\hline 2-Nitrophenol & 136 & 1 & 0.7 & Surrogate recovery is $<10 \%$ & 1 & 0.7 \\
\hline 4,6-Dinitro-2-methylphen & 136 & 1 & 0.7 & Surrogate recovery is $<10 \%$ & 1 & 0.7 \\
\hline 4-Chloro-3-methylphenol & 136 & 1 & 0.7 & Surrogate recovery is $<10 \%$. & 1 & 0.7 \\
\hline 4-Methylphenol & 136 & 1 & 0.7 & Surrogate recovery is $<10 \%$ & 1 & 0.7 \\
\hline 4-Nitrophenol & 136 & 1 & 0.7 & Surrogate recovery is $<10 \%$. & 1 & 0.7 \\
\hline Pentachlorophenol & 136 & 1 & 0.7 & Surrogate recovery is $<10 \%$. & 1 & 0.7 \\
\hline Phenol & 136 & 1 & $\overline{0.7}$ & Surrogate recovery is $<10 \%$ & 1 & 0.7 \\
\hline
\end{tabular}

Environmental Media: Surfiace Water

Class: Inorganics

\begin{tabular}{|c|c|c|c|c|c|c|}
\hline \multirow{2}{*}{ Ciass: Hara } & \multirow{2}{*}{$\begin{array}{c}\text { Totsil } \\
\text { Samaples }\end{array}$} & \multicolumn{2}{|c|}{ Total Rejected } & \multirow[b]{2}{*}{ Reason for Rejection } & \multicolumn{2}{|c|}{ Rejected for Reason } \\
\hline & & Samples & Percen & & Samples & Percent \\
\hline Bervllium & 10 & 1 & 10.0 & Blank has a megative value $5 \times$ 's the IDL & 1 & 10.0 \\
\hline Selenium & 10 & 1 & 10.0 & Recovery less than $30 \%$. & 1 & 10.0 \\
\hline
\end{tabular}


Table E.2

Bear Creek Valley OU2 RI FA

Summary of Rejected Amalytes

STORAGE YARD 200

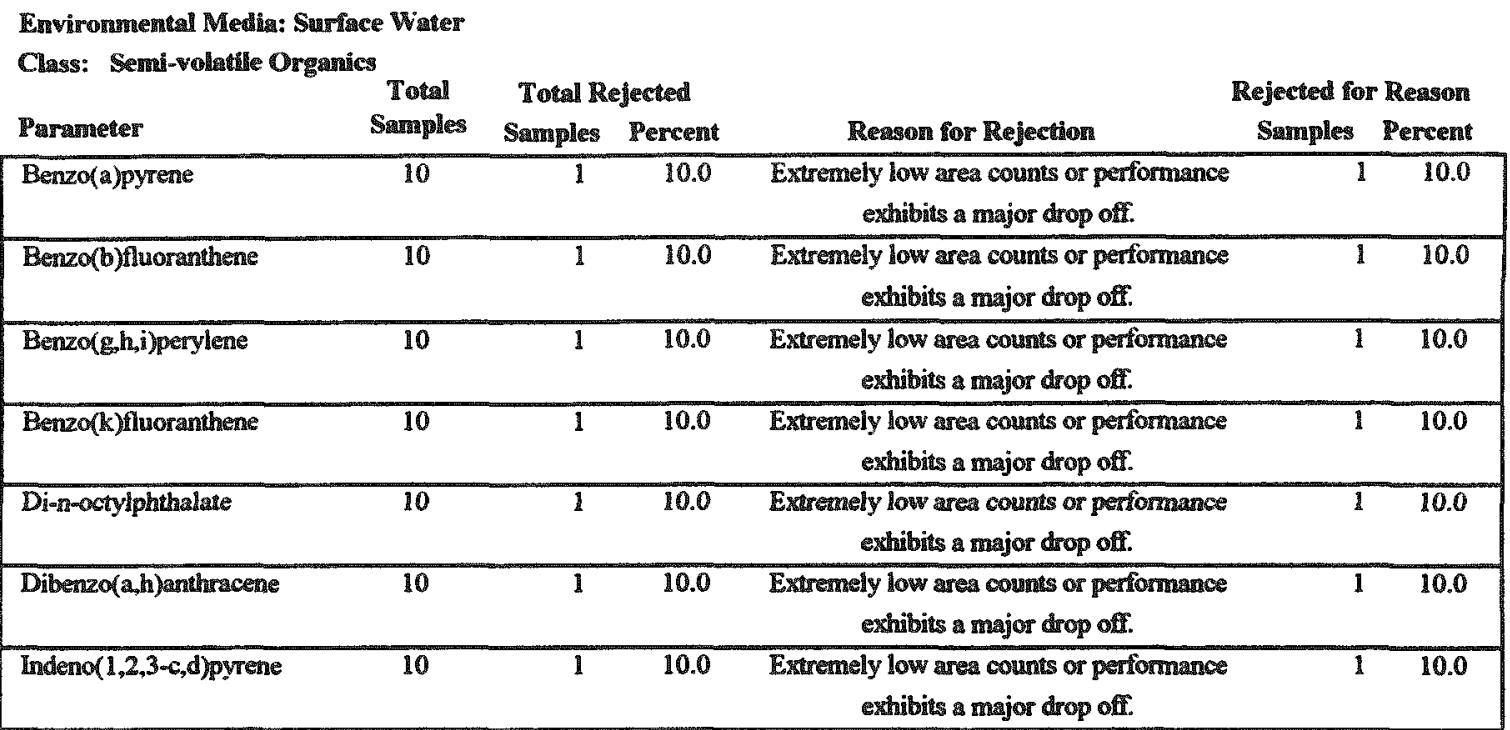

Evvironmental Media: Grovendwater

Class: Imorganics

Parameter

Tด1

Total Refected

Samples Sarmiles percent

\begin{tabular}{|lrrr}
\hline Arsenic & 6 & 4 & 66.6 \\
\hline Selenium & 6 & 4 & 66.6
\end{tabular}

Reascor for Rejection Recovery less than $30 \%$. Recovery less than $30 \%$.
Rejected for Reason

Samples Percent

\begin{tabular}{ll}
6.6 & Recovery less than $30 \%$ \\
\hline 6.6 & Recovery less than $30 \%$
\end{tabular}

$4+66.6$


Table E.3. Bear Creek Operable Unit 2 - Data Quality Assessment Laboratory Duplicate Evaluation - Average Relative Percent Difference (RPD)

\begin{tabular}{|c|c|c|c|c|c|c|c|c|}
\hline \multirow[b]{2}{*}{ Analysis } & \multicolumn{4}{|c|}{ Soil } & \multicolumn{4}{|c|}{ Water } \\
\hline & $\begin{array}{c}\text { Average } \\
\text { RPD }\end{array}$ & $\begin{array}{l}\text { Minimum } \\
\text { RPD }\end{array}$ & $\begin{array}{l}\text { Maximum } \\
\text { RPD }\end{array}$ & $\mathbb{N}$ & $\begin{array}{c}\text { Average } \\
\text { RPD }\end{array}$ & $\begin{array}{l}\text { Minimum } \\
\text { RPD }\end{array}$ & $\begin{array}{c}\text { Maximum } \\
\text { RPD }\end{array}$ & $\mathbb{N}$ \\
\hline Mercury (AV) & & & & & 1.9 & 1.9 & 1.9 & 1 \\
\hline Mercury (CV) & 8.5 & 0.2 & 17.2 & 13 & & & & \\
\hline Arsenic (F) & 26.9 & 0.6 & 80.2 & 14 & & & & \\
\hline Lead (F) & 30.3 & 3.8 & 151.8 & 8 & 24.6 & 20.5 & 29.1 & 3 \\
\hline Selenium $(F)$ & 181.5 & 144.4 & 200.0 & 3 & & & & 0 \\
\hline Aluminum (P) & 13.8 & 0.7 & 42.2 & 24 & 14.4 & 1.1 & 21.8 & 4 \\
\hline Barium $(\mathbb{P})$ & 48.9 & 8.2 & 80.2 & 3 & 0.4 & 0.4 & 0.4 & 1 \\
\hline Calcium (P) & 59.8 & 7.2 & 170.0 & 14 & 0.9 & 0.5 & 1.2 & 2 \\
\hline Chromium (P) & 16.4 & 1.6 & 55.9 & 23 & 4.9 & 0.6 & 9.2 & 2 \\
\hline Cobalt (P) & 95.2 & 68.3 & 122.1 & 2 & & & & \\
\hline Copper $(\mathbb{P})$ & 22.9 & 1.7 & 106.4 & 10 & 2.4 & 2.4 & 2.4 & 1 \\
\hline Iron $(P)$ & 13.7 & 0.1 & 55.2 & 24 & 1.2 & 0.5 & 2.6 & 4 \\
\hline Lead $(P)$ & 12.7 & 0.3 & 46.1 & 16 & & & & \\
\hline Magnesium (P) & 24.6 & 0.1 & 119.1 & 24 & 1.2 & 0.5 & 2.9 & 4 \\
\hline Manganese (P) & 35.0 & 0.8 & 151.6 & 24 & 1.2 & 0.4 & 2.1 & 4 \\
\hline Nickel (P) & 51.0 & 14.1 & 134.0 & 4 & & & & \\
\hline Vanadium (P) & 6.3 & 0.3 & 16.5 & 8 & & & & \\
\hline Zinc $(\mathbb{P})$ & 13.6 & 0.9 & 55.8 & 24 & 1.2 & 0.8 & 1.5 & 2 \\
\hline $4,4^{\prime}-\mathrm{DDT}$ & 18.1 & -31.0 & 200.0 & 28 & 14.8 & -13.0 & 60.0 & 4 \\
\hline Aldrin & 13.8 & -25.0 & 133.0 & 28 & 16.0 & -16.0 & 75.0 & 4 \\
\hline Dieldrin & 12.5 & -31.0 & 128.0 & 28 & 5.5 & -13.0 & 24.0 & 4 \\
\hline Endrin & 23.3 & -62.0 & 200.0 & 28 & 3.8 & -13.0 & 23.0 & 4 \\
\hline Gamma-BHC (lindane) & 11.5 & -24.0 & 128.0 & 28 & 4.3 & -16.0 & 20.0 & 4 \\
\hline Heptachlor & 10.5 & -20.0 & 117.0 & 28 & 6.0 & -14.0 & 35.0 & 4 \\
\hline
\end{tabular}


Table E.3 (continued)

\begin{tabular}{|c|c|c|c|c|c|c|c|c|}
\hline \multirow[b]{2}{*}{ Analysis } & \multicolumn{4}{|c|}{ Soil } & \multicolumn{4}{|c|}{ Water } \\
\hline & $\begin{array}{l}\text { Average } \\
\text { RPD }\end{array}$ & $\begin{array}{l}\text { Minimum } \\
\text { RPD }\end{array}$ & $\begin{array}{l}\text { Maximum } \\
\text { RPD }\end{array}$ & $\mathbf{N}$ & $\begin{array}{l}\text { Average } \\
\text { RPD }\end{array}$ & $\begin{array}{l}\text { Minimum } \\
\text { RPD }\end{array}$ & $\begin{array}{l}\text { Maximum } \\
\text { RPD }\end{array}$ & $\mathbf{N}$ \\
\hline 1,2,4-Trichlorobenzene & 18.2 & 0.0 & 75.0 & 19 & 4.0 & 1.0 & 9.0 & 4 \\
\hline 1,4-Dichlorobenzene & 17.8 & 0.0 & 60.0 & 19 & 6.5 & 2.0 & 12.0 & 4 \\
\hline 2,4-Dinitrotoluene & 22.2 & 3.0 & 128.0 & 19 & 5.0 & 0.0 & 11.0 & 4 \\
\hline 2-Chlorophenol & 20.3 & 0.0 & 97.0 & 19 & 8.8 & 5.0 & 16.0 & 4 \\
\hline 4-Chloro-3-methylphenol & 23.1 & 0.0 & 126.0 & 19 & 22.8 & 1.0 & 81.0 & 4 \\
\hline 4-Nitrophenol & 25.0 & 0.0 & 155.0 & 19 & 7.3 & 2.0 & 12.0 & 4 \\
\hline Acenaph thene & 18.6 & 2.0 & 80.0 & 19 & 38.5 & 0.0 & 146.0 & 4 \\
\hline n-nitroso-di-n-prop. (1) & 24.5 & 1.0 & 131.0 & 19 & 3.5 & 0.0 & 10.0 & 4 \\
\hline Pentachlorophenol & 26.6 & 0.0 & 93.0 & 19 & 4.5 & 1.0 & 12.0 & 4 \\
\hline Phenol & 22.8 & 0.0 & 113.0 & 19 & 20.0 & 0.0 & 67.0 & 4 \\
\hline Pyrene & 24.7 & 1.0 & 84.0 & 19 & 51.0 & 1.0 & 188.0 & 4 \\
\hline 1,1-Dichloroethene & 6.2 & -9.0 & 20.0 & 18 & 3.8 & 1.0 & 8.0 & 4 \\
\hline Benzene & 4.7 & -3.0 & 13.0 & 18 & 2.5 & 0.0 & 7.0 & 4 \\
\hline Chlorobenzene & 5.7 & -1.0 & 15.0 & 18 & 2.5 & 1.0 & 4.0 & 4 \\
\hline Toluene & 6.1 & -3.0 & 20.0 & 18 & 1.8 & 1.0 & 3.0 & 4 \\
\hline Trichloroethene & 5.2 & -4.0 & 15.0 & 18 & 2.0 & 0.0 & 6.0 & 4 \\
\hline
\end{tabular}


Table E.4. Bear Creek Operable Unit 2 - Data Quality Assessment Laboratory Control Sample Evaluation - Average Percent Recovery (\%Rec)

\begin{tabular}{|c|c|c|c|c|c|c|c|c|}
\hline \multirow[b]{2}{*}{ Analysis } & \multicolumn{4}{|c|}{ Soil } & \multicolumn{4}{|c|}{ Water } \\
\hline & $\begin{array}{c}\text { Average } \\
\% \text { Rec }\end{array}$ & $\begin{array}{c}\text { Minimum } \\
\text { \% Rec }\end{array}$ & $\begin{array}{c}\text { Maximum } \\
\text { \%Rec }\end{array}$ & $\mathbb{N}$ & $\begin{array}{c}\text { Average } \\
\text { \%Rec }\end{array}$ & $\begin{array}{c}\text { Minimum } \\
\text { \%Rec }\end{array}$ & $\begin{array}{c}\text { Maximum } \\
\% \operatorname{Rec}\end{array}$ & $\mathbf{N}$ \\
\hline Aluminum & 91.4 & 80.8 & 123.6 & 24 & 97.2 & 90.9 & 108.7 & 17 \\
\hline Antimony & 114.0 & 78.8 & 145.7 & 24 & 96.7 & 21.9 & 108.6 & 17 \\
\hline Arsenic & 104.0 & 84.2 & 119.8 & 24 & 99.9 & 83.8 & 109.1 & 17 \\
\hline Barium & 89.2 & 81.1 & 94.7 & 24 & 94.0 & 89.5 & 98.6 & 17 \\
\hline Beryllium & 88.8 & 79.5 & 95.9 & 24 & 91.2 & 86.8 & 99.0 & 17 \\
\hline Cadmium & 93.0 & 83.5 & 101.9 & 24 & 93.4 & 88.8 & 102.5 & 17 \\
\hline Calcium & 92.6 & 82.0 & 104.8 & 24 & 95.0 & 90.5 & 101.6 & 17 \\
\hline Chromium & 81.1 & 73.1 & 105.4 & 24 & 92.5 & 81.8 & 106.3 & 17 \\
\hline Cobalt & 90.0 & 80.6 & 98.3 & 24 & 92.2 & 87.1 & 98.8 & 17 \\
\hline Copper & 89.1 & 79.1 & 102.3 & 24 & 94.0 & 88.8 & 98.3 & 17 \\
\hline Cyanide & 83.8 & 65.8 & 94.0 & 14 & & & & \\
\hline Iron & 75.7 & 66.3 & 102.0 & 24 & 92.3 & 88.3 & 102.8 & 17 \\
\hline Lead & 90.0 & 76.9 & 137.1 & 24 & 102.6 & 89.7 & 115.2 & 17 \\
\hline Magnesium & 82.8 & 71.8 & 108.9 & 24 & 95.2 & 90.2 & 103.0 & 17 \\
\hline Manganese & 88.0 & 78.3 & 102.5 & 24 & 94.8 & 89.3 & 102.6 & 17 \\
\hline Mercury & 92.5 & 73.7 & 131.4 & 27 & & & & \\
\hline Nickel & 90.2 & 80.8 & 99.5 & 24 & 92.3 & 87.3 & 101.4 & 17 \\
\hline Potassium & 91.3 & 84.3 & 101.2 & 24 & 93.9 & 88.0 & 99.2 & 17 \\
\hline Selenium & 95.3 & 83.7 & 115.1 & 24 & 106.4 & 96.0 & 116.8 & 16 \\
\hline Silver & 92.0 & 84.9 & 101.5 & 24 & 89.7 & 83.0 & 98.4 & 17 \\
\hline Sodium & 97.6 & 84.9 & 121.1 & 24 & 94.6 & 88.8 & 99.8 & 17 \\
\hline Thallium & 98.2 & 66.0 & 121.0 & 24 & 98.7 & 85.4 & 109.5 & 17 \\
\hline Vanadium & 87.8 & 78.4 & 103.1 & 24 & 94.6 & 90.1 & 100.1 & 17 \\
\hline Zinc & 85.6 & 77.7 & 94.2 & 24 & 95.6 & 85.6 & 108.2 & 17 \\
\hline
\end{tabular}


Table E.5. Bear Creek Operable Unit 2 - Data Quality Assessment Matrix Spike/Matrix Spike Duplicate Sample Evaluation Average Percent Recovery (\%Rec)

\begin{tabular}{|c|c|c|c|c|c|c|c|c|}
\hline \multirow[b]{2}{*}{ Analysis } & \multicolumn{4}{|c|}{ Soil } & \multicolumn{4}{|c|}{ Water } \\
\hline & $\begin{array}{c}\text { Average } \\
\text { \%Rec }\end{array}$ & $\begin{array}{c}\text { Minimum } \\
\% \text { Rec }\end{array}$ & $\begin{array}{c}\text { Maximum } \\
\text { \%Rec }\end{array}$ & $\mathrm{N}$ & $\begin{array}{c}\text { Average } \\
\text { \%Rec }\end{array}$ & $\begin{array}{c}\text { Minimum } \\
\% \operatorname{Rec}\end{array}$ & $\begin{array}{c}\text { Maximum } \\
\text { \%Rec }\end{array}$ & $\mathbb{N}$ \\
\hline Mercury (AV) & & & & & 85.2 & 68.4 & 96.6 & 3 \\
\hline Cyanide (CA) & 86.0 & 0.0 & 99.7 & 14 & 90.1 & 81.1 & 97.0 & 4 \\
\hline Mercury (CV) & 106.2 & 68.2 & 261.2 & 18 & & & & \\
\hline Antimony (F) & 21.6 & 11.5 & 34.1 & 24 & 78.4 & 50.6 & 102.3 & 4 \\
\hline Arsenic (F) & 80.6 & -117.4 & 632.6 & 21 & 52.3 & 5.2 & 92.7 & 4 \\
\hline Lead $(F)$ & 677.4 & 677.4 & 677.4 & 1 & 92.6 & 74.2 & 108.2 & 3 \\
\hline Selenium $(\mathrm{F})$ & 8.4 & -176.0 & 127.0 & 24 & 42.4 & 0.0 & 118.9 & 4 \\
\hline Thallium (F) & 99.3 & 32.6 & 115.2 & 24 & 78.1 & 67.5 & 98.6 & 4 \\
\hline Aluminum (P) & & & & & 210.8 & 165.6 & 256.0 & 2 \\
\hline Barium $(P)$ & 92.1 & 51.6 & 121.2 & 24 & 93.7 & 91.6 & 95.8 & 4 \\
\hline Beryllium (P) & 91.0 & 77.9 & 102.4 & 24 & 95.4 & 92.2 & 99.7 & 4 \\
\hline Cadmium (P) & 81.9 & 37.6 & 93.0 & 24 & 92.5 & 87.0 & 98.9 & 4 \\
\hline Chromium (P) & 100.9 & 38.3 & 246.8 & 24 & 94.9 & 92.2 & 97.2 & 4 \\
\hline Cobalt (P) & 88.4 & -37.3 & 171.8 & 24 & 91.9 & 90.5 & 94.1 & 4 \\
\hline Copper (P) & 82.6 & -181.9 & 116.1 & 24 & 95.0 & 91.3 & 99.5 & 4 \\
\hline Iron $(P)$ & & & & & 103.2 & 103.2 & 103.2 & 1 \\
\hline Lead $(\mathbf{P})$ & 95.9 & 69.3 & 180.8 & 16 & & & & \\
\hline Manganese (P) & 126.5 & 97.9 & 155.1 & 2 & 92.5 & 90.8 & 94.6 & 3 \\
\hline Nickel (P) & 82.8 & -88.8 & 103.3 & 24 & 92.9 & 90.8 & 97.7 & 4 \\
\hline Silver (P) & 85.4 & 51.9 & 108.6 & 24 & 93.8 & 89.4 & 98.3 & 4 \\
\hline Vanadium (P) & 91.7 & 56.3 & 155.4 & 24 & 93.4 & 91.2 & 96.5 & 4 \\
\hline Zinc $(\mathbb{P})$ & 94.1 & 38.5 & 223.5 & 23 & 96.5 & 91.2 & 108.3 & 4 \\
\hline
\end{tabular}


Table E.5 (continued)

\begin{tabular}{|c|c|c|c|c|c|c|c|c|}
\hline \multirow[b]{2}{*}{ Analysis } & \multicolumn{4}{|c|}{ Soil } & \multicolumn{4}{|c|}{ Water } \\
\hline & $\begin{array}{l}\text { Average } \\
\text { \% Rec }\end{array}$ & $\begin{array}{c}\text { Minimum } \\
\text { \%Rec }\end{array}$ & $\begin{array}{c}\text { Maximum } \\
\text { \% Rec }\end{array}$ & $\mathbb{N}$ & $\begin{array}{l}\text { Average } \\
\% \text { Rec }\end{array}$ & $\begin{array}{c}\text { Minimum } \\
\text { \%Rec }\end{array}$ & $\begin{array}{c}\text { Maximum } \\
\text { \% Rec }\end{array}$ & $\mathbf{N}$ \\
\hline Cyanide (CA) & 101.8 & 101.8 & 101.8 & 1 & & & & \\
\hline Aluminum $(\mathbb{P})$ & & & & & 95.7 & 95.0 & 96.3 & 2 \\
\hline Barium (P) & 96.0 & 92.9 & 99.5 & 3 & & & & \\
\hline Chromium (P) & 103.5 & 97.8 & 109.7 & 7 & & & & \\
\hline Cobalt (P) & 102.2 & 87.8 & 116.6 & 2 & & & & \\
\hline Copper (P) & 94.6 & 90.4 & 98.7 & 2 & & & & \\
\hline Lead (P) & 96.9 & 94.2 & 99.5 & 2 & & & & \\
\hline Manganese (P) & 90.2 & 90.2 & 90.2 & $\mathbb{1}$ & & & & \\
\hline Nickel (P) & 100.4 & 87.9 & 107.1 & 3 & & & & \\
\hline Vanadium $(P)$ & 110.7 & 104.2 & 120.2 & 3 & & & & \\
\hline Zinc (P) & 99.7 & 83.8 & 112.0 & 9 & & & & \\
\hline $4,4^{\prime}-\mathrm{DDT}$ & 42.5 & 0.0 & 112.0 & 28 & 74.8 & 46.0 & 86.0 & 4 \\
\hline Aldrin & 48.7 & 0.0 & 198.0 & 28 & 74.8 & 50.0 & 92.0 & 4 \\
\hline Dieldrin & 57.3 & 0.0 & 242.0 & 28 & 88.3 & 86.0 & 95.0 & 4 \\
\hline Endrin & 63.7 & 0.0 & 383.0 & 28 & 90.0 & 84.0 & 100.0 & 4 \\
\hline Gamma-BHC (lindane) & 39.1 & 0.0 & 97.0 & 28 & 78.5 & 69.0 & 82.0 & 4 \\
\hline Heptachlor & 41.8 & 0.0 & 122.0 & 28 & 80.5 & 74.0 & 92.0 & 4 \\
\hline $4,4^{\prime}-\mathrm{DDT}$ & 37.3 & 0.0 & 91.0 & 28 & 90.5 & 86.0 & 95.0 & 4 \\
\hline Aldrin & 44.3 & 0.0 & 139.0 & 28 & 92.8 & 71.0 & 110.0 & 4 \\
\hline Dieldrin & 59.2 & 0.0 & 251.0 & 28 & 97.3 & 81.0 & 110.0 & 4 \\
\hline Endrin & 69.0 & 0.0 & 431.0 & 28 & 100.3 & 86.0 & 114.0 & 4 \\
\hline Gamma-BHC (lindane) & 37.6 & 0.0 & 86.0 & 28 & 88.3 & 67.0 & 100.0 & 4 \\
\hline Heptachlor & 39.6 & 0.0 & 100.0 & 28 & 91.5 & 72.0 & 110.0 & 4 \\
\hline
\end{tabular}


Table E.5 (continued)

\begin{tabular}{|c|c|c|c|c|c|c|c|c|}
\hline \multirow[b]{2}{*}{ Analysis } & \multicolumn{4}{|c|}{ Soil } & \multicolumn{4}{|c|}{ Water } \\
\hline & $\begin{array}{l}\text { Average } \\
\text { \%Rec }\end{array}$ & $\begin{array}{l}\text { Minimum } \\
\text { \%Rec }\end{array}$ & $\begin{array}{l}\text { Maximum } \\
\text { \%Rec }\end{array}$ & $\mathrm{N}$ & $\begin{array}{l}\text { Average } \\
\% \text { Rec }\end{array}$ & $\underset{\% \operatorname{Rec}}{\text { Minimum }}$ & $\begin{array}{c}\text { Maximum } \\
\text { \%Rec }\end{array}$ & $\mathrm{N}$ \\
\hline 1,1-Dichloroethene & 110.1 & 52.0 & 197.0 & 18 & 103.0 & 89.0 & 114.0 & 4 \\
\hline Benzene & 98.2 & 74.0 & 143.0 & 18 & 97.5 & 89.0 & 105.0 & 4 \\
\hline Chlorobenzene & 99.9 & 84.0 & 141.0 & 18 & 95.0 & 89.0 & 98.0 & 4 \\
\hline Toluene & 101.9 & 81.0 & 160.0 & 18 & 97.0 & 90.0 & 101.0 & 4 \\
\hline Trichloroethene & 98.4 & 78.0 & 122.0 & 18 & 94.3 & 87.0 & 98.0 & 4 \\
\hline 1, 1-Dichloroethene & 111.0 & 49.0 & 214.0 & 18 & 101.0 & 93.0 & 112.0 & 4 \\
\hline Benzene & 99.2 & 66.0 & 147.0 & 18 & 99.8 & 95.0 & 105.0 & 4 \\
\hline Chlorobenzene & 100.3 & 81.0 & 134.0 & 18 & 97.5 & 93.0 & 100.0 & 4 \\
\hline Toluene & 103.9 & 76.0 & 155.0 & 18 & 98.8 & 92.0 & 102.0 & 4 \\
\hline Trichloroethene & 98.4 & 72.0 & 119.0 & 18 & 96.0 & 92.0 & 98.0 & 4 \\
\hline
\end{tabular}




\section{E-18}

Table E.6

Summary of Field Duplicate Relative Percent Differences (RPD)

Bear Creek Valley OU2 RI FA

\begin{tabular}{|c|c|c|c|c|c|c|}
\hline Parameter & $\begin{array}{c}\text { Method of } \\
\text { RPD } \\
\text { Calculation }\end{array}$ & Units & $\begin{array}{c}\text { Average } \\
\text { RPD }\end{array}$ & $\begin{array}{l}\text { RPD } \\
\text { Lower }\end{array}$ & $\begin{array}{l}\text { Limits } \\
\text { Upper }\end{array}$ & $\begin{array}{l}\text { Number } \\
\text { Results } \\
\text { Within } \\
\text { Limits }\end{array}$ \\
\hline 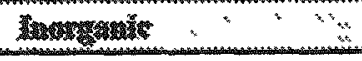 & $\therefore$ & \multicolumn{2}{|c|}{ (1) } & (x) & is & \\
\hline Alsmmum & Rel \% Difference & $\%$ & 19.05 & 6.95 & 62.51 & $12 / 20$ \\
\hline Antimony & Abs Difference & $\mathrm{MG} / \mathrm{KG}$ & 0.09 & 0.03 & 0.25 & $5 / 6$ \\
\hline \multirow[t]{2}{*}{ Arsenc } & Abs. Difference & $\mathrm{MG} / \mathrm{KG}$ & 2.30 & 0.07 & 060 & $0 / 2$ \\
\hline & Rel \% Difference & $\%$ & 55.48 & 13.54 & 121.87 & $13 / 20$ \\
\hline \multirow[t]{2}{*}{ Barum } & Abs Dufference & $\mathrm{MG} / \mathrm{KG}$ & 24.97 & 8.08 & 72.75 & $8 / 13$ \\
\hline & Rel \% Difference & $\%$ & 10.19 & 2.45 & 2201 & $13 / 20$ \\
\hline Bervllum & Abs Difference & $\mathrm{MG} / \mathrm{KG}$ & 0.24 & 006 & 0.52 & $17 / 20$ \\
\hline Cadmum & Abs Difference & $\mathrm{MG} / \mathrm{KG}$ & 076 & 0.21 & 1.92 & $1 / 2$ \\
\hline \multirow[t]{2}{*}{ Calcum } & Abs. Difference & MGRG & 1464.33 & 452.51 & 4072.57 & $2 / 3$ \\
\hline & Rel \% Difference & $\%$ & 67.47 & 16.37 & 147.31 & $15 / 20$ \\
\hline Chromum & Rel. \% Difference & $\%$ & 13.58 & 4.82 & 43.36 & $12 / 20$ \\
\hline Cobalt & Abs. Difference & $\mathrm{MG} / \mathrm{KG}$ & 2.80 & 0.70 & 6.28 & $16 / 20$ \\
\hline \multirow[t]{2}{*}{ Copper } & Abs. Difference & MG/KG & 5.46 & 1.08 & 9.69 & $2 / 3$ \\
\hline & Rel. \% Difference & $\%$ & 36.03 & 15.19 & 136.75 & $13 / 20$ \\
\hline Iron & Rel. \% Difference & $\%$ & 16.07 & 3.92 & 35.28 & $14 / 20$ \\
\hline Lead & Rel. \% Difference & $\%$ & 23.78 & 7.03 & 63.27 & $13 / 20$ \\
\hline \multirow[t]{2}{*}{ Magnesium } & Abs. Difference & $\mathrm{MG} / \mathrm{KG}$ & 1327.15 & 259.69 & 2337.18 & $10 / 13$ \\
\hline & Rel. \% Difference & $\%$ & 23.18 & 6.08 & 54.73 & $16 / 20$ \\
\hline Manganese & Rel. \% Difference & $\%$ & 46.06 & 13.63 & 122.64 & $12 / 20$ \\
\hline \multirow{2}{*}{ Mercury } & Abs. Difference & MG/KG & 3.51 & 1.61 & 14.48 & $1 / 3$ \\
\hline & Rel. \% Difference & $\%$ & 79.34 & 21.36 & 192.25 & $11 / 19$ \\
\hline \multirow[t]{2}{*}{ Nackel } & Abs. Difference & $\mathrm{MG} / \mathrm{KG}$ & 7.17 & 1.12 & 10.05 & $5 / 8$ \\
\hline & Rel. \% Difference & $\%$ & 14.26 & 5.84 & 52.58 & $11 / 20$ \\
\hline \multirow[t]{2}{*}{ Potassium } & Abs. Difference & $\mathrm{MG} / \mathrm{KG}$ & 418.05 & 90.57 & 815.12 & $17 / 19$ \\
\hline & Rel. \% Difference & $\%$ & 689 & 0.00 & 0.00 & $17 / 20$ \\
\hline Selenum & Abs. Difference & MG/KG & 0.99 & 0.30 & 2.73 & $1 / 2$ \\
\hline Silver & Abs. Difference & $\mathrm{MG} / \mathrm{KG}$ & 0.45 & 0.19 & 1.74 & $8 / 16$ \\
\hline Sodum & Abs. Difference & MG/RG & 27.50 & 8.93 & 8040 & $7 / 11$ \\
\hline Thalihum & Abs Difference & MG/KG & 0.06 & 0.02 & 0.19 & $4 / 6$ \\
\hline \multirow[t]{2}{*}{ Vanadum } & Abs. Drfference & $\mathrm{MG} / \mathrm{KG}$ & 537 & 1.12 & 10.09 & $5 / 7$ \\
\hline & Rel. \% Difference & $\%$ & 17.36 & 4.99 & 44.87 & $14 / 20$ \\
\hline Zne & Rel. \% Difference & $\%$ & 3491 & 10.85 & 97.64 & $14 / 20$ \\
\hline
\end{tabular}

- In calcalating the RPD, rejected nesuls were mot inctuded.

Aralytes that were mot detected in ihe ampiple or duplicate were not

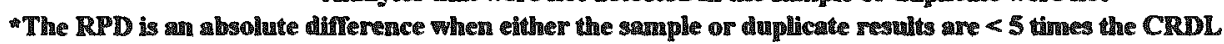

Report: D001.1

Page 1 


\section{E-19}

Table E.6

Summary of Field Duplicate Relative Percent Differences (RPD) Bear Creek Valley OU2 RI FA

\begin{tabular}{|c|c|c|c|c|c|c|}
\hline Parameter & $\begin{array}{l}\text { Method of } \\
\text { RPD } \\
\text { Calculation }^{*}\end{array}$ & Units & $\begin{array}{c}\text { Average } \\
\text { RPD }\end{array}$ & \multicolumn{2}{|c|}{ RPD Limits } & $\begin{array}{l}\text { Number } \\
\text { Results } \\
\text { Within } \\
\text { Limits }\end{array}$ \\
\hline Qnescinte & $"$ & \multicolumn{2}{|c|}{ 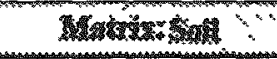 } & 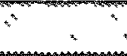 & $\because 9$ & 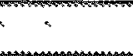 \\
\hline 1,1,2-Trichlora-1,2,2-Trifluoro & Abs Difference & $\mathrm{UG} / \mathrm{KG}$ & 1400 & 3.41 & 30.69 & $2 \cdot 3$ \\
\hline 1,2-Dichloroethene & Abs Difference & UGKG & 33.50 & 7.17 & 6450 & $2 / 2$ \\
\hline 2,4-Dunitrotoluene & Abs Difference & UGKG & 62.50 & 017 & 150 & $0 / 2$ \\
\hline 2-Butanone & Abs Difference & UGRG & 2700 & 0.33 & 3.00 & $0 / 2$ \\
\hline 2-Hexanone & Abs. Dufference & UG/KG & 2.00 & NA & NA & 1 \\
\hline 4-Natrophenol & Abs Difference & $\mathrm{UG} / \mathrm{KG}$ & 104.00 & 8.67 & 7800 & $1 / 2$ \\
\hline Acenaphthene & Abs Difference & UG/KG & 27.00 & NA & NA & 1 \\
\hline Acenaphthylene & Abs Difference & $\mathrm{UG} / \mathrm{KG}$ & 120.00 & NA & NA & 1 \\
\hline \multirow[t]{2}{*}{ Acetone } & Abs. Difference & UG/KG & 249.75 & 67.68 & 609.08 & $3 / 4$ \\
\hline & Rel \% Difference & $\%$ & 5.18 & 018 & 1.59 & $3 / 6$ \\
\hline Anthracene & Abs Dufference & UGKG & 50.00 & NA & NA & 1 \\
\hline Aroclor -1254 & Rel \% Difference & $\%$ & 26.66 & NA & $\mathrm{NA}$ & 1 \\
\hline \multirow[t]{2}{*}{ Aroclor- 1260} & Abs. Difference & UG/KG & 50.40 & 14.05 & 126.41 & $4 / 5$ \\
\hline & Rel. \% Difference & $\%$ & 62.06 & 18.95 & 170.51 & $8 / 12$ \\
\hline Benzene & Abs. Difference & UG/KG & 1.00 & NA & NA & 1 \\
\hline Benzo(a)anthracene & Abs. Dufference & UGKG & 164.00 & 46.62 & 419.58 & $2 / 3$ \\
\hline Benzo(a)pyrene & Abs. Difference & UGRG & 131.33 & 39.11 & 351.95 & $2 / 3$ \\
\hline Benzo(b)fluoranthene & Abs. Difference & UG/KG & 135.66 & 40.13 & 361.13 & $2 / 3$ \\
\hline Benzo(g,h, $)$ perylene & Ahs. Difference & UG/KG & 52.66 & 10.35 & 93.12 & $3 / 3$ \\
\hline Benzo(k)fluoranthene & Abs Defference & UG/RG & 180.66 & 6471 & 582.35 & $2 / 3$ \\
\hline Butylbenzylphthalate & Abs Difference & UGKG & 90.50 & 9.83 & 88.50 & $1 / 2$ \\
\hline Carbazole & Abs Difference & UG/KG & & NA & NA & 1 \\
\hline Carbon Dssulfide & Abs. Difference & UG/KG & 7.66 & 2.67 & 24.04 & $1 / 3$ \\
\hline Chrysene & Abs. Difference & UG/KG & 14900 & 5278 & 475.05 & $2: 4$ \\
\hline Di-n-butylphthalate & Abs. Difference & UG/KG & 3300.00 & NA & NA & 1 \\
\hline Dibenzo(a,h)anthracene & Abs. Difference & UG/RG & 20.00 & NA & $\mathrm{NA}$ & 1 \\
\hline Dibenzofuran & Abs. Difference & UG/KG & 7.00 & NA & NA & 1 \\
\hline Dimethyiphihalate & Abs. Difference & UG/KG & 460.00 & NA & NA & 1 \\
\hline Ethylbenzene & Abs. Difference & UG/KG & 5.00 & NA & NA & 1 \\
\hline Fluoranthene & Abs. Difference & UG/KG & 209.83 & 103.87 & 93483 & $2 / 6$ \\
\hline Fluorene & Abs. Difference & UGKG & 11.00 & NA & NA & 1 \\
\hline Indeno $(1,2.3-6, d)$ pyrene & Abs. Difference & UG/KG & 43.00 & 902 & 81.20 & $2 / 3$ \\
\hline Methylene Chloride & Abs. Difference & UGKG & 85.33 & 14.40 & 129.62 & $2 / 3$ \\
\hline
\end{tabular}

- In calculating the RPD, rejected results were not included.

Anslytes that were mot deterted in the sample or uluplicate were not

-The RPD is an absolute difierence when either the sample or duplicate results are $<5$ times the CRDL

Report: D001.1 
Table E.6

Summary of Field Duplicate Relative Percent Differemces (RPD)

Bear Creek Valley OU2 RI FA

\begin{tabular}{|c|c|c|c|c|c|c|}
\hline Parameter & $\begin{array}{c}\text { Method of } \\
\text { RPD } \\
\text { Calculation } \\
\text { Rel \% Difference }\end{array}$ & $\begin{array}{c}\text { Units } \\
\%\end{array}$ & $\begin{array}{l}\text { Average } \\
\text { RPD } \\
571\end{array}$ & $\begin{array}{l}\text { RPD } \\
\text { Lower } \\
000\end{array}$ & $\begin{array}{r}\text { Limits } \\
\text { Upper } \\
000\end{array}$ & $\begin{array}{l}\text { Number } \\
\text { Results } \\
\text { Within } \\
\text { Limits } \\
\qquad 2 / 4\end{array}$ \\
\hline Pentachiorophenol & Abs Difference & UG/NG & 7850 & 250 & 22.50 & $0 / 2$ \\
\hline Phenanthrene & Abs Dufference & $\mathrm{UG} / \mathrm{KG}$ & 163.33 & 4219 & 37974 & $3 / 3$ \\
\hline Pyrene & Abs. Difference & UG/KG & 230.66 & 101.19 & 91072 & $3 / 6$ \\
\hline Tetrachloroethene & Abs Difference & UG/RG & 59450 & 197.17 & 177450 & $1 / 2$ \\
\hline Toluene & Abs Difference & UG/RG & 28.00 & 4.33 & 39.00 & $1 / 2$ \\
\hline Trichloroethene & Abs Difference & UGRG & 8700 & NA & NA & 1 \\
\hline Xvlene, Total & Abs Difference & UG/KG & 900 & NA & NA & 1 \\
\hline bis(2-Ethylhexyl)phthalate & Abs Dufference & UG/KG & 127.25 & 56.73 & $510 \$ 3$ & $2 / 4$ \\
\hline \multicolumn{7}{|c|}{ 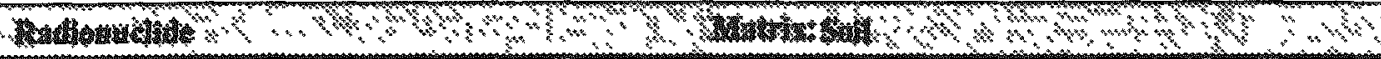 } \\
\hline Gross Alpha & Abs Difference & PCUG & 3.81 & 1.06 & 9.51 & $12 / 15$ \\
\hline Gross Beta & Abs. Difference & PCI/G & 3.15 & 0.78 & 6.99 & $18 / 20$ \\
\hline $\mathrm{U} .234$ & Abs. Difference & $\mathrm{PCI} / \mathrm{G}$ & 048 & 0.23 & 2.04 & $7 / 20$ \\
\hline U-235 & Abs. Dufference & PCIG & 0.09 & 0.04 & 0.35 & $6 / 12$ \\
\hline \multirow[t]{2}{*}{ U.238 } & Abs Difference & PCI/G & 017 & 0.04 & 0.37 & $14 / 18$ \\
\hline & Rel \% Difference & $\%$ & 41.64 & 12.99 & 116.95 & $15 / 20$ \\
\hline \multicolumn{7}{|c|}{ 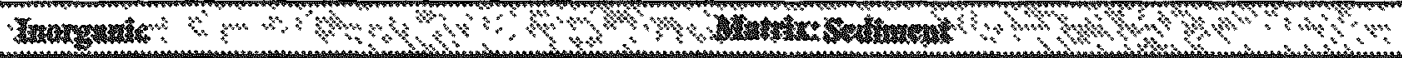 } \\
\hline Alumunum & Rel \% Difference & $\%$ & 0.71 & NA & NA & 1 \\
\hline Antumony & Abs Difference & $\mathrm{MG} / \mathrm{KG}$ & 0.30 & NA & NA & 1 \\
\hline Arsenc & Rel \% Difference & $\%$ & 15.92 & $\mathrm{NA}$ & NA & 1 \\
\hline Banum & Abs Difference & MG/KG & 2.80 & NA & NA & 1 \\
\hline Beryllium & Abs Difference & MG/KG & 008 & NA & NA & 1 \\
\hline Calcum & Abs. Difference & $\mathrm{MG} / \mathrm{KG}$ & 404.00 & NA & NA & 1 \\
\hline Chromium & Rel \% Difference & $\%$ & 10.48 & NA & NA & 1 \\
\hline Cobalt & Abs Difference & MG/RG & & NA & NA & 1 \\
\hline Copper & Rel \% Dufference & $\%$ & 964 & NA & NA & 1 \\
\hline Iron & Rel. \% Difference & $\%$ & & NA & NA & 1 \\
\hline Lead & Rel. \% Difference & $\%$ & 3.33 & NA & NA & 1 \\
\hline Magnesıum & Abs Difference & MG/KG & 19.00 & NA & NA & 1 \\
\hline Manganese & Rel. \% Difference & 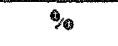 & 22.75 & NA & NA & 1 \\
\hline Mercury & Abs Difference & $\mathrm{MG} / \mathrm{KG}$ & 0.10 & NA & NA & 1 \\
\hline Nickel & Abs. Difference & $\mathrm{MG} / \mathrm{KG}$ & 0.80 & NA & NA & 1 \\
\hline Potassum & Abs. Difference & $\mathrm{MG} / \mathrm{KG}$ & 33.00 & NA & NA & 1 \\
\hline Silver & Abs. Difference & MG/KG & 001 & NA & NA & 1 \\
\hline
\end{tabular}

- In calculating the RPD, rejected resullts were mot inchuded.

Analytes that were jot detected in the saanple or duplicate were mot

-The RPD is an absolute difference when either the samaple or duplicate results are $<5$ times the CROL 
Table $\mathrm{E}_{\mathrm{E}} 6$

Summary of Field Duplicate Relative Percent Differences (RPD) Bear Creek Valley OU2 RI FA

\begin{tabular}{|c|c|c|c|c|c|c|}
\hline Parameter & $\begin{array}{c}\text { Method of } \\
\text { RPD } \\
\text { Calculntion }\end{array}$ & Unies & Average & $\begin{array}{r}\text { Lower } \\
\text { Lower }\end{array}$ & $\begin{array}{l}\text { Limits } \\
\text { Upper }\end{array}$ & $\begin{array}{l}\text { Number } \\
\text { Results } \\
\text { Within } \\
\text { Limits }\end{array}$ \\
\hline Vanadium & Rel \% Dufference & 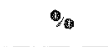 & 018 & $\mathrm{NA}$ & NA & 1 \\
\hline Zanc & Rel \% Difference & $\%$ & 16.27 & NA & $\mathrm{NA}$ & 1 \\
\hline \multicolumn{7}{|c|}{ V1) } \\
\hline 4-Methyl-2-pentanone & Abs Difference & UG/KG & 4.00 & $\mathrm{NA}$ & NA & 1 \\
\hline Acetone & Abs. Difference & $\mathrm{UG} / \mathrm{KG}$ & 5100 & NA & NA & 1 \\
\hline Aroclor -1260 & Abs Difference & UG/KG & 31.00 & NA & $\mathrm{NA}$ & 1 \\
\hline Naphthalene & Abs. Dufference & $\mathrm{UG} / \mathrm{KG}$ & 53.00 & $\mathrm{NA}$ & NA & 1 \\
\hline \multicolumn{7}{|c|}{ 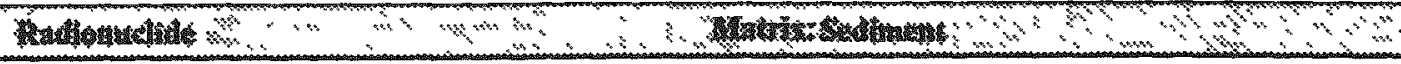 } \\
\hline Gross Alpha & Abs Difference & PCUG & 3.53 & NA & NA & 1 \\
\hline Gross Beta & Abs. Difference & $\mathrm{PCW}$ & 2.60 & NA & NA & 1 \\
\hline U.234 & Abs. Difference & $\mathrm{PCl} / \mathrm{G}$ & 2.08 & NA & NA & 1 \\
\hline U.235 & Abs. Difference & $\mathrm{PCVG}$ & 0.04 & $\mathrm{NA}$ & NA & 1 \\
\hline U-238 & Abs. Difierence & $\mathrm{PCl} / \mathrm{G}$ & 0.24 & NA & NA & 1 \\
\hline \multicolumn{7}{|c|}{ 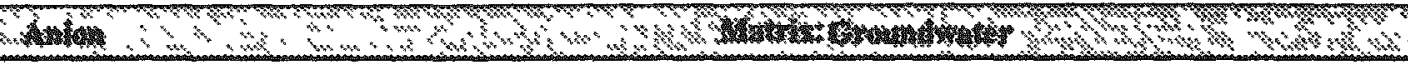 } \\
\hline Chionde & Rel \% Difference & $\%$ & 5.70 & NA & NA & 1 \\
\hline Sulfate & Abs. Difference & MG/L & 8.70 & NA & NA & 1 \\
\hline \multicolumn{7}{|c|}{ (1) } \\
\hline Alumunum & Rel. \% Difference & $\%$ & 16.92 & NA & $\mathrm{NA}$ & 1 \\
\hline Barum & Abs. Difierence & UGL & 24.00 & NA & NA & 1 \\
\hline Calcrum & Rel. \% Difierence & $\%$ & 4.02 & $\mathrm{NA}$ & NA & 1 \\
\hline Chromum & Abs. Dufference & UGL & 0.80 & NA & NA & 1 \\
\hline Cobalt & Abs. Difference & $\mathrm{UG} / \mathrm{L}$ & 5.10 & NA & NA & 1 \\
\hline Copper & Abs. Differance & UG/L & 0.10 & NA & $\mathrm{NA}$ & 1 \\
\hline Cyande & Abs Dufterence & UG/L & 1370 & NA & NA & 1 \\
\hline Iron & Rel. \% Dufference & $\%$ & 6.14 & NA & NA & 1 \\
\hline Lead & Rel $\%$ Difference & $\%$ & & NA & $\mathrm{NA}$ & 1 \\
\hline Magnesium & Rel. \% Difference & $\%$ & 3.24 & NA & NA & 1 \\
\hline Manganese & Rel \%Difterence & $\%$ & 3.22 & NA & NA & 1 \\
\hline Mercury & Abs. Difference & UG/L & 0.15 & NA & NA & 1 \\
\hline Nickel & Ab Difference & UG/L & 200 & NA & NA & 1 \\
\hline Potassium & Abs Difference & UG/L & 200.00 & NA & NA & 1 \\
\hline Sodtum & Rel \% Difference & $\%$ & 4.37 & NA & $\mathrm{NA}$ & 1 \\
\hline Vanadiurn & Abs Difference & UG/L & 0.30 & NA & NA & 1 \\
\hline Zane & Abs. Difference & UGL & 5.50 & NA & NA & 1 \\
\hline
\end{tabular}

- ir calcalating the RPD, rejected results were not meluded.

Andytes that were not detected hin the ample or dupplicate were not

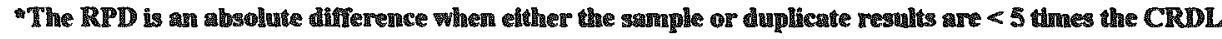

Report: D001.1 
E-22

Table E.6

Summary of Field Duplicate Relative Percent Differences (RPD)

Bear Creek Valley OU2 RI FA

\begin{tabular}{|c|c|c|c|c|c|c|}
\hline Parmeter & $\begin{array}{l}\text { Method of } \\
\text { RPD } \\
\text { Calculation" }\end{array}$ & Units & $\begin{array}{l}\text { Average } \\
\text { RPD }\end{array}$ & $\begin{array}{l}\text { RPD } \\
\text { Lower }\end{array}$ & $\begin{array}{l}\text { Limits } \\
\text { Upper }\end{array}$ & $\begin{array}{l}\text { Number } \\
\text { Results } \\
\text { Within } \\
\text { Limits }\end{array}$ \\
\hline 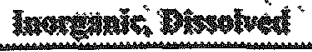 & $\because$ & \multicolumn{4}{|c|}{ (1) } & $\cdots$ \\
\hline Antumony & Abs Difference & UG/L & 4.10 & NA & NA & 1 \\
\hline Barum & Abs. Difference & $\mathrm{UG} / \mathrm{L}$ & 21.00 & NA & $\mathrm{NA}$ & 1 \\
\hline Calcum & Rel \% Difference & $\%$ & 2.76 & NA & NA & 1 \\
\hline Cobalt & Abs. Difference & UG/L & 3.20 & NA & NA & 1 \\
\hline Lead & Abs. Difference & UG/L & 2.00 & NA & NA & 1 \\
\hline Magnestum & Rel \% Difference & $\%$ & 3.22 & NA & NA & 1 \\
\hline Manganese & Rel \% Difference & $\%$ & 1.61 & NA & NA & 1 \\
\hline Mercury & Abs. Difference & UGL & 0.01 & NA & NA & 1 \\
\hline Nickel & Abs Difference & UG/L & 1.30 & NA & NA & 1 \\
\hline Potassium & Abs. Dufference & UG/ & 300.00 & NA & NA & 1 \\
\hline Sodum & Rel. \% Dufference & $\%$ & 3.53 & NA & NA & 1 \\
\hline \multicolumn{7}{|c|}{ Inta } \\
\hline Alkalinity & Rel. \% Difference & $\%$ & 5.67 & NA & NA & 1 \\
\hline Total Dissolved Solids & Rel \% Difference & $\%$ & & NA & NA & 1 \\
\hline Total Suspended Solids & Rel. \% Difference & $\%$ & 6.92 & NA & NA & 1 \\
\hline Turbidity & Rel. \% Dufference & $\%$ & 4.56 & NA & NA & 1 \\
\hline \multicolumn{7}{|c|}{ 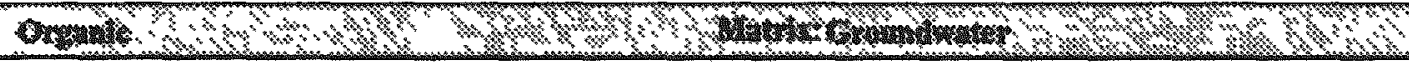 } \\
\hline Butylbenzylphthalate & Abs. Difference & UG/L & 2.00 & NA & NA & 1 \\
\hline Di-n-butylphthaiate & Abs. Duference & UG/L & 3.00 & NA & NA & 1 \\
\hline Diethvighthalate & Abs Difference & UGR & 2.00 & NA & NA & 1 \\
\hline Tetrachloroethene & Abs Difference & UG/L & & NA & $\mathrm{NA}$ & 1 \\
\hline bis(2-Ethylhexyl)phthalate & Abs. Difference & UG/L & 3.00 & NA & NA & 1 \\
\hline \multicolumn{7}{|c|}{ (1) } \\
\hline Gross Alpha & Abs. Dufference & PCIL & 16.10 & NA & NA & 1 \\
\hline Gross Beta & Rel. \% Dufference & $\%$ & 4.64 & NA & NA & 1 \\
\hline U-234 & Abs. Difference & PCI & 0.56 & NA & $\mathrm{NA}$ & 1 \\
\hline U.235 & Abs. Difference & PCIL & 0.39 & NA & NA & 1 \\
\hline U.238 & Abs. Difference & $\mathrm{PCI}$ & 0.11 & NA & $\mathrm{NA}$ & 1 \\
\hline \multicolumn{7}{|c|}{ 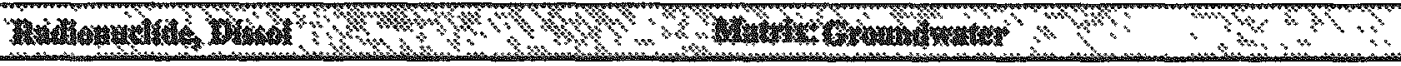 } \\
\hline Gross Beta & Rel. \% Difference & $\%$ & 10.31 & NA & NA & 1 \\
\hline$U_{-234}$ & Abs. Difference & $\mathrm{PCl} / \mathrm{L}$ & 047 & NA & NA & 1 \\
\hline U.23 & Abs. Drference & $\mathrm{PCL} /$ & 0.03 & NA & NA & 1 \\
\hline
\end{tabular}

-In calculating the RPD, rejected resulis were not inchaded.

Analytes that were mot detected in the sampilte or dupllcate were mot

-The RPD is an absolute difference when either the sample or duplicate results are < 5 tumes the CRDL

Report. D001.1 
Table E.7

Bear Creek Valley OU2 RI FA

QC Sample Summary

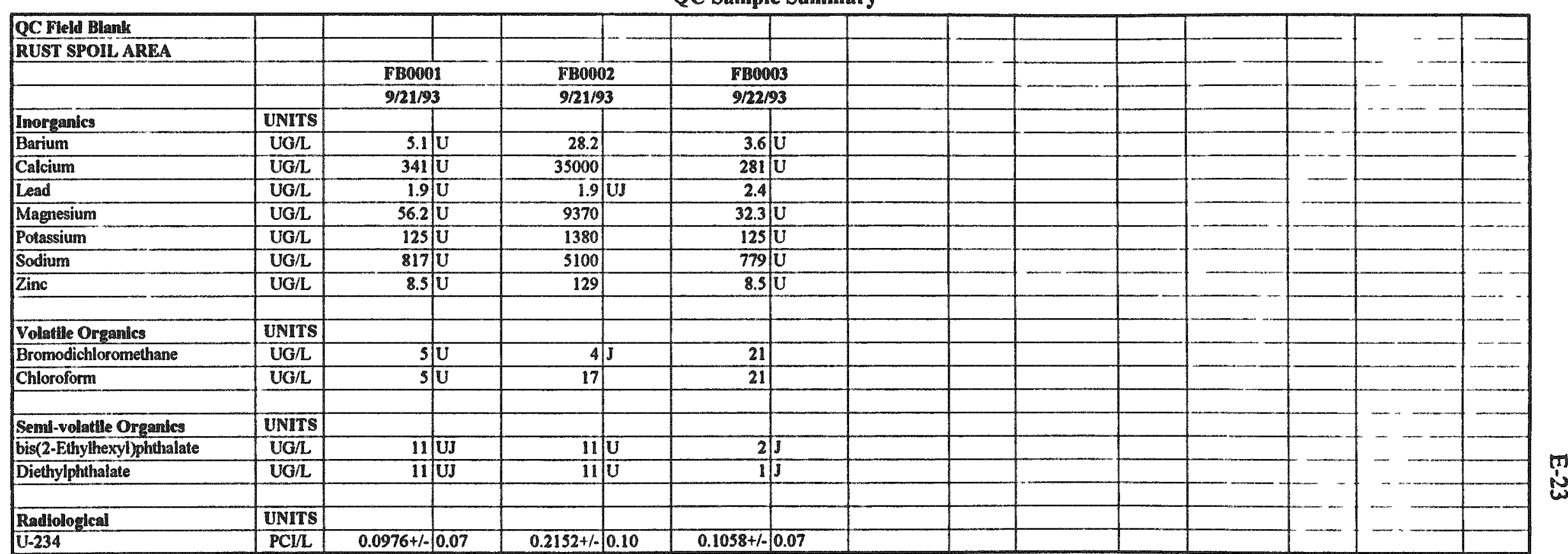


Table E.7

Bear Creek Valley OU2 RI FA

QC Sample Summary

\begin{tabular}{|c|c|c|c|c|c|c|c|c|c|c|c|c|c|c|c|}
\hline \multirow{2}{*}{\multicolumn{16}{|c|}{$\begin{array}{l}\text { QC Tield BIank } \\
\text { STORAGR YARD } 200\end{array}$}} \\
\hline & & & & & & & & & & & & & & & \\
\hline & & \multicolumn{2}{|c|}{ FBOOOA } & \multicolumn{2}{|c|}{ FBO000 } & \multicolumn{2}{|c|}{ FBQ006 } & \multicolumn{2}{|c|}{ Fro007 } & \multicolumn{2}{|c|}{ FBO008 } & \multicolumn{2}{|c|}{ FB0009 } & \multicolumn{2}{|c|}{ FiBO010 } \\
\hline & & \multicolumn{2}{|c|}{$10 / 26 / 93$} & \multicolumn{2}{|c|}{$11 / 3 / 93$} & \multicolumn{2}{|c|}{$11 / 4 / 93$} & \multicolumn{2}{|c|}{$11 / 10 / 93$} & \multicolumn{2}{|c|}{$11 / 11 / 93$} & \multicolumn{2}{|c|}{$11 / 18 / 93$} & \multicolumn{2}{|c|}{$11 / 17193$} \\
\hline Inor reanics & UNITS & & & & & & & & & & & & & & \\
\hline Alumi:的的能 & $\mathrm{UG} / \mathrm{L}$ & 44.2 & $\mathrm{U}$ & 73 & $\mathrm{U}$ & 171 & & 31.3 & U & 15.5 & U & 1020 & & 171 & U \\
\hline Barium & $\mathrm{VG} / \mathrm{L}$ & 3.6 & U & 1.2 & 0 & 28.4 & & 3.6 & $U$ & 3.6 & U & 10.4 & & 25 & \\
\hline Beryllium & UG/L & 0.2 & $\bar{U}$ & 0.2 & U & 0.2 & $\mathrm{U}$ & 0.35 & $\bar{U}$ & 0.2 & $\mathrm{U}$ & 0.2 & $\bar{U}$ & 0.2 & U \\
\hline Calcium & VOA. & 24.2 & U & 39 & u & 38200 & & 24.2 & $U$ & 174 & & 970 & U & 33700 & \\
\hline Chromium & UGL & 4.1 & $\mathrm{U}$ & 2.1 & U & 9.4 & & 4.1 & $\mathrm{U}$ & 4.1 & $\mathrm{U}$ & 4.1 & $\mathrm{U}$ & 5.5 & $\mathrm{U}$ \\
\hline Copper & $\mathrm{UG} / \mathrm{L}$ & 2.9 & U & 2.5 & $\mathrm{U}$ & 10 & & 2.9 & $\mathrm{U}$ & 2.9 & $U$ & 2.9 & U & 9.5 & $U$ \\
\hline Iron & Ual & 29 & U & 35 & $\mathrm{U}$ & 100 & $\mathrm{U}$ & 53.8 & U & 57.9 & $\mathrm{U}$ & 90.1 & & 980 & \\
\hline Lead & UGR & 3 & $J$ & 2.5 & $\mathrm{U}$ & 2.5 & UJ & 10.8 & U & 2.5 & $\mathrm{U}$ & 2.5 & 11 & 2.8 & \\
\hline Magnesium & UG/L & \begin{tabular}{l|l|}
32.3 \\
\end{tabular} & U & 59.8 & U & 10500 & & 65.1 & $U$ & 32.3 & U & 144 & U & 9960 & \\
\hline Manganese & UG/L & 1.4 & U & 1.7 & $\mathrm{U}$ & 12.5 & & 1.9 & & 1.3 & U & 29.2 & & 31.6 & \\
\hline Mercury & UG/L & 0.2 & $\mathrm{U}$ & 0.2 & U & 0.64 & & 0.2 & U & 0.2 & $\mathrm{U}$ & 0.2 & U & $\overline{0.2}$ & U \\
\hline Nickel & UG/L & 3.9 & U & 3.4 & U & 3.9 & U & 3.9 & U & 3.9 & $\mathrm{U}$ & 3.9 & $\mathrm{U}$ & 3.9 & $\mathrm{U}$ \\
\hline Potassium & UGL & 125 & $\bar{U}$ & 131 & $U$ & 1740 & & 127 & & 255 & & 125 & u & 1500 & \\
\hline Selenium & UG/L & 2.4 & $\mathbf{u}^{-}$ & 3.2 & & 2.7 & $\mathrm{U}$ & 2.7 & $\mathrm{U}$ & 4.5 & & 4.1 & $\mathrm{U}$ & 2.4 & $\mathrm{U}$ \\
\hline Sodium & UG/L & 129 & $\mathrm{U}$ & 114 & $\mathrm{U}$ & 5740 & & 225 & U & 477 & & 255 & u & $\overline{5080}$ & \\
\hline Thallium & UG/L & 3.8 & UJ & 3.8 & $\mathrm{U}$ & 3.8 & $\mathrm{U}$ & 3.8 & $U$ & 2.3 & $\mathrm{U}$ & 2.3 & $\mathrm{U}$ & $3 . \overline{8}$ & U \\
\hline Zine & UG/L & 8.5 & $\mathrm{U}$ & 4.4 & $\mathrm{U}$ & 116 & & 8.5 & U & 8.6 & & 9.7 & & 202 & \\
\hline & & & & & & & & & & & & & & & \\
\hline Volatile Organles & UNITS & & & & & & & & & & & & & & \\
\hline Acetone & UG/L & 10 & $\mathbf{U}$ & 10 & U & 10 & UJ & 10 & U & NA & & 10 & $\mathrm{U}$ & 10 & $U$ \\
\hline Bromodichloromethane & UGL & 5 & $\mathrm{U}$ & 5 & U & 5 & 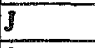 & 1 & $J$ & NA & & 5 & U & 5 & \\
\hline Chloroform & UG/L & 2 & $\mathrm{~J}$ & 6 & & 16 & 1 & 6 & & NA & & 4 & $\mathrm{U}$ & 15 & \\
\hline Methylene Chloride & UG/L & 5 & & 5 & $\bar{U}$ & 5 & UI & 5 & U & NA & & 5 & U & 3 & $J$ \\
\hline Toluene & UG/L & 5 & $u$ & 5 & U & 5 & UJ & 5 & & NA & & 5 & $\mathrm{U}$ & 5 & $\mathrm{U}$ \\
\hline & & & & & & & & & & & & $t=$ & & 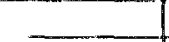 & . \\
\hline Semil-volatile Organics & UNITS & & & & & & & & & & & & & & \\
\hline bis(2-Ethylhexyl)phohalate & UG/L & 2 & $\mathrm{~J}$ & 11 & $\mathrm{U}$ & 11 & $u$ & 10 & $\mathrm{U}$ & NA & & 11 & $\mathrm{u}$ & 10 & $U$ \\
\hline Butylbenzylphthalate & UGL & 11) & $\mathrm{U}$ & 11 & $\mathrm{U}$ & 11 & U & 10 & U & NA & & 11 & U & 10 & $\mathrm{U}$ \\
\hline & & & & & & & & & & & & & & - & 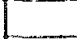 \\
\hline Radliological & UNITS & & & & & & & & & & & & & & \\
\hline Gross Beta & $\mathrm{PCI} / \mathrm{L}$ & $0.00 \mathrm{E}+00+1$. & 1.77 & $3.33 \mathrm{E}+00+1$ & 2.08 & $4.40 \mathrm{E}+00+1-$ & 2.15 & $0.00 \mathrm{E}+00+1$ & 2.25 & $0.00 \mathrm{E}+00+1$. & 2.20 & $0.00 \mathrm{E}+00+1-$ & 1.75 & $463 E+00+1-$ & 2.22 \\
\hline 0.234 & $\mathrm{PCI/L}$ & $1.41 E-02+1$ & 0.05 & $1.28 \mathrm{E}-01+1-$ & 0.15 & $7.96 \mathrm{E}-01+1-$ & $0.52 \mathrm{~J}$ & $6.04 \mathrm{E}-02+1$ & 0.12 & $5.20 \mathrm{E}-\overline{02+1}$ & 0.09 & $1.79 \mathrm{E}-01+1 /$ & 010 & $247 \mathrm{E}-01+1$ & 0.11 \\
\hline & & & & & & & & & & & & & & - & \\
\hline & & & & & & & & & & & & & & & \\
\hline
\end{tabular}


Table E.7

Bear Creek Valley OU2 RI TA

QC Sample Summary

\begin{tabular}{|c|c|c|c|c|c|c|c|c|c|c|c|c|c|c|c|}
\hline \multicolumn{16}{|l|}{ STORAGE YARD 200} \\
\hline & & \multicolumn{2}{|c|}{ FBPOCI } & \multicolumn{2}{|c|}{ FBO014 } & \multicolumn{2}{|c|}{ FBO015 } & \multicolumn{2}{|c|}{ FBo016 } & \multicolumn{2}{|c|}{ FBO017 } & \multicolumn{2}{|c|}{ Fono18 } & \multicolumn{2}{|c|}{ FBO019 } \\
\hline & & \multicolumn{2}{|c|}{$11 / 23 / 93$} & \multicolumn{2}{|c|}{$12 / 3 / 93$} & \multicolumn{2}{|c|}{$12 / 3 / 93$} & \multicolumn{2}{|c|}{$12 / 9 / 93$} & \multicolumn{2}{|c|}{$12 / 9 / 93$} & \multicolumn{2}{|c|}{$12 / 16 / 93$} & \multicolumn{2}{|c|}{$12 / 16 / 93$} \\
\hline Inorganies & UNITS & & & & & & & & & & & & & & \\
\hline Aluminum & UG/L & 33.1 & $\mathrm{U}$ & 35.6 & $\mathrm{U}$ & 89 & $U$ & 29.611 & $\mathrm{U}$ & 151 & $\mathrm{U}$ & 73.6 & & 18.5 & \\
\hline Barium & $\mathrm{UO} / \mathrm{L}$ & 0.88 & $\mathrm{U}$ & 3.6 & $\mathrm{U}$ & 27.2 & & 0.811 & $\mathrm{U}$ & 0.8 & $\mathrm{U}$ & 27.3 & & $5 . \overline{9}$ & U \\
\hline Beryllium & UG/L & 0.1 & $\mathbf{U}$ & 0.2 & $\mathrm{U}$ & 0.2 & $\mathrm{U}$ & 0.31 & U & 0.3 & $\mathrm{U}$ & 0.37 & & 0.1 & U \\
\hline Calcium & $\mathrm{UG} / \mathrm{L}$ & 27.8 & $\mathrm{U}$ & 24.2 & U & 32600 & & $83.41 \mathrm{C}$ & $\mathrm{U}$ & 83.4 & $\mathrm{U}$ & 32900 & & 67.1 & Ii \\
\hline Chromium & $\mathrm{UG} / \mathrm{L}$ & 9.7 & $\mathrm{U}$ & 4.1 & 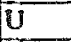 & 4.1 & $\mathrm{U}$ & 3.51 & $\mathrm{U}$ & 2.5 & $\mathrm{u}$ & $\$ .6$ & U & 3.6 & 10 \\
\hline Copper & UG/L & 1.8 & $\mathrm{U}$ & 2.9 & U & 4.7 & & 1.61 & U & 1.6 & $\mathrm{U}$ & 28 & & 5 & U \\
\hline Iron & UG/L & 14.7 & $\mathrm{U}$ & 39.3 & $\mathrm{U}$ & 64.2 & & 1610 & $\mathrm{U}$ & 13.4 & $\mathrm{U}$ & 28.9 & $\mathrm{U}$ & 9.9 & U \\
\hline lead & UG/L & 2.5 & $\mathbf{U}$ & 9.8 & & 2.4 & & 1 & & $1]$ & U & 1.3 & $\mathrm{U}$ & 1.5 & U \\
\hline Magnesium & UG/L & 32.2 & U & 50.3 & U & 9520 & & 32.20 & U & 32.2 & $\mathrm{U}$ & 9960 & & 44.5 & I \\
\hline Manganese & $\mathrm{UG} / \mathrm{L}$ & 0.8 & $\mathrm{U}$ & 1.3 & $\mathrm{U}$ & 2.9 & $\mathrm{U}$ & 0.8 & $\mathrm{U}$ & 0.8 & $\mathrm{U}$ & 1.2 & $\mathrm{U}$ & $-\ldots$ & U \\
\hline Mercury & UG/L & 0.2 & $\mathrm{U}$ & 0.2 & $\mathrm{U}$ & 0.2 & U & 0.21 & $\mathrm{U}$ & 0.2 & U & 0.2 & $\mathrm{u}^{-}$ & $-\infty$ & U \\
\hline Nickel & UG/L & 4.4 & U & 3.9 & $U$ & 4 & & $4 . 4 \longdiv { 1 }$ & $U$ & 4.4 & 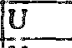 & 4.7 & U & - & U \\
\hline Potassium & UG/L & 119 & & 215 & & 1630 & & 58.8 & $\mathrm{U}$ & 58.8 & $\mathrm{U}$ & 1620 & & 129 & U \\
\hline Selenium & UG/L & 2.7 & UJ & 2.7 & UJ & 2.7 & $\mathrm{U}$ & $4 . 1 \longdiv { 1 }$ & $\mathrm{U}$ & 4.1 & $\mathrm{U}$ & 2.7 & US & 2.7 & $\mathrm{U}$ \\
\hline Sodium & UG/L & 47.7 & & 295 & $U$ & 5240 & & 68.2 & & 39.6 & $\mathrm{U}$ & 6200 & & 59.6 & $U$ \\
\hline Thallium & $\mathrm{UG} / \mathrm{L}$ & 3.8 & $\mathrm{U}$ & 1.3 & $\mathrm{U}$ & 1.3 & $U$ & 1.3 & U & 1.3 & $\mathrm{U}$ & 2 & & 1.8 & U \\
\hline \multirow[t]{2}{*}{ Zine } & $\mathrm{UG} / \mathrm{L}$ & 4.6 & U & 8.5 & $\mathrm{U}$ & 113 & & 4.6 & U & 4.6 & is & 122 & & 13.6 & \\
\hline & & & & & & & & & & & & & & & \\
\hline Volattile Organles & UNITS & & & & & & & & & & & & & & \\
\hline Acetone & $\mathrm{Ua} / \mathrm{L}$ & 10 & $\mathrm{U}$ & NA & & 10 & 0 & NA & & 88 & & 20 & & NA & \\
\hline Bromodichloromethane & UG/L & 5 & $U$ & NA & & 5 & & NA & & 5 & U & 6 & & NA & \\
\hline Chloroform & $\mathrm{UG} / \mathrm{L}$ & 4 & $\mathrm{U}$ & NA & & 20 & & NA & & 1. & $\mathrm{~J}$ & 23 & & NA & \\
\hline Methylene Chloride & UG/L & 5 & $\mathrm{u}$ & NA & & 3 & $U$ & NA & & 8 & US & 5 & $\mathrm{u}$ & NA & \\
\hline \multirow[t]{2}{*}{ Toluene } & UG/L & 5 & $\mathrm{U}$ & NA & & 5 & U & NA & & & $\mathrm{U}$ & & U & NA & \\
\hline & & & & & & & & & & & & & & - & \\
\hline Semi-volatile Orgamics & UNITS & & & & & & & & & & & & & & \\
\hline bis(2-Ethylhexyl)phthalate & UG/L & 11 & $\mathbf{U}$ & NA & & 10 & 0 & NA & & & $\mathrm{J}$ & 9 & $\mathrm{U}$ & NA & \\
\hline \multirow[t]{2}{*}{ Butylbenzylphthalate } & $\mathrm{UG} / \mathrm{L}$ & 11) & $\mathrm{U}$ & NA & & 3 & $\mathrm{~J}$ & NA & & 13 & $\mathrm{U}$ & 9 & U & $\mathrm{NA}$ & \\
\hline & & & & & & & & & & & & & & & \\
\hline Radiological & UNITS & & & & & & & & & & & & & & \\
\hline Gross Beta & $\mathrm{PCI} / \mathrm{L}$ & $0.00 \mathrm{E}+00+1-$ & 1.78 & $0.00 \mathrm{E}+00+1-$ & 1.86 & $4.62 \mathrm{E}-01+1-$ & 1.99 & $7.38 \mathrm{E}-01+1-1$ & 2.05 & $0.00 \mathrm{E}+00+1-$ & 1.97 & $1.08 E+01+1-$ & 3.00 & $0.00 \mathrm{E}+00+1$ & 2.39 \\
\hline U.234 & $\mathrm{PCI} / \mathrm{L}$ & $2.68 \mathrm{E}-01+1-$ & 0.11 & $2.30 \mathrm{E}-01+1-1$ & 0.43 & $1.99 \mathrm{E}-01+10$ & 0.14 & $1.10 \mathrm{E}-01+1-$ & 0.09 & $3.09 \mathrm{E}-01+1$. & 0.15 & $3.74 \mathrm{E}-01+1$ & 0.15 & $2.32 \mathrm{E}-01+1-$ & 0.10 \\
\hline & & & & & & & & & & & & & & & \\
\hline & & & & & & & & & & & & & & & \\
\hline
\end{tabular}


Table E.7

Bear Creek Valley OU2 RI FA

QC Sample Summary

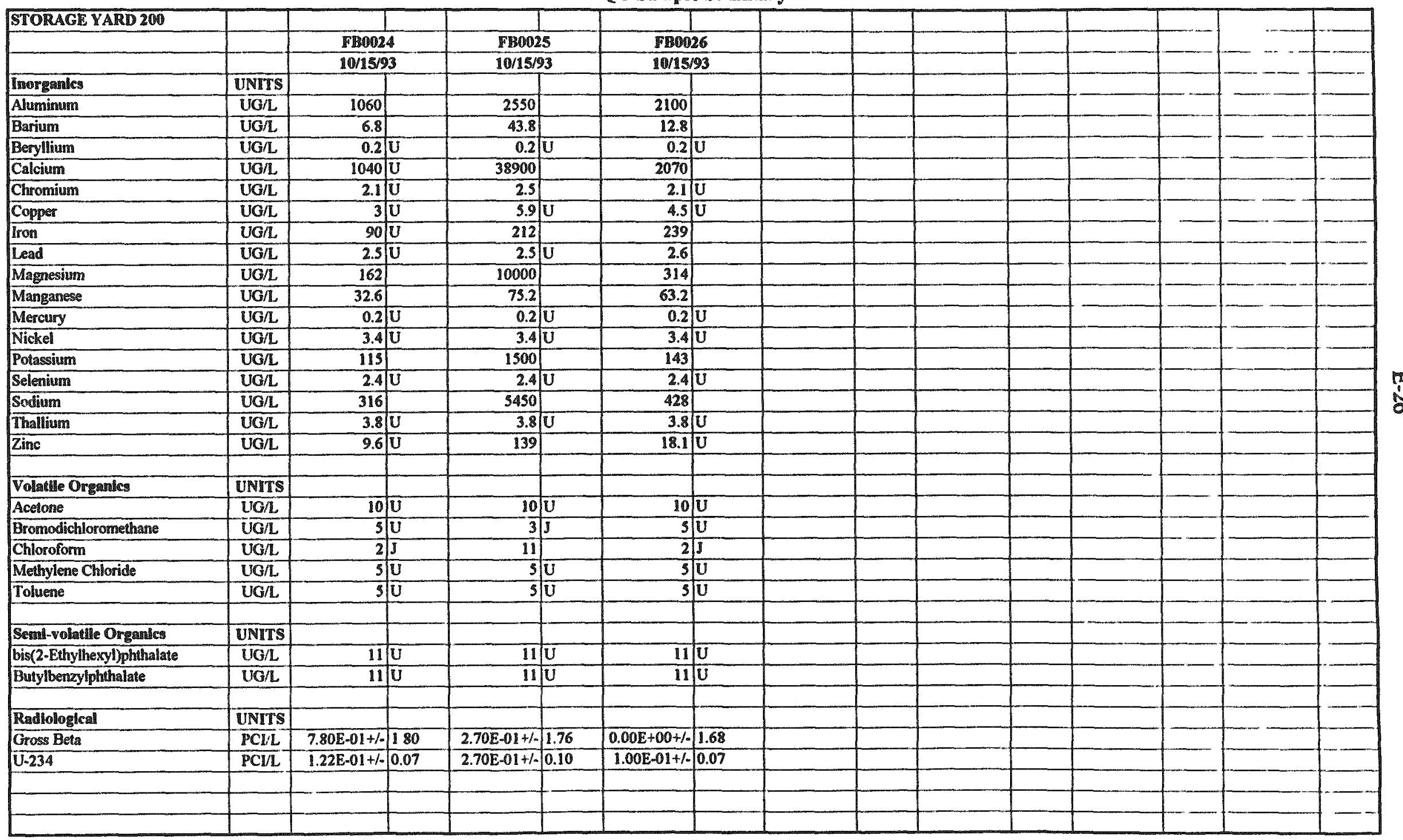


Table E.7

Bear Creek Valley OU2 RI FA

QC Sample Summary

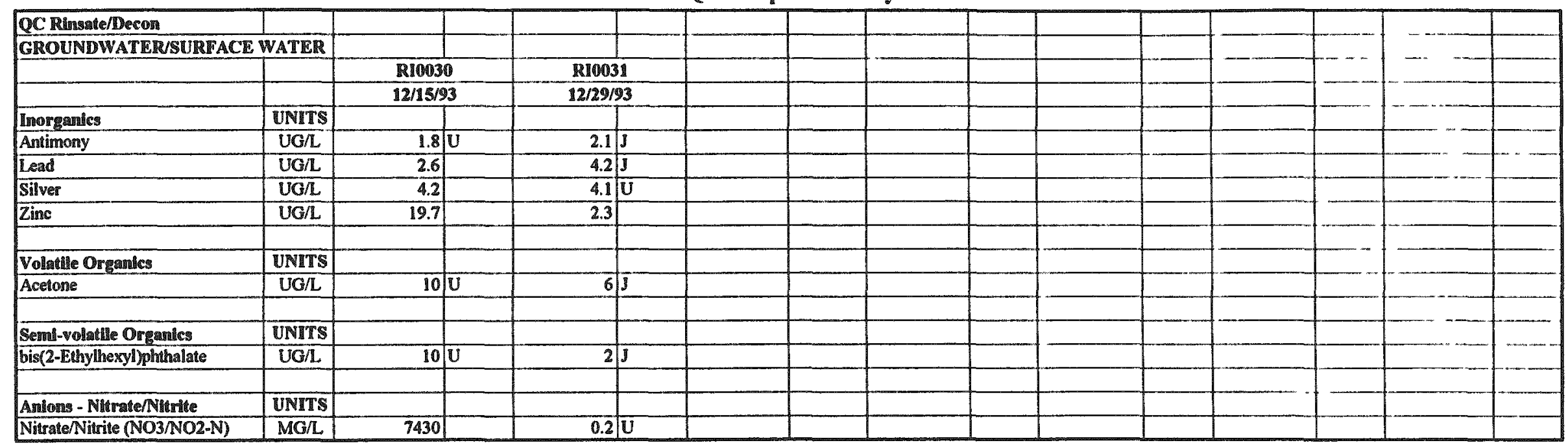


Table E.7

Bear Creek Valley OU2 RI FA

QC Sample Summary

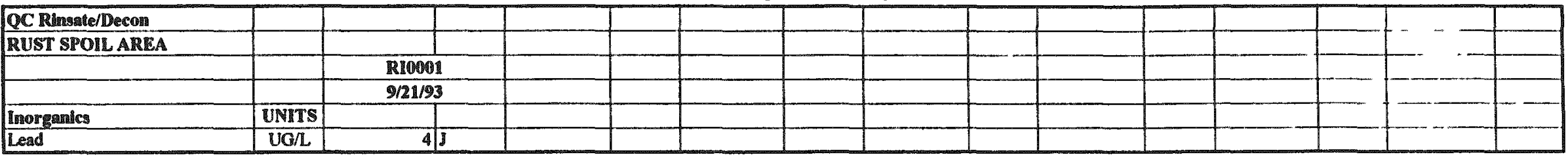


Table E.7

Bear Creek Valley OU2 RI FA

QC Sample Summary

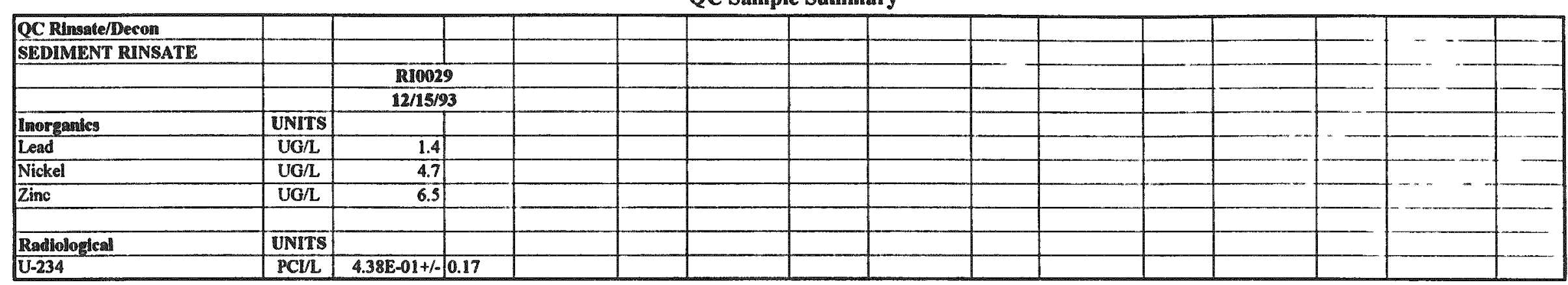


Table E.7

Bear Creek Valley OU2 RI FA

QC Sample Summary

\begin{tabular}{|c|c|c|c|c|c|c|c|c|c|c|c|c|c|c|c|}
\hline \multirow{3}{*}{\begin{tabular}{|l|} 
QC Rinsate/Decon \\
STORAGE YARD 200
\end{tabular}} & & & & & & & & & & & & & & & \\
\hline & & & & & & & & & & & & & & & \\
\hline & & \multicolumn{2}{|c|}{ RI0004 } & \multicolumn{2}{|c|}{ R10005 } & \multicolumn{2}{|c|}{ RIoov6 } & \multicolumn{2}{|c|}{ R10007 } & \multicolumn{2}{|l|}{ RI0008 } & \multicolumn{2}{|c|}{ RIO009 } & \multicolumn{2}{|c|}{ R10010 } \\
\hline & & \multicolumn{2}{|c|}{$10 / 26 / 93$} & \multicolumn{2}{|c|}{$11 / 4 / 93$} & \multicolumn{2}{|c|}{$11 / 10 / 93$} & \multicolumn{2}{|c|}{$11 / 17 / 93$} & \multicolumn{2}{|c|}{$11 / 23 / 93$} & \multicolumn{2}{|c|}{$12 / 3 / 93$} & \multicolumn{2}{|l|}{$12 / 7 / 93$} \\
\hline Inorganics & UNITS & & & & & & & & & & & & & & \\
\hline Aluminum & UG/L & 42.5 & $\bar{U}$ & 209 & & 3490 & & 1180 & & 698 & & 245 & & $\overline{24.7} \overline{\mathrm{C}}$ & $\mathrm{U}$ \\
\hline Barium & UG/L & 3.6 & U & 3.6 & $U$ & 16.2 & & 7.1 & & $4.2 \mathrm{C}$ & $\bar{U}$ & 3.6 & $\mathrm{U}$ & 0.88 & $\bar{U}$ \\
\hline Beryllium & UG/L & 0.2 & $\mathrm{U}$ & 0.21 & $\mathrm{U}$ & 0.36 & U & 0.21 & $\mathrm{U}$ & 0.21 & $\mathrm{U}$ & 0.2 & U & 0.3 & $\mathrm{U}$ \\
\hline Calcium & $\mathrm{UG} / \mathrm{L}$ & 91.2 & $\bar{U}$ & 243 & & 3330 & & 1480 & & 586 & & 195 & & 83.41 & $\mathrm{u}$ \\
\hline Chromium & UG/L & 4.1 & $\mathbf{U}$ & 13.3 & & 11.1 & & $4.1 \mathrm{~d}$ & U & 19.10 & $\mathrm{U}$ & 50.9 & & 8.2 & $\mathrm{U}$ \\
\hline Copper & UG/L & 2.9 & $\mathrm{U}$ & 2.9 & U & 5.1 & & $3.61 \mathrm{c}$ & U & $3.61 \mathrm{c}$ & $\bar{U}$ & 4 & & 1.8 & $\mathrm{u}$ \\
\hline Iron & UG/L & 48.1 & $\mathrm{U}$ & 132 & U & 354 & & 103 & & $121 \mathrm{l}$ & $\bar{U}$ & 647 & & 71.4 & u \\
\hline Lead & $\mathrm{UG} / \mathrm{L}$ & 2.5 & $\mathrm{U}$ & 2.5 & $\mathrm{U}$ & 4.4 & $\mathrm{U} / \mathrm{J}$ & $\begin{array}{c}2.50 \\
0\end{array}$ & $\mathrm{U}$ & $2.5 \pi$ & $\mathrm{U}$ & 13.3 & & 2.5 & \\
\hline Magnesium & UG/L & 32.3 & U & 38 & U & 302 & & 220 & & 129 & $\mathrm{U}$ & 50.4 & $\mathrm{U}$ & 55 & $\mathrm{U}$ \\
\hline Manganese & UO/L & 2.6 & 0 & 7.9 & & 99.8 & & 40.9 & & 18.4 & & 21.5 & & 2.1 & 0 \\
\hline Nickel & UG/L & 3.9 & $U$ & 3.9 & U & 6 & & 3.97 & $\mathrm{U}$ & 4.41 & $\mathrm{U}$ & 6.4 & & 4.4 & $\mathrm{U}$ \\
\hline Potassium & UG/L & 125 & $\mathrm{U}$ & 164 & & 223 & & $125 \mathrm{C}$ & $\mathrm{U}$ & 179 & & 125 & $\mathrm{U}$ & 59.7 & \\
\hline Selenium & UG/L & 2.4 & U & 2.7 & Us & 2.7 & UI & 2.61 & U & 41 & & 2.7 & U & 4.1 & $\mathrm{U}$ \\
\hline Sodium & UG/L & 170 & U & 90.7 & U & 832 & U & 467 & & 190 & & 198 & $\mathrm{u}$ & 62.8 & \\
\hline Zinc & UGh & 8.5 & $\mathrm{U}$ & 19.1 & & 27.3 & & 14.7 & & 101 & $\mathbf{U}$ & 10.6 & & 4.8 & $\bar{U}$ \\
\hline & & & & & & & & & & & & & & & \\
\hline Volatile Organics & UNITS & & & & & & & & & & & & & & \\
\hline Chloroform & $\mathrm{UG} / \mathrm{L}$ & 2 & I & & $\mathrm{U}$ & NA & & 4 & 1 & 3 & $\mathrm{U}$ & NA & & $\overline{\mathrm{NA}}$ & \\
\hline Methylene Chloride & UG/L & 4 & $\mathrm{~J}$ & 5 & $\mathrm{U}$ & NA & & & $\mathrm{U}$ & & $\mathbf{U}$ & NA & & NA & \\
\hline & & & & & & & & & & & & & & _ & \\
\hline Semil-volutalle Organlics & UNITS & & & & & & & & & & & & & & \\
\hline Diethylphthalate & UG/L & 11 & $\mathrm{U}$ & 10 & U & NA & & 117 & $\mathrm{U}$ & 121 & $\mathrm{U}$ & NA & & NA & \\
\hline & & & & & & & & & & & & & & & \\
\hline Pestledes and PCRs & UNITS & & & & & & & & & & & & & & \\
\hline Heptachlor & UG/L & 0.054 & $\mathrm{U}$ & 0.054 & $\mathrm{U}$ & 0.057 & $\mathbf{U}$ & 0.054 & U & 0.052 & $\mathrm{U}$ & 0.052 & $\mathrm{U}$ & $\mathrm{NA}$ & \\
\hline & & & & & & & & & 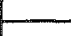 & & & & & of & \\
\hline Radiological & UNITS & & & & & & & & & & & & & & \\
\hline Gross Beta & $\overline{\mathrm{PCI} / \mathrm{L}}$ & $0.00 \mathrm{E}+00+1$. & 1.84 & $2.54 \mathrm{E}+00+1-$ & 2.04 & $0.00 \mathrm{E}+00+\%$ & 2.27 & $8.80 \mathrm{E}-01+\%$ & 1.83 & $1.48 \mathrm{E}+00+1-$ & 1.89 & $0.00 \mathrm{E}+00+1$. & 1.92 & $4.61 E+00+1$ & 2.15 \\
\hline U.234 & $\mathrm{BCL} / \mathrm{L}$ & $2.37 \mathrm{E}-01+1$. & 0.10 & $3.15 \mathrm{E}-01+/-$ & 0.22 & $2.89 \mathrm{E}-01+1$ & 0.20 & $3.18 \mathrm{E}-01+1$. & 0.11 & $2.83 \mathrm{E}-01+/-$ & 0.11 & $7.29 \mathrm{E}+00+1$. & 0.84 & $1.07 \mathrm{E}-01+1-$ & 0.09 \\
\hline U.235 & $\mathrm{PCL} / \mathrm{L}$ & $7.98 \mathrm{E}-02+\%$ & 0.06 & $6.78 \mathrm{E}-02+1-$ & $0.13 \mathrm{~J}$ & $3.13 E-02+/$ & $0.05 \mathrm{~J}$ & $2.07 \mathrm{E}-01+1 / 0$ & 0.09 & $2.93 \mathrm{E}-02+1-$ & 0.04 & $1.91 \mathrm{E}=01+1-$ & 0.12 & $3.34 \mathrm{E}-02+1$. & 0.07 \\
\hline 1.238 & $\mathrm{PCL} / \mathrm{L}$ & $1.15 \mathrm{E}-01+1$. & 0.07 & $1.02 \mathrm{E}-01+1-$ & 0.15 & $0.00 \mathrm{E}+00+1-$ & 0.55 & $1.46 \mathrm{E}-01+1$. & 0.07 & $2.05 \mathrm{E}-01+1-$ & 0.09 & $5.93 E+00+1 \%$ & $\overline{0.73}$ & $5 . \overline{68 \mathrm{E}-02+1-}$ & 0.07 \\
\hline & & & & & & & & & & & & & & $\ldots \ldots$ & \\
\hline & & & & & & & & & & & & & & & \\
\hline
\end{tabular}


Table E.7

Bear Creek Valley OU2 RI FA

QC Sample Summary

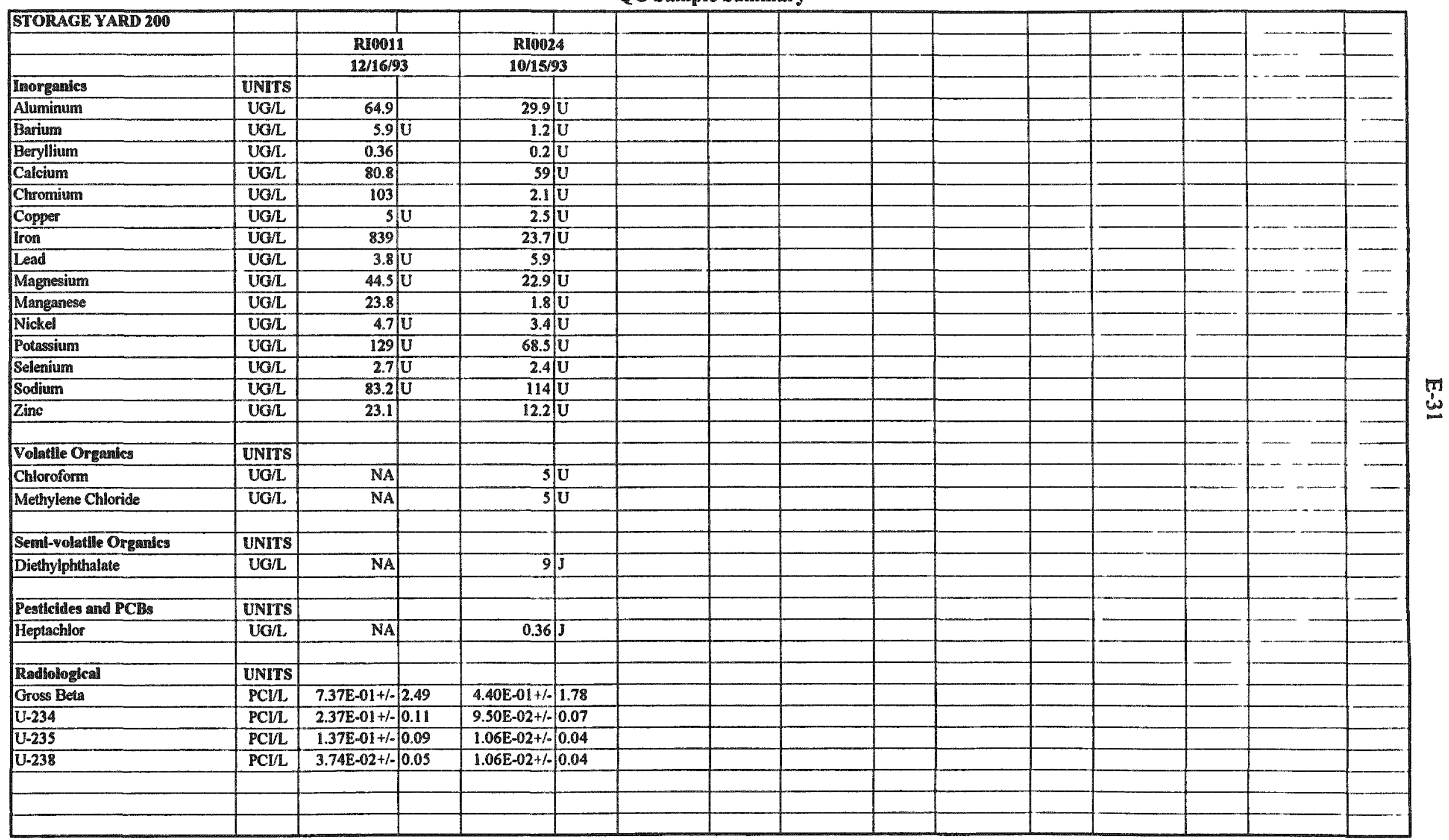


Table E.7

Bear Creck Valley OU2 RI TA

QC Sample Summary

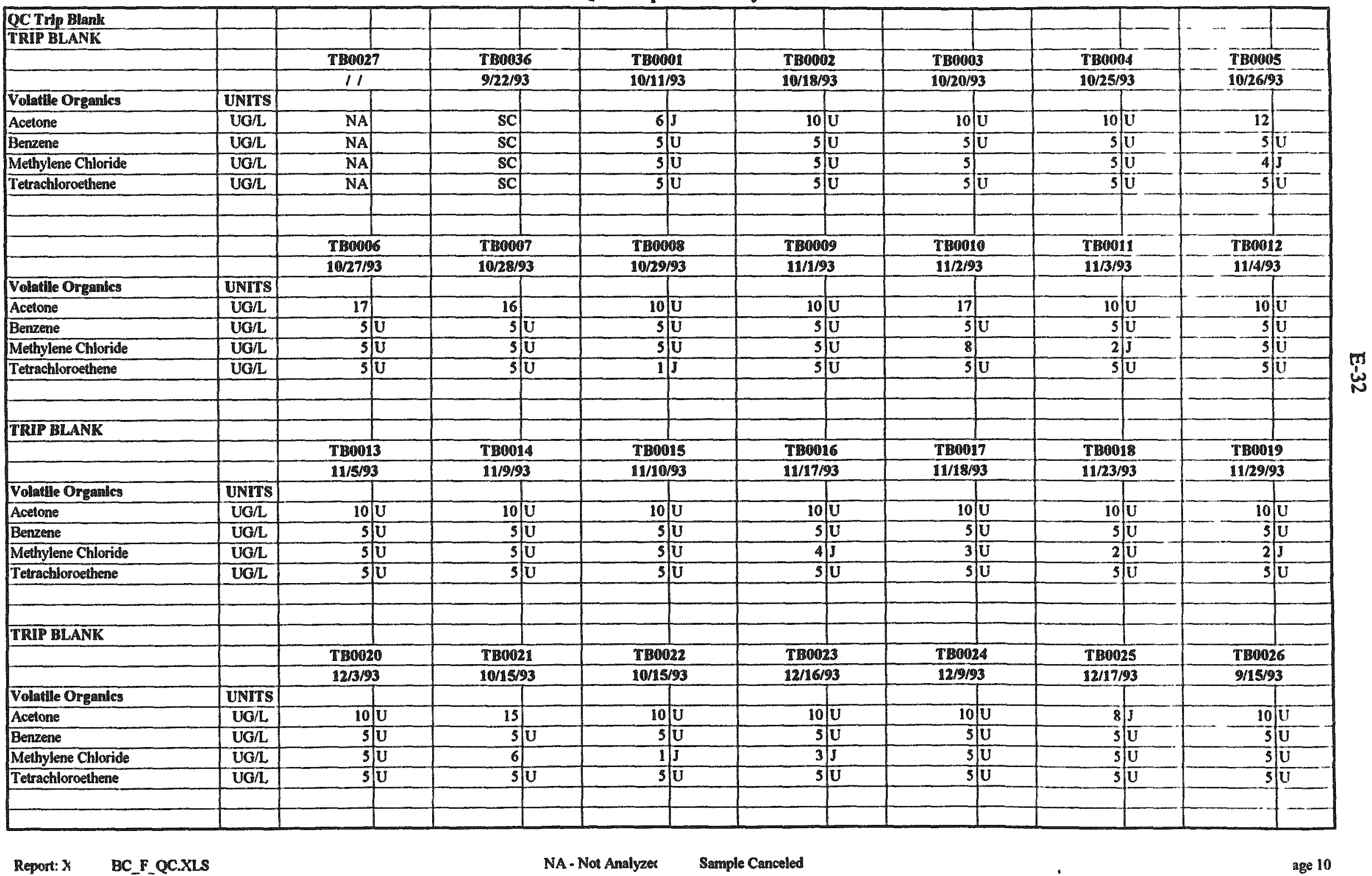


Table E.7

Bear Creek Valley OU2 RI FA

QC Sample Summary

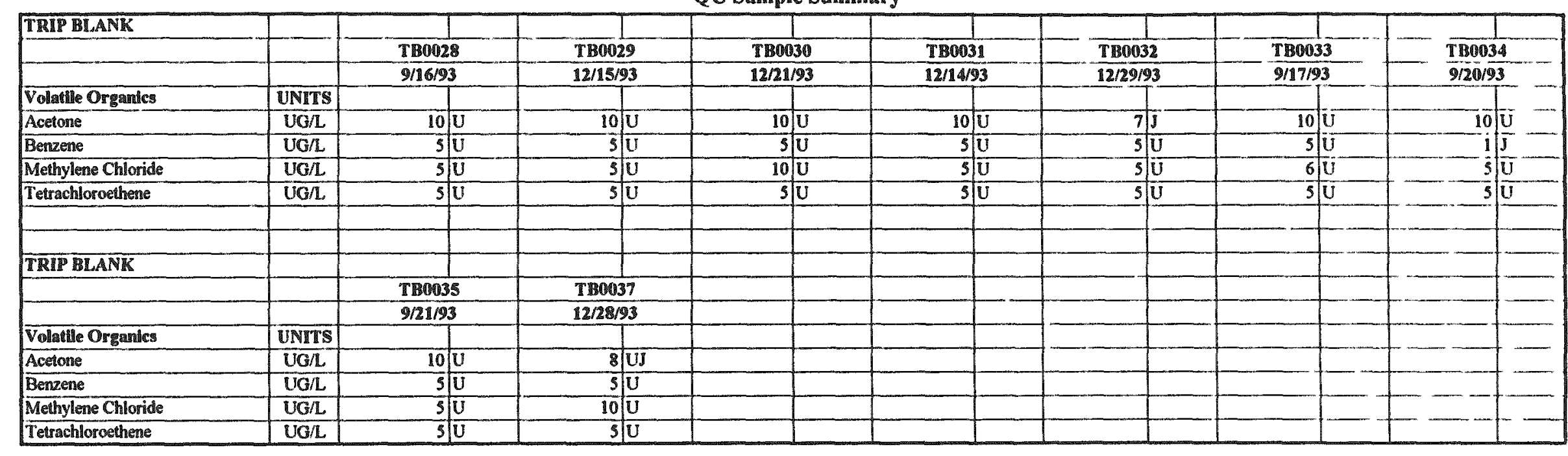





\section{DISTRIBUTION}

1. V. J. Brumback

2. M. F. P. Delozier

3. J. T. Fowler

4. J. D. Gass

5. C. D. Goins

6. I. T. Grumski

7. C. S. Haase

8. P. J. Halsey

9. P. H. Hight

10. J. A. Hodgins

11. L. M. Houlberg

12. J. B. Hunt

13. W. K. Jago

14. C. W. Kimbrough

15. H. L. King

16-17. A. K. Lee/DOE-OSTI

18. J. M. Loar

19-21. D. M. Matteo

22. M. McKinney

23. L.W. McMahon

24. T. I. Nakamoto

25. H. C. Newsom/D. C. White

26. B. Nourse

27-28. P. T. Owen

29. G. E. Rymer

30. C. M. Smith

31. R. J. Spence

32. G. W. Suter

33. T. S. Tison

34. R. R. Turner

35. C. S. Walker

36. D. Watson

37. R. W. Weigel

38. R. K. White

39. Y-12 Technical Library

40-42. ER Document Management Center

43. Y-12 ER Document Center

44. Y-12 Central Files

45. P. Delfonzo, ENSERCH, 111 Union Valley Road, Oak Ridge, TN 37830

46. J. A. Archer, Jacobs Engineering, 800 Oak Ridge Turnpike, Oak Ridge, TN 37830

47. J. Pfeffer, Jacobs Engineering, 800 Oak Ridge Turnpike, Oak Ridge, TN 37830

48. J. Darden, MK-Ferguson of Oak Ridge, P.O. Box 2011, Oak Ridge, TN 37831

49. A. Bailey, Science Applications International Corporation, P.O. Box 2501, 800 Oak Ridge Turnpike, Oak Ridge, TN 37831

50-59. S. L. Lankford, DOE Oak Ridge Operations Office, P.O. Box 2001, Oak Ridge, TN 37831-8541

60-61. R. L. Nace, Branch Chief, Nonenrichment Facilities, Oak Ridge Program Division, Office of Eastern Area Programs, Office of Environmental Restoration, EM-423, Trevion 2, U.S. Department of Energy, Washington, DC 20585

62. R. C. Sleeman, DOE Oak Ridge Operations Office, P.O. Box 2001, Oak Ridge, TN $37831-8541$

63-64. H. M. Thron, Chief, Enrichment Facilities, Oak Ridge Program Division, Office of Eastern Area Programs, Office of Environmental Restoration, EM-423, Trevion 2, U.S. Department of Energy, Washington, DC 20585 


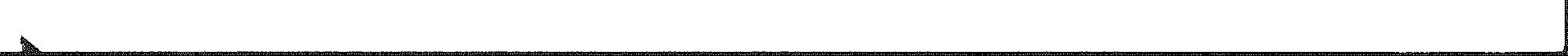



